DEAR21-96MC33091

PILOT-SCALE TESTING REPORT

$$
\text { TOPICAL }
$$

\title{
ORGANIC-CONTAMINANT DESTRUCTION UNIT ECO LOGIC Process \\ Gas Phase Chemical Reduction
}

Treatment of Napalm at the Army's Edgewood Research and

Development, Engineering Center (ERDEC)

Toxic Test Chamber Facilities, Aberdeen Proving Ground, Maryland

\section{Submitted to:}

US Department of Energy

Federal Energy Technology Center

3610 Collins Ferry Road

Morgantown, WV 26507

Submitted by:

Science Applications International Corporation

411 Hackensack Avenue, 3rd Floor

Hackensack, NJ 07601

and

ELI Eco Logic International Inc.

(ECO LOGIC)

- 143 Dennis Street

Rockwood, Ontario

Canada - NOB 2KO

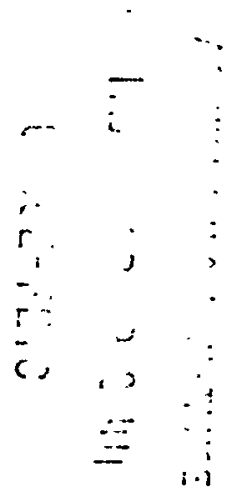

April 17, 1998 


\section{EXECUTIVE SUMMARY}

The Eco Logic Process is a mobile, gas-phase chemical reduction process with demonstrated experience in the destruction of high and low strength organic wastes such as polychlorinated biphenyls (PCBs), polyaromatic hydrocarbons (PAHs), dioxins and furans (PCDDs and PCDFs), hexachloride wastes, organophosphorus pesticides, chemical warfare agents, and other organic compounds. The Process involves the gas-phase reduction of organic compounds at temperatures of approximately $850^{\circ} \mathrm{C}$ and ambient pressure. Chlorinated hydrocarbons such as PCBs and PCDD/Fs are chemically reduced to methane and hydrochloric acid, while non-chlorinated compounds such as napalm are reduced to methane and minor amounts of other light hydrocarbons. All outputs from the Process, including product gas, can be held and tested to determine their compliance with regulatory criteria, prior to reuse in the system or disposal. The Eco Logic Process has undergone regulatory testing at both pilot- and full-scale, with results of $99.9999 \%$ (6 nines) destruction and removal efficiency (DRE) for materials tested.

In June 1997, the Eco Logic Process was used to perform pilot-scale treatment of neat styrenated gasoline (napalm) in aluminum containers. The treatability study was conducted as part of a contract awarded to Science Applications International Corporation (SAIC) and Eco Logic by the Department of Energy's Federal Energy Technology Center (FETC) in Morgantown, West Virginia to conduct treatability studies on complex hazardous wastes, energetics and low level mixed wastes. The testing took place at the US Army's Edgewood Research Development, Engineering Center (ERDEC) toxic test chamber facility located at Aberdeen Proving Ground, Maryland. Three test runs were conducted, each processing a specific amount of napalm. The first run, or optimization run, was performed to determine optimal treatment conditions. Two additional test runs then are performed using the optimized treatment conditions. Samples of system outputs were collected for analysis after completion of the test runs.

The technical objectives of the pilot-scale treatability studies were successfully achieved and the results demonstrate the ability of the Eco Logic Process to completely desorb napalm safely and effectively. Analysis of the scrubber water before and after tertiary treatment as well as analysis of the treated napalm container, indicate the Eco Logic Process achieved optimum system conditions required to volatilize napalm from the aluminum containers. Continuous monitoring of the product gas resulting from the processing of the desorbed napalm indicate that destruction products are consistent with other nonchlorinated contaminants treated by the Eco Logic Process and should be fully treatable by the full-scale Eco Logic Process. 


\section{DISCLAIMER}

This report was prepared as an account of work sponsored by an agency of the United States Government. Neither the United States Government nor any agency thereof, nor any of their employees, make any warranty, express or implied, or assumes any legal liability or responsibility for the accuracy, completeness, or usefulness of any information, apparatus, product, or process disclosed, or represents that its use would not infringe privately owned rights. Reference herein to any specific commercial product, process, or service by trade name, trademark, manufacturer, or otherwise does not necessarily constitute or imply its endorsement, recommendation, or favoring by the United States Government or any agency thereof. The views and opinions of authors expressed herein do not necessarily state or reflect those of the United States Government or any agency thereof. 


\section{DISCLAIMER}

Portions of this document may be illegible in electronic image products. Images are produced from the best available original document. 


\section{TABLE OF CONTENTS}

EXECUTIVE SUMMARY $\ldots \ldots \ldots \ldots \ldots \ldots \ldots \ldots \ldots \ldots$ i

1. INTRODUCTION $\ldots \ldots \ldots \ldots \ldots \ldots \ldots \ldots \ldots \ldots \ldots \ldots \ldots \ldots \ldots \ldots \ldots$

$1.1 \quad$ Technology Overview $\ldots \ldots \ldots \ldots \ldots \ldots \ldots \ldots \ldots \ldots \ldots \ldots \ldots \ldots$

1.2 Waste Processing at Commercial-Scale $\ldots \ldots \ldots \ldots \ldots \ldots \ldots \ldots \ldots$

1.2 .1 Process Outputs . . . . . . . . . . . . . . . . . . . . 6

1.3 Summary of Previous Experience $\ldots \ldots \ldots \ldots \ldots \ldots \ldots \ldots 6 . \ldots \ldots$

1.3.1 Demonstration-Scale Testing, Hamilton Harbour, Ontario, Canada $\ldots .88$

1.3.2 Demonstration-Scale Operations, Bay City, Michigan ......... 8

1.3.3 Full-Scale Applications of the Eco Logic Process . . . . . . . . . 10

2. PILOT-SCALE TESTING $\ldots \ldots \ldots \ldots \ldots \ldots \ldots \ldots \ldots \ldots \ldots \ldots \ldots \ldots$

2.1 Pilot-Scale System Description $\ldots \ldots \ldots \ldots \ldots \ldots \ldots \ldots \ldots \ldots \ldots \ldots$

$2.2 \quad$ Pilot-Scale Test Operations . . . . . . . . . . . . . . . . . . . 13

2.2.1 Goals and Objectives of Pilot-Scale Testing $\ldots \ldots \ldots \ldots \ldots \ldots \ldots 13$

2.2.2 Test Preparation and System Integrity $\ldots \ldots \ldots \ldots \ldots \ldots \ldots \ldots \ldots \ldots$

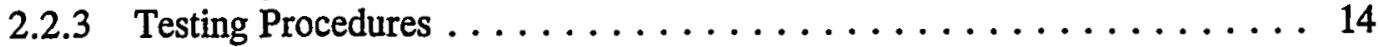

$2.3 \quad$ Process Operating Conditions $\ldots \ldots \ldots \ldots \ldots \ldots \ldots \ldots \ldots \ldots \ldots \ldots$

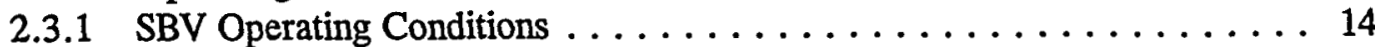

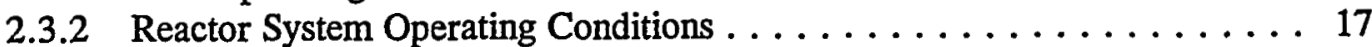

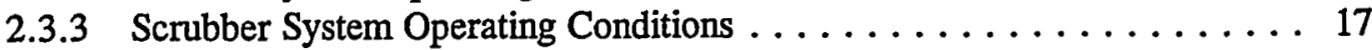

$2.4 \quad$ Sampling and Monitoring Procedures $\ldots \ldots \ldots \ldots \ldots \ldots \ldots \ldots \ldots \ldots \ldots$

2.4.1 Sampling Methods ...................... 18

2.4.2 General Sampling/Quality Control Procedures ............ 19

2.4 .3 Liquid Sample Collection $\ldots \ldots \ldots \ldots \ldots \ldots \ldots \ldots \ldots \ldots$

2.4.4 Napalm Aluminum Container Sampling .............. 19

2.4.5 Process Performance Monitoring Via On-Line Instrumentation . . . . . . 19

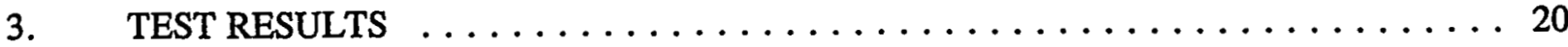

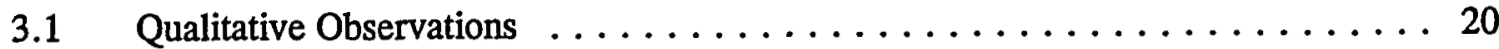

3.2 Chemical Analysis of System Input and Outputs $\ldots \ldots \ldots \ldots \ldots \ldots \ldots 20$

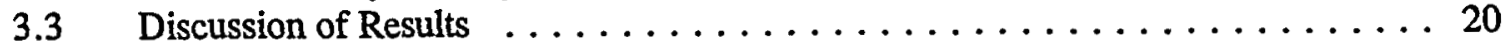

3.3.1 Napalm Destruction Chemistry in the Eco Logic Process ........ 25

3.3.2 Comparison of Test Results to Target Performance Goals ......... 27

Appendix A PROCESS DATA SHEETS

Appendix B CHAIN OF CUSTODY FORMS

Appendix C GRAPHICAL REPRESENTATION OF ON-LINE DATA

Appendix D ANALYTICAL DATA SETS FOR NAPALM TREATABILITY TEST RUNS 


\section{LIST OF FIGURES}

Figure 1

Figure 2

Figure 3

Figure 4

Figure 5

Figure 6

Figure 7

Table 1

Table 2

Table 3

Table 4

Table 5

Table 6

Table 7

Table 8

Table 9

Table 10

Table 11

ECO LOGIC PROCESS REACTIONS . . . . . . . . . . . . 2

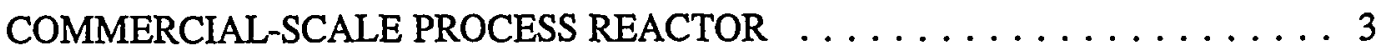
COMMERCIAL-SCALE PROCESS SCHEMATIC . . . . . . . . . . . 4 SCHEMATIC OF A TYPICAL COMMERCIAL-SCALE SITE LAYOUT $\ldots \ldots \ldots 7$ SCHEMATIC OF PILOT-SCALE SYSTEM . . . . . . . . . . . . . 12 VISUAL OBSERVATION OF NAPALM IN CONTAINER BEFORE TESTING . 20 VISUAL OBSERVATION OF NAPALM IN CONTAINER AFTER TESTING . . 20

\section{LIST OF TABLES}

HAMILTON HARBOUR PERFORMANCE TEST RESULTS $\ldots \ldots \ldots \ldots \ldots$. USEPA SITE PROGRAM TEST RESULTS . . . . . . . . . . . . . 9 STACK TEST DATA FOR COMMERCIAL-SCALE TREATMENT OF DDT . . 11 SUMMARY OF COMMERCIAL-SCALE DRE AND DE VALUES FOR PCBS . 11 TREATABILITY-SCALE STARTUP AND SHUTDOWN PROCEDURES . . . . 15 SUMMARY OF NAPALM TREATABILITY STUDY OPERATING CONDITIONS16 SUMMARY OF SAMPLING PROGRAM FOR NAPALM $\ldots \ldots \ldots \ldots \ldots 18$ PRE-RUN SCRUBBER WATER RESULTS . . . . . . . . . . . . 22 SCRUBBER WATER EFFLUENT WATER RESULTS . . . . . . . . . . . 23 POST CARBON TREATED SCRUBBER EFFLUENT WATER RESULTS . . . 24 TREATED NAPALM CONTAINER RESULTS $\ldots \ldots \ldots \ldots \ldots \ldots \ldots$ 
LIST OF TABLES

LIST OF FIGURES

\section{LIST OF APPENDICES}


1. INTRODUCTION

This report describes the Eco Logic Process and discusses the procedures and results of a pilot-scale treatability study on neat styrenated gasoline (napalm) contained in aluminum containers. The study was conducted as part of a contract which was awarded to Science Applications International Corporation (SAIC) and Eco Logic by the Department of Energy's Federal Energy Technology Center (FETC) in Morgantown, West Virginia to conduct treatability studies on complex hazardous wastes, energetics and low level mixed wastes.

Napalm stockpiles are stored in sealed aluminum canisters measuring 12 feet in length and 8 feet in diameter. Due to its high viscosity, napalm is difficult to handle. To minimize handling, Eco Logic proposes to treat the napalm in storage canisters. This would be accomplished by puncturing or removing the lids or valves and volatilizing the napalm from the container during treatment in the Sequencing Batch Vaporizer (SBV). The simulation of full-scale treatment of the aluminum canisters containing napalm was accomplished by placing pre-weighed bulk samples of napalm in aluminum cans, without lids, and treating them in the pilot-scale SBV.

Three treatability tests on neat napalm were performed between June 23 and June 25, 1997 at the US Army's Edgewood Research Development, Engineering Center (ERDEC) toxic test chamber facility located at Aberdeen Proving Ground, Maryland. Project planning activities, set-up activities, on-site treatability study operations, treatability study results and interpretation of these results are discussed in detail in the following sections.

\subsection{Technology Overview}

The Eco Logic system for processing contaminated bulk solids includes a Sequencing Batch Vaporizer (SBV), a gas phase chemical reduction reactor (GPCR), a gas scrubber system, a gas compression and storage system and process control and monitoring systems. The SBV acts as a front-end unit to introduce contaminants into the reactor. It is a large autoclave-type chamber that operates in a hydrogen atmosphere at temperatures of $600^{\circ} \mathrm{C}$. All organic contaminants contained in the material being treated are volatilized and the resultant organic vapors are swept directly into the GPCR reactor by the hydrogen-rich sweep gas. Examples of material suitable for SBV treatment include personal protective equipment, electrical equipment, miscellaneous large equipment, contaminated drums and other material.

The gas stream from the SBV, consisting of sweep gas, steam and desorbed organic contaminants, is further heated in the GPCR reactor to a temperature of approximately $900^{\circ} \mathrm{C}$. Chlorinated hydrocarbons are chemically reduced to methane and hydrogen chloride $(\mathrm{HCl})$, while non-chlorinated hydrocarbons, such as napalm are reduced substantially to methane and minor amounts of other light hydrocarbons.

Figure 1 shows some examples of the reduction reactions for the destruction of a variety of contaminants using the Eco Logic Process. Unlike oxidation reactions, the efficiency of these reduction reactions is enhanced by the presence of water, which acts as a heat transfer and reducing agent, as well as a source of hydrogen. Therefore, dewatering of input waste is unnecessary. The water shift reactions shown produce hydrogen, carbon monoxide and carbon dioxide from methane and water. These reactions are used at higher efficiencies in a catalytic steam reformer (used in the full-scale system) to generate hydrogen for reuse in the system by subjecting scrubbed methane-rich product gas to high temperatures in the presence of a catalyst. 


\section{Figure 1 Eco Logic PROCESS REACTIONS}

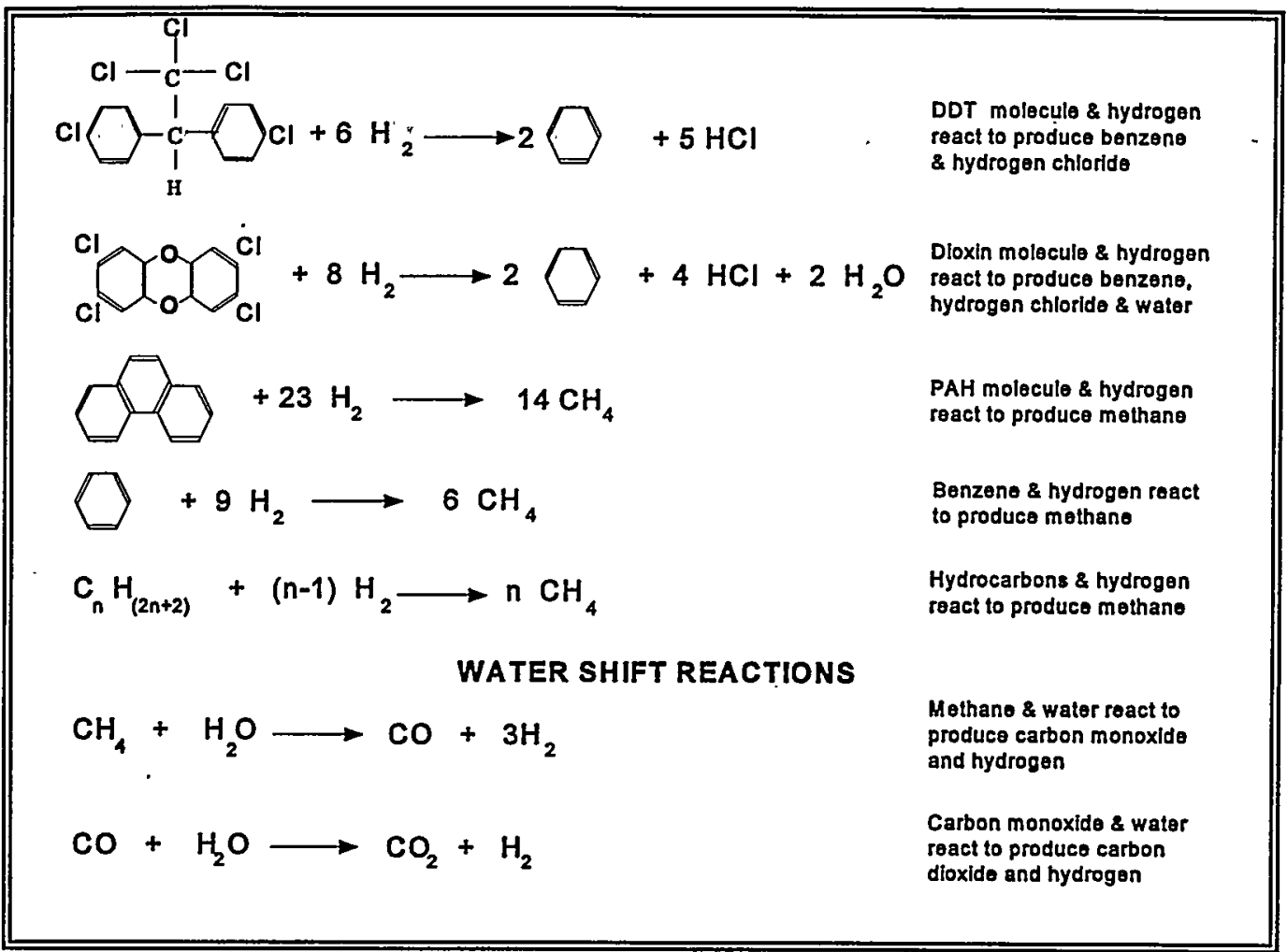

\subsection{Waste Processing at Commercial-Scale}

Figure 2 is a schematic of the SE25 commercial-scale reactor where the destruction of organiccontaminated waste, through its breakdown into re-usable or disposable products, takes place. Thermal desorption of contaminants from solid phases is accomplished with the SBV or the Thermal Reduction Mill (TRM). The reactor waste input streams from the SBV, TRM and vaporized liquid wastes are injected through several ports mounted tangentially near the top of the reactor. Special nozzles are used to atomize liquid wastes with steam and hydrogen to accelerate liquid vaporization. The gas mixture swirls around a. central stainless steel tube, and is heated by 18 vertical radiant tube heaters with internal electric heating elements. By the time it reaches the bottom of the reactor, the gas mixture has reached a temperature of at least $850^{\circ} \mathrm{C}$. The process reactions take place from the bottom of the central tube onwards, and takes less than one second to complete.

Figure 3 shows the process flow diagram for the commercial-scale system. Process gas leaving the reactor is scrubbed to remove $\mathrm{HCl}$, water, heat, fine particulate, aromatic compounds and carbon dioxide. The first stage of the scrubber can be operated to recover hydrochloric acid, which avoids neutralization with caustic. For low strength wastes such as contaminated soil and sediment, the first stage of the scrubber is operated as a caustic scrubber for removal of $\mathrm{HCl}$ by neutralization.

The second stage of the scrubbing drops the temperature of the gas to remove water and completes the removal of $\mathrm{HCl}$ by caustic packed tower scrubbing. Particulate matter, which may have entered the reactor as dissolved or suspended solids in the original waste, is removed in both the first and second stages of the scrubber by continuous filtering. Heat is removed using plate heat exchangers and is transferred to the cooling water system. 
Figure 2 COMMERCIAL-SCALE PROCESS REACTOR

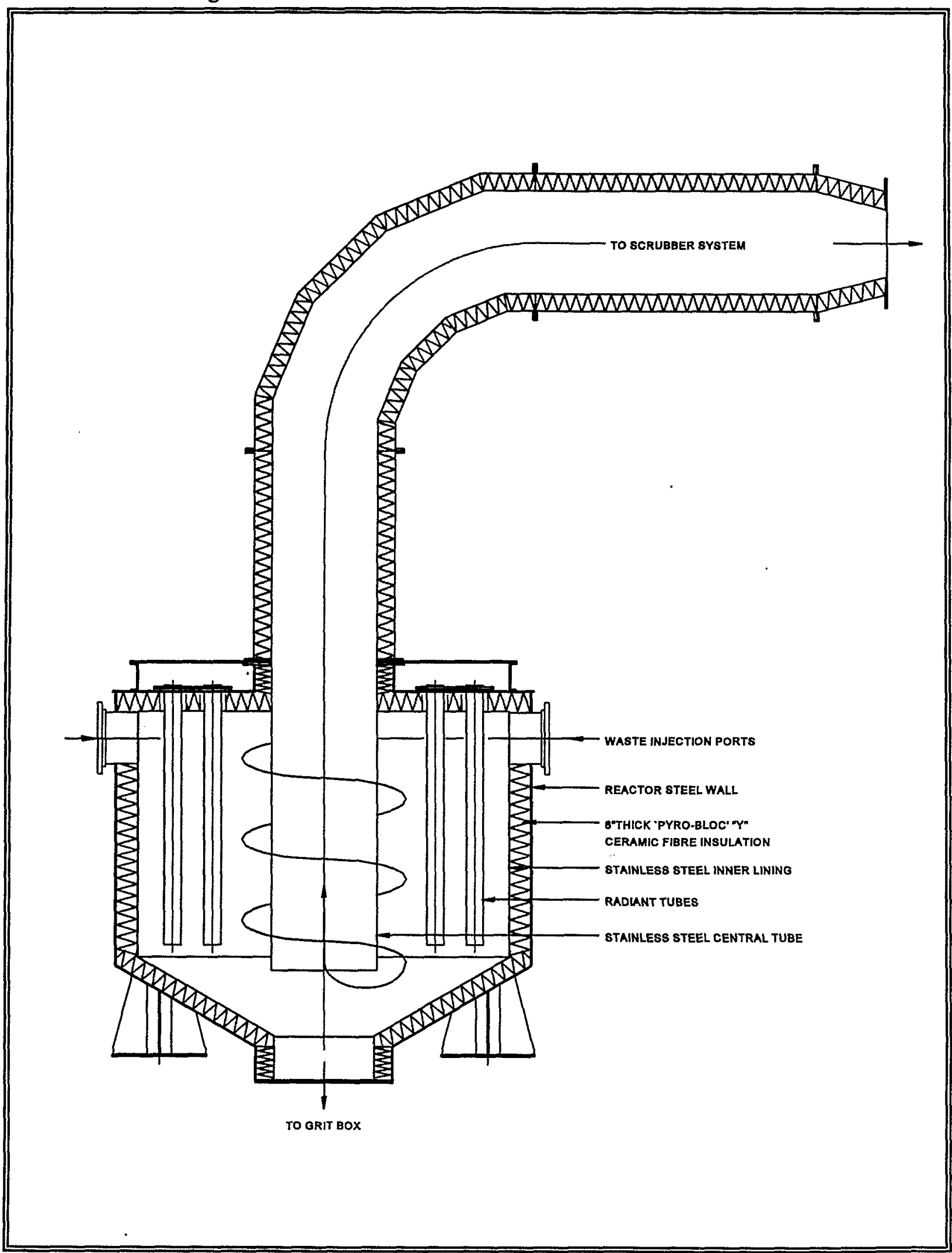


Figure 3 Commercial-Scale Process Schematic

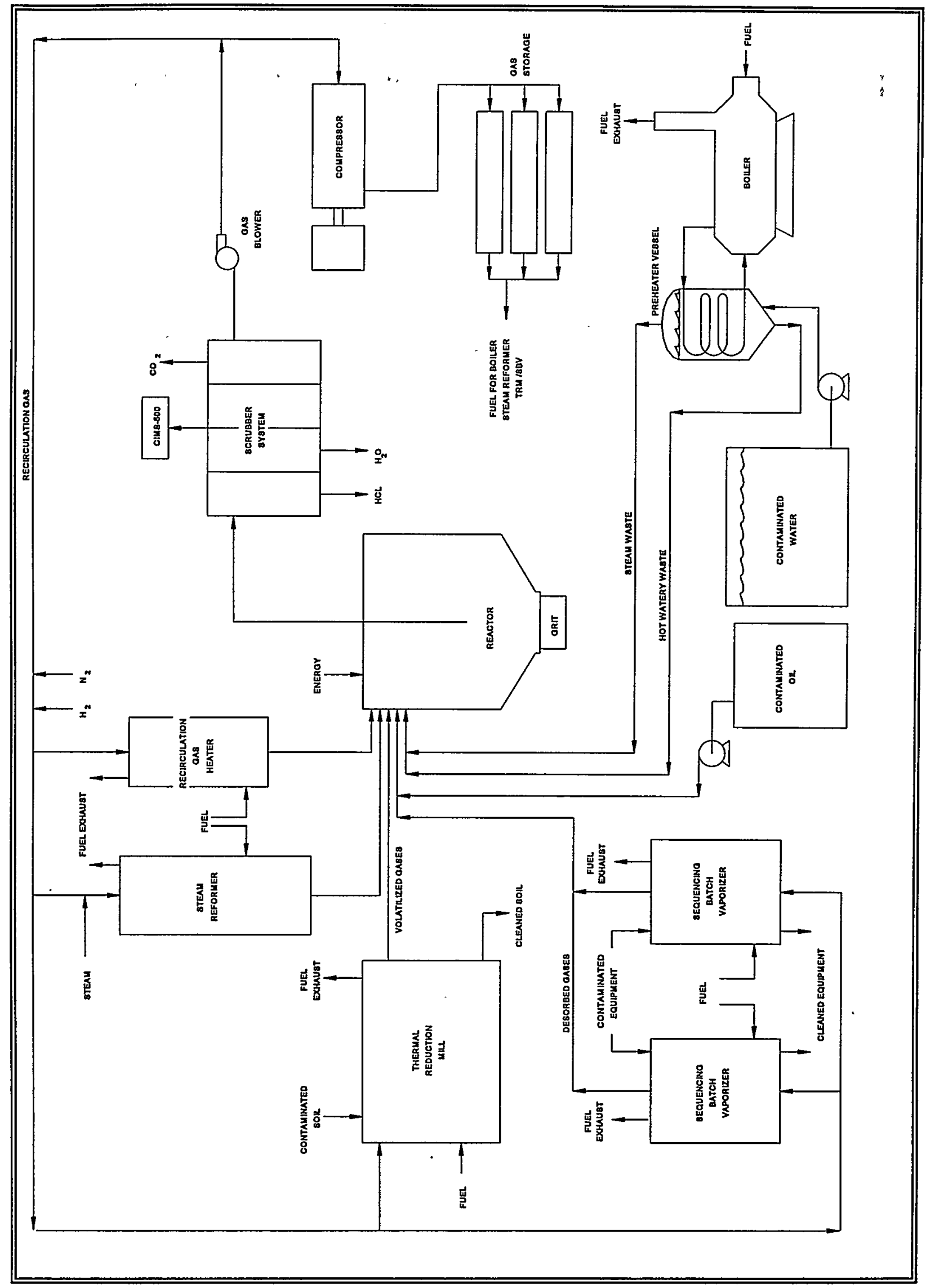


The third stage of scrubbing removes low levels of benzene and naphthalene from the gas stream by neutral oil washing. The rich oil is pumped to a stripper where benzene and naphthalene are removed and sent to the inlet of the catalytic steam reformer for conversion to hydrogen and carbon dioxide. The fourth scrubbing stage removes carbon dioxide using monoethanolamine (MEA) absorption. The rich MEA is pumped to a stripper where the carbon dioxide is removed and is sent to the common boiler/catalytic steam reformer stack.

The scrubber water from the stage-two scrubber leg returns to the covered section of the scrubber tank through a drop-tube that extends well below the water surface. This acts as a seal against air infiltration and as an emergency pressure relief mechanism. There will be no gas release if a short-term pressure surge forces gas out of the bottom of this tube since a check valve allows the gas to re-enter the system once the pressure returns to normal. The entire treatment loop operates within 1 psi of atmospheric pressure.

As solid waste is processed through the system, water is generated as an output. Scrubber decant water is filtered and carbon-treated to remove any trace of organic contamination and is then stored for analysis prior to discharge. Spent activated carbon can be regenerated on-site in the SBV and the minor amount of scrubber particulate produced can also be processed through the SBV or TRM.

The cooled and scrubbed process gas is a clean dry mixture of hydrogen, methane, carbon monoxide, and other light hydrocarbons. Some of the process gas is reheated and recirculated back into the reactor. Process gas is also recirculated through the SBV as sweep gas, through the TRM as sweep gas, and through the catalytic steam reformer for hydrogen generation. Excess process gas is removed from the system, compressed, and stored. This stored "product gas" is chemically tested and subsequently used as fuel to heat ancillary system components.

Storage of the product gas under pressure permits the continuous analysis of the gas to determine that it does not contain contaminants of concern. It can then be used as supplemental fuel in the boiler, catalytic steam reformer, or auxiliary burner, all of which use natural gas as their primary fuel. A micro gas chromatograph (micro-GC) is used to analyze the gas and provides a continuous record of product gas quality. The micro-GC readings are monitored by the process control system and the use of product gas as a fuel is stopped automatically should it not meet the established quality criteria. This eliminates the potential for releases to the environment as the gas can simply be reprocessed.

The process gas is also analyzed by the Chemical Ionization Mass Spectrometer (CIMS) and other gas analyzers prior to compression and storage. The CIMS is capable of accurately monitoring up to 10 organic compounds every few seconds at concentrations ranging from percent levels down to ppb levels. It is used as part of the Eco Logic process as a diagnostic tool to monitor the concentrations of certain compounds indicative of the process destructive efficiency. The compounds selected for monitoring depend on the waste being processed. The CIMS readings are monitored by the process control system, such that if alarm limits are exceeded, a message is sent directly to the operator. The process control system monitors and controls over 300 operating parameters of the process. Set point values and ranges for critical operating parameters are given high priority alarms to ensure the system performs at optimum conditions. These parameters include operating temperatures and pressures for the SBV, TRM, reactor, scrubbers, and ancillary equipment; residence time of the process gas through the reactor; and concentrations of gases and contaminants within the process and product gas streams. There are numerous secondary alarms also linked to the process control system to alert operators to deviations from normal operating ranges. These parameters include tank levels, scrubber $\mathrm{pH}$, pump and vessel pressures, gas and liquid florets, instrument air pressure, valve positions and on/off status of motors, pumps and valves. 
Figure 4 presents a "footprint" diagram showing how a full-scale SE25 unit would typically be deployed at a site. Most of the system components are mounted on standard-bed highway trailers for ease of mobility. The reactor trailer houses the reactor, the electrical heating control system, the scrubber system, the recirculation gas blower, the recirculation gas heater and the water waste preheated vessel. A second trailer contains the main power distribution room, the boiler and catalytic steam reformer. Gas cooling capacity for the scrubbing system is recirculated through evaporative coolers, and scrubber stripping operations are carried out in a container situated near the boiler. The product gas compression and storage system is also skid-mounted to allow flexibility in site layout. The SBV is a skid mounted unit, while the TRM is housed on a separate trailer. The process control system, gas analyzer systems, and the command center are housed in a standard office trailer.

\subsubsection{Process Outputs}

The outputs created from the destruction of napalm includes scrubber water, product gas and the treated aluminum canisters.

Water During normal operations, water is generated as scrubber decant water, which may contain trace levels of low molecular weight hydrocarbons (benzene and naphthalene). The water is treated with granular activated carbon (GAC), stored on-site, and tested to ensure compliance with local discharge limitations prior to discharge to the municipal sewer system. Alternatively, the treated process water can be re-used on-site in the evaporative coolers.

Product Gas Excess product gas is generated during the destruction of complex organic contaminants, such as napalm. The composition of the product gas during full-scale operations is as follows: hydrogen (65-75\%), methane (10-20\%), and carbon monoxide (5-15\%), with trace amounts of lighter organic compounds (e.g., ethylene and benzene). The gas is compressed and stored in tanks for analysis, after which it can be used on-site as supplementary fuel to heat system components.

Processed Solids The aluminum canisters used to contain the napalm will remain following treatment in the SBV. The canisters are chemically tested for waste-specific contaminants to verify removal of organic contaminants. Once analysis has verified that the canisters are decontaminated, it can be transported to an off-site disposal area or offered to scrap metal companies for recycling.

\subsection{Summary of Previous Experience}

SAIC and Eco Logic have a high degree of confidence that the Eco Logic Process can be optimized for the destruction of neat styrenated napalm in aluminum canisters. This confidence is based on experience and success with bench-, laboratory-, pilot- and full-scale applications of the Process treating hazardous organic waste. Development of the Eco Logic Process has progressed to the stage where two full-scale sites (St. Catharines, Ontario and Kwinana, Western Australia) have treated hazardous organic wastes.

Since 1986, extensive research, development, test evaluation and product engineering has been conducted with the aim of developing an efficient, non-incineration technology for destroying organic wastes in a wide range of matrices including sediment, soil, leachate, sludge, bulk solids, high- and low-strength liquids. Much of this work was supported by the Governments of Canada (Environment Canada and the Department of National Defense) and the Unites States of America (U.S. Environmental Protection Agency - USEPA). The goal was to develop a commercially-viable chemical process that could deal with aqueous wastes and also destroy concentrated organic wastes. 
Figure 4 SCHEMATIC OF A TYPICAL COMMERCIAL-SCALE SITE LAYOUT

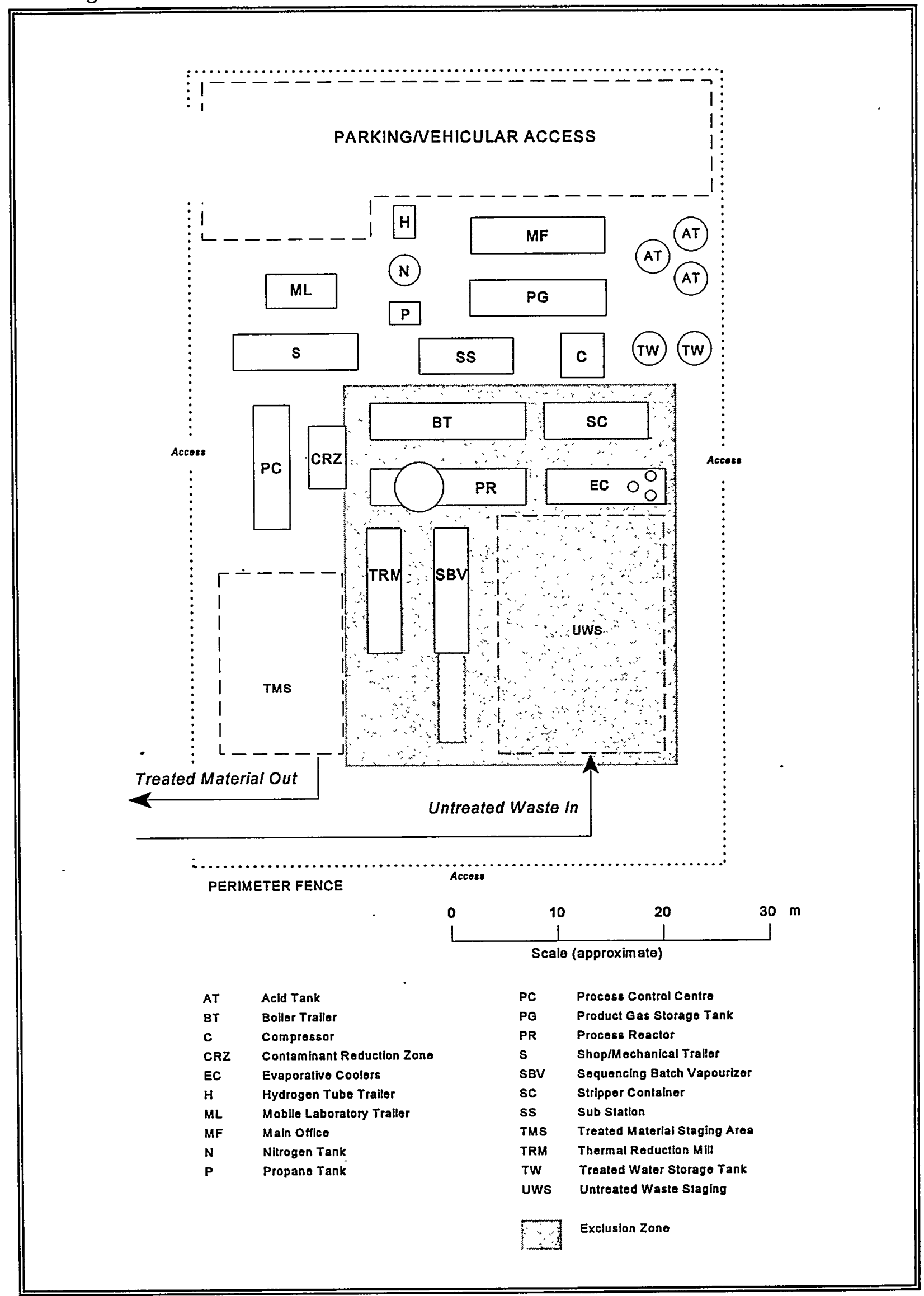


Development of the technology began at bench-scale in 1987 and tests at laboratory-scale have been conducted since 1988. In 1991, a demonstration-scale hazardous waste unit was built and tested extensively at sites in Canada and the United States. Eco Logic has successfully scaled-up the technology to develop two full-scale units, which have been operating in Australia and Canada.

The technology has been demonstrated and proven to be a highly efficient and publicly acceptable means of destroying high strength organic waste streams on-site. Due to the highly versatile and non-selective nature of the Eco Logic Process there are many potential applications. These include destruction of PCBs, dioxins, pesticides, and solvents as well as the treatment of industrial and municipal sewage sludges, chemical warfare agents, radioactive mixed waste, and energetic material.

Details of demonstration studies and full-scale operations are provided in the following sections.

\subsubsection{Demonstration-Scale Testing, Hamilton Harbour, Ontario, Canada}

The demonstration-scale unit employing the Eco Logic Process was first tested at Hamilton Harbour, Ontario, Canada, in the summer of 1991. The demonstration was supported by Environment Canada and the Ontario Ministry of the Environment.

The waste processed during these tests was coal-tar contaminated harbor sediment with PAH concentrations averaging $30,000 \mathrm{mg} / \mathrm{kg}$ (dry weight basis). Three test runs were performed on the contaminated sediment with the third run being spiked with PCBs at a nominal concentration of $500 \mathrm{mg} / \mathrm{kg}$. The harbor sediment was injected directly into the gas-phase chemical reduction reactor as a 5-10\% solids slurry.

PAH destruction and removal efficiencies of $99.9999 \%$ were calculated (Table 1), based on the total organic input and the PAHs analyzed in the boiler stack emissions. The concentration of PCBs in air emissions, liquid outputs and processed solids were all below the detection limits resulting in a PCB Destruction and Removal Efficiency (DRE) of at least 99.9999\%.

Table 1. HAMILTON HARBOUR PERFORMANCE TEST RESULTS

\begin{tabular}{||c|c|c|c|c|c|c|c||}
\hline \hline Run & $\begin{array}{c}\text { Target } \\
\text { Analytes }\end{array}$ & $\begin{array}{c}\text { Conc. in } \\
\text { Waste } \\
(\mathrm{mg} / \mathrm{kg})\end{array}$ & $\begin{array}{c}\text { Decant } \\
\text { Water } \\
\text { Conc. } \\
(\mu \mathrm{g} / \mathrm{kg})\end{array}$ & $\begin{array}{c}\text { Grit Conc. } \\
(\mathrm{mg} / \mathrm{kg})\end{array}$ & $\begin{array}{c}\text { Sludge } \\
\text { Conc. } \\
(\mathrm{mg} / \mathrm{kg})\end{array}$ & $\begin{array}{c}\text { Stack Gas } \\
\text { Conc. } \\
\left(\mu \mathrm{g} / \mathrm{m}^{3}\right)\end{array}$ & $\begin{array}{c}\text { DRE } \\
(\%)\end{array}$ \\
\hline P1 & PAHs & 21,000 & 483 & 1.67 & 32.8 & 0.27 & 99.9999 \\
P2 & PAHs & 30,000 & 680 & 7.76 & 56.1 & 0.23 & 99.9999 \\
P3 & PAHs & 30,000 & 423 & 0.37 & 4.3 & 0.14 & 99.9999 \\
P4 & PCBs & 500 & ND & ND & ND & ND & 99.9999 \\
\hline
\end{tabular}

DRE $=$ (Total Input - Stack Emissions)/(Total Input)

ND $=$ Non Detect

\subsubsection{Demonstration-Scale Operations, Bay City, Michigan, USA}

In 1992, Eco Logic relocated the demonstration-scale unit to a site in Bay City, Michigan. This demonstration was performed under the auspices of the USEPA's Superfund Innovative Technology Evaluation (SITE) Program and was co-sponsored by Environment Canada, the Ontario Ministry of the Environment and the City of Bay City. 
The wastes processed included oily PCB-contaminated water, high-strength PCB oil, and PCBcontaminated soil. As part of the demonstration, Eco Logic constructed a prototype thermal desorption unit (TDU), which was the fore-runner of the current Thermal Reduction Mill, and also demonstrated the capability to compress and store the product gas generated. The results for the test program, confirmed by the USEPA, are shown in Table 2. The SITE Program Project Bulletins, Technology Evaluation Report, and Applications Analysis Reports have now been published.

The waste oil was obtained from beneath the Bay City landfill. Analyses by the USEPA showed levels of $25 \%$ PCBs and percent levels of other chlorinated solvents. The contaminated soil was obtained from installation of the sump wells used to collect the oil, and the contaminated water was groundwater from the landfill. The test matrix called for three water/oil tests, three oil tests, and three soil tests.

Table 2. USEPA SITE PROGRAM TEST RESULTS

\begin{tabular}{|c|c|c|c|c|c|}
\hline \multicolumn{6}{|c|}{ Water/Oil and High-Strength Oil Tests } \\
\hline Run & Waste Type & Contaminant & Concentration ( $\mathrm{mg} / \mathrm{kg}$ ) & Target $D R E / D E$ & Achieved \\
\hline 1 & $\begin{array}{l}\text { Water/Oil } \\
\text { Tracer }\end{array}$ & $\begin{array}{c}\text { PCBs } \\
\text { Perchloroethene }\end{array}$ & $\begin{array}{l}4,800 \\
4,670\end{array}$ & $\begin{array}{c}99.9999 \\
99.99\end{array}$ & $\begin{array}{l}\text { Yes } \\
\text { Yes }\end{array}$ \\
\hline 2 & $\begin{array}{l}\text { Water/Oil } \\
\text { Tracer - }\end{array}$ & $\begin{array}{c}\text { PCBs } \\
\text { Perchloroethene }\end{array}$ & $\begin{array}{l}2,450 \\
2,360\end{array}$ & $\begin{array}{c}99.9999 \\
99.99\end{array}$ & $\begin{array}{l}\text { Yes } \\
\text { Yes }\end{array}$ \\
\hline 3 & $\begin{array}{l}\text { Water/Oil } \\
\text { Tracer }\end{array}$ & $\begin{array}{c}\text { PCBs } \\
\text { Perchloroethene }\end{array}$ & $\begin{array}{l}5,950 \\
6,100\end{array}$ & $\begin{array}{c}99.9999 \\
99.99\end{array}$ & $\begin{array}{l}\text { Yes } \\
\text { Yes }\end{array}$ \\
\hline 4 & $\begin{array}{c}\text { Oil } \\
\text { Tracer }\end{array}$ & $\begin{array}{c}\text { PCBs } \\
\text { Perchloroethene }\end{array}$ & $\begin{array}{c}254,000 \\
33,000\end{array}$ & $\begin{array}{c}99.9999 \\
99.99\end{array}$ & $\begin{array}{l}\text { Yes } \\
\text { Yes }\end{array}$ \\
\hline 5 & $\begin{array}{c}\text { Oil } \\
\text { Tracer }\end{array}$ & $\begin{array}{c}\text { PCBs } \\
\text { Perchloroethene }\end{array}$ & $\begin{array}{c}254,000 \\
26,000\end{array}$ & $\begin{array}{c}99.9999 \\
99.99\end{array}$ & $\begin{array}{l}\text { Yes } \\
\text { Yes }\end{array}$ \\
\hline 6 & $\begin{array}{c}\text { Oil } \\
\text { Tracer }\end{array}$ & $\begin{array}{c}\text { PCBs } \\
\text { Perchloroethene }\end{array}$ & $\begin{array}{c}254,000 \\
26,000\end{array}$ & $\begin{array}{c}99.9999 \\
99.99\end{array}$ & $\begin{array}{l}\text { Yes } \\
\text { Yes }\end{array}$ \\
\hline \multicolumn{6}{|c|}{ Soil Tests } \\
\hline Run & Waste Type & Contaminant & Concentration (mg/kg) & \multicolumn{2}{|c|}{ Desorption Efficiency (\%) } \\
\hline 1 & $\begin{array}{c}\text { Soil } \\
\text { Tracer } \\
\text { Tracer }\end{array}$ & $\begin{array}{c}\text { PCBs } \\
\text { HCB } \\
\text { OCDD }\end{array}$ & $\begin{array}{c}538 \\
12,4000 \\
0.744\end{array}$ & \multicolumn{2}{|l|}{$\begin{array}{l}94 \\
72 \\
40\end{array}$} \\
\hline 2 & $\begin{array}{c}\text { Soil } \\
\text { Tracer } \\
\text { Tracer }\end{array}$ & $\begin{array}{c}\text { PCBs } \\
\text { HCB } \\
\text { OCDD }\end{array}$ & $\begin{array}{c}718 \\
24,800 \\
1.49\end{array}$ & \multicolumn{2}{|c|}{$\begin{array}{c}99 \\
99.99 \\
99.8 \\
\end{array}$} \\
\hline
\end{tabular}

The water/oil tests were to be nominally $4,000 \mathrm{mg} / \mathrm{kg}$ PCBs, based on injecting the water and oil in a 100:1 ratio through the atomizing nozzle; perchloroethene was added as a tracer compound. The oil tests were designed to process the high-strength oil at higher throughputs while demonstrating the ability to compress and store the product gas generated. Steam was added through a separate port, but liquid water was not co-injected with the PCB oil. Again, perchloroethene was added as a tracer compound. After oil waste processing, the stored gas was directed to the boiler for about 24 hours, and stack testing by the USEPA contractor was conducted. The target DRE for the PCBs was $99.9999 \%$, and this was achieved for all six tests. The target destruction efficiency (DE) for the perchloroethene was $99.99 \%$ and this was also 
achieved for all six tests. The SITE Program analytical results for the input concentrations of the water/oil mixture and the high-strength oil are shown in Table 2.

Soils with various contamination levels were mixed to produce a relatively homogeneous quantity of soil with a nominal $1,000 \mathrm{mg} / \mathrm{kg}$ PCB concentration. The soil test runs were conducted after construction and commissioning of the new TDU was completed. During the first TDU test, contaminated soil was processed with a desorption efficiency of $94 \%$, resulting in a processed soil PCB concentration of 30 $\mathrm{mg} / \mathrm{kg}$. The soil residence time inside the TDU was increased for the second run, and a desorption removal efficiency of $99 \%$ was achieved according to the SITE program results.

The tracer compound used for the soil tests was hexachlorobenzene (HCB), which was spiked at significantly higher concentrations that the PCBs. The hexachlorobenzene was contaminated with significant levels of octachlorodibenzo-p-dioxin (OCDD). The desorption efficiencies achieved for the HCB and OCDD for Test 2 (Table 2) were $99.99 \%$ and $99.8 \%$, respectively.

Due to TSCA permit restrictions, only two runs were performed for the third test condition. It should be noted that the performance of the TDU is independent of the destruction process. The reactor destruction efficiencies for the desorbed contaminants were high for both TDU runs.

An additional component of the test program was a 72-hour endurance test aimed at demonstrating the continuous operation capabilities of the Eco Logic Process. The equipment operated perfectly and the 72hour test was concluded successfully.

\subsubsection{Full-Scale Applications of the Eco Logic Process}

With the information and successful results obtained from demonstration trials, Eco Logic began development of a full-scale unit, termed the SE25 ELI Destructor. Several improvements were made over the previous units, including a Sequencing Batch Vaporizer (SBV) for preprocessing solids such as drums and bulk metal objects, a Thermal Reduction Mill (TRM) for preprocessing soils and sediments, and a catalytic steam reformer for recovering hydrogen from the product gas.

The first SE25 ELI Destructor is currently operational in Western Australia. The first waste stream treated was an obsolete solution of the DDT pesticide and the solvent toluene. Table 3 provides stack testing data from the treatment of this material. The results show that the DRE exceeded six nines (99.9999) for DDT. Following this project, the unit was used to process PCB-contaminated electrical equipment.

The second SE25 ELI Destructor has recently completed treatment operations at the General Motors of Canada Limited (GMCL) facility in St. Catharines, Ontario, Canada. This engine plant and foundry was being transferred to foreign interests and a variety of PCB-contaminated material required remediation. The treated materials included PCB oil and watery waste, electrical equipment (ballasts, capacitors and tools) and granular solids such as soils, concrete and sub-base.

The Eco Logic treatment system achieved a DRE of at least seven nines (99.99999) for PCBs in all tests, which satisfied Eco Logic's DRE objective for PCB treatment of six nines (Table 4). The Eco Logic treatment system achieved a DRE of a least six nines (99.9999) for chlorobenzenes in all tests, which satisfies Eco Logic DRE objective for chlorobenzene treatment of six nines (Table 4). The results of the performance testing demonstrated the efficiency of the Eco Logic Process to treat high-strength PCBs and chlorobenzenes in concentrated form with negligible environmental impact. 
Table 3. STACK TEST DATA FOR COMMERCIAL-SCALE TREATMENT OF DDT

\begin{tabular}{||l|l|l||}
\hline Input & Quantity & Units \\
\hline Duration of Run & 81 & minutes \\
\hline DDT Feed & 127.5 & $\mathrm{~L}$ \\
\hline DDT Concentration & 30.3 & $\%$ \\
\hline DDT Mass Input & 39.7915 & $\mathrm{~kg}$ \\
\hline Output & & \\
\hline Gas Flow Rate & 0.76 & $\mathrm{~m}^{3} / \mathrm{sec}$ \\
\hline Total Gas Flow & 3694 & $\mathrm{Nm}^{3}$ \\
\hline DDT Concentration & $\leq 1.7$ & $\mu \mathrm{g} / \mathrm{Nm}^{3}$ \\
\hline DDT Mass Output & $\leq 0.00000628$ & $\mathrm{~kg}$ \\
\hline \multicolumn{2}{|c|}{ DRE $\leq(39.7951-0.00000628) / 39.7951 \geq 99.999984$} \\
\hline
\end{tabular}

Table 4. SUMMARY OF COMMERCIAL-SCALE DRE AND DE VALUES FOR PCBs

\begin{tabular}{||l|c|c|c|c||}
\hline \hline Parameter & Units & Test 1 & Test 2 & Test 3 \\
\hline DRE for PCBs & $\%$ & 99.9999996 & 99.9999985 & 99.9999997 \\
\hline DRE for CBs & $\%$ & 99.9999842 & 99.9999985 & 99.9999977 \\
\hline DE for PCBs & $\%$ & 99.9999996 & 99.9999985 & 99.9999808 \\
\hline DE for CBs & $\%$ & 99.9999836 & 99.9999972 & 99.9999971 \\
\hline
\end{tabular}

\section{PILOT-SCALE TESTING}

\subsection{Pilot-Scale System Description}

The pilot-scale unit used for the treatment of napalm was set up in the ERDEC facility at the Aberdeen Proving Ground in Maryland. This unit and its predecessors have been used by Eco Logic over the past ten years to perform waste-specific treatability studies for many commercial and government clients. Figure 5 presents a generalized process schematic of the treatability-scale unit. The system includes an SBV, a gas phase chemical reduction reactor, a two-stage scrubbing system and a product gas compression and storage system with continuous emission monitoring equipment.

The SBV is an autoclave-type chamber into which the bulk waste is placed. The SBV is sealed and heated to $600^{\circ} \mathrm{C}$. The SBV off-gas (sweep gas and desorbed organic compounds from the SBV) is further heated in the reactor to about $900^{\circ} \mathrm{C}$.

Once the contaminant gas enters the reactor, the contaminants react with hydrogen and steam]over a period of several seconds. Under these conditions, the complex organic contaminants (including napalm) are reduced primarily to methane at high efficiency. As the reduced gas mixture exits the reactor, it is quenched quickly with a dilute scrubber solution of sodium hydroxide An identical second scrubber serves 


\section{Figure 5 SCHEMATIC OF PILOT-SCALE SYSTEM}

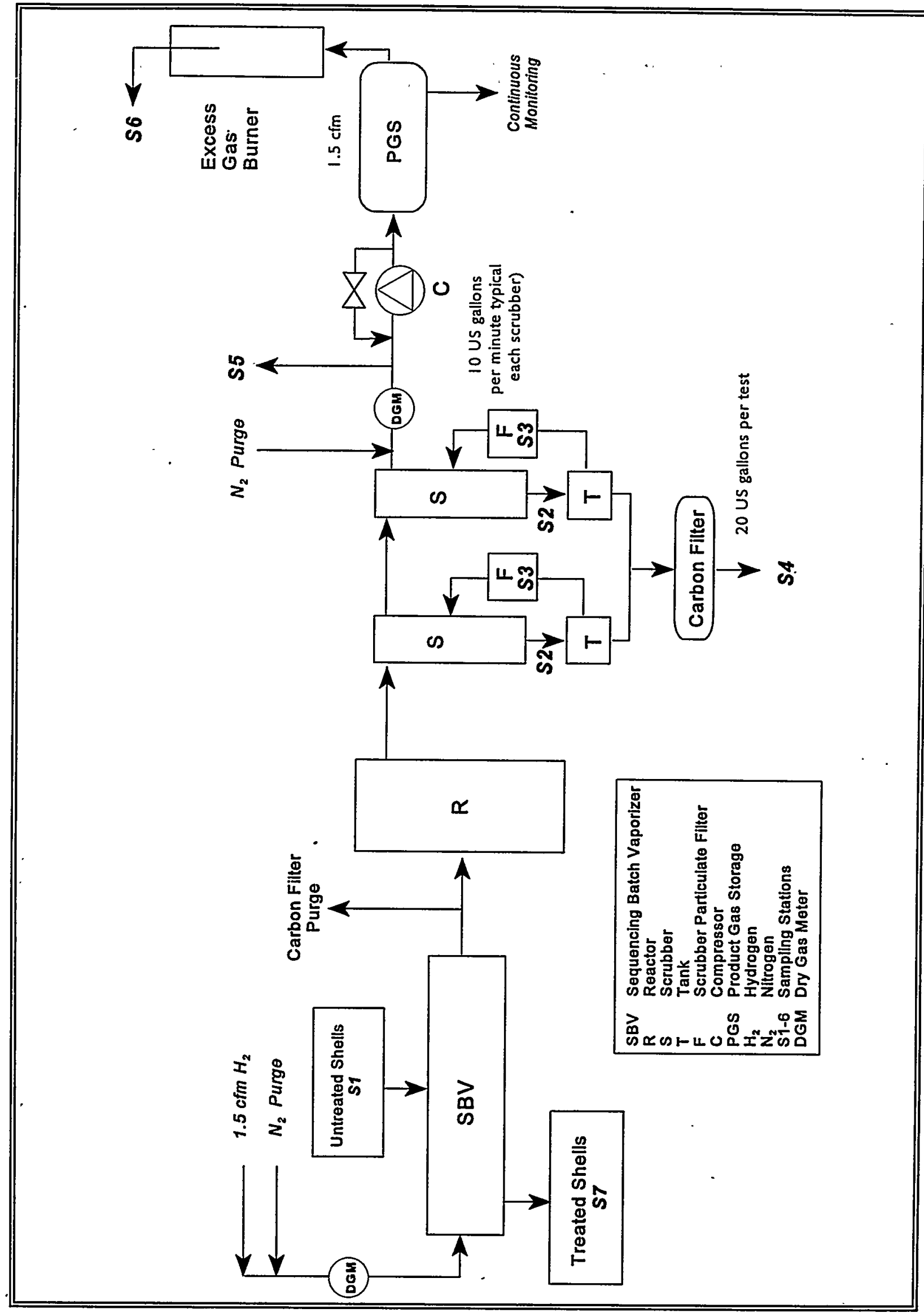


as a backup to the first. The scrubbers consist of spray nozzles mounted at the top and the middle of the scrubber columns with reservoirs beneath. A pump on each scrubber draws scrubber solution from the reservoir and supplies the spray nozzles. The pipes leading to the spray nozzles are equipped.with cooling jackets to maintain the temperature of scrubbing solutions below $35^{\circ} \mathrm{C}$.

After the gas passes through the scrubbers, it is sent to a sampling system manifold or a bypass, and ultimately through an excess gas flare (EGF). The test setup employs instrumentation that ensures all relevant data is collected and the process is properly monitored and controlled. Instrumentation includes temperature thermocouples at numerous locations, gas pressure gauges and flow meters, and liquid pressure and level gauges. The gas monitoring system consists of an oxygen analyser, for purging and safety reasons, a Nova multi-gas analyser for monitoring $\mathrm{H}_{2}, \mathrm{CH}_{4}, \mathrm{CO}$, and $\mathrm{CO}_{2}$ and a CIMS for monitoring trace levels of hydrocarbon products, such as benzene and toluene. At the end of each test run, the scrubber effluent is collected and analysed prior to off-site disposal. The particulate filters that are in use continuously during the test run are removed and replaced for the next test run.

The pilot-scale system is operated as a once-through system with hydrogen as the sweep gas (the full-scale system uses a catalytic steam reformer to convert product gas to hydrogen for reuse). The product gas is compressed, stored and analysed prior to being sent to the excess gas flare. If the on-line analysis (S6) of the product gas, as measured by the CIMS shows levels of selected compounds indicative of incomplete destruction, the gas filtering is discontinued, the waste feed halted, and the hydrogen input is shut off as the system recirculation valve is opened. This would allow gas to recirculate until the concentrations decrease to an acceptable level. It has never been necessary to use this recirculation capability for any of the treatability tests carried out to date.

\section{$2.2 \quad$ Pilot-Scale Test Operations}

\subsubsection{Goals and Objectives of Pilot-Scale Testing}

The primary goal of the napalm pilot-scale treatability testing program was to demonstrate the ability of the Eco Logic process to treat the napalm safely and effectively. The study was undertaken with the following objectives:

- to determine optimum system conditions required to volatilize napalm from the aluminum containers;

- $\quad$ to verify SBV safety during treatment of neat napalm.

- $\quad$ to qualitatively verify the destruction of napalm in the reactor.

\subsubsection{Test Preparation and System Integrity Testing}

The components of the pilot-scale unit, including the SBV, reduction reactor, scrubber system, compressor, product gas storage tank and instrumentation, were set up within ERDEC's toxic test chambers at Aberdeen Proving Ground in Maryland. Commissioning of the Eco Logic's pilot-scale treatability test unit involved a standard set of set-up procedures and requirements: .

- connection of propane, hydrogen, and nitrogen tanks,

- flushing of all fluid piping to ensure that the lines were free of particulate or other foreign objects,

- assembly of all components and connection to the remote process control system,

- checking of all piping and vessels for leaks, 
- manual check of all valves in the system,

- manual check of all electrically powered pumps and motors,

- $\quad$ pressure testing of all gas piping,

- connection, calibration and testing of all system control loops and instrumentation.

\subsubsection{Testing Procedure}

Three test runs on neat napalm were conducted over a three day period. The amount of napalm processed was 236.86 grams, 200.74 grams and 224.20 grams during test runs 1,2 and 3, respectively. The duration of each test run was between 2.5 hours 3 hours.

The unit operation team for napalm testing was comprised of three Eco Logic staff members (Operations Supervisor, System Operator, and Technician). Daily activities involved approximately three hours of operations planning and equipment set-up, 2.5 - 3 hours of operation, and two hours of sampling and equipment shut-down. To maximize time spent treating napalm, routine system maintenance was performed during start-up and shut-down.

Operation of the unit involved a standard set of start-up, normal operation and shut-down protocols. Table 5 lists the start-up and shut-down procedures used by the project team. Prior to testing, the process control computer and monitoring equipment were calibrated with all sampling apparatus ready. During the actual performance of the tests, certain operational parameters were manually logged every ten minutes by the operators. The critical operational parameters included reactor pressure, reactor temperature, scrubber temperature, scrubber $\mathrm{pH}$, oxygen concentration, dry gas flow rates of the hydrogen to the SBV and flow of product gas to the burner. These data points complimented the suite of process control compounds continuously monitored by the on-line instruments. All events that occurred during a test (e.g., manual increase in flow rate, test stop, etc.) were recorded by the operators in the operating log book and the process control data sheets. Process data log sheets are provided in Appendix A.

\subsection{Process Operating Conditions}

An optimization test with napalm was conducted to determine appropriate operating conditions of the SBV specific to the treatment of napalm. The input flow rate of $\mathrm{H}_{2}$, the rate of temperature increase and the $\mathrm{pH}$ control of the scrubbers were also determined during this optimization test run. Performance tests (Test Run 2 and 3) utilized the operating condition parameters identified during the optimization run. Critical parameters of the process operation and outputs, for all three tests, are summarized in Table 6.

\subsubsection{SBV Operating Conditions}

The napalm was loaded and sealed within the SBV prior to system purging and heating. The aluminum containers, containing napalm, were placed in the center of the SBV with a thermocouple inserted directly into the napalm. The SBV starting temperature for all three test runs was $30^{\circ} \mathrm{C}$ which was increased to a maximum inner temperature of 600,725 and $625^{\circ} \mathrm{C}$ for test runs 1,2 and 3 respectively.

Pre-heated hydrogen was input to the SBV to provide a saturated hydrogen environment. The hydrogen and volatilized organic compounds were then swept from the SBV and directed to the pre-heated reactor vessel to complete the destruction of the organic contaminants. 
Table 5. TREATABILITY-SCALE STARTUP AND SHUTDOWN PROCEDURES

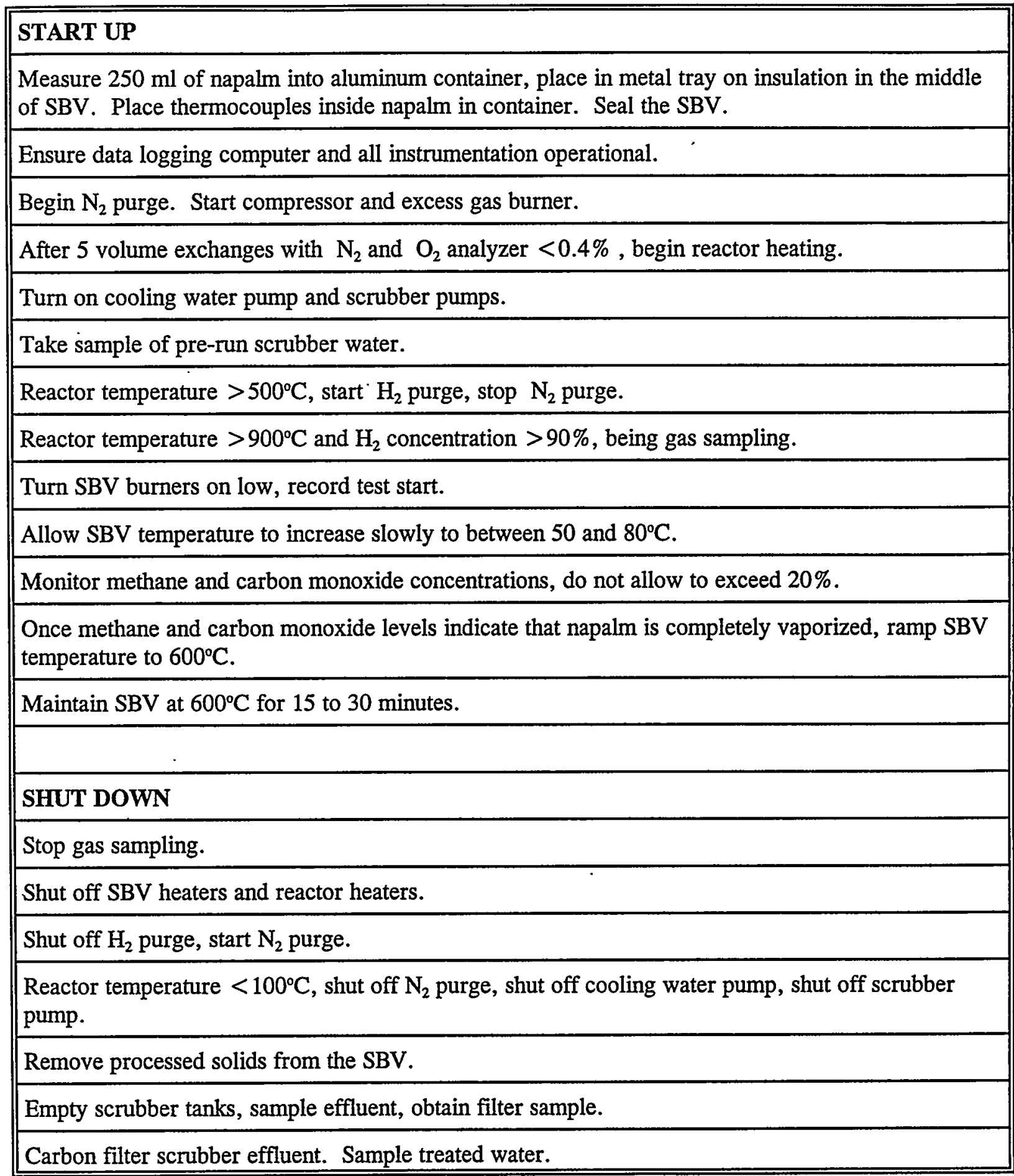


Table 6. SUMMARY OF NAPACM TREATABILITY STUDY OPERATING CONDITIONS

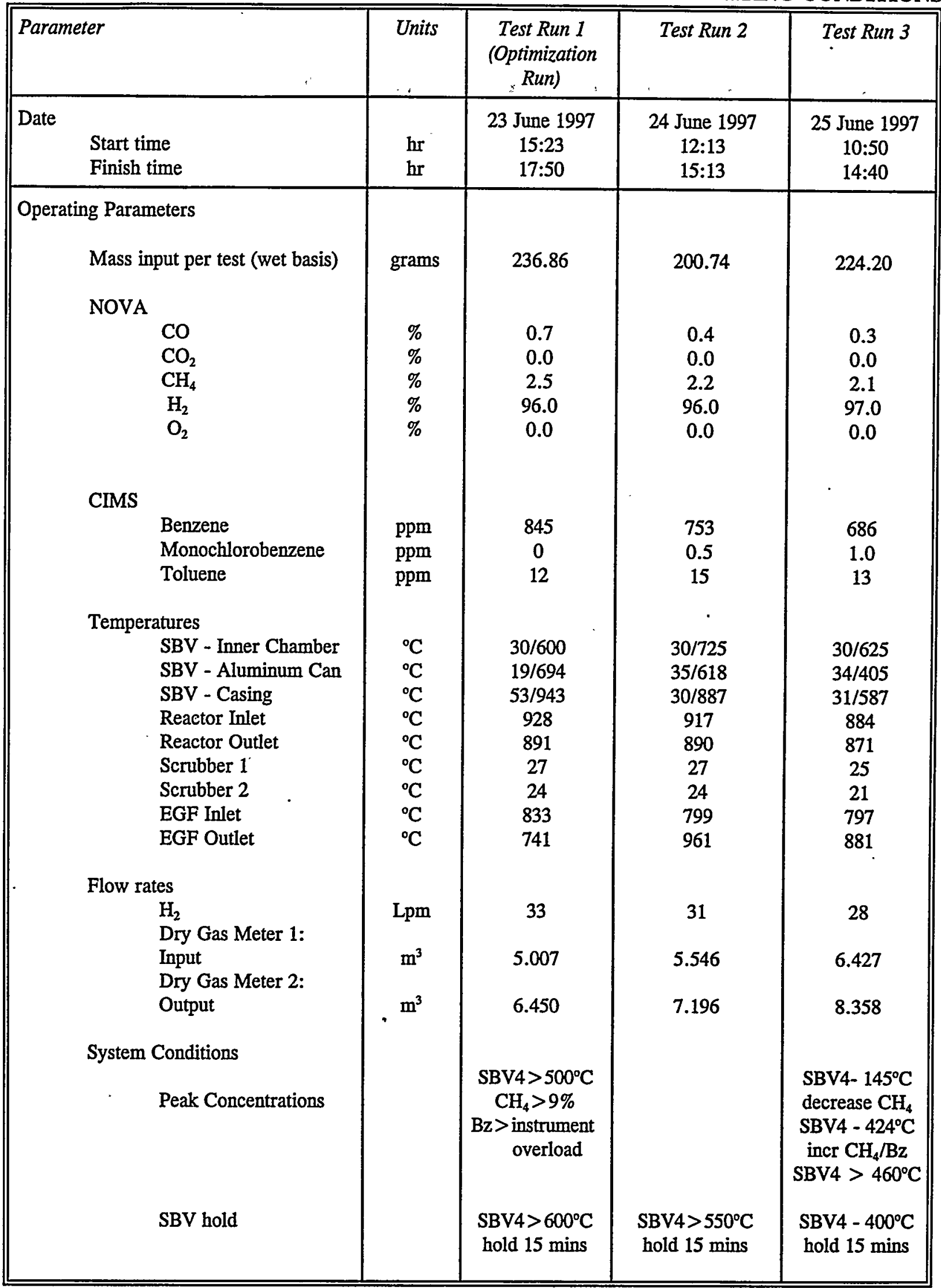




\subsubsection{Reactor System Operating Conditions}

The reactor was operated at an average temperature of $928^{\circ} \mathrm{C}, 917{ }^{\circ} \mathrm{C}$ and $884^{\circ} \mathrm{C}$ for Tests 1,2 and 3 , respectively. The design of the reactor ensures that the residence time is greater than 1 second for complete destruction of the organic contaminants. The operating pressure averaged $0.27,0.22$ and 0.19 psig for Tests 1,2 , and 3, respectively. The hydrogen input flow rate was constant at 33, 31 and 28 liters per minute for Tests 1,2 and 3 , respectively, which resulted in average reactor hydrogen concentrations of $92 \%$ for Test $1,96.0 \%$ for Test 2 and $97.0 \%$ for Test 3 . The input flow of hydrogen was a minimum requirement to ensure proper purging of contaminants from the SBV. Lower concentrations of hydrogen in the reactor have been demonstrated to produce effective destruction efficiencies. The concentration is always maintained above $50 \%$ on a dry basis.

\subsubsection{Scrubber System Operating Conditions}

The scrubbers provide removal of particulate and soluble organic contaminants from the product gas. The design of the scrubber towers is such that the product gas is thoroughly wetted from both the top and middle spray inputs. The flow of the product gas is concurrent in the first scrubber and counter-current in the second scrubber. Initial dosing of the scrubber solution for each test ensured that a $\mathrm{pH}$ greater than 10 was maintained.

The scrubbers were charged with 35 liters of fresh caustic solution ( $\mathrm{pH} 10)$ prior to commencing each test. The scrubber solutions were cooled in-line with water jacket heat exchangers and a supply of cold water. The average temperature for Scrubber No. 1 was $27^{\circ} \mathrm{C}$ for Runs 1 and 2 and $25^{\circ} \mathrm{C}$ for Run 3 . The average temperature for Scrubber No. $2 \mathrm{w}$ as $24^{\circ} \mathrm{C}$ for Runs 1 and 2 and $21^{\circ} \mathrm{C}$ for Run 3 . At the completion of each test run, the scrubber solutions were sampled, carbon filtered, sampled and stored in drums for disposal.

\section{$2.4 \quad$ Sampling and Monitoring Procedures}

The sampling program for the napalm treatability study and analytical methods are summarized in Table 7 and discussed below. These sampling locations are shown on Figure 5. The testing program allowed for verification of desorption efficiencies and decontamination of the aluminum containers.

Sampling activities were undertaken by personnel experienced in the operation and sampling associated with the pilot-scale unit. All samples were homogenized, where appropriate, to ensure they were representative of the matrix being tested. Representative scrubber solution samples were taken from actively mixed tanks.

Untreated Napalm (S1): The amount of napalm processed was based on the known amount of napalm weighed into the aluminum container.

Pre-Run Scrubber Water: Samples of the scrubber water were collected as background samples prior to each test run. These samples were analyzed for volatile organic compounds (VOCs) and total recoverable petroleum hydrocarbons (TRPH).

Scrubber Effluent (S2): At the end of each run, samples of the untreated scrubber effluent were collected and subjected to analysis of TRPH, semi-volatile organic compounds (SVOCs) and VOCs.

Scrubber Effluent Particulate (S3): Particulate was removed from the recirculating scrubber solution during the run using $1 \mu \mathrm{m}$ cartridge filters. The particulate filters were collected after each run and the dry weight determined. 
Scrubber Effluent (post-tertiary treatment)(S4): After the scrubber effluent passed through the activated carbon system, scrubber effluent samples (post-tertiary treatment) were collected and analyzed for TRPH, SVOCs and VOCs.

Scrubber Product Gas (S5): The scrubbed product gas was continuously analyzed for benzene and toluene, using the CIMS. The NOVA multi-gas analyzer was used for bulk gas analyses of hydrogen, methane, $\mathrm{CO}_{2}$, and $\mathrm{CO}$.

Treated Container (S6): Following SBV treatment, wipe samples from the interior surface of the aluminum container or SBV tray were collected and analyzed for TRPH and VOCs.

\subsubsection{Sampling Methods}

Sampling schedule, methodology and parameters (summarized in Table 7) were presented and accepted in the test plan for this treatability study titled Demonstration Test Plant for Testing on Napalm at the US Army's Edgewood Research Development, Engineering Center (ERDEC) Toxic Test Chamber Facilities, June 23, 1997. Analyses of all parameters in solid and liquid samples were performed by Triangle Laboratories using EPA methodologies.

Table 7. SUMMARY OF SAMPLING PROGRAM FOR NAPALM

\begin{tabular}{|c|c|c|c|c|c|}
\hline $\begin{array}{c}\text { Parameter and } \\
\text { Location (see } \\
\text { Figure 4) }\end{array}$ & $\begin{array}{l}\text { Sampling/ } \\
\text { Monitorin } \\
\text { g Method }\end{array}$ & $\begin{array}{l}\text { Frequency of } \\
\text { Sampling }\end{array}$ & Analytes & $\begin{array}{l}\text { Analytical } \\
\text { Method }\end{array}$ & Sampling Method \\
\hline $\begin{array}{l}\text { Untreated } \\
\text { napalm (S1) }\end{array}$ & $\begin{array}{l}\text { To be } \\
\text { based on } \\
\text { weighed } \\
\text { amount of } \\
\text { napalm }\end{array}$ & NA & NA & NA & NA \\
\hline $\begin{array}{l}\text { Scrubber } \\
\text { Effluent (\$2) }\end{array}$ & $\begin{array}{l}\text { grab } \\
\text { sample }\end{array}$ & $\begin{array}{l}1 \text { grab sample } \\
\text { per test run }\end{array}$ & $\begin{array}{l}\text { TRPH } \\
\text { SVOCs } \\
\text { VOCs }\end{array}$ & $\begin{array}{l}\text { EPA } 8330 \\
\text { EPA } 8270 \\
\text { EPA } 8260\end{array}$ & $\begin{array}{l}\text { Comp./grab } \\
\text { SW-846 }\end{array}$ \\
\hline $\begin{array}{l}\text { Scrubber } \\
\text { Effluent } \\
\text { Particulate } \\
\text { (S3) }\end{array}$ & $\begin{array}{l}\text { grab } \\
\text { sample }\end{array}$ & $\begin{array}{l}1 \text { grab sample } \\
\text { per test run } \\
\text { (if available) }\end{array}$ & dry weight & NA & NA \\
\hline $\begin{array}{l}\text { Scrubber } \\
\text { Effluent (post } \\
\text { tertiary } \\
\text { treatment) (S4) }\end{array}$ & $\begin{array}{l}\text { grab } \\
\text { sample }\end{array}$ & $\begin{array}{l}1 \text { grab sample } \\
\text { per test run }\end{array}$ & $\begin{array}{l}\text { TRPH } \\
\text { SVOCs } \\
\text { VOCs }\end{array}$ & $\begin{array}{l}\text { EPA } 8330 \\
\text { EPA } 8270 \\
\text { EPA } 8260\end{array}$ & $\begin{array}{l}\text { Comp./grab } \\
\text { SW } 846\end{array}$ \\
\hline $\begin{array}{l}\text { Scrubbed } \\
\text { Product Gas } \\
\text { (S5) }\end{array}$ & $\begin{array}{l}\text { CIMS, } \\
\text { NOVA }\end{array}$ & $\begin{array}{l}\text { Continuous } \\
\text { monitoring }\end{array}$ & $\begin{array}{l}\text { benzene, } \\
\text { toluene, } \mathrm{H}_{2} \text {, } \\
\mathrm{CH}_{4}, \mathrm{CO}, \mathrm{CO}_{2}\end{array}$ & & \\
\hline $\begin{array}{l}\text { Treated } \\
\text { napalm } \\
\text { container (S6) }\end{array}$ & $\begin{array}{l}\text { wipe } \\
\text { sample }\end{array}$ & $\begin{array}{l}1 \text { wipe sample } \\
\text { per test run }\end{array}$ & $\begin{array}{l}\text { TRPH } \\
\text { SVOCs }\end{array}$ & $\begin{array}{l}\text { EPA } 8330 \\
\text { EPA } 8270\end{array}$ & wipe sampling \\
\hline
\end{tabular}




\subsubsection{General Sampling/Quality Control Procedures}

Quality Control measures for process sampling includes a check on the following procedures:

- cleaning of the sampling equipment

- $\quad$ sample collection procedures

- $\quad$ recording observations on pre-formatted data sheets

In order to prevent cross contamination of the samples, the sampling staff ensured that there was no personal or environmental contamination of the surrounding areas during sampling and storage. The controlled provision of clean supplies and sample bottles minimized the possibility of sample contamination.

Samplers recorded all observations on pre-formatted chain-of-custody forms. Copies of the chain of custody forms are provided in Appendix B.

\subsubsection{Liquid Sample Collection}

Liquid samples of treated and untreated scrubber water were obtained by grab sampling. Samplers wore latex gloves and other personal protective equipment (PPE). Liquid grab samples were poured directly into a $1 \mathrm{~L}$ amber glass bottles provided by the subcontracted analytical laboratory, and appropriately labeled. Samples were then logged and stored in the refrigerator at $4^{\circ} \mathrm{C}$ before being transported to the laboratory for analysis.

\subsubsection{Napalm Aluminum Container Sampling}

Samples of the interior of the aluminum container were obtained by wipe sampling. Stainless steel tweezers were used to hold a gauze pad soaked with hexane. The gauze pad was wiped over the entire surface of the SBV tray (Test Run 2) or the aluminum container (Test Run 3). Wipe samples were placed in glass containers provided by the analytical laboratory, and appropriately labeled. Samples were then logged in and stored in the refrigerator at $4^{\circ} \mathrm{C}$ before being transported to the laboratory for analysis.

\subsubsection{Process Performance Monitoring Via On-Line Instrumentation}

The concentration of specific compounds within the scrubbed product were continuously monitored using the CIMS, NOVA Bulk Gas Analyzer and NOVA $\mathrm{O}_{2}$ Analyzer. The gas was sampled from the exit of the Scrubber No. 1 and from the product gas storage tank.

The CIMS is capable of accurately monitoring up to ten organic compounds every few seconds at concentrations ranging from parts per billion $(\mathrm{ppb})$ to percent levels. For this treatability study, the CIMS was configured to measure benzene, monochlorobenzene and toluene in the product gas at the exit of the scrubber. Measurement of intermediate reduction products allows continuous indication of destruction efficiency as well as detection of unwanted compounds in the product gas before being directed to the excess gas flare. Data from the CIMS can also be directly connected to alarm limits and operator indicators.

The NOVA Bulk Gas Analyzer was used to continuously monitor the concentrations of $\mathrm{CO}, \mathrm{CO}_{2}, \mathrm{CH}_{4}$, and $\mathrm{H}_{2}$. The reducing hydrogen atmosphere is maintained at more than 50 percent hydrogen (dry basis) to prevent the formation of polyaromatic hydrocarbons (PAHs). Hydrogen concentration is thus a critical operating parameter. In addition, levels of $\mathrm{CO}, \mathrm{CO}_{2}, \mathrm{CH}_{4}$, and $\mathrm{H}_{2}$ are measured as final products of reduction to monitor the process reactions. The concentration of oxygen in the system is also a critical operating parameter which is continuously monitored by the NOVA $\mathrm{O}_{2}$ analyzer. This allows for the safe operation of the hydrogen reduction process. 
The CIMS and NOVA analyzers were linked to the data logging system. The system was equipped with appropriate alarm indicators to ensure safe and efficient operation of the Eco Logic Process.

Each instrument was zeroed and calibrated before and after each test run using certified calibration gases. The on-line data are provided graphically in Appendix $\mathbf{C}$.

3.

\section{TEST RESULTS}

\section{1}

\section{Qualitative Observations}

Visual observations of the contents of the aluminum container before and after SBV treatment are presented in Figures 1 and 2. Pre-treatment samples indicated a quantity of untreated liquid napalm contained in the aluminum container (see Figure 1). After Test Runs 1 and 2, the aluminum container melted into the SBV tray during the treatment process and there was no visible sign of napalm in the SBV. After altering the SBV operating procedures to monitor operating temperature in the SBV instead of in the aluminum container for Test Run 3, the aluminum container remains intact and there is no quantity of liquid napalm remaining (see Figure 2).

\subsection{Chemical Analysis of System Input and Outputs}

Tables 8 to 11 summarize key analytical data for solid and liquid outputs from Test Runs 2 and 3 . Sampling locations (S1 through S6) are illustrated in Figure 5 and described in more detail in Table 7. All data is reported to two significant figures. The complete data sets provided in Appendix D.

\subsection{Discussion of Results}

The results presented in Section 3.1 were interpreted to evaluate the performance of the Process in terms of the objectives of the study, as described in Section 2.2.1.

Low levels of volatile organic compounds were detected in the pre-run scrubber water collected to determine background concentrations of these compounds. Following treatment of napalm, levels of VOCs in the untreated scrubber effluent are of the same magnitude as those reported in the pre-run scrubber water. Following tertiary carbon treatment of the scrubber effluent, levels of VOCs and TRPH are generally reduced to less than the method detection limit.

Semi-volatile organic compounds (SVOCs) in untreated scrubber water were analyzed for test run 2 only. SVOCs concentrations ranged from non-detect to $72 \mathrm{ppb}$, with the exception of naphthalene which was detected at a concentration of $540 \mathrm{ppb}$. However, following tertiary carbon treatment all but three SVOCs, were reduced to less than the detection limit, including naphthalene. The concentrations of the detected SVOCs ranged from 1.3 to $8.1 \mathrm{ppb}$ and 1.8 to $4.6 \mathrm{ppb}$ for test runs 2 and 3 respectively (Table 10).

It should be noted that in the full-scale SE25 system, the VOCs and SVOCs would be removed in the third leg of the four stage scrubbing system that involves neutral oil washing. The rich oil is pumped to a stripper where VOCs and SVOCs such as benzene and naphthalene are removed and sent to the inlet of the catalytic steam reformer for conversion to hydrogen and carbon dioxide.

Total recoverable petroleum hydrocarbons (TRPH) was not detected in the post-treatment scrubber water samples. Levels of TRPH of the treated napalm container were less than the detection limit of $100 \mu \mathrm{g} / \mathrm{wipe}$ for both test runs. 


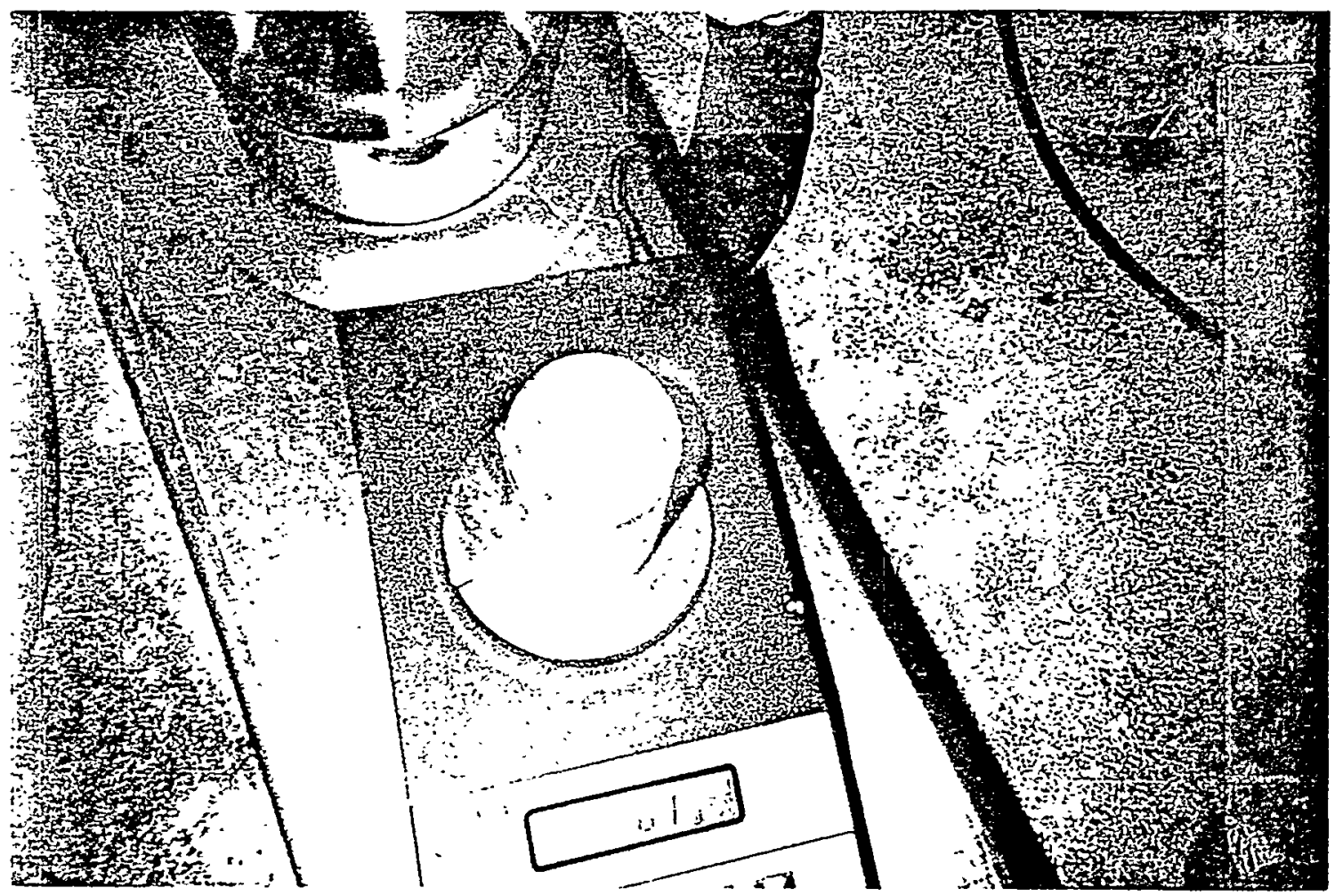

FIGURE 6 - VISUAL: OBSERVATION OF NAPALM IN CONTAINER BEFORE TESTING

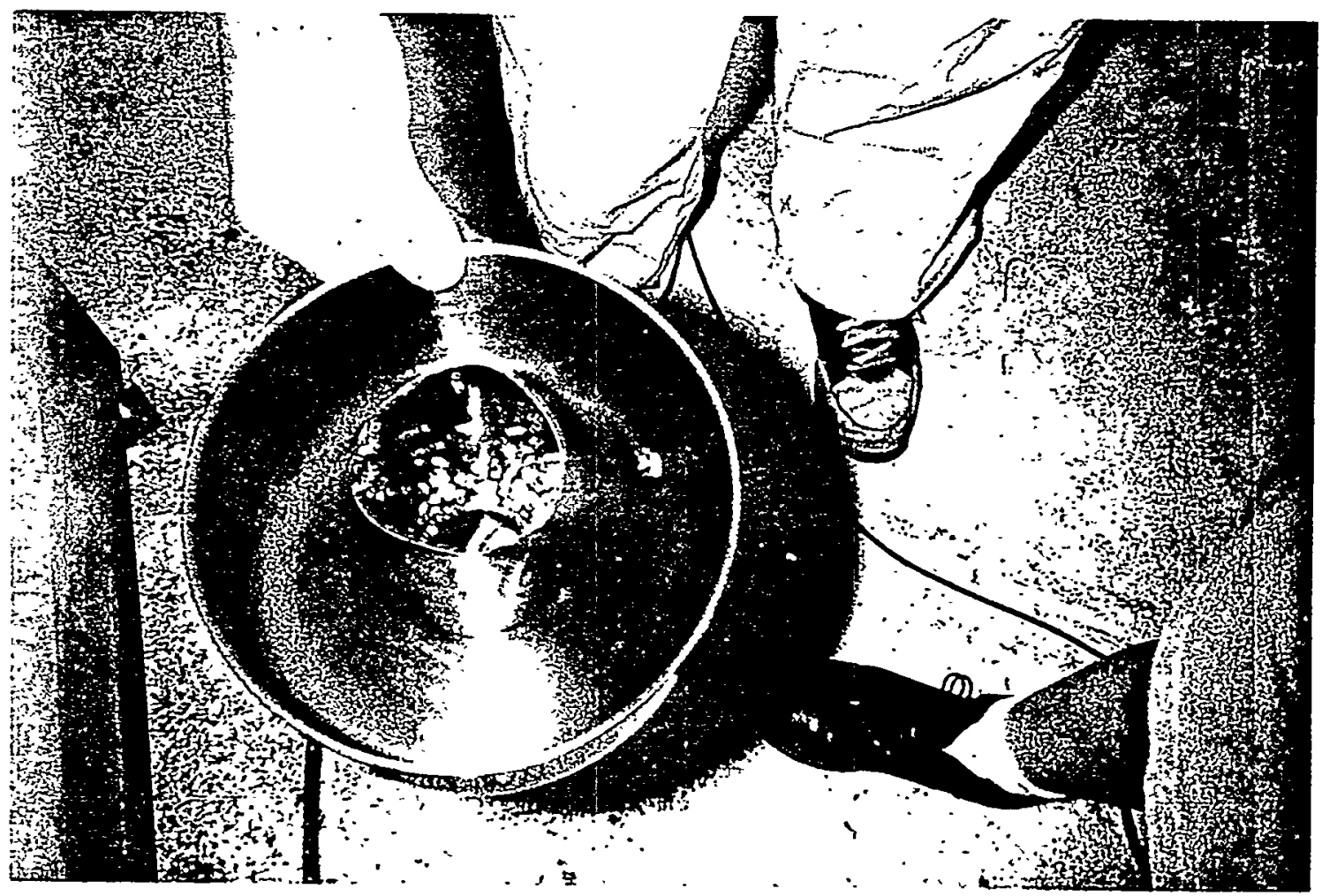

FIGURE 7 . VISUAL OBSERVATION OF NAPALM IN CONTAINER AFTER TESTING 
Table 8 PRE-RUN SCRUBBER WATER $(\mu \mathrm{g} / \mathrm{L})$

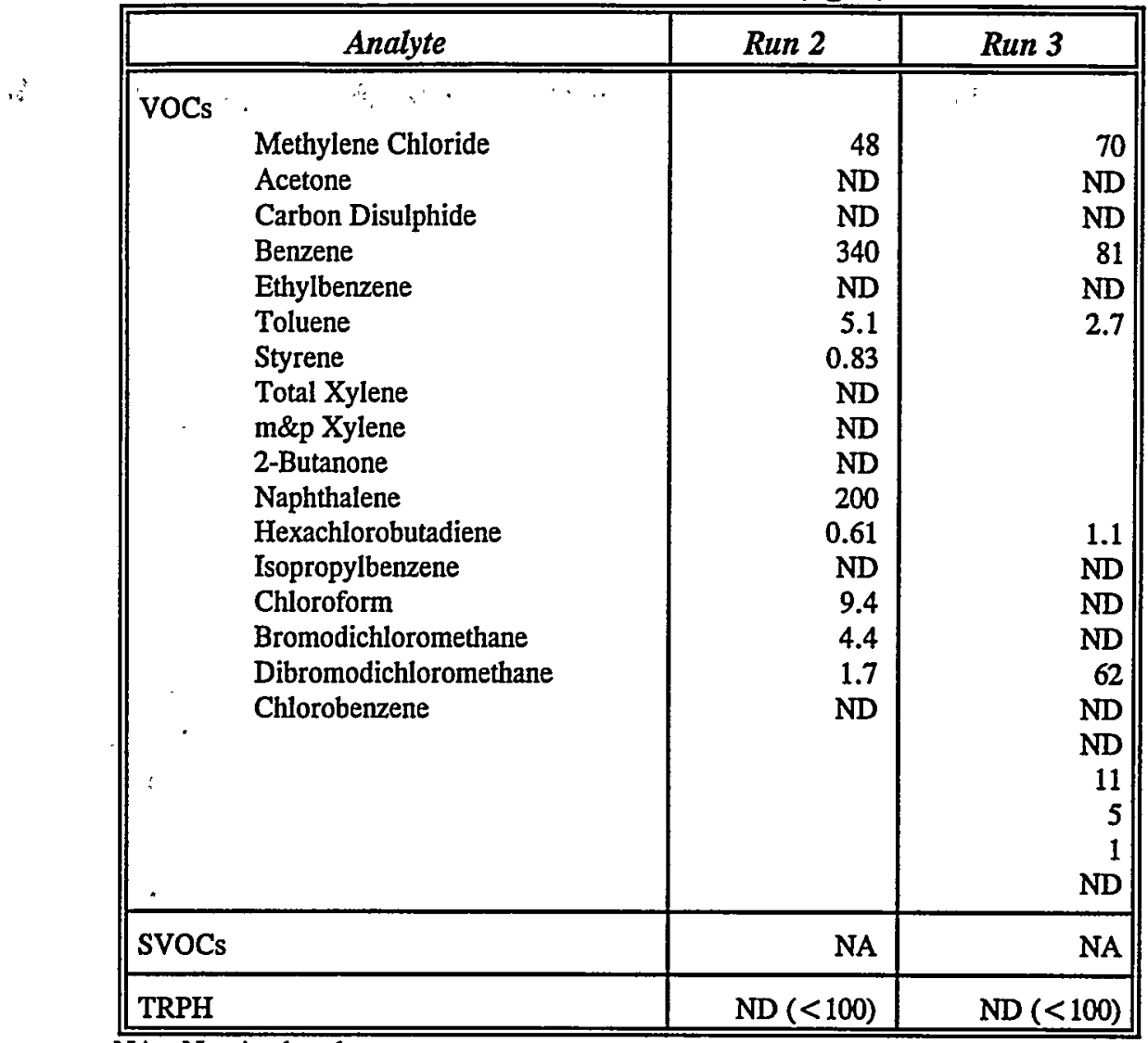

NA - Not Analysed

ND - Not Detected 
Table 9 SCRUBBER EFFLUENT WATER (S2) $(\mu \mathrm{g} / \mathrm{L})$

\begin{tabular}{|c|c|c|c|}
\hline Analyte & & Run 2 & Run 3 \\
\hline \multicolumn{4}{|l|}{ VOCs } \\
\hline & Methylene Chloride & 160 & 130 \\
\hline & Acetone & ND & ND \\
\hline & Carbon Disulfide & ND & ND \\
\hline & Benzene & 480 & 300 \\
\hline & Ethylbenzene & 4.7 & 2.7 \\
\hline & Toluene & 92 & 50 \\
\hline & Styrene & 66 & 52 \\
\hline & Total Xylene & ND & ND \\
\hline & m\&p-xylene & 0.66 & ND \\
\hline & 2-Butanone & ND & ND \\
\hline & Naphthalene & 710 & 550 \\
\hline & Hexachlorobutadiene & ND & ND \\
\hline & Isopropylbenzene & 0.73 & 0.48 \\
\hline & Chloroform & ND & 1.6 \\
\hline & Bromodichloromethane & ND & ND \\
\hline & Dibromochloromethane & ND & ND \\
\hline & Chlorobenzene & ND & ND \\
\hline \multirow[t]{37}{*}{ SVOCs } & & NA & \\
\hline & Naphthalene & & 540 \\
\hline & Dibenzofuran & & 0.97 \\
\hline & Fluorene & & 28 \\
\hline & Phenanathrene & & 72 \\
\hline & Anthracene & & 11 \\
\hline & Di-n-butyl phthalate & & - 4.3 \\
\hline & Fluoranthene & & 4.8 \\
\hline & Pyridine & & 1.1 \\
\hline & Pyrene & & 1.6 \\
\hline & Phenol & & 1.8 \\
\hline & Aniline & & 4.3 \\
\hline & 2-methylphenol & & 0.52 \\
\hline & 4-methylphenol & & 0.69 \\
\hline & Acetophenone & & 9.9 \\
\hline & 2,4-Dimethylphenol & & 0.33 \\
\hline & 2-Methylnaphthalene & & 1.3 \\
\hline & Acenaphthalene & & 17 \\
\hline & Acenaphthene & & 2.3 \\
\hline & Di-n-octylphthalate & & ND \\
\hline & 1,2,4-Trichlorobenzene & & ND \\
\hline & Diethylphthalate & & ND \\
\hline & Benzo(a)anthracena & & ND \\
\hline & Chrysene & & ND \\
\hline & Bis(2-ethylhexyl)phthalate & & ND \\
\hline & Dibenz(a,h)anthracene & & ND \\
\hline & Benzo(ghl)perylene & & ND \\
\hline & Benzo(b)fluoranthene & & ND \\
\hline & Benzo(k)fluoranthrene & & ND \\
\hline & Benzo(a)pyrene & & ND \\
\hline & Indeno(1,2,3-cd)pyrene & & $\mathrm{ND}$ \\
\hline & Butylbenzylphthalate & & ND \\
\hline & $\mathrm{N}$-nitrosodiphenylamine & & ND \\
\hline & N-nitrosodimethylamine & & ND \\
\hline & Benzyl alcohol & & ND \\
\hline & 1,2,4,5-Tetrachlorobenzene & & ND \\
\hline & Benzoic Acid & & ND \\
\hline TRPH & & ND $(<100)$ & $\mathrm{ND}(<100)$ \\
\hline
\end{tabular}

NA - Not Analysed

ND - Not Detected 
Table 10 POST CARBON TREATED SCRUBBER EFFLUENT WATER (S4) $(\mu \mathrm{g} / \mathrm{L})$

\begin{tabular}{|c|c|c|c|}
\hline & Analyte & Run 1 & Run 2 \\
\hline . & $\begin{array}{l}\text { Methylene Chloride } \\
\text { Acetone } \\
\text { Carbon Disulfide } \\
\text { Benzene } \\
\text { Ethylbenzene } \\
\text { Toluene } \\
\text { Styrene } \\
\text { Total Xylene } \\
\text { m\&p-xylene } \\
\text { 2-Butanone } \\
\text { Naphthalene } \\
\text { Hexachlorobutadiene } \\
\text { Isopropylbenzene } \\
\text { Chloroform } \\
\text { Bromodichloromethane } \\
\text { Dibromochloromethane } \\
\text { Chlorobenzene }\end{array}$ & $\begin{array}{r}73 \\
\text { ND } \\
\text { ND } \\
\text { ND } \\
\text { ND } \\
\text { ND } \\
\text { ND } \\
\text { ND } \\
\text { ND } \\
71 \\
\text { ND } \\
\text { ND } \\
\text { ND } \\
\text { ND } \\
\text { ND } \\
\text { ND } \\
\text { ND }\end{array}$ & $\begin{array}{r}75 \\
\text { ND } \\
\text { ND } \\
0.74 \\
\text { ND } \\
\text { ND } \\
\text { ND } \\
\text { ND } \\
\text { ND } \\
55 \\
5.5 \\
\text { ND } \\
\text { ND } \\
\text { ND } \\
\text { ND } \\
\text { ND } \\
\text { ND }\end{array}$ \\
\hline SVOCs & $\begin{array}{l}\text { Naphthalene } \\
\text { Dibenzofuran } \\
\text { Fluorene } \\
\text { Phenanathrene } \\
\text { Anthracene } \\
\text { Di-n-butyl phthalate } \\
\text { Fluoranthene } \\
\text { Pyridine } \\
\text { Pyrene } \\
\text { Phenol } \\
\text { Aniline } \\
\text { 2-methylphenol } \\
\text { 4-methylphenol } \\
\text { Acetophenone } \\
\text { 2,4-Dimethylphenol } \\
\text { 2-Methylnaphthalene } \\
\text { Acenaphthalene } \\
\text { Acenaphthene } \\
\text { Di-n-octylphthalate } \\
\text { 1,2,4-Trichlorobenzene } \\
\text { Diethylphthalate } \\
\text { Benzo(a)anthracena } \\
\text { Chrysene } \\
\text { Bis(2-ethylhexyl)phthalate } \\
\text { Dibenz(a,h)anthracene } \\
\text { Benzo(ghl)perylene } \\
\text { Benzo(b)fluoranthene } \\
\text { Benzo(k)fluoranthrene } \\
\text { Benzo(a)pyrene } \\
\text { Indeno(1,2,3-cd)pyrene } \\
\text { Butylbenzylphthalate } \\
\text { N-nitrosodiphenylamine } \\
\text { N-nitrosodimethylamine } \\
\text { Benzyl alcohol } \\
\text { 1,2,4,5-Tetrachlorobenzene } \\
\text { Benzoic Acid }\end{array}$ & $\begin{array}{l}\text { ND } \\
\text { ND } \\
\text { ND } \\
\text { ND } \\
8.1 \\
\text { ND } \\
\text { ND } \\
\text { ND } \\
2.8 \\
\text { ND } \\
\text { ND } \\
\text { ND } \\
1.3 \\
\text { ND } \\
\text { ND } \\
\text { ND } \\
\text { ND } \\
\text { ND } \\
\text { ND } \\
\text { ND } \\
\text { ND } \\
\text { ND } \\
\text { ND } \\
\text { ND } \\
\text { ND } \\
\text { ND } \\
\text { ND } \\
\text { ND } \\
\text { ND } \\
\text { ND } \\
\text { ND } \\
\text { ND }\end{array}$ & $\begin{array}{l}\text { ND } \\
\text { ND } \\
\text { ND } \\
\text { ND } \\
4.6 \\
\text { ND } \\
\text { ND } \\
\text { ND } \\
2.4 \\
\text { ND } \\
\text { ND } \\
\text { ND } \\
1.8 \\
\text { ND } \\
\text { ND } \\
\text { ND } \\
\text { ND } \\
\text { ND } \\
\text { ND } \\
\text { ND } \\
\text { ND } \\
\text { ND } \\
\text { ND } \\
\text { ND } \\
\text { ND } \\
\text { ND } \\
\text { ND } \\
\text { ND } \\
\text { ND } \\
\text { ND } \\
\text { ND } \\
\text { ND }\end{array}$ \\
\hline TRPH & & $\mathrm{ND}(<100)$ & $\mathrm{ND}(<100)$ \\
\hline
\end{tabular}


Table 11 TREATED NAPALM CONTAINER ( $\mu \mathrm{g} / \mathrm{wipe})$

\begin{tabular}{|c|c|c|}
\hline Analytes & Run 1 & Run 2 \\
\hline \hline TRPH & $\mathrm{ND}(<100 \mu \mathrm{g} /$ wipe $)$ & $\mathrm{ND}(<100 \mu \mathrm{g} /$ wipe $)$ \\
\hline
\end{tabular}

\subsubsection{Napalm Destruction Chemistry in the Eco Logic Process}

Napalm is composed of gasoline and polystyrene. Gasoline is largely saturated aliphatic hydrocarbons but also contains some aromatics such as benzene, toluene and xylenes. The polystyrene is a polymer of styrene (vinylbenzene) units.

The heating of gasoline type molecules (e.g., hexanes, heptanes, octanes, etc.) to $\sim 500^{\circ} \mathrm{C}$ should cause primarily evaporation or even boiling, depending on the time/temperature profile in the SBV. Since the unit is considered "open" and there is a high flow rate of inert gas, these volatile organic compounds will be swept out of the SBV largely unchanged. Small portions may decompose by free radical reactions as noted later for the Reactor.

Polystyrene, on the other hand, must depolymerize to become volatile. The initial product of polystyrene decomposition will be styrene monomer. In the presence of hydrogen gas or in the presence of other hydrogen sources such as the gasoline, the depolymerization of polystyrene may also produce some species such as ethylbenzene by hydrogenation, or toluene or benzene by free radical cleavage of the benzene at the side chain and hydrogen abstraction from other molecules. At $-500^{\circ} \mathrm{C}$ in an overwhelming flow of a non-oxidizing atmosphere such as hydrogen, there should be minimal further decomposition of the styrene species initially produced. Consequently, the styrene and the gasoline hydrocarbons will be swept out of the SBV.

Only minimal thermal decomposition to carbon char or polymer tars should occur as hydrogen is "stolen" by other molecules of the gases. Recombination of the initially generated free radicals can result in longer and/or branched chain hydrocarbons as well as single and multi-ring compounds (such as benzene again, naphthalene, anthracene, etc.), but conversion to such products should be quite small. Again, high dilution with the carrier gas, hydrogen, probably favors ring formation. Any cooler surfaces between the SBV and the Reactor could encourage vapors to condense and may lead to recombination (condensation) reactions to produce tars.

In SBV:

Gasoline $\left(\mathrm{C}_{\mathrm{n}} \mathrm{H}_{2 \mathrm{n}+2}\right.$ hydrocarbons) $-\ldots \mathrm{C}_{\mathrm{n}} \mathrm{H}_{2 \mathrm{n}+1}$ (radical) $-\longrightarrow \mathrm{C}_{2} \mathrm{H}_{4}, \mathrm{C}_{3} \mathrm{H}_{6}$, etc. and ultimately methane

Polystyrene: $\mathrm{C}_{6} \mathrm{H}_{5}\left[-\mathrm{CH}=\mathrm{CH}_{2}-\right]_{\mathrm{n}} \longrightarrow \mathrm{C}_{6} \mathrm{H}_{5}-\mathrm{CH}=\mathrm{CH}_{2}, \mathrm{C}_{6} \mathrm{H}_{5}-\mathrm{CH}_{2}-\mathrm{CH}_{3}$, etc.

When any of these carbon-hydrogen compounds enter and strike the much higher temperature surfaces of the Reactor, $-900^{\circ} \mathrm{C}$, additional cleavage and decomposition will occur, probably by various free radical mechanisms. These mechanisms will lead primarily to shorter chain aliphatic species such as ethane, propane, butanes and pentanes, and ultimately to methane. These lower hydrocarbons are the primary products expected; however, significant portions of the longer chain gasoline hydrocarbons and the styrenebased aromatics may survive the short contact time in the reactor. In addition, more char may form by hydrogen abstraction. Tars may also form, but they would be apt to be converted to a mixture of char (carbon) and volatile short chain hydrocarbons, and ultimately to more methane. 
The chemical equations shown below are given as samples or representations of what are much more complex chain reactions with various initiation, branching, recombination and termination options.

For Styrene (once depolymerization and volatilization have occurred)

Polystrene: $\mathrm{C}_{6} \mathrm{H}_{5}\left[-\mathrm{CH}=\mathrm{CH}_{2}-\right]_{n}--->>$ Styrene monomer: $\mathrm{C}_{6} \mathrm{H}_{5} \mathrm{CH}=\mathrm{CH}_{2}$

$\mathrm{C}_{6} \mathrm{H}_{5}-\mathrm{CH}=\mathrm{CH}_{2}--->>\mathrm{C}_{6} \mathrm{H}_{5}-\mathrm{CH}-\mathrm{H}_{2} \mathrm{C} \cdot$ or $\mathrm{C}_{6} \mathrm{H}_{5}-\mathrm{HC} \cdot-\mathrm{CH}_{3}$ (free radicals) $--->$

$$
\mathrm{C}_{6} \mathrm{H}_{6}+\mathrm{CH}_{2}=\mathrm{CH}_{2}, \mathrm{C}_{6} \mathrm{H}_{6}-\mathrm{CH}_{3}+\mathrm{CH}_{4} \text {, etc. }
$$

For free radical thermal decomposition of gasoline using octane as representative of gasoline:

Octane: $\mathrm{C}_{8} \mathrm{H}_{18}$ OR $\mathrm{H}_{3} \mathrm{C}-\left[\mathrm{CH}_{2}\right]_{6}-\mathrm{CH}_{3}$

$\mathrm{H}_{3} \mathrm{C}-\left[\mathrm{CH}_{2}\right]_{6}-\mathrm{CH}_{3} \longrightarrow-\cdots \mathrm{H}_{3} \mathrm{C}-\left[\mathrm{CH}_{2}\right]_{\mathrm{x}}-\mathrm{CH}_{3}$ where "x" is a number $<6$

One or more free radical intermediates such as the following may exist. They may abstract another hydrogen fróm another compound, may lose a hydrogen, may couple to pair electrons, etc. These reactions give rise to the mixture of products.

Hydrogen radical loss:

$$
\mathrm{H}_{3} \mathrm{C}-\left[\mathrm{CH}_{2}\right]_{x}-\mathrm{CH}_{3} \longrightarrow \mathrm{H}_{3} \mathrm{C}-\left[\mathrm{CH}_{2}\right]_{x}-\mathrm{CH}_{2} \quad+\mathrm{H} \cdot
$$

Second hydrogen loss to yield olefin:

$\mathrm{H}_{3} \mathrm{C}-\left[\mathrm{CH}_{2}\right]_{x}-\mathrm{CH}_{2} \cdot->>\mathrm{H}_{3} \mathrm{C}-\left[\mathrm{CH}_{2}\right]_{x=}=\mathrm{CH}_{2}+\mathrm{H} \cdot$

Loss of radical organic group, chain shortening:

$\mathrm{H}_{3} \mathrm{C}-\left[\mathrm{CH}_{2}\right]_{\mathrm{x}}-\mathrm{CH}_{2} \cdot \longrightarrow \mathrm{H}_{3} \mathrm{C}-\left[\mathrm{CH}_{2}\right]_{x-1}=\mathrm{CH}_{2}+\mathrm{CH}_{3} \cdot$

Continued abstraction of hydrogen can lead to the formation of hydrogen-poor carbon, i.e., char and fused ring structures.

Coupling of two organic radicals:

$2 \mathrm{H}_{3} \mathrm{C}-\left[\mathrm{CH}_{2}\right]_{x}-\mathrm{CH}_{2}-\longrightarrow \mathrm{H}_{3} \mathrm{C}-\left[\mathrm{CH}_{2}\right]_{x}-\mathrm{CH}_{2}-\mathrm{H}_{3} \mathrm{C}-\left[\mathrm{CH}_{2}\right]_{x}-\mathrm{CH}_{3} \longrightarrow \mathrm{H}_{3} \mathrm{C}-\left[\mathrm{CH}_{2}\right]_{x}-\mathrm{CH}_{2}$

If the coupling is "encouraged" by the correct temperature/time and concentrations, the result can be tar formation.

Eventually, given sufficient time at elevated temperature, enough $\mathrm{C}-\mathrm{H}$ bonds, and perhaps an outside source of hydrogen, the result is chain breakage down to the simplest molecules such as ethane, ethylene, and methane.

$$
\mathrm{H}_{3} \mathrm{C}-\left[\mathrm{CH}_{2}\right]_{6}-\mathrm{CH}_{3}->->->->\mathrm{H}_{3} \mathrm{C}-\mathrm{CH}_{3}, \mathrm{H}_{2} \mathrm{C}=\mathrm{CH}_{2} \text {, and } \mathrm{CH}_{4}
$$

While the mechanisms may be slightly different for polystyrene, including both ring cleavage and ring fusion of the benzene to naphthalene, etc., essentially the same series of reactions can and do occur. 
If there is any source of oxygen, such as moisture in the napalm or from the addition of steam, then the whole series of "steam reforming" reactions may take place, primarily at the elevated temperature in the Reactor. These reactions will convert methane to carbon monoxide and then, if enough moisture/steam is present, to carbon dioxide. The more complex aliphatic, saturated ring, and aromatic ring hydrocarbons, will also tend to decompose by this route, but because of their lower volatility, may tend to accumulate.

$$
\begin{aligned}
& \mathrm{H}_{3} \mathrm{C}-\left[\mathrm{CH}_{2}\right]_{6}-\mathrm{CH}_{3} \\
& \text { or } \\
& \mathrm{C}_{6} \mathrm{H}_{5} \mathrm{CH}=\mathrm{CH}_{2}
\end{aligned} \quad+8 \mathrm{H}_{2} \mathrm{O} \cdots>8 \mathrm{CO}+\sim 16 \mathrm{H}_{2}
$$

with the final carbonaceous product, methane, the reaction is simply:

$\mathrm{CH}_{4}+\mathrm{H}_{2} \mathrm{O}-\mathrm{CO}+\mathrm{H}_{2}$

and then, in both cases if sufficient water vapor is present:

$8 \mathrm{CO}+8 \mathrm{H}_{2} \mathrm{O} \longrightarrow 8 \mathrm{CO}_{2}+8 \mathrm{H}_{2}$

\subsection{2}

\section{Comparison of Test Results to Target Performance Goals}

The overall objectives of the study were achieved during the treatability study and the following conclusions can be drawn from the qualitative and quantitative results:

- Napalm was completely destroyed

- $\quad$ based on visual observations

- $\quad$ based on data results that report non-detectable results for TRPH on wipe samples

- $\quad$ based on TRPH reported as non-detect in scrubber samples

- Napalm can be processed directly from the aluminum containers and the containers remain intact.

- $\quad$ Test Run 3, at a lower SBV temperature, indicates that this can be accomplished with aluminum containers remaining intact

- Demonstrate that the process can be operated safely and effectively for napalm treatment

- $\quad$ No upset conditions were reported during napalm treatment verifying the ability of the Eco Logic Process to treat napalm safely and effectively. 


\section{APPENDIX A}

PROCESS DATA LOG SHEETS 


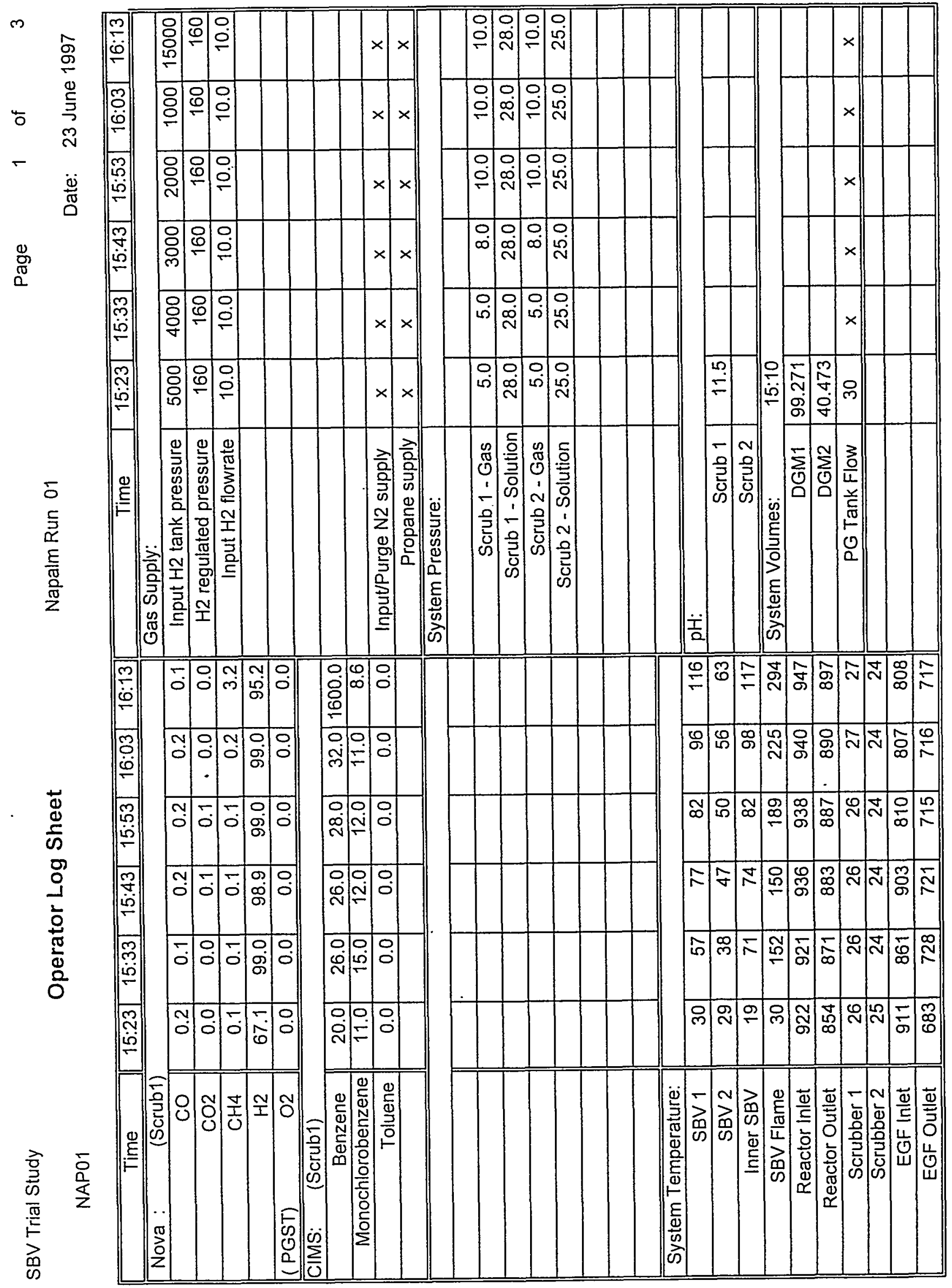




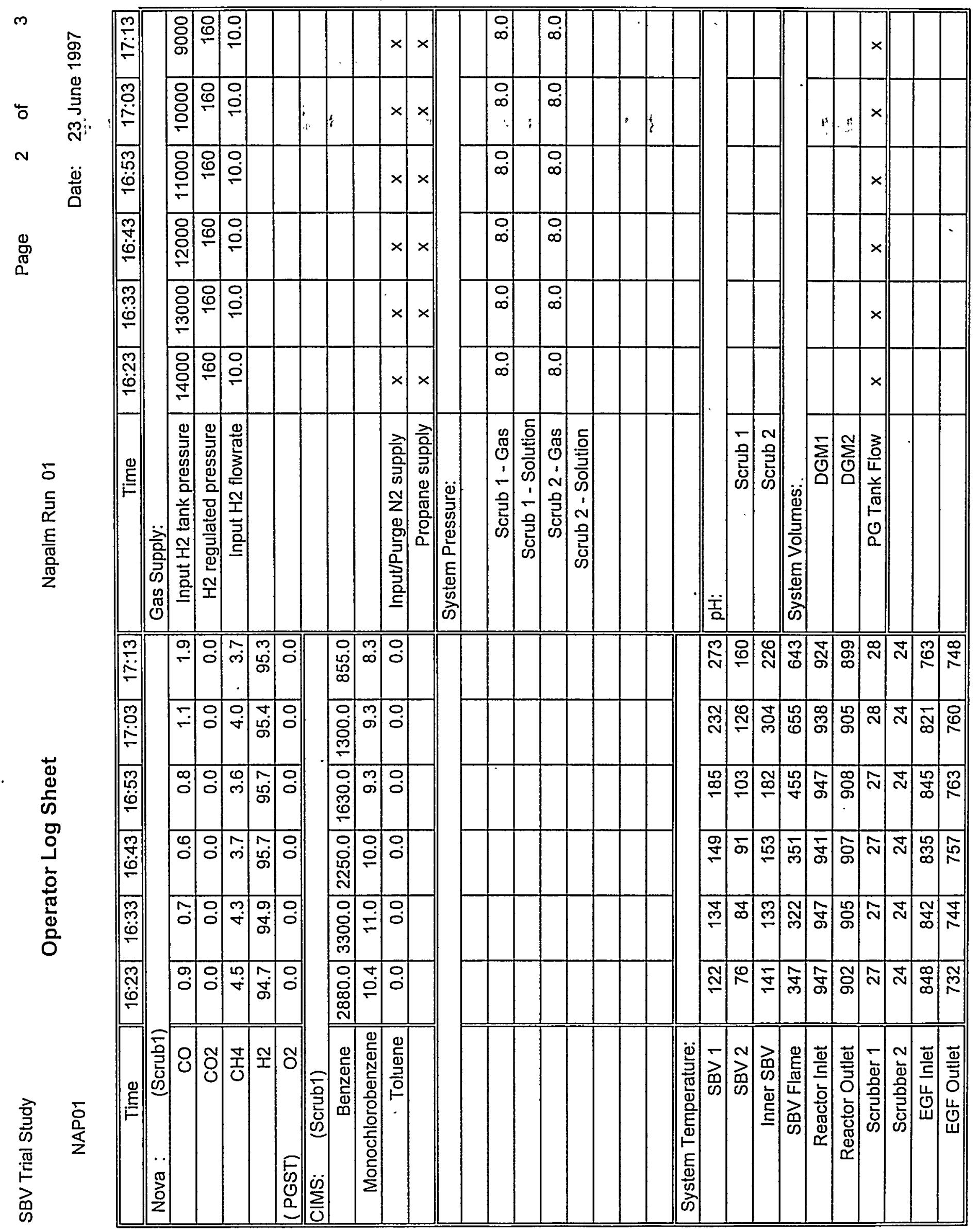




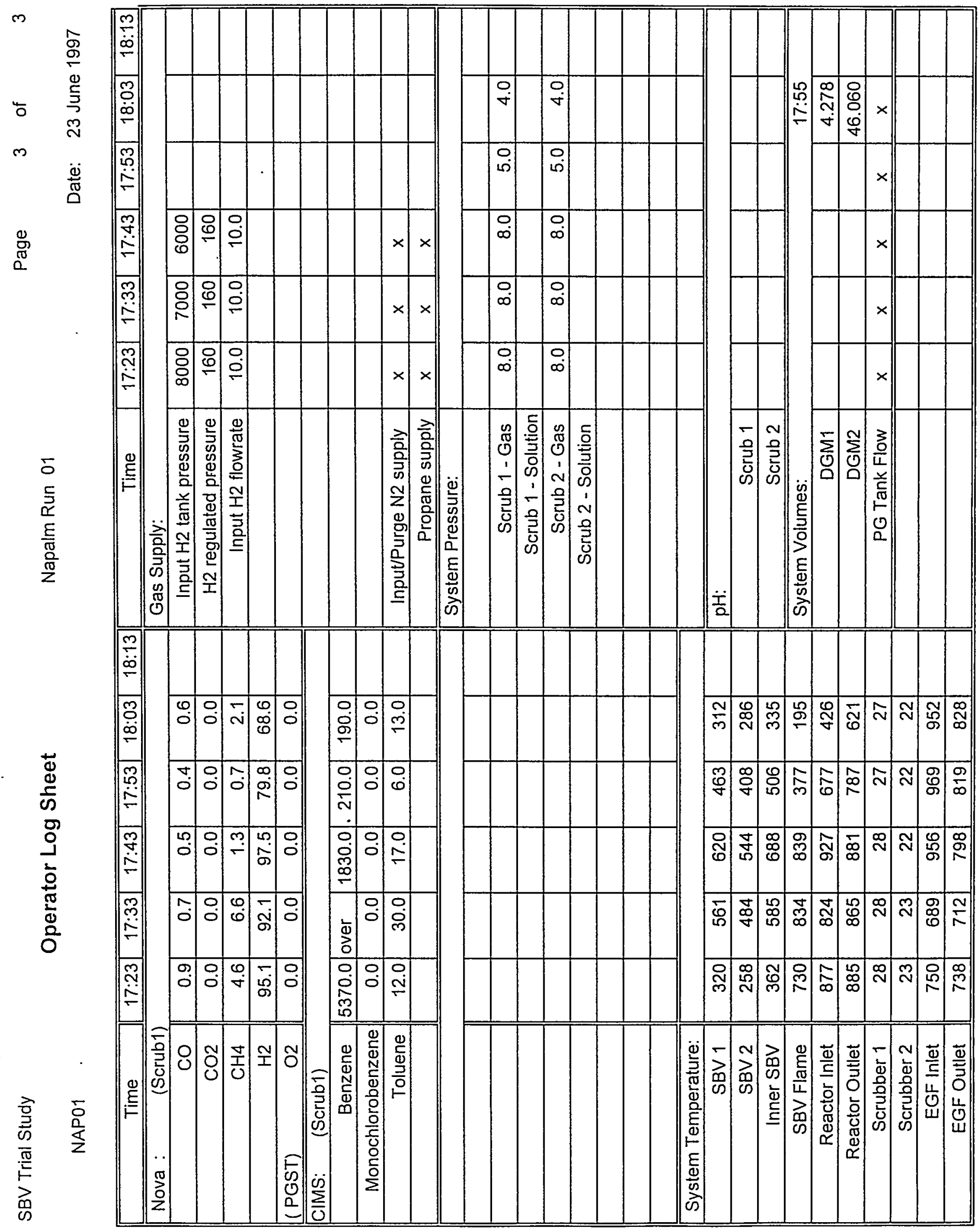




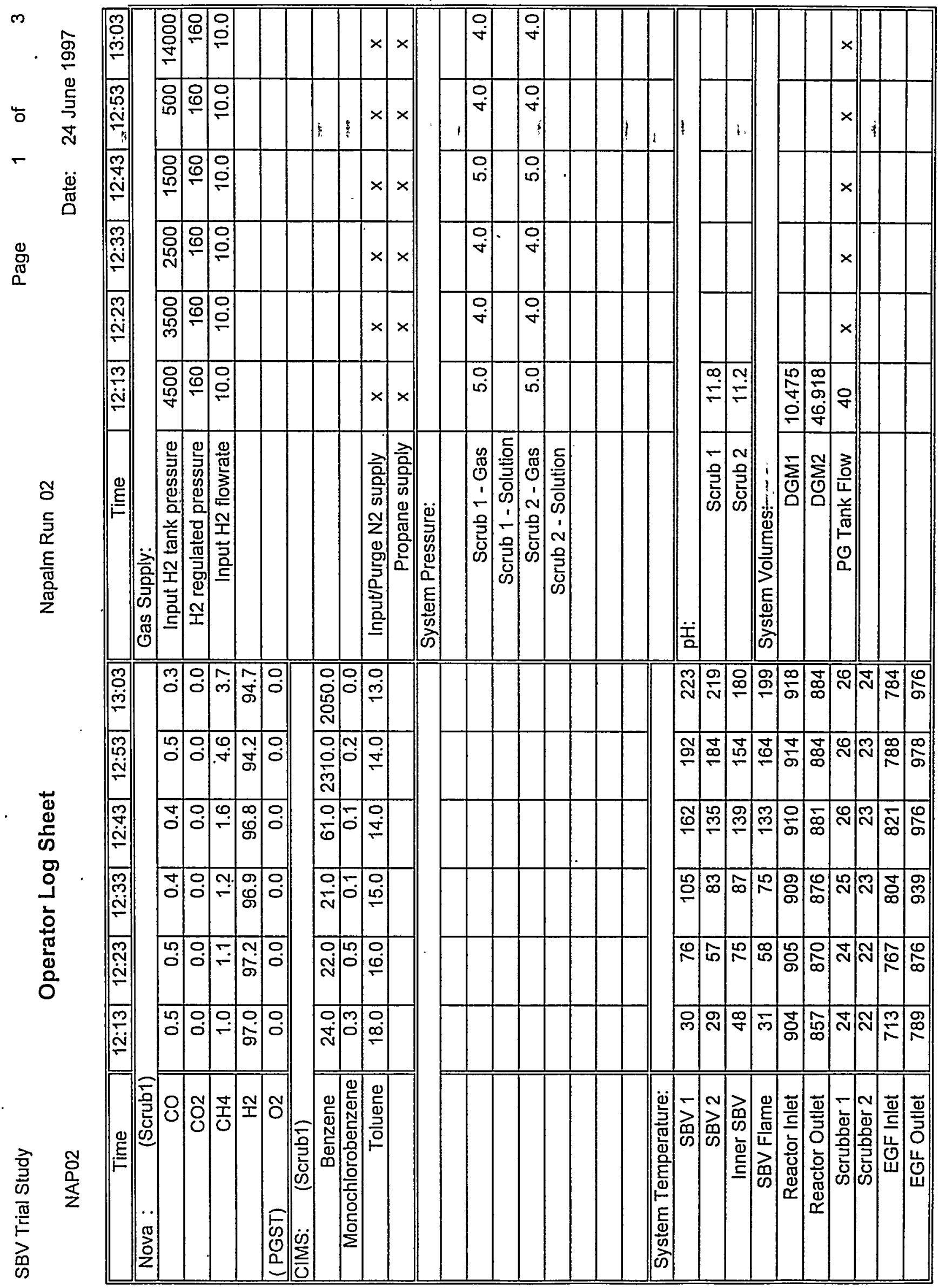




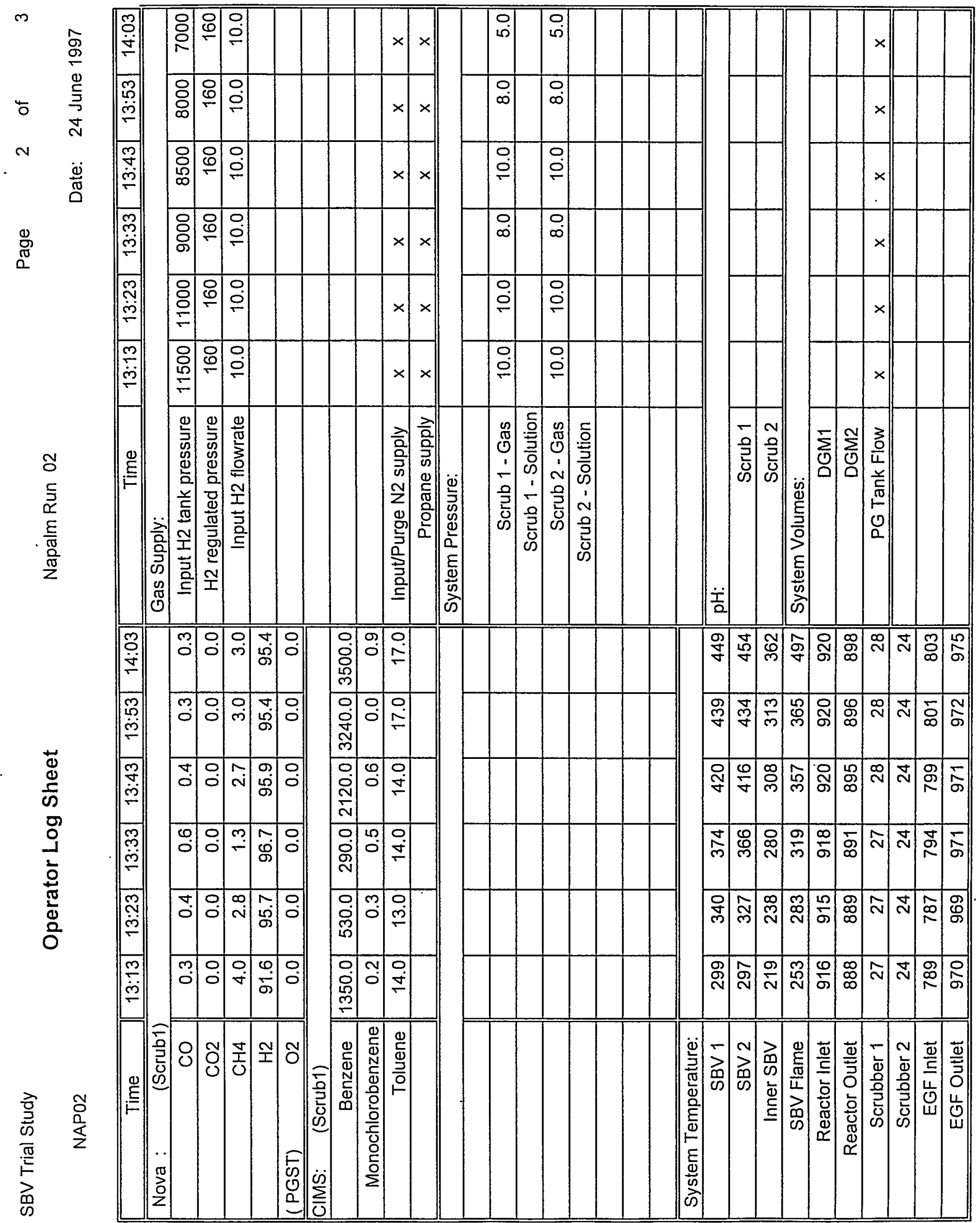




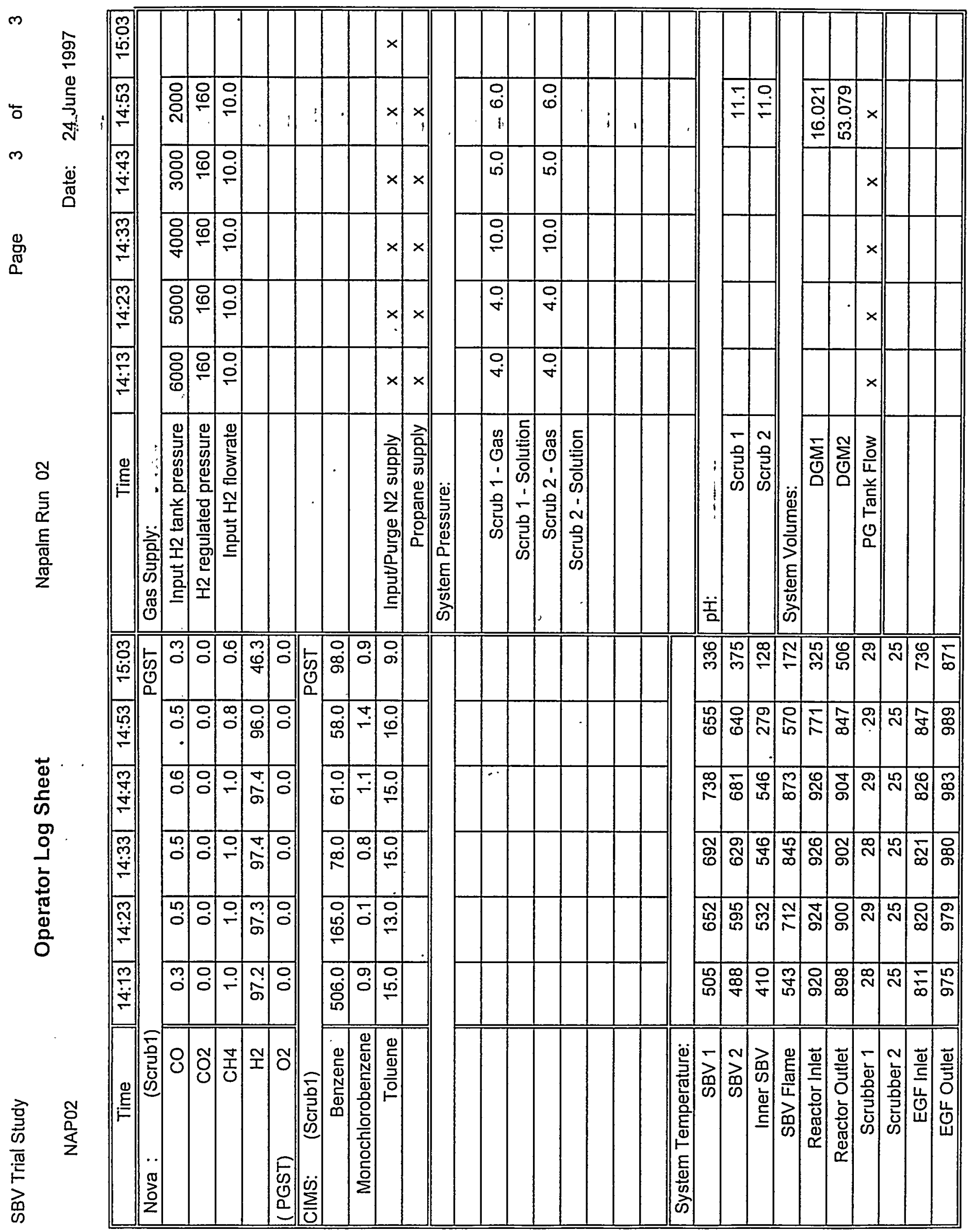




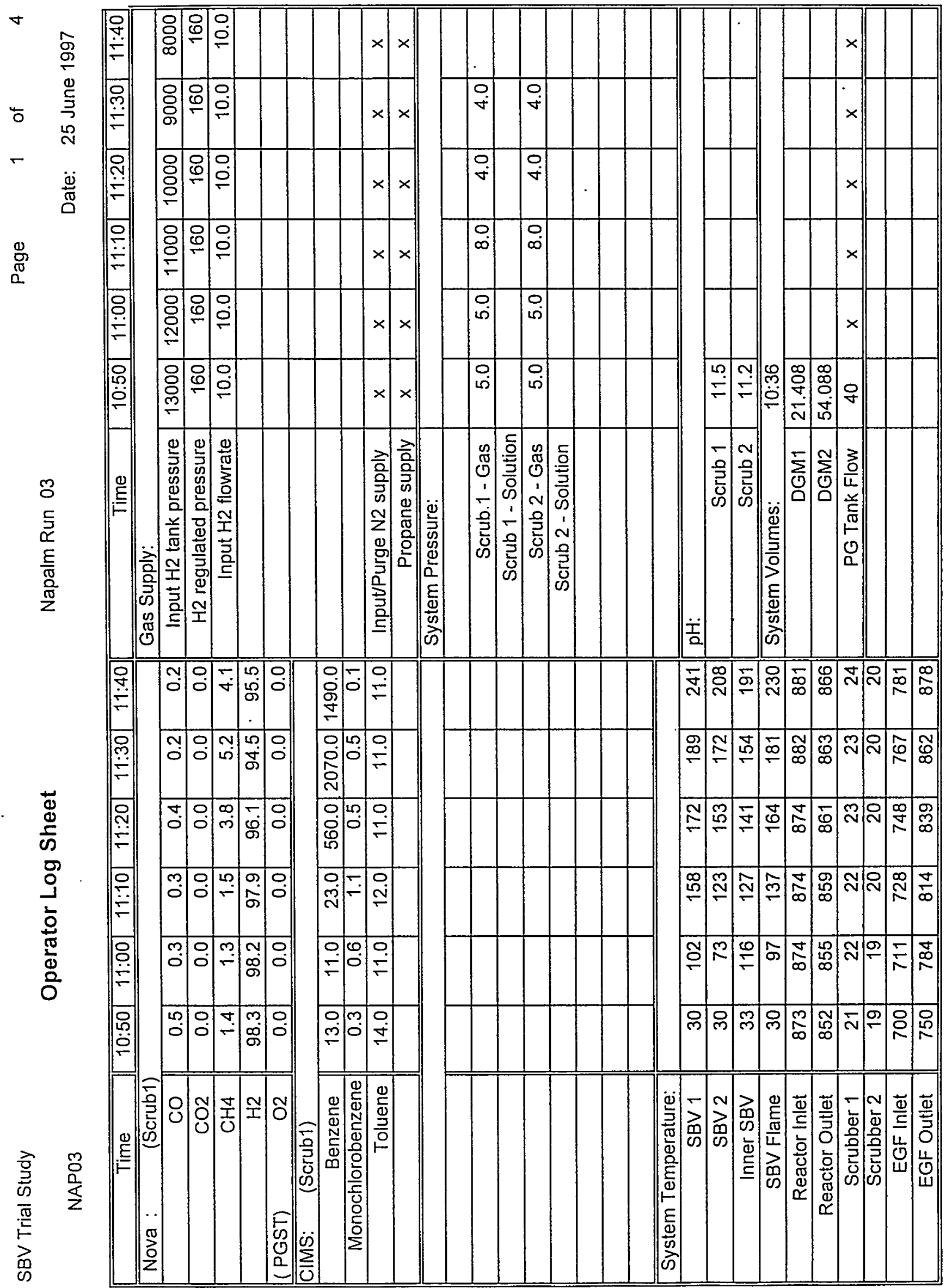




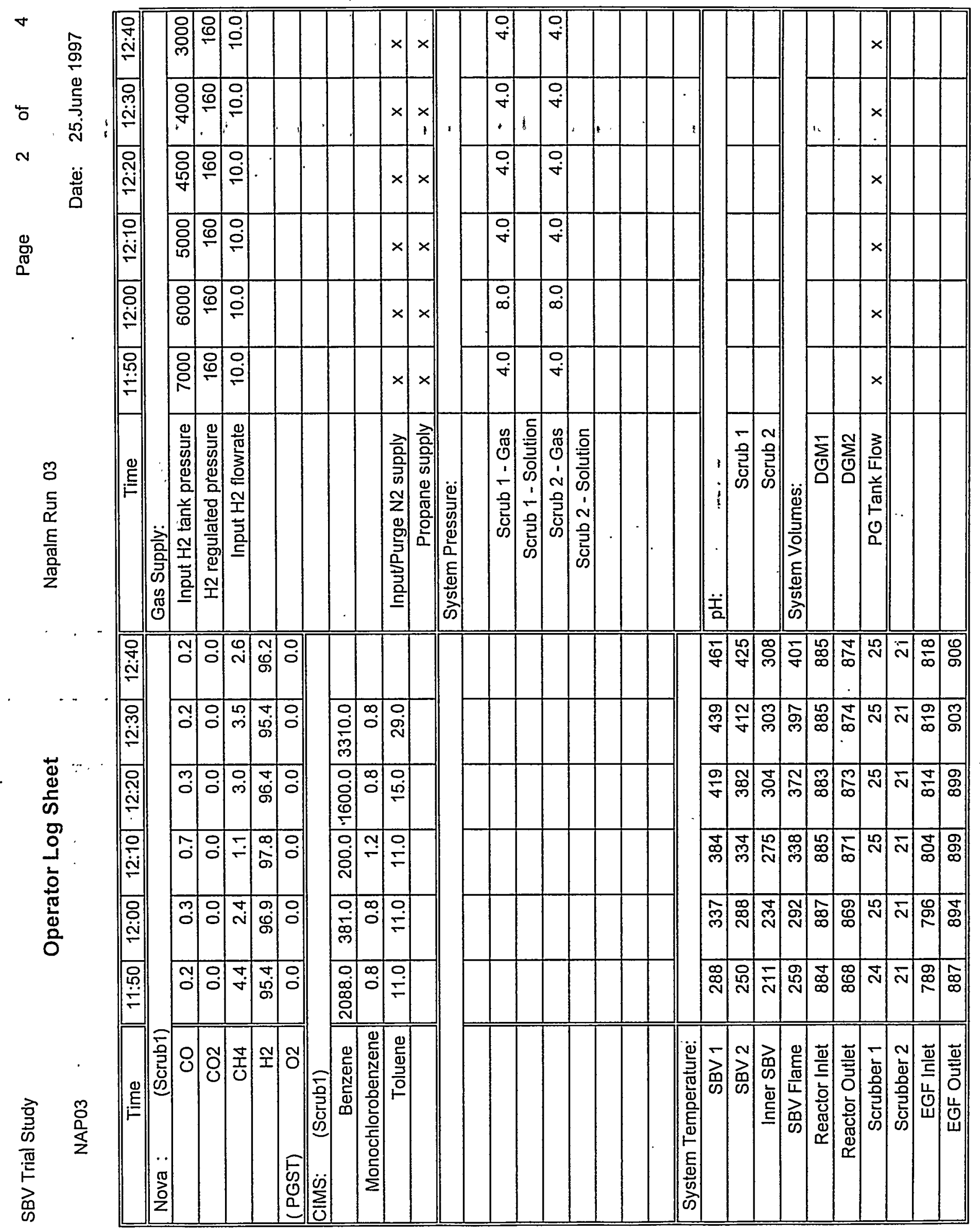




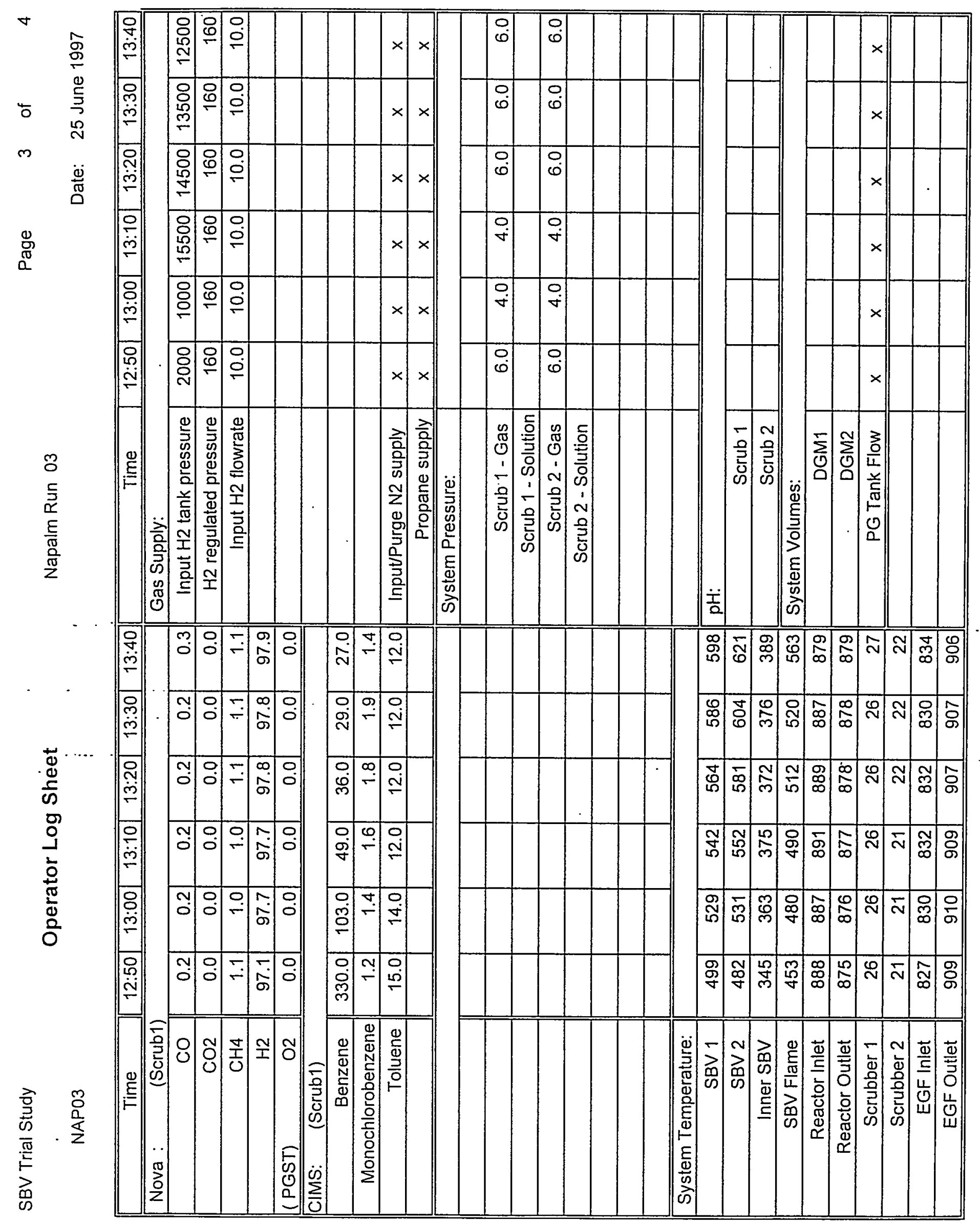




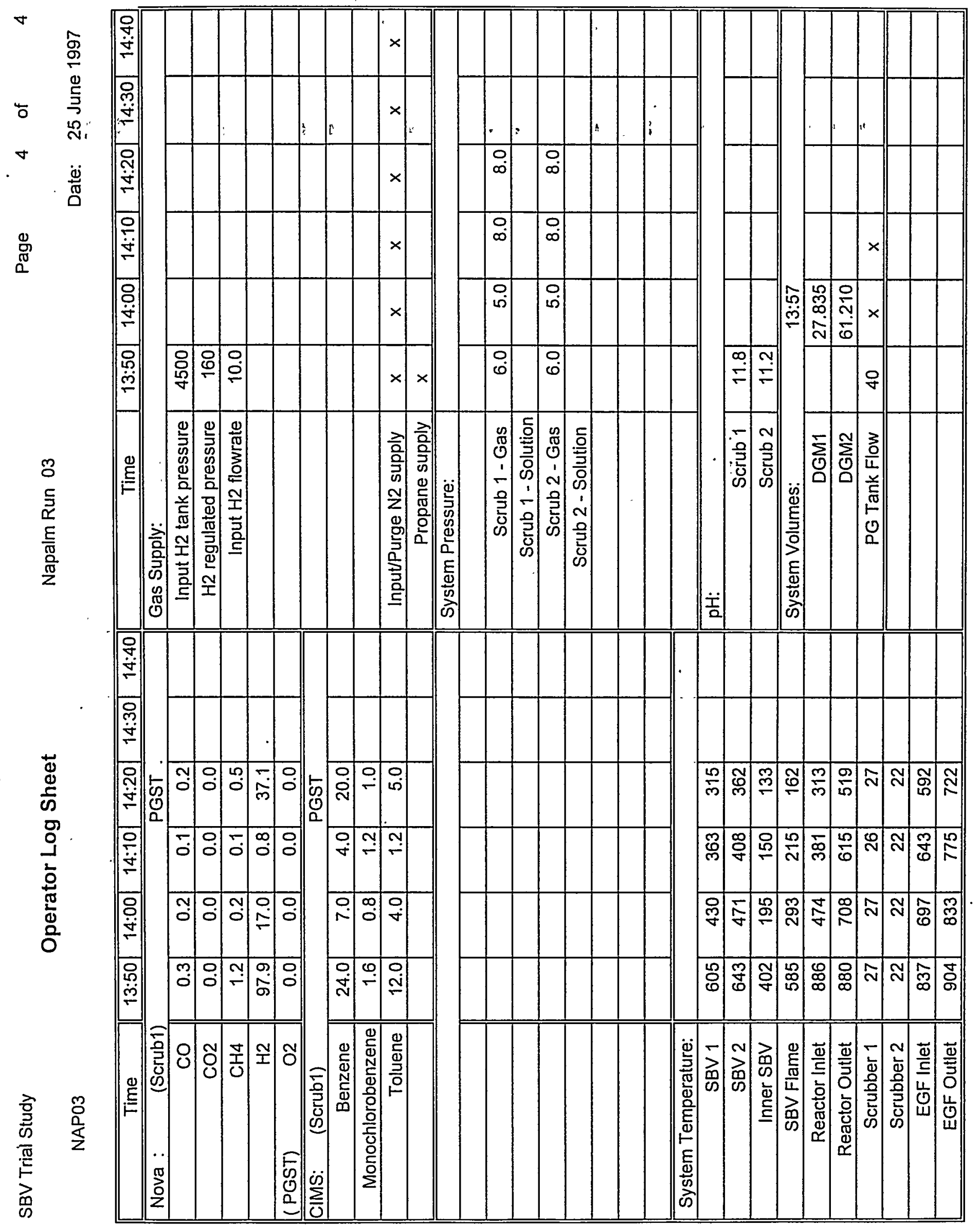




\section{APPENDIX B}

CHAIN OF CUSTODY FORMS 


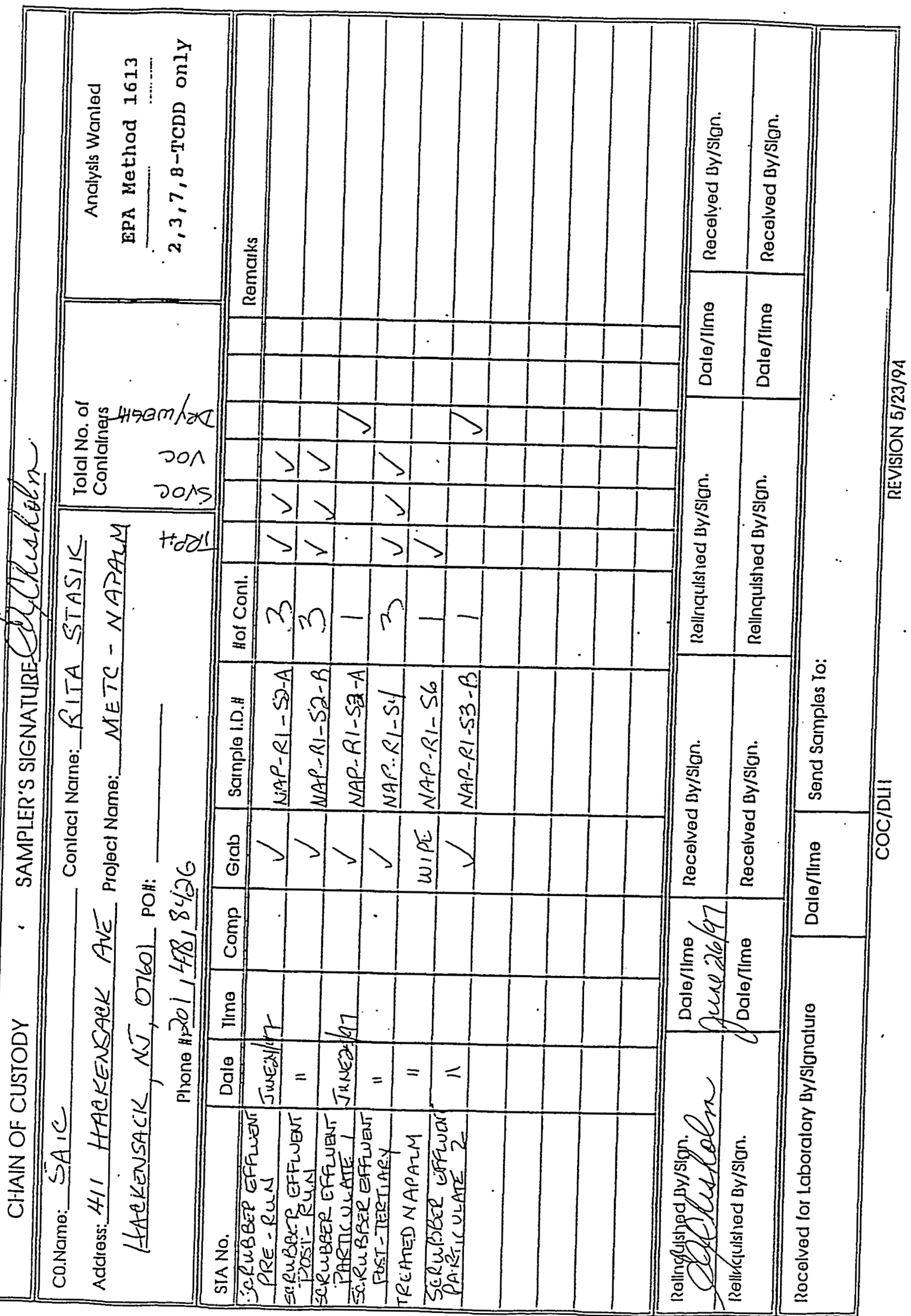




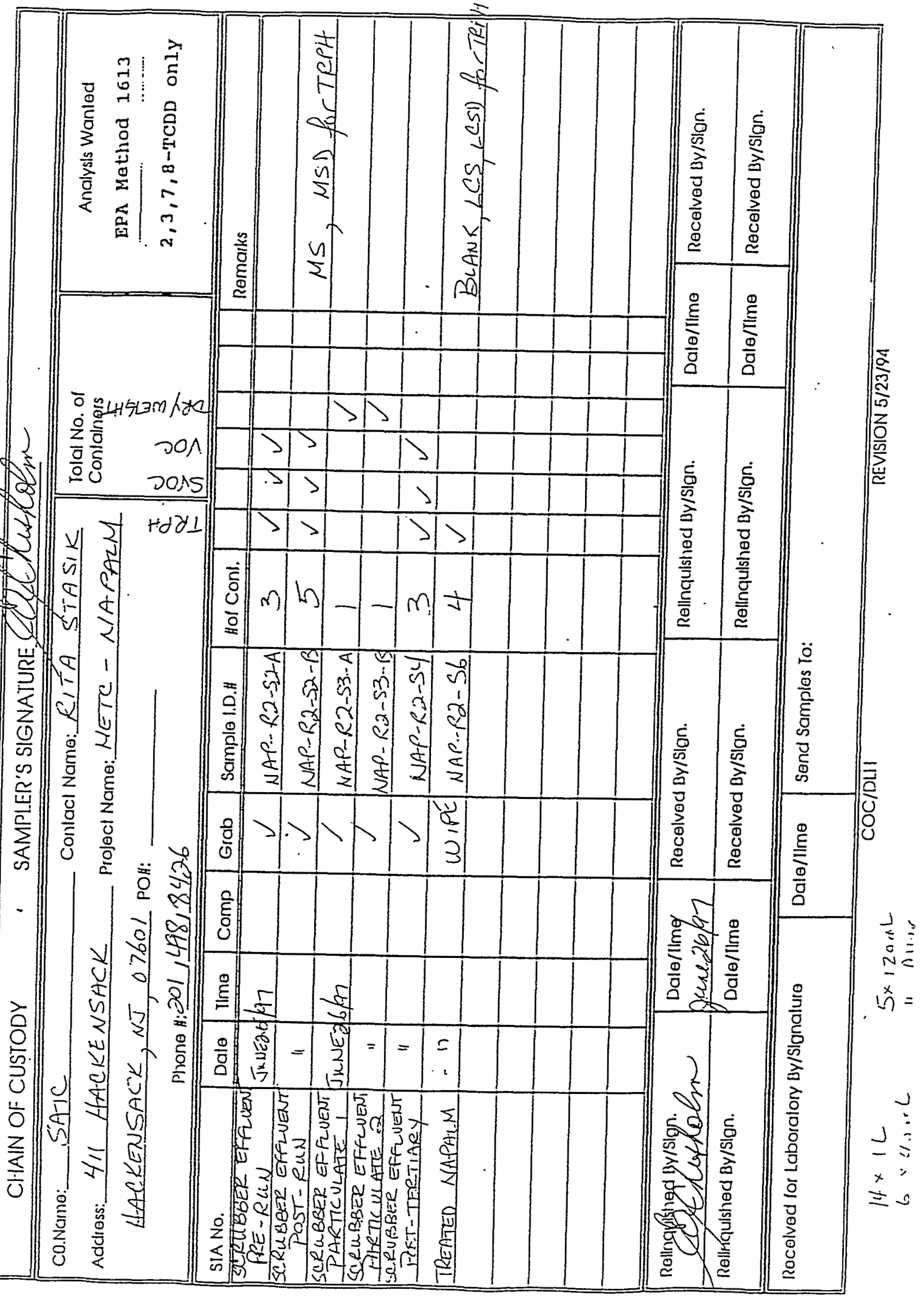




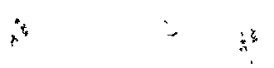

APPENDIX C

GRAPHICAL REPRESENTATION OF ON-LINE DATA 


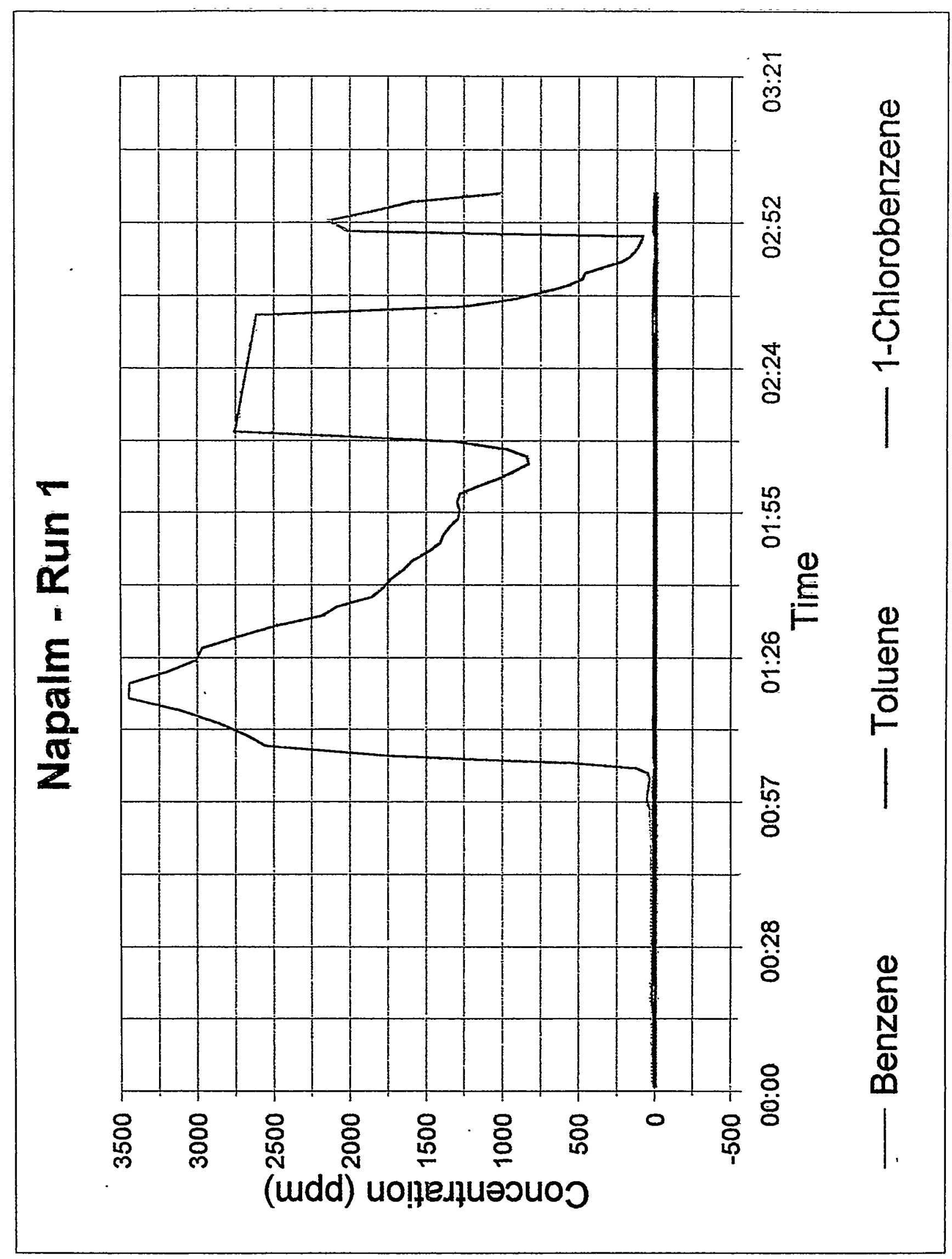




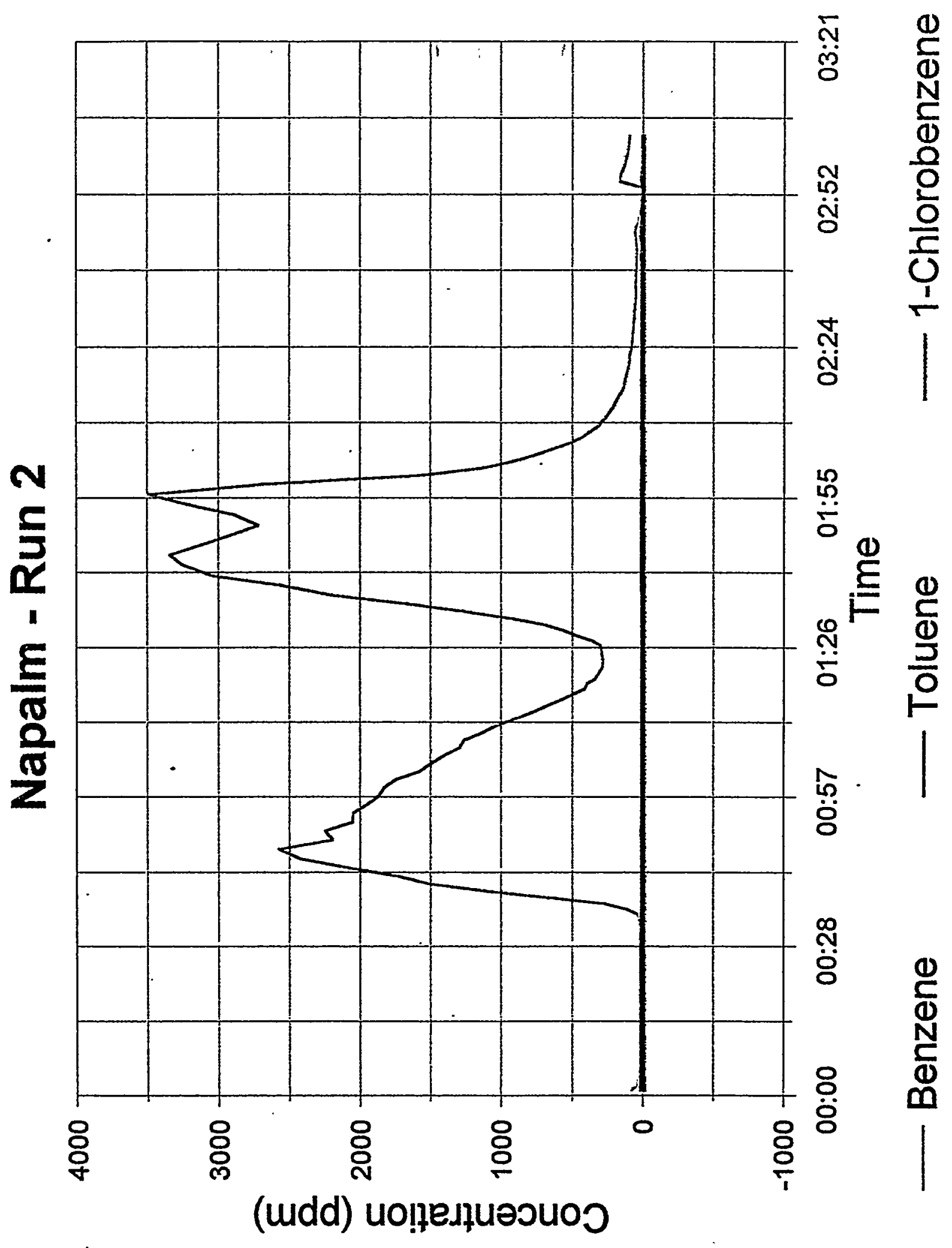




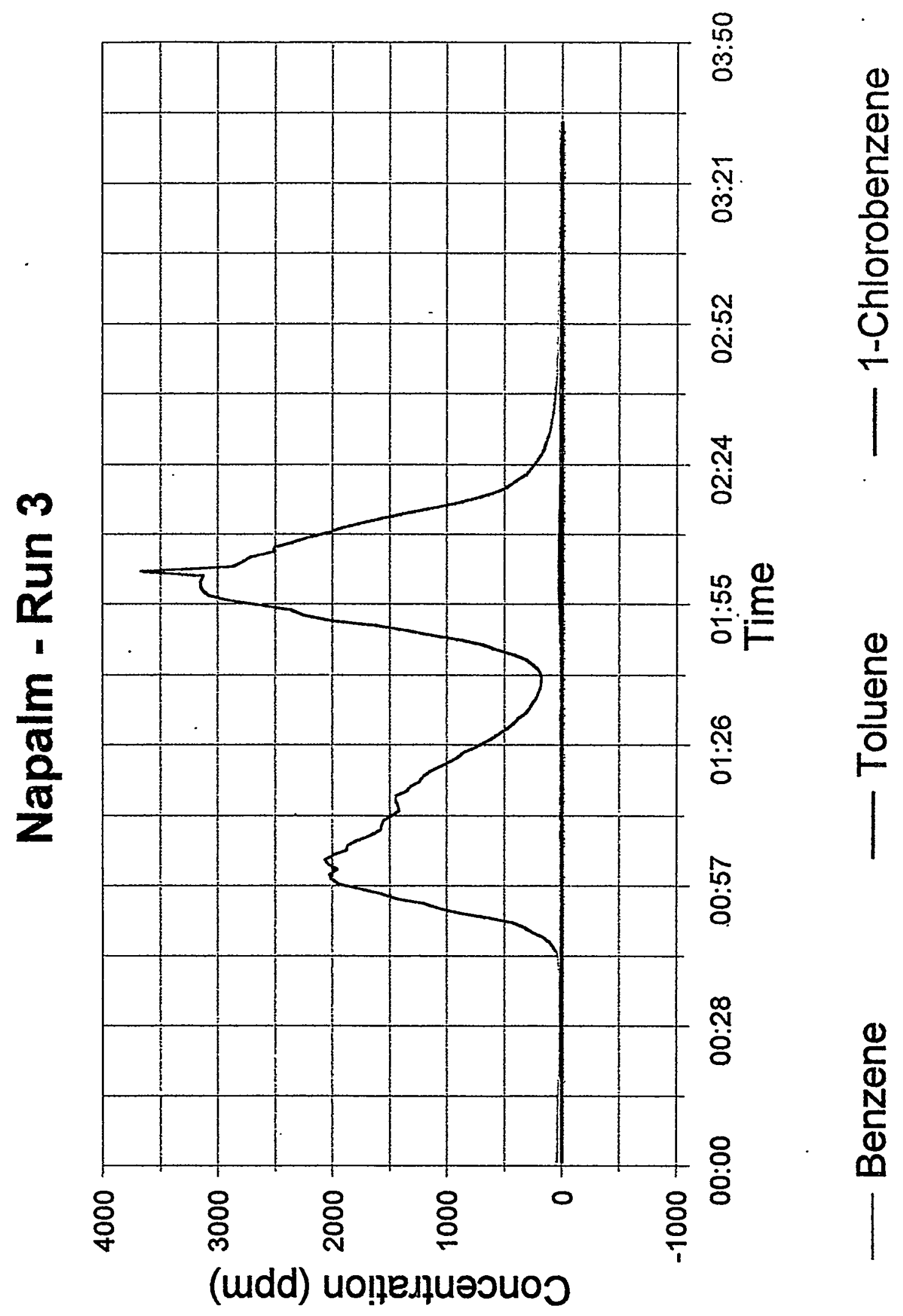




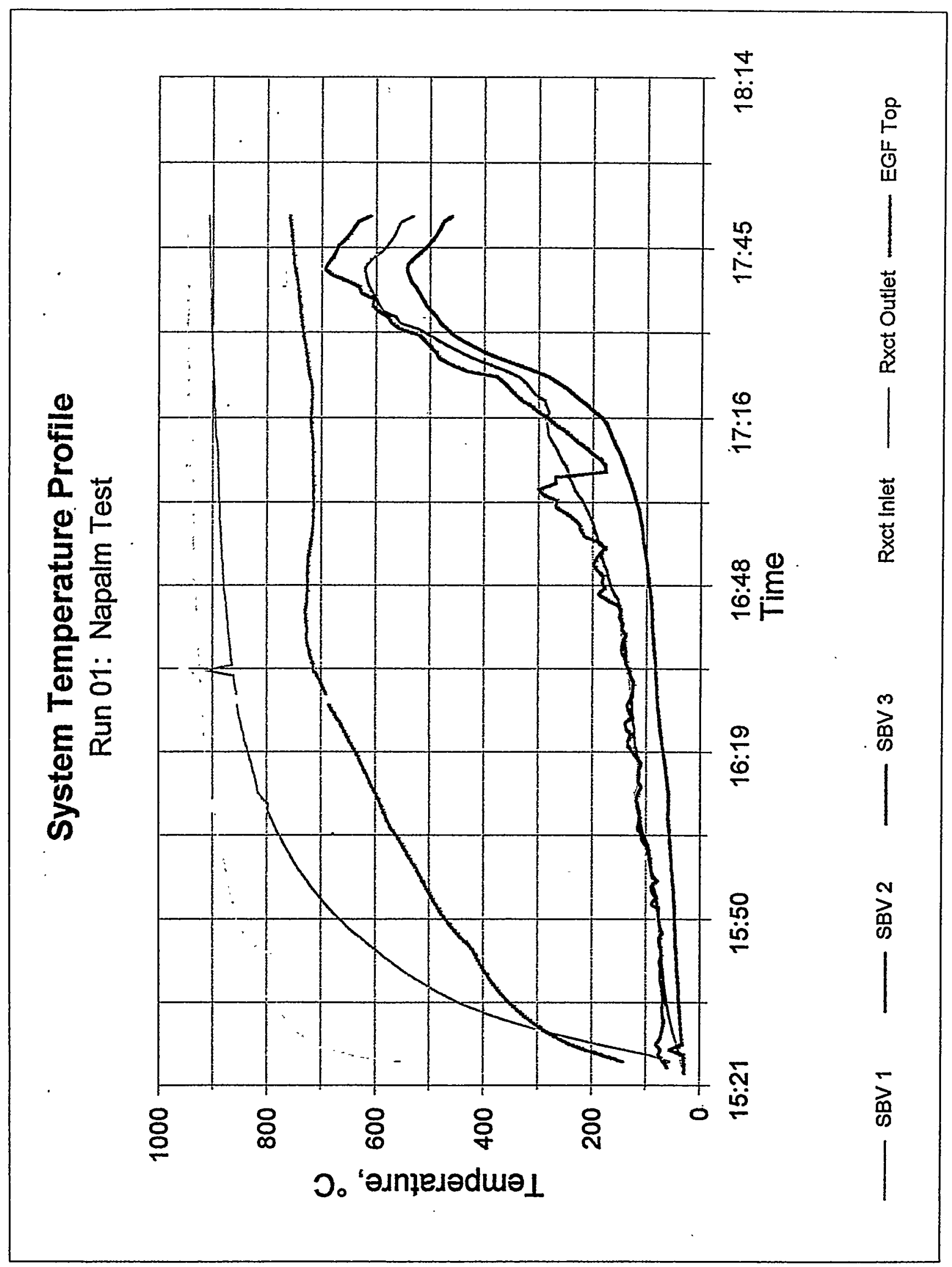




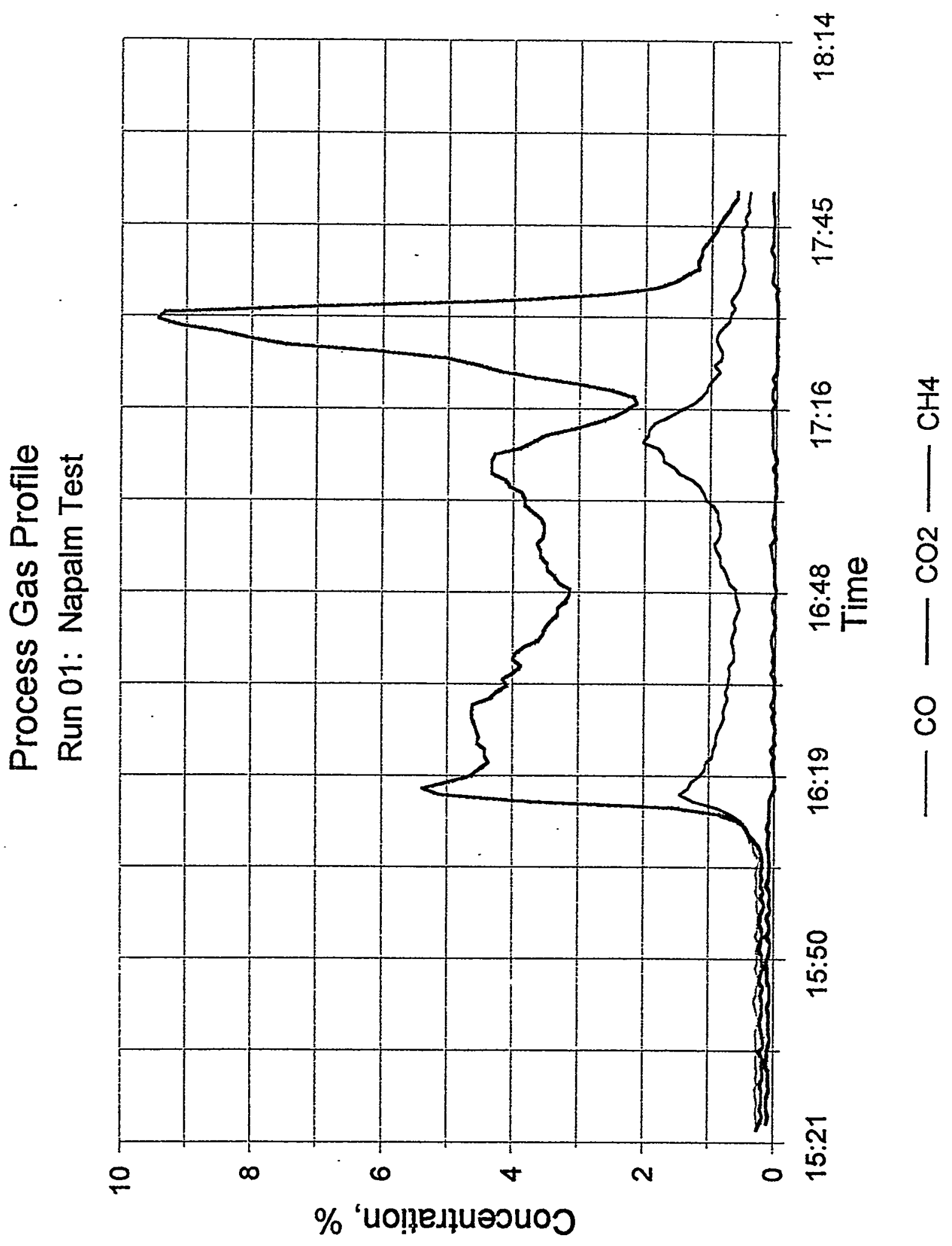




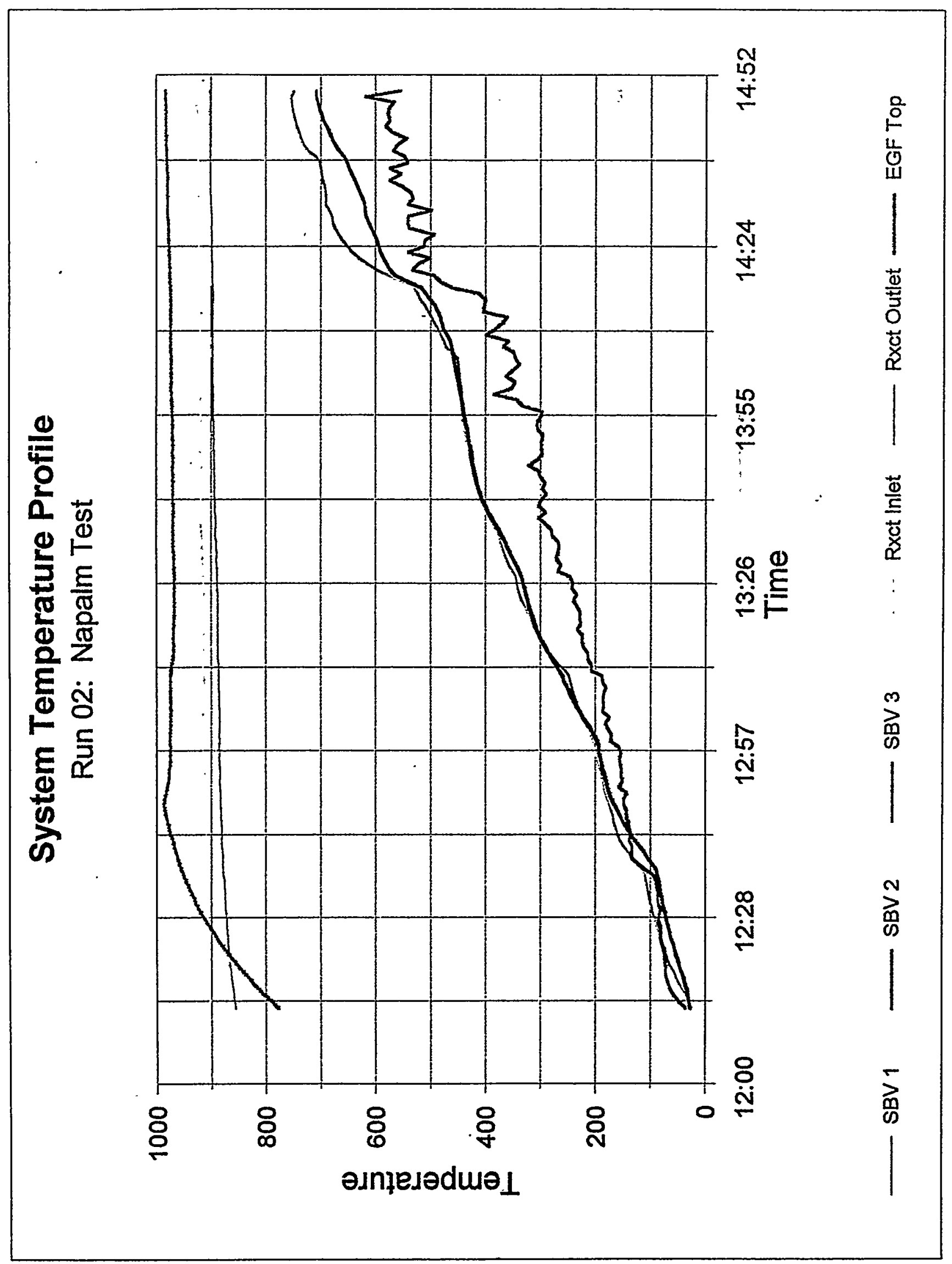




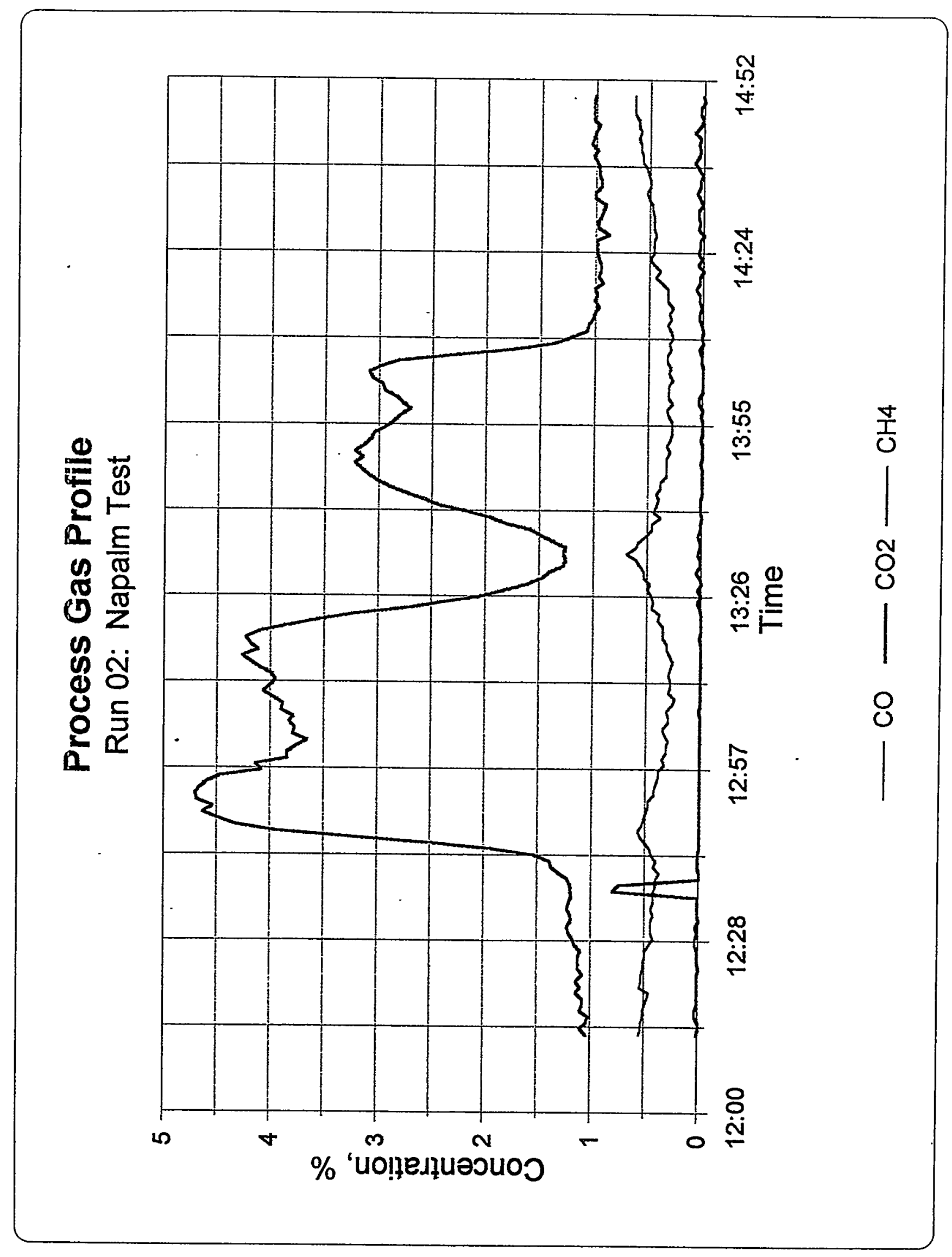




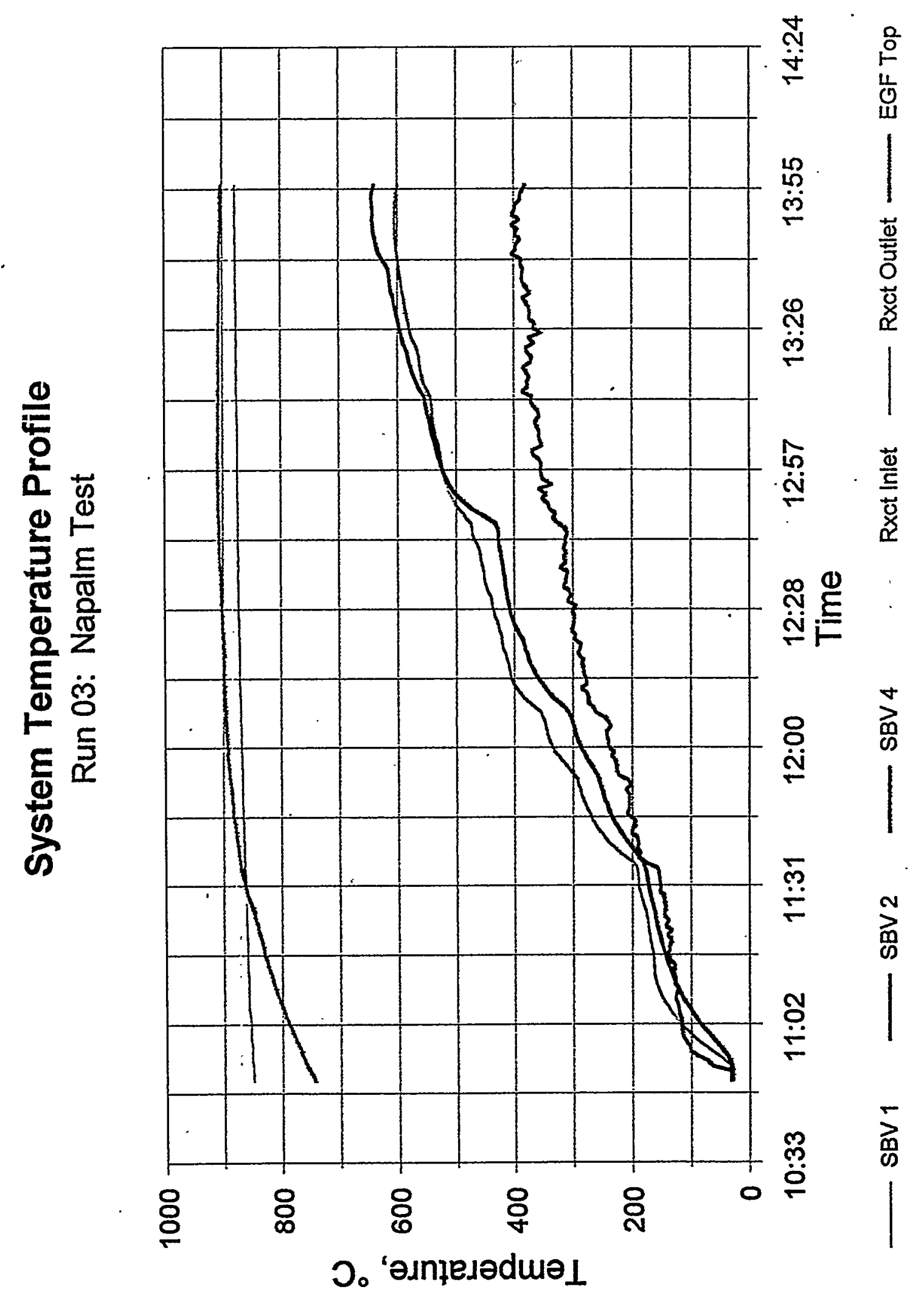




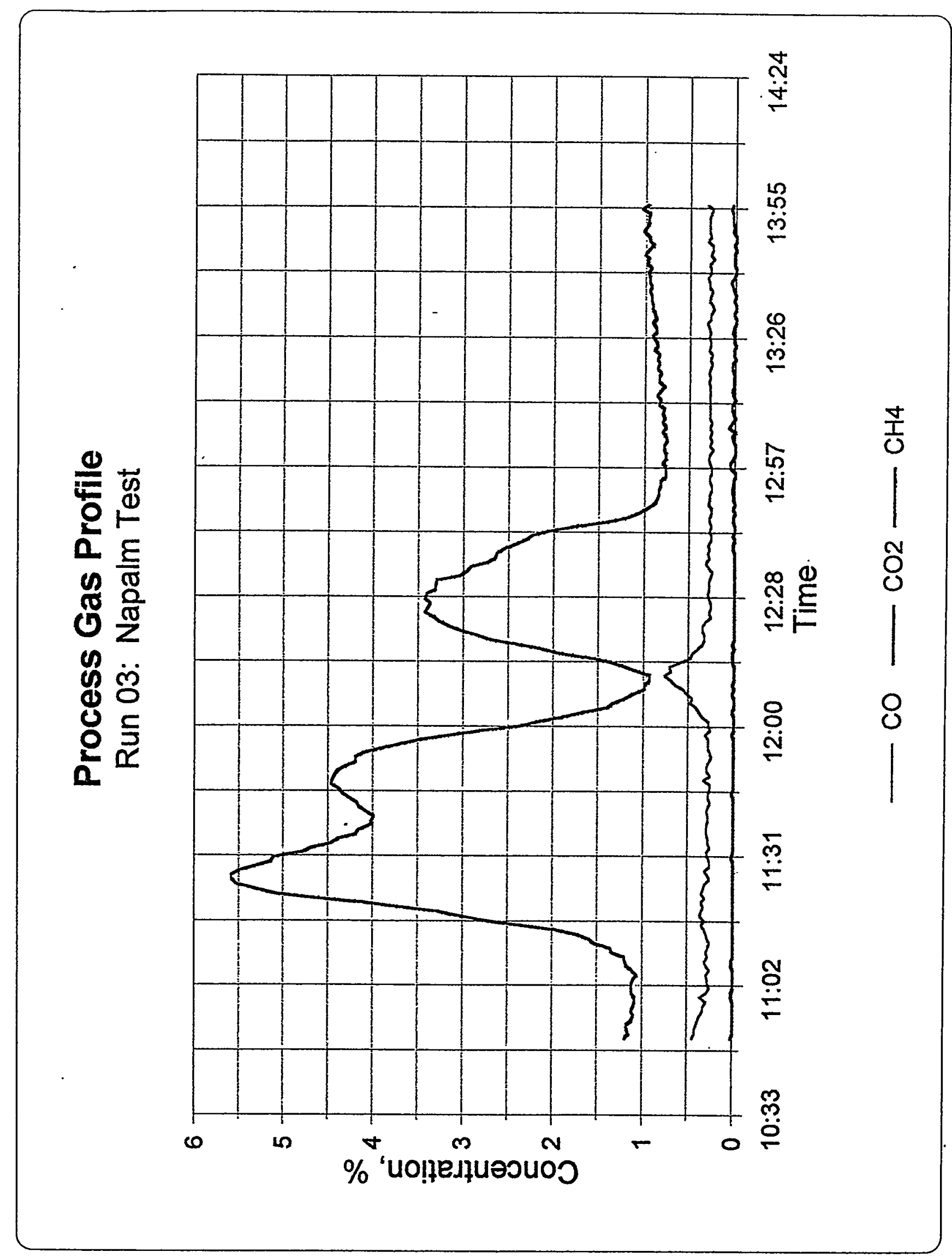




$$
\begin{aligned}
& -3091 \\
& 1-7 \text { ioner lift. } \\
& 546
\end{aligned}
$$

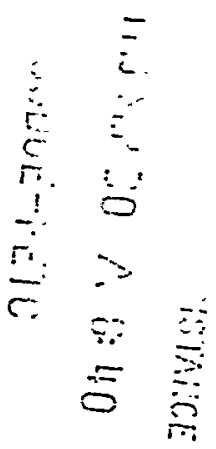

\section{APPENDIX D}

\section{ANALYTICAL DATA SETS FOR TREATABILITY}

TEST RUNS 


\section{DATA 産 CHEM \\ A SOREKSON COUPAKY}

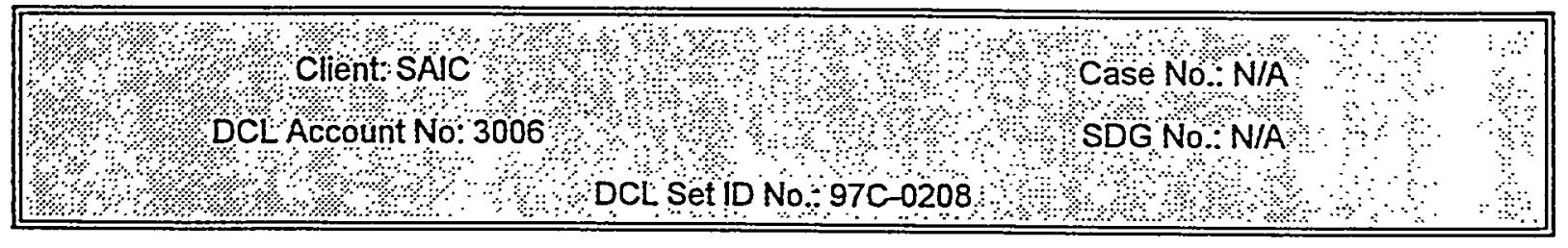

Page Nos. (Please Check:)

From To Lab Client

1. Inventory Sheet (Do not number)

2. Client Chain-of-Custody Records

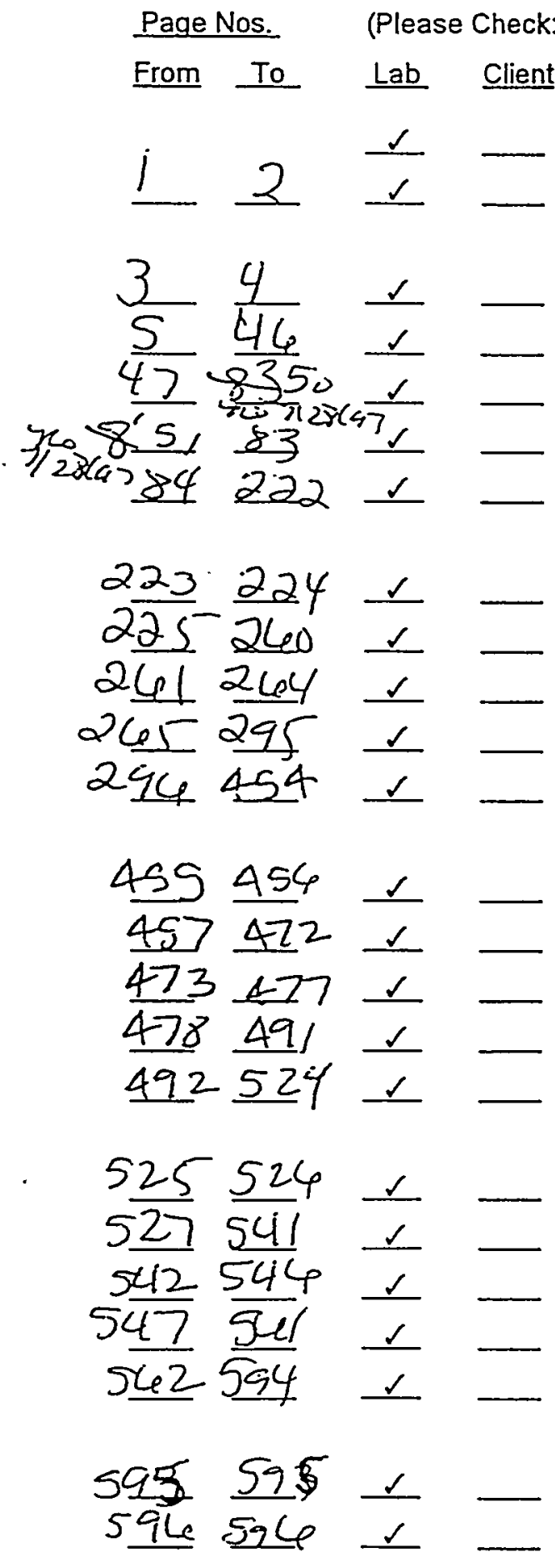

3. Volatiles Anaylsis

Case Narrative

Analytical Forms

Sample Tracking Documentation

Analytical Documentation

Raw Data

4. Semivolatles Analysis

Case Narrative

Analytical Forms

Sample Tracking Documentation

Analytical Documentation

Raw Data

5. Total Petroleum Hydrocarbons Analysis-Water

Case Narrative

Analytical Forms

Sample, Tracking Documentation

Analytical Documentation

Raw Data

6. Total Petroleum Hydrocarbons Analysis-Wipes

Case Narrative

Analytical Forms

Sample Tracking Documentation

Analytical Documentation

Raw Data

7. Shipping/Receiving Documents

Airbill (No. Of Shipments 1 )

DCL Cooler Receipt Cinecklist

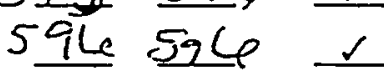


Page Nos. (Please Check:)

From To Lab Client

8. Comments:

Completed by (DataChem Laboratories):

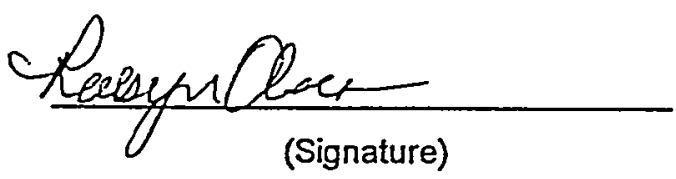

$\frac{\text { Robyn Olsen / Document Control }}{\text { (Print Name \& Title) }} \frac{07 / 28 / 97}{\text { (Date) }}$

Audited by (Client):

(Signature)

(Print Name \& Title)

(Date) 


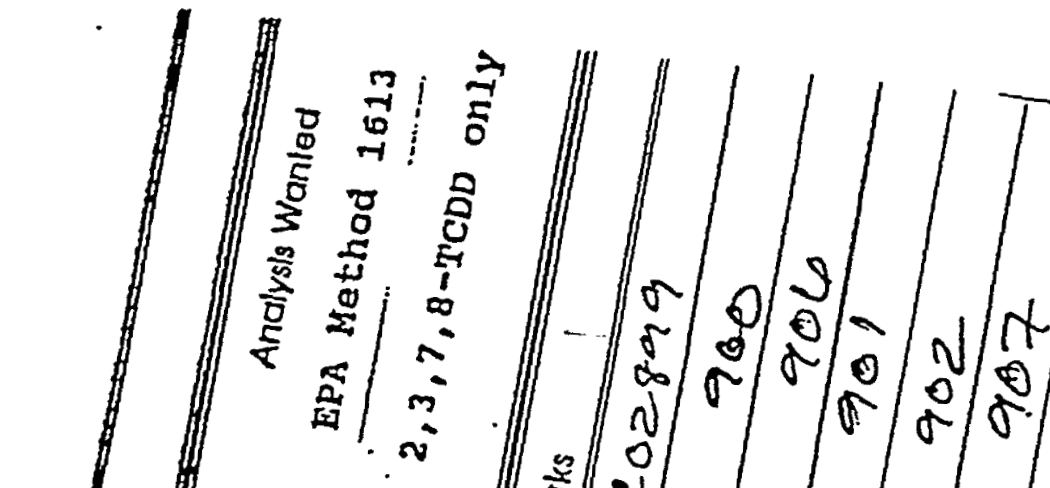

(3) 部就

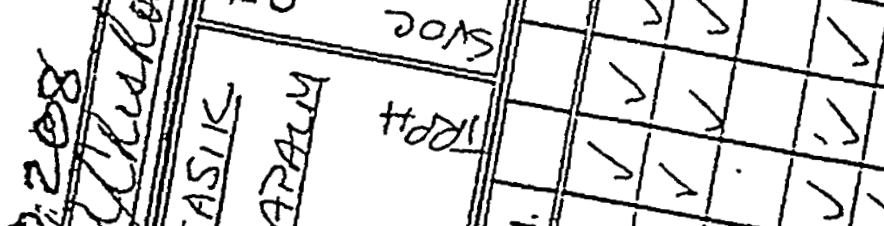

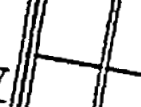

11
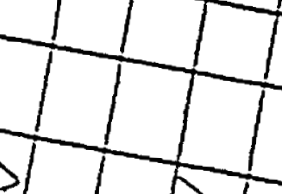
(i) if

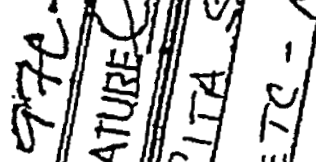

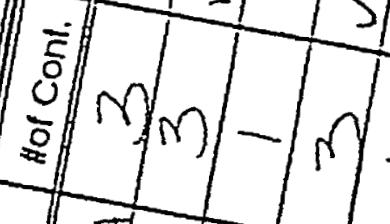

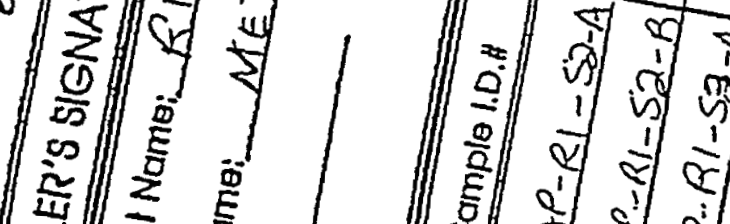

2.

敦高喜

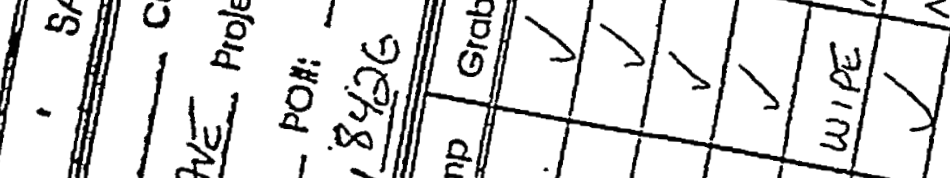
*े

辛

(ㄴ)

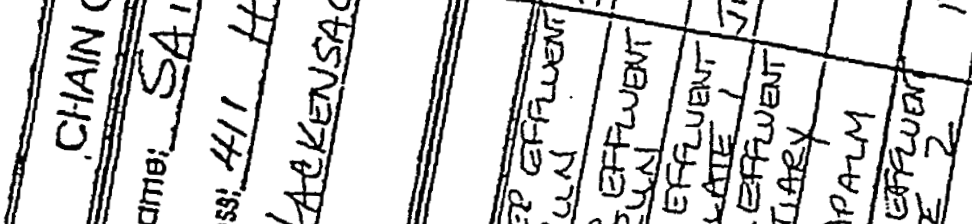

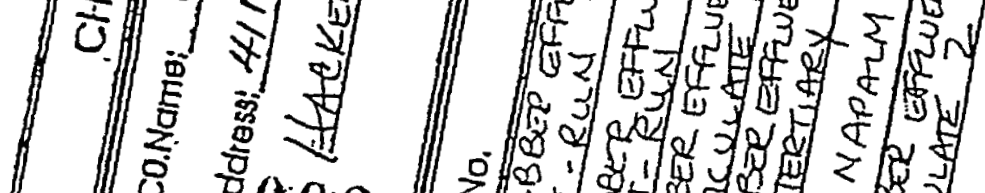

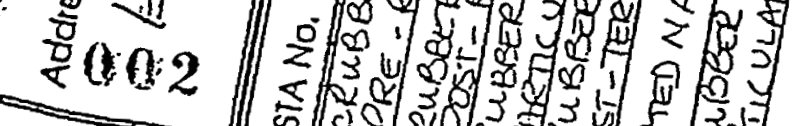

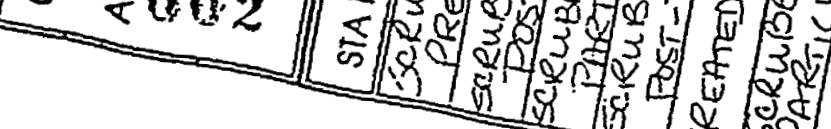




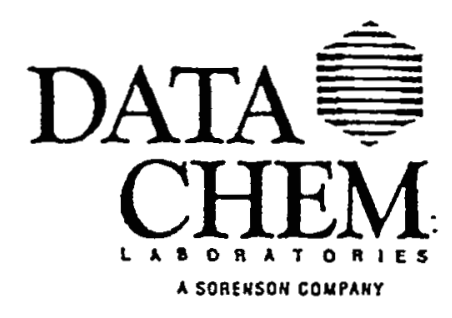

Case Narrative

Method: 8260A

Analysis: VOA

Preparation SOP \#: NA

Analysis SOPH: OV-SW-8260A Rev 0

Lot/SDG\#: NA

DCL Set ID \#(s): 97C-0208-01
Client: SAIC

Account \#: 3006

Matrix: Water

Analysis / Method: Method 8260A is an EPA SW846 method (DCL SOP OV-SW-8260A Revision 0 - herein referred to as the "method") used in the analysis of water samples for volatile organics by GC/MS purge and trap techniques.

General Set Information: DataChem Laboratories received six water samples for VOA analysis. The samples were analyzed within holding times. Recoveries of target analytes are reported on the Sample Analysis Data Sheet in units of ug/L.

Sample Preparation: This method has no extraction procedure for the water matrix. The sample preparation date is the same as the date of analysis. Five milliliters of water sample was spiked with $2.5 \mathrm{uL}$ of Internal Standard and $2.5 \mathrm{uL}$ of Surrogate Solution and purged.

Instrument Calibration: The GC/MS was hardware tuned to meet the criteria for a $50 \mathrm{ng}$ purging of 4-Bromofluorobenzene as specified in the method. This tune check is valid for 12 hours.

Initial and Continuiní Calibration Verification: The five point initial calibration curve which was analyzed prior to sample analysis met the specified criteria in the method. System performance check compounds (SPCC) are checked for a minimum response factor. These compounds are chloromethane $(0.100)$, bromoform $(>0.100), 1,1$-dichloroethane $(0.100)$, chlorobenzene (0.300), and 1,1,2,2-tetrachloroethane (0.300). Response factors for the calibration check compounds (toluene, 1,1-dichloroethene, chloroform, 1,2-dichloropropane, ethylbenzene, and vinyl chloride) from the initial calibration curve are used to calculate percent relative standard deviations (\%RSD). For the initial calibration standards, the \%RSD for the calibration check compounds (CCC) must be less than $30 \%$.

$063_{\mathrm{A}}$ continuing calibration standard (CCS) which is used in the validation of the initial calibration was also analyzed prior to sample analysis. The CCS met the method criteria as specified. The response factors of the SPCC's met the minimum criteria as specified in the method. The CCC's were less than $20 \%$ drift from the target based on the initial calibration curve. 
Method Blank Analysis: Method blanks (BL-136531-1, 2) were prepared using Barnstead reagent water spiked with 2.5 uL of Internal Standard and $2.5 \mathrm{uL}$ of Surrogate Solution and analyzed prior to sample analysis. The blanks were free of volatile organic contaminants within the specifications of the method.

MS / MSD Analysis: Matrix spike/matrix spike duplicate analyses were performed for sample 97C02901 (NAP-R1-S4). The MS compounds (1,1-dichloroethene, benzene, trichloroethene, toluene and chlorobenzene) were spiked at a concentration of $50 \mathrm{ug} / \mathrm{L}$. All recoveries met established QC acceptance criteria.

Laboratory Control Sample Analysis: A laboratory control sample (QC-136531-1) was analyzed for each analytical batch. The LCS compounds (same as the MS/MSD) were spiked at a concentration of $50 \mathrm{ug} / \mathrm{L}$. All recoveries met established QC acceptance criteria.

Data Qualifier Codes: A "J" qualifier indicates that the result is greater than the MDL but less than the CRDL or that the value is an estimate based on a relative response factor of one. Analytes found in field samples which also appear in the method blanks are reported with a " $B$ " qualifier in the flag column.

NC/CAR: None required.

Miscellaneous Comments: All surrogate recoveries were within established QC limits. Instrument designation is HP5971-L. Samples 97C02897 (NAP-R2-S2-B), 97C02899 (NAPR1-S2-A), and 97C02900 (NAP-R1-S2-B) were additionally analyzed as dilutions in order to bring benzene and or naphthalene within the linear range of the curve. The cooler temperatures were measured and found to be out of the acceptable range upon their receipt at DCL. Consult the CRIR contained in the work order for more detailed information.

\section{Sample Calculations:}

Relative Response Factor:

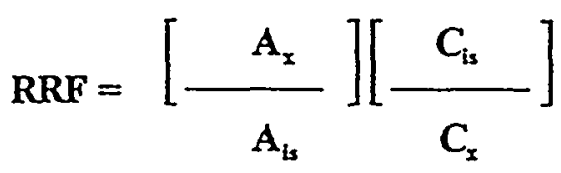

where $A_{\alpha}$ is the area of the characteristic ion for the compound to be measured, $A_{i s}$ is the area of the characteristic ion for the intemal standard, $C_{i s}$ is the concentration of the internal standard, and $\mathbf{C}_{\mathbf{x}}$ is the concentration of the compound to be measured. Analytes which do not meet the minimum 15\% RSD in the initial calibration are calculated based on a linear regression from the five point curve using the equation:

$$
y=a x+b
$$

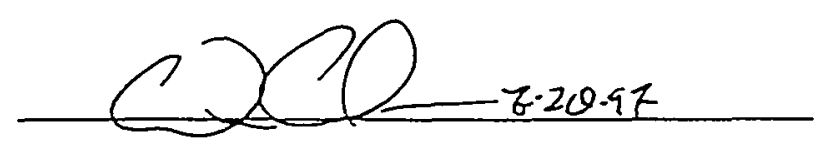

Christopher Q. Coleman 

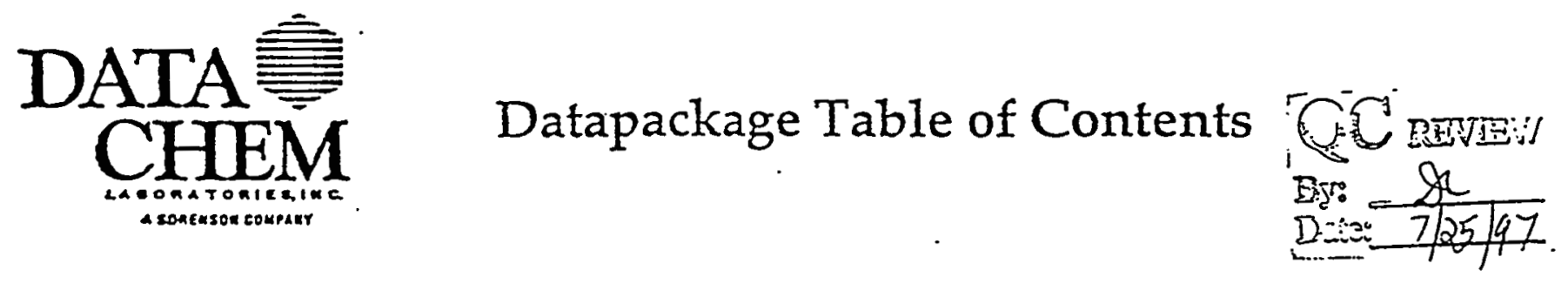

Information pertaining to this datapackage is divided into the four categories listed below. A Case Narrative immediately precedes this Table of Contents and contains pertinent information about this datapackage.

Analytical Results ............... Yellow Sample Tracking Documentation ......... Pink Analytical Documentation ............. Blue Raw Data ...................... Green

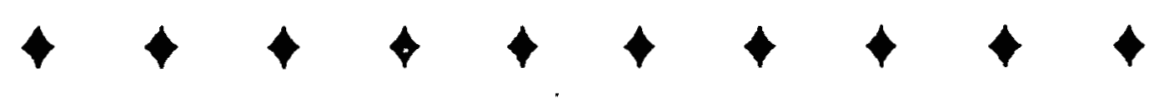

\section{Analytical Results}

005 


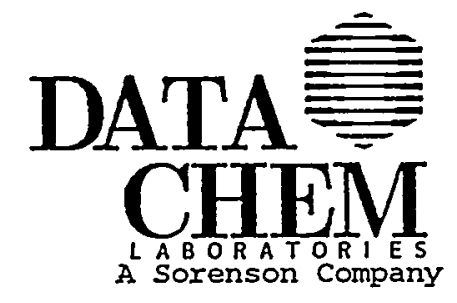

SAIC

Attention: Rita M. Schmon-Stasik 11251 Roger Bacon Drive Reston, VA 20190
COVER PAGE

ANALYTICAI, REPORT FOR

SAIC

Phone(201) 498-8496 Fax(201) 489-1592
Form COVER-V1.3

0721971109263

Page 1

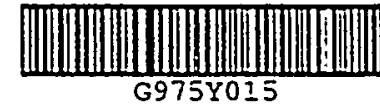

DCL Report Group. : : 97C-0208-01

Date Printed.... : 21-JUL-97 11::J

Project Protocol : P975BG02

Client Ref Number.: Not Provicied

Release Number....: Not Proviced

Analysis Method(s): $8260 \mathrm{~A}$

\begin{abstract}
- Client
Sample Name

Method Blank Method Blank LCS

NAP-R2-S2-A

NAP-R2-S2-B

NAP-R2-S4

NAP-R1-S2-A

NAP-R1-S2-B

NAP-R1-S4

NAP-RI-S4

NAP-R1-S4
\end{abstract}

\begin{tabular}{l} 
Laboratory \\
Sample Name \\
\hline BL-136531-1 \\
BL-136531-2 \\
QC-136531-1 \\
$97 \mathrm{C} 02896$ \\
$97 \mathrm{C0} 2897$ \\
$97 \mathrm{C} 02898$ \\
$97 \mathrm{C} 02899$ \\
$97 \mathrm{C} 02900$ \\
$97 \mathrm{C} 02901$ \\
$97 \mathrm{C0} 2901 \mathrm{MS}$ \\
97C02901MSD
\end{tabular}

Date

Sampled

NA

NA

NA

25-JUN-97

25-JUN-97

26-JUN-97

24-JUN-97

24-JUN-97

25-JUN-97

25-JUN-97

25-JUN-97
Date

Received

NA

NA

NA

27-JUN-97

27-JUN-97

27-JUN-97

27-JUN-97

27-JUN-97

27-JUN-97

27-JUN-97

27-JUN-97

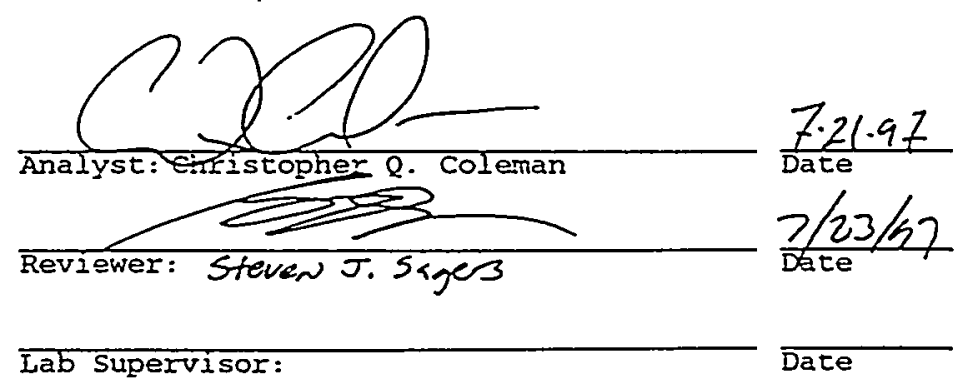

960 West LeVoy Drive / Salt Iake City, Utah 84123-2547 Phone (801) 266-7700 


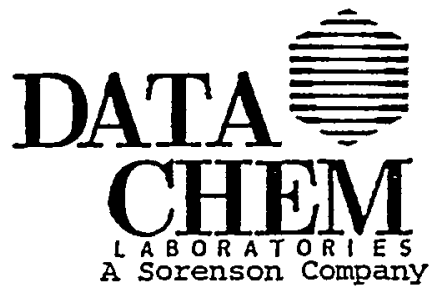

A Sorenson Company
FORM H (TYPE I)

SINGLE METHOD ANALYSES

SAMPLE GROUP COMMENTS
Form RLIMS63H-V1.3

$0721971109263:$

Page 2

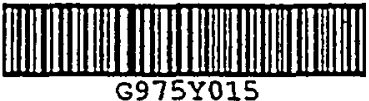

DCL Report Group. : : 97C-0208-01

Date Printed.....: 21-JUL-97 11:10

Release Number....: Not Provided

\section{Sample Group Comments}

OC and LCS data included in set 97C-0208-01.

Surrogate and matrix spike compounds are spiked at $50 \mathrm{ug} / \mathrm{L}$.

The QC and sample data for this set are within acceptable parameters.

The samples were analyzed by GC/MS according to method 8260A (OV-SW-8260A $R$ O).

\section{General Information}

The DCL QC Database maintains all numerical figures which are input from the pertinent data source. These data have not been rounded to significant figures nor have they been moisture corrected. Reports generated from the system, however, list data which have been rounded to the number of significant figures requested by the client or deemed appropriate for the method. This may create minor discrepancies between data which appear on the QC Sunmary Forms (Forms B-G) and those that would be calculated from rounded analytical results. Additionally, if a moisture correction is performed, differences will be observed between the QC data and the surrogate data reported on Form $A$ (or other report forms) and corresponding data reported on QC Sumary Forms. In these cases, the Form A will indicate the "Report Basis" as well as the moisture value used for making the correction.

Report generation options: $\mathrm{X}$

\section{Result Symbol Definitions}

ND - Not Detected above the MDL or IDL (LID or MDC for radiochemistry).

** - No result could be reported, see sample comments for details.

\section{Qualifier Symbol Definitions}

U - Not Detected above the MDL or IDL (LLD or MDC for radiochemistry). - For radiochemistry the nuclide was not identified by the Canberra Nuclear NID program, activity values reported are calculated using the Canberra Nuclear MINACT program.

$B$ - For organic analysis the qualifier indicates that this analyte was found in the method blank. For inorganic analysis the qualifier signifies the value is between the IDL and PQL.

$J$ - The qualifier indicates that the value is between the MDL and the PQL. It is also used for indicating an estimated value for tentatively identified compounds in mass spectrometry where a $1: 1$ response is assumed. 


\section{DATA \\ FORM A (TYPE I) SINGLE METHOD ANALYSES} ctan

A Sorenson Company
Form RLIMS63A-V1.. Page 3

072197110926 :

SAMPLE ANALYSIS DATA SHEET

\section{Page}

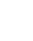

$5976600 \mathrm{C}$
Date Printed.......: 21-JUL-97 11:10

Client Name......... SAIC

Client Ref Number.... Not Provided

Sampling Site....... Not Applicable

Release Number....... Not Provided

Date Received....... Not Applicable

DCI Preparation Group: Not Applicable Date Prepared....... Not Applicable Preparation Method... : Not Applicable Aliquot Weight/Volume: $5.0 \mathrm{~mL}$

Net Weight/Volume....: Not Required
Client Sample Name: BL-136531-1

DCL Sample Name... : BL-136531-1

DCL Report Group.. : 97C-0208-01

Matrix......... WATER

Date Sampled..... . Not Applicable

Reporting Units...: ug/L

DCL Analysis Group: G976M004

Analysis Method...: 8260A

Instrument Type...: GC/MS VO

Instrument ID... . : 5971-L

Column Type...... D : 624

Q Primary

$\square$ Confinmation

Analytical Results

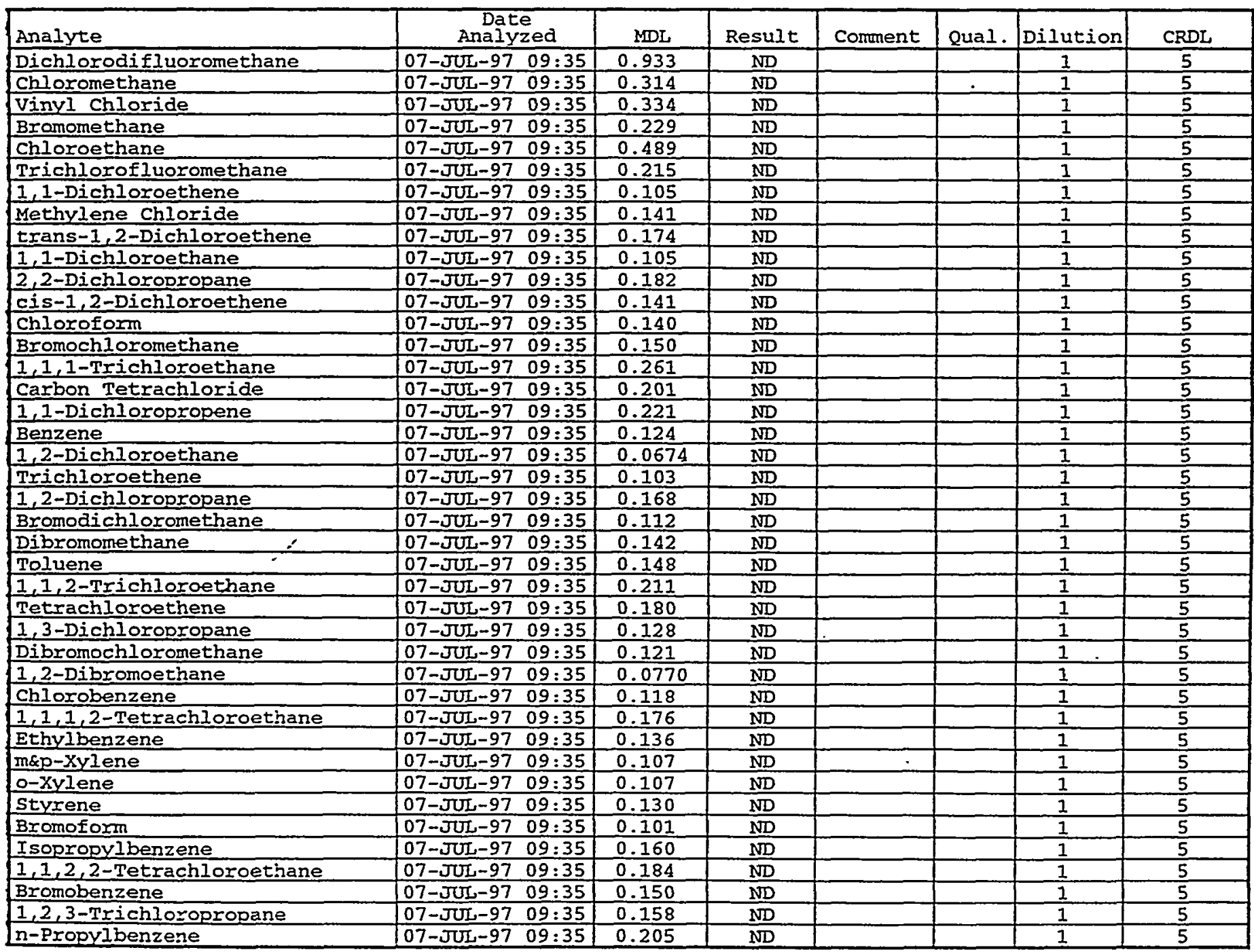

008
960 West LeVoy Drive / Salt Lake City, Utah 84123-2547

?hone (801) 266-7700

EAX (801) 268-9992
Web Page: www.datachem.com

E-mail: labedatachem.com 


\section{DATA \\ FORM A (TYYPE I) \\ SINGLE METHOD ANALYSES} CHEM

A Sorenson Company
SAMPLE ANALYSIS DATA SHEET
Form RLIMS63A-V1.3 0721971109263:

Page 4

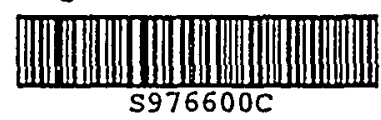

Date Printed........ : 21-JU-97 11:10

DCL Sample Name...: BL-136531-1

Client Name......... SAIC

DCL Report Group... 97C-0208-01

\section{Analytical Results}

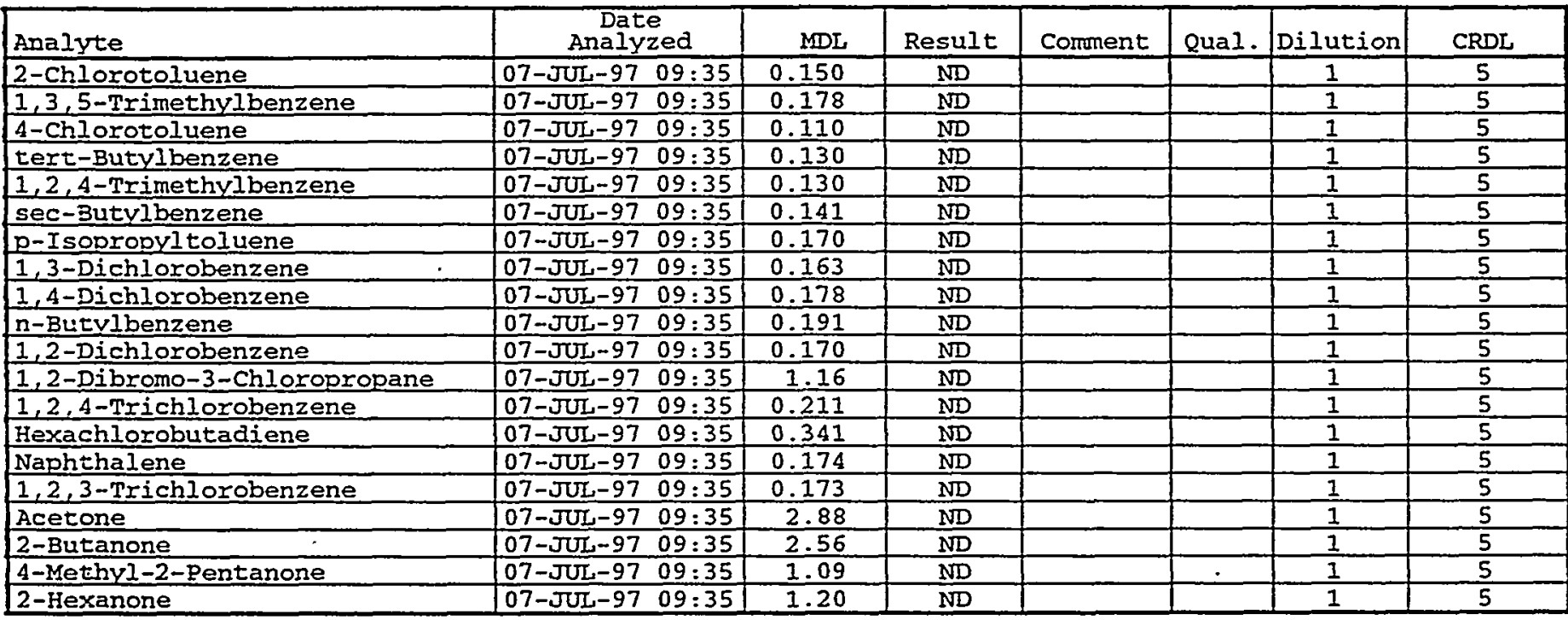

\section{Surrogate Recoveries}

\begin{tabular}{|l|c|c|c|}
\hline Analyte & Result & $\begin{array}{c}\text { Spiked } \\
\text { Amount }\end{array}$ & $\begin{array}{c}\text { Percent } \\
\text { Recovery }\end{array}$ \\
\hline 1,2-Dichloroethane-d4 & 42.2 & 50.0 & 84.4 \\
\hline 4-Bromofluorobenzene & 44.8 & 50.0 & 89.7 \\
\hline Toluene-d8 & 46.9 & 50.0 & 93.7 \\
\hline
\end{tabular}

009
960 West LeVoy Drive / Salt Lake City, Utah 84123-2547 Phone (801) 266-7700 FAX (801) 268-9992
Web Page: www.datachem.com

E-mail: labedatachem.com 


\section{DATA 童 CHEM \\ A Sorenson Company \\ SAMPLE ANALYSIS DATA SHEET}

Form RIIMS63A-V1.: Page 5 072197110926

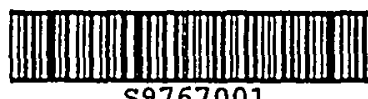

\$9767001
Date Printed........: 21-JUT-97 11:10

client Name........ SAIC

Client Ref Number.... Not Provided

Sampling Site....... Not Applicable

Release Number...... Not Provided

Date Received....... Not Applicable

DCL Preparation Group: Not Applicable Date Prepared........ Not Applicable Preparation Method...: Not Applicable Aliquot Weight/Volume: $5.0 \mathrm{~mL}$ Net Weight/Volume.... : Not Reguired
Client Sample Name: BL-136531-2 DCL Sample Name... : BL-136531-2 DCL Report Group. . : 97C-0208-01

Matrix........ WATER

Date Sampled..... Not Applicable Reporting Units...: ug/L

DCI Analysis Group: G976M004 Analysis Method...: 8260A Instrument Type...: GC/MS vo Instrument ID....: 5971-L Colunn Type......: DB 624

Q $\square$ Confinmation

Analytical Results

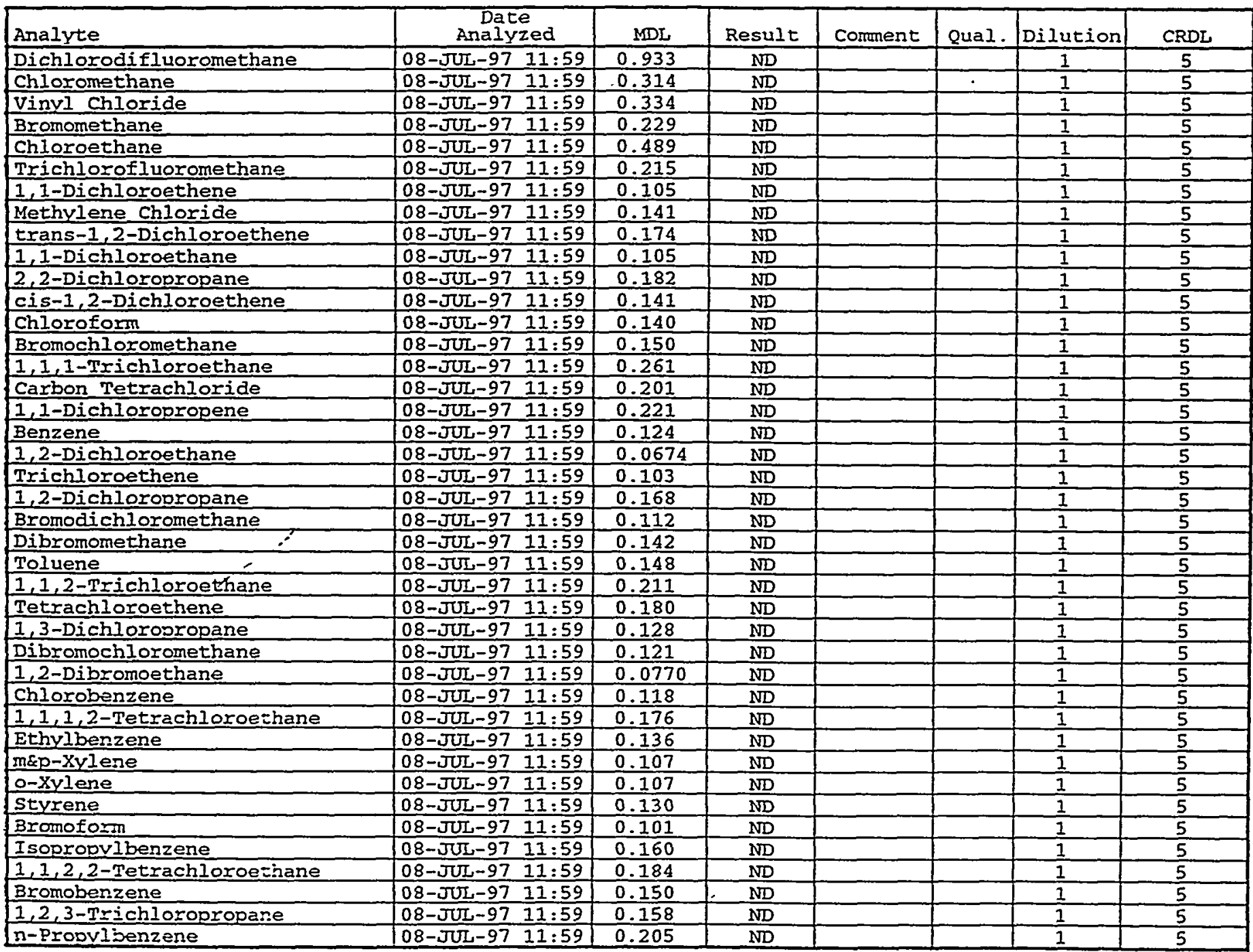

กิ10

960 West LeVoy Drive / Salt Lake City, Utah 84123-2547

Phone (801) 266-7700

FAX (801) 268-9992

Web Page: www.datachem.com

E-mail: labedatachem.com 


\section{DATA \\ FORM A (TYPE I) SINGLE METHOD ANALYSES} CHEM

A Sorenson Company
Form RLIMS63A-V1. $072197110926 \Xi$

Page 6

SAMPLE ANALYSIS DATA SHEET

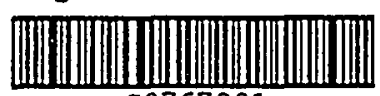

DCL Sample Name...: BL-136531-2

DCL Report Group..: 97C-0208-01

Date Printed.......: 21-JUL-97 11:10

: SAIC

Analytical Results

\begin{tabular}{|c|c|c|c|c|c|c|c|}
\hline Analyte & $\begin{array}{c}\text { Date } \\
\text { Aralyzed }\end{array}$ & $\mathrm{MDL}$ & Result & Comment & Qual. & Dilution & CRDL \\
\hline 2-Chlorotoluene & 08-JUL-97 11:59 & 0.150 & ND & & & 1 & 5 \\
\hline 1,3,5-Trimethylbenzene & $08-\pi U L-97 \quad 11: 59$ & 0.178 & ND & & & 1 & 5 \\
\hline 4-Chlorotoluene & $08-\pi U L-97 \quad 11: 59$ & 0.110 & ND & & & 1 & 5 \\
\hline cert-Butylbenzene & $08-\pi U L-97 \quad 11: 59$ & 0.130 & ND & & & 1 & 5 \\
\hline 1,2,4-Trimethylbenzene & $08-\pi U L-97 \quad 11: 59$ & 0.130 & ND & & & 1 & 5 \\
\hline sec-ButyIbenzene & 08-JUL-97 $11: 59$ & 0.141 & ND & & & 1 & 5 \\
\hline D-Isopropyltoluene & $08-J U L-97 \quad 11: 59$ & 0.170 & ND & & & 1 & 5 \\
\hline 1,3-Dichlorobenzene & $08-\pi \pi L-97 \quad 11: 59$ & 0.163 & ND & & & 1 & 5 \\
\hline 1,4-Dichlorobenzene & $08-\pi U L-97 \quad 11: 59$ & 0.178 & ND & & & 1 & 5 \\
\hline$n$-Butylbenzene & $08-\pi \pi L-97 \quad 11: 59$ & 0.191 & ND & & & 1 & 5 \\
\hline 1,2-Dichlorobenzene & $08-J U L-97 \quad 11: 59$ & 0.170 & ND & & & 1 & 5 \\
\hline 1,2-Dibromo-3-Chloropropane & $08-\pi U L-97 \quad 11: 59$ & 1.16 & $\mathrm{ND}$ & & & 1 & 5 \\
\hline 1,2,4-Trichlorobenzene & $08-J U L-97 \quad 11: 59$ & 0.211 & ND & & & 1 & 5 \\
\hline Hexachlorobutadiene & $08-\pi \pi L-97 \quad 11: 59$ & 0.341 & ND & & & 1 & 5 \\
\hline Naphthalene & 08-JUL-97 $11: 59$ & 0.174 & ND & & & 1 & 5 \\
\hline 1,2,3-Trichlorobenzene & $08-J U L-97 \quad 11: 59$ & 0.173 & ND & & & 1 & 5 \\
\hline Acetone & $08-J U L-97 \quad 11: 59$ & 2.88 & ND & & & 1 & 5 \\
\hline 2-Butanone & $08-J U L-97 \quad 11: 59$ & 2.56 & ND & & & 1 & 5 \\
\hline 4-Methyl-2-Pentanone & 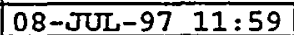 & 1.09 & ND & & - & 1 & 5 \\
\hline 2-Hexanone & $08-\pi 0 L-97 \quad 11: 59$ & 1.20 & ND & & & 1 & 5 \\
\hline
\end{tabular}

\section{Surxogate Recoveries}

\begin{tabular}{|l|c|c|c|}
\hline Analyte & Result & $\begin{array}{c}\text { Spiked } \\
\text { Amount }\end{array}$ & $\begin{array}{c}\text { Percent } \\
\text { Recovery }\end{array}$ \\
\hline 1,2 -Dichloroethane-d4 & 47.4 & 50.0 & 94.9 \\
\hline 4-Bromofluorobenzene & 47.1 & 50.0 & 94.1 \\
\hline Toluene-d8 & 49.2 & 50.0 & 98.4 \\
\hline
\end{tabular}




\section{FORM A (TYPE I) \\ DATA SINGLE METHOD ANATYSES

Form RLIMS63A-V1.3

0723971703412 .

Page 7

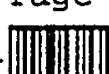

Date Printed....... : 23-JUL-97 16:58

Client Name......... SAIC

Client Ref Number....: Not Provided

Sampling site....... Not Applicable

Release Number....... Not Provided

Date Received....... Not Applicable

DCL Preparation Group: Not Applicable Date Prepared....... Not Applicable Preparation Method... Not Applicable Aliquot Weight/Volume: $5.0 \mathrm{~mL}$ Net Weight/Volume.... Not Required
Client Sample Name: QC-136531-1 DCL Sample Name...: QC-136531-1 DCI Report Group.. : 97C-0208-01

Matrix......... : WATER Date Sampled..... Not Applicable Reporting Units...: ug/L

DCL Analysis Group: G976M004 Analysis Method...: 8260A Instrument Type...: GC/MS Vo Instrument ID....: 5971-L Column Type......: DB 624

Q

$\square$ Confinmation

Analytical Results

\begin{tabular}{|c|c|c|c|c|c|c|c|}
\hline Analyte & $\begin{array}{c}\text { Date } \\
\text { Analyzed } \\
\end{array}$ & MDL & Result & Comment & Qual. & Dilution & CRDL \\
\hline 1,1-Dichloroethene & $07-\pi u L-97 \quad 10: 07$ & 0.105 & 47. & & & 1 & 5 \\
\hline Benzene & $\begin{array}{ll}07-\pi U L-97 & 10: 07\end{array}$ & 0.124 & 43 & & . & 1 & 5 \\
\hline Trichloroethene & $07-\pi U L-97 \quad 10: 07$ & 0.103 & 49. & & & 1 & 5 \\
\hline Chlorobenzene & $07-\pi U L-97 \quad 10: 07$ & 0.118 & 46. & & & 1 & 5 \\
\hline
\end{tabular}

\section{Surrogate Recoveries}

\begin{tabular}{|l|c|c|c|}
\hline Analyte & Result & $\begin{array}{c}\text { Spiked } \\
\text { Amount }\end{array}$ & $\begin{array}{c}\text { Percent. } \\
\text { Recovery }\end{array}$ \\
\hline 1,2-Dichloroethane-d4 & 41.9 & 50.0 & 83.9 \\
\hline 4-Bromofluorobenzene & 44.6 & 50.0 & 89.3 \\
\hline Toluene-d8 & 46.5 & 50.0 & 92.9 \\
\hline
\end{tabular}




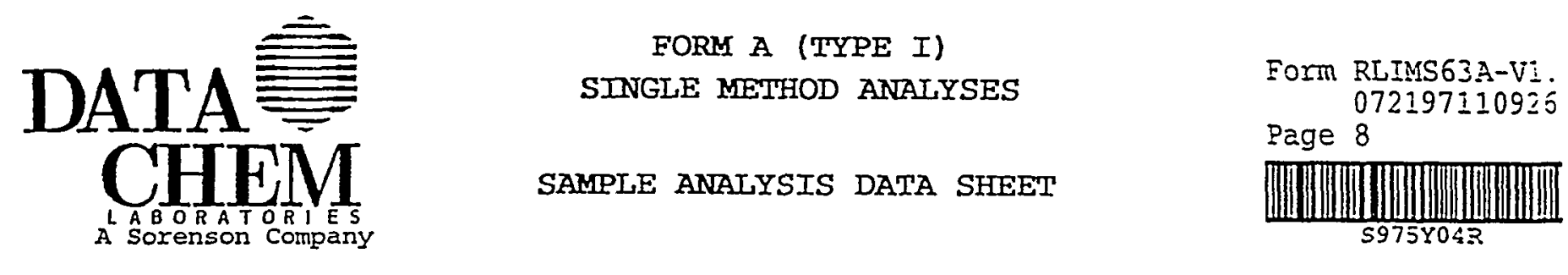

Date Printed......: 21-JUL-97 11:10

Client Sample Niame: NAP-R2-S2-A

DCL Sample Name... : 97C02896

Client Name........ SAIC

client Ref Number.... Not Provided

Sarmling Site....... Not Provided

Release Number...... Not Provided

DCL Report Group.. : 97C-0208-01

Matrix......... WATER

Date Sampled.....: 25-JUN-97 00:00

Reporting Units...: ug/L

Date Received......: 27-JUN-97 00:00

Report Basis..... : As Received $\square$ Dries

DCI Preparation Group: Not Applicable Date Prepared....... Not Applicable Preparation Method...: Not Applicable Aliquot Weight/Volume: $5.0 \mathrm{~mL}$ Net Weight/Volume.... : Not Required

DCL Analysis Group: G976\$004

Analysis Mechod...: 8260A

Instrument Type...: GC/MS vo

Instrument ID....: 5971-L

Column Type.....: D3 624

[Primary

口confirmation

Analytical Results

\begin{tabular}{|c|c|c|c|c|c|c|c|}
\hline Analyte & $\begin{array}{c}\text { Date } \\
\text { Analyzed }\end{array}$ & $\mathrm{MDL}$ & Result & Comment & Qual. & Dilusion & CRDL \\
\hline Dichlorodifluoromethane & $07-\pi \pi-97 \quad 14: 20$ & 0.933 & $\mathrm{ND}$ & & & 1 & 5 \\
\hline Chloromethane & $07-J U L-97 \quad 14: 20$ & 0.314 & ND & & . & $\overline{1}$ & 5 \\
\hline Vinyl Chloride & 07-JUL-97 14:20 & 0.334 & $\mathrm{ND}$ & & & 1 & 5 \\
\hline Chloroethane & $07-\pi U L-97 \quad 14: 20$ & 0.489 & $\mathrm{ND}$ & & & 1 & 5 \\
\hline Trichlorofluoromethane & $07-\pi U L-97 \quad 14: 20$ & 0.215 & ND & & & 1 & 5 \\
\hline 1,1-Dichloroethene & $07-J U L-97 \quad 14: 20$ & 0.105 & $\mathrm{ND}$ & & & 1 & 5 \\
\hline 2,2-Dichloropropane & $07-\pi U_{L}-97 \quad 14: 20$ & 0.182 & $\mathrm{ND}$ & & & 1 & 5 \\
\hline cis-1,2-Dichloroethene & $07-\pi U=-97 \quad 14: 20$ & 0.141 & ND & & & 1 & 5 \\
\hline Chloroform & $07-J U L-97 \quad 14: 20$ & 0.140 & 11. & & & $I$ & 5 \\
\hline Bromochloromethane & $07-\pi U L-97 \quad 14: 20$ & 0.150 & ND & & & 1 & 5 \\
\hline $1,1,1$-Trichloroethane & $07-J U L-97 \quad 14: 20$ & 0.261 & ND & & & 1 & 5 \\
\hline Carbon Tetrachloride & $07-\pi \sqrt{2}-97 \quad 14: 20$ & 0.201 & $\mathrm{ND}$ & & & 1 & 5 \\
\hline Bromodichloromethane & $07-J U L-97 \quad 14: 20$ & 0.112 & 5.0 & & & 1 & 5 \\
\hline Dibromomethane & $07-J U L-97 \quad 14: 20$ & 0.142 & ND & & & 1 & 5 \\
\hline Toluene & $07-5 U_{L}-97 \quad 14: 20$ & 0.148 & 2.7 & & $\mathbf{J}$ & 1 & 5 \\
\hline 1,1,2-Trichloroethane & $07-\pi \sqrt{L}-97 \quad 14: 20$ & 0.211 & ND & & & 1 & 5 \\
\hline Tetrachloroethene & $07-J U L-97 \quad 14: 20$ & 0.180 & ND & & & 1 & 5 \\
\hline 1,3-Dichloropropane & $07-J_{L}-97 \quad 14: 20$ & 0.128 & ND & & & 1 & 5 \\
\hline Dibromochloromethane & $07-J U L-97 \quad 14: 20$ & 0.121 & 1.9 & & $\mathbf{J}$ & 1 & 5 \\
\hline 1,2-Dibromoethane & $07-\pi L_{L}-97 \quad 14: 20$ & 0.0770 & ND & & & 1 & 5 \\
\hline Chlorobenzene & $07-J U L-97 \quad 14: 20$ & 0.118 & ND & & & 1 & 5 \\
\hline $1,1,1,2$-Tetrachloroethane & $07-$ JUL $-97 \quad 14: 20$ & 0.176 & ND & & & 1 & 5 \\
\hline Ethylbenzene & $07-\pi \pi L-97 \quad 14: 20$ & 0.136 & ND & & & 1 & 5 \\
\hline m\&p-Xylene & $07-J U L-97 \quad 14: 20$ & 0.107 & $\mathrm{ND}$ & & & 1 & 5 \\
\hline o-xylene & $07-J U L-97 \quad 14: 20$ & 0.107 & $\mathrm{ND}$ & & & 1 & 5 \\
\hline
\end{tabular}

913

960 West LeVoy Drive

Phone (801) 266-7700

EAX (80I) 268-9992
Iake City, Utah 84123-2547

web Page: www.datachem.com

E-mail: labedatachem.com 


\section{DATA \\ FORM A (TYPE I) \\ SINGLE MEIHOD ANALYSES} CHEM

A Sorenson Company
Form RLIMS63A-VI. $07219711092 E$

Page 9

SAMPLE ANALYSIS DATA SHEET

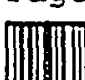

DCL Sample Name. . : 97c02896

DCL Report Group.. : 97C-0208-01 client Name........ SAIC

: 21-JUL-97 11:10

Analytical Results

\section{Surrogate Recoveries}

\begin{tabular}{|l|c|c|c|}
\hline Analyte & Result & $\begin{array}{c}\text { Spiked } \\
\text { Amount }\end{array}$ & $\begin{array}{c}\text { Percent } \\
\text { Recovery }\end{array}$ \\
\hline 1.2 -Dichloroethane-d4 & 41.6 & 50.0 & 83.3 \\
\hline 4-Bromofluorobenzene & 45.3 & 50.0 & 90.5 \\
\hline Toluene-d8 & 47.9 & 50.0 & 95.9 \\
\hline
\end{tabular}




\section{DATA \\ FORM A (TYPE I) SINGLE METHOD ANALYSES} CHEM

A Sorenson Company
Form RLIMS63A-V1.3 0721971109263

Page 10

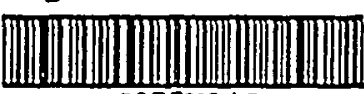

S975Y04S
Date Printed....... : 21-JUL-97 11:10

Client Name......... SAIC

Client Ref Number.... Not Provided

Sampling Site........ Not Provided

Release Number....... Not Provided

Date Received.....: 27-JUN-97 00:00

DCI Preparation Group: Not Applicable Date Prepared....... . Not Applicable Preparation Method...: Not Applicable Aliquot Weight/Volume: $5.0 \mathrm{~mL}$ Ner Weight/Volume.... : Not Required
Client Sample Name: NAP-R2-S2-B

DCI Sample Name... : 97C02897

DCL Report Group. . : 97C-0208-01

Matrix........: WATER

Date Sampled....: 25-JUN-97 00:00

Reporting Units...: ug/L

Report Basis...... 因As Received $\square$ Dried

DCL Analysis Group: G976M004

Analysis Method...: 8260A

Instrument Type...: GC/MS VO

Instrument ID....: 5971-L

Colum Type......: DB 624

Q

$\square$ Confirmation

Analytical Results

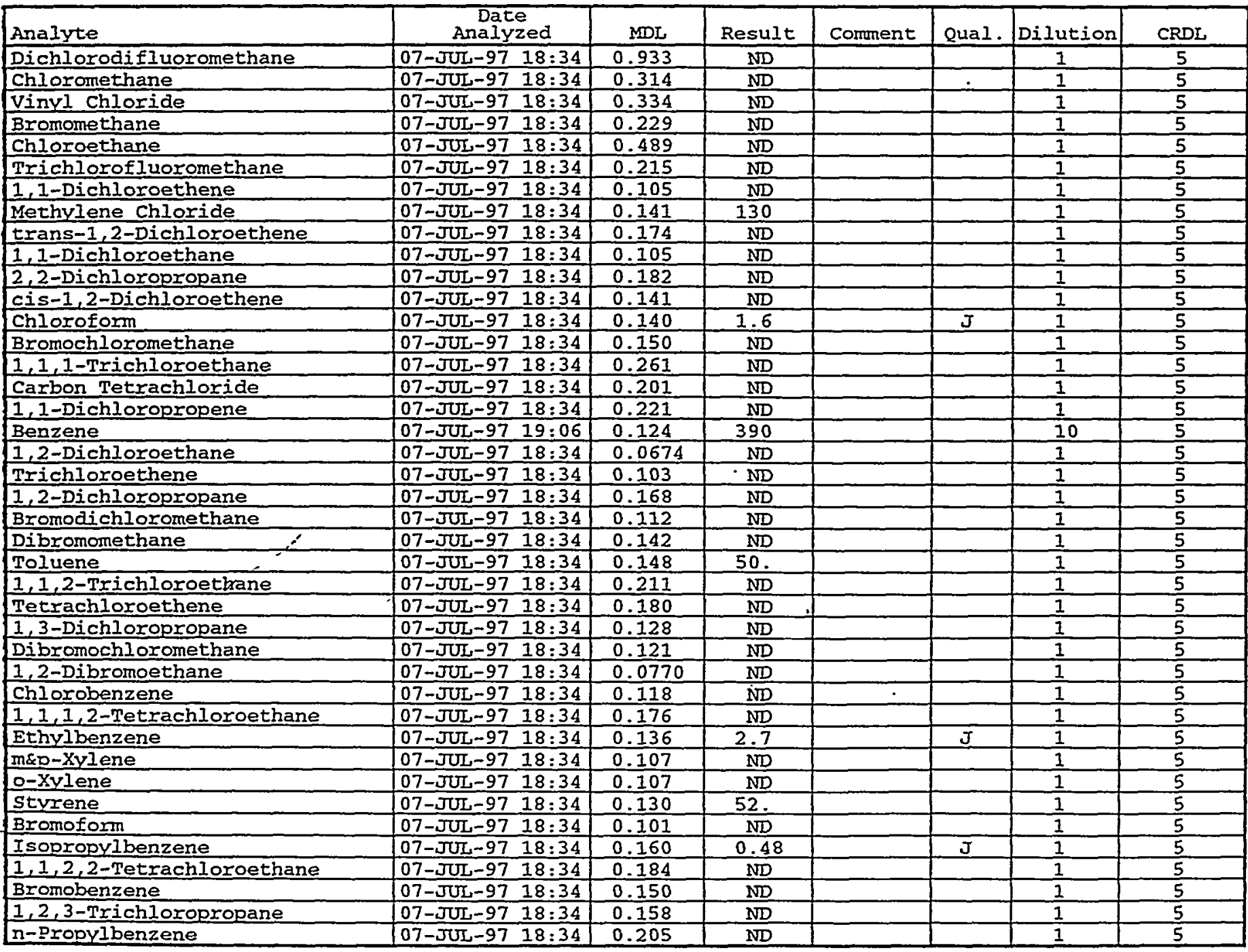




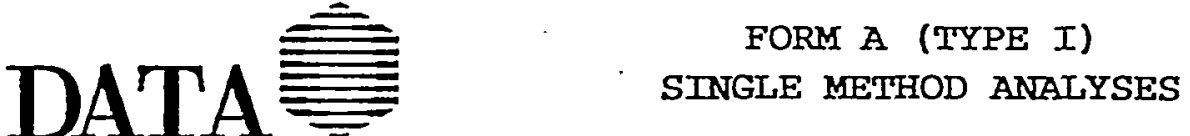 CHEM \\ SAMPLE ANALYSIS DATA SHEET}

Form RLIMS63A-V1.. $072197110926:$

Page 11

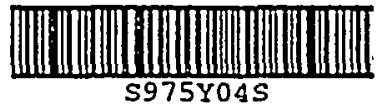

DCL Sample Name... : 97C02897

DCL Report Group.. : 97C-0208-01

client Name......... SAIC

Analytical Results

\begin{tabular}{|c|c|c|c|c|c|c|c|}
\hline Analyte & $\begin{array}{c}\text { Date } \\
\text { Analyzed }\end{array}$ & $\mathrm{MDL}$ & Result & Comment & Qual. & Dilution & CRDL \\
\hline 2-Chlorotoluene & 07-JUL-97 $18: 34$ & 0.150 & ND & & & .1 & 5 \\
\hline 1,3,5-Trimethylbenzene & $07-$ JUL-97 $18: 34$ & 0.178 & ND & & & 1 & 5 \\
\hline 4-Chlorotoluene & 07-JUL-97 18:34 & 0.110 & ND & & & 1 & 5 \\
\hline $1,2,4$-Trimethylbenzene & $07-$ JUL-97 $18: 34$ & 0.130 & $\mathrm{ND}$ & & & 1 & 5 \\
\hline sec-Butylbenzene & $07-\pi U L-97 \quad 18: 34$ & 0.141 & ND & & & 1 & 5 \\
\hline p-Isopropyltoluene & $07-\pi U L-97 \quad 18: 34$ & 0.170 & ND & & & 1 & 5 \\
\hline n-Butylbenzene & $07-\pi U L-97 \quad 18: 34$ & 0.191 & ND & & & 1 & 5 \\
\hline 1,2-Dichlorobenzene & $07-J U L-97 \quad 18: 34$ & 0.170 & ND & & & 1 & 5 \\
\hline 1,2-Dibromo-3-Chloropropane & $07-\pi / 4-97 \quad 18: 34$ & 1.16 & ND & & & 1 & 5 \\
\hline $1,2,4$-Trichlorobenzene & $07-$ JUL-97 $18: 34$ & 0.211 & ND & & & 1 & 5 \\
\hline Hexachlorobutadiene & $07-\pi U L-97 \quad 18: 34$ & 0.341 & $\mathrm{ND}$ & & & 1 & 5 \\
\hline Naphthalene & $07-\pi U L-97 \quad 19: 06$ & 0.174 & 550 & & & 10 & 5 \\
\hline 1,2,3-Trichlorobenzene & $07-\mathrm{JUL}-97 \quad 18: 34$ & 0.173 & ND & & & 1 & 5 \\
\hline
\end{tabular}

Tentatively Identified Compound Results

\begin{tabular}{|c|c|c|c|c|c|}
\hline Analyte (Retention Time) & $\begin{array}{c}\text { Date } \\
\text { Analyzed }\end{array}$ & Result & Comment & Qual. & Dilution \\
\hline Indene $(22.75)$ & $07-\pi U L-97 \quad 18: 34$ & 43. & & $\mathrm{~J}$ & 1 \\
\hline
\end{tabular}

\section{Surrogate Recoveries}

\begin{tabular}{|l|c|c|c|}
\hline Analyte & Result & $\begin{array}{c}\text { Spiked } \\
\text { Amount }\end{array}$ & $\begin{array}{c}\text { Percent } \\
\text { Recovery }\end{array}$ \\
\hline 1,2-Dichloroethane-d4 & 43.0 & 50.0 & 86.0 \\
\hline 4-Bromofluorobenzene & 46.8 & 50.0 & 93.6 \\
\hline Toluene-d8 & 49.2 & 50.0 & 98.3 \\
\hline
\end{tabular}




\section{Dent \\ DATA SINGLE METHOD ANALYSES CHEM \\ A Sorenson Company \\ Form RLIMS63A-V1.3 0721971109263: \\ Page 12 \\ SAMPLE ANAUYSIS DATA SHEET

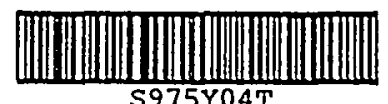 \\ S975Y04T}

Date Printed....... : 21-JUL-97 11:10

Client Name.......... SAIC

Client Ref Number....: Not Provided

Sampling site....... Not Provided

Release Number....... Not Provided

Date Received.....: 27-JUN-97 00:00

DCI, Preparation Group: Not Applicable Date Prepared........ Not Applicable Preparation Method... : Not Applicable Aliquot Weight/Volume: $5.0 \mathrm{~mL}$ Ner Weight/Volume.... : Not Required
Client Sample Name: NAP-R2-S4

DCL Sample Name...: 97C02898

DCL Report Group.. : 97C-0208-01

Matrix........ : WATER

Date sampled.....: 26-JUN-97 00:00

Reporting Units...: ug/I

Report Basis..... : As Received $\square \mathrm{D}$ ried

DCL Analysis Group: G976M004

Analysis Method...: 8260A

Instrument Type...: GC/MS vo

Instrument ID. . . : 5971-L

Column Type......: DB 624

(x) Primary

$\square$ Confinmation

Analytical Results

\begin{tabular}{|c|c|c|c|c|c|c|c|}
\hline Inalyte & $\begin{array}{c}\text { Date } \\
\text { Analyzed } \\
\end{array}$ & $\mathrm{MDL}$ & Result & Comment & Qual. & Dilution & CRDL \\
\hline Dichlorodifluoromethane & $07-J U L-97 \quad 15: 24$ & 0.933 & $\mathrm{ND}$ & & & 1 & 5 \\
\hline Chloromethane & $07-\pi \pi-97 \quad 15: 24$ & 0.314 & ND & & - & 1 & 5 \\
\hline Vinyl Chloride & $07-\pi U L-97 \quad 15: 24$ & 0.334 & ND & & & 1 & 5 \\
\hline Bromomethane & $07-\pi U \pi-97 \quad 15: 24$ & 0.229 & ND & & & 1 & 5 \\
\hline Chloroethane & $07-\pi U L-97 \quad 15: 24$ & 0.489 & $\mathrm{ND}$ & & & 1 & 5 \\
\hline Mrichlorofluoromethane & $07-\pi U L-97.15: 24$ & 0.215 & ND & & & 1 & 5 \\
\hline 1,1-Dichloroethene & $07-J U L-97 \quad 15: 24$ & 0.105 & ND & & & 1 & 5 \\
\hline Methylene Chloride & $07-\pi U L-97 \quad 15: 24$ & 0.141 & 75. & & & 1 & 5 \\
\hline trans $-1,2-D i c h l o r o e t h e n e$ & $07-\pi u L-97 \quad 15: 24$ & 0.174 & ND & & & 1 & 5 \\
\hline 1,1-Dichloroethane & $07-\pi U L-97 \quad 15: 24$ & 0.105 & $\mathrm{ND}$ & & & 1 & 5 \\
\hline 2,2-Dichloropropane & $07-J U L-97 \quad 15: 24$ & 0.182 & ND & & & $I$ & 5 \\
\hline cis-1,2-Dichloroethene & 07-JUL-97 $15: 24$ & 0.141 & $\mathrm{ND}$ & & & 1 & 5 \\
\hline Chloroform & 07-JUL-97 15:24 & 0.140 & $\mathrm{ND}$ & & & 1 & 5 \\
\hline Bromochloromethane & \begin{tabular}{|ll}
$07-J U L-97$ & $15: 24$ \\
\end{tabular} & 0.150 & ND & & & $I$ & 5 \\
\hline 1,1,1-Trichloroethane & 07-JUL-97 $\quad 15: 24$ & 0.261 & ND & & & 1 & 5 \\
\hline Carbon Tetrachloride & \begin{tabular}{|ll}
$07-\pi U L-97$ & $15: 24$ \\
\end{tabular} & 0.201 & ND & & & 1 & 5 \\
\hline 1,1-Dichloropropene & 07-JUL-97 15:24 & 0.221 & ND & & & 1 & 5 \\
\hline Benzene & $07-J U L-97 \quad 15: 24$ & 0.124 & 0.74 & & $\mathrm{~J}$ & 1 & 5 \\
\hline 1,2-Dichloroethane & $07-J U L-97 \quad 15: 24$ & 0.0674 & ND & & & 1 & 5 \\
\hline Trichloroethene & 07-JUL-97 15:24 & 0.103 & ND & & & 1 & 5 \\
\hline 1,2-Dichloropropane & $07-\pi J-97 \quad 15: 24$ & 0.168 & ND & & & 1 & 5 \\
\hline Bromodichloromethane & $07-\pi U L-97 \quad 15: 24$ & 0.112 & ND & & & 1 & 5 \\
\hline Dibromomethane & $07-\pi U L-97 \quad 15: 24$ & 0.142 & ND & & & 1 & 5 \\
\hline Toluene & 07-JUL-97 $15: 24$ & 0.148 & ND & & & 1 & 5 \\
\hline 1,1,2-Trichloroethane & $07-J U L-97 \quad 15: 24$ & 0.211 & ND & & & 1 & 5 \\
\hline Tetrachloroethene & $07-\pi U L-97 \quad 15: 24$ & 0.180 & $\mathrm{ND}$ & & & 1 & 5 \\
\hline 1,3-Dichloxopropane & 07-JUL-97 $15: 24$ & 0.128 & ND & & & 1 & 5 \\
\hline Dibromochloromethane & $07-\pi U L-97 \quad 15: 24$ & 0.121 & ND & & & 1 & 5 \\
\hline 1,2-Dibromoethane & $07-\pi J L-97 \quad 15: 24$ & 0.0770 & ND & & & 1 & 5 \\
\hline Chlorobenzene & $07-\pi U L-97 \quad 15: 24$ & 0.118 & ND & & & 1 & 5 \\
\hline $1,1,1,2$-Tetrachloroethane & $07-J U L-97 \quad 15: 24$ & 0.176 & ND & & & 1 & 5 \\
\hline Ethylbenzene & $07-\pi \pi /-97 \quad 15: 24$ & 0.136 & ND & & & 1 & 5 \\
\hline m\&D-Xylene & $07-\pi U L-97 \quad 15: 24$ & 0.107 & ND & & & 1 & 5 \\
\hline $0-x y$ lene & $07-\pi U L-97 \quad 15: 24$ & 0.107 & ND & & & 1 & 5 \\
\hline Styrene & $07-J U L-97 \quad 15: 24$ & 0.130 & ND & & & 1 & 5 \\
\hline Bxomoform & $07-\pi U L-97 \quad 15: 24$ & 0.101 & ND & & & 1 & 5 \\
\hline Isopropylbenzene & 07-JUL-97 $15: 24$ & 0.160 & ND & & & 1 & 5 \\
\hline $1,1,2,2$-Tetrachloroetine & \begin{tabular}{|ll}
$07-\pi U 1-97$ & $15: 24$
\end{tabular} & 0.184 & ND & & & 1 & 5 \\
\hline$B=$ omobenzene & $07-\pi U L-97 \quad 15: 24$ & 0.150 & ND & & & 1 & 5 \\
\hline 1,2,3-Trichloropropane & $07-\pi U L-97 \quad 15: 24$ & 0.158 & ND & & & 1 & 5 \\
\hline$n$-Propylbenzene & $07-\pi U L-97 \quad 15: 24$ & 0.205 & ND & & & 1 & 5 \\
\hline
\end{tabular}

\section{$9+1 ?$}

960 West LeVoy Drive / Salt Lake City, Utah 84123-2547

?hone (801) 266-7700

IAX (801) 268-9992
Web Page: www.datachem.com

E-mail: labedatachem.com 


\section{DATA CHEM \\ A Sorenson Company}

FORM A (TYPE I)

STNGLE METHOD ANALYSES

\section{SAMPLE ANALYSIS DATA SHEET}

: 21- $-\pi 4-97 \quad 11: 10$

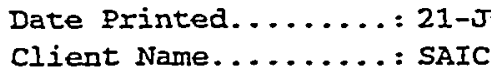

Form RLIMS63A-VI.: 0721971109262

Page 13

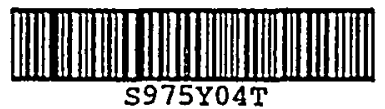

DCL Sample Name. . : 97C02898

DCL Report Group.. : 97C-0208-01

Analytical Results

\begin{tabular}{|c|c|c|c|c|c|c|c|}
\hline Analyte & $\begin{array}{c}\text { Date } \\
\text { Analyzed }\end{array}$ & MDL, & Result & Comment & Qual. & Dilution & CRDL \\
\hline 2-Chlorotoluene & 07-JUL-97 $15: 24$ & 0.150 & ND & & & 1 & 5 \\
\hline $1,3,5$-Trimethylbenzene & 07-JUL-97 $15: 24$ & 0.178 & $\mathrm{ND}$ & & & 1 & 5 \\
\hline 4-Chlorotoluene & $07-J U L-97 \quad 15: 24$ & 0.110 & ND & & & 1 & 5 \\
\hline tert-Butylbenzene & 07-JUL-97 15:24 & 0.130 & $\mathrm{ND}$ & & & 1 & 5 \\
\hline $1,2,4$-Trimethylbenzene & $07-J U L-97 \quad 15: 24$ & 0.130 & ND & & & 1 & 5 \\
\hline sec-Butylbenzene & 07-JUL-97 $15: 24$ & 0.141 & ND & & & 1 & 5 \\
\hline p-Isopropyltoluene & $07-\pi U L-97 \quad 15: 24$ & 0.170 & $\mathrm{ND}$ & & & 1 & 5 \\
\hline 1,3-Dichlorobenzene & $07-J U L-97 \quad 15: 24$ & 0.163 & $\mathrm{ND}$ & & & 1 & 5 \\
\hline 1,4-Dichlorobenzene & 07-JUL-97 15:24 & 0.178 & ND & & & 1 & 5 \\
\hline n-Butylbenzene & $07-\pi U L-97 \quad 15: 24$ & 0.191 & $\mathrm{ND}$ & & & 1 & 5 \\
\hline 1,2-Dichlorobenzene & $07-J U L-97 \quad 15: 24$ & 0.170 & $\mathrm{ND}$ & & & 1 & 5 \\
\hline 1,2-Dibromo-3-Chloropropane & 07-JUL-97 $15: 24$ & 1.16 & $\mathrm{ND}$ & & & 1 & 5 \\
\hline 1,2,4-Trichlorobenzene & $07-J U L-97 \quad 15: 24$ & 0.211 & ND & & & 1 & 5 \\
\hline Hexachlorobutadiene & 07-JUL-97 $15: 24$ & 0.341 & ND & & & 1 & 5 \\
\hline Naphthalene & 07-JUL-97 $15: 24$ & 0.174 & 5.5 & & & 1 & 5 \\
\hline $1,2,3$-Trichlorobenzene & 07-JUL-97 15:24 & 0.173 & ND & & & 1 & 5 \\
\hline Acetone & 07-JUL-97 15:24 & 2.88 & ND & & & 1 & 5 \\
\hline 2-Butanone & 07-JUL-97 $15: 24$ & 2.56 & 55. & & & 1 & 5 \\
\hline 4-Methyl-2-Pentanone & 07-JUL-97 $15: 24$ & 1.09 & ND & & $\cdot$ & 1 & 5 \\
\hline 2-Hexanone & 07-JUL-97 15:24 & 1.20 & ND & & & 1 & 5 \\
\hline
\end{tabular}

Surrogate Recoveries

\begin{tabular}{|l|c|c|c|}
\hline Aralyte & Result & $\begin{array}{c}\text { Spiked } \\
\text { Amount }\end{array}$ & $\begin{array}{c}\text { Percent } \\
\text { Recovery }\end{array}$ \\
\hline 1.2-Dichloroethane-d4 & 43.2 & 50.0 & 86.3 \\
\hline 4-Bromofluorobenzene & 44.0 & 50.0 & 88.0 \\
\hline Toluene-d8 & 49.0 & 50.0 & 97.9 \\
\hline
\end{tabular}




\section{DATA CHEM \\ A Sorenson Company}

FORM A (TYPE I) SINGLE METHOD ANALYSES
Form RLIMS63A-V1.3 072197110926

Page 14

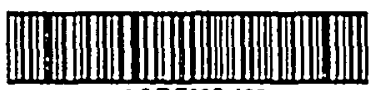

S975Y04V
Date Printed......: 21-JuL-97 11:10

Client Name......... SAIC

client Ref Number.... Not Provided

Sampling Site........ Not Provided

Release Number....... Not Provided

Date Received......: 27-JUN-97 00:00

DCL Preparation Group: Not Applicable Date Prepared....... Not Applicable Preparation Method... Not Applicable Aliquot Weight/Volume: $5.0 \mathrm{~mL}$ Net Weight/Volume.... : Not Required
Client Sample Name: NAP-R1-S2-A

DCL Sample Name. . : 97c02899

DCL Report Group.. : 97C-0208-01

Matrix......... WATER

Date Sampled.....: 24-JUN-97 00:00

Reporting Units...: ug/L

Report Basis.....: 团As Received $\square$ Dried

DCL Analysis Group: G976M004

Analysis Method...: 8260A

Instrument Type...: GC/MS VO

Instrument ID. . . : 5971-L

Column Type......: DB 624

[Q Primary

$\square$ Confinmation

Analytical Results

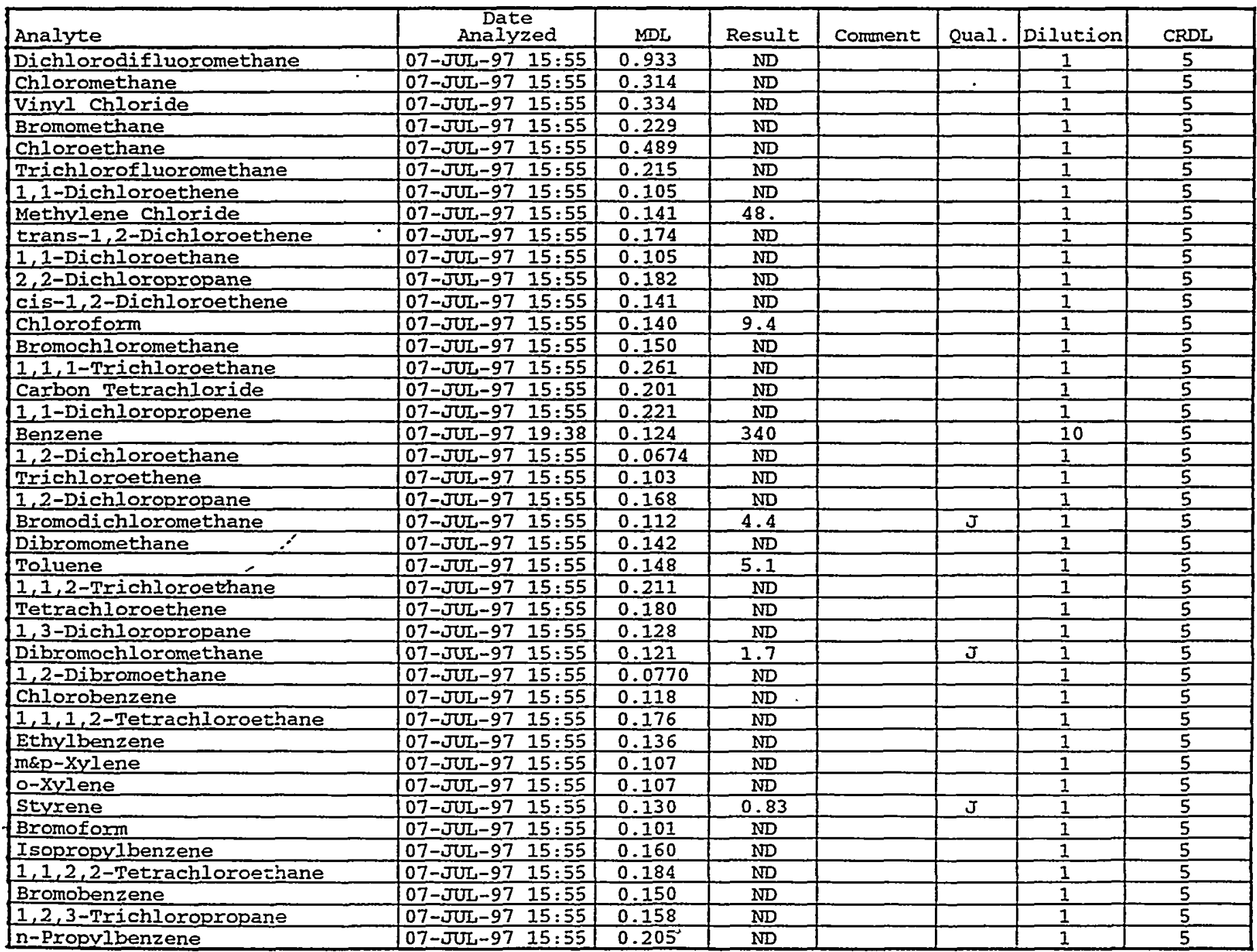




\section{DATA \\ FORM A (TYPE I) SINGLE METHOD ANALYSES} CHEM A Sorenson Company

SAMPLE ANALYSIS DATA SHEET
Form RLIMS63A-V1.3

0721971109263

Page 15

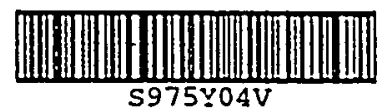

Date Printed.......: 21-JUL-97 11:10

DCL Sample Name... : 97C02899

Client Name........ SAIC

DCL Report Group... : 97C-0208-01

Analytical Results

\begin{tabular}{|c|c|c|c|c|c|c|c|}
\hline Analyte & $\begin{array}{c}\text { Date } \\
\text { Analyzed } \\
\end{array}$ & $\mathrm{MDL}$ & Result & Comment & Qual. & Dilution & CRDL \\
\hline 2-Chlorotoluene & 07-JUL-97 $15: 55$ & 0.150 & $\mathrm{ND}$ & & & 1 & 5 \\
\hline 1,3,5-Trimethylbenzene & 07-JUL-97 $15: 55$ & 0.178 & $\mathrm{ND}$ & & & 1 & 5 \\
\hline 4-Chlorotoluene & $07-\pi U L-97 \quad 15: 55$ & 0.110 & ND & & & 1 & 5 \\
\hline tert-Butylbenzene & $07-\pi U \pi-97 \quad 15: 55$ & 0.130 & ND & & & 1 & 5 \\
\hline $1,2,4$-Trimethylbenzene & 07-JUL-97 $15: 55$ & 0.130 & ND & & & 1 & 5 \\
\hline sec-Butylbenzene & $07-J u=-97 \quad 15: 55$ & 0.141 & ND & & & 1 & 5 \\
\hline P-I sopropyltoluene & $07-J U L-97 \quad 15: 55$ & 0.170 & ND & & & 1 & 5 \\
\hline 1,3-Dichlorobenzene & 07-JUL-97 $15: 55$ & 0.163 & ND & & & 1 & 5 \\
\hline 1,4-Dichlorobenzene & 07-JUL-97 $15: 55$ & 0.178 & ND & & & 1 & 5 \\
\hline n-Butylbenzene & 07-JUL-97 $15: 55$ & 0.191 & ND & & & 1 & 5 \\
\hline 1,2-Dichlorobenzene & $07-\pi U L-97 \quad 15: 55$ & 0.170 & ND & & & 1 & 5 \\
\hline 1,2-Dibromo-3-Chloropropane & $07-\pi U 1-97 \quad 15: 55$ & 1.16 & ND & & & 1 & 5 \\
\hline 1,2,4-Trichlorobenzene & 07-JUL-97 $15: 55$ & 0.211 & ND & & & 1 & 5 \\
\hline Hexachlorobutadiene & $07-\pi U L-97 \quad 15: 55$ & 0.341 & 0.61 & & $\mathbf{J}$ & 1 & 5 \\
\hline Naphthalene & $07-\pi U L-97 \quad 15: 55$ & 0.174 & 200 & & & $I$ & 5 \\
\hline $1,2,3$-Trichlorobenzene & 07-JUL-97 $15: 55$ & 0.173 & ND & & & 1 & 5 \\
\hline Acetone & $07-J U L-97 \quad 15: 55$ & 2.88 & $\mathrm{ND}$ & & & 1 & 5 \\
\hline 2-Butanone & $07-\pi U L-97 \quad 15: 55$ & 2.56 & ND & & & 1 & 5 \\
\hline 4-Methyl-2-Pentanone & 07-JUL-97 $15: 55$ & 1.09 & ND & & . & 1 & 5 \\
\hline 2-Fexanone & 07-JUL-97 15:55 & 1.20 & ND & & & 1 & 5 \\
\hline
\end{tabular}

Tentatively Identified Compound Results

\begin{tabular}{|c|c|c|c|c|c|}
\hline Analyte (Retention Time) & $\begin{array}{c}\text { Date } \\
\text { Analyzed } \\
\end{array}$ & Result & Comment & Qual. & Dilution \\
\hline Indene $(22.74)$ & $07-\pi U L-97 \quad 15: 55$ & 6.5 & & $\mathrm{~J}$ & 1 \\
\hline
\end{tabular}

\section{Surrogate Recoveries}

\begin{tabular}{|l|c|c|c|}
\hline Analyte & Result & $\begin{array}{c}\text { Spiked } \\
\text { Amount }\end{array}$ & $\begin{array}{c}\text { Percent } \\
\text { Recovery }\end{array}$ \\
\hline 1,2 -Dichloroethane-d4 & 43.2 & 50.0 & 86.5 \\
\hline 4-Bromofluorobenzene & 45.1 & 50.0 & 90.1 \\
\hline Toluene-d8 & 48.5 & 50.0 & 97.0 \\
\hline
\end{tabular}




\section{DATA CHEM \\ A Sorenson Company}

FORM A (TYPE I)

SINGLE METHOD ANALYSES

SAMPLE ANALYSIS DATA SHEET
Form RIIMS63A-V1:

Page 16

$072397171413^{\circ}$

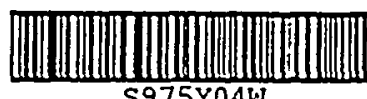

S975Y046
Date Printed.......: 23-JUL-97 17:12

Client Name......... SAIC

Client Ref Number.... Not Provided Sampling site....... Not Provided

Release Number....... Not Provided

Date Received.....: 27-JUN-97 00:00

DCL Preparation Group: Not Applicable Date Prepared....... Not Applicable Preparation Method... : Not Applicable Aliquot Weight/Volume: $5.0 \mathrm{~mL}$ Net Weight/Volume... . Not Required
Client Sample Name: NAP-R1-S2-B

DCL Sample Name...: 97C02900

DCL Report Group.. : 97C-0208-01

Matrix........ WATER

Date Sampled.....: 24-JUN-97 00:00

Reporting Units...: ug/L

Report Basis.....: $[$ As Received $\square$ Dried

DCL Analysis Group: G976M004

Analysis Method...: 8260A

Instrument Type...: GC/MS vo

Instrument ID. . . . : 5971-L

Colunn Type......: DB 624

图 Drimary

$\square$ Confirmation

Analytical Results

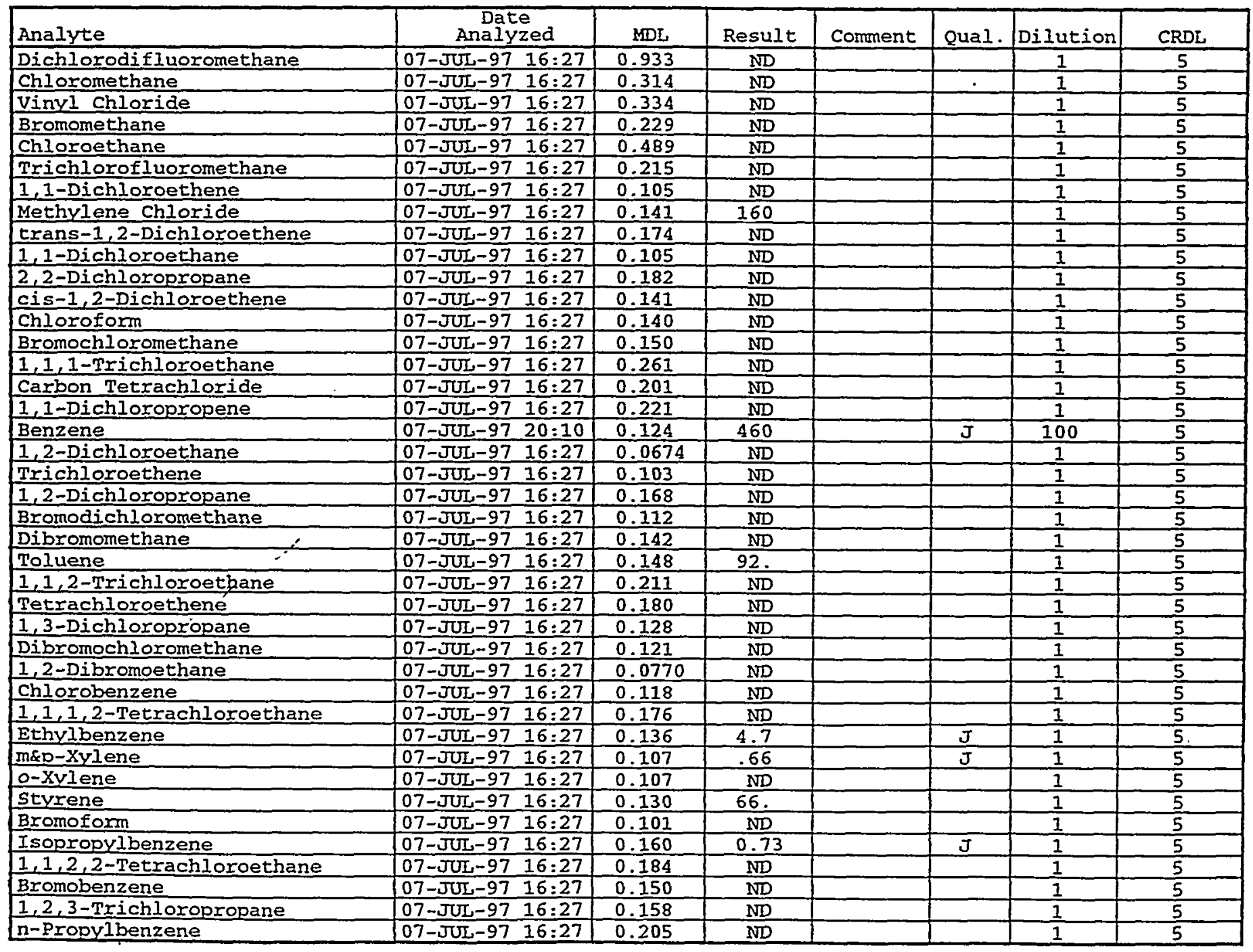

021
960 West LeVoy Drive / Salt Lake City, Utah 84123-2547 Phone (801) 266-7700 FAX (801) 268-9992
Web Page: www.datachem.com

E-mail: lab@datachem.com 


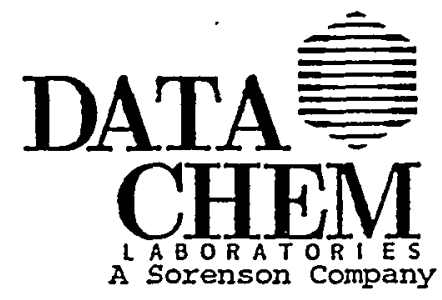

Date Printed.......: 21-JUL-97 11:10

Client Name......... SAIC
FORM A (TYPE I)

SINGLE METHOD ANALYSES

SAMPLE ANALYSIS DATA SHEET
Form RLIMS63A-V1. 072197110926 .

Page 17

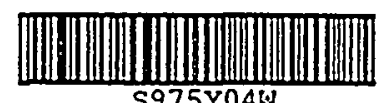

DCL Sample Name... : 97C02900

DCL Report Group.. : 97C-0208-01

Analytical Results

\begin{tabular}{|c|c|c|c|c|c|c|c|}
\hline Analyte & $\begin{array}{c}\text { Date } \\
\text { Analyzed }\end{array}$ & MDL & Result & Comment & Qual. & Dilution & CRDL \\
\hline 2-Chlorotoluene & $07-\pi \pi L-97 \quad 16: 27$ & 0.150 & ND & & & 1 & 5 \\
\hline $1,3,5$-Trimethy] benzene & $07-J U L-97 \quad 16: 27$ & 0.178 & ND & & & 1 & 5 \\
\hline 4-Chlorotoluene & \begin{tabular}{|cc}
$07-\pi \pi-97$ & $16: 27$
\end{tabular} & 0.110 & ND & & & 1 & 5 \\
\hline tert-ButyIbenzene & $07-J U L-97 \quad 16: 27$ & 0.130 & ND & & & 1 & 5 \\
\hline $1,2,4$-Trimethylbenzene & $07-J U L-97 \quad 16: 27$ & 0.130 & ND & & & 1 & 5 \\
\hline sec-Butylbenzene & $07-\pi U L-97 \quad 16: 27$ & 0.141 & ND & & & 1 & 5 \\
\hline p-Isopropyltoluene & $07-\pi U L-97 \quad 16: 27$ & 0.170 & ND & & & 1 & 5 \\
\hline 1,3-Dichlorobenzene & \begin{tabular}{|ll}
$07-J U L-97$ & $16: 27$
\end{tabular} & 0.163 & ND & & & $\overline{1}$ & 5 \\
\hline 1,4-Dichlorobenzene & $07-J U L-97 \quad 16: 27$ & 0.178 & ND & & & 1 & 5 \\
\hline n-Butylbenzene & $07-\pi U_{L}-97 \quad 16: 27$ & 0.191 & ND & & & 1 & 5 \\
\hline 1,2-Dichlorobenzene & $07-J U L-97 \quad 16: 27$ & 0.170 & $\mathrm{ND}$ & & & 1 & 5 \\
\hline 1,2-Dibromo-3-Chloropropane & $\begin{array}{ll}07-\pi U L-97 & 16: 27\end{array}$ & 1.16 & $\mathrm{ND}$ & & & $\overline{1}$ & 5 \\
\hline 1,2,4-Trichlorobenzene & $07-J U L-97 \quad 16: 27$ & 0.211 & ND & & & 1 & 5 \\
\hline Hexachlorobutadiene & $07-\pi U L-97 \quad 16: 27$ & 0.341 & $\mathrm{ND}$ & & & 1 & 5 \\
\hline Naphthalene & $07-\pi U L-97 \quad 20: 10$ & 0.174 & 710 & & & 100 & 5 \\
\hline 1,2,3-Trichlorobenzene & $07-J U L-97 \quad 16: 27$ & 0.173 & $\mathrm{ND}$ & & & 1 & 5 \\
\hline Acetone & $07-\pi U L-97 \quad 16: 27$ & 2.88 & ND & & & 1 & 5 \\
\hline 2-Butanone & $07-\pi \pi L-97 \quad 16: 27$ & 2.56 & ND & & & 1 & 5 \\
\hline 4-Methyl-2-Pentanone & $07-\pi U L-97 \quad 16: 27$ & 1.09 & ND & & & 1 & 5 \\
\hline 2-Hexanone & $07-\pi U L-97 \quad 16: 27$ & 1.20 & $\mathrm{ND}$ & & & 1 & 5 \\
\hline
\end{tabular}

Tentatively Identified Compound Results

\begin{tabular}{|c|c|c|c|c|c|}
\hline Analyte (Retention Time) & $\begin{array}{c}\text { Date } \\
\text { Analyzed }\end{array}$ & Result & Comment & Qual. & Dilution \\
\hline Indene $(22.74)$ & $07-\pi \pi-97 \quad 16: 27$ & 43. & & $\mathrm{~J}$ & 1 \\
\hline
\end{tabular}

\section{Surrogate Recoveries}

\begin{tabular}{|l|c|c|c|}
\hline Analyte & Result & $\begin{array}{c}\text { Spiked } \\
\text { Amount }\end{array}$ & $\begin{array}{c}\text { Percent } \\
\text { Recovery }\end{array}$ \\
\hline 1,2 -Dichloroethane-d4 & 41.8 & 50.0 & 83.7 \\
\hline 4-Bromofluorobenzene & 43.0 & 50.0 & 86.0 \\
\hline Toluene-d8 & 47.1 & 50.0 & 94.3 \\
\hline
\end{tabular}




\section{DATA

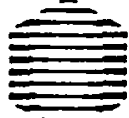 \\ FORM A (TYPE I) SINGLE METHOD ANALYSES} CHEM

A Sorenson Company
SAMPLE ANALYSIS DATA SHEET
Form RLIMS63A-V1.3 0721971109263:

Page 18

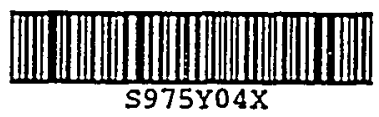

Date Printed....... 21- $\pi \pi-97$ 11:10

client Name........ SAIC

Client Ref Number....: Not Provided Sampling site....... Not Provided Release Number....... Not Provided

Date Received......: 27-JUN-97 00:00

DCL Preparation Group: Not Applicable Date Prepared....... Not Applicable Preparation Mechod... Not Applicable Aliquot Weight/Volume: $5.0 \mathrm{~mL}$ Net Weight/Volume.... Not Required
Client Sample Name: NAP-RI-S4

DCL Sample Name. . : 97C02901

DCI Report Group. . : 97C-0208-01

Matrix........ : WATER

Date Sampled.....: 25-JUN-97 00:00

Reporting Units...: ug/L

Report Basis...... : 因As Received $\square$ Dried

DCL Analysis Group: G976M004

Analysis Method...: 8260A

Instrument Type...: GC/MS Vo

Instrument ID. . . : 5971-L

Column TYpe......: DB 624

[X Primary

$\square$ Confirmation

Analytical Results

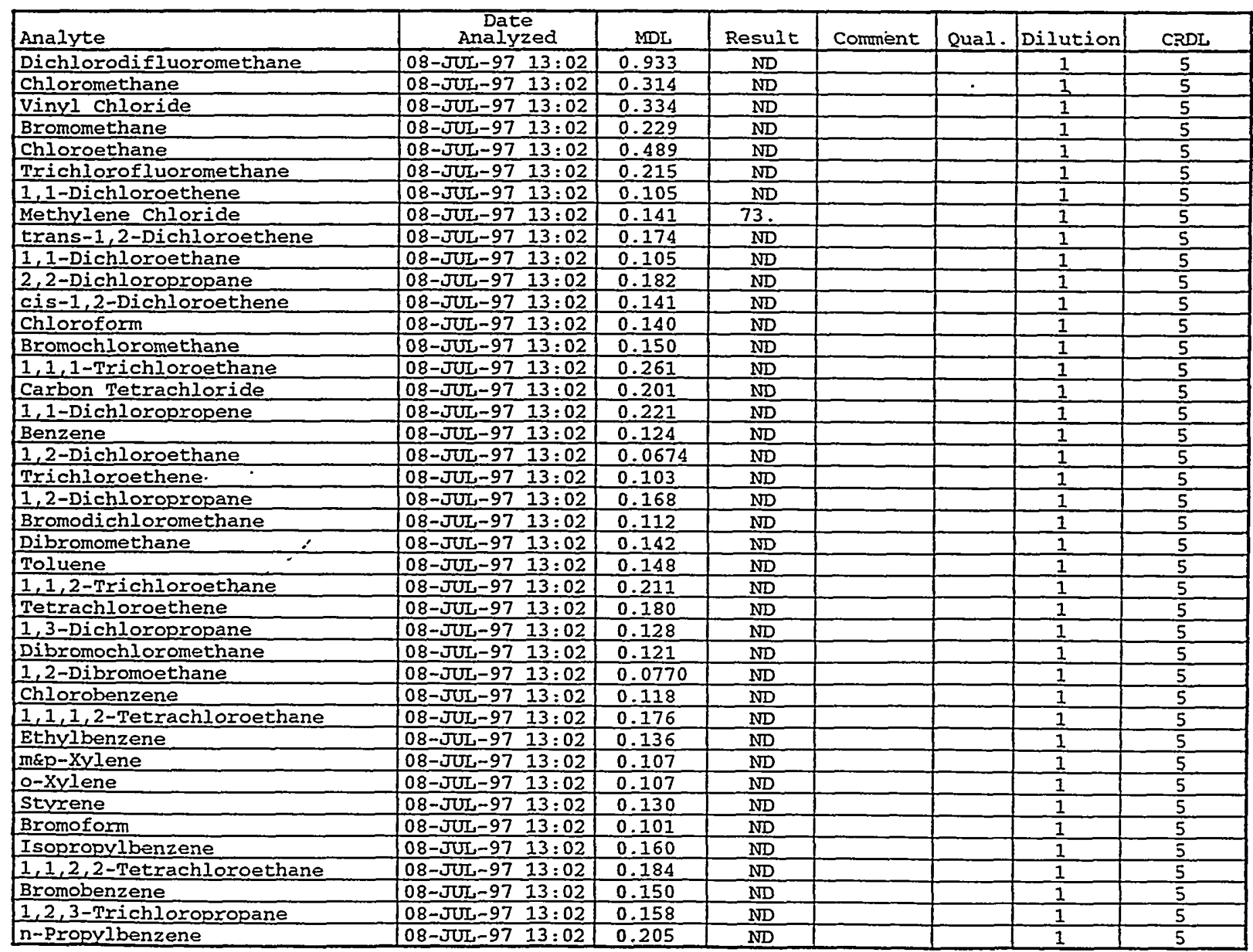




\section{DATA CHEM \\ $A$ Soxenson Company}

FORM A (TYPE I)

SINGLE METHOD ANALYSES

SAMPLE ANALYSIS DATA SHEET

Form RIIMS63A-V1.:

Page 19

$072197110926 ?$

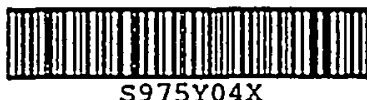

Date Printed........: 21-JUL-97 11:10

DCL Sample Name...: 97C02901

client Name......... SAIC

DCL Report Group... : 97C-0208-01

Analytical Results

\begin{tabular}{|c|c|c|c|c|c|c|c|}
\hline Analyte & $\begin{array}{c}\text { Date } \\
\text { Analyzed }\end{array}$ & MDL & Result & Comment & Qual. & Dilution & CRDL \\
\hline 2-Chlorotoluene & $08-\pi U L-97 \quad 13: 02$ & 0.150 & $\mathrm{ND}$ & & & 1 & 5 \\
\hline 1,3,5-Trimethylbenzene & $08-\pi U L-97 \quad 13: 02$ & 0.178 & ND & & & 1 & 5 \\
\hline 4-Chlorotoluene & $08-\pi U L-97 \quad 13: 02$ & 0.110 & $\mathrm{ND}$ & & & 1 & 5 \\
\hline 1,2,4-Trimethylbenzene & $08-\mathrm{JUL}-97 \quad 13: 02$ & 0.130 & ND & & & 1 & 5 \\
\hline sec-Butylbenzene & $08-\pi U L-97 \quad 13: 02$ & 0.141 & ND & & & 1 & 5 \\
\hline p-Isopropyltoluene & $08-J U L-97 \quad 13: 02$ & 0.170 & ND & & & 1 & 5 \\
\hline n-Butylbenzene & $08-\pi U L-97 \quad 13: 02$ & 0.191 & ND & & & 1 & 5 \\
\hline 1,2-Dichlorobenzene & $08-J U L-97 \quad 13: 02$ & 0.170 & ND & & & 1 & 5 \\
\hline 1,2-Dibromo-3-Chloropropane & $08-\pi U L-97 \quad 13: 02$ & 1.16 & ND & & & 1 & 5 \\
\hline 1,2,4-Trichlorobenzene & $08-\sqrt{U L}-97 \quad 13: 02$ & 0.211 & ND & & & 1 & 5 \\
\hline Hexachlorobutadiene & $08-\pi U L-97 \quad 13: 02$ & 0.341 & ND & & & 1 & 5 \\
\hline Naphthalene & 08 -JUL-97 $13: 02$ & 0.174 & ND & & & 1 & 5 \\
\hline 1,2,3-Trichlorobenzene & $08-\pi U L-97 \quad 13: 02$ & 0.173 & ND & & & 1 & 5 \\
\hline
\end{tabular}

\section{Surrogate Recoveries}

\begin{tabular}{|l|c|c|c|}
\hline Analyte & Result & $\begin{array}{c}\text { Soiked } \\
\text { Amount }\end{array}$ & $\begin{array}{c}\text { Percent } \\
\text { Recovery }\end{array}$ \\
\hline $1,2-$ Dichloroethane-d4 & 46.6 & 50.0 & 93.2 \\
\hline 4-Bromofluorobenzene & 46.3 & 50.0 & 92.7 \\
\hline Toluene-d8 & 48.5 & 50.0 & 97.1 \\
\hline
\end{tabular}




\section{DATA} CHEM

$A^{L}$ Sorenson Company

\section{SAMPLE ANALYSIS DATA SHEET}

Form RLIMS63A-V1.3

0721971109263:

Page 20

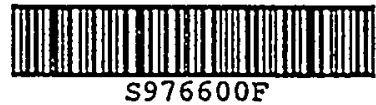

Client Sample Name: NAP-R1-S4

DCL Sample Name... : 97C02901MS

DCL Report Group.. : 97C-0208-01

Matrix.........: WATER

Date Sampled.....: 25-JUN-97 00:00

Reporting Units... : ug/L

Report Basis...... : 冈 As Received $\square$ Dried

DCL Analysis Group: G976M004

Analysis Method...: 8260A

Instrument Type...: GC/MS vo

Instrument ID....: 5971-L

Column Type......: DB 624

[ive Primary

$\square$ Confirmation

Analytical Results

\begin{tabular}{|c|c|c|c|c|c|c|c|}
\hline Analyte & $\begin{array}{c}\text { Date } \\
\text { Analyzed }\end{array}$ & $\mathrm{MDL}$ & Result & Conment & Qual. & Dilution & CRDL \\
\hline 1,1-Dichloroethene & $07-\pi U L-97 \quad 17: 31$ & 0.105 & 45. & & & 1 & 5 \\
\hline Benzene & 07-JUL-97 $17: 31$ & 0.124 & 42 . & & . & 1 & 5 \\
\hline Trichloroethene & $07-J U L-97 \quad 17: 31$ & 0.103 & 47. & & & 1 & 5 \\
\hline Toluene & $07-\overline{J U L}-97 \quad 17: 31$ & 0.148 & 43. & & & 1 & 5 \\
\hline Chlorobenzene & 07-जUL-97 $17: 31$ & 0.118 & 44. & & & 1 & 5 \\
\hline
\end{tabular}

\section{Surrogate Recoveries}

\begin{tabular}{|l|c|c|c|}
\hline Analyte & Result & $\begin{array}{c}\text { Spiked } \\
\text { Amount }\end{array}$ & $\begin{array}{c}\text { Percent } \\
\text { Recovery }\end{array}$ \\
\hline 1,2-Dichloroethane-d4 & 42.8 & 50.0 & 85.7 \\
\hline 4-Bromofluorobenzene & 45.2 & 50.0 & 90.4 \\
\hline Toluene-d8 & 48.5 & 50.0 & 96.9 \\
\hline
\end{tabular}




\section{DATA

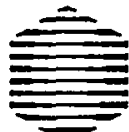 \\ FORM A (TYPE I) SINGLE METHOD ANALYSES} CHEM A Sorenson Company
Form RLIMS63A-V1.: $072197110926 \vdots$ Page 21

SAMPLE ANALYSIS DATA SHEET
S976600G
Date Printed.......: 21-JUL-97 11:10

Client Name......... SAIC

Client Ref Number.... Not Provided Sampling Site........ Not Provided Release Number....... : Not Provided

Date Received......: 27-JUN-97 00:00

DCI Preparation Group: Not Applicable Date Prepared....... Not Applicable Preparation Method...: Not Agplicable Aliquot Weight/Volume: $5.0 \mathrm{~mL}$ Net Weight/Volume.... Not Required
Client Sample Name: NAP-RI-S4 DCL Sample Name...: 97C02901MSD DCL Report Group..: 97C-0208-01

Matrix......... WATER

Date Sampled.....: 25-JUN-97 00:00

Reporting Units... : ug/L

Report Basis..... : . As Received $\square$ Dried

DCL Analysis Group: G976M004

Analysis Method...: $8260 \mathrm{~A}$

Instrument Type...: GC/MS Vo

Instrument ID....: 5971-L

Column Type.....: DB 624

[] Primary

$\square$ Confirmation

Analytical Results

\begin{tabular}{|c|c|c|c|c|c|c|c|}
\hline Analyte & $\begin{array}{c}\text { Date } \\
\text { Analyzed } \\
\end{array}$ & $\mathrm{MDL}$ & Result & Comment & Qual. & Dilution & CRDL \\
\hline 1,1-Dichloroethene & $07-J U L-97 \quad 18: 02$ & 0.105 & 46. & & & 1 & 5 \\
\hline Benzene & $07-\pi U L-97 \quad 18: 02$ & 0.124 & 42. & & . & 1 & 5 \\
\hline Trichloroethene & $07-J u L-97 \quad 18: 02$ & 0.103 & 48. & & & 1 & 5 \\
\hline Toluene & \begin{tabular}{|ll}
$07-J U L-97$ & $18: 02$ \\
\end{tabular} & 0.148 & 44. & & & 1 & 5 \\
\hline Chlorobenzene & $07-\pi U_{L}-97 \quad 18: 02$ & 0.118 & 45 . & & & 1 & 5 \\
\hline
\end{tabular}

\section{Surrogate Recoveries}

\begin{tabular}{|l|c|c|c|}
\hline Analyte & Result & $\begin{array}{c}\text { Spiked } \\
\text { Amount }\end{array}$ & $\begin{array}{c}\text { Percent } \\
\text { Recovery }\end{array}$ \\
\hline 1.2-Dichloroethane-c4 & 42.5 & 50.0 & 85.1 \\
\hline 4-Bromofluorobenzene & 46.7 & 50.0 & 93.4 \\
\hline Toluene-d8 & 48.6 & 50.0 & 97.2 \\
\hline
\end{tabular}




\section{DATA CHEM A Sorenson Company}

FORM B (TYPE I)

SINGLE MEIHOD ANALYSES

QUALITY CONTROL DATA SHEET

IAABORATORY CONTROL SAMPLE (LCS)
Form RLIMS63B-V1.3

07219711092632

Page 22

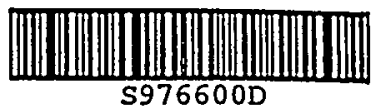

Client Name........ SAIC

Release Number....... Not Provided

Matrix.......... WATER

Reporting Units...... ug/L

DCI Preparation Group: Not Applicable Date Prepared........ Not Applicable Preparation Method... : Not Applicable
DCL Sample Name...: QC-136531-1

Date Printed.....: 21-JU-97 11:10

DCL Analysis Group: G976M004 Analysis Method...: SW 8260 Instrument Type...: GC/MS Vo Instrument ID. . . : 5971-L Column Type......: DB 624

$$
\therefore \quad \square \text { Confirmation }
$$

QC Limit Type..... : Method

Analytical Results

\begin{tabular}{|c|c|c|c|c|c|c|}
\hline Analyte & $\begin{array}{c}\text { Date } \\
\text { Analyzed } \\
\end{array}$ & Target & Result & $\begin{array}{c}\text { Percent } \\
\text { Recovery }\end{array}$ & $\begin{array}{c}Q C \\
\text { Limits }\end{array}$ & $\begin{array}{l}\text { QC } \\
\text { Flag }\end{array}$ \\
\hline 1,1-Dichloroethene & $07-\pi U L-97 \quad 10: 07$ & 50.0 & 47.3 & 94.5 & $1.00 / 234$. & \\
\hline Benzene & $07-\pi U t-97 \quad 10: 07$ & 50.0 & 42.8 & 85.7 & $37.0 / 151$. & \\
\hline Chlorobenzene & $07-\pi U L-97 \quad 10: 07$ & 50.0 & 46.0 & 91.9 & $37.0 / 160$. & \\
\hline Trichloroethene & $107-\pi 04-97 \quad 10: 07$ & 50.0 & 48.7 & 97.4 & $71.0 / 157$ & \\
\hline
\end{tabular}




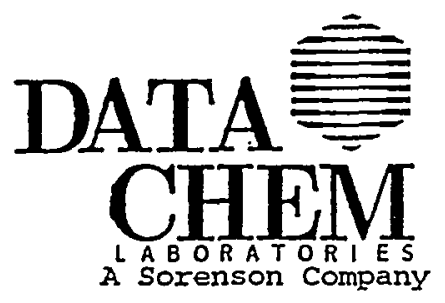

Client Name........: SAIC

Release Number....... Not Provided

Matrix.......... : WATER

Reporting Units.....: ug/L

DCI Preparation Group: Not Applicable Date Prepared........ Not Applicable Preparation Method... : Not Applicable

Analytical Results

\begin{tabular}{|c|c|c|c|c|c|c|c|}
\hline Analyte & $\begin{array}{c}\text { Date } \\
\text { Analyzed }\end{array}$ & $\begin{array}{l}\text { Sample } \\
\text { Result }\end{array}$ & $\begin{array}{l}\text { Spiked } \\
\text { Result }\end{array}$ & $\begin{array}{l}\text { Spike } \\
\text { Added }\end{array}$ & $\begin{array}{l}\text { Percent } \\
\text { Recovery }\end{array}$ & $\begin{array}{c}Q C \\
\text { Limits }\end{array}$ & $\begin{array}{l}\text { QC } \\
\text { Elag }\end{array}$ \\
\hline 1,1-Dichloroethene & $\mid 07-\pi U L-97 \quad 17: 31$ & ND & 45.3 & 50.0 & 90.5 & $1.00 / 234$. & \\
\hline Benzene & $\mid 07-J U L-97 \quad 17: 31$ & ND & 41.6 & 50.0 & 83.2 & $37.0 / 151$. & \\
\hline Chlorobenzene & $07-J U L-97 \quad 17: 31$ & ND & 43.8 & 50.0 & 87.7 & $37.0 / 160$ & \\
\hline Toluene & $\mid \begin{array}{ll}07-\pi U L-97 & 17: 31\end{array}$ & ND & 42.8 & 50.0 & 85.7 & $47.0 / 150$. & \\
\hline Trichloroethene & $\mid 07-J U L-97 \quad 17: 31$ & ND & 47.2 & 50.0 & 94.3 & $71.0 / 157$ & \\
\hline
\end{tabular}

\section{QUALITY CONTROI DATA SHEET} MATRTX SPIKE SAMPLE MATRIX SPIKE DUPLICATE SAMPLE
Form RLIMS63F-V1.3

Page 23

07219711092632

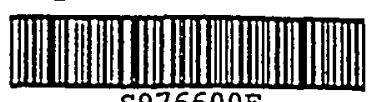

DCL Sample Name... : 97C02901MS

Date Printed.....: 21-JUL-97 11:10

DCL Analysis Group: G976M004

Analysis Method...: SW 8260

Instrument Type...: GC/MS Vo

Instrument ID. . . . : 5971-L

Column Type....... DB 624

$$
\therefore \quad \square \text { Primary }
$$

QC Limit Type.....: Method

Analytical Results

\begin{tabular}{|c|c|c|c|c|c|c|c|c|}
\hline Analyte & $\begin{array}{c}\text { Date } \\
\text { Analyzed }\end{array}$ & $\begin{array}{c}\text { Duplicate } \\
\text { Result }\end{array}$ & $\begin{array}{l}\text { Percent } \\
\text { Recovery }\end{array}$ & Mean & Range & RPD & $\begin{array}{c}\overline{Q C} \\
\text { Limits }\end{array}$ & $\begin{array}{l}\text { QC } \\
\text { Flag }\end{array}$ \\
\hline 1,1-Dichloroethene & 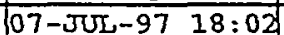 & 46.4 & 92.7 & 45.8 & 1.12 & 2.4 & $0.00 / 50.0$ & \\
\hline Benzene & $07-\pi U L-97 \quad 18: 02$ & 42.4 & 84.8 & 42.0 & 0.800 & 1.9 & $0.00 / 50.0$ & \\
\hline Chlorobenzene & 07-JUL-97 $18: 02$ & 45.2 & 90.3 & 44.5 & 1.33 & 3.0 & $0.00 / 50.0$ & \\
\hline Toluene & $107-\mathrm{JUL}-97 \quad 18: 02$ & 44.4 & 88.7 & 43.6 & 1.52 & 3.5 & $0.00 / 50.0$ & \\
\hline Trichloroethene & $\mid 07-\pi U-97 \quad 18: 02$ & 48.5 & 97.0 & 47.8 & 1.35 & 2.8 & $0.00 / 50.0$ & \\
\hline
\end{tabular}




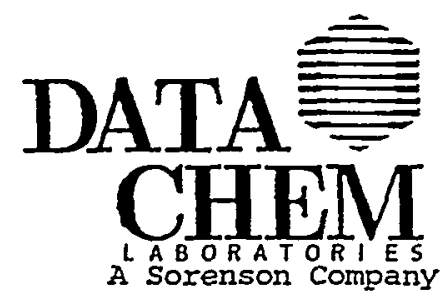

client Name........: SAIC

Release Number....... Not Provided

Matrix........... WATER

Reporting Units.....: ug/L

Surrogate Recoveries

\begin{tabular}{|c|c|c|c|c|c|c|c|c|c|}
\hline Surr. ID & \multicolumn{3}{|c|}{$\frac{1,2 \text {-Dichloroethane-d4 }}{16.0 / 114}$} & \multicolumn{3}{|c|}{$\frac{\text { 4-Bromofluorobenzene }}{86.0 / 115}$} & \multicolumn{3}{|c|}{$\frac{\text { Toluene-d8 }}{88.0 / 110 .}$} \\
\hline $\begin{array}{l}\text { DCL Sample } \\
\text { Number }\end{array}$ & $\begin{array}{l}\text { Analyce } \\
\text { Result }\end{array}$ & $\begin{array}{l}\text { Spiked } \\
\text { Amount }\end{array}$ & $\begin{array}{c}8 \\
\text { Rec. }\end{array}$ & $\begin{array}{l}\text { Analyce } \\
\text { Result }\end{array}$ & $\begin{array}{l}\text { Spiked } \\
\text { Amount }\end{array}$ & Rec. & $\begin{array}{l}\text { Analyce } \\
\text { Result }\end{array}$ & $\begin{array}{l}\text { Spikea } \\
\text { Amount }\end{array}$ & $\operatorname{Rec}$ \\
\hline 97002896 & 41.6 & 50.0 & 83.3 & 45.3 & 50.0 & 90.5 & 47.9 & 50.0 & 95.9 \\
\hline 97002897 & 43.0 & 50.0 & 86.0 & 46.8 & 50.0 & 93.6 & 49.2 & 50.0 & 98.3 \\
\hline $97 C 02898$ & 43.2 & 50.0 & 86.3 & 44.0 & 50.0 & 88.0 & 49.0 & 50.0 & 97.9 \\
\hline $97 C 02899$ & 43.2 & 50.0 & 86.5 & 45.1 & 50.0 & 90.1 & 48.5 & 50.0 & 97.0 \\
\hline $97 \mathrm{CO} 2900$ & 41.8 & 50.0 & 83.7 & 43.0 & 50.0 & 86.0 & 47.1 & 50.0 & 94.3 \\
\hline 97002901 & 46.6 & 50.0 & 93.2 & 46.3 & 50.0 & 92.7 & 48.5 & 50.0 & 97.1 \\
\hline $97 \mathrm{C} 02901 \mathrm{MS}$ & 42.8 & 50.0 & 85.7 & 45.2 & 50.0 & 90.4 & 48.5 & 50.0 & 96.9 \\
\hline $97 \mathrm{CD} 2901 \mathrm{MSD}$ & 42.5 & 50.0 & 85.1 & 46.7 & 50.0 & 9 & 48.6 & 50.0 & 97.2 \\
\hline 97002872 & 42.5 & 50.0 & 85.0 & 46.4 & 50.0 & 92.7 & 47.8 & 50.0 & 95.6 \\
\hline $97 \mathrm{U} 02873$ & 40.9 & 50.0 & 81.8 & 44.7 & 50.0 & 89.4 & 46.8 & 50.0 & 93.5 \\
\hline 97002874 & 41.8 & 50.0 & 83.5 & 45.8 & 50.0 & 91.6 & 47.2 & 50.0 & 94.5 \\
\hline 97002951 & 41.2 & 50.0 & 82.4 & 45.3 & 50.0 & 90.6 & 46.7 & 50.0 & 93.5 \\
\hline 97002952 & 41.5 & 50.0 & 83.0 & 45.5 & 50.0 & 91.1 & 47.3 & 50.0 & 94.6 \\
\hline $97 U 02953$ & 41.3 & 50.0 & 82.6 & 45.1 & 50.0 & 90.1 & 46.3 & 50.0 & 92.7 \\
\hline 97002954 & 41.8 & 50.0 & 83.6 & 45.6 & 50.0 & 91.2 & 47.4 & 50.0 & 94.7 \\
\hline $3 L-136531-1$ & 42.2 & 50.0 & 84.4 & 44.8 & 50.0 & 89.7 & 46.9 & 50.0 & 93.7 \\
\hline$B L-136531-2$ & 47.4 & 50.0 & 94.9 & 47.1 & 50.0 & 94.1 & 49.2 & 50.0 & 98.4 \\
\hline OC-136531-1 & 41.9 & 50.0 & 83.9 & 44.6 & 50.0 & 89.3 & 46.5 & 50.0 & 92.9 \\
\hline
\end{tabular}

Form RLIMS63G-V1.3

Page 24

07219711092632

\section{QUALITY CONITROI DATA SHEET SURROGATE SUMMARY}

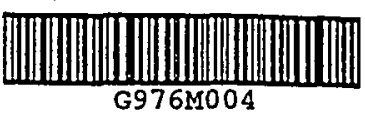

Date Printed.....: 21-JUL-97 11:10

DCL Analysis Group: G976M004

Analysis Method...: Si 8260

DCL Prep Groip.... Not Applicable Preparation Nethod: Not Applicable

QC Iimit Type. . . . : Method 
Form 4 Equivalut

DataChem Laboratories

LIMS - Samole Master System

Analysis Group Report

Date: 20-JUL-1997 12:24

User: COLEMAN

Analysis Run Name: G976M004

Samples: 18

\begin{tabular}{|c|c|c|c|}
\hline Pos & $\begin{array}{l}\text { Laboratory } \\
\text { Sample Name }\end{array}$ & $\begin{array}{l}\text { Field Sample } \\
\text { Name } 1\end{array}$ & $\begin{array}{l}\text { Field Sample } \\
\text { Name } 2\end{array}$ \\
\hline 1 & BL-136531-1 & BL-136531-1 & \\
\hline 2 & BL-136531-2 & BL-136531-2 & \\
\hline & QC-136531-1 & QC $-136531-1$ & \\
\hline 4 & $97 C 02896$ & NAP $-\mathrm{R} 2-\mathrm{S} 2-\mathrm{A}$ & \\
\hline 5 & $97 C 02897$ & NAP-R2-S2-B & \\
\hline & $97 C 02898$ & NAP-R2-S4 & \\
\hline 7 & $97 C 02899$ & $\mathrm{NAP}-\mathrm{R} 1-\mathrm{S} 2-\mathrm{A}$ & \\
\hline B & $97 C 02900$ & NAP-R1-S2-B & \\
\hline 9 & $97 C 02901$ & NAP-R1-S4 & \\
\hline 10 & $97 \mathrm{CO} 2901 \mathrm{MS}$ & NAP-R1-S4 & \\
\hline 11 & 97C02901MSD & NAP-R1-S4 & \\
\hline 12 & 97U02872 & AW3TRIP02 & 00059 \\
\hline 13 & 97002873 & AW3RNSWO3 & 00068 \\
\hline 14 & $97 U 02874$ & AW3FBLK02 & 00070 \\
\hline 15 & 97002951 & AW3TRIP03 & 00071 \\
\hline 16 & 97U02952 & AW3RNSW0 4 & 00076 \\
\hline 17 & 97U02953 & AW2TRIP04 & 00092 \\
\hline & 97002954 & AW2 RNSHO 4 & 00093 \\
\hline
\end{tabular}

Page: 1

RLIMS15-V 1.2

Group ID: G976M004

\begin{tabular}{lll} 
Laboratory & $\begin{array}{l}\text { Laboratory } \\
\text { Sample ID }\end{array}$ & $\begin{array}{l}\text { Accnt. } \\
\text { Number }\end{array}$ \\
\hline S976600C & $97 \mathrm{C}-0208-01$ & 03006 \\
S9767001 & $97 \mathrm{C}-0208-01$ & 03006 \\
S976600D & $97 \mathrm{C}-0208-01$ & 03006 \\
S975Y04R & $97 \mathrm{C}-0208-01$ & 03006 \\
S975Y04S & $97 \mathrm{C}-0208-01$ & 03006 \\
S975Y04T & $97 \mathrm{C}-0208-01$ & 03006 \\
S975Y04V & $97 \mathrm{C}-0208-01$ & 03006 \\
S975Y04W & $97 \mathrm{C}-0208-01$ & 03006 \\
S975Y04X & $97 \mathrm{C}-0208-01$ & 03006 \\
S976600E & $97 \mathrm{C}-0208-01$ & 03006 \\
S976600G & $97 \mathrm{C}-0208-01$ & 03005 \\
S975S091 & $97 \mathrm{U}-0194-06$ & 03402 \\
S975S092 & $97 \mathrm{U}-0194-06$ & 03402 \\
S975S093 & $97 \mathrm{U}-0194-06$ & 03402 \\
S975V08M & $97 \mathrm{U}-0199-07$ & 03402 \\
S975V08N & $97 \mathrm{U}-0199-07$ & 03402 \\
S975V08P & $97 \mathrm{U}-0199-07$ & 03402 \\
S975V080 & $97 \mathrm{U}-0199-07$ & 03402
\end{tabular}


Form S Equivalent

$\mathrm{BFB}$

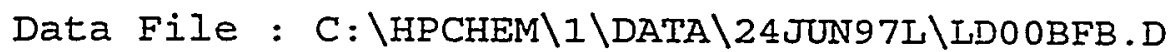

Acq Time : 06/24/97 14:05

Sample : $50 \mathrm{ng} \mathrm{BFB}$

Misc : 2.0 uL of 39 IS7676

Operazor: CQC

Inst : 5971-L

Multiplr: 1.00

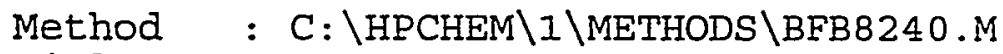
Title :

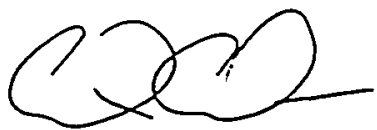

6.24. 97

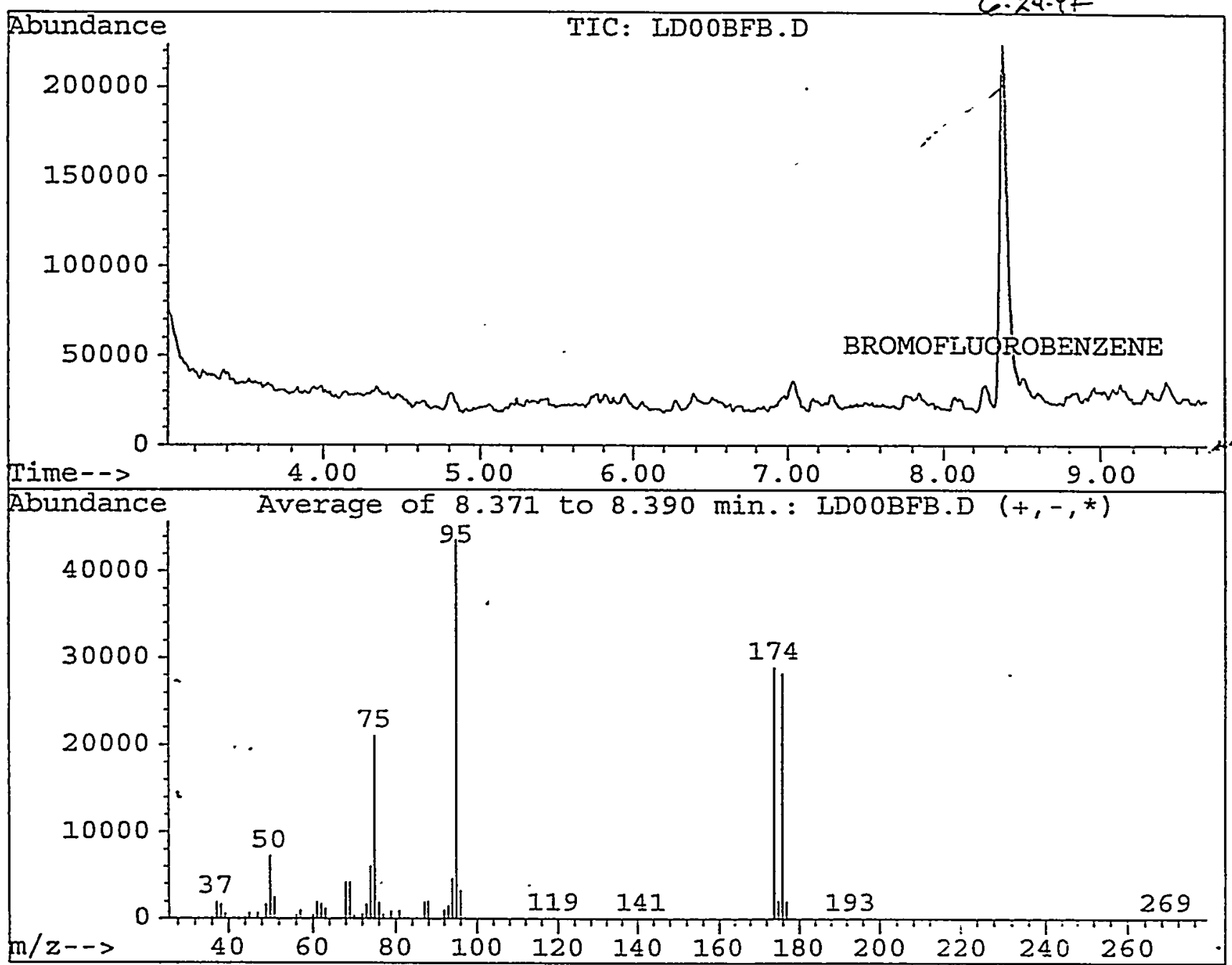

Peak Apex is scan: 555

Average of 3 Scans: 554, 555, 556 minus Background scan: $5 \leqslant 9$

\begin{tabular}{|c|c|c|c|c|c|c|}
\hline $\begin{array}{c}\text { Target } \\
\text { Mass }\end{array}$ & $\begin{array}{l}\text { Rel. to } \\
\text { Mass }\end{array}$ & $\begin{array}{l}\text { Lower } \\
\text { Limit\% }\end{array}$ & $\begin{array}{l}\text { Upper } \\
\text { Iimit\% }\end{array}$ & $\begin{array}{l}\text { Rel. } \\
\text { Abn: }\end{array}$ & $\begin{array}{l}\text { Rar: } \\
\text { Abr: }\end{array}$ & $\begin{array}{c}\text { Result } \\
\text { Pass/Fail }\end{array}$ \\
\hline $\begin{array}{r}50 \\
75 \\
95 \\
96 \\
173 \\
174 \\
175 \\
176 \\
177\end{array}$ & $\begin{array}{r}95 \\
95 \\
95 \\
95 \\
174 \\
95 \\
174 \\
174 \\
176\end{array}$ & $\begin{array}{r}15 \\
30 \\
100 \\
5 \\
0 \\
50 \\
5 \\
95 \\
5\end{array}$ & $\begin{array}{r}40 \\
60 \\
100 \\
9 \\
2 \\
100 \\
9 \\
101 \\
9\end{array}$ & $\begin{array}{r}16.65 \\
48.36 \\
100.00 \\
7.48 \\
0.00 \\
66.43 \\
7.44 \\
97.58 \\
7.33\end{array}$ & $\begin{array}{r}7259 \\
21=09 \\
43 \leqslant 53 \\
3254 \\
0 \\
28997 \\
2 \Xi 58 \\
28296 \\
2073\end{array}$ & $\begin{array}{l}\text { PASS } \\
\text { PASS } \\
\text { PASS } \\
\text { PASS } \\
\text { PASS } \\
\text { PASS } \\
\text { PASS } \\
\text { PASS } \\
\text { PASS }\end{array}$ \\
\hline
\end{tabular}


Data File : C: \HPCHEM \I\DATA 24 JUN97L LDOOBFB.D

$\begin{array}{llll}\text { Inject Time: } & 06 / 24 / 97 \text { I4:05 } & \text { Operator:CQC } \\ \text { Sample } & : 50 \mathrm{ng} \mathrm{BFB} & \text { Inst } & \text { :5971-L } \\ \text { Misc } & : & 2.0 \mathrm{uL} \text { of 39IS7676 } & \end{array}$

Average of 8.371 to $8.390 \mathrm{~min}$ : LDOOBFB.D

$50 \mathrm{ng}$ BFB

Modified:added subtracted scaled

$\begin{array}{rrrrrrrr}\mathrm{m} / \mathrm{z} & \text { abund. } & \mathrm{m} / \mathrm{z} & \text { abund. } & \mathrm{m} / \mathrm{z} & \text { abund. } & \mathrm{m} / \mathrm{z} & \text { abund. } \\ 36.00 & 299 & 51.05 & 2516 & 69.00 & 4216 & 82.90 & 228 \\ 37.10 & 2001 & 55.10 & 45 & 70.05 & 421 & 84.10 & 125 \\ 38.10 & 1696 & 56.05 & 504 & 72.00 & 545 & 85.05 & 179 \\ 39.10 & 602 & 57.05 & 1009 & 73.00 & 1720 & 87.00 & 1962 \\ 41.10 & 115 & 58.00 & 17 & 74.05 & 6087 . & 87.95 & 2020 \\ 42.10 & 72 & 60.00 & 449 & 75.05 & 21109 & 92.00 & 994 \\ 44.00 & 188 & 61.00 & 1990 & 76.05 & 1931 & 93.00 & 1487 \\ 45.05 & 670 & 62.00 & 1776 & 77.05 & 527 & 93.95 & 4649 \\ 47.05 & 713 & 63.00 & 1230 & 78.95 & 922 & 95.00 & 43653 \\ 49.05 & 1698 & 67.10 & 131 & 80.95 & 991 & 96.05 & 3264 \\ 50.05 & 7269 & 68.00 & 4248 & 82.00 & 132 & 96.95 & 136\end{array}$

Average of 8.371 to $8.390 \mathrm{~min}$.: LDOOBFB.D $50 \mathrm{ng} \mathrm{BFB}$

Modified:added subtracted scaled

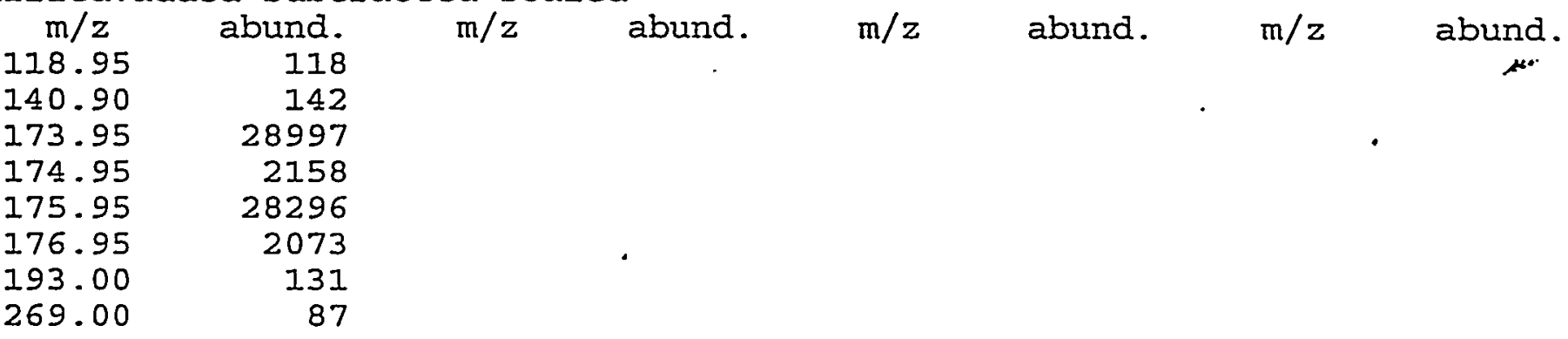


Form $S$ Equivalurt

BFB

Data File : C: \HPCHEM \I\DATA $\backslash 07 J U L 97 L \backslash L E 90 B F B . D$

Acq Time : 07/07/97 08:32

Sample : $50 \mathrm{ng} \mathrm{BFB}$

Misc : $2.0 \mathrm{uI}$ of 39157676

Operator: CQC

Inst : 5971-I

Multiplr: 1.00

Method : C: \HPCHEM $\backslash I \backslash M E T H O D S \backslash B F B 8240 . M$

Title :
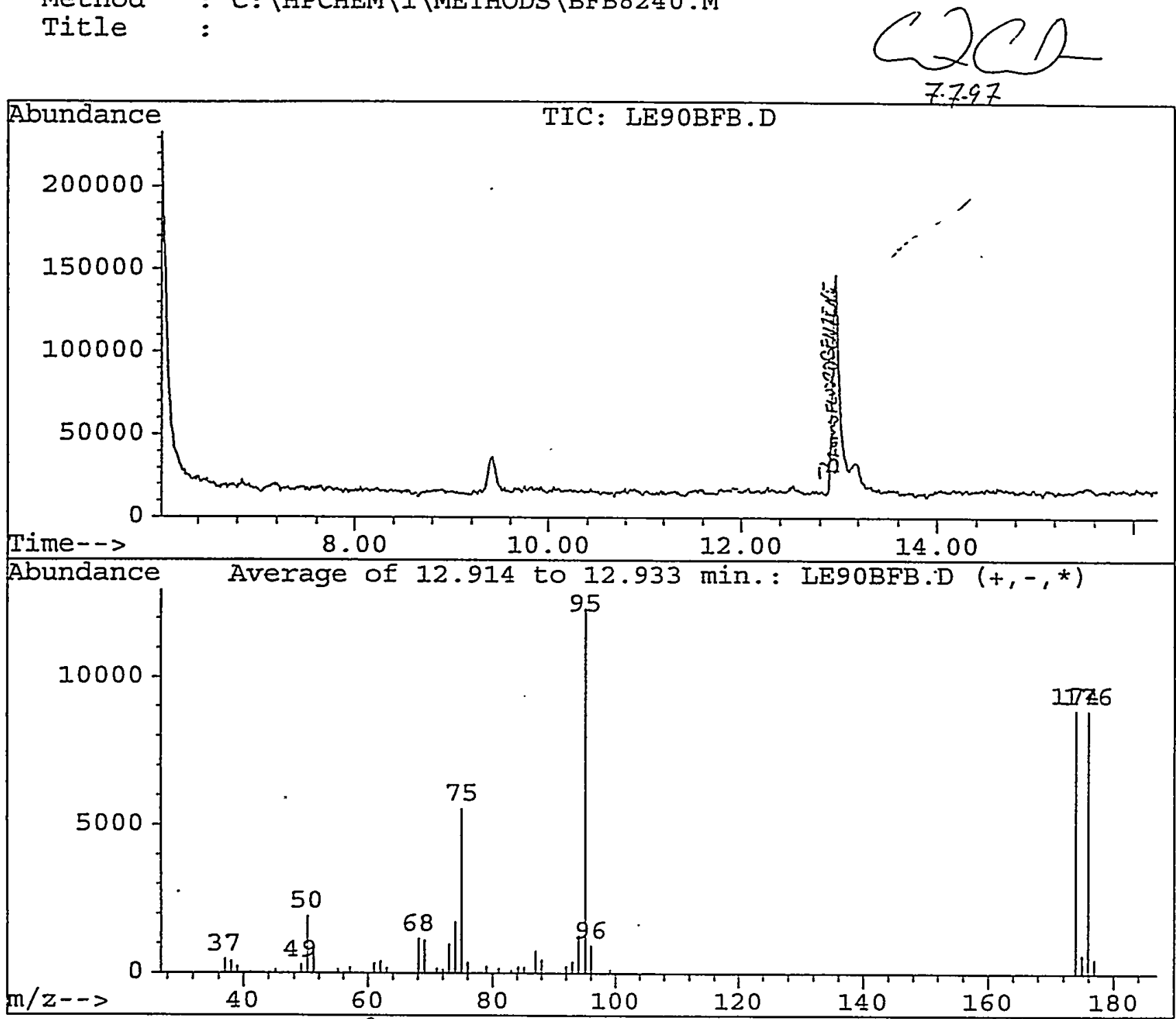

Peak Apex is scan: $7 \frac{7.47}{76} 719$

Averaye of cians: $714,715,716$ minu background sciin: 705

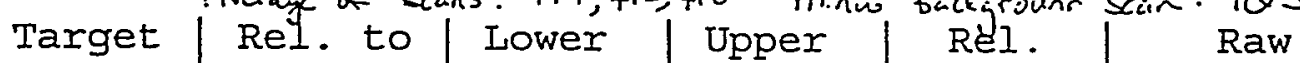

\begin{tabular}{l|l|l|l|l|l|l} 
Mass & Mass & Limit\% & Limit\% & Abn\% & Abn & Result
\end{tabular}

\begin{tabular}{|r|r|r|r|r|r|r|}
50 & 95 & 15 & 40 & 15.68 & 1936 & PASS \\
75 & 95 & 30 & 60 & 45.04 & 5561 & PASS \\
95 & 95 & 100 & 100 & 100.00 & 12347 & PASS \\
96 & 95 & 5 & 9 & 7.65 & 944 & PASS \\
173 & 174 & 0 & 2 & 0.00 & 0 & PASS \\
174 & 95 & 50 & 100 & 72.33 & 8931 & PASS \\
175 & 174 & 5 & 9 & 7.45 & 665 & PASS \\
176 & 174 & 95 & 101 & 99.80 & 8913 & PASS \\
177 & 176 & 5 & 9 & 5.77 & 514 & PASS
\end{tabular}

50 ng BEB 5971-I $07 / 07 / 97 \quad 08: 32 \quad$ PX125

6.31A Re 7/28)(9.) 
Average of 12.914 to $12.933 \mathrm{~min}$.: LE9OBFB.D $50 \mathrm{ng} \mathrm{BFB}$

Modified:added subtracted scaled

$\mathrm{m} / \mathrm{z}$

37.00

38.05

39.05

40.05

41.10

43.10

44.05

45.05

49.05

50.05

51.05 abund.

$476 \quad 55.10$

$401 \quad 56.10$

$228 \quad 57.10$

$19 \quad 58.00$

$67 \quad 61.00$

$65 \quad 62.00$

$57 \quad 63.00$

$138 \quad 68.05$

29169.05

1936

673

71.05

72.05 abund. $\mathrm{m} / \mathrm{z}$

$146 \quad 73.05$

$43 \quad 74.05$

$223 \quad 75.05$

$54 \quad 76.00$

$350 \quad 78.95$

$411 \quad 80.95$

$184 \quad 83.00$

$1170 \quad 84.10$

$1118 \quad 85.10$

$164 \quad 87.00$

$134 \quad 88.00$ abund.

984

1740

5561

365

242

173

120

243

217

765

473 $\mathrm{m} / \mathrm{z}$

92.05

93.05

94.00

95.05

96.00

99.05

173.95

174.95

175.95

176.95 abund. 256

398

1316

12347

944

143

8931

665

8913 
Form 5 Equivalent

$\mathrm{BFB}$

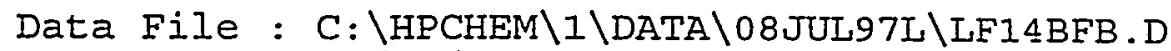

Acq Time: 07/08/97 09:13

Sample : $50 \mathrm{ng} \mathrm{BFB}$

Misc $: 2.0$ uL of 39 IS7676

Operator: CQC

Inst : 5971-L

Multiplr: 1.00

Method : C: $\backslash$ HPCHEM $\backslash I \backslash M E T H O D S \backslash B F B 8240 . M$

Title :

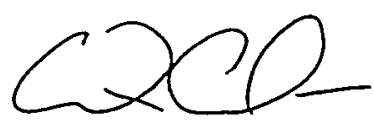

7.8 .97

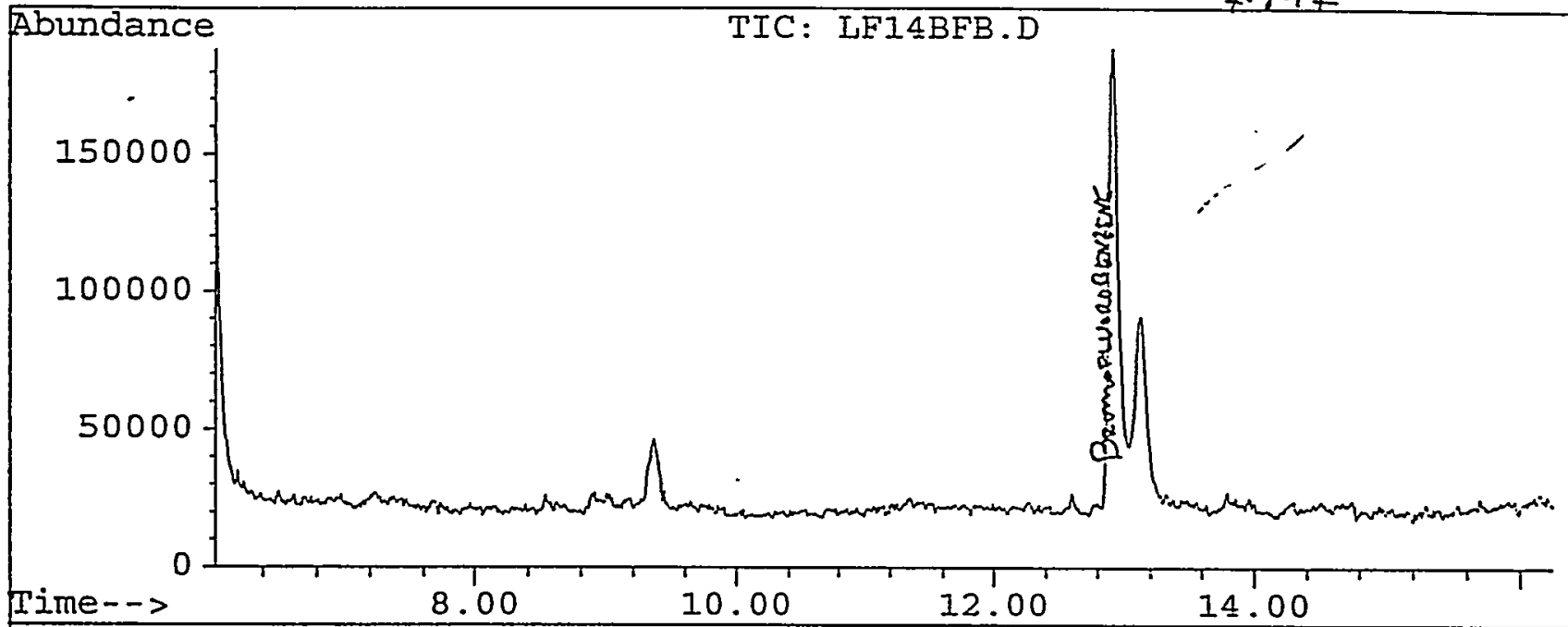

Abundance Scan $710(12.879 \mathrm{~min}):$ LF14BFB.D (-)

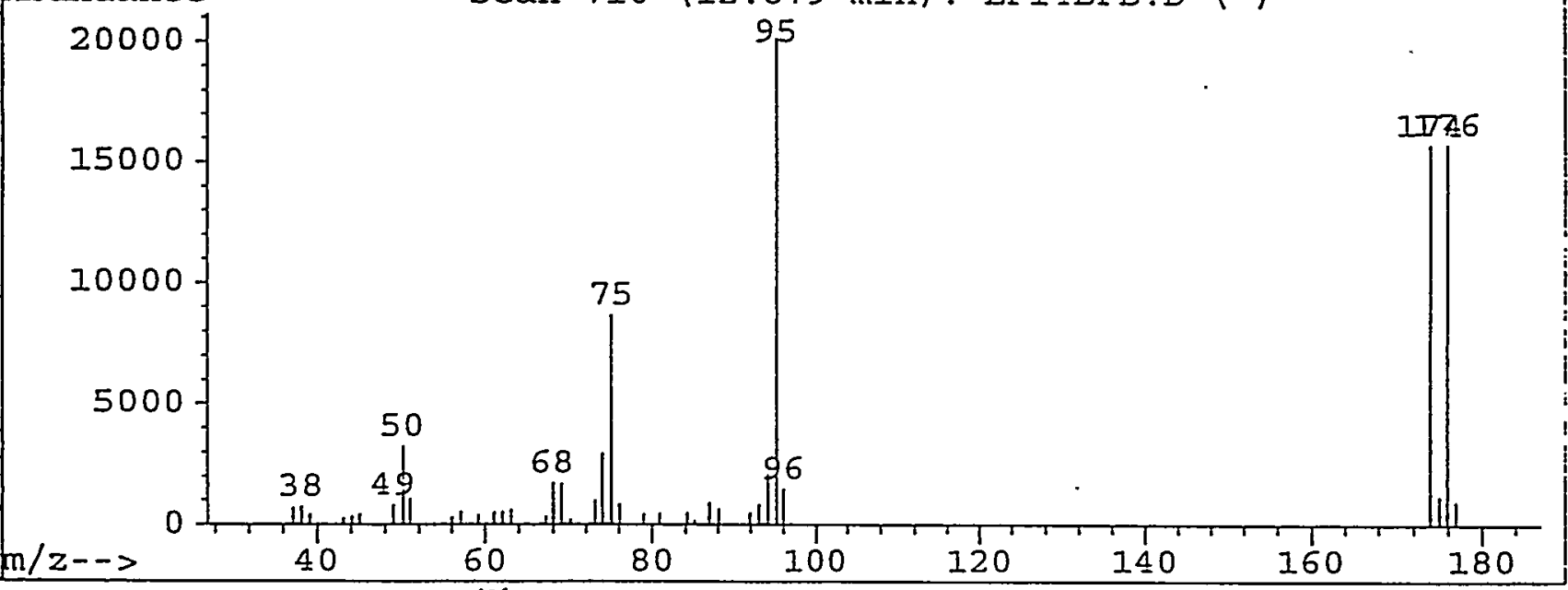

Peak Apex is scan: $\frac{7.757}{605} 713$ Scuntaka: 710 Mims backyround scun: 665

\begin{tabular}{|c|c|c|c|c|c|c|}
\hline $\begin{array}{c}\text { Target } \\
\text { Mass }\end{array}$ & $\begin{array}{l}\text { Rel. to } \\
\text { Mass }\end{array}$ & $\begin{array}{l}\text { Lower } \\
\text { Limit\% }\end{array}$ & $\begin{array}{l}\text { Upper } \\
\text { Iimit\% }\end{array}$ & $\begin{array}{l}\text { Rel. } \\
\text { Abn: }\end{array}$ & $\begin{array}{l}\text { Raw } \\
\text { Abn }\end{array}$ & $\begin{array}{r}\text { Resu] } \\
\text { Pass/Fo }\end{array}$ \\
\hline 50 & 95 & 15 & 40 & 16.09 & 3244 & PAS \\
\hline 75 & 95 & 30 & 60 & 43.09 & 8686 & PASS \\
\hline 95 & 95 & 100 & 100 & 100.00 & 20160 & PASS \\
\hline 96 & 95 & 5 & 9 & 7.33 & 1477 & PAS \\
\hline 173 & 174 & 0 & 2 & 0.00 & 0 & PAS \\
\hline 174 & 95 & 50 & 100 & 78.21 & 15768 & PAS \\
\hline 175 & 174 & 5 & 9 & 7.61 & 1200 & PAS \\
\hline 176 & 174 & 95 & 101 & 100.27 & 15811 & PASS \\
\hline 177 & 176 & 5 & 9 & 6.29 & 995 & PASS \\
\hline
\end{tabular}

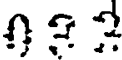

177 
Scan $710(12.879 \mathrm{~min}):$ LF14BFB.D

Modified: subtracted

$\mathrm{m} / \mathrm{z}$ abund.

37.15

38.15

39.10

40.05

42.10

43.10

44.05

45.00

49.00

50.10

51.00
687

735

436

60

11

284

337

459

812

3244

1066 $\mathrm{m} / \mathrm{z}$

55.05

56.05

57.10

59.15

60.05

61.05

62.05

63.05

67.10

68.00

69.00 abund. $\mathrm{m} / \mathrm{z}$

$93 \quad 70.10$

$329 \quad 73.10$

$529 \quad 74.00$

$423 \quad 75.10$

$80^{\circ} \quad 76.10$

$518 \quad 78.95$

$535 \quad 80.85$

$597 \quad 84.25$

$365 \quad 85.10$

$1732 \quad 86.95$

$1703 \quad 88.05$ abund. $\mathrm{m} / \mathrm{z}$ abund.

$230 \quad 91.90$

$1010 \quad 93.00$

$2977 \quad 94.10$

$8686 \quad 95.10$

$847 \quad 96.00$

$456 \quad 173.95$

$468 \quad 175.05$

$511 \quad 175.95$

$192 \quad 176.95$

924

676
507

855

2110

20160

1477

15768

1200

15811

995 


\section{Form 6 Eguibulat \\ Response Factor Report 5971-L}

Method

Title

: C: \HPCHEM \I\METHODS\L826ARWA.M

Last Update: Wed Jun 25 09:02:09 1997

Response via : Continuing Calibration

Calibration Files

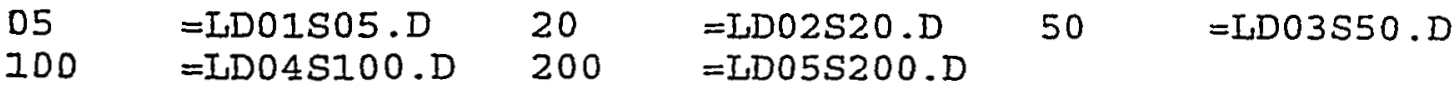

Compound

$05 \quad 20 \quad 50 \quad 100 \quad 200 \quad$ Avg

$\div$ RSD

1) I CIOI Flourobenzene

$0.456 \quad 0.368 \quad 0.393 \quad 0.39$

2)

COO2 dichlorodifluor

3) $\mathrm{T}$

$\mathrm{COO} 3$ chloromethane

$\begin{array}{llll}0.173 & 0.142 & 0.167\end{array}$

0.396

0.387

0.400

8.30

4) $T$

$0.2660 .236 \quad 0.253$

0.1720 .179

0.167

8.55

5) $\mathrm{T}$

c005 bromomethane

$\begin{array}{llll}0.380 & 0.273 & 0.28\end{array}$

$\begin{array}{ll}0.254 & 0.249 \\ 0.268 & 0.258\end{array}$

0.252

4.31

5) $T$

C006 chloroethane

$\begin{array}{llll}0.161 & 0.143 & 0.147\end{array}$

0.1420 .135

0.292

17.10

7) $T$

C007 trichlorofluorometha

$0.661 \quad 0.562$

0.605

0.6110 .602

146

6.47

B)

C071 acrolein

9) $\mathrm{T} \operatorname{C008} 1,1$-dichloroethene

$\begin{array}{llll}0.320 & 0.266 & 0.293\end{array}$

5.81

C063 freon 113

11) $\mathrm{T}$

C009 acetone

12) $T$

COI0 carbon disulfide

13) $T$

CO1I methylene chloride

$\begin{array}{llll}0.118 & 0.057 & 0.057 & 0.1\end{array}$

0.289

0.000

$-1.00$

14)

C072 acrylonitrile

$\begin{array}{llll}0.823 & 0.705 & 0.779\end{array}$

0.0580 .060

0.290

6.67

C012 trans-1,2-Dichloroet

$\begin{array}{llll}0.355 & 0.277 & 0.293\end{array}$

$-1.00$

15)

16)

C305 methyl-t-butyl-ether

$\begin{array}{llll}0.088 & 0.069 & 0.073 & 0.1\end{array}$

0.778

40

38.72

17) $T$

C013 cis-1,2-dichloroethe

0.355

0.298

0.328

$\begin{array}{llll}0.872 & 0.693 & 0.75\end{array}$

0.764

0

0.0360 .0340 .034

0.377

0.0360 .034

$\begin{array}{llll}0.237 & 0.194 & 0.211\end{array}$

$0.743 \quad 0.5890 .622$

(1)

0

$$
0
$$

0.

$\begin{array}{lllllll}0.577 & 0.464 & 0.518 & 0.539 & 0.537 & 0.527\end{array}$

$\begin{array}{lllllll}1.131 & 0.924 & 1.002 & 0.990 & 0.965 & 1.002\end{array}$

$\begin{array}{lllllll}0.475 & 0.425 & 0.460 & 0.451 & 0.439 & 0.450\end{array}$

$\begin{array}{llllll}0.431 & 0.354 & 0.381 & 0.380 & 0.370 & 0.383\end{array}$

$\begin{array}{llllll}0.354 & 0.302 & 0.328 & 0.330 & 0.316 & 0.326\end{array}$

\section{0}

$\begin{array}{llllllll}0.606 & 0.522 & 0.569 & 0.581 & 0.568 & 0.569 & 5.34\end{array}$

35) $T$

$\begin{array}{llllllll}0.440 & 0.350 & 0.363 & 0.379 & 0.340 & 0.374 & 10.62\end{array}$

\section{7) I CIO2 Chlorobenzene-d5 \\ 38) T C028 trans-1,3-dichloropr \\ 39) T C029 1,1,2-trichloroethan \\ 40) \\ 4I) (3) 32}$$
\text { 0. }
$$

$\begin{array}{lllllll}705 & 0.608 & 0.681 & 0.687 & 0.686 & 0.673\end{array}$

$\begin{array}{llllll}0.705 & 0.608 & 0.681 & 0.687 & 0.686 & 0.673 \\ 0.582 & 0.467 & 0.504 & 0.496 & 0.485 & 0.507\end{array}$

$\begin{array}{llllllll}0.233 & 0.222 & 0.258 & 0.271 & 0.263 & 0.249\end{array}$

$\begin{array}{lllllll}0.764 & 0.652 & 0.719 & 0.721 & 0.700 & 0.711\end{array}$

$\begin{array}{llllll}1.353 & 1.270 & 1.279 & 1.264 & 1.265 & 1.286\end{array}$
5.59

8.74

8.27

$5: 72$

2.92

(\#) = Out of Range 
Response Factor Report 5971-I

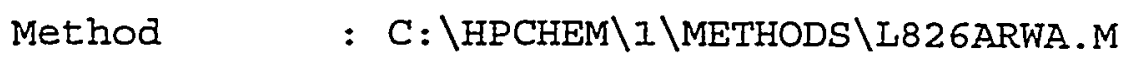

Title

: VOA COMPOUND LIST

Last Update : Wed Jun 25 09:02:09 1997

Response via : Continuing Calibration

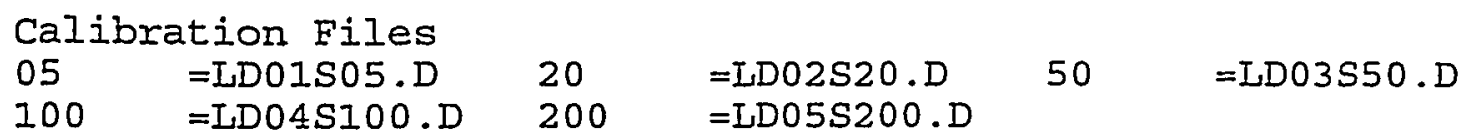

\begin{tabular}{|c|c|c|c|c|c|c|c|c|c|c|}
\hline \multicolumn{4}{|c|}{ Compound } & 05 & 20 & 50 & 100 & 200 & avg & SD \\
\hline \\
\hline 43) & & C03I & Toluene & 1.007 & 0.836 & 0.910 & 0.910 & 0,900 & 0.913 & 6.7 \\
\hline 44) & & $\mathrm{CO} 32$ & 1,3-dichloropropane & 0.865 & 0.728 & 0.799 & 0.792 & 0.775 & 0.792 & 6.2 \\
\hline 45) & $\mathrm{T}$ & $\mathrm{CO33}$ & dibromochloromethane & 37 & 0.733 & 0.810 & 0.817 & 0.795 & 0.798 & \\
\hline 46) & $\mathrm{T}$ & $\mathrm{CO34}$ & bromoform & & 0.528 & 0.599 & 0.627 & 0.621 & 0.591 & \\
\hline 47) & $\mathrm{T}$ & $\cos 5$ & tetrachloroethene & 0. & 0 . & 651 & 0.617 & 0.581 & 610 & \\
\hline 48) & $T$ & $\mathrm{C} 036$ & chlorobenzene & 1.312 & 1.075 & 1.163 & 1.146 & 1.131 & 1.165 & \\
\hline 49) & & $\mathrm{CO} 37$ & $1,1,1,2$-tetrachloroe & 0.691 & 0.569 & 0.622 & 0.624 & 0.621 & 0.625 & \\
\hline 50) & $T$ & $\operatorname{co3} 8$ & ethylber & & 0.490 & 0.538 & 0.528 & 0.516 & 0.530 & \\
\hline 51) & & $\operatorname{co39}$ & $m, p-x y$ & 38 & 0.605 & 0.667 & 0.648 & 0.630 & 0.658 & \\
\hline 52) & $T$ & $\mathrm{CO} 40$ & o-xylene & & 0.5 & 0.6 & 0.628 & 0.613 & 0.640 & \\
\hline 53) & & C250 & total xylene & & 0.6 & 0.660 & 0.6 & 0.628 & 0.6 & \\
\hline 54) & $\mathrm{T}$ & $\mathrm{CO41}$ & styrene & 1.235 & 1.045 & 1.150 & 1.133 & 1.105 & 1.134 & .0 \\
\hline 55 & & $\mathrm{CO} 42$ & isopropylbenzene & 2.129 & 1.773 & 1.946 & 1.893 & 1.847 & 1.918 & \\
\hline
\end{tabular}

56) I CI03 1,4-Dichlorobenzene- $\ldots$

CS10 4-bromofluorobenzene $1.561 \quad 1.265 \quad 1.297 \quad 1.295 \quad 1.263 \quad 1.336$

58) $C 0431,1,2,2$-tetxachloroe 1.4821 .2091 .3111 .3341 .2351 .314

$\begin{array}{lllllll}1.065 & 0.875 & 0.954 & 0.954 & 0.921 & 0.956\end{array}$

60) T C045 1,2,3-trichloropropa $1.391 \quad 1.108 \quad 1.258 \quad 1.318 \quad 1.228 \quad 1.261$

61) C046 n-propylbenzene $\quad 3.9653 .303 \quad 3.6623 .6583 .4723 .612$

62) C047.1,3,5-trimethylbenze $2.657 \quad 2.1642 .355 \quad 2.306 \quad 2.201 \quad 2.336$

63) C048 2-chlorotoluene $\quad 2.360 \quad 3.303 \quad 3.662 \quad 3.658 \quad 3.472 \quad 3.291 \quad 16$.

64) Co49 4-chlorotoluene $\quad 2.9912 .4772 .695 \quad 2.672 \quad 2.5512 .677$

65) Co50 tert-butylbenzene $\quad 3.567 \quad 2.892 \quad 3.171 \quad 3.1573 .002 \quad 3.158$

66) Co51'1,2,4-trimethylbenze $2.713 \quad 2.164 \quad 2.367 \quad 2.3132 .203 \quad 2.352$

67) Co52 sec-Butylbenzene $\quad \begin{array}{llllll}3.868 & 3.158 & 3.473 & 3.424 & 3.229 & 3.430\end{array}$

68) C053 p-Isopropyltoluene $\quad 3.3942 .7713 .0162 .9852 .8272 .999$

69) Co54 1,3-Dichlorobenzene 1.8621 .5171 .6921 .6851 .6351 .678

70) Co55 1,4-Dichlorobenzene $1.8621 .5171 .8091 .7981 .731 \quad 1.743$

71) Co56 n-Butylbenzene $\quad 3.1202 .597 \quad 2.8572 .8902 .7432 .841$

72) C057 1,2-Dichlorobenzene $1.8561 .496 \quad 1.616 \quad 1.613 \quad 1.547 \quad 1.626$

73) $\quad \begin{array}{lllllllll}\mathrm{C} 058 & 1,2-D i b r o m o-3-c h l o r o & 0.280 & 0.246 & 0.288 & 0.299 & 0.277 & 0.278\end{array}$

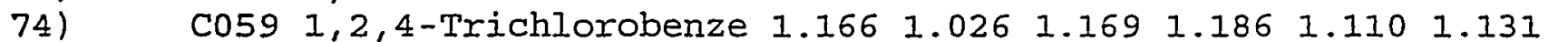

75)

76)

77)

c060 Hexachlorobutadiene

C061 Naphthalene

$\begin{array}{llllll}0.917 & 0.714 & 0.815 & 0.900 & 0.830 & 0.835\end{array}$

$2.315 \quad 1.801 \quad 2.1342 .188 \quad 1.900 \quad 2.067$

8.15

7.34

8.38

6.84

8.34

6.45

7.34

8.12

9.25

8.09

8.14

7.41

7.74

6.81

8.49

7.17

5.81 .

C062 1,2,3-Trichlorobenze $1.0210 .9691 .082 \quad 1.1040 .9501 .025 \quad 6.57$

936

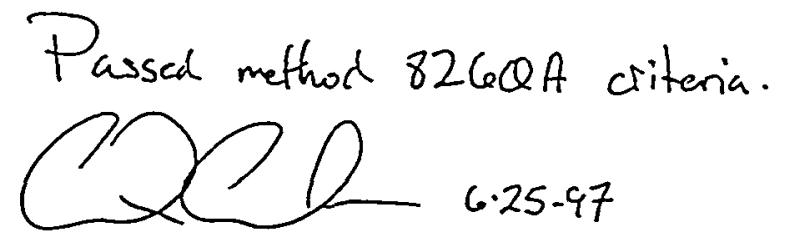

$(\#)=$ Out of Range 


$$
\text { Form } 7 \text { Eqnivalat }
$$

\section{A CONTINUING CALIBRATION REPORT}

SW-846, 8260A, Revision 1

Continuing Calibration File: LE91S50.D

Initial Calibration Method:

L826ARWA

\begin{tabular}{rccccc}
\multicolumn{2}{c}{ Calibration Check Compounds: } & \multicolumn{4}{c}{$\%$ Drift must be $<20 . \%$} \\
& RT & CC Amnt & CC Target & $\%$ Drift & \\
C004 vinyl chloride & 4.58 & 42.07 & 50.00 & 15.9 & OK \\
C008 1,1-dichloroethene & 7.78 & 48.55 & 50.00 & 2.9 & OK \\
C016 chloroform & 11.50 & 48.44 & 50.00 & 3.7 & OK \\
C023 1.2-dichloropropane & 13.76 & 40.80 & 50.00 & 18.4 & OK \\
C031 Toluene & 15.46 & 46.73 & 50.00 & 6.5 & OK \\
C038 ethylbenzene & 17.98 & 48.22 & 50.00 & 3.6 & OK
\end{tabular}

SPCC compounds:

C003 chloromethane C014 1,1-dichloroethane

c034 bromoform

C036 chlorobenzene

c043 1,1,2,2-tetrachloroethane
CC RFs must be $>I=0.1$

CC RF

0.161

0.482

OK

OK

CC RFs must be $>0.1$

0.713 - OK

CC RFs must be $>I=0.3$

1.123

1.009

OK

OK 


\section{A CONTINUING CALIBRATION REPORT (cont'd) \\ SW-846, 8260A, Revision 1}

Continuing Calibration File: LE91S50.D

07/07/97

Initial Calibration Method: L826ARWA

\begin{tabular}{|c|c|c|c|c|c|}
\hline Surrogate Compounds: & $\mathrm{RT}$ & CC Amnt & $\begin{array}{c}\text { CC Target } \\
\text { CC RF }\end{array}$ & $\%$ Drift & \\
\hline CS15 1,2-dichloroethane-d4 & 12.28 & 45.72 & 50.00 & 8.6 & \\
\hline $\operatorname{cs} 05$ toluene-d8 & 15.35 & 47.30 & 50.00 & 5.4 & \\
\hline CS10 4-bromofluorobenzene & 19.79 & 42.44 & 50.00 & 15.1 & \\
\hline Target Compounds: & RT & CC Amint & CC Target & $\%$ Drift & \\
\hline COO2 dichlorodifluoromethane & 3.54 & 48.09 & 50.00 & 3.8 & \\
\hline C003 chloromethane & 4.19 & 48.39 & 50.00 & 3.2 & \\
\hline Co04 vinyl chloride & 4.58 & 42.07 & 50.00 & 15.9 & \\
\hline C005 bromomethane & 5.59 & 40.96 & 50.00 & 18.1 & \\
\hline C006 chloroethane & 5.90 & 41.37 & 50.00 & 17.3 & \\
\hline C007 trichlorofluoromethane & 6.63 & 51.05 & 50.00 & -2.1 & \\
\hline C008 1,1-dichloroethene & 7.78 & 48.55 & 50.00 & 2.9 & \\
\hline C009 acetone & 7.92 & 38.47 & 50.00 & 23.1 & warning \\
\hline C010 carbon disulfide & 8.14 & 45.02 & 50.00 & 10.0 & \\
\hline C011 methylene chloride & 8.75 & 45.55 & 50.00 & 8.9 & \\
\hline C072 acrylonitrile & 9.19 & 34.42 & 50.00 & $31.2^{\circ}$ & warning \\
\hline C012 trans-1,2-Dichloroethene & 9.27 & 48.71 & 50.00 & 2.6 & \\
\hline C305 methyl-t-butyl-ether & 9.32 & 45.12 & 50.00 & 9.8 & \\
\hline C013 cis-1,2-dichloroethene & 10.98 & 48.76 & 50.00 & 2.5 & \\
\hline C055 total-1,2-dichloroethene & 10.98 & 97.49 & 100.00 & 2.5 & \\
\hline C014 1,1-dichloroethane & 10.00 & 42.82 & 50.00 & 14.4 & \\
\hline C015 2,2-dichloropropane & 10.98 & 47.05 & 50.00 & 5.9 & \\
\hline C110 2-butanone & 11.01 & 46.60 & 50.00 & 6.8 & \\
\hline Cl01 bromochloromethane & 11.37 & 54.30 & 50.00 & -8.6 & \\
\hline C016 chloroform & 11.50 & 48.44 & 50.00 & 3.1 & \\
\hline C017 1.1,1-Trichloroethane & 11.81 & 50.30 & 50.00 & -0.6 & \\
\hline C018 1,1-dichloropropene & 12.07 & 46.09 & 50.00 & 7.8 & \\
\hline Co19 1,2-dichloroethane & 12.40 & 45.11 & 50.00 & 9.8 & \\
\hline C020 carbon tetrachloride & 12.09 & 52.06 & 50.00 & -4.1 & \\
\hline C021 benzene & 12.40 & 44.53 & 50.00 & 10.9 & \\
\hline CO22 trichloroethene & 13.43 & 53.36 & 50.00 & -6.7 & \\
\hline C023 1,2-dichloropropane & 13.76 & 40.80 & 50.00 & 18.4 & \\
\hline CO24 dibromomethane & 13.94 & 49.07 & 50.00 & 1.9 & \\
\hline C025 bromodichloromethane & 14.18 & 48.07 & 50.00 & 3.9 & \\
\hline CO27 cis-1,3-dichloropropene & 14.90 & 45.84 & 50.00 & 8.3 & \\
\hline C205 4-methyl-2-pentanone & 15.11 & 47.26 & 50.00 & 5.5 & \\
\hline C028 trans-1,3-dichloropropene & 15.77 & 44.74 & 50.00 & 10.5 & \\
\hline Co29 1,1,2-trichloroethane & 16.07 & 47.24 & 50.00 & 5.5 & \\
\hline C210 2-hexanone & 16.46 & 40.46 & 50.00 & 19.1 & \\
\hline C030 1,2-Dibromoethane & 16.96 & 48.16 & 50.00 & 3.7 & \\
\hline C031 Toluene & 15.46 & 46.73 & 50.00 & 6.5 & \\
\hline C032 1,3-dichloropropane & 16.36 & 43.55 & 50.00 & 12.9 & \\
\hline C033 dibromochloromethane & 16.75 & 51.31 & 50.00 & -2.6 & \\
\hline $\mathrm{C034}$ bromoform & 19.21 & 60.33 & 50.00 & -20.7 & \\
\hline
\end{tabular}




\section{A CONTINUING CALIBRATION REPORT (cont'd) \\ SW-846, 8260A, Revision 1}

Continuing Calibration File: LE91S50.D

$07 / 07 / 97$

Initial Calibration Method:

L826ARWA

Target Compounds (cont'd): C035 tetrachloroethene

c036 chlorobenzene

RT

16.38

17.80

C037 1,1,1,2-tetrachloroethane

C038 ethylbenzene

C039 m,p-xylene

c040 o-xylene

C250 total xylene

C041 styrene

C042 isopropylbenzene

C043 1,1,2,2-tetrachloroethane

C044 bromobenzene

C045 1,2,3-trichloropropane

C046 n-propylbenzene

C047 1,3,5-trimethylbenzene C048 2-chlorotoluene c049 4-chlorotoluene C050 tert-butylbenzene

co51 1,2,4-trimethylbenzene C052 sec-Butylbenzene C053 p-Isopropyltoluene C054 1,3-Dichloroberizene Co55 1,4-Dichlorobenzene C056 n-Butylbenzene

C057 1,2-Dichlorobenzene

C058 1.2-Dibromo-3-chloropropane Co59 1,2,4-Trichlorobenzene

C060 Hexachlorobutadiene C061 Naphthalene

C062 1,2,3-Trichlorobenzene
17.92

17.98

18.17

18.87

18.17

18.88

19.50

19.99

20.07

20.07

20.24 .

20.55

20.24

20.60

21.16

21.24

21.55

21.81

21.77

21.92

22.55

22.59

23.73

24.87

25.12

25.22

25.57

$\begin{array}{ccc}\text { CC Amnt } & \text { CC Target } & \text { \% Drift } \\ 56.49 & 50.00 & -13.0 \\ 48.18 & 50.00 & 3.6 \\ 51.34 & 50.00 & -2.7 \\ 48.22 & 50.00 & 3.6 \\ 96.42 & 100.00 & 3.6, \\ 48.58 & 50.00 & 2.8 \\ 144.95 & 150.00 & 3.4 \\ 47.68 & 50.00 & 4.6 \\ 47.58 & 50.00 & 4.8 \\ 38.37 & 50.00 & 23.3 \\ 49.84 & 50.00 & 0.3 \\ 38.38 & 50.00 & 23.2 \\ 41.47 & 50.00 & 17.1 \\ 41.93 & 50.00 & 16.1 \\ 42.62 & 50.00 & 14.8 \\ 41.71 & 50.00 & 16.6 \\ 44.58 & 50.00 & 10.8 \\ 42.04 & 50.00 & 15.9 \\ 41.92 & 50.00 & 16.2 \\ 43.85 & 50.00 & 12.3 \\ 49.04 & 50.00 & 1.9 \\ 50.95 & 50.00 & -1.9 \\ 41.80 & 50.00 & 16.4 \\ 48.43 & 50.00 & 3.1 \\ 41.10 & 50.00 & 17.8 \\ 54.40 & 50.00 & -8.8 \\ 57.65 & 50.00 & -15.3 \\ 46.94 & 50.00 & 6.1 \\ 56.30 & 50.00 & -12.6 \\ & & \end{array}$




$$
\text { Fon } 7 \text { Esuiralut }
$$

\section{A CONTINUING CALIBRATION REPORT}

SW-846, 8260A, Revision 1

Continuing Calibration File: LF15S50.D

$07 / 08 / 97$

Initial Calibration Method:

L826ARWA

\begin{tabular}{rccccc}
\multicolumn{2}{c}{ Calibration Check Compounds: } & \multicolumn{3}{c}{$\%$ Drift must be $<20 . \%$} \\
& RT & CC Amnt & CC Target & $\%$ Drift & \\
C004 vinyl chloride & 4.55 & 41.25 & 50.00 & 17.5 & OK \\
C008 1,1-dichloroethene & 7.73 & 52.42 & 50.00 & -4.8 & OK \\
C016 chloroform & 11.46 & 53.70 & 50.00 & $-7.4-$ & OK \\
C023 1,2-dichloropropane & 13.70 & 42.24 & 50.00 & 15.5 & OK \\
C031 Toluene & 15.40 & 50.31 & 50.00 & -0.6 & OK \\
C038 ethylbenzene & 17.92 & 53.97 & 50.00 & -7.9 & OK
\end{tabular}

SPCC compounds:

C003 chloromethane

c014 1,1-dichloroethane

C034 bromoform

C036 chlorobenzene

C043 1,1,2,2-tetrachloroethane

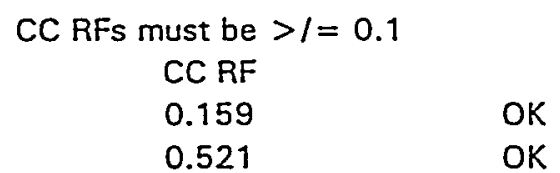

CC RFs must be $>0.1$

0.836

OK

CC RFs must be $>I=0.3$

1.232

0.930

OK

OK 


\section{A CONTINUING CALIBRATION REPORT (cont'd)}

SW-846, 8260A, Revision 1

Continuing Calibration File: LF15S50.D

$07 / 08 / 97$

Initial Calibration Method:

L826ARWA

Surrogate Compounds: $\quad \mathrm{RT} \quad \mathrm{CC}$ Amnt $\mathrm{CC}$ Target $\%$ Drift

CS15 1,2-dichloroethane-d4 CSO5 toluene-d8

CS10 4-bromofluorobenzene

Target Compounds:

$\mathrm{COO} 2$ dichlorodifluoromethane C003 chloromethane

C004 vinyl chloride

c005 bromomethane

c006 chloroethane

C007 trichlorofluoromethane

C008 1,1-dichloroethene c009 acetone

c010 carbon disulfide

C011 methylene chloride

C072 acrylonitrile

C012 trans-1,2-Dichloroethene

C305 methyl-t-butyl-ether

C013 cis-1,2-dichloroethene

C055 total-1,2-dictiloroethene

C014 1,1-dichloroethane

C015 2,2-dichloropropane

c110 2-butanone

Cl01 bromochloromethane

c016 chloroform

C017 1,1,1-Trichloroethane

C018 1,1-dichloropropene

Co19 1,2-dichloroethane

C020 carbon tetrachloride

C021 benzene

C022 trichloroethene

C023 1,2-dichloropropane

C024 dibromomethane

C025 bromodichloromethane

C027 cis-1,3-dichloropropene

C205 4-methyl-2-pentanone

C028 trans-1,3-dichloropropene

C029 1,1,2-trichloroethane

C210 2-hexanone

c030 1,2-Dibromoethane

C031 Toluene

C032 1,3-dichloropropane

C033 dibromochloromethane

C034 bromoform
12.23

15.30

19.72

RT

3.51

4.15

4.55

5.55

5.87

6.58 .

7.73

7.88

8.10

8.71

9.15

9.24

9.29

10.95

10.95

9.96

10.94

10.97

11.32

11.46

11.77

12.03

12.35

12.05

12.35

13.37

13.70

13.89

14.13

14.84

15.06

15.71

16.02

16.41

16.90

15.40

16.30

16.70

19.15
49.47

49.52

43.54

CC Amnt 46.98

47.65

41.25

45.54

45.97

59.69

52.42

37.18

44.42

46.09

37.23

52.52

45.43

52.88

105.46

46.34

52.42

38.30

56.42

53.70

59.13

48.67

50.92

61.72

45.28

56.18

42.24

52.73

53.68

46.77

41.00

47.42

49.36

34.47

52.00

50.31

43.48

56.20

70.79
CC RF

50.00

50.00

50.00

\subsection{0}

CC Target

50.00

50.00

50.00

50.00

50.00

50.00

50.00

50.00

50.00

50.00

50.00

50.00

50.00

50.00

100.00

50.00

50.00

50.00

50.00

50.00

50.00

50.00

50.00

50.00

50.00

50.00

50.00

50.00

50.00

50.00

50.00

50.00

50.00

50.00

50.00

50.00

50.00

50.00

50.00
1.1

1.0

12.9<smiles>[3H][IH]</smiles>

\% Drift

6.0

4.7

17.5

8.9

8.1

$-19.4$

$-4.8$

25.6

11.2

7.8

$25.5^{\circ}$

$-5.0$

9.1

$-5.8$

$-5.5$

7.3

$-4.8$

23.4

$-12.8$

$-7.4$

$-18.3$

2.7

$-1.8$

-23.4 warning

9.4

$-12.4$

15.5

$-5.5$

$-7.4$

6.5

18.0

5.2

1.3

31.1

$-4.0$

$-0.6$

13.0

$-12.4$

-41.6 warning warning

warning

warning

warning 
8260A CONTINUING CALIBRATION REPORT (cont'd)

SW-846, 8260A, Revision 1

Continuing Calibration File: LF15S50.D

$07 / 08 / 9.7$

Initial Calibration Method: L826ARWA

\begin{tabular}{|c|c|c|c|c|c|}
\hline $\begin{array}{l}\text { Target Compounds (cont' } d \text { ): } \\
\text { c035 tetrachloroethene }\end{array}$ & $\begin{array}{c}\text { RT } \\
16.33\end{array}$ & $\begin{array}{l}\text { CC Amnt } \\
68.77\end{array}$ & $\begin{array}{l}\text { CC Target } \\
50.00\end{array}$ & $\begin{array}{l}\% \text { Drift } \\
-37.5\end{array}$ & warning \\
\hline C036 chlorobenzene & 17.74 & 52.84 & 50.00 & -5.7 & \\
\hline C037 1.1.1.2-tetrachloroethane & 17.87 & 54.64 & 50.00 & -9.3 & \\
\hline C038 ethylbenzene & 17.92 & 53.97 & 50.00 & -7.9 & \\
\hline c039 m,p-xylene & 18.12 & 109.12 & 100.00 & -9.1 & \\
\hline C040 o-xylene & 18.82 & 54.36 & 50.00 & -8.7 & \\
\hline C250 total xylene & 18.12 & 164.41 & 150.00 & -9.6 & \\
\hline C041 styrene & 18.83 & 53.33 & 50.00 & -6.7 & \\
\hline C042 isopropylbenzene & 19.45 & 55.49 & 50.00 & -11.0 & \\
\hline C043 1,1,2,2-tetrachloroethane & 19.93 & 35.39 & 50.00 & 29.2 & \\
\hline C044 bromobenzene & 20.02 & 56.02 & 50.00 & -12.0 & \\
\hline C045 1,2,3-trichloropropane & 20.03 & 35.43 & 50.00 & 29.1 & warning \\
\hline C046 n-propylbenzene & 20.19 & 44.10 & 50.00 & 11.8 & \\
\hline Co47 1,3,5-trimethylbenzene & 20.50 & 47.96 & 50.00 & 4.1 & \\
\hline C048 2-chlorotoluene & 20.19 & 45.33 & 50.00 & 9.3 & \\
\hline C049 4-chlorotoluene & 20.55 & 44.58 & 50.00 & 10.8 & \\
\hline C050 tert-butylbenzene & 21.10 & 47.53 & 50.00 & 4.9 & \\
\hline C051 1,2,4-trimethylbenzene & 21.19 & 47.69 & 50.00 & $4.6^{\circ}$ & \\
\hline C052 sec-Butylbenzene & 21.50 & 46.78 & 50.00 & 6.4 & \\
\hline C053 p-Isopropyltoluene & 21.76 & 46.84 & 50.00 & 6.3 & \\
\hline C054 1,3-Dichlorobenzene & 21.71 & 50.98 & 50.00 & -2.0 & \\
\hline C055 1,4-Dichlorobenzene & 21.88 & 52.65 & 50.00 & -5.3 & \\
\hline C056 n-Butylbenzene & 22.51 & 44.12 & 50.00 & 11.8 & \\
\hline C057 1,2-Dichlorobenzene & 22.55 & 49.76 & 50.00 & 0.5 & \\
\hline Co5B 1.2-Dibromo-3-chloropropane & 23.69 & 39.49 & 50.00 & 21.0 & warning \\
\hline Co59 1,2,4-Trichlorobenzene & $24.84^{\circ}$ & 64.65 & 50.00 & -29.3 & warning \\
\hline C060 Hexachlorobutadiene & 25.08 & 71.00 & 50.00 & -42.0 & warning \\
\hline C061 Naphthalene & 25.18 & 42.92 & 50.00 & 14.2 & \\
\hline C062 1,2,3-Trichlorobenzene & 25.53 & 64.22 & 50.00 & -28.4 & warning \\
\hline
\end{tabular}


Form 8 Equivalent

\section{INTERNAL STANDARD AREA SUMMARY}

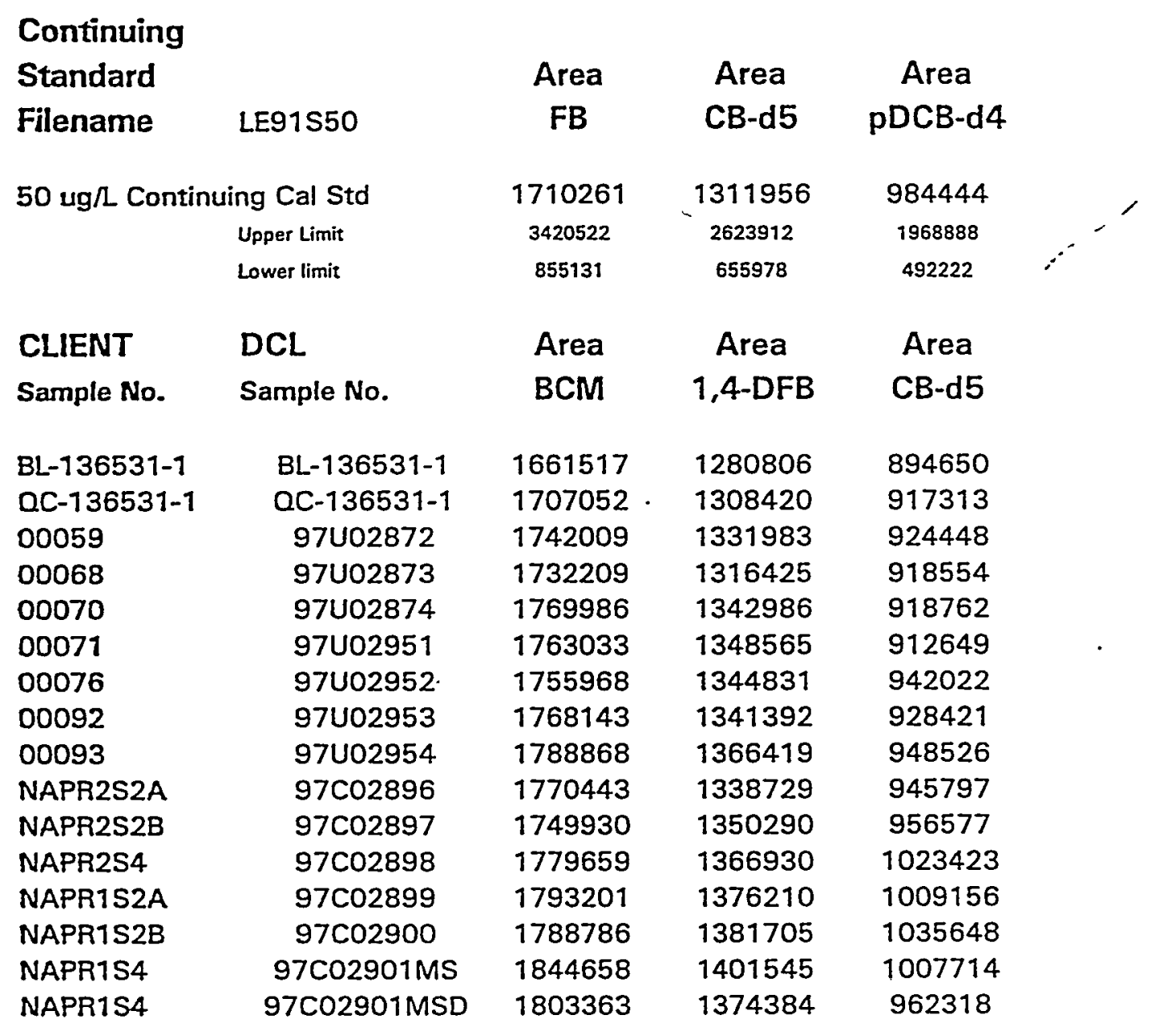

LIMITS: Areas should be within 50 to 200 percent of the corresponding area in the calibration. 


\section{Form 8 Equivalent \\ INTERNAL STANDARD RETENTION-TIME SUMMMARY}

\begin{tabular}{|c|c|c|c|c|c|}
\hline Continuing & . & & & & \\
\hline Standard & . & RT & RT & RT & \\
\hline Filename & LE91S50 & $\mathrm{BCM}$ & $1,4-D F B$ & CB-d5 & \\
\hline 50 ug/L Contin & ing Cal Std & 12.83 & 17.75 & 21.88 & \\
\hline & Upper Limit & 13.33 & 18.25 & 22.38 & \\
\hline & Lower limit & 12.33 & 17.25 & 21.38 & \\
\hline CLIENT & $\mathrm{DCL}$ & $\mathrm{RT}$ & $\mathrm{RT}$ & $\mathrm{RT}$ & \\
\hline Sample No. & Sample No. & FB & CB-d5 & pDCB-d4 & \\
\hline BL-136531-1 & BL-136531-1 & 12.83 & 17.75 & 21.89 & \\
\hline$Q C-136531-1$ & QC-136531-1 & 12.82 & 17.73 & 21.87 & \\
\hline 00059 & $97 \cup 02872$ & 12.83 & 17.74 & 21.88 & \\
\hline 00068 & $97 \cup 02873$ & 12.83 & 17.75 & 21.88 & \\
\hline 00070 & $97 \cup 02874$ & 12.82 & 17.74 & 21.88 & \\
\hline 00071 & $97 \cup 02951$ & 12.82 & 17.74 & 21.87 & . \\
\hline 00076 & $97 \cup 02952$ & 12.83 & 17.74 & 21.87 & \\
\hline 00092 & $97 \cup 02953$ & 12.83 & 17.74 & 21.88 & \\
\hline 00093 & $97 \cup 02954$ & 12.82 & 17.74 & 21.88 & \\
\hline NAPR2S2A & $97 C 02896$ & 12.82 & 17.74 & 21.87 & \\
\hline NAPR2S2B & $97 C 02897$ & 12.82 & 17.74 & 21.88 & \\
\hline NAPR2S4 & $97 C 02898$ & 12.83 & 17.75 & 21.88 & \\
\hline NAPR1S2A & $97 C 02899$ & 12.82 & 17.74 & 21.88 & \\
\hline NAPR1S2B & $97 C 02900$ & 12.82 & 17.73 & 21.87 & \\
\hline NAPR1S4 & 97C02901MS & 12.82 & 17.74 & 21.88 & \\
\hline NAPR1S4 & 97C02901MSD & 12.82 & 17.75 & 21.88 & \\
\hline
\end{tabular}

LIMITS: Retention times should be within 30 seconds of the corresponding RT in the calibration. 


\section{Form 8 Equivalent \\ INTERNAL STANDARD AREA SUMMARY}

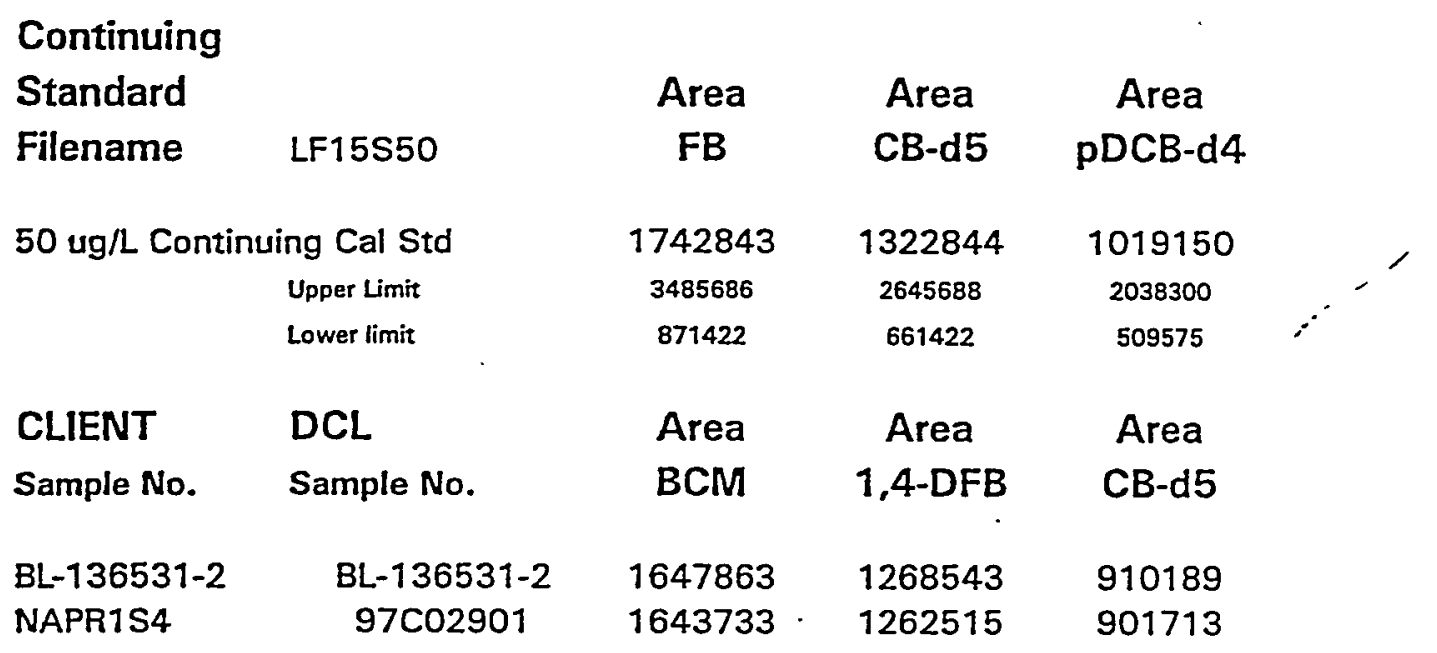

LIMITS: Areas should be within 50 to 200 percent of the corresponding area in the calibration.

045 
Form 8 Equivalent

\section{INTERNAL STANDARD RETENTION-TIME SUMMARY}

Continuing .

Standard

Filename

LF15S50

RT

RT

RT

50 ug/L Continuing Cal Std

BCM

1,4-DFB

CB-d5

50 ug/L Continuing Cal St
\[ \begin{array}{r}\text { Upper Limit } \\ \text { Lower limit }\end{array} \]

12.78
13.28
12.28

17.69
18.19
17.19

21.83

22.33

21.33

\section{CLIENT DCL}

Sample No.

Sample No.

RT

RT

RT

FB

CB-d5

pDCB-d4

BL-136531-2

BL-136531-2

12.78

17.70

21.84

NAPR1S4

$97 \mathrm{C02901}$

12.79

17.70

21.83

LIMITS: Retention times should be within 30 seconds of the corresponding RT in the calibration.

$0 \frac{8}{x} 6$ 

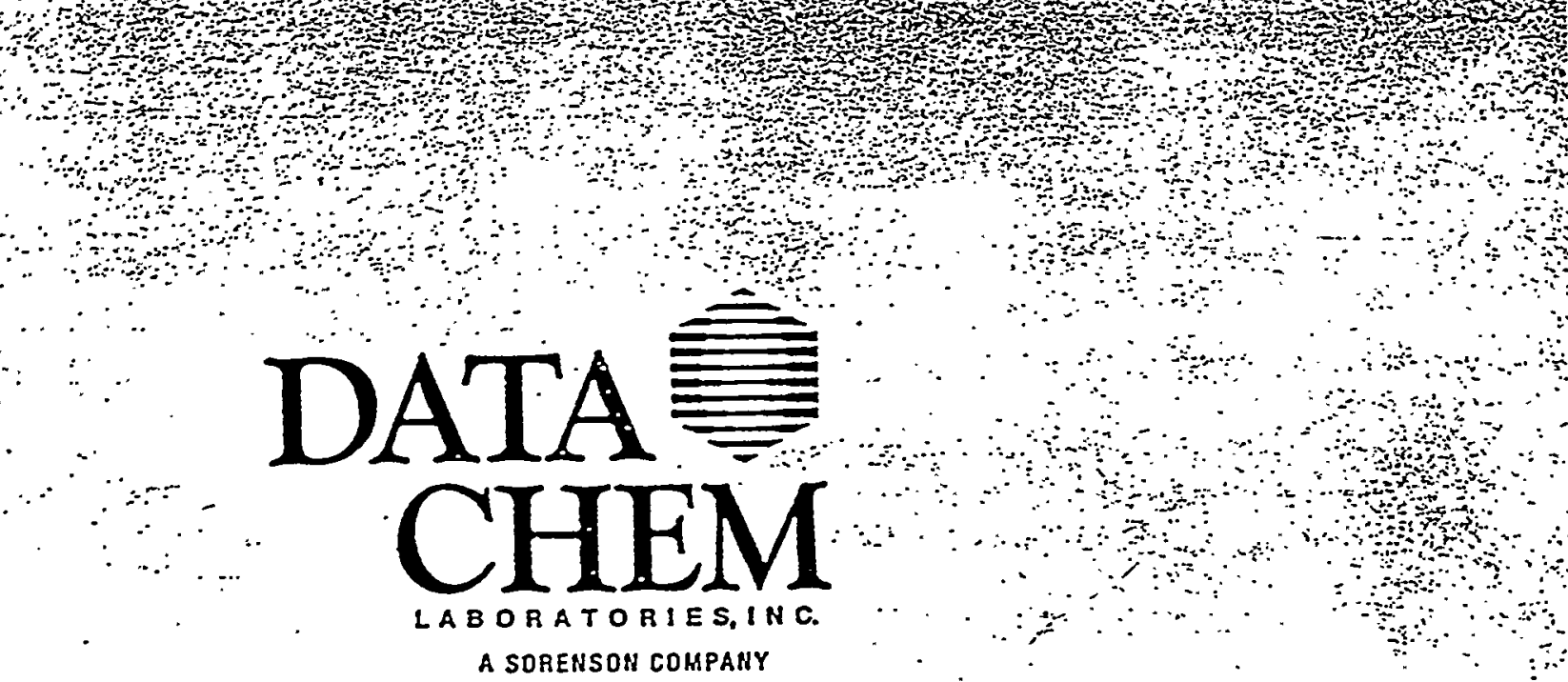

A SORENSON COMPANY

\section{Sample Tracking Documentation}




\section{DataChem Laboratories \\ LIMS - Sample Master System \\ Analysis Group Report}

Date: 20-JUL-1997 12:24

User: COLEMAN

Analysis Run Name: G976M004

Samples: 18

\begin{tabular}{rll} 
& Laboratory & Field Sample \\
Pos & Sample Name & Name 1 \\
\hline 1 & BL-136531-1 & BL-136531-1 \\
2 & BL-136531-2 & BL-136531-2 \\
3 & QC-136531-1 & QC-136531-1 \\
4 & 97 C02896 & NAP-R2-S2-A \\
5 & $97 C 02897$ & NAP-R2-S2-B \\
6 & 97 C02898 & NAP-R2-S4 \\
7 & $97 C 02899$ & NAP-R1-S2-A \\
8 & $97 C 02900$ & NAP-R1-S2-B \\
9 & $97 C 02901$ & NAP-R1-S4 \\
10 & $97 C 02901 M S$ & NAP-R1-S4 \\
11 & $97 C 02901 M S D$ & NAP-R1-S4 \\
12 & $97 U 02872$ & AW3TRIP02 \\
13 & $97 U 02873$ & AW3RNSW03 \\
14 & $97 U 02874$ & AW3FBLK02 \\
15 & $97 U 02951$ & AW3TRIP03 \\
16 & $97 U 02952$ & AW3RNSW04 \\
17 & $97 U 02953$ & AW2TRIP04 \\
18 & $97 U 02954$ & AW2RNSW04
\end{tabular}

Field Sample Name 2

00059
00068
00070
00071
00076
00092
00093

Page: 1

RLIMS15-V1.2

Group ID: G976:004

\begin{tabular}{lll}
$\begin{array}{l}\text { Laboratory } \\
\text { Sample ID }\end{array}$ & $\begin{array}{l}\text { Laboratory } \\
\text { Group Name }\end{array}$ & $\begin{array}{l}\text { Accnt. } \\
\text { Number }\end{array}$ \\
\hline S976600C & $97 C-0208-01$ & 03006 \\
S9767001 & $97 C-0208-01$ & 03006 \\
S976600D & $97 C-0208-01$ & 03006 \\
S975Y04R & $97 C-0208-01$ & 03006 \\
S975Y04S & $97 C-0208-01$ & 03006 \\
S975Y04T & $97 C-0208-01$ & 03006 \\
S975Y04V & $97 C-0208-01$ & 03006 \\
S975Y04W & $97 C-0208-01$ & 03006 \\
S975Y04X & $97 C-0208-01$ & 03006 \\
S976600E & $97 C-0208-01$ & 03006 \\
S976600G & $97 C-0208-01$ & 03006 \\
S975S091 & $97 U-0194-06$ & 03402 \\
S975S092 & $97 U-0194-06$ & 03402 \\
S975SO93 & $97 U-0194-06$ & 03402 \\
S975V08M & $97 U-0199-07$ & 03402 \\
S975V08N & $97 U-0199-07$ & 03402 \\
S975V08P & $97 U-0199-07$ & 03402 \\
S975V080 & $97 U-0199-07$ & 03402
\end{tabular}



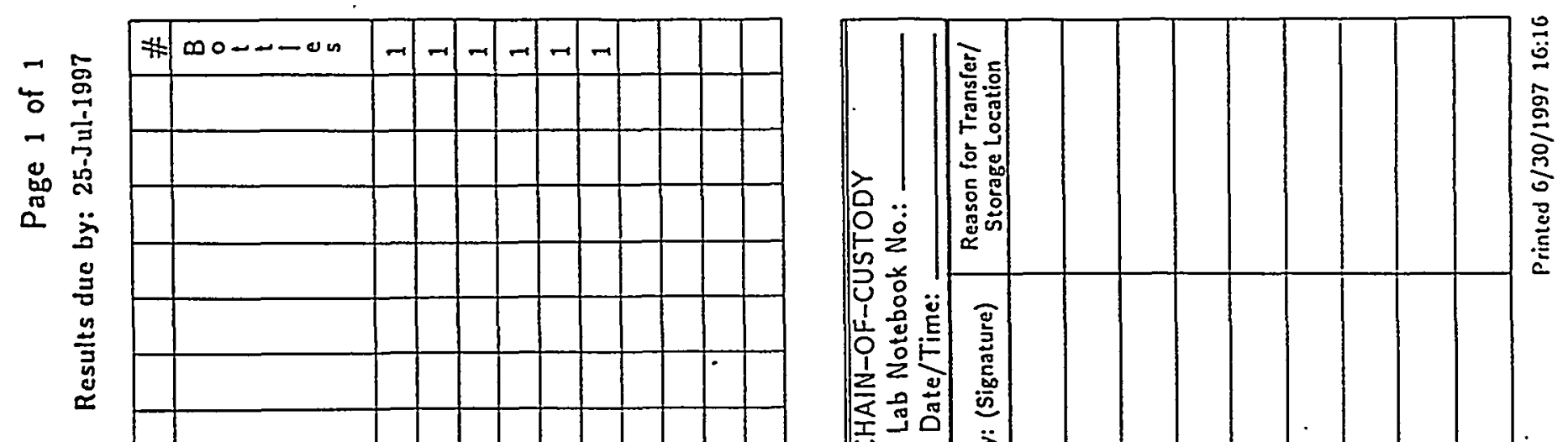

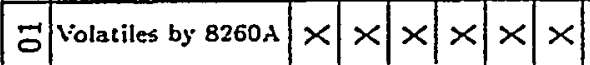
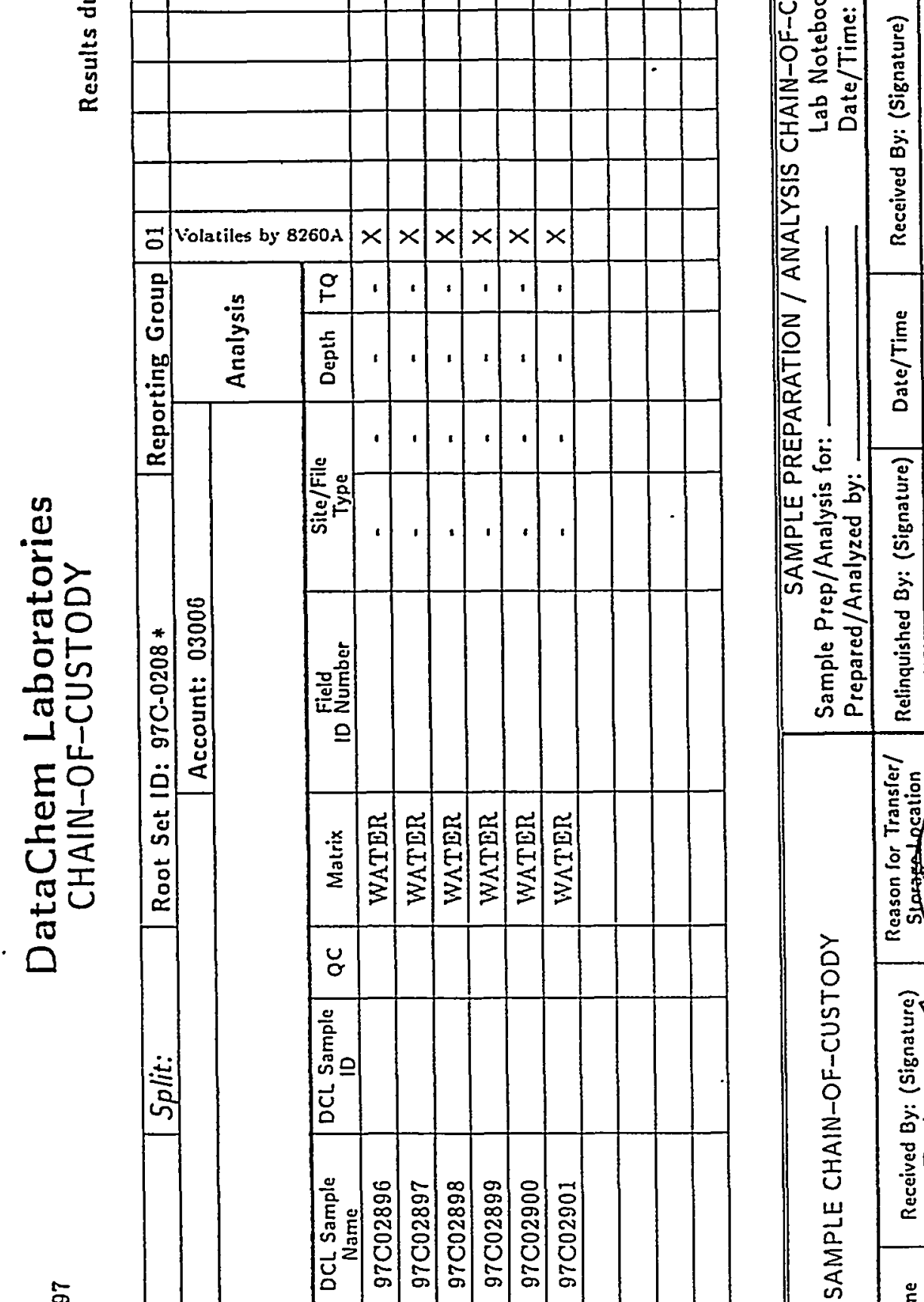

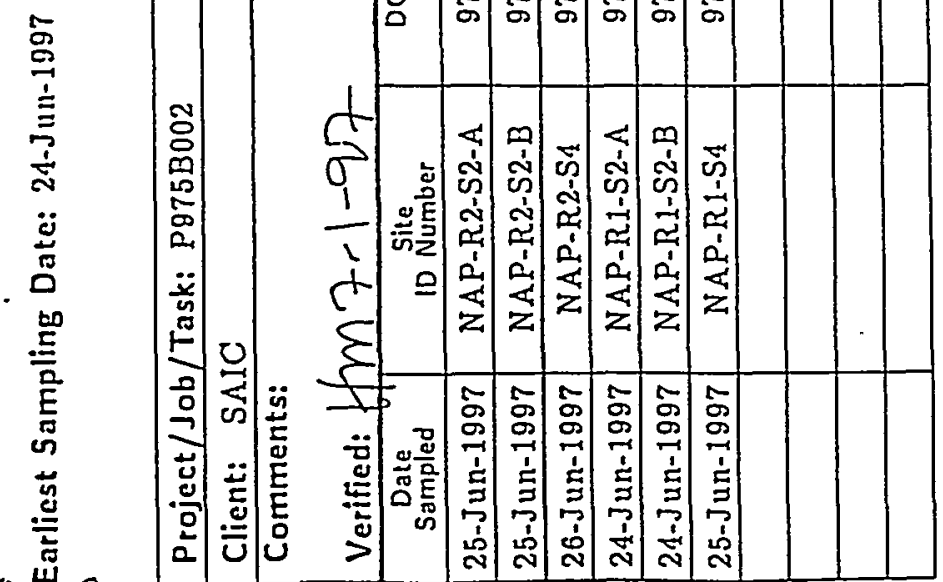
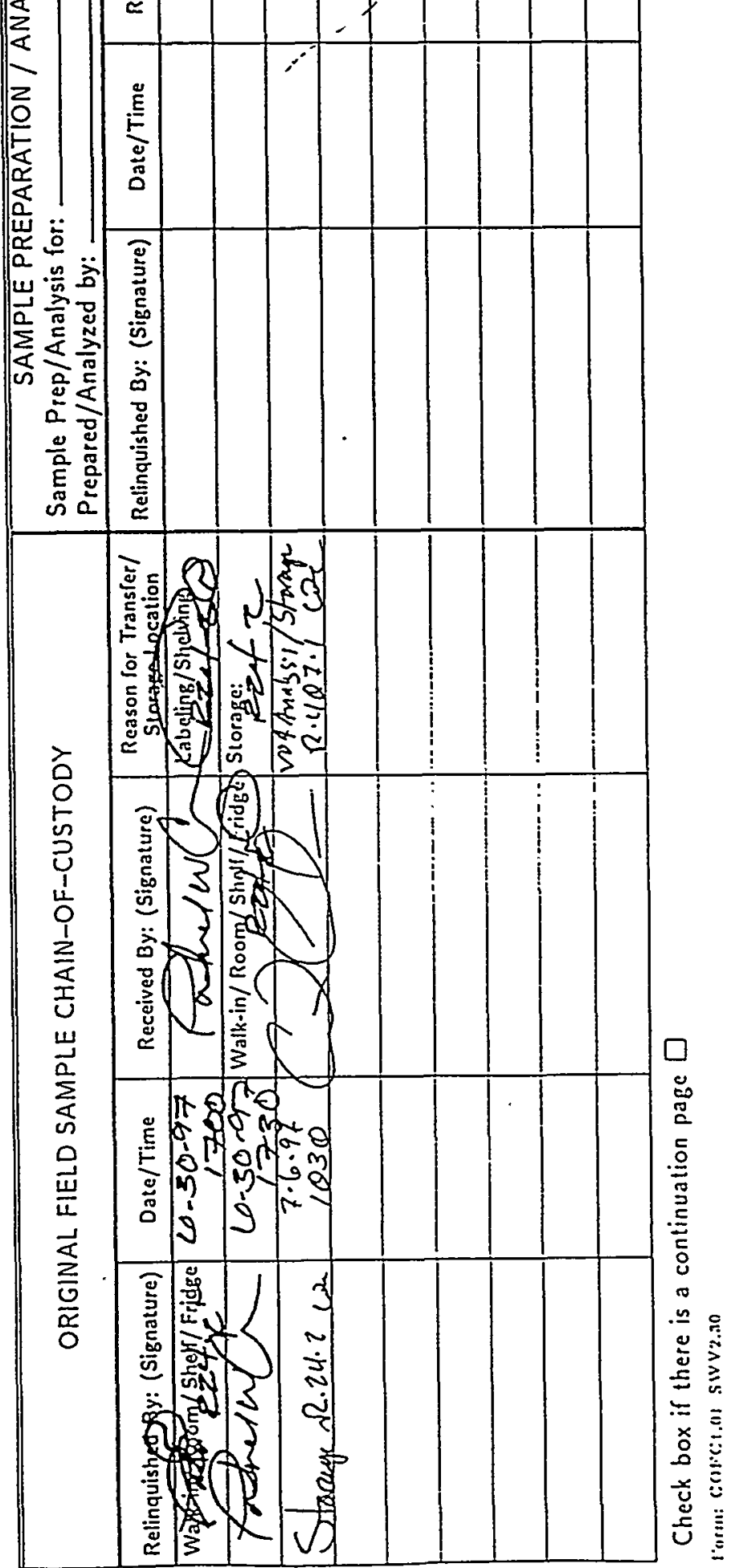


\section{Sample Work Order}

- Elearance:

DCL Root Set ID: 97C-0208*

$\therefore$.ject Manager: Carol M. Bell

DCL Lab. Name: 97C02896-97C02901

-lient: SAIC

Account: 03006

Total \# Samples: 6

Sample Entry: Patrick W. Corum

Project/Task: P975B002

Section: FG

Date Received: 27-Jun-1997

Earliest Sampling Date: 24-Jun-1997

Date for Mailing Report: 25-Jul-1997

Preparation Type:

Date for Verbal Report: 25-Jul-1997

\begin{tabular}{|c|c|c|c|c|c|c|c|c|}
\hline $\begin{array}{l}\text { Rep. } \\
\text { Group }\end{array}$ & $\begin{array}{c}\text { FG Section } \\
\text { Analytes Requested } \\
\end{array}$ & $\begin{array}{c}\text { Latest } \\
\text { Prep. Date }\end{array}$ & $\begin{array}{c}\text { Latest } \\
\text { Anal. Date } \\
\end{array}$ & $\begin{array}{l}\text { No. of } \\
\text { Samp. }\end{array}$ & $\begin{array}{l}\text { Storage } \\
\text { Location }\end{array}$ & $\begin{array}{c}\text { Analysis/Prep. } \\
\text { Method }\end{array}$ & Inst. & Matrix \\
\hline 01 & Volatiles by $8260 \mathrm{~A}$ & & 9-Jul-1997c & 6 & & $8260 \mathrm{~A}$ & GC/MS vo & WATER \\
\hline 02 & 625/8270 Water List & 2-Jul-1997c & Prept $40 \mathrm{~d}$ & 4 & & $8270 / 3510$ & GC/MS SV & WATER \\
\hline
\end{tabular}

${ }^{c}$ Based on date of collection

Special Instructions:

Section Manager: Reed A. Hendricks

Other Sections Receiving Sample Portions: ZI, FC 

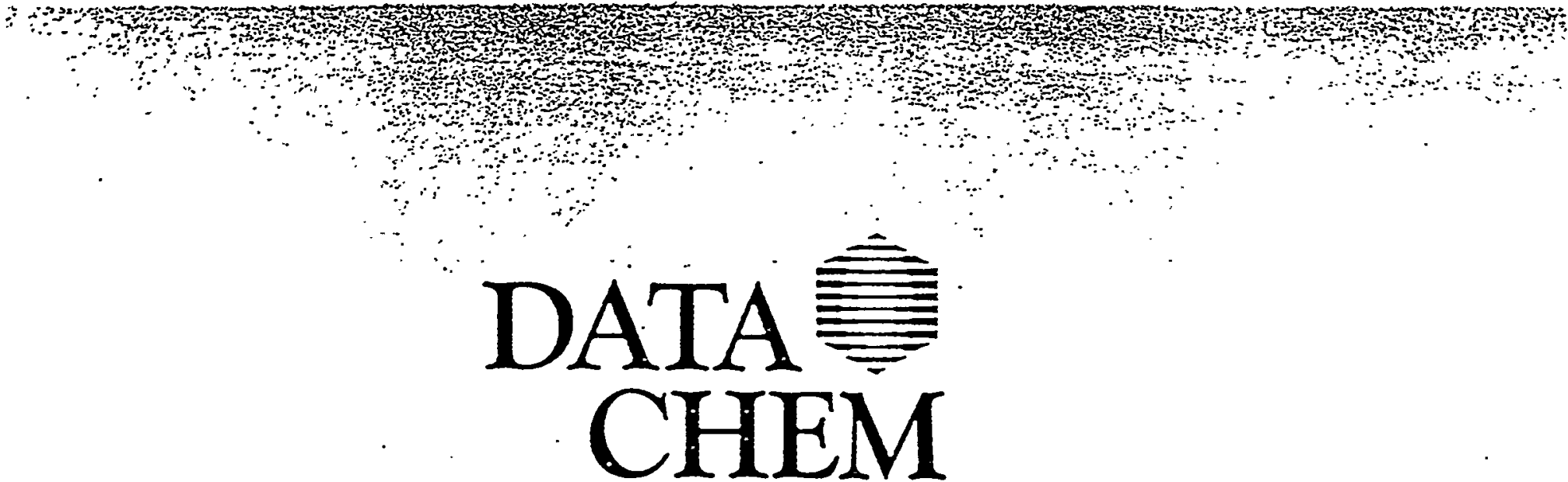

LABORATORIES, INC. A SORENSON COMPANY

\section{Analytical Documentation}

$6 s^{\prime}$ 


\section{WORKING STANDARDS}

\section{STANDARD PREPARATION LOGBOOK NO.142WS}

Page No. 090

142 WS 28290 DCL WORKING STANDARD SOLUTION IDENTIFICATION NUMBER

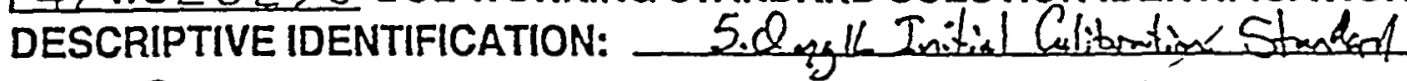

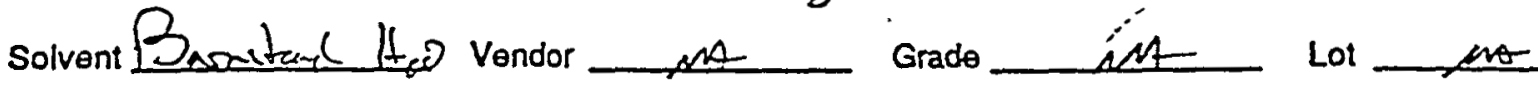

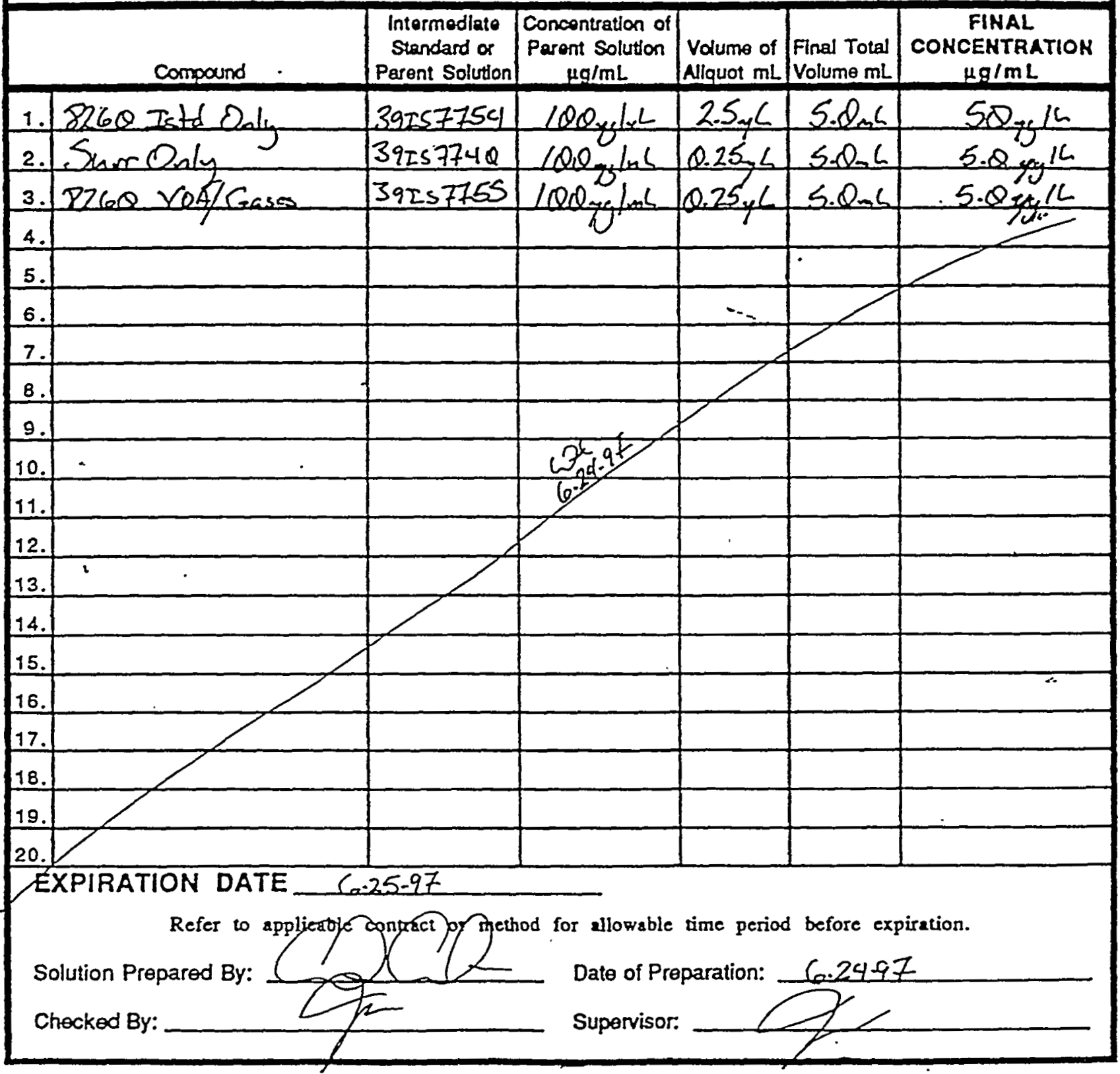

058 Comments: 


\section{WORKING STANDARDS}

\section{STANDARD PREPARATION LOGBOOK NO.1 42WS}

\section{WS 28292 DCL WORKING STANDARD SOLUTION IDENTIFICATION NUMBER}

DESCRIPTIVE IDENTIFICATION: 50 igll Initial Calitation Stand Solvent Bamstad $H_{0} 2$ vendor $M A$

\begin{tabular}{|c|c|c|c|c|c|c|}
\hline & Compound & $\begin{array}{c}\text { Intermedlato } \\
\text { Standard or } \\
\text { Parent Solution }\end{array}$ & $\begin{array}{c}\text { Concentratton of } \\
\text { Parent Solution } \\
\mu \mathrm{g} / \mathrm{mL}\end{array}$ & $\begin{array}{l}\text { Volume of } \\
\text { Allguot } \mathrm{mL}\end{array}$ & $\begin{array}{l}\text { Final Total } \\
\text { Volume mL }\end{array}$ & $\begin{array}{c}\text { FINAL } \\
\text { CONCENTRATION } \\
\mu \mathrm{g} / \mathrm{mL}\end{array}$ \\
\hline 1. & 82600 Istrlosin & 35957754 & $1000 \times 4-a b$ & 2.5 .6 & S.0.1 & 58 ralc \\
\hline 2. & Sured Ody & 39557740 & 100 calach & $2.5 \%$ & $500-2$ & $58 \times 16$ \\
\hline 3. & $8268 \mathrm{vod} /$ Gases & $39 \pi 57755$ & 10094 & 2.5 .6 & $50 \mathrm{C}$ & 50.16 \\
\hline 4. & & & & & & 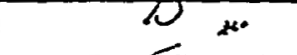 \\
\hline \multicolumn{7}{|c|}{5.} \\
\hline \multicolumn{7}{|c|}{6.} \\
\hline \multicolumn{7}{|l|}{7.} \\
\hline \multicolumn{7}{|c|}{8.} \\
\hline \multicolumn{7}{|c|}{9.} \\
\hline \multicolumn{7}{|c|}{10.} \\
\hline \multicolumn{7}{|c|}{11.} \\
\hline \multicolumn{7}{|c|}{12.} \\
\hline \multicolumn{7}{|c|}{13.} \\
\hline \multicolumn{7}{|c|}{14.} \\
\hline \multicolumn{7}{|c|}{15.} \\
\hline 16. & & & & & & \% \\
\hline \multicolumn{7}{|c|}{17.} \\
\hline \multicolumn{7}{|c|}{18.} \\
\hline \multicolumn{7}{|c|}{19.} \\
\hline 20. & Z & & & & & \\
\hline \multicolumn{7}{|c|}{ EXPIRATION DATE $\quad 6.25 .97$} \\
\hline & $\begin{array}{l}\text { lution Prepared By: } \\
\text { recked By: }\end{array}$ & & $\begin{array}{l}\text { lod for allowable } \\
\text { - Date of Pre } \\
\text { Supervisor: }\end{array}$ & $\begin{array}{l}\text { time period } \\
\text { paration: }\end{array}$ & $\begin{array}{r}\text { before } \\
6.24 \\
\end{array}$ & \\
\hline
\end{tabular}

Comments:

060 


\section{WORKING STANDARDS}

\section{STANDARD PREPARATION LOGBOOK NOI $42 W S$}

Page No. 093

142 WS 28293 DCL WORKING STANDARD SOLUTION IDENTIFICATION NUMBER DESCRIPTIVE IDENTIFICATION: - 100 is il Initial Culitation Standard Solvent Beonstad Hag vendor Mt Grade_MA Lot L as.

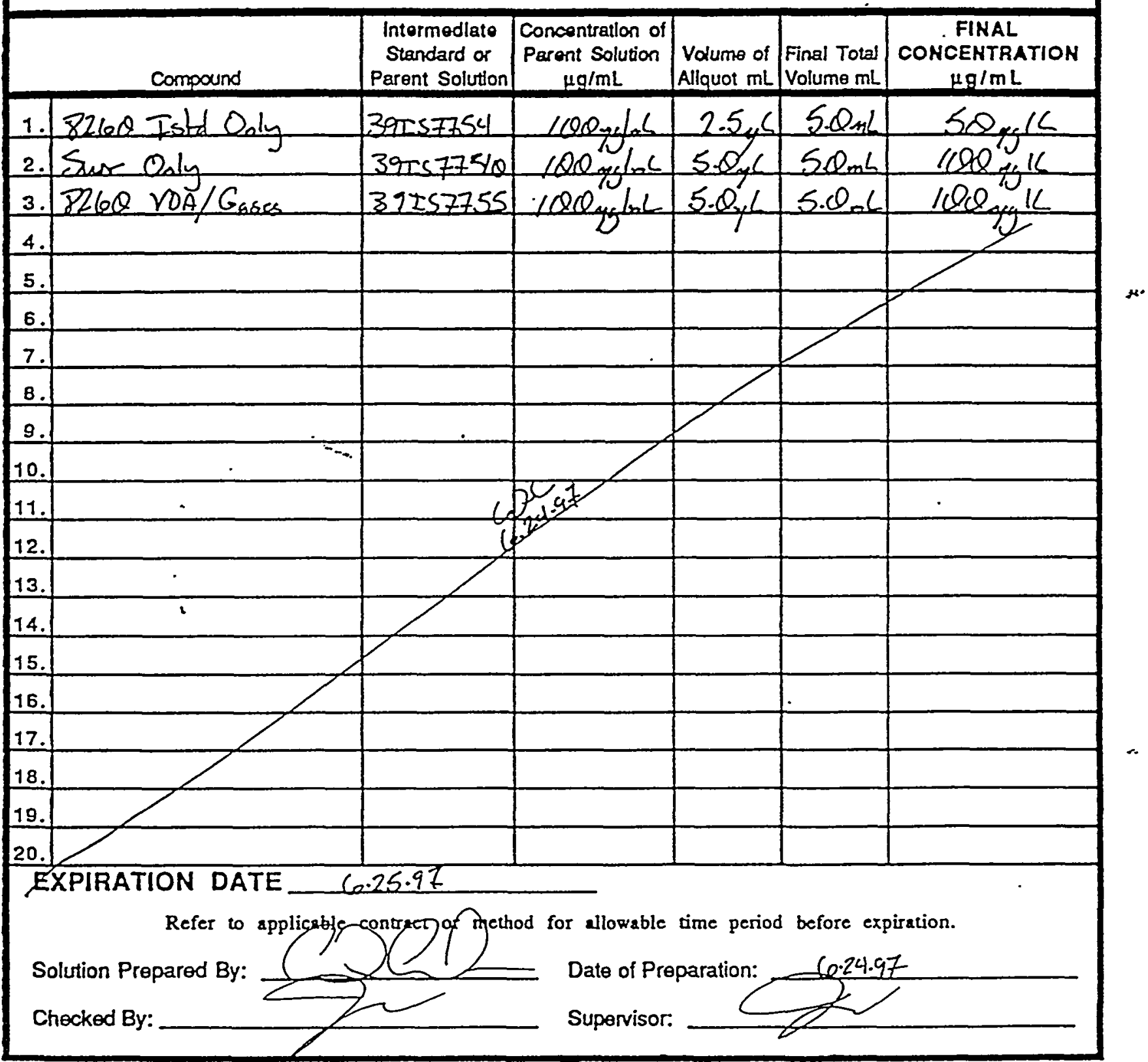

Comments:

063 


\section{WORKING STANDARDS}

\section{STANDARD PREPARATION LOGBOOK NO.142WS}

\section{Page No. 094}

142 WS28294 DCL WORKING STANDARD SOLUTION IDENTIFICATION NUMBER

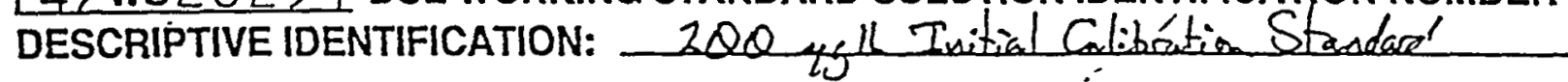

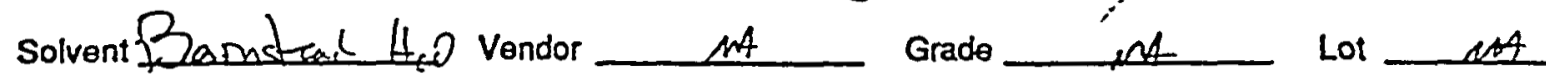

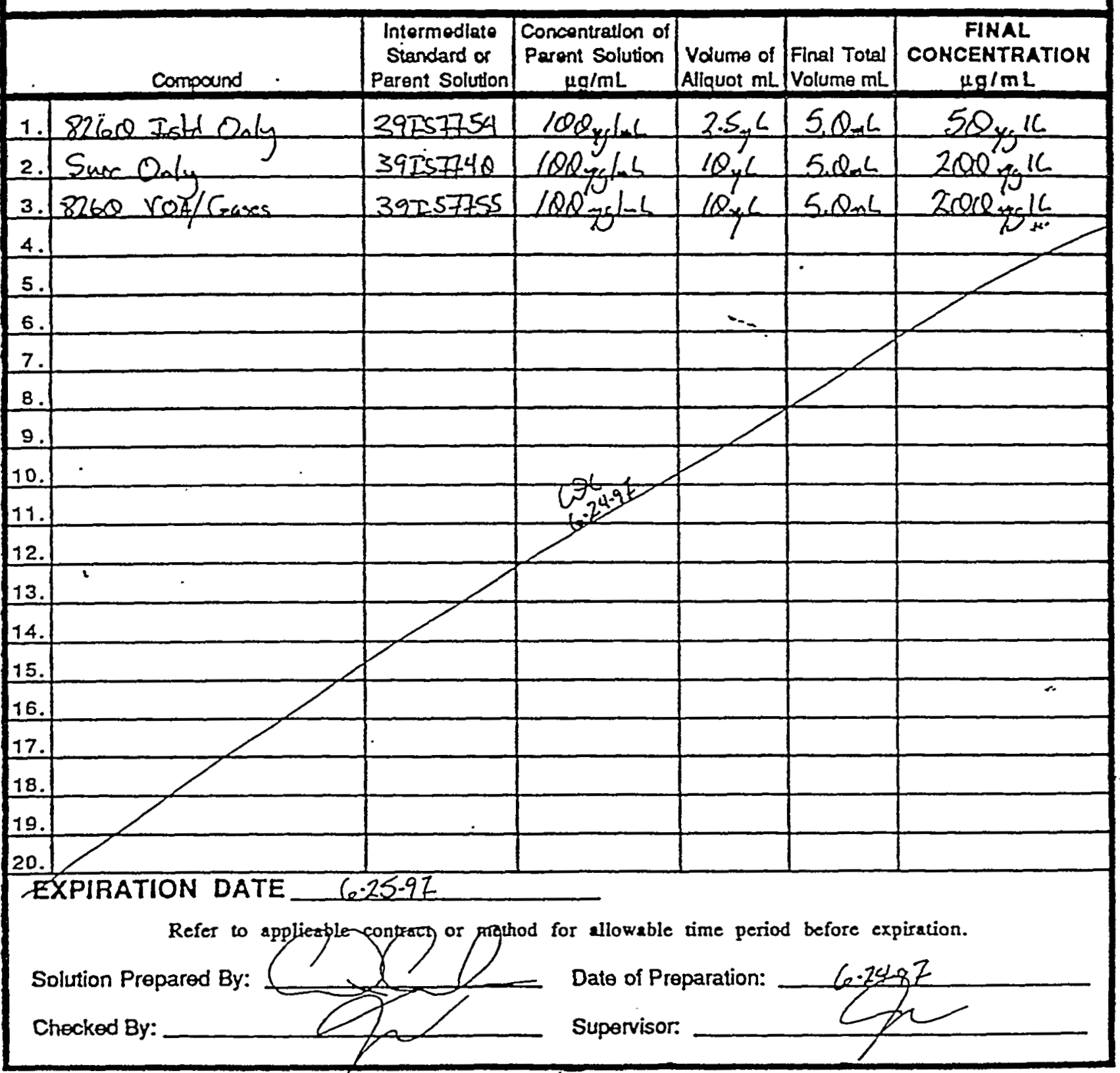




\section{WORKING STANDARDS}

\section{STANDARD PREPARATION LOGBOOK NO1 42WS}

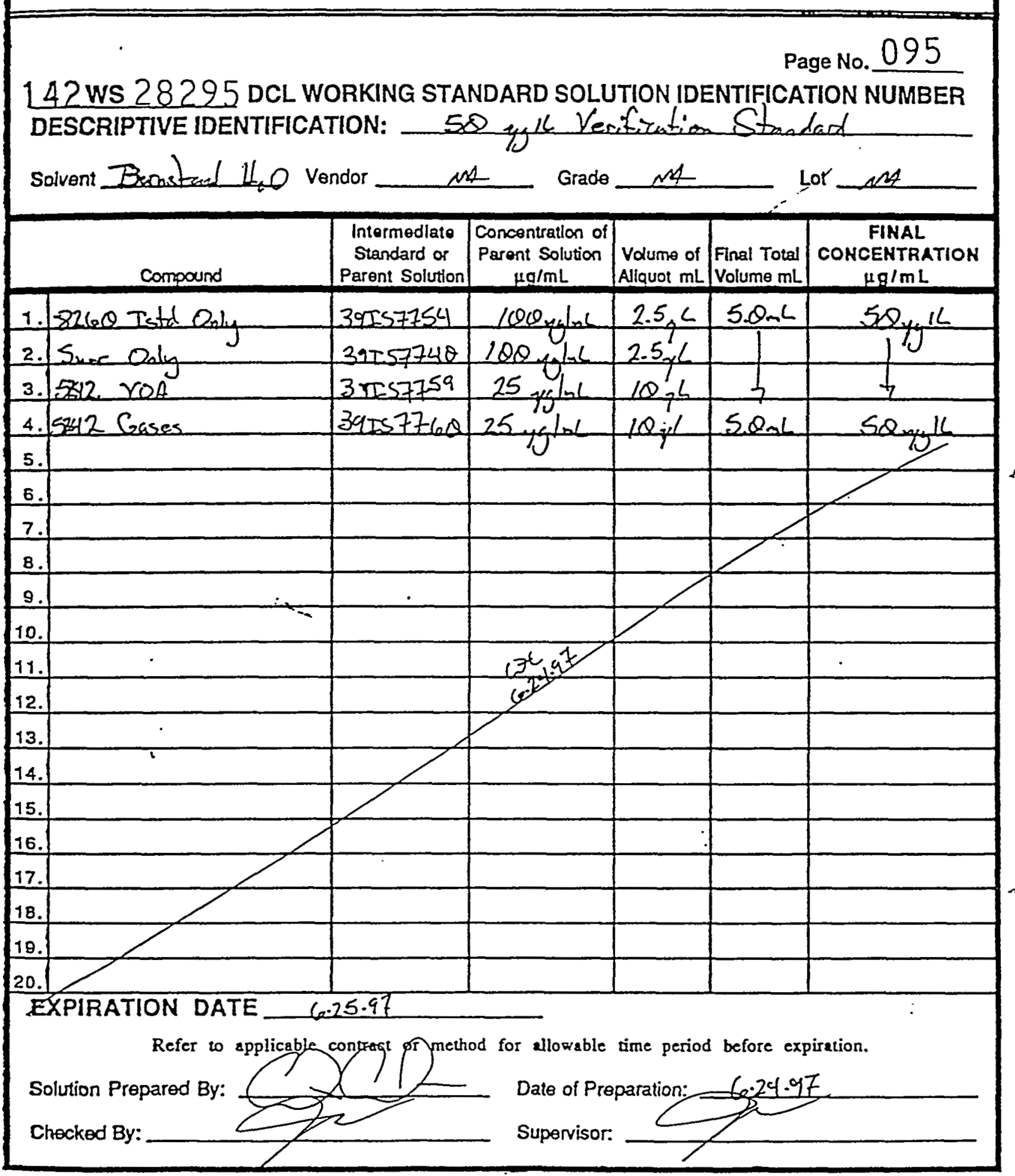

Comments:

$0 ? 3$ 


\section{STANDARD SOLUTIONS OF INTERMEDIATE CONCENTRATION}

\section{STANDARD PREPARATION LOGBOOK NO.39 IS}

Page No. 076

39 IS 7676 DCL INTERMEDIATE STANDARD SOLUTION IDENTIFICATION NUMBER DESCRIPTIVE IDENTIFICATION: BFB

Solvent MeOH Vendor Bd) Grade PAT,' Lot BN58Ge

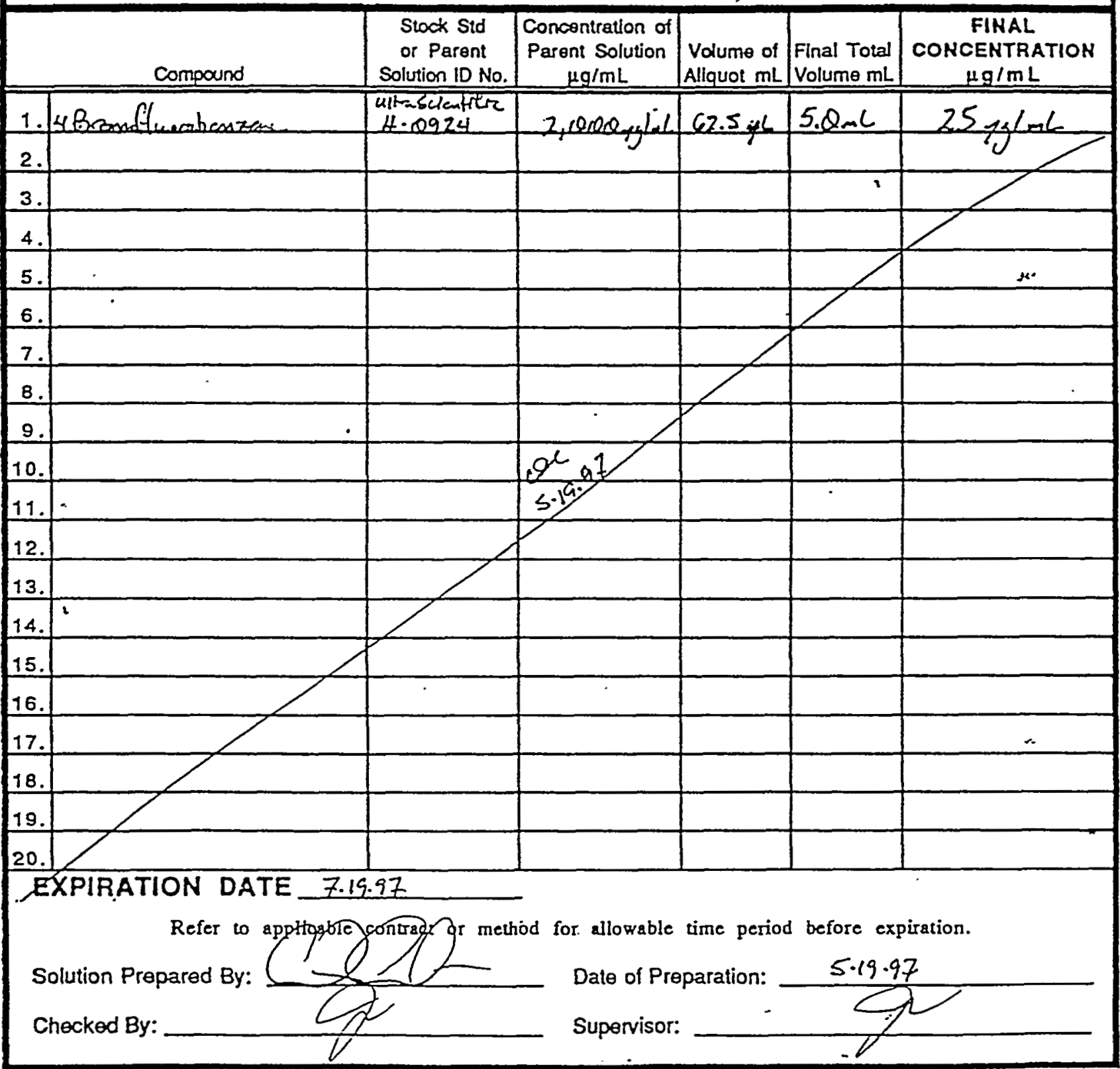

064

Comments: 


\section{A2LA Certified Reference Material}

4-Bromoflúorobenzene Solution

Catalog Number: STS-110

Citrification Date: $11 / 22 / 94$
Lot Number: $\mathrm{H}-0924$ A2LA Certification Number: US0160

Page:

This stancard has been certified tor accuracy, homogeneity, and stability according to American Association for LEboratory

Accreditation (A2LA) sdecifications.

\section{Component}

A-toromof Luorobenzene

Solvent: methanol
Certified

True Concentration*

$$
200 i .5 \mathrm{ug} / \mathrm{mL}
$$

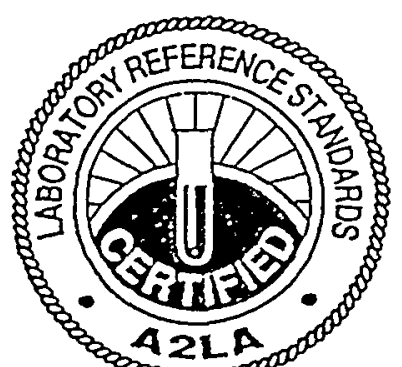

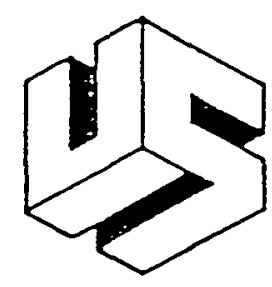

ULTRA SCIENTIFIC

250 Smith Street

North Kingstown, RI 02852 (401) 294-9400 


\section{STANDARD SOLUTIONS OF INTERMEDIATE CONCENTRATION}

\section{STANDARD PREPARATION LOGBOOK NO.39 IS}

Page No. 154 39 IS 7754 DCL INTERMEDIATE STANDARD SOLUTION IDENTIFICATION NUMBER DESCRIPTIVE IDENTIFICATION: ISTD OnLY $8260 \mathrm{~A}$

solvent Meoff vendor BetJ Grad PAT Lot Bo $\$ 84$

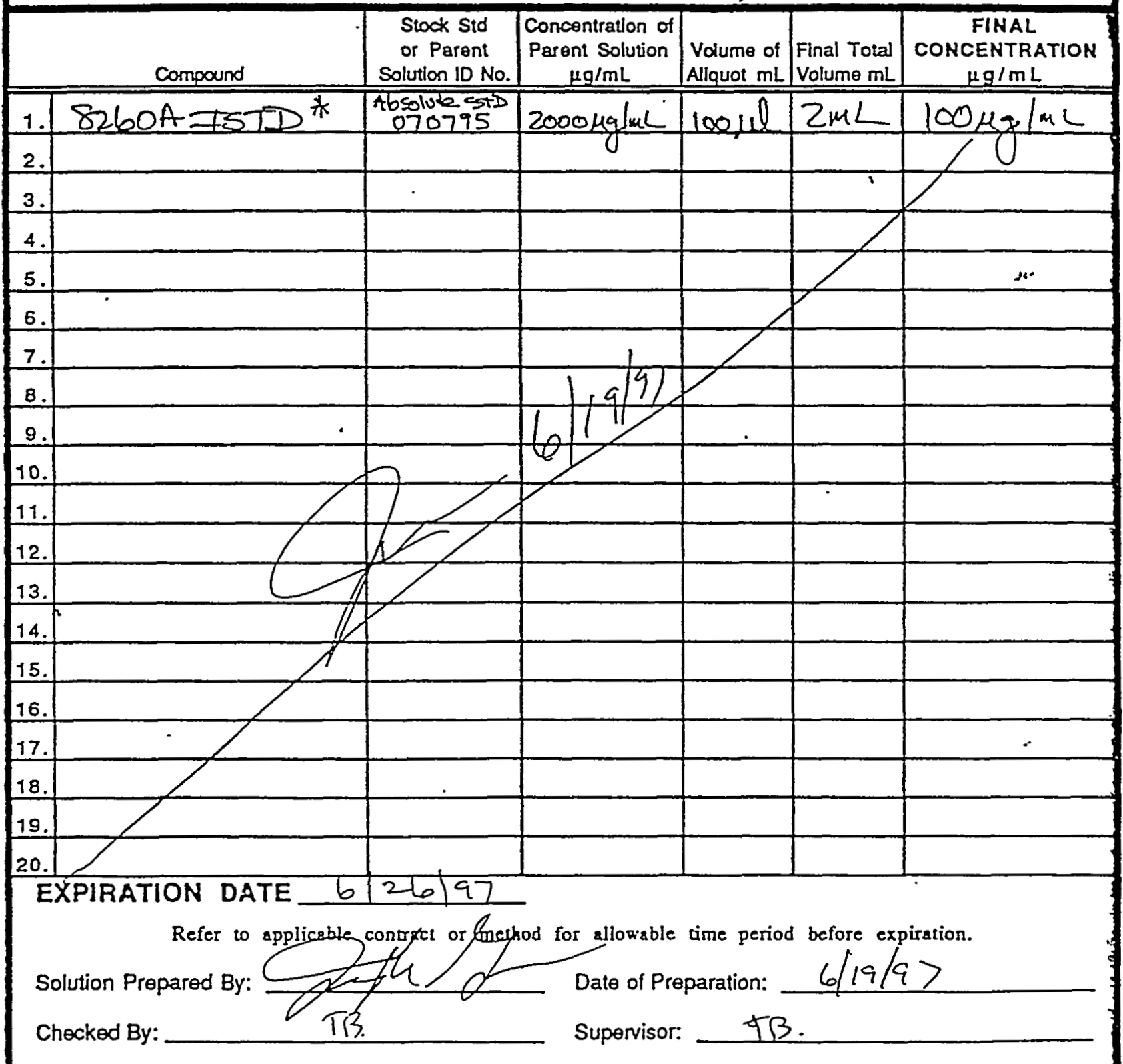

comments: $*$-Part 22013 


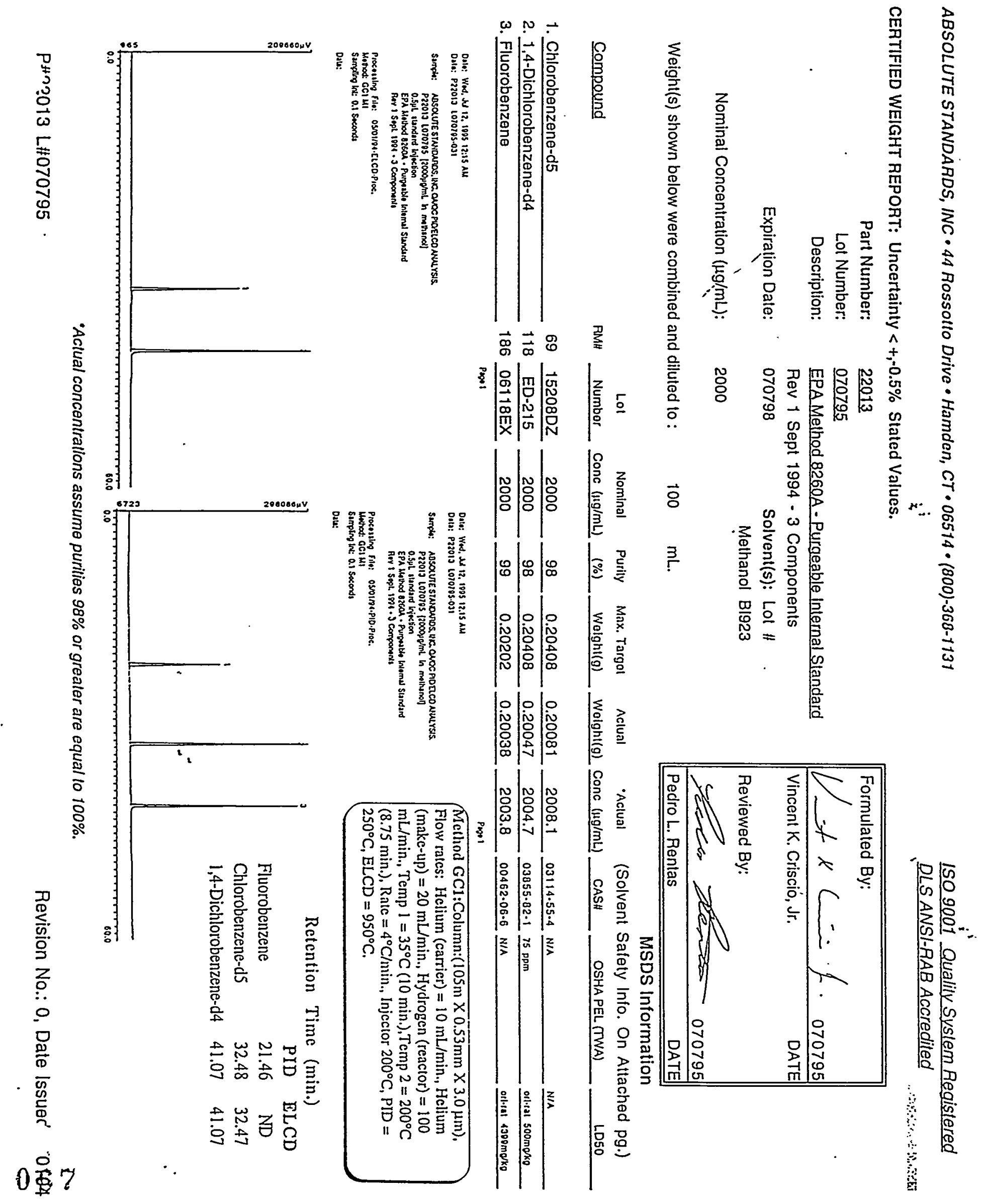




\section{STANDARD SOLUTIONS OF INTERMEDIATE CONCENTRATION}

\section{STANDARD PREPARATION LOGBOOK NO.39 IS}

39 IS 7740 DCL INTERMEDIATE STANDARD SOLUTION IDENTIFICATION NUMBER DESCRIPTIVE IDENTIFICATION: Surr Ohly

Solvent MeOH vendor BfCI Grade PQT, Lot BO $\$ 84$

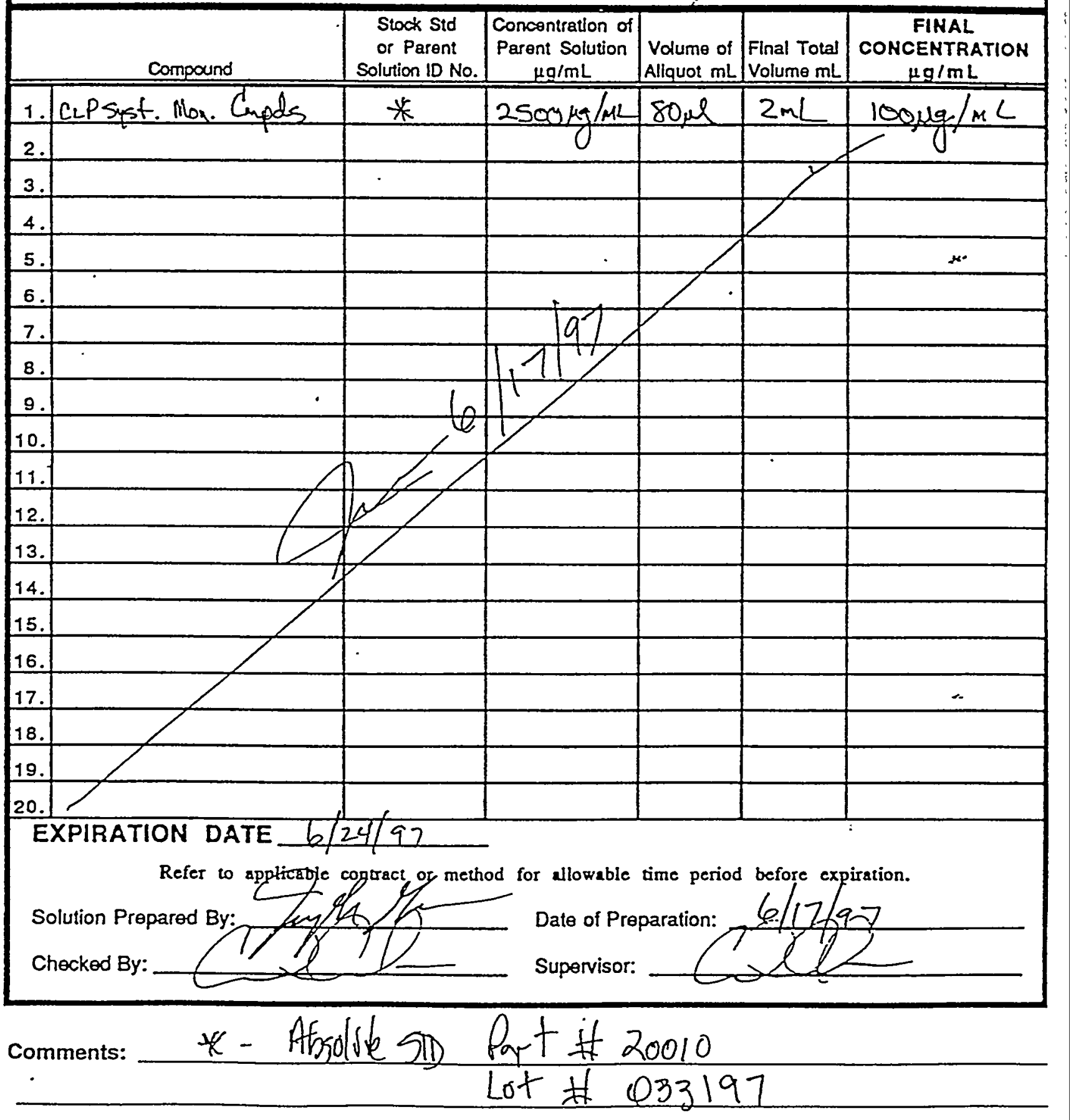




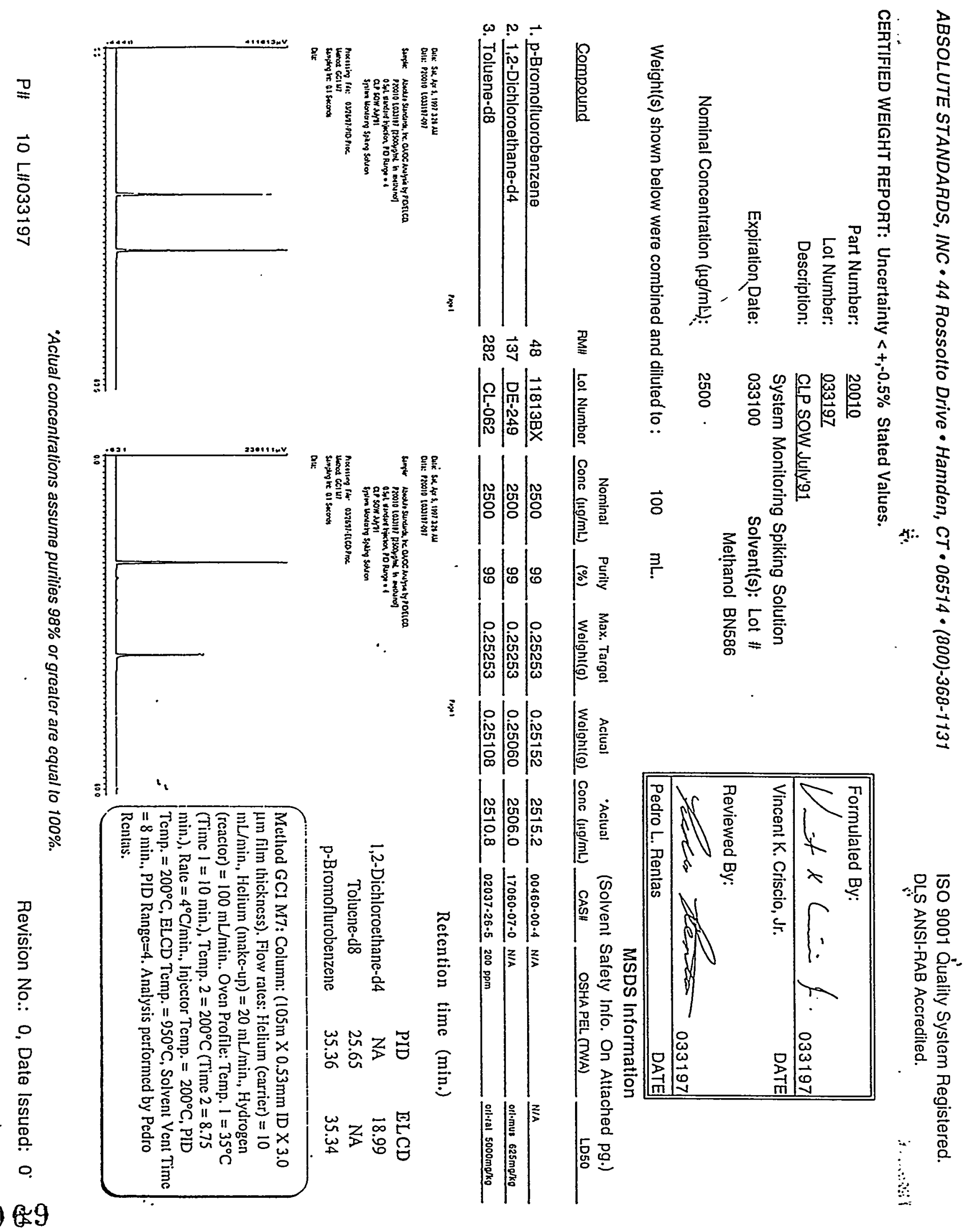




\section{STANDARD SOLUTIONS OF INTERMEDIATE CONCENTRATION}

\section{STANDARD PREPARATION LOGBOOK NO.39 IS}

39 IS 7755 DCL INTERMEDIATE STANDARD SOLUTION IDENTIFICATION NUMBER DESCRIPTIVE IDENTIFICATION: $8260 \mathrm{~A}$ VOA-t6ASES

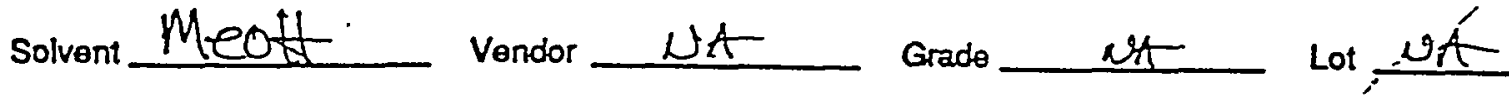

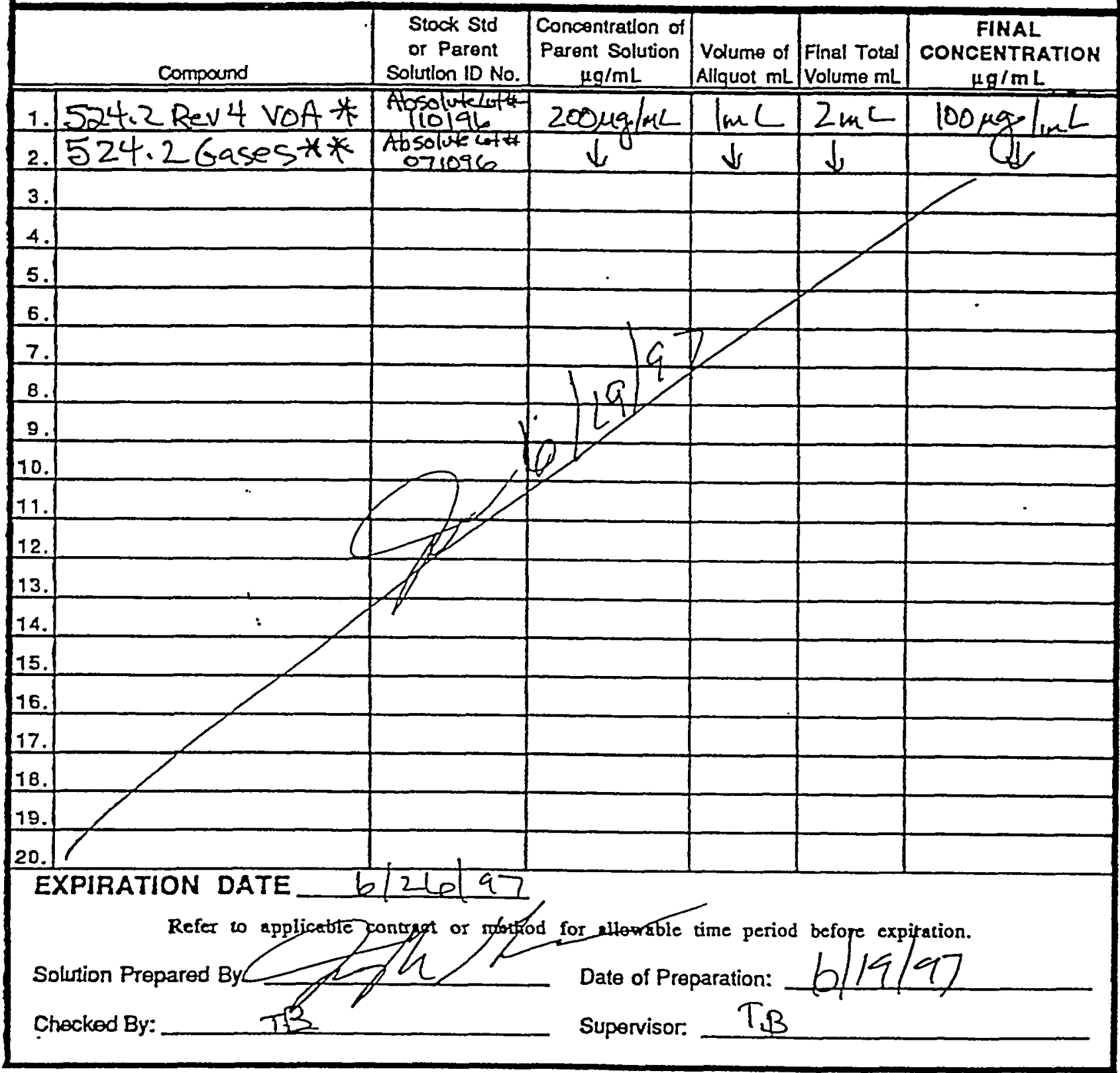

Comments: if - Peff $3300 \mathrm{~L}$

$0 \% 0$ sicpert 30002 
CERTIEDD WEIGHT REPORT: Uncertainty $<+,-0.5 \%$ Stated Values.

Part Number: $\underline{33001}$

Lot Number: 110196

Description: EPA Method 524.2 -Revision 4.0

78 Components

Expiration Date: $110199 \quad$ Solvent(s): Lot \#

Methanol BM957 (90\%)

Nominal Concentration ( $\mu \mathrm{g} / \mathrm{mL}$ ): 200

Water $\mathrm{H} 36273(10 \%)$

Volume(s) shown below were combined and diluted to:

$100 \mathrm{~mL}$.

Note: Part"s's 32001 and 34002 diluted 1/10.

\section{Da. Inital Initias}

Compound

1. Bromodichloromethane

2. Dibromochloromethane

3. 1.1-Dichloroethene

4. cis-1,2-Dichloroethene

5. trans-1,2-Dichloroethene

6. Methylene chloride

7. Bromochloromethane

B. Bromolorm

9. Carbon tetrachloride

10. Chlorolorm

11. Dibromomethane

12. 1.1-Dichloroethane

13. 2,2-Dichloropropane

14. Tetrachloroethene

15. 1,1.1-Trichloroethane

16. 1.2-Dibromo-3-chloropropane

17. 1,2-Dibromoethane

18. 1,2-Dichlomethane

19. 1.2-Dichloropropane

20. 1.3-Dichloropropane

21. 1,1-Dichloropropene

22. cis-1,3-Dichloropropene

23. trans-1,3-Dichloropropene

24. Hexactilorobutadiene

25. 1.1.1,2-Tetrachloroethane

25. 1,1.2.2-Tetrachloroethane

27. 1.1,2-Trichloroethane

28. Trichloroethene

29. 1.2.3-Trichloropropane

30. Benzene

31. Bromobenzene

32. n-Butyl benzene

33. Ethyl benzene

34. D-Isopropyl toluene

35. Naphthalene

36. Styrene

37. Toluene

38. 1,2.3-Trichlorobenzene

39. 1,2,4-Trichlorobenzene

40. 1,2,4-Trimethylbenzene

41. 1,3.5-Trimethylbenzene (Mesitylene)

42. m-Xylene

43. tert-Butyl benzene

44. sec-Butyl benzene

45. Chlorobenzene

46. 2-Chlorotoluene

47. 4-Chiorotoluene

48. 1,2-Dichlombenzene

49. 1.3-Dichlorobenzene

Pant Number Lot Number Factor Vol. (mL) Conc.(ug/mL)

$32001 \frac{103096}{103096} \frac{0.10}{0.10}-10.00 \frac{2002.7}{10.00}$ $32001 \frac{103096}{103096} \frac{0.10}{0.10} \frac{10.00}{10.00}-\frac{2002.7}{2002.8}$ $32001 \frac{103096}{103096} \frac{.0 .10}{0.10} \frac{10.00}{10.00} \frac{2002.8}{2007.3}$ $32001 \frac{103096}{0.10}-\frac{10.00}{103096}-\frac{2007.4}{2004.4}$ $32001 \frac{103096 \quad 0.10 \quad 10.00 \quad 2003.6}{103096}$ $32001 \frac{103096}{0.10} 10.00 \frac{0004.5}{10.00}$ $32001 \frac{103096}{103096} \frac{0.10}{0.10}-\frac{10.00}{10.00}-\frac{2004.5}{2008.1}$ $32001 \frac{103096}{0.10} \frac{10.00}{2010.7}$ $32001 \frac{103096}{0.10}-\frac{10.00}{2009.6}$ $32001-\frac{103096}{10.10}-\frac{10.00}{103090}-\frac{2009.3}{0.10}$ $32001 \frac{103096}{0.10} \frac{10.00}{2010.0}$ $32001 \frac{103096}{0.10} 10.00 \frac{0009.1}{103096}$ $32001 \frac{103096}{30.10} \frac{10.00}{10009.5}$ $32001 \frac{103096}{103096} \frac{0.10}{10.00} 2010.7$ $32001 \frac{103096-0.10-10.00-2009.6}{103090}$ $32001 \frac{103096}{0.10}-10.002010 .0$ $32001 \frac{103096}{0.10} \frac{10.00}{2008.8}$ $32001 \frac{103096}{0.10} \frac{10.00}{2009.2}$ $32001 \frac{103096}{10.10} \frac{0.10}{10.00} \frac{10008.5}{10.00}$ $32001 \frac{103096}{0.10} \frac{10.00}{2009.2}$ $32001 \frac{103096}{0.10} 10.00 \quad 2000.4$ $32001+103096-0.10 \frac{10.00}{2021.8}$ $32001 \frac{103096}{0.10} \frac{10.00}{2022.9}$ $32001-\frac{103096}{0.10}-\frac{10.00}{2018.3}$ $32001 \frac{103095}{0.10} 10.002010 .1$ $32001 \frac{103096}{0.10} \frac{10.00}{2009.3}$ $32001-\frac{103090}{103096}-\frac{0.10}{0.10} \frac{10.00}{10.00} \frac{2016.9}{10.00}$ $32001 \frac{103096}{103096} \frac{0.10}{103096} \frac{10.00}{2009.7}$ $32001 \frac{103096}{0.10} 10.00 \frac{2008.1}{103090}$ $\frac{32001}{32001} \frac{103096}{103096} \frac{0.10}{0.10} \frac{10.00}{10.00} \frac{2005.2}{2002.0}$ $32001 \frac{103096}{0.10}-10.00-2001.2$ $32001 \frac{103096}{0.10} \frac{10.00}{103096} \frac{2005.8}{20.800 .6}$ $32001 \frac{103096}{0.10} 10.002005 .2 \frac{200.5}{103096}$ $32001 \frac{103096}{0.10} \frac{10.00}{2001.0} 200.1$ $32001 \frac{103096}{0.10} \frac{10.00}{2004.0} \frac{200.4}{103096}$ $32001 \frac{10309}{103096} \frac{0.10}{0.10} \frac{10.00}{10.00} \frac{2004.0}{2002.7} \frac{200.4}{200.3}$ $32001 \frac{103096}{0.10} \frac{10.00}{2001.6}-200.2$ $32001 \frac{103096}{0.10} 10.00 \frac{2005.5}{200.5}$ $32001 \frac{103096}{0.10} \frac{10.00}{2001.5} 200.2$ $32001 \frac{103096}{10309} \frac{0.10}{0.10 .00} \frac{2002.1}{200.2}$ $32001 \frac{103096}{0.10} \frac{10.00-2001.0-200.1}{103096}$ $32001 \frac{103096}{0.10} \frac{10.00}{2009.1} 200.9$ $32001 \frac{103096}{10.10} \frac{10.00}{2008.9} 200.9$ $32001 \frac{103096}{0.10} \frac{10.00}{2008.6} 200.9$ $32001 \frac{103096}{0.10} \frac{10.00}{2008.4} 200.8$ $32001 \frac{103096}{0.10} 10.00 \frac{0010.5}{2009}$ $32001 \frac{103096}{103096} \frac{0.10}{0.10} \frac{10.00}{10.00} \frac{2010.5}{2009.6}$ $32001 \quad 103096 \quad 0.10 \quad 10.00 \quad 2019.5$
Formulated By:

$V \rightarrow$ it $\times C$ C

Vincent K. Criscio, Jr. DATE

Reviewed By:

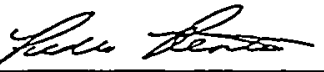
DATE 110196

MSDS Information

Final (Solvent Safety Info. On Attached pg.)

$200.3 \quad 00075-274 \mathrm{wi}^{2}$

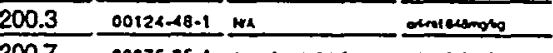

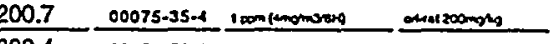

00.4 00156-50-5 wa watizangho

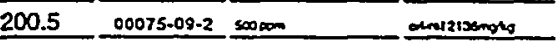

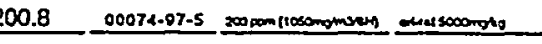

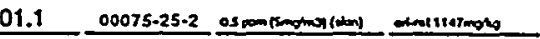

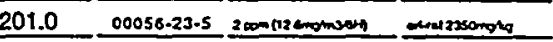

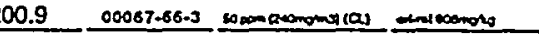

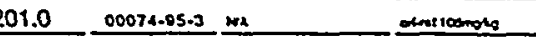

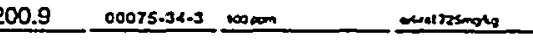
$201.1-\frac{0504-20.7}{00127-18 \alpha} \frac{\mathrm{KL}}{\mathrm{NO}}$

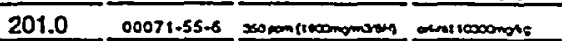

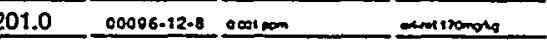

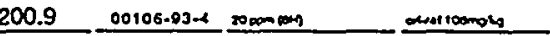

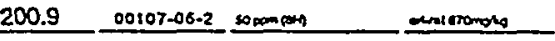

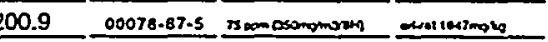
00.9 00142-28-0 $\mathrm{kt}$ 00.0 00563.58-6 $\mathrm{NL}$ $02.2 \frac{10061.00 \mathrm{t}-5}{100 \mathrm{mat}}$

$02.3 \frac{10061.02 .6}{\mathrm{wat}} \mathrm{wa}$

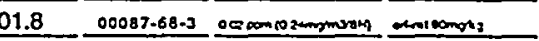
01.0 00630-20.6 $\mathrm{wer}$

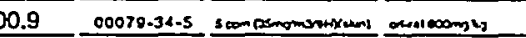

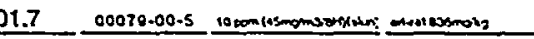

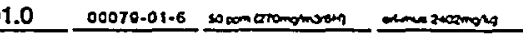

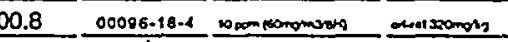

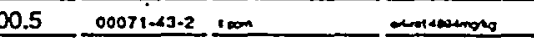
$00108-86.1 \mathrm{wa}$ $\cos 2000000$ $00104.51-8$ wax wa

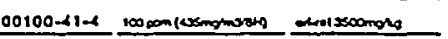

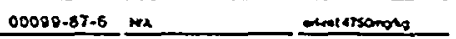

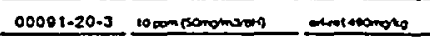

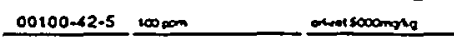

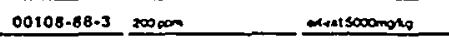
$00087.61 .6 \mathrm{HL}$ H.

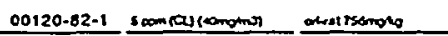

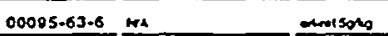
$00100-67.8 \mathrm{WA}$

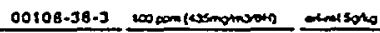
00098-06.6 NA

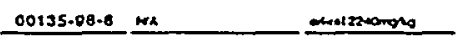

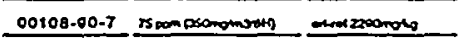

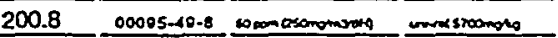

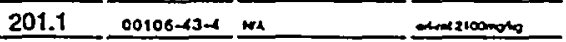

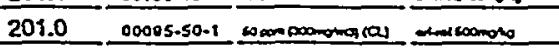
202.0 00541-73-1 $\mathrm{me}$ 


\begin{tabular}{|c|c|c|c|c|c|c|c|c|c|}
\hline Cempound & Pant Number & Lot Number. & $\begin{array}{c}\text { Da. } \\
\text { Factor }\end{array}$ & $\begin{array}{l}\text { Initial } \\
\text { Vor. (mL) }\end{array}$ & $\begin{array}{c}\text { Initial } \\
\text { Conc.(ug/mL) }\end{array}$ & $\begin{array}{c}\text { Final } 1 \\
\text { Conciug/mL) } \\
\end{array}$ & $\begin{array}{l}\text { (Solvent Safety Info. On A } \\
\text { cas OSHAPEL (TWA) }\end{array}$ & $\begin{array}{l}\text { ttached pg.) } \\
\text { Loso } \\
\end{array}$ & \\
\hline 50. 1.4-Dichlorobenzene & 32001 & 103096 & 0.10 & 10.00 & 2008.3 & 200.8 & 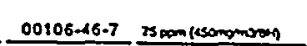 & andsoonoso & \\
\hline 51. Isopropylbenzene & 32001 & 103096 & 0.10 & 10.00 & 2009.4 & 200.9 & 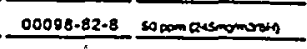 & arrelesonoto & \\
\hline 52. n-Propylbenzene & 32001 & 103096 & 0.10 & 10.00 & 2008.4 & 200.8 & $00103.65 .1 \mathrm{~ms}$ & 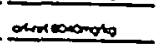 & \\
\hline 53. o-Xylene & 32001 & 103096 & 0.10 & 10.00 & 2019.4 & 201.9 & 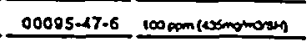 & onsixinges & \\
\hline 54. p-Xylene & 32001 & 103096 & 0.10 & 10.00 & 2009.5 & 201.0 & 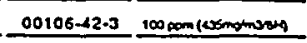 & $\operatorname{argsen}$ & \\
\hline 55. Acetone & 34002 & 121494 & 0.10 & 10.00 & 2004.5 & 200.4 & 00057.64 .1750000 & mowersonode & \\
\hline 56. Acrylonitrile & 34002 & 121494 & 0.10 & 10.00 & 2005.6 & 200.6 & 00107-13-1 m & dutnareo & \\
\hline 57. Allyl chloride (3-Chloropropene) & 34002 & 121494 & 0.10 & 10.00 & 2003.4 & 200.3 & 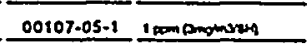 & antroconds & \\
\hline 58. 2-Butanone (Methyl ethyl ketone) & 34002 & 121494 & 0.10 & 10.00 & 2001.3 & 200.1 & 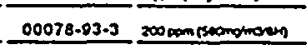 & andmimons & \\
\hline 59. Carbon disulphide & 34002 & 121494 & 0.10 & 10.00 & 2003.9 & 200.4 & 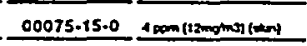 & 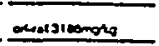 & \\
\hline 60. Chloroacetonitrile & 34002 & 121494 & 0.10 & 10.00 & 2007.0 & 200.7 & $00107-18-2 \mathrm{~km}$ & $\operatorname{anc} 120 \sin 00$ & \\
\hline 61. 1-Chlorobutane & 34002 & 121494 & 0.10 & 10.00 & 2007.1 & 200.7 & $00108-69-3 \mathrm{kNA}$ & andiarodes & \\
\hline 62. Irans-1,4-Dichloro-2-butene & 34002 & 121494 & 0.10 & 10.00 & 2007.8 & 200.8 & $00110.57 .5 \mathrm{mat}$ & Nes & \\
\hline 63. 1.1-Dichloropropanone-2 & 34002 & 121494 & 0.10 & 10.00 & 2008.4 & 200.8 & $00513.88 .2,18 \mathrm{sm}^{-}$ & sas & \\
\hline 64. Diethyl ether (Ethyl ether) & 34002 & 121494 & 0.10 & 10.00 & 2003.8 & 200.4 & $00080-29-7000$ & andizisongen & \\
\hline 65. Ethyl methacrylate & 34002 & 121494 & 0.10 & 10.00 & 2004.8 & 200.5 & 00097.63 .2 & asainesonong & \\
\hline 66. 2-Hexanone & 34002 & 121494 & 0.10 & 10.00 & 2008.2 & 200.8 & 00591.78 .6 rosponssionomsens & androonons & \\
\hline 67. Hexachloroethane & 34002 & 121494 & 0.10 & 10.00 & 2003.6 & 200.4 & 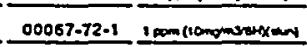 & araturoses & \\
\hline 68. Methacrylonitrile & 34002 & 121494 & 0.10 & 10.00 & 2005.4 & 200.5 & 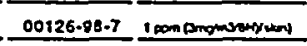 & astirondes & \\
\hline 69. Methyl acrylate & 34002 & 121494 & 0.10 & 10.00 & 2003.2 & 200.3 & 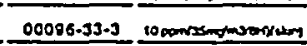 & oratzrimons & \\
\hline 70. lodomethane (Methyl iodide) & 34002 & 121494 & 0.10 & 10.00 & 2004.9 & 200.5 & 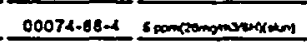 & $=$ & \\
\hline 71. Methyl methacrylate & 34002 & 121494 & 0.10 & 10.00 & 2007.8 & 200.8 & 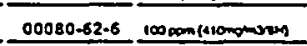 & anctersimose & \\
\hline 72. Methyl tert-butyl ether (MTBE) & 34002 & 121494 & 0.10 & 10.00 & 2004.2 & 200.4 & 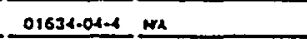 & dancenos & \\
\hline 73. 1+Methyt-2-pentanone (Methyl isobory l ketone) & 34002 & 121494 & 0.10 & 10.00 & 2003.9 & 200.4 & $00108-10-1$ 10000mA10mpmisen & 200000000000 & \\
\hline 74. Nitrobenzene & 34002 & 121494 & 0.10 & 10.00 & 2004.4 & 200.4 & $00008.05 .3 \div 3 \sin s \sin p \sin x+1$ & asostrompong & \\
\hline 75. 2-Nitropropane & 34002 & 121494 & 0.10 & 10.00 & 2001.6 & 200.2 & 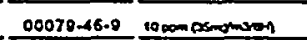 & mastronsens & \\
\hline 76. Pentachloroethane & 34002 & 121494 & 0.10 & 10.00 & 2003.5 & 200.4 & $00076-01-7$ WA & $\kappa$ & \\
\hline 77. Propionitrile & 34002 & 121494 & 0.10 & 10.00 & 2003.0 & 200.3 & $00107-12.0 \mathrm{Nad}$ & 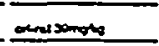 & \\
\hline 78. Tetrahydrofuran & 34002 & 121494 & 0.10 & 10.00 & 2000.9 & 200.1 & 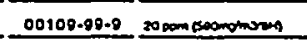 & sostzsoonode & \\
\hline
\end{tabular}



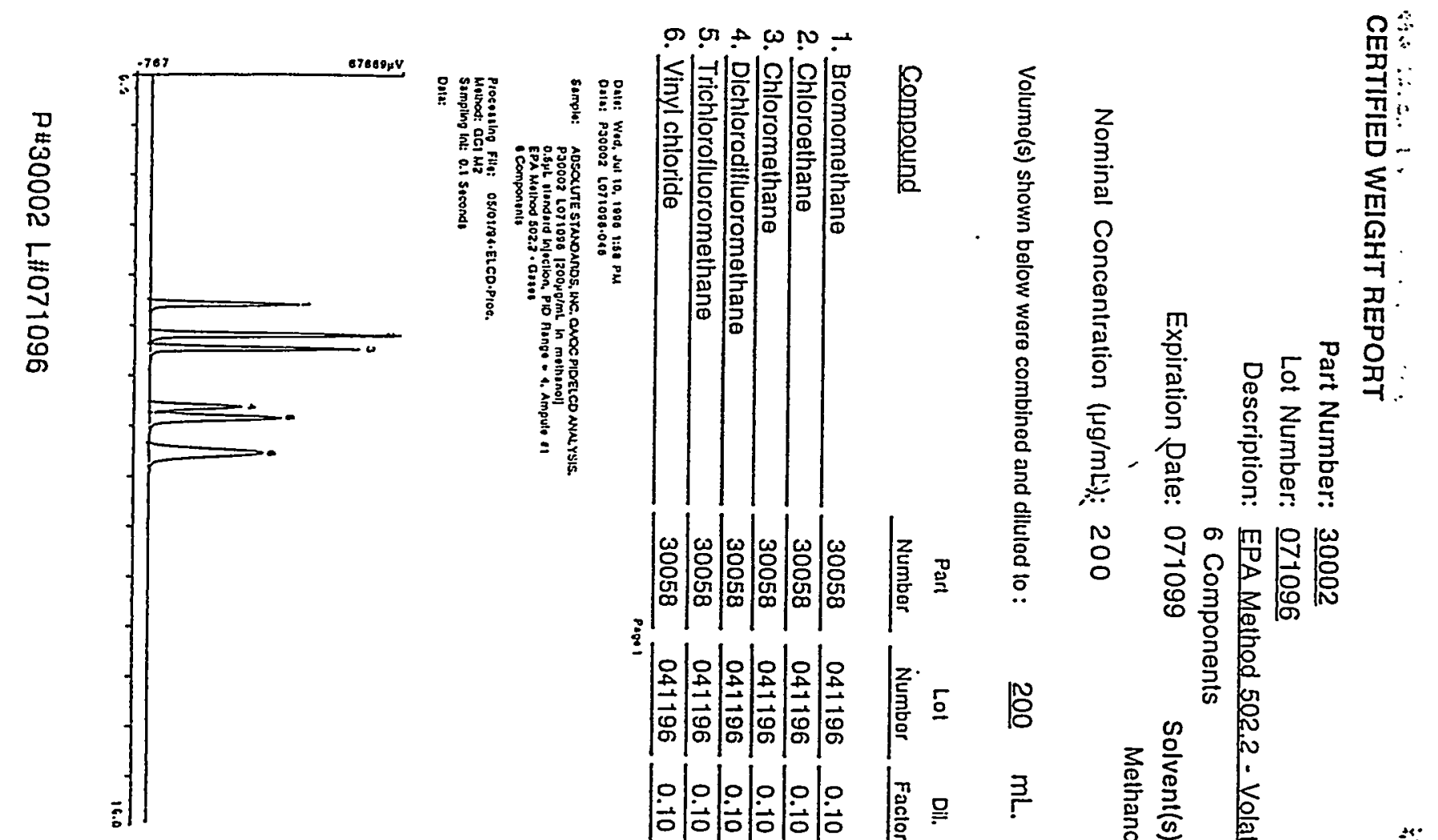

|

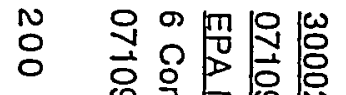

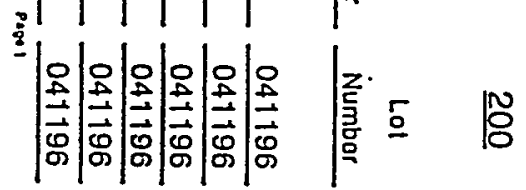

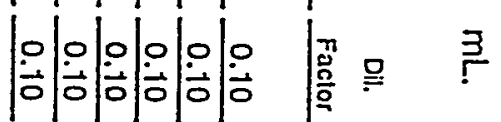
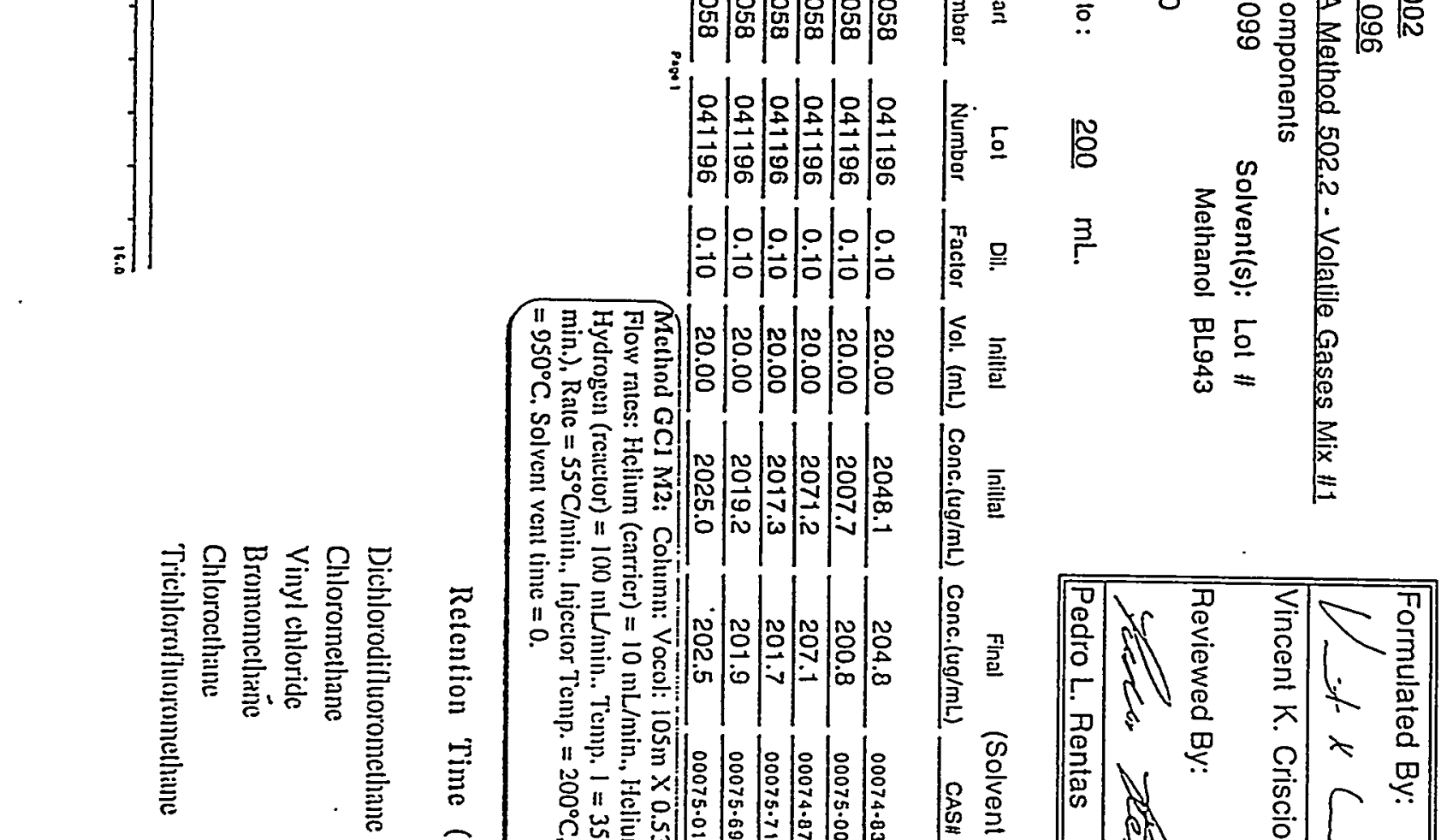

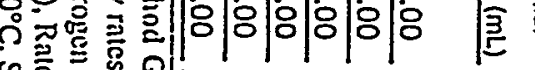
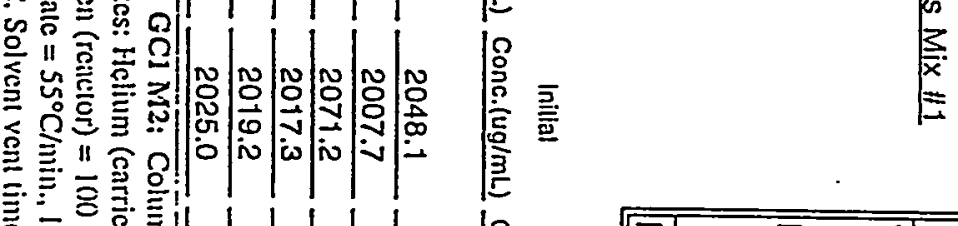

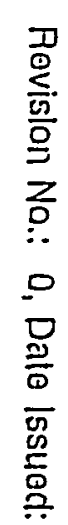

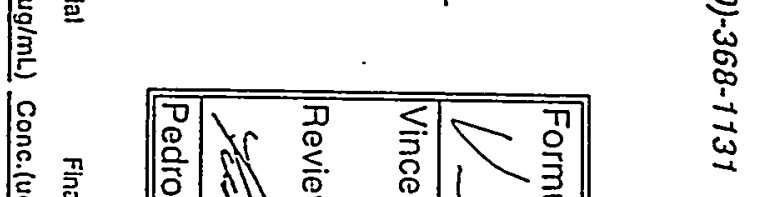

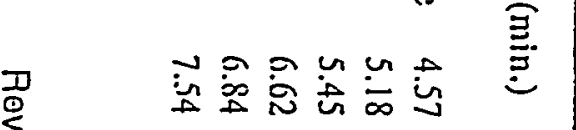

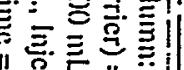

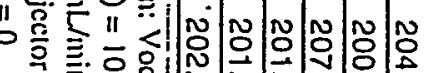

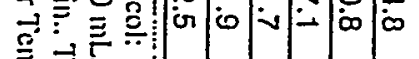

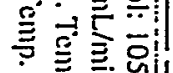

11

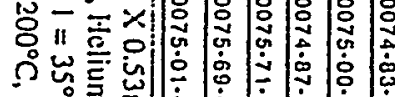

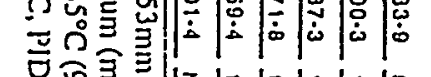

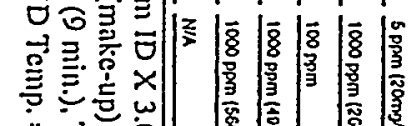
i1 $\rightarrow 1$ il

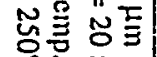
กำ寻

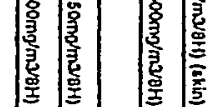

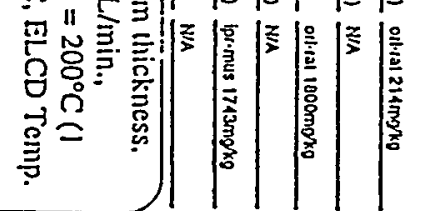

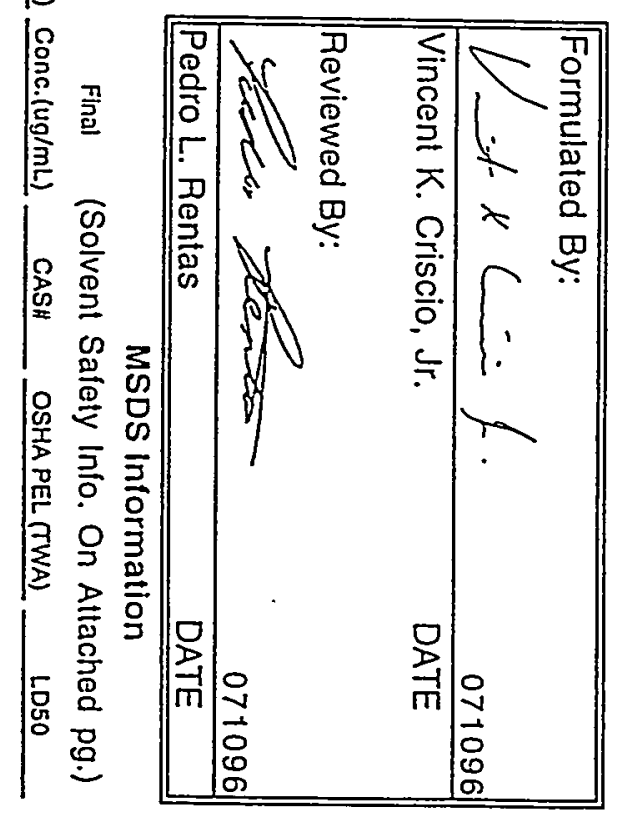

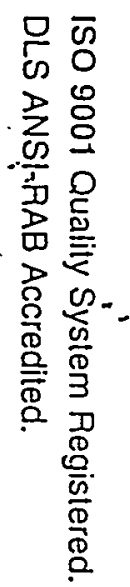




\section{STANDARD SOLUTIONS OF INTERMEDIATE CONCENTRATION}

\section{STANDARD PREPARATION LOGBOOK NO.39 IS}

39 is 7759 DCL INTERMEDIATE STANDARD SOLUTION IDENTIFICATION NUMBER DESCRIPTIVE IDENTIFICATION: 524.2 NOR QCS

Solvent MeOH Vendor B+J Grade P+T LOTOSY

\begin{tabular}{|c|c|c|c|c|c|c|}
\hline & Compound & $\begin{array}{c}\text { Stock Std } \\
\text { of Parent } \\
\text { Solutiton ID No. } \\
\end{array}$ & $\begin{array}{c}\text { Concentration of } \\
\text { Parent Solution } \\
\mu \mathrm{g} / \mathrm{mL}\end{array}$ & $\begin{array}{l}\text { Volume of } \\
\text { Allauot } \mathrm{mL}\end{array}$ & $\begin{array}{l}\text { Final Total } \\
\text { Volume mL }\end{array}$ & $\begin{array}{c}\text { FINAL } \\
\text { CONCENTRATION } \\
\mu \mathrm{g} / \mathrm{mL} \\
\end{array}$ \\
\hline 1. & 524.2 -GASES SOPQCS & $\not$ & 200 & .250 & 2. & 25. \\
\hline 2. & $T B .6 .23 .97$ & & & & & $\leftarrow$ \\
\hline 3. & & & & & & \\
\hline 4. & & & & & & \\
\hline 5. & & & & & $\angle$ & \\
\hline 6. & 1 & & & $\triangle$ & & \\
\hline 7. & & & & 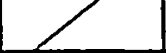 & & \\
\hline 8. & & & & & & \\
\hline 9. & & & & & & \\
\hline 10. & & & & & & \\
\hline 11. & & & 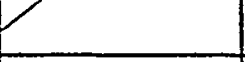 & & & \\
\hline 12. & & $\angle$ & $K_{B} .6 .23 .97$ & & & \\
\hline 13. & 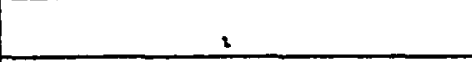 & 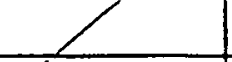 & & & & \\
\hline 14. & $:$ & & & & & \\
\hline 15. & & & & & & \\
\hline 16. & & & & & & \\
\hline 17. & & & & & & \\
\hline 18. & 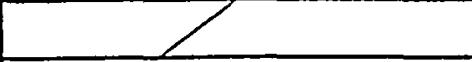 & & & & & \\
\hline 19. & & & & & & \\
\hline 20. & & & & & & \\
\hline & 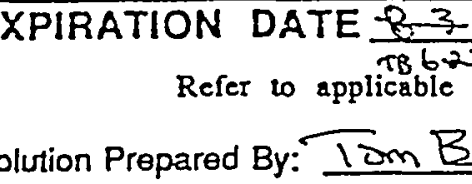 & $\begin{array}{l}8.23 .97 \\
\text { ontract or meth } \\
\text { osed }\end{array}$ & od for allowable & time period & $\begin{array}{l}\text { before ex } \\
6.23 .\end{array}$ & qiration. \\
\hline & høcked By: & 79 & & & 78 & \\
\hline
\end{tabular}

Comments: * Absolute Stdo. Ime.

$$
\text { PAirt } 30001 \text { Lot } 122396
$$


CERTIFIED WEIGHT REPORT: Uncertainty $<+,-0.5 \%$ Stated Values.

Part Number: 30001

Lot Number: 122396

Description: EPAMethod 5022-Liauids 54 Components

Expiration Date: 122399 Solvent(s): Lot \# Methanol BK414

Nominal Concentration ( $\mu \mathrm{g} / \mathrm{mL}): 200$

Vohme(s) shown below were combined and diluted to:

$200 \mathrm{~mL}$.

Pant Lot Dil. Initiat

Compoung

1. Bromodichloromethane

2. Dibromochloromethane

3. 1.1-Dichloroethene

4. cis-1.2-Dichloroethene

5. trans-1.2-Dichloroethene

6. Methylene chloride

7. Bromochloromethane

B. Bromoform

9. Carbon tetrachloride

10. Chlorolorm

11. Dibromomethane

12. 1.1-Dichloroethane

13. 2.2.Dichloropropane

14. Tetrachloroethene

16. 1.2.0ibromo-3-chloropropane

17. 1.2. Dibromoethane

18. 1.2-Dichloroethane

19. 1.2-Dichloropropane

20. 1.3-Dichloropropane

21. 1.1-Dichloropropene

22. cis-1,3-Dichloropropene

23. trans-1,3-Dichloropropene

24. Hexachlorobutadiene

25. 1.1.1.2-Tetrachloroethane

26. 1.1.2.2-Tetrachloroethane

27. 1.1.2-Trichloroethane

28. Trichloroethene

29. 1.2.3-Trichloropropane

30, Benzene

31. Bromobenzehe

32. n-Bulvl benzene

33. Ethyl benzene

34. D-Isopropyl toluene

35. Naphthalene

36. Styrene

37. Toluene

38. 1,2,3-Trichloroben2ene

39. 12.4-Trichlorobenzene

40. 1.2.4-Trimethylbenzene

41. 1.3.5-Trimethylbenzene (Mesitylene)

42. m-Xylene

43. tert-Butyl benzene

44. sec-Butyl benzene

45. Chlorobenzene

46. 2-Chiorotoluene

47. 4-Chlorotoluene

48. 1,2-Dichlorobenzene

49. 1.3-Dichlorobenzene

50. 1.4-Dichlorobenzene

51. Isopropylbenzene

52. n-Propylbenzene

53. o-Xylene

54. p-xylene

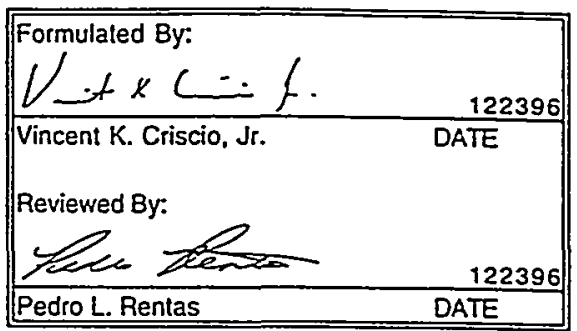

MSDS Information

Final (Solvent Safety Info. On Attached pg.)

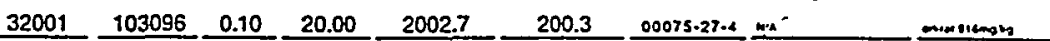
$32001 \frac{103096}{0.10} \frac{0.00}{20002.8}-200.3 \frac{00122.28 .1}{2007}$

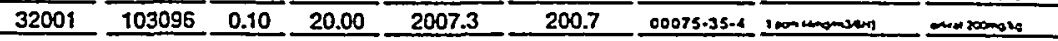
$32001 \frac{103096}{0.10} \frac{20.00}{2004.4} 200.4 \frac{00156 \cdot 59 \cdot 2}{20.12}$

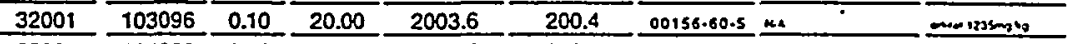
$32001 \frac{103096}{0.10} \frac{0.00}{2004.5} \frac{200.5}{200.8075 .09 \cdot 2} \mathrm{som}$

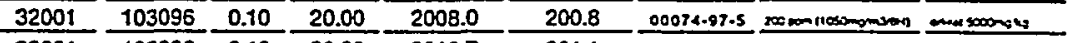

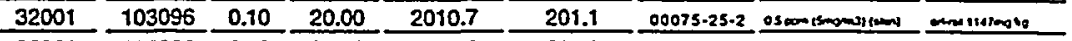

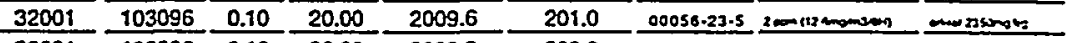

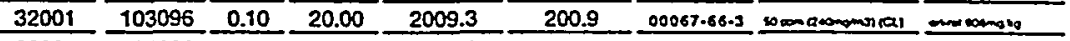
$32001 \frac{103096}{0.10} 20.00 \frac{2010.0}{201.0} 00074-25.3 \mathrm{wat}$

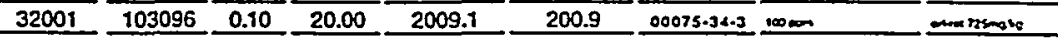
$32001 \frac{103096}{0.10} \frac{0.1000}{20009.5} 201.0 \frac{00594.20 .7}{200}$

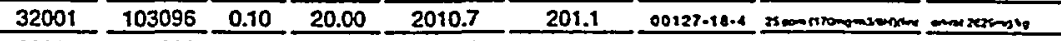

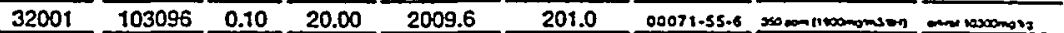
$32001 \frac{103096}{0.10} \frac{20.00}{2010.0} \frac{201.0}{0.10} \frac{0096.12 .8}{0.001000}$

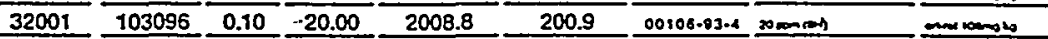
$32001 \frac{103096}{0.10} 20.00 \frac{2009.2}{200.9} 00107.08 .2$ Donren

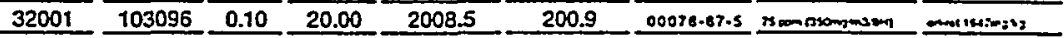

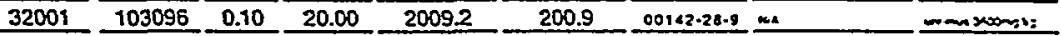

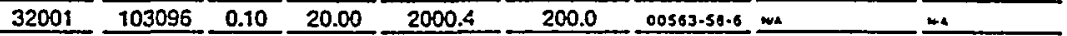
$32001 \frac{103096}{0.10} \frac{0.00}{20.00} \frac{20021.8}{202.2} \frac{10061.01-5}{m}$ $32001 \frac{103096}{0.10} \frac{0.00}{20.00} \frac{2021.8}{2022.9} \frac{202.3}{10061.02 .6} \mathrm{kin}$

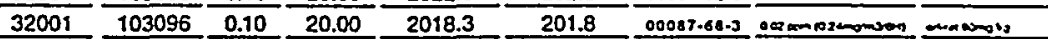

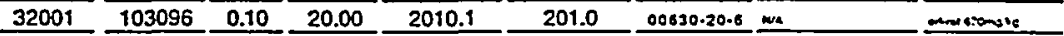

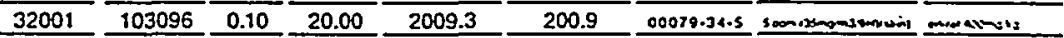

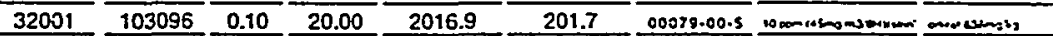
$32001 \frac{103096}{0.10} \frac{20.00}{2009.7} 201.0$ 00079.01.6 scomrionmsinn

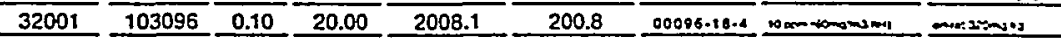

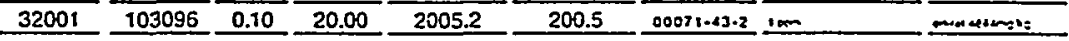
$32008 \frac{103096}{10309} \frac{0.10}{20.00} \frac{2002.0}{20.00} \frac{20.2}{200198.66 .1} \mathrm{me}$ $32001 \quad 103096 \quad 0.10 \quad 20.00 \quad 20012 \quad 200.120010451 .0 \mathrm{mad}$

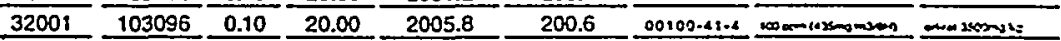
$32001 \frac{103096}{0.10} \frac{0.00}{20.00} \frac{2005.2}{200.5} 0.0099 .87 .6 \mathrm{~m}$

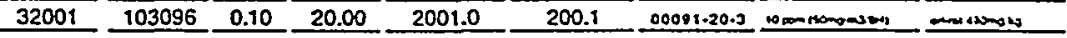
$32001 \frac{103096}{10.10} \frac{20.00}{2004.0} \frac{200.4}{200.00100 .42 .5} \frac{0000 \mathrm{~m}}{20027}$ $32001 \frac{103096}{0.10} \frac{20.00}{2002.7} \frac{200.3}{2002} \frac{00108-88-3}{200000}$ $32001 \frac{103096}{0.10} \frac{20.00}{2001.6} \frac{200.2}{2000087.61 \cdot 8} \mathrm{mat}$ $32001 \frac{103096}{10.10} 20.00 \frac{2005.5}{200.5} 00120.82-1$. $32001 \frac{103096}{0.10} \frac{0.00}{20001.5} 200.2000005-63.6 \mathrm{man}$ $32001 \frac{103096}{0.10} \frac{20.00}{2002.1} \frac{200.2}{0.00108 .67 .8} \mathrm{~kat}$ $32001 \frac{103096}{10096} \frac{0.10}{20.00} \frac{2001.0}{200.1} \frac{200.1}{20090108-38.3}$

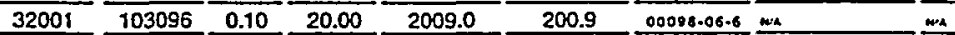
$32001 \frac{103096}{0.10} 20.002008 .9200 .900135 .08 .8$ wa

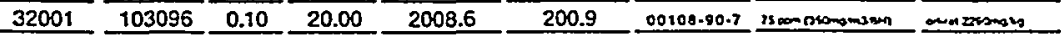

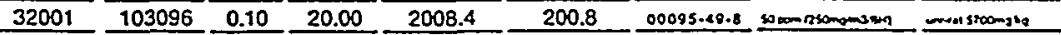
$32001 \frac{103096}{0.10} \frac{0.00}{20.00} \frac{2010.5}{201.1} \frac{0106.43 .4}{20.4}$

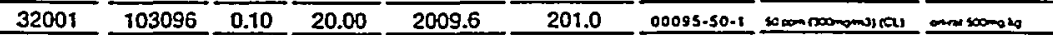

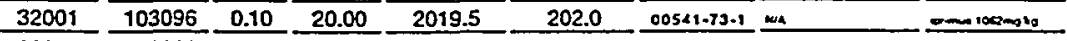

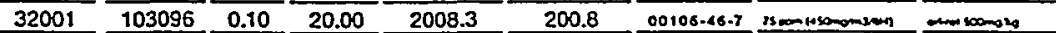
$32001 \frac{103096}{0.10} \frac{20.00}{2009.4} 200.9$ D0002-82.8

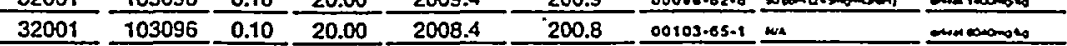
$32001 \frac{103096}{0.10} \frac{0.00}{2019.4} 201.9$ 00095.47.6

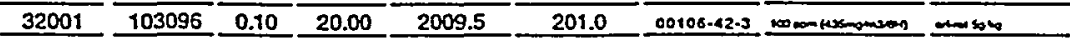




\section{STANDARD SOLUTIONS OF INTERMEDIATE CONCENTRATION}

\section{STANDARD PREPARATION LOGBOOK NO.39 IS}

Page No. 160 39 IS 7760 DCL INTERMEDIATE STANDARD SOLUTION IDENTIFICATION NUMBER DESCRIPTIVE IDENTIFICATION: 524.2 GASES QCS

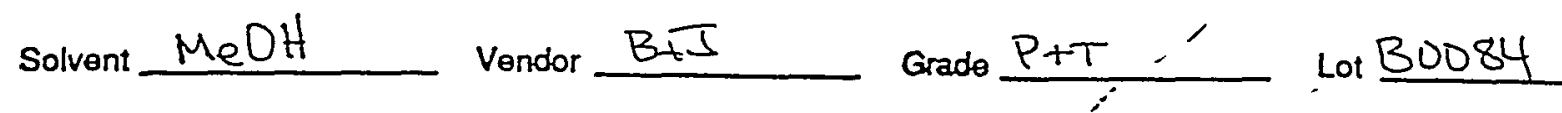

\begin{tabular}{|c|c|c|c|c|c|c|}
\hline \multicolumn{2}{|r|}{ Compound } & $\begin{array}{c}\text { Stock Std } \\
\text { or Parent } \\
\text { Solution ID No. } \\
\end{array}$ & $\begin{array}{c}\text { Concentration of } \\
\text { Parent Solution } \\
\mu \mathrm{g} / \mathrm{mL}\end{array}$ & $\begin{array}{l}\text { Volume of } \\
\text { Allquot } \mathrm{mL}\end{array}$ & $\begin{array}{l}\text { Final Total } \\
\text { Volume } \mathrm{mL}\end{array}$ & $\begin{array}{c}\text { FINAL } \\
\text { CONCENTRATION } \\
\mu \mathrm{g} / \mathrm{mL}\end{array}$ \\
\hline 1. & 524.2 GASES-QCS & 迷 & 200 & .250 & 2 & 25 \\
\hline \multicolumn{7}{|c|}{2.} \\
\hline \multicolumn{7}{|c|}{3.} \\
\hline \multicolumn{7}{|c|}{4.} \\
\hline 5. & & & & & & $\because$ \\
\hline \multicolumn{7}{|c|}{6.} \\
\hline \multicolumn{7}{|c|}{7.} \\
\hline \multicolumn{7}{|c|}{8.} \\
\hline \multicolumn{7}{|c|}{9.} \\
\hline \multicolumn{7}{|l|}{10.} \\
\hline \multicolumn{7}{|c|}{13.623 .97} \\
\hline \multicolumn{7}{|l|}{12.} \\
\hline \multicolumn{7}{|c|}{13.} \\
\hline \multicolumn{7}{|l|}{14.} \\
\hline \multicolumn{7}{|l|}{15.} \\
\hline \multicolumn{7}{|l|}{16.} \\
\hline 17. & & & & & & $\therefore$ \\
\hline \multicolumn{7}{|l|}{18.} \\
\hline \multicolumn{7}{|c|}{19.} \\
\hline 20. & & & & & & \\
\hline & $\begin{array}{l}\text { KPIRATION DATE } 6.3 \\
\text { Refer to applicable } \\
\text { Iution Prepared, By: } 6 \mathrm{~m}\end{array}$ & ontract or meth & $\begin{array}{l}\text { od for allowable } \\
\text { Date of Pre }\end{array}$ & $\begin{array}{l}\text { time period } \\
\text { paration: }\end{array}$ & $\begin{array}{l}\text { before exp } \\
6.23 .9\end{array}$ & $\begin{array}{l}\text { iration. } \\
7\end{array}$ \\
\hline & ecked By: & Zapr & Suporvisor: & & & 792 \\
\hline
\end{tabular}

Comments: *Ultra Scientifie

$$
\text { DWM-584 Lort L-D147 }
$$




\section{Certificate of Analysis}

Voc lias Mixture

Catalog Number: $5 W M-584$

Lot Number: L0147

Page: 1

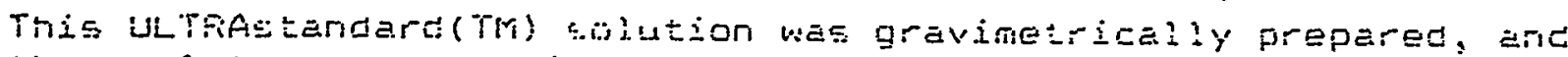

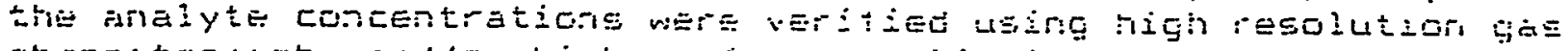
ahromitogrephy ancior hish pertornenge ziquit chromazography. The solution was prepared th the rominal conconstatian stated on the Jo\% label. The true vèue for ectí analyte, cetermlned gravimetrically, is listed Selow.

Component

bronamethane

enloroethane

chloromethane

Lichlorceitiuoromethorie

triztiorofiugromethane

vimy! chlories

So!-fut: methano! .
Weight/mL*

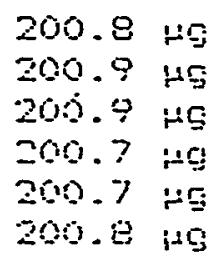

1

* All weights are traceable through N.I.S.T. Test No. 732/221797 


\section{STANDARD SOLUTIONS OF INTERMEDIATE CONCENTRATION}

\section{STANDARD PREPARATION LOGBOOK NO. 39 IS}

Page No. 147

39 IS 7747 DCL INTERMEDIATE STANDARD SOLUTION IDENTIFICATION NUMBER DESCRIPTIVE IDENTIFICATION:

Solven HeOH Vendor B\&T Grado_\&\&T Lot \$10084

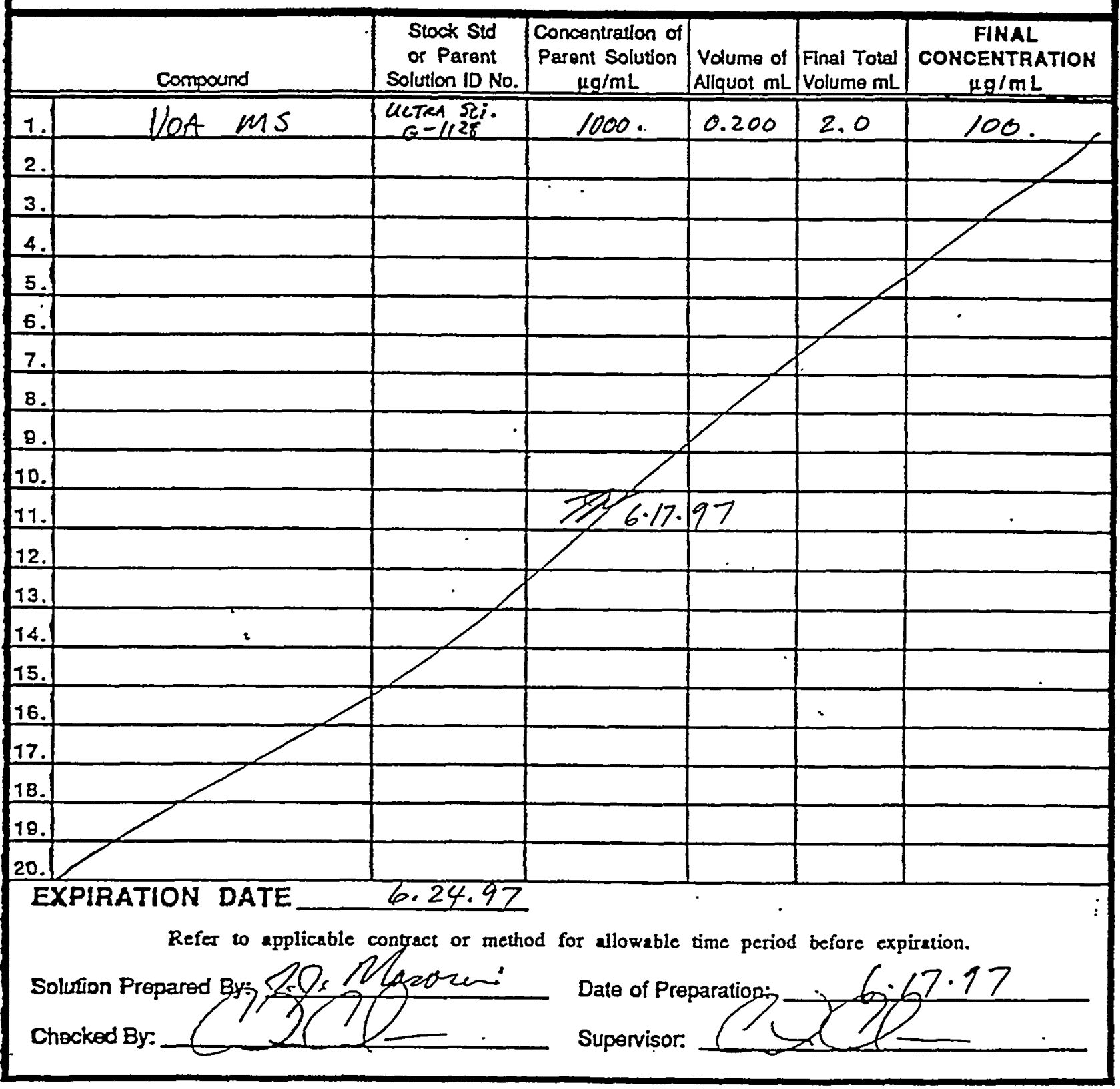

Comments:

078 


\section{A2LA Certified Reference Material}

Volatiles Matrix Spiking Solution

Catalog Number: CLP-100

Certification Date: 02/14/94
Lot Number: $\quad 6-1128$

A2LA Certification Number: USO119

Page:

1

This standard has been certified for accuracy, homogeneity, and stability according to American Association for Laboratory Accredițation (A2LA) specifications.

Component

benzene

chlorobenzene

1,1-dichloroethene

toluene

trichloroethene

Solvent: methanol
Certified

True Concentration*

$1000.4 \mu \mathrm{g} / \mathrm{mL}$ $1000.9 \mu \mathrm{H} / \mathrm{mL}$ $1001.0 \mu \mathrm{\mu} / \mathrm{mL}$ $1000.8 \mathrm{\mu g} / \mathrm{mL}$ $1000.9 \mu \mathrm{\mu g} / \mathrm{mL}$

2

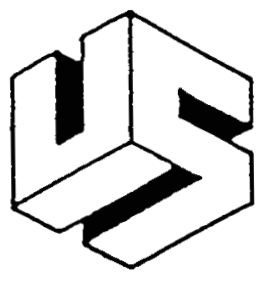

ULTRA SCIENTIFIC

250 Smith Street North Kingstown, RJ 02852 (401) $294-9400$

* Calceppopeced from gravimetric and volumetric measurements performed during preparation. 


\section{STANDARD SOLUTIONS OF INTERMEDIATE CONCENTRATION}

\section{STANDARD PREPARATION LOGBOOK NO.39 IS}

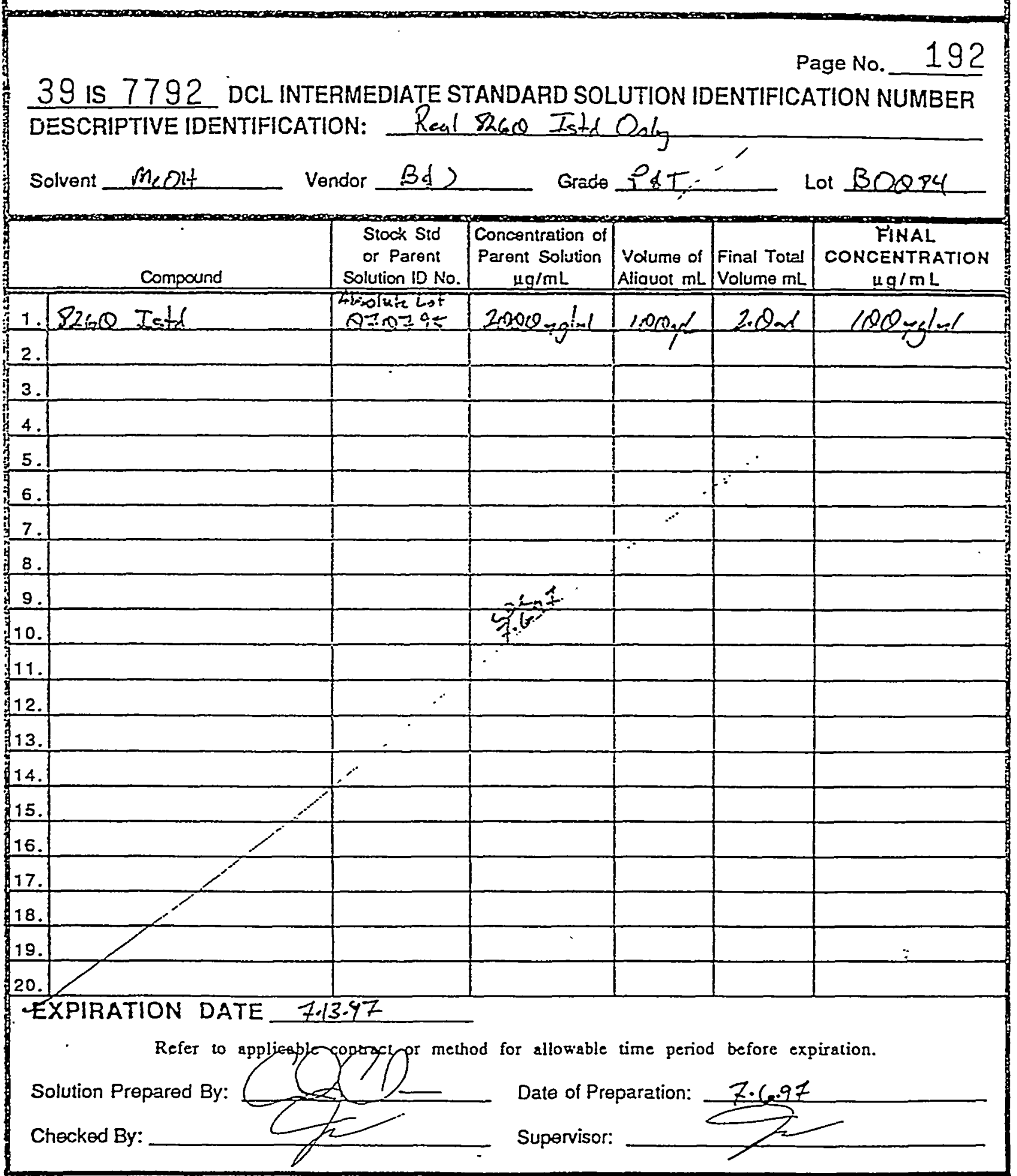

Comments: 


\section{STANDARD SOLUTIONS OF INTERMEDIATE CONCENTRATION}

\section{STANDARD PREPARATION LOGBOOK NO.39 IS}

\section{Page No. 182}

39 IS 7782 DCL INTERMEDIATE STANDARD SOLUTION IDENTIFICATION NUMBER DESCRIPTIVE IDENTIFICATION:

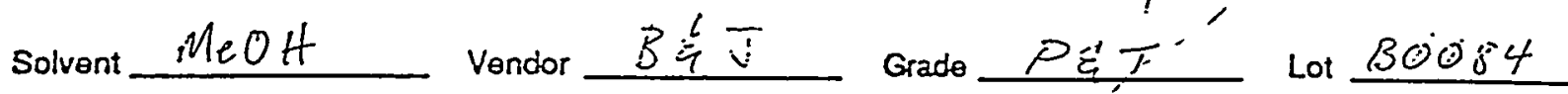

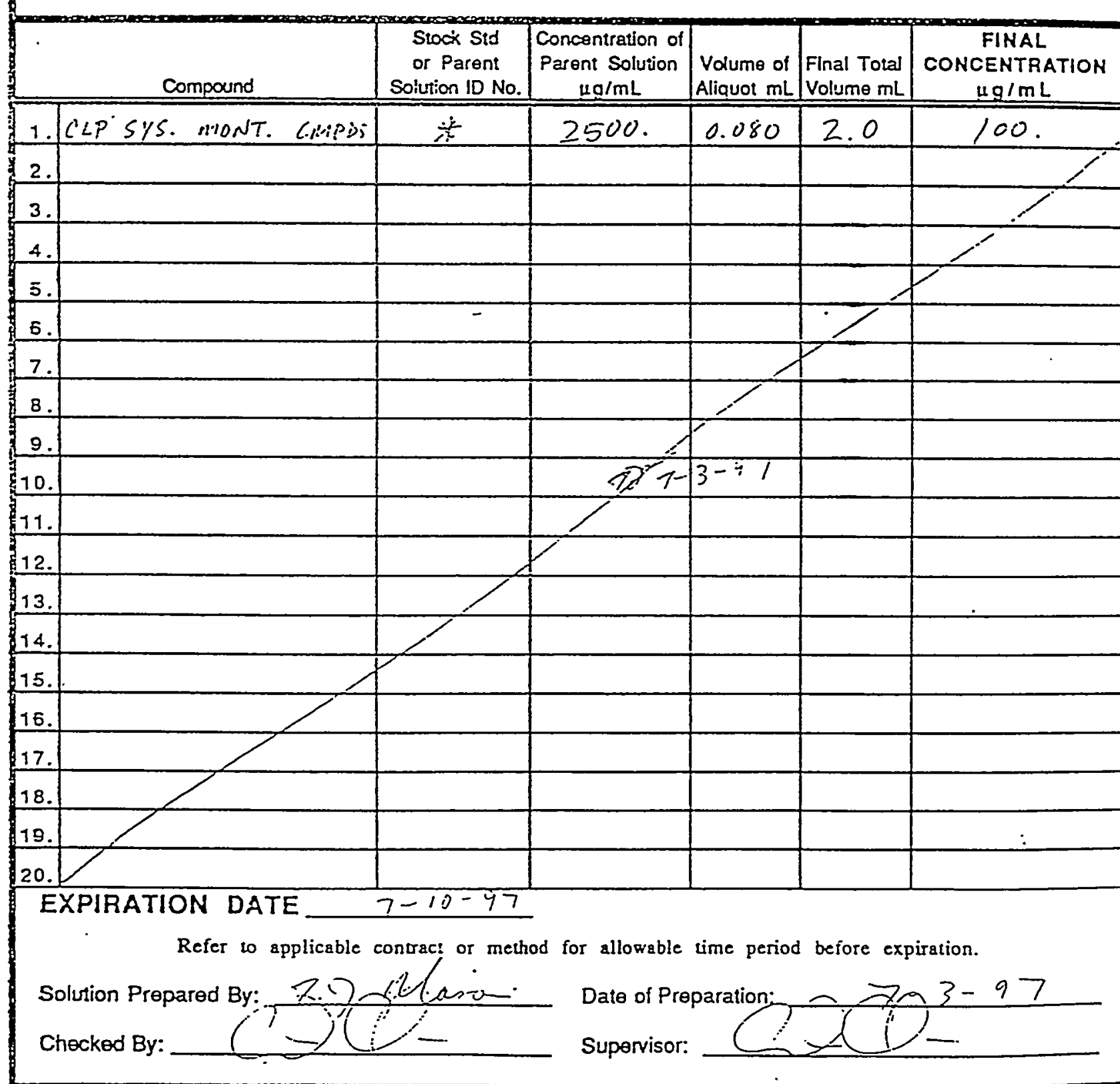

Comments: \# ABSOLUTE STD PIT,T \#20010

$$
107 \# 033197
$$




\section{STANDARD SOLUTIONS OF INTERMEDIATE CONCENTRATION}

\section{STANDARD PREPARATION LOGBÓOK NO.39 IS}

Page No. 193

39 IS 7793 DCL INTERMEDIATE STANDARD SOLUTION IDENTIFICATION NUMBER DESCRIPTIVE IDENTIFICATION: Real 8260 VOA/Gases

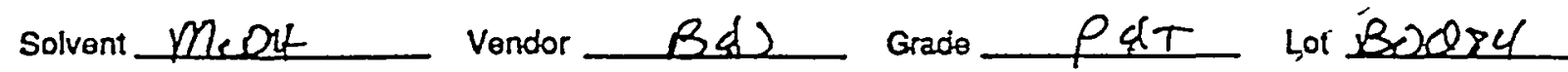

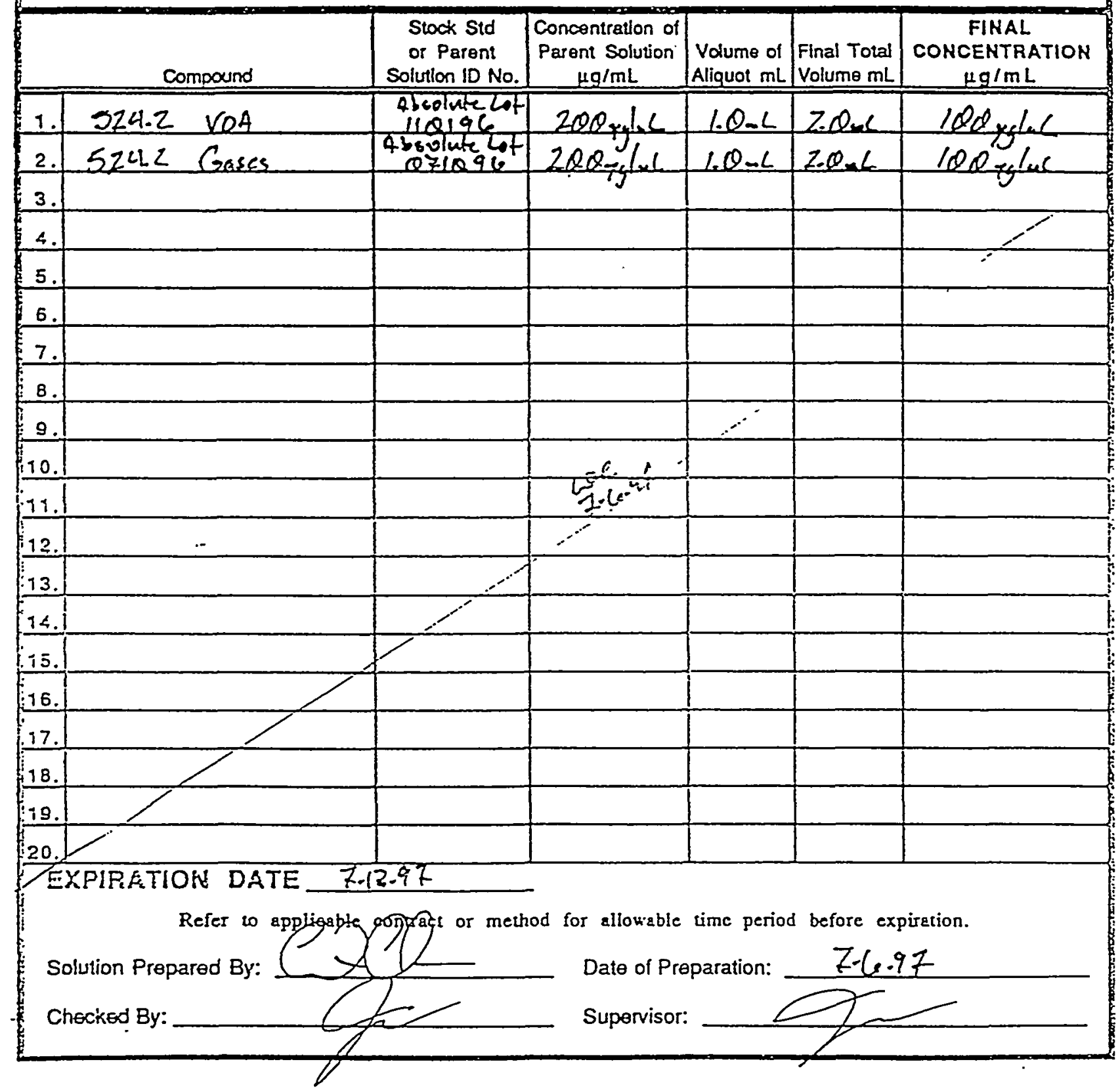

Comments: 


\section{STANDARD SOLUTIONS OF INTERMEDIATE CONCENTRATION}

\section{STANDARD PREPARATION LOGBOOK NO.39 IS}

39 IS 7789 DCL INTERMEDIATE STANDARD SOLUTION IDENTIFICATION NUMBER DESCRIPTIVE IDENTIFICATION:

$100.7 \mathrm{~ms}$

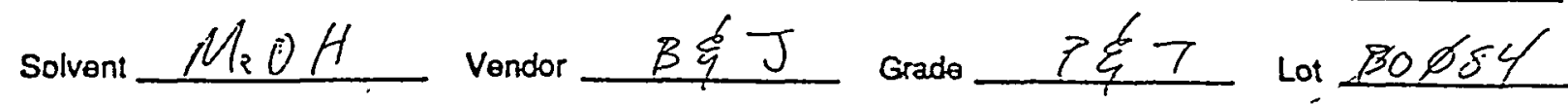

\begin{tabular}{|c|c|c|c|c|c|c|}
\hline Compound & $\begin{array}{c}\text { Stock Sid } \\
\text { or Parent } \\
\text { Solution ID No. }\end{array}$ & $\begin{array}{c}\text { Concentration of } \\
\text { Parent Solution } \\
\mu \mathrm{g} / \mathrm{mL}\end{array}$ & $\begin{array}{c}\text { Folume of } \\
\text { Aliquot } \mathrm{mL}\end{array}$ & $\begin{array}{c}\text { Flnal Total } \\
\text { Volume } \mathrm{mL}\end{array}$ & $\begin{array}{c}\text { CONCENTRATION } \\
\mu \mathrm{g} / \mathrm{mL}\end{array}$ \\
\hline
\end{tabular}

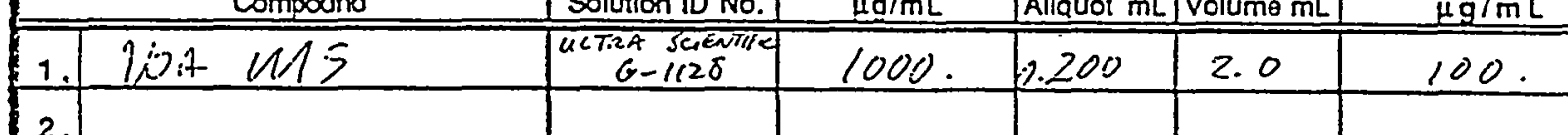

2.

3.

4.

5.

6.

7.

8.

9.

10.

11.

12.

:13.

15.

15.

16.

17.

18.

19.

20.

EXPIRATION DRTE

Refer to applicable contract of method for allowable time period before expiration.

Solution Prepared By: Date of Preparation:

Comments: 


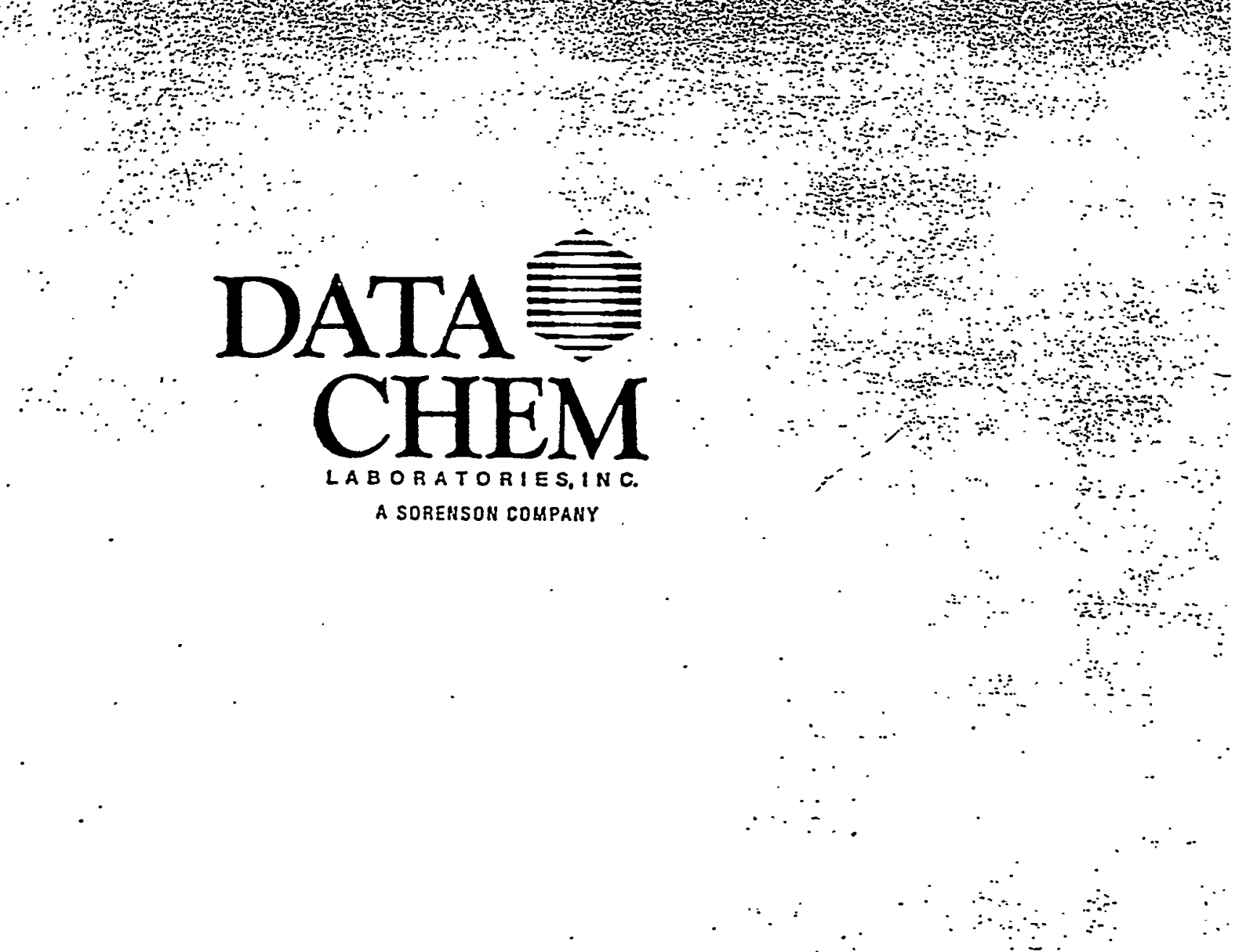

\section{Raw Data}


Quantitation Report

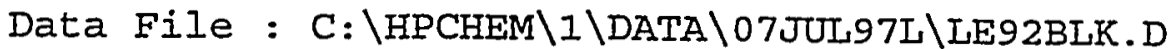

Acq Time : 07/07/97 09:35

Sample : BL-136531-1

Misc : 2.5 uL of 39IS7792, 828260 WATER

Quant Time: Jul 7 10:06 1997

Operator: CQC

Inst : 5971-L

Multiplr: 1.00

Method : C: \HPCHEM \1\METHODS\L826ARWA.M

Title

: VOA COMPOUND IIST

Last Update : Wed Jun 25 12:48:54 1997.

Response via : Multiple Level Calibration

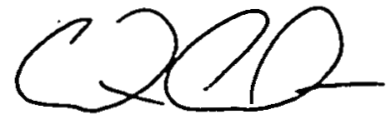

7.797

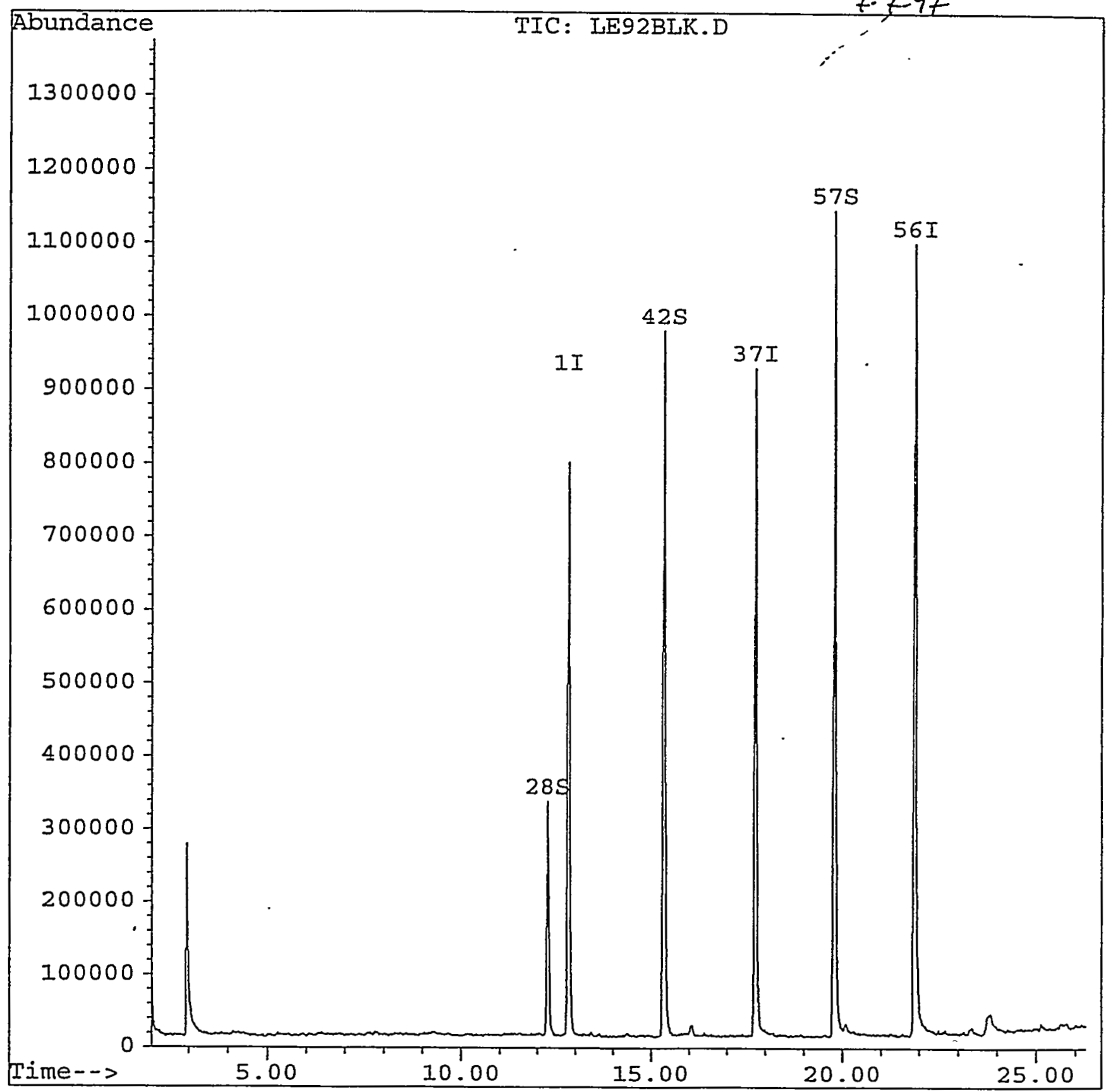

- 085 


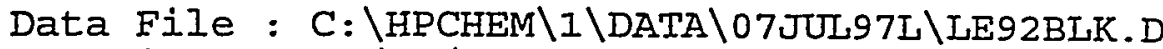

Acq Time : 07/07/97 09:35

Sample : BL-136531-1

Misc : 2.5 uL of 39IS7792, 82 8260 WATER

Quant Time: Jul 7 10:06 1997

Operator: $\mathrm{CQC}$

Inst : 5971-I

Multiplr: 1.00

Method

Title

: C: $\backslash$ HPCHEM \I\METHODS \L826ARWA.M

Last Update : Wed Jun 25 12:48:54 1997

Response via : Multiple Level Calibration

Internal standards

1) CIO1 Flourobenzene

37) CIO2 ChIorobenzene-d5

56) CIO3 1,4-Dichlorobenzene-d

System Monitoring Compounds

28) Cs15 1,2-dichloroethane-d4

42) CSO5 toluene-d8

57) CS10 4-bromofluorobenzene

12.27

15.34

19.78

17.75

21.89

117

152

1

1661517

$\begin{array}{lr}65 & 453417 \\ 98 & 1543829 \\ 95 & 1071903\end{array}$

Target Compounds

2) $\mathrm{C} 002$ dichlorodifluorometha

3) $\mathrm{C003}$ chloromethane

4) C004 vinyl chloride

5) C005 bromomethane

6) C006 chloroethane

7) C007 trichlorofluoromethan

8) $\mathrm{CO71}$ acrolein

9) $\operatorname{Co08} 1,1$-dichloroethene

10) $\mathrm{C} 063$ freon 113

11) co09 acetone

12) C010 carbon disulfide

13) C011 methylene chloride

14) $\mathrm{C} 072$ acrylonitrile

15) $\mathrm{C} 012$ trans-1,2-Dichloroeth

16) C305 methyl-t-butyl-ether

17) $\mathrm{C} 013 \mathrm{cis}-1,2-d i c h l o r o e t h e n$

18) $\mathrm{C} 55$ total-1,2-dichloroeth

19) C014 1,1-dichloroethane

20) C015 2,2-dichloropropáne

21) C110 2-butanone

22) CIOI bromochloromethane

23) $\mathrm{C} 016$ chloroform

24) $\mathrm{C} 017$ 1,1,1-Trichloroethane

25) C018 1,1-dichloropropene

26) Co19 1,2-dichloroethane

27) $\mathrm{C} 020$ carbon tetrachloride

29) C021 benzene

30) Co22 trichloroethene

31) C023 1,2-dichloropropane

32) C024 dibromomethane

33) C025 bromodichloromethane

34) C026 2-chloroethylvinyleth

35) $\mathrm{C} 027 \mathrm{cis-1,3-dichloroprope}$
$0.00 \quad 85$

$0.00 \quad 50$

$0.00 \quad 62$

$0.00 \quad 94$

$0.00 \quad 64$

$0.00 \quad 101$

$0.00 \quad 56$

$0.00 \quad 96$

$0.00 \quad 151$

$0.00 \quad 43$

$0.00 \quad 76$

$0.00 \quad 84$

$0.00 \quad 53$

0.0096

$0.00 \quad 73$

$0.00 \quad 96$

$0.00 \quad 96$

$0.00 \quad 63$

$0.00 \quad 77$

$0.00 \quad 72$

$0.00 \quad 128$

$0.00 \quad 83$

$0.00 \quad 97$

$0.00 \quad 75$

$0.00 \quad 62$

$0.00 \quad 117$

$0.00 \quad 78$

$0.00 \quad 130$

$0.00 \quad 63$

$0.00 \quad 93$

$0.00 \quad 83$

0.0063

$0.00 \quad 75$
1280806

894650

Response Conc Units Area\%

$50.00 \mathrm{ug} / \mathrm{L}$

0.00

$50.00 \mathrm{ug} / \mathrm{L}$

0.00

0.00

$50.00 \mathrm{ug} / \mathrm{L}$

$\%$ Recovery

$42.22 \mathrm{ug} / \mathrm{L} \quad 84.44 \%$

$46.86 \mathrm{ug} / \mathrm{L} \quad 93.72 \%$

$44.83 \mathrm{ug} / \mathrm{L}$

$89.67 \%$

Qvalue

Not Detected

Not Detected

Not Detected

Not Detected

Not Detected

Not Detected

Not Detected

Not Detected

Not Detected

Not Detected

Not Detected

Not Detected

Not Detected

Not Detected

Not Detected

Not Detected

Not Detected

Not Detected

Not Detected

Not Detected

Not Detected

Not Detected

Not Detected

Not Detected

Not Detected

Not Detected

Not Detected

Not Detected

Not Detected

Not Detected

Not Detected

Not Detected

Not Detected

$(\mathrm{NR})=$ Not Reported $(\mathrm{SP})=$ Spiked analyte $(\mathrm{m})=$ manual integration

(*) Does not meet EPA spectral criteria (False Hit) PX125 
Data File : C: \HPCHEM \I\DATA $07 J U L 97 L \backslash L E 92 B L K . D$

$\begin{array}{llll}\text { Acq Time } & : 07 / 07 / 97 \text { 09:35 } & & \text { Operator: CQC } \\ \text { Sample } & : \text { BL-136531-1 } & \text { Inst } \\ \text { Misc } & : 2.5 \text { uL of 39IS7792,82 } 8260 \text { WATER } & \text { Multiplr: } 1.00 \\ \text { Ouant Time: Jul } 710: 061997 & \end{array}$

Method : C: \HPCHEM \I\METHODS \L826ARWA.M

Title : VOA COMPOUND LIST

Last Update : Wed Jun 25 12:48:54 1997

Response via : Multiple Level Calibration

Compound
R.T. QIon Response Conc Unit Qvalue

36) C205 4-methyl-2-pentanone

38) C028 trans-1,3-dichloropro

$0.00 \quad 43$

$0.00 \quad 75$

$0.00 \quad 97$

40) C210 2-hexanone

$0.00 \quad 43$

$0.00 \quad 107$

$0.00 \quad 92$

$0.00 \quad 76$

$0.00 \quad 129$

$0.00 \quad 173$

$0.00 \quad 164$

$0.00 \quad 112$

$0.00 \quad 131$

$0.00 \quad 106$

$0.00 \quad 106$

0.00106

0.00106

$0.00 \quad 104$

$0.00 \quad 105$

$0.00 \quad 83$

0.00156

$0.00 \quad 75$

$0.00 \quad 91$

$0.00 \quad 105$

$0.00 \quad 91$

$0.00 \quad 91$

$0.00 \quad 119$

$0.00 \quad 105$

0.00105

0.00119

$0.00 \quad 146$

0.00146

$0.00 \quad 91$

$0.00 \quad 146$

0.0075

$0.00 \quad 180$

$0.00 \quad 225$

$0.00 \quad 128$

$0.00 \quad 180$
Not Detected

Not Detected

Not Detected

Not Detected

Not Detected

Not Detected

Not Detected

Not Detected

Not Detected

Not Detected

Not Detected

Not Detected

Not Detected

Not Detected

Not Detected

Not Detected

Not Detected

Not Detected

Not Detected

Not Detected

Not Detected

Not Detected

Not Detected

Not Detected

Not Detected

Not Detected

Not Detected

Not Detected

Not Detected

Not Detected

Not Detected

Not Detected

Not Detected

Not Detected

Not Detected

Not Detected

Not Detected

Not Detected

\section{7}

Data Processed By:

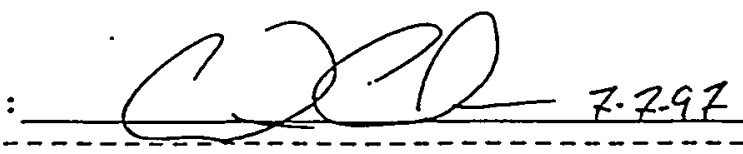

$(\mathrm{NR})=$ Not Reported $(\mathrm{SP})=$ Spiked analyte $(\mathrm{m})=$ manual integration

(*) Does not meet EPA spectral criteria (False Hit) PXI25 
Quantitation Report

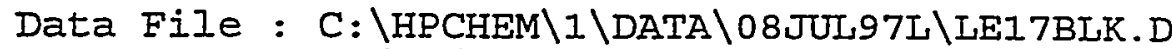

Acq Time : 07/08/97 11:59

Sample : BI-136531-2

MisC : 2.5 uI of 39IS7792, 82

Quant Time: Jul 8 12:43 1997

Operator: $\mathrm{CQC}$

Inst : 5971-I

Multiplr: 1.00

Method : C: \HPCHEM \I\METHODS \L826ARWA.M

Title : VOA COMPOUND LIST

Iast Update : Tue Jul 08 12:41:59 1997

Response via : Multiple Level Calibration

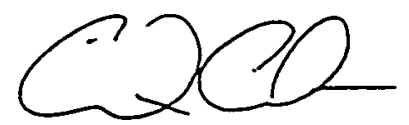

$7 \cdot 8.97$

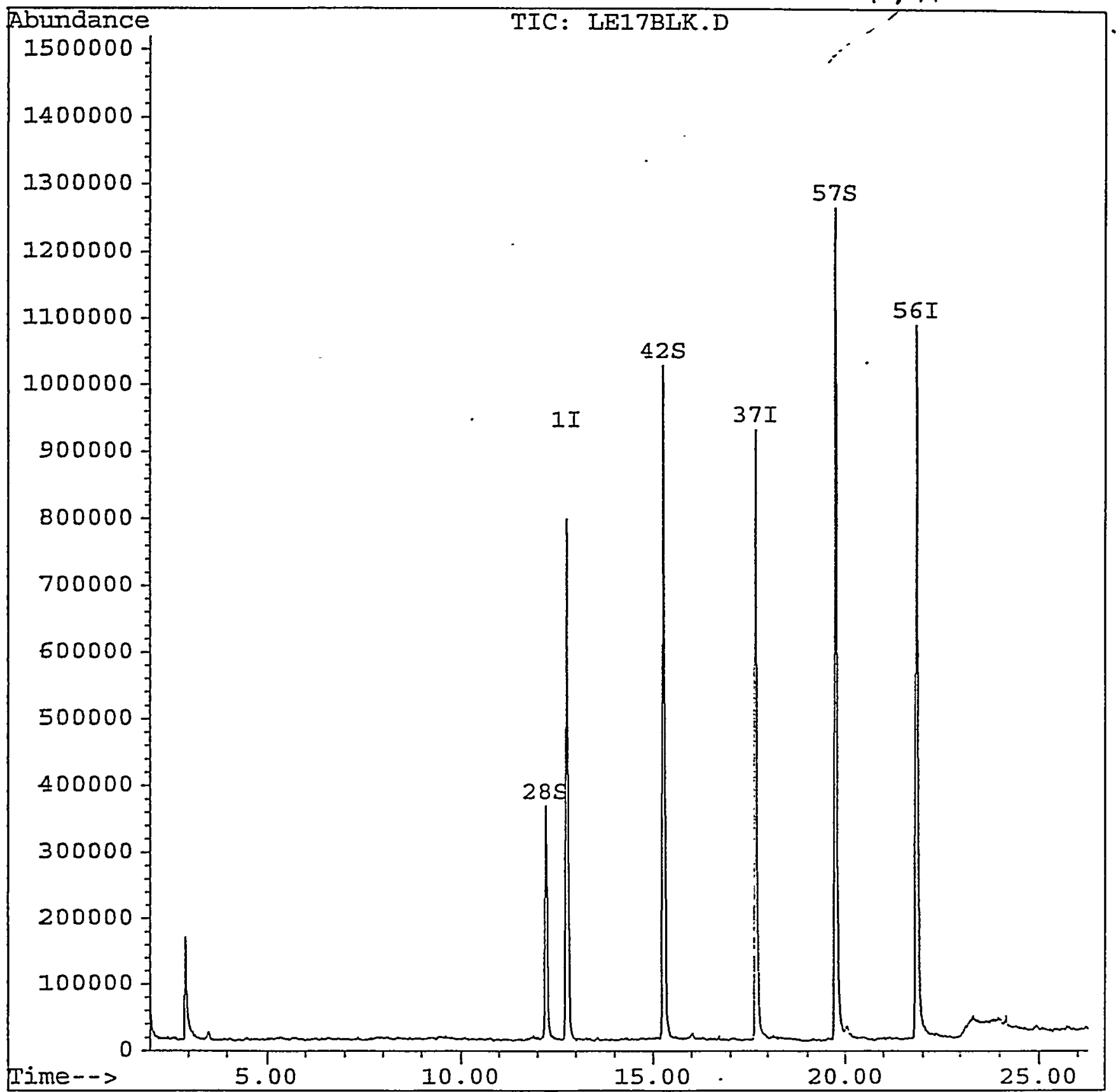

088 


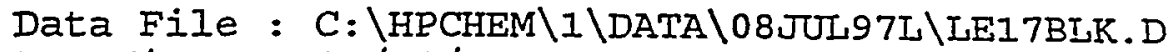

Acq Time : 07/08/97 11:59

Sample : BL-136531-2

Misc : 2.5 uL of 39IS7792, 82

Quant Time: JuI $812: 431997$

Operator: CQC

Inst : 5971-L

Multiplr: 1.00

Method

Title

: C: \HPCHEM \I\METHODS \L826ARWA.M

Iast Upaate : Tue JuI 08 12:41:59 1997

Response via : Multiple Level Calibration

Internal standards

R.T. QIon Response

Conc Units

Area:

1) CIOI Flourobenzene

37) CI02 Chlorobenzene- $d 5$

12.78

96

1647863

$56.00 \mathrm{ug} / \mathrm{L}$

0.00

56) CI03 1,4-Dichlorobenzene-d

17.70

117

$1268543:-$

$50.00 \mathrm{ug} / \mathrm{L}$

$-0.05$

$21.84 \quad 152 \quad 910189$

$50.00 \mathrm{ug} / \mathrm{I}$

$-0.03$

System Monitoring Compounds

28) CS15 1,2-dichloroethane-d4

42) Cs05 toluene-d8

$12.23 \quad 65 \quad 505259$

57) CSIO 4-bromofluorobenzene

15.29

98

1605665

19.73

95

1144425

Target Compounds

2) $\mathrm{COO2}$ dichlorodifluorometha

3) $\mathrm{COO3}$ chloromethane

$0.00 \quad 85$

$0.00 \quad 50$

4) 0004 vinyl chloride

0.00

62

5) C005 bromomethane

0.00

94

6) 0006 chloroethane

0.00

64

7) $\mathrm{C007}$ trichlorofluoromethan

0.00

101

B) 0071 acrolein

$0.00 \quad 56$

9) $\operatorname{Co0} 81,1$-dichloroethene

$0.00 \quad 96$

10) $\mathrm{C} 063$ freon 113

$0.00 \quad 151$

II) C009 acetone

$0.00 \quad 43$

12) COI0 carbon disulfide

0.00

76

$0.00 \quad 84$

13) Co1I methylene chloride

0.00

53

14) C072 acrylonitrile

0.00

96

16) C305 methyl-t-butyl-ether

0.00

73

17) $\mathrm{C} 013 \mathrm{cis}-1,2-d i c h l o r o e t h e n$

0.00

96

18) C055 total-1,2-dichloroeth

$0.00 \quad 96$

19) $\mathrm{C} 014$ I,I-dichloroethane

0.0063

20) C015 2,2-dichloropropane

0.00

77

$0.00 \quad 72$

21) C110 2-butanone

0.00

128

22) CI01 bromochloromethane

23) $\mathrm{C} 016$ chloroform

0.00

83

$0.00 \quad 97$

24) $\mathrm{C} 17$ 1,1,1-Trichloroethane

0.00

75

26) Co19 I,2-dichloroethane

0.00

62

$0.00 \quad 117$

27) C020 carbon tetrachloride

0.00

78

$0.00 \quad 130$

30) C022 trichloroethene

0.00

63

31) C023 1,2-dichloropropane

32) C024 dibromomethane

0.00

93

33) C025 bromodichloromethane

34) $\mathrm{C} 026$ 2-chloroethylvinyleth

0.00

83

C027 cis-1,3-dichloroprope

$0.00 \quad 63$

$0.00 \quad 75$

\%Recovery

$47.44 \mathrm{ug} / \mathrm{L} \quad 94.87 \%$

$49.21 \mathrm{ug} / \mathrm{L} \quad 98.41 \%$

$47.05 \mathrm{ug} / \mathrm{I}$

$94.10 \%$

Not Detected

Qvalue

Not Detected

Not Detected

Not Detected

Not Detected

Not Detected

Not Detected

Not Detected

Not Detected

Not Detected

Not Detected

Not Detected

Not Detected

Not Detected

Not Detected

Not Detected

Not Detected

Not Detected

Not Detected

Not Detected

Not Detected

Not Detected

Not Detected

Not Detected

Not Detected

Not Detected

Not Detected

Not Detected

Not Detected

Not Detected

Not Detected

Not Detected

Not Detected

$(\mathrm{NR})=$ Not Reported $(\mathrm{SP})=$ Spiked analyte $(\mathrm{m})=$ manual integration

(*) Does not meet EPA spectral criteria (False Hit) PX125 Page 1 
Data File : C: \HPCHEM \I\DATA

Acq Time : 07/08/97 11:59

Sample : BL-136531-2

Misc : 2.5 uL of 39IS7792, 82

Quant Time: Jul $8 \quad 12: 431997$

Method : C: \HPCHEM \I\METHODS\I826ARWA.M

Title : VOA COMPOUND LIST

Iast Update : Tue Jul 08 12:41:59 1997

Response via : Multiple Level Calibration
Operator: CQC

Inst : 5971-L

Multiplr: 1.00
Compound

R.T. QIon Response Conc Unit Qvalue

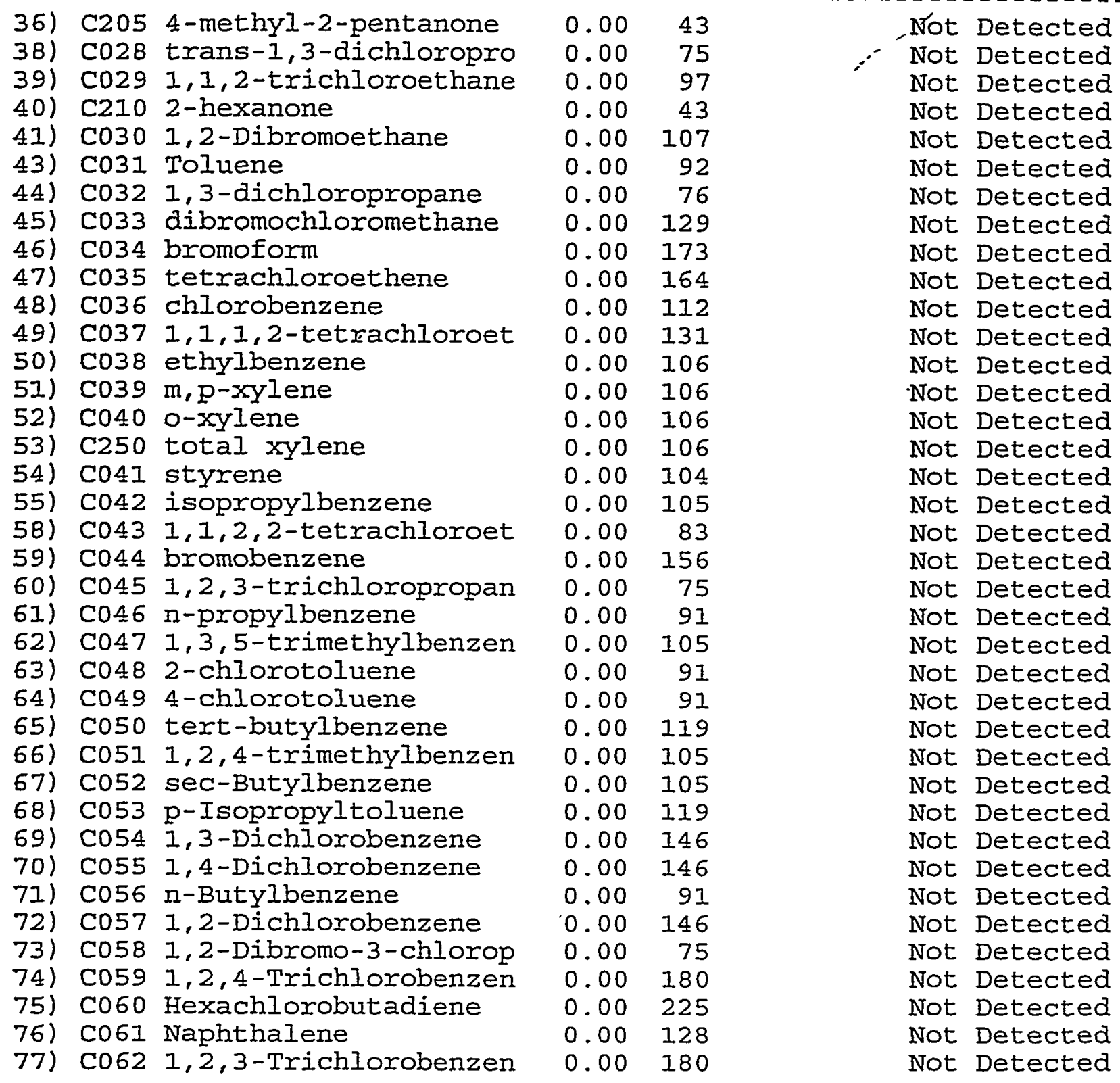

090

Data Processed By:

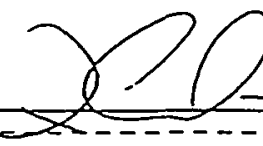

7.P.4Z

$(N R)=$ Not Reported $(S P)=$ Spiked analyte $(\mathrm{m})=$ manual integration

(*) Does not meet EPA spectral criteria (False Hit) PX125 
Quantitation Report

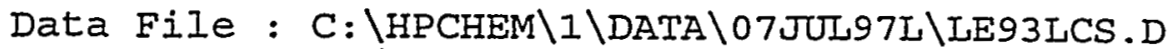

Acq Time : 07/07/97 10:07

Sample : QC-136531-1

Misc : 2.5 uL of 39IS7792, 82, 898260 WATER

Quant Time: Jul 7 10:35 1997

Operator: $\mathrm{COC}$

Inst : 5971-L

Multiplr: 1.00

Method : C: \HPCHEM \I\METHODS\L826ARWA.M

Title : VOA COMPOUND LIST

Last Update : Wed Jun 25 12:48:54 1997

Response via : Multiple Level Calibration
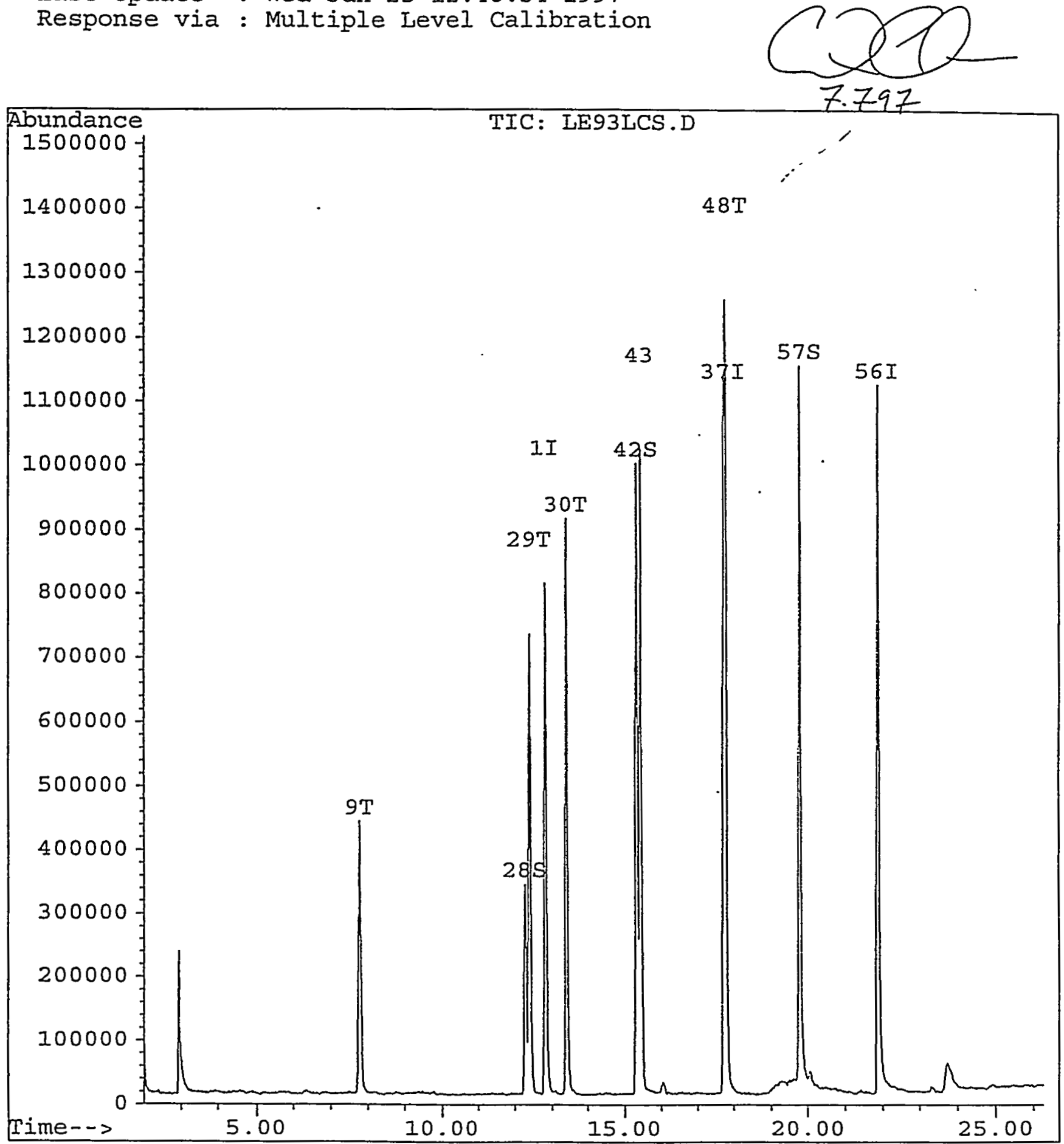

091 
Data File : C: \HPCHEM \I\DATA $\backslash 07 J U L 97 L \backslash L E 93 L C S . D$

Acq Time : 07/07/97 10:07

Sample : QC-136531-1

Misc : 2.5 uL of 39IS7792, 82, 898260 WATER

Quant Time: Jul 7 10:35 1997
Operator: CQC

Inst : 5971-L Multiplr: 1.00

Method

Title

: C: \HPCHEM \I\METHODS \L826ARWA.M

Last Update : Wed Jun 25 12:48:54 1997

Response via : Multiple Level Calibration

Internal Standards

1) CIOI Elourobenzene

37) CI02 Chlorobenzene-d5

56) CI03 1,4-Dichlorobenzene-d

System Monitoring Compounds

28) CSI5 1,2-dichloroethane-d4

42) Cs05 toluene-d8

57) CSI0 4-bromofluorobenzene

Target Compounds

2) $\mathrm{C002}$ dichlorodifluorometha

3) $\mathrm{C003}$ chloromethane

4) 0004 vinyl chloride

5) C005 bromomethane

6) $\mathrm{C006}$ chloroethane

7) $\mathrm{C007}$ trichlorofluoromethan

8) 6071 acrolein

9) $\mathrm{C} 008$ 1,I-dichloroethene

10) 0063 freon 113

11) $\operatorname{co09}$ acetone

12) $C 010$ carbon disulfide

13) COII methylene chloride

14) $\mathrm{C} 072$ acrylonitrile

15) $\mathrm{C} 012$ trans-1,2-Dichloroeth

16) $C 305$ methyl-t-butyl-ether

17) $\operatorname{co13}$ cis-1,2-dichloroethen

18) $\mathrm{C} 055$ total-1,2-dichloroeth

19) $\operatorname{Co14} 1,1$-dichloroethane

20) C015 2,2-dichloropropane

21) $C 110$ 2-butanone

22) $\mathrm{CIOI}$ bromochloromethane

23) $\mathrm{C} 016$ chloroform

24) $\operatorname{CoI} 7$ 1,1,1-Txichloroethane

25) $\operatorname{Co18~1,1-dichloropropene~}$

26) $\operatorname{col9} 1,2$-dichloroethane

27) 0020 carbon tetrachloride

29) C021 benzene

30) C022 trichloroethene

31) C023 1,2-dichloropropane

32) C024 dibromomethane

33) C025 bromodichloromethane

34) C026 2-chloroethylvinyleth

35) C027 cis-1,3-dichloroprope
R.T. QIon Response

Conc Units.

Area\%
12.82

17.73

21.87
1707052

1308420

917313

152

12.27

15.34

19.77

65

98

95

462847
1563803
1094490

462847
1563803

1094490
$0.00 \quad 85$

$0.00 \quad 50$

$0.00 \quad 62$

0.00

0.00

0.00

0.00

7.77

0.00

0.00

0.00

0.00

0.00

0.00

0.00

0.00

0.00

0.00

0.00

0.00

0.00

0.00

0.00

0.00

0.00

0.00

12.40

13.42

0.00

0.00

0.00

0.00

0.00

1465513

748091
$50.00 \mathrm{ug} / \mathrm{L}$

$50.00 \mathrm{ug} / \mathrm{L}$

$50.00 \mathrm{ug} / \mathrm{L}$
0.00

$-0.02$

$-0.01$
94

64

101

56

96

151

43

76

84

53

96

73

96

96

63

77

72

128

83

97

75

62

117

78

130

63

93

83

63

75
468435

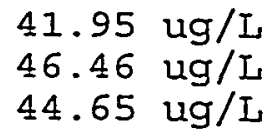

$\%$ Recovery

$83.89 \%$

$92.93 \%$

$89.30 \%$

Qvalue

Not Detected

Not Detected

Not Detected

- Not Detected

Not Detected

Not Detected

Not Detected
$47.26 \mathrm{ug} / \mathrm{L} \#$

Not Detected

Not Detected

Not Detected

Not Detected

Not Detected

Not Detected

Not. Detected

Not Detected

Not Detected

Not Detected

Not Detected

Not Detected

Not Detected

Not Detected

Not Detected

Not Detected

Not Detected

Not Detected

$42.83 \mathrm{ug} / \mathrm{L} \#$

$48.69 \mathrm{ug} / \mathrm{L} \#$

Not Detected

855

Not Detected

Not Detected

Not Detected

Not Detected

$(\mathrm{NR})=$ Not Reported $(\mathrm{SP})=$ Spiked analyte $(\mathrm{m})=$ manual integration

(*) Does not meet EPA spectral criteria (False Hit) PXI25 Page 1 


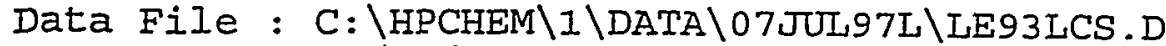

Acq Time : 07/07/97 10:07

Sample : QC-136531-1

Misc

Quant

$: 2.5$ UL of 39

Method

Jul $710: 351997$

Title

: C: \HPCHEM \I\METHODS \L826ARWA.M

Last Update : Wed Jun 25 12:48:54 1997

Response via : Multiple Level Calibration

Compound

\begin{tabular}{|c|c|c|c|c|c|c|c|}
\hline & \multicolumn{2}{|c|}{ Compound } & R.T. & QIon & Response & Conc & : Unit \\
\hline 36) & C205 & 4-methyl-2-pentanone & $0 \cap 0$ & 43 & & & \\
\hline 38$)$ & $\mathrm{CO} 28$ & trans-1,3-dichloropro & 0.00 & 75 & & Not & Detected \\
\hline 39) & $\mathrm{C} 029$ & 1,1,2-trichloroethane & 0.00 & 97 & & Not & Detected \\
\hline 40) & C210 & 2-hexanone & 0.00 & 43 & & Not & Detected \\
\hline 41) & $\mathrm{CO} 30$ & 1,2-Dibromoethane & 0.00 & 107 & & Not & Detected \\
\hline 43) & $\mathrm{CO31}$ & Toluene & 15.46 & 92 & 1066768 & 44.6 & $6 \mathrm{ug} / \mathrm{L}$ \\
\hline 44) & $\mathrm{CO} 32$ & 1,3-dichloropropane & 0.00 & 76 & & Not & Detected \\
\hline 45) & $\mathrm{CO} 33$ & dibromochloromethane & 0.00 & 129 & & Not & Detected \\
\hline 46) & $\mathrm{CO} 34$ & bromoform & 0.00 & 173 & & Not & Detected \\
\hline 47) & $\mathrm{CO35}$ & tetrachloroethene & 0.00 & 164 & & Not & Detected \\
\hline 48) & $\mathrm{C} 036$ & chlorobenzene & 17.79 & 112 & 1401777 & 45.9 & $6 \mathrm{ug} / \mathrm{L}$ \\
\hline 49) & $\mathrm{C037}$ & $1,1,1,2$-tetrachloroet & 0.00 & 131 & & Not & Detecte \\
\hline 50) & $\mathrm{CO} 38$ & ethylbenzene & 0.00 & 106 & & Not & Detected \\
\hline 51) & C039 & $\mathrm{m}, \mathrm{p}$-xylene & -0.00 & 106 & & Not & Detected \\
\hline 52) & $\mathrm{CO40}$ & o-xylene & 0.00 & 106 & & Not & Detected \\
\hline 53) & $\mathrm{C} 250$ & total xylene & 0.00 & 106 & & Not & Detected \\
\hline 54) & $\mathrm{C} 041$ & styrene & 0.00 & 104 & & Not & Detected \\
\hline 55) & $\mathrm{C} 042$ & isopropylbenzene & 0.00 & 105 & & Not & Detecte \\
\hline 58) & $\mathrm{CO} 43$ & $1,1,2,2$-tetrachloroet & 0.00 & 83 & & Not & Det \\
\hline 59) & $\mathrm{CO} 44$ & bromobenzene & 0.00 & 156 & & Not & ecte \\
\hline 60) & $\mathrm{C} 045$ & 1,2,3-trichloropropan & 0.00 & 75 & & Not & Det \\
\hline 61) & $\mathrm{C} 046$ & n-propylbenzene & 0.00 & 91 & & Not & Detected \\
\hline 62) & $\mathrm{C} 047$ & $1,3,5$-trimethylbenzen & 0.00 & 105 & & Not & Detecte \\
\hline 63) & $\mathrm{C} 048$ & 2-chlorotoluene & 0.00 & 91 & & Not & Detected \\
\hline 64) & $\mathrm{C} 049$ & 4-chlorotoluene & 0.00 & 91 & & Not & Detecte \\
\hline 65) & $\mathrm{C} 050$ & tert-butylbenzenẹ & 0.00 & 119 & & Not & Detected \\
\hline 66) & $\cos 1$ & $1,2,4$-trimethylbenzen & 0.00 & 105 & & Not & Detected \\
\hline 67) & $\mathrm{CO52}$ & sec-Butylbenzene & 0.00 & 105 & & Not & Detected \\
\hline 68) & $\mathrm{CO53}$ & p-Isopropyltoluene & 0.00 & 119 & & Not & Detected \\
\hline 69) & C054 & I, 3-Dichlo & 0.00 & 146 & & Not & Detected \\
\hline 70$)$ & C055 & 1,4-Dichlor & 0.00 & .146 & & Not & Detected \\
\hline 7I) & $\mathrm{C} 056$ & n-Butylbenzene & 0.00 & 91 & & Not & Detecte \\
\hline 72) & C057 & I,2-Dichlorobenzene & 0.00 & 146 & & Not & Detected \\
\hline 73) & $\mathrm{C} 058$ & 1,2-Dibromo-3-chlorop & 0.00 & 75 & & Not & Detected \\
\hline 74) & $\cos 9$ & 1,2,4-Trichlorobenzen & 0.00 & 180 & & Not & Detected \\
\hline 75) & $\mathrm{C} 060$ & Hexachlorobutadiene & 0.00 & 225 & & Not & Detected \\
\hline 76) & C06I & Naphthalene & 0.00 & 128 & & Not & Detected \\
\hline 77) & $\mathrm{C} 062$ & 1,2,3-Trichlorobenzen & 0.00 & 180 & & Not & Delecte \\
\hline
\end{tabular}

Operator: $\mathrm{CQC}$

Inst : 5971-L

Multiplr: 1.00
8260 WATER

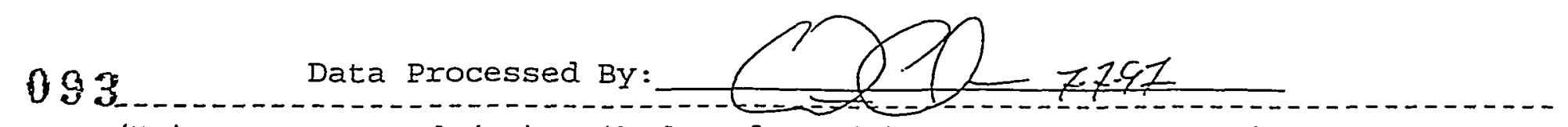

$(\mathrm{NR})=$ Not Reported $(\mathrm{SP})=$ Spiked analyte $(\mathrm{m})=$ manual integration

(*) Does not meet EPA spectral criteria (False Hit) PX125 
Quantitation Report

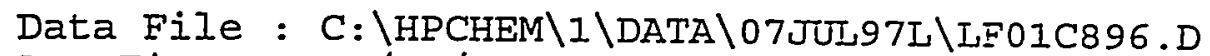

Acq Time : 07/07/97 14:20

Sample : $97 \mathrm{C02896}$ NAP-R2-S2-A

Misc : 97C-0208-01 8260 WATER

Quant Time: Jul 7 14:52 1997

Operator: CQC

Inst : 5971-I

Multiplr: 1.00

Method : C: \HPCHEM \I\METHODS\L826ARWA.M

Title : VOA COMPOUND IIST

Last Update : Mon Jul 07 12:48:10 1997

Response.via : Multiple Level Calibration

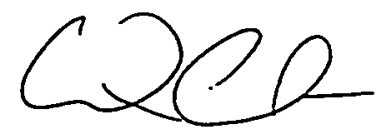

7.797

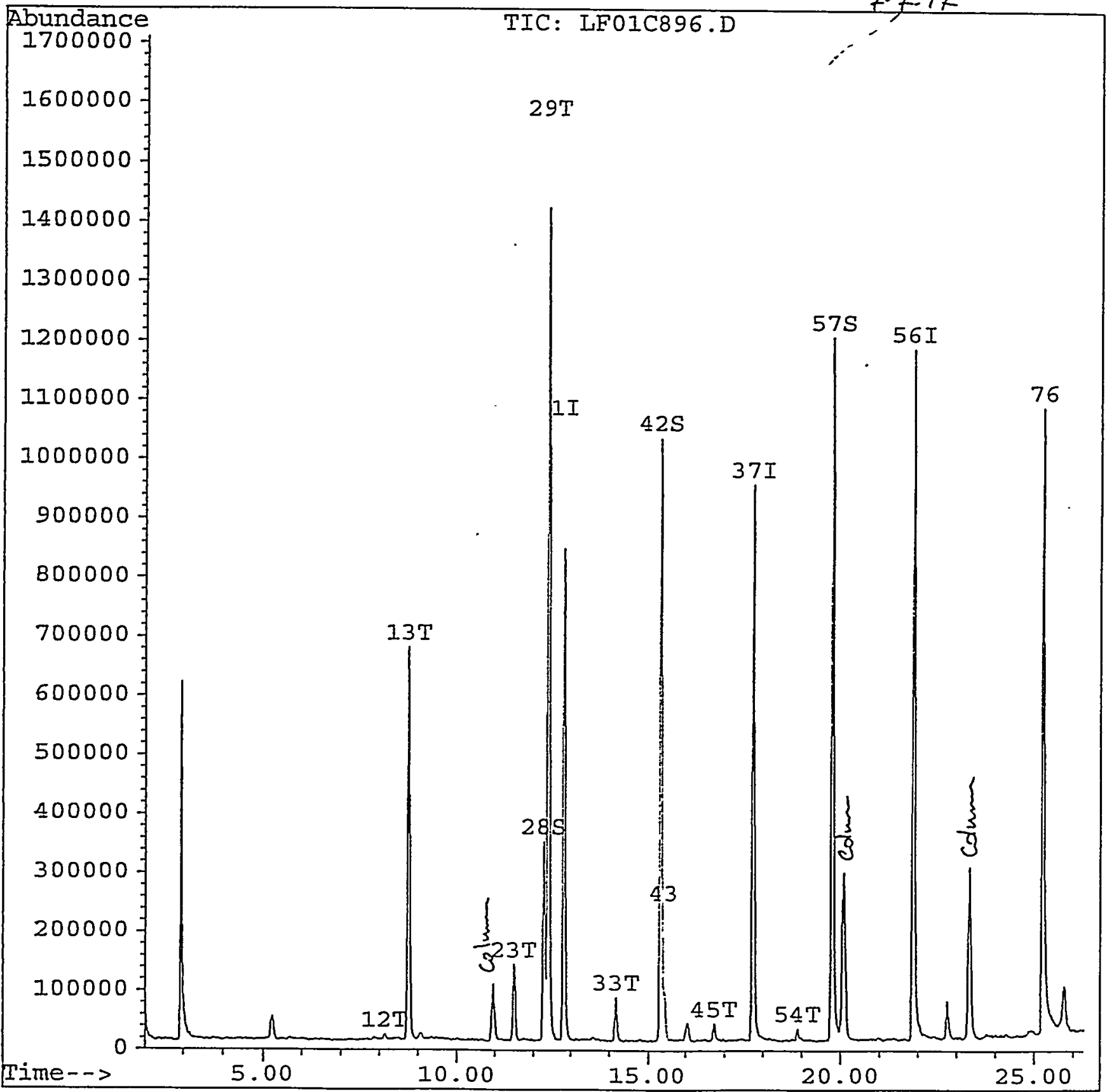

094 
Quantitation Report

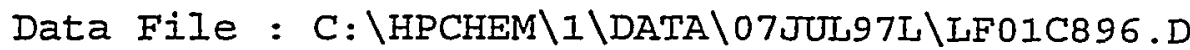

Acq Time : 07/07/97 14:20

Sample : 97C02896 NAP-R2-S2-A

MisC : 97C-0208-01 8260 WATER

Quant Time: Jul 7 14:52 1997

Operator: $\mathrm{CQC}$

Inst : 5971-L

Multiplr: 1.00

Method

Title

: C: \HPCHEM \I\METHODS \L826ARWA.M

Last Update : Mon Jul 07 12:48:10 1997

Response via : Multiple Ievel Calibration

Internal standards

1) CI01 Flourobenzene

37) CI02 Chlorobenzene-d5

56) CI03 1,4-Dichlorobenzene-d

System Monitoring Compounds

28) CS15 1,2-dichloroethane-d4

42) CSO5 toluene-d8

57) CS10 4-bromofluorobenzene

Target Compounds

2) C002 dichlorodifluorometha

3) $\mathrm{COO3}$ chloromethane

4) $\mathrm{COO4}$ vinyl chloride

5) C005 bromomethane

6) $\mathrm{CO06}$ chloroethane

7) $\mathrm{C007}$ trichlorofluoromethan

B) $C 071$ acrolein

9) $\operatorname{COOB~1,1-dichloroethene~}$

10) $C 063$ freon 113

11) co09 acetone

12) C010 carbon disulfide

13) COI1 methylene chloride

14) C072 acrylonitrile

15) $\mathrm{C} 012$ trans-1,2-Dichloroeth

16) C305 methyl-t-butyl-ether

17) $\mathrm{C013}$ cis-1,2-dichloroethen

18) C055 total-1,2-dichloroeth

19) CO14 1,1-dichloroethane

20) C015 2,2-dichloropropane

21) C110 2-butanone

22) CIOI bromochloromethane

23) 0016 chloroform

24) $\operatorname{Col7} 1,1,1$-Trichloroethane

25) $\operatorname{Co18~1,1-dichloropropene~}$

26) Co19 1,2-dichloroethane

27) $\mathrm{C} 020$ carbon tetrachloride

29) C02I benzene

30) Co22 trichloroethene

31) C023 1,2-dichloropropane

32) 0024 dibromomethane

33) C025 bromodichloromethane

34) $\mathrm{C} 026$ 2-chloroethylvinyleth

35) c027 cis-1,3-dichloroprope
R.T. QIOn

12.82

17.74

21.87

96

117

152

1770443

1338729

$945797^{\circ}$

$50.00 \mathrm{ug} / \mathrm{I}$

$50.00 \mathrm{ug} / \mathrm{I}$

$50.00 \mathrm{ug} / \mathrm{I}$

0.00

0.00
12.27

15.34

19.78
65

98

95

476521

1650671

1144211
$41.64 \mathrm{ug} / \mathrm{I}$

$47.93 \mathrm{ug} / \mathrm{L}$

$45.27 \mathrm{ug} / \mathrm{L}$
\%Recovery

$83.28 \%$

$95.87 \%$

$90.54 \%$
Qvalue

Not Detected

Not Detected

Not Detected

Not Detected

Not Detected

Not Detected

Not Detected

Not Detected

Not Detected

Not Detected

$1.12 \mathrm{ug} / \mathrm{I}$

8.1376

30630

8.75

740242

0.00

84

0.00

53

0.00

96

0.00

73

0.00

96

0.00

96

0.00

63

0.00

77

$0.00 \quad 128$

$11.50 \quad 83$

$0.00 \quad 97$

234126

$70.10 \mathrm{ug} / \mathrm{L} \#$

Not Detected

Not Detected

Not Detected

Not Detected

Not Detected

Not Detected

Not Detected

Not Detected

Not Detected

$10.60 \mathrm{ug} / \mathrm{s} \#$

Not Detected

Not Detected

Not Detected

Not Detected

$0.00 \quad 62$

$0.00 \quad 117$

12.3978

$0.00 \quad 130$

2886945

$81.34 \mathrm{ug} / \mathrm{L} \#$

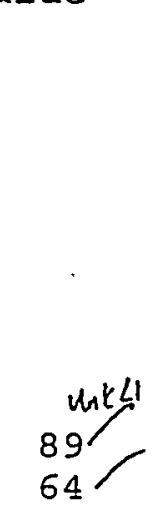

$0.00 \quad 63$

Not Detected

Not Detected

Not Detected

$14.16 \quad 83 \quad 115850$

5.05. ug/L

Not Detected

Not Detected

0.0063

$0.00 \quad 75$

$(\mathrm{NR})=$ Not Reported $(\mathrm{SP})=$ Spiked analyte $(\mathrm{m})=$ manual integration

(*) Does not meet EPA spectral criteria (False Hit) PX125 Page 1 
Quantitation Report

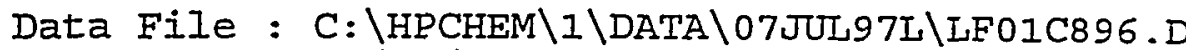

$\begin{array}{llll}\text { Acq Time } & : & 07 / 07 / 97 & 14: 20 \\ \text { Sample } & : 97 C 02896 & \text { NAP-R2-S2-A } \\ \text { Misc } & : 97 C-0208-01 & 8260 \text { WATER }\end{array}$

MisC : 97C-0208-0I 8260 WATER

Quant Time: Jul 7 14:52 1997

Operator: $\mathrm{CQC}$

Inst : 5971-I

Multiplr: 1.00

Method

Title

: C: \HPCHEM \I\METHODS \L826ARWA.M

Iast Update : Mon JuI 07 12:48:10 1997

Response via : Multiple Level Calibration

Compound

36) C205 4-methyl-2-pentanone
38) C028 trans-1,3-dichloropro
39) C029 1,1,2-trichloroethane
40) C210 2-hexanone
41) C030 1,2-Dibromoethane
43) C031 Toluene
44) C032 1,3-dichloropropane
45) C033 dibromochloromethane
46) C034 bromoform
47) C035 tetrachloroethene
48) C036 chlorobenzene
49) C037 1,1,1,2-tetrachloroet
50) C038 ethylbenzene
51) C039 m,p-xylene
52) C040 o-xylene
53) C250 total xylene
54) C041 styrene
55) C042 isopropylbenzene
58) C043 1,1,2,2-tetrachloroet
59) C044 bromobenzene
60) C045 1,2,3-trichloropropan
61) C046 n-propylbenzene
62) C047 1,3,5-trimethylbenzen
63) C048 2-chlorotoluene
64) C049 4-chlorotoluene
65) C050 tert-butylbenzene
66) C051 1,2,4-trimethylbenzen
67) C052 sec-Butylbenzene
68) C053 p-Isopropyltoluene
69) C054 1,3-Dichlorobenzene
70) C055 1,4-Dichlorobenzene
$71)$ C056 n-Butylbenzene
72) C057 1,2-Dichlorobenzene
73) C058 I,2-Dibromo-3-chlorop
74) C059 1,2,4-Trichlorobenzen
75) C060 Hexachlorobutadiene
76) C061 Naphthalene
77) C062 1,2,3-Trichlorobenzen

R.T. QIon Response Conc Unit Qvalue

$\begin{array}{rrrr}0.00 & 43 & & \text { Not Detected } \\ 0.00 & 75 & & \text { Not Detected } \\ 0.00 & 97 & \text { Not Detected } \\ 0.00 & 43 & \text { Not Detected } \\ 0.00 & 107 & & \text { Not Detected } \\ 15.45 & 92 & 64779 & 2.65 \text { ug/I \# } \\ 0.00 & 76 & & \text { Not Detected } \\ 16.75 & 129 & 40049 & 1.87 \text { ug/I } \\ 0.00 & 173 & & \text { Not Detected } \\ 0.00 & 164 & \text { Not Detected } \\ 0.00 & 112 & \text { Not Detected } \\ 0.00 & 131 & \text { Not Detected } \\ 0.00 & 106 & \text { Not Detected } \\ 0.00 & 106 & \text { Not Detected } \\ 0.00 & 106 & \text { Not Detected } \\ 0.00 & 106 & \text { Not Detected } \\ 18.89 & 104 & 33713 & \text { 1.11 ug/I \# } \\ 0.00 & 105 & & \text { Not Detected } \\ 0.00 & 83 & \text { Not Detected } \\ 0.00 & 156 & \text { Not Detected } \\ 0.00 & 75 & \text { Not Detected } \\ 0.00 & 91 & \text { Not Detected } \\ 0.00 & 105 & \text { Not Detected } \\ 0.00 & 91 & \text { Not Detected } \\ 0.00 & 91 & \text { Not Detected } \\ 0.00 & 119 & \text { Not Detected } \\ 0.00 & 105 & \text { Not Detected } \\ 0.00 & 105 & \text { Not Detected } \\ 0.00 & 119 & \text { Not Detected } \\ 0.00 & 146 & \text { Not Detected } \\ 0.00 & 146 & \text { Not Detected } \\ 0.00 & 91 & \text { Not Detected } \\ 0.00 & 146 & \text { Not Detected } \\ 0.00 & 75 & \text { Not Detected } \\ 0.00 & 180 & \text { Not Detected } \\ 0.00 & 225 & \text { Not Detected } \\ 25.22 & 128 & 2439185 & 62.37 \text { ug/I } \\ 0.00 & 180 & & \text { Not Detected } \\ & & \end{array}$



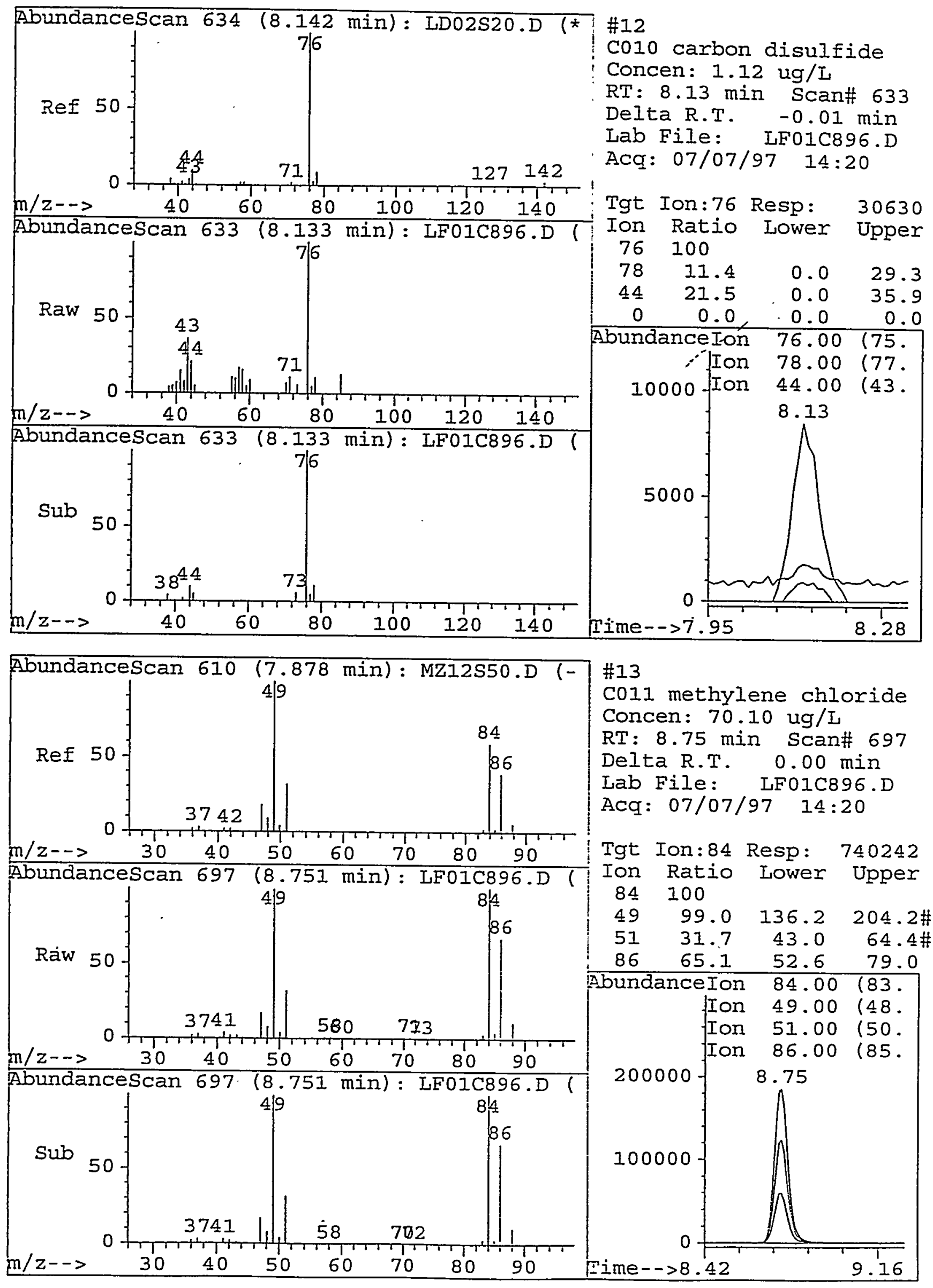


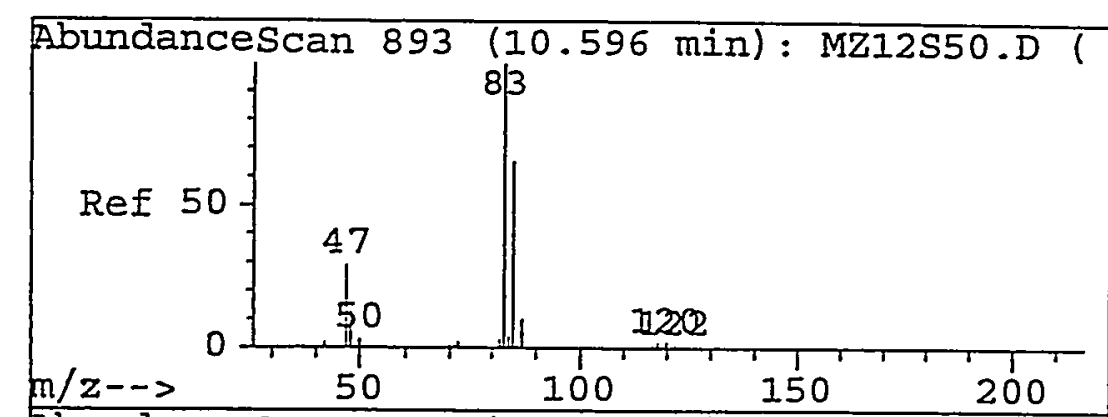

\#23

C016 chloroform

Concen: $10.60 \mathrm{ug} / \mathrm{L}$

RT: $11.50 \mathrm{~min}$ Scan\# 982

Delta R.T. $0.08 \mathrm{~min}$

Lab File: LF01C896.D

Acq : 07/07/97 14:20

\begin{tabular}{lcccc}
$\mathrm{m} / \mathrm{z}-\mathrm{z}$ & 50 & 100 & 150 & 200 \\
\hline Abundancescan & 982 & $(11.500$ & $\mathrm{min})$ & $\mathrm{TF} 01 \mathrm{C} 896$
\end{tabular}

Tgt Ion:83 Resp: 234126

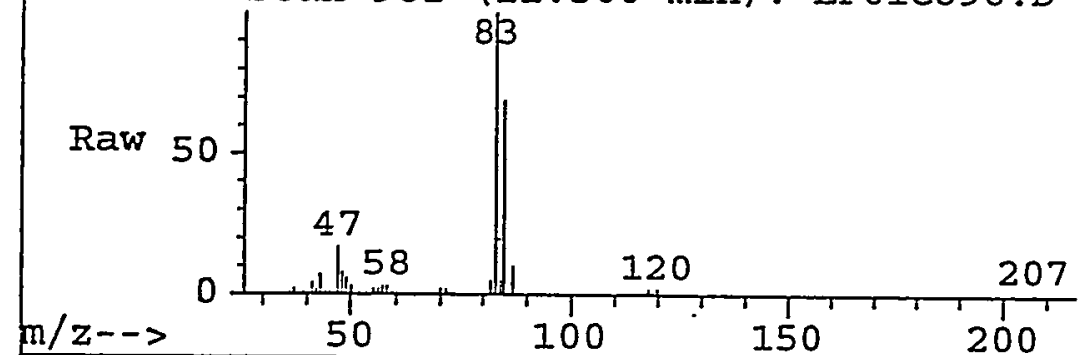

Ion Ratio Lower Upper

83100

$\begin{array}{llll}85 & 64.9 & 53.0 & 79.6\end{array}$

$47 \quad 17.4 \quad 23.0 \quad 34.4 \#$

Abundancescan 982 (11.500 min): LF01C896.D

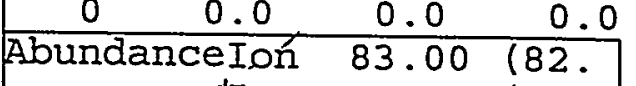

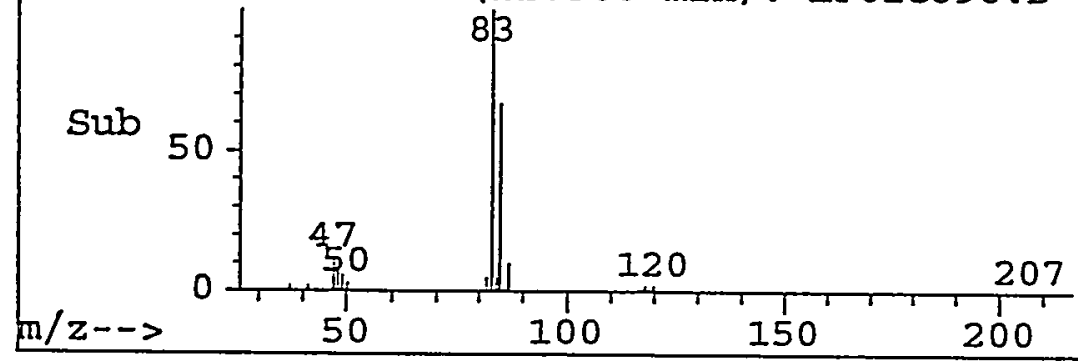

Time- $>11.24$

$85.00 \quad(84$.

60000 Ion $\begin{gathered}47.00 \quad(46.5 \\ 11.50\end{gathered}$
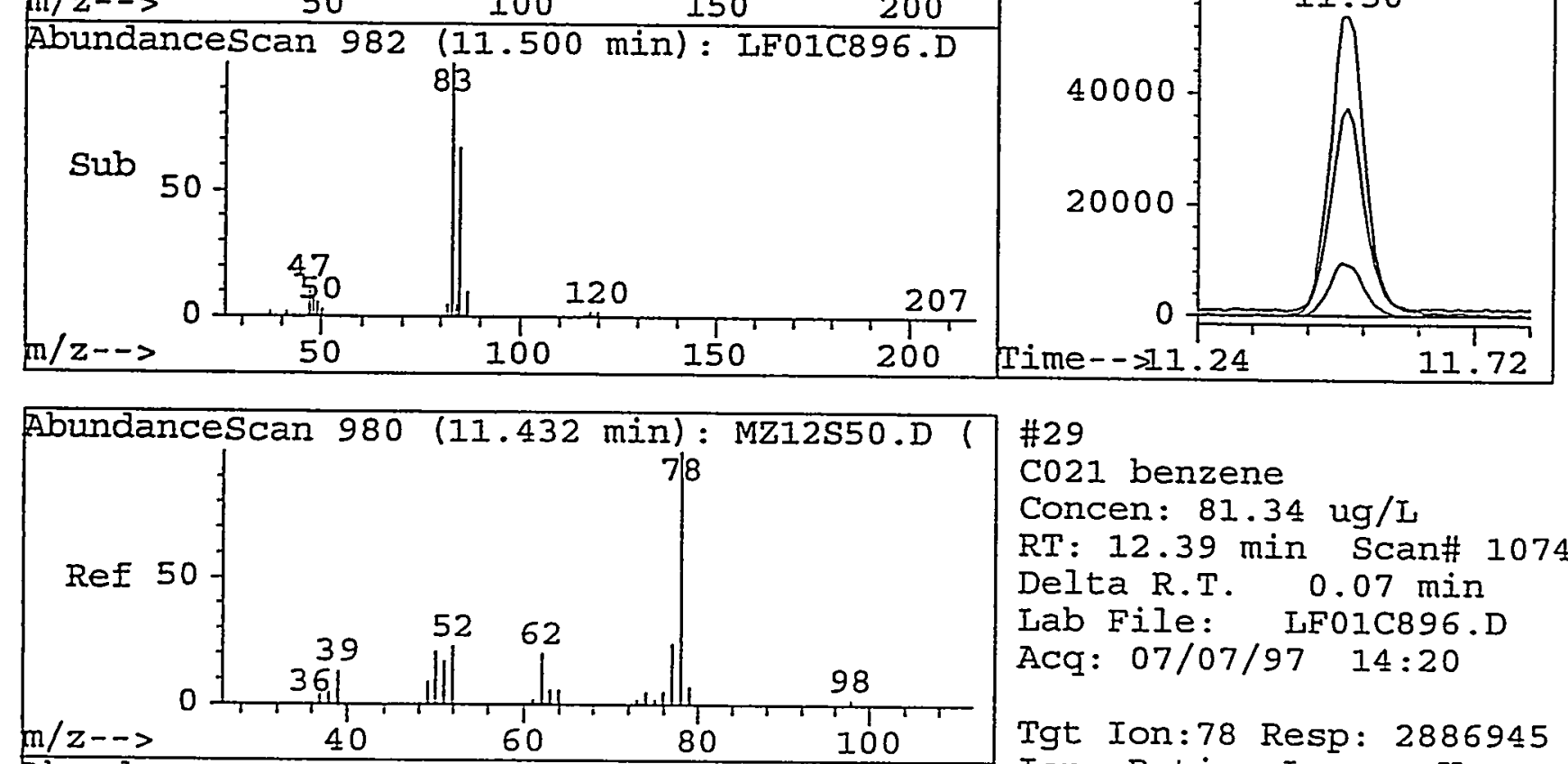

Abundancescan 1074 (12.388 min): IF01C896.D

\section{\#29}

C021 benzene

Concen: $81.34 \mathrm{ug} / \mathrm{L}$

RT: $12.39 \mathrm{~min}$ Scan\# 1074

Delta R.T. $0.07 \mathrm{~min}$

Lab File: LF01C896.D

Acq: 07/07/97 14:20

Tgt Ion:78 Resp: 2886945

Ion Ratio Lower Upper

$78 \quad 100$

$52 \quad 14.0 \quad 17.1 \quad 25.7 \#$

$\begin{array}{llll}0 & 0.0 & 0.0 & 0.0\end{array}$

Raw 507

Abundancescan 1074 (12.388 min): IF01C896.D

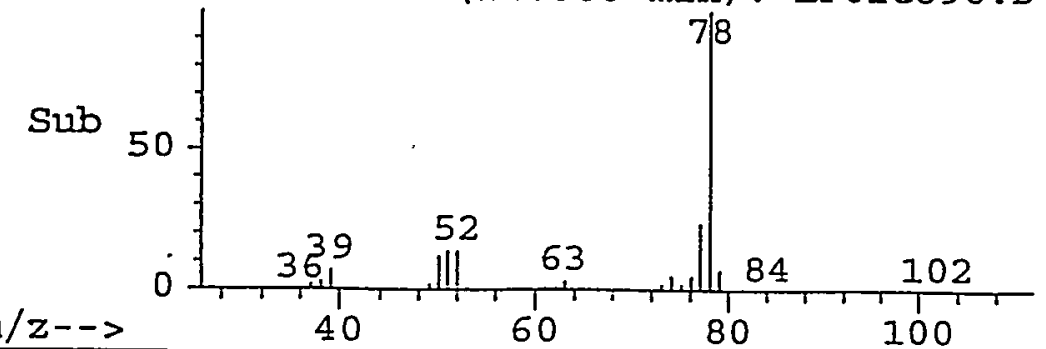

\begin{tabular}{|cccc}
0 & 0.0 & 0.0 & 0.0 \\
\hline AbundanceIon & 78.00 & $(77$.
\end{tabular}

800000 tion $52.00 \quad(51$.

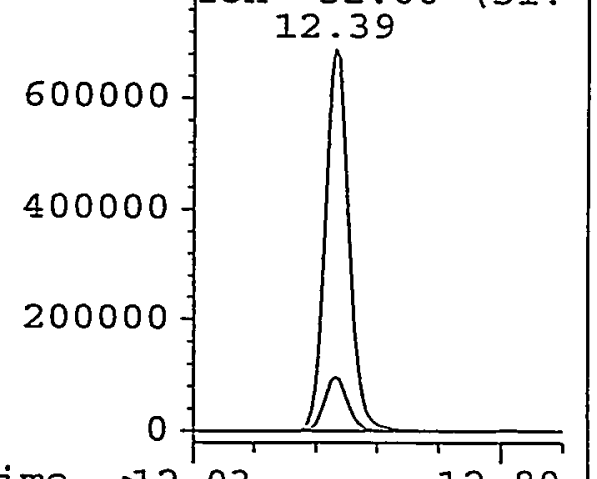

Time- $>12.03$

12.80 


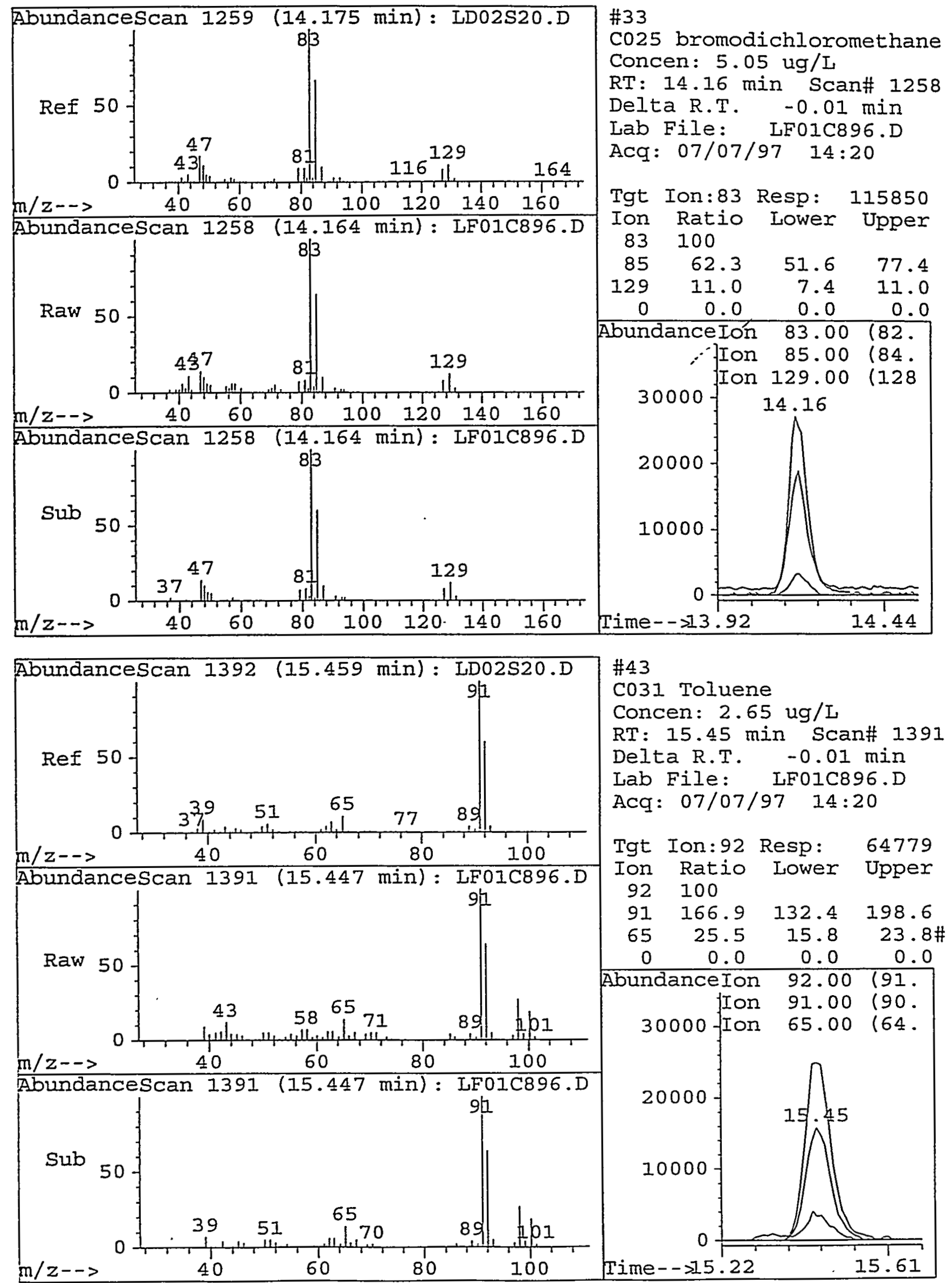




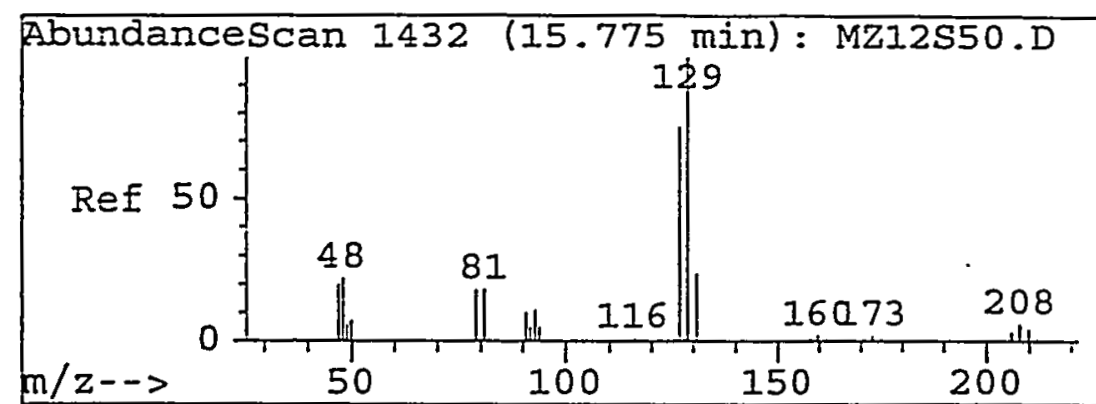

Abundancescan 1526. (16.750 min): LF01C896.D

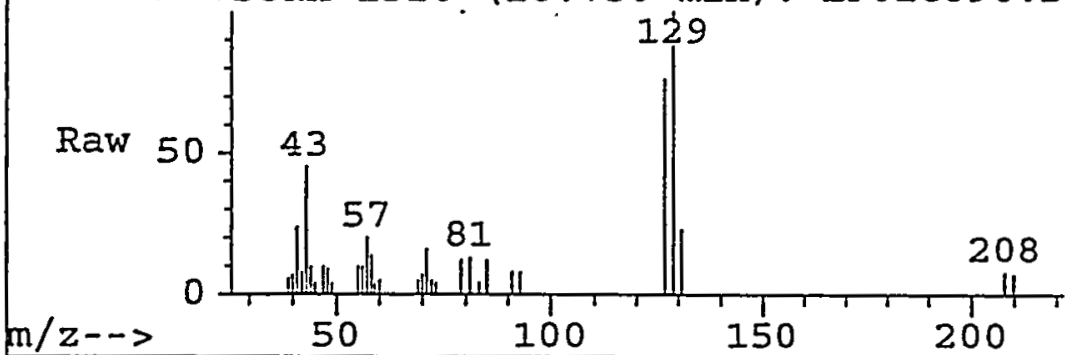

Abundancescan 1526 (16.750 min): LF01C896.D
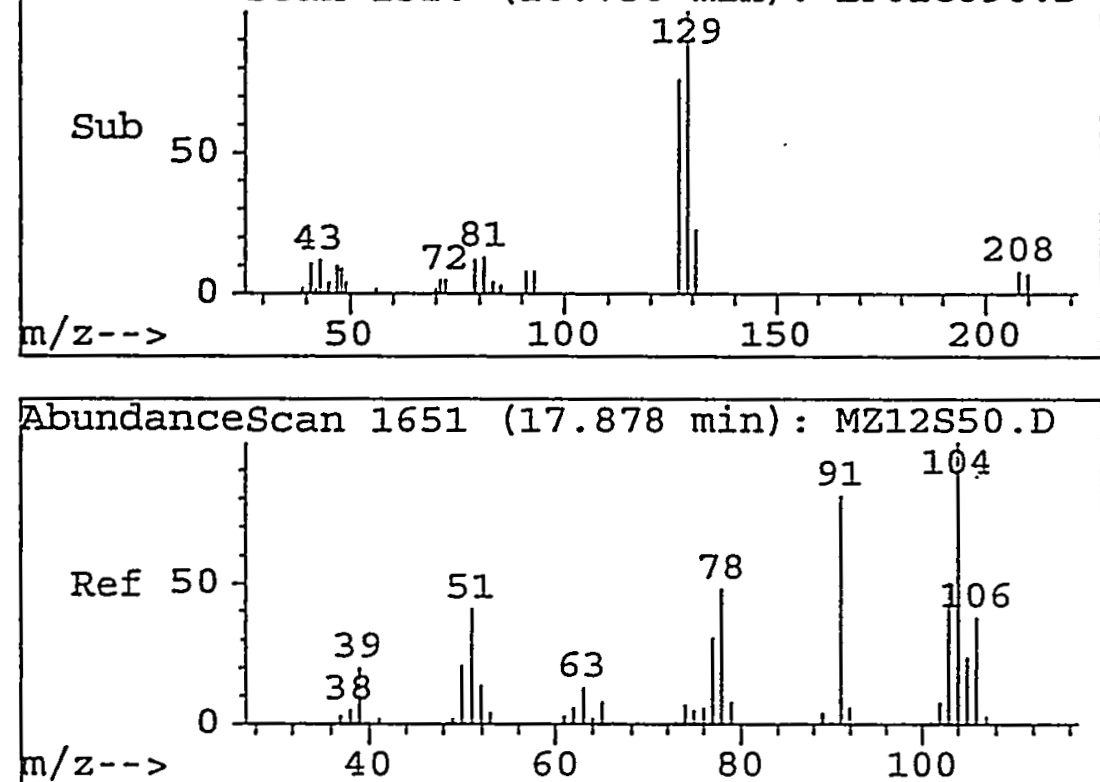

Abundancescan 1748 (18.892 min) : LF01C896.D

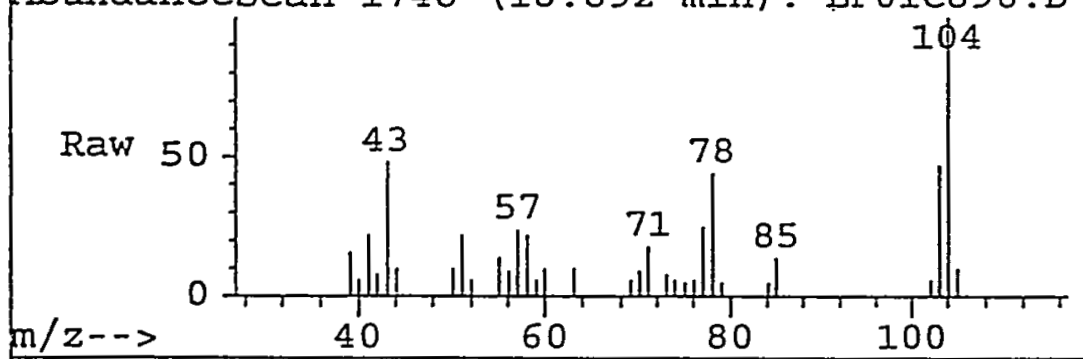

Abundancescan $1748(18.892 \mathrm{~min}):$ LF01C896.D

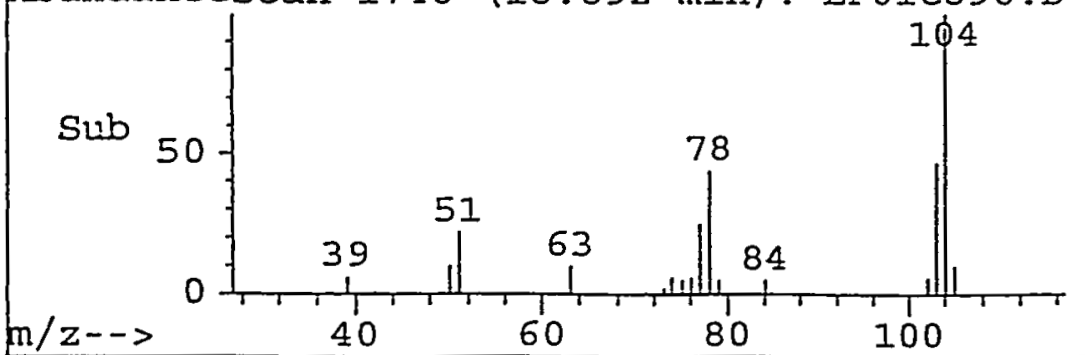

$\# 45$

C033 dibromochloromethane Concen: $1.87 \mathrm{ug} / \mathrm{I}$

RT: $16.75 \mathrm{~min}$ Scan\# 1526

Delta R.T. $0.04 \mathrm{~min}$

Lab File: LF01C896.D

Acq : 07/07/97 14:20

Tgt Ion:129 Resp: 40049

Ion Ratio Lower Upper

129100

$\begin{array}{llll}208 & 5.8 & 4.4 & 6.6\end{array}$

$206 \quad 0.0 \quad 1.9 \quad 2.9 \#$

$0 \quad 0.0,0.0 \quad 0.0$

AbundanceIon 129.00 (128

$\therefore$ Ion $208.00 \quad(207$

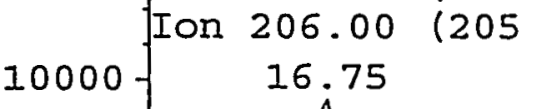

Time-->16.53

0

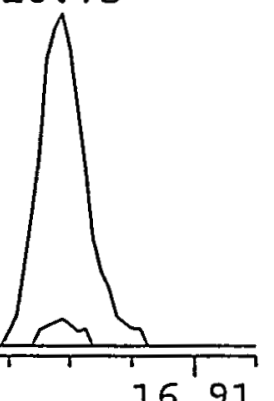

\#54

C04I styrene

Concen: $1.11 \mathrm{ug} / \mathrm{L}$

RT: $18.89 \mathrm{~min}$ Scan\# 1748

Delta R.T. $\quad-0.04 \mathrm{~min}$

Lab File: LF01C896.D

Acq : 07/07/97 14:20

Tgt Ion:104 Resp: 33713

Ion Ratio Lower Upper

104100

$\begin{array}{llll}103 & 48.6 & 42.1 & 63.1\end{array}$

$\begin{array}{llll}78 & 45.6 & 38.4 & 57.6\end{array}$

$\begin{array}{llll}51 & 20.8 & 35.4 & 53.0 \#\end{array}$

AbundanceIon 104.00 (103

10000 IIon $103.00 \quad(102$

Ion $78.00 \quad(77$.

Ion $51.00 \quad(50$.

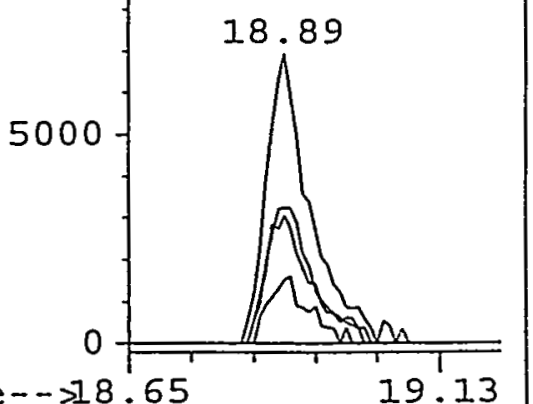

Time-->18.65 19.13 


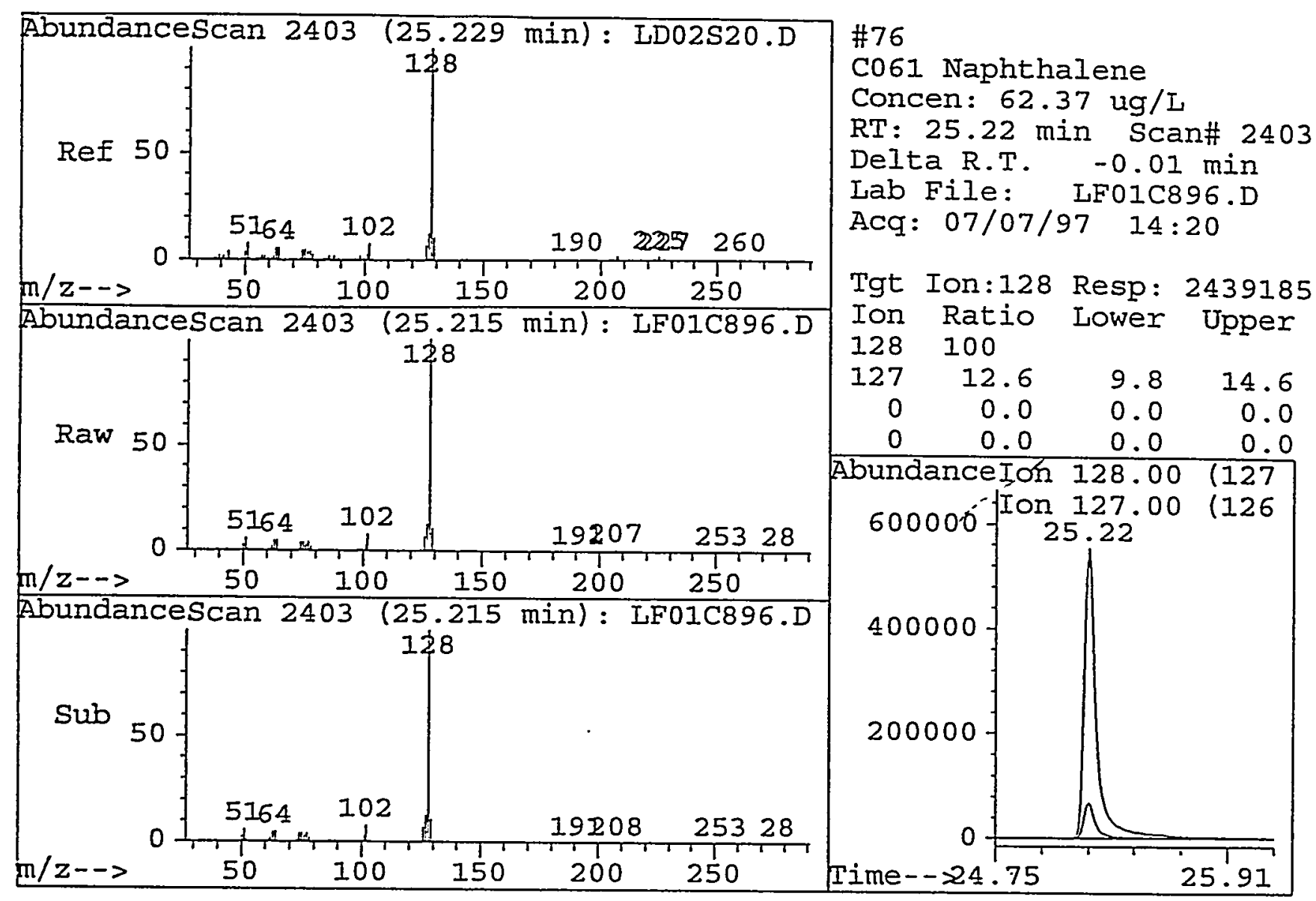

101 


\section{Quantitation Report}

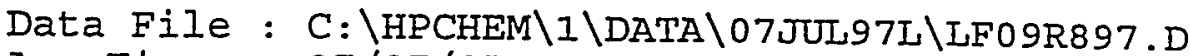

Acq Time : 07/07/97 18:34

Sample : 97C02897 NAP-R2-S2-B

Misc : 97C-0208-01

Quant Time: Jul $2013: 141997$

Operator: CQC

Inst : 5971-L Multiplr: 1.00

\footnotetext{
Method

Title

: J : \L\METHODS \L826ARWA.M

Last Update : Wed Jun 25 09:14:05 1997

Response via : Multiple Level Calibration
}

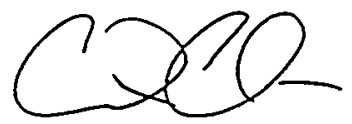

$7 \cdot 40.47$

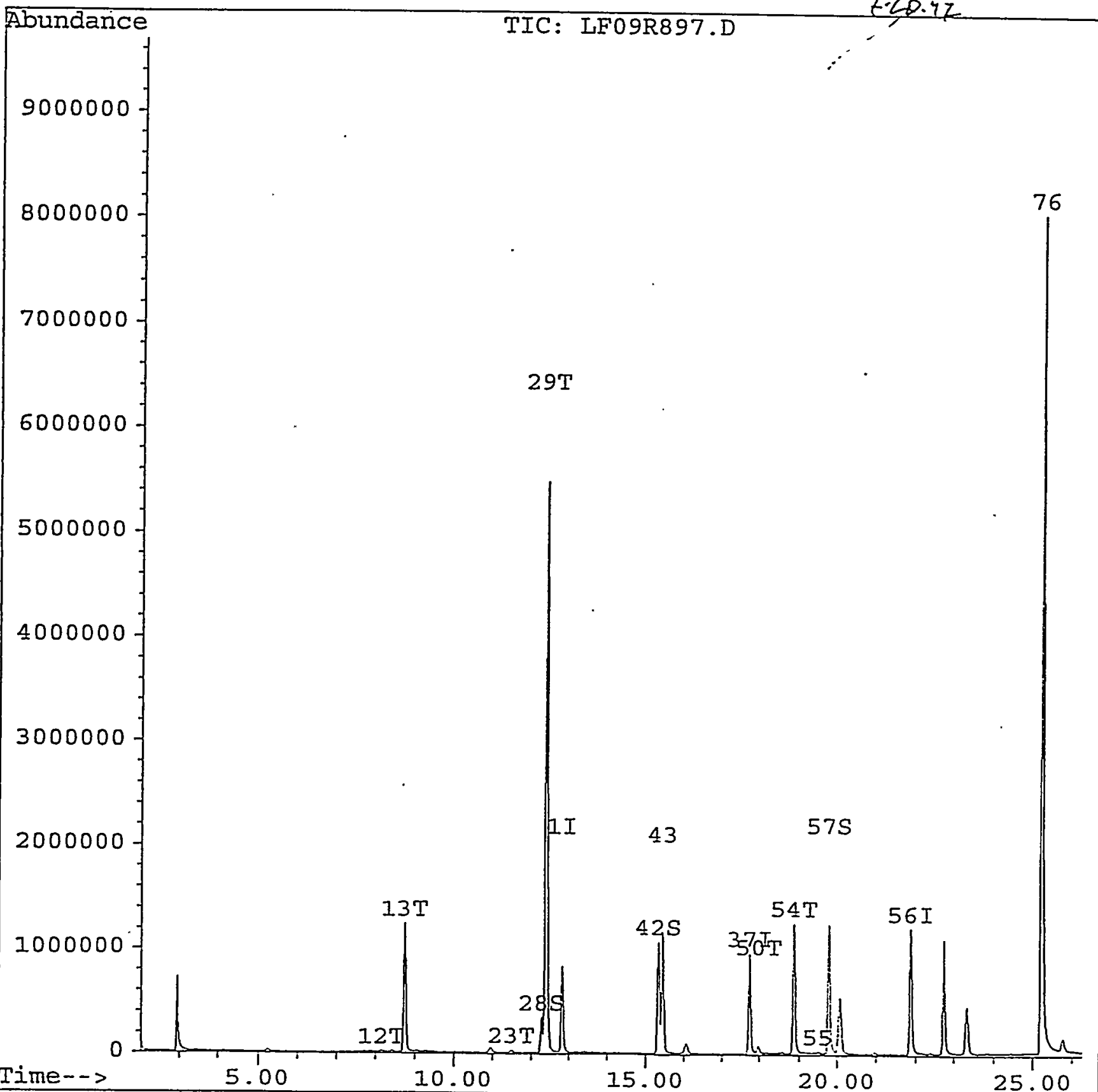

102 
Data File : C: $\backslash$ HPCHEM $\backslash 1 \backslash D A T A \backslash 07 J U L 97 L \backslash L F 09 R 897 . D$

Acq Time : 07/07/97 18:34

Sample : 97C02897 NAP-R2-S2-B

Misc : 97C-0208-01

Quant Time: Jul 20 13:14 1997

Method : J: \L\METHODS \L826ARWA.M

Title : VOA COMPOUND LIST

Last Update : Wed Jun 25 09:14:05 1997

Response via : Multiple Ievel Calibration
Operator: CQC

Inst : 5971-L

Multiplr: 1.00

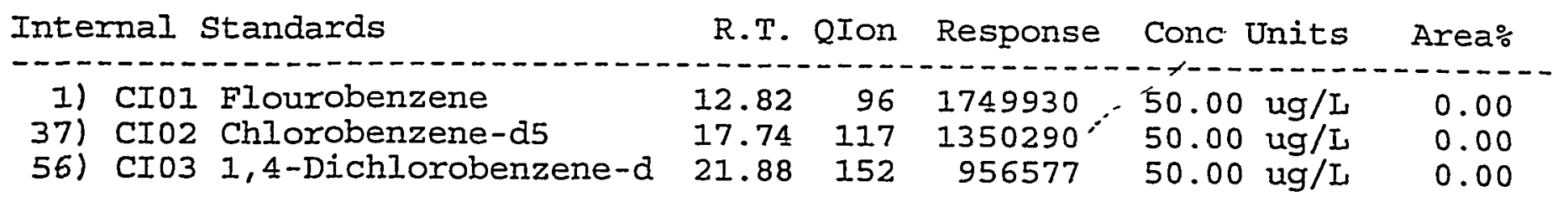

System Monitoring Compounds

28) CS15 1,2-dichloroethane-d4

42) CS05 toluene-d8

57) CSI0 4-bromofluorobenzene

Target Compounds

2) $\mathrm{COO2}$ dichlorodifluorometha

3) $\mathrm{C003}$ chloromethane

4) 0004 vinyl chloride

5) C005 bromomethane

6) 0006 chloroethane

7) $\mathrm{C007}$ trichlorofluoromethan

B) $\mathrm{CO} 71$ acrolein

9) $\operatorname{Co08} 1,1$-dichloroethene

10) $\mathrm{C} 063$ freon 113

11) C009 acetone

12) CO10 carbon disulfide

13) COII methylene chloride

14) $\mathrm{C} 072$ acrylonitrile

15) $\mathrm{CO12}$ trans-1,2-Dichloroeth

16) C305 methyl-t-butyl-ether

17) $\mathrm{COI3}$ cis-1,2-dichloroethen

1B) C055 total-1,2-dichloroeth

19) C014 1,1-dichloroethane

20) COI5 2,2-dichloropropane

2I) CIIO 2-butanone

22) CIOI bromochloromethane

23) $\mathrm{CO16}$ chloroform

24) C017 1,1,1-Trichloroethane

25) C018 1,1-dichloropropene

26) C019 1,2-dichloroethane

27) C020 carbon tetrachloride

29) C02I benzene

30) $\mathrm{C} 022$ trichloroethene

31) $\mathrm{CO23} 1,2$-dichloropropane

32) C024 dibromomethane

33) C025 bromodichloromethane

34) C026 2-chloroethylvinyleth

35) C027 cis-1,3-dichloroprope
12.27

15.34

19.77

65

98

95

486213

1707499

1196681

$0.00 \quad 85$

$0.00 \quad 50$

$0.00 \quad 62$

$0.00 \quad 94$

$0.00 \quad 64$

$0.00 \quad 101$

$0.00 \quad 56$

$0.00 \quad 96$

$0.00 \quad 151$

0.00

8.13

8.74

0.00

0.00

0.00

0.00

0.00

0.00

0.00

0.00

0.00

11.50

0.00

0.00

0.00

0.00

12.40

0.00

0.00

0.00

0.00

0.00

0.00
151
43

76

84

53

96

73

96

96

63

77

72

128

83

97

75

62

117

$\begin{array}{ll}78 & 11317359\end{array}$

130

63

93

83

63

75

\section{5}

1358367

34161
$\%$ Recovery

$42.99 \mathrm{ug} / \mathrm{L} \quad 85.97 \%$

$49.16 \mathrm{ug} / \mathrm{L} \quad 98.32 \%$

$46.81 \mathrm{ug} / \mathrm{I} \quad 93.63 \%$
$(\mathrm{NR})=$ Not Reported

(*) Does not meet
Qvalue

Not Detected

Not Detected

Not Detected

Not Detected

Not Detected

Not Detected

Not Detected

Not Detected

Not Detected

Not Detected

$0.77 \mathrm{ug} / \mathrm{L}$

$130.14 \mathrm{ug} / \mathrm{I} \#$

Not Detected

Not Detected

Not Detected

Not Detected

Not Detected

Not Detected

Not Detected

Not Detected

Not Detected

$1.56 \mathrm{ug} / \mathrm{L}$ \#

Not Detected

87

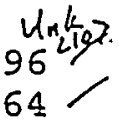

Not Detected

Not Detected

Not Detected

$322.62 \mathrm{ug} / \mathrm{L}$

Not Detected

Not Detected

Not Detected

Not Detected

Not Detected

Not Detected
$1000 \% 14$ 
Quantitation Report

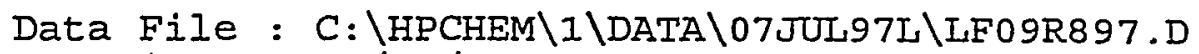

Acq Time : 07/07/97 18:34

Sample : 97C02897 NAP-R2-S2-B

Misc : 97C-0208-01

Quant Time: Jul 20 13:14 1997

Operator: CQC

Inst : 5971-L Multiplr: 1.00

Method : J: \L\METHODS \L826ARWA.M

Title : VOA COMPOUND LIST

Last Update : Wed Jun 25.09:14:05 1997

Response via : Multiple Level Calibration

Compound

\begin{tabular}{|c|c|c|c|c|c|}
\hline 36) & C205 & 4-methyl-2-pentanone & 0.00 & 43 & \\
\hline 38) & $\mathrm{CO} 28$ & trans-i, 3 -dichloropro & 0.00 & 75 & \\
\hline 39) & $\mathrm{CO} 29$ & 1,1,2-trichloroethane & 0.00 & 97 & \\
\hline 40) & $\mathrm{C} 210$ & 2-hexanone & 0.00 & 43 & \\
\hline 41) & $\mathrm{CO} 30$ & 1,2-Dibromoethane & 0.00 & 107 & \\
\hline 43) & $\operatorname{CO3I}$ & Toluene & 15.45 & 92 & 1239205 \\
\hline 44) & $\mathrm{CO} 32$ & 1,3-dichloropropane & 0.00 & 76 & \\
\hline 45) & $\mathrm{CO} 33$ & dibromochloromethane & 0.00 & 129 & \\
\hline 46) & $\cos 4$ & bromoform & 0.00 & 173 & \\
\hline 47) & $\mathrm{CO} 35$ & tetrachloroethene & 0.00 & 164 & \\
\hline 48) & $\mathrm{C036}$ & chlorobenzene & 0.00 & 112 & \\
\hline 49) & $\cos 7$ & $1,1,1,2$-tetrachloroet & 0.00 & 131 & - \\
\hline 50) & $\cos 8$ & ethylbenzene & 17.97 & 106 & 38116 \\
\hline 51) & $\cos 9$ & $\mathrm{~m}, \mathrm{p}$-xylene & 0.00 & 106 & \\
\hline 52) & $\mathrm{CO} 40$ & o-xylene & 0.00 & 106 & \\
\hline 53) & C250 & total xylene & 0.00 & 106 & \\
\hline 54) & $\mathrm{CO} 41$ & styrene & 18.87 & 104 & 1592812 \\
\hline 55) & $\mathrm{CO} 42$ & isopropylbenzene & 19.50 & 105 & 24922 \\
\hline 58) & $\mathrm{C043}$ & $1,1,2,2$-tetrachloroet & 0.00 & 83 & \\
\hline 59) & $\mathrm{CO} 44$ & bromobenzene & 0.00 & 156 & \\
\hline 60) & C045 & 1,2,3-trichloropropan & 0.00 & 75 & \\
\hline 61) & $\operatorname{Co4} 6$ & n-propylbenzene & 0.00 & 91 & \\
\hline 62) & $\mathrm{CO47}$ & $1,3,5$-trimethylbenzen & 0.00 & 105 & \\
\hline 63) & $\mathrm{CO48}$ & 2-chlorotoluene & 0.00 & 91 & \\
\hline 64) & $\operatorname{Co49}$ & 4-chlorotoluene & 0.00 & 91 & \\
\hline 65) & $\operatorname{Co50}$ & tert-butylbenzene & 0.00 & 119 & \\
\hline 66) & C051 & $1,2,4$-trimethylbenzen & 0.00 & 105 & \\
\hline 67) & C052 & sec-Butylbenzene & 0.00 & 105 & \\
\hline 68) & $\mathrm{CO53}$ & p-Isopropyltoluene & 0.00 & 119 & \\
\hline 69) & $\operatorname{Co54}$ & 1,3-Dichlorobenzene & 0.00 & 146 & \\
\hline 70) & C055 & I, 4-Dichlorobenzene & 0.00 & 146 & \\
\hline 71) & C056 & n-Butylbenzene & 0.00 & 91 & \\
\hline 72) & $\operatorname{Co57}$ & 1,2-Dichlorobenzene & 0.00 & 146 & \\
\hline 73) & $\mathrm{CO58}$ & 1,2-Dibromo-3-chlorop & 0.00 & 75 & \\
\hline 74) & $\mathrm{C059}$ & $1,2,4$-Trichlorobenzen & 0.00 & 180 & \\
\hline 75) & $\mathrm{CO} 60$ & Hexachlorobutadiene & 0.00 & 225 & \\
\hline 76) & C061 & Naphthalene & 25.22 & 128 & 15694262 \\
\hline 77) & $\mathrm{CO} 62$ & $1,2,3$-Trichlorobenzen & 0.00 & 180 & \\
\hline
\end{tabular}

R.T. QIon Response Conc Unit Qvalue

Not Detected

Not Detected

Not Detected

Not Detected

Not Detected

$50.28 \mathrm{ug} / \mathrm{I}$

Not Detected

Not Detected

Not Detected

Not Detected

Not Detected

Not Detected

$2.66 \mathrm{ug} / \mathrm{L}$

Not Detected

Not Detected

Not Detected

$52.03 \mathrm{ug} / \mathrm{L} \#$

$0.48 \mathrm{ug} / \mathrm{L}$

Not Detected

Not Detected

Not Detected

Not Detected

Not Detected

Not Detected

Not Detected

Not Detected

Not Detected

Not Detected

Not Detected

Not Detected

Not Detected

Not Detected

Not Detected

Not Detected

Not Detected

Not Detected

$395.79 \mathrm{ug} / \mathrm{L}$ m ${ }_{7 x+12}^{\alpha}$ oDilus Not Detected 


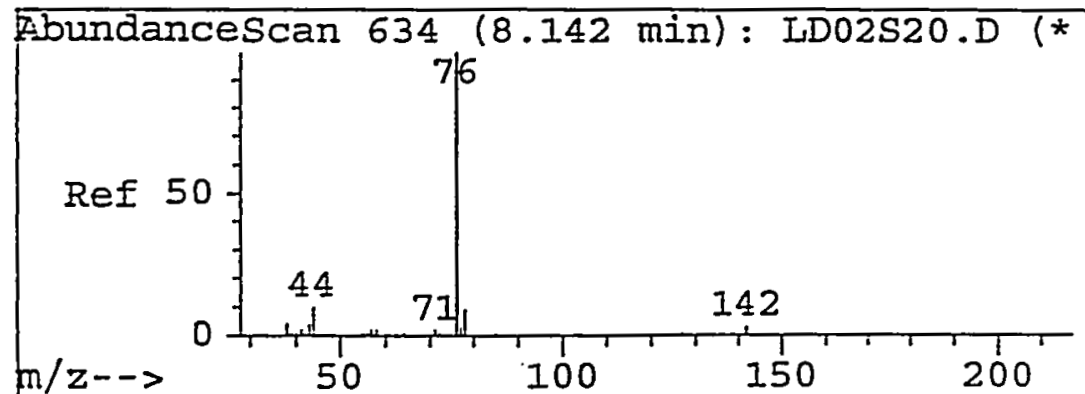

bundancescan 633 (8.133 min): LF09R897.D (

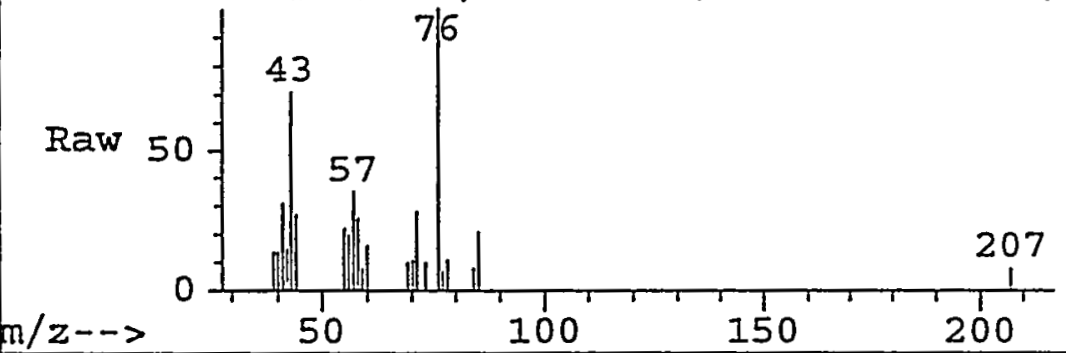

Abundancescan $633(8.133 \mathrm{~min}):$ LF09R897.D (

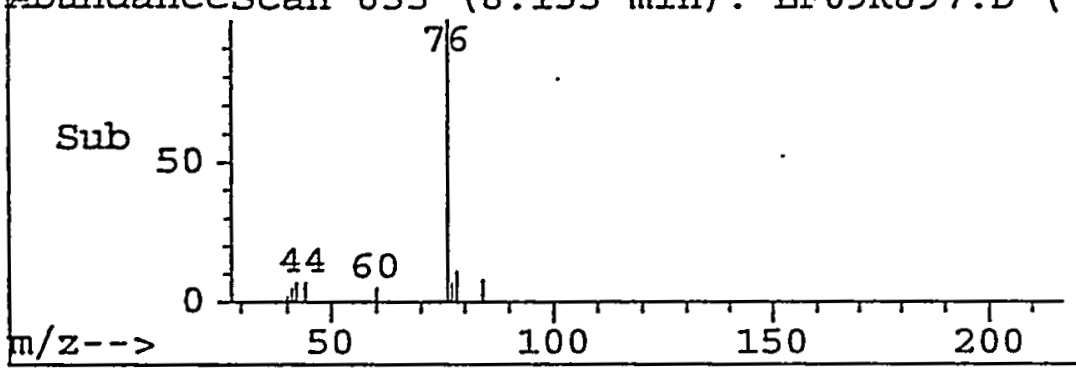

Abundancescan $610(7.878 \mathrm{~min}):$ MZ12S50.D (-

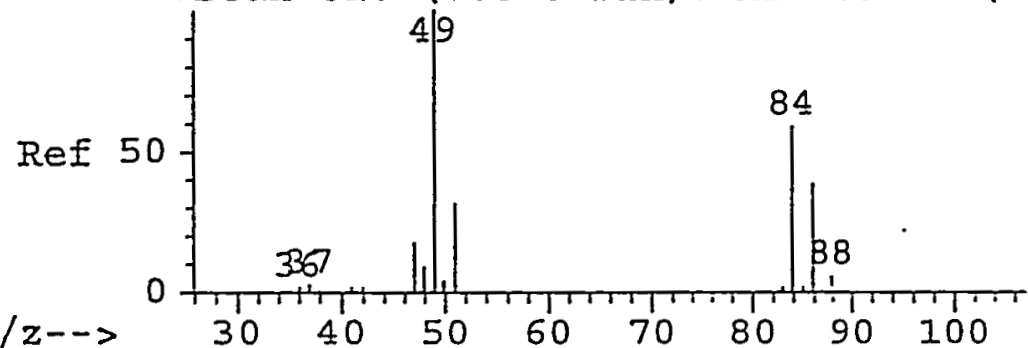

Abundancescan $696(8.741 \mathrm{~min}):$ LF09R897.D (

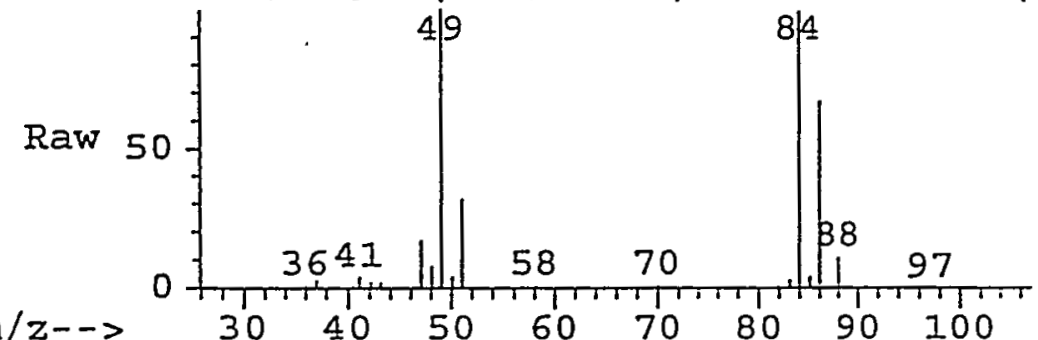

$\begin{array}{lllllllll}30 & 40 & 50 & 60 & 70 & 80 & 90 & 100\end{array}$

Aiundancescan 696 (8.741 min) : LF09R897.D (

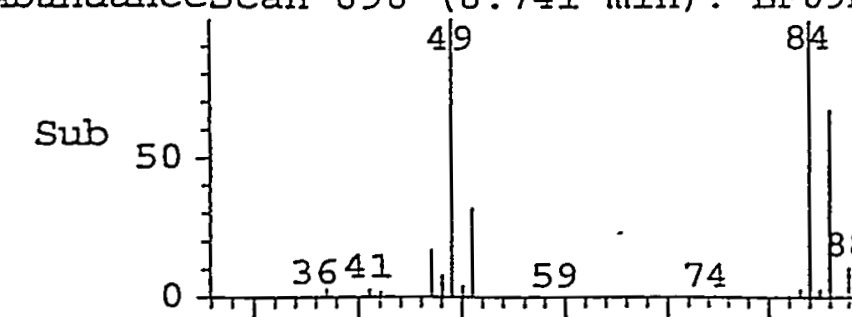
30
\#12

COI0 carbon disulfide

Cozcen: $0.77 \mathrm{ug} / \mathrm{L}$

$\mathrm{RT}: 8.13 \mathrm{~min}$ Scan 633

Delta R.T. $\quad-0.01 \mathrm{~min}$

Lab File: LF09R897.D

Acq: 07/07/97 18:34

Tgt Ion:76 Resp: 20805

Ion Ratio Lower Upper

$76 \quad 100$

$\begin{array}{llll}78 & 8.6 & 0.0 & 29.3\end{array}$

$4 \leqslant \quad 18.0 \quad 0.0 \quad 35.9$

$0 \quad 0.0<0.0 \quad 0.0$

AbundanceIon 76.00 (75.
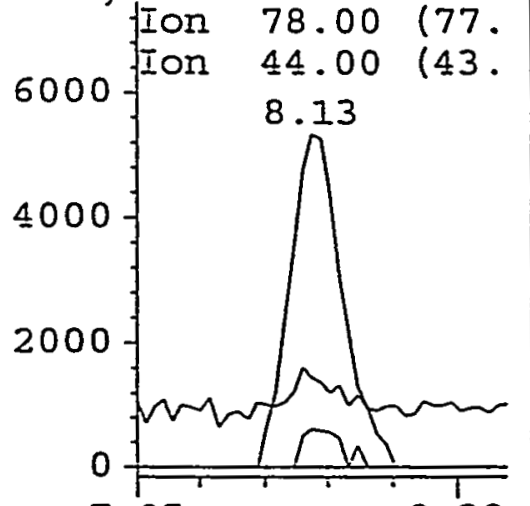

Time-->7.95

8.28
\#13

C011 methylene chloride Concen: $130.14 \mathrm{ug} / \mathrm{L}$ RT: 8.74 min Scan: 696 Delta R.T. $\quad-0.01$ min Lab File: IF09R897.D Aco: 07/07/97 18:34

Tgt Ion: 8 \& Resp: 1358367

Ion Ratio Iower Upper $84 \quad 100$

$49 \quad 100.2 \quad 136.2 \quad 204.2 \#$ $\begin{array}{llll}51 & 31.8 & 43.0 & 64.4 \#\end{array}$ $\begin{array}{llll}86 & 66.3 & 52.6 & 79.0\end{array}$ Abundanceion 84.00 (83. IIon $49.00 \quad(48$. $\begin{array}{rrr}\text { Ion } & 51.00 & (50 . \\ & \text { Ion } 86.00 & (85 .\end{array}$ (1)

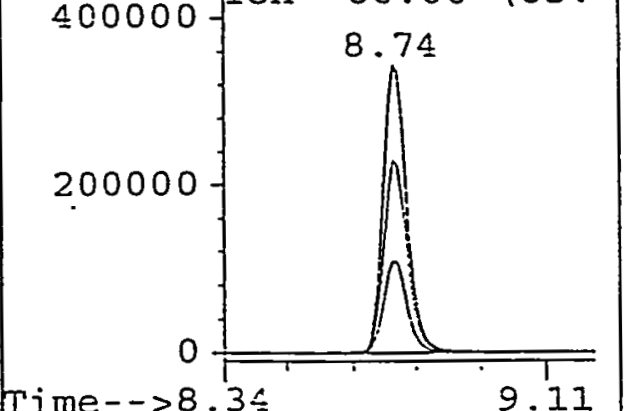




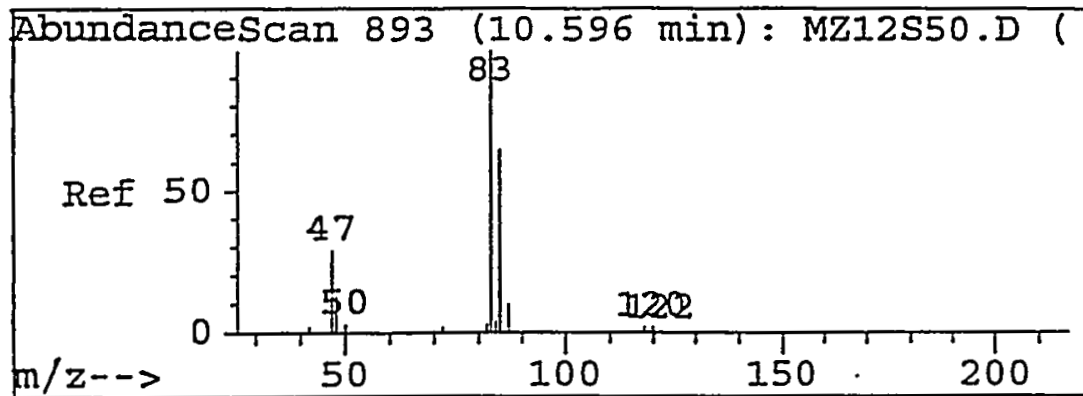

Abundancescan 982 (11.500 min): IF09R897.D

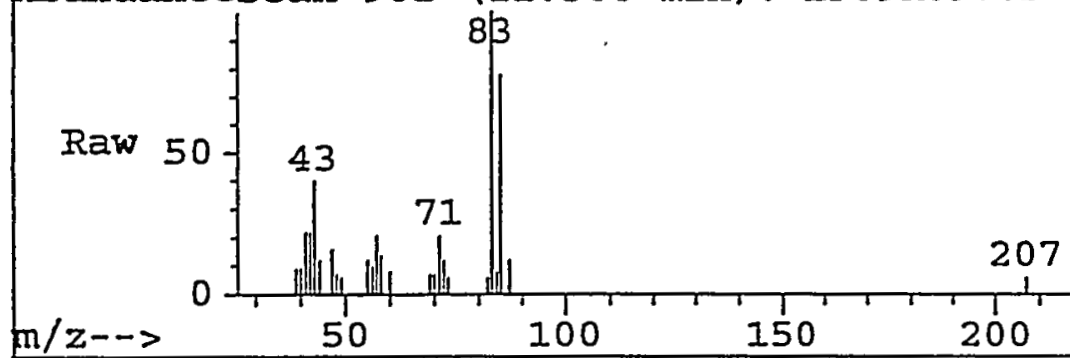

Abundancescan 982 (11.500 min): LF09R897.D
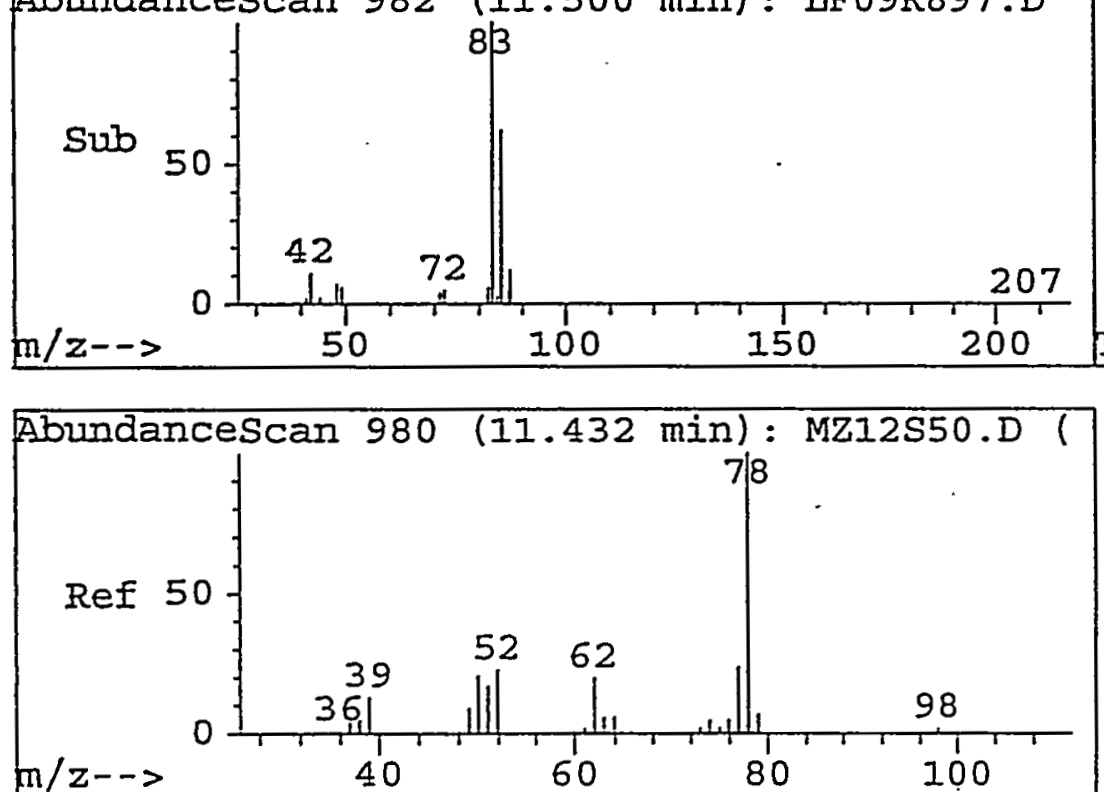

Abundancescan 1075 (12.398 min): LF09R897.D

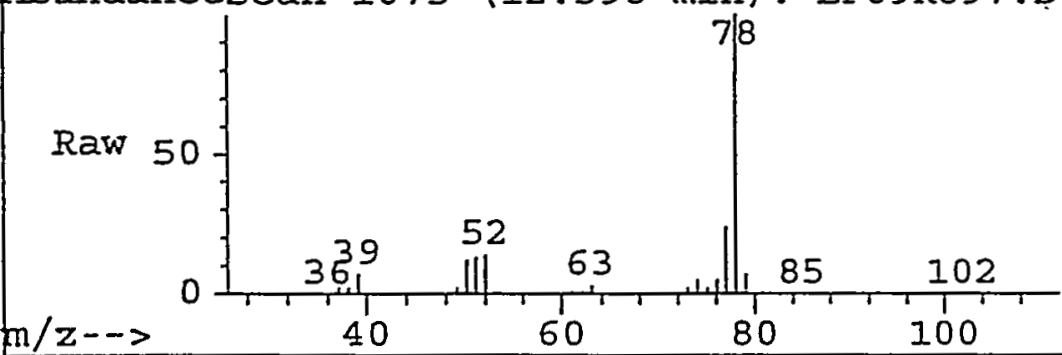

Abundancescan $1075(12.398 \mathrm{~min}):$ LF09R897.D

$106 / 2-m$

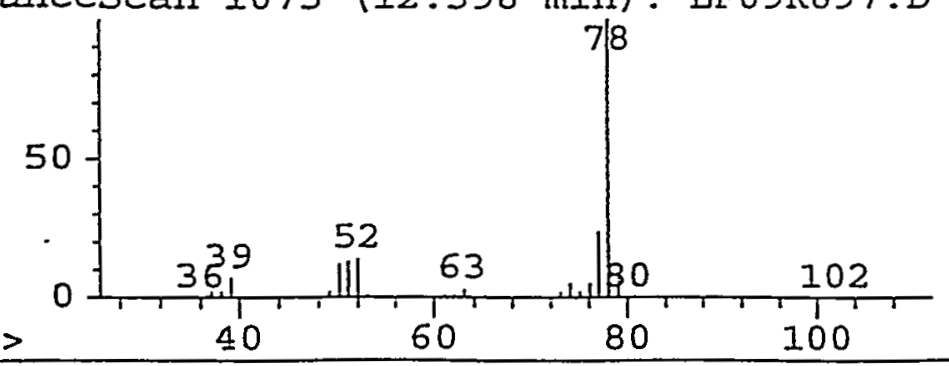

\#23

C016 chloroform

Concen: $1.56 \mathrm{ug} / \mathrm{L}$

$\mathrm{RT}: 11.50 \mathrm{~min}$ Scan\# 982

Delta R.T. $0.08 \mathrm{~min}$

Lab File: LF09R897.D

Acq: $07 / 07 / 97 \quad 18: 34$

Tgt Ion:83 Resp: 34161

Ion Ratio Lower Upper

83100

$\begin{array}{llll}85 & 72.3 & 53.0 & 79.6\end{array}$

$47 \quad 15.8 \quad 23.0 \quad 34.4 \#$

$0 \quad 0.0<0.0 \quad 0.0$

Abundancelón $83.00 \quad(82$.

10000 IIon $85.00 \quad(84$.

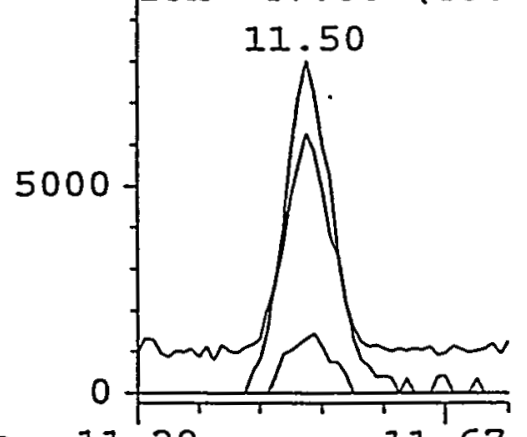

Time-->11.29

11.67

\section{\#29}

C021 benzene

Concen: $322.62 \mathrm{ug} / \mathrm{L}$

RT: $12.40 \mathrm{~min}$ Scan\# 1075

Delta R.T. $\quad-0.00 \mathrm{~min}$

Lab File: LF09R897.D

ACq : 07/07/97 18:34

Tgt Ion:78 Resp:11317359

Ion Ratio Lower Upper

$78 \quad 100$

$\begin{array}{llll}52 & 13.9 & 11.1 & 16.7\end{array}$

$\begin{array}{llll}0 & 0.0 & 0.0 & 0.0\end{array}$

$\begin{array}{llll}0 & 0.0 & 0.0 & 0.0\end{array}$

AbundanceIon $78.00 \quad(77$.

3000000 fIon 52.00 (51.

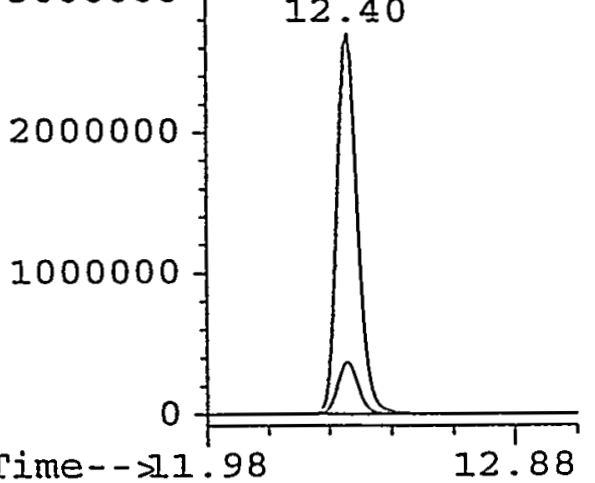




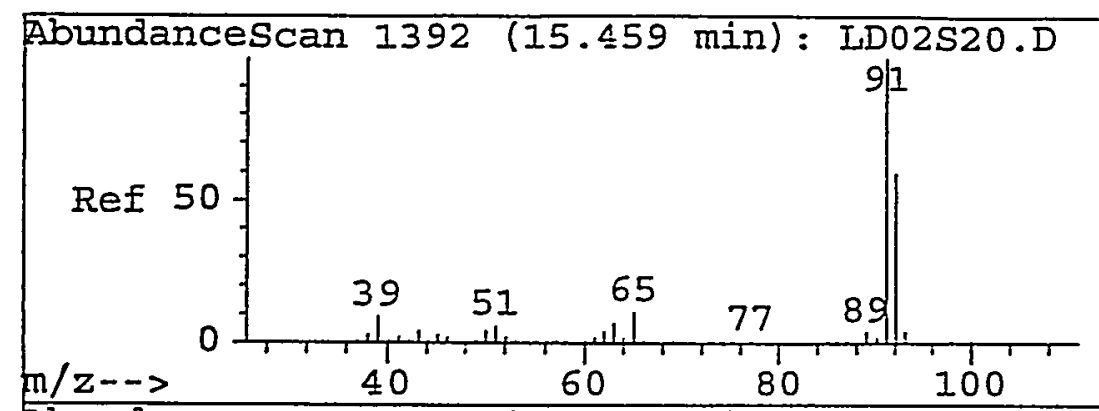

Abundancescan 1391 (15.447 min): LF09R897.D

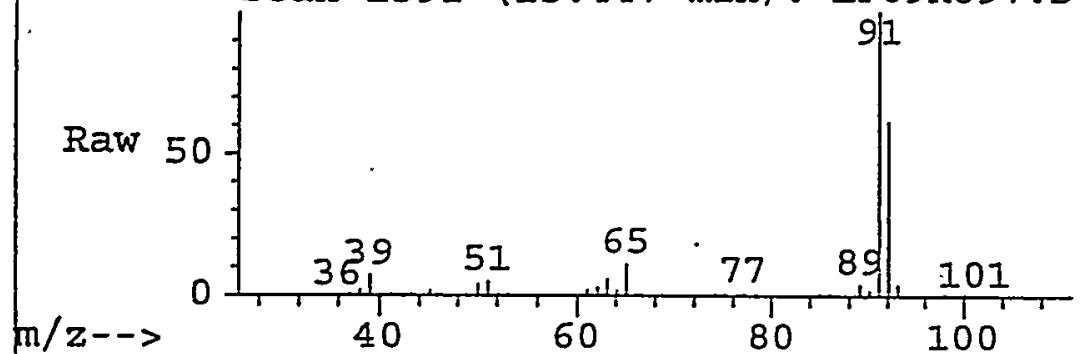

Abundancescan 1391 (15.447 min): IF09R897.D

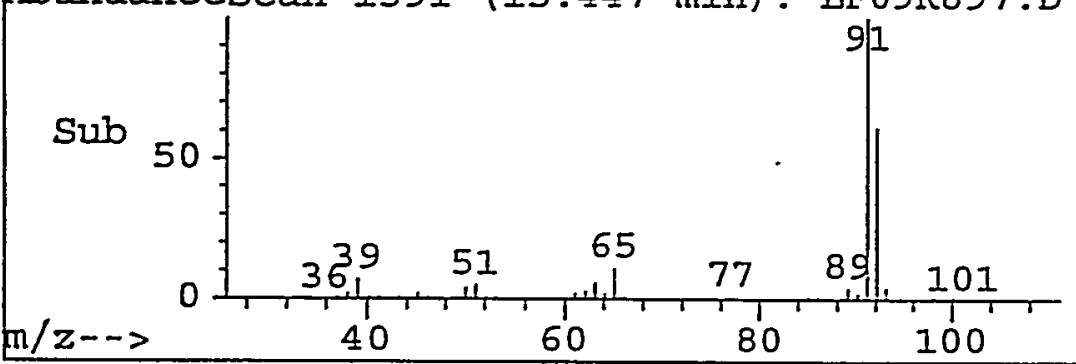

\#43

C031 Toluene

Concen: $50.28 \mathrm{ug} / \mathrm{L}$

RT: $15.45 \mathrm{~min}$ Scan\# 1391

Delta R.T. $\quad-0.01 \mathrm{~min}$

Lab File: IF09R897.D

Acq: $07 / 07 / 97 \quad 18: 34$

Tgt Ion:92 Resp: 1239205

Ion Ratio Lower Upper

92100

$\begin{array}{llll}91 & 164.8 & 132.4 & 198.6\end{array}$

$\begin{array}{llll}65 & 17.8 & 15.8 & 23.8\end{array}$

AbundanceIón 92.00 (91.

$\therefore$ Ion 91.00190 .

600000 IIon $65.00 \quad 164$.

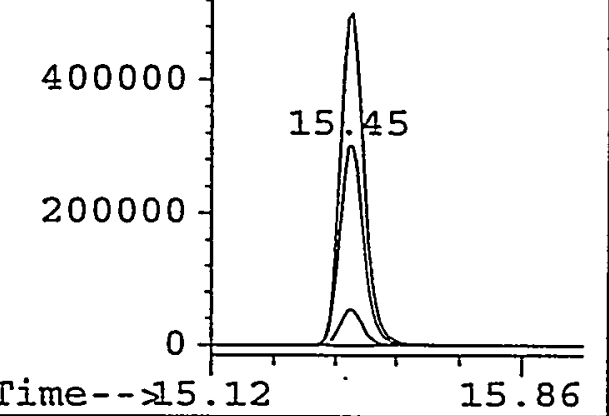

Time- $>15.12$

15.86

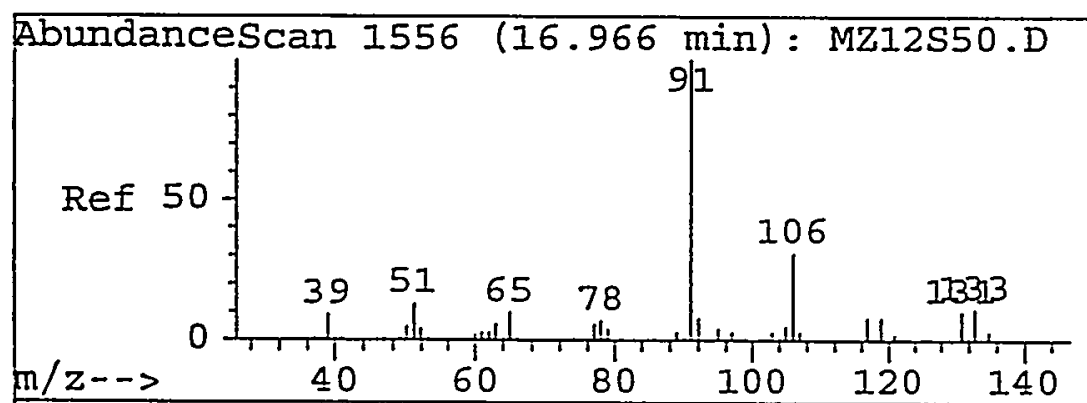

Abundancescan 1652 (17.966 min) : LF09R897.D

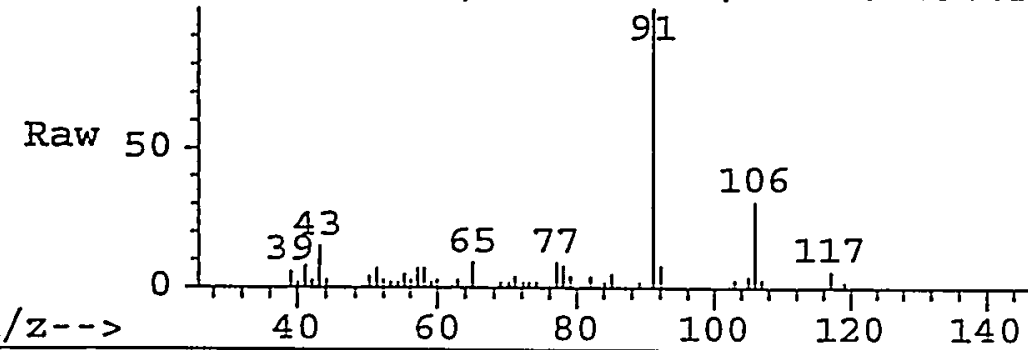

Abundancescan 1652 (17.966 min): LF09R897.D

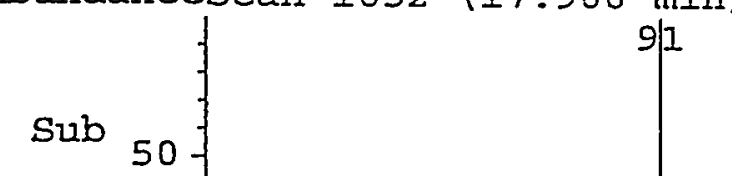

(2)

107

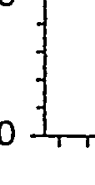

\section{6}

$\begin{array}{llll}39 & 51 & 65 & 77\end{array}$

$40 \quad 60$

$60^{1}$
117

$\mathrm{m} / \mathrm{z}--\mathrm{s}$

1
80
\#50

C038 ethylbenzene

Concen: $2.66 \mathrm{ug} / \mathrm{L}$

RT: $17.97 \mathrm{~min}$ Scan\# 1652

Delta R.T. $\quad-0.00 \mathrm{~min}$

Lab File: LF09R897.D

Acq: 07/07/97 $18: 34$

Tgt Ion:106 Resp: 38116

Ion Ratio Lower Upper

106100

$\begin{array}{llll}91 & 337.7 & 266.1 & 399.1\end{array}$

$\begin{array}{llll}0 & 0.0 & 0.0 & 0.0\end{array}$

$\begin{array}{llll}0 & 0.0 & 0.0 & 0.0\end{array}$

AbundanceIon 106.00 (105

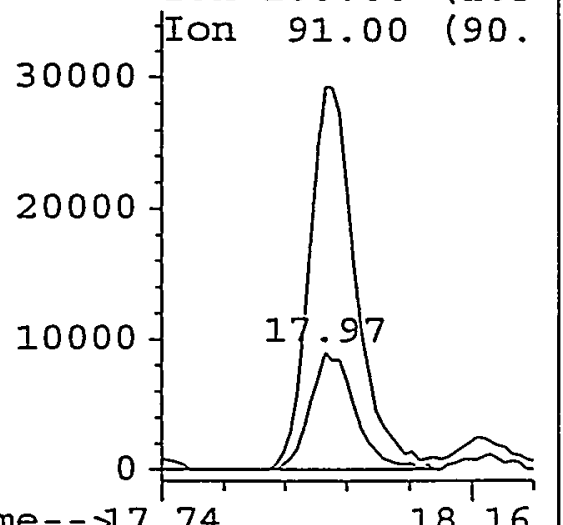




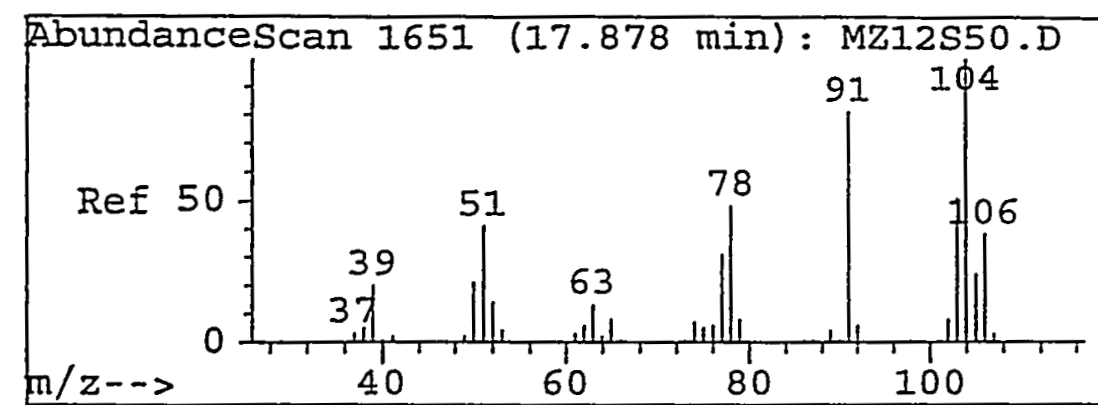

AbundanceScan 1746 (18.873 min): IF09R897.D

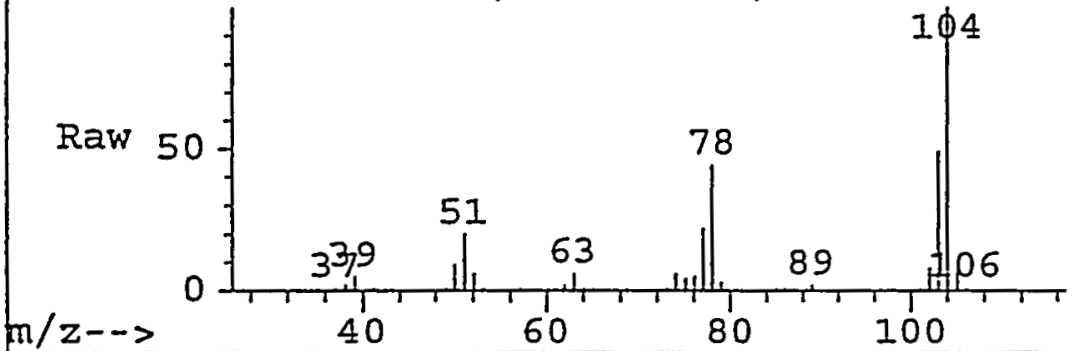

Abundancescan 1746 (18.873 $\mathrm{min}):$ LF09R897.D
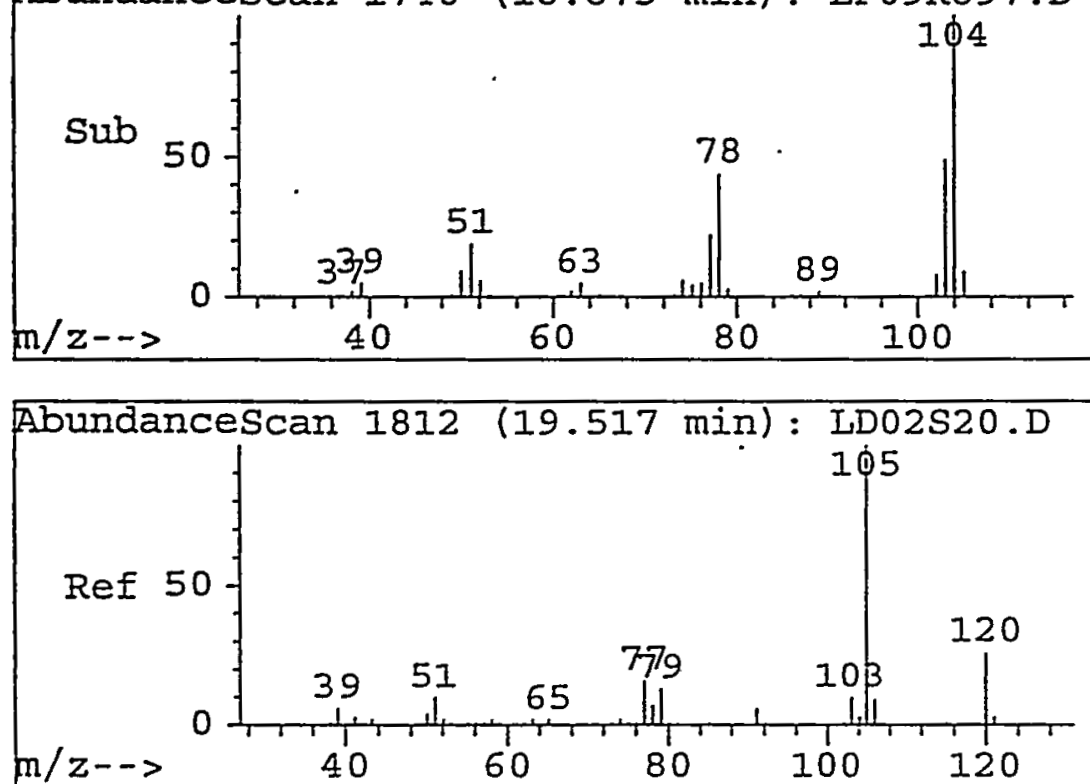

Abundancescan 1811 (19.501 min): LF09R897.D

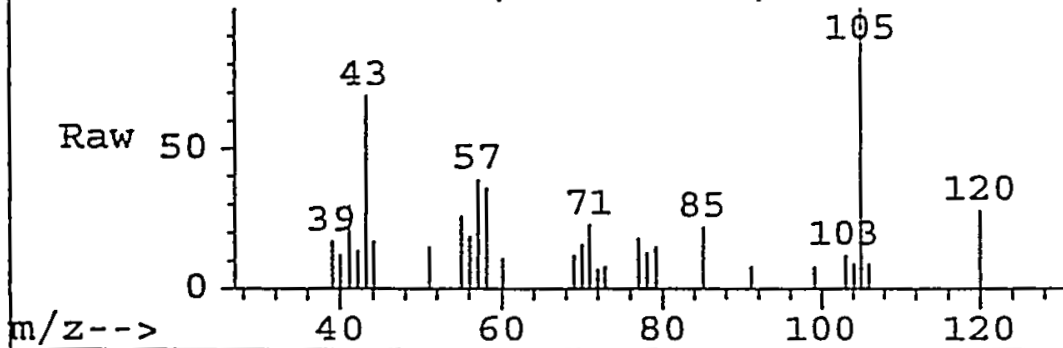

Abundancescan 1811 (19.501 min): LF09R897.D

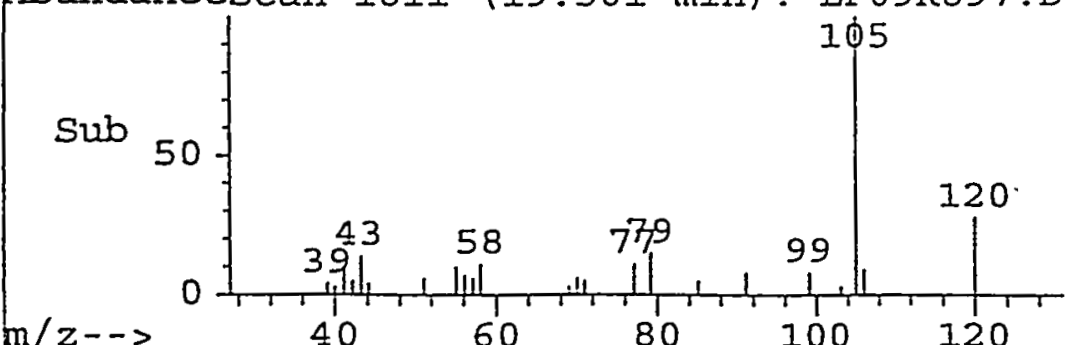

\#54

C041 styrene

Concen: $52.03 \mathrm{ug} / \mathrm{L}$

RT: $18.87 \mathrm{~min}$ Scan\# 1746

Delta R.T. $\quad-0.06 \mathrm{~min}$

Lab File: LF09R897.D

Acg: 07/07/97 18:34

Tgt Ion:104 Resp: 1592812

Ion Ratio Lower Upper

104100

$\begin{array}{llll}103 & 47.8 & 42.1 & 63.1\end{array}$

$\begin{array}{llll}78 & 44.2 & 38.4 & 57.6\end{array}$

$5120.0<35.453 .0 \#$

Abundanceion 104.00 (103

600000 -Ion $103.00 \quad(102$

Ion $78.00 \quad 77$.

Ion $51.00 \quad(50$.

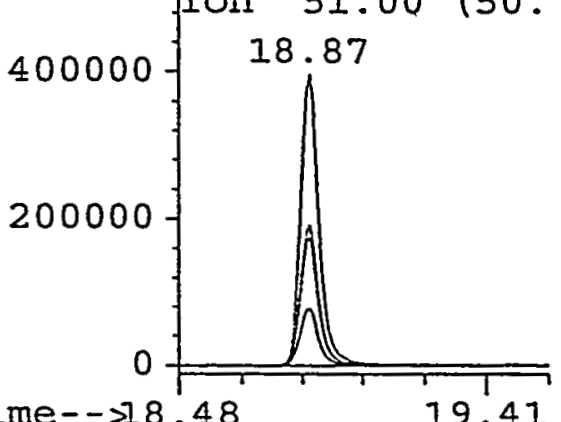

Time- $>18.48$

19.41

\#55

C042 isopropylbenzene

Concen: $0.48 \mathrm{ug} / \mathrm{L}$

$\mathrm{RT}: 19.50 \mathrm{~min}$ Scan\# 1811

Delta R.T. $\quad-0.02 \mathrm{~min}$

Lab File: LF09R897.D

Acq: 07/07/97 18:34

Tgt Ion:105 Resp: 24922

Ion Ratio Lower Upper

105100

$\begin{array}{llll}120 & 25.3 & 20.0 & 30.0\end{array}$
0
0.0
0.0
0.0

$\begin{array}{ccc}0.0 & 0.0 & 0.0 \\ \text { Abundanceion } 105.00 & (104\end{array}$

6000 fIon 120.00 (119
19.50

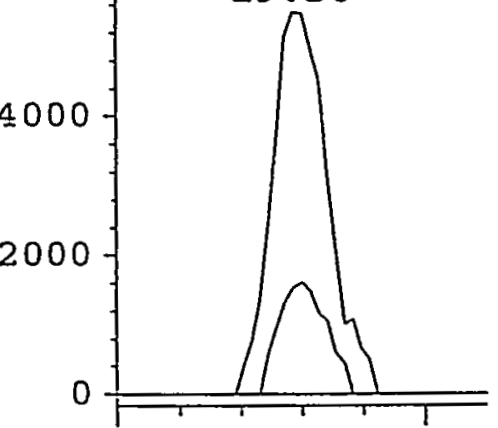

Time- $>19.30$ 


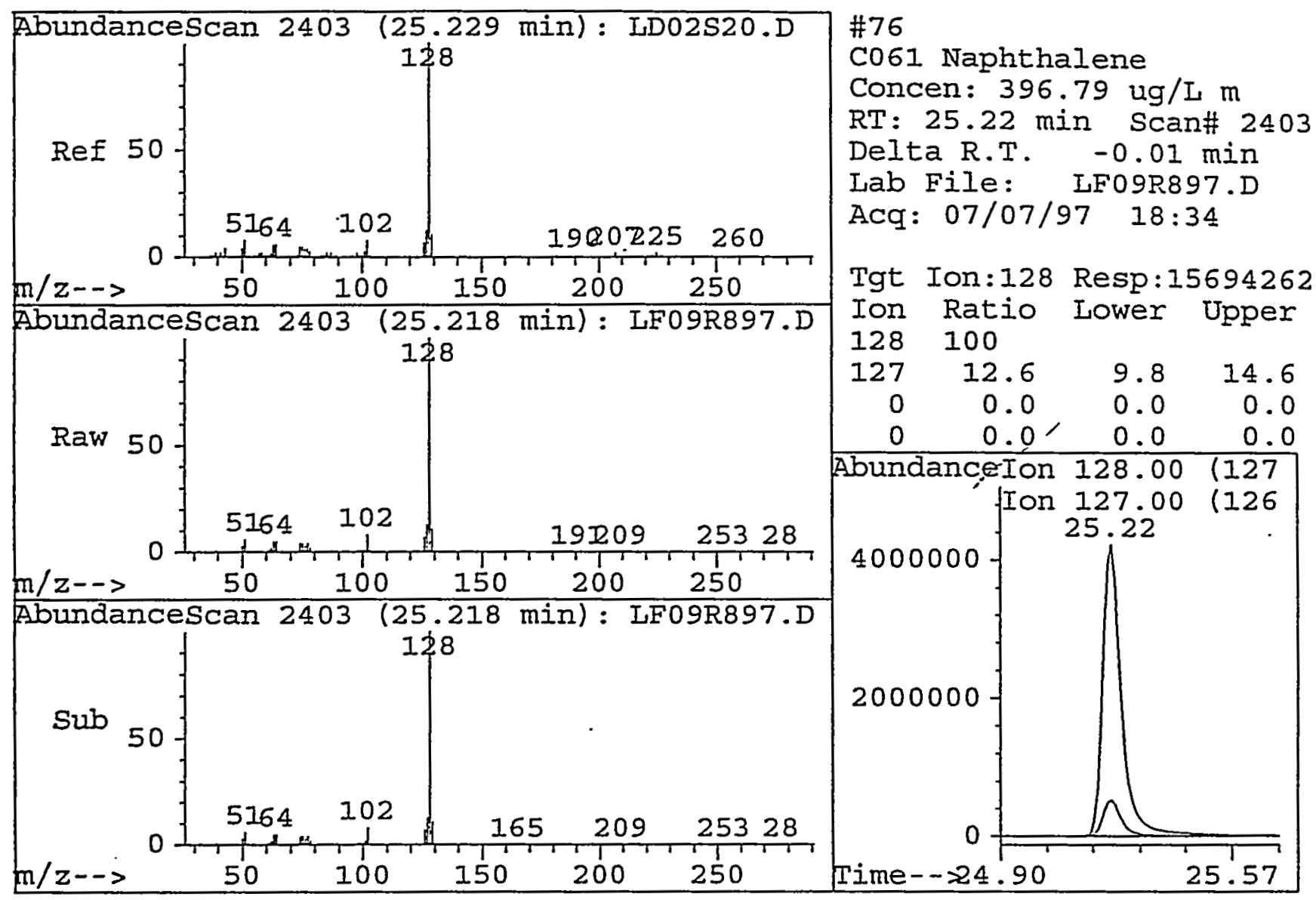


Quantitation Report

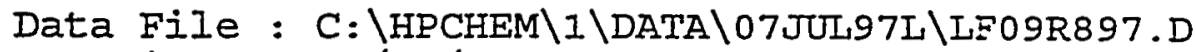

Acq Time : 07/07/97 18:34

Sample : 97C02897 NAP-R2-S2-B

Misc : 97C-0208-01

Quant Time: Jul 20 13:14 1997

Operator: $\mathrm{CQC}$

Inst : 5971-L Multiplr: 1.00

Method : J:\I\METHODS \I826ARWA.M

Title : VOA COMPOUND IIST

Last Update : Wed Jun 25 09:14:05 1997

Response via : Multiple Level Calibration

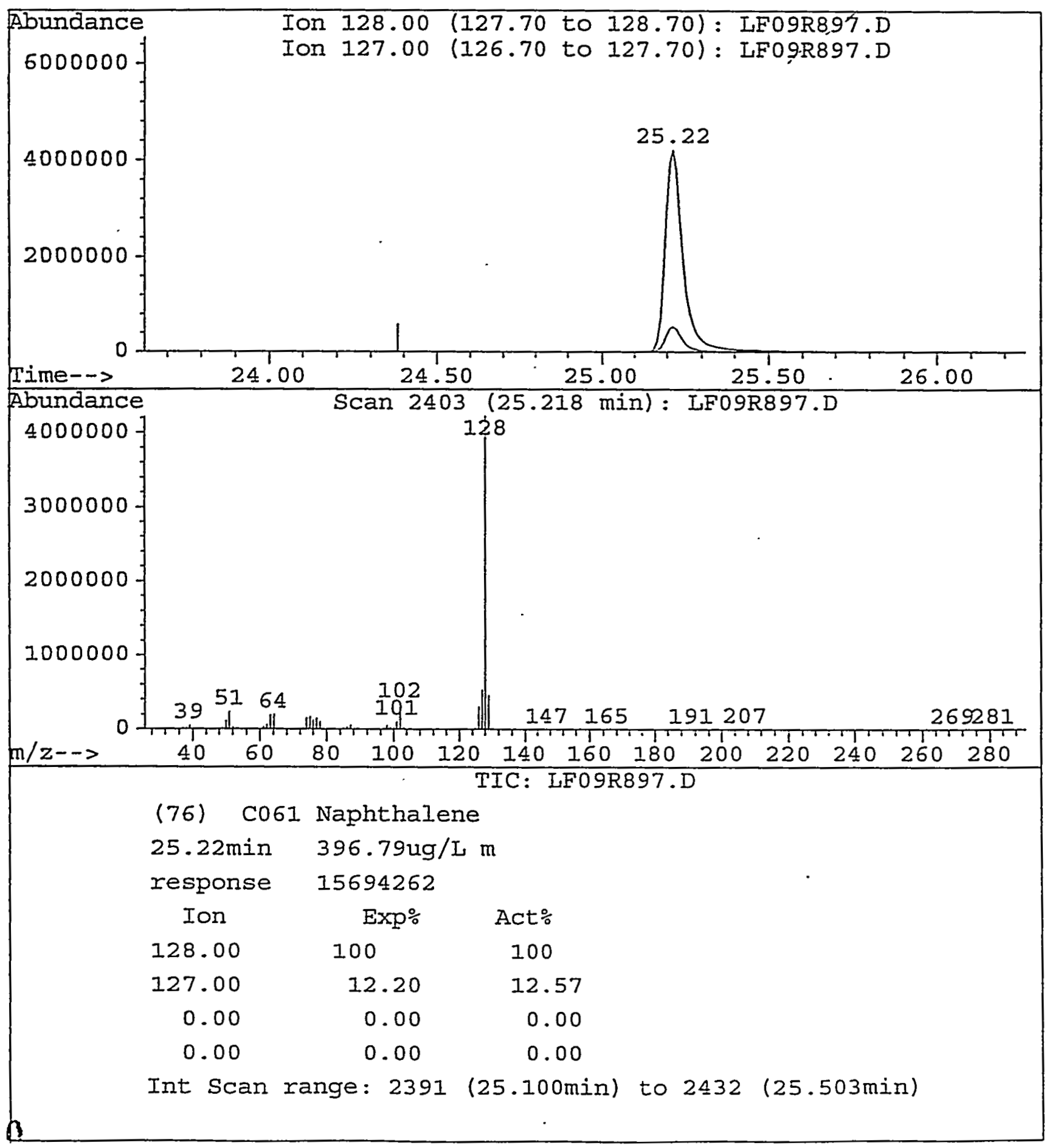




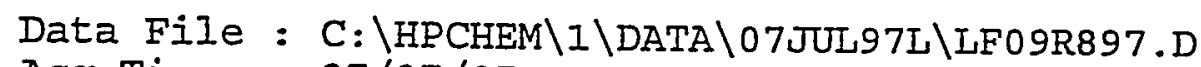

Acq Time : 07/07/97 18:34

Sample : 97C02897 NAP-R2-S2-B

Misc : 97C-0208-01

Operator: CQC

Inst : 5971-L

Multiplr: 1.00

Method : J: \I\METHODS \L826ARWA.M

Title : VOA COMPOUND IIST

Iibrary : C: \DATABASE\NBS75K.I

R.T. Conc Area Relative to ISTD ISTD Area

$22.75 \quad 42.97 \mathrm{ug} / \mathrm{L} \quad 4290493$ CI03 1,4-Dichlorobenzene-d4 4992661

Hit\# of 9

Tentative ID

Ref\# CAS\#

Qual

Indene-

2-Benzene, 1-ethynyl-4-methyl-

3 Benzene, 1-propynyl-

4 Benzene, 1,2-propadienyl-

5 IH-Indene, 1-chloro-2,3-dihydro-

$64387000095-13-6 \quad 95.00$

$3388000766-97-2 \quad 91.00$

$64385000673-32-5 \quad 91.00$

$3387002327-99-3 \quad 91.00$

$10202 \quad 035275-62-8 \quad 43.00$

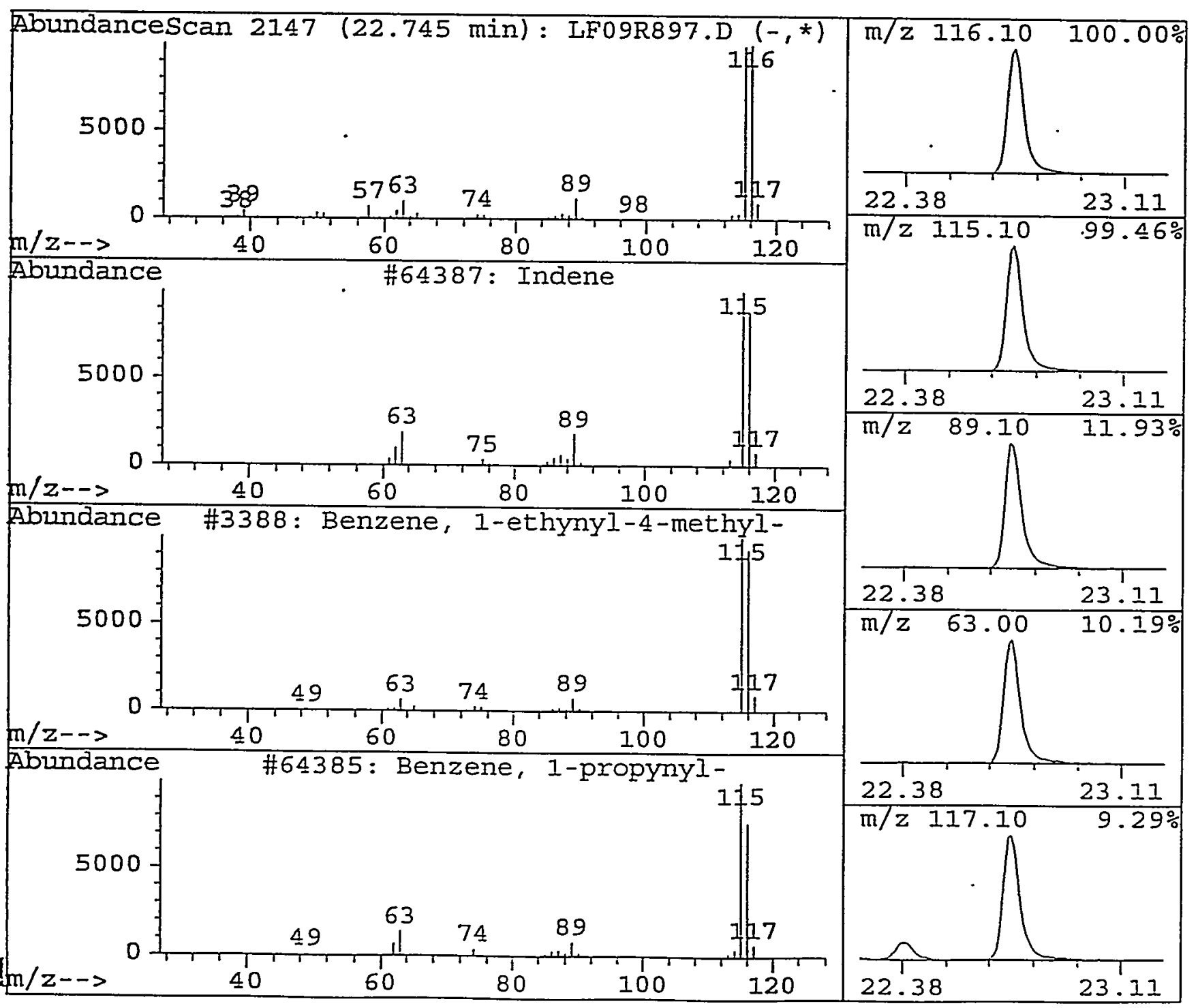




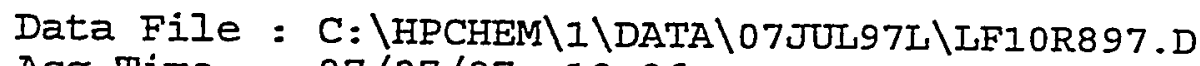

Acq Time : 07/07/97 19:06

Sample : 97C02897 NAP-R2-S2-B

Misc : 97C-0208-01 1:10 Dilution

Quant Time: Jul $20 \quad 13: 28 \quad 1997$

Operator: CQC

Inst : 5971-L Multiplr: 1.00

Method : J: \I\METHODS \I826ARWA.M

Title : VOA COMPOUND LIST.

Last Update : Wed Jun 25 09:14:05 1997

Response via : Multiple Level Calibration
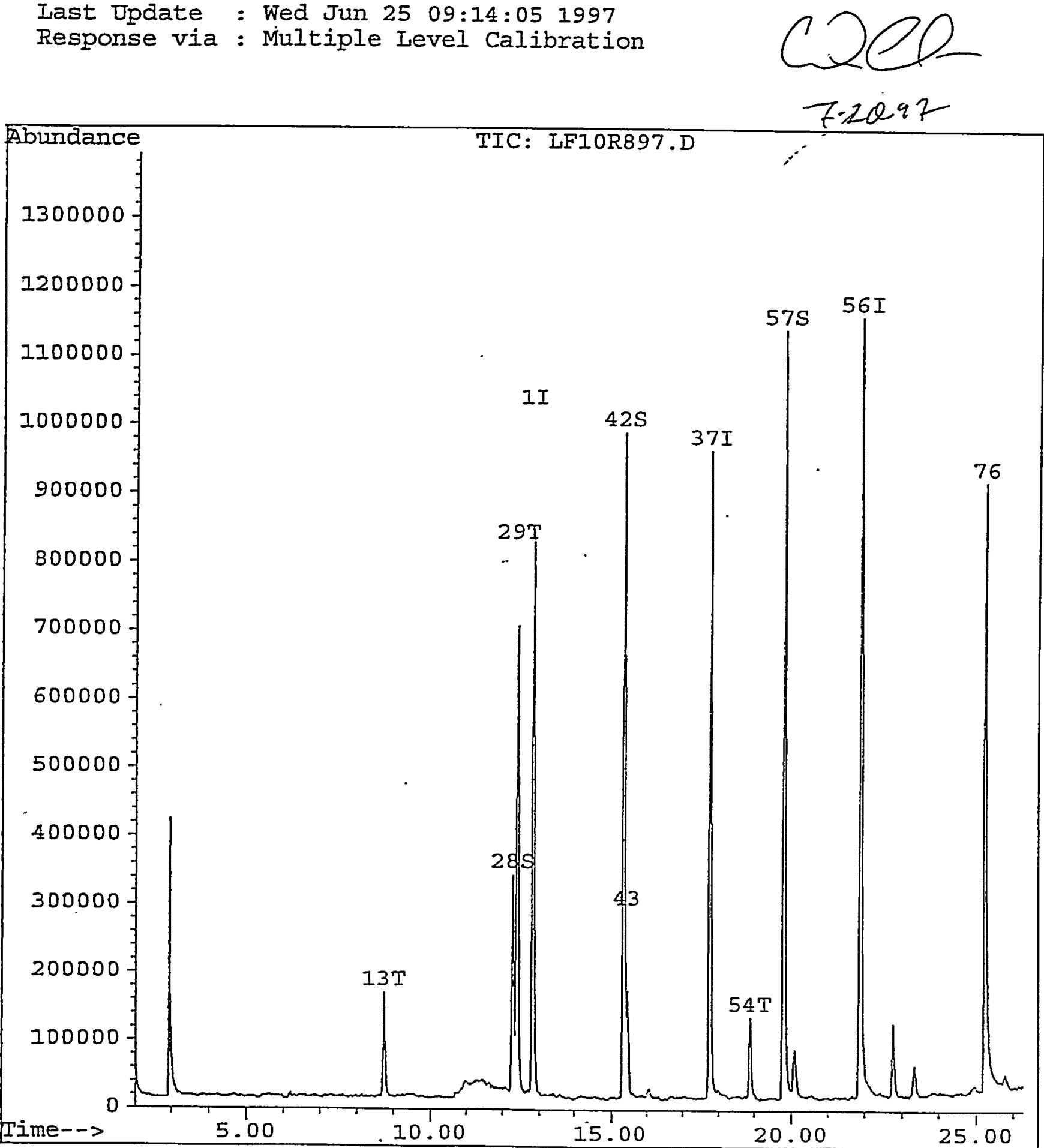

112 
Quantitation Report

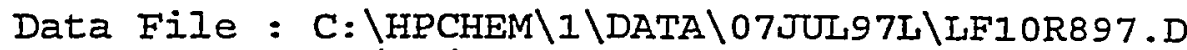

Acq Time : 07/07/97 19:06

Sample : 97C02897 NAP-R2-S2-B

Misc : 97C-0208-01 1:10 Dilution

Quant Time: JuI 20 13:28 1997

Operator: CQC

Inst : 5971-I

Multiplr: 1.00

Method : J: \I\METHODS \I826ARWA.M

Title : VOA COMPOUND IIST

Last Update : Wed Jun 25 09:14:05 1997

Response via : Multiple Level Calibration

Internal standards

1) CIO1 Flourobenzene

37) CI02 Chlorobenzene- $d 5$

56) CI03 1,4-Dichlorobenzene-d

System Monitoring Compounds

28) CS15 1,2-dichloroethane-d4

42) CS05 toluene-d8

57) CSIO 4-bromofluorobenzene

Target Compounds

2) $\mathrm{C002}$ dichlorodifluorometha

3) $\mathrm{C003}$ chloromethane

4) $\mathrm{COO} 4$ vinyl chloride

5) C005 bromomethane

6) $\mathrm{COO5}$ chloroethane

7) $\mathrm{C007}$ trichlorofluoromethan

B) $\mathrm{C} 071$ acrolein

9) $\mathrm{C008} 1,1$-dichloroethene

10) $\mathrm{C} 063$ freon 113

11) $\operatorname{co09}$ acetone

12) C010 carbon disulfide

13) Co11 methylene chloride

14) $\mathrm{C} 072$ acrylonitrile

15) $\mathrm{C} 012$ trans-1,2-Dichloroeth

16) C305 methyl-t-butyl-ether

17) $\mathrm{C} 013 \mathrm{cis}-1,2-$ dichloroethen

18) C055. total-1,2-dichloroeth

19) $\mathrm{C} 0141,1$-dichloroethane

20) C015 2,2-dichloropropane

21) CIIO 2-butanone

22) CIO1 bromochloromethane

23) $\mathrm{C} 016$ chloroform

24) $\mathrm{CD17} 1,1,1$-Trichloroethane

25) $\operatorname{COI8~1,1-dichloropropene~}$

26) $\operatorname{col} 9$ 1,2-dichloroethane

27) $\mathrm{C020}$ carbon tetrachloride

29) $\mathrm{C} 21$ benzene

30) Co22 trichloroethene

31) C023 1,2-dichloropropane

32) $\mathrm{C024}$ dibromomethane

33) C025 bromodichloromethane

34) 0026 2-chloroethylvinyleth

35) $\mathrm{C} 27 \mathrm{cis}-1,3$-dichloroprope
R.T. QIon
12.82

17.74

21.88
96

117

152
1765095

943294

12.27

15.34

19.78
65

98

95

$\leqslant 43022$

1563102

1077491
1340261
$38.83 \mathrm{ug} / \mathrm{I}$
$45.34 \mathrm{ug} / \mathrm{I}$
$42.74 \mathrm{ug} / \mathrm{I}$
$77.66 \%$
$90.68 \%$
$85.49 \%$

$\div$ Recovery

$50.00 \mathrm{ug} / \mathrm{L} \quad 0.00$

$50.00 \mathrm{ug} / \mathrm{I} \quad 0.00$

$50.00 \mathrm{ug} / \mathrm{I}$

0.01

Qvalue

Not Detected

Not Detected

Not Detected

Not Detected

Not Detected

Not Detected

Not Detected

Not Detected

Not Detected

Not Detected

Not Detected

0.0076

8.7484

$0.00 \quad 53$

166406

$0.00 \quad 96$

0.0073

0.0096

0.0096

$0.00,63$

$0.00 \quad 77$

0.0072

$0.00 \quad 128$

0.0083

$0.00 \quad 97$

0.0075

$0.00 \quad 62$

$0.00 \quad 117$

12.3978

$0.00 \quad 130$

1380894 $15.81 \mathrm{ug} / \mathrm{L} \#$ Not Detected

Not Detected

Not Detected

Not Detected

Not Detected

Not Detected

Not Detected

Not Detected

Not Detected

Not Detected

Not Detected

Not Detected

Not Detected

Not Detected

$39.03 \mathrm{ug} / \mathrm{L}$

Not Detected

Not Detected

Not Detected

Not Detected

Not Detected

Not Detected

$(\mathrm{NR})=$ Not Reported $(\mathrm{SP})=$ Spiked analyte $(\mathrm{m})=$ manual integration

(*) Does not meet EPA spectral criteria (Ealse jit) PX125 Page 1 
Quantitation Report

Data File : C: \HPCHEM \I\DATA 07 JUL97L\LF10R897.D

$\begin{array}{lll}\text { Acq Time } & : 07 / 07 / 97 \quad 19: 06 \\ \text { Sample } & : 97 C 02897 \quad \text { NAP-R2-S2-B } \\ \text { Misc } & : 97 C-0208-01 \quad 1: 10 \text { Dilution }\end{array}$

Operator: $\mathrm{CQC}$

Inst : 5971-L

Quant Time: JuI $2013: 281997$

Multiplr: 1.00

Method : J: \I\METHODS \L826ARWA.M

Title : VOA COMPOUND IIST

Last Update : Wed Jun 25 09:14:05 1997

Response via : Multiple Level Calibration

Compound

\begin{tabular}{|c|c|c|}
\hline 36) & C205 & 4-methyl-2-pentanone \\
\hline 3B) & $\mathrm{C} 028$ & trans-i, 3-dichloropro \\
\hline 39) & C029 & 1,1,2-trichloroethane \\
\hline 40) & C210 & 2-hexanone \\
\hline 41) & $\mathrm{CO} 30$ & 1,2-Dibromoethane \\
\hline 43) & $\operatorname{co3} 1$ & Toluene \\
\hline 44) & $\mathrm{CO} 32$ & 1,3-dichloropropane \\
\hline 45) & $\mathrm{C033}$ & dibromochloromethane \\
\hline 46) & $\mathrm{C034}$ & bromoform \\
\hline 47) & $\mathrm{C035}$ & tetrachloroethene \\
\hline 48) & $\mathrm{C036}$ & chlorobenzene \\
\hline 49) & $\mathrm{CO} 37$ & $1,1,1,2$-tetrachloroet \\
\hline 50) & $\mathrm{CO} 38$ & ethylbenzene \\
\hline 51) & $\operatorname{co3} 9$ & $m, p-x y l e n e$ \\
\hline 52) & $\mathrm{CO} 40$ & o-xylene \\
\hline 53) & $\mathrm{C} 250$ & total xylene \\
\hline 54) & $\mathrm{CO41}$ & styrene \\
\hline 55) & $\mathrm{CO} 42$ & isopropylbenzene \\
\hline 58) & $\mathrm{CO} 43$ & $1,1,2,2$-tetrachloroet \\
\hline 59) & $\mathrm{CO} 44$ & bromobenzene \\
\hline 60) & $C 0 \leqslant 5$ & 1,2,3-trichloropropan \\
\hline 61) & $\mathrm{C} 046$ & n-propylbenzene \\
\hline 62) & $C 047$ & $1, \overline{3}, 5$-trimethylbenzen \\
\hline 63) & $\mathrm{CO} 48$ & 2-chlorotoluene \\
\hline 64) & $\mathrm{CO} 49$ & 4-chlorotoluene \\
\hline 65) & C050 & tert-butylbenzene \\
\hline 66) & C051 & I, 2,4-trimethylbenzen \\
\hline 67) & $\mathrm{CO52}$ & sec-Butylbenzene \\
\hline 68) & $\cos 3$ & p-Isopropyltoluene \\
\hline 69) & C054 & 1,3-Dichlorobenzene \\
\hline 70) & C055 & 1,4-Dichlorobenzene \\
\hline 71) & C056 & n-Butylbenzene \\
\hline 72) & C057 & 1,2-Dichlorobenzene \\
\hline 73 & $\mathrm{CO58}$ & 1,2-Dibromo-3-chlorop \\
\hline 74) & $\cos 5$ & $1,2,4$-Trichlorobenzen \\
\hline 75) & $\mathrm{CO} 60$ & Hexachlorobutadiene \\
\hline 76) & CD61 & Naphthalene \\
\hline & $\mathrm{CD} 62$ & $1,2,3$-Trichlorobenzen \\
\hline
\end{tabular}

R.T. QIon Response Conc Unit Qvalue

\begin{tabular}{|c|c|c|c|c|c|}
\hline 0.00 & 43 & & Not & Detected & \\
\hline 0.00 & 75 & & Not & Detected & \\
\hline 0.00 & 97 & & Not & Detected & \\
\hline 0.00 & 43 & & Not & Detected & \\
\hline 0.00 & 107 & & Not & Detected & \\
\hline 15.45 & 92 & 148408 & 6.0 & 7 ug/L & 98 1/2 \\
\hline 0.00 & 76 & & Not & Detected & \\
\hline 0.00 & 129 & & Not & Detected & \\
\hline 0.00 & 173 & & Not & Detected & \\
\hline 0.00 & 164 & & Not & Detected & \\
\hline 0.00 & 112 & & Not & Detected & \\
\hline 0.00 & 131 & & Not & Detected & \\
\hline 0.00 & 106 & & Not & Detected & \\
\hline 0.00 & 106 & & Not & Detected & \\
\hline 0.00 & 106 & & Not & Detected & \\
\hline 0.00 & 106 & & Not & Detected & \\
\hline 18.88 & 104 & 169611 & 5.5 & $58 \mathrm{ug} / \mathrm{L} \#$ & 83 is \\
\hline 0.00 & 105 & & Not & Detected & \\
\hline 0.00 & 83 & & Not & Detected & \\
\hline 0.00 & 156 & & Not & Detected & \\
\hline 0.00 & 75 & & Not & Detected & \\
\hline 0.00 & 91 & & Not & Detected & \\
\hline 0.00 & 105 & & Not & Detected & \\
\hline 0.00 & 91 & & Not & Detected & \\
\hline 0.00 & 91 & & Not & Detected & \\
\hline 0.00 & 119 & & Not & Detected & \\
\hline 0.00 & 105 & & Not & Detected & \\
\hline 0.00 & 105 & & Not & Detected & \\
\hline 0.00 & 119 & & Not & Detected & \\
\hline 0.00 & 146 & & Not & Detected & \\
\hline 0.00 & 146 & & Not & Detected & \\
\hline 0.00 & 91 & & Not & Detected & \\
\hline 0.00 & 146 & & Not & Detected & \\
\hline 0.00 & 75 & & Not & Detected & \\
\hline 0.00 & 180 & & Not & Detected & \\
\hline 0.00 & 225 & & Not & Detected & \\
\hline 25.23 & 128 & 2134238 & 54.7 & $22 \mathrm{ug} / \mathrm{L}$ & 99 \\
\hline 0.00 & 180 & & Not & Detected & \\
\hline
\end{tabular}

114 Data Processed By: 7.28-96 $(\mathrm{NR})=$ Not Reported $(\mathrm{SP})=$ Spiked analyte $(\mathrm{m})=$ manual integration

(*) Does not meet EPA spectral criteria (False Hit) PX125 Page 2 


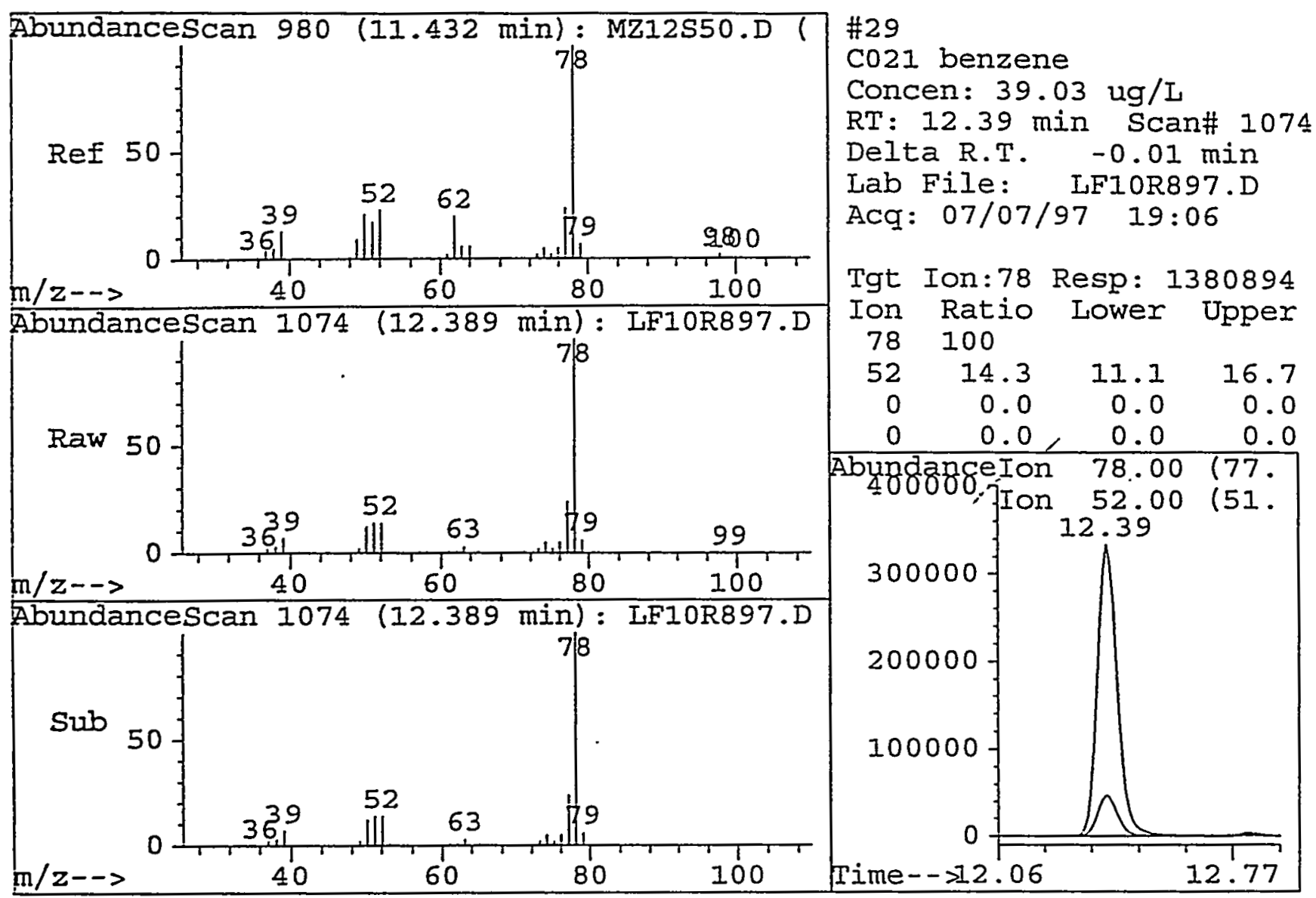




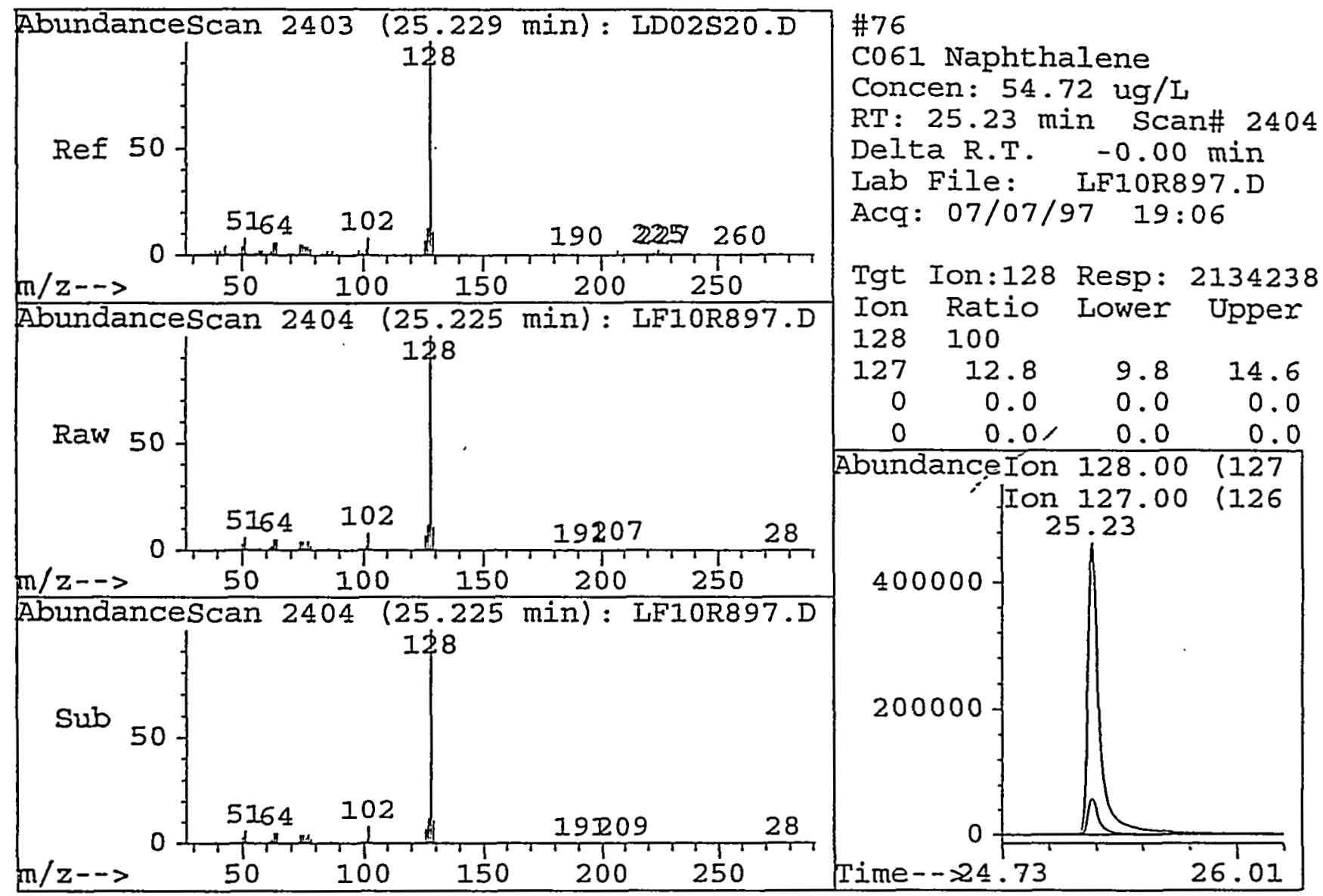

\section{6}


Quantitation Report

Data File : C: \HPCHEM \I\DATA $\backslash 07 J U L 97 L \backslash L F 03 C 898 . D$

$\begin{array}{llll}\text { Acq Time } & \text { : } & 07 / 07 / 97 & 15: 24 \\ \text { Sample } & : 97 C 02898 & \text { NAP-R2-S4 } \\ \text { Misc } & : 97 C-0208-01 & 8260 \text { WATER } \\ \text { Quant Time: Jul 7 15:52 } & 1997\end{array}$

Operator: $\mathrm{CQC}$

Inst : 5971-L

Multiplr: 1.00

Method : C: \HPCHEM \I\METHODS \L826ARWA.M

Title : VOA COMPOUND IIST

Last Update : Mon Jul 07 15:11:47 1997

Response via : Multiple Level Calibration
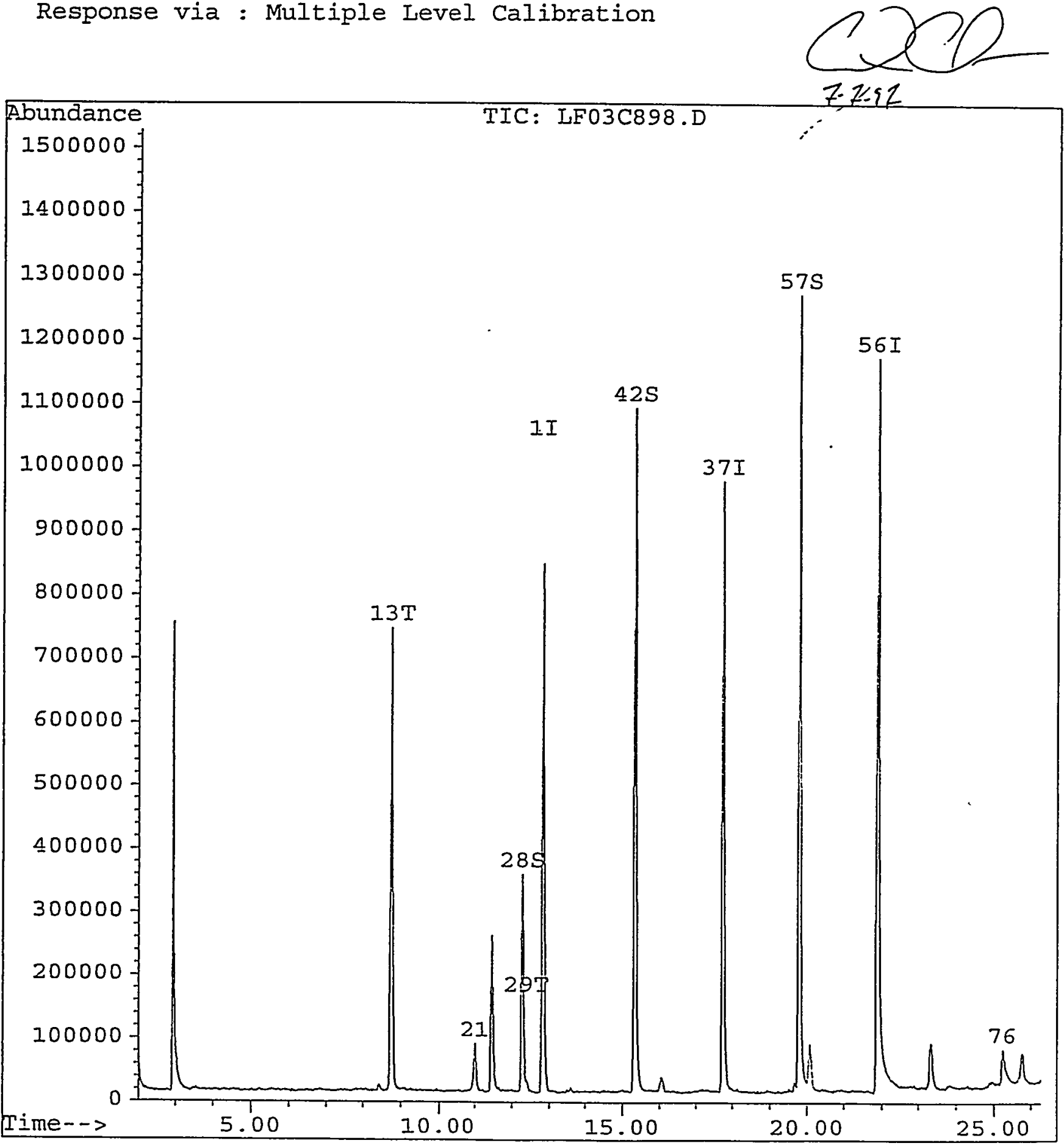


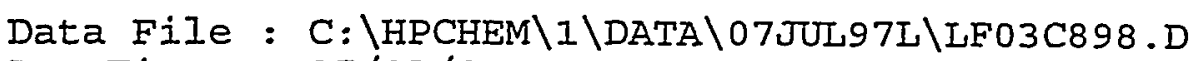

Acq Time : 07/07/97 15:24

Sample : 97C02898 NAP-R2-S4

Misc : 97C-0208-01 8260 WATER

Quant Time: Jul 7 15:52 1997

Operator: CQC

Inst : 5971-L

Multiplr: 1.00

Method : C: \HPCHEM \I\METHODS \L826ARWA.M

Title : VOA COMPOUND LIST

Last Update : Mon Jul 07 15:11:47 1997

Response via : Multiple Level Calibration

Internal standards

1) CI01 Flourobenzene
57) CI02 Chlorobenzene-d5

System Monitoring Compounds

28) CS15 1,2-dichloroethane-d4

42) CS05 toluene-d8

57) CS10 4-bromofluorobenzene

Target Compounds

2) $\mathrm{C} 002$ dichlorodifluorometha

3) $\mathrm{C003}$ chloromethane

4) 0004 vinyl chloride

5) C005 bromomethane

6) $\mathrm{C006}$ chloroethane

7) $\mathrm{C007}$ trichlorofluoromethan

8) 0071 acrolein

9) 0008 1,1-dichloroethene

10) $\mathrm{C} 063$ freon 113

11) 0009 acetone

12) 0010 carbon disulfide

13) C011 methylene chloride

14) $\mathrm{C} 072$ acrylonitrile

15) C012 trans-1,2-Dichloroeth

16) C305 methyl-t-butyl-ether

17) $\mathrm{C} 013 \mathrm{cis-1}, 2$-dichloroethen

18), C055 total-1,2-dichloroeth

19) C014 1,1-dichloroethane

20) C015 2,2-dichloropropane

21) CI10 2-butanone

22) CIOI bromochloromethane

23) $\mathrm{C} 016$ chloroform

24) $\operatorname{Col7~1,1,1-Trichloroethane~}$

25) $\mathrm{C} 018$ 1,1-dichloropropene

26) C019 1,2-dichloroethane

27) C020 carbon tetrachloride

29) C021 benzene

30) C022 trichloroethene

31) C023 1,2-dichloropropane

32) C024 dibromomethane

33) C025 bromodichloromethane

34) C026 2-chloroethylvinyleth

35) C027 cis-1,3-dichloroprope
R.T. QIon

12.83

17.75

21.88

96
117
152

152

12.28

15.35

19.78

65

98

95

496388

1721813

1202817

$0.00 \quad 85$

$0.00 \quad 50$

$0.00 \quad 62$

$0.00 \quad 94$

$0.00 \quad 64$

0.00101

$0.00 \quad 56$

$0.00 \quad 96$

$0.00 \quad 151$

$0.00 \quad 43$

$0.00 \quad 76$

$8.75 \quad 84$

$0.00 \quad 53$

$0.00 \quad 96$

0.0073

$0.00 \quad 96$

$0.00 \quad 96$

$0.00 \quad 63$

$0.00 \quad 77$

$11.01 \quad 72$

$0.00 \quad 128$

$0.00 \quad 83$

$0.00 \quad 97$

$0.00 \quad 75$

$0.00 \quad 62$

$0.00 \quad 117$

$12.40 \quad 78$

$0.00 \quad 130$

$0.00 \quad 63$

$0.00 \quad 93$

$0.00 \quad 83$

$0.00 \quad 63$

$0.00 \quad 75$

1023423

26323
Response Conc Units Area\%

1779659

$50.00 \mathrm{ug} / \mathrm{L}$

0.00

1366930

$50.00 \mathrm{ug} / \mathrm{L}$

0.00

$50.00 \mathrm{ug} / \mathrm{L}$

0.01

$\div$ Recovery

$43.15 \mathrm{ug} / \mathrm{L} \quad 86.30 \%$

$48.97 \mathrm{ug} / \mathrm{I} \quad 97.94 \%$

$43.98 \mathrm{ug} / \mathrm{Is}$

$87.96 \%$

Qvalue

Not Detected

Not Detected

Not Detected

Not Detected

Not Detected

Not Detected

Not Detected

Not Detected

Not Detected

Not Detected

Not Detected

$79626675.01 \mathrm{ug} / \mathrm{I}$ \#

Not Detected

Not Detected

Not Detected

Not Detected

Not Detected

Not Detected

Not Detected

$6279354.96 \mathrm{ug} / \mathrm{L} \#$

Not Detected

Not Detected

Not Detected

Not Detected

Not Detected

Not Detected

$0.74 \mathrm{ug} / \mathrm{L}$

Not Detected

Not Detected

Not Detected

Not Detected

Not Detected

Not Detected 


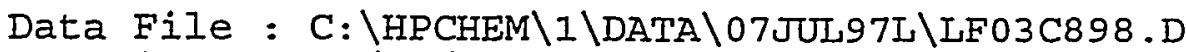

Acq Time : 07/07/97 15:24

Sample : 97C02898 NAP-R2-S4

MisC : 97C-0208-01 8260 WATER

Quant Time: Jul 7 15:52 1997

Operator: $\mathrm{CQC}$

Inst : 5971-L

Multiplr: 1.00

Method $\cdot \quad$ : C: \HPCHEM \I\METHODS\I826ARWA.M

Title : VOA COMPOUND LIST

Last Update : Mon Jul 07 15:11:47 1997

Response via : Multiple Level Calibration

Compound

R.T. QIon Response Conc Unit Qvalue

36) C205 4-methyl-2-pentanone

38) C028 trans-1,3-dichloropro

39) Co29 1,1,2-trichloroethane

40) C210 2-hexanone

41) C030 1,2-Dibromoethane

43) $\mathrm{C} 31$ Toluene

44) C032 1,3-dichloropropane

45) $\mathrm{C0} 33$ dibromochloromethane

46) $\mathrm{C034}$ bromoform

47) C035 tetrachloroethene

48) $\mathrm{C} 036$ chlorobenzene

49) $\mathrm{C} 037$ 1, 1, 1,2-tetrachloroet

50) $\operatorname{Co38}$ ethylbenzene

51) C039 m,p-xylene

52) co40 o-xylene

53) C250 total xylene

54) 0041 styrene

55) $\mathrm{C} 042$ isopropylbenzene

58) $\mathrm{C} 043$ I, 1,2,2-tetrachloroet

59) C044 bromobenzene

60) C045 1,2,3-trichloropropan

61) $\mathrm{C} 046 \mathrm{n}$-propylbenzene

62) $\mathrm{C047} 1,3,5$-trimethylbenzen

63) C048 2-chlorotoluene

64) Co49 4-chlorotoluene

65) C050 text-butylbenzene

66) C05I I,2,4-trimethylbenzen

67) C052 sec-Butylbenzene

68) C053 p-Isopropyltoluene

69) $\mathrm{C} 054$ I,3-Dichlorobenzene

70) C055 1,4-Dichlorobenzene

71) 0056 n-Butylbenzene

72) 0057 1,2-Dichlorobenzene

73) $\mathrm{C} 058$ 1,2-Dibromo-3-chlorop

74) C059 1,2,4-Trichlorobenzen

75) C060 Hexachlorobutadiene

76) C061 Naphthalene

77) C062 1,2,3-Trichlorobenzen
$0.00 \quad 43$

$0.00 \quad 75$

$0.00 \quad 97$

$0.00 \quad 43$

$0.00 \quad 107$

$0.00 \quad 92$

$0.00 \quad 76$

$0.00 \quad 129$

$0.00 \quad 173$

$0.00 \quad 164$

$0.00 \quad 112$

$0.00 \quad 131$

$0.00 \quad 106$

$0.00 \quad 106$

$0.00 \quad 106$

$0.00 \quad 106$

$0.00 \quad 104$

$0.00 \quad 105$

$0.00 \quad 83$

$0.00 \quad 156$

$0.00 \quad 75$

0.0091

$0.00 \quad 105$

$0.00 \quad 91$

$0.00 \quad 91$

$0.00 \quad 119$

$0.00 \quad 105$

$0.00 \quad 105$

$0.00 \quad 119$

$0.00 \quad 146$

$0.00 \quad 146$

$0.00 \quad 91$

$0.00 \quad 146$

$0.00 \quad 75$

$0.00 \quad 180$

$0.00 \quad 225$

$25.25 \quad 128$

$0.00 \quad 180$
Not Detected

Not Detected

Not Detected

Not Detected

Not Detected

Not Detected

Not Detected

Not Detected

Not Detected

Not Detected

Not Detected

Not Detected

Not Detected

Not Detected

Not Detected

Not Detected

Not Detected

Not Detected

Not Detected

Not Detected

Not Detected

Not Detected

Not Detected

Not Detected

Not Detected

Not Detected

Not Detected

Not Detected

Not Detected

Not Detected

Not Detected

Not Detected

Not Detected

Not Detected

Not Detected

Not Detected

$5.52 \mathrm{ug} / \mathrm{L}$

Not Detected 


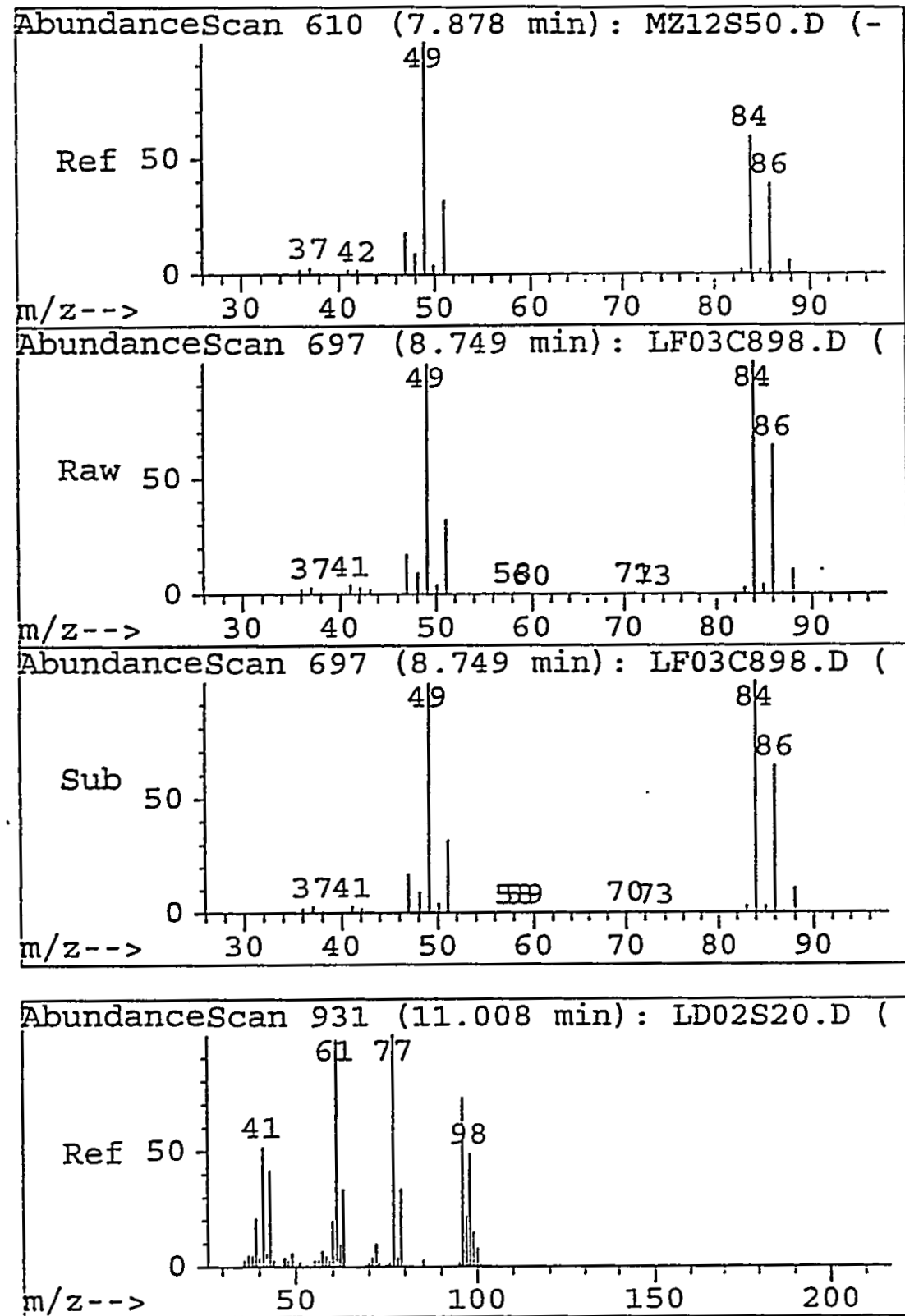

Abundancescan 931 (11.006 min): IF03C898.D

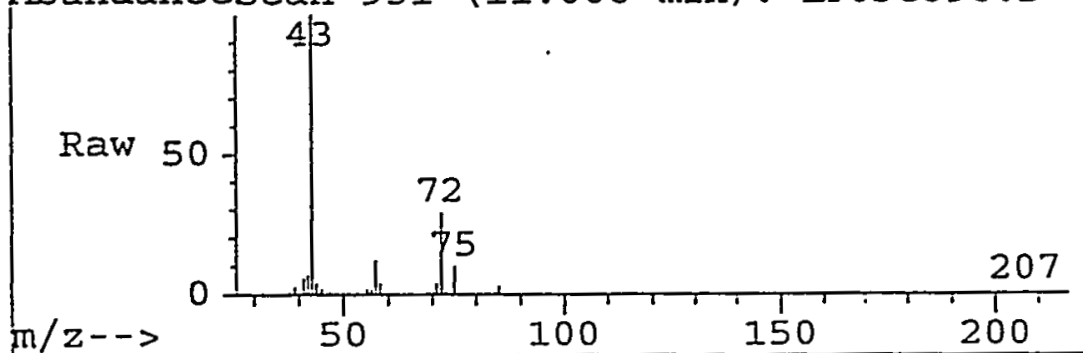

Abundancescan 931 (11.006 min): LF03C898.D

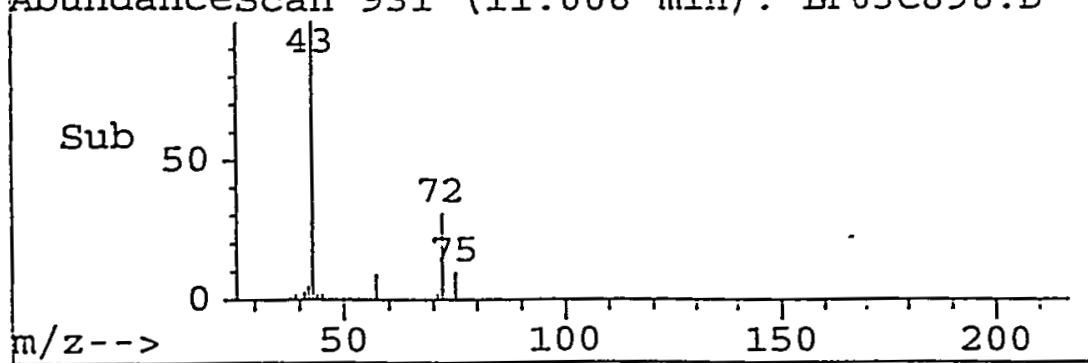

\#13

C011 methylene chloride Concen: $75.01 \mathrm{ug} / \mathrm{L}$

$\mathrm{RT}: 8.75 \mathrm{~min}$ Scan\# 697

Delta R.T. $\quad-0.00 \mathrm{~min}$

Lab File: LF03C898.D

Acq: $07 / 07 / 97$ 15:24

Tgt Ion:84 Resp: 796266

Ion Ratio Lower Upper

84100

$\begin{array}{llll}49 & 99.5 & 136.2 & 204.2 \#\end{array}$

$51 \quad 32.0 \quad 43.0 \quad 64.4 \#$

$86 \quad 65.5,52.6 \quad 79.0$

Abundanceion $84.00 \quad(83$.

300000 tion $49.00 \quad(48$.

Time- $->8.38$ Ion 81.00150.

\#21

C110 2-butanone

Concen: $54.96 \mathrm{ug} / \mathrm{I}$

RT: $11.01 \mathrm{~min}$ Scan\# 931

Delta R.T. $\quad-0.00 \mathrm{~min}$

Lab File: LF03C898.D

Acq : $07 / 07 / 97 \quad 15: 24$

Tgt Ion:71.95 Resp: 62793

Ion Ratio Lower Upper

72100

$43 \quad 312.0 \quad 462.2 \quad 693.2 \#$
0
0.0
0.0
0.0

$\begin{array}{llll}0 & 0.0 & 0.0 & 0.0\end{array}$

AbundanceIon 71.95 (71.

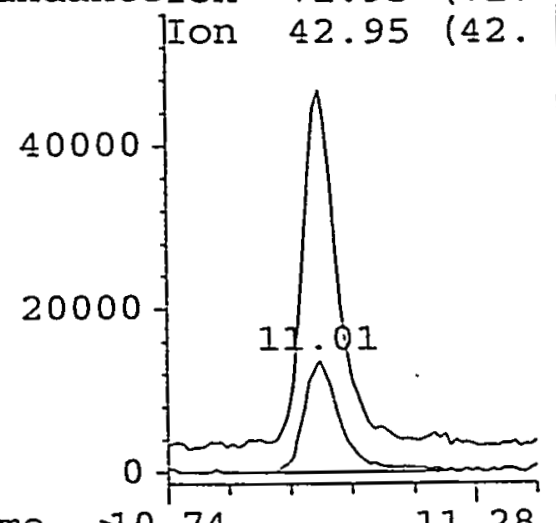

Time-- $>10.74$ 


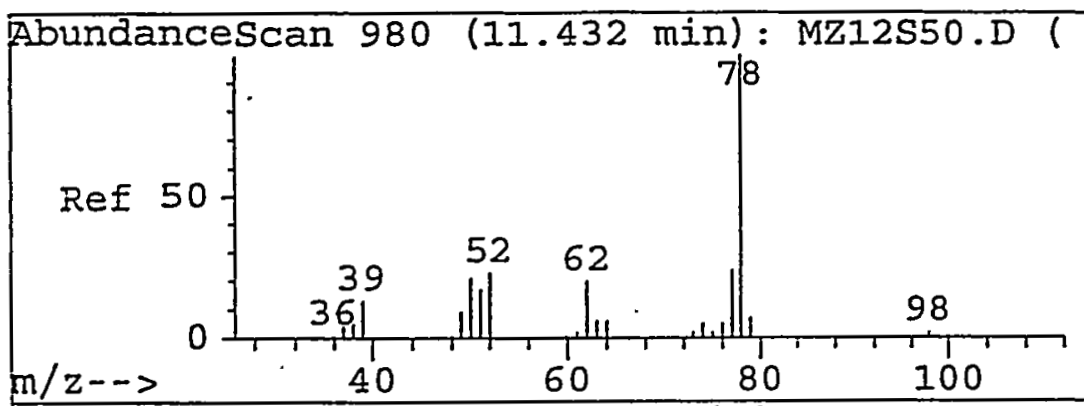

Abundancescan 1075 (12.396 min): LF03C898.D

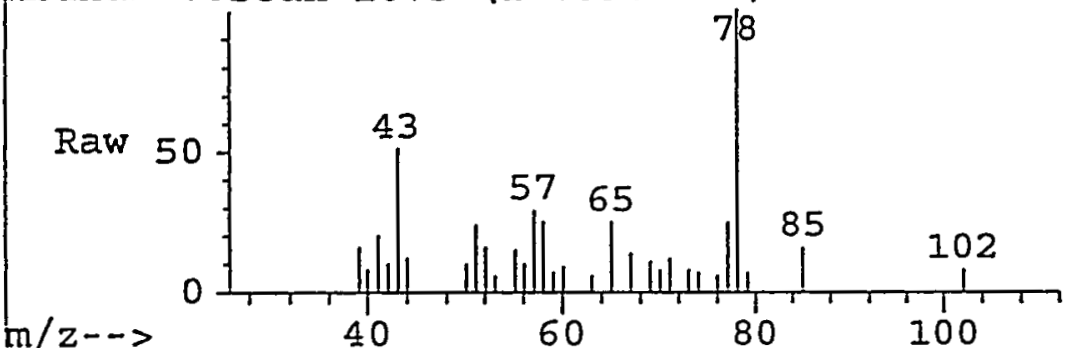

Abundancescan 1075 (12.396 min): LF03C898.D

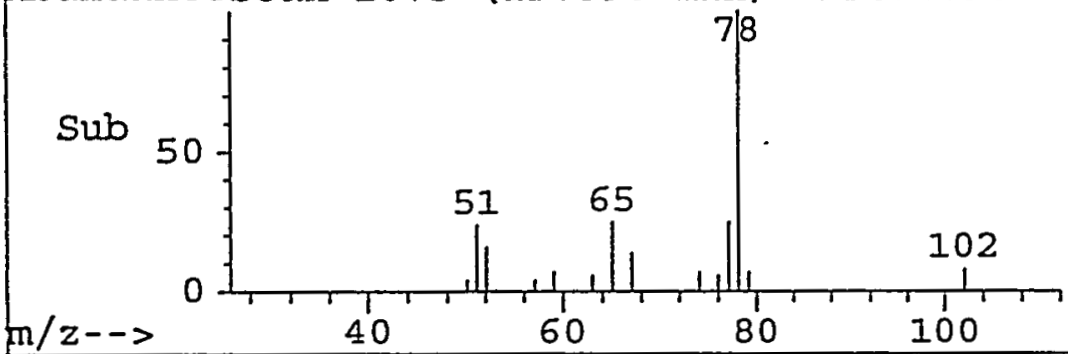

Time-->12.21
C021 benzene

Concen: $0.74 \mathrm{ug} / \mathrm{L}$

RT: $12.40 \mathrm{~min}$ Scan\# 1075

Delta.R.T. $\quad-0.00 \mathrm{~min}$

Lab File: LF03C898.D

Acq : $07 / 07 / 97 \quad 15: 24$

Tgt Ion:78 Resp: 26323

Ion Ratio Lower Upper

78100

$\begin{array}{llll}52 & 14.3 & 11.1 & 16.7\end{array}$

$\begin{array}{rrrr}14.3 & 0.0 & 0.0 & 0.0\end{array}$

$\begin{array}{llll}0 & 0.0^{\prime} & 0.0 & 0.0\end{array}$

ÁbundançeIon $78.00 \quad(77$.

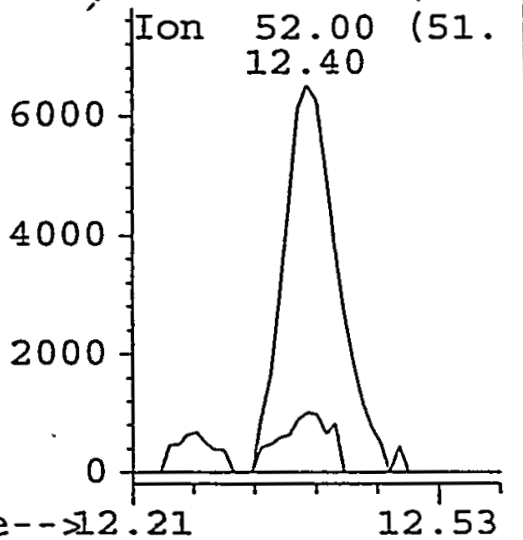

Abundancescan $2403(25.229 \mathrm{~min}):$ ID02S20.D

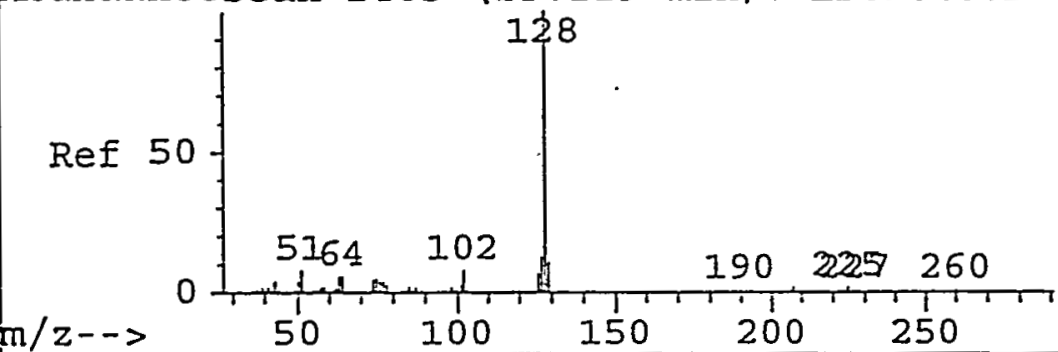

Abundancescan $2408(25.247 \mathrm{~min}):$ LF03C898.D

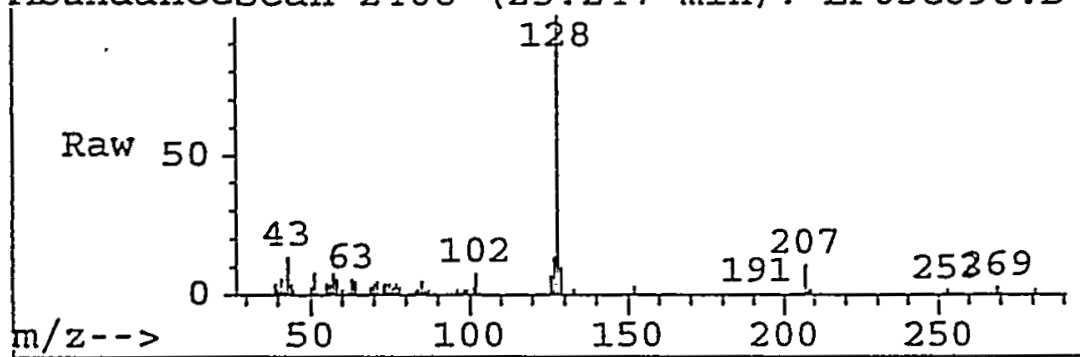

Abundancescan $2408(25.247 \mathrm{~min}):$ LF03C898.D

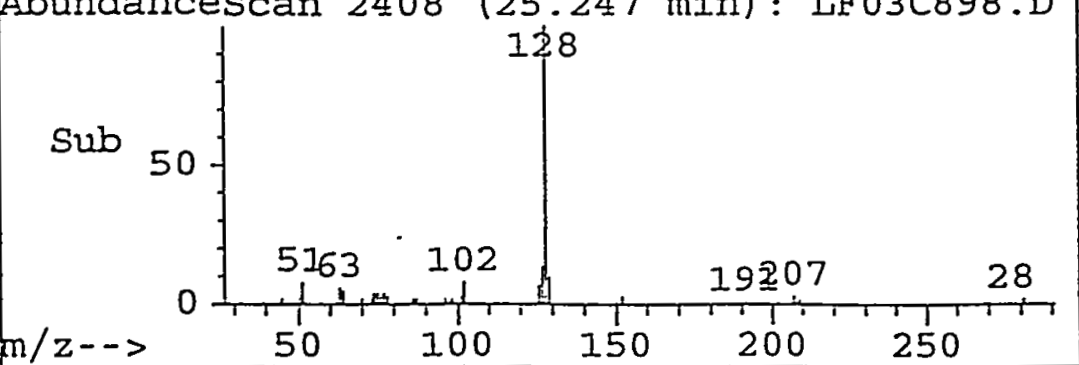

\#76

C061 Naphthalene Concen: $5.52 \mathrm{ug} / \mathrm{L}$

$\mathrm{RT}: 25.25 \mathrm{~min}$ Scan\# 2408

Delta R.T. $0.02 \mathrm{~min}$

Lab File: LF03C898.D

Acq : 07/07/97 15:24

Tgt Ion: 128 Resp: 233586

Ion Ratio Lower Upper

128100

$\begin{array}{llll}127 & 13.0 & 9.8 & 14.6\end{array}$

$\begin{array}{llll}0 & 0.0 & 0.0 & 0.0\end{array}$

\begin{tabular}{cccc}
0 & 0.0 & 0.0 & 0.0 \\
\hline AbundanceIon & 128.00 & $(127$
\end{tabular}

30000 fIon $127.00 \quad(126$

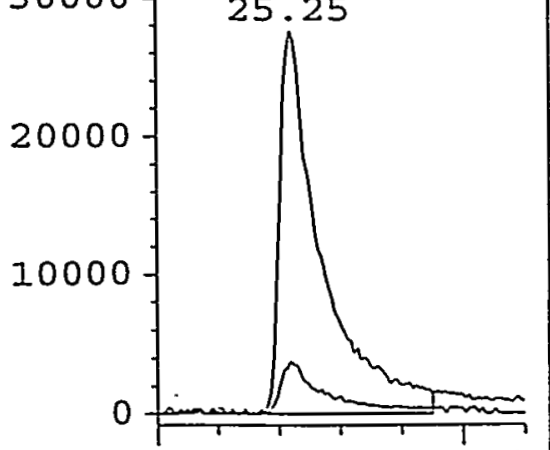

Time- -24.85 


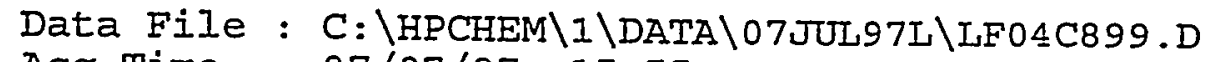

Acq Time : 07/07/97 15:55

Sample : 97C02899 NAP-RI-S2-A

MisC : 97C-0208-01 8260 WATER

Quant Time: Jul 7 16:25 1997

Operator: CQC

Inst : 5971-I

Multiplr: 1.00

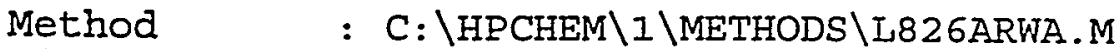

Title : VOA COMPOUND IIST

Last Update : Mon Jul 07 15:11:47 1997

Response via : Multiple Level Calibration
}

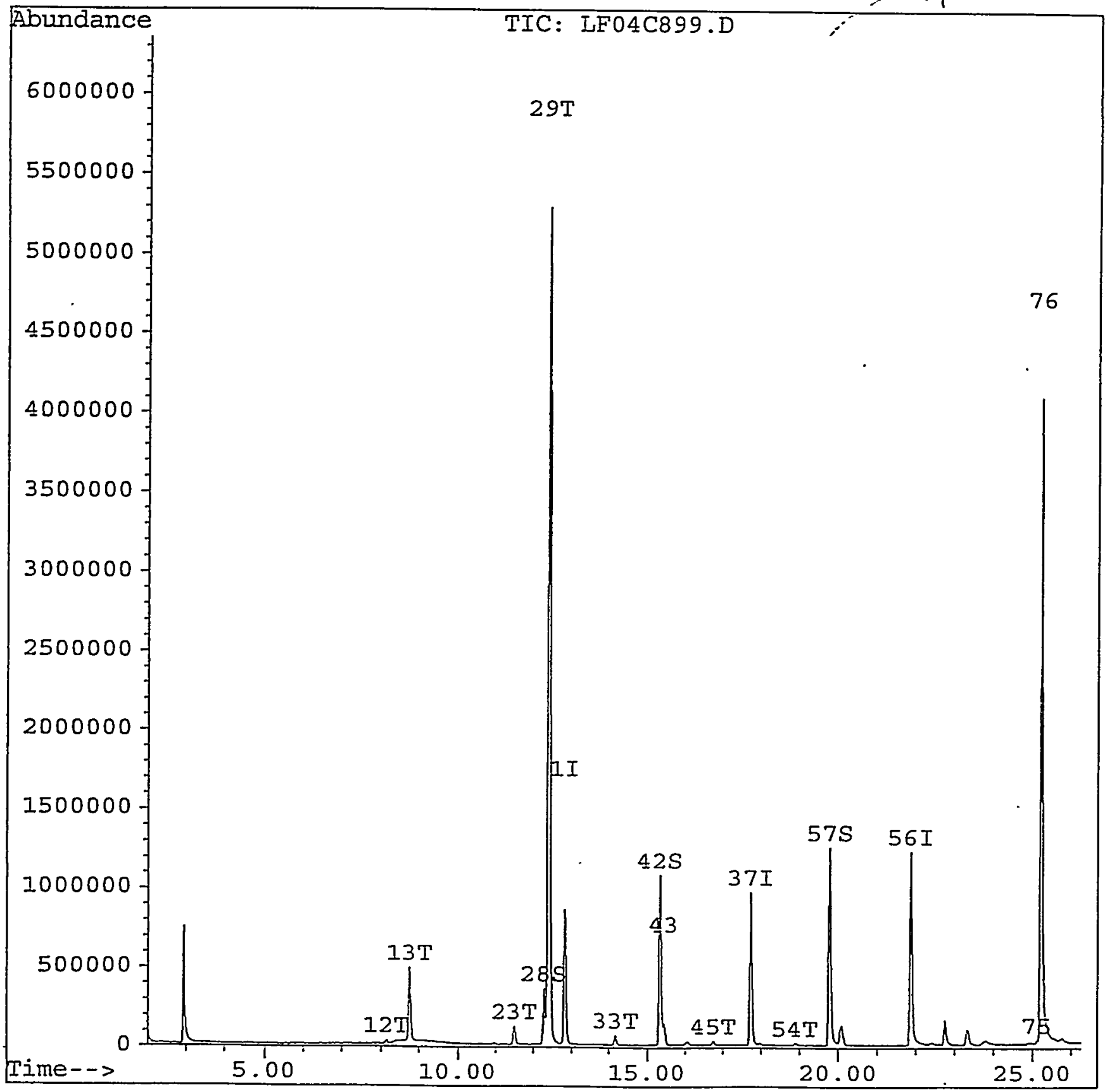




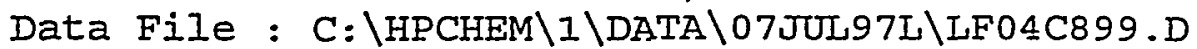

$\begin{array}{llll}\text { Acq Time } & \text { 07/07/97 } 15: 55 \\ \text { Sample } & \text { N7C02899 } & \text { NAP-R1-S2-A } \\ \text { Misc } & 97 C-0208-01 & 8260 \text { WATER } \\ \text { Quant Time: Jul } 716: 251997 & \end{array}$

Operator: $\mathrm{CQC}$

Inst : 5971-L Multiplr: 1.00

Method $\quad: C: \backslash H P C H E M \backslash I \backslash M E T H O D S \backslash I 826 A R W A . M$

Title

: VOA COMPOUND LIST

Last Update : Mon JuI 07 15:11:47 1997

Response via : Multiple Level Calibration

Internal Standards

1) CIOI Flourobenzene

37) CIO2 Chlorobenzene-d5

56) CI03 1,4-Dichlorobenzene-d

System Monitoring Compounds

28) CS15 1,2-dichloroethane-d4

42) $\operatorname{cs} 05$ toluene-d8

57) CS10 4-bromofluorobenzene

Target Compounds

2) $\mathrm{C002}$ dichlorodifluorometha

3) $\mathrm{COO3}$ chloromethane

4) C004 vinyl chloride

5) 0005 bromomethane

6) $\mathrm{C006}$ chloroethane

7) $\mathrm{C007}$ trichlorofluoromethan

B) C071 acrolein

9) $\mathrm{C} 008$ 1,1-dichloroethene

10) $C 063$ freon 113

11) 0009 acetone

12) C010 carbon disulfide

13) C011 methylene chloride

14) $\mathrm{C} 072$ acrylonitrile

15) $\mathrm{C} 012$ trans-1,2-Dichloroeth

16) C305 methyl-t-butyl-ether

17) $\mathrm{C} 013$ Cis-1,2-dichloroethen

18) 0055 total-1,2-dichloroeth

19) C0I4 1,1-dichloroethane

20) C015 2,2-dichloropropane

21) C110 2-butanone

22) CIOI bromochloromethane

23) $\mathrm{C} 016$ chloroform

24) 0017 1,1,1-Trichloroethane

25) $\mathrm{C} 018$ 1,1-dichloropropene

26) C019 1,2-dichloroethane

27) $\mathrm{C} 020$ carbon tetrachloride

29) C02I benzene

30) C022 trichloroethene

31) C023 1,2-dichloropropane

32) 0024 dibromomethane

33) C025 bromodichloromethane

34) 0026 2-chloroethylvinyleth

35) C027 cis-1,3-dichloroprope
R.T. QIon

12.82

17.74

21.88

117

152

1376210

1009156

12.27

15.34

19.78

$\begin{array}{rr}65 & 501241 \\ 98 & 1717669 \\ 95 & 1215416\end{array}$

$95 \quad 1215416$

$0.00 \quad 85$

$0.00 \quad 50$

0.00

62

0.00

94

0.00

64

$0.00 \quad 101$

$0.00 \quad 56$

$0.00 \quad 96$

$0.00 \quad 151$

0.00

43

8.13

76

8.75

0.00

84

0.00

53

0.00

96

0.00

73

0.00

96

0.00

63

0.00

77

$0.00 \quad 72$

0.00

128

11.50

83

0.00

97

0.00

75

0.00

62

0.00

117

12.39

78

0.00

130

0.00

63
93

0.00

14.17

83

0.00

63

0.00
72447

508927

10994273

101876
Conc Units

$50.00 \mathrm{ug} / \mathrm{L}$

$50.00 \mathrm{ug} / \mathrm{L}$

$50.00 \mathrm{ug} / \mathrm{L}$

$-0.02$

0.00

$\because$ Recovery

$43.24 \mathrm{ug} / \mathrm{L}$

$86.49 \%$

$48.52 \mathrm{ug} / \mathrm{L}$

$97.04 \%$

$45.07 \mathrm{ug} / \mathrm{L}$

$90.14 \%$

Qvalue

Not Detected

Not Detected

Not Detected

Not Detected

Not Detected

Not Detected

Not Detected

Not Detected

Not Detected

Not Detected

$2.62 \mathrm{ug} / \mathrm{L}$

$47.58 \mathrm{ug} / \mathrm{L} \#$

Not Detected

Not Detected

Not Detected

Not Detected

Not Detected

Not Detected

Not Detected

Not Detected

Not Detected

$9.38 \mathrm{ug} / \mathrm{I} \mathrm{\#}$

Not Detected

Not Detected

Not Detected

Not Detected

$305.84 \mathrm{ug} / \mathrm{L}$

Not Detected

Not Detected

Not Detected

$4.38 \mathrm{ug} / \mathrm{L}$

Not Detected

Not Detected

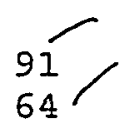

93

100 Dil

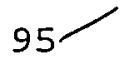


Data File : C: \HPCHEM \I\DATA 07JUL97L\IF04C899.D

Acq Time : 07/07/97 15:55

Sample : 97C02899 NAP-RI-S2-A

Misc : 97C-0208-01 8260 WATER

Quant Time: Jul 7 16:25 1997

Operator: CQC

Inst : 5971-L

Multiplr: 1.00

Method : C: \HPCHEM IIMETHODS \L826ARWA.M

Title : VOA COMPOUND LIST

Last Update : Mon Jul 07 15:11:47 1997

Response via : Multiple Level Calibration

Compound

R.T. QIon Response Conc Unit Qvalue

36) C205 4-methyl-2-pentanone

38) $\mathrm{C} 28$ trans-1,3-dichloropro

39) C029 1,1,2-trichloroethane

40) C2I0 2-hexanone

41) 0030 1,2-Dibromoethane

43) 0031 Toluene

44) $\mathrm{CO} 32$ 1,3-dichloropropane

45) $\mathrm{C033}$ dibromochloromethane

46) $\mathrm{C} 034$ bromoform

47) C035 tetrachloroethene

48) C036 chlorobenzene

49) $\operatorname{Co37} 1,1,1,2$-tetrachloroet

50) $\mathrm{C} 038$ ethylbenzene

51) $\mathrm{CO} 39 \mathrm{~m}, \mathrm{p}$-xylene

52) Co40 o-xylene

53) C250 total xylene

54) C04I styrene

55) $\mathrm{C} 042$ isopropylbenzene

. 58) $C 0431,1,2,2$-tetrachloroet

59) $\mathrm{C} 044$ bromobenzene

60) C045 1,2,3-trichloropropan

61) 046 n-propylbenzene

62) $\mathrm{C047} 1,3$,5-trimethylbenzen

63) C048 2-chlorotoluene

64) Co49 4-chlorotoluene

65) C050 tert-butylbenzene

65) $\cos 1$ 1,2,4-trimethyibenzen

57) 0052 sec-Butylbenzene

68) C053 p-Isopropyltoluene

69) C054 1,3-Dichlorobenzene

70) C055 1,4-Dichlorobenzene

71) C056 n-Butylbenzene

72) C057 1,2-Dichlorobenzene

73) C058 1,2-Dibromo-3-chlorop

74) $\operatorname{Co59} 1,2,4-T r i c h l o r o b e n z e n$

75) C060 Hexachlorobutadiene

76) C061 Naphthalene

77) C062 1,2,3-Trichlorobenzen
0.004

$0.00 \quad 75$

$0.00 \quad 97$

$0.00 \quad 43$

$0.00 \quad 107$

$15.45 \quad 92$

0.0076

$16.75 \quad 129$

$0.00 \quad 173$

$0.00 \quad 164$

$0.00 \quad 112$

$0.00 \quad 131$

$0.00 \quad 106$

$0.00 \quad 106$

0.00106

$0.00 \quad 106$

$18.89 \quad 104$

$0.00 \quad 105$

$0.00 \quad 83$

$0.00 \quad 156$

$0.00 \quad 75$

$0.00 \quad 91$

$0.00 \quad 105$

$0.00 \quad 91$

0.0091

0.00119

$0.00 \quad 105$

$0.00 \quad 105$

$0.00 \quad 119$

$0.00 \quad 146$

$0.00 \quad 146$

$0.00 \quad 91$

$0.00 \quad 146$

$0.00 \quad 75$

$0.00 \quad 180$

$25.12 \quad 225$

$25.22 \quad 128$

$0.00 \quad 180$
Not Detected

Not Detected

Not Detected

Not Detected

Not Detected

$1271215.06 \mathrm{ug} / \mathrm{I}$

Not Detected

$37176 \quad 1.69 \mathrm{ug} / \mathrm{I}$ \#

Not Detected

Not Detected

Not Detected

Not Detected

Not Detected

Not Detected

Not Detected

Not Detected

$258140.83 \mathrm{ug} / \mathrm{L}$ \#

Not Detected

Not Detected

Not Detected

Not Detected

Not Detected

Not Detected

Not Detected

Not Detected

Not Detected

Not Detected

Not Detected

Not Detected

Not Detected

Not Detected

Not Detected

Not Detected

Not Detected

Not Detected

10250

$0.61 \mathrm{ug} / \mathrm{L}$

8481550
$203.26 \mathrm{ug} / \mathrm{L}$

Not Detected

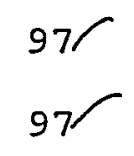

84

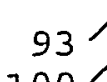

100

121 Data Processed By: 1 7.7.97 $(\mathrm{NR})=$ Not Reported $(\mathrm{SP})=$ Spiked analyte $(\mathrm{m})$ = manual integration

(*) Does not meet EPA spectral criteria (False Hit) PX125 Page 2 


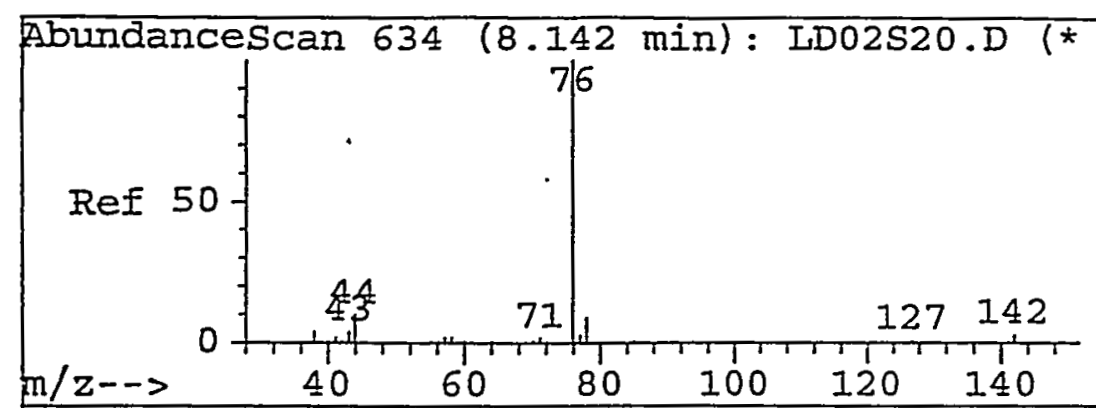

Abundancescan $633(8.133 \mathrm{~min}):$ LF04C899.D (

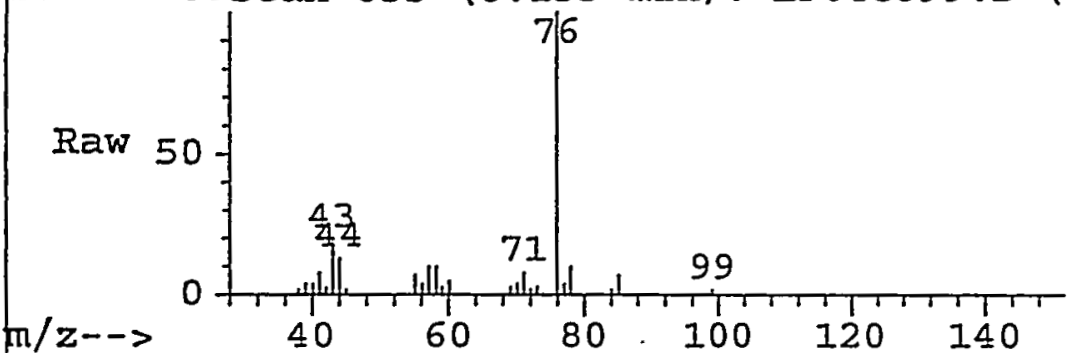

Abundancescan $633(8.133 \mathrm{~min}):$ LF04C899.D (
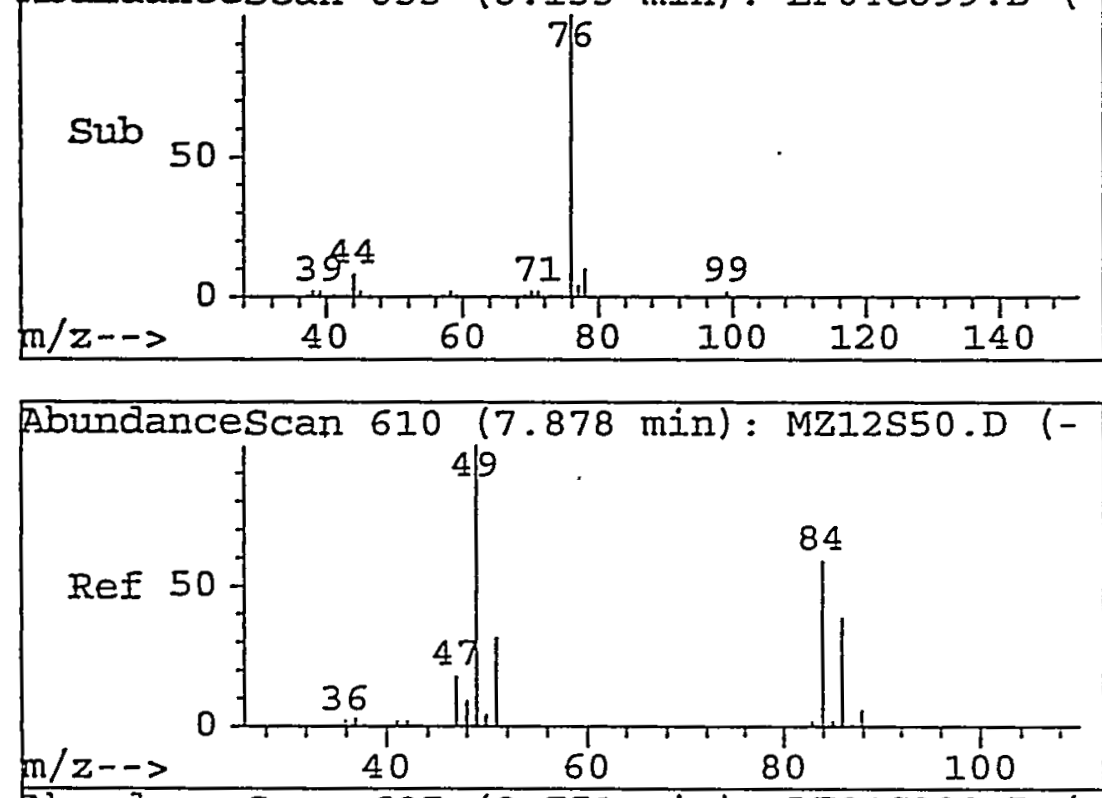

Abundancescan 697 (8.751 min): LF04C899.D (

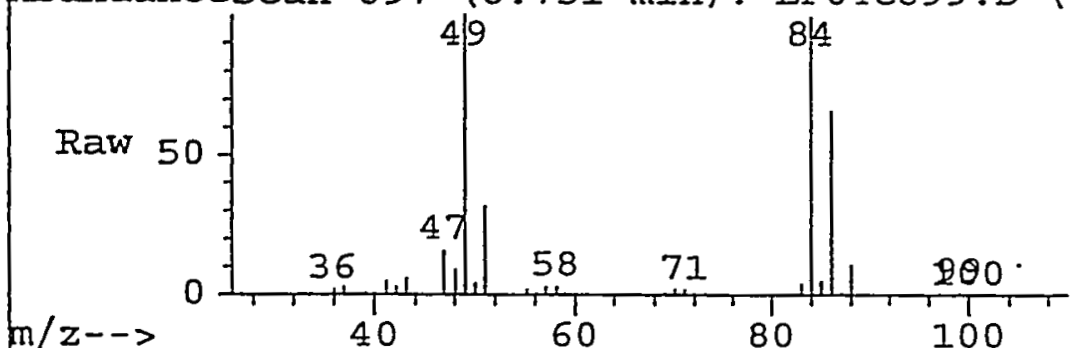

Abundancescan 697 (8.751 min): LF04C899.D (

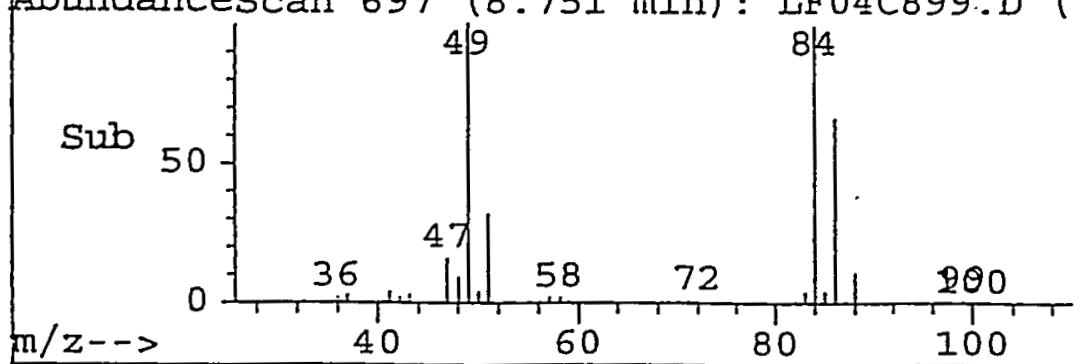

\#12

C010 carbon disulfide

Concen: $2.62 \mathrm{ug} / \mathrm{L}$

RT: 8.13 min Scan\# 633

Delta R.T. $\quad-0.01 \mathrm{~min}$

Lab File: LF04C899.D

Acq : 07/07/97 $15: 55$

Tgt Ion:76 Resp: 72447

Ion Ratio Lower Upper

76100

$\begin{array}{llll}78 & 9.3 & 0.0 & 29.3\end{array}$

$\begin{array}{llll}44 & 10.0 & 0.0 & 35.9\end{array}$

$\begin{array}{rcr}0.0 & 0.0 & 0.0 \\ \text { AbundanceIon } & 76.00 & (75.0\end{array}$

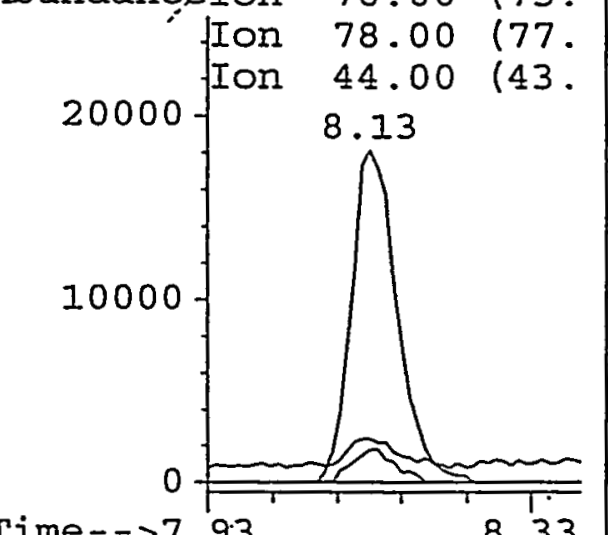

Time- $>7.93$

8.33

\#13

C011 methylene chloride

Concen: $47.58 \mathrm{ug} / \mathrm{I}$

RT: $8.75 \mathrm{~min}$ Scan\# 697

Delta R.T. $0.00 \mathrm{~min}$

Lab File: LF04C899.D

Acq : 07/07/97 15:55

Tgt Ion:84 Resp: 508927

Ion Ratio Lower Upper

84100

$49 \quad 100.6 \quad 136.2 \quad 204.2 \#$

$\begin{array}{llll}51 & 32.6 & 43.0 & 64.4 \#\end{array}$

$\begin{array}{llll}86 & 67.3 & 52.6 & 79.0\end{array}$

AbundanceIon $84.00 \quad(83$.

200000 -Ion 49.00 (48.

Ion $51.00(50$.

150000 IIon $86.00(85$.

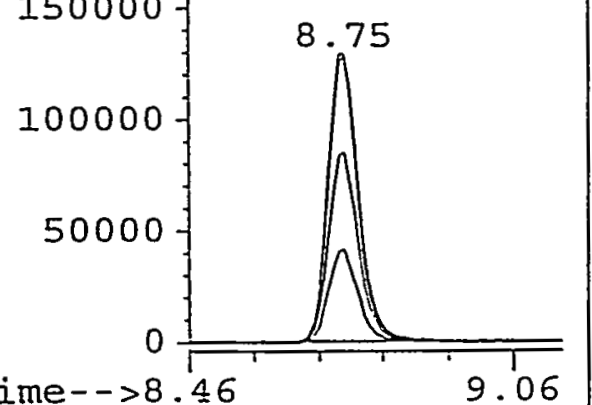

$\pm ?$ 


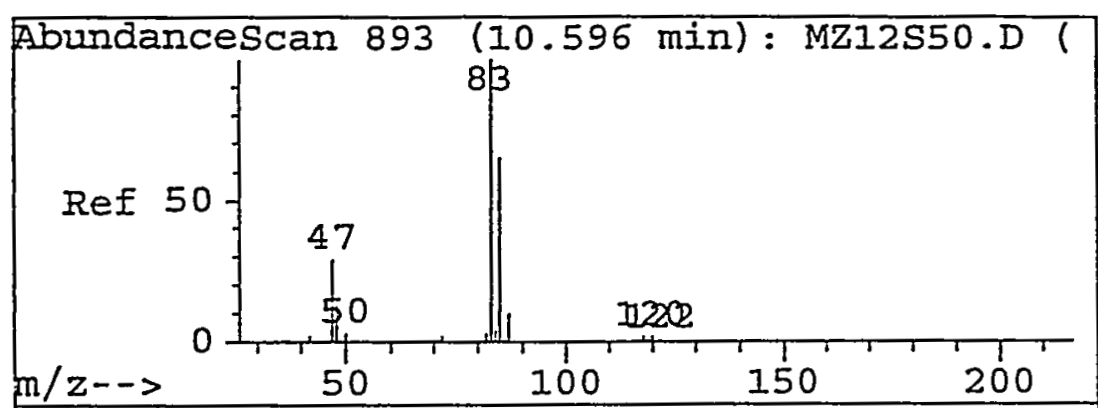

Abundancescan $982(11.501 \mathrm{~min}):$ LF04C899.D

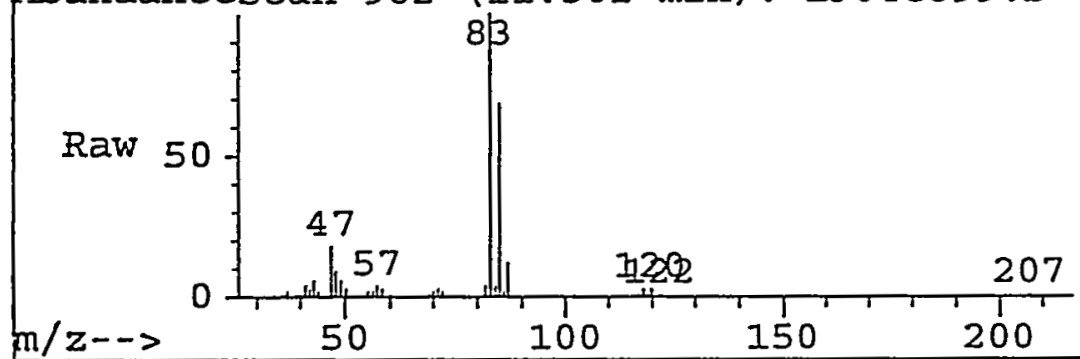

Abundancescan $982(11.501 \mathrm{~min}):$ LF04C899.D Sub 50

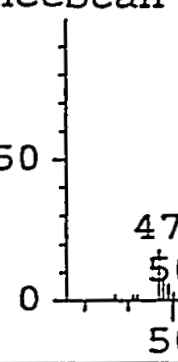

\#23

C016 chloroform Concen: $9.38 \mathrm{ug} / \mathrm{L}$ RT: $11.50 \mathrm{~min}$ Scan\# 982 Delta R.T. $0.08 \mathrm{~min}$ Lab File: LF04C899.D Acq : $07 / 07 / 97 \quad 15: 55$

Tgt Ion:83 Resp: 209808 Ion Ratio Lower Upper 83100

$\begin{array}{llll}85 & 66.0 & 53.0 & 79.6\end{array}$

$47 \quad 17.4 \quad 23.0 \quad 34.4 \#$

$00.0<0.0 \quad 0.0$

Abundancé-Ión $83.00 \quad(82$. 60000 Ion 85.00 (84.

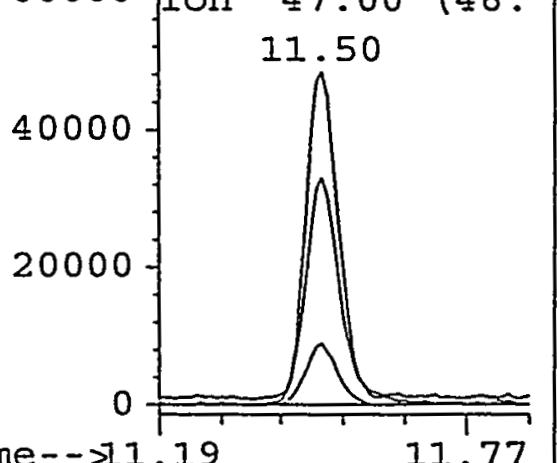

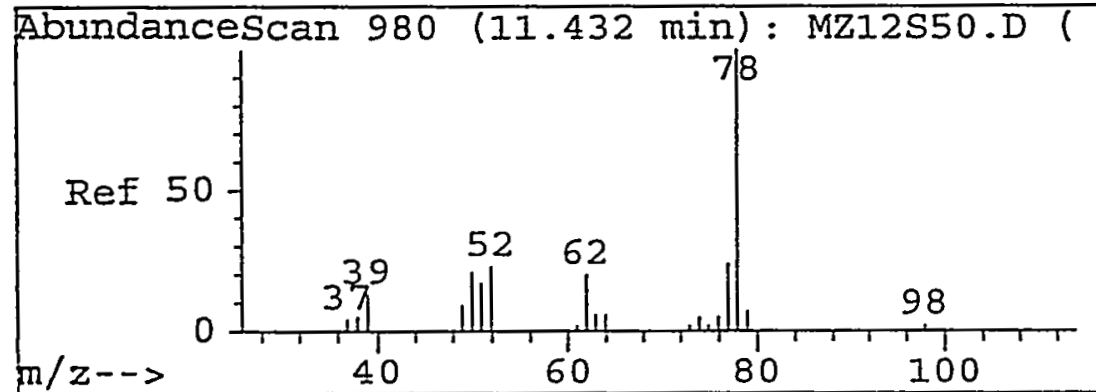

Abundancescan 1074 (12.389 min): LF04C899.D

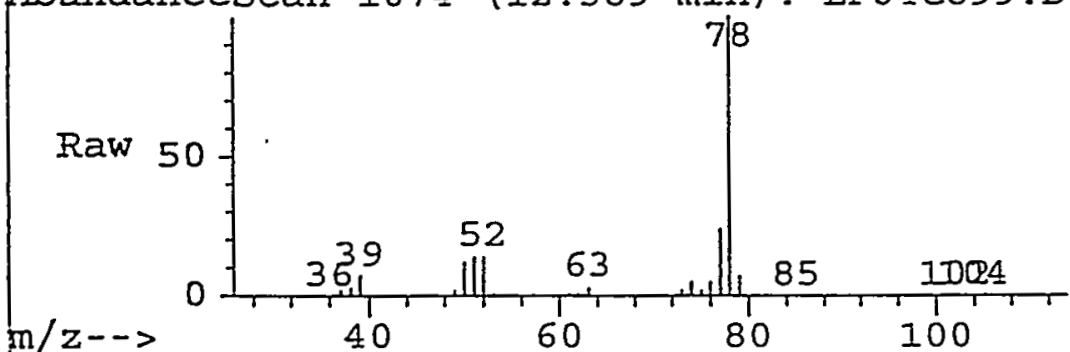

AbundanceScan 1074 (12.389 min): LF04C899.D

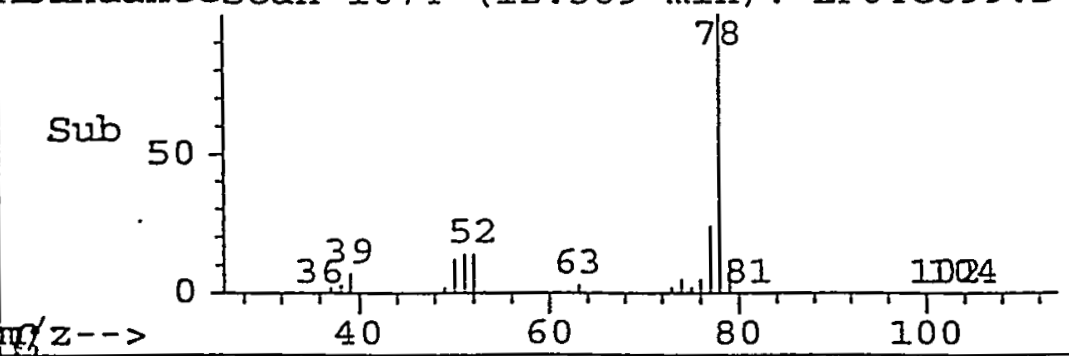

\#29

C021 benzene

Concen: $305.84 \mathrm{ug} / \mathrm{L}$

$\mathrm{RT}: 12.39 \mathrm{~min}$ scan\# 1074

Delta R.T. $\quad-0.01 \mathrm{~min}$

Lab File: LF04C899.D

Acq : 07/07/97 15:55

Tgt Ion:78 Resp:10994273

Ion Ratio Lower Upper

78100

$\begin{array}{llll}52 & 14.0 & 11.1 & 16.7\end{array}$

$\begin{array}{llll}0 & 0.0 & 0.0 & 0.0\end{array}$

$\begin{array}{llll}0 & 0.0 & 0.0 & 0.0\end{array}$

AbundanceIon 78.00177 .

3000000 fIon 52.00 (51.

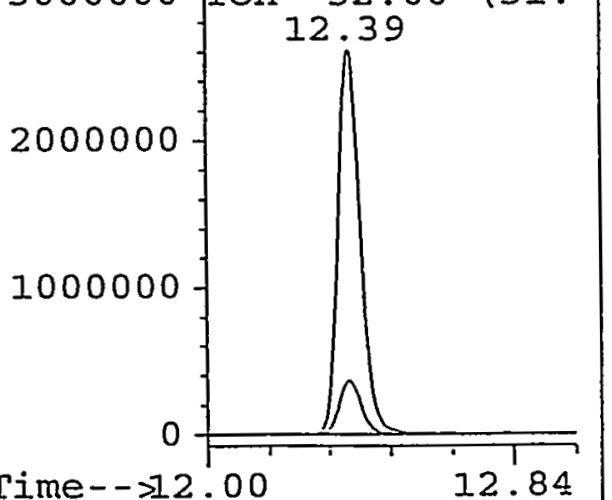



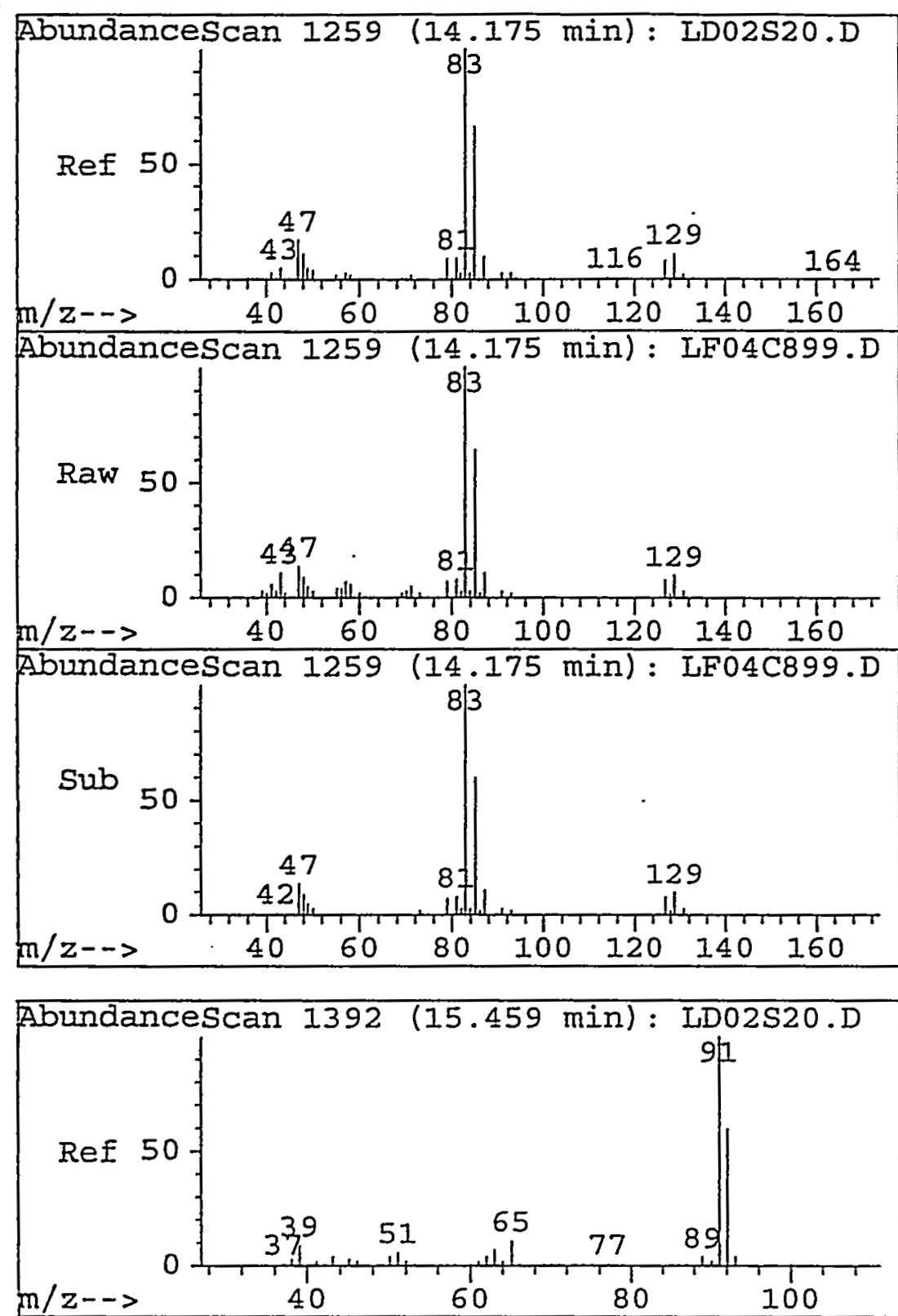

Abundancescan 1391 (15.448 min): LF04C899.D

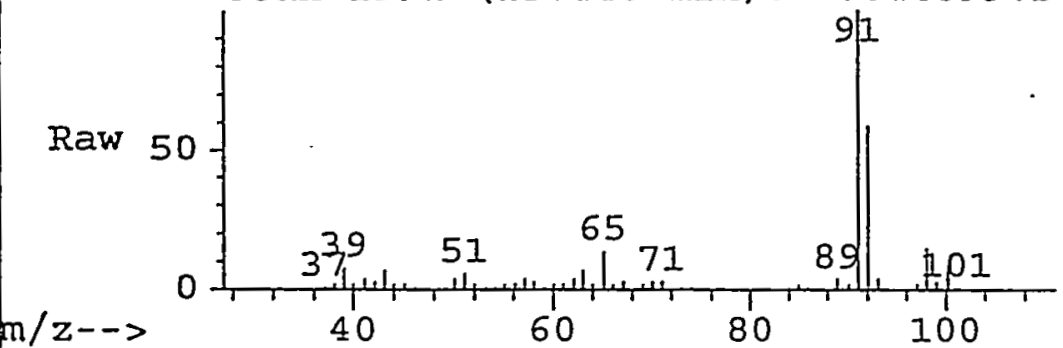

Abundancescan 1391 (15.448 min) : LF04C899.D

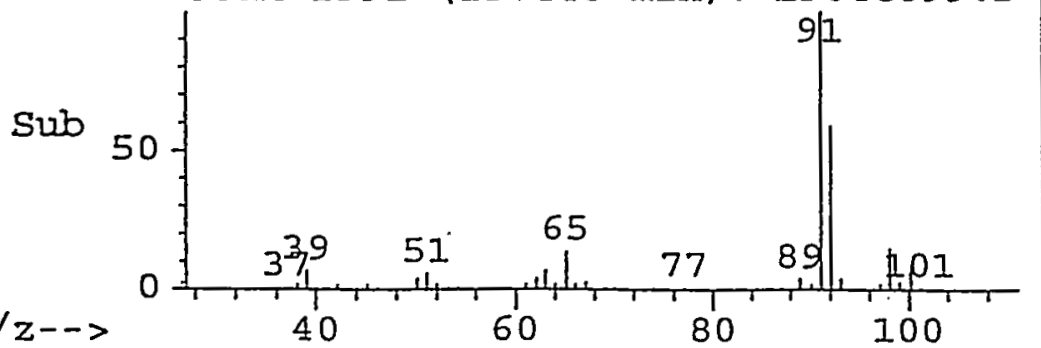

\#33

C025 bromodichloromethane Concen: $4.38 \mathrm{ug} / \mathrm{I}$

RT: $14.17 \mathrm{~min}$ Scan\# 1259

Delta R.T. $\quad-0.00 \mathrm{~min}$

Lab File: LF04C899.D

Acq: $07 / 07 / 97 \quad 15: 55$

Tgt Ion:83 Resp: 101876

Ion Ratio Lower Upper

83100

$\begin{array}{llll}85 & 60.0 & 51.6 & 77.4\end{array}$

$129 \quad 10.4 \quad 7.4 \quad 11.0$

$0 \quad 0.0<0.0 \quad 0.0$

Abundancéión $83.00 \quad(82$.

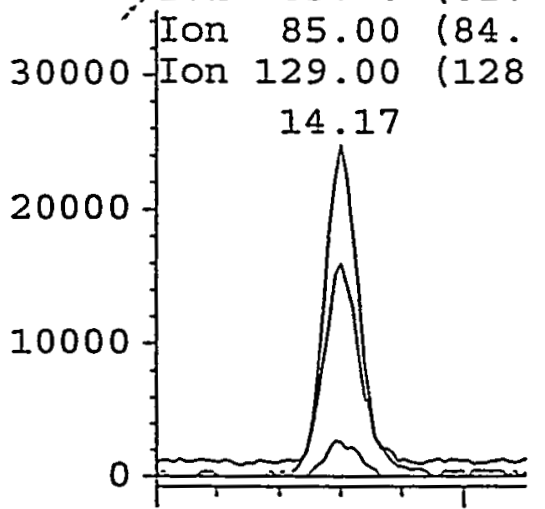

Time-- $>13.87$

14.38

C031 Toluene

Concen: $5.06 \mathrm{ug} / \mathrm{I}$

$\mathrm{RT}: 15.45 \mathrm{~min}$ Scan\# 1391

Delta R.T. $\quad-0.01 \mathrm{~min}$

Iab File: LF04C899.D

Acq : 07/07/97 15:55

Tgt Ion:92 Resp: 127121

Ion Ratio Lower Upper

92100

$\begin{array}{llll}91 & 169.0 & 132.4 & 198.6\end{array}$

$\begin{array}{llll}65 & 21.5 & 15.8 & 23.8\end{array}$

$\begin{array}{llll}0 & 0.0 & 0.0 & 0.0\end{array}$

AbundanceIon 92.00 (91.

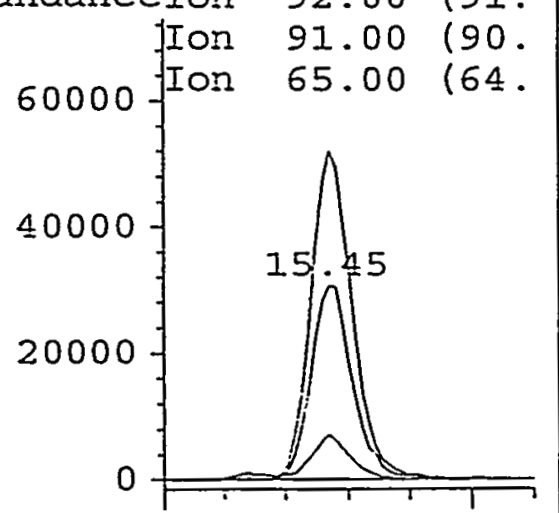

Time-->15.21 $\quad 15.66$ 


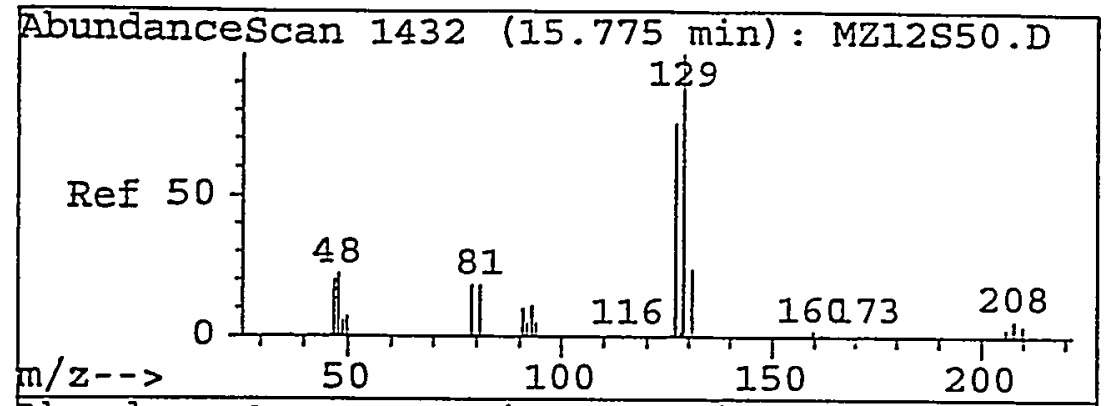

Abundancescan $1526(16.751 \mathrm{~min}):$ LF04C899.D

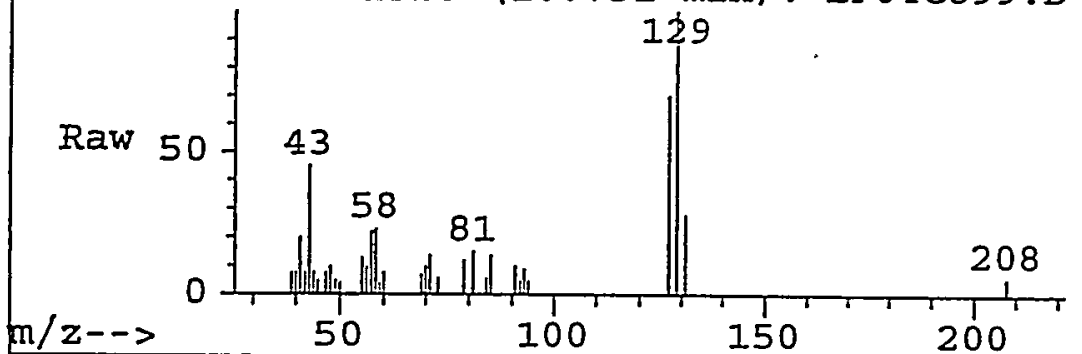

Abundancescan 1526 (16.751 min) : LF04C899.D

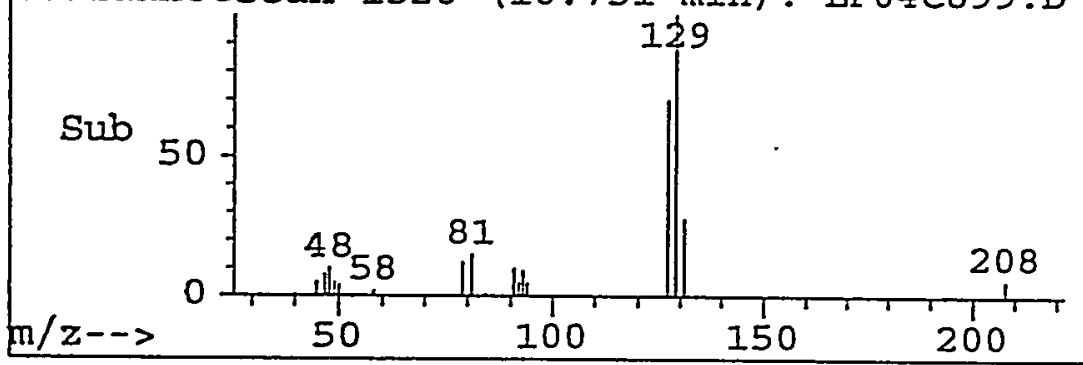

\#45

C033 dibromochloromethane Concen: $1.69 \mathrm{ug} / \mathrm{L}$

RT: $16.75 \mathrm{~min}$ Scan\# 1526

Delta R.T. $0.04 \mathrm{~min}$

Lab File: LF04C899.D

Acq : $07 / 07 / 97 \quad 15: 55$

Tgt Ion:129 Resp: 37176

Ion Ratio Lower Upper

129100

$\begin{array}{llll}208 & 6.0 & 4.4 & 6.6\end{array}$

$206 \quad 0.0,1.9 \quad 2.9 \#$

$\begin{array}{cccc}0 & 0.0 & 0.0 & 0.0 \\ \text { Abundancelon } & 129.00 & (128\end{array}$

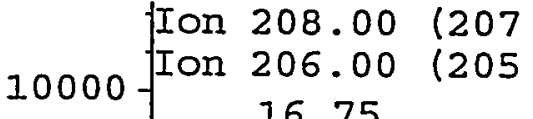

Time-->16.54

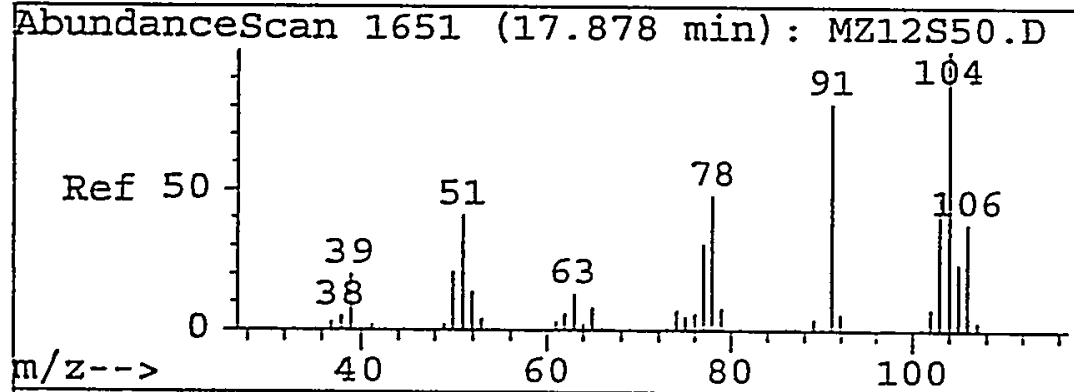

Abundancescan $1748(18.893 \mathrm{~min}):$ LF04C899.D

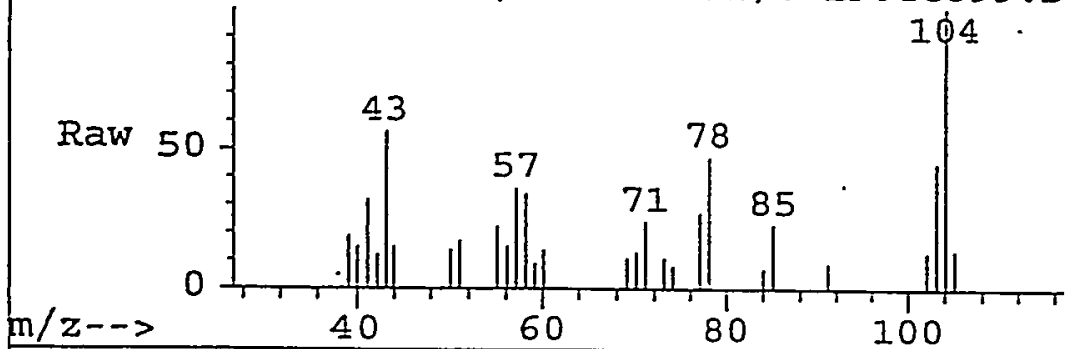

Abundancescan 1748 (18.893 min): LF04C899.D

$128 \mathrm{~m} / \mathrm{zm}$

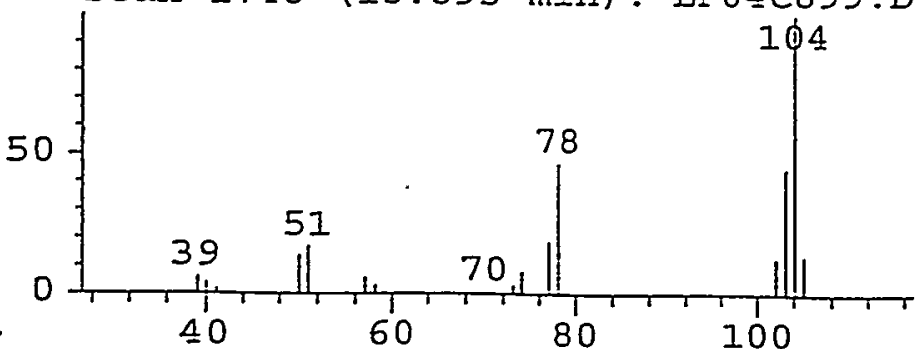

\#54

C041 styrene

Concen: $0.83 \mathrm{ug} / \mathrm{L}$

RT: $18.89 \mathrm{~min}$ Scan\# 1748

Delta R.T. $\quad-0.04 \mathrm{~min}$

Lab File: LF04C899.D

Acq : 07/07/97 15:55

Tgt Ion:104 Resp: 25814

Ion Ratio Lower Upper

104100

$\begin{array}{llll}103 & 44.5 & 42.1 & 63.1\end{array}$

$\begin{array}{llll}78 & 45.8 & 38.4 & 57.6\end{array}$

$51 \quad 20.5 \quad 35.4 \quad 53.0 \#$

AbundanceIon 104.00 (103

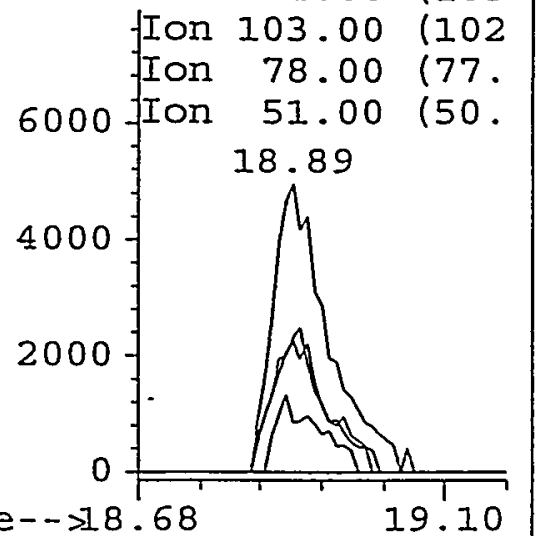




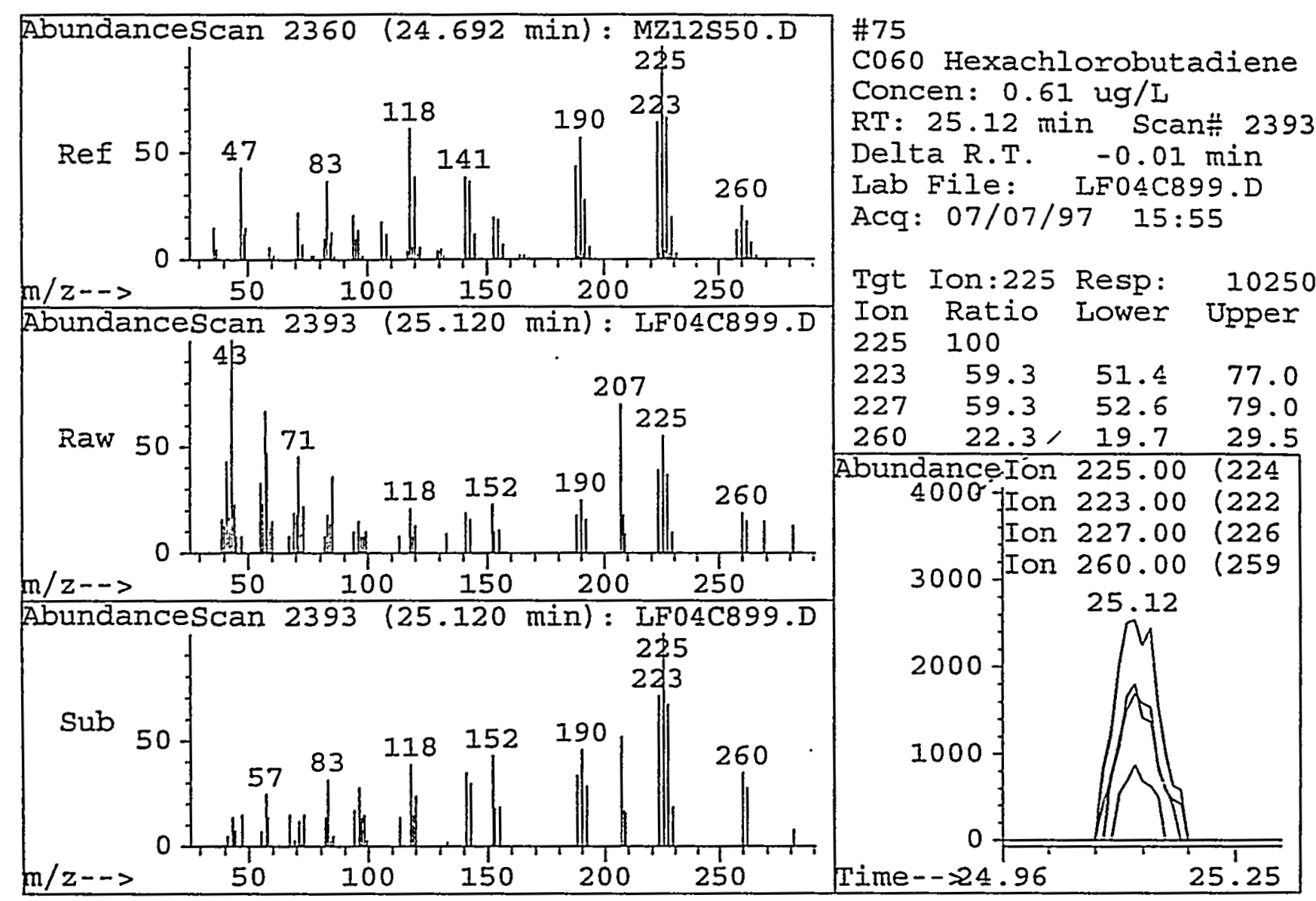

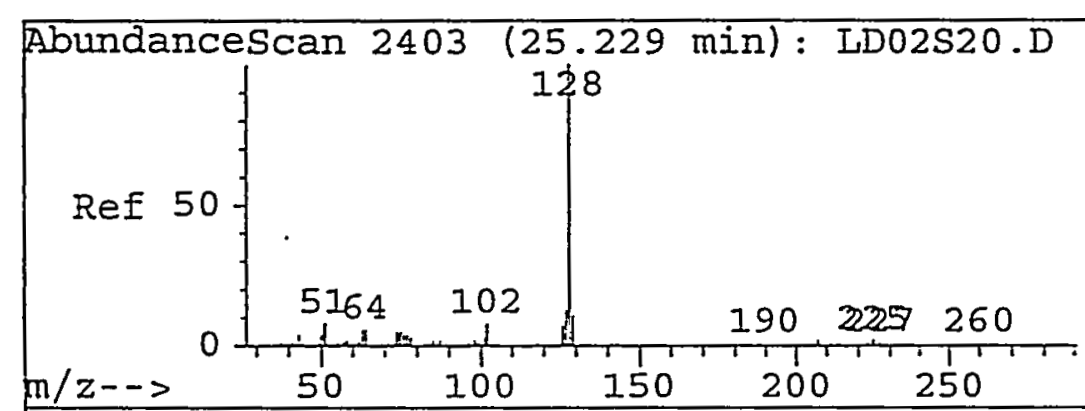

Abundancescan $2403(25.217 \mathrm{~min}):$ LF04C899.D

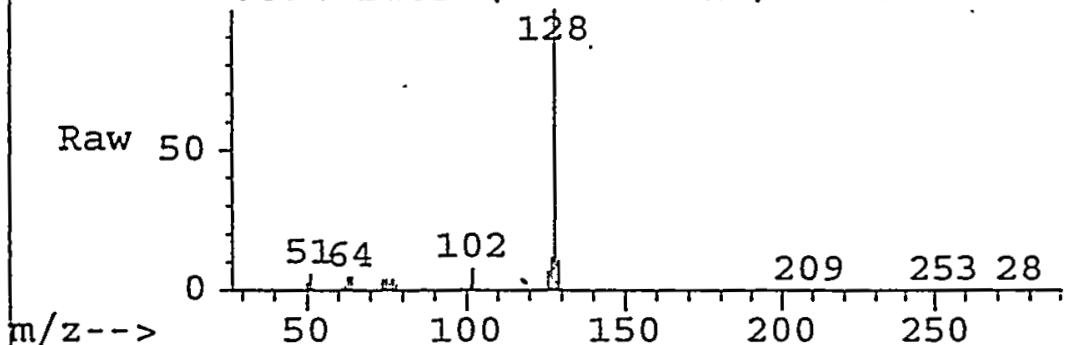

AbundanceScan $2403(25.217 \mathrm{~min}):$ LF04C899.D

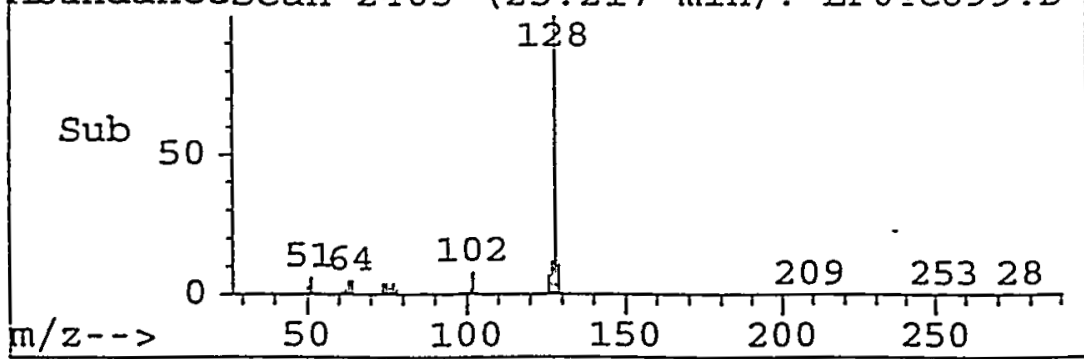

\#76

C061 Naphthalene

Concen: $203.26 \mathrm{ug} / \mathrm{L}$

$\mathrm{RT}: 25.22 \mathrm{~min}$ Scan\# 2403

Delta R.T. $\quad-0.01 \mathrm{~min}$

Lab File: LF04C899.D

Acq : $07 / 07 / 97 \quad 15: 55$

Tgt Ion: 128 Resp: 8481550

Ion Ratio Lower Upper

128100

$\begin{array}{rrrr}127 & 12.2 & 9.8 & 14.6 \\ 0 & 0.0 & 0.0 & 0.0 \\ 0 & 0.0 & 0.0 & 0.0\end{array}$

AbundanceIon $128.00 \quad(127$

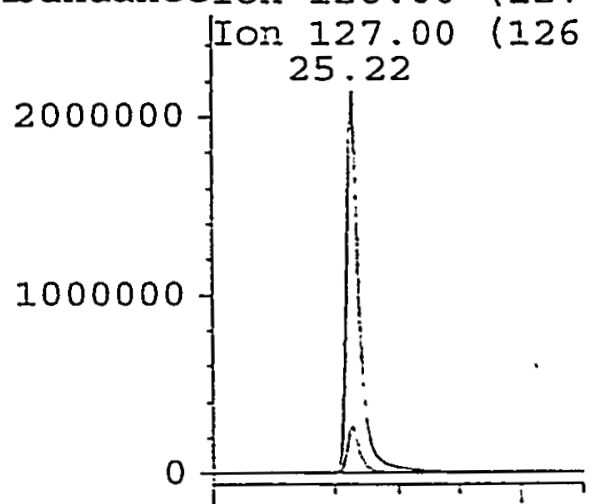

Time- $-24.57 \quad 25.99$ 


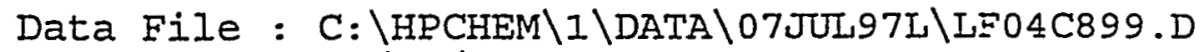

$\begin{array}{lll}\text { Acq Time } & : 07 / 07 / 97 & 15: 55 \\ \text { Sample } & : 97 C 02899 & \text { NAP-R1-S2-A } \\ \text { Misc } & : 97 C-0208-01 & 8260 \text { WATER }\end{array}$

Operator: $\mathrm{CQC}$ Inst : 597I-I Multiplr: 1.00

Method : C: \HPCHEM \I\METHODS \L826ARWA.M

Title : VOA COMPOUND LIST

Library : C:\DATABASE\NBS75K.L

R.T. Conc Area Relative to ISTD ISTD Area

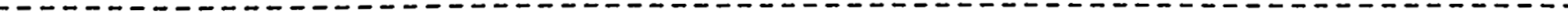

$22.74 \quad 6.47 \mathrm{ug} / \mathrm{I}$

Hit\# of 9

1 Indene

2 Benzene, 1-ethynyl-4-methyl-

3 Benzene, 1-propynyl-

4 Benzene, 1,2-propadienyl-

5 Tetracyclo $[2.2 .1 .0(2,6) .0(3,5)]$ hept
CI03 1,4-Dichlorobenz'ene-d4 5249965 Tentative ID Ref\# ¿CAS\#

Qual
$64387000095-13-695.00$

$3388000766-97-2 \quad 90.00$

$64385000673-32-5 \quad 87.00$

$3387002327-99-3 \quad 37.00$

$3384000000-00-037.00$

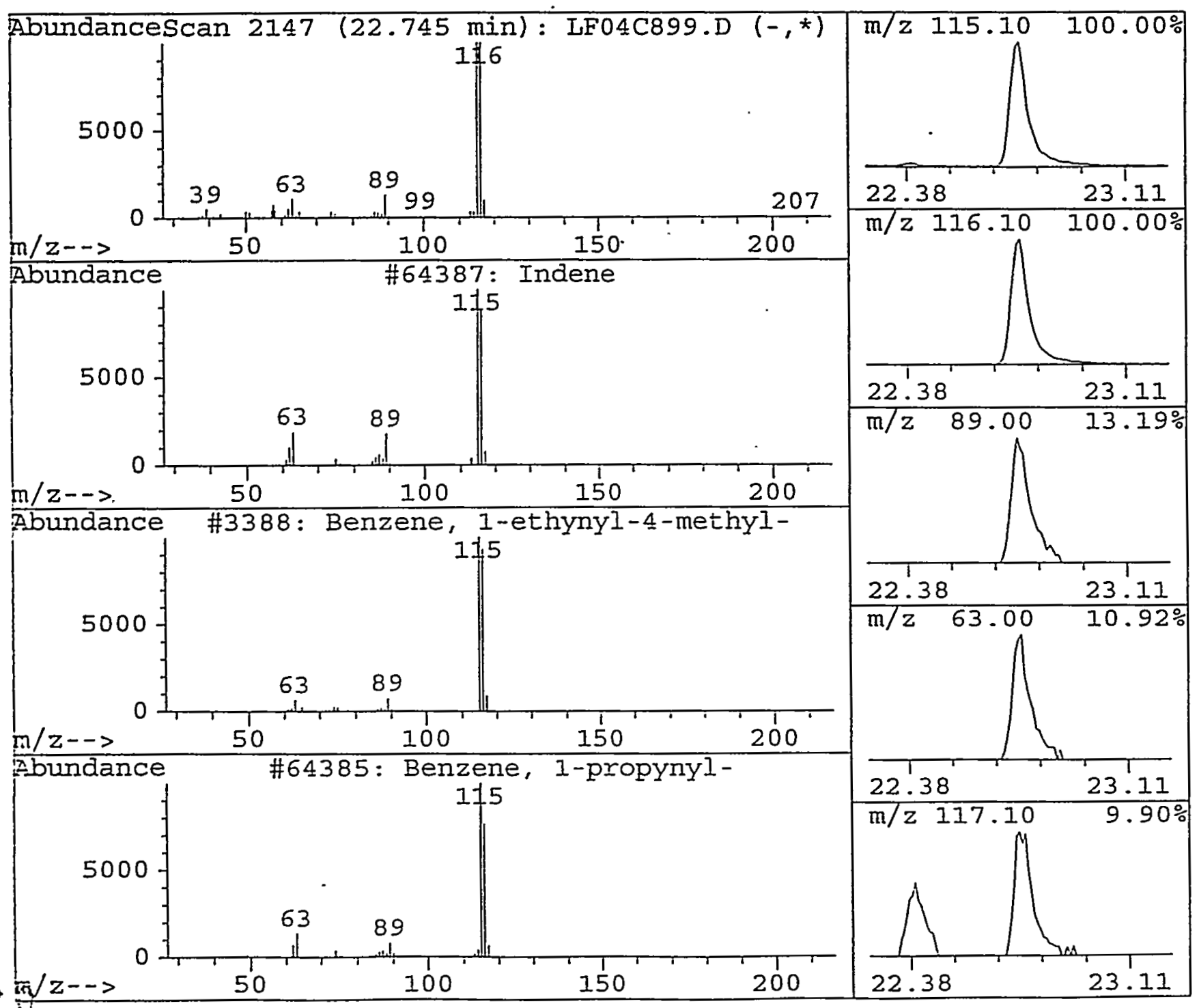


Quantitation Report

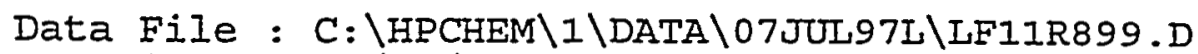

Acq Time : 07/07/97 19:38

Sample : 97C02899 NAP-RI-S2-A

Misc : 97C-0208-01 1:10 Dilution

Operator: CQC

Inst : 5971-I

Quant Time: Jul 20 13:31 1997

Multiplr: 1.00

Method : J: \I\METHODS \L826ARWA.M

Title : VOA COMPOUND IIST

Iast Update : Wed Jun 25 09:14:05 1997

Response via : Multiple Level Calibration

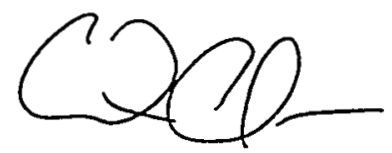

7.20 .97

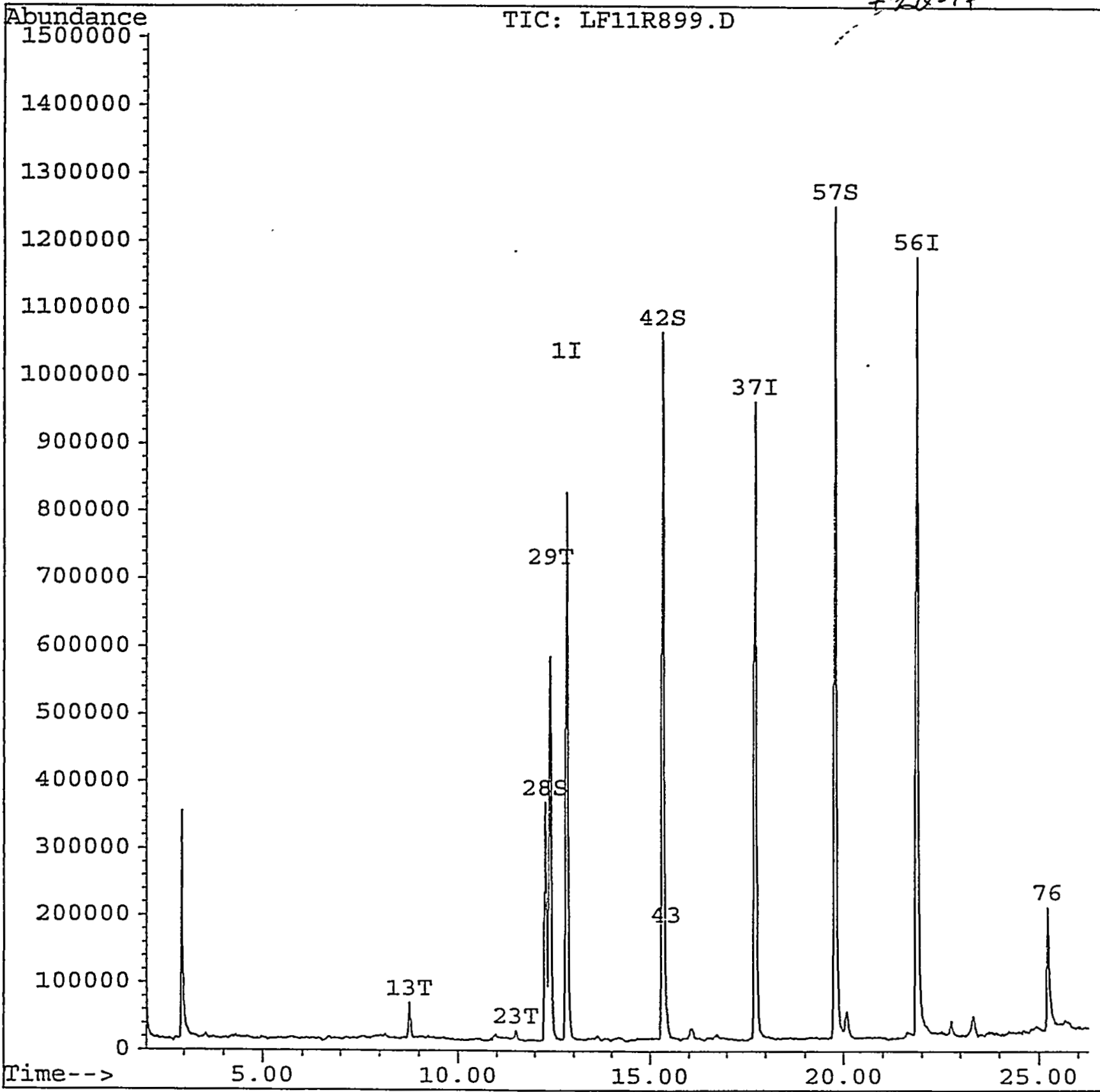

131 
Quantitation Report

Data File : C:\HPCHEM IIDATA 07 JUL97L\LFIIR899.D

Acq Time : 07/07/97 19:38

Sample : 97C02899 NAP-R1-S2-A

Misc : 97C-0208-01 1:10 Dilution

Quant Time: Jul $20 \quad 13: 311997$

Operator: $\mathrm{CQC}$

Inst : 597I-L

Multiplr: 1.00

Method : J: \L\METHODS \L826ARWA.M

Title : VOA COMPOUND IIST

Last Update : Wed Jun 25 09:14:05 1997

Response via : Multiple Level Calibration

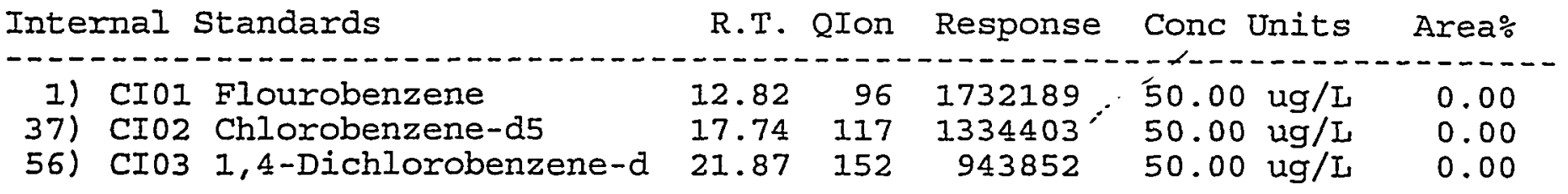

System Monitoring Compounds

28) CSI5 1,2-dichloroethane-d4

42) Cs05 toluene-d8

57) CSI0 4-bromofluorobenzene

Target Compounds

2) $\mathrm{C} 002$ dichlorodifluorometha

3) $\mathrm{C003}$ chloromethane

4) 0004 vinyl chloride

5) C005 bromomethane

6) 0006 chloroethane

7) $\mathrm{C007}$ trichlorofluoromethan

B) 0071 acrolein

9) C008 1,1-dichloroethene

10) $\mathrm{C} 063$ freon 113

11) c009 acetone

12) C010 carbon disulfide

13) C011 methylene chloride

14) $\mathrm{C} 072$ acrylonitrile

15) $\mathrm{C} 012$ trans-1,2-Dichloroeth

16) C305 methyl-t-butyl-ether

17) $\mathrm{CO13}$ cis-1,2-dichloroethen

18) C055 total-1,2-dichloroeth

19) C014 1,1-dichloroethane

20) Co15 2,2-dichloropropane

21) C110 2-butanone

22) CI01 bromochloromethane

23) $\mathrm{COI} 6$ chloroform

24) $\operatorname{CoI7~1,1,1-Trichloroethane~}$

25) C018 1,I-dichloropropene

26) C019 1,2-dichloroethane

27) C020 carbon tetrachloride

29) CO2I benzene

30) C022 trichloroethene

31) C023 1,2-dichloropropane

32) $\mathrm{C} 24$ dibromomethane

33) C025 bromodichloromethane

34) C026 2-chloroethylvinyleth

132
12.27

15.34

19.78

65

488189

$98 \quad 1705456$

$95 \quad 1191284$

$0.00 \quad 85$

$0.00 \quad 50$

$0.00 \quad 62$

$0.00 \quad 94$

$0.00 \quad 64$

$0.00 \quad 101$

$0.00 \quad 56$

$0.00 \quad 96$

$0.00 \quad 151$

$0.00 \quad 43$

$0.00 \quad 76$

$8.74 \quad 84$

$0.00 \quad 53$

$0.000^{\circ} 96$

$0.00 \quad 73$

$0.00 \quad 96$

$0.00 \quad 96$

$0.00 \quad 63$

$0.00 \quad 77$

$0.00 \quad 72$

$0.00 \quad 128$

$11.51 \quad 83$

$0.00 \quad 97$

$0.00 \quad 75$

$0.00 \quad 62$

$0.00 \quad 117$

$12.40 \quad 78$

$0.00 \quad 130$

$0.00 \quad 63$

$0.00 \quad 93$

$0.00 \quad 83$

$0.00 \quad 63$

$0.00 \quad 75$
$\%$ Recovery

$43.60 \mathrm{ug} / \mathrm{L} \quad 87.20 \%$

$49.69 \mathrm{ug} / \mathrm{L} \quad 99.37 \%$

$47.23 \mathrm{ug} / \mathrm{L} \quad 94.46 \%$

Qvalue

Not Detected

Not Detected

Not Detected

Not Detected

Not Detected

Not Detected

Not Detected

Not Detected

Not Detected

Not Detected

Not Detected

$628776.09 \mathrm{ug} / \mathrm{L} \#$

Not Detected

Not Detected

Not Detected

Not Detected

Not Detected

Not Detected

Not Detected

Not Detected

Not Detected

$235471.09 \mathrm{ug} / \mathrm{L} \#$

Not Detected

Not Detected

Not Detected

Not Detected

1164091

$33.52 \mathrm{ug} / \mathrm{L}$

Not Detected

Not Detected

Not Detected

Not Detected

Not Detected

Not Detected

100

$57 \mathrm{NR}$

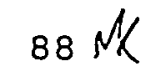

$(\mathrm{NR})=$ Not Reported $(\mathrm{SP})=$ Spiked analyte $(\mathrm{m})=$ manual integration

(*) Does not meet EPA spectral criteria (False Hit) PX125 
Quantitation Report

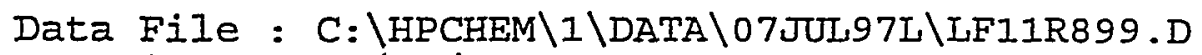

Acq Time : 07/07/97 19:38

Sample : 97C02899 NAP-R1-S2-A

Misc : 97C-0208-01 1:10 Dilution

Operator: CQC

Quant Time: JuI 20 13:31 1997

Inst : 5971-L

Multiplr: 1.00

Method : J:\L\METHODS \L826ARWA.M

Title : VOA COMPOUND LIST

Last Update : Wed Jun 25 09:14:05 1997

Response via : Multiple Level Calibration

Compound

R.T. QIon Response Conc. Unit Qvalue

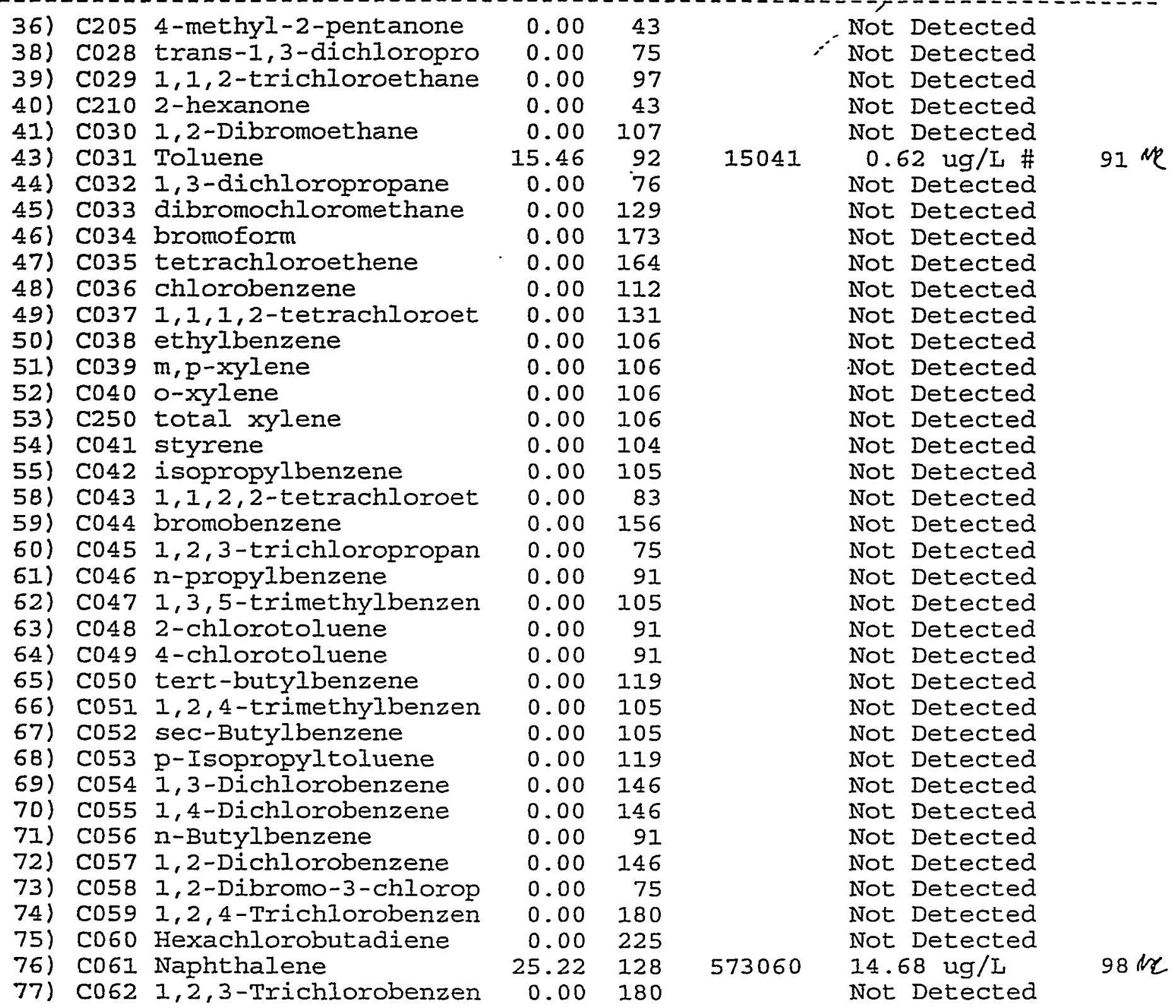

Data Processed By:

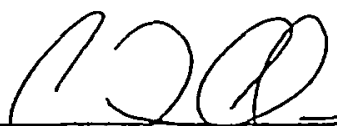

Zr20.9.7

$(\mathrm{NR})=$ Not Reported $(\mathrm{SP})=$ Spiked analyte $(\mathrm{m})=$ manual integration

(*) Does not meet EPA spectral criteria (False Hit) PXI25 $\quad$ Page 2 


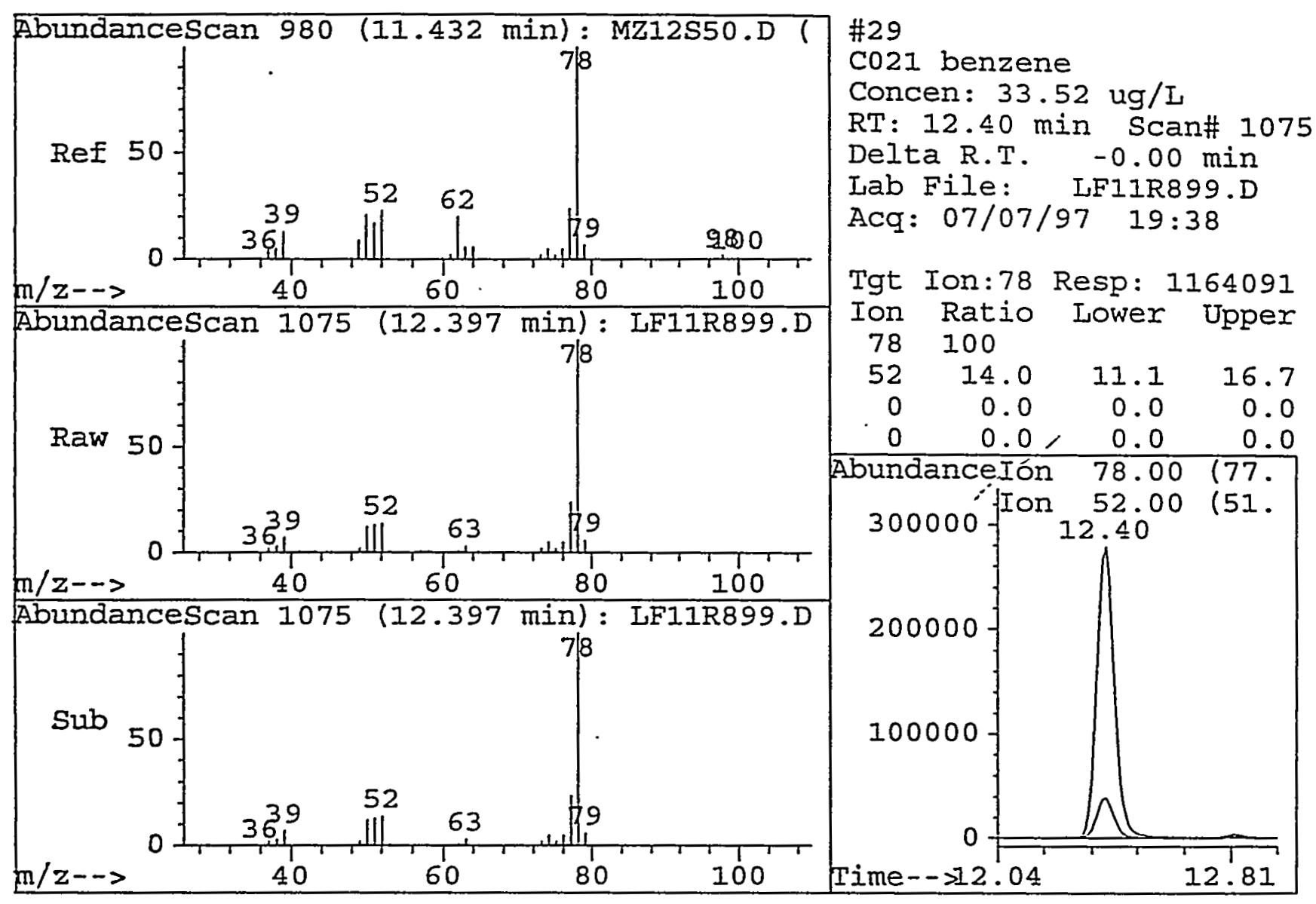

134 


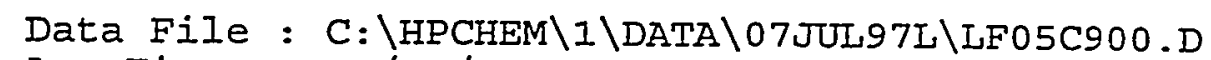

Acq Time : 07/07/97 16:27

Sample : 97C02900 NAP-R1-S2-B

Misc : 97C-0208-01 8260 WATER

Quant Time: Jul 20 13:02 1997

Operator: $\mathrm{CQC}$

Inst : 5971-L

Multiplr: 1.00

Method : J: \L\METHODS \L826ARWA.M

Title : VOA COMPOUND LIST

Last Update : Wed Jun 25 09:14:05 1997

Response via : Multiple Level Calibration

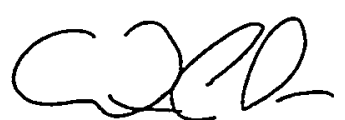

- $2-28-92$

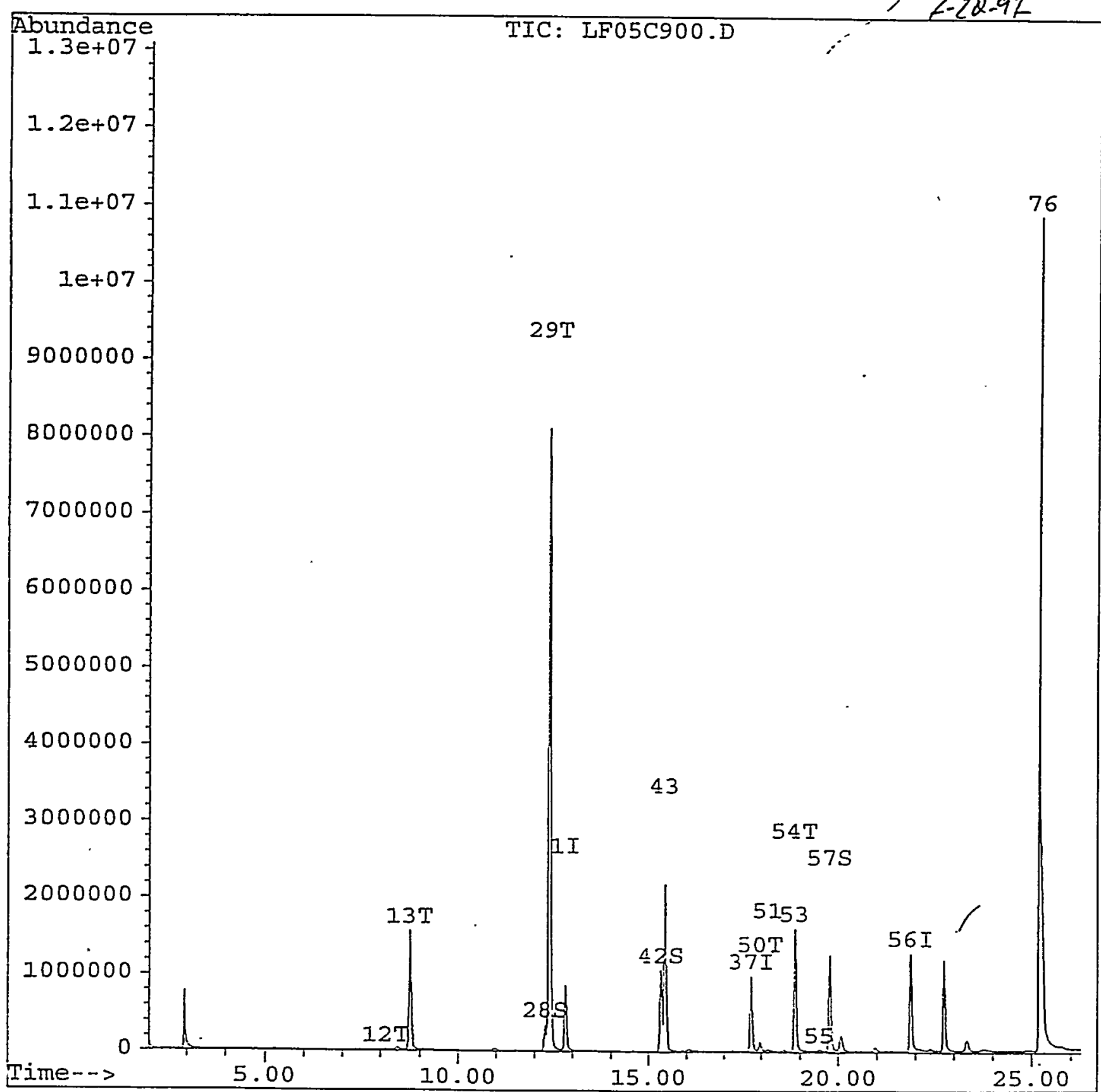


Data File : C: \HPCHEM \1\DATA $107 \pi U L 97 L \backslash L F 05 C 900 . D$

Acq Time: 07/07/97 16:27

Sample : 97C02900 NAP-R1-S2-B

MisC : 97C-0208-01 8260 WATER

Quant Time: Jul 20 13:02 1997

Method : J:\I\METHODS \L826ARWA.M

Title : VOA COMPOUND IIST

Last Update : Wed Jun 25 09:14:05 1997

Response via : Multiple Level Calibration
Operator: $\mathrm{CQC}$

Inst : 5971-L

Multiplr: 1.00
Internal standards

1) $\mathrm{CIOI}$ Flourobenzene

37) CIO2 Chlorobenzene-d5

56) CIO3 1,4-Dichlorobenzene-d

System Monitoring Compounds

28) CS15 1,2-dichloroethane-d4

42) CS05 toluene-d8

57) CSIO 4-bromofluorobenzene

Target Compounds

2) $\mathrm{COO2}$ dichlorodifluorometha

3) $\mathrm{COO3}$ chloromethane

4) $\mathrm{C004}$ vinyl chloride

5) C005 bromomethane

6) C006 chloroethane

7) $\mathrm{C007}$ trichlorofluoromethan

B) $C 071$ acrolein

9) $\operatorname{CoOB} 1,1$-dichloroethene

10) $\mathrm{C} 063$ freon 113

11) Co09 acetone

12) C010 carbon disulfide

13) COII methylene chloride

14) C072 acrylonitrile

15) C012 trans-1,2-Dichloroeth

16) C305 methyl-t-butyl-ether

17) $\mathrm{C} 013$ cis-1,2-dichloroethen

18) C055 total-I,2-dichloroeth

19) COI4 I,1-dichloroethane

20) C015 2,2-dichloropropane

21) C110 2-butanone

22) CIOI bromochloromethane

23) $\mathrm{C} 016$ chloroform

24) $\operatorname{Co17} 1,1,1-T r i c h l o r o e t h a n e$

25) $\operatorname{CO18~1,1-dichloropropene~}$

26) Co19 1,2-dichloroethane

27) C020 carbon tetrachloride

29) C021 benzene

30) C022 trichloroethene

31) C023 1,2-dichloropropane

32) C024 dibromomethane

33) C025 bromodichloromethane

34) C026 2-chloroethylvinyleth

35) C027 cis-1,3-dichloroprope
R.T. QIon

$\begin{array}{rrr}12.82 & 96 & 1788786 \\ 17.73 & 117 & 1381705 \\ 21.87 & 152 & 1035648 \\ & & \\ 12.27 & 65 & 483729 \\ 15.34 & 98 & 1675405 \\ 19.77 & 95 & 1190696\end{array}$

$0.00 \quad 85$

$0.00 \quad 50$

$0.00 \quad 62$

$0.00 \quad 94$

$0.00 \quad 64$

$0.00 \quad 101$

$0.00 \quad 56$

0.0096

$0.00 \quad 151$

$0.00 \quad 43$

$8.13 \quad 76$

8.74

16366

0.00

84

0.00

53

1729399

0.00

96

$0.00 \quad 96$

0.0096

$0.00 \quad 63$

$0.00 \quad 77$

$0.00 \quad 72$

$0.00 \quad 128$

$0.00 \quad 83$

$0.00 \quad 97$

$0.00 \quad 75$

$0.00 \quad 62$

$0.00 \quad 117$

12.40

17
78

0.00130

0.0063

$0.00 \quad 93$

0.0083

$0.00 \quad 63$

$0.00 \quad 75$
Response Conc Units Area\%

$50.00 \mathrm{ug} / \mathrm{I} \quad 0.00$

$50.00 \mathrm{ug} / \mathrm{I} \quad-0.02$

$50.00 \mathrm{ug} / \mathrm{I} \quad 0.00$

$\div$ Recovery

$41.84 \mathrm{ug} / \mathrm{L} \quad 83.67 \%$

$47.14 \mathrm{ug} / \mathrm{I} \quad 94.28 \%$

$43.02 \mathrm{ug} / \mathrm{I} \quad 86.04 \%$

Not Detected

Not Detected

Not Detected

Not Detected

Not Detected

Not Detected

Not Detected

Not Detected

Not Detected

Not Detected

$0.59 \mathrm{ug} / \mathrm{I}$

$162.09 \mathrm{ug} / \mathrm{I} \#$

Not Detected

Not Detected

Not Detected

Not Detected

Not Detected

Not Detected

Not Detected

Not Detected

Not Detected

Not Detected

Not Detected

Not Detected

Not Detected

Not Detected

$470.91 \mathrm{ug} / \mathrm{L}$

Not Detected

Not Detected

Not Detected

Not Detected

Not Detected

Not Detected
Qvalue

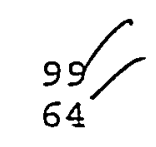

100Dil

$(N R)=$ Not Reported $(S P)=$ Spiked analyte $(m)=$ manual integration

(*) Does not meet EPA spectral criteria (False Hit) PXI25 Page 1 
Quantitation Report

Data File : C: \HPCHEM \IDATA $107 J U L 97 L \backslash L F 05 C 900 . D$

Acq Time : 07/07/97 16:27

Sample : 97C02900 NAP-R1-S2-B

Misc : 97C-0208-01 8260 WATER

Quant Time: Jul 20 13:02 1997

Operator: $\mathrm{CQC}$

Inst : 5971-I

Multiplr: 1.00

Method : J: \I\METHODS \L826ARWA.M

Title : VOA COMPOUND IIST

Last Update : Wed Jun 25 09:14:05 1997

Response via : Multiple Level Calibration

Compound

\begin{tabular}{|c|c|c|}
\hline $36 !$ & $\mathrm{C} 205$ & 4-methyl-2-pentanone \\
\hline 38) & $\mathrm{C} 028$ & trans-1,3-dichloropro \\
\hline 39) & C029 & $1,1,2$-trichloroethane \\
\hline 40$)$ & $\mathrm{C} 210$ & 2-hexanone \\
\hline 41) & $\mathrm{CO} 30$ & 1,2-Dibromoethane \\
\hline 3) & $\operatorname{Co31}$ & Toluene \\
\hline 44) & $\mathrm{CO32}$ & 1,3-dichloropropane \\
\hline 45) & $\mathrm{C033}$ & dibromochloromethane \\
\hline 46) & $\mathrm{C} 034$ & bromoform \\
\hline 47) & C035 & tetrachloroethene \\
\hline 48) & $\mathrm{C036}$ & chlorobenzene \\
\hline 49) & C037 & $1,1,1,2$-tetrachloroet \\
\hline 50) & $\mathrm{CO} 38$ & ethylbenzene \\
\hline 51) & $\operatorname{co39}$ & $\mathrm{m}, \mathrm{p}$-xylene \\
\hline 52) & $c 0 \leqslant 0$ & $0-x y l e n e$ \\
\hline 53) & C250 & total xylene \\
\hline 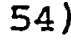 & $\mathrm{CO41}$ & styrene \\
\hline 55) & $\mathrm{C} 042$ & isopropylbenzene \\
\hline 5B. & $\mathrm{CO} 43$ & $1,1,2,2$-tetrachloroet \\
\hline 59) & $\mathrm{CO} \triangle 4$ & bromobenzene \\
\hline 60) & $\mathrm{CO} 45$ & 1,2;3-trichloropropan \\
\hline 61) & $C 046$ & n-propylbenzene \\
\hline 62) & $\mathrm{C} 047$ & $1,3,5$-trimethylbenzen \\
\hline 63) & $\mathrm{CO} 48$ & 2-chlorotoluene \\
\hline 64) & $\mathrm{CO} 49$ & 4-chlorotoluene \\
\hline 65) & $\mathrm{C} 050$ & tert-butylbenzene \\
\hline 66) & $\cos 1$ & $1,2,4$-trimethylbenzen \\
\hline 67) & $\mathrm{CO52}$ & sec-Butylbenzene \\
\hline 68) & $\mathrm{CO53}$ & p-Isopropyltoluene \\
\hline 69) & C054 & 1,3-Dichlorobenzene \\
\hline 0) & $\operatorname{CO55}$ & 1,4-Dichlorobenzene \\
\hline 71) & $\mathrm{C} 056$ & n-Butylbenzene \\
\hline 72) & $\cos 7$ & 1,2-Dichlorobenzene \\
\hline 73) & $\mathrm{COSB}$ & 1,2-Dibromo-3-chlorop \\
\hline 4) & $\cos 9$ & $1,2,4$-Trichlorobenzen \\
\hline 5) & $\mathrm{C060}$ & Hexachlorobutadiene \\
\hline 7 & C06I & Naphthalene \\
\hline & & 1,2,3-Trichlorobenzen \\
\hline
\end{tabular}

R.T. QIon Response Conc Unit Qvalue

$\begin{array}{lll}0.00 & 43 & \text { Not Detected } \\ 0.00 & 75 & \text { Not Detected } \\ 0.00 & 97 & \text { Not Detected } \\ 0.00 & 43 & \text { Not Detected }\end{array}$

$0.00 \quad 107$

$15.45 \quad 92$

$0.00 \quad 76$

2315525

$0.00 \quad 129$

$0.00 \quad 173$

0.00164

$0.00 \quad 112$

$0.00 \quad 131$

$17.97 \quad 106$

$18.18 \quad 106$

$0.00 \quad 106$

$18.85 \quad 106$

$18.86 \quad 104$

$19.51 \quad 105$

$0.00 \quad 83$

$0.00 \quad 156$

$0.00 \quad 75$

$0.00 \quad 91$

$0.00 \quad 105$

$0.00 \quad 91$

$0.00 \quad 91$

$0.00 \quad 119$

$0.00 \quad 105$

$0.00 \quad 105$

$0.00 \quad 119$

$0.00 \quad 146$

$0.00 \quad 146$

$0.00 \quad 91$

$0.00 \quad 146$

$0.00 \quad 75$

$0.00 \quad 180$

$0.00 \quad 225$

$25.20 \quad 128 \quad 11790777$

68225

11985

15251

2053872

38417

$0.00 \quad 180$
Not

Not Detected

$91.81 \mathrm{ug} / \mathrm{I}$

Not Detected

Not Detected

Not Detected

Not Detected

Not Detected

Not Detected

$4.66 \mathrm{ug} / \mathrm{L}$

$.0 .66 \mathrm{ug} / \mathrm{I}$

Not Detected

$0.84 \mathrm{ug} / \mathrm{L} \#$

$65.57 \mathrm{ug} / \mathrm{L} \#$

$0.72 \mathrm{ug} / \mathrm{L}$

Not Detected

Not Detected

Not Detected

Not Detected

Not Detected

Not Detected

Not Detected

Not Detected

Not Detected

Not Detected

Not Detected

Not Detected

Not Detected

Not Detected

Not Detected

Not Detected

Not Detected

Not Detected

$275.34 \mathrm{ug} / \mathrm{L}$ mirient oDih Not Detected

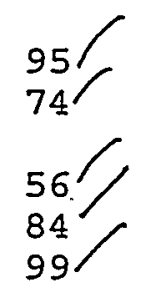




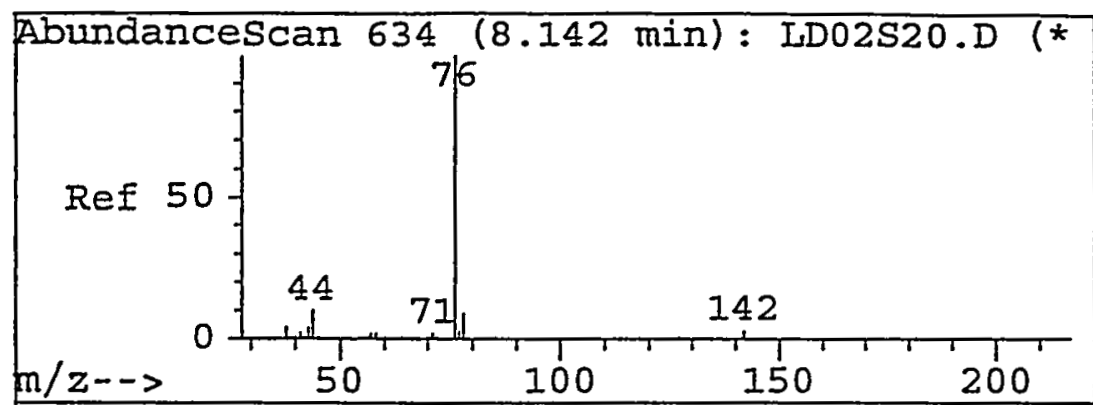

Abundancescan $633(8.133 \mathrm{~min}):$ LF05C900.D (

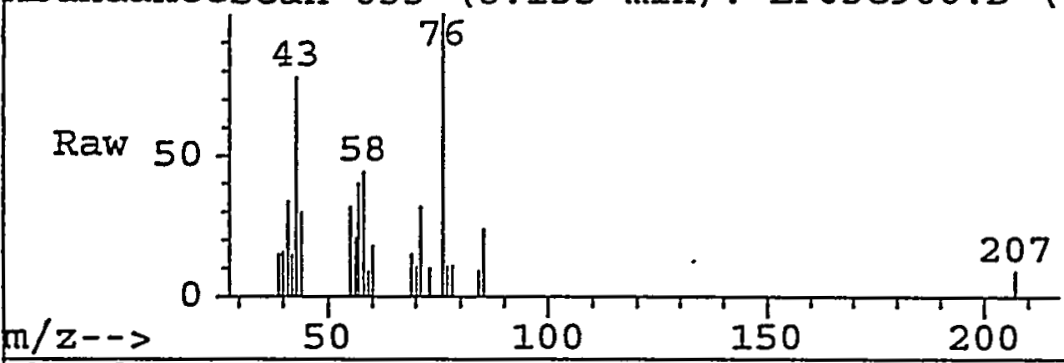

Abundancescan $633(8.133 \mathrm{~min}):$ LF05C900.D (
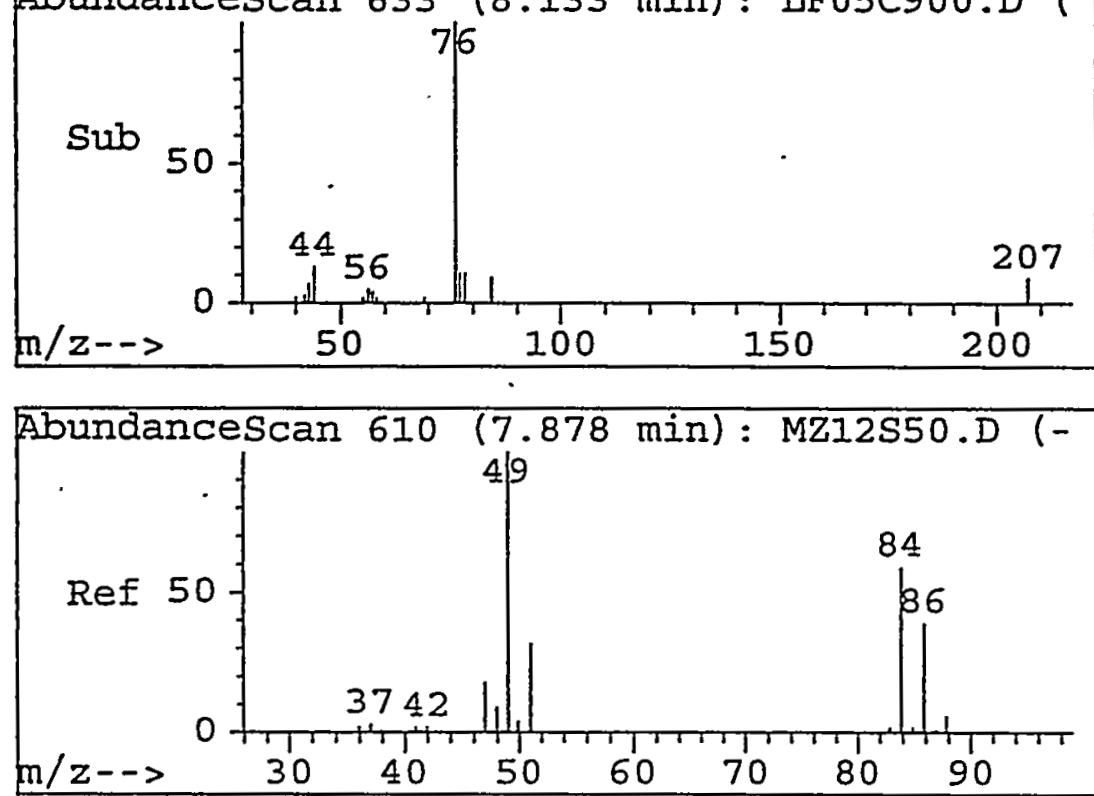

Abundancescan 696 (8.741 min): LF05C900.D (

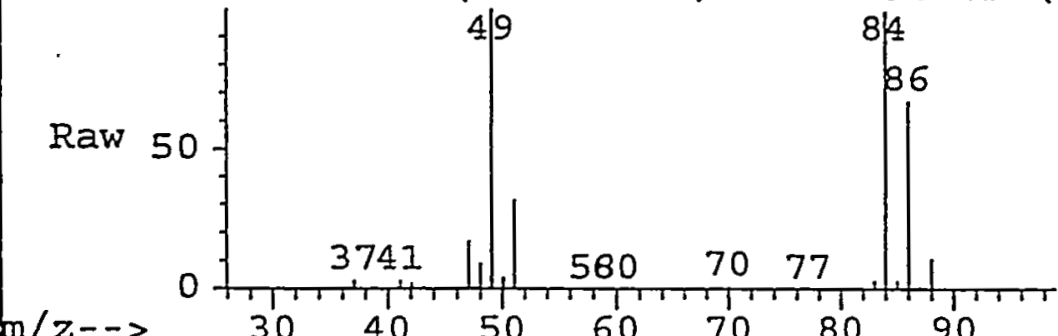

Abundancescan $696(8.741 \mathrm{~min}):$ LF05C900.D (

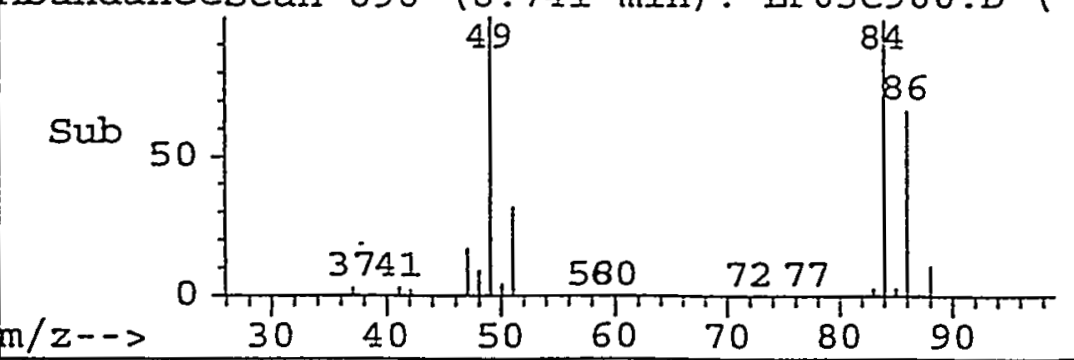

\#12

COI0 carbon disulfide

Concen: $0.59 \mathrm{ug} / \mathrm{I}$

$\mathrm{RT}: 8.13 \mathrm{~min}$ Scan\# 633

Delta R.T. $\quad-0.01 \mathrm{~min}$

Lab File: LF05C900.D

Acq: 07/07/97 16:27

Tgt Ion:76 Resp: 16366

Ion Ratio Lower Upper

$76 \quad 100$

$\begin{array}{llll}78 & 10.3 & 0.0 & 29.3\end{array}$

$\begin{array}{llll}44 & 16.2 & 0.0 & 35.9\end{array}$

$00.0 / 0.0 \quad 0.0$

Abundancéón 76.00 (75.

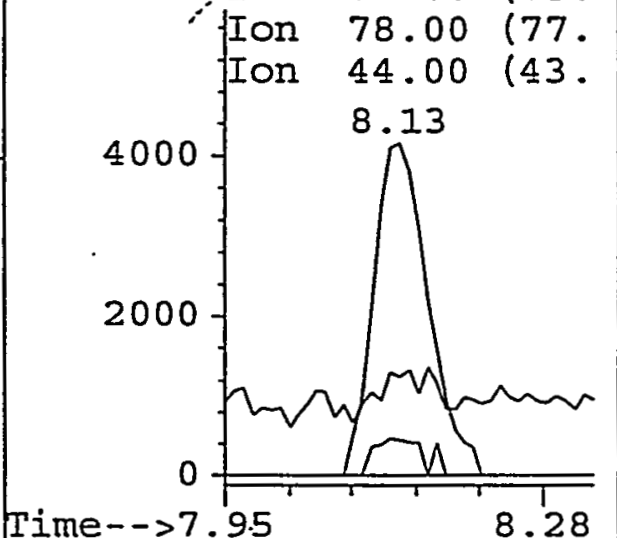

\#13

C011 methylene chloride

Concen: $162.09 \mathrm{ug} / \mathrm{L}$

RT: $8.74 \mathrm{~min}$ Scan\# 696

Delta R.T. $\quad-0.01 \mathrm{~min}$

Lab File: LF05C900.D

Acq : $07 / 07 / 97 \quad 16: 27$

Tgt Ion:84 Resp: 1729399

Ion Ratio Lower Upper

84100

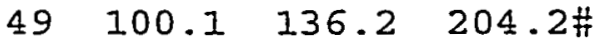

$51 \quad 32.0 \quad 43.0 \quad 64.4 \#$

$\begin{array}{llll}86 & 66.5 & 52.6 & 79.0\end{array}$

AbundanceIon $84.00 \quad(83$.

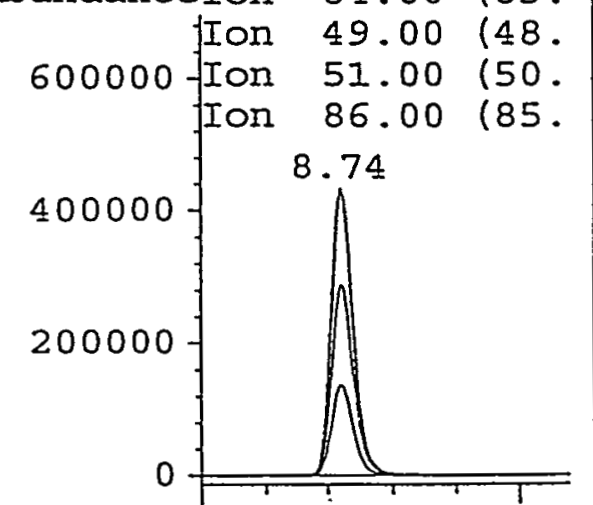

Time- $->8.37$ 


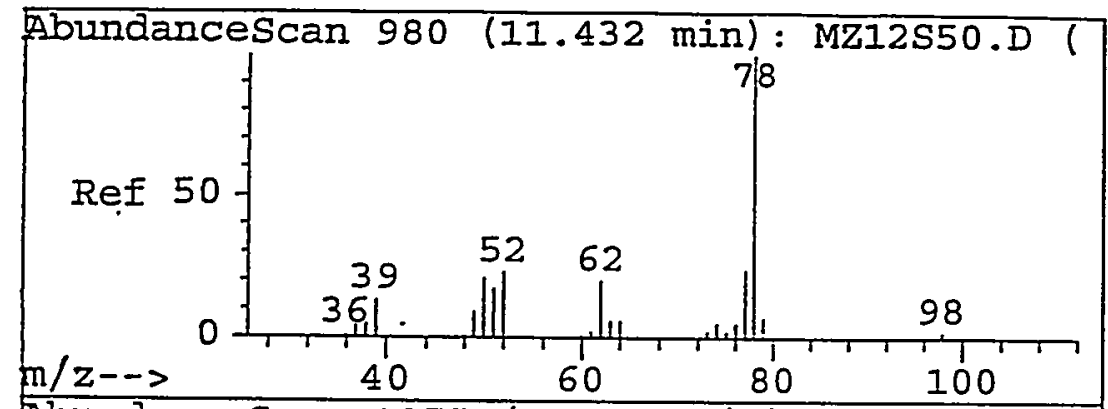

Abundancescan 1075 (12.397 $\mathrm{min}):$ LF05C900.D

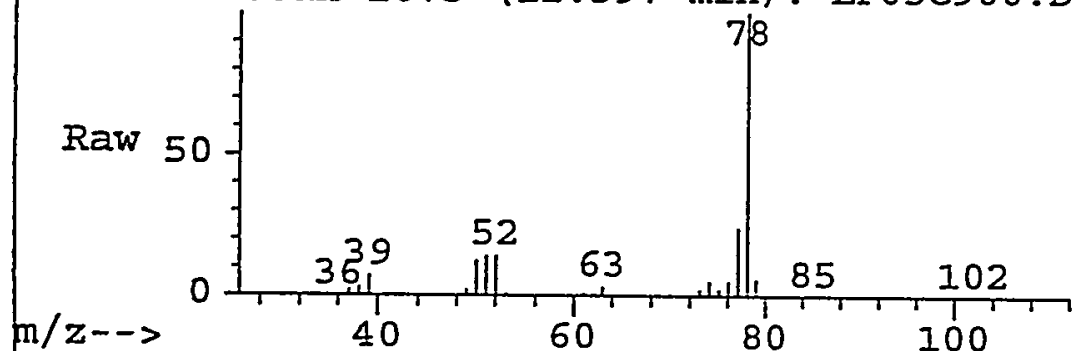

abundancescan 1075 (12.397 min): LF05C900.D
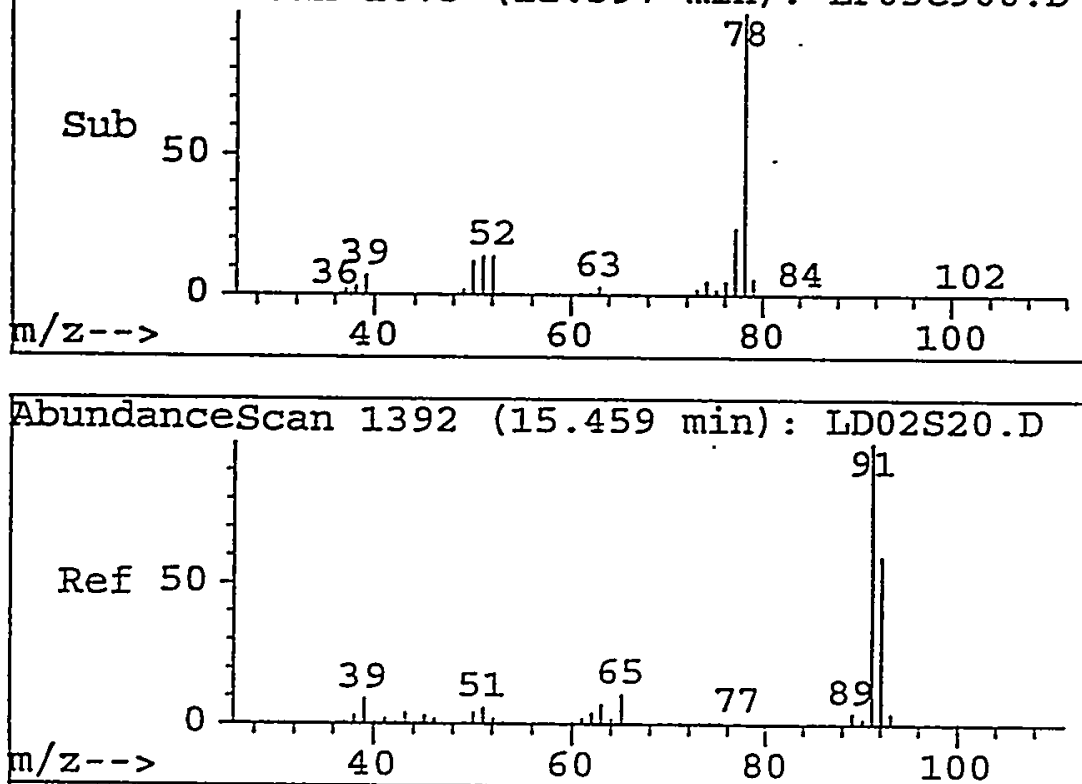

Abundancescan 1391 (15.447 min): LF05C900.D

$m / z-->$

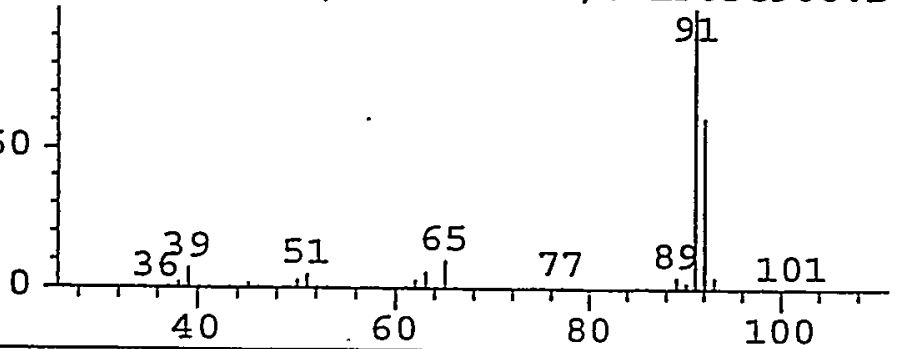

Abundancescan 1391 (15.447 min): LF05C900.D

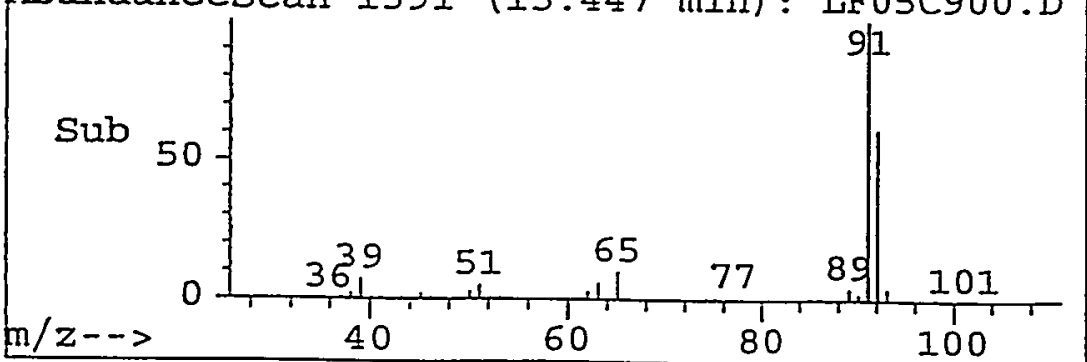

\#29

C021 benzene

Concen: $470.91 \mathrm{ug} / \mathrm{L}$

RT: $12.40 \mathrm{~min}$ scan\# 1075

Delta R.T. $\quad-0.00 \mathrm{~min}$

Lab File: LF05C900.D

Acq: 07/07/97 16:27

Tgt Ion:78 Resp:16886259

Ion Ratio Lower Upper

78100

$\begin{array}{llll}52 & 13.8 & 11.1 & 16.7\end{array}$

$\begin{array}{llll}0 & 0.0 & 0.0 & 0.0\end{array}$

$\begin{array}{llll}0 & 0.0 & 0.0 & 0.0\end{array}$

Abundancelon 78.00 (77.

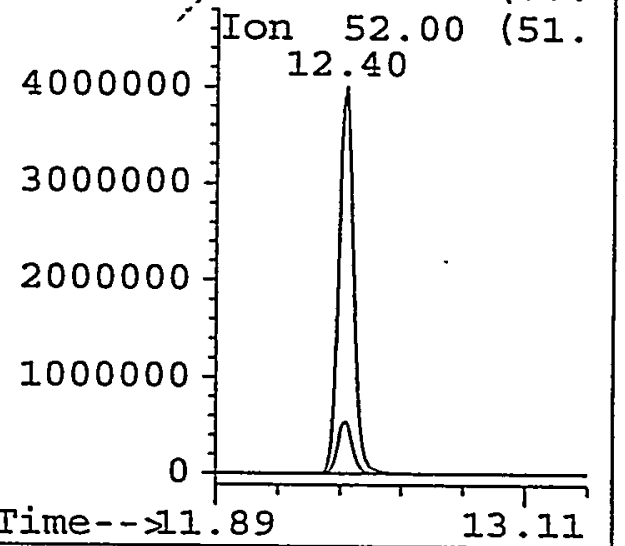

Time-->11.89

13.11

\#43

C031 Toluene

Concen: $91.81 \mathrm{ug} / \mathrm{L}$

$\mathrm{RT}: 15.45 \mathrm{~min}$ Scan\# 1391

Delta R.T. $\quad-0.01 \mathrm{~min}$

Lab File: LF05C900.D

Acq : 07/07/97 16:27

Tgt Ion:92 Resp: 2315525

Ion Ratio Lowex Upper

92100

$\begin{array}{llll}91 & 165.2 & 132.4 & 198.6\end{array}$

$\begin{array}{llll}65 & 17.3 & 15.8 & 23.8\end{array}$

$\begin{array}{rrrr}0 & 0.0 & 0.0 & 0.0\end{array}$

AbundanceIon 92.00 (91.

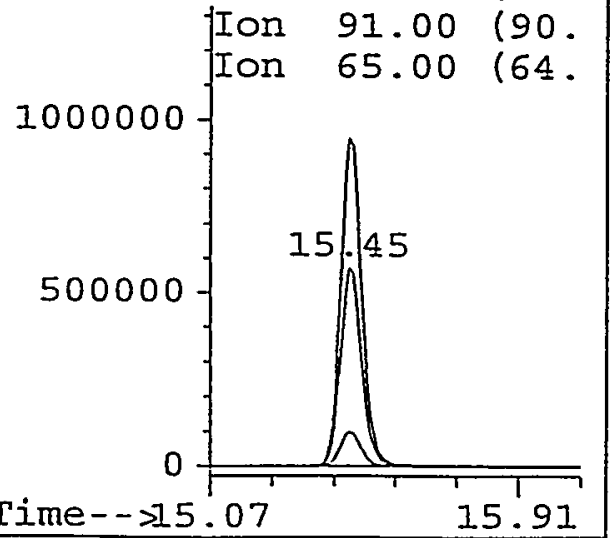




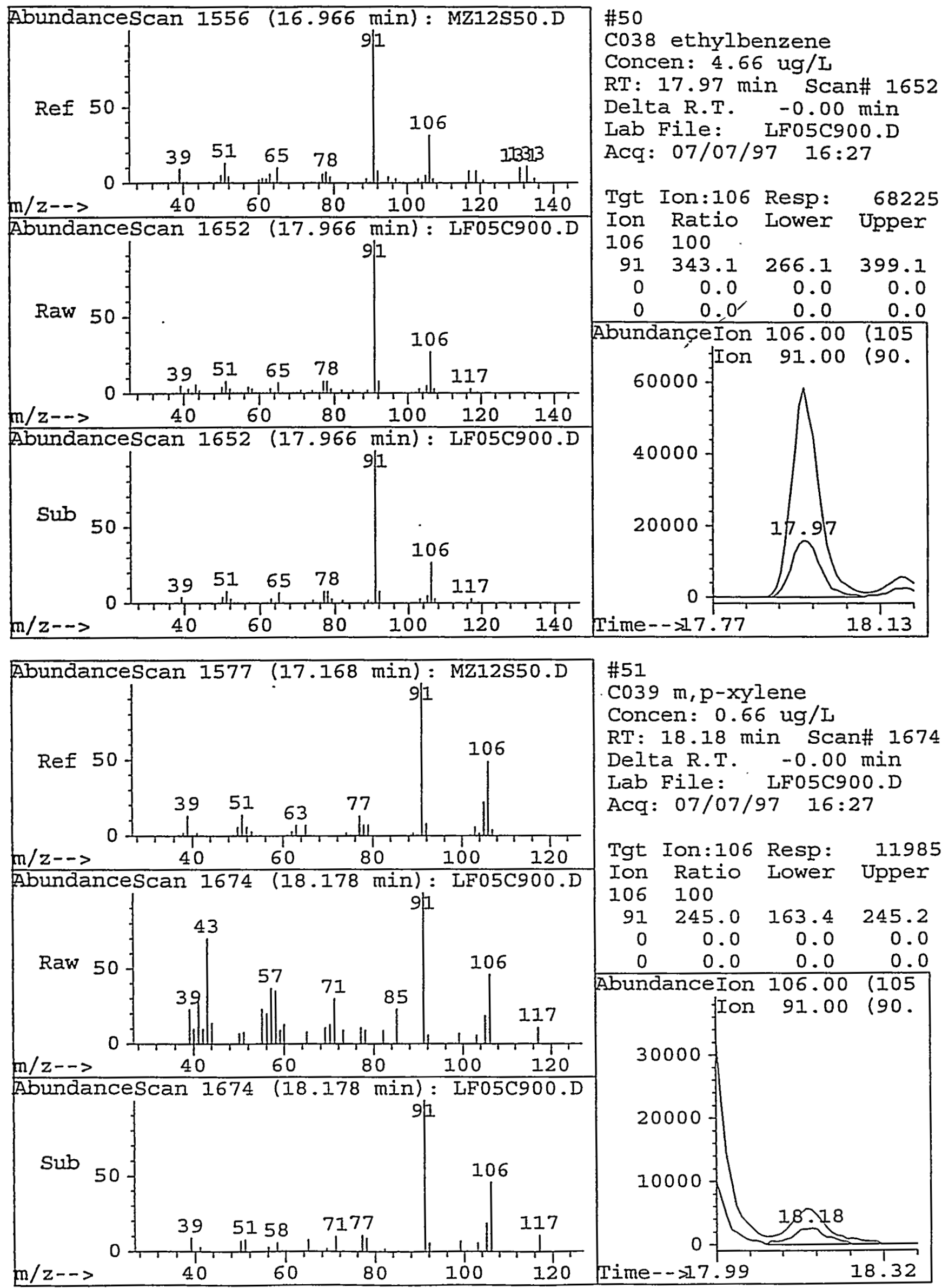




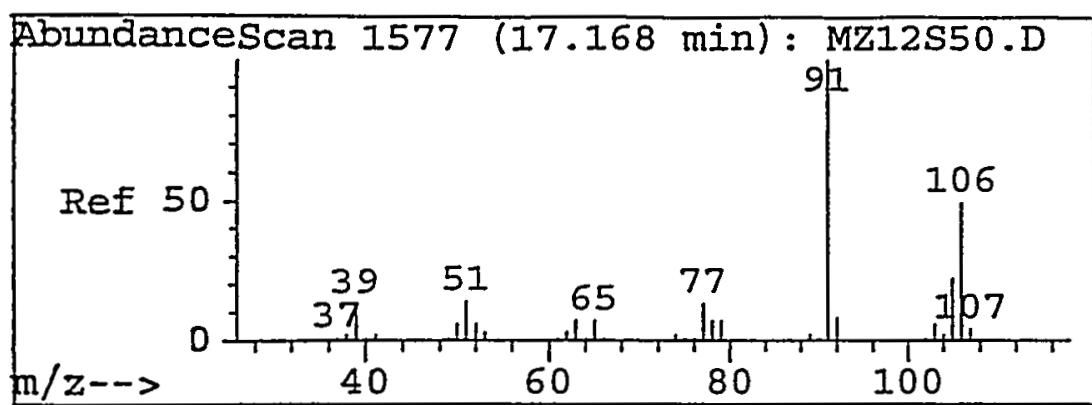

abundancescan 1744 (18.854 min): LF05C900.D

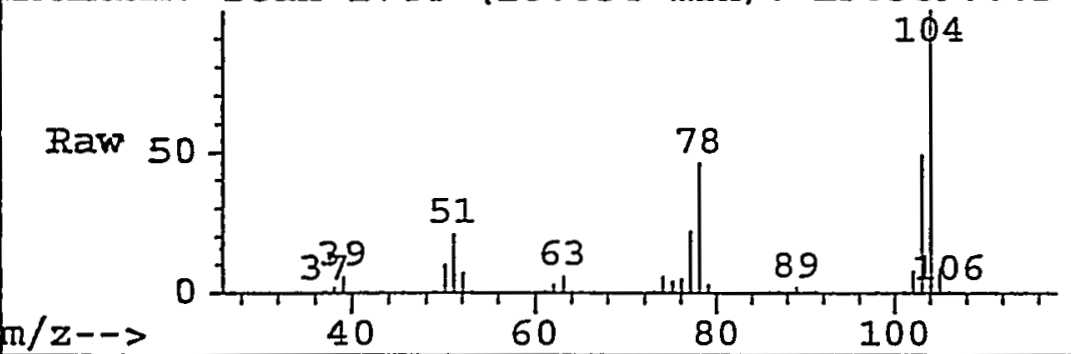

Abundancescan $1744(18.854 \mathrm{~min}):$ LF05C900.D
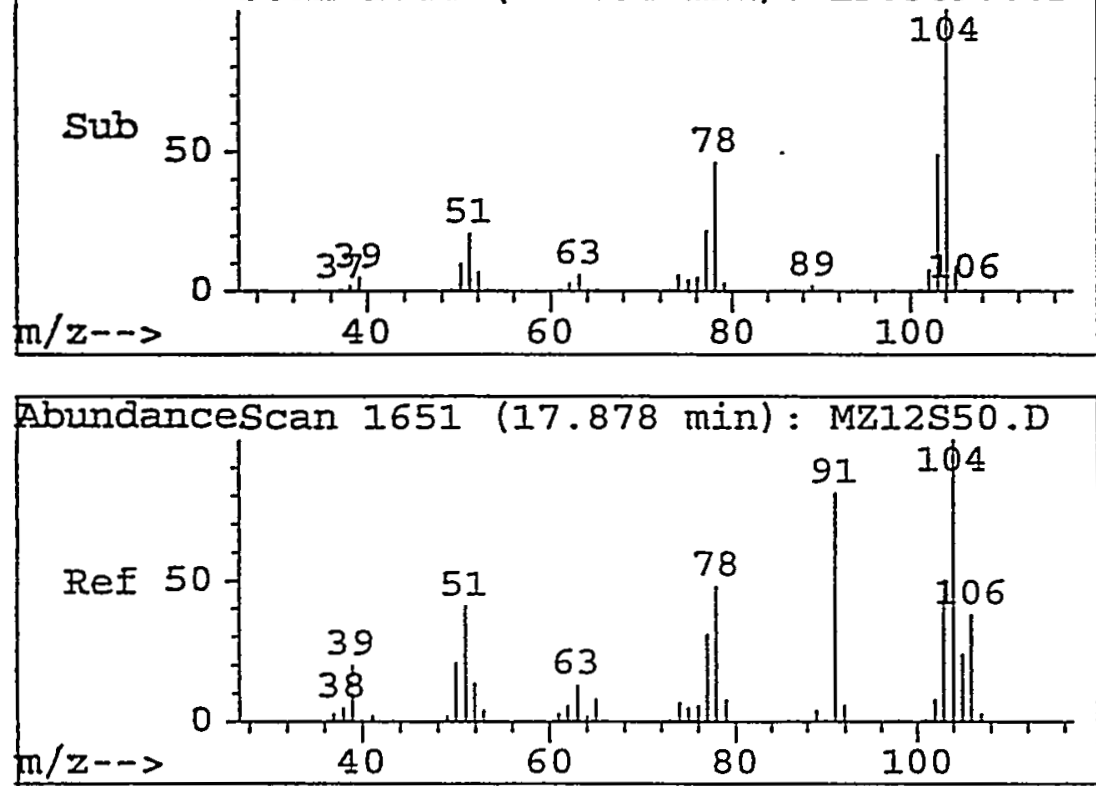

Abundancescan 1745 (18.864 min): LF05C900.D

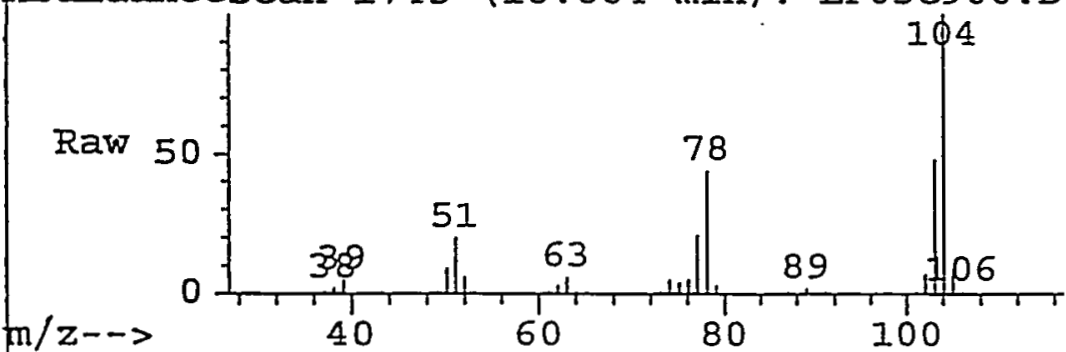

Abundancescan 1745 (18.864 min): LF05C900.D

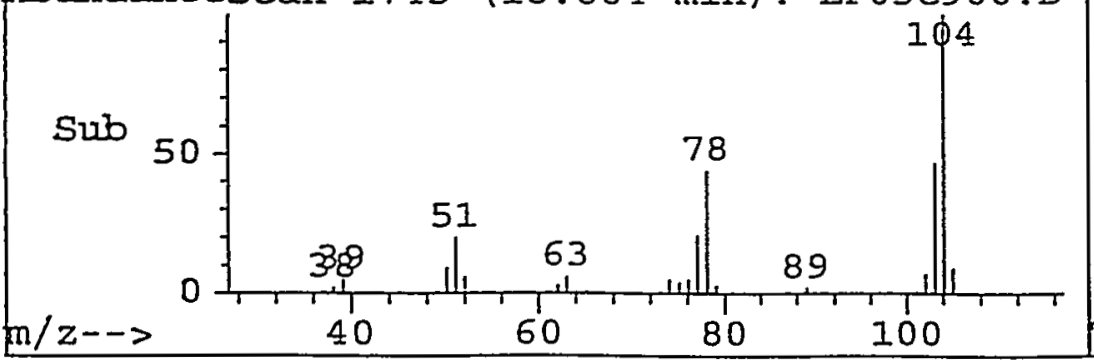

\#53

C250 total xylene Concen: $0.84 \mathrm{ug} / \mathrm{L}$ RT: $18.85 \mathrm{~min}$ Scan\# 1744

Delta R.T. $\quad-0.06 \mathrm{~min}$ Lab File: LF05C900.D

Acq: $07 / 07 / 97 \quad 16: 27$

Tgt Ion:106.05 Resp: 15251

Ion Ratio Lower Upper 106100

$91 \quad 147.2 \quad 174.1 \quad 261.1 \#$
0
0.0
0.0
0.0

AbundanceIon 106.05 (105

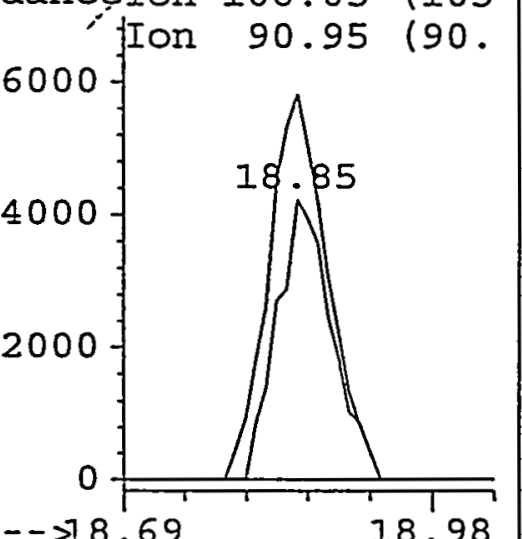

Time-- $>18.69$

18.98

\#54

C041 styrene

Concen: $65.57 \mathrm{ug} / \mathrm{L}$

$\mathrm{RT}: 18.86 \mathrm{~min}$ Scan\# 1745

Delta R.T. $\quad-0.07 \mathrm{~min}$

Lab File: LF05C900.D

Acq: $07 / 07 / 97 \quad 16: 27$

Tgt Ion:104 Resp: 2053872

Ion Ratio Lower Upper

104100

$\begin{array}{llll}103 & 47.7 & 42.1 & 63.1\end{array}$

$\begin{array}{llll}78 & 44.3 & 38.4 & 57.6\end{array}$

$51 \quad 20.0 \quad 35.4 \quad 53.0 \#$

AbundanceIon 104.00 (103

800000 IIon $103.00 \quad(102$

Ion 78.00177.

600000 Ion $51.00(50$. 18.86

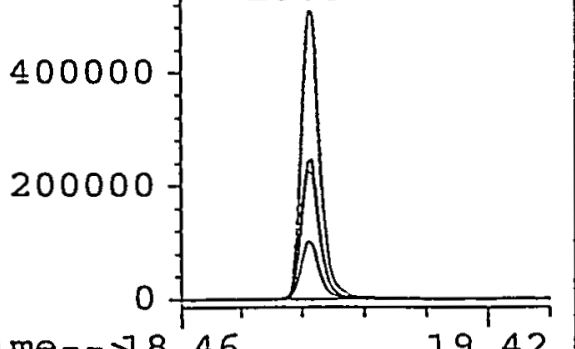

Time-->18. 46 19.42 


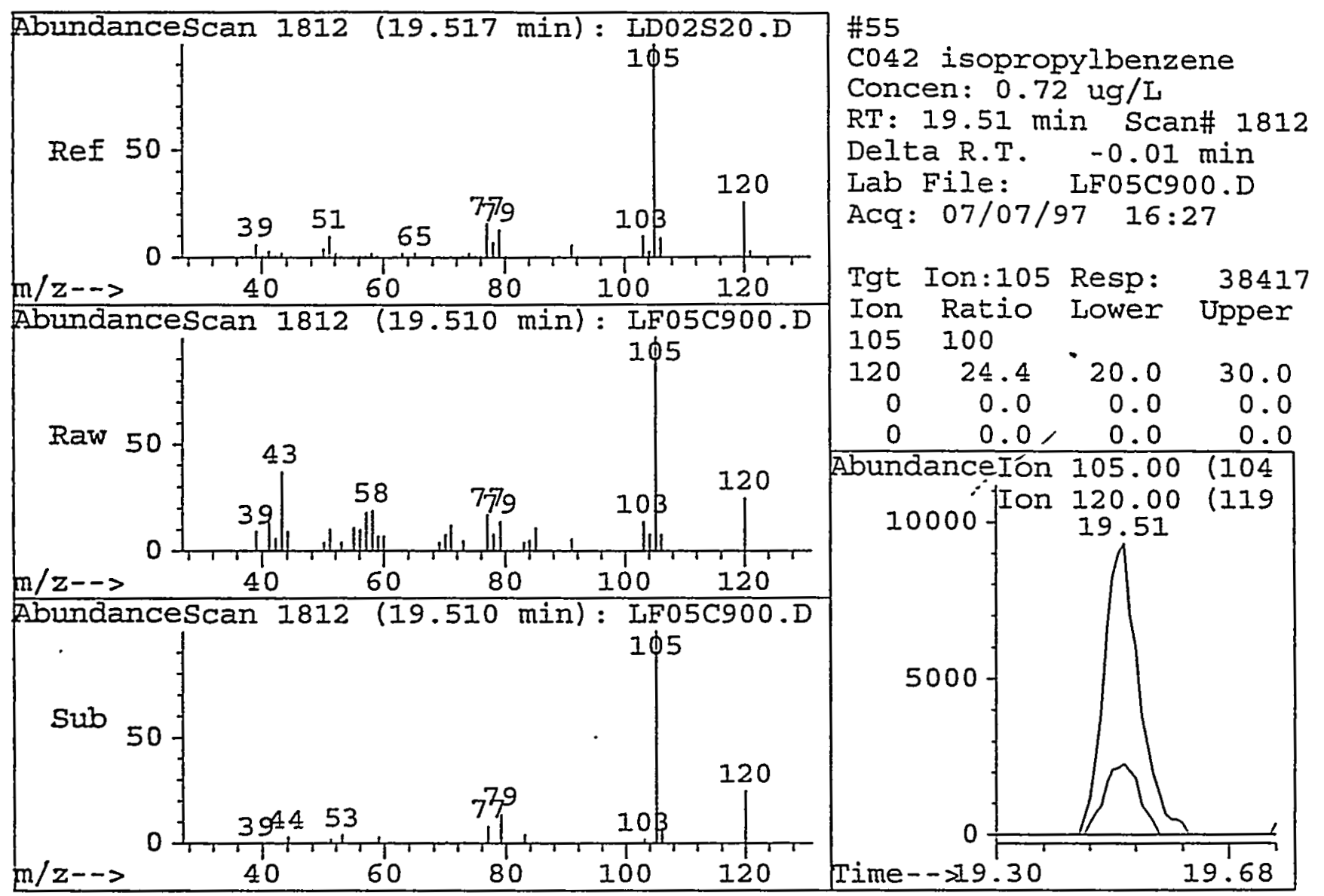




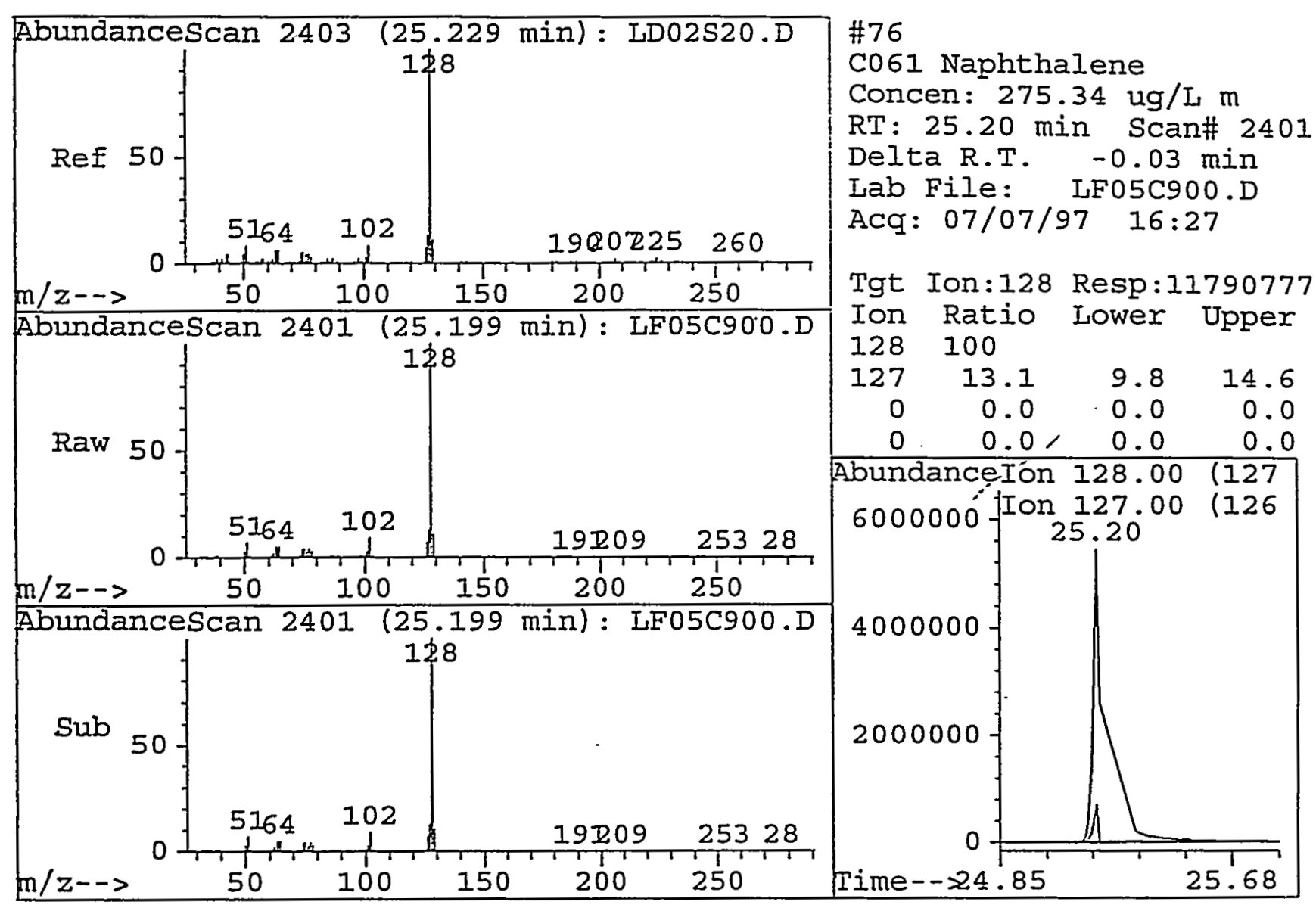


Quantitation Report

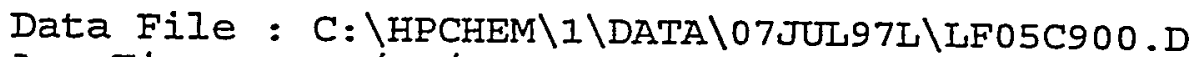

Acq Time : 07/07/97 16:27

Sample : 97C02900 NAP-R1-S2-B

Misc : 97C-0208-01 8260 WATER

Quant Time: Jul 20 13:02 1997

Operator: $\mathrm{CQC}$

Inst : 5971-I Multiplr: 1.00

Method : J:\I\METHODS \I826ARWA.M

Title : VOA COMPOUND LIST

Iast Update, : Wed Jun 25 09:14:05 1997

Response via : Multiple Level Calibration

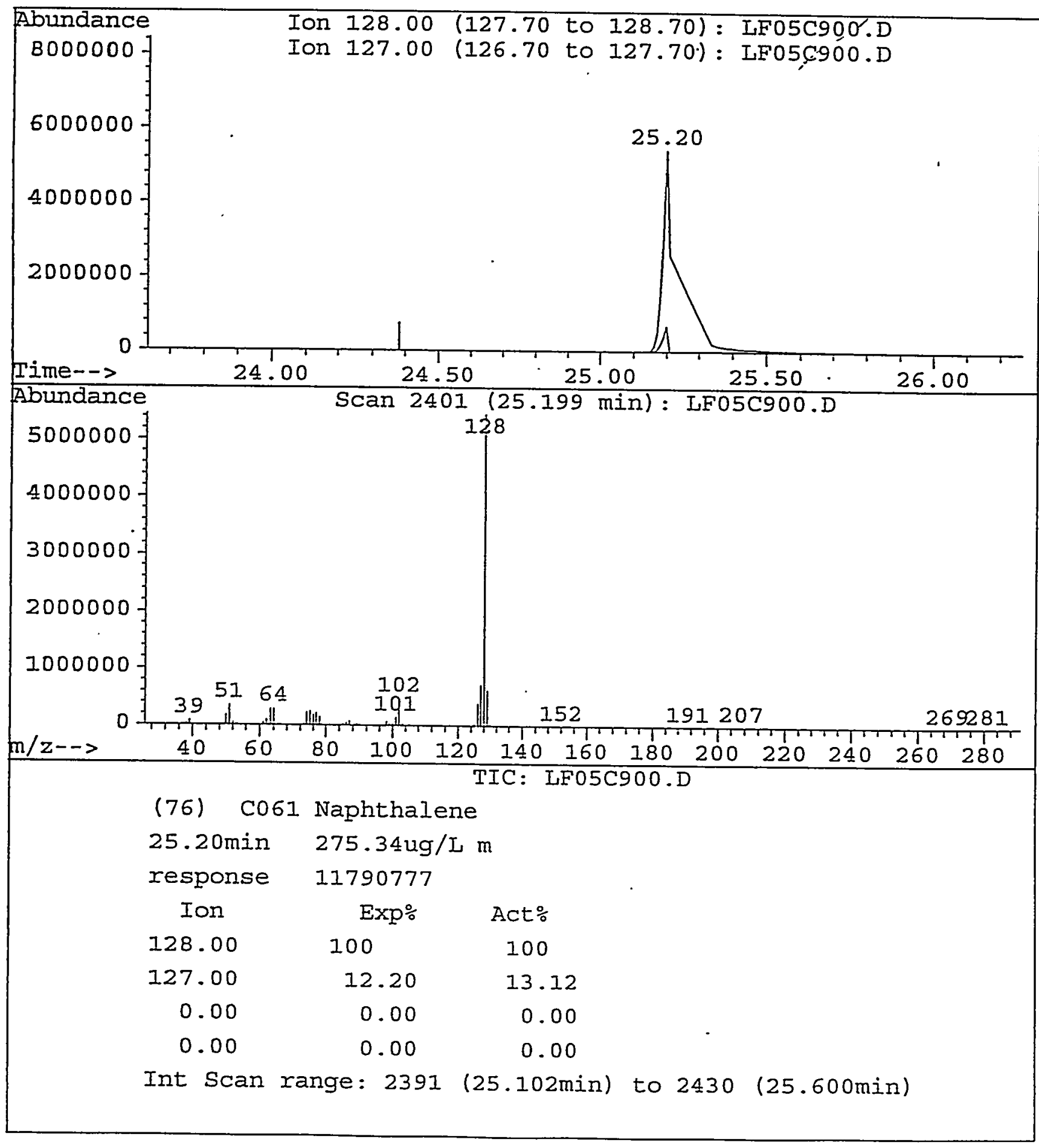




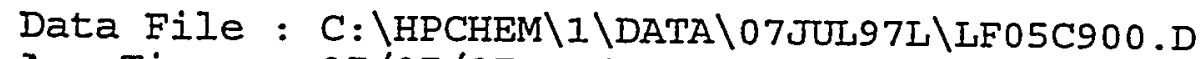
Acq Time
: 07/07/97 16:27
Sample
: 97002900
NAP-R1-S2-B
Misc
: 97C-0208-01 8260 WATER

Operator: CQC

Inst : 5971-L

Multiplr: 1.00

Method : J: \L\METHODS \L826ARWA.M

Title : VOA COMPOUND. LIST

Library : C:\DATABASE\NBS75K.I

R.T. Conc

Area

Relative to ISTD

ISTD Area

$22.74 \quad 42.83 \mathrm{ug} / \mathrm{L}$

4606945

CI03 1,4-Dichlorobentene-d4 5378317

Hit\# of 9

Tentative ID

Ref\# CAS\#

Qual

Indene

2 Benzene, 1-ethynyl-4-methyl-

3 Benzene, 1-propynyl-

4 IH-Indene, 1-chloro-2,3-dihydro-

5 Benzene, 1,2-propadienyl-

$64387000095-13-695.00$

$3388000766-97-2 \quad 91.00$

$64385000673-32-5 \quad 91.00$

$10202035275-62-8 \quad 43.00$

$3387002327-99-3 \quad 37.00$

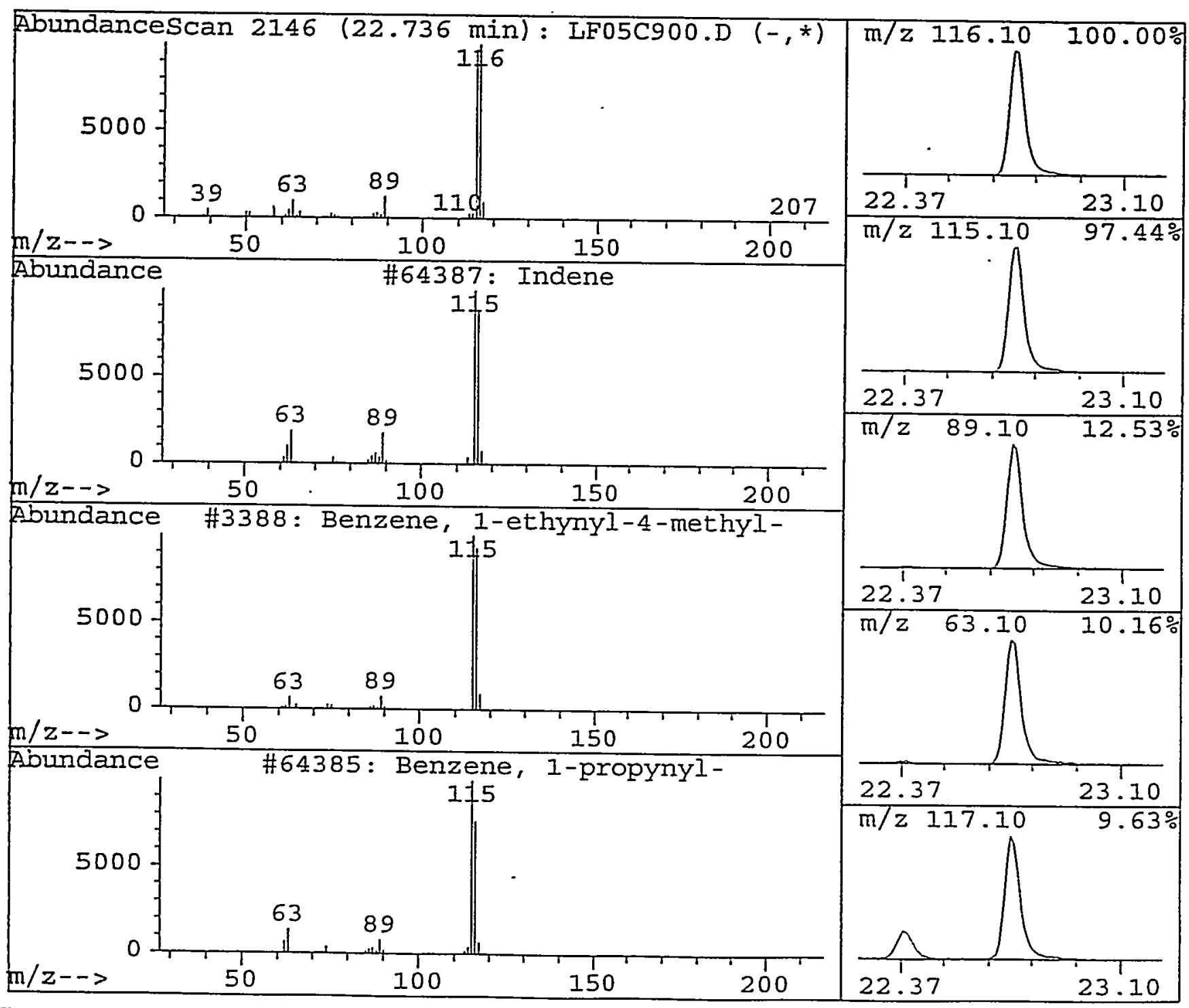

145 
Quantitation Report

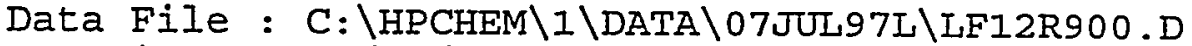

Acq Time : 07/07/97 20:10

Sample : 97C02900 NAP-R1-S2-B

Misc : 97C-0208-01 1:100 Dilution

Quant Time: JuI $2013: 331997$

Operator: $\mathrm{CQC}$

Inst : 5971-I

Multiplr: 1.00

Method $\cdot: \mathrm{J}: \backslash L \backslash M E T H O D S \backslash L 826 A R W A . M$

Title : VOA COMPOUND LIST

Last Update : Wed Jun 25 09:14:05 1997

Response via : Multiple Level Calibration

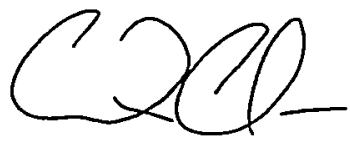

$7 \cdot 20.92$

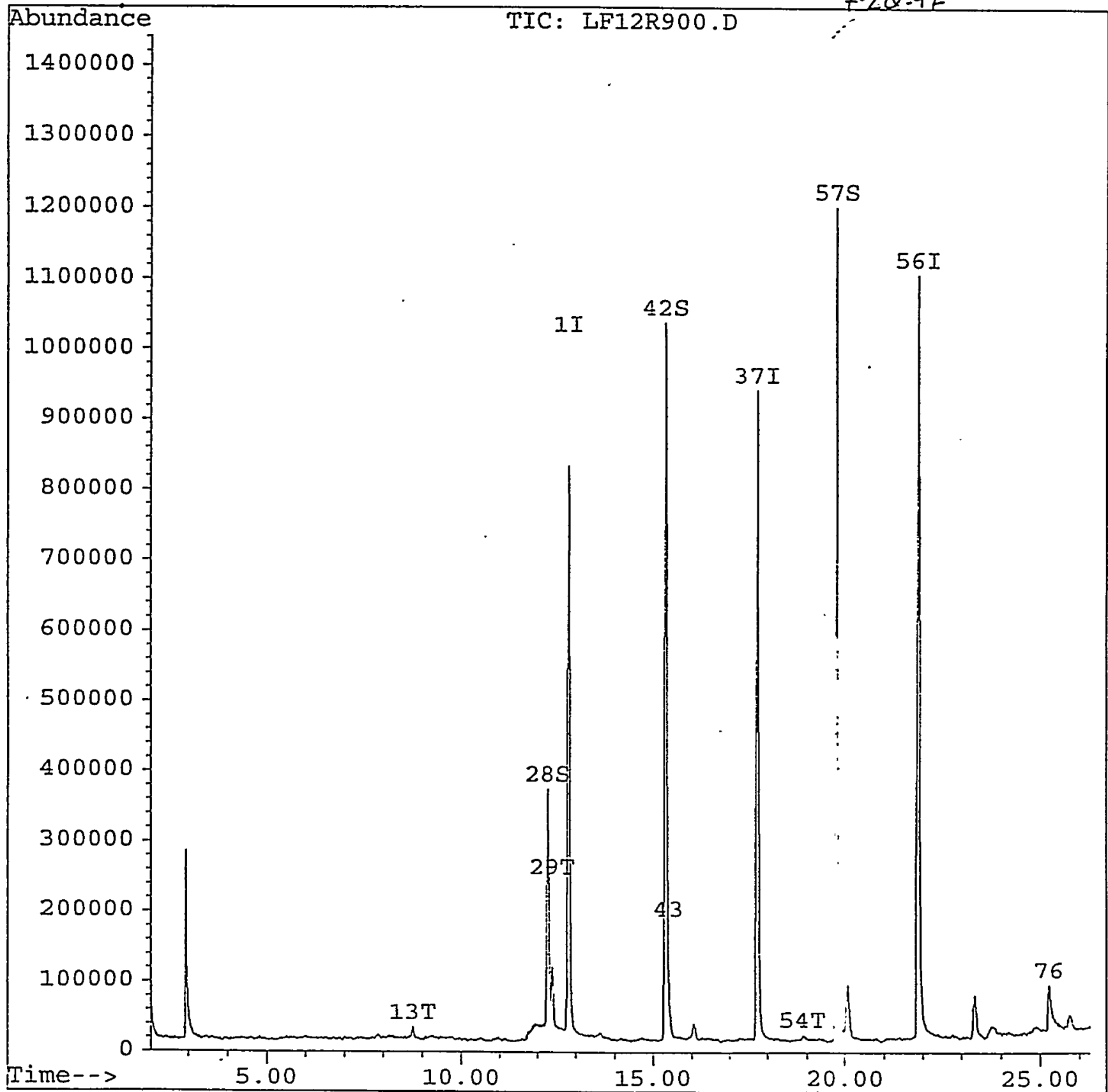

146 


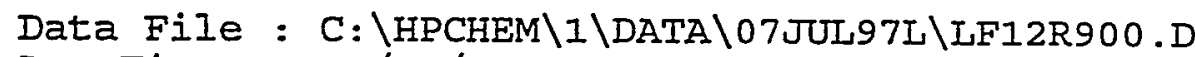

Acq Time : 07/07/97 20:10

Sample : 97C02900 NAP-RI-S2-B

Misc : 97C-0208-01 1:100 Dilution

Quant Time: Jul 20 13:33 1997

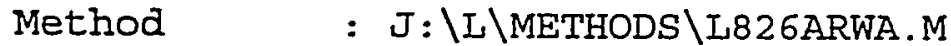

Title : VOA COMPOUND LIST

Last Update : Wed Jun 25 09:14:05 1997

Response via : Multiple Level Calibration
Operator: CQC

Inst $\quad: 5971-\mathrm{L}$

Multiplr: 1.00

Internal standards

R.T. QIon

1) CIOI Flourobenzene

37) CIO2 Chlorobenzene-d5

56) CI03 1,4-Dichlorobenzene-d

System Monitoring Compounds

28) CS15 1,2-dichloroethane-d4

42) Cs05 toluene-d8

57) CSI0 4-bromofluorobenzene

Target Compounds

2) C002 dichlorodifluorometha

3) $\mathrm{CO03}$ chloromethane

4) $\mathrm{COD4}$ vinyl chloride

5) C005 bromomethane

6) 0006 chloroethane

7) $\mathrm{C007}$ trichlorofluoromethan

B) $\mathrm{CO71}$ acrolein

9) C008 1,1-dichloroethene

10) $\mathrm{C} 063$ freon 113

11) C009 acetone

12) C010 carbon disulfide

13) Coll methylene chloride

14) Co72 acrylonitrile

15) $\mathrm{C} 012$ trans-1,2-Dichloroeth

16) $C 305$ methyl-t-butyl-ether

17) $\mathrm{C} 013 \mathrm{cis}-1,2$-dichloroethen

18) $\mathrm{C} 055$ total-1,2-dichloroeth

19) CO14 1,1-dichloroethane

20) C015 2,2-dichloropropane

21) CII0 2-butanone

22) CIOI bromochloromethane

23) $\mathrm{C} 016 \mathrm{chloroform}$

24) $\operatorname{Co17}$ I,I,I-Trichloroethane

25) $C 018$ 1,1-dichloropropene

26) Co19 1,2-dichloroethane

27) $\mathrm{C} 020$ carbon tetrachloride

29) C021 benzene

30) C022 trichloroethene

31) $\mathrm{C} 023$ 1,2-dichloropropane

32) C024 dibromomethane

33) C025 bromodichloromethane

34) C026 2-chloroethylvinyleth
12.27

15.34

19.78

65

98

471047

1652438

$95 \quad 1149791$

$0.00 \quad 85$

0.00

50

0.00

0.00

0.00

0.00

0.00

0.00

0.00

0.00

0.00

8.75

0.00

0.00

0.00

0.00

0.00

0.00

0.00

0.00

0.00

0.00

0.00

0.00

0.00

0.00

12.39

0.00

0.00

0.00

0.00

0.00

0.00
Response

1718402

1308661

915719

$50.00 \mathrm{ug} / \mathrm{L}$

$50.00 \mathrm{ug} / \mathrm{I}$

$50.00 \mathrm{ug} / \mathrm{I}$

$\%$ Recovery

$42.41 \mathrm{ug} / \mathrm{L} \quad 84.82 \%$

$49.09 \mathrm{ug} / \mathrm{L} \quad 98.18 \%$

$46.99 \mathrm{ug} / \mathrm{L}$

$93.97 \%$

Qvalue

Not Detected

Not Detected

Not Detected

Not Detected

Not Detected

Not Detected

Not Detected

Not Detected

Not Detected

Not Detected

Not Detected

$2.18 \mathrm{ug} / \mathrm{L} \#$

Not Detected

Not Detected

Not Detected

Not Detected

Not Detected

Not Detected

Not Detected

Not Detected

Not Detected

Not Detected

Not Detected

Not Detected

Not Detected

Not Detected

157311

$4.57 \mathrm{ug} / \mathrm{L}$

Not Detected

Not Detected

Not Detected

Not Detected

Not Detected

Not Detected 


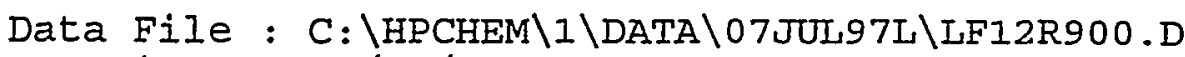

Acq Time : 07/07/97 20:10

Sample : 97C02900 NAP-R1-S2-B

Misc : 97C-0208-01 1:100 Dilution

Quant Time: Jul 20 13:33 1997

Method : J: \I\METHODS \L826ARWA.M

Title : VOA COMPOUND LIST

Last Update : Wed Jun 25 09:14:05 1997

Response via : Multiple Level Calibration
Operator: CQC

Inst : 5971-L

Multiplr: 1.00
Compound

R.T. QIon Response Conc Unit Qvalue

36) C205 4-methyl-2-pentanone

38) C028 trans-1,3-dichloropro

39) C029 1,1,2-trichloroethane

40) C210 2-hexanone

41) $\operatorname{Co30} 1,2-$ Dibromoethane

43) C031 Toluene

44) C032 1,3-dichloropropane

45) C033 dibromochloromethane

46) $\mathrm{C} 034$ bromoform

47) C035 tetrachloroethene

48) $\mathrm{C} 036$ chlorobenzene

49) $\operatorname{Co37} 1,1,1,2$-tetrachloroet

50) 0038 ethylbenzene

51) C039 m,p-xylene

52) C040 o-xylene

53) C250 total xylene

54) C041 styrene

55) C042 isopropylbenzene

58) $\mathrm{C} 043$ 1,1,2,2-tetrachloroet

59) C044 bromobenzene

60) C045 1,2,3-trichloropropan

61) $\mathrm{C} 046 \mathrm{n}$-propylbenzene

62) $\mathrm{C} 047$ 1,3,5-trimethylbenzen

63) $\mathrm{C} 048$ 2-chlorotoluene

64) 0494 -chlorotoluene

65) C050 tert-butylbenzene

66) C05I 1,2,4-trimethylbenzen

67) C052 sec-Butylbenzene

68) C053 p-Isopropyltoluene

69) C054 1,3-Dichlorobenzene

70) C055 1,4-Dichlorobenzene

71) C056 n-Butylbenzene

72) $C 057$ 1,2-Dichlorobenzene

73) C058 1,2-Dibromo-3-chlorop

74) C059 1,2,4-Trichlorobenzen

75) C060 Hexachlorobutadiene

76) C061 Naphthalene

77) C062 1,2,3-Trichlorobenzen
$0.00 \quad 43$

$0.00 \quad 75$

$0.00 \quad 97$

$0.00 \quad 43$

$0.00 \quad 107$

$15.45 \quad 92$

$0.00 \quad 76$

0.00129

$0.00 \quad 173$

$0.00 \quad 164$

$0.00 \quad 112$

$0.00 \quad 131$

$0.00 \quad 106$

$0.00 \quad 106$

$0.00 \quad 106$

$0.00 \quad 106$

$18.90 \quad 104$

$.0 .00 \quad 105$

$0.00 \quad 83$

$0.00 \quad 156$

$0.00 \quad 75$

$0.00 \quad 91$

$0.00 \quad 105$

$0.00 \quad 91$

$0.00 \quad 91$

$0.00 \quad 119$

$0.00 \quad 105$

$0.00 \quad 105$

$0.00 \quad 119$

0.00146

$0.00 \quad 146$

$0.00 \quad 91$

$0.00 \quad 146$

$0.00 \quad 75$

$0.00 \quad 180$

$0.00 \quad 225$

$25.23 \quad 128$

$0.00 \quad 180$

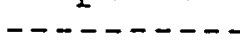

Not Detected

Not Detected

Not Detected

Not Detected

Not Detected

$197980.83 \mathrm{ug} / \mathrm{L} \#$

Not Detected

Not Detected

Not Detected

Not Detected

Not Detected

Not Detected

Not Detected

Not Detected

Not Detected

Not Detected

$175590.59 \mathrm{ug} / \mathrm{L} \#$

Not Detected

Not Detected

Not Detected

Not Detected

Not Detected

Not Detected

Not Detected

Not Detected

Not Detected

Not Detected

Not Detected

Not Detected

Not Detected

Not Detected

Not Detected

Not Detected

Not Detected

Not Detected

Not Detected

268315

$7.09 \mathrm{ug} / \mathrm{L}$

Not Detected
$91 \mathrm{Nz}$

$83^{-K}$

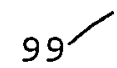




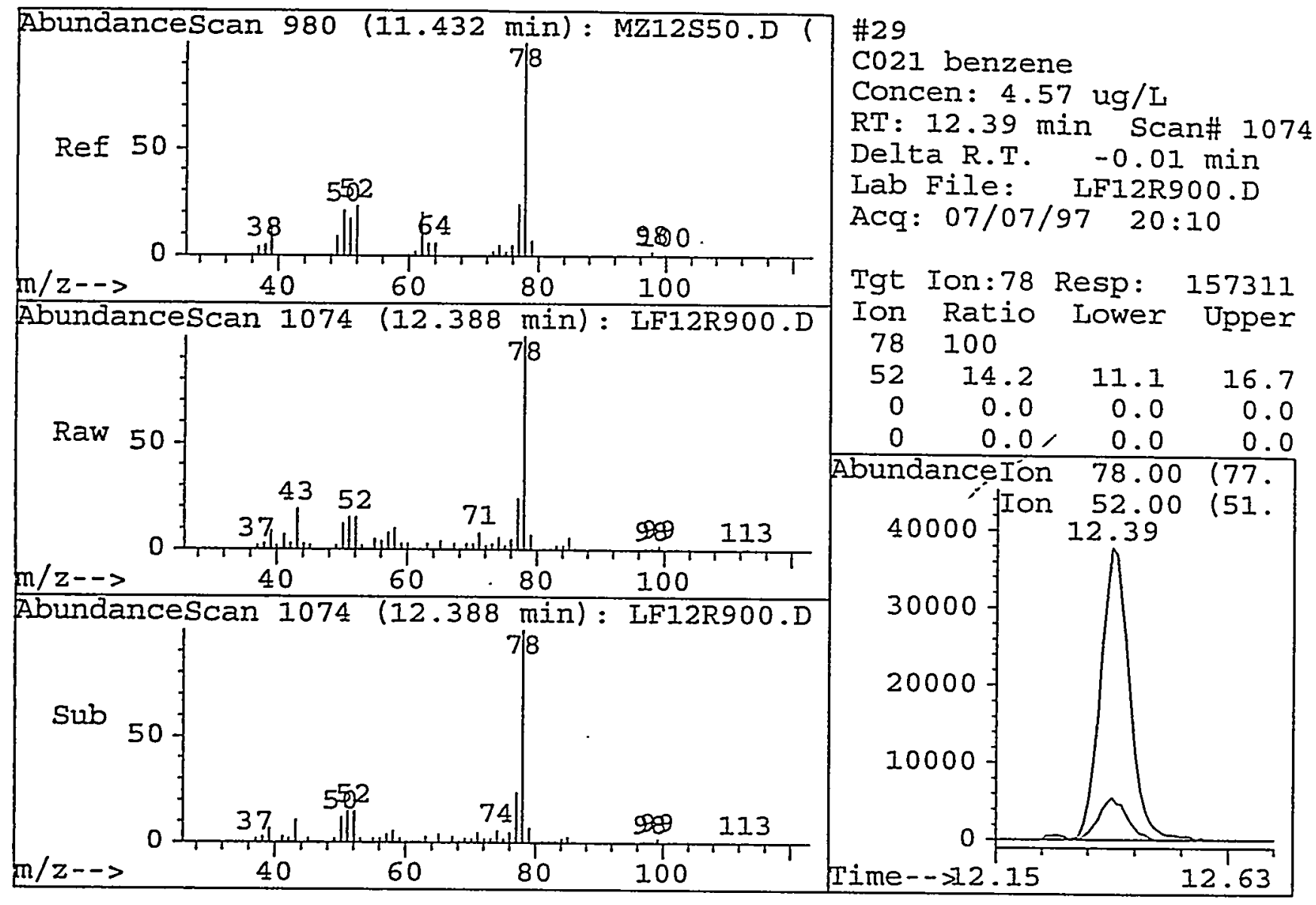




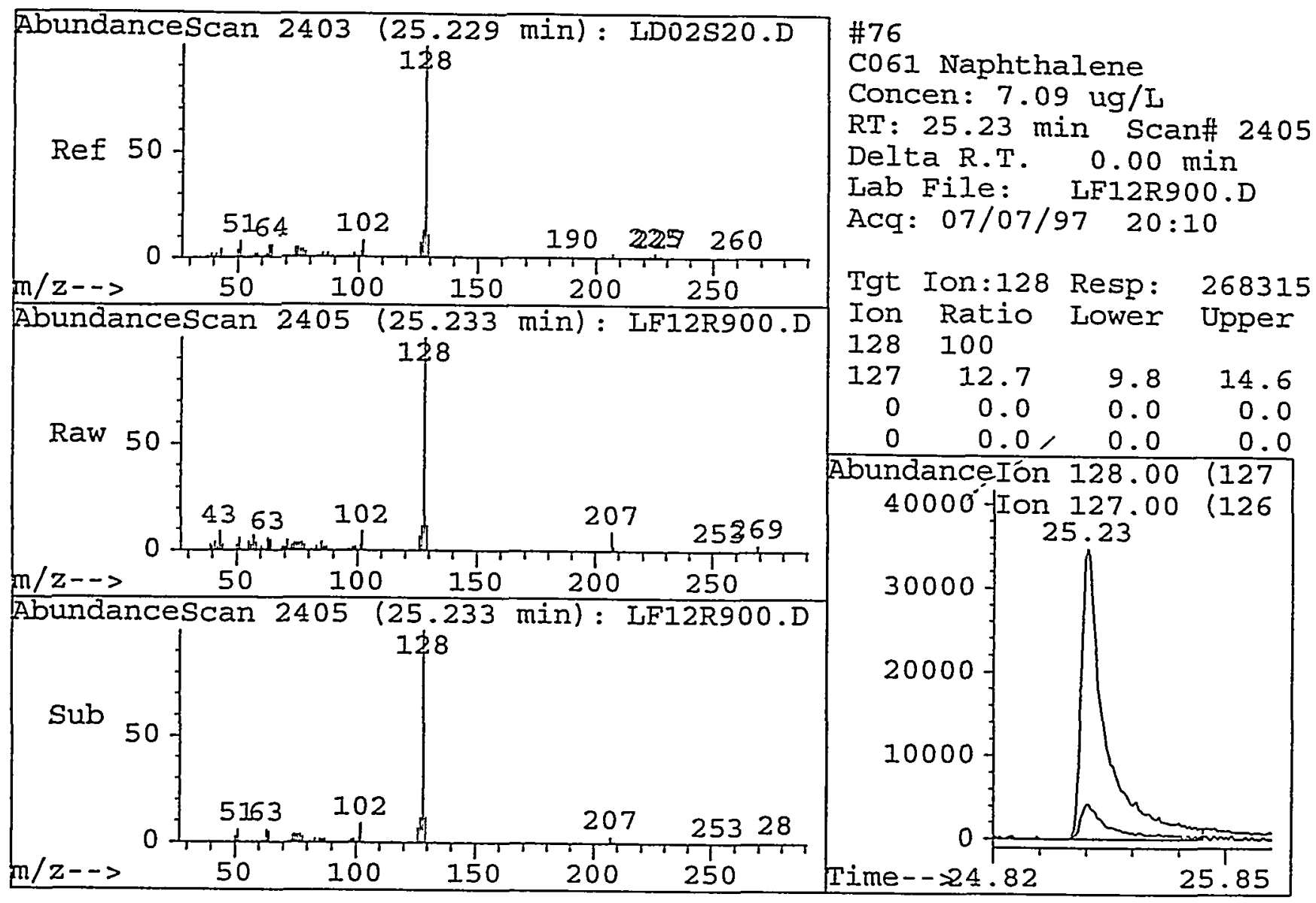


Quantitation Report

Data File : C:\HPCHEM \I\DATA 08 JUL97L\LF19R90I.D

Acq Time : 07/08/97 13:02

Sample : 97C02901 NAP-R1-S4

Misc : 97C-0208-01

Quant Time: Jul 8 14:26 1997

Operator: $\mathrm{CQC}$

Inst : 5971-L

Multiplr: 1.00

Method : C: \HPCHEM \I\METHODS \L826ARWA.M

Title : VOA COMPOUND IIST

Last Update : Tue Jul 08 12:41:59 1997

Response via : Multiple Level Calibration
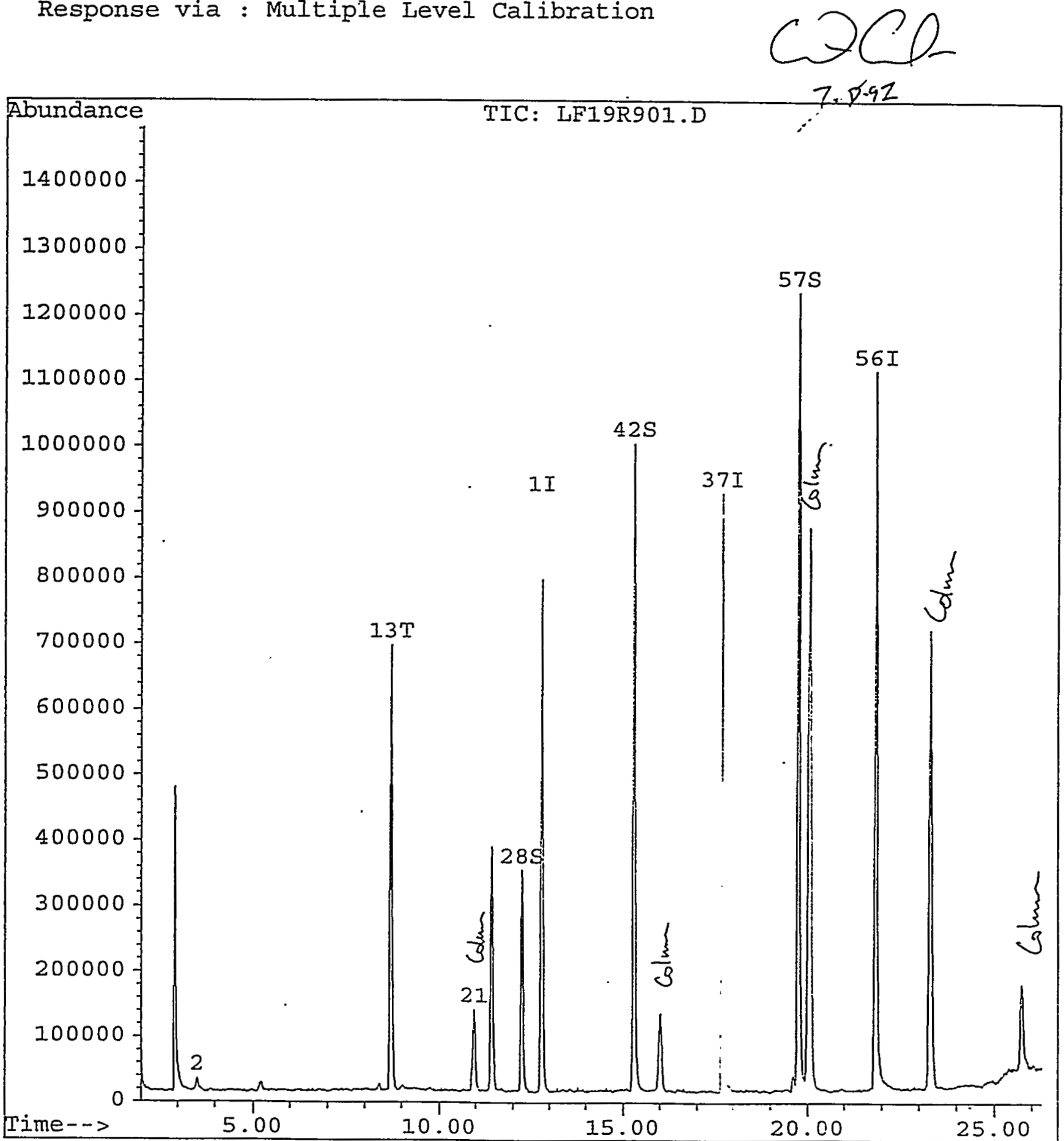

151 


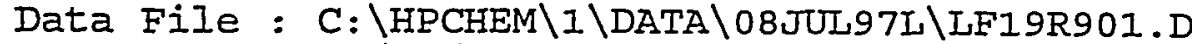

Acq Time : 07/08/97 13:02

Sample : 97C02901 NAP-RI-S4

MisC : 97C-0208-01

Quant Time: Jul 8 14:26 1997

Method : C: \HPCHEM IIMETHODS \I826ARWA.M

Title : VOA COMPOUND IIST

Last Update : Tue JuI 08 12:41:59 1997

Response via : Multiple Level Calibration
Operator: CQC

Inst : 5971-L

Multiplr: 1.00

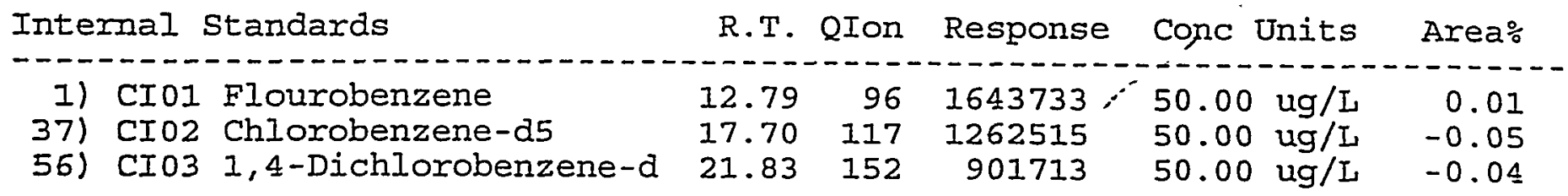

System Monitoring Compounds

2B) CS15 1,2-dichloroethane-d4

42) CS05 toluene-d8

57) CS10 4-bromofluorobenzene

Target Compounds

2) $\mathrm{COO} 2$ dichlorodifluorometha

3) $\mathrm{C003}$ chloromethane

4) $\mathrm{COO} 4$ vinyl chloride

5) C005 bromomethane

6) 0006 chloroethane

7) $\mathrm{C007}$ trichlorofluoromethan

8) 0071 acrolein

9) $\operatorname{COOB} 1,1$-dichloroethene

10) $\mathrm{C} 063$ freon 113

11) Co09 acetone

12) $C 010$ carbon disulfide

13) COII methylene chloride

14) C072 acrylonitrile

15) $\mathrm{CO12}$ trans-1,2-Dichloroeth

16) C305 methyl-t-butyl-ether

17) $\mathrm{C} 013 \mathrm{cis-1,2-dichloroethen}$

18) $\mathrm{C} 055$ total-I,2-dichloroeth

19) $\mathrm{CO} 14$ 1,1-dichloroethane

20) $\operatorname{Co15} 2,2$-dichloropropane

2I) CIID 2-butanone

22) CIOI bromochloromethane

23) $\mathrm{C} 016$ chloroform

24) $\mathrm{C} 017$ 1,1,1-Trichloroethane

25) $\operatorname{Co18~1,1-dichloropropene~}$

25) C019 1,2-dichloroethane

27) $\mathrm{C} 020$ carbon tetrachloride

29) C021 benzene

30) C022 trichloroethene

31) C023 1,2-dichloropropane

32) C024 dibromomethane

12.24

15.31

19.74

65

98

95

495198

1575953

1116425

3.52

0.00

62

0.00

0.00

0.00101

0.00

0.0096

$0.00 \quad 151$

0.00

0.00

8.71

0.00

0.00

0.00

0.00

0.00

0.00

0.00

10.96

0.00

0.00

0.00

0.00

0.00

0.00

0.00

0.00

0.00

0.00

0.00

0.00

0.00
85

94

64

56

43

76

51060

718776

$$
53
$$

96

73

96

96

63

77

72

128

83

97

75

62

117

78

130

63

93

83

63 $\because$ Recovery

$46.61 \mathrm{ug} / \mathrm{I} \quad 93.22 \%$

$48.53 \mathrm{ug} / \mathrm{I} \quad 97.05 \%$

$46.33 \mathrm{ug} / \mathrm{I} \quad 92.66 \%$

33) $\mathrm{C} 25$ bromodichloromethane

35) 0027 cis-1,3-dichloroprope 
Quantitation Report

Data File : C:\HPCHEM \I\DATA 08JUL97L\LF19R90I.D

Acq Time: 07/08/97 13:02

Sample : 97C02901 NAP-R1-S4

Misc : 97C-0208-01

Operator: $\mathrm{CQC}$

Inst : 5971-I

Quant Time: Jul 8 14:26 1997

Multiplr: 1.00

Method : C: \HPCHEM $\backslash I \backslash M E T H O D S \backslash L 826 A R W A . M$

Title : VOA COMPOUND LIST

Last Update : Tue Jul 08 12:41:59 1997

Response via : Multiple Level Calibration

Compound

R.T. QIon Response Conc Unit Qvalue

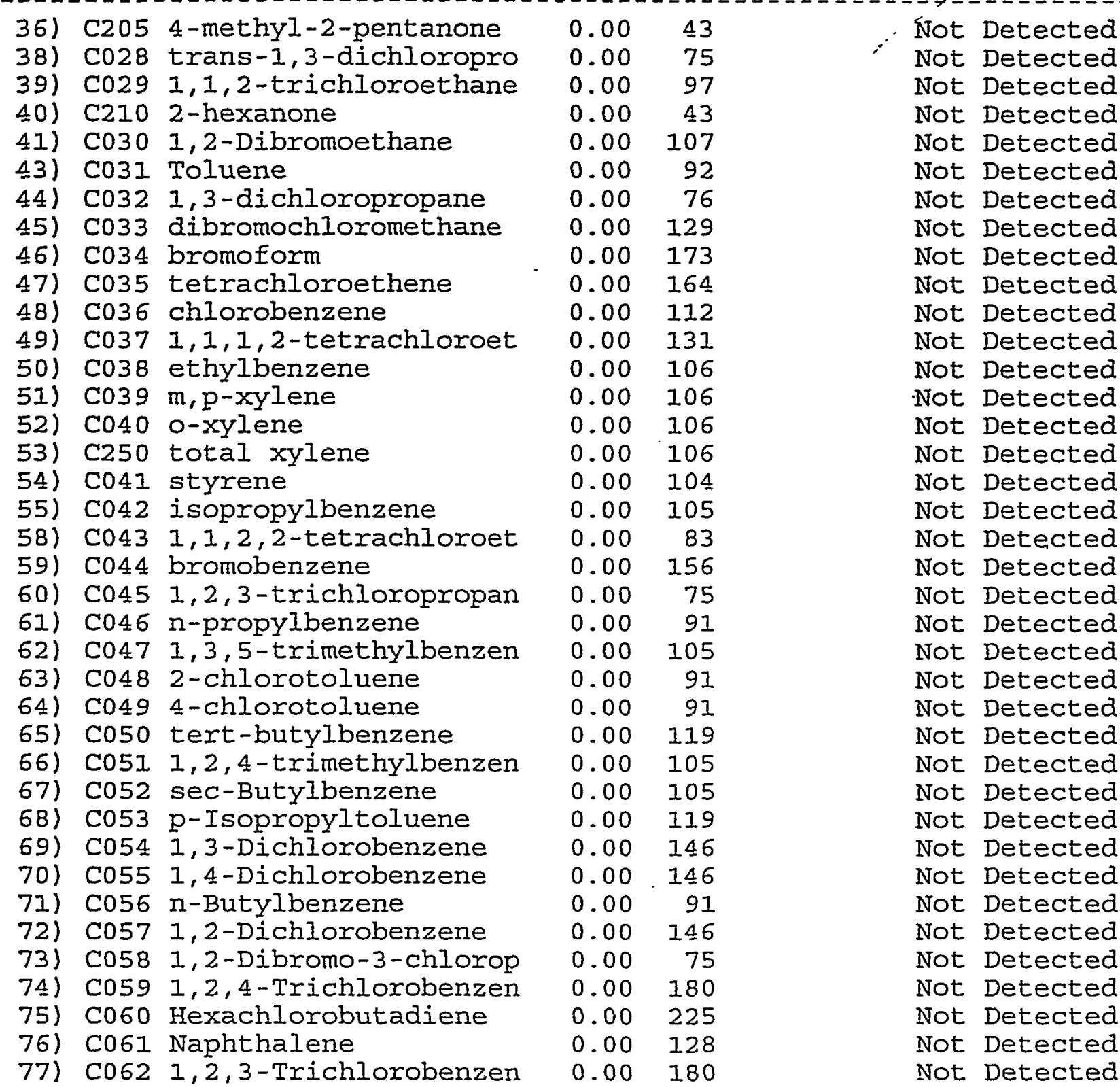



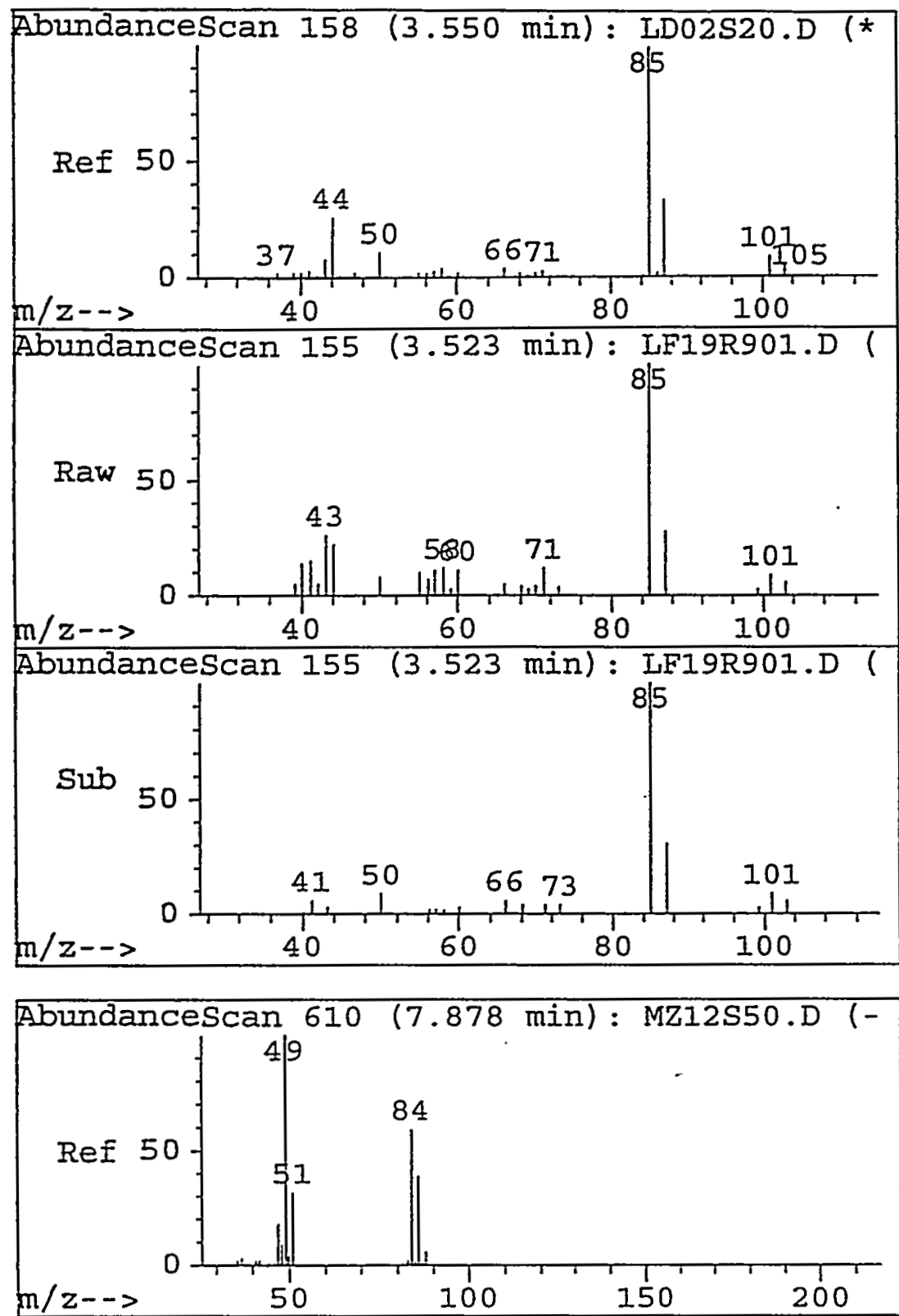

Abundancescan $693(8.714 \mathrm{~min}):$ LFI9R901.D (

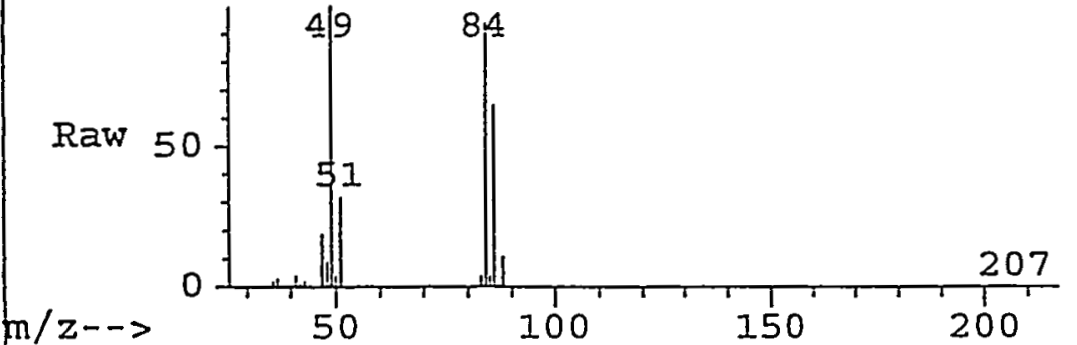

Abundancescan 693 (8.714 min): LF19R901.D (

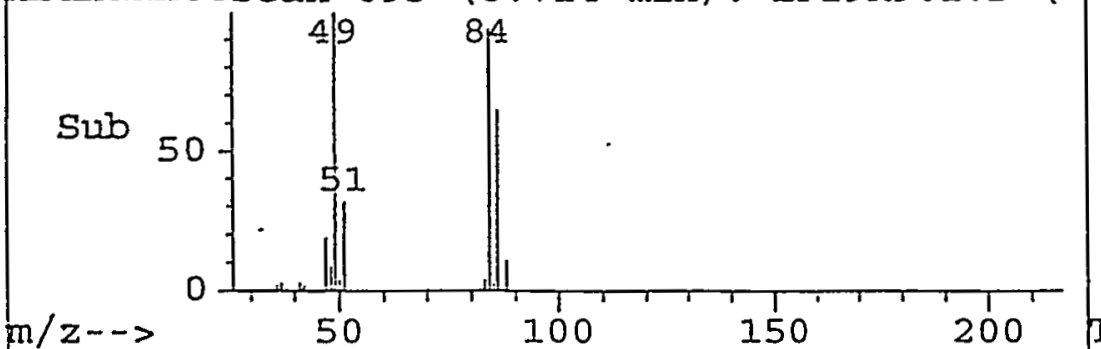

\#2

C002 dichlorodifluoromethane

Concen: $3.99 \mathrm{ug} / \mathrm{I}$

RT: $3.52 \mathrm{~min}$ Scan\# 155

Delta R.T. $\quad-0.03 \mathrm{~min}$

Lab File: LF19R901.D

Acq: 07/08/97 13:02

Tgt Ion:85 Resp: 51060

Ion Ratio Lower Upper

85100

$\begin{array}{llll}87 & 31.6 & 27.6 & 41.4\end{array}$

$\begin{array}{llll}101 & 7.1 & 7.0 & 10.6\end{array}$

$1034.0,4.5 \quad 6.7 \#$

Abundanceión $85.00(84$.

iI Ion $87.00 \quad(86$.

15000 Ion $101.00 \quad(100$

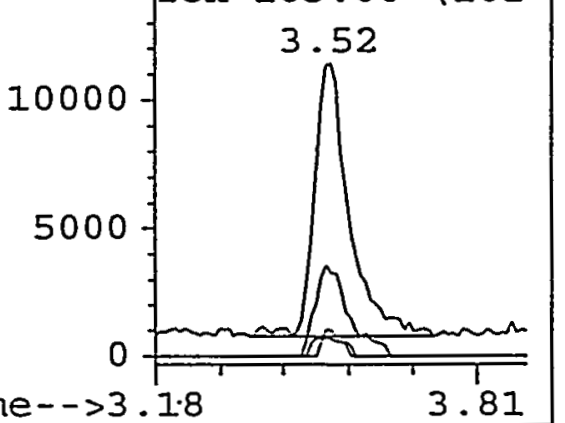

Time- $->3.1 \cdot 8$

3.81

$\# 13$

C01I methylene chloride

Concen: $73.31 \mathrm{ug} / \mathrm{L}$

$\mathrm{RT}: 8.71 \mathrm{~min}$ scan\# 693

Delta R.T. $\quad-0.04 \mathrm{~min}$

Lab File: IFI9R901.D

Acq: $07 / 08 / 97$ 13:02

Tgt Ion:84 Resp: 718776

Ion Ratio Lower Upper

84100

$49 \quad 104.5 \quad 136.2 \quad 204.2 \#$

$\begin{array}{llll}51 & 33.6 & 43.0 & 64.4 \#\end{array}$

$\begin{array}{llll}86 & 67.2 & 52.6 & 79.0\end{array}$

AbundanceIon 84.00 (83.

300000 IIon $49.00 \quad(48$.

Ion $51.00 \quad(50$.

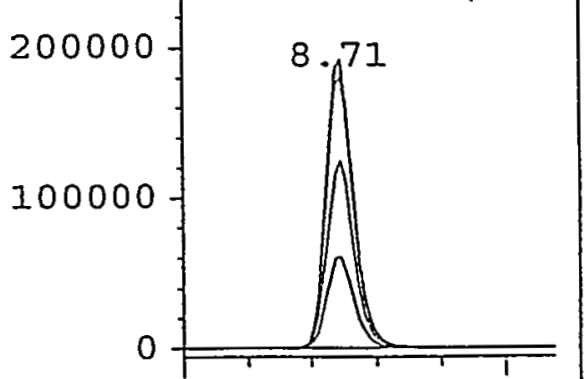

Time- $\rightarrow 8.39$ 


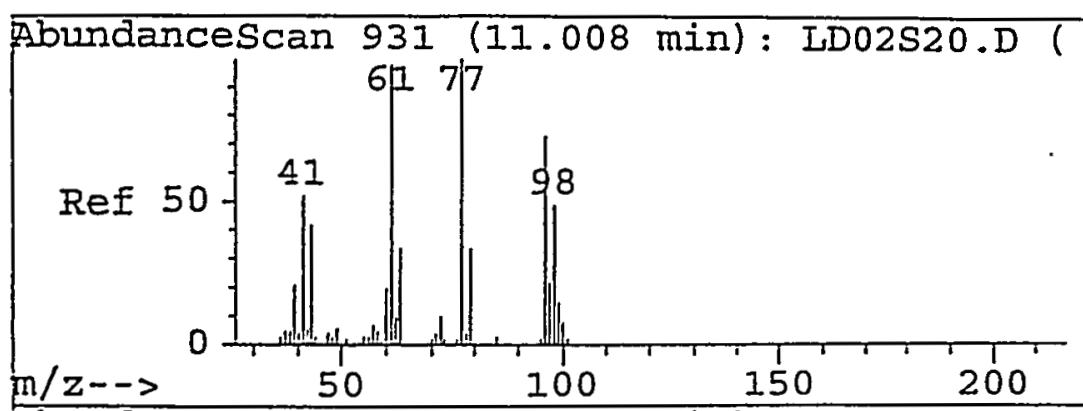

Abundancescan 926 (10.963 min): LF19R901.D

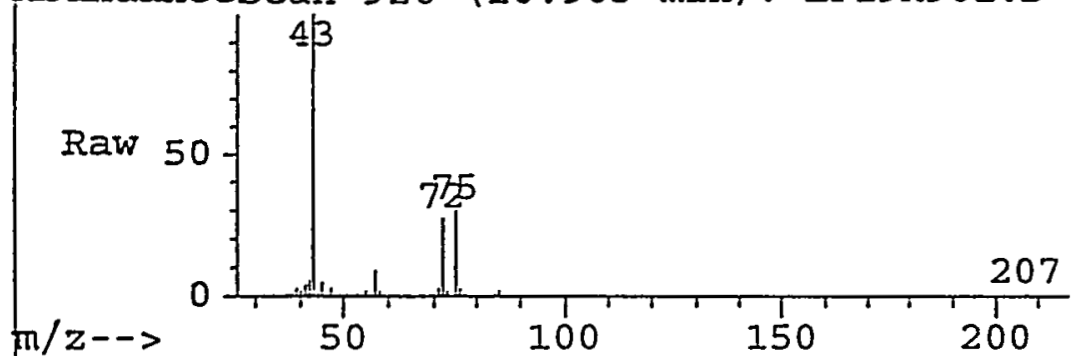

Abundancescan 926 (10.963 min): LF19R901.D

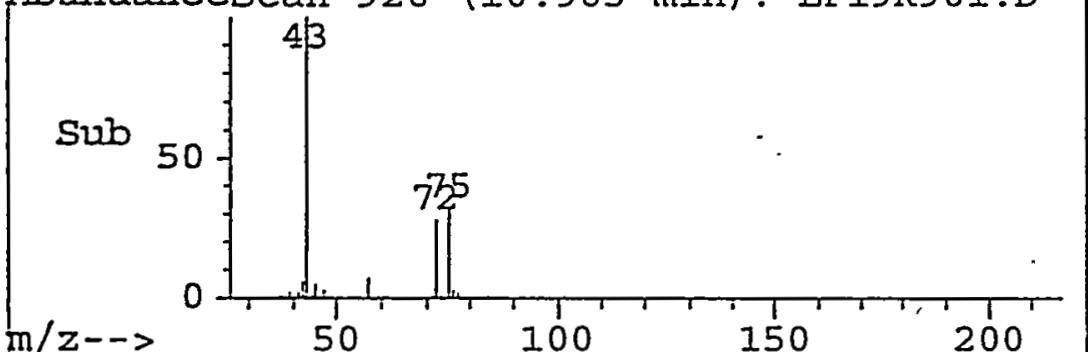

\#21

C110 2-butanone

Concen: $71.06 \mathrm{ug} / \mathrm{L}$

$\mathrm{RT}: 10.96 \mathrm{~min}$ Scan\# 926

Delta R.T. $\quad-0.05 \mathrm{~min}$

Lab File: LF19R901.D

Acq : 07/08/97 13:02

Tgt Ion:71.95 Resp: 74980

Ion Ratio Lower Upper

72100

$43 \quad 352.6 \quad 462.2 \quad 693.2 \#$

$\begin{array}{llll}0 & 0.0 & 0.0 & 0.0\end{array}$

$0 \quad 0.0,0.0 \quad 0.0$

Abundancelón 71.95 (71.

fIon 42.95 (42.

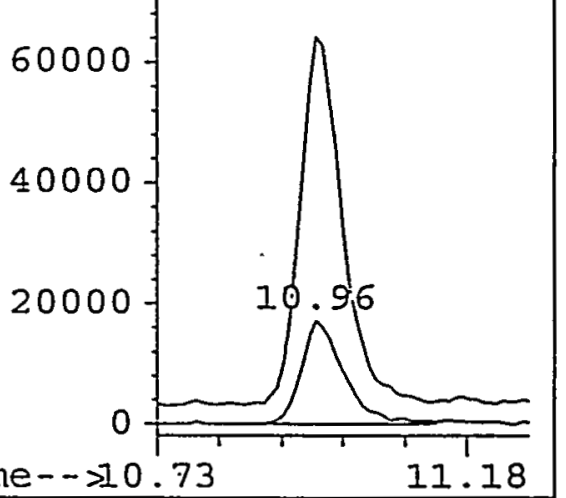




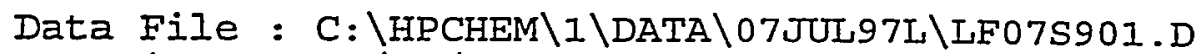

Acq Time : 07/07/97 17:31

Sample : 97C02901MS NAP-RI-S4

MisC : 97C-0208-0I 8260 WATER

Operator: CQC

Inst : 5971-L

Quant Time: Jul 20 13:05 1997

Multiplr: 1.00

Method : J: \I\METHODS \L826ARWA.M

Title : VOA COMPOUND IIST

Last Update : Wed Jun 25 09:14:05 1997

Response via : Multiple Level Calibration

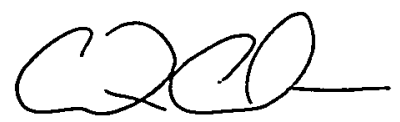

$7 \cdot 20.97$

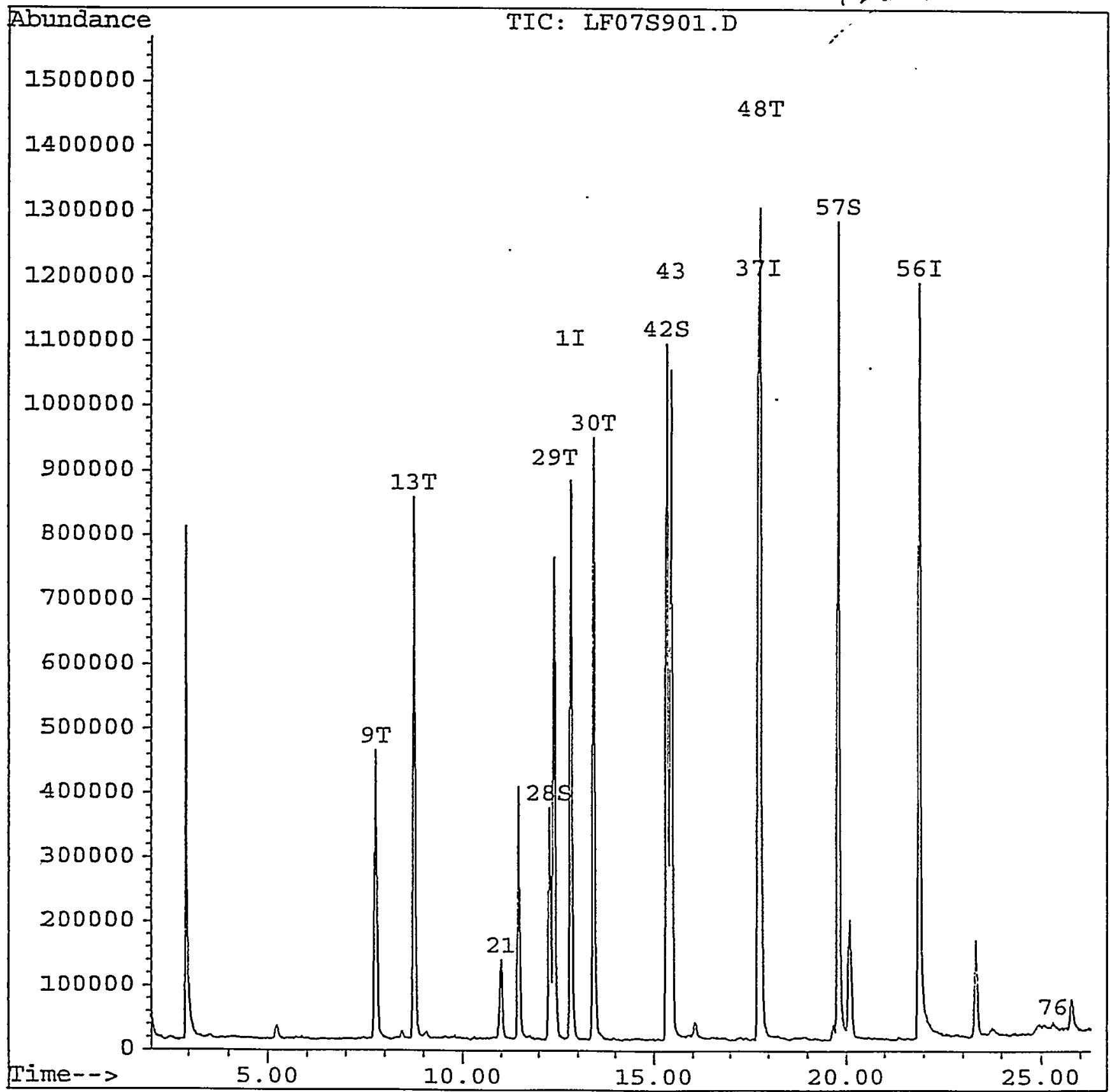

156 


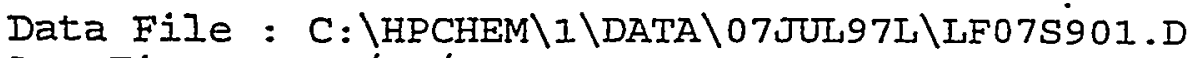

Acq Time : 07/07/97 17:31

Sample : 97C02901MS NAP-RI-S4

Misc : 97C-0208-01 8260 WATER

Quant Time: Jul 20 13:05 1997

Method : J: \I\METHODS \L826ARWA.M

Title' : VOA COMPOUND IIST

Last Update : Wed Jun 25 09:14:05 1997

Response via : Multiple Level Calibration
Operator: CQC

Inst : 5971-I

Multiplr: 1.00
Internal Standards

I) CIOI Flourobenzene

37) CIO2 Chlorobenzene-d5

56) CI03 1,4-Dichlorobenzene-d

System Monitoring Compounds

28) CS15 1,2-dichloroethane-d4

42) CS05 toluene-d8

57) CS10 4-bromofluorobenzene

Target Compounds

2) $\mathrm{COO} 2$ dichlorodifluorometha

3) $\mathrm{C003}$ chloromethane

4) C004 vinyl chloride

5) 0005 bromomethane

6) $\mathrm{C006}$ chloroethane

7) $\mathrm{C007}$ trichlorofluoromethan

B) C071 acrolein

9) C008 1,1-dichloroethene

10) $\mathrm{C} 063$ freon 113

11) co09 acetone

12) $\mathrm{CO10}$ carbon disulfide

13) C011 methylene chloride

14) $\mathrm{C} 072$ acrylonitrile

15) $\mathrm{C} 012$ trans-1,2-Dichloroeth

16) C305 methyl-t-butyl-ether

17) $\mathrm{CO13}$ cis-1,2-dichloroethen

18) $\mathrm{C} 055$ total-1,2-dichloroeth

19) C014 1,1-dichloroethane

20) C015 2,2-dichloropropane

21) CII0 2-butanone

22) CIOI bromochloromethane

23) $\mathrm{C} 016$ chloroform

24) $\operatorname{Co17} 1,1,1-T r i c h l o r o e t h a n e$

25) $\mathrm{CO18}$ I,1-dichloropropene

25) C019 1,2-dichloroethane

27) $\mathrm{CO20}$ carbon tetrachloride

29) CO2I benzene

30) $\mathrm{C} 022$ trichloroethene

31) $\mathrm{C023}$ 1,2-dichloropropane

32) C024 dibromomethane

33) C025 bromodichloromethane

34) C026 2-chloroethylvinyleth

35) C027 cis-1,3-dichloroprope

R.T. QIon

Response

1844658

1401545

1007714

Conc Units

Area\%

12.82

17.74

21.88
96

117

152
$50.00 \mathrm{ug} / \mathrm{L}$

$50.00 \mathrm{ug} / \mathrm{L}$

$50.00 \mathrm{ug} / \mathrm{L}$

$\%$ Recovery

12.27

15.34

19.78

65

98

95

510795

1747056

1216853

$0.00 \quad 85$

$0.00 \quad 50$

0.0062

0.0094

0.0064

$0.00 \quad 101$

0.00

7.77

0.00

0.00

0.00

8.75

0.00

0.00

0.00

0.00

0.00

0.00

0.00

11.00

0.00

0.00

0.00

0.00

0.00

0.00

12.40

13.42

0.00

0.00

0.00

0.00

0.00
56

96

151

43

76

84

53

96

73

96

96

63

77

72

128

83

97

75

62

117

78

130

63

93

83

63

75
$42.84 \mathrm{ug} / \mathrm{L}$ $48.46 \mathrm{ug} / \mathrm{L}$ $45.19 \mathrm{ug} / \mathrm{L}$

$85.68 \%$

$96.92 \%$

$90.37 \%$
0.00

0.00

0.01
Not Detected $45.25 \mathrm{ug} / \mathrm{L} \#$ Not Detected Not Detected Not Detected $940498 \quad 85.48 \mathrm{ug} / \mathrm{L} \#$ Not Detected Not Detected Not Detected Not Detected Not Detected Not Detected Not Detected $8646473.02 \mathrm{ug} / \mathrm{L} \#$ Not Detected Not Detected Not Detected Not Detected Not Detected Not Detected $41.62 \mathrm{ug} / \mathrm{I}$ $47.15 \mathrm{ug} / \mathrm{L} \#$ Not Detected Not Detected Not Detected Not Detected Not Detected
1539066

782847
$78 P$

$63 \mu$

$13 N$

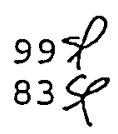

$(\mathrm{NR})=$ Not Reported $(\mathrm{SP})=$ Spiked analyte $(\mathrm{m})=$ manual integration

(*) Does not meet EPA spectral criteria (False Hit) PX125 
Data File : C: \HPCHEM \I\DATA $107 \pi U L 97 L \backslash L F 075901 . D$

Acq Time : 07/07/97 17:31

Sample : 97C02901MS NAP-RI-S4

MisC : 97C-0208-01 8260 WATER

Quant Time: Jul 20 13:05 1997

Operator: $\mathrm{CQC}$

Enst : 5971-L

Multiplr: 1.00

Method : J: IL\METHODS \L826ARWA.M

Title : VOA COMPOUND LIST

Last Update : Wed Jun 25 09:14:05 1997

Response via : Multiple Level Calibration

Compound

R.T. QIon Response Conc Unit Qvalue

\begin{tabular}{|c|c|c|c|c|c|c|c|c|}
\hline 36) & C205 & 4-methyl-2-pentanone & 0.00 & 43 & & Not & Detected & \\
\hline 38) & $\mathrm{CO28}$ & trans-I,3-dichloropro & 0.00 & 75 & & Not & Detected & \\
\hline 39) & C029 & 1,1,2-trichloroethane & 0.00 & 97 & & Not & Detected & \\
\hline 40$)$ & C210 & 2-hexanone & 0.00 & 43 & & Not & Detected & \\
\hline 41) & $\mathrm{CO} 30$ & 1,2-Dibromoethane & 0.00 & 107 & & Niot & Detected & \\
\hline 43) & $\operatorname{Co31}$ & Toluene & 15.46 & 92 & 1096187 & $\leq 2.8$ & $85 \mathrm{ug} / \mathrm{I}$ & 9980 \\
\hline 44) & $\mathrm{CO} 32$ & 1,3-dichloropropane & 0.00 & $76^{\circ}$ & & Niot & Detected & \\
\hline 45) & $\mathrm{CO} 33$ & dibromochloromethane & 0.00 & 129 & & Not & Detected & \\
\hline 46) & $\mathrm{CO} 34$ & bromoform & 0.00 & 173 & & Not & Detected & \\
\hline 47) & $\mathrm{CO35}$ & tetrachloroethene & 0.00 & 164 & & Not & Detected & \\
\hline 48) & $\mathrm{C} 036$ & chlorobenzene & 17.79 & 112 & 1432106 & $\leq 3.8$ & $84 \mathrm{ug} / \mathrm{L}$ & 965 \\
\hline 49) & $\operatorname{Co37}$ & $1,1,1,2$-tetrachloroet & 0.00 & 131 & & Niot & Detected & \\
\hline 50) & $\operatorname{co3} 8$ & ethylbenzene & 0.00 & 106 & & liot & Detected & \\
\hline 51) & $\operatorname{co39}$ & $\mathrm{m}, \mathrm{p}$-xylene & 0.00 & 106 & & Not & Detected & \\
\hline 52) & $\mathrm{CO} 40$ & o-xylene & 0.00 & 106 & & Not & Detected & \\
\hline 53) & $\mathrm{C} 250$ & total xylene & 0.00 & 106 & & Not & Detected & \\
\hline 54) & $C O 4 I$ & styrene & 0.00 & 104 & & Not & Detected & \\
\hline 55) & $\mathrm{CO} 42$ & isopropylbenzene & 0.00 & 105 & & Not & Detected & \\
\hline 58) & $\mathrm{CO} 43$ & $1,1,2,2$-tetrachloroet & 0.00 & 83 & & Not & Detected & \\
\hline 59) & $\mathrm{CO} 44$ & bromobenzene & 0.00 & 156 & & Not & Detected & \\
\hline 60) & $\mathrm{CO45}$ & 1,2,3-trichloropropan & 0.00 & 75 & & Not & Detected & \\
\hline 6I) & $\mathrm{C} 046$ & n-propylbenzene & 0.00 & 91 & & Not & Detected & \\
\hline 62) & $\mathrm{C} 047$ & $1,3,5$-trimethylbenzen & 0.00 & 105 & & Not & Detected & \\
\hline 63) & $\mathrm{CO48}$ & 2-chlorotoluene & 0.00 & 91 & & Not & Detected & \\
\hline 64) & CO49 & 4-chlorotoluene & 0.00 & 91 & & Not & Detected & \\
\hline 65) & $\mathrm{C050}$ & tert-butylbenzene & 0.00 & 119 & & Not & Detected & \\
\hline 66) & C05I & $1,2,4$-trimethylbenzen & 0.00 & 105 & & Not & Detected & \\
\hline 67) & $\mathrm{C} 052$ & sec-Butylbenzene & 0.00 & 105 & & Not & Detected & \\
\hline 68) & $\mathrm{C} 053$ & p-Isopropyltoluene & 0.00 & 119 & & Not & Detected & \\
\hline 69) & $\mathrm{CO54}$ & I,3-Dichlorobenzene & 0.00 & 146 & & Not & Detected & \\
\hline 70) & $\mathrm{C} 055$ & 1,4-Dichlorobenzene & 0.00 & 146 & & Not & Detected & \\
\hline 71) & $\mathrm{C} 056$ & n-Butylbenzene & 0.00 & $9 I$ & & Not & Detected & \\
\hline 72) & $\cos 7$ & 1,2-Dichlorobenzene & 0.00 & 146 & & Not & Detected & \\
\hline 73) & $\mathrm{CO58}$ & 1,2-Dibromo-3-chlorop & 0.00 & 75 & & Not & Detected & \\
\hline 74) & C059 & $1,2,4$-Trichlorobenzen & 0.00 & 180 & & Not & Detected & \\
\hline 75) & C060 & Hexachlorobutadiene & 0.00 & 225 & & Not & Detected & \\
\hline 76) & C061 & Naphthalene & 25.30 & 128 & 68263 & I. & $64 \mathrm{ug} / \mathrm{L}$ & $95 N$ \\
\hline & $\mathrm{C0} 62$ & $1,2,3$-Trichlorobenzen & 0.00 & 180 & & Not & Detected & \\
\hline
\end{tabular}

158 Data Processed By:

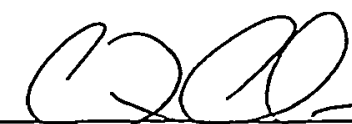

$(\mathrm{NR})=$ Not Reported $(\mathrm{SP})=$ Spiked analyte $(\mathrm{m})=$ manual integration

(*) Does not meet EPA spectral criteria (False Hit) PXI25 Page 2 
Quantitation Report

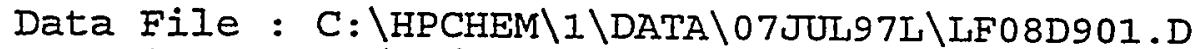

Acq Time : 07/07/97 18:02

Sample : 97C02901MSD NAP-R1-S4

Misc : 97C-0208-01 8260 WATER

Quant Time: Jul 20 13:08 1997

Operator: CQC

Inst : 5971-L

Multiplr: 1.00

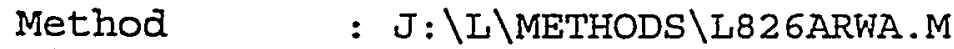

Title : VOA COMPOUND IIST

Last Update : Wed Jun 25 09:14:05 1997

Response via : Multiple Level Calibration

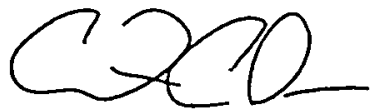

$7 \cdot 20-97$

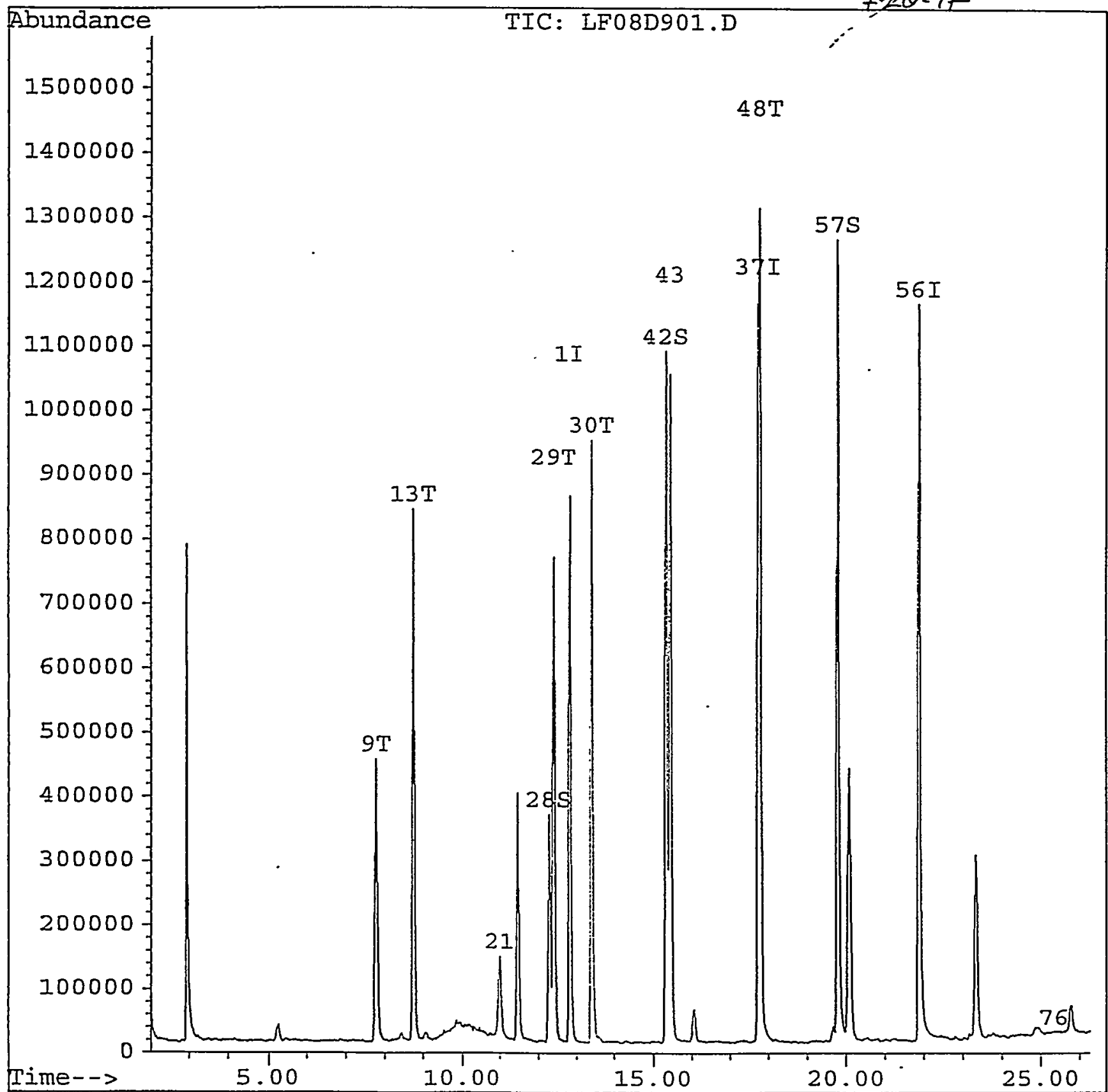


Data File : C: \HPCHEM \I\DATA \07JUL97L\LF08D90I.D

Acq Time : 07/07/97 18:02

Sample : 97C02901MSD NAP-RI-S4

Misc : 97C-0208-01 8260 WATER

Quant Time: Jul 20 13:08 1997
Operator: CQC

Inst : 5971-I

Multiplr: 1.00

Method : J: \I\METHODS \I825ARWA.M

Title : VOA COMPOUND LIST

Last Update : Wed Jun 25 09:14:05 1997

Response via : Multiple Level Calibration

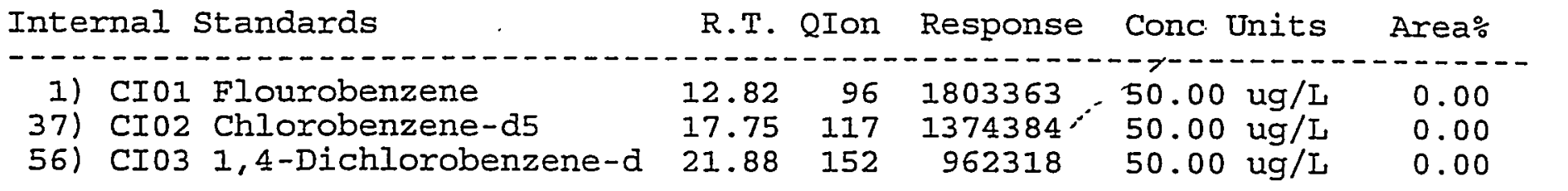

System Monitoring Compounds

28) CSI5 1,2-dichloroethane-d4

42) CS05 toluene-d8

57) CSI0 4-bromofluorobenzene

Target Compounds

2) Co02 dichlorodifluorometha

3) $\mathrm{CO03}$ chloromethane

4) $\mathrm{COO4}$ vinyl chloride

5) C005 bromomethane

6) C006 chloroethane

7) C007 trichlorofluoromethan

8) $C 071$ acrolein

9) $\mathrm{C008}$ 1,1-dichloroethene

10) $\mathrm{C} 063$ freon 113

11) Coog acetone

12) C010 carbon disulfide

13) C011 methylene chloride

14) C072 acrylonitrile

15) $\mathrm{C} 012$ trans-1,2-Dichloroeth

16) C305 methyl-t-butyl-ether

17) $\mathrm{C} 013$ cis-1,2-dichloroethen

I8) $\mathrm{C} 055$ total-1,2-dichloroeth

19) C014 1,1-dichloroethane

20) Co15 2,2-dichloropropane

21) C110 2-butanone

22) CIOI bromochloromethane

23) $\mathrm{C} 016$ chloroform

24) $\mathrm{C} 017$ 1, 1,1-Trichloroethane

25) $\operatorname{Co18~1,1-dichloropropene~}$

26) Co19 1,2-dichloroethane

27) $\mathrm{C} 20$ carbon tetrachloride

29) C021 benzene

30) C022 trichloroethene

31) $\mathrm{CO23} \mathrm{1,2-dichloropropane}$

32) Co24 dibromomethane

33) CO25 bromodichloromethane

34) $\mathrm{C} 026$ 2-chloroethylvinyleth

35) C027 cis-1,3-dichloroprope
12.27

15.34

19.78

$65 \quad 495902$

$98 \quad 1718725$

$95 \quad 1201585$

\begin{tabular}{|c|c|c|}
\hline 0.00 & 85 & \\
\hline 0.00 & 50 & \\
\hline 0.00 & 62 & \\
\hline 0.00 & 94 & \\
\hline 0.00 & 64 & \\
\hline 0.00 & 101 & \\
\hline 0.00 & 56 & \\
\hline 7.77 & 96 & 485540 \\
\hline 0.00 & 151 & \\
\hline 0.00 & 43 & \\
\hline 0.00 & 76 & \\
\hline 8.74 & 84 & 910105 \\
\hline 0.00 & 53 & \\
\hline 0.00 & 96 & \\
\hline 0.00 & 73 & \\
\hline 0.00 & 96 & \\
\hline 0.00 & 96 & \\
\hline 0.00 & 63 & \\
\hline 0.00 & 77 & \\
\hline 11.00 & 72 & 82691 \\
\hline 0.00 & 128 & \\
\hline 0.00 & 83 & \\
\hline 0.00 & 97 & \\
\hline 0.00 & 75 & \\
\hline 0.00 & 62 & \\
\hline 0.00 & 117 & \\
\hline 12.39 & 78 & 1533531 \\
\hline 13.42 & 130 & 787273 \\
\hline 0.00 & 63 & \\
\hline 0.00 & 93 & \\
\hline 0.00 & 83 & \\
\hline 0.00 & 63 & \\
\hline 0.00 & 75 & \\
\hline
\end{tabular}

$\%$ Recovery

$42.54 \mathrm{ug} / \mathrm{L} \quad 85.09 \%$

$48.62 \mathrm{ug} / \mathrm{L} \quad 97.23 \%$

$46.72 \mathrm{ug} / \mathrm{L} \quad 93.45 \%$

Not Detected

Not Detected

Not Detected

Not Detected

Not Detected

Not Detected

Not Detected

$46.37 \mathrm{ug} / \mathrm{L}$ \#

Not Detected

Not Detected

Not Detected

$84.61 \mathrm{ug} / \mathrm{L} \#$

Not Detected

Not Detected

Not Detected

Not Detected

Not Detected

Not Detected

Not Detected

$71.43 \mathrm{ug} / \mathrm{L}$ \#

Not Detected

Not Detected

Not Detected

Not Detected

Not Detected

Not Detected

$42.42 \mathrm{ug} / \mathrm{L}$

$48.51 \mathrm{ug} / \mathrm{L} \#$

Not Detected

Not Detected

Not Detected

Not Detected

Not Detected

Qvalue 


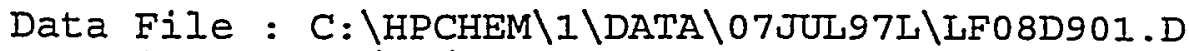

Acq Time : 07/07/97 18:02

Sample : 97C02901MSD NAP-RI-S4

MisC : 97C-0208-01 8260 WATER

Quant Time: Jul 20 13:08 1997

Operator: $\mathrm{CQC}$

Inst : 5971-L

Multiplr: 1.00

Method : J: \I\METHODS \L826ARWA.M

Title : VOA COMPOUND LIST

Last Update : Wed Jun 25 09:14:05 1997

Response via : Multiple Level Calibration

Compound

\begin{tabular}{|c|c|c|c|c|c|}
\hline 36) & $\mathrm{C} 205$ & 4-methyl-2-pentanone & 0.00 & 43 & \\
\hline 38) & $\mathrm{CO} 28$ & trans-1,3-dichloropro & 0.00 & 75 & \\
\hline 39) & C029 & 1,1,2-trichloroethane & 0.00 & 97 & \\
\hline 40) & $\mathrm{C} 210$ & 2-hexanone & 0.00 & 43 & \\
\hline 41) & $\cos 0$ & 1,2-Dibromoethane & 0.00 & 107 & \\
\hline 43) & $\cos 1$ & Toluene & 15.46 & 92 & 1113057 \\
\hline 44) & $\mathrm{CO} 32$ & 1,3-dichloropropane & 0.00 & 76 & \\
\hline 45) & $\mathrm{CO33}$ & dibromochloromethane & 0.00 & 129 & \\
\hline 46) & C034 & bromoform & 0.00 & 173 & \\
\hline 47) & C035 & tetrachloroethene & 0.00 & 164 & \\
\hline 48) & $\mathrm{C} 036$ & chlorobenzene & 17.79 & 112 & 1447042 \\
\hline 49) & $\cos 7$ & $1,1,1,2$-tetrachloroet & 0.00 & 131 & \\
\hline 50) & $\cos 8$ & ethylbenzene & 0.00 & 106 & \\
\hline 51) & $\cos 9$ & $\mathrm{~m}, \mathrm{p}-\mathrm{xy}$ lene & 0.00 & 106 & \\
\hline 52) & $\mathrm{CO} 40$ & o-xylene & 0.00 & 106 & \\
\hline 53) & $\mathrm{C} 250$ & total xylene & 0.00 & 106 & \\
\hline 54) & $\mathrm{CO41}$ & styrene & 0.00 & 104 & \\
\hline 55) & $\mathrm{CO} 42$ & isopropylbenzene & 0.00 & 105 & \\
\hline 58) & $\mathrm{C} 043$ & $1,1,2,2$-tetrachloroet & 0.00 & 83 & \\
\hline 59) & $\mathrm{CO44}$ & bromobenzene & 0.00 & 156 & \\
\hline 60) & $\mathrm{C} 045$ & 1,2,3-trichloropropan & 0.00 & 75 & \\
\hline 6I) & $\mathrm{C} 046$ & n-propylbenzene & 0.00 & 91 & \\
\hline 62) & $\mathrm{CO} 47$ & $1, \overline{3}, 5$-trimethylbenzen & 0.00 & 105 & \\
\hline 63) & $\mathrm{CO} 48$ & 2-chlorotoluene & 0.00 & 91 & \\
\hline 64) & $\mathrm{CO49}$ & 4-chlorotoluene & 0.00 & 91 & \\
\hline 65) & $\mathrm{CO50}$ & tert-butylbenzene & 0.00 & 119 & \\
\hline 66) & $\cos 1$ & $1,2,4$-trimethylbenzen & 0.00 & 105 & \\
\hline 67) & $\mathrm{C} 052$ & sec-Butylbenzene & 0.00 & 105 & \\
\hline 68) & $\mathrm{C} 053$ & p-Isopropyltoluene & 0.00 & 119 & \\
\hline 69) & $\mathrm{C} 054$ & 1,3-Dichlorobenzene & 0.00 & 146 & \\
\hline 70) & C055 & 1,4-Dichlorobenzene & 0.00 & 146 & \\
\hline 71) & C056 & n-Butylbenzene & 0.00 & 91 & \\
\hline 72) & $\mathrm{C} 057$ & 1,2-Dichlorobenzene & 0.00 & 146 & \\
\hline 73) & C058 & 1,2-Dibromo-3-chlorop & 0.00 & 75 & \\
\hline 74) & C059 & 1,2,4-Trichlorobenzen & 0.00 & 180 & \\
\hline 75) & $\mathrm{C} 060$ & Hexachlorobutadiene & 0.00 & 225 & \\
\hline 76) & C061 & Naphthalene & 25.33 & 128 & 29470 \\
\hline 77) & $\mathrm{C} 062$ & $1,2,3$-Trichlorobenzen & 0.00 & 180 & \\
\hline
\end{tabular}

R.T. QIon Response Conc Unit Qvalue Not Detected Not Detected Not Detected Not Detected Not Detected $44.37 \mathrm{ug} / \mathrm{L}$ Not Detected Not Detected Not Detected Not Detected $45.17 \mathrm{ug} / \mathrm{L}$ Not Detected Not Detected Not Detected Not Detected Not Detected Not Detected Not Detected Not Detected Not Detected Not Detected Not Detected Not Detected Not Detected Not Detected Not Detected Not Detected Not Detected Not Detected Not Detected Not Detected Not Detected Not Detected Not Detected Not Detected Not Detected $0.74 \mathrm{ug} / \mathrm{L}$ Not Detected

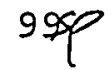

$96 \$$

$96 N K$ 
Response Factor Report 5971-I

Method

Title

Last Update

Response via
: C: \HPCHEM \I\METHODS \L826ARWA.M

: VOA COMPOUND IIST

: Wed Jun 25 09:02:09 1997

Continuing Calibration
Calibration Files

$05=$ LD01S05.D

100

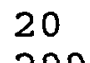

200
$=$ LD02S20.D $=\mathrm{LD} 05 \mathrm{~S} 200 . \mathrm{D}$
50

$=\mathrm{LD} 03550 . \mathrm{D}$

Compound

05

20

50

100

200 Avg

$\%$ RSD

1) I CIOI Flourobenzene

$\begin{array}{llll}\text { ISTD } & & & \\ 93 & 0.396 & 0.387 & 0.400\end{array}$

2) $\mathrm{COO2}$ dichlorodifluorometh

3) $\mathrm{T}$

C003 chloromethane

$\begin{array}{llll}0.456 & 0.368 & 0.393 & 0.396\end{array}$

0.1730 .142

0.167

0.254

0.179

0.167

0.2660 .236

0.253

$0.380 \quad 0.273$

0.280

$\begin{array}{lll}0.161 & 0.143\end{array}$

0.147

0.268

0.258

0.252

5) $T$

C006 chloroethane

0.6610 .562

0.605

0.611

0.135

0.292

0.146

8.30

C071 acrolein

B)

9) $T$

10)

COOB 1,1-dichloroethene

C063 freon 113

11) $\mathrm{T}$

12) $\mathrm{T}$

co09 acetone

13) $\mathrm{T}$

C010 carbon disulfide

C011 methylene chloride

C072 acrylonitrile

$0.320 \quad 0.266$

0.293

0.289

0.602

0.608

8.55

4.31

17.10

C012 t trans-1,2-Dichloroet

15)

16)

17) $\mathrm{T}$

C305 methyl-t-butyl-ether

$\begin{array}{llllll}0.118 & 0.057 & 0.057 & 0.058 & 0.060\end{array}$

0.000

6.47

5.81

C013 cis-1,2-dichloroethe

$0.8230 .705 \quad 0.2$

$0.3550 .277 \quad 0.2$

0.779

0.778

$0.088 \quad 0.069$

0.073

0.288

0.764

0.000

$-1.00$

$0.3550 .298 \quad 0.3$

$0.073 \cdot 0.069$

0.070

6.67

$0.8720 .693 \quad 0.7$

0.328

0.326

$\begin{array}{lll}0.377 & 0.319\end{array}$

0.346

$\begin{array}{lll}0.764 & 0.728\end{array}$

0.070
0.770

$-1.00$

18)

C055 total-1; $2=$ dichloroet

0.3660 .308

0.337

0.344

0.336

0.298

38.72

19) $\mathrm{T}$

C014 1,1-dichloroethane

0.6330 .523

0.572

0.5990 .494

0.335

0

.326

0.074
0.324

5.53

C015 2,2-dichloropropane

21)

CI10`2-butanone

$0.0360 .034 \quad 0$.

0.536

0.533

0.532

0.763

10.53

CI01 bromochloromethane

0.2370 .194

0.211

0.034

0.518

0.344

6.45

8.82

C016 chloroform

$\begin{array}{llll}0.743 & 0.589 & 0.622\end{array}$

0.208

$$
0
$$

0.334

6.10

24)

25)

26) $T$

C017, 1, 1, 1-Trichloroethan

C018'1,1-dichloropropene

0.6370 .537

0.587

0.598

0.5540 .455

0.499

0.584

0.4110 .353

0.383

0.385

0.562

6.27

7.83

7.29

27) $T$

C020 carbon tetrachloride

$\begin{array}{ll}0.577 & 0.46 \\ 0.356 & 0.311\end{array}$

CS15 1,2-dichloroethane-d

C021 benzene

29) $\mathrm{T}$

30) $\mathrm{T}$

C022 trichloroethene

31) $T$

32) $T$

33) $T$

34) $T$

C023 1,2-dichloropropane

C024 dibromomethane

0.518

0.539

0.201

0.536

4.28

7.68

$\begin{array}{lllllll}1.131 & 0.924 & 1.002 & 0.990 & 0.965 & 1.323\end{array}$

0.4750 .4250 .4600 .4510 .4391 .002

$\begin{array}{lllllll}0.475 & 0.425 & 0.460 & 0.451 & 0.439\end{array}$

0.450

11.14

C025 bromodichloromethane

$\begin{array}{lll}0.431 & 0.354 & 0.381\end{array}$

0.380

0.370

0.383

$\begin{array}{lllllll}0.354 & 0.302 & 0.328 & 0.330 & 0.316 & 0.326\end{array}$

C027 cis-1,3-dichloroprop
C205 4-methyl-2-pentanone

$0.606 \quad 0.522 \quad 0.569$

0.653

0.648

0.000

36)

0.4400 .350

0.363

0.5810 .568

0.569

0.3790 .340

0.374

6.18

7.28

5.43

7.87

5.80

7.77

4. 24

7.52

5.90

$6.53^{-}$

$-1.00$

5.34

10.62

\section{7) I CIO2 Chlorobenzene-d5}

38) $T$

39) $T$

- 40$)$

41)

- 1 造方 $s$

C028 trans-1,3-dichloropr

C029 1,1,2-trichloroethan

C210 2-hexanone

C030 1,2-Dibromoethane

CS05 toluene-d8

-..-.-.-.-.-- ISTD

$\begin{array}{llllllll}0.705 & 0.608 & 0.681 & 0.687 & 0.686 & 0.673\end{array}$

$\begin{array}{lllllll}0.582 & 0.467 & 0.504 & 0.496 & 0.485 & 0.507\end{array}$

$\begin{array}{lllllll}0.233 & 0.222 & 0.258 & 0.271 & 0.263 & 0.249\end{array}$

5.59

8.74

$\begin{array}{lllllll}0.764 & 0.652 & 0.719 & 0.721 & 0.700 & 0.711\end{array}$

8.27

$1.3531 .270 \quad 1.279 \quad 1.264 \quad 1.265 \quad 1.286$

5.72

2.92

(\#) = Out of Range 
Response Factor Report 5971-I

Method $\quad$ C: $\backslash$ HPCHEM $\backslash I \backslash M E T H O D S \backslash L 826 A R W A . M$

Title : VOA COMPOUND IIST

Iast Update : Wed Jun 25 09:02:09 1997

Response via : Continuing Calibration

Calibration Files

$05=$ LD01S05.D $20 \quad=L D 02 S 20 . \mathrm{D} \quad 50 \quad=L D 03550 . \mathrm{D}$

$100=L D 04 S 100 . \mathrm{D} 200=\mathrm{LD} 05 \mathrm{~S} 200 \mathrm{D}$

Compound $\quad 05 \quad 20 \quad 50 \quad 100 \quad 200 \quad$ Avg $\%$ RSD

\begin{tabular}{|c|c|c|c|c|c|c|c|c|c|c|}
\hline 43) & & $\mathrm{C} 031$ & Toluene & 1.007 & 0.836 & 0.910 & 0.910 & $0 \% 900$ & 0.913 & 6.72 \\
\hline & & & 1,3-dichloropropane & & & & & & & \\
\hline & $\mathbf{T}$ & $\mathrm{C} 033$ & dibromochloromethane & & 0.733 & & & & & \\
\hline & $T$ & $\mathrm{C} 034$ & bromoform & & & & & & & \\
\hline & $T$ & & Ioroethene & & & & & & & \\
\hline & $T$ & $\mathrm{C} 036$ & chlorobenzene & & & & & & & \\
\hline & & $\cos 7$ & $1,1,1,2$-tetrachloroe & & & & & & & \\
\hline & $T$ & $\mathrm{C} 038$ & ethylbenzene & & & & & & & \\
\hline & & $\cos 9$ & $\mathrm{~m}, \mathrm{p}$-xylene & & & & & 530 & & \\
\hline & $T$ & $\mathrm{CO} 40$ & o-xylene & & & & & & & \\
\hline & & C250 & total xylene & & & & & & & \\
\hline & $T$ & $\mathrm{C} 041$ & styrene & 1.235 & 1.045 & 1.150 & 1.133 & & & \\
\hline & & $\mathrm{CO} 42$ & isopropylbenzene & 2.129 & 1.773 & 1.946 & 1.893 & 1.847 & 1.918 & \\
\hline
\end{tabular}

56) I CI03 1,4-Dichlorobenzene-

57) $S$

CS10 4-bromofluorobenzene

$\begin{array}{lllllll}1.561 & 1.265 & 1.297 & 1.295 & 1.263 & 1.336\end{array}$

9.50

58) $\mathrm{T}$

C043 1, 1,2,2-tetrachloroe

1.4821 .209

$\begin{array}{ll}1.263 & 1.336 \\ 1.235 & 1.314\end{array}$

8.15

C044 bromobenzene

1.0650 .875

0.964

0.954

7.34

60) $T$

C045 1,2,3-tîjehloropropa

C046 n-propylbenzene

$\begin{array}{llllll}1.391 & 1.108 & 1.258 & 1.318 & 1.228 & 1.261\end{array}$

8.38

61)

C0 47 1,3,5-trimethylbenze

$\begin{array}{llllll}3.965 & 3.303 & 3.662 & 3.658 & 3.472 & 3.612\end{array}$

6.84

62)

C048'2-chlorotoluene

2

C049 4-chlorotoluene

$2.3603 .303 \quad 3.662 \quad 3.6583 .4723 .3$

8.34

64)

C050 tert-butylbenzene

$\begin{array}{lllllll}2.360 & 3.303 & 3.662 & 3.658 & 3.472 & 3.291\end{array}$

16.45

65)

$\operatorname{Cos} 1,1,2,4$-trimethylbenze

$\begin{array}{lllll}3.567 & 2.892 & 3.171 & 3.157\end{array}$

7.34

66)

67)

C052 sec-Butylbenzene

$2.5672 .8923 .1713 .1573 .002 \quad 3.158$

8.12

68)

C053 p-Isopropyltoluene

3.8683 .1582 .3672 .3132 .2032 .352

9.25

C054 1,3-Dichlorobenzene

$\begin{array}{lllllll}3.868 & 3.158 & 3.473 & 3.424 & 3.229 & 3.430\end{array}$

8.09

69)

70)

C055 1,4-Dichlorobenzene

C056 n-Butylbenzene

$\begin{array}{lllllll}3.394 & 2.771 & 3.016 & 2.985 & 2.827 & 2.999\end{array}$

8.14

$1.8621 .517 \quad 1.6921 .6851 .6351 .678$

7.41

$\begin{array}{llllll}1.862 & 1.517 & 1.809 & 1.798 & 1.731 & 1.743\end{array}$

7.74

72)

73)

C057 1,2-Dichlorobenzene

$\begin{array}{lllllll}3.120 & 2.597 & 2.857 & 2.890 & 2.743 & 2.841\end{array}$

6.81

C058 1,2-Dibromo-3-chloro

$1.856 \quad 1.496 \quad 1.616 \quad 1.613 \quad 1.547 \quad 1.626$

8. 49

$\begin{array}{llllllll}1 & 1.286 & 1.496 & 1.616 & 1.613 & 1.547 & 1.626 & 8.49\end{array}$

74)

75)

76)

C059 1,2,4-Trichlorobenze

5.81

C060 Hexachlorobutadiene

1.0261 .1691 .1861 .1101 .131

C061 Naphthalene

$\begin{array}{lllllll}0.917 & 0.714 & 0.815 & 0.900 & 0.830 & 0.835\end{array}$

$9.67 \cdots$

C062 1,2,3-Trichlorobenze

2.3151 .8012 .134

2.1881 .9002 .067

10.24

77)

1.0210 .9691 .0821 .1040

0.950

1.025

6.57

163

Passed method 8260 A criteria.

(\#) = Out of Range

L826ARWA.M

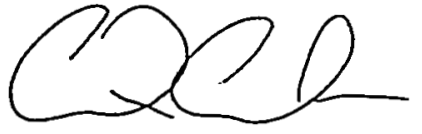

$6 \cdot 25 \cdot 97$ 


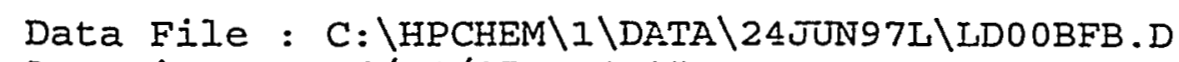

Acq Time : 06/24/97 14:05

Sample : $50 \mathrm{ng}$ BFB

MisC : 2.0 uL of 39157676

Operator: CQC Inst : 5971-L Multiplr: 1.00

Method : C: \HPCHEM $\backslash I \backslash M E T H O D S \backslash B E B 8240 . M$ Title :

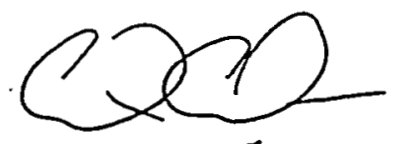

6.24.97

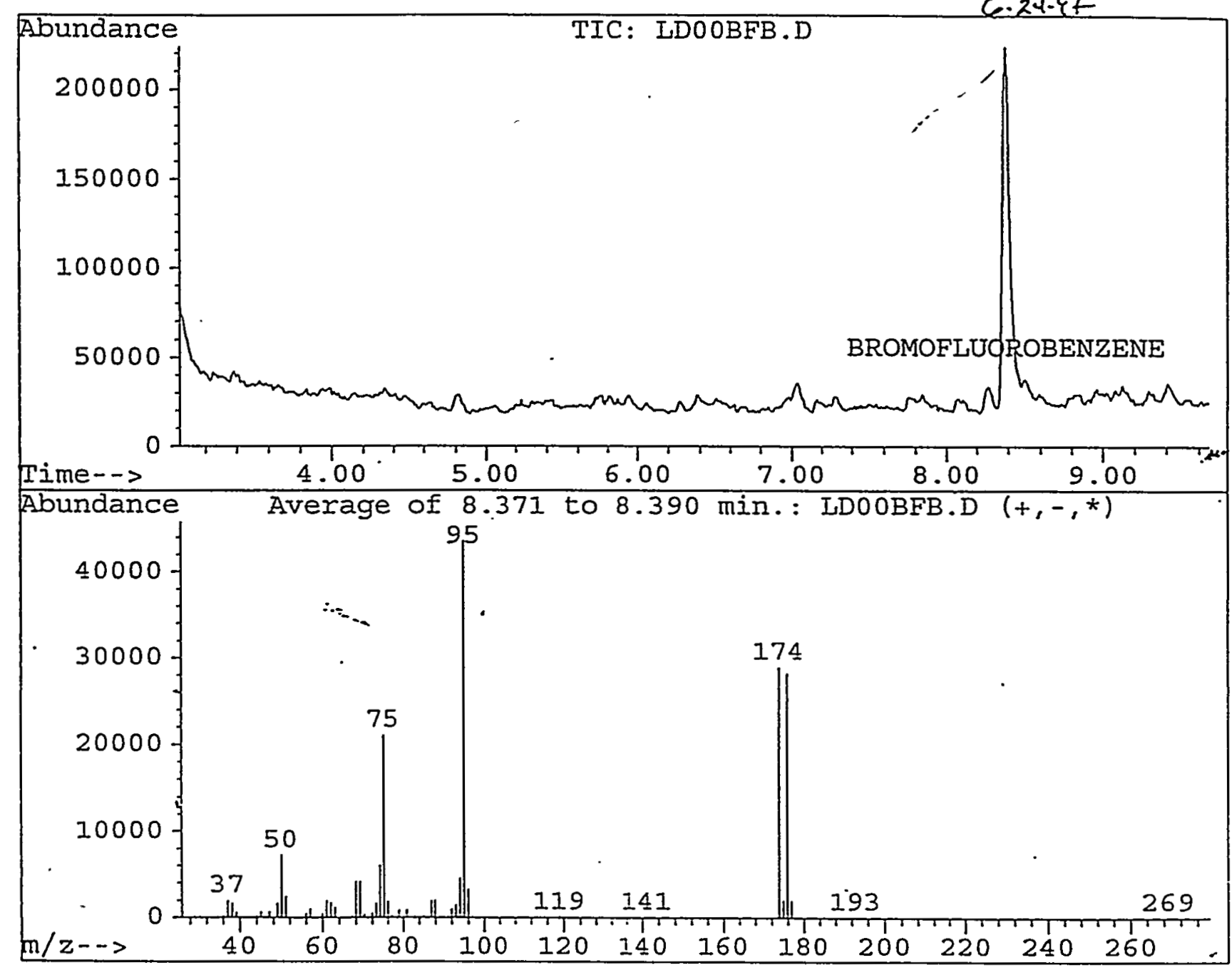

Peak Apex is scan: 555

Average of 3 Scans: 554, 555, 555 minus Background scan: 549

\begin{tabular}{|c|c|c|c|c|c|c|}
\hline $\begin{array}{c}\text { Target } \\
\text { Mass }\end{array}$ & $\begin{array}{l}\text { Rel. to } \\
\text { Mass }\end{array}$ & $\begin{array}{l}\text { Lower } \\
\text { Limit\% }\end{array}$ & $\begin{array}{l}\text { Upper } \\
\text { Limiこ\% }\end{array}$ & $\begin{array}{l}\text { Rel. } \\
\text { Abn: }\end{array}$ & $\begin{array}{l}\text { Raw } \\
\text { Abn }\end{array}$ & $\begin{array}{c}\text { Result } \\
\text { Pass/Fail }\end{array}$ \\
\hline 50 & 95 & 15 & 40 & 16.65 & 7269 & PASS \\
\hline 75 & 95 & 30 & 60 & 48.36 & 21109 & PASS \\
\hline 95 & 95 & 100 & 100 & 100.00 & 43653 & PASS \\
\hline 96 & 95 & 5 & 9 & 7.48 & 3264 & PASS \\
\hline 173 & 174 & 0 & 2 & 0.00 & 0 & PASS \\
\hline 174 & 95 & 50 & 100 & 66.43 & 28997 & PASS \\
\hline 175 & 174 & 5 & 9 & 7.44 & 2158 & PASS \\
\hline 176 & 174 & 95 & 101 & 97.58 & 28296 & PASS \\
\hline 177 & 176 & 5 & 9 & 7.33 & 2073 & PASS \\
\hline
\end{tabular}




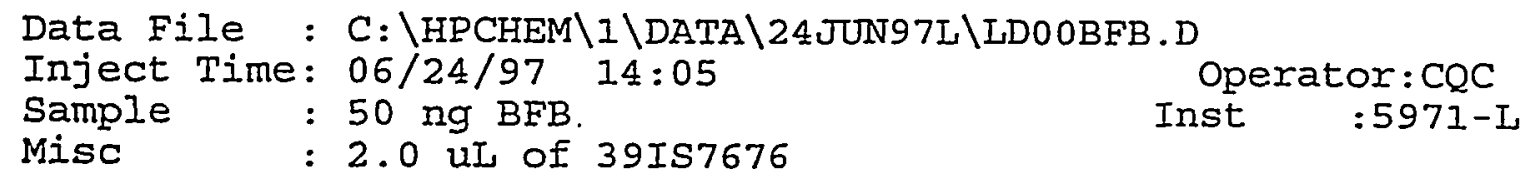

Average of 8.371 to $8.390 \mathrm{~min}$.: LDOOBFB.D $50 \mathrm{ng} \mathrm{BFB}$

Modified:added subtracted scaled

$\begin{array}{rrrrrrrr}\mathrm{m} / \mathrm{z} & \text { abund. } & \mathrm{m} / \mathrm{z} & \text { abund. } & \mathrm{m} / \mathrm{z} & \text { abund. } & \mathrm{m} / \mathrm{z} & \text { abund. } \\ 36.00 & 299 & 51.05 & 2516 & 59.00 & 4216 & 82.90 & 228 \\ 37.10 & 2001 & 55.10 & 45 & 70.05 & 421 & 84.10 & 125 \\ 38.10 & 1696 & 56.05 & 504 & 72.00 & 545 & 85.05 & 179 \\ 39.10 & 602 & 57.05 & 1009 & 73.00 & 1720 & 87.00 & 1962 \\ 41.10 & 115 & 58.00 & 17 & 74.05 & 6087 . & 87.95 & 2020 \\ 42.10 & 72 & 60.00 & 449 & 75.05 & 21109 & 92.00 & 994 \\ 44.00 & 188 & 61.00 & 1990 & 76.05 & 1931 & 93.00 & 1487 \\ 45.05 & 670 & 62.00 & 1776 & 77.05 & 527 & 93.95 & 4649 \\ 47.05 & 713 & 63.00 & 1230 & 78.95 & 922 & 95.00 & 43653 \\ 49.05 & 1698 & 67.10 & 131 & 80.95 & 991 & 96.05 & 3264 \\ 50.05 & 7269 & 68.00 & 4248 & 82.00 & 132 & 96.95 & 136\end{array}$

Average of 8.371 to $8.390 \mathrm{~min}$ : LDOOBFB.D $50 \mathrm{ng} \mathrm{BFB}$

Modified:added subtracted scaled

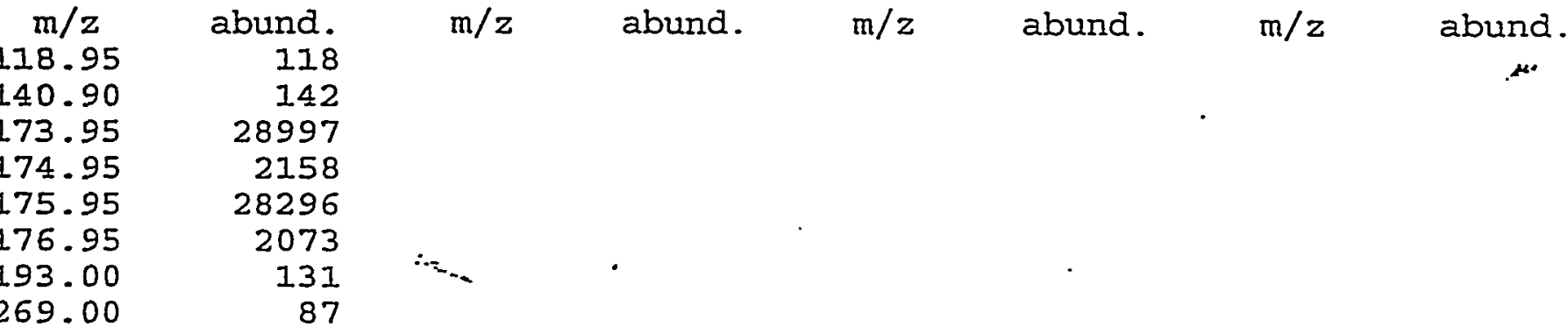


Quantitation Report

Data File : C: \HPCHEM \I\DATA 24 JUN97L\LD01S05.D

Acq Time : 06/24/97 14:27

Sample : $5.0 \mathrm{ug} / \mathrm{L}$ Initial cal std

Misc : 142WS28290

Quant Time: Jun 25 8:35 1997

Operator: CQC

Inst : 5971-L

Multiplr: 1.00

Method : C: \HPCHEM \I\METHODS \L826ARWA.M

Title : VOA COMPOUND IIST

Last Update : Wed Jun 25 08:33:27 1997

Response via : Single Level Calibration

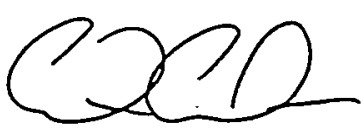

$6.25-97$

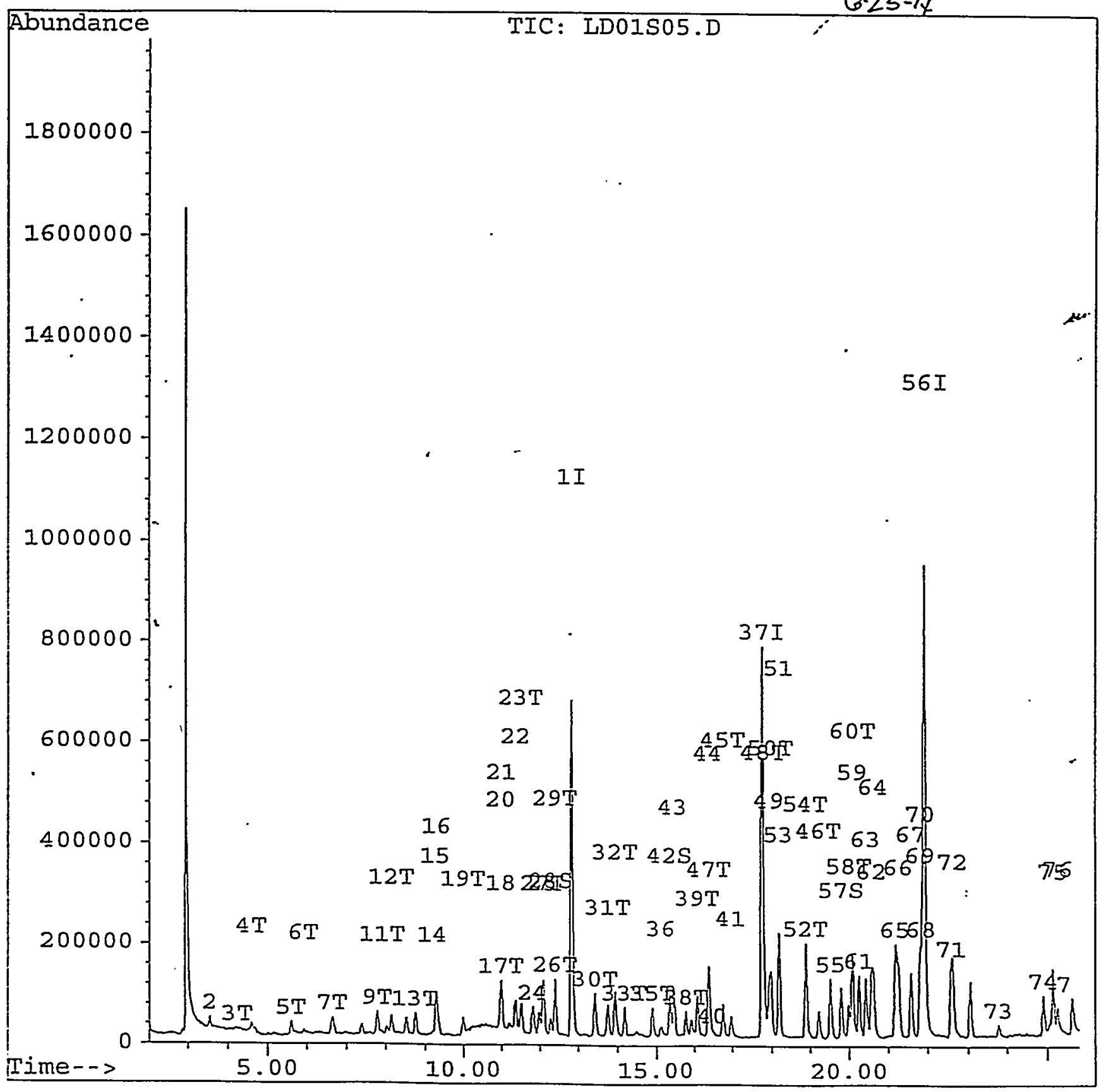

$1 \subseteq 6$ 


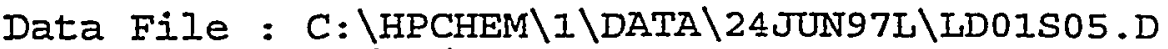

Acq Time : 06/24/97 14:27

Sample : $5.0 \mathrm{ug} / \mathrm{I}$ Initial Cal std

Misc : 142WS28290

Operator: CQC

Quant Time: Jun 25 8:35 1997

Inst : 5971-L

Multiplr: 1.00

Method : C: \HPCHEM \I\METHODS \L826ARWA.M

Title : VOA COMPOUND IIST

Iast Update : Wed Jun 25 08:33:27 1997

Response via : LD01S05.

Internal Standards

R.T. QIon Response Conc Units Area\%

1) CIO1 Flourobenzene

37) CI02 Chlorobenzene- $\mathrm{d5}$

56) CI03 1,4-Dichlorobenzene-d

System Monitoring Compounds

28) CS15 1,2-dichloroethane-d4

42) CSO5 toluene-d8

57) CS10 4-bromofluorobenzene

Target Compounds

2) $\mathrm{COO2}$ dichlorodifluorometha

3) $\mathrm{CO03}$ chloromethane

4) C004 vinyl chloride

5) C005 bromomethane

6) $\mathrm{COO} 6$ chloroethane

7) $\mathrm{C007}$ trichlorofluoromethan

B) $\mathrm{C} 071$ acrolein

9) $\operatorname{Co0} 8$ 1,1-dichloroethene

10) $\mathrm{C} 063$ freon 113

11) co09-acetone

12) $C 010$ carbon disulfide

13) Co11 methylene chloride

14) C072 acrylonitrile

15) C012'trans-1,2-Dichloroeth

16) C305 methyl-t-butyl-ether

17) 0013 cis-1,2-dichloroethen

IB) $\mathrm{C} 055$ total-1,2-dichloroeth

19) $\operatorname{Co14} 1,1$-dichloroethane

20) $\operatorname{Col5} 2,2$-dichloropropane

21) CII0 2-butanone

22) CIO1 bromochloromethane

23) $\mathrm{C} 016 \mathrm{chloroform}$

24) $\operatorname{Co17} 1,1,1$-Trichloroethane

25) $\operatorname{Co18~1,1-dichloropropene~}$

26) 0019 1,2-dichloroethane

27) C020 carbon tetrachloride

29) $\mathrm{C} 021$ benzene

30) C022 trichloroethene

31) C023 1,2-dichloropropane

32) Co24 dibromomethane

33) C025 bromodichloromethane

34) C026 2-chloroethylvinyleth

35) C027 cis-1,3-dichloroprope
12.28

15.35

19.80

$\begin{array}{rrr}12.83 & 96 & 1349535 \\ 17.75 & 117 & 980362 \\ 21.90 & 152 & 637818 \\ & & \\ 12.28 & 65 & 48098 \\ 15.35 & 98 & 132607 \\ 19.80 & 95 & 99584\end{array}$

3.56

4.23

85

61589

4.59

5.59

5.92

6.65

0.00

7.78

0.00

7.91

8.15

8.76

9.20

9.28

9.33

10.98

10.98

10.00

10.98

11.02

11.36

11.51

11.81

12.08

12.41

12.09

12.40

13.43

13.76

13.94

14.18

0.00

14.90
50

62

23396

35885

51274

21719

89184

101

56

96

151

43

76

84

53

96

73

96

96

63

77

72

128

83

97

75

62

117

78

130

63

93

83

63

75
43149

15973

111055

47954

11857

47926

117681

50833

98700

85489

80869

4799

31930

100309

85992

74765

55497

77904

152658

64058

58135

47737

96411

81754
$50.00 \mathrm{ug} / \mathrm{L} \quad 100.00$

$50.00 \mathrm{ug} / \mathrm{I} \quad 100.00$

$50.00 \mathrm{ug} / \mathrm{L} \quad 100.00$

$\because$ Recovery

$5.00 \mathrm{ug} / \mathrm{I}$

$10.00 \%$

$5.00 \mathrm{ug} / \mathrm{L}$

$10.00 \%$

$5.00 \mathrm{ug} / \mathrm{L}$

$10.00 \%$

$5.00 \mathrm{ug} / \mathrm{L} \#$

Qvalue

$5.00 \mathrm{ug} / \mathrm{L}$

$5.00 \mathrm{ug} / \mathrm{I}$

$5.00 \mathrm{ug} / \mathrm{I} \#$

$5.00 \mathrm{ug} / \mathrm{L}$

$5.00 \mathrm{ug} / \mathrm{I} \#$

Not Detected

$5.00 \mathrm{ug} / \mathrm{L}$

Not Detected

$5.00 \mathrm{ug} / \mathrm{I} \#$

$5.00 \mathrm{ug} / \mathrm{I}$

$5.00 \mathrm{ug} / \mathrm{I}$

$5.00 \mathrm{ug} / \mathrm{L} \#$

$5.00 \mathrm{ug} / \mathrm{I}$

$5.00 \mathrm{ug} / \mathrm{L}$

$5.00 \mathrm{ug} / \mathrm{L}$

$10.00 \mathrm{ug} / \mathrm{L}$ m6.2547 96

$5.00 \mathrm{ug} / \mathrm{I} \# \quad 94$

$5.00 \mathrm{ug} / \mathrm{L} \#-72$

$5.00 \mathrm{ug} / \mathrm{L} \# \quad 25$

$5.00 \mathrm{ug} / \mathrm{L} \# \quad 70$

$5.00 \mathrm{ug} / \mathrm{L} \# \quad 92$

$5.00 \mathrm{ug} / \mathrm{I}$

$5.00 \mathrm{ug} / \mathrm{L}$

$5.00 \mathrm{ug} / \mathrm{L} \#$

$5.00 \mathrm{ug} / \mathrm{L}$

$5.00 \mathrm{ug} / \mathrm{L} \#$

$5.00 \mathrm{ug} / \mathrm{L}$

$5.00 \mathrm{ug} / \mathrm{L}$

$5.00 \mathrm{ug} / \mathrm{L}$

$5.00 \mathrm{ug} / \mathrm{I}$

Not Detected

$5.00 \mathrm{ug} / \mathrm{I} \#$

95

98

97

98

96

89

60

91

66

79

90

92

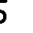

$(\mathrm{NR})=$ Not Reported $(\mathrm{SP})=$ Spiked analyte $(\mathrm{m})=$ manual integration

(*) Does not meet EPA spectral criteria (False Hit) 


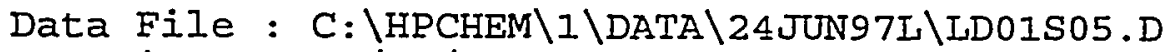

Acq Time : 06/24/97 14:27

Sample : $5.0 \mathrm{ug} / \mathrm{L}$ Initial Cal sta

Misc : 142WS28290

Quant Time: Jun $258: 351997$

Operator: CQC

Inst : 5971-L Multiplr: 1.00

Method : C: \HPCHEM II\METHODS \I826ARWA.M

Title

: VOA COMPOUND LIST

Iast Update : Wed Jun 25 08:33:27 1997

Response via : LD01S05.

Compound

36) C205 4-methyl-2-pentanone
38) C028 trans-1,3-dichloropro
39) C029 1,1,2-trichloroethane
40) C210 2-hexanone
41) C030 1,2-Dibromoethane
43) C031 Toluene
44) C032 1,3-dichloropropane
45) C033 dibromochloromethane
46) C034 bromoform
47) C035 tetrachloroethene
48) C036 chlorobenzene
49) C037 1,1,1,2-tetrachloroet
50) C038 ethylbenzene
51) C039 m,p-xylene
52) C040 o-xylene
53) C250 total xylene
54) C041 styrene
55) C042 isopropylbenzene
58) C043 1,1,2,2-tetrachloroet
59) C044-bromobenzene
60) C045 1,2,3-trichloropropan
61) C046 n-propylbenzene
62) C047 1,3,5-trimethylbenzen
63) C048 2-chlorotoluene
64) C049 4-chlorotoluene
65) C050 tert-butylbenzene
66) C051 1,2,4-trimethylbenzen
67) C052 sec-Butylbenzene
58) C053 p-Isopropyltoluene
69) C054 1,3-Dichlorobenzene
70) C055 1,4-Dichlorobenzene
71) C056 n-Butylbenzene
72) C057 1,2-Dichlorobenzene
73) C058 1,2-Dibromo-3-chlorop
74) C059 1,2,4-Trichlorobenzen
75) C060 Hexachlorobutadiene
76) C061 Naphthalene
77) C062 1,2,3-Trichlorobenzen

R.T. QIon

$15.12 \quad 43$

15.78

16.07

16.49

16.96

15.47

16.35

16.76

19.22

16.39

17.81

17.93

17.99

18.19

18.87

18.19

18.89

19.53

19.99

20.09

20.09

20.26

20.57

20.43

20.62

21.17

21.26

21.57

21.83

21.79

21.79

22.57

22.62

23.76

24.90

25.14

25.25

25.60

43
75
97
43
107
92
76
129
173
164
112
131
106
106
106
106
104
105
83
156
75
91
105
91
91
119
105
105
119
146
146
91
146
75
180
225
128
180

180
Response

$594420^{\circ}$
69094
57060
22852
74939
98746
84762
82091
56799
59446
128603
67745
56617
144797

144797

70467

214811

121049

208694

94556

67950

88748

252924

169441

150543

190743

227530

173011

246725

216492

118753

118753

198978

118386

17834

74382

58480

147645

65153
Conc Unit Qvalue

$5.00 \mathrm{ug} / \mathrm{L} \#-96$

$5.00 \mathrm{ug} / \mathrm{L} \# \quad 77$

$5.00 \mathrm{ug} / \mathrm{I} \quad 93$

$5.00 \mathrm{ug} / \mathrm{L} \# \quad 97$

$5.00 \mathrm{ug} / \mathrm{L} \# \quad 97$

$5.00 \mathrm{ug} / \mathrm{L} \quad 99$

$5.00 \mathrm{ug} / \mathrm{L} \quad 89$

$5.00 \mathrm{ug} / \mathrm{L} \quad 100$

$5.00 \mathrm{ug} / \mathrm{L} \# \quad 99$

$5.00 \mathrm{ug} / \mathrm{I} \quad 92$

$5.00 \mathrm{ug} / \mathrm{I} \quad 92$

$5.00 \mathrm{ug} / \mathrm{L}$. 98

$5.00 \mathrm{ug} / \mathrm{L} \quad 89$

$10.00 \mathrm{ug} / \mathrm{L} \quad 89$

$5.00 \mathrm{ug} / \mathrm{L} \quad 91$

$15.00 \mathrm{ug} / \mathrm{I} \mathrm{m}_{6.2647}^{0 \mathrm{x}} 91$

$5.00 \mathrm{ug} / \mathrm{L} \quad 93$

$5.00 \mathrm{ug} / \mathrm{L} \quad 97$

$5.00 \mathrm{ug} / \mathrm{L} \quad 96$

$5.00 \mathrm{ug} / \mathrm{L} \quad 97$

$5.00 \mathrm{ug} / \mathrm{L} \quad 93$

$5.00 \mathrm{ug} / \mathrm{L} \quad 97$

$5.00 \mathrm{ug} / \mathrm{L} \quad 94$

$5.00 \mathrm{ug} / \mathrm{L} \quad 93$

$5.00 \mathrm{ug} / \mathrm{L} \quad 96$

$5.00 \mathrm{ug} / \mathrm{L}$. $\quad 99$

$5.00 \mathrm{ug} / \mathrm{L} \quad 80$

$5.00 \mathrm{ug} / \mathrm{L} \quad 95$

$5.00 \mathrm{ug} / \mathrm{L} \quad \cdot 98$

$5.00 \mathrm{ug} / \mathrm{L} \quad 94$

$5.00 \mathrm{ug} / \mathrm{L} \quad 95$

$5.00 \mathrm{ug} / \mathrm{L} \# \quad 91$

$5.00 \mathrm{ug} / \mathrm{I} \quad 98$

$5.00 \mathrm{ug} / \mathrm{I} \quad 87$

$5.00 \mathrm{ug} / \mathrm{L} \quad 97$

$5.00 \mathrm{ug} / \mathrm{L} \quad 96$

$5.00 \mathrm{ug} / \mathrm{L} \quad 99$

$5.00 \mathrm{ug} / \mathrm{L} \quad 97$

168. Data Processed By:

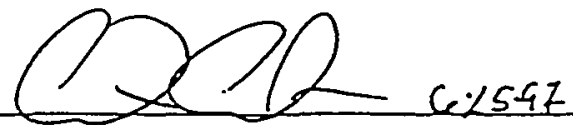

$(N R)=$ Not Reported $(S P)=S p i k e d$ analyte $(m)=$ manual integration

(*) Does not meet EPA spectral criteria (False Hit) PX125 Page 2 
Quantitation Report

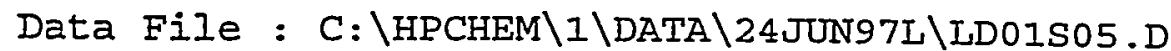

Acq Time : 06/24/97 $14: 27$

Sample : $5.0 \mathrm{ug} / \mathrm{I}$ Initial Cal std

Misc : $142 \mathrm{WS} 28290$

Quant Time: Jun 25 8:34 1997

Operator: CQC Inst : 5971-L Multiplr: 1.00

Method : C: \HPCHEM \I\METHODS \L826ARWA.M

Title : VOA COMPOUND IIST

Last Update : Wed Jun 25 08:33:27 1997

Response via : Single Level Calibration

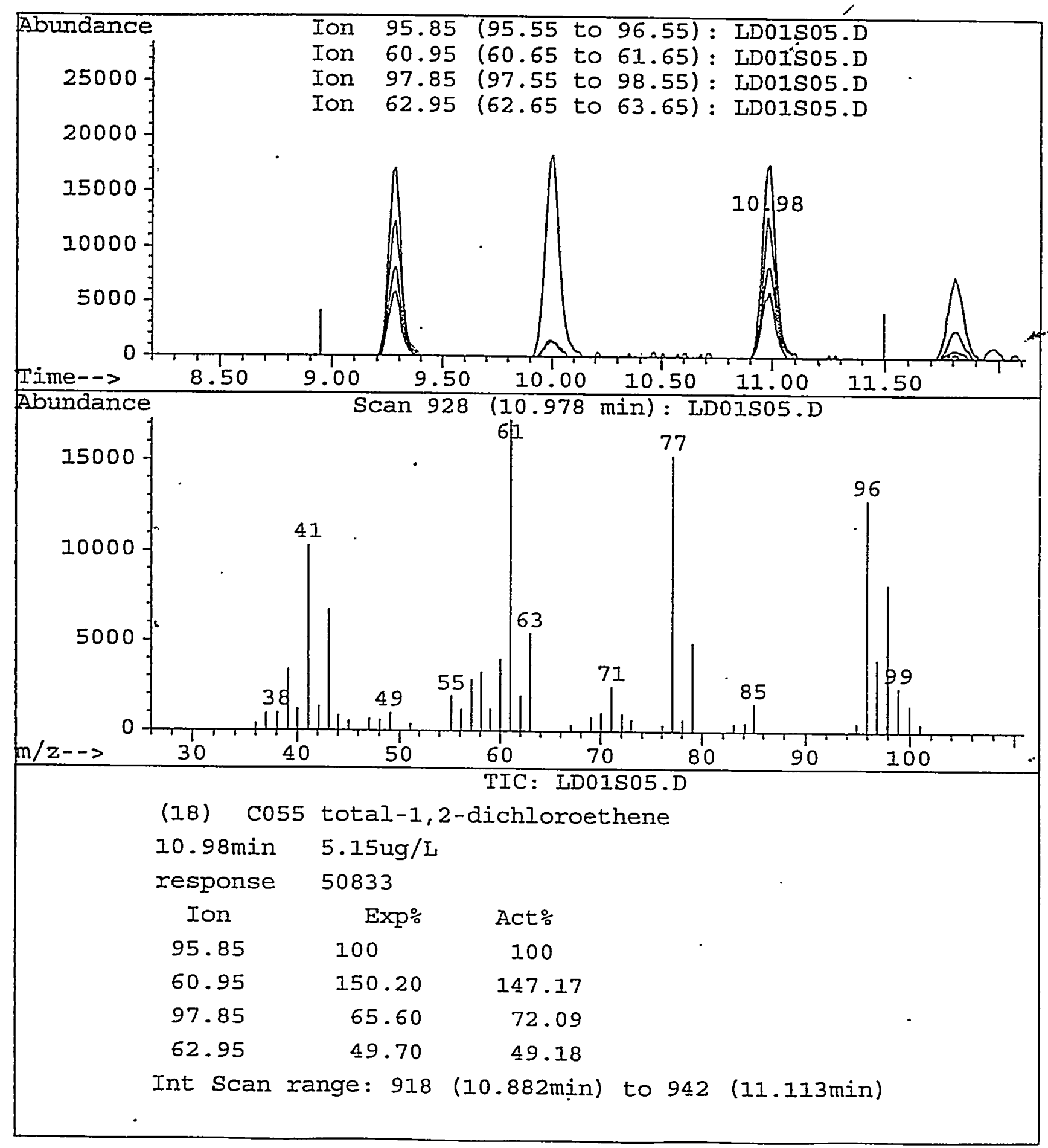


Quantitation Report

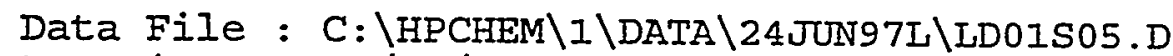

Acq Time : 06/24/97 14:27

Sample : $5.0 \mathrm{ug} / \mathrm{I}$ Initial cal std

Misc : $142 W S 28290$

Quant Time: Jun 25 8:34 1997

Operator: CQC

Inst : 5971-L

Multiplr: 1.00

Method : C: \HPCHEM \I\METHODS\L826ARWA.M

Title : VOA COMPOUND LIST

Last Update : Wed Jun 25 08:33:27 1997

Response via : Single Level Calibration

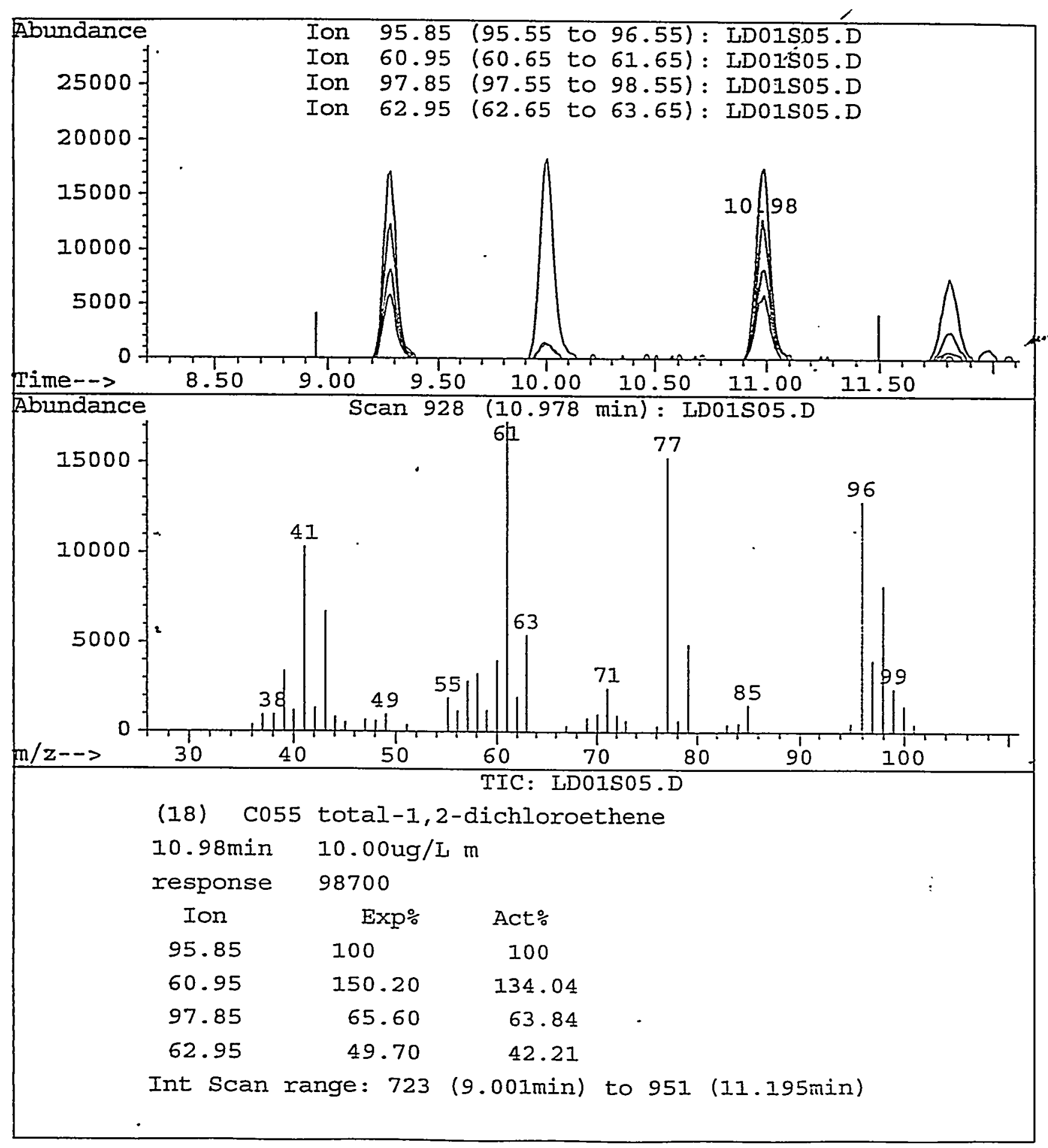


Quantitation Report

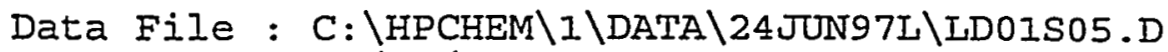

Acq Time : 06/24/97 14:27

Sample : $5.0 \mathrm{ug} / \mathrm{L}$ Initial Cal std

Misc : 142WS28290

Quant Time: Jun 25 8:34 1997

Operator: CQC

Inst : 5971-L Multiplr: 1.00

Method : C: \HPCHEM \I\METHODS\L826ARWA.M

Title : VOA COMPOUND IIST

Last Update : Wed Jun 25 08:33:27 1997

Response via : Single Level Calibration

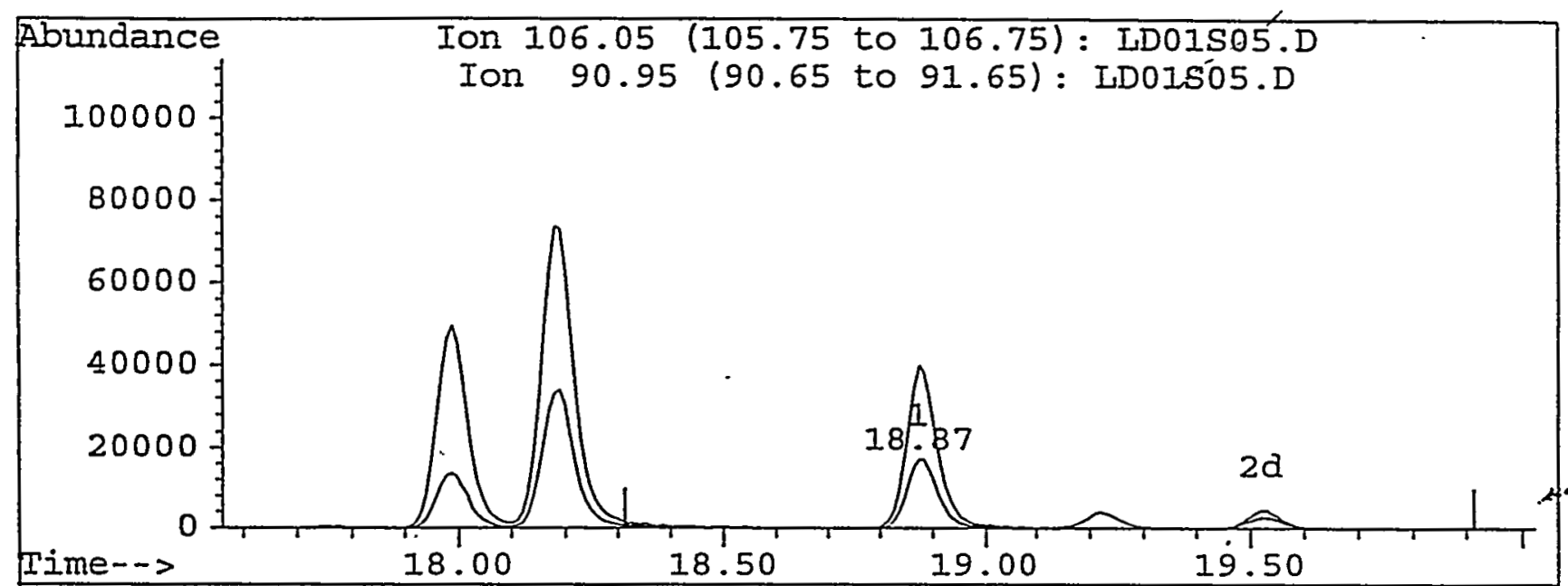

Abundance Scan $1746(18.874 \mathrm{~min}):$ ID01S05.D

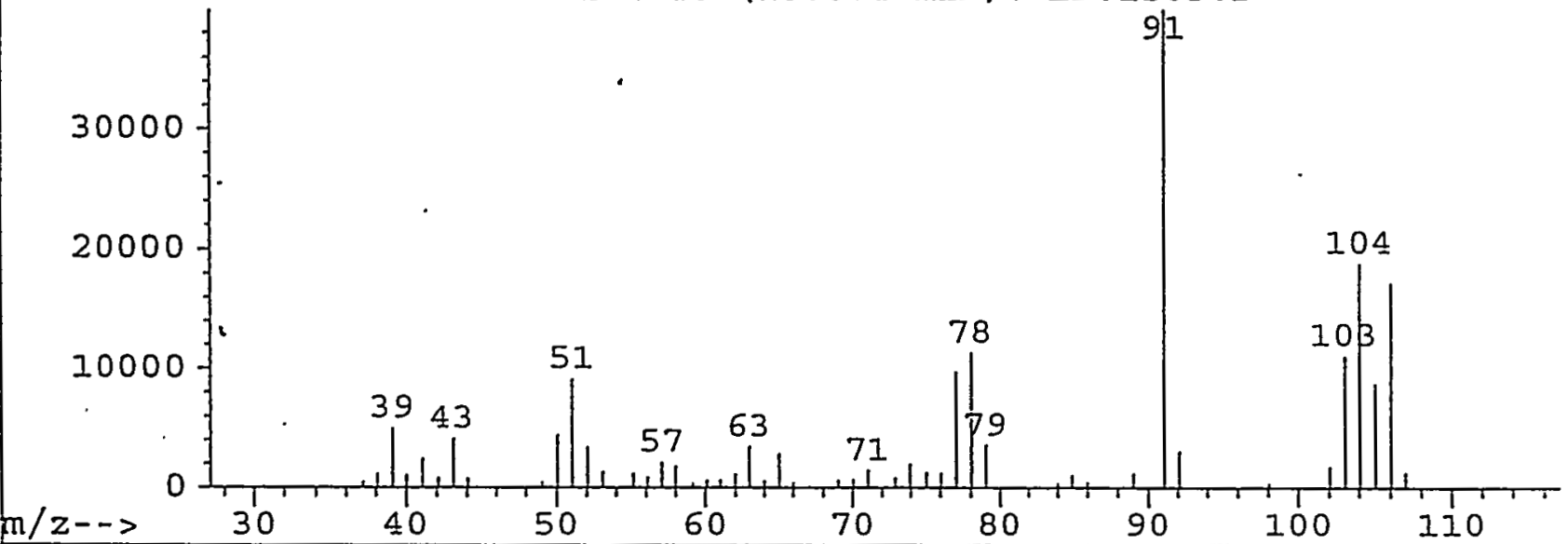

$m / z-->$ $30 \quad 40$ TIC: LD01S05.D

(53) C250 total xylene

$18.87 \mathrm{~min} \quad 4.92 \mathrm{ug} / \mathrm{I}$

response 70467

Ion

106.05

Exp\%

Act $\%$

90.95

100

100

0.00

217.60

231.42

0.00

0.00

0.00

Int Scan range: 1737 (18.787min) to 1761 (19.019min) 


\section{Quantitation Report}

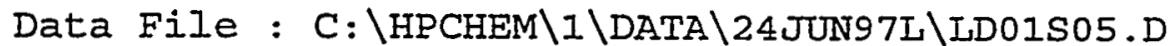

Acq Time : 06/24/97 14:27

Sample : $5.0 \mathrm{ug} / \mathrm{L}$ Initial Cal std

Misc : 142WS28290

Quant Time: Jun 25 8:35 1997

Operator: CQC

Inst : 5971-L Multiplr: 1.00

Method : C: \HPCHEM\I\METHODS\I826ARWA.M

Title : VOA COMPOUND LIST

Last Update : Wed Jun 25 08:33:27 1997

Response via : Single Level Calibration

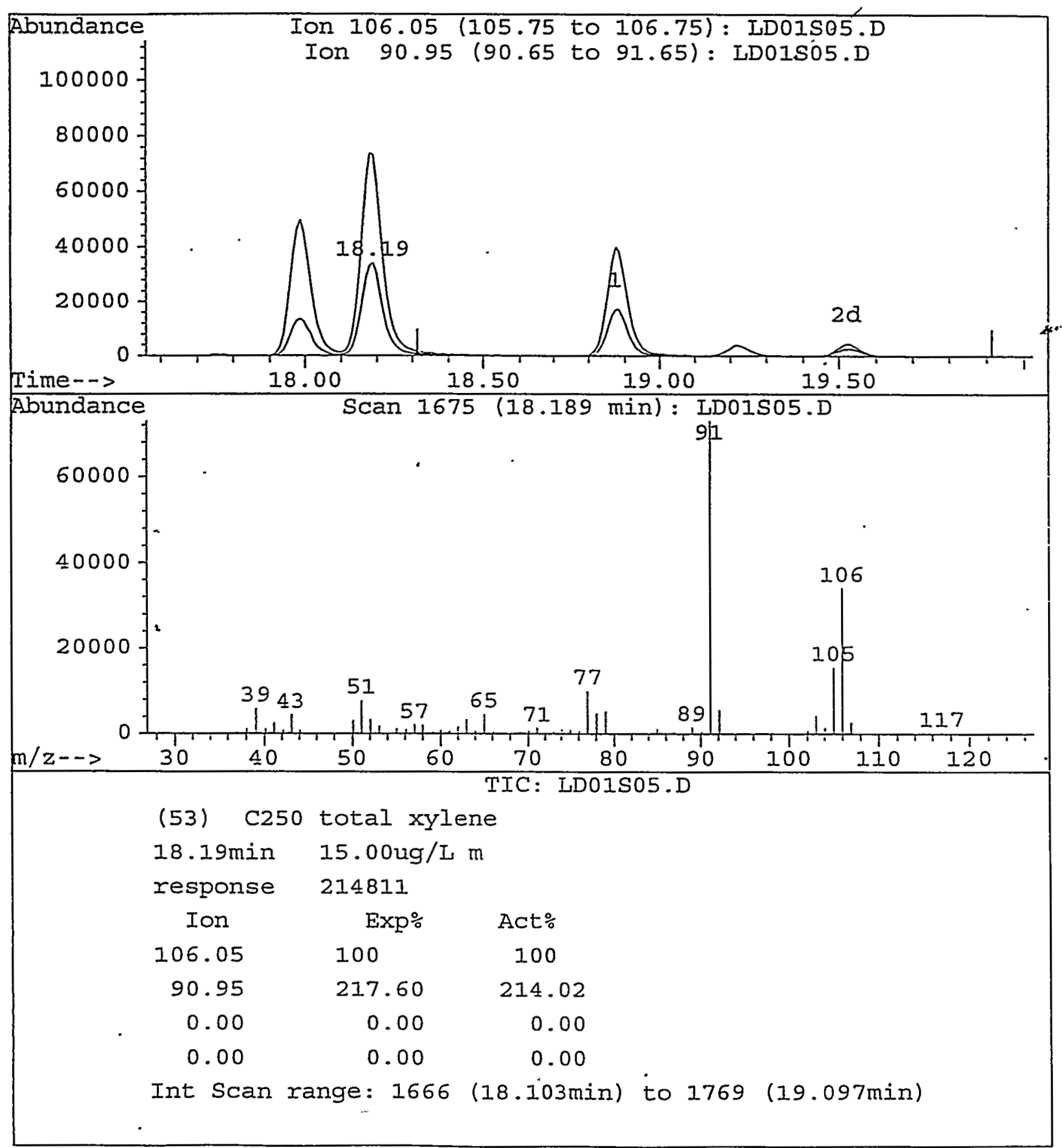


Quantitation Report

Data File : C: $\backslash$ HPCHEM $\backslash I \backslash D A T A \backslash 24 J U N 97 L \backslash L D 02 S 20 . D$

Acq Time : 06/24/97 14:59

Sample : $20 \mathrm{ug} / \mathrm{I}$ Initial Cal std

Misc : 142WS28291

Quant Time: Jun 25 8:39 1997

Operator: CQC

Inst : $5971-\mathrm{I}$

Multiplr: 1.00

Method : C: \HPCHEM\I\METHODS \L826ARWA.M

Title : VOA COMPOUND LIST

Last Update : Wed Jun 25 08:37:44 1997

Response via : Single Level Calibration
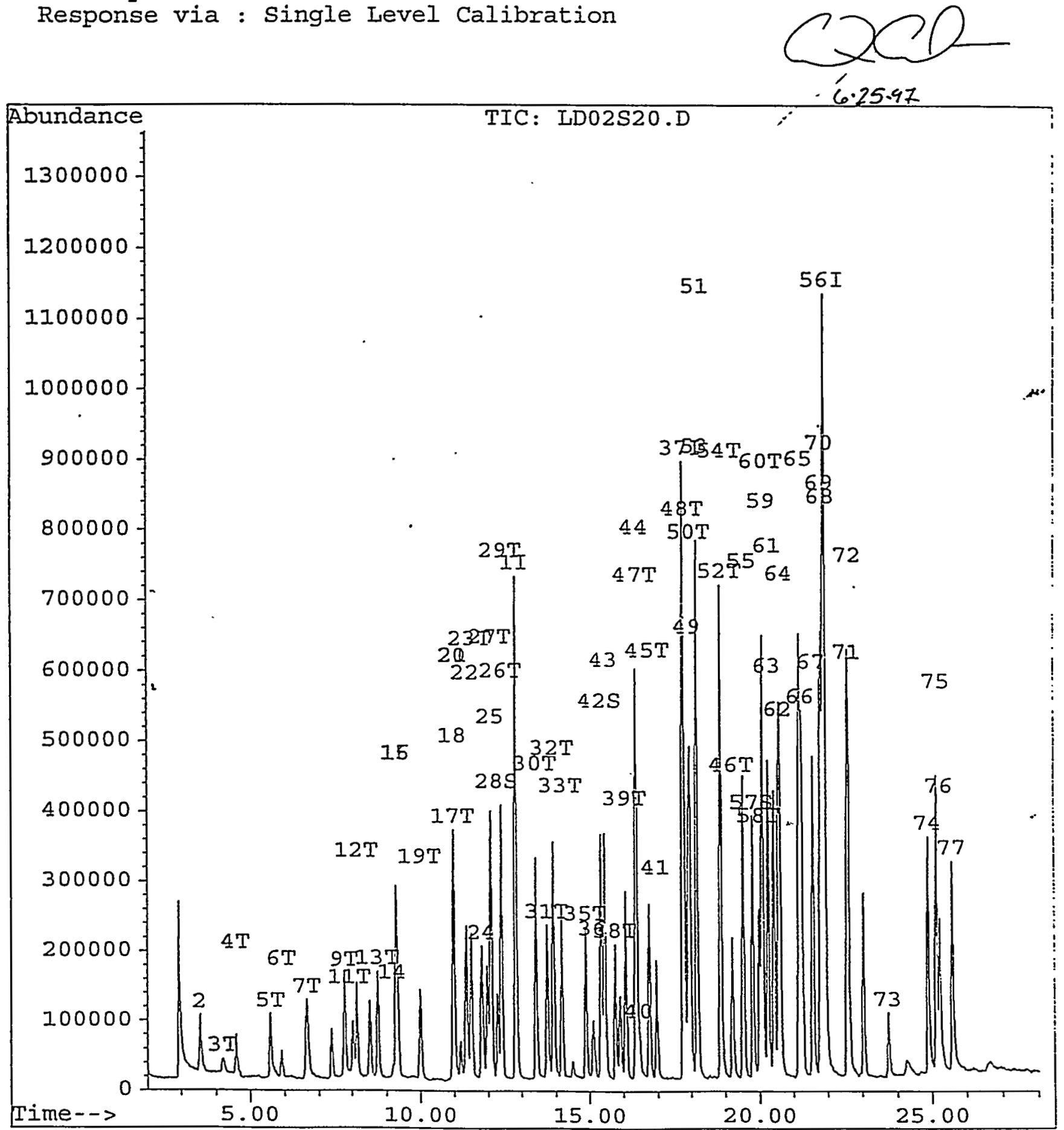
Quantitation Report

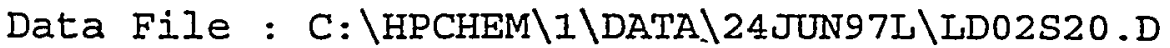

Acq Time : 06/24/97 14:59

Sample : $20 \mathrm{ug} / \mathrm{L}$ Initial Cal std

Misc : 142WS28291

Quant Time: Jun 25 8:39 1997

Operator: $\mathrm{CQC}$

Inst : 5971-L

Multiplr: 1.00

Method : C: \HPCHEM \I\METHODS \L826ARWA.M

Title : VOA COMPOUND LIST

Last Update : Wed Jun 25 08:37:44 1997

Response via : LD02S20.

Internal standards

1) $\mathrm{CIOI}$ Flourobenzene

37) CI02 Chlorobenzene-d5

56) CI03 1,4-Dichlorobenzene-d

System Monitoring Compounds

28) CS15 1,2-dichloroethane-d4

42) CS05 toluene-d8

57) CSI0 4-bromofluorobenzene

Target Compounds

2) $\mathrm{C002}$ dichlorodifluorometha

3) $\mathrm{C003}$ chloromethane

4) C004 vinyl chloride

5) C005 bromomethane

6) C006 chloroethane

7) $\mathrm{C007}$ trichlorofluoromethan

8) C071 acrolein

9) $\mathrm{C} 008$ 1,1-dichloroethene

10) $\mathrm{C} 063$ freon 113

11) Co09-acetone

12) C010 carbon disulfide

13) C011 methylene chloride

14) C072 acrylonitrile

15) $\mathrm{C} 012$ trans-1,2-Dichloroeth

16) C305 methyl-t-butyl-ether

17) $\mathrm{C} 013 \mathrm{cis}-1,2-$ dichloroethen

18) $\mathrm{C} 55$ total-1,2-dichloroeth

19) C014 1,1-dichloroethane

20) $\mathrm{C} 015$ 2,2-dichloropropane

21) CI10 2-butanone

22) CI01 bromochloromethane

23) $\mathrm{C} 016$ chloroform

24) $\operatorname{Col} 11,1,1$-Trichloroethane

25) $\operatorname{co18} 1,1$-dichloropropene

26) C019 1,2-dichloroethane

27) C020 carbon tetrachloride

29) C021 benzene

30) C022 trichloroethene

31) C023 1,2-dichloropropane

32) C024 dibromomethane

33) Co25 bromodichloromethane

34) C026 2-chloroethylvinyleth

35) C027 cis-1,3-dichloroprope

R.T. QIon

12.82

17.75

21.89

96

117

152

$14353470^{\circ}$

1058621

695083

12.27

15.34

19.79
65

98

95

\subsection{5}

4.21

4.59

5.59

5.91

6.64

0.00

7.78

0.00

7.92

8.14

8.75

9.18

9.27

9.32

10.98

10.98

9.99

10.98

11.01

11.37

11.50

11.81

12.07

12.40

12.09

12.40

13.42

13.75

13.93

14.18

0.00

14.89

85

50

62

94

64

101

56

96

151

43

76

84

53

96

73

96

96

63

77

72

128

83

97

75

62

117

78

130

63

93

83

63

75

\section{2 \\ 537721 \\ 351676}

211409

81706

135420

156766

82355

322502

152898

32466

404618

159054

39697

170894

397621

183060

353630

300018

283534

19492

111407

337944

308138

260978

202942

266163

530478

244098

203135

173207

343173

299818.
Conc Units

Area:

$50.00 \mathrm{ug} / \mathrm{I} \quad 100.00$

$50.00 \mathrm{ug} / \mathrm{I} \quad 100.00$

$50.00 \mathrm{ug} / \mathrm{I} \quad 100.00$

$\because$ Recovery

$20.00 \mathrm{ug} / \mathrm{I} \quad 40.00 \%$

$20.00 \mathrm{ug} / \mathrm{L} \quad 40.00 \%$

$20.00 \mathrm{ug} / \mathrm{L}$

$40.00 \%$

Qvalue

$20.00 \mathrm{ug} / \mathrm{L} \quad 98$

$20.00 \mathrm{ug} / \mathrm{I}$. 98

$20.00 \mathrm{ug} / \mathrm{I} \quad 99$

$20.00 \mathrm{ug} / \mathrm{I} \quad 95$

$20.00 \mathrm{ug} / \mathrm{L} \quad 96$

$20.00 \mathrm{ug} / \mathrm{I} \quad 98$

Not Detected

$20.00 \mathrm{ug} / \mathrm{L}$

89

Not Detected

$20.00 \mathrm{ug} / \mathrm{I} \# \quad 80$

$20.00 \mathrm{ug} / \mathrm{L} \quad 91$

$20.00 \mathrm{ug} / \mathrm{L} \# \quad 71$

$20.00 \mathrm{ug} / \mathrm{I} \quad 89$

$20.00 \mathrm{ug} / \mathrm{I} \quad 90$

$20.00 \mathrm{ug} / \mathrm{I} \# \quad 88$

$20.00 \mathrm{ug} / \mathrm{L} \quad 88$

$40.00 \mathrm{ug} / \mathrm{L} \mathrm{m}_{6.25 .97}^{06} 98$

$20.00 \mathrm{ug} / \mathrm{L}$ \# 96

$20.00 \mathrm{ug} / \mathrm{L} \quad \# \quad \ldots .75$

$20.00 \mathrm{ug} / \mathrm{L} \# 32$

$20.00 \mathrm{ug} / \mathrm{I} \# \quad 68$

$20.00 \mathrm{ug} / \mathrm{I} \# \quad 94$.

$20.00 \mathrm{ug} / \mathrm{I} \quad 99$

$20.00 \mathrm{ug} / \mathrm{L} \# \quad 85$

$20.00 \mathrm{ug} / \mathrm{L} \quad 99$

$20.00 \mathrm{ug} / \mathrm{L} \quad 98$

$20.00 \mathrm{ug} / \mathrm{I} \# \quad 88$

$20.00 \mathrm{ug} / \mathrm{L} \quad 89$

$20.00 \mathrm{ug} / \mathrm{I} \quad 97$

$20.00 \mathrm{ug} / \mathrm{L} \quad 91$

$20.00 \mathrm{ug} / \mathrm{I} \quad 98$

Not Detected

$20.00 \mathrm{ug} / \mathrm{L} \#$ 
Data File : C: \HPCHEM \I\DATA $24 \pi U N 97 L \backslash L D 02 S 20 . D$

$\begin{array}{ll}\text { Acq Time } & : 06 / 24 / 97 \text { 14:59 } \\ \text { Sample } & : 20 \mathrm{ug} / \mathrm{L} \text { Initial Cal std } \\ \text { Misc } & : 142 W 528291\end{array}$

Quant Time: Jun 25 8:39 1997

Operator: CQC

Inst : 5971-I Multiplr: 1.00

Method : C: \HPCHEM \I\METHODS \L826ARWA.M

Title : VOA COMPOUND LIST

Last Update : Wed Jun 25 08:37:44 1997

Response via : LDO2S20.

Compound
R.T. QIOI

15.11

15.76

16.07

16.46

16.96

15.46

16.35

16.74

19.22

16.39

17.80

17.92

17.98

18.17

18.87

18.17

18.89

19.52

19.99

20.08

20.09

20.25

20.56

20.25

20.61

21.16

21.25

21.56

21.82

21.78

21.78

22.56

22.60

23.74

24.89

25.13

43
75
97
43
107
92
76
129
173
164
112
131
106
106
106
106
104
105
83
156
75
91
105
91
91
119
105
105
119
146
146
91
146
75
180
225
128
180

$25.23 \quad 128$

77) $\operatorname{Cos} 2$ 1,2,3-Trichlorobenzen

Response

Conc Unit

Qvalue

200693
257448
197798
94198
276051
353906
308151
310222
223457
252034
455343
240794
207689

$20.00 \mathrm{ug} / \mathrm{L} \#$

97

$20.00 \mathrm{ug} / \mathrm{I} \#$

$20.00 \mathrm{ug} / \mathrm{L}$

$20.00 \mathrm{ug} / \mathrm{I} \#$

$20.00 \mathrm{ug} / \mathrm{I}$

$20.00 \mathrm{ug} / \mathrm{L}$

$20.00 \mathrm{ug} / \mathrm{I} \mathrm{\#}$

$20.00 \mathrm{ug} / \mathrm{L}$

$20.00 \mathrm{ug} / \mathrm{L} \#$

$20.00 \mathrm{ug} / \mathrm{I}$

$20.00 \mathrm{ug} / \mathrm{L}$

$20.00 \mathrm{ug} / \mathrm{I}$

$.20 .00 \mathrm{ug} / \mathrm{I}$

$40.00 \mathrm{ug} / \mathrm{L}$

512473

251080

$20.00 \mathrm{ug} / \mathrm{I}$

769615

442660

750838

336220

243412

308167

918399

$60.00 \mathrm{ug} / \mathrm{L}$ (3e 9

$20.00 \mathrm{ug} / \mathrm{L} \mathrm{m}_{6.2547} 92$

$20.00 \mathrm{ug} / \mathrm{L} \quad 97$

$20.00 \mathrm{ug} / \mathrm{L} \# \quad 98$

$20.00 \mathrm{ug} / \mathrm{L} \quad 97$

$20.00 \mathrm{ug} / \mathrm{L} \quad 94$

$20.00 \mathrm{ug} / \mathrm{L} \# \quad 56$

601596

918399

688827

804096

601602

878039

770555

421713

421713

721942

415979

68323

285149

198426

500774

$20.00 \mathrm{ug} / \mathrm{L}$

$20.00 \mathrm{ug} / \mathrm{L}$

$20.00 \mathrm{ug} / \mathrm{L}$

$20.00 \mathrm{ug} / \mathrm{L}$

$20.00 \mathrm{ug} / \mathrm{L}$

$20.00 \mathrm{ug} / \mathrm{L}$

$20.00 \mathrm{ug} / \mathrm{I}$

$20.00 \mathrm{ug} / \mathrm{L}$

$20.00 \mathrm{ug} / \mathrm{I}$

$20.00 \mathrm{ug} / \mathrm{L} \#$

$20.00 \mathrm{ug} / \mathrm{L}$

$20.00 \mathrm{ug} / \mathrm{I}$

$20.00 \mathrm{ug} / \mathrm{I}$

$20.00 \mathrm{ug} / \mathrm{I}$

$20.00 \mathrm{ug} / \mathrm{I}$

269379

$20.00 \mathrm{ug} / \mathrm{I}$

92

45

96

97

78

95

97

95

96

89.

99

86

97

97

98

98 
Quantitation Report

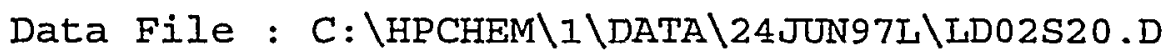

Acq Time : 06/24/97 14:59

Sample : $20 \mathrm{ug} /$ I Initial Cal std

Misc : 142WS28291

Quant Time: Jun 25 8:38 1997

Operator: $\mathrm{CQC}$

Inst : 5971-L Multiplr: 1.00

\footnotetext{
Method

: C: \HPCHEM \I\METHODS \L826ARWA.M

Title

: VOA COMPOUND LIST

Last Update : Wed Jun 25 08:37:44 1997

Response via : Single Level Calibration
}

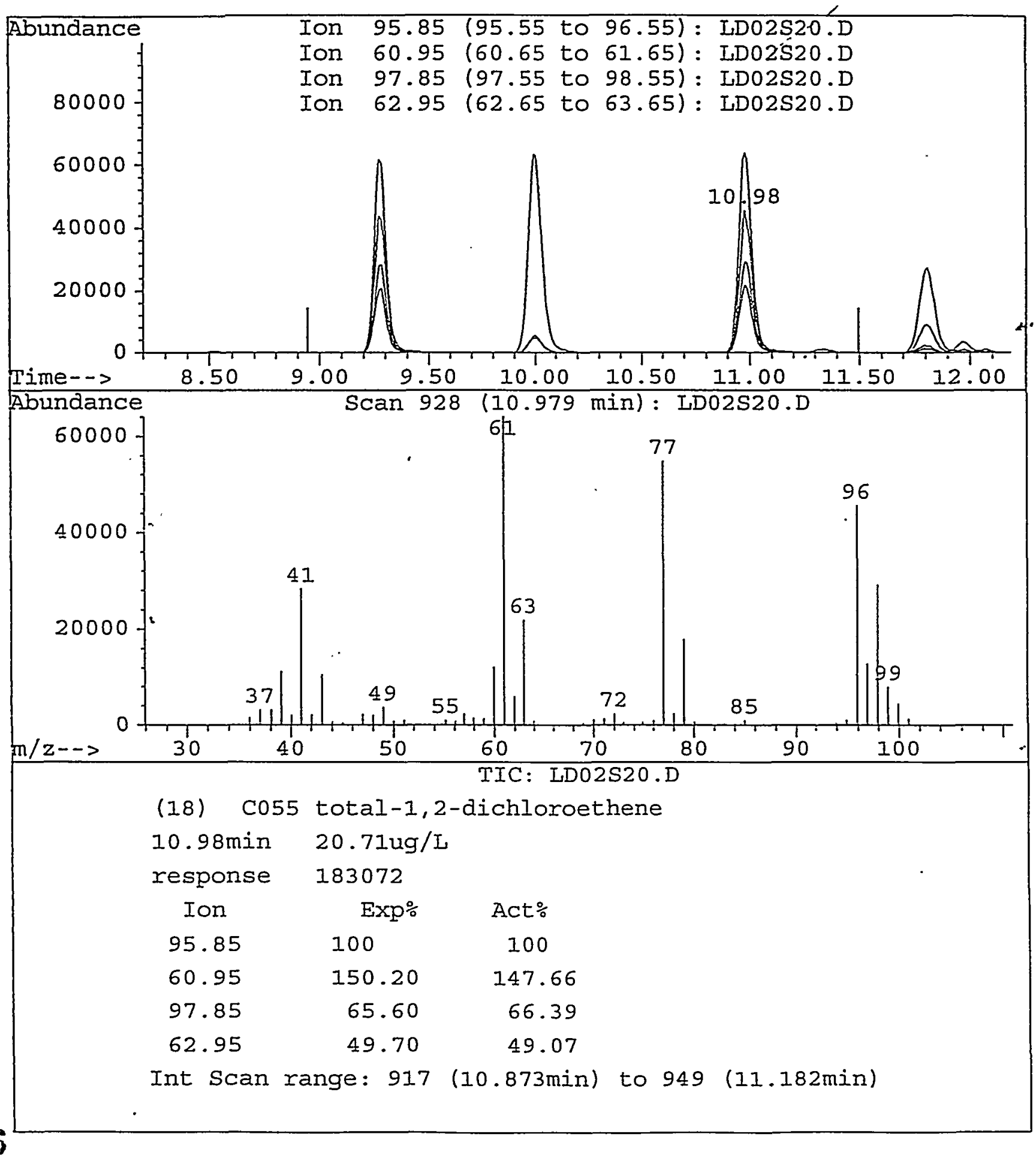


Data File : C: \HPCHEM \I\DATA 24 JUN97L\ID02S20.D

Acq Time : 06/24/97 14:59

Sample : $20 \mathrm{ug} / \mathrm{L}$ Initial Cal std

Misc : I42WS2829I

Quant Time: Jun 25 8:39 1997

Operator: CQC

Inst : 5971-L Multiplr: 1.00

Method : C: \HPCHEM \I\METHODS \L826ARWA.M

Title : VOA COMPOUND LIST

Iast Update : Wed Jun 25 08:37:44 1997

Response via : Single Level Calibration

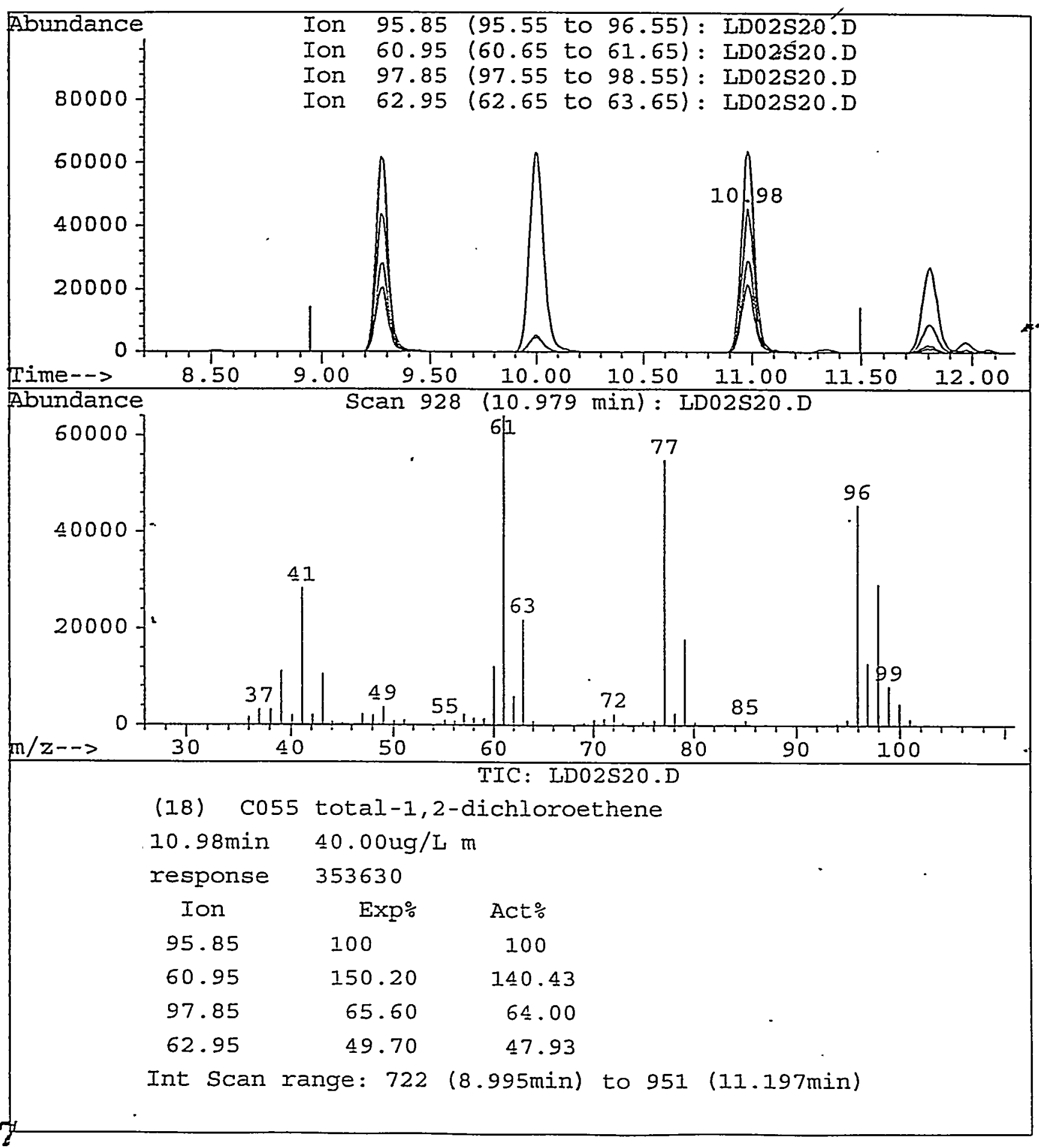


Quantitation Report

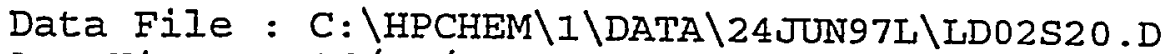

Acq Time : 06/24/97 14:59

Sample : $20 \mathrm{ug} / \mathrm{L}$ Initial Cal std

Misc : 142WS28291

Quant Time: Jun 25 8:39 1997

Operator: $\mathrm{CQC}$

Inst : 5971-L

Multiplr: 1.00

Method : C: \HPCHEM \I\METHODS \I826ARWA.M

Title : VOA COMPOUND LIST

Last Update : Wed Jun 25 08:37:44 1997

Response via : Single Level Calibration

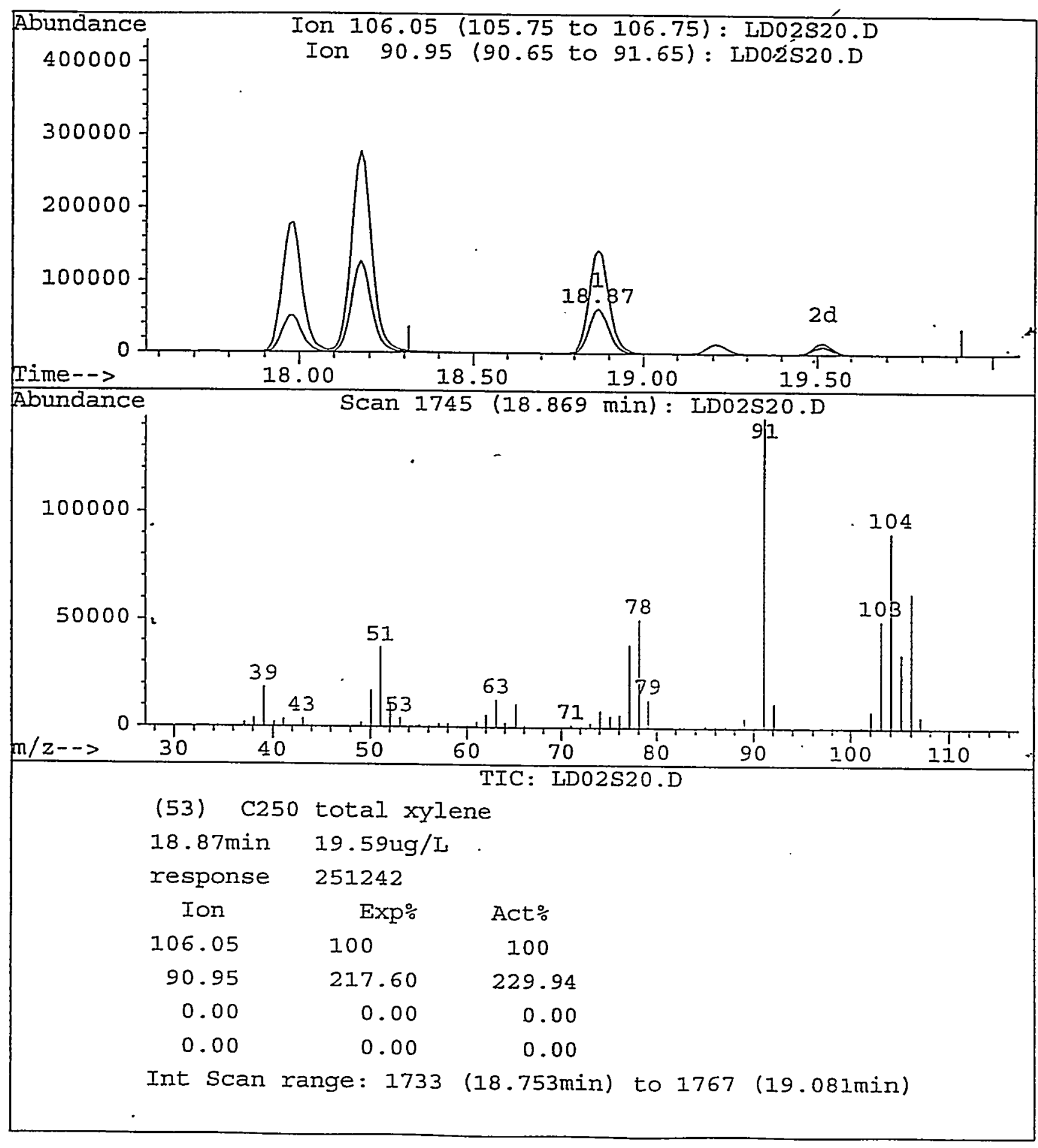


Quantitation Report

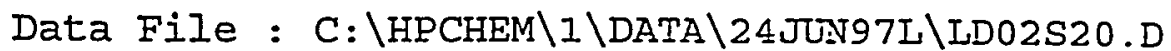

Acq Time : 06/24/97 14:59

Sample : $20 \mathrm{ug} / \mathrm{L}$ Initial Cal std

Misc : 142WS28291

Quant Time: Jun 25 8:39 1997

Operator: CQC

Inst : 5971-L Multiplr: 1.00

Method : C: \HPCHEM \I\METHODS \L826ARWA.M

Title

: VOA COMPOUND LIST

Last Update : Wed Jun 25 08:37:44 1997

Response via : Single Level Calibration

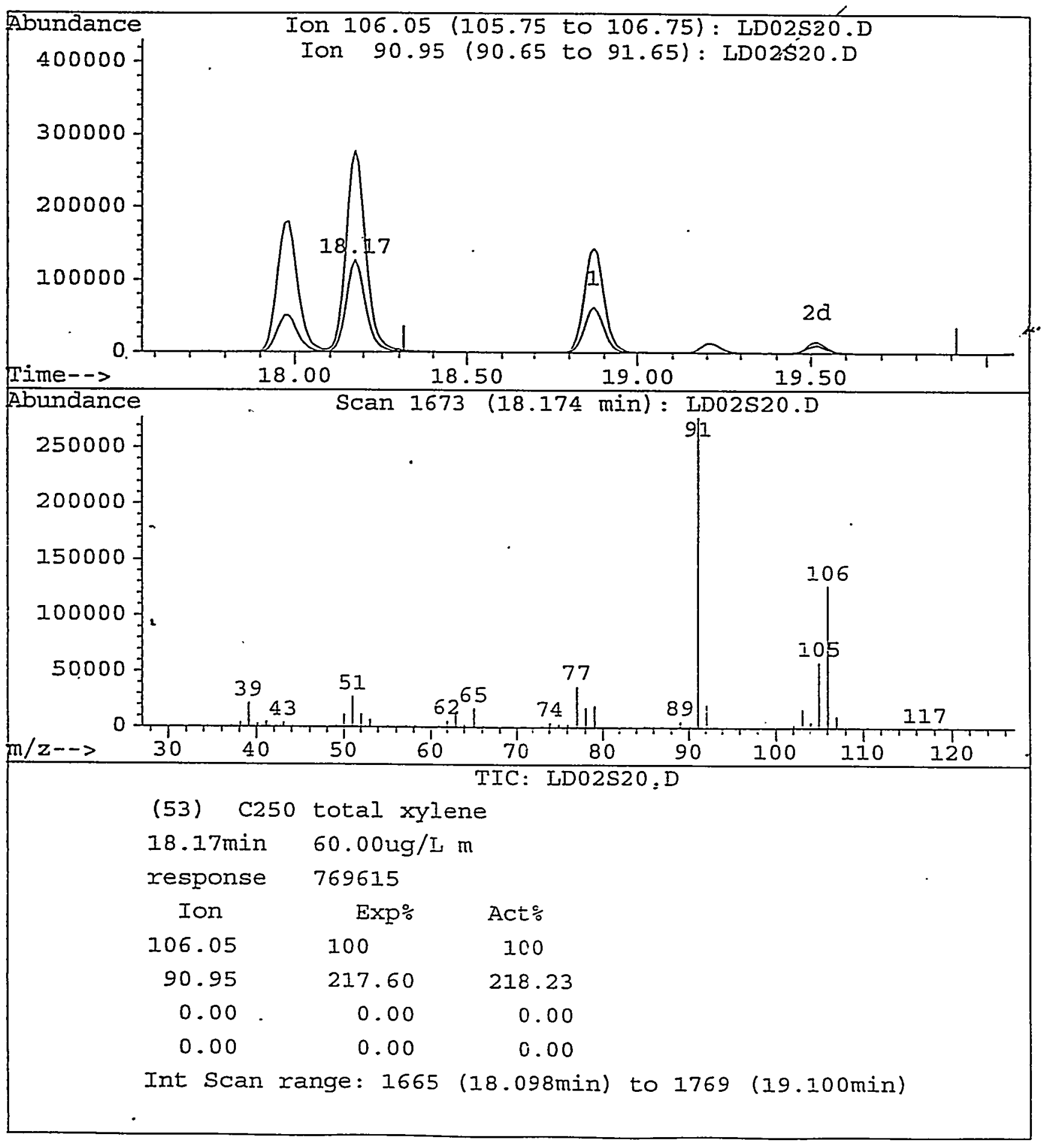


Quantitation Report

Data File : C: \HPCHEM\I\DATA $24 J U N 97 L \backslash L D 03550 . D$

Acq Time : 06/24/97 15:32

Sample : $50 \mathrm{ug} / \mathrm{L}$ Initial Cal std

Misc : 142WS28292

Quant Time: Jun 25 8:44 1997

Operator: $\mathrm{CQC}$

Inst : 5971-L

Multiplr: 1.00

Method : C: \HPCHEM IIMETHODS\L826ARWA.M

Title : VOA COMPOUND LIST

Last Update : Wed Jun 25 08:42:04 1997

Response via : Single Level Calibration

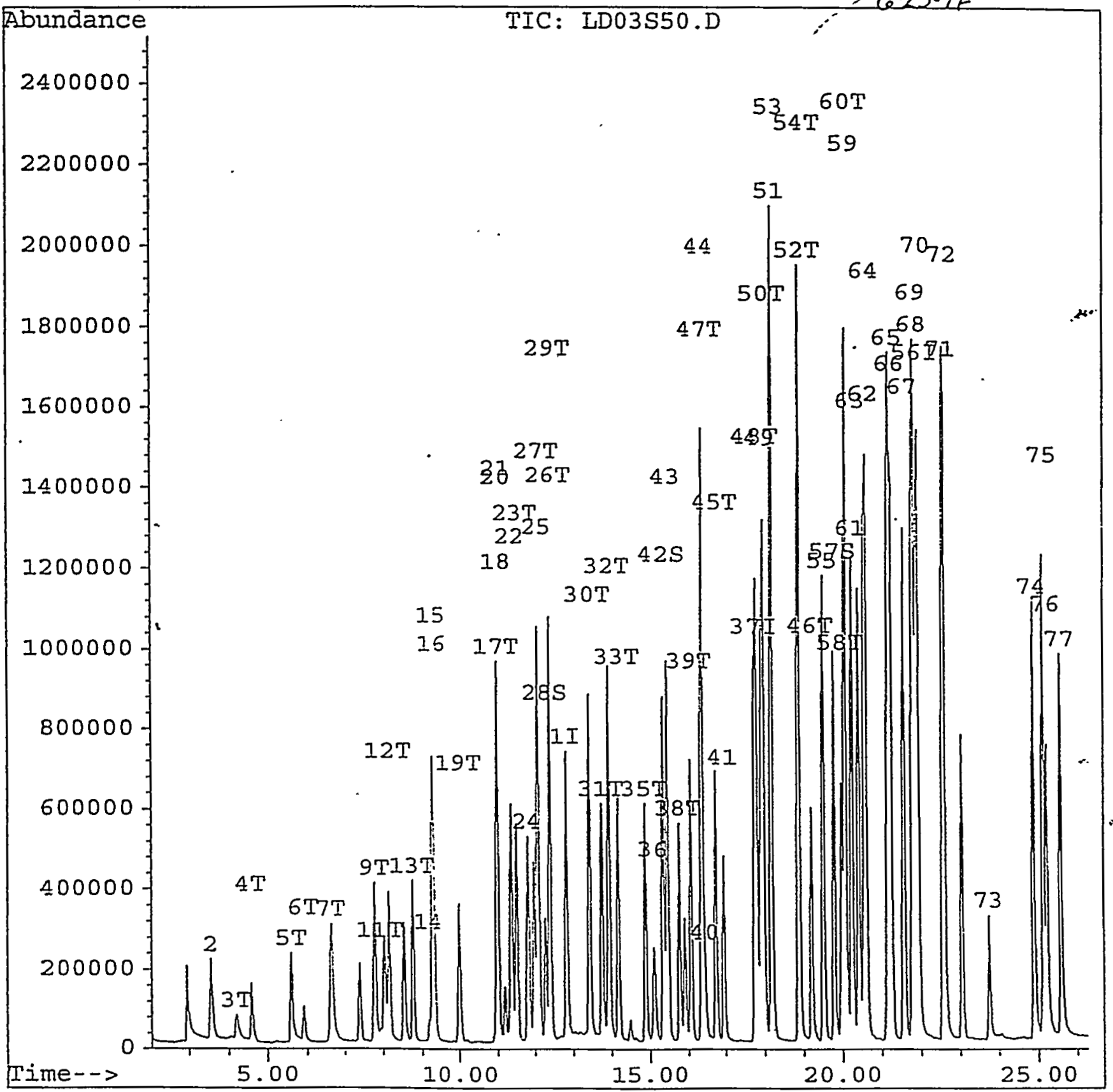

180 
Quantitation Report

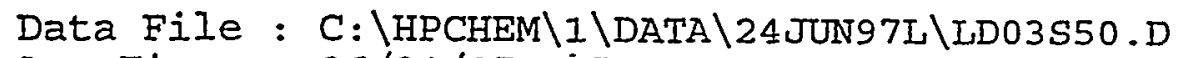

Acq Time: 06/24/97 15:32

Sample : $50 \mathrm{ug} / \mathrm{L}$ Initial Cal std

Misc : 142WS28292

Quant Time: Jun 25 8:44 1997

Operator: CQC

Inst : 5971-L

Multiplr: 1.00

Method : C: \HPCHEM II\METHODS \L826ARWA.M

Title : VOA COMPOUND LIST

Last Update : Wed Jun 25 08:42:04 1997

Response via : LD03550.

Internal standards

$\begin{array}{rrrr}\text { 1) CI01 Flourobenzene } & 12.83 & 96 & 1420814 \\ \text { 37) CI02 ChIorobenzene-d5 } & 17.74 & 117 & 1039370 \\ \text { 56) CI03 1,4-Dichlorobenzene-d } & 21.88 & 152 & 683127 \\ \text { System Monitoring Compounds } & & & \\ \text { 28) CS15 1,2-dichloroethane-d4 } & 12.28 & 65 & 449932 \\ \text { 42) CSO5 toluene-d8 } & 15.35 & 98 & 1329141 \\ \text { 57) CS10 4-bromofluorobenzene } & 19.78 & 95 & 886205\end{array}$

Target Compounds

2) $\mathrm{C002}$ dichlorodifluorometha

3) $\mathrm{COO3}$ chloromethane

4) 2004 vinyl chloride

5) C005 bromomethane

6) 0006 chloroethane

7) $\mathrm{C007}$ trichlorofluoromethan

B) $\mathrm{C071}$ acrolein

9) 0008 1,1-dichloroetherie

10) $\mathrm{C} 063$ freon 113

11) C009.acetone

12) COI0 carbon disulfide

13) Co11 methylene chloride

14) C072 acrylonitrile

15) C012 trans-1,2-Dichloroeth

16) C305 methyl-t-butyl-ether

17) $\mathrm{C} 013 \mathrm{cis}-1,2-$ dichloroethen

18) C055 total-1,2-dichloroeth

19) C014 1,1-dichloroethane

20) C015 2,2-dichloropropane

21) C110 2-butanone

22) CIOI bromochloromethane

23) $\mathrm{C} 016$ chloroform

24) $\operatorname{Co17} 1,1,1$-Txichloroethane

25) $\operatorname{Co18~1,1-dichloropropene~}$

26) Co19 1,2-dichloroethane

27) $\mathrm{C} 20$ carbon tetrachloride

29) C021 benzene

30) Co22 trichloroethene

31) $\mathrm{CO23} \mathrm{1,2-dichloropropane}$

32) C024 dibromomethane

33) C025 bromodichloromethane

34) C026 2-chloroethylvinyleth

1351 C027 cis-1,3-dichloroprope
R.T. QIon Response Conc Units Area\%

$\begin{array}{rrrrr}12.83 & 96 & 1420814 & 50.00 \mathrm{ug} / \mathrm{I} & 100.00 \\ 17.74 & 117 & 1039370 & 50.00 \mathrm{ug} / \mathrm{L} & 100.00 \\ 21.88 & 152 & 683127 & 50.00 \mathrm{ug} / \mathrm{I} & 100.00\end{array}$

$\because$ Recovery

$50.00 \mathrm{ug} / \mathrm{L} \quad 100.00 \%$

$50.00 \mathrm{ug} / \mathrm{L} \quad 100.00 \%$

$50.00 \mathrm{ug} / \mathrm{L} \quad 100.00 \%$

$3.55 \quad 85 \quad 558025$

$4.20 \quad 50 \quad 237852$

$4.58 \quad 62 \quad 360021$

$5.59 \quad 94 \quad 398318$

$5.90 \quad 64 \quad 209189$

$6.64 \quad 101 \quad 859880$

$0.00 \quad 56$

7.7896

$0.00 \quad 151$

$7.92 \quad 43$

$8.13 \quad 76$

8.7584

9.1953

9.27

9.32

96

10.98

10.98

10.00

10.97

11.00

11.36

11.49

11.80

12.07

12.39

12.09

12.39

13.42

13.74

13.94

14.18

0.00

14.88
416972

80907

1106685

416634

103968

465660

1078636

491650

956664

812117

761896

47641

299177

883554

833503

709118

544560

736171

1423202

653958

541752

465907

906748

807761
Qvalue

$50.00 \mathrm{ug} / \mathrm{I} \quad 98$

$50.00 \mathrm{ug} / \mathrm{I} \quad \mu 100$

$.50 .00 \mathrm{ug} / \mathrm{L} \quad 98$

$50.00 \mathrm{ug} / \mathrm{I} \quad 98$

$50.00 \mathrm{ug} / \mathrm{I} \quad 96$

$50.00 \mathrm{ug} / \mathrm{I} \quad 99$

Not Detected

$50.00 \mathrm{ug} / \mathrm{L}$

Not Detected

$50.00 \mathrm{ug} / \mathrm{I} \quad 90$

$50.00 \mathrm{ug} / \mathrm{L} \# \quad 72$

$50.00 \mathrm{ug} / \mathrm{I} \quad 91$

$50.00 \mathrm{ug} / \mathrm{I} \quad 89$

$50.00 \mathrm{ug} / \mathrm{I} \quad 88$

$100.00 \mathrm{ug} / \mathrm{I} \mathrm{m} \underset{6.25 .4799}{\mathrm{cos}} 9$

$50.00 \mathrm{ug} / \mathrm{I} \# \quad 96$

$50.00 \mathrm{ug} / \mathrm{I} \# \quad .74$

$50.00 \mathrm{ug} / \mathrm{L} \# \quad 17$

$50.00 \mathrm{ug} / \mathrm{I} \# \quad 69$

$50.00 \mathrm{ug} / \mathrm{L} \# 37$

$50.00 \mathrm{ug} / \mathrm{I} \quad 100$

$50.00 \mathrm{ug} / \mathrm{L}$ \# 78

$50.00 \mathrm{ug} / \mathrm{L} \quad 98$

$50.00 \mathrm{ug} / \mathrm{L} \quad 97$

$50.00 \mathrm{ug} / \mathrm{L} \# \quad 88$

$50.00 \mathrm{ug} / \mathrm{L} \quad 89$

$50.00 \mathrm{ug} / \mathrm{I} \quad 95$

$50.00 \mathrm{ug} / \mathrm{L} \quad 91$

$50.00 \mathrm{ug} / \mathrm{L} \quad 99$

Not Detected

$50.00 \mathrm{ug} / \mathrm{I} \#$
$50.00 \mathrm{ug} / \mathrm{I} \# \quad 69$

$50.00 \mathrm{ug} / \mathrm{L} \# \quad 87$ 


\section{Quantitation Report}

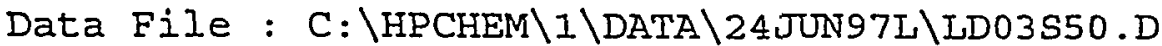

Acq Time : 06/24/97 15:32

Sample : $50 \mathrm{ug} / \mathrm{L}$ Initial Cal std

Misc : $142 W S 28292$

Quant Time: Jun 25 8:44 1997

Operator: CQC

Inst : $5971-\mathrm{L}$

Multiplr: 1.00

\footnotetext{
Method

Title

: C: \HPCHEM \I\METHODS \L826ARWA.M

Last Update : Wed Jun 25 08:42:04 1997

Response via : LD03550.
}

Compound

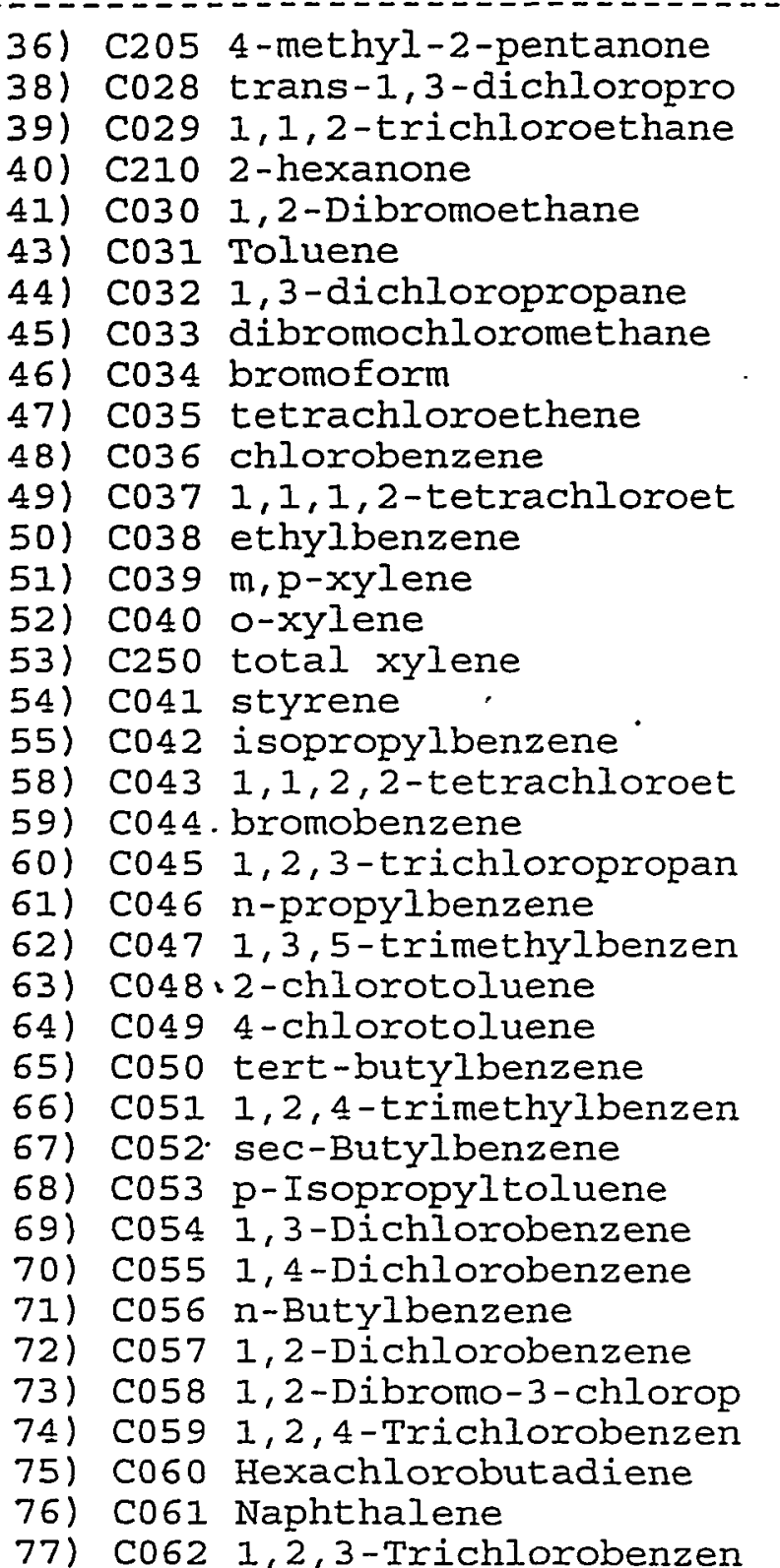

R.T. QIon Response Conc Unit Qvalue

$15.11 \quad 43$

15.76

16.06

16.46

16.95

15.45

16.35

16.75

19.20

16.38

17.79

17.92

17.98

18.17

18.87

18.17

18.88

19.51

19.98

20.08

20.08

20.24

20.55

20.24

20.60

21.16

21.24

21.56

21.81

21.77

21.93

22.55

22.59

23.72

24.87

25.12

25.22

25.57
75

97

43

107

92

76

129

173

164

112

131

106

106

106

106

104

105

83

156

75

91

105

91

91

119

105

105

119

146

146

91

146

75

180

225

128

180
$516134,-$

708164

523420

268109

746971

945780

830855

841416

622574

677047

1208899

646715

559491

1387373

675349

2059265

1195002

2022203

895838

658500

859087

2501688

1608812

2501688

1841286

2166228

1617244

2372185

2060595

1155794

1235772

1951480

1103987

196843

798725

556913

1457794

738908
$50.00 \mathrm{ug} / \mathrm{L} \#$ $50.00 \mathrm{ug} / \mathrm{L} \#$ $50.00 \mathrm{ug} / \mathrm{L}$ $50.00 \mathrm{ug} / \mathrm{L} \#$ $50.00 \mathrm{ug} / \mathrm{L}$ $50.00 \mathrm{ug} / \mathrm{L}$ $50.00 \mathrm{ug} / \mathrm{I}$ \# $50.00 \mathrm{ug} / \mathrm{L}$ $50.00 \mathrm{ug} / \mathrm{L} \#$ $50.00 \mathrm{ug} / \mathrm{L}$ $50.00 \mathrm{ug} / \mathrm{L}$ $50.00 \mathrm{ug} / \mathrm{L}$ $50.00 \mathrm{ug} / \mathrm{L}$ $i 00.00 \mathrm{ug} / \mathrm{L}$ $50.00 \mathrm{ug} / \mathrm{L}$ $150.00 \mathrm{ug} / \mathrm{L} \mathrm{m}_{\text {(z) }}^{\text {(zs)t }}$ $50.00 \mathrm{ug} / \mathrm{L}$ $50.00 \mathrm{ug} / \mathrm{L}$ $50.00 \mathrm{ug} / \mathrm{L} \#$ $50.00 \mathrm{ug} / \mathrm{L}$ $50.00 \mathrm{ug} / \mathrm{L}$ $50.00 \mathrm{ug} / \mathrm{L} \#$ $50.00 \mathrm{ug} / \mathrm{I}$ $50.00 \mathrm{ug} / \mathrm{L}$ \# $50.00 \mathrm{ug} / \mathrm{L}$ $50.00 \mathrm{ug} / \mathrm{L}$ $50.00 \mathrm{ug} / \mathrm{L}$ $50.00 \mathrm{ug} / \mathrm{L}$ $50.00 \mathrm{ug} / \mathrm{L}$ $50.00 \mathrm{ug} / \mathrm{L}$ $50.00 \mathrm{ug} / \mathrm{L}$ $50.00 \mathrm{ug} / \mathrm{I} \#$ $50.00 \mathrm{ug} / \mathrm{L}$ $50.00 \mathrm{ug} / \mathrm{L} \#$ $50.00 \mathrm{ug} / \mathrm{I}$ $50.00 \mathrm{ug} / \mathrm{L}$ $50.00 \mathrm{ug} / \mathrm{L}$ $50.00 \mathrm{ug} / \mathrm{L}$
93 75 96 89 100

97

84

98

99

92

98

92

91

90

93

93

93

97

98

98

93

56

92

45

97

97

77

95

96

95

96

88

99

83

98

97

100

100

182

Data Processed By:

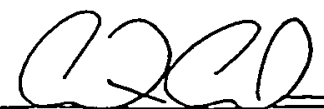

6.25 .97

$(\mathrm{NR})=$ Not Reported $(\mathrm{SP})=$ Spiked analyte $(\mathrm{m})=$ manual integration

(*) Does not meet EPA spectral criteria (False Hit) 
Quantitation Report

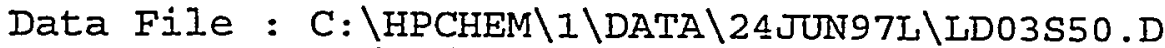

Acq Time : 06/24/97 15:32

Sample : $50 \mathrm{ug} / \mathrm{I}$ Initial Cal std

Misc : I42WS28292

Quant Time: Jun 25 8:43 1997

Operator: CQC

Inst : 5971-L Multiplr: 1.00

Method : C: \HPCHEM \I\METHODS \L826ARWA.M

Title : VOA COMPOUND LIST

Last Update : Wed Jun 25 08:42:04 1997

Response via : Single Level Calibration

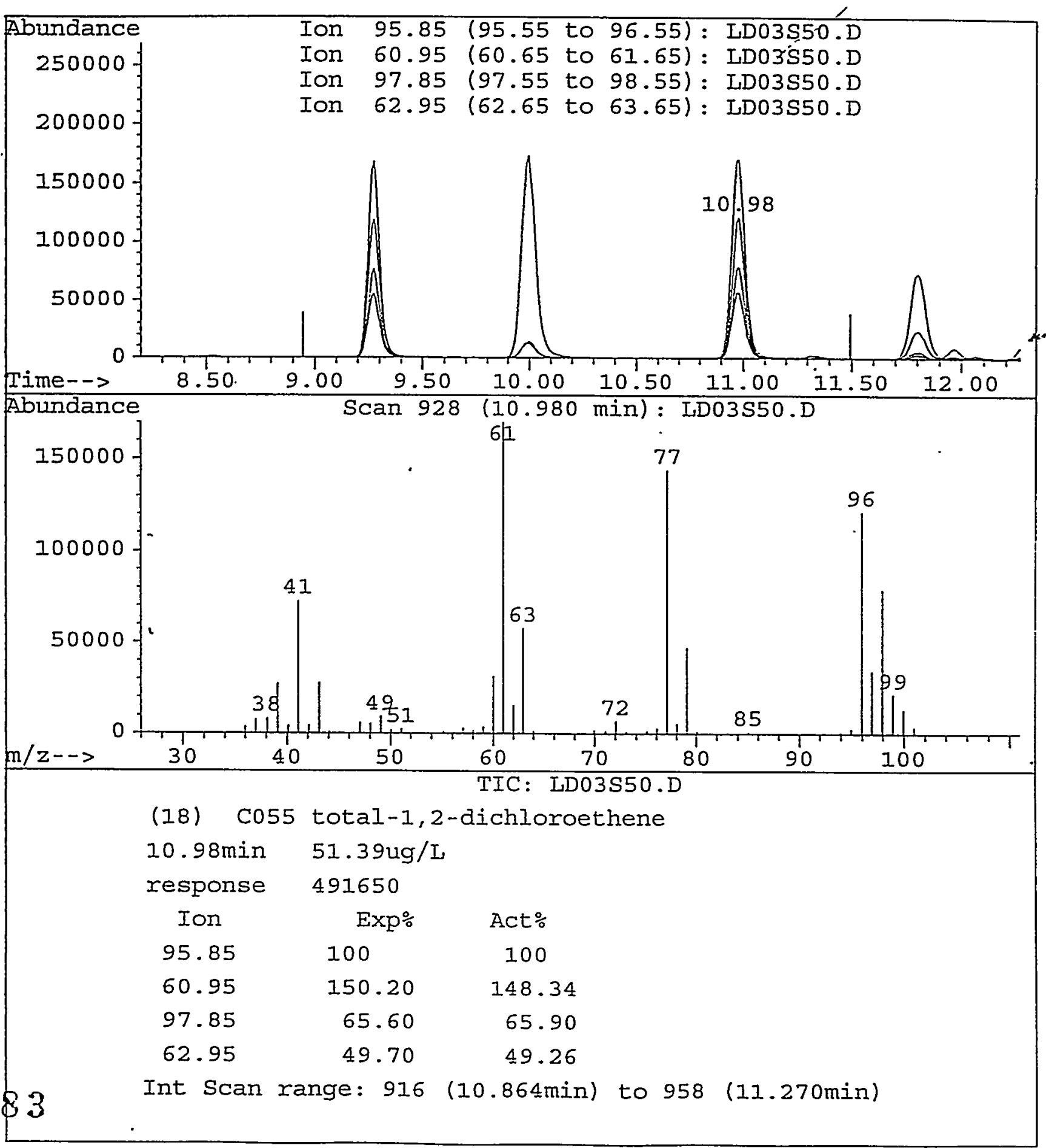




\section{Quantitation Report}

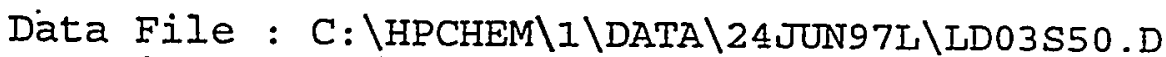

Acq Time : 06/24/97 15:32

Sample : $50 \mathrm{ug} /$ I Initial Cal std

Misc : 142WS28292

Quant Time: Jun 25 8:43 1997

Operator: $\mathrm{CQC}$

Inst : 5971-L Multiplr: 1.00

Method : C: \HPCHEM \I\METHODS \I826ARWA.M

Title

: VOA COMPOUND LIST

Last Update : Wed Jun 25 08:42:04 1997

Response via : Single Level Calibration

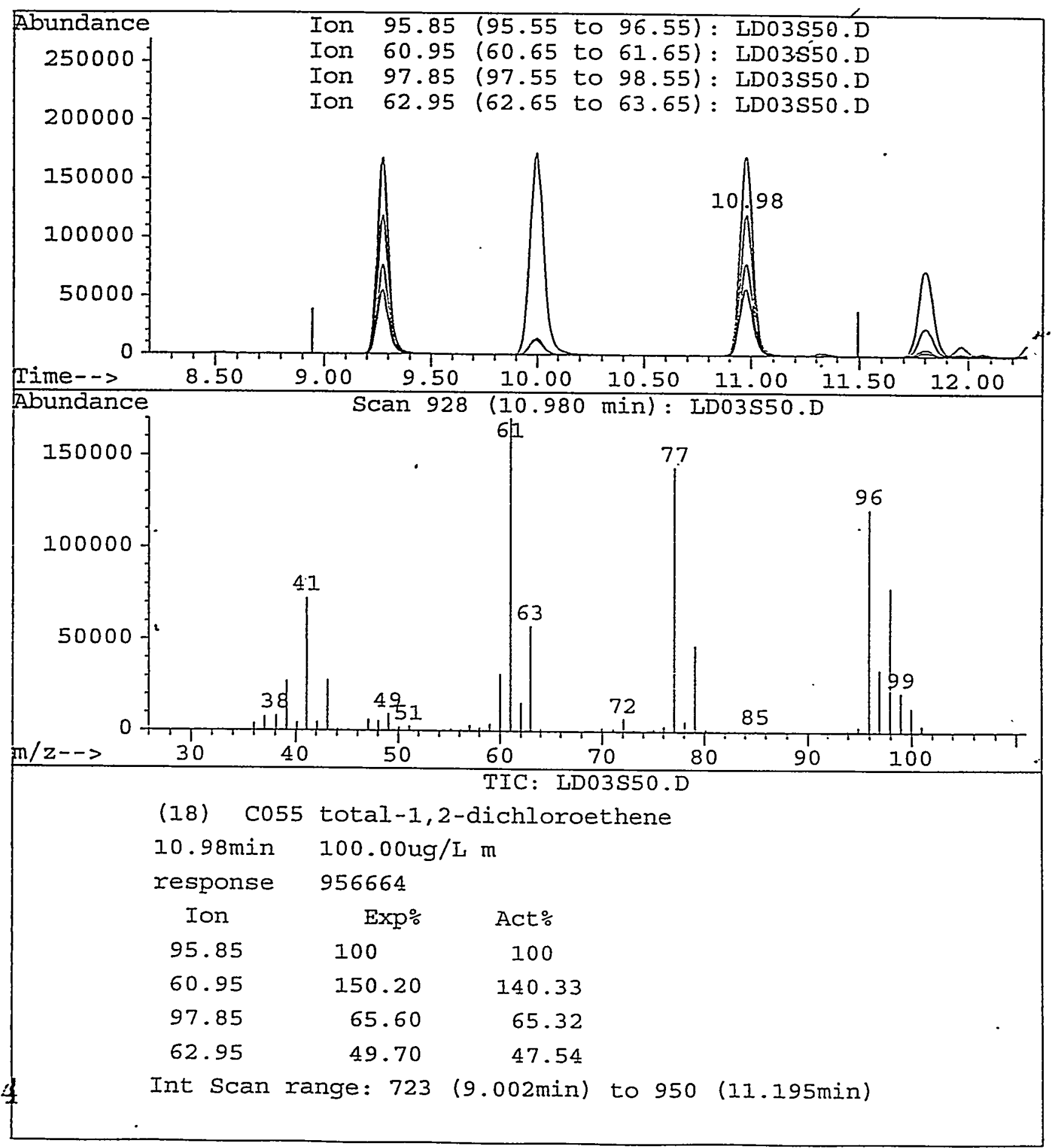




\section{Quantitation Report}

Data File : C: \HPCHEM \I\DATA $\backslash 24 \pi N 97 I \backslash L D 03550 . D$

Acq Time : 06/24/97 15:32

Sample : $50 \mathrm{ug} /$ I Initial Cal std

MisC : 142WS28292

Quant Time: Jun 25 8:43 1997

Operator: CQC

Inst : 5971-L Multiplr: 1.00

Method : C: \HPCHEM \I\METHODS \L826ARWA.M

Title : VOA COMPOUND LIST.

Last Update : Wed Jun 25 08:42:04 1997

Response via : Single Level Calibration

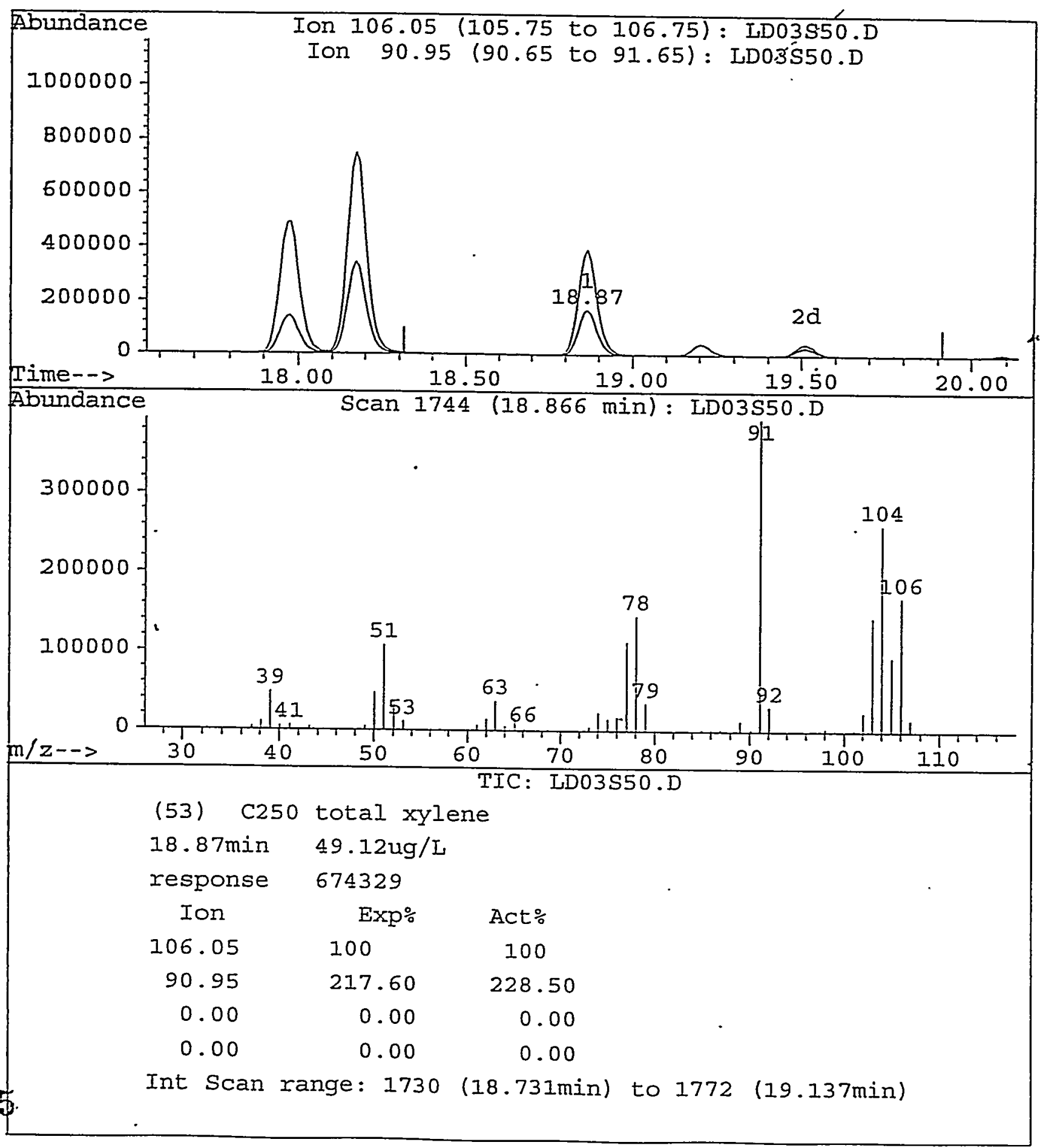


Quantitation Report

Data File : C: \HPCHEM \I\DATA $24 J U N 97 L \backslash L D 03550 . D$

Acq Time : 06/24/97 15:32

Sample : $50 \mathrm{ug} / \mathrm{L}$ Initial Cal std

Misc : 142WS28292

Quant Time: Jun 25 8:44 1997

Operator: CQC

Inst : 5971-I

Multiplr: 1.00

Method : C: \HPCHEM \I\METHODS \L826ARWA.M

Title : VOA COMPOUND LIST

Last Update : Wed Jun 25 08:42:04 1997

Response via : Single Level Calibration

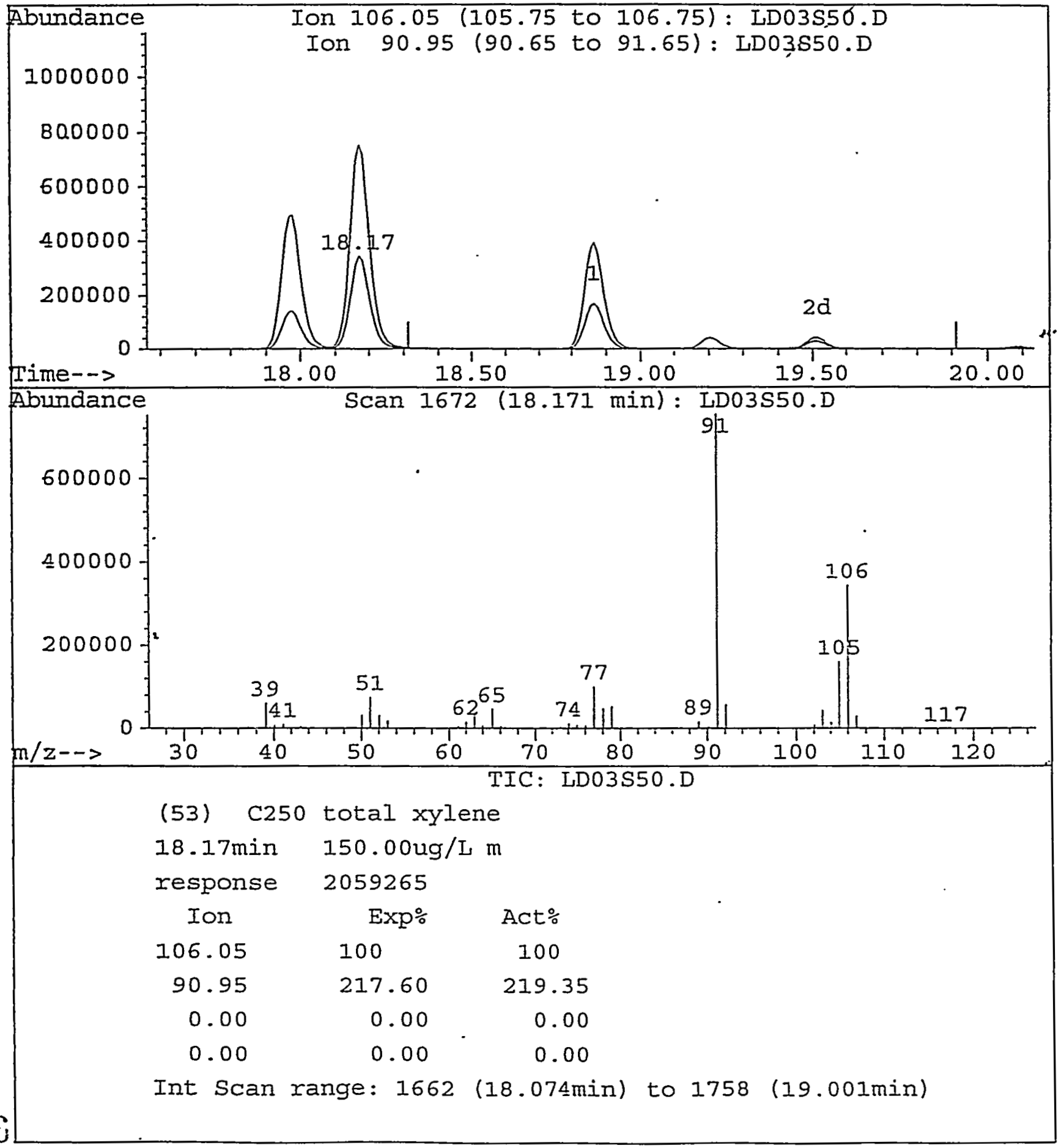


Quantitation Report

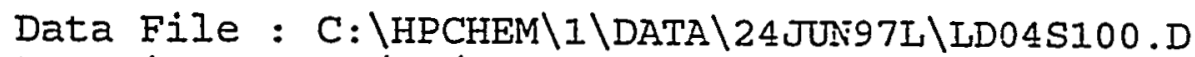
Acq Time : 06/24/97 16:04
Sample : $100 \mathrm{ug} / \mathrm{L}$ Initial Cal std
Misc : 142WS28293
Quant Time: Jun 25 8:52 1997
Method : C: \HPCHEM II IMETHODS \L826ARWA.M
Title : VOA COMPOUND LIST
Last Update : Wed Jun 25 08:50:18 1997
Response via : Single Level Calibration

Operator: CQC

Inst : 5971-I

Multiplr: 1.00
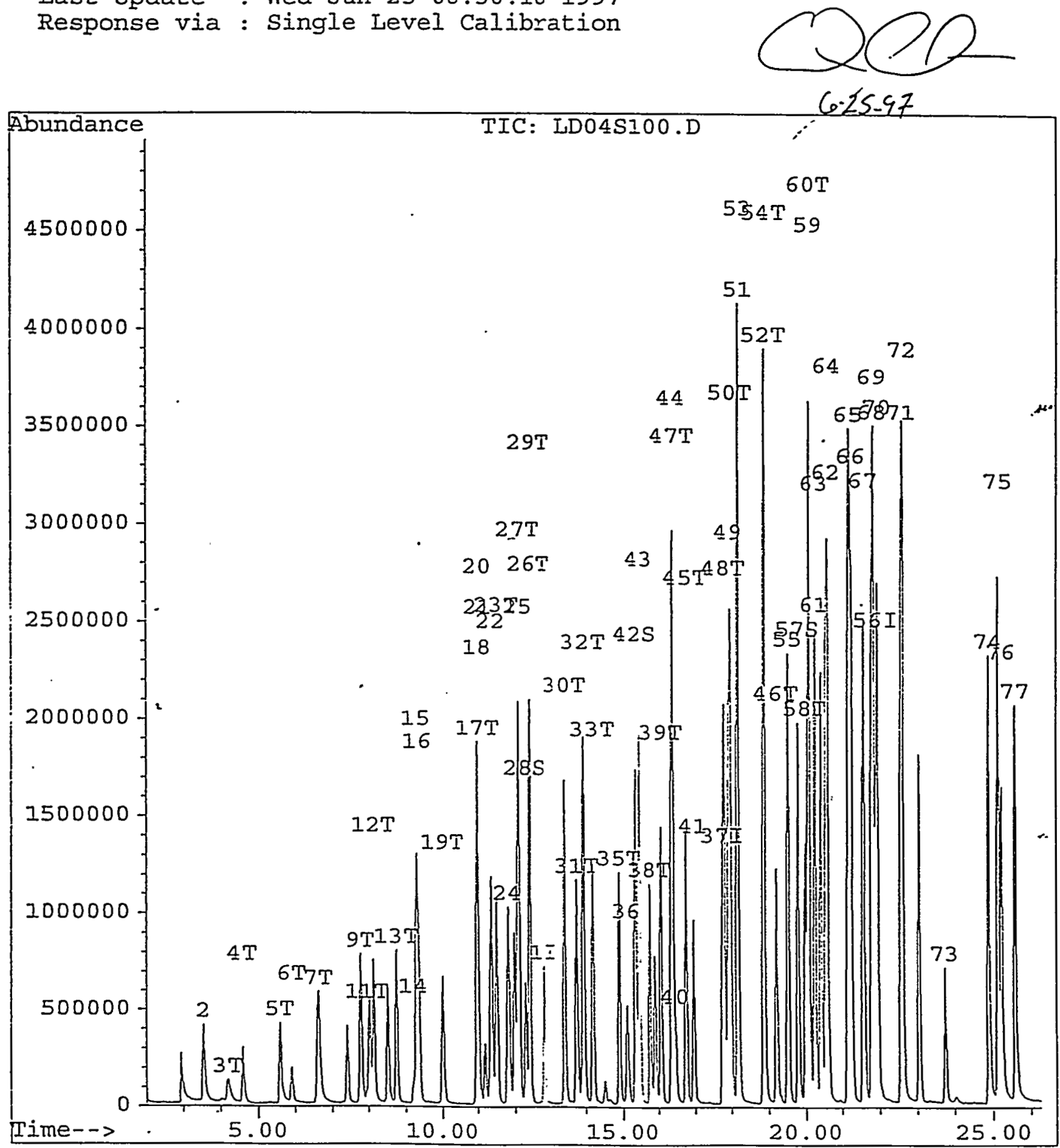
Data File : C:\HPCHEM I \DATA 24 JUN97L\LDO4SI00.D

Acq Time : 06/24/97 16:04

Sample : $100 \mathrm{ug} / \mathrm{I}$ Initial Cal std

MisC : 142WS28293

Quant Time: Jun 25 8:52 1997
Operator: $\mathrm{CQC}$

Inst : 5971-I

Multiplr: 1.00

Method : C: \HPCHEM \I\METHODS \L826ARWA.M

Title : VOA COMPOUND LIST

Last Update : Wed Jun 25 08:50:18 1997

Response via : LD04S100

Internal standards

37) CIO1 Flourobenzene

System Monitoring Compounds

28) CS15 1,2-dichloroethane-d4

42) CSO5 toluene-d8

57) CSI0 4-bromofluorobenzene

Target Compounds

2) C002 dichlorodifluorometha

3) $\mathrm{C003}$ chloromethane

4) C004 vinyl chloride

5) C005 bromomethane

6) C006 chloroethane

7) $\mathrm{C007}$ trichlorofluoromethan

8) 0071 acrolein

9) $\operatorname{Co0} 8$ 1,1-dichloroetherie

10) 0063 freon 113

11) Co09 acetone

12) C010 carbon disulfide

13) COII methylene chloride

14) C072 acrylonitrile

15) $\mathrm{C} 012$,trans $-1,2-\mathrm{Dich}$ loroeth

16) C305 methyl-t-butyl-ether

17) $\mathrm{C} 013 \mathrm{cis}-1,2-d i c h l o r o e t h e n$

18) $\mathrm{C} 055$ total-1,2-dichloroeth

19) $\mathrm{C} 014$ I,I-dichloroethane

20) C015 2,2-dichloropropane

21) C110 2-butanone

22) CI01 bromochloromethane

23) 0016 chloroform

24) $\operatorname{Co17}$ 1,1,1-Trichloroethane

25) $\operatorname{Co18~1,1-dichloropropene~}$

26) C019 1,2-dichloroethane

27) C020 carbon tetrachloride

29) CO2I benzene

30) Co22 trichloroethene

31) C023 1,2-dichloropropane

32) Co24 dibromomethane

33) C025 bromodichloromethane

34) Co26 2-chloroethylvinyleth

35) C027 cis-1,3-dichloroprope
R.T. QIon

12.83

17.75

21.88

\section{6}

117

152
12.28

15.34

19.78

65

98

95

3.55

4.20

4.58

5.59

5.90

6.62

0.00

7.78

0.00

7.95

8.13

8.75

9.20

9.27

9.33

10.98

10.98

10.00

10.97

11.01

11.36

11.50

11.80

12.07

12.39

12.09

12.39

13.42

13.75

13.94

14.18

0.00

14.89

\section{5}

50

62

94

64

101

56

96

151

43

76

84

53

96

73

96

96

63

77

72

128

83

97

75

62

117

78

130

63

93

83

63

75
Response

$14106020^{\circ}$

1048285

685640

Conc Units

$\begin{array}{ll}50.00 \mathrm{ug} / \mathrm{I} & 100.00 \\ 50.00 \mathrm{ug} / \mathrm{I} & 100.00 \\ 50.00 \mathrm{ug} / \mathrm{I} & 100.00\end{array}$

$\div$ Recovery

895125 2650459

1775398

$100.00 \mathrm{ug} / \mathrm{L}$ $100.00 \mathrm{ug} / \mathrm{L}$ $200.00 \%$ $100.00 \mathrm{ug} / \mathrm{L}$ $200.00 \%$ $200.00 \%$

Qvalue

1118186

484265

717153

756563

401467

1724187

814201

164720

2194284

813642

207062

918406

2156449

969290

1887517

1555810

1503807

95744

585652

1686808

1648342

1395071

1085735

1521426

2792024

1272677

1072533

930450

1841954

$100.00 \mathrm{ug} / \mathrm{L}$

$100.00 \mathrm{ug} / \mathrm{L}$

$100.00 \mathrm{ug} / \mathrm{L}$

$100.00 \mathrm{ug} / \mathrm{I}$

$100.00 \mathrm{ug} / \mathrm{L}$

$100.00 \mathrm{ug} / \mathrm{L}$

Not Detected

$100.00 \mathrm{ug} / \mathrm{L}$

Not Detected

$100.00 \mathrm{ug} / \mathrm{I} \#$

$100.00 \mathrm{ug} / \mathrm{I}$

$100.00 \mathrm{ug} / \mathrm{I} \#$

$100.00 \mathrm{ug} / \mathrm{L}$

$100.00 \mathrm{ug} / \mathrm{L}$

$100.00 \mathrm{ug} / \mathrm{I} \#$

$100.00 \mathrm{ug} / \mathrm{I}$

$200.00 \mathrm{ug} / \mathrm{L} \mathrm{m}_{G-2547}^{C F} 99$

$100.00 \mathrm{ug} / \mathrm{I}$

$100.00 \mathrm{ug} / \mathrm{I}$

$100.00 \mathrm{ug} / \mathrm{L} \#$

$100.00 \mathrm{ug} / \mathrm{L} \#$

$100.00 \mathrm{ug} / \mathrm{L} \#$

$100.00 \mathrm{ug} / \mathrm{L}$

$100.00 \mathrm{ug} / \mathrm{L}$ \#

$100.00 \mathrm{ug} / \mathrm{L}$

$100.00 \mathrm{ug} / \mathrm{L}$

$100.00 \mathrm{ug} / \mathrm{L} \#$

$100.00 \mathrm{ug} / \mathrm{L}$

$100.00 \mathrm{ug} / \mathrm{L}$

$100.00 \mathrm{ug} / \mathrm{L}$

$100.00 \mathrm{ug} / \mathrm{L}$

Not Detected

1639587
98

H. 99

98

98

97

99

88

70

90

73

93

89

87

88

97

73

15

69

94

$100^{\circ}$

77

98

97

88

89

95

90

99

73

$(\mathrm{NR})=$ Not Reported $(\mathrm{SP})=$ Spiked analyte $(\mathrm{m})=$ manual integration

(*) Does not meet EPA spectral criteria (False Hit) PX125 


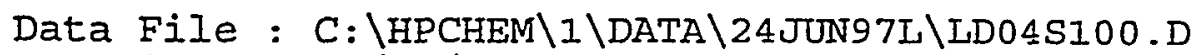

Acq Time : 06/24/97 16:04

Sample : $100 \mathrm{ug} / \mathrm{I}$ Initial Cal std

Misc : I42WS28293

Quant Time: Jun 25 8:52 1997
Operator: $\mathrm{CQC}$

Inst : 5971-L

Multiplr: 1.00

Method : C: \HPCHEM \I\METHODS \L826ARWA.M

Title : VOA COMPOUND LIST

Last Update : Wed Jun 25 08:50:18 1997

Response via : LD04S100

Compound
R.T. QIon Response Conc Unit Qvalue

\begin{tabular}{|c|c|c|c|c|c|c|}
\hline 15.11 & 43 & 1069528 & 100.00 & $\mathrm{ug} / \mathrm{L}$ & \# & 93 \\
\hline 15.76 & 75 & 1439331 & 100.00 & $\mathrm{ug} / \mathrm{I}$ & \# & 75 \\
\hline 16.07 & 97 & 1040592 & 100.00 & $\mathrm{ug} / \mathrm{L}$ & & 96 \\
\hline 16.46 & 43 & 567438 & 100.00 & $\mathrm{ug} / \mathrm{L}$ & $\#$ & 84 \\
\hline 16.96 & 107 & 1511148 & 100.00 & $\mathrm{ug} / \mathrm{I}$ & & 100 \\
\hline 15.46 & 92 & 1908527 & 100.00 & $\mathrm{ug} / \mathrm{I}$ & & 97 \\
\hline 16.35 & 76 & 1661522 & 100.00 & $\mathrm{ug} / \mathrm{L}$ & $\#$ & 84 \\
\hline 16.74 & 129 & 1713808 & 100.00 & $\mathrm{ug} / \mathrm{I}$ & & 98 \\
\hline 19.21 & 173 & 1313676 & 100.00 & $u g / I$ & \# & 99 \\
\hline 16.39 & 164 & 1292870 & 100.00 & $u g / L$ & & 93 \\
\hline 17.80 & 112 & 2402451 & 100.00 & $u g / I$ & & 98 \\
\hline 7.91 & 131 & 1307334 & 100.00 & $\mathrm{ug} / \mathrm{I}$ & טג & 91 \\
\hline 7.97 & 106 & 1106334 & 100.00 & $u g / I$ & & 90 \\
\hline 8.17 & 106 & 2717295 & 200.00 & $u g / L$ & & 89 \\
\hline 8.86 & 106 & 1316565 & 100.00 & ug/L & & 91 \\
\hline 18.17 & 106 & 4068197 & 300.00 & $\mathrm{ug} / \mathrm{I}$ & $m<x$ & 91 \\
\hline 18.88 & 104 & 2374373 & 100.00 & $\mathrm{ug} / \mathrm{I}$ & & 93 \\
\hline 19.51 & 105 & 3969780 & 100.00 & $\mathrm{ug} / \mathrm{I}$ & & 96 \\
\hline 19.98 & 83 & 1829041 & 100.00 & $\mathrm{ug} / \mathrm{I}$ & \# & 98 \\
\hline 20.07 & 156 & 1308500 & 100.00 & $\mathrm{ug} / \mathrm{I}$ & & 98 \\
\hline 20.08 & 75 & 1806680 & 100.00 & $\mathrm{ug} / \mathrm{I}$ & & 92 \\
\hline 20.25 & 91 & 5016370 & 100.00 & $\mathrm{ug} / \mathrm{I}$ & \# & 56 \\
\hline 20.56 & 105 & 3161779 & 100.00 & $\mathrm{ug} / \mathrm{I}$ & & 92 \\
\hline 20.25 & 91 & 5016370 & 100.00 & $\mathrm{ug} / \mathrm{I}$ & \# & 45 \\
\hline 20.60 & 91 & 3663732 & 100.00 & $\mathrm{ug} / \mathrm{I}$ & & 96 \\
\hline 21.17 & 119 & 4329384 & 100.00 & $\mathrm{ug} / \mathrm{I}$ & & 96 \\
\hline 21.24 & 105 & 3172185 & 100.00 & $\mathrm{ug} / \mathrm{I}$ & & 77 \\
\hline 21.56 & 105 & 4695158 & 100.00 & $\mathrm{ug} / \mathrm{I}$ & \# & 95 \\
\hline 21.82 & 119 & 4092768 & 100.00 & $\mathrm{ug} / \mathrm{I}$ & . & 96 \\
\hline 21.78 & 146 & 2310488 & 100.00 & $\mathrm{ug} / \mathrm{I}$ & & 95 \\
\hline 21.93 & 146 & 2466109 & 100.00 & ug/I & & 96 \\
\hline 2.56 & 91 & 3963062 & 100.00 & $u g / L$ & \# & 89 \\
\hline 22.60 & 146 & 2212393 & 100.00 & $\mathrm{ug} / \mathrm{L}$ & & 90 \\
\hline 23.73 & 75 & 410013 & 100.00 & $\mathrm{ug} / \mathrm{I}$ & \# & 81 \\
\hline 24.88 & 180 & 1626580 & 100.00 & $\mathrm{ug} / \mathrm{I}$ & & 99 \\
\hline 5.12 & 225 & 1234787 & 100.00 & $\mathrm{ug} / \mathrm{I}$ & & 97 \\
\hline 25.22 & 128 & 2999838 & 100.00 & $\mathrm{ug} / \mathrm{L}$ & & 99 \\
\hline 5.58 & 180 & 1513337 & 100.00 & $\mathrm{ug} / \mathrm{I}$ & & 99 \\
\hline
\end{tabular}




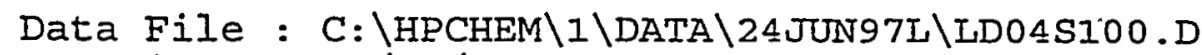

Acq Time : 06/24/97 16:04

Sample : $100 \mathrm{ug} / \mathrm{L}$ Initial Cal std

Misc : 142WS28293

Quant Time: Jun 25 8:51 1997

Operator: $\mathrm{CQC}$ Inst : 5971-L Multiplr: 1.00

Method : C: \HPCHEM \I\METHODS \I826ARWA.M

Title : VOA COMPOUND LIST

Last Update : Wed Jun 25 08:50:18 1997

Response via : Single Level Calibration

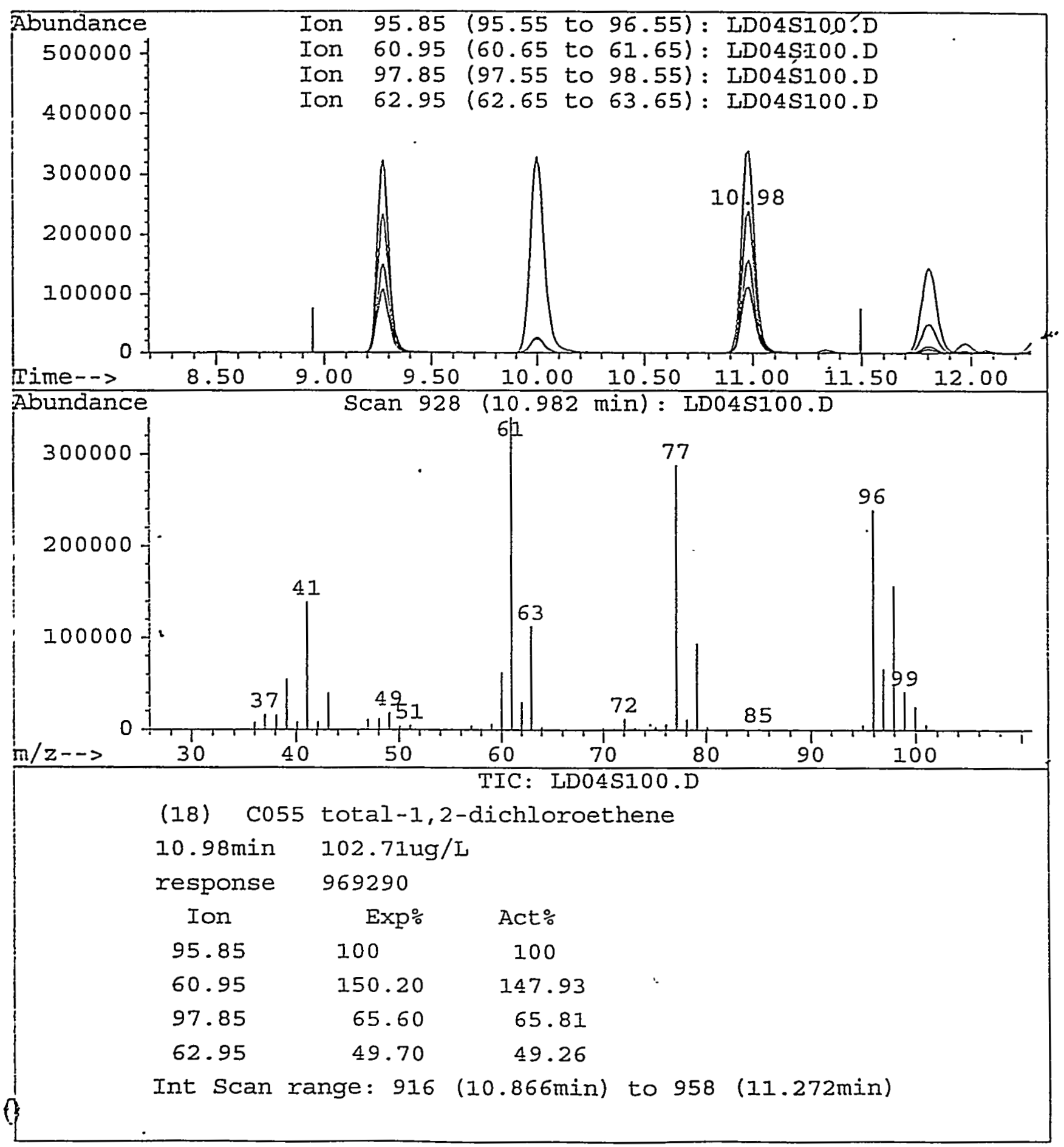




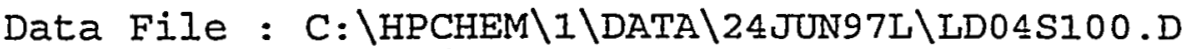

Acq Time : 06/24/97 16:04

Sample : $100 \mathrm{ug} / \mathrm{L}$ Initial Cal std

Misc : 142WS28293

Quant Time: Jun 25 8:51 1997

Operator: CQC

Inst : 5971-L

Multiplr: 1.00

Method : C: \HPCHEM \I\METHODS $\backslash$ L826ARWA.M

Title : VOA COMPOUND IIST

Last Update : Wed Jun 25 08:50:18 1997

Response via : Single Level Calibration

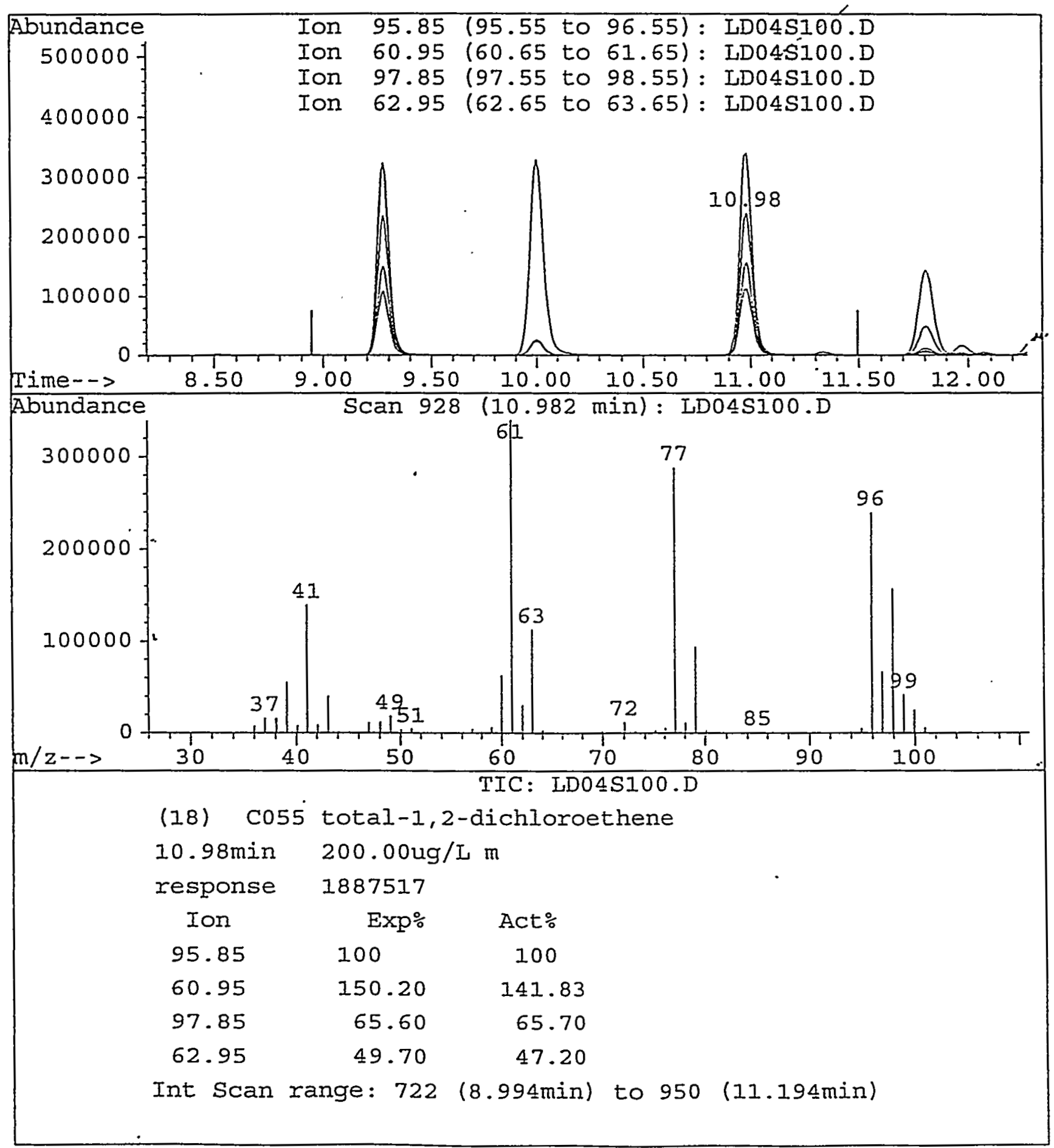


Quantitation Report

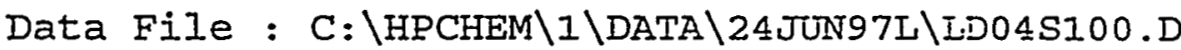

Acq Time : 06/24/97 16:04

Sample : $100 \mathrm{ug} / \mathrm{I}$ Initial Cal std

Misc : 142WS28293

Quant Time: Jun 25 8:51 1997

Operator: $\mathrm{CQC}$ Inst : 5971-L Multiplr: 1.00

Method : C: \HPCHEM \I\METHODS \L826ARWA.M

Title

: VOA COMPOUND LIST

Last Update : Wed Jun 25 08:50:18 1997

Response via : Single Level Calibration

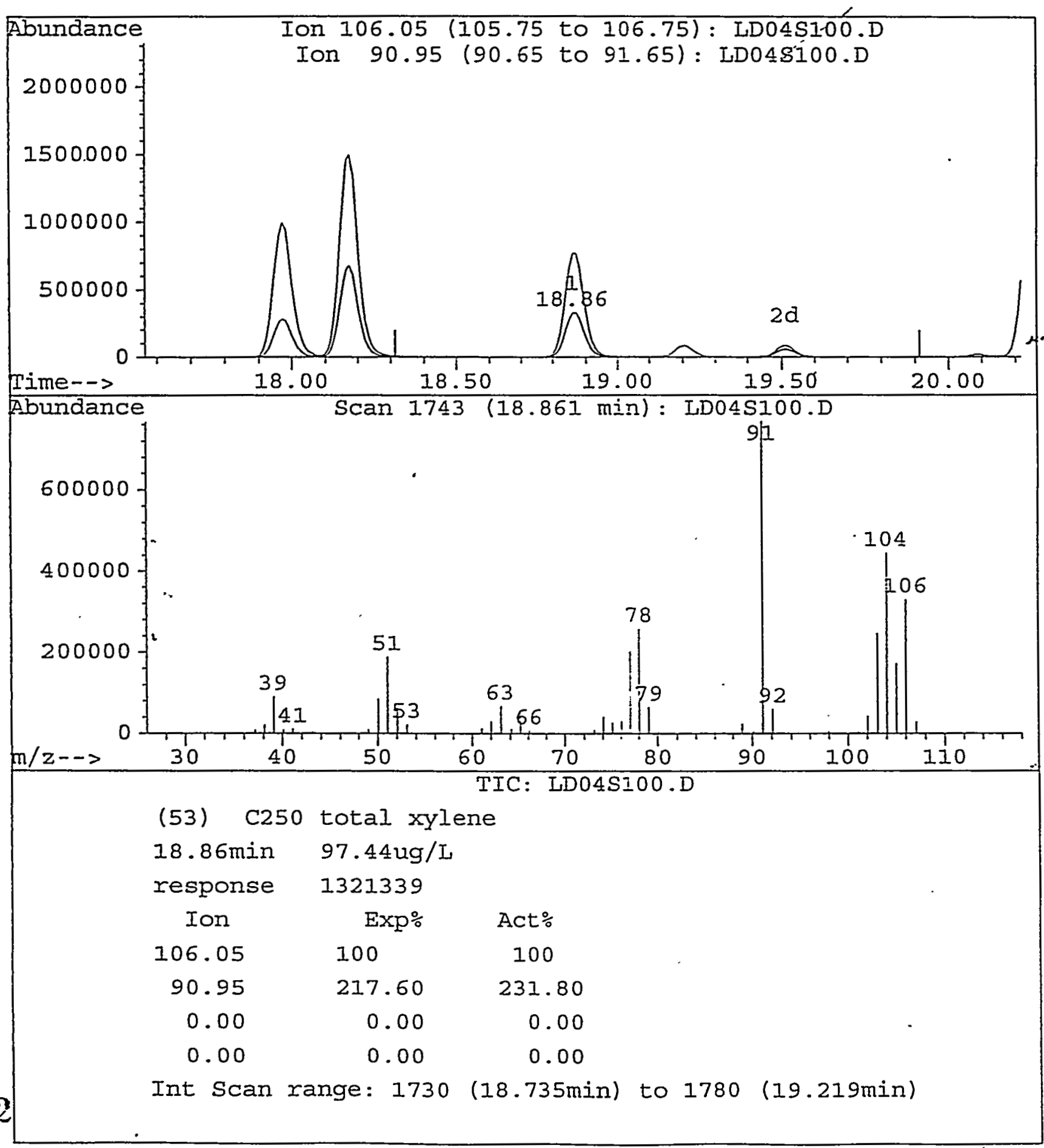




\section{Quantitation Report}

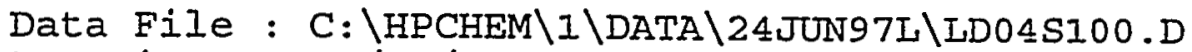

Acq Time : 06/24/97 16:04

Sample : $100 \mathrm{ug} / \mathrm{I}$ Initial Cal std

Misc : 142WS28293

Quant Time: Jun 25 8:52 1997

Operator: CQC

Inst : 5971-L Multiplr: 1.00

Method : C: $\backslash$ HPCHEM $\backslash I \backslash M E T H O D S \backslash I 826 A R W A . M$

Title

: VOA COMPOUND IIST

Last Update : Wed Jun 25 08:50:18 1997

Response via : Single Level Calibration

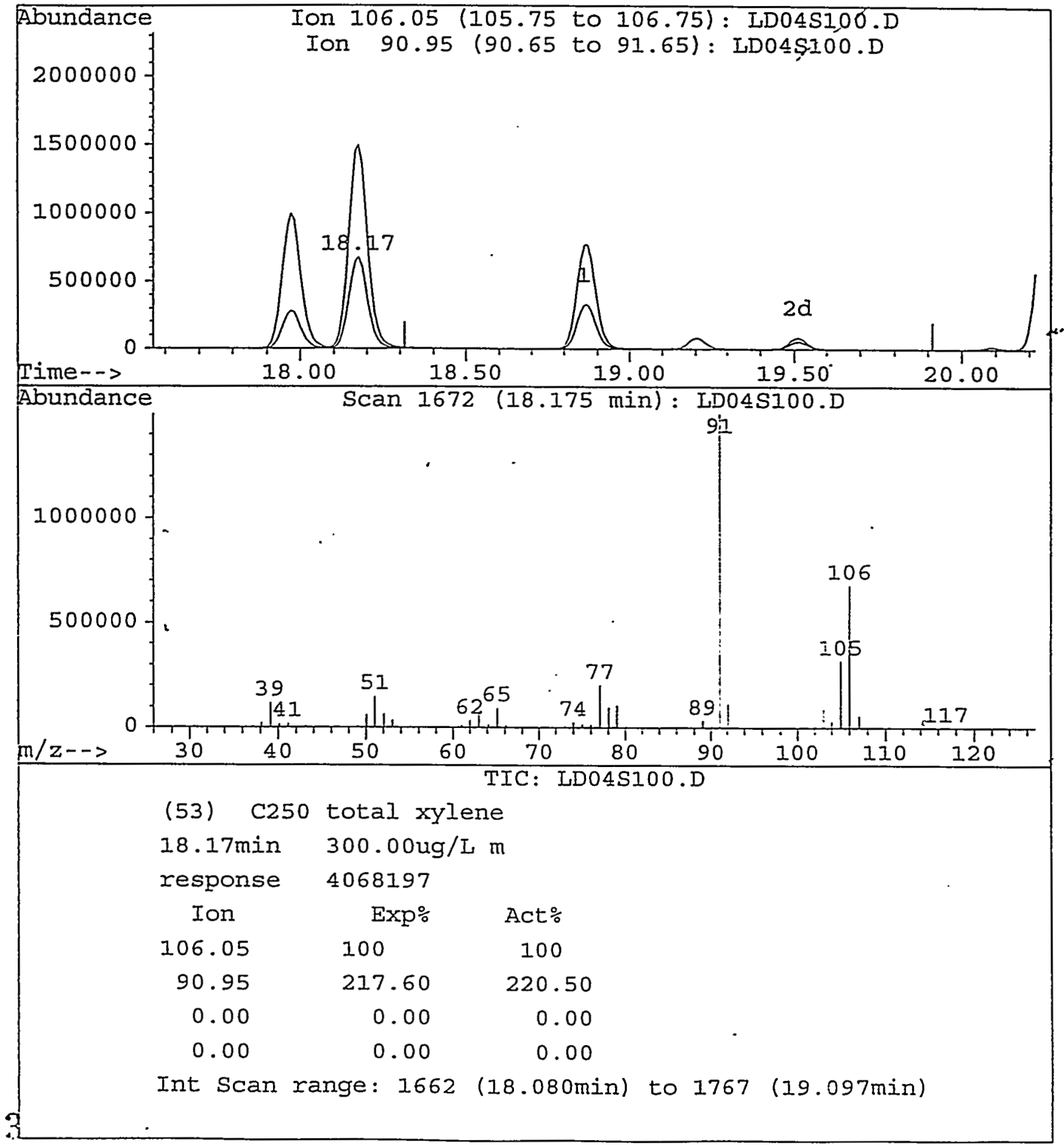


Quantitation Report

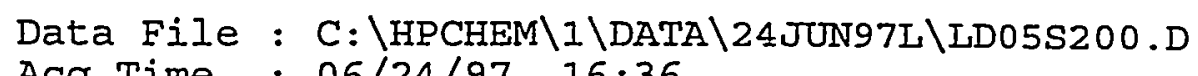

Acq Time : 06/24/97 $16: 36$

Sample : $200 \mathrm{ug} / \mathrm{L}$ Initial Cal std

Operator: CQC

Misc : 142WS28294

Quant Time: Jun 25 8:59 1997

Inst : 5971-I

Multiplr: 1.00

Method : C: \HPCHEM \I\METHODS\L826ARWA.M

Title : VOA COMPOUND LIST

Last Update : Wed Jun 25 08:57:37 1997

Response via : Single Level Calibration

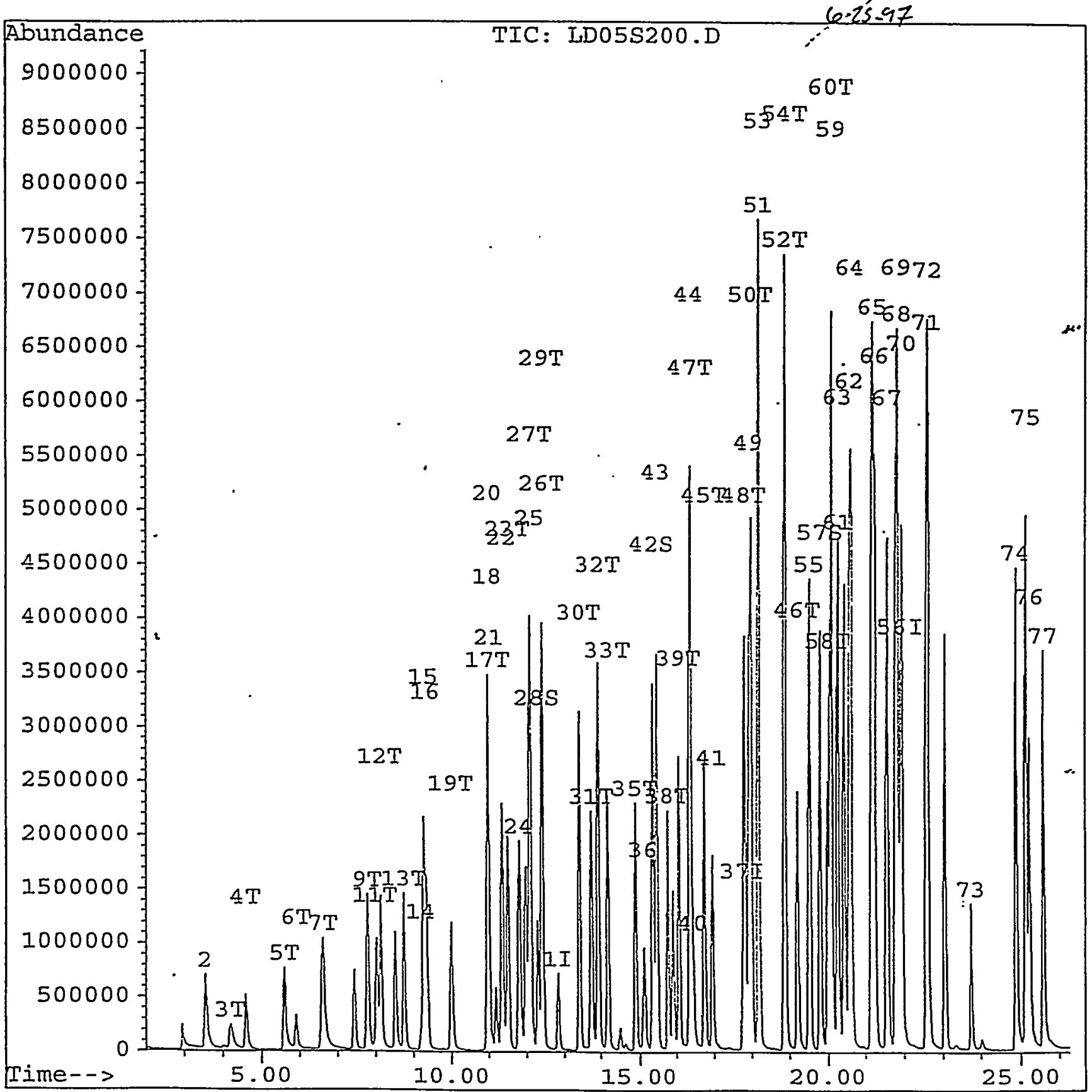

194 


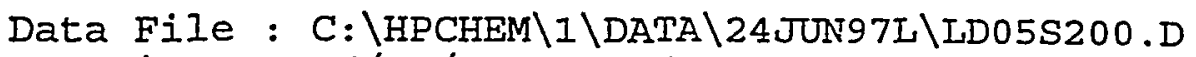

Acq Time : 06/24/97 16:36

Sample : $200 \mathrm{ug} / \mathrm{L}$ Initial Cal std

Misc : 142WS28294

Quant Time: Jun 25 8:59 1997

Method : C: \HPCHEM \I\METHODS\L826ARWA.M

Title : VOA COMPOUND IIST

Iast Update : Wed Jun 25 08:57:37 1997

Response via : LD05S200
Operator: $\mathrm{CQC}$

Inst : 5971-L

Multiplr: 1.00
Internal standards

\section{1) CIOI Flourobenzene}

37) CI02 Chlorobenzene-d5

56) CI03 1,4-Dichlorobenzene-d

System Monitoring Compounds

28) CS15 1,2-dichloroethane-d4

42) CS05 toluene-d8

57) CS10 4-bromofluorobenzene.

Target Compounds

2) $\mathrm{C002}$ dichlorodifluorometha

3) $\mathrm{C003}$ chloromethane

4) C004 vinyl chloride

5) C005 bromomethane

5) 0006 chloroethane

7) $\mathrm{C007}$ trichlorofluoromethan

8) 6071 acrolein

9) 0008 1,1-dichloroetherie

10) 0063 freon 113

11) Co09-acetone

12) C010 carbon disulfide

13) Co11 methylene chloride

14) C072 acrylonitrile

15) CO12.trans-1,2-Dichloroeth

16) C305 methyl-t-butyl-ether

17) $\mathrm{C} 013$ cis-1,2-dichloroethen

18) C055 total-1,2-dichloroeth

19) $\mathrm{C} 014$ 1,1-dichloroethane

20) Co15 2,2-dichloropropane

21) C110 2-butanone

22) CI01 bromochloromethane

23) $\mathrm{C} 016$ chloroform

24) $\mathrm{C} 017$ 1,1,1-Trichloroethane

25) $\operatorname{Co18~1,1-dichloropropene~}$

26) C019 1,2-dichloroethane

27) C020 carbon tetrachloride

29) C021 benzene

30) C022 trichloroethene

31) C023 1,2-dichloropropane

32) C024 dibromomethane

33) C025 bromodichloromethane

34) C026 2-chloroethylvinyleth

35) $\mathrm{C} 27 \mathrm{cis}-1,3-$ dichloroprope
R.T. QIon

$\begin{array}{rrc}12.82 & 96 & 1390397 \\ 17.75 & 117 & 1018652 \\ 21.88 & 152 & 685496\end{array}$

12.27

15.35

65

1750814

19.79

98

5155393

3.55

4.21

4.59

5.60

5.90

6.61

0.00

7.77

0.00

7.97

8.13

8.74

9.21

9.27

9.33

10.97

10.97

9.99

10.97

11.02

11.36

11.51

11.80

12.07

12.40

12.09

12.40

13.42

13.75

13.93

14.18

0.00

14.89
3462310

95

85

50

62

94

64

101

56

96

151

43

76

84

53

96

73

96

96

63

77

72

128

83

97

75

62

117

78

130

63

93

83

63

75
2153637
994994
1383028
1434043
753206
3348744

1576566

331080

4248392

1541282

381218

1754473

4046414

1870544

3623974

2960579

2880235

175335

1120471

3158078

3184801

2689748

2089828

2985331

5367707

2440397

2055657

1757150

3547251

3160975
Conc Units

Area:

$\begin{array}{ll}50.00 \mathrm{ug} / \mathrm{L} & 100.00 \\ 50.00 \mathrm{ug} / \mathrm{I} & 100.00 \\ 50.00 \mathrm{ug} / \mathrm{I} & 100.00\end{array}$

$\%$ Recovery

$200.00 \mathrm{ug} / \mathrm{L} \quad 400.00 \%$

$200.00 \mathrm{ug} / \mathrm{L} \quad 400.00 \%$

$200.00 \mathrm{ug} / \mathrm{L} \quad 400.00 \%$

Qvalue

$200.00 \mathrm{ug} / \mathrm{I}$

98

$200.00 \mathrm{ug} / \mathrm{I} \quad 99$

$200.00 \mathrm{ug} / \mathrm{L} \quad 98$

$200.00 \mathrm{ug} / \mathrm{L} \quad 99$

$200.00 \mathrm{ug} / \mathrm{L} \quad 96$

$200.00 \mathrm{ug} / \mathrm{L}$

Not Detected

$200.00 \mathrm{ug} / \mathrm{L}$

Not Detected

$200.00 \mathrm{ug} / \mathrm{L} \mathrm{\#}$

$200.00 \mathrm{ug} / \mathrm{L}$

$200.00 \mathrm{ug} / \mathrm{L} \#$

$200.00 \mathrm{ug} / \mathrm{L}$

$200.00 \mathrm{ug} / \mathrm{L}$

$200.00 \mathrm{ug} / \mathrm{I}$ \#

$200.00 \mathrm{ug} / \mathrm{L}$

$400.00 \mathrm{ug} / \mathrm{L} \mathrm{m}$ ces 98

$200.00 \mathrm{ug} / \mathrm{I}_{6.1547} 97$

$200.00 \mathrm{ug} / \mathrm{L} \#-73$

$200.00 \mathrm{ug} / \mathrm{I} \# \quad 15$

$200.00 \mathrm{ug} / \mathrm{I} \#$

$200.00 \mathrm{ug} / \mathrm{L} \#$

$200.00 \mathrm{ug} / \mathrm{L}$

$200.00 \mathrm{ug} / \mathrm{I}$ \#

$200.00 \mathrm{ug} / \mathrm{I}$

$200.00 \mathrm{ug} / \mathrm{I}$

$200.00 \mathrm{ug} / \mathrm{I} \#$

$200.00 \mathrm{ug} / \mathrm{L}$

$200.00 \mathrm{ug} / \mathrm{L}$

$200.00 \mathrm{ug} / \mathrm{L}$

$200.00 \mathrm{ug} / \mathrm{L}$

Not Detected

$200.00 \mathrm{ug} / \mathrm{L} \#$
89

75

90

73

96

89

87

88

7

69

99

77

98

97

88

89

96

90

99

73

$(\mathrm{NR})=$ Not Reported $(\mathrm{SP})=$ Spiked analyte $(\mathrm{m})=$ manual integration

(*) Does not meet EPA spectral criteria (False Hit) 
Quantitation Report

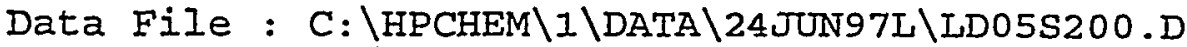

Acq Time : 06/24/97 16:36

Sample : $200 \mathrm{ug} / \mathrm{L}$ Initial Cal Std

Misc : 142WS28294

Quant Time: Jun 25 8:59 1997

Operator: $\mathrm{CQC}$

Inst : 5971-L

Multiplr: 1.00

Method : C: \HPCHEM II \METHODS \L826ARWA.M

Title : VOA COMPOUND LIST

Last Update : Wed Jun 25 08:57:37 1997

Response via : LD05S200

Compound

36) C205 4-methyl-2-pentanone

38) C028 trans-1,3-dichloropro

39) Co29 1,1,2-trichloroethane

40) C210 2-hexanone

41) $\operatorname{Co3} 0$ 1,2-Dibromoethane

43) C031 Toluene

44) $\cos 2$ 1,3-dichloropropane

45) C033 dibromochloromethane

46) $\mathrm{C034}$ bromoform

47) C035 tetrachloroethene

48) 0036 chlorobenzene

49) $\operatorname{Co3} 71,1,1,2$-tetrachloroet

50) C038 ethylbenzene

51) $C 039 \mathrm{~m}, \mathrm{p}$-xylene

52) C040 o-xylene

53) C250 total xylene

54) C041 styrene

55) 0042 isopropylbenzene.

58) $\mathrm{C} 043$ 1,1,2,2-tetrachloroet

59) $\mathrm{CO} 44$.bromobenzene

60) $C 045$ 1,2,3-trichloropropan

61) $\mathrm{C} 046$ n-propylbenzene

62) $\operatorname{co47} 1,3,5$-trimethylbenzen

63) $\mathrm{C} 048.2$-chlorotoluene

64) C049 4-chlorotoluene

65) C050 tert-butylbenzene

66) C051 1,2,4-trimethylbenzen

67) 0052 sec-Butylbenzene

68) C053 p-Isopropyltoluene

69) C054 1,3-Dichlorobenzene

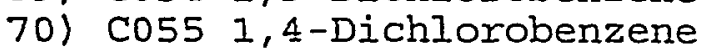

71) 0056 n-Butylbenzene

72) $\mathrm{C057}$ 1,2-Dichlorobenzene

73) $\mathrm{C} 058$ I,2-Dibromo-3-chlorop

74) C059 1,2,4-Trichlorobenzen

75) C060 Hexachlorobutadiene

76) C061 Naphthalene

77) $\operatorname{Co621,2,3-Trichlorobenzen~}$

R.T. QIon Response Conc Unit Qvalue

15.11

15.76

16.07

16.46

16.95

15.45

16.35

16.75

19.21

16.39

17.79

17.92

17.98

18.18

18.87

18.18

18.89

19.52

19.98

20.07

20.08

20.25

20.56

20.25

20.60

21.17

21.24

21.56

21.82

21.78

21.93

22.55

22.60

23.72

24.88

25.13

25.22

25.57

(10 188

$43 \quad 1889520$

75

97

43

107

92

76

129

173

164

112

131

106

106

106

106

104

105 .

83

156

75

91

105

91

91

119

105

105

119

146

146

91

146

75

180

225

128

180
2795508

1974378

1071976

2851364

3668129

3158975

3238786

2529756

2365707

4609351

2529465

2100503

5130373

2497225

7670990

4503074

7527586

3387216

2525771

3366783

9521463

.6035648

9521463

6993792

8231087

6041826

8854830

7751707

4483189

4745354

7521586

4242407

760294

3042895

2276694

5208863

2605530
Cone

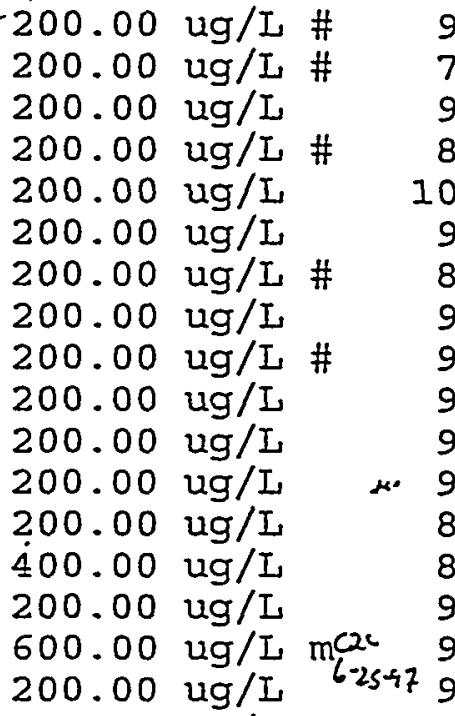

$200.00 \mathrm{ug} / \mathrm{L} \quad 96$

$200.00 \mathrm{ug} / \mathrm{L} \# \quad 98$

$200.00 \mathrm{ug} / \mathrm{L} \quad 98$

$200.00 \mathrm{ug} / \mathrm{L} \quad 92$

$200.00 \mathrm{ug} / \mathrm{L}$ \# 56

$200.00 \mathrm{ug} / \mathrm{I} \quad 92$

$200.00 \mathrm{ug} / \mathrm{I} \# \quad 45$

$200.00 \mathrm{ug} / \mathrm{L} \quad 96$

$200.00 \mathrm{ug} / \mathrm{L} \quad 97$

$200.00 \mathrm{ug} / \mathrm{L} \quad 77$

$200.00 \mathrm{ug} / \mathrm{I} \# \quad 95$

$200.00 \mathrm{ug} / \mathrm{L} \approx 96$

$200.00 \mathrm{ug} / \mathrm{L} \quad 95$

$200.00 \mathrm{ug} / \mathrm{I} \quad 96$

$200.00 \mathrm{ug} / \mathrm{L} \# \quad 88$

$200.00 \mathrm{ug} / \mathrm{I} \quad 99$

$200.00 \mathrm{ug} / \mathrm{I} \# \quad 80$

$200.00 \mathrm{ug} / \mathrm{L} \quad 99$

$200.00 \mathrm{ug} / \mathrm{L} \quad 97$

$200.00 \mathrm{ug} / \mathrm{L} \quad 99$

$200.00 \mathrm{ug} / \mathrm{L} \quad 99$ 


\section{Quantitation Report}

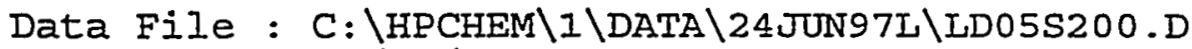

Acq Time : 06/24/97 16:36

Sample : $200 \mathrm{ug} / \mathrm{I}$ Initial Cal std

Misc : 142WS28294

Quant Time: Jun 25 8:58 1997

Operator: CQC

Inst : 5971-I

Multiplr: 1.00

Method : C: \HPCHEM IIMETHODS \L826ARWA.M

Title : VOA COMPOUND IIST

Iast Update : Wed Jun 25 08:57:37 1997

Response via : Single Level Calibration

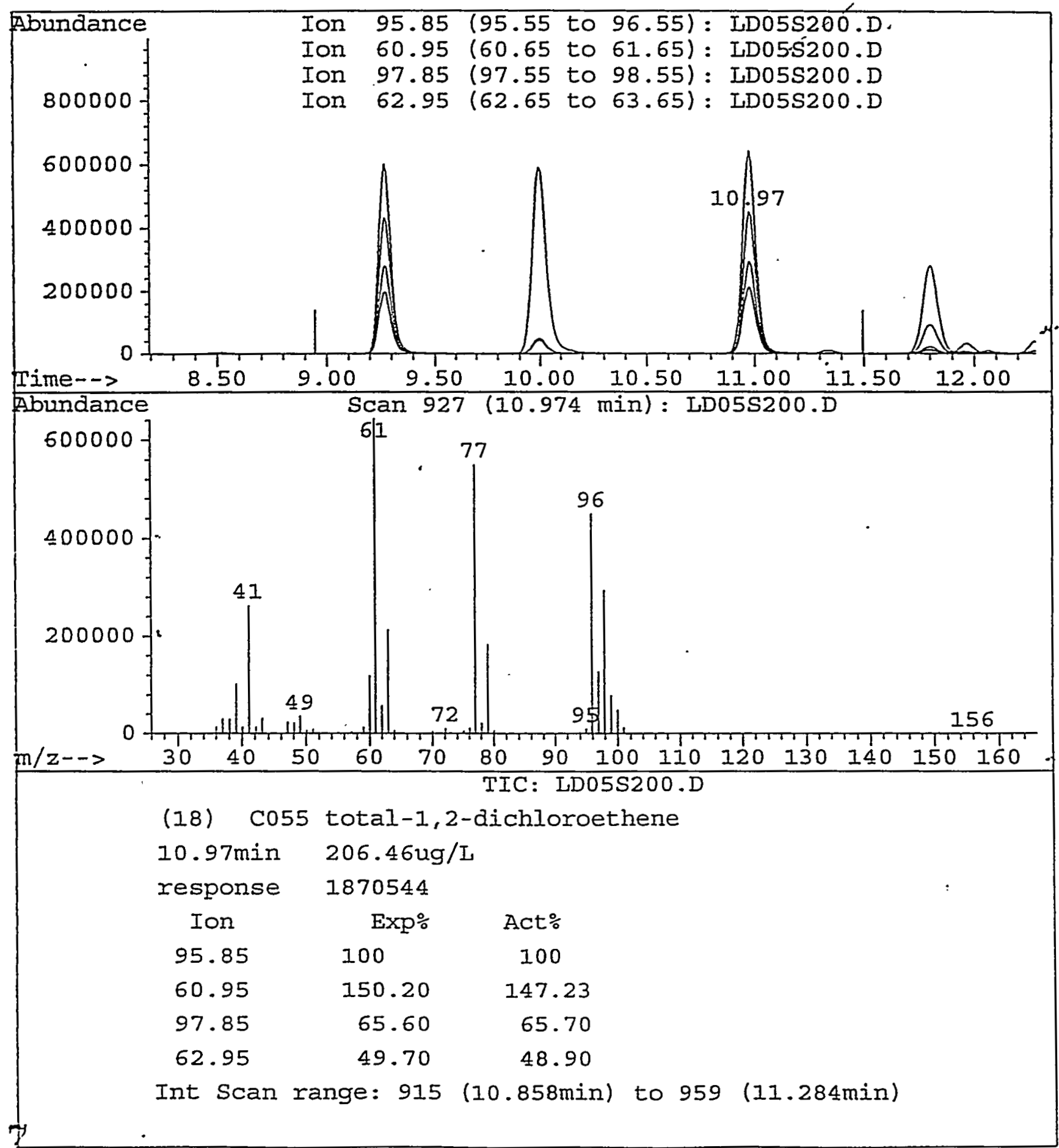


Data File : C: \HPCHEM \IDATA $\backslash 24 J U N 97 L \backslash I D 05 S 200 . D$

Acq Time : 06/24/97 16:36

Sample : $200 \mathrm{ug} / \mathrm{L}$ Initial Cal std

MisC : I42WS28294

Quant Time: Jun 25 8:58 1997

Operator: CQC Inst : 5971-L Multiplr: 1.00

Method : C: \HPCHEM \I\METHODS \L826ARWA.M

Title : VOA COMPOUND LIST

Last Update : Wed Jun 25 08:57:37 1997

Response via : Single Level Calibration

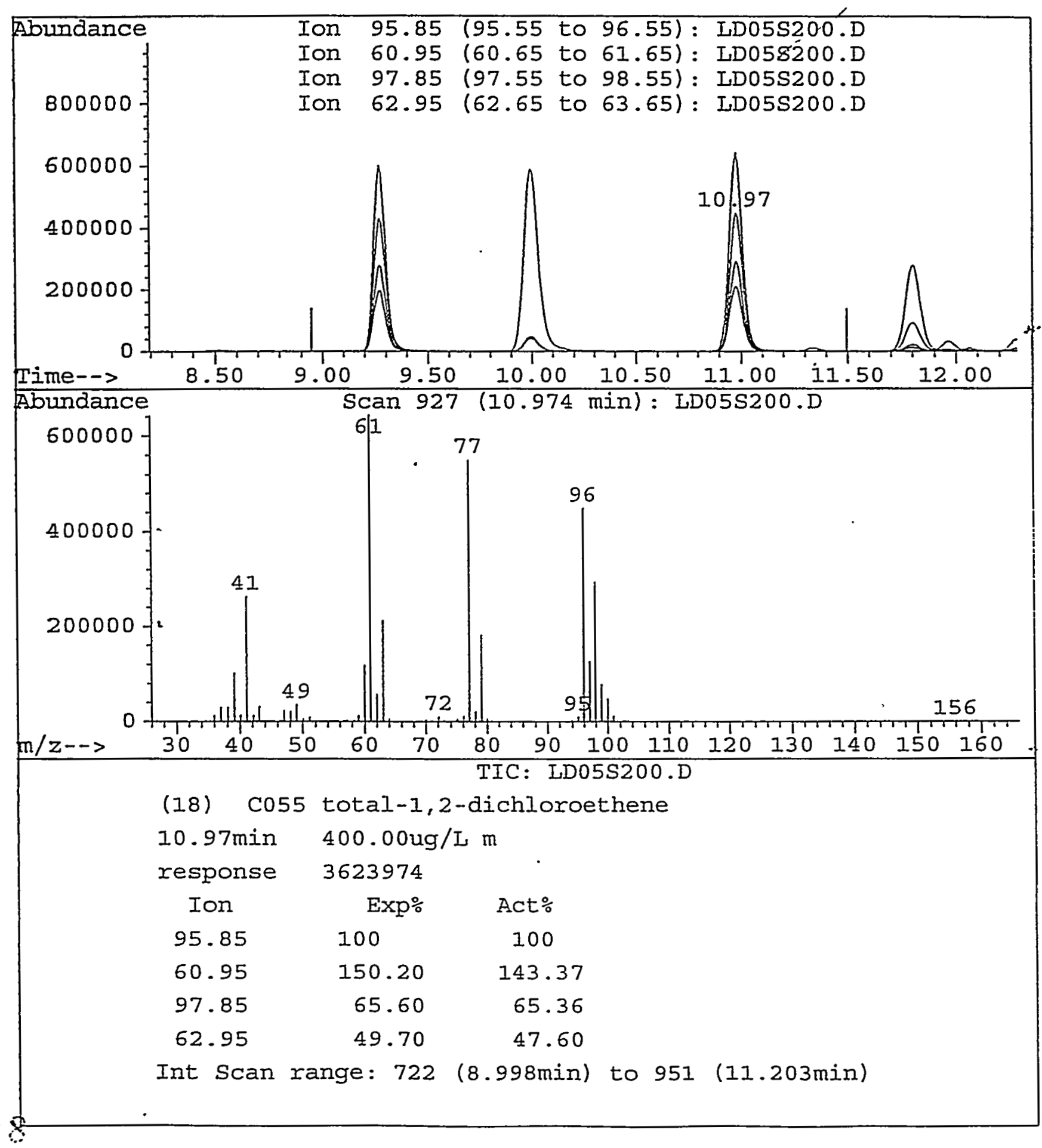


Quantitation Report

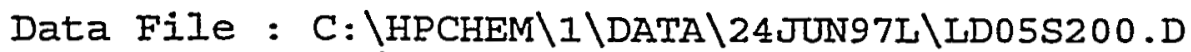

Acq Time: 06/24/97 $16: 36$

Sample : $200 \mathrm{ug} / \mathrm{L}$ Initial Cal std

Misc : 142WS28294

Quant Time: Jun 25 8:59 1997

Operator: CQC

Inst : 5971-L

Multiplr: 1.00

Method : C: \HPCHEM IIMETHODS \I826ARWA.M

Title : VOA COMPOUND IIST

Last Update : Wed Jun 25 08:57:37 1997

Response via : Single Level Calibration

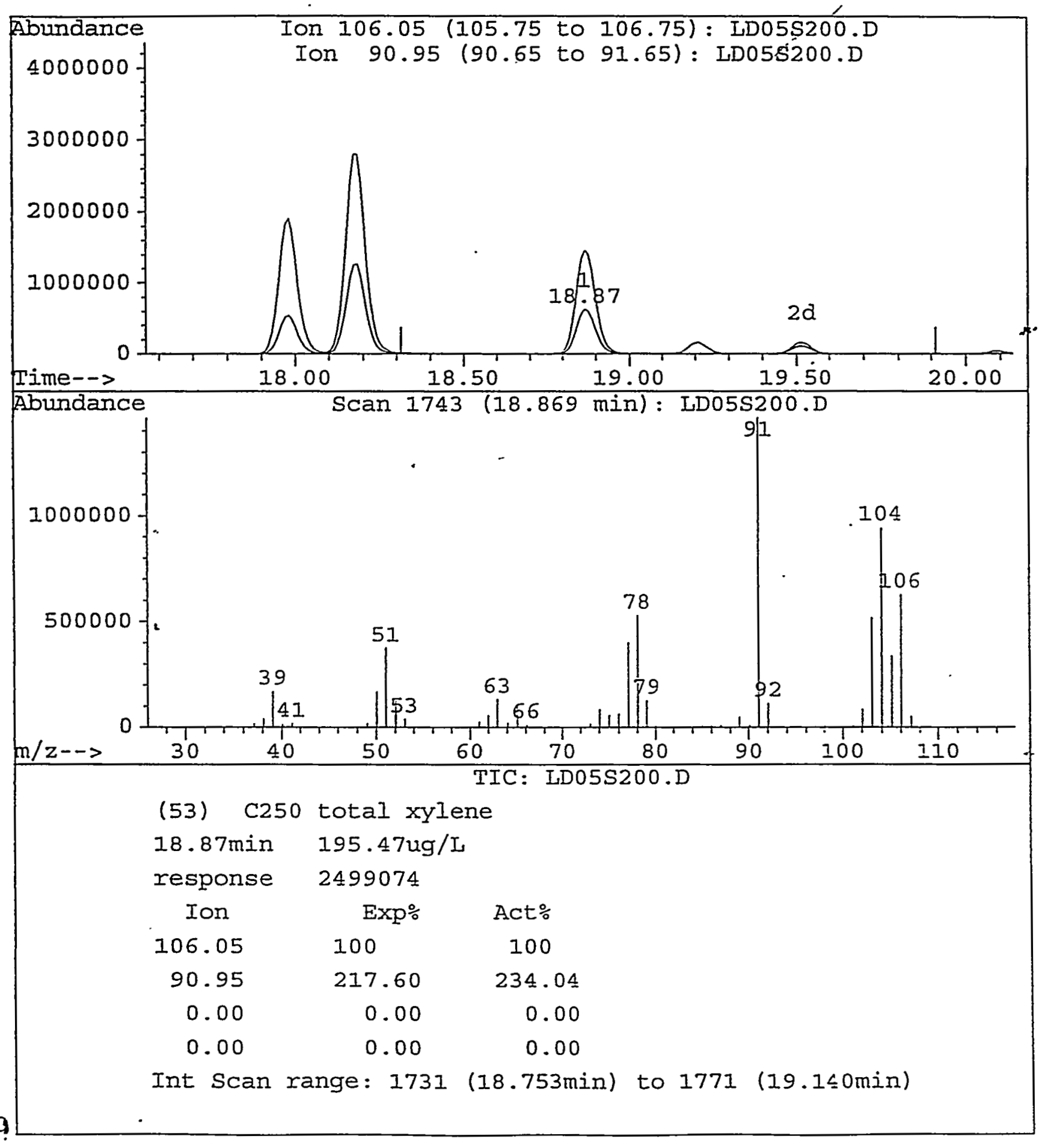


Quantitation Report

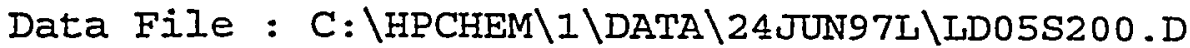

Acq Time : 06/24/97 16:36

Sample : $200 \mathrm{ug} / \mathrm{L}$ Initial Cal std

Misc : 142WS28294

Quant Time: Jün 25 8:59 1997

Operator: CQC

Inst : 5971-I

Multiplr: 1.00

Method : C: \HPCHEM \I\METHODS\I826ARWA.M

Title : VOA COMPOUND LIST

Last Update : Wed Jun 25 08:57:37 1997

Response via : Single Level Calibration

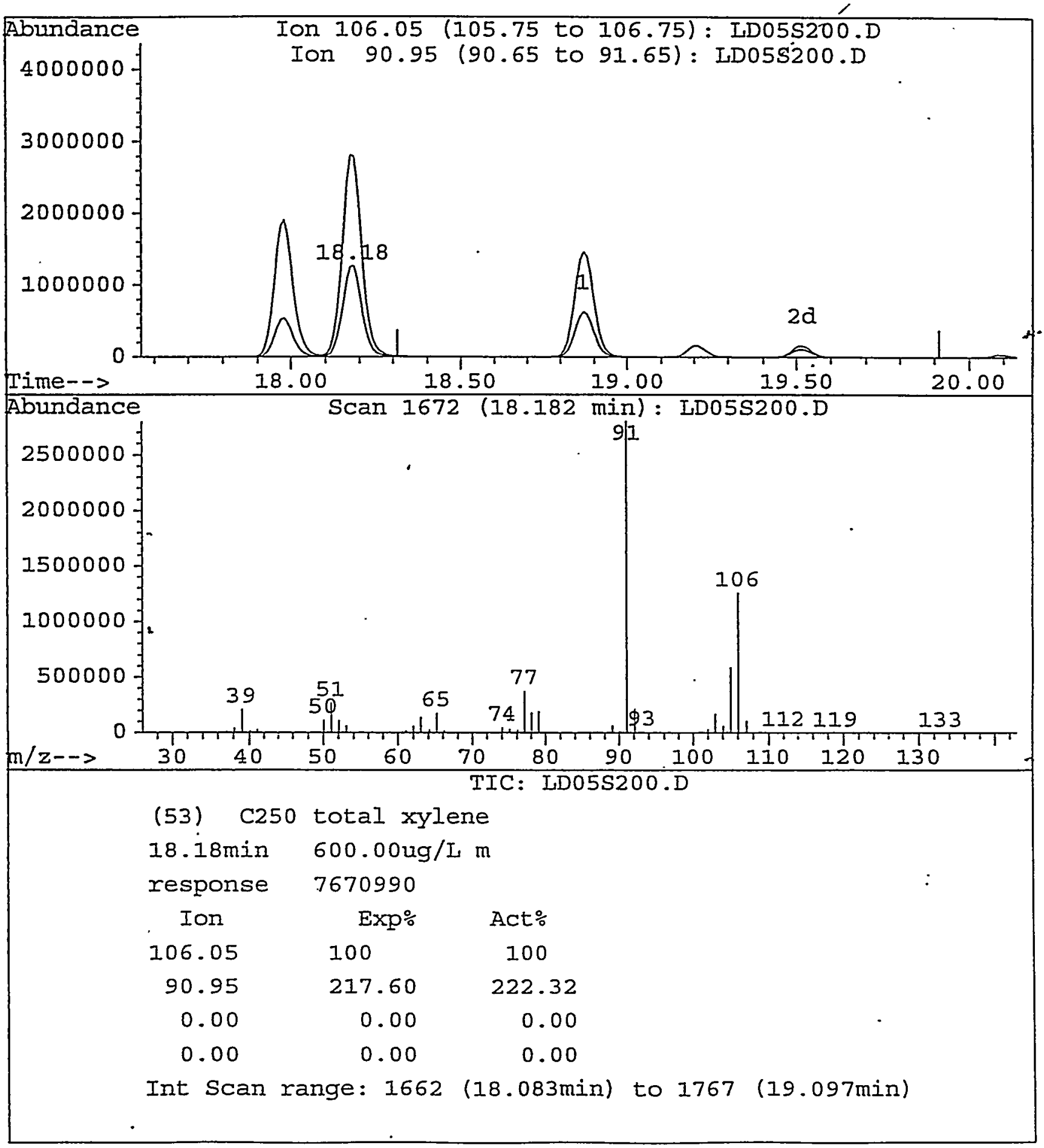




\section{A CONTINUING CALIBRATION REPORT \\ SW-846, 8260A, Revision 1}

Continuing Calibration File: LE91S50.D

07/07/97

Initial Calibration Method:

L826ARWA

\begin{tabular}{|c|c|c|c|c|c|}
\hline Calibration Check $\mathrm{C}$ & mpounds: & $\% \mathrm{Dr}$ & $t$ must be $<$ & $0 . \%$ & \\
\hline & RT & CC Amnt & CC Target & $\%$ Drift & \\
\hline C004 vinyl chloride & 4.58 & 42.07 & 50.00 & 15.9 & o \\
\hline C008 1.1-dichloroethene & 7.78 & 48.55 & 50.00 & 2.9 & o \\
\hline C016 chloroform & 11.50 & 48.44 & 50.00 & $3: 11$ & \\
\hline CO23 1,2-dichloropropane & 13.76 & 40.80 & 50.00 & 18.4 & \\
\hline C031 Toluene & 15.46 & 46.73 & 50.00 & 6.5 & \\
\hline C038 ethylbenzene & 17.98 & 48.22 & 50.00 & 3.6 & \\
\hline SPCC C & mpounds: & & & & \\
\hline & & CC R & $\begin{array}{l}\text { must be }> \\
\text { CC RF }\end{array}$ & $=0.1$ & \\
\hline C003 chloromethane & - & & 0.161 & & \\
\hline C014 1,1-dichloroethane & & & 0.482 & & \\
\hline & & $\mathrm{CC}$ & Fs must be & 0.1 & \\
\hline C034 bromoform & & & 0.713 & - & \\
\hline & & CC R & 5 must be $>$ & $=0.3$ & \\
\hline C036 chlorober & & & 1.123 & & \\
\hline 1,1,2,2-tetrachloroethane & & & 1.009 & & \\
\hline
\end{tabular}




\title{
8260A CONTINUING CALIBRATION REPORT (cont'd) SW-846, 8260A, Revision 1
}

Continuing Calibration File: LE91S50.D

$07 / 07 / 97$

\author{
Initial Calibration Method: L826ARWA
}

\begin{tabular}{|c|c|c|c|c|c|}
\hline Surrogate Compounds: & RT & CC Amnt & $\begin{array}{c}\text { CC Target } \\
\text { CC RF }\end{array}$ & $\%$ Drift & \\
\hline CS15 1,2-dichloroethane-d4 & 12.28 & 45.72 & 50.00 & 8.6 & \\
\hline CS05 toluene-d8 & 15.35 & 47.30 & 50.00 & 5.4 & \\
\hline CS10 4-bromofluorobenzene & 19.79 & 42.44 & 50.00 & 15.1 & 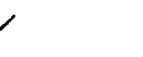 \\
\hline Target Compounds: & RT & CC Amnt & CC Target & $\%$ Drift & \\
\hline C002 dichlorodifluoromethane & 3.54 & 48.09 & 50.00 & 3.8 & \\
\hline C003 chloromethane & 4.19 & 48.39 & 50.00 & 3.2 & \\
\hline C004 vinyl chloride & 4.58 & 42.07 & 50.00 & 15.9 & \\
\hline C005 bromomethane & 5.59 & 40.96 & 50.00 & 18.1 & \\
\hline C006 chloroethane & 5.90 & 41.37 & 50.00 & 17.3 & \\
\hline C007 trichlorofluoromethane & 6.63. & 51.05 & 50.00 & -2.1 & \\
\hline COOB 1,1-dichloroethene & 7.78 & 48.55 & 50.00 & 2.9 & \\
\hline Co09 acetone & 7.92 & 38.47 & 50.00 & 23.1 & warning \\
\hline C010 carbon disulfide & 8.14 & 45.02 & 50.00 & 10.0 & \\
\hline C011 methylene chloride & 8.75 & 45.55 & 50.00 & 8.9 & \\
\hline C072 acrylonitrile & 9.19 & 34.42 & 50.00 & $31.2^{\circ}$ & warning \\
\hline C012 trans-1,2-Dichloroethene & 9.27 & 48.71 & 50.00 & 2.6 & \\
\hline C305 methyl-t-butyl-ether & 9.32 & 45.12 & 50.00 & 9.8 & \\
\hline C013 cis-1,2-dichloroethene & 10.98 & 48.76 & 50.00 & 2.5 & \\
\hline C055 total-1,2-dichloroethene & 10.98 & 97.49 & 100.00 & 2.5 & \\
\hline C014 1,1-dichloroethane & 10.00 & 42.82 & 50.00 & 14.4 & \\
\hline C015 2,2-dichloropropane & 10.98 & 47.05 & 50.00 & 5.9 & \\
\hline C110 2-butanone & 11.01 & 46.60 & 50.00 & 6.8 & \\
\hline Cl01 bromochloromethane & 11.37 & 54.30 & 50.00 & -8.6 & \\
\hline C016 chloroform & 11.50 & 48.44 & 50.00 & 3.1 & \\
\hline C017 1,1,1-Trichloroethane & 11.81 & 50.30 & 50.00 & -0.6 & \\
\hline C018 1,1-dichloropropene & 12.07 & 46.09 & 50.00 & 7.8 & \\
\hline Co19 1,2-dichloroethane & 12.40 & 45.11 & 50.00 & 9.8 & \\
\hline C020 carbon tetrachloride & 12.09 & 52.06 & 50.00 & -4.1 & \\
\hline Co21 benzene & 12.40 & 44.53 & 50.00 & 10.9 & \\
\hline $\mathrm{CO} 22$ trichloroethene & 13.43 & 53.36 & 50.00 & -6.7 & \\
\hline C023 1,2-dichloropropane & 13.76 & 40.80 & 50.00 & 18.4 & \\
\hline C024 dibromomethane & 13.94 & 49.07 & 50.00 & 1.9 & \\
\hline C025 bromodichloromethane & 14.18 & 48.07 & 50.00 & 3.9 & \\
\hline C027 cis-1,3-dichloropropene & 14.90 & 45.84 & 50.00 & 8.3 & \\
\hline C205 4-methyl-2-pentanone & 15.11 & 47.26 & 50.00 & 5.5 & \\
\hline C028 trans-1,3-dichloropropene & 15.77 & 44.74 & 50.00 & 10.5 & \\
\hline Co29 1,1,2-trichloroethane & 16.07 & 47.24 & 50.00 & 5.5 & \\
\hline C210 2-hexanone & 16.46 & 40.46 & 50.00 & 19.1 & \\
\hline C030 1,2-Dibromoethane & 16.96 & 48.16 & 50.00 & 3.7 & \\
\hline C031 Toluene & 15.46 & 46.73 & 50.00 & 6.5 & \\
\hline C032 1,3-dichloroproparie & 16.36 & 43.55 & 50.00 & 12.9 & \\
\hline C033 dibromochloromethane & 16.75 & 51.31 & 50.00 & -2.6 & \\
\hline C034 bromoform & 19.21 & 60.33 & 50.00 & -20.7 & warning \\
\hline
\end{tabular}


8260A CONTINUING CALIBRATION REPORT (cont'd)

SW-846, 8260A, Revision 1

Continuing Calibration File: LE91S50.D

$07 / 07 / 97$

Initial Calibration Method: L826ARWA

Target Compounds (cont'd):

C035 tetrachloroethene

C036 chlorobenzene

C037 1,1,1,2-tetrachloroethane

C038 ethyibenzene

C039 m,p-xylene

c040 o-xylene

C250 total xylene C041 styrene

C042 isopropylbenzene

C043 1,1,2,2-tetrachloroethane

C044 bromobenzene

C045 1,2,3-trichloropropane C046 n-propylbenzene

C047 1,3,5-trimethylbenzene C048 2-chlorotoluene C049 4-chlorotoluene C050 tert-butylbenzene

Co51 1,2,4-trimethylbenzene C052 sec-Butylbenzene C053 p-isopropyltoluene Co54 1,3-Dichlorobenzene Co55 1,4-Dichlorobenzene C056 n-Butylbenzene

Co57 1,2-Dichlorobenzene

C058 1,2-Dibromo-3-chloropropane Co59 1,2,4-Trichlorobenzene C060 Hexachlorobutadiene C061 Naphthalene C062 1,2,3-Trichlorobenzene

$\begin{array}{cccc}\text { RT } & \text { CC Amnt } & \text { CC Target } & \text { \% Drift } \\ 16.38 & 56.49 & 50.00 & -13.0 \\ 17.80 & 48.18 & 50.00 & 3.6 \\ 17.92 & 51.34 & 50.00 & -2.7 \\ 17.98 & 48.22 & 50.00 & 3.6 \\ 18.17 & 96.42 & 100.00 & 3,6 \\ 18.87 & 48.58 & 50.00 & 2.8 \\ 18.17 & 144.95 & 150.00 & 3.4 \\ 18.88 & 47.68 & 50.00 & 4.6 \\ 19.50 & 47.58 & 50.00 & 4.8 \\ 19.99 & 38.37 & 50.00 & 23.3 \\ 20.07 & 49.84 & 50.00 & 0.3 \\ 20.07 & 38.38 & 50.00 & 23.2 \\ 20.24 & 41.47 & 50.00 & 17.1 \\ 20.55 & 41.93 & 50.00 & 16.1 \\ 20.24 & 42.62 & 50.00 & 14.8 \\ 20.60 & 41.71 & 50.00 & 16.6 \\ 21.16 & 44.58 & 50.00 & 10.8 \\ 21.24 & 42.04 & 50.00 & 15.9 \\ 21.55 & 41.92 & 50.00 & 16.2 \\ 21.81 & 43.85 & 50.00 & 12.3 \\ 21.77 & 49.04 & 50.00 & 1.9 \\ 21.92 & 50.95 & 50.00 & -1.9 \\ 22.55 & 41.80 & 50.00 & 16.4 \\ 22.59 & 48.43 & 50.00 & 3.1 \\ 23.73 & 41.10 & 50.00 & 17.8 \\ 24.87 & 54.40 & 50.00 & -8.8 \\ 25.12 & 57.65 & 50.00 & -15.3 \\ 25.22 & 46.94 & 50.00 & 6.1 \\ 25.57 & 56.30 & 50.00 & -12.6\end{array}$




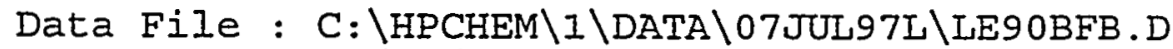

Acq Time : 07/07/97 08:32

Sample : $50 \mathrm{ng} \mathrm{BFB}$

Misc

: 2.0 uL of 39 IS7676
Operator: $\mathrm{CQC}$

Inst : 5971-I Multiplr: 1.00

Method : C: \HPCHEM \I\METHODS \BFB8240.M Title :

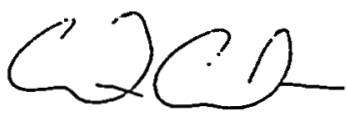

7.7 .67

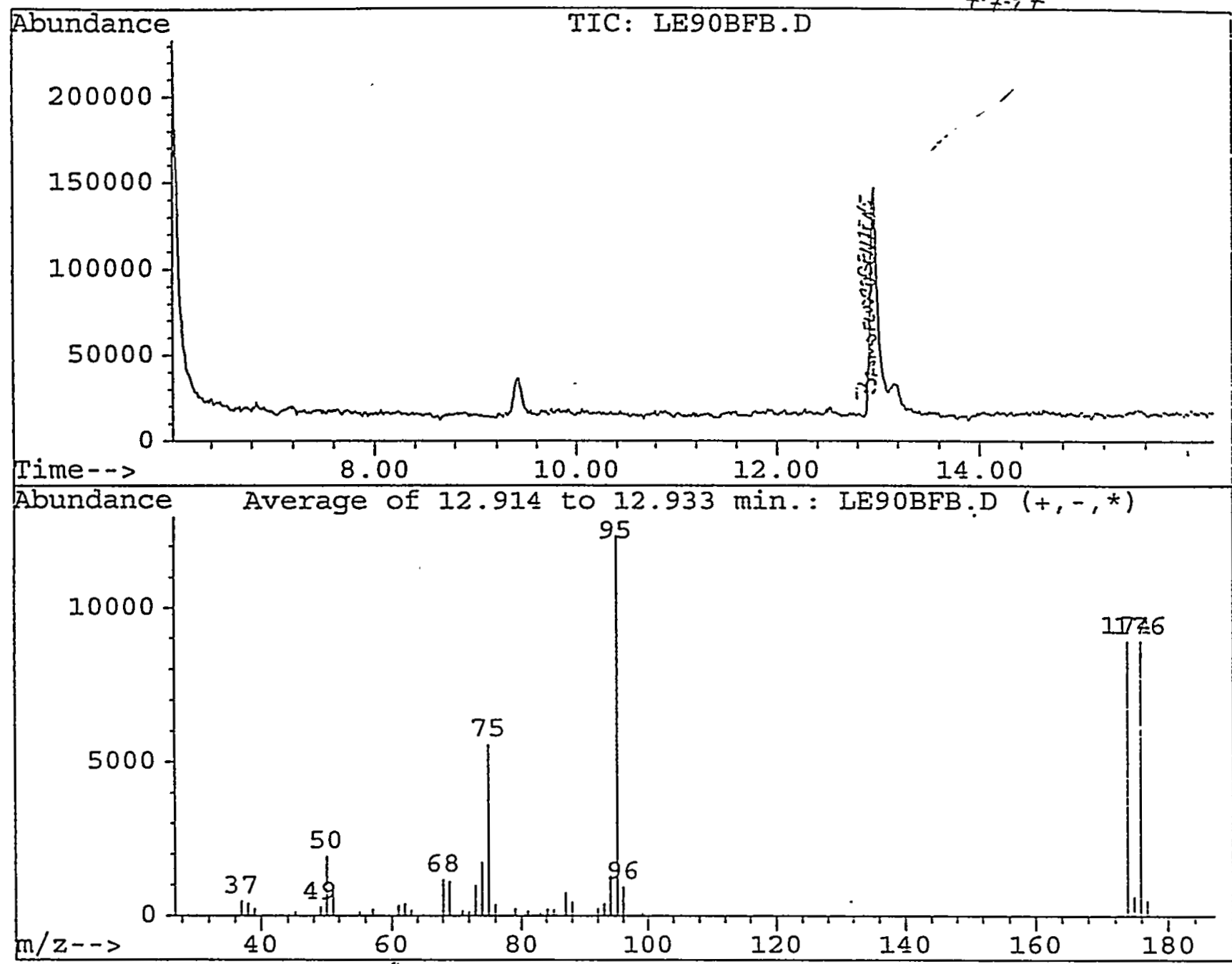

Peak Apex is scan: $7.765-719$ Averaye of sians: $714,715,716$ mine back ground sxion: 705 \begin{tabular}{l|l|l|l|l|l} 
Target & Rel. to & Lower & Upper & Rel. & Raw
\end{tabular} Mass $\mid$ Mass $\mid$ Limit\% $\mid$ Limit\% Abn\% Abn

Result Pass/Fail

\begin{tabular}{|r|r|r|r|r|r|r|}
50 & 95 & 15 & 40 & 15.68 & 1936 & PASS \\
75 & 95 & 30 & 60 & 45.04 & 5561 & PASS \\
95 & 95 & 100 & 100 & 100.00 & 12347 & PASS \\
96 & 95 & 5 & 9 & 7.65 & 944 & PASS \\
173 & 174 & 0 & 2 & 0.00 & 0 & PASS \\
174 & 95 & 50 & 100 & 72.33 & 8931 & PASS \\
175 & 174 & 5 & 9 & 7.45 & 665 & PASS \\
176 & 174 & 95 & 101 & 99.80 & 8913 & PASS \\
177 & 176 & 5 & 5 & 5.77 & 514 & PASS
\end{tabular}


Average of 12.914 to $12.933 \mathrm{~min}$ : LE9OBFB.D

50 ng BFB

$\mathrm{m} / \mathrm{z}$ added subtracted scaled

37.00

38.05

39.05

40.05

41.10

43.10

44.05

45.05

49.05

$\mathrm{m} / \mathbf{z}$

$476 \quad 55.10$

$401 \quad 56.10$

$228 \quad 57.10$

$19 \quad 58.00$

$67 \quad 61.00$

$65 \quad 62.00$

$57 \quad 63.00$

$138 \quad 68.05$

$291 \quad 69.05$

50.05

1936

71.05

51.05

$673 \quad 72.05$

abund. $\mathrm{m} / \mathrm{z}$

146

43

223

54

350

411

184

1170

1118

164

134
73.05

74.05

75.05

76.00

78.95

80.95

83.00

84.10

85.10

87.00

88.00 abund. 984

1740

5561

365

242

173

120

243

217

765

473

$\mathrm{m} / \mathrm{z}$
92.05
93.05
94.00
95.05
96.00
99.05
173.95
174.95
175.95
176.95

abund.

256

398

1316

12347

944

143

8931

665

8913

514 


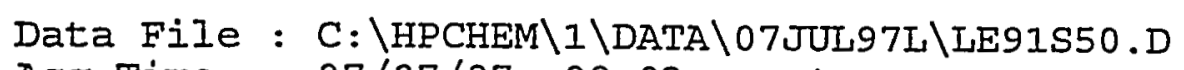

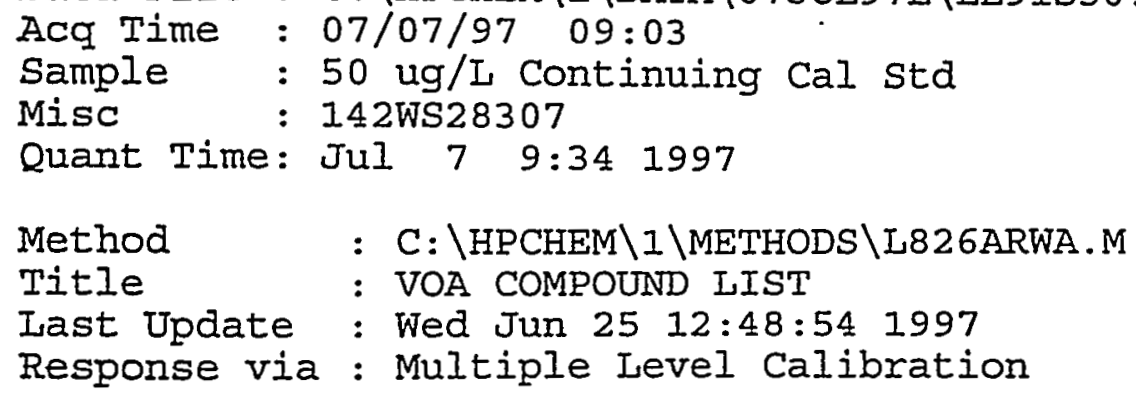

Method : C: $\backslash$ HPCHEM IIMETHODS \L826ARWA.M

Title : VOA COMPOUND LIST

Last Update : Wed Jun 25 12:48:54 1997

Response via : Multiple Level Calibration

Operator: CQC

Inst : 5971-L

Multiplr: 1.00

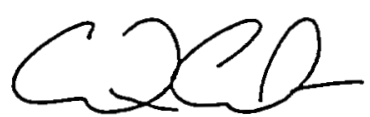

7797

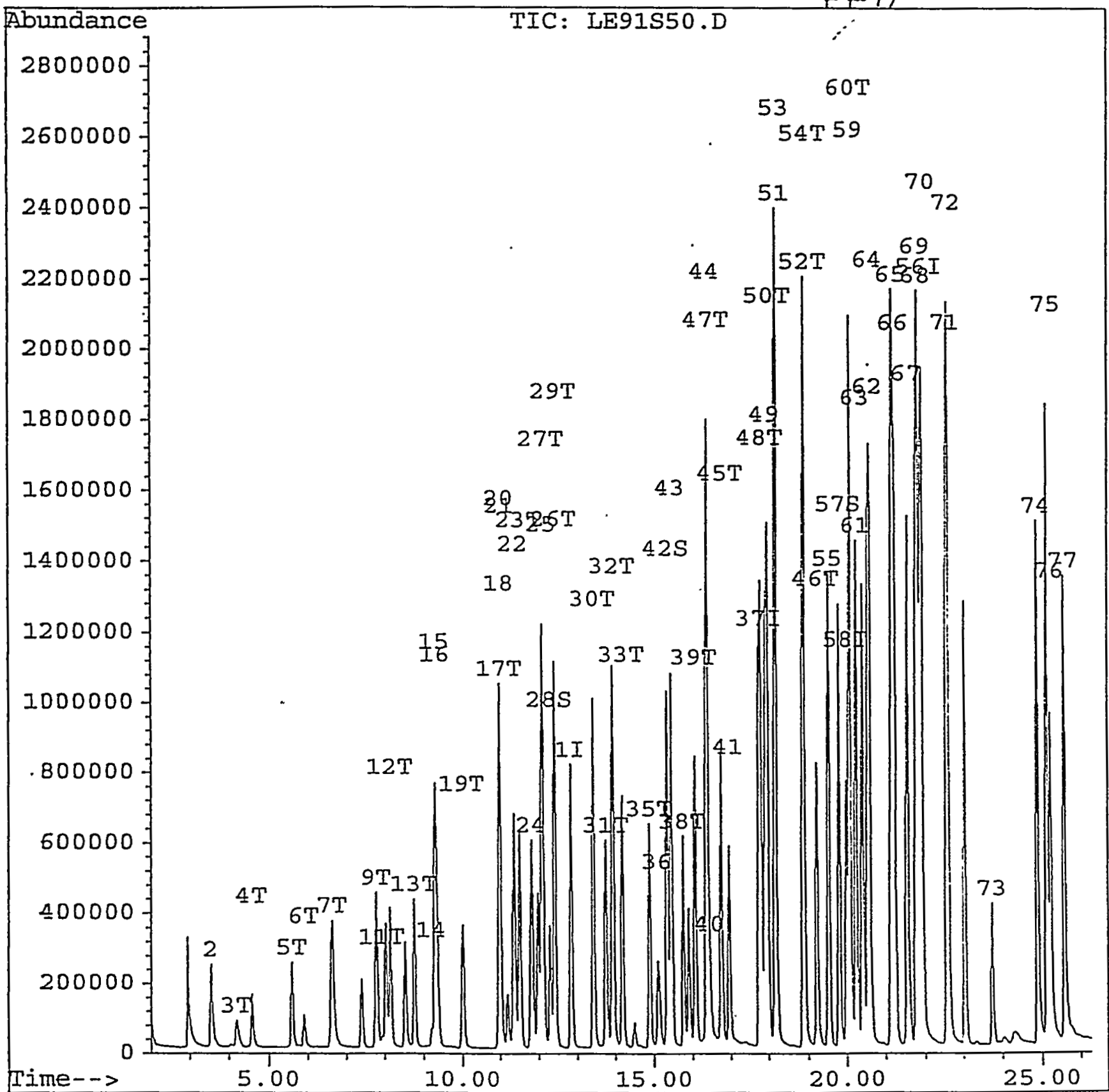

206 
Data File : C: \HPCHEM \1\DATA $\backslash 07 \pi U L 97 L \backslash L E 91550 . D$

Acq Time : 07/07/97 09:03

Sample : $50 \mathrm{ug} / \mathrm{L}$ Continuing Cal std

Misc : 142WS28307

Quant Time: Jul 7 9:34 1997

Operator: $\mathrm{CQC}$

Inst : 5971-L

Multiplr: 1.00

Method : C: \HPCHEM \I\METHODS $\backslash$ L826ARWA.M

Title : VOA COMPOUND LIST

Last Update : Wed Jun 25 12:48:54 1997

Response via : Multiple Level Calibration

Internal standards

1) CIOI Flourobenzene

37) CIO2 Chlorobenżene-d5

56) CIO3 1,4-Dichlorobenzene-d
R.T. QIon Response

$\begin{array}{rrr}12.83 & 96 & 1710261 \\ 17.75 & 117 & 1311956 \\ 21.88 & 152 & 984444 \\ & & \\ 12.28 & 65 & 505390 \\ 15.35 & 98 & 1596272 \\ 19.79 & 95 & 1116588\end{array}$

12.83

17.75
System Monitoring Compounds

28) CS15 1,2-dichloroethane-d4

42) Cso5 toluene-d8

57) CS10 4-bromofluorobenzene
Target Compounds.

2) $\mathrm{C002}$ dichlorodifluorometha

3) $\mathrm{CO03} \cdot \mathrm{chloromethane}$

4) $\mathrm{COO4}$ vinyl chloride

5) C005 bromomethane

6) 0006 chloroethane

7) $\mathrm{C007}$ trichlorofluoromethan

B) $\operatorname{C071}$ acrolein

9) $\operatorname{co0} 8$ 1,1-dichloroethene

10) $\mathrm{C} 063$ Ereon 113

11) co09 acetone

12) C010 carbon disulfide

13) C011 methylene chloride

14) $\mathrm{C} 072$ acrylonitrile

15) $\mathrm{CO12}$ trans-1,2-Dichloroeth

16) $C 305$ methyl-t-butyl-ether

17) $\mathrm{C} 013$ cis-1,2-dichloroethen

18) C055 total-I,2-dichloroeth

19) C014 1,1-dichloroethane

20) $\operatorname{Co15} 2,2$-dichloropropane

21) C110 2-butanone

22) CIOI bromochloromethane

23) $\mathrm{C} 016 \mathrm{chloroform}$

24) $\operatorname{Col7~1,1,1-Trichloroethane~}$

25) C018 1,1-dichloropropene

26) $\operatorname{Co19} 1,2$-dichloroethane

27) C020 carbon tetrachloride

29) CO21 benzene

30) C022 trichloroethene

31) C023 1,2-dichloropropane

32) C024 dibromomethane

33) C025 bromodichloromethane

34) C026 2-chloroethylvinyleth

35) $\mathrm{C} 27$ cis-1,3-dichloroprope
$3.54 \quad 85 \quad 640068$

$4.19 \quad .50$

4.58

5.59

5.90

6.63

0.00

7.78

0.00

7.92

8.14

8.75

9.19

9.27

9.32

10.98

10.98

10.00

10.98

11.01

11.37

11.50

11.81

12.07

12.40

12.09

12.40

13.43

13.76

13.94

14.18

0.00

14.90
52

94

64

101

56

96

151

43

76

84

53

96

73

96

96

63

77

72

128

83

97

75

62

117

78

130

63

93

83

63

75
275981

362031

365707

206426

1061982

482149

77896

1185150

464679

87629

540341

1177645

574259

1114207

823603

862663

51166

390117

1033675

783711

588986

938380

1526579

821365

534662

546836

1065895

892596
1003924
Conc Units

Area:

$\begin{array}{ll}50.00 \mathrm{ug} / \mathrm{L} & 0.00 \\ 50.00 \mathrm{ug} / \mathrm{L} & 0.00 \\ 50.00 \mathrm{ug} / \mathrm{L} & 0.00\end{array}$

$(\mathrm{NR})=$ Not Reported $(\mathrm{SP})=$ Spiked analyte $(\mathrm{m})=$ manual integration

(*) Does not meet EPA spectral criteria (False Hit) PX125 1

$\%$ Recovery

$45.72 \mathrm{ug} / \mathrm{L} \quad 91.43 \%$

$47.30 \mathrm{ug} / \mathrm{L} \quad 94.60 \%$

$42.44 \mathrm{ug} / \mathrm{L}$

$84.89 \%$

Qvalue

$48.09 \mathrm{ug} / \mathrm{L} \quad 99$

$48.39 \mathrm{ug} / \mathrm{L} \quad 99$

$42.07 \mathrm{ug} / \mathrm{I} \quad 98$

$40.96 \mathrm{ug} / \mathrm{L} \quad 99$

$41.37 \mathrm{ug} / \mathrm{L} \quad 95$

$51.05 \mathrm{ug} / \mathrm{L} \# \quad 98$

Not Detected

$48.55 \mathrm{ug} / \mathrm{L} \#$

Not Detected

$38.47 \mathrm{ug} / \mathrm{I} \#$

79

$45.02 \mathrm{ug} / \mathrm{L}$

$45.55 \mathrm{ug} / \mathrm{L}$ \#

$34.42 \mathrm{ug} / \mathrm{L}$

$48.71 \mathrm{ug} / \mathrm{L} \#$

$45.12 \mathrm{ug} / \mathrm{L} \#$

$48.76 . \mathrm{ug} / \mathrm{L} \#$

$97.49 \mathrm{ug} / \mathrm{L} \mathrm{m}$ zex 88

$2.82 \mathrm{ug} / \mathrm{I}$ \# 95

$47.05 \mathrm{ug} / \mathrm{L} \# \quad 69$

$46.60 \mathrm{ug} / \mathrm{L} \# \quad 1$

$54.30 \mathrm{ug} / \mathrm{工} \# 52$

$48.44 \mathrm{ug} / \mathrm{L} \# \quad 93$

$50.30 \mathrm{ug} / \mathrm{I} \quad 100$

$46.09 \mathrm{ug} / \mathrm{I} \# \quad 75$

$45.11 \mathrm{ug} / \mathrm{I}$ \# 97

$52.06 \mathrm{ug} / \mathrm{L} \quad 97$

$44.53 \mathrm{ug} / \mathrm{I} \# \quad 85$

$53.36 \mathrm{ug} / \mathrm{I} \# \quad 84$

$40.80 \mathrm{ug} / \mathrm{I} \quad 95$

$49.07 \mathrm{ug} / \mathrm{L} \# 73$.

$48.07 \mathrm{ug} / \mathrm{L} \# \quad 99$

Not Detected

$45.84 \mathrm{ug} / \mathrm{I}$ \# 90

\section{3} 6 9 4

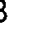

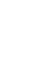

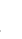
3 5 , . s. 9 69

9

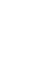


Data File : C:\HPCHEM \I\DATA 07JUL97L\LE91S50.D

Acq Time : 07/07/97 09:03

Sample : $50 \mathrm{ug} / \mathrm{L}$ Continuing Cal std

Misc : 142WS28307

Operator: CQC

Quant Time: Jul 7 9:34 1997

Inst : 5971-L

Nultiplr: 1.00

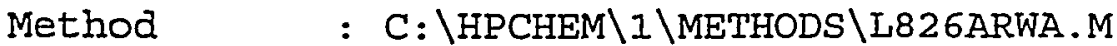

Title : VOA COMPOUND IIST

Last Update : Wed Jun 25 12:48:54 1997

Response via : Multiple Level Calibration

Compound
R.T. QIon Response Conc Unit Qvalue

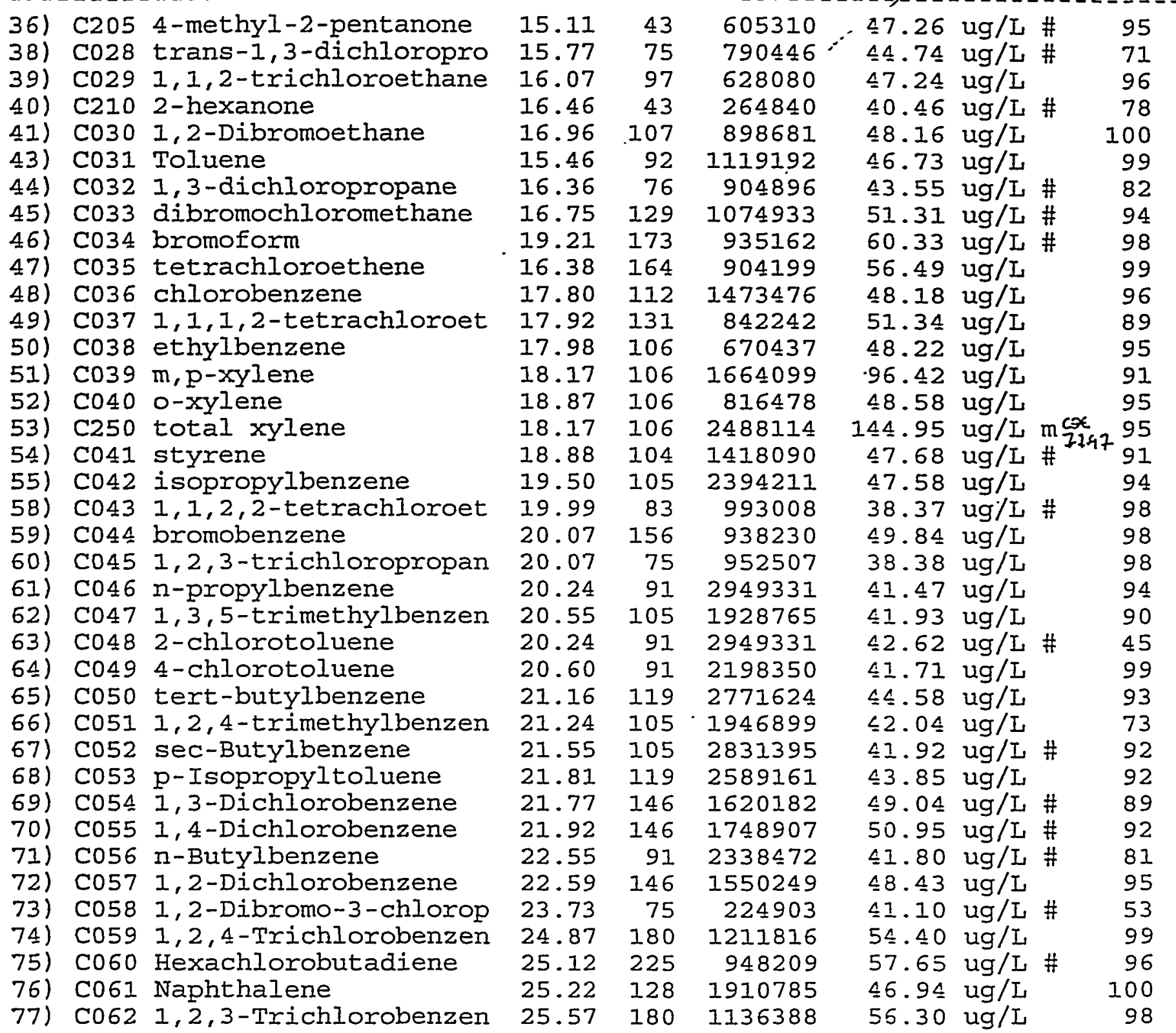


Data File : C: \HPCHEM \I\DATA $107 J U L 97 L \backslash L E 91550 . D$

Acq Time : 07/07/97 09:03

Sample : $50 \mathrm{ug} / \mathrm{L}$ Continuing Cal std

Misc : I42WS28307

Quant Time: Jul 7 9:30 1997

Operator: CQC Inst : 5971-L Multiplr: 1.00

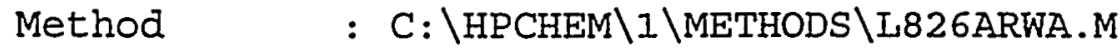

Title : VOA COMPOUND LIST

Last Update : Wed Jun 25 12:48:54 1997

Response via : Multiple Level Calibration

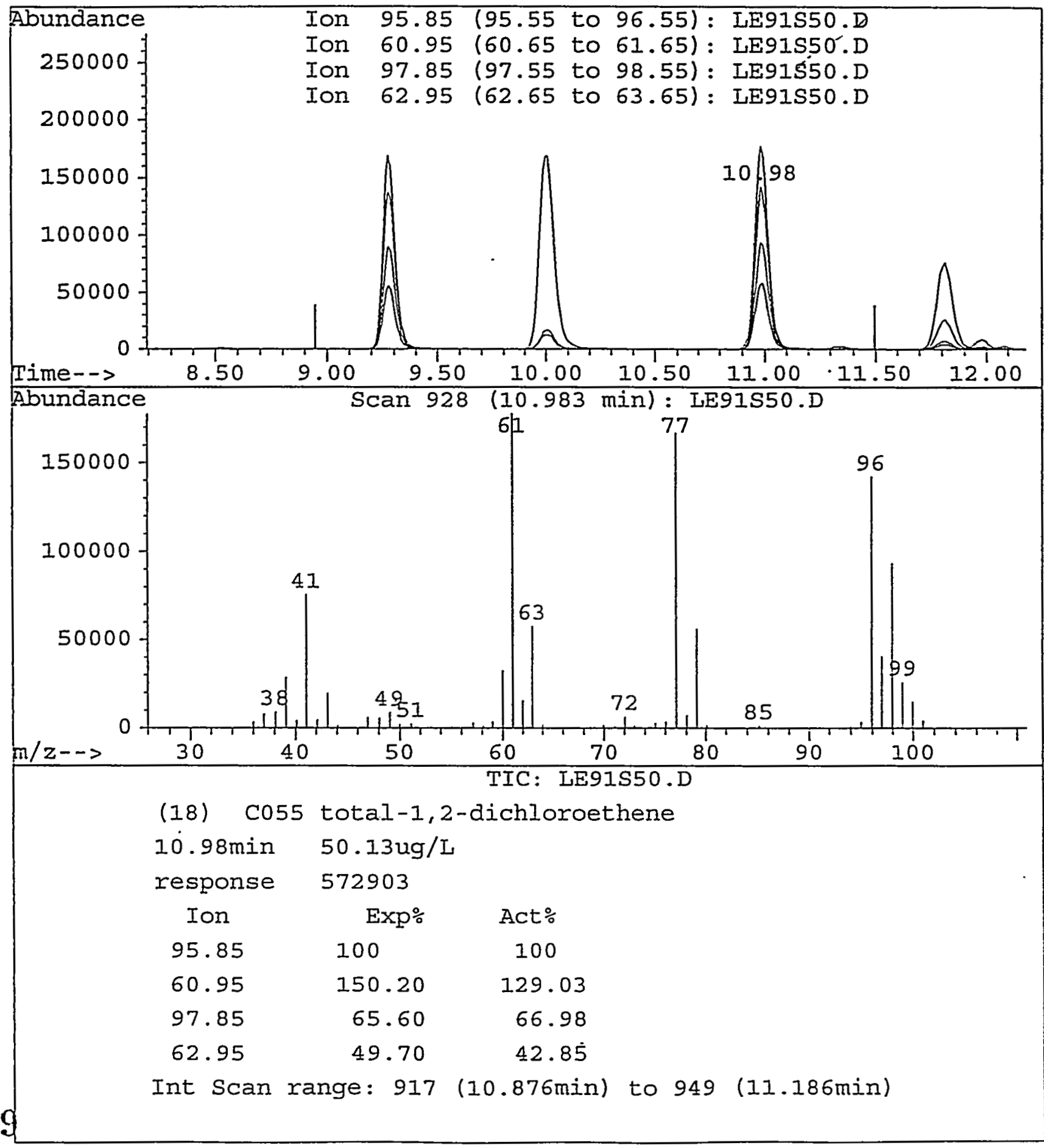




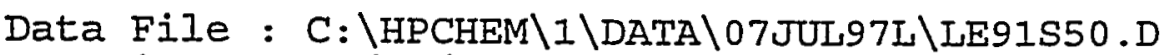

Acq Time : 07/07/97 09:03

Sample : $50 \mathrm{ug} / \mathrm{I}$ Continuing Cal std

Misc : 142WS28307

Operator: CQC

Quant Time: JuI 7 9:33 1997

Inst : 5971-I

Multiplr: 1.00

Method : C: \HPCHEM \I\METHODS \L826ARWA.M

Title : VOA COMPOUND LIST

Last Update : Wed Jun 25 12:48:54 1997

Response via : Multiple Level Calibration

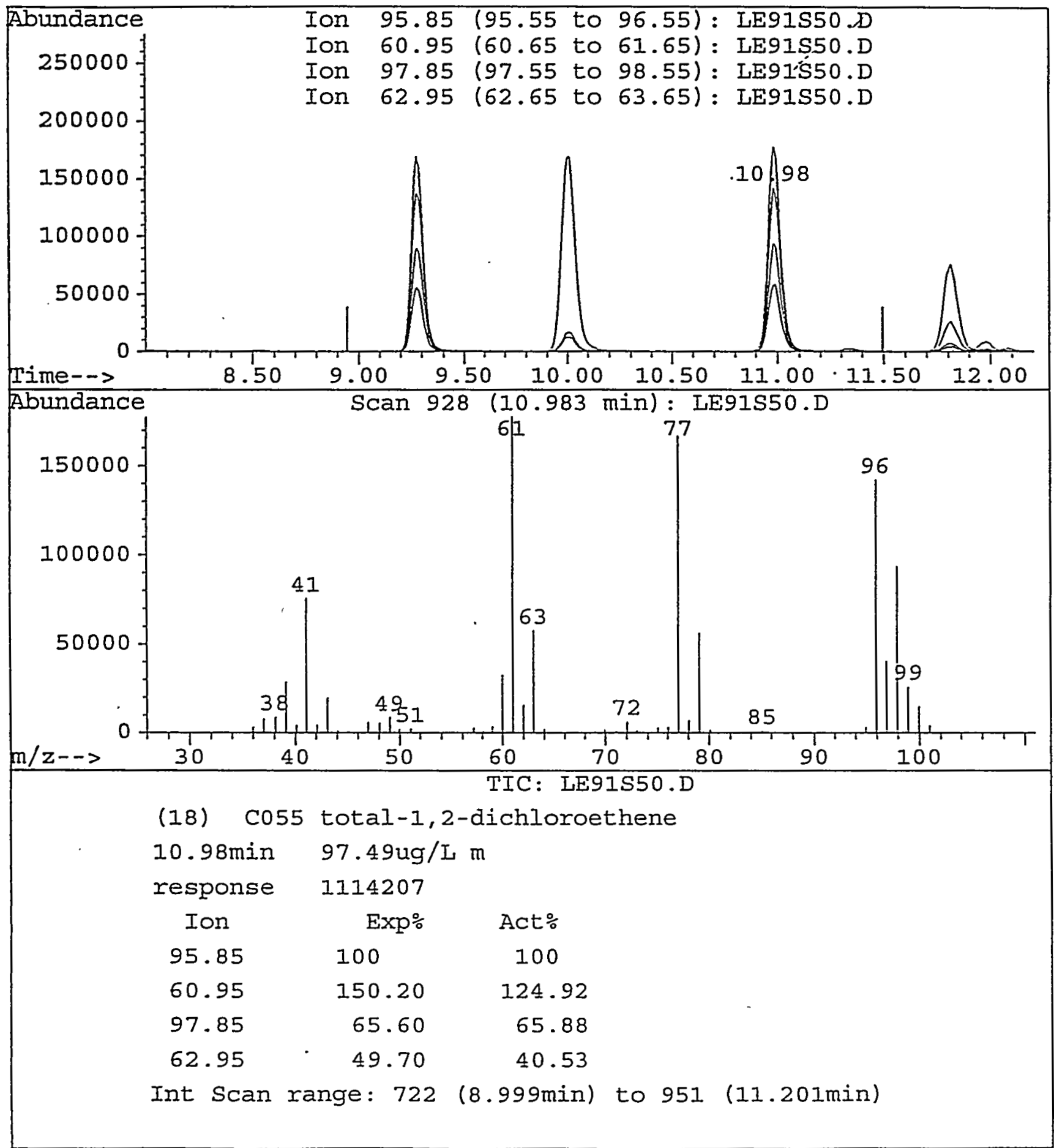

210 


\section{Quantitation Report}

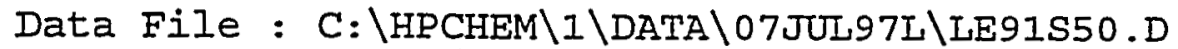

Acq Time : 07/07/97 09:03

Sample : $50 \mathrm{ug} / \mathrm{I}$ Continuing Cal std

Misc : 142WS28307

Quant Time: Jul 7 9:33 1997

Operator: CQC Inst : 5971-L Multiplr: 1.00

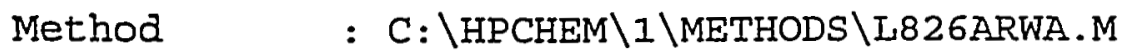

Title : VOA COMPOUND LIST

Iast Update : Wed Jun 25 12:48:54 1997

Response via : Multiple Level Calibration

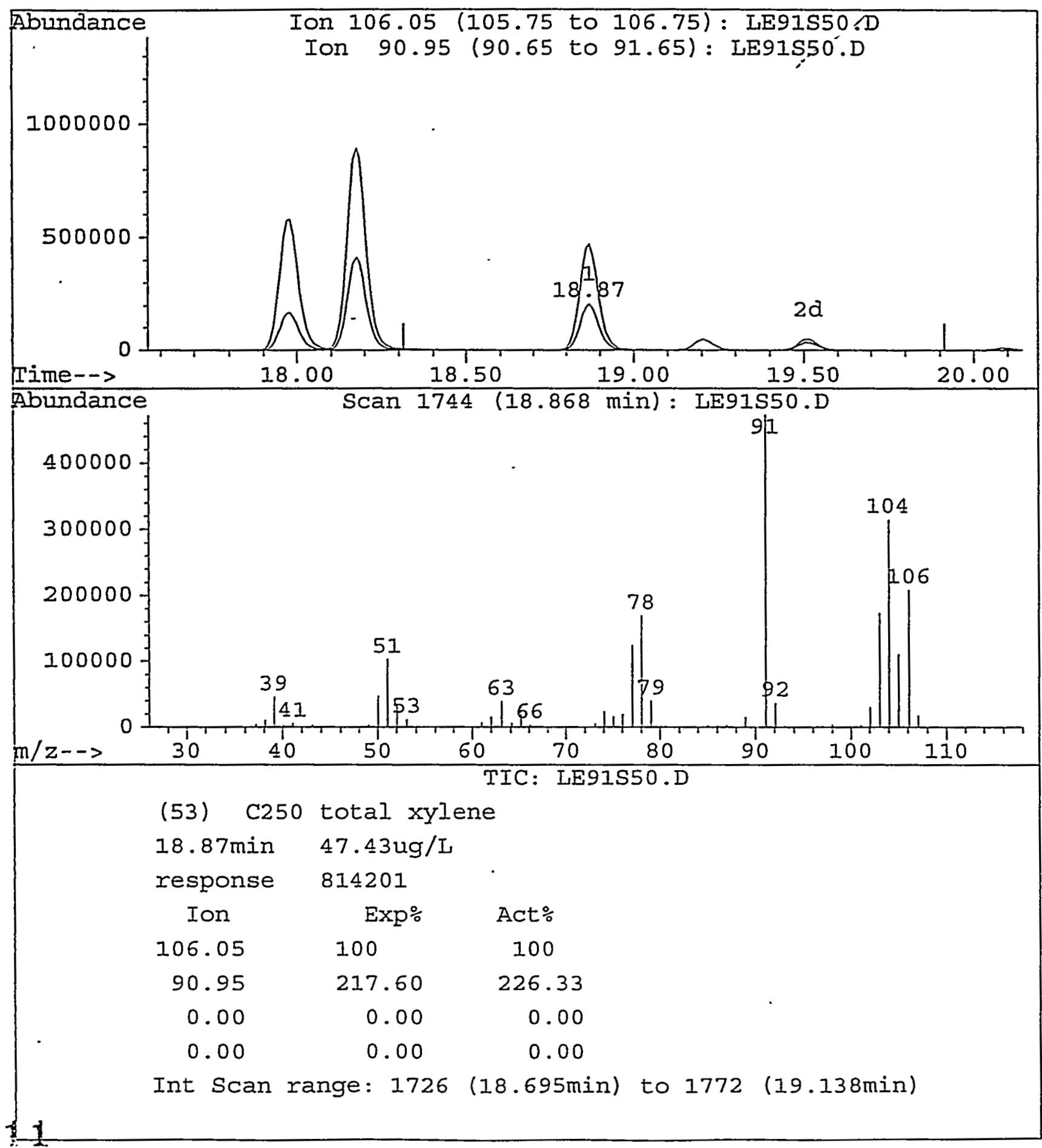


Quantitation Report

Data File : C: $\backslash$ HPCHEM \I\DATA $07 \pi U L 97 L \backslash L E 91550 . D$

Acq Time : 07/07/97 09:03

Sample : $50 \mathrm{ug} / \mathrm{I}$ Continuing Cal std

Misc : 142WS28307

Quant Time: Jul 7 9:34 1997

Operator: $\mathrm{CQC}$

Inst : 5971-I

Multiplr: 1.00

Method : C: \HPCHEM I \METHODS $\backslash$ I.826ARWA.M

Title : VOA COMPOUND LIST

Last Update : Wed Jun 25 12:48:54 1997

Response via : Multiple Level Calibration

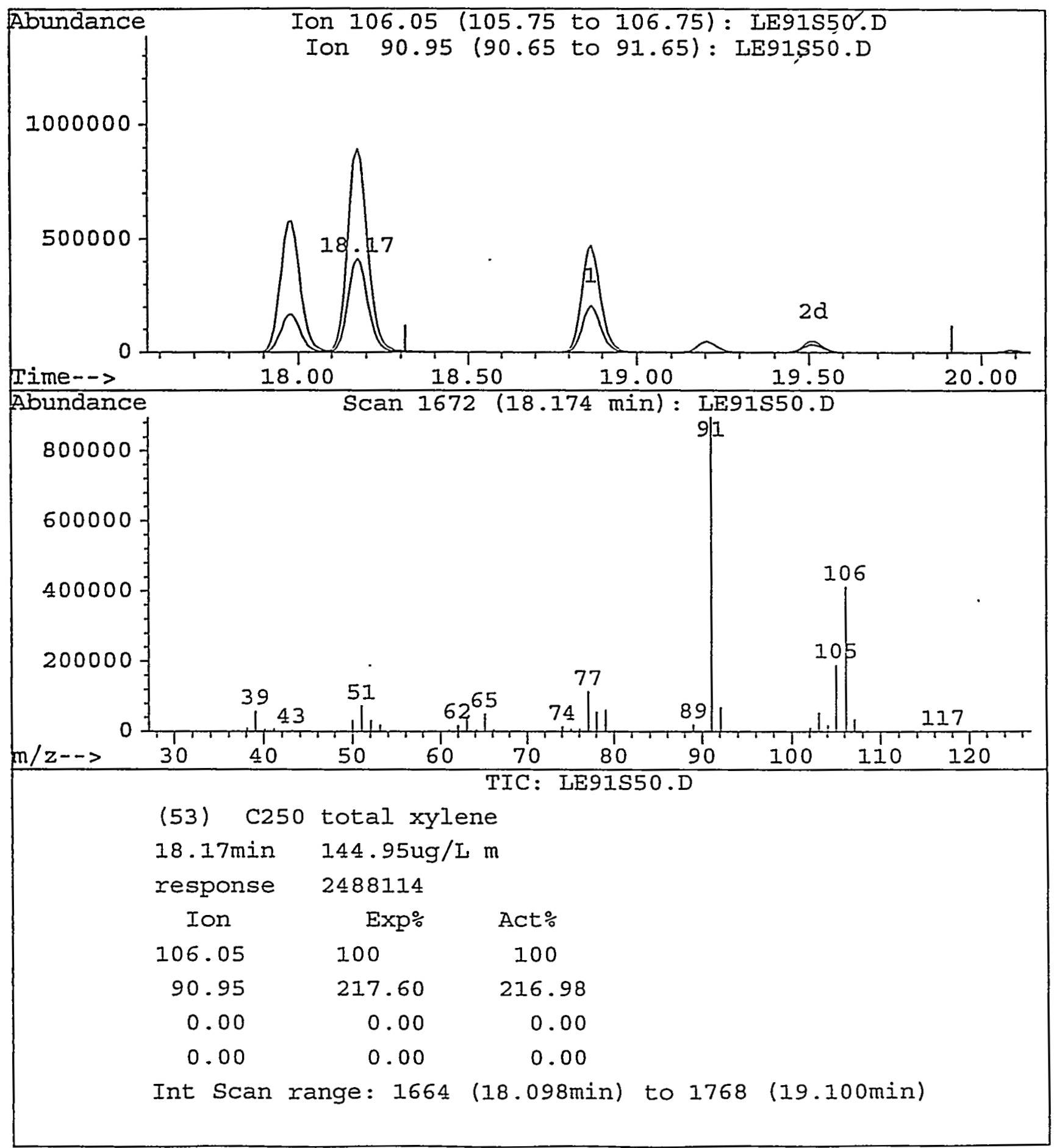

$2 \stackrel{2}{2}$

LE91S50.D L826ARWA.M Mon Jul 07 09:34:27 1997 


\section{A CONTINUING CALIBRATION REPORT}

SW-846, 8260A, Revision 1

Continuing Calibration File: LF15S50.D

$07 / 08 / 97$

Initial Calibration Method: $\quad$ L826ARWA

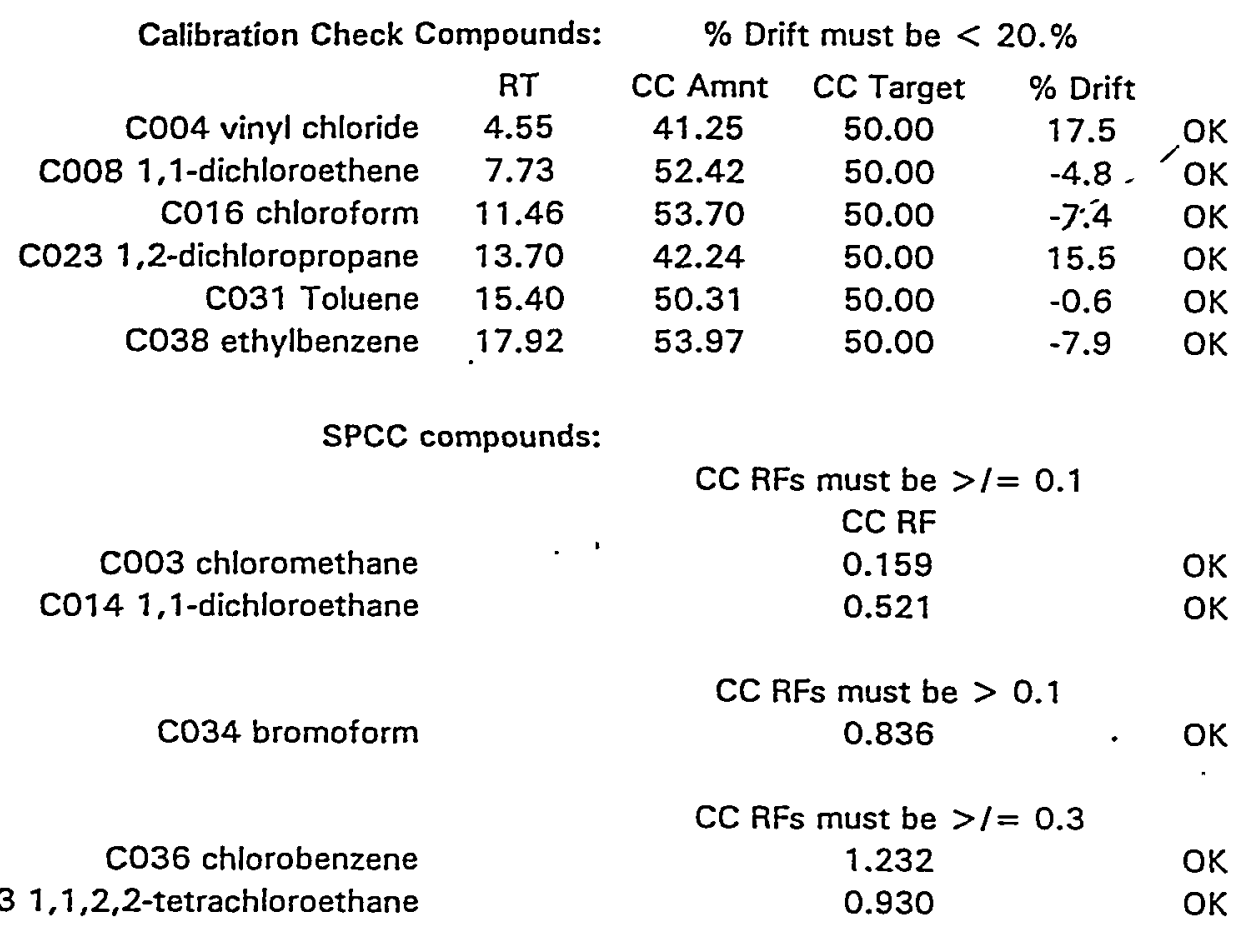




\section{A CONTINUING CALIBRATION REPORT (cont'd) SW-846, 8260A, Revision 1 \\ Continuing Calibration File: LF15S50.D}

$07 / 08 / 97$

Initial Calibration Method:- L826ARWA

\begin{tabular}{|c|c|c|c|c|c|}
\hline Surrogate Compounds: & RT & CC Amnt & $\begin{array}{c}\text { CC Target } \\
\text { CC RF }\end{array}$ & $\%$ Drift & \\
\hline CS15 1,2-dichloroethane-d4 & 12.23 & 49.47 & 50.00 & 1.1 & \\
\hline $\operatorname{cs} 05$ toluene-d8 & 15.30 & 49.52 & 50.00 & 1.0 & \\
\hline CS10 4-bromofluorobenzene & 19.72 & 43.54 & 50.00 & 12.9 & , \\
\hline Target Compounds: & RT & CC Amnt & CC Target & $\%$ Drift & \\
\hline COO2 dichlorodifluoromethane & 3.51 & 46.98 & 50.00 & 6.0 & \\
\hline C003 chloromethane & 4.15 & 47.65 & 50.00 & 4.7 & \\
\hline C004 vinyl chloride & 4.55 & 41.25 & 50.00 & 17.5 & \\
\hline C005 bromomethane & 5.55 & 45.54 & 50.00 & 8.9 & \\
\hline C006 chloroethane & 5.87 & 45.97 & 50.00 & 8.1 & \\
\hline $\mathrm{COO} 7$ trichlorofluoromethane & 6.58 & 59.69 & 50.00 & -19.4 & \\
\hline C008 1,1-dichloroethene & $7.73^{-}$ & 52.42 & 50.00 & -4.8 & \\
\hline C009 acetone & 7.88 & 37.18 & 50.00 & 25.6 & warning \\
\hline C010 carbon disulfide & 8.10 & 44.42 & 50.00 & 11.2 & \\
\hline C011 methylene chloride & 8.71 & 46.09 & 50.00 & 7.8 & \\
\hline C072 acrylonitrile & 9.15 & 37.23 & 50.00 & 25.5 & warning \\
\hline C012 trans-1,2-Dichloroethene & 9.24 & 52.52 & 50.00 & -5.0 & \\
\hline C305 methyl-t-butyl-ether & 9.29 & 45.43 & 50.00 & 9.1 & \\
\hline C013 cis-1,2-dichloroethene & 10.95 & 52.88 & 50.00 & -5.8 & \\
\hline C055 total-1,2-dichloroethene & 10.95 & 105.46 & 100.00 & -5.5 & \\
\hline C014 1,1-dichloroethane & 9.96 & 46.34 & 50.00 & 7.3 & \\
\hline C015 2,2-dichloropropane & 10.94 & 52.42 & 50.00 & -4.8 & \\
\hline C110 2-butanone & 10.97 & 38.30 & 50.00 & 23.4 & warning \\
\hline Cl01 bromochloromethane & 11.32 & 56.42 & 50.00 & -12.8 & \\
\hline C016 chloroform & 11.46 & 53.70 & 50.00 & -7.4 & \\
\hline C017 1,1,1-Trichloroethane & 11.77 & 59.13 & 50.00 & -18.3 & \\
\hline C018 1.1-dichloropropene & 12.03 & 48.67 & 50.00 & 2.7 & \\
\hline C019 1,2-dichloroethane & 12.35 & 50.92 & 50.00 & -1.8 & \\
\hline C020 carbon tetrachloride & 12.05 & 61.72 & $50.00^{\circ}$ & -23.4 & warning \\
\hline C021 benzene & 12.35 & 45.28 & 50.00 & 9.4 & \\
\hline C022 trichloroethene & 13.37 & 56.18 & 50.00 & -12.4 & \\
\hline C023 1.2-dichloropropane & 13.70 & 42.24 & 50.00 & 15.5 & \\
\hline C024 dibromomethane & 13.89 & 52.73 & 50.00 & -5.5 & \\
\hline C025 bromodichloromethane & 14.13 & 53.68 & 50.00 & -7.4 & \\
\hline C027 cis-1,3-dichloropropene & 14.84 & 46.77 & 50.00 & 6.5 & \\
\hline C205 4-methyl-2-pentanone & 15.06 & 41.00 & 50.00 & 18.0 & \\
\hline C028 trans-1,3-dichloropropene & 15.71 & 47.42 & 50.00 & 5.2 & \\
\hline C029 1,1,2-trichloroethane & 16.02 & 49.36 & 50.00 & 1.3 & \\
\hline C210 2-hexanone & 16.41 & 34.47 & 50.00 & 31.1 & warning \\
\hline C030 1,2-Dibromoethane & 16.90 & 52.00 & 50.00 & -4.0 & \\
\hline C031 Toluene & 15.40 & 50.31 & 50.00 & -0.6 & \\
\hline C032 1,3-dichloropropane & 16.30 & 43.48 & 50.00 & 13.0 & \\
\hline C033 dibromochloromethane & 16.70 & 56.20 & 50.00 & -12.4 & \\
\hline c034 bromoform & 19.15 & 70.79 & 50.00 & -41.6 & warning \\
\hline
\end{tabular}

214 
8260A CONTINUING CALIBRATION REPORT (cont'd)

SW-846, 8260A, Revision 1

Continuing Calibration File: LF15S50.D

07/08/97

Initial Calibration Method:

L826ARWA

Target Compounds (cont'd):

C035 tetrachloroethene

C036 chlorobenzene

$\begin{array}{ccccc}\text { RT } & \text { CC Amnt } & \text { CC Target } & \text { \% Drift } & \\ 16.33 & 68.77 & 50.00 & -37.5 & \text { warning } \\ 17.74 & 52.84 & 50.00 & -5.7 & \\ 17.87 & 54.64 & 50.00 & -9.3 & \\ 17.92 & 53.97 & 50.00 & -7.9 & \\ 18.12 & 109.12 & 100.00 & -9.1 & \\ 18.82 & 54.36 & 50.00 & -8.7 & \\ 18.12 & 164.41 & 150.00 & -9.6 & \\ 18.83 & 53.33 & 50.00 & -6.7 & \\ 19.45 & 55.49 & 50.00 & -11.0 & \\ 19.93 & 35.39 & 50.00 & 29.2 & \\ 20.02 & 56.02 & 50.00 & -12.0 & \\ 20.03 & 35.43 & 50.00 & 29.1 & \text { warning } \\ 20.19 & 44.10 & 50.00 & 11.8 & \\ 20.50 & 47.96 & 50.00 & 4.1 & \\ 20.19 & 45.33 & 50.00 & 9.3 & \\ 20.55 & 44.58 & 50.00 & 10.8 & \\ 21.10 & 47.53 & 50.00 & 4.9 & \\ 21.19 & 47.69 & 50.00 & 4.6 . & \\ 21.50 & 46.78 & 50.00 & 6.4 & \\ 21.76 & 46.84 & 50.00 & 6.3 & \\ 21.71 & 50.98 & 50.00 & -2.0 & \\ 21.88 & 52.65 & 50.00 & -5.3 & \\ 22.51 & 44.12 & 50.00 & 11.8 & \\ 22.55 & 49.76 & 50.00 & 0.5 & \\ 23.69 & 39.49 & 50.00 & 21.0 & \text { warning } \\ 24.84 & 64.65 & 50.00 & -29.3 & \text { warning } \\ 25.08 & 71.00 & 50.00 & -42.0 & \text { warning } \\ 25.18 & 42.92 & 50.00 & 14.2 & \\ 25.53 & 64.22 & 50.00 & -28.4 & \text { warning }\end{array}$

C037 1,1,1,2-tetrachloroethane C038 ethylbenzene C039 m,p-xylene CO40 o-xylene C250 total xylene C041 styrene C042 isopropylbenzene

C043 1,1,2,2-tetrachloroethane C044 bromobenzene

C045 1,2,3-trichloropropane C046 n-propylbenzene

C047 1,3,5-trimethylbenzene C048 2-chlorotoluene C049 4-chlorotoluene C050 tert-butylbenzene

C051 1,2,4-trimethylbenzene C052 sec-Butylbenzene C053 p-Isopropyltoluene C054 1,3-Dichlorobenzene C055 1,4-Dichlorobenzene C056 n-Butylbenzene C057 1,2-Dichlorobenzene C058 1,2-Dibromo-3-chloropropane Co59 1,2,4-Trichlorobenzene C060 Hexachlorobutadiene C061 Naphthalene C062 1,2,3-Trichlorobenzene 
BFB

Data File : C: \HPCHEM \I\DATA $08 \pi U L 97 L \backslash L F 14 B F 3 . D$

Acq Time : 07/08/97 09:13

Sample : $50 \mathrm{ng}$ BFB

MisC $\quad: 2.0$ uL of $39 I S 7676$

Operator: CQC

Inst : 5971-I

Multiplr: 1.00

Method : C: \HPCHEM $\backslash I \backslash M E T H O D S \backslash B F B 8240 . M$

Title :

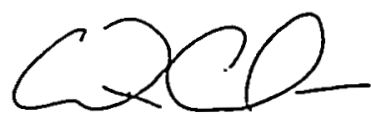

7.8 .97

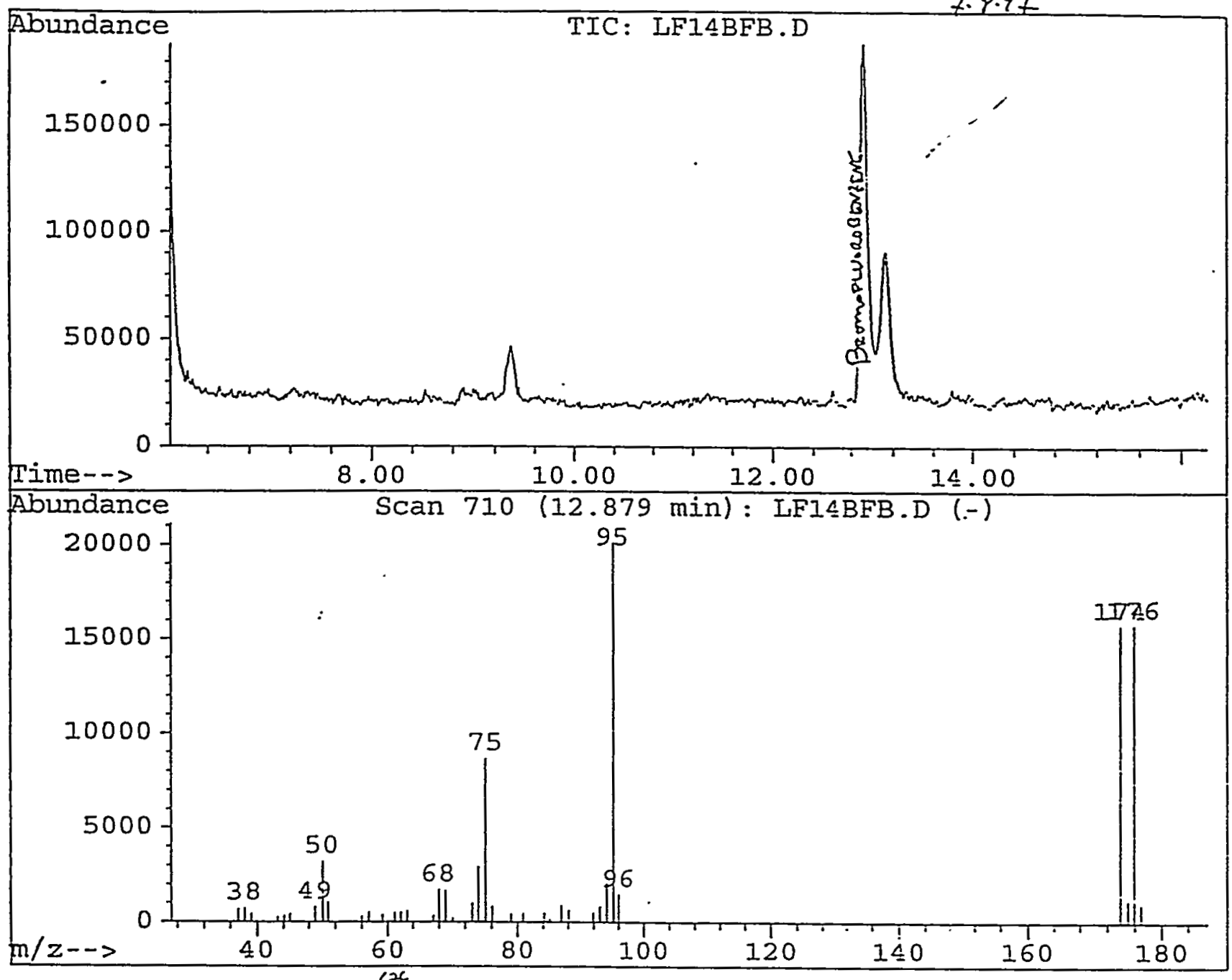

Peak Apex is scan: $\frac{7.947}{6.65} 713$

Scuataka: 710 Mims tackyround sean: 665

\begin{tabular}{c|c|l|l|l|l|l|l} 
Target & Rel. to & Lower & Upper & Rel. & Raw & Result \\
Mass & Mass & Limit\% & Limit\% & Abn\% & Ab: & Pass/Fail
\end{tabular}

\begin{tabular}{|r|r|r|r|r|r|r|r|}
50 & 95 & 15 & 40 & 16.09 & $32 \leq 4$ & PASS \\
75 & 95 & 30 & 60 & 43.09 & 8536 & PASS \\
95 & 95 & 100 & 100 & 100.00 & $20 \pm 50$ & PASS \\
96 & 95 & 5 & 9 & 7.33 & $1 \leq 77$ & PASS \\
173 & 174 & 0 & 2 & 0.00 & 0 & PASS \\
174 & 95 & 50 & 100 & 78.21 & -15758 & PASS \\
175 & 174 & 5 & 9 & 7.61 & 1200 & PASS \\
176 & 174 & 95 & 101 & 100.27 & 15811 & PASS \\
177 & 176 & 5 & 9 & 6.29 & 995 & PASS
\end{tabular}

216

50 ng BFB 5971-L 07/08/97 09:13 PX125 
Scan 710 (12.879 min): LF14BFB.D $50 \mathrm{ng} \mathrm{BFB}$

Modified: subtracted

$\mathrm{m} / \mathrm{z}$ abund.

37.15

38.15

39.10

40.05

42.10

43.10

44.05

45.00

49.00

50.10

51.00 $\mathrm{m} / \mathrm{z}$

687

735

436

60

11

284

337

459

812

3244

1066
55.05

56.05

57.10

59.15

60.05

61.05

62.05

63.05

67.10

68.00

69.00 abund. 93

329

529

423

80

518

535

597

365

1732

1703 $\mathrm{m} / \mathrm{z}$

70.10

73.10

74.00

75.10

76.10

78.95

80.85

84.25

85.10

86.95

88.05 abund. $\mathrm{m} / \mathrm{z}$ abund.

$230 \quad 91.90$

$1010 \quad 93.00$

$2977 \quad 94.10$

$8686 \quad 95.10$

$847 \quad 96.00$

$456 \quad 173.95$

$468 \quad 175.05$

$511 \quad 175.95$

$192 \quad 176.95$

924

676
507

855

2110

20160

1477

15768

1200

15811

995 
Quantitation Report

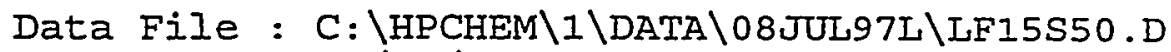

Acq Time : 07/08/97 10:55

Sample : $50 \mathrm{ug} / \mathrm{L}$ Continuing Cal std

Operator: $\mathrm{CQC}$

MisC : 142WS28308

Quant Time: Jul 8 12:28 1997

Inst : 5971-L

Multiplr: 1.00

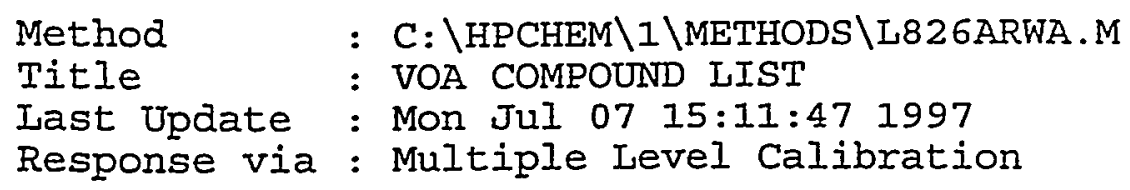

Method

Title

: C: $\backslash$ HPCHEM $\backslash 1 \backslash$ METHODS \L826ARWA.M

Last Update : Mon Jul 07 15:11:47 1997

Response via : Multiple Level Calibration

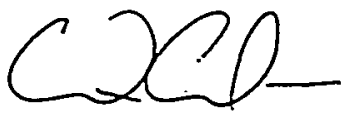

$7 \cdot 9 \cdot 92$

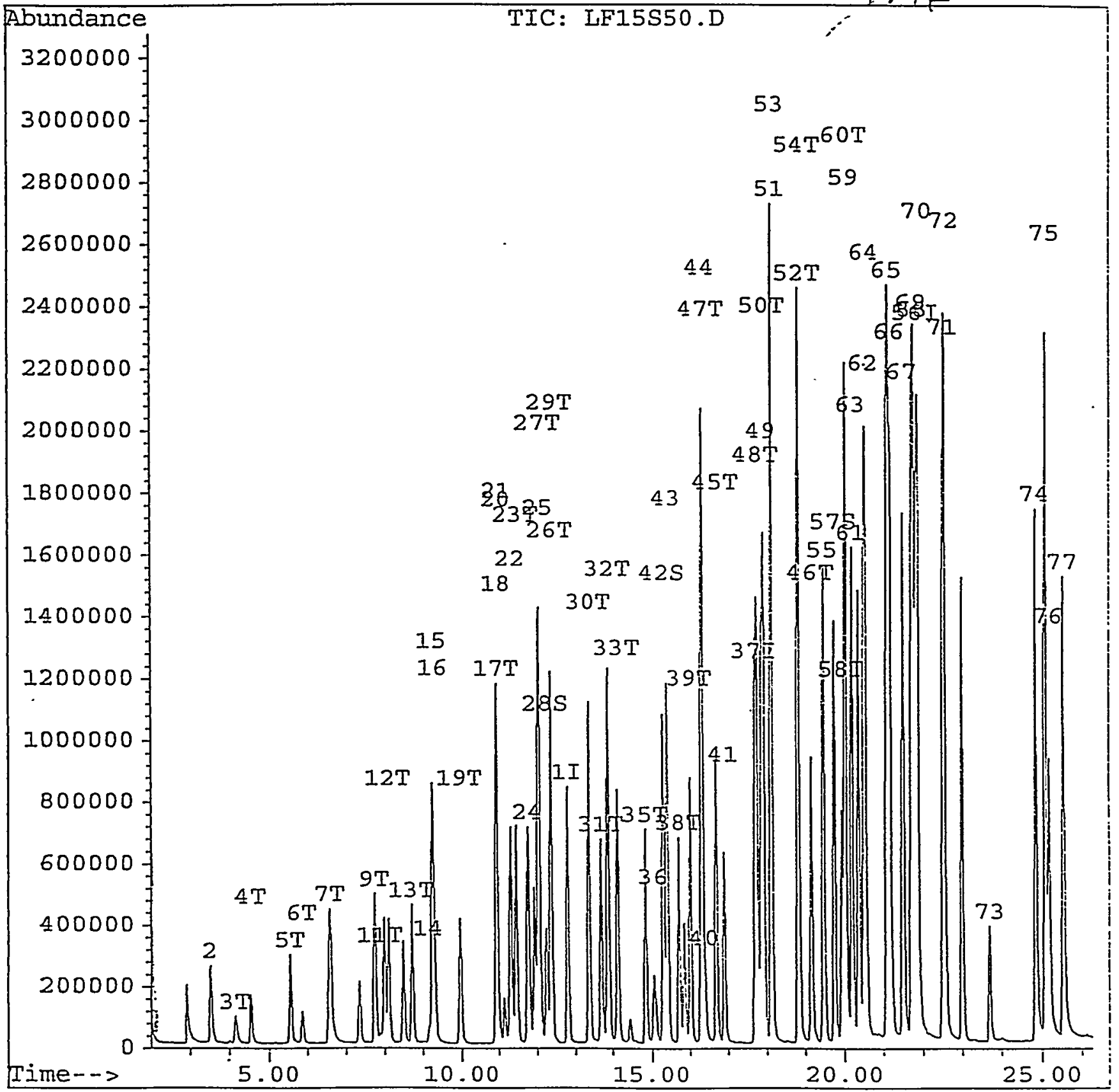




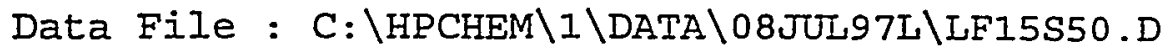

Acq Time : 07/08/97 10:55

Sample : $50 \mathrm{ug} / \mathrm{L}$ Continuing Cal std

Misc : 142 WS28308

Quant Time: Jul 8 12:28 1997

Operator: CQC

Inst : 5971-I

Multiplr: 1.00

Method : C: \HPCHEM \I\METHODS\L826ARWA.M

Title : VOA COMPOUND IIST

Iast Update : Mon JuI 07 15:11:47 1997

Response via : Multiple Level Calibration

Internal standards

1) CIOI Flourobenzene

37) CI02 Chlorobenzene-d5

56) CI03 1,4-Dichlorobenzene-d

System Monitoring Compounds

28) CS15 1,2-dichloroethane-d4

42) Cs05 toluene-d8

57) CSIO 4-bromofluorobenzene

Target Compounds

2) $\mathrm{C002}$ dichlorodifluorometha

3) $\mathrm{COO3}$ chloromethane

4) $\mathrm{C004}$ vinyl chloride

5) $\mathrm{C} 005$ bromomethane

6) $\mathrm{C} 006$ chloroethane

7) $\mathrm{C007}$ trichlorofluoromethan

8) 0071 acrolein

9) C008 1,1-dichloroethene

10) 0063 freon 113

11) 0009 acetone

12) C010 carbon disulfide

13) Col1 methylene chloride

14) C072 acrylonitrile

15) C012 trans-1,2-Dichloroeth

16) C305 methyl-t-butyl-ether

17) $\mathrm{CO13}$ cis-1,2-dichloroethen

18) C055 total-1,2-dichloroeth

19) C014 1,1-dichloroethane

20) Col5 2,2-dichloropropane

21) C110 2-butanone

22) CIOI bromochloromethane

23) $\mathrm{C} 016$ chloroform

24) C017 1,1,1-Trichloroethane

25) C018 1,1-dichloropropene

26) C019 1,2-dichloroethane

27) C020 carbon tetrachloride

29) 0221 benzene

30) C022 trichloroethene

31) C023 1,2-dichloropropane

32) 024 dibromomethane

33) C025 bromodichloromethane

34) C026 2-chloroethylvinyleth

35) C027 cis-1,3-dichloroprope

R.T. QIon

Response

12.78

96

1742843

17.69

117

1322844

1019150

21.83

152

12.23

15.30

19.72
$65 \quad 557352$

$98 \quad 1684963$

95

1185898
3.51

4.15

4.55

5.55

5.87

6.58

0.00

7.73

0.00

7.88

8.10

8.71

9.15

9.24

9.29

10.95

10.95

9.96

10.94

10.97

11.32

11.46

11.77

12.03

12.35

12.05

12.35

13.37

13.70

13.89

14.13

0.00

14.84

85
50
62
94
64
101
56
96
151
43
76
84
53
96
73
96
96
63
77
72
128
83
97
75
62
117
78
130
63
93
83
63
75

85

50

62

94

64

101

56

96

151

43

76

84

53

96

73

96

96

63

77

72

128

83

97

75

62

117

78

130

63

93

83

75
637188

276900

361808

414339

233748

1265340

530429

76709

1191547

479103

96576

593645

1208548

634572

1228235

908272

979419

42850

413060

1167810

1202655

843454

677523

1133746

1582054

881274

564070

598851

1212789

927925
Conc Units

Area:

$$
\begin{array}{ll}
50.00 \mathrm{ug} / \mathrm{L} & -0.04 \\
50.00 \mathrm{ug} / \mathrm{L} & -0.06 \\
50.00 \mathrm{ug} / \mathrm{L} & -0.04
\end{array}
$$

$\%$ Recovery

$49.47 \mathrm{ug} / \mathrm{L} \quad 98.95 \%$

$49.52 \mathrm{ug} / \mathrm{L} \quad 99.04 \%$

$43.54 \mathrm{ug} / \mathrm{L}$

$87.09 \%$

Qvalue

$46.98 \mathrm{ug} / \mathrm{L} \quad 99$

$47.65 \mathrm{ug} / \mathrm{L} \quad 99$

$41.25 \mathrm{ug} / \mathrm{L} \quad 98$

$.45 .54 \mathrm{ug} / \mathrm{L} \quad 98$

$45.97 \mathrm{ug} / \mathrm{L} \quad 96$

$59.69 \mathrm{ug} / \mathrm{L}$ \# 98

No Calib \#

$52.42 \mathrm{ug} / \mathrm{L} \#$

Not Detected

$37.18 \mathrm{ug} / \mathrm{L} \stackrel{\#}{\mathrm{t}} \quad 79$

$44.42 \mathrm{ug} / \mathrm{L} \quad 92$

$46.09 \mathrm{ug} / \mathrm{L} \# \quad 66$

$37.23 \mathrm{ug} / \mathrm{L} \quad 92$

$52.52 \mathrm{ug} / \mathrm{L} \# \quad 80$

$45.43 \mathrm{ug} / \mathrm{L}$ \# 87

$52.88 \mathrm{ug} / \mathrm{L} \# \quad 78$

$105.46 \mathrm{ug} / \mathrm{L} \mathrm{m}_{3 \times \times 47}^{\sec } 89$

$46.34 \mathrm{ug} / \mathrm{L} \# 95$

$52.42 \mathrm{ug} / \mathrm{L} \# \quad 74$

$38.30 \mathrm{ug} / \mathrm{L} \# \quad 10$

$56.42 \mathrm{ug} / \mathrm{L} \# \quad 52$

$53.70 \mathrm{ug} / \mathrm{L} \# \quad 95$

$59.13 \mathrm{ug} / \mathrm{L} \quad 98$

$48.67 \mathrm{ug} / \mathrm{I} \# \quad 79$

$50.92 \mathrm{ug} / \mathrm{L} \# \quad 98$

$61.72 \mathrm{ug} / \mathrm{L} \quad 98$

$45.28 \mathrm{ug} / \mathrm{L} \quad 95$

$56.18 \mathrm{ug} / \mathrm{L} \quad 87$

$42.24 \mathrm{ug} / \mathrm{L} \quad 97$

$52.73 \mathrm{ug} / \mathrm{L} \# \quad 66$

$53.68 \mathrm{ug} / \mathrm{L} \# \quad 99$

Not Detected

$46.77 \mathrm{ug} / \mathrm{L} \#$ 


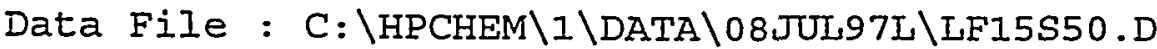

Acq Time : 07/08/97 10:55

Sample : $50 \mathrm{ug} / \mathrm{L}$ Continuing Cal std

Misc : 142 WS28308

Quant Time: Jul $8 \quad 12: 28 \quad 1997$

Operator: $\mathrm{CQC}$

Inst : 5971-L Multiplr: 1.00

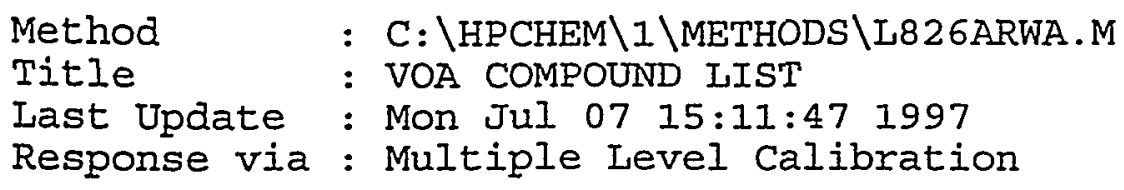

Compound

R.T. QIon Response Conc Unit Qvalue

36) C205 4-methyl-2-pentanone

38) C028 trans-1,3-dichloropro

39) C029 1,1,2-trichloroethane

40) C210 2-hexanone

41) C030 1,2-Dibromoethane

43) C031 Toluene

44) C032 1,3-dichloropropane

45) C033 dibromochloromethane

46) $\mathrm{C} 034$ bromoform

47) C035 tetrachloroethene

48) C036 chlorobenzene

49) $\operatorname{Co37} 1,1,1,2$-tetrachloroet

50) $\mathrm{C} 038$ ethylbenzene

51) C039 m,p-xylene

52) c040 o-xylene

53) C250 total xylene

54) C04I styrene

55) 0042 isopropylbenzene

58) $\mathrm{C} 043$ 1, 1,2,2-tetrachloroet

59) C044 bromobenzene

60) C045 1,2,3-trichloropropan

61) $\mathrm{C} 046 \mathrm{n}$-propylbenzene

62) 0047 1,3,5-trimethylbenzen

63) C048 2-chlorotoluene

64) C049 4-chlorotoluene

65) C050 tert-butylbenzene

66) C051 1,2,4-trimethylbenzen

67) C052 sec-Butylbenzene

68) C053 p-Isopropyltoluene

69) C054 1,3-Dichlorobenzene

70) C055 1,4-Dichlorobenzene

71) C056 n-Butylbenzene

72) $C 057$ 1,2-Dichlorobenzene

73) C058 1,2-Dibromo-3-chlorop

74) C059 1,2,4-Trichlorobenzen

75) C060 Hexachlorobutadiene

76) C061 Naphthalene

77) $\mathrm{C} 062$ 1,2,3-Trichlorobenzen
$15.06 \quad 43$

$15.71 \quad 75$

16.02

16.41

16.90

15.40

16.30

16.70

19.15

16.33

17.74

17.87

17.92

18.12

18.82

18.12

18.83

19.45

19.93

20.02

20.03

20.19

20.50

20.19

20.55

21.10

21. 19

21.50

21.76

21.71

21.88

22.51

22.55

23.69

24.84

25.08

25.18

25.53
97

43

107

92

76

129

173

164

112

131

106

106

106

106

104

105

83

156

75

91

105

91

91

119

105

105

119

146

146

91

146

75

180

225

128

180
535130

$844795^{\circ}$

661721

227492

978399

1214822

910862

1187159

1106331

1109876

1629312

903882

756612

1898811

921138

2845525

1599255

2815169

948234

1091633

910332

3247109

2284037

3247109

2432539

3059308

2286609

3270887

2862807

1743918

1871021

2555069

1648821

223760

1490887

1208838

1808580

1341850
$-41.00 \mathrm{ug} / \mathrm{L} \#$ $47.42 \mathrm{ug} / \mathrm{L} \#$ $49.36 \mathrm{ug} / \mathrm{L}$ $34.47 \mathrm{ug} / \mathrm{L} \#$ $52.00 \mathrm{ug} / \mathrm{L} \#$ $50.31 \mathrm{ug} / \mathrm{L}$ $43.48 \mathrm{ug} / \mathrm{L} \#$ $56.20 \mathrm{ug} / \mathrm{I} \#$ $70.79 \mathrm{ug} / \mathrm{L} \#$ $68.77 \mathrm{ug} / \mathrm{L}$ $52.84 \mathrm{ug} / \mathrm{L}$ $54.64 \mathrm{ug} / \mathrm{L}$ $53.97 \mathrm{ug} / \mathrm{L}$ $109.12 \mathrm{ug} / \mathrm{L}$ $54.36 \mathrm{ug} / \mathrm{L}$

$54.36 \mathrm{ug} / \mathrm{L}$ we 97 $164.41 \mathrm{ug} / \mathrm{L} \mathrm{m}_{7-8.47}$ $53.33 \mathrm{ug} / \mathrm{I} \#$ $55.49 \mathrm{ug} / \mathrm{L}$ $35.39 \mathrm{ug} / \mathrm{I} \#$ $56.02 \mathrm{ug} / \mathrm{L}$ $35.43 \mathrm{ug} / \mathrm{L}$ $44.10 \mathrm{ug} / \mathrm{L}$ $47.96 \mathrm{ug} / \mathrm{L}$ $45.33 \mathrm{ug} / \mathrm{L} \#$ $44.58 \mathrm{ug} / \mathrm{L}$ \# $47.53 \mathrm{ug} / \mathrm{L}$ $47.69 \mathrm{ug} / \mathrm{L}$ $46.78 \mathrm{ug} / \mathrm{I}$ \# $46.84 \mathrm{ug} / \mathrm{L}$ $50.98 \mathrm{ug} / \mathrm{L} \#$ $52.65 \mathrm{ug} / \mathrm{L}$ $44.12 \mathrm{ug} / \mathrm{I} \#$ $49.76 \mathrm{ug} / \mathrm{L}$ $39.49 \mathrm{ug} / \mathrm{I}$ \# $64.65 \mathrm{ug} / \mathrm{L}$ $71.00 \mathrm{ug} / \mathrm{L}$ \# $42.92 \mathrm{ug} / \mathrm{L}$ $64.22 \mathrm{ug} / \mathrm{L}$
94

74

93

68

100

99

84

93

98

94

94

90

98

94

98

91

98

99

98

95

96

45

45

96

81

95

95

90

92

84

96

58

96

94

99

97 


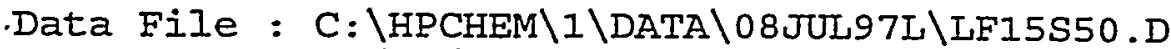

Acq Time : 07/08/97 10:55

Sample : $50 \mathrm{ug} / \mathrm{L}$ Continuing Cal std

Misc : 142 WS28308

Quant Time: Jul 8 12:27 1997

Operator: CQC

Inst : 5971-I Multiplr: 1.00

Method : C: \HPCHEM $\backslash I \backslash M E T H O D S \backslash I 826 A R W A . M$

Title

: VOA COMPOUND LIST

Last Update : Mon Jul 07 15:11:47 1997

Response via : Multiple Level Calibration

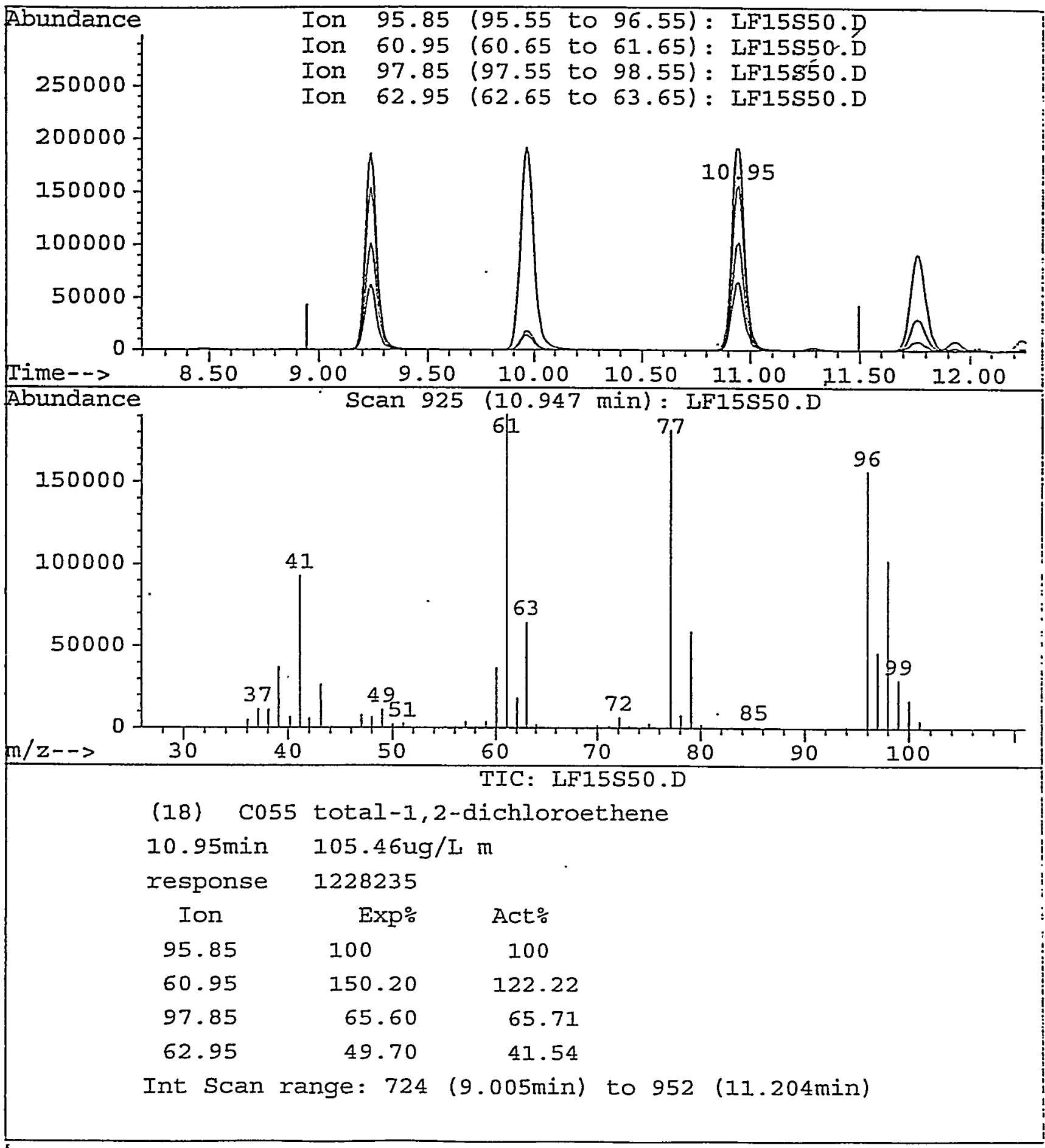


Quantitation Report

Data File : C: \HPCHEM IIDATA 08JUL97L\LFI5S50.D

Acq Time : 07/08/97 10:55

Sample : $50 \mathrm{ug} / \mathrm{L}$ Continuing Cal std

Misc : 142WS28308

Quant Time: JuI 8 12:28 1997

Operator: CQC

Inst : 5971-L

Mutiplr: 1.00

Method : C: \HPCHEM \I\METHODS \L826ARWA.M

Title : VOA COMPOUND LIST

Last Update : Mon Jul 07 15:11:47 1997

Response via : Multiple Level Calibration

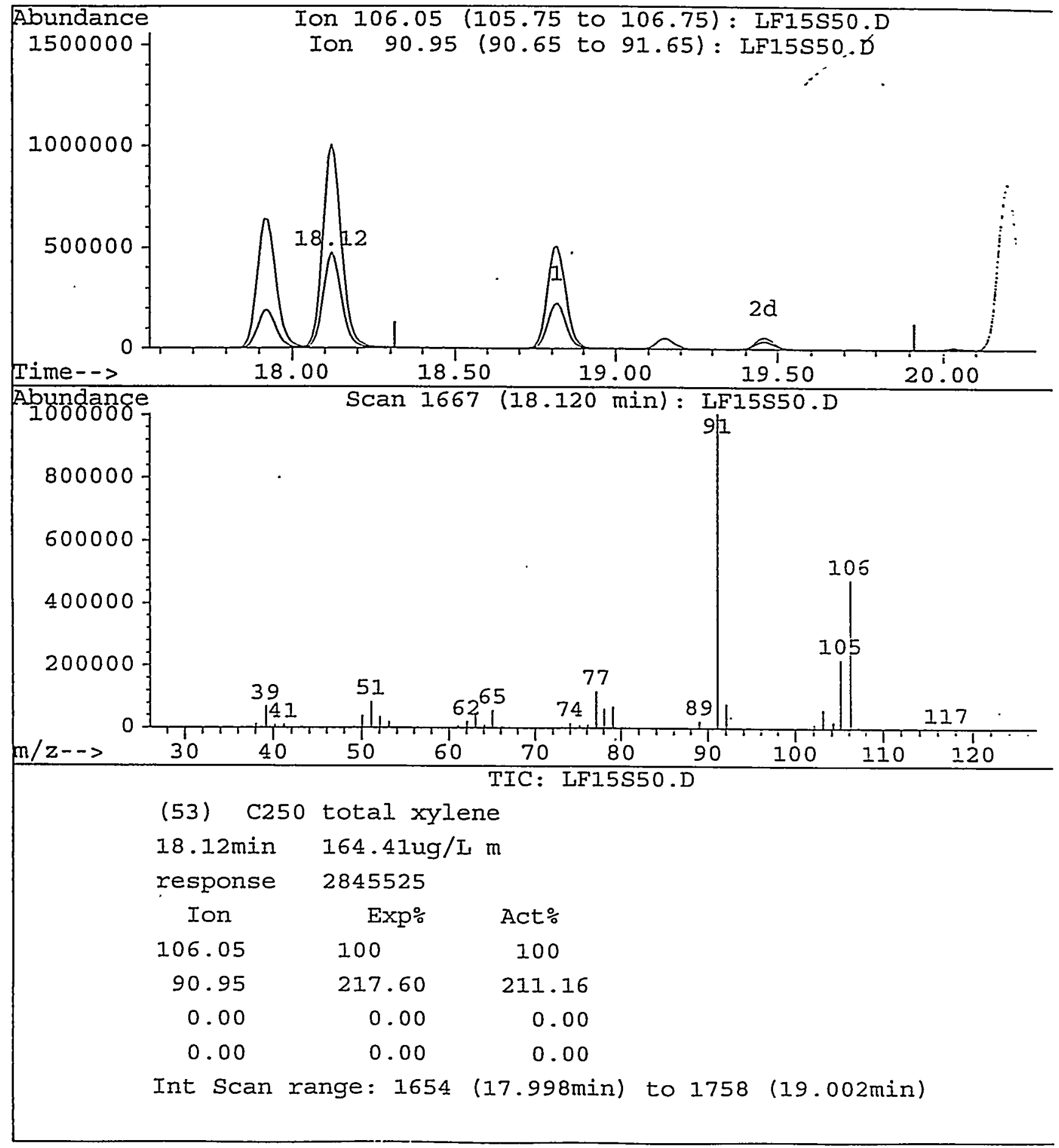




\section{DATA CHEM \\ a SCREKSOH COMPAKY}

\section{Case Narrative}

Method: $8270 \mathrm{~B}$

Analysis: Semivolatiles by GC/MS

Preparation SOP \#: OE-SW-3510

Analysis SOP \#: OS-SW-8270B

DCL Set ID: 97C-0208-02
Client: SAIC

Account: 3006

Matrix: Water

General Set Information: There are three samples in this set.

Method Summary: This method involves the solvent extraction of the sample and analysis of the extract by gas chromatography/mass spectrometry to determine the concentration of semivolatile organic compounds.

Sample Preparation: All samples were prepared in accordance with published procedures. All samples had a final volume of one milliliter.

Holding Times: The holding times were met for both preparation and analysis.

Dilution(s): A 1:10 dilution was required for sample 97C02897 (NAP-R2-S2-B) for naphthalene.

Method and Sample QC data: The method blank was clean of target analytes above the CRDL. All samples met internal standard area and surrogate recovery criteria.

MS/MSD Analysis: An MS/MSD was not performed for this set. Two LCS's were performed. Based on MS/MSD criteria, the percent recovery criteria was met for this method, however, the RPD's for 4-nitrophenol and pentachlorophenol were high.

Instrument QC: The DFTPP tunes met method requirements. Instrument initial and continuing calibrations were within quality control limits.

Flagging Codes: N/A

NC/CAR: None required.

Miscellaneous Comments: No problems were encountered during analysis. The bottle for sample $97 \mathrm{C} 02896$ (NAP-R2-S2-A) was broken so the analysis was not performed for this sample. The ice in the cooler was melted and the temperature was out of range. A ClientRelated Information Report is enclosed with this data pack pertaining to the broken bottle and temperature problems. Additional compounds were reported as found. 


\section{Sample Calculation}

Method 8270B Water

The raw data Quan-list amount, in $\mathrm{ug} / \mathrm{ml}$ is determined by the following equation.

$$
Q_{a m t}=\frac{\left(A_{a g}\right)\left(C_{18}\right)}{\left(A_{1 \delta}\right)(R F)}
$$

Where

$Q_{c z}=$ Quan-list amount (ug per $\mathrm{ml}$ of extract)

$A_{c a}=$ Area of analyte

$\mathrm{C}_{\mathrm{is}}=$ Concentration of internal standard $(\mathrm{ug} / \mathrm{ml})$

$A_{1 s}=$ Area of internal standard

$R F=$ response factor *

* Hote: Response factors are calculated by a first or second order regression fit of the initial calibration curve.

The final reported concentration, in $u g / L$, is calculated in the following manner.

$$
C_{a n}=\frac{\left(Q_{\text {amt }}\right)(F V)(D F)}{\left(A M T_{\text {ext }}\right)}
$$

Where

$C_{a s}=$ Concentration of the analyte (ug/L)

$Q_{a=:}=$ Quan-list amount (ug per ml of extract)

$\mathrm{FV}=$ Final extract volume (ml).

$D F=$ Dilution factor ( 1 if no dilutions performed)

AMT $_{\text {ext }}=$ Amount extracted (usually $1.0 \mathrm{~L}$ )

Example caluclations.

Set ID No.:97C-0208-02 DCL Sample No.: 97602897

Client sample No.: NAP-82-52-B Target analyte naphthalem

Quan-list amount:

Final reported concentration:

$$
c_{a n}=\frac{(54.181) u g / m I(1) m I(10)}{(1) L}=540
$$

$-$ 


\section{Datapackage Table of Contents}

Information pertaining to this datapackage is divided into the four categories listed below. A Case Narrative immediately precedes this Table of Contents and contains pertinent information about this datapackage.

Analytical Results

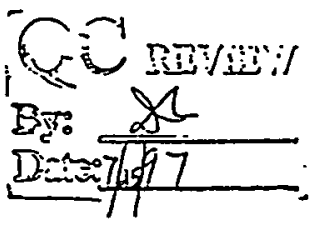
Sample Tracking Documentation ......... Pifik Analytical Documentation ............. Blue Raw Data Green

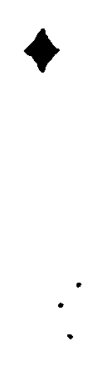

\section{Analytical Results}




\section{DATA CHEM A Sorenson Company}

SAIC

Attention: Art Shattuck 411 Hackensack Avenue Hackensack, NJ 07601
COVER PAGE

ANALYTICAL REPORT FOR SAIC

Phone(201) 498-8496 Fax(201) 489-1592
Form COVER-V1.3

Page 1

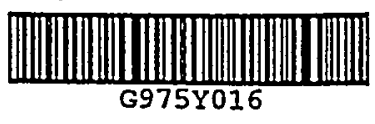

DCL Report Group. . : 97C-0208-02

Date Printed.....: 22-JUL-97 19:08

Project Protocol \#: P975B002

Client Ref Number.: Not Provided Release Number....: Not Provided Analysis'Method(s) : $8270 \mathrm{~B}$

Client
Sample Name
Method Blank
LCS
LCS
NAP-R2-S2-B
NAP-R2-S4
NAP-R1-S4

\begin{tabular}{l} 
Laboratory \\
Sample Name \\
\hline BL-136532-1 \\
QC-136532-1 \\
QC-136532-2 \\
$97 C 02897$ \\
$97 \mathrm{C} 02898$ \\
$97 \mathrm{C} 02901$
\end{tabular}

Date

Sampled

NA

NA

NA

25-JUN-97

$26-J U N-97$

25-JUN-97
Date

Received

NA

NA

NA

27-JUN-97

27-JUN-97

27-JUN-97
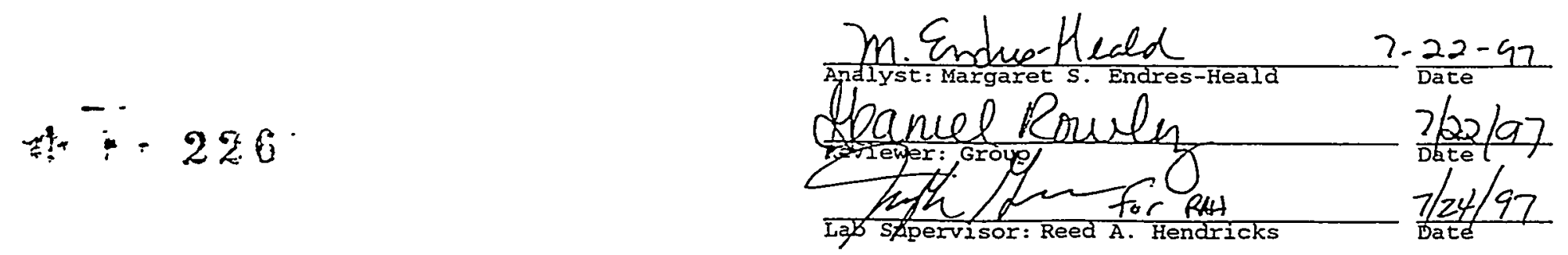

960 West LeVoy Drive / Salt Lake City, Utah 84123-2547 


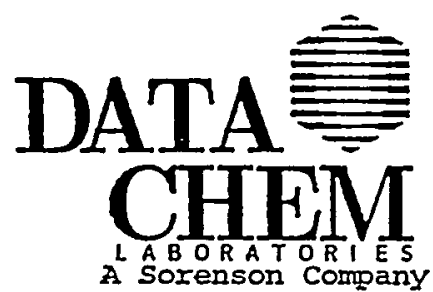

A Sorenson Company
FORM H (TYPE I)

SINGLE METHOD ANALYSES

SAMPLE GROUP COMMENTS
Form RLIMS63H-V1.3

07229719104524

Page 2

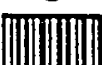

G975Y016

client Name...: SAIC

DCL Report Group.. : 97C-0208-02

Date Printed.....: 22-JUL-97 19:08

Release Number....: Not Provided

\section{General Information}

The DCI QC Database maintains all numerical figures which are input from, the pertinent data source. These data have not been rounded to significant figures nor have they been moisture corrected. Reports generated from the system, however, list data which have been rounded to the number of significant figures requested by the client or deemed appropriate for the method. This may create minor discrepancies between data which appear on the QC Summary Forms (Forms B-G) and those that would be calculated from rounded analytical results. Additionally, if a moisture correction is performed, differences will be observed between the $Q C$ data and the surrogate data reported on Form $A$ (or other report forms) and corresponding data reported on QC Sumnary Forms. In these cases, the Form A will indicate the "Report Basis" as well as the moisture value used for making the correction.

Report generation options : $\mathbf{M X}$

\section{Result Symol Definitions}

ND - Not Detected above the MDL or IDL (ILD or MDC for radiochemistry).

** - No result could be reported, see sample comments for details.

\section{Qualifier Symbol Definitions}

U - Not Detected above the MDL or IDL (ILD or MDC for radiochemistry). For radiochemistry the nuclide was not identified by the Canberra Nuclear NID program, activity values reported are calculated using the Canberra Nuclear MINACT program.

$B$ - For organic analysis the qualifier indicates that this analyte was found in the method blank. For inorganic analysis the qualifier signifies the value is between the IDL and PQL.

$J$ - The qualifier indicates that the value is between the MDL and the PQL. It is also used for indicating an estimated value for tentatively identified compounds in mass spectrometry where a $1: 1$ response is assumed.

\section{7}




\section{DATA \\ FORM A (TYPE I) STNGLE METHOD ANALYSES} CHEM

is Sorenson Company
SAMPLE ANALYSIS DATA SHEET
Form: RLIMS63A-V1.3 07229719104526 Page 3

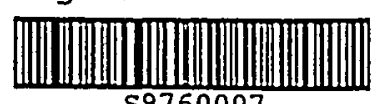

Client Sample Nane: BL-136532-1

DCL Sample Name. . : : BL-136532-1

DCL Report Group..: 97C-0208-02

Matrix......... : WATER

Date Sampled...... Not Applicable

Reporting Units... ug/L

DCL Analysis Group: G9760008

Analysis Method...: $8270 \mathrm{~B}$

Instrument Type...: GC/MS SV

Instrument ID....: $5972-\mathrm{N}$

Column Type.....: DB5,30n $\times .32 \mathrm{~mm}$

8 Primary

$\square$ Confizmation

Analytical Results

\begin{tabular}{|c|c|c|c|c|c|c|c|}
\hline Analyte & $\begin{array}{c}\text { Date } \\
\text { Analyzed } \\
\end{array}$ & MDI & Result & Comment & Qual. & Dilution & CRDL \\
\hline Pyridine & $15-\pi \Omega-97 \quad 11: 55$ & 0.47 & $\mathrm{ND}$ & & & & 10 \\
\hline N-nitrosodimethylamine & $15-J U L-97 \quad 11: 55$ & 0.23 & ND & & & & 10 \\
\hline 2-Picoline & $15-\pi U L-97 \quad 11: 55$ & 3.4 & ND & & & & 10 \\
\hline Methyl methanesulfonate & $15-\pi U L-97 \quad 11: 55$ & 0.24 & ND & & & & 10 \\
\hline Ethyl methanesulfonate & $15-\pi U L-97 \quad 11: 55$ & 1.5 & ND & & & & 10 \\
\hline Phenol & $15-\pi U L-97 \quad 11: 55$ & 0.62 & ND & & & & 10 \\
\hline Aniline & $15-\pi \pi-97 \quad 11: 55$ & 0.26 & ND & & & & 10 \\
\hline 2-Chlorophenol & $15-\pi U L-97 \quad 11: 55$ & 0.27 & ND & & & & 10 \\
\hline 1,3-Dichlorobenzene & $15-\pi U L-97 \quad 11: 55$ & 0.36 & ND & & & & 10 \\
\hline 1,4-Dichlorobenzene & $15-\pi U L-97 \quad 11: 55$ & 0.32 & ND & & & & 10 \\
\hline Benzyl Alcohol & 15-JUL-97 11:55 & 0.29 & ND & & & & 20 \\
\hline 1,2-Dichlorobenzene & $15-\pi U L-97 \quad 11: 55$ & 0.32 & ND & & & & 10 \\
\hline 2-Methylphenol & $15-\pi U L-97 \quad 11: 55$ & 0.20 & ND & & & & 10 \\
\hline Bis (2-chloroisopropyl) ether & $15-\pi / L-97 \quad 11: 55$ & 0.35 & ND & & & & 10 \\
\hline 4-Methylphenol & $15-\pi U L-97 \quad 11: 55$ & 0.18 & ND & & & & 10 \\
\hline Hexachloroethane & $15-\pi U L-97 \quad 11: 55$ & 0.35 & ND & & 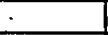 & & 10 \\
\hline Dis $(2$-chloroethyl)ether & 15-JUL-97 11:55 & 0.29 & ND & & & & 10 \\
\hline Acetophenone & 15-JUL-97 $11: 55$ & 0.12 & ND & & & & 10 \\
\hline Nitrobenzene & $15-J U L-97 \quad 11: 55$ & 0.36 & ND & & & & 10 \\
\hline N-nitrosopiperidine & $15-\pi U L-97 \quad 11: 55$ & 1.3 & ND & & & & 10 \\
\hline Isophorone & $15-\pi \Omega-97 \quad 11: 55$ & 0.22 & ND & & & & 10 \\
\hline 2-Nitrophenol & $15-$ JUL-97 $11: 55$ & 0.24 & ND & & & & 10 \\
\hline 2,4-Dimethylphenol & $15-\pi U L-97 \quad 11: 55$ & 0.11 & ND & & & & 10 \\
\hline Benzoic acid & $15-\pi U L-97 \quad 11: 55$ & 6.6 & ND & & & & 50 \\
\hline Bis (2-chloroethoxy) methane & $15-\pi U L-97 \quad 11: 55$ & 0.25 & ND & & & & 10 \\
\hline 2,4-Dichlorophenol & $15-\pi U L-97 \quad 11: 55$ & 0.16 & ND & & & & 10 \\
\hline A. A. -dimethylphenethylamine & $15-J U L-97 \quad 11: 55$ & 4.1 & ND & & & & 50 \\
\hline $1,2,4-$ Trichlorobenzene & $15-J U L-97 \quad 11: 55$ & 1.1 & ND & & & & 10 \\
\hline Naphthalene & $15-\pi U L-97 \quad 11: 55$ & 0.29 & ND & & & & 10 \\
\hline A-Chloroaniline & $15-\pi U L-97 \quad 11: 55$ & 0.77 & ND & & & & 20 \\
\hline 2,6-Dichlorophenol & $15-\pi U L-97 \quad 11: 55$ & 0.50 & ND & & & & 10 \\
\hline Fexachlorobutadiene & $15-\pi U L-97 \quad 11: 55$ & 0.30 & $\mathrm{ND}$ & & & & 10 \\
\hline N-nitroso-di-n-propylamine & 15-JUL-97 $11: 55$ & 0.27 & ND & & & & 10 \\
\hline 4-Chloro-3-methylphenol & $15-\pi U L-97 \quad 11: 55$ & 0.28 & ND & & & & 20 \\
\hline 2-Methylnaphthalene & $15-\pi U L-97 \quad 11: 55$ & 0.29 & ND & & & & 10 \\
\hline $1,2,4,5$-Tetrachlorobenzene & $15-\pi u-97 \quad 11: 55$ & 4.3 & ND & & & & 10 \\
\hline Hexachlorocyclopentadiene & $15-\pi U L-97 \quad 11: 55$ & 0.17 & $\mathrm{ND}$ & & & & 10 \\
\hline 2,4,6-Trichlorophenol & $15-J U L-97 \quad 11: 55$ & 0.17 & ND & & & & 10 \\
\hline 2,4,5-Trichlorophenol & $15-\pi U L-97 \quad 11: 55$ & 0.19 & ND & & & & 10 \\
\hline 2-Chloronaphthalene & $15-\pi U L-97 \quad 11: 55$ & 0.12 & ND & & & & 10 \\
\hline 1-Chloronaphthalene & $15-J U L-97 \quad 11: 55$ & 0.22 & ND & & & & 10 \\
\hline
\end{tabular}

$2 \% 8$ 


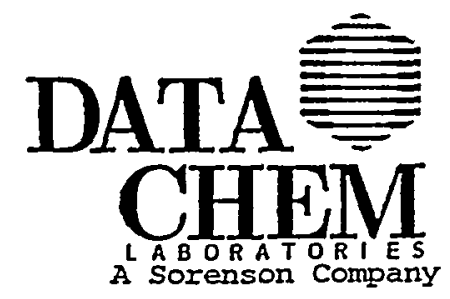

Date Printed........: 22-JUL-97 19:22

Client Name......... SAIC
FORM A (TYPE I)

SINGLE METHOD ANALYSES
Form RLIMS63A-V1.3

07229719242029

Page 4

SAMPIE ANALYSIS DATA SHEET

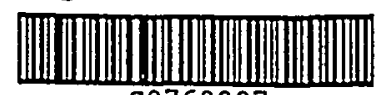

59760007
DCL Sample Name... : BL-136532-1

DCL Report Group. .: 97C-0208-02

Analytical Results

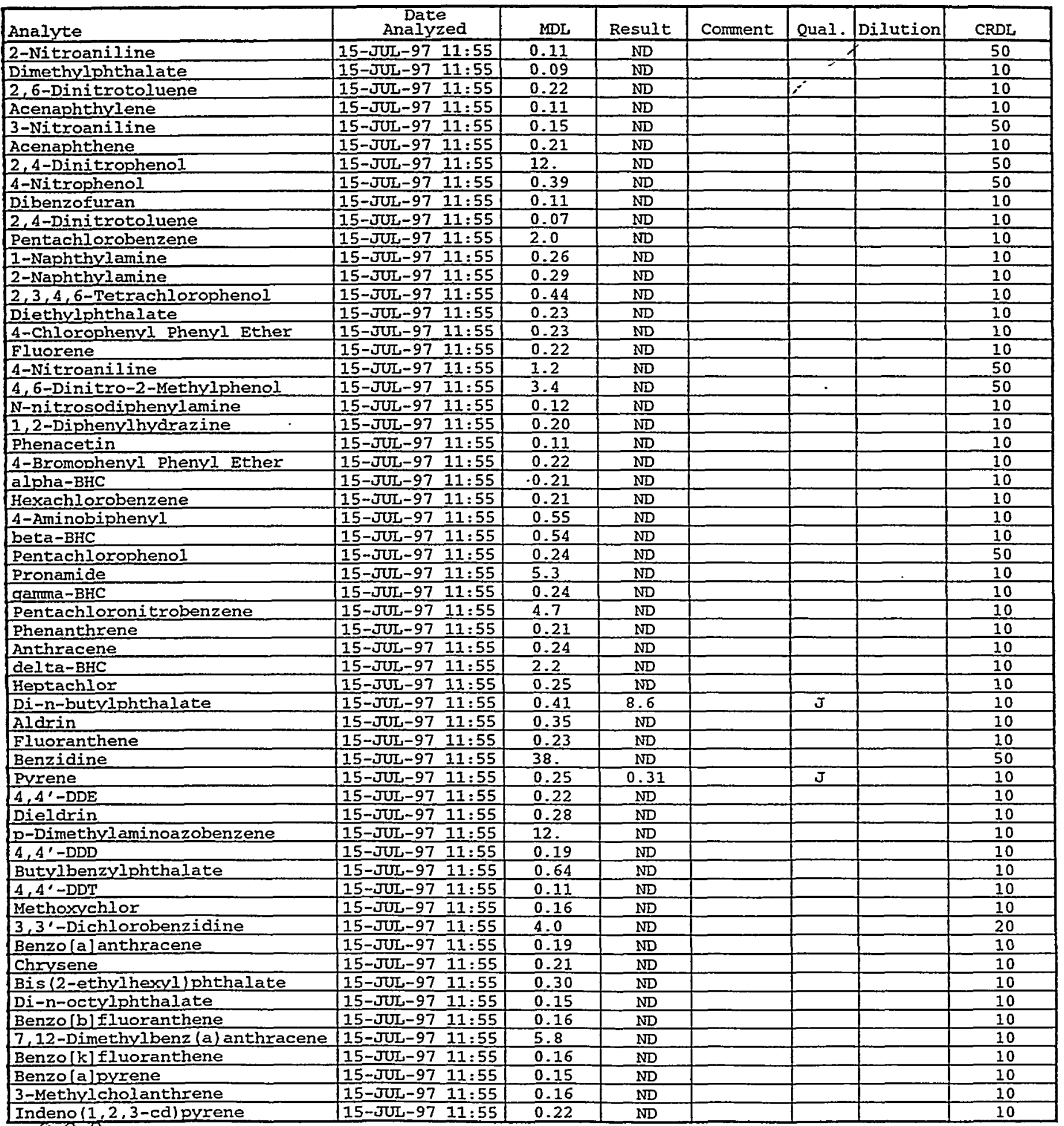
229

960 West LeVoy Drive / Salt Lake City, Utah 84123-2547

Phone (801) 266-7700

FAY (Rח1) J6R-999?

Web Page: www.datachem.com

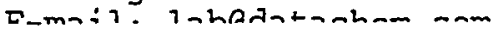




\section{Dy \\ DATA SINGLE METHOD ANALYSES}

SAMPIE ANATYSIS DATA SHEET
Form RLIMS63A-V1.3

Page 5

07229719104524

5

59760007

Date Printed....... 22-JUL-97 19:08

DCL Sample Name. . . : BL-136532-1

Client Name......... SAIC

DCL Report Group. . : 97C-0208-02

Analytical Results

\begin{tabular}{|c|c|c|c|c|c|c|c|}
\hline Analyte & $\begin{array}{c}\text { Date } \\
\text { Analyzed } \\
\end{array}$ & MDL & Result & Comment & Qual. & Dilution & CRDL, \\
\hline Dibenz $[a, h]$ anthracene & 15-JUL-97 $11: 55$ & 0.28 & $\mathrm{ND}$ & & 1 & & 10 \\
\hline Benzo[ahilpervlene & $15-J U L-97 \quad 11: 55$ & 0.32 & ND & & 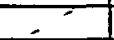 & & 10 \\
\hline
\end{tabular}

Tentatively Identified Compound Results

\begin{tabular}{|c|c|c|c|c|c|}
\hline Analyte (Retention Time) & $\begin{array}{c}\text { Date } \\
\text { Analyzed }\end{array}$ & Result & Comment & Qual. & Dilution \\
\hline Oxvhydrocarbon $(4.16)$ & 15-JUL-97 $11: 55$ & 5.1 & & $\mathrm{~J}$ & \\
\hline
\end{tabular}

Surrogate Recoveries

\begin{tabular}{|l|c|c|c|}
\hline Analyte & Result & $\begin{array}{c}\text { Spiked } \\
\text { Amount }\end{array}$ & $\begin{array}{c}\text { Percent } \\
\text { Recovery }\end{array}$ \\
\hline $2,4,6$-Tribromophenol & 60.9 & 100. & 60.9 \\
\hline 2-Fluorobiphenyl & 73.2 & 100. & 73.2 \\
\hline 2-Fluorophenol & 47.1 & 100. & 47.1 \\
\hline Nitrobenzene-d5 & 81.1 & 100. & 81.1 \\
\hline Phenol-d5 & 34.8 & 100. & 34.8 \\
\hline Terohenyl-d14 & 91.7 & 100. & 91.7 \\
\hline
\end{tabular}



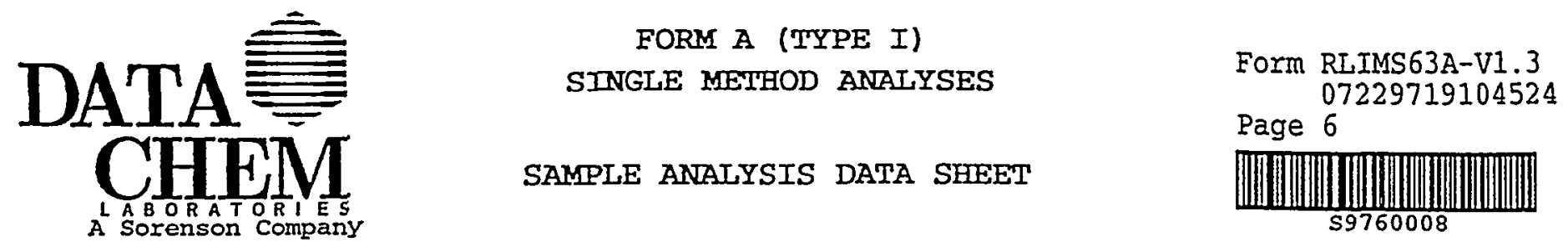

Date Printed.......: 22-JUL-97 19:08

Client Sample Name: QC-136532-1

DCL Sample Name...: : QC-136532-1

Client Name........ SAIC

DCL Report Group..: 97C-0208-02

client Ref Number....: Not Provided.

Sampling site....... Not Applicable

Matrix......... : WATER

Release Number. . . . . . Not Provided

Date Sampled...... Not Applicable

Reporting Units... $/$ ug/L

Date Received........ Not Applicable

DCL Preparation Group: G9760008

Date Prepared......: 01-JUL-97 00:00

DCL Analysis Group: G9760008

Preparation Method. . : 3510

Aliquot Weight/Volume: $1000 \mathrm{MLS}$

Net Weight/Volume.... : Not Required

Analysis Method...: $8270 \mathrm{~B}$

Instrument Type...: GC/MS SV

Instrument ID....: 5972-N

Column Type......: DB5,30m $\times .32 \mathrm{~mm}$

Q Primary

$\square$ Confirmation

Analytical Results

\begin{tabular}{|c|c|c|c|c|c|c|c|}
\hline Analyte & $\begin{array}{c}\text { Date } \\
\text { Analyzed }\end{array}$ & $\mathrm{MDL}$ & Result & Comment & Qual. & Dilution & CRDL \\
\hline Phenol & $15-\pi U L-97 \quad 12: 29$ & 0.62 & 34. & & & & 10 \\
\hline 2-Chlorophenol & $15-J U L-97 \quad 12: 29$ & 0.27 & 60. & & . & & 10 \\
\hline 1,4-Dichlorobenzene & 15-JUL-97 12:29 & 0.32 & 78. & & & & 10 \\
\hline 1,2,4-Trichlorobenzene & $15-\pi J[-97 \quad 12: 29$ & 1.1 & 66. & & & & 10 \\
\hline N-nitroso-di-n-propylamine & $15-J U L-97 \quad 12: 29$ & 0.27 & 140 & & & & 10 \\
\hline 4-Chloro-3-methylphenol & $15-\pi U L-97 \quad 12: 29$ & 0.28 & 86. & & & & 20 \\
\hline Acenaphthene & $15-J_{L}-97 \quad 12: 29$ & 0.21 & 92. & & & & 10 \\
\hline 4-Nitrophenol & $15-J U L-97 \quad 12: 29$ & 0.39 & 13. & & & & 50 \\
\hline 2,4-Dinitrotoluene & $15-\pi J 5-97 \quad 12: 29$ & 0.07 & 93. & & & & 10 \\
\hline Pentachlorophenol & $15-\pi U L-97 \quad 12: 29$ & 0.24 & 33. & & & & 50 \\
\hline Pvrene & $15-\pi U-97 \quad 12: 29$ & 0.25 & 93. & & & & 10 \\
\hline
\end{tabular}

\section{Surrogate Recoveries}

\begin{tabular}{|l|c|c|c|}
\hline Analyte & Result & $\begin{array}{c}\text { Spiked } \\
\text { Anount }\end{array}$ & $\begin{array}{c}\text { Percent } \\
\text { Recovery }\end{array}$ \\
\hline 2,4, -Tribromophenol & 45.0 & 100. & 45.0 \\
\hline 2-Fluorobiphenyl & 81.1 & 100. & 81.1 \\
\hline 2-Fluorophenol & 33.8 & 100. & 33.8 \\
\hline Nitrobenzene-d5 & 89.9 & 100. & 89.9 \\
\hline Phenol-d5 & 33.0 & 100. & 33.0 \\
\hline Terphenyl-d14 & 98.9 & 100. & 98.9 \\
\hline
\end{tabular}




\section{DATA

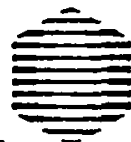 \\ FORM A (TYPE I) SINGLE MEIHOD ANALYSES} CHEM

A Sorenson Company

Daze Printed........ 22-JUL-97 19:08

client Name.......... SAIC

Client Ref Number.... Not Provided

Samling Site....... Not Applicable

Release Number....... Not Provided

Date Received....... : Not Applicable

DCL Preparation Group: G9760008

Date Prepared.......: 01-JUL-97 00:00

Preparation Method... : 3510

Aliquot Weight/Volume: 1000 Mrs

Net Weight/Volume.... . Not Required
SAMPLE ANALYSIS DATA SHEET
Form RLIMS63A-V1.3 07229719104524 Page 7

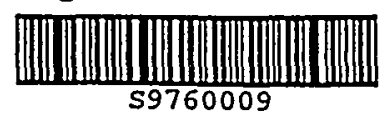

Client Sample Name: QC-136532-2

DCL Sample Name... : QC-136532-2

DCL Report Group.. : 97C-0208-02

Matrix......... : WATER

Date Sampled...... Not Applicable

Reporting Units... $y$ ug/L<smiles>[CH][CH]</smiles>

DCL Analysis Group: G9760008

Analysis Method...: 8270B

Instrument Type...: GC/MS SV

Instrument ID. . . . : 5972-N

Column Type.....: DB5,30m x.32m

$\otimes$ Primary

Confirmation

\section{Analytical Results}

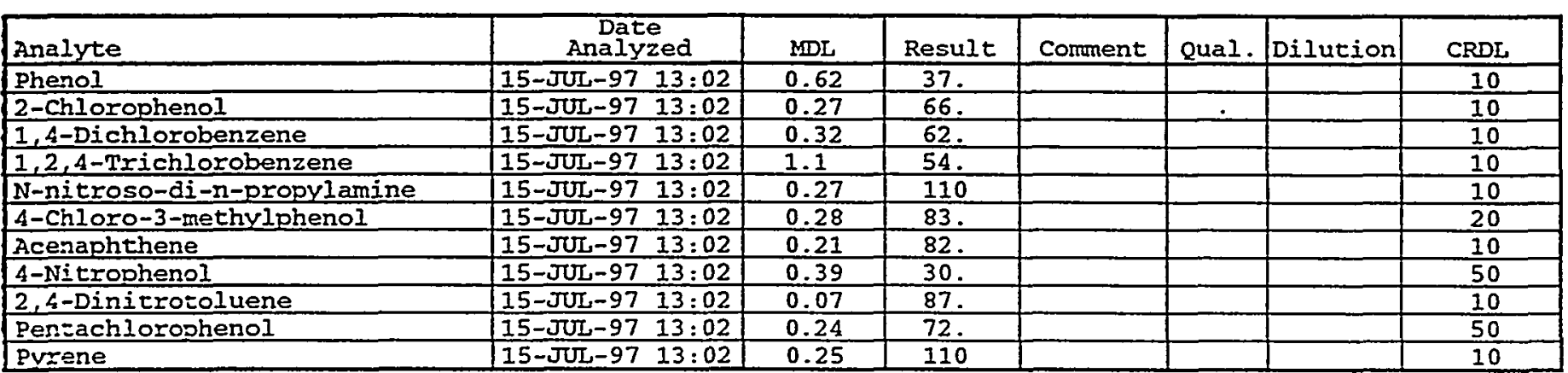

\section{Surrogate Recoveries}

\begin{tabular}{|l|c|c|c|}
\hline Analyte & Result & $\begin{array}{c}\text { Spiked } \\
\text { Amount }\end{array}$ & $\begin{array}{c}\text { Percent } \\
\text { Recovery }\end{array}$ \\
\hline 2,4.6-Tribromophenol & 65.7 & 100. & 65.7 \\
\hline 2-Eluorobipienyl & 71.0 & 100. & 71.0 \\
\hline 2-Eluorophenol & 43.6 & 100. & 43.6 \\
\hline Nitrobenzene-d5 & 75.9 & 100. & 75.9 \\
\hline Phenol-d5 & 34.5 & 100. & 34.5 \\
\hline Tezohenvl-d14 & 112. & 100. & 112. \\
\hline
\end{tabular}




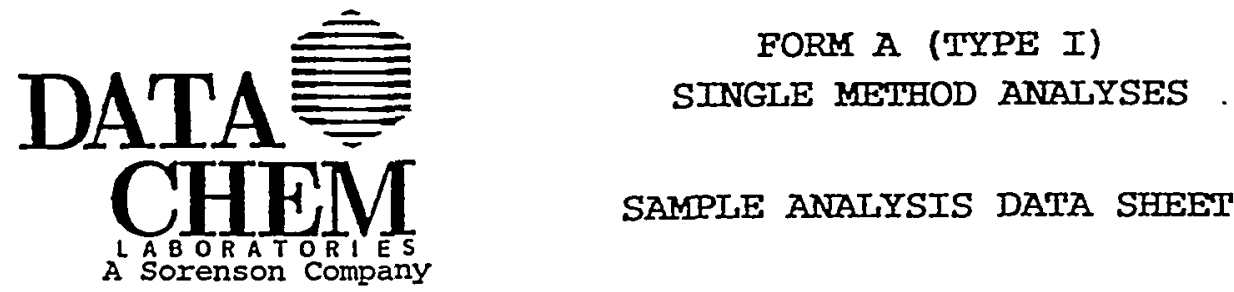

Form RLIMS63A-V1.3 07229719241108 Page 8

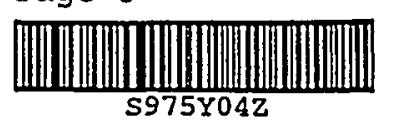

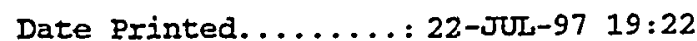

Client Name......... SAIC

Client Ref Number.... . Not Provided

Sampling Site....... Not Provided

Release Number....... Not Provided

Date Received......: 27-JUN-97 00:00

DCI Preparation Group: G9760008

Date Prepared.......: 01-JUL-97 00:00

Preparation Method. . : 3510

Aliquot Weight/Volume: $1000 \mathrm{kmS}$

Net Weight/Volume.... : Not Required
Client Sample Name: NAP-R2-S2-B

DCL Sample Name...: 97C02897

DCL Report Group..: 97C-0208-02

Matrix........: WATER

Date Sampled.....: 25-JUN-97 00:00

Reporting Units.. A ug/L

Report Basis...... : $\square$ As Received $\square$ Dried

DCI Analysis Group: G9760008

Analysis Method...: 8270B

Instrument Type...: GC/MS SV

Instrument ID. . . : : 5972-N

Column Type......: DB5,30m $\times .32 \pi m$

ㅁ. Primary

$\square$ Confirmation

Analytical Results

\begin{tabular}{|c|c|c|c|c|c|c|c|}
\hline Analyte & $\begin{array}{l}\text { Date } \\
\text { Analyzed }\end{array}$ & MDL & Result & Comment & Qual. & Dilution & CRDL \\
\hline Pyridine & $15-\pi U=-97 \quad 13: 36$ & 0.47 & 1.1 & & $\mathrm{~J}$ & & 10 \\
\hline N-nitrosodimethylamine & $15-\mathrm{JUL}-97 \quad 13: 36$ & 0.23 & ND & & $\cdot$ & & 10 \\
\hline 2-Picoline & $15-J U L-97 \quad 13: 36$ & 3.4 & $\mathrm{ND}$ & & & & 10 \\
\hline Methyl methanesulfonate & $15-J U L-97 \quad 13: 36$ & 0.24 & ND & & & & 10 \\
\hline Ethyl methanesulfonate & $15-J U L-97 \quad 13: 36$ & 1.5 & ND & & & & 10 \\
\hline Phenol & $15-J U L-97 \quad 13: 36$ & 0.62 & 1.8 & & $\mathrm{~J}$ & & 10 \\
\hline Aniline & 15-JUL-97 $13: 36$ & 0.26 & 4.3 & & $\mathrm{~J}$ & & 10 \\
\hline 2-Chlorophenol & $15-\pi U L-97 \quad 13: 36$ & 0.27 & $\mathrm{ND}$ & & & & 10 \\
\hline 1,3-Dichlorobenzene & $15-J U L-97 \quad 13: 36$ & 0.36 & ND & & & & 10 \\
\hline 1,4-Dichlorobenzene & $15-J U L-97 \quad 13: 36$ & 0.32 & $\mathrm{ND}$ & & & & 10 \\
\hline Benzyl Alcohol & $15-J U L-97 \quad 13: 36$ & 0.29 & ND & & & & 20 \\
\hline 1,2-Dichlorobenzene & $15-\pi U L-97 \quad 13: 36$ & 0.32 & $\mathrm{ND}$ & & & & 10 \\
\hline 2-Methvlphenol & 15-JUL-97 $13: 36$ & 0.20 & 0.52 & & $\mathrm{I}$ & & 10 \\
\hline Bis (2-chloroisopropyl) ether & 15-JUL-97 $13: 36$ & 0.35 & $\mathrm{ND}$ & & & & 10 \\
\hline 4-Methylphenol & $15-\pi U L-97 \quad 13: 36$ & 0.18 & 0.69 & & $\mathrm{~J}$ & & 10 \\
\hline Hexachloroethane & $15-J U L-97 \quad 13: 36$ & 0.35 & ND & & & & 10 \\
\hline Bis (2-chloroethyl) ether & $15-\pi U L-97 \quad 13: 36$ & 0.29 & ND & & & & 10 \\
\hline Acetophenone & $15-J U L-97 \quad 13: 36$ & 0.12 & 9.9 & & $J$ & & 10 \\
\hline Nitroberzene & 15-JUL-97 $13: 36$ & 0.36 & ND & & & & 10 \\
\hline N-nitrosopiperidine & $15-\pi U L-97 \quad 13: 36$ & 1.3 & ND & & & & 10 \\
\hline Isophorone & $15-\pi U L-97 \quad 13: 36$ & 0.22 & $\mathrm{ND}$ & & & & 10 \\
\hline 2-Nitrophenol & $15-J U L-97 \quad 13: 36$ & 0.24 & ND & & & & 10 \\
\hline 2,4-Dimethylphenol & 15 -JUL-97 $13: 36$ & 0.11 & 0.33 & & $\mathrm{~J}$ & & 10 \\
\hline Benzoic acid & $15-\pi U 1-97 \quad 13: 36$ & 6.6 & ND & & & & 50 \\
\hline Bis (2-chloroethoxy) methane & $15-J U L-97 \quad 13: 36$ & 0.25 & ND & & & & 10 \\
\hline 2,4-Dichlorophenol & $15-J U L-97 \quad 13: 36$ & 0.16 & ND & & & & 10 \\
\hline A.,A.-dimethylphenethylamine & 15-JUL-97 $13: 36$ & 4.1 & ND & & & & 50 \\
\hline $1,2,4$-Trichlorobenzene & $15-J U L-97 \quad 13: 36$ & 1.1 & $\mathrm{ND}$ & & & & 10 \\
\hline Napithalene & $16-\pi U L-97 \quad 07: 03$ & 2.9 & 540 & & & 10 & 100 \\
\hline 4-Chloroaniline & $15-J U L-97 \quad 13: 36$ & 0.77 & $\mathrm{ND}$ & & & & 20 \\
\hline 2,6-Dichlorophenol & $15-J 05-97 \quad 13: 36$ & 0.50 & ND & & & & 10 \\
\hline Hexachlorobutadiene & 15-JUL-97 $13: 36$ & 0.30 & $\mathrm{ND}$ & & & & 10 \\
\hline N-nitroso-di-n-propylamine & $15-\pi U L-97 \quad 13: 36$ & 0.27 & $\mathrm{ND}$ & & & & 10 \\
\hline 4-Ciloro-3-methylphenol & 15-JUL-97 $13: 36$ & 0.28 & ND & & & & 20 \\
\hline 2-Methylnaphthalene & 15-JUL-97 $13: 36$ & 0.29 & 1.3 & & $\mathbf{J}$ & & 10 \\
\hline $1,2,4,5$-Tetrachlorobenzene & 15-JUL-97 $13: 36$ & 4.3 & ND & & & & 10 \\
\hline Hexachlorocyclopentadiene & $15-\pi U L-97 \quad 13: 36$ & 0.17 & ND & & & & 10 \\
\hline $2,4,6$-Trichlorophenol & $15-\pi U L-97 \quad 13: 36$ & 0.17 & ND & & & & 10 \\
\hline 2,4,5-Trichlorophenol & 15-JUL-97 $\quad 13: 36$ & 0.19 & ND & & & & 10 \\
\hline 2-Chloromaphthalene & 15-JUL-97 $13: 36$ & 0.12 & ND & & & & 10 \\
\hline I-Cinloronaphthalene & $15-J U L-97 \quad 13: 36$ & 0.22 & ND & & & & 10 \\
\hline
\end{tabular}

233

960 West LeVoy Drive / Salt Lake City, Utah 84123-2547

Phone (801) 266-7700

FAX (8ח1) 26R-900)
Web Page: www.datachem.com

F_msij: ishAdstsahnm nam 


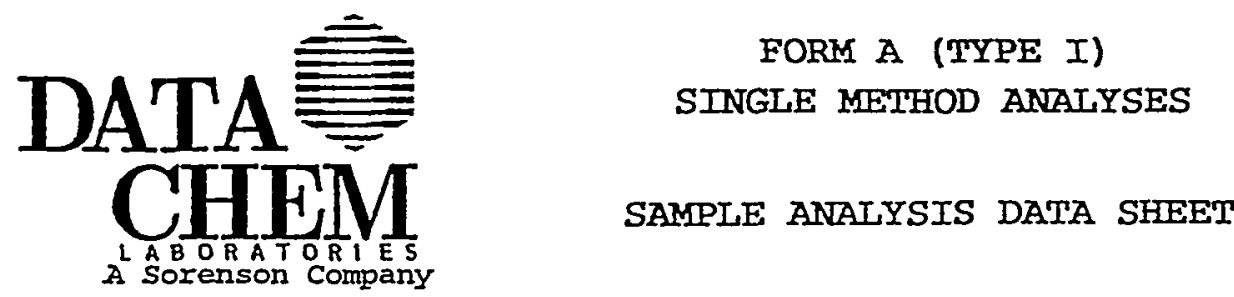

Form RLIMS63A-V1.3 07229719104524

Page 9

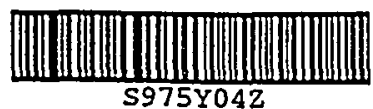

DCL Sample Name. . : 97C02897

DCI Report Group. . : 97C-0208-02

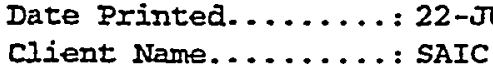

\section{Analytical Results}

\begin{tabular}{|c|c|c|c|c|c|c|c|}
\hline Analyte & $\begin{array}{c}\text { Date } \\
\text { Analyzed }\end{array}$ & MDL & Result & Comment & Qual. & Dilution & CRDL \\
\hline 2-Nitroaniline & $15-\pi U L-97 \quad 13: 36$ & 0.11 & ND & & & & 50 \\
\hline Dimethylphthalate & $15-J U L-97 \quad 13: 36$ & 0.09 & $\mathrm{ND}$ & & & & 10 \\
\hline 2,6-Dinitrotoluene & $15-\pi U L-97 \quad 13: 36$ & 0.22 & ND & & $\therefore$ & & 10 \\
\hline Acenaphthylene & $15-\pi U L-97 \quad 13: 36$ & 0.11 & 17. & & & & 10 \\
\hline 3-Nitroaniline & $15-$ JUL-97 $13: 36$ & 0.15 & ND & & & & 50 \\
\hline Acenaphthene & $15-\pi U L-97 \quad 13: 36$ & 0.21 & 2.3 & & $\mathrm{~J}$ & & 10 \\
\hline 2,4-Dinitrophenol & $15-\pi 04-97 \quad 13: 36$ & 12. & ND & & & & 50 \\
\hline 4-Nitrophenol & $15-J U L-97 \quad 13: 36$ & 0.39 & ND & & & & 50 \\
\hline Dibenzofuran & $15-J \widetilde{L}-97 \quad 13: 36$ & 0.11 & 0.97 & & $\mathrm{~J}$ & & 10 \\
\hline 2,4-Dinitrotoluene & $15-\pi U L-97 \quad 13: 36$ & 0.07 & ND & & & & 10 \\
\hline Pentachlorobenzene & $15-J U L-97 \quad 13: 36$ & 2.0 & $\mathrm{ND}$ & & & & 10 \\
\hline 1-Naphthylamine & $15-\pi u L-97 \quad 13: 36$ & 0.26 & ND & & & & 10 \\
\hline 2-Naphthylamine & $15-\pi J L-97 \quad 13: 36$ & 0.29 & ND & & & & 10 \\
\hline $2,3,4,6$-Tetrachlorophenol & $15-J U L-97 \quad 13: 36$ & 0.44 & $\mathrm{ND}$ & & & & 10 \\
\hline Diethylphthalate & $15-\pi U L-97 \quad 13: 36$ & 0.23 & ND & & & & 10 \\
\hline 4-Chlorophenyl Phenyl Ether & $15-\pi U L-97 \quad 13: 36$ & 0.23 & $\mathrm{ND}$ & & & & 10 \\
\hline Fluorene & $15-\pi U L-97 \quad 13: 36$ & 0.22 & 26. & & & & 10 \\
\hline 4-Nitroaniline & $15-\pi U L-97 \quad 13: 36$ & 1.2 & $\mathrm{ND}$ & & & & 50 \\
\hline 4,6-Dinitro-2-Methylphenol & $15-J U L-97 \quad 13: 36$ & 3.4 & ND & & & & 50 \\
\hline N-nitrosodiphenylamine & $15-\pi U L-97 \quad 13: 36$ & 0.12 & ND & & & & 10 \\
\hline 1,2-Diphenylhydrazine & $15-\pi U L-97 \quad 13: 36$ & 0.20 & ND & & & & 10 \\
\hline phenacetin & $15-\pi U L-97 \quad 13: 36$ & 0.11 & ND & & & & 10 \\
\hline 4-Bromophenyl Phenyl Ether & $15-$ JuL-97 $13: 36$ & 0.22 & ND & & & & 10 \\
\hline aloha-BHC & $15-\sqrt{U L}-97 \quad 13: 36$ & 0.21 & ND & & & & 10 \\
\hline Hexachlorobenzene & $15-\pi u 5-97 \quad 13: 36$ & 0.21 & $\mathrm{ND}$ & & & & 10 \\
\hline 4-AminobiphenvI & $15-\pi U L-97 \quad 13: 36$ & 0.55 & ND & & & & 10 \\
\hline beta-BHC & $15-3 U L-97 \quad 13: 36$ & 0.54 & ND & & & & 10 \\
\hline Pentachlorophenol & $15-\pi U L-97 \quad 13: 36$ & 0.24 & ND & & & & 50 \\
\hline Pronamide & $15-\pi U L-97 \quad 13: 36$ & 5.3 & ND & & & & 10 \\
\hline garma-BHC & $15-\pi U L-97 \quad 13: 36$ & 0.24 & ND & & & & 10 \\
\hline Pentachloronitrobenzene & $15-\pi U L-97 \quad 13: 36$ & 4.7 & $\mathrm{ND}$ & & & & 10 \\
\hline Phenanthrene & $15-\pi U L-97 \quad 13: 36$ & 0.21 & 72 & & & & 10 \\
\hline Anthracene & $15-\pi 04-97 \quad 13: 36$ & 0.24 & 11. & & & & 10 \\
\hline delta-BHC & $15-\pi U L-97 \quad 13: 36$ & 2.2 & $\mathrm{ND}$ & & & & 10 \\
\hline Hestachlor & $15-\pi J L-97 \quad 13: 36$ & 0.25 & $\mathrm{ND}$ & & & & 10 \\
\hline Di-n-butylphthalate & $15-J U L-97 \quad 13: 36$ & 0.41 & 4.3 & & $\mathrm{JB}$ & & 10 \\
\hline Aldrin & $15-\pi U L-97 \quad 13: 36$ & 0.35 & ND & & & & 10 \\
\hline Fluoranthene & $15-5 U L-97 \quad 13: 36$ & 0.23 & 4.8 & & $\mathrm{~J}$ & & 10 \\
\hline Benzidine & $15-$ JUL-97 $13: 36$ & 38. & ND & & & & 50 \\
\hline Pyzene & $15-\pi U L-97 \quad 13: 36$ & 0.25 & 1.6 & & JB & & 10 \\
\hline $4,4^{\prime}-D D E$ & $15-\pi U L-97 \quad 13: 36$ & 0.22 & ND & & & & 10 \\
\hline Dieldrin & $15-\pi U L-97 \quad 13: 36$ & 0.28 & ND & & & & 10 \\
\hline p-Dimethylaminoazobenzene & $15-$ JUL-97 $13: 36$ & 12. & ND & & & & 10 \\
\hline $4,4^{\prime}-D D D$ & $15-\pi U L-97 \quad 13: 36$ & 0.19 & ND & & & & 10 \\
\hline Butylbenzylphthalate & $15-\pi U L-97 \quad 13: 36$ & 0.64 & ND & & & & 10 \\
\hline $4,4^{\prime}-D D T$ & $15-\pi U L-97 \quad 13: 36$ & 0.11 & ND & & & & 10 \\
\hline Mechosychlor & $15-\pi U L-97 \quad 13: 36$ & 0.16 & ND & & & & 10 \\
\hline 3,3'-Dichlorobenzidine & $15-\pi U L-97 \quad 13: 36$ & 4.0 & ND & & & & 20 \\
\hline Benzo[a]anthracene & $15-\pi U L-97 \quad 13: 36$ & 0.19 & ND & & & & $\frac{20}{10}$ \\
\hline Chrusene & $15-\pi U L-97 \quad 13: 36$ & 0.21 & ND & & & & 10 \\
\hline Bis(2-ethulhexyl)phthalate & $15-\pi 04-97 \quad 13: 36$ & 0.30 & ND & & & & 10 \\
\hline Di-n-octylphthalate & $15-\pi \sqrt{1}-97 \quad 13: 36$ & 0.15 & ND & & & & 10 \\
\hline Benzo[b]fluoranthene & $15-J U L-97 \quad 13: 36$ & 0.16 & ND & & & & 10 \\
\hline 7,12-Dimethvibenz (a) anthracene & $15-\pi U L-97 \quad 13: 36$ & 5.8 & ND & & & & 10 \\
\hline Benzo $[\mathrm{k}]$ fluoranthene & $15-\mathrm{JUL}-97 \quad 13: 36$ & 0.16 & ND & & & & 10 \\
\hline Benzola lpyrene & $15-\pi U L-97 \quad 13: 36$ & 0.15 & ND & & & & 10 \\
\hline 3-Methylcholanthrene & $15-\pi U L-97 \quad 13: 36$ & 0.16 & ND & & & & 10 \\
\hline Indeno $(1,2,3-c d)$ pyrer & $15-\pi U L-97 \quad 13: 36$ & 0.22 & ND & & & & 10 \\
\hline
\end{tabular}

234 


\section{Defl \\ DATA SINGLE METHOD ANALYSES \\ Form RLIMS63A-V1.3 \\ CHEM \\ SAMPLE ANALYSIS DATA SHEET \\ Page 10 \\ A Sorenson Company

Date Printed.......: 22-JUL-97 19:08

DCL Sample Name. . : 97c02897

client Name......... SAIC

DCL Report Group..: 97C-0208-02

Analytical Results

\begin{tabular}{|c|c|c|c|c|c|c|c|}
\hline Analyte & $\begin{array}{c}\text { Date } \\
\text { Analyzed }\end{array}$ & $\mathrm{MDL}$ & Result & Comment & Qual. & Dilution & CRDL \\
\hline Dibenz $[a, h]$ anthracene & $15-\pi U L-97 \quad 13: 36$ & 0.28 & ND & & & 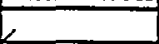 & 10 \\
\hline Benzo(ghi)perylene & 15-JUL-97 $13: 36$ & 0.32 & ND & & $\overline{-}$ & & 10 \\
\hline
\end{tabular}

Tentatively Identified Compound Results

\begin{tabular}{|c|c|c|c|c|c|}
\hline Analyte (Retention Time) & $\begin{array}{c}\text { Date } \\
\text { Analyzed }\end{array}$ & Result & Comment & Qual. & Dilution \\
\hline Aromatic MW $104(4.13)$ & $15-\pi U L-97 \quad 13: 36$ & 69. & & $\mathrm{~J}$ & \\
\hline Indene or isomer $(5.41)$ & $15-$ JUL-97 $13: 36$ & 65. & & $\mathrm{~J}$ & \\
\hline Benzenemethanol, dimethyl or i $(5.69)$ & 15 -JUL-97 $13: 36$ & 63. & & $\mathrm{~J}$ & \\
\hline Benzothiazole or similar compo $(7.03)$ & $15-\pi U L-97 \quad 13: 36$ & 8.9 & & $\mathrm{~J}$ & \\
\hline Biohenyl or similar compound (8.42) & $15-\pi U L-97 \quad 13: 36$ & 56. & & $\mathrm{~J}$ & \\
\hline Dibenzothiophene or similar co(11.89) & $15-J U L-97 \quad 13: 36$ & 5.6 & & $\mathrm{~J}$ & \\
\hline 2-Mercaptobenzothiazole or $\operatorname{sim}(13.39)$ & $15-J U L-97 \quad 13: 36$ & 14. & & $\mathrm{~J}$ & \\
\hline 2-Phenylnaphthalene or isomer $(13.53)$ & $15-$ JUL-97 $13: 36$ & 6.2 & & $\mathrm{~J}$ & \\
\hline
\end{tabular}

\section{Surrogate Recoveries}

\begin{tabular}{|l|c|c|c|}
\hline Analyte & Result & $\begin{array}{c}\text { Spiked } \\
\text { Amount }\end{array}$ & $\begin{array}{c}\text { Percent } \\
\text { Recovery }\end{array}$ \\
\hline 2,4,6-Tribromophenol & 63.0 & 100. & 63.0 \\
\hline 2-Fluorobiphenvl & 75.6 & 100 & 75.6 \\
\hline 2-Eluorophenol & 40.5 & 100. & 40.5 \\
\hline Nitrobenzene-d5 & 95.5 & 100. & 95.5 \\
\hline Phenol-d5 & 34.8 & 100. & 34.8 \\
\hline Terohenvl-d14 & 90.2 & 100. & 90.2 \\
\hline
\end{tabular}

$22: 3$ 


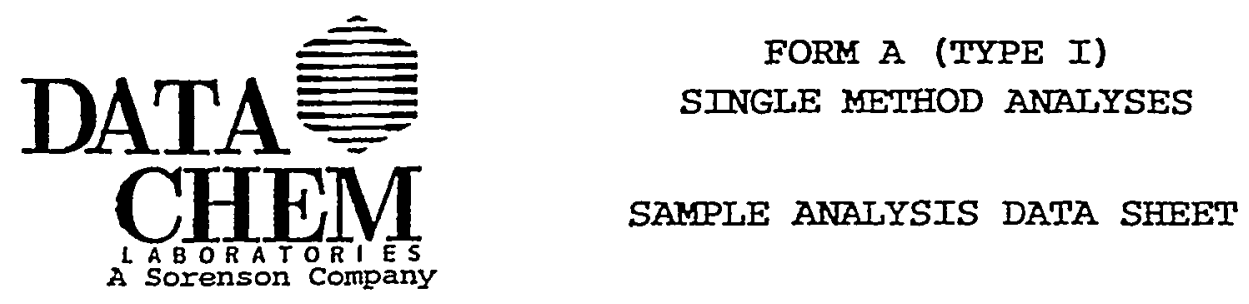

Form RLIMS63A-V1.3

Page 11 07229719104524

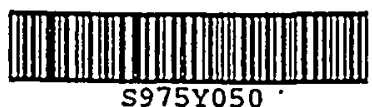

Client Sample Name: NAP-R2-S4

DCL Sample Name. . : 97C02898

DCL Report Group... : 97C-0208-02

Matrix......... WATER

Date Sampled.....: 26-JUN-97 00:00

Reporting Units...: ug/L

Report Basis.....: $\square$ As Received $\square$ Dried $\therefore$

DCL Analysis Group: G9760008

Analysis Method...: $8270 \mathrm{~B}$

Instrument Type...: GC/MS SV

Instrument ID. . . : :5972-N

Column Type.....: DB5.30m $\times .32 \mathrm{~mm}$

冈 Primary

$\square$ Confirmation

Analytical Results

\begin{tabular}{|c|c|c|c|c|c|c|c|}
\hline Analyte & $\begin{array}{c}\text { Date } \\
\text { Analyzed }\end{array}$ & MDL & Result & Comment & Qual. & Dilution & CRDL \\
\hline Pvridine & $15-J U L-97 \quad 14: 10$ & 0.47 & $\mathrm{ND}$ & & & & 10 \\
\hline N-nitrosodimethylamine & $15-\pi U 2-97 \quad 14: 10$ & 0.23 & ND & & & & 10 \\
\hline 2-picoline & $15-\pi \pi L-97 \quad 14: 10$ & 3.4 & ND & & & & 10 \\
\hline Methyl methanesulfonate & $15-\pi U L-97 \quad 14: 10$ & 0.24 & ND & & & & 10 \\
\hline Ethyl methanesulfonate & 15-JUL-97 $14: 10$ & 1.5 & ND & & & & 10 \\
\hline Phenol & $15-\pi \pi-97 \quad 14: 10$ & 0.62 & 2.4 & & $\mathrm{~J}$ & & 10 \\
\hline Aniline & $15-J U L-97 \quad 14: 10$ & 0.26 & ND & & & & 10 \\
\hline 2-Chlorophenol & $15-\mathrm{JUL}-97 \quad 14: 10$ & 0.27 & ND & & & & 10 \\
\hline 1,3-Dichlorobenzene & $15-J U L-97 \quad 14: 10$ & 0.36 & ND & & & & 10 \\
\hline 1,4-Dichlorobenzene & $15-\pi U_{L}-97 \quad 14: 10$ & 0.32 & $\mathrm{ND}$ & & & & 10 \\
\hline Benzyl Alcohol & $15-J U L-97 \quad 14: 10$ & 0.29 & ND & & & & 20 \\
\hline 1,2-Dichlorobenzene & $15-\mathrm{JUL}-97 \quad 14: 10$ & 0.32 & ND & & & & 10 \\
\hline 2-Methylphenol & $15-\pi \Omega-97 \quad 14: 10$ & 0.20 & ND & & & & 10 \\
\hline Bis(2-chloroisopropyl) ether & 15-JUL-97 $14: 10$ & 0.35 & ND & & & & 10 \\
\hline 4-Methylphenol & $15-\pi \tilde{L}-97 \quad 14: 10$ & 0.18 & ND & & & & 10 \\
\hline Hexachloroethane & $15-\mathrm{JUL}-97 \quad 14: 10$ & 0.35 & $\mathrm{ND}$ & & & & 10 \\
\hline Bis (2-chloroethyl) ether & $15-\pi U_{L}-97 \quad 14: 10$ & 0.29 & ND & & & & 10 \\
\hline Acetophenone & $15-J U L-97 \quad 14: 10$ & 0.12 & 1.8 & & $\mathrm{~J}$ & & 10 \\
\hline Nitrobenzene & $15-\pi U-97 \quad 14: 10$ & 0.36 & $\mathrm{ND}$ & & & & 10 \\
\hline N-nitrosopiperidine & $15-\pi U L-97 \quad 14: 10$ & 1.3 & ND & & & & 10 \\
\hline Isophorone & $15-\pi U L-97 \quad 14: 10$ & 0.22 & ND & & & & 10 \\
\hline 2-Nitrophenol & $15-\pi U L-97 \quad 14: 10$ & 0.24 & ND & & & & 10 \\
\hline 2,4-Dimethvlphenol & 15-JUL-97 $14: 10$ & 0.11 & ND & & & & 10 \\
\hline Benzoic acid & $15-J U L-97 \quad 14: 10$ & 5.6 & ND & & & & 50 \\
\hline Bis (2-chloroethoxy) methane & $15-$ JUL-97 $14: 10$ & 0.25 & ND & & & & 10 \\
\hline 2, A-Dichlorophenol & $15-\pi U L-97 \quad 14: 10$ & 0.16 & $\mathrm{ND}$ & & & & 10 \\
\hline A. A.-dimethylphenethylamine & $15-\pi L-97 \quad 14: 10$ & 4.1 & ND & & & & 50 \\
\hline 1,2,4-Trichlorobenzene & $15-\pi U L-97 \quad 14: 10$ & 1.1 & ND & & & & 10 \\
\hline Naphthalene & $15-\pi U L-97 \quad 14: 10$ & 0.29 & ND & & & & 10 \\
\hline 4-Chloroaniline & 15-JUL-97 $14: 10$ & 0.77 & ND & & & & 20 \\
\hline 2,6-Dichlorophenol & $15-\pi U-97 \quad 14: 10$ & 0.50 & ND & & & & 10 \\
\hline Hexachlorobutadiene & 15-JUL-97 $14: 10$ & 0.30 & ND & & & & 10 \\
\hline N-nitroso-di-n-propylamine & $15-\pi \pi-97 \quad 14: 10$ & 0.27 & ND & & & & 10 \\
\hline 4-Cinloro-3-methylphenol & $15-\pi U L-97 \quad 14: 10$ & 0.28 & ND & & & & 20 \\
\hline 2-Nethylnaphthalene & $15-J U L-97 \quad 14: 10$ & 0.29 & ND & & & & 10 \\
\hline $1,2,4,5$-Tetrachlorobenzene & $15-J U L-97 \quad 14: 10$ & 4.3 & ND & & & & 10 \\
\hline Hexachlorocvclopentadiene & 15-JUL-97 $14: 10$ & 0.17 & ND & & & & 10 \\
\hline 2,4,6-Trichlorophenol & 15-JUL-97 $14: 10$ & 0.17 & ND & & & & 10 \\
\hline 2,4,5-Trichlorophenol & $15-\pi U L-97 \quad 14: 10$ & 0.19 & ND & & & & 10 \\
\hline 2-Chloronaphthalene & $15-\pi U L-97 \quad 14: 10$ & 0.12 & ND & & & & 10 \\
\hline 1-Chloronaphthalene & $15-$ JUL-97 $14: 10$ & 0.22 & ND & & & & 10 \\
\hline
\end{tabular}

$2 ? 0$ 


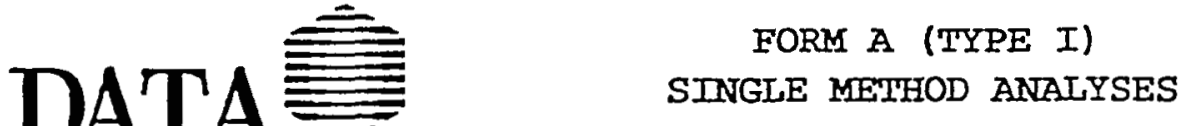
CHEM A A Borenson Company
SAMPLE ANAIYSIS DATA SHEET
Form RLIMS63A-V1.3 07229719104524

Page 12

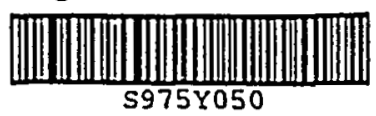

Date Printed........ 22-JUL-97 19:08

client Nane........ SAIC
DE: Sample Name. . : 97C02898

DE: Report Group.. : 97C-0208-02

Analytical Results

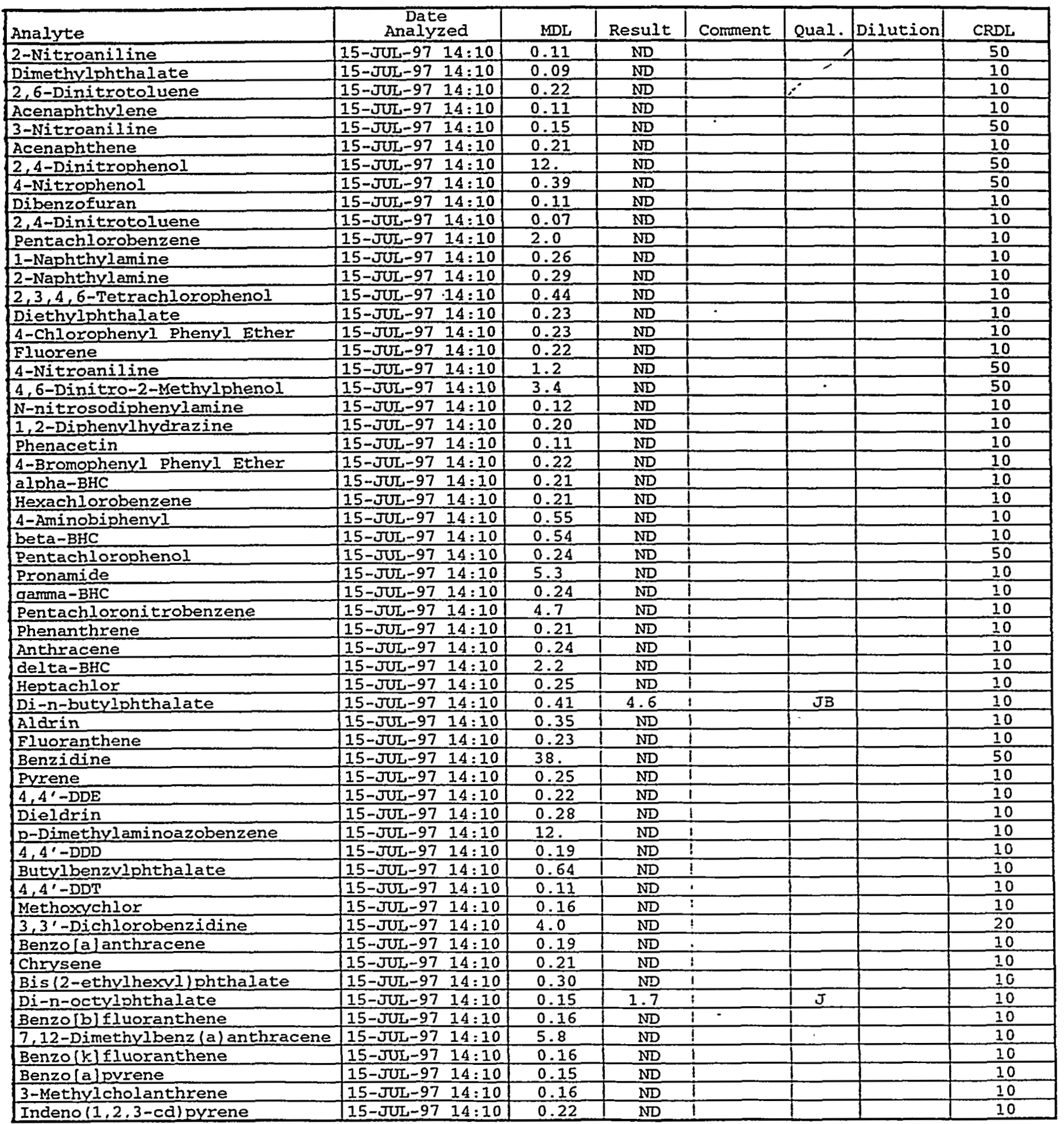

$2 ? ?$

960 West LeVoy Drive

Phone (801) 266-7700

FAX (801) 2.68-999?
Salt Lake City, utah 8\$123-2547

Web Page: www.ảatachem.com

F.-ma;1. 1 a-3Aatarin=m anm 


\section{DATA CHEM \\ A Sorenson Company}

FORM A (TYPE I)

SINGLE METHOD ANALYSES

SAMPLE ANALYSIS DATA SHEET
Form RLIMS63A-V1.3 07229719104524

Page 13

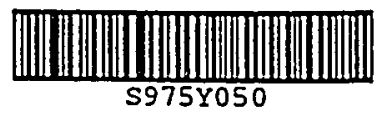

DCL Sample Name...: 97002898

DCL Report Group... 97C-0208-02

Client Name........ SAIC

Analytical Results

\begin{tabular}{|c|c|c|c|c|c|c|c|}
\hline Analyte & $\begin{array}{c}\text { Date } \\
\text { Analyzed } \\
\end{array}$ & MDL & Result & Comment & Qual. & Dilution & CRDL \\
\hline Dibenz $[a, h]$ anthracene & $15-\pi U L-97 \quad 14: 10$ & 0.28 & ND & & & & 10 \\
\hline Benzo[ghi]perylene & $15-J U L-97 \quad 14: 10$ & 0.32 & ND & & - & & 10 \\
\hline
\end{tabular}

Tentatively Identified Compound Results

\begin{tabular}{|c|c|c|c|c|c|}
\hline Aralyte (Retention Time) & $\begin{array}{c}\text { Date } \\
\text { Analyzed } \\
\end{array}$ & Result & Comment & Qual. & Dilution \\
\hline Cyclohexanone $(4.16)$ & $15-J U L-97 \quad 14: 10$ & 140 & & $\mathrm{~J}$ & \\
\hline
\end{tabular}

\section{Surrogate Recoveries}

\begin{tabular}{|l|c|c|c|}
\hline Analyte & Result & $\begin{array}{c}\text { Spiked } \\
\text { Amount }\end{array}$ & $\begin{array}{c}\text { Percent } \\
\text { Recovery }\end{array}$ \\
\hline 2,4,6-Tribromophenol & 39.2 & 100. & 39.2 \\
\hline 2-Fluorobiphenyl & 87.0 & 100. & 87.0 \\
\hline 2-Eluorophenol & 34.2 & 100. & 34.2 \\
\hline Nitrobenzene-d5 & 89.1 & 100. & 89.1 \\
\hline Phenol-d5 & 36.9 & 100. & 36.9 \\
\hline Terohenyl-d14 & 99.8 & 100. & 99.8 \\
\hline
\end{tabular}

238 


\section{Dy \\ DATA \\ SINGLE METHOD ANALYSES} CHEM

A Sorenson Company
SAMPLE ANALYSIS DATA SHEET
Form RLIMS63A-V1.3 07229719104524

Page 14

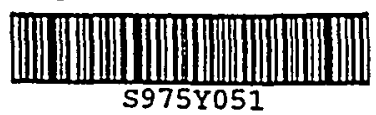

Date Printed.......: 22-JUL-97 19:08

Client Name......... SAIC

Client Ref Number.... : Not Provided

Sampling Site....... Not Provided

Release Number....... Not Provided

Date Received......: 27-JUN-97 00:00

DCL Preparation Group: G9760008

Date Prepared.......: 01-JUL-97 00:00

Preparation Method. . . : 3510

Aliquot Weight/Volume: 1000 Mus

Net Weight/Volume. . . . : Not Required
Client Sample Name: NAP-R1-S4

DCL Sample Name...: 97C02901

DCL Report Group. . : 97C-0208-02

Matrix........ : WATER

Date Sampled.....: 25-JUN-97 00:00

Reporting Units... ug/L

Report Basis.....: $\square$ As Received $\square$ Dried

DCL Analysis Group: G9760008

Analysis Method...: 8270B

Instrument Type...: GC/MS SV

Instrument ID....:5972-N

Column Type......: DB5,30m $\times .32 \mathrm{~mm}$

圆 Primary

DConfimation

\section{Analytical Results}

\begin{tabular}{|c|c|c|c|c|c|c|c|}
\hline Analyte & $\begin{array}{c}\text { Date } \\
\text { Analyzed }\end{array}$ & $\mathrm{MDL}$ & Result & Comment & Qual. & Dilution & CRDL \\
\hline Pyridine & $15-\pi U L-97 \quad 14: 43$ & 0.47 & ND & & & & 10 \\
\hline $\mathrm{N}$-nitrosodimethylamine & $15-\pi U L-97 \quad 14: 43$ & 0.23 & ND & & & & 10 \\
\hline 2-Picoline & $15-\pi U L-97 \quad 14: 43$ & 3.4 & ND & & & & 10 \\
\hline Ethyl methanesulfonate & 15-JUL-97 $14: 43$ & 1.5 & $\mathrm{ND}$ & & & & 10 \\
\hline Phenol & $15-\pi U L-97 \quad 14: 43$ & 0.62 & 2.8 & & $\mathbf{J}$ & & 10 \\
\hline Aniline & 15-JUL-97 $14: 43$ & 0.26 & ND & & & & 10 \\
\hline 1,4-Dichlorobenzene & $15-\pi U L-97 \quad 14: 43$ & 0.32 & ND & & & & 10 \\
\hline Benzyl Alcohol & $15-\pi U L-97 \quad 14: 43$ & 0.29 & ND & & & & 20 \\
\hline 1,2-Dichlorobenzene & $15-\pi U L-97 \quad 14: 43$ & 0.32 & ND & & & & 10 \\
\hline 2-Methvlphenol & 15-JUL-97 14:43 & 0.20 & ND & & & & 10 \\
\hline Bis(2-chloroisopropyl) ether & $15-\pi U L-97 \quad 14: 43$ & 0.35 & ND & & & & 10 \\
\hline 4-Methvlphenol & $15-\mathrm{JUL}-97 \quad 14: 43$ & 0.18 & ND & & & & 10 \\
\hline Hexachloroethane & $15-\pi \pi L-97 \quad 14: 43$ & 0.35 & ND & & & & 10 \\
\hline 2-Nitrophenol & 15-JUL-97 14:43 & 0.24 & ND & & & & 10 \\
\hline 2, 4-Dimethylphenol & $15-\pi U L-97 \quad 14: 43$ & 0.11 & ND & & & & 10 \\
\hline Benzoic acid & $15-\pi U L-97 \quad 14: 43$ & 6.6 & ND & & & & 50 \\
\hline Bis $(2-$ chloroethoxy) methane & $15-\pi U L-97 \quad 14: 43$ & 0.25 & ND & & & & 10 \\
\hline 2,4-Dichlorophenol & $15-\pi U L-97 \quad 14: 43$ & 0.16 & ND & & & & 10 \\
\hline A.,A.-dimethylphenethylamine & 15-JUL-97 $14: 43$ & 4.1 & ND & & & & 50 \\
\hline $1,2,4$-Trichlorobenzene & 15-JUL-97 $14: 43$ & 1.1 & ND & & & & 10 \\
\hline Naphthalene & 15-JUL-97 $14: 43$ & 0.29 & ND & & & & 10 \\
\hline 4-Chloroaniline & $15-\pi \mathrm{J}-97 \quad 14: 43$ & 0.77 & ND & & & & 20 \\
\hline 2,6-Dichlorophenol & $15-\pi U L-97 \quad 14: 43$ & 0.50 & ND & & & & 10 \\
\hline Hexachlorobutadiene & $15-\pi U L-97 \quad 14: 43$ & 0.30 & ND & & & & 10 \\
\hline N-nitroso-di-n-propylamine & $15-J U L-97 \quad 14: 43$ & 0.27 & ND & & & & 10 \\
\hline 4-Chloro-3-methylphenol & $15-J U L-97 \quad 14: 43$ & 0.28 & ND & & & & 20 \\
\hline
\end{tabular}

239
960 West LeVoy Drive / Salt Lake City, Utah 84123-2547

Phone (801) 266-7700

FAX (801) 268-9992
Web Page: www.datachem.com

F.-mail. iahoratarhem $\mathrm{nnm}$ 


\section{DATA CHEM \\ A Sorenson Company}

FORM A (TYPE I) SINGLE METHOD ANALYSES

SAMPLE ANAIYSIS DATA SHEET
Form RLIMS63A-V1.3 07229719104524

Page 15

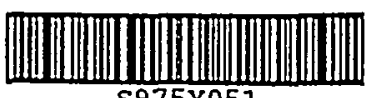

DCL Sample Name. . : 97C02901

DCL Report Group... : 97C-0208-02
Date Printed....... : 22-JUL-97.19:08

Client Name.........: SAIC

\section{Analytical Results}

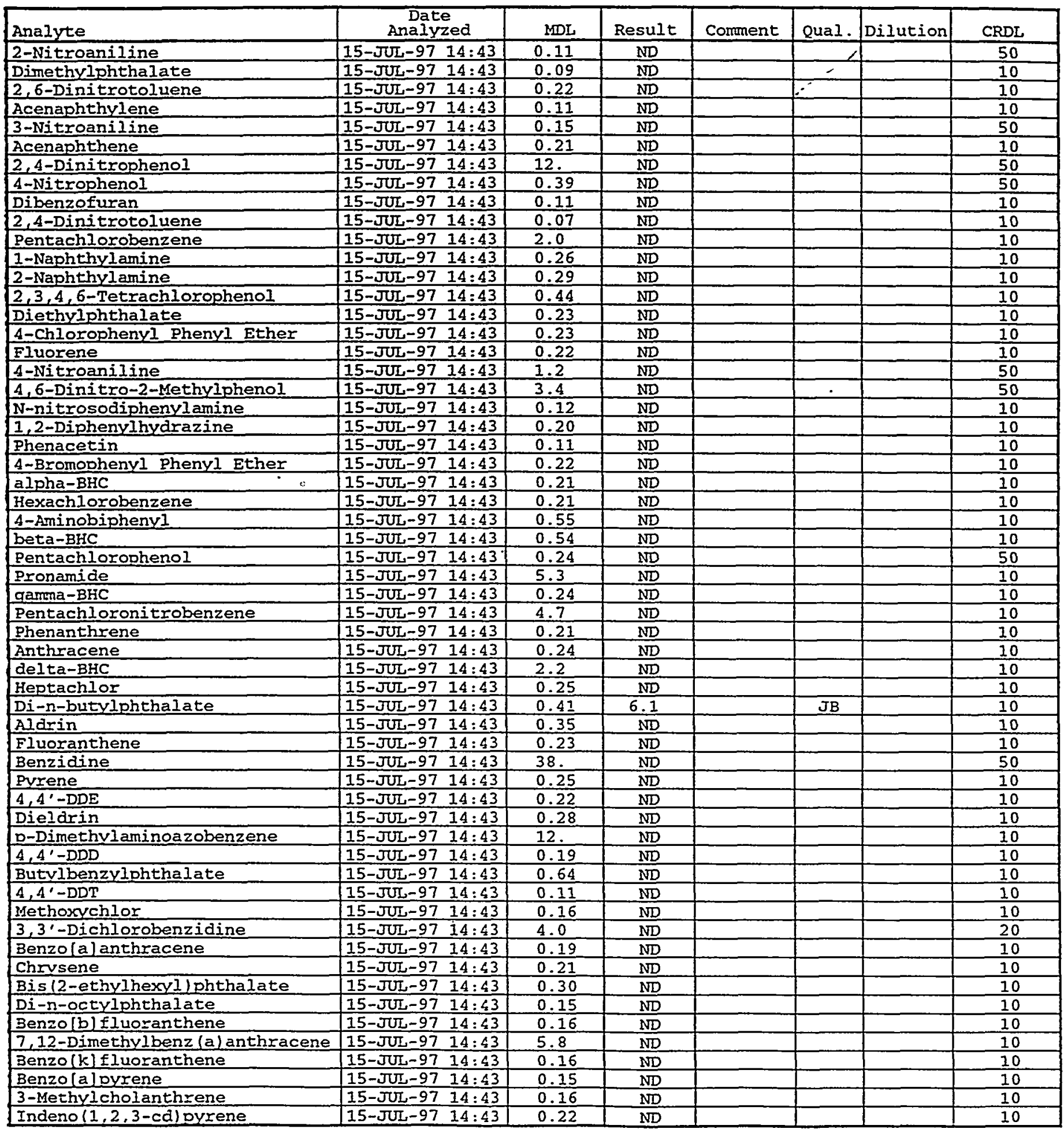




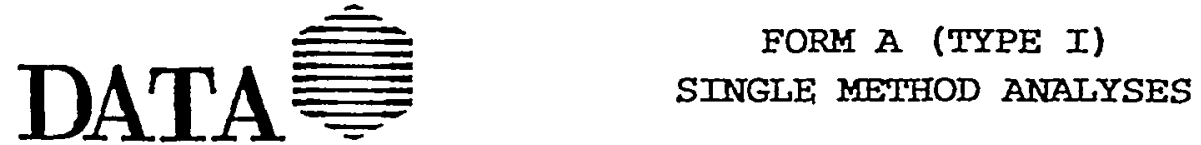
C A Sorenson Company

Date Printed.......: 22-JUL-97 19:08

Client Name........ SAIC
Form RLIMS63A-V1.3

07229719104524

Page 16

SAMPLE ANALYSIS DATA SHEET

DCL Sample Name. . : 97C02901

DCL Report Group.. : 97C-0208-02

Analytical Results

\begin{tabular}{|c|c|c|c|c|c|c|c|}
\hline Analyte & $\begin{array}{c}\text { Date } \\
\text { Analyzed }\end{array}$ & MDL & Result & Corment & Qual. & Dilution & CRDL \\
\hline Dibenz $[a, h]$ anthracene & $15-\pi U L-97 \quad 14: 43$ & 0.28 & $\mathrm{ND}$ & & & & 10 \\
\hline Benzo(ghi)perylene & $15-\pi U L-97 \quad 14: 43$ & 0.32 & ND & & & & 10 \\
\hline
\end{tabular}

Tentatively Identified Compound Results

\begin{tabular}{|c|c|c|c|c|c|}
\hline Analyte (Retention Time) & $\begin{array}{c}\text { Date } \\
\text { Anaiyzed }\end{array}$ & Result & Comment & Qual. & Dilution \\
\hline Cvclohexanone (4.17) & $15-J U L-97 \quad 14: 43$ & 150 & & $\mathrm{~J}$ & \\
\hline
\end{tabular}

\section{Surrogate Recoveries}

\begin{tabular}{|l|c|c|c|}
\hline Analyte & Result & $\begin{array}{c}\text { Spiked } \\
\text { Amount }\end{array}$ & $\begin{array}{c}\text { Percent } \\
\text { Recovery }\end{array}$ \\
\hline 2,4,6-Tribromophenol & 73.0 & 100. & 73.0 \\
\hline 2-Eluorobiphenyl & 75.4 & 100. & 75.4 \\
\hline 2-Fluorophenol & 47.7 & 100. & 47.7 \\
\hline Nitrobenzene-d5 & 73.0 & 100. & 73.0 \\
\hline Phenol-d5 & 34.5 & 100. & 34.5 \\
\hline Terphenyl-d14 & 88.1 & 100. & 88.1 \\
\hline
\end{tabular}




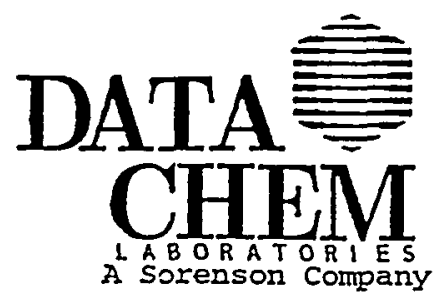

Client Name........ SAIC

Release Number....... Not Provided

Matrix........... WATER

Reporting Units.....: ug/L

DCI Preparation Group: 69760008

Date Prepared.......: 01-JUL-97 00:00

Preparation Method... : 3510

Analytical Results

\begin{tabular}{|c|c|c|c|c|c|c|}
\hline Analyte & $\begin{array}{c}\text { Date } \\
\text { Analyzed }\end{array}$ & Target & Result & $\begin{array}{c}\text { Percent } \\
\text { Recovery }\end{array}$ & $\begin{array}{c}Q C \\
\text { Linits }\end{array}$ & $\begin{array}{c}\text { QC } \\
\text { Elag }\end{array}$ \\
\hline $1,2,4$-Trichlorobenzene & $15-3 U L-97 \quad 12: 29$ & 100 & 65.8 & 65.8 & $44.0 / 142$ & \\
\hline 1,4-Dichlorobenzene & $15-\pi \pi-97 \quad 12: 29$ & 100. & 78.3 & 78.3 & $20.0 / 124$ & \\
\hline 2,4-Dinitrotoluene & $15-\pi U L-97 \quad 12: 29$ & 100 . & 93.2 & 93.2 & $39.0 / 139$. & \\
\hline 2-Chlorophenol & $15-\pi U L-97 \quad 12: 29$ & 100. & 59.8 & 59.8 & $23.0 / 134$ & \\
\hline 4-Chloro-3-methylphenol & $15-\pi U L-97 \quad 12: 29$ & 100. & 86.3 & 86.3 & $22.0 / 147$. & \\
\hline 4-Nitrophenol & $15-\pi U \pi-97 \quad 12: 29$ & 100. & 12.6 & 12.6 & $0.00 / 132$ & \\
\hline Acenaphthene & $15-J U L-97 \quad 12: 29$ & 100. & 92.2 & 92.2 & $47.0 / 145$ & \\
\hline N-Nitroso-dipropylamine & $15-\pi U L-97 \quad 12: 29$ & 100. & 138. & 138. & $0.00 / 230$ & \\
\hline Pentachlorophenol & $15-\pi \pi-97 \quad 12: 29$ & 100. & 33.2 & 33.2 & $14.0 / 176$ & \\
\hline Phenol & $15-\pi \pi L-97 \quad 12: 29$ & 100. & 34.3 & 34.3 & $5.00 / 112$ & \\
\hline Dyrene & $15-\pi U L-97 \quad 12: 29$ & 100. & 93.1 & 93.1 & $52.0 / 115$. & \\
\hline
\end{tabular}

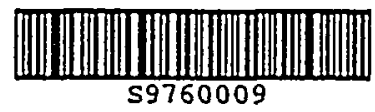

DCL Sample Name. . : QC-136532-2

Analytical Results

\begin{tabular}{|c|c|c|c|c|c|c|c|c|}
\hline Analyte & $\begin{array}{c}\text { Date } \\
\text { Analyzed }\end{array}$ & $\begin{array}{c}\text { Duplicate } \\
\text { Result }\end{array}$ & $\begin{array}{l}\text { Percent } \\
\text { Recovery }\end{array}$ & Mean & Range & RPD & $\underset{\text { Iimits }}{Q C}$ & Flag \\
\hline 1,2,4-Trichlorobenzene & $15-\pi \pi-97 \quad 13: 02$ & 54.1 & 54.1 & 60.0 & 11.7 & 20. & $0.00 / 28.0$ & \\
\hline I,4-Dichlorobenzene & $15-J U L-97 \quad 13: 02$ & 62.2 & 62.2 & 70.3 & 16.1 & 23. & $0.00 / 28.0$ & \\
\hline 2,4-Dinitrotoluene & $15-\pi U L-97 \quad 13: 02$ & 87.4 & 87.4 & 90.3 & 5.71 & 6.3 & $0.00 / 38.0$ & \\
\hline 2-Chlorophenol & $15-J U L-97 \quad 13: 02$ & 66.2 & 66.2 & 63.0 & 6.45 & 10. & $0.00 / 40.0$ & \\
\hline 4-Chloro-3-methylphenol & $15-\pi U L-97 \quad 13: 02$ & 83.2 & 83.2 & 84.7 & 3.02 & 3.6 & $0.00 / 42.0$ & \\
\hline A-Nicrophenol & $15-\pi \sqrt{2}-97 \quad 13: 02$ & 30.1 & 30.1 & 21.4 & 17.5 & 82. & $0.00 / 50.0$ & * \\
\hline Acenaphthene & $15-\pi U L-97 \quad 13: 02$ & 82.0 & 82.0 & 87.1 & 10.2 & 12. & $0.00 / 31.0$ & \\
\hline N-NiEroso-dipropvlamine & $15-\pi U-97 \quad 13: 02$ & 114. & 114 . & 126 . & 24.6 & 20 . & $0.00 / 38.0$ & \\
\hline Pentachlorophenol & $15-J U L-97 \quad 13: 02$ & 72.2 & 72.2 & 52.7 & 39.0 & 74. & $0.00 / 50.0$ & 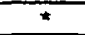 \\
\hline ghenol & $15-\pi U L-97 \quad 13: 02$ & 36.7 & 36.7 & 35.5 & 2.32 & 6.5 & $0.00 / 42.0$ & \\
\hline Zyrene & $15-\pi U L-97 \quad 13: 02$ & 109. & 109. & 101. & 15.9 & 16. & $0.00 / 31.0$ & \\
\hline
\end{tabular}




\section{DATA CHEM \\ L A B OR A T OR I ES
A Sorenson Company}

client Name........ SAIC

Release Number....... Not Provided

Iatrix. . . . . . . . : WATER

Reporting Units...... ug/L

\section{Surrogate Recoveries}

\begin{tabular}{|c|c|c|c|c|c|c|c|c|c|}
\hline $\begin{array}{l}\text { QC Limits } \\
\text { DCL Sample } \\
\text { Number }\end{array}$ & $\begin{array}{l}\text { Analyte } \\
\text { Result }\end{array}$ & $\begin{array}{l}\text { Spiked } \\
\text { Amount }\end{array}$ & $\operatorname{Rec}^{\frac{6}{6}}$ & $\begin{array}{l}\text { Analyte } \\
\text { Result }\end{array}$ & $\begin{array}{l}\text { Splkea } \\
\text { Amount }\end{array}$ & Rec. & $\begin{array}{l}\text { Analyte } \\
\text { Result }\end{array}$ & $\begin{array}{l}\text { Splked } \\
\text { Amount }\end{array}$ & $\begin{array}{c}8 \\
\text { Rec. }\end{array}$ \\
\hline 97002897 & 63.0 & 100. & 63.0 & 75.6 & 100 & 75.6 & 40.5 & 100 & 40.5 \\
\hline $97 C 02898$ & 39.2 & 100. & 39.2 & 87.0 & 100. & 87.0 & 34.2 & 100. & 34.2 \\
\hline $97 \mathrm{C02901}$ & 73.0 & 100 . & 73.0 & 75.4 & 100 . & 75.4 & 47.7 & 100. & 47.7 \\
\hline $\mathrm{BL}-136532-1$ & 60.9 & 100. & 60.9 & 73.2 & 100. & 73.2 & 47.1 & 100. & 47.1 \\
\hline Qc-136532-1 & 45.0 & 100. & 45.0 & 81.1 & 100. & 81.1 & 33.8 & 100. & 33.8 \\
\hline$Q c-136532-2$ & 65.7 & 100. & 65.7 & 71.0 & 100. & 71.0 & 43.6 & 100. & 43.6 \\
\hline
\end{tabular}

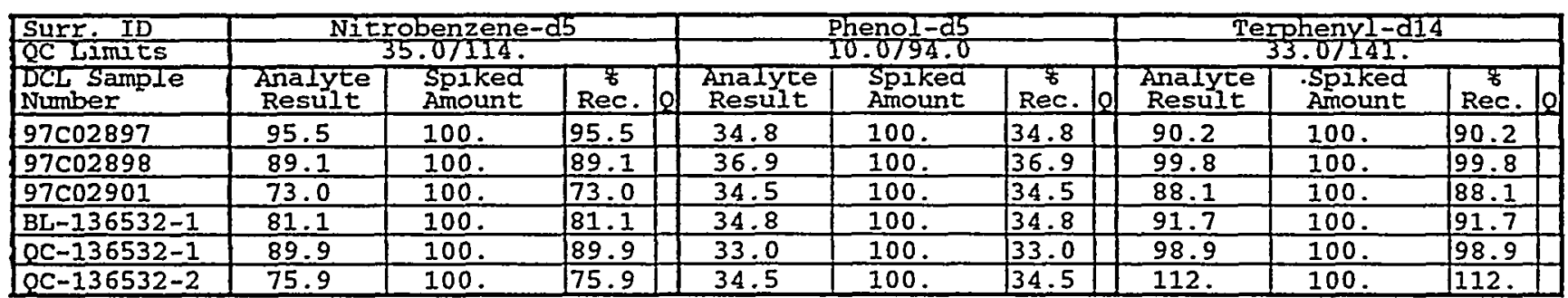

Form RLIMS63G-V1.3 07229719104524 Page 18

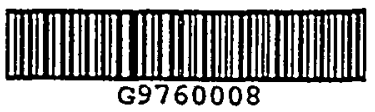

Prep Group ... : G9760008

Preparation Method: 3510

QC Limit Type:.... . Method 


\section{Form 4 Equivalent \\ DataChem Laboratories \\ LIMS - Sample Master System \\ Client Group Report}

Date: 22-JUL-1997 19:12

User: ENDRES

Page: 1

RLIVS15-V1.2

Client Group Name: 97C-0208-02

Group ID: G975Y016

Samples: 6

\begin{tabular}{|c|c|c|}
\hline Pos & $\begin{array}{l}\text { Laboratory } \\
\text { Sample Name }\end{array}$ & $\begin{array}{l}\text { Field Sample } \\
\text { Name } 1\end{array}$ \\
\hline & & \\
\hline & $\begin{array}{l}B L-136532-1 \\
Q C-136532-1 \\
Q C-136532-2 \\
97 C 02897 \\
97 C 02898 \\
97 C 02901\end{array}$ & $\begin{array}{l}B L-136532-1 \\
Q C-136532-1 \\
Q C-136532-2 \\
\text { NAP-R2-S2-B } \\
\text { NAP-R2-S4 } \\
\text { NAP-R1-S4 }\end{array}$ \\
\hline
\end{tabular}

Field Sample Name 2

Laboratory

Sample ID

59760007

S9760008

S9760009

S975Y04Z

S975Y050

S975Y051

\begin{tabular}{ll} 
Labozatory & Accnt. \\
Group Name & Number \\
\hdashline $97 c-0208-02$ & 03006 \\
$97 \mathrm{C}-0208-02$ & 03006 \\
$97 \mathrm{C}-0208-02$ & 03006 \\
$97 \mathrm{C}-0208-02$ & 03006 \\
$97 \mathrm{C}-0208-02$ & 03006 \\
$97 \mathrm{C}-0208-02$ & 03006
\end{tabular}


Form S Equiralents

DFTPP 625 Results

C: \HPCHEM \I\DATA II9JUN97N\NQFOITUN.D
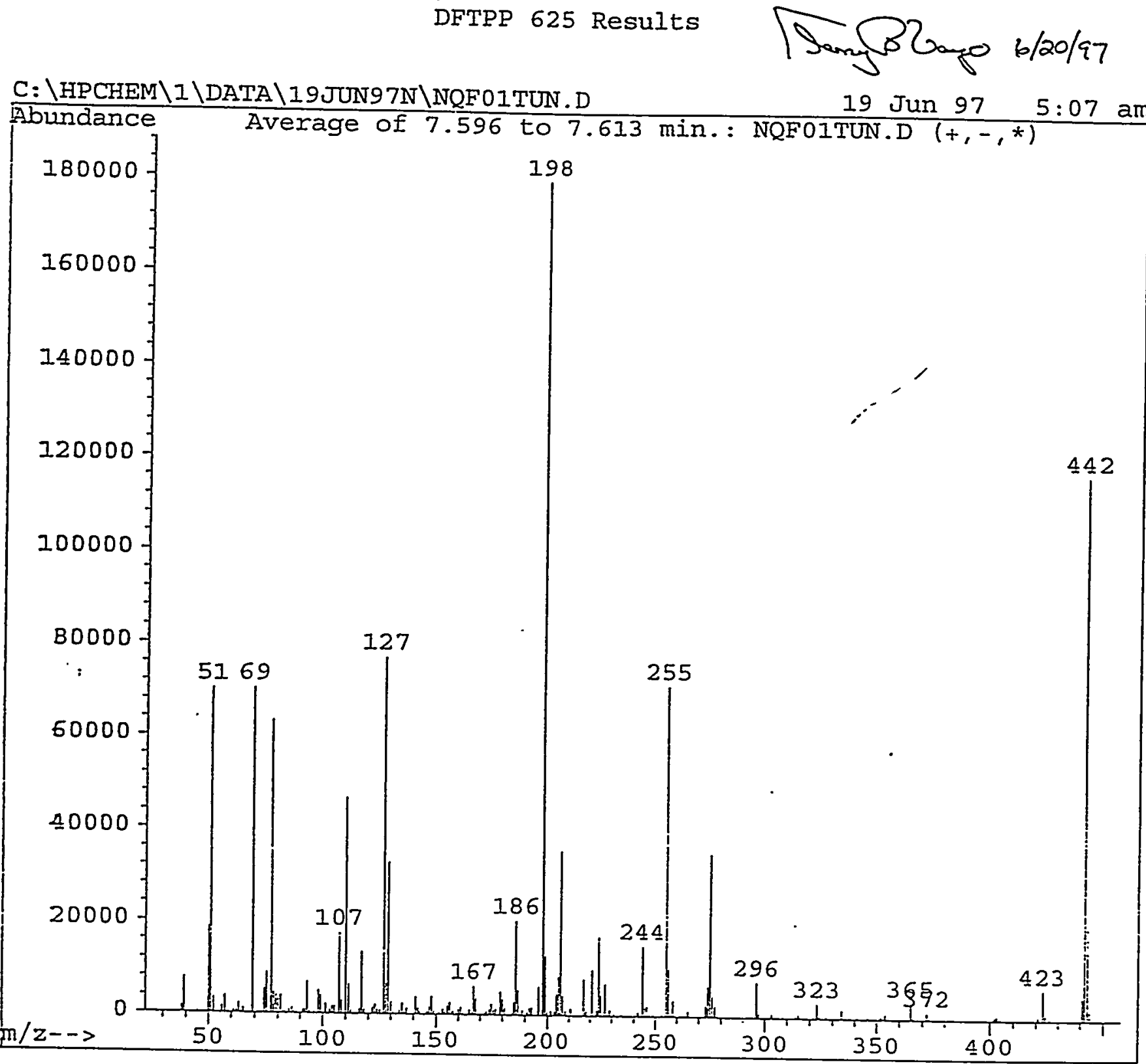

Peak Apex is scan: 296.

Average of 3 scans: 295,296,297 minus background scan 290

\begin{tabular}{|c|c|c|c|c|c|}
\hline $\begin{array}{l}\text { Target } \\
\text { Mass }\end{array}$ & $\begin{array}{c}\text { Comparison } \\
\text { Mass }\end{array}$ & $\begin{array}{l}\text { Lower } \\
\text { Iimit, \% }\end{array}$ & $\begin{array}{l}\text { Upper } \\
\text { Limit, \% }\end{array}$ & $\begin{array}{l}\text { Relative } \\
\text { Abundance, } \%\end{array}$ & $\begin{array}{c}\text { Result } \\
\text { Pass/Fail }\end{array}$ \\
\hline $\begin{array}{r}51 \\
68 \\
69 \\
70 \\
127 \\
197 \\
198 \\
199 \\
275 \\
365 \\
441 \\
442 \\
443\end{array}$ & $\begin{array}{r}198 \\
69 \\
198 \\
69 \\
198 \\
198 \\
198 \\
198 \\
198 \\
198 \\
443 \\
198 \\
442\end{array}$ & $\begin{array}{r}30 \\
0 \\
0 \\
0 \\
40 \\
0 \\
100 \\
5 \\
10 \\
1 \\
0 \\
40 \\
17\end{array}$ & $\begin{array}{r}60 \\
2 \\
100 \\
2 \\
60 \\
1 \\
100 \\
9 \\
30 \\
100 \\
100 \\
100 \\
23\end{array}$ & $\begin{array}{r}39.2 \\
0.0 \\
39.3 \\
0.0 \\
43.0 \\
0.0 \\
100.0 \\
7.2 \\
19.6 \\
2.0 \\
22.7 \\
65.3 \\
18.8\end{array}$ & $\begin{array}{l}\text { PASS } \\
\text { PASS } \\
\text { PASS } \\
\text { PASS } \\
\text { PASS } \\
\text { PASS } \\
\text { PASS } \\
\text { PASS } \\
\text { PASS } \\
\text { PASS } \\
\text { PASS } \\
\text { PASS } \\
\text { PASS }\end{array}$ \\
\hline
\end{tabular}


Data File:

Date Acquired:

Operator ID:

Name :

Misc:

Cal Title:

Last Cal Update: Wed Apr 09 20:45:09 1997

EXP. $11 / 7 / 97$
NQEOITUN . D

06/19/97 05:07

TV

50 NG DFTPP (136WS27184)

EXP. 11/7/97
Instrument: $5972-\mathrm{N}$

Acq Method: DFTPP625

DA Method: DFTPP625.M

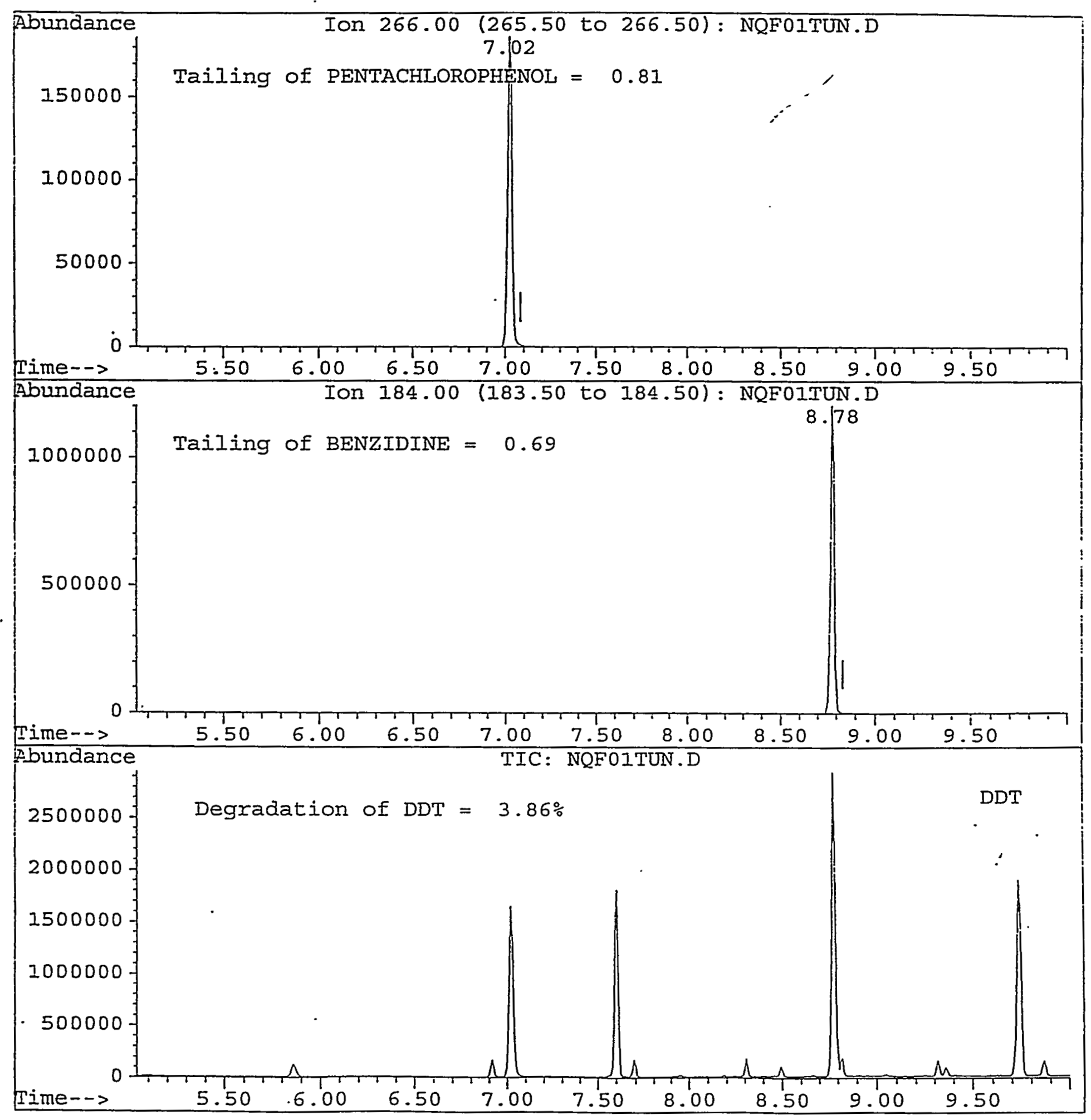

246 
Average of 7.596 to $7.613 \mathrm{~min}$.: NQFOITUN.D 50 NG DETPP (136WS27184)

Modified:added subtracted scaled

$\begin{array}{crcrc}\mathrm{m} / \mathrm{z} & \text { abund. } & \mathrm{m} / \mathrm{z} & \text { abund. } & \mathrm{m} / \mathrm{z} \\ 36.10 & 225 & 55.90 & 1678 & 74.90 \\ 36.70 & 186 & 56.95 & 3888 & 77.00 \\ 37.10 & 273 & 60.95 & 696 & 77.95 \\ 37.95 & 1661 & 62.00 & 771 & 78.90 \\ 39.00 & 7774 & 63.00 & 2208 & 79.90 \\ 39.85 & 172 & 64.00 & 337 & 80.90 \\ 44.90 & 350 & 64.95 & 1242 & 81.95 \\ 50.00 & 18753 & 66.95 & 151 & 83.00 \\ 51.00 & 70257 & 68.95 & 70378 & 84.95 \\ 51.90 & 3470 & 73.05 & 475 & 85.95 \\ 52.85 & 101 & 74.00 & 5447 & 86.90\end{array}$

Average of 7.596 to $7.613 \mathrm{~min}$.: NQFO1TUN.D

50 NG DETPP (136WS27184)

Modified:added subtracted scaled

$\begin{array}{crcrc}\mathrm{m} / \mathrm{z} & \text { abund. } & \mathrm{m} / \mathrm{z} & \text { abund. } & \mathrm{m} / \mathrm{z} \\ 102.95 & 981 & 117.95 & 1136 & 130.90 \\ 103.90 & 1669 & 118.85 & 101 & 131.95 \\ 104.95 & 1744 & 119.80 & 108 & 133.90 \\ 106.95 & 17421 & 121.90 & 1474 & 134.95 \\ 107.95 & 3076 & 122.90 & 2062 & 135.95 \\ 109.95 & 46613 & 123.95 & 1116 & 137.00 \\ 110.95 & 6525 & 124.95 & 939 & 139.70 \\ 111.95 & 806 & 126.90 & 77115 & 140.00 \\ 112.95 & 118 & 128.00 & 6777 & 141.00 \\ 116.05 & 1295 & 128.90 & 32704 & 142.00 \\ 116.95 & 13525 & 129.90 & 2845 & 142.80\end{array}$

Average of 7.596 to $7.613 \mathrm{~min} .:$ NQEOITUN.D

50 NG DFTPP (136WS27184)

Modified:added subtracted scaled

$\begin{array}{crcrc}\mathrm{m} / \mathrm{z} & \text { abund. } & \mathrm{m} / \mathrm{z} & \text { abund. } & \mathrm{m} / \mathrm{z} \\ 155.95 & 2699 & 167.90 & 3612 & 179.90 \\ 157.00 & 784 & 168.90 & 464 & 180.90 \\ 157.85 & 833 & 169.90 & 396 & 182.00 \\ 158.95 & 506 & 170.70 & 272 & 184.00 \\ 159.95 & 1264 & 171.90 & 572 & 185.00 \\ 160.95 & 1672 & 172.95 & 870 & 186.00 \\ 161.90 & 460 & 173.90 & 1450 & 187.00 \\ 163.90 & 258 & 174.95 & 2488 & 188.00 \\ 164.95 & 1272 & 176.00 & 1237 & 188.85 \\ 166.00 & 723 & 176.90 & 1414 & 189.85 \\ 166.90 & 6217 & 178.90 & 5173 & 190.90\end{array}$

Average of 7.596 to $7.613 \mathrm{~min}$. : NQFOITUN.D

50 NG DFTPP (136WS27184)

Modified:added subtracted scaled

$\begin{array}{crrrrrrr}\mathrm{m} / \mathrm{z} & \text { abund. } & \mathrm{m} / \mathrm{z} & \text { abund. } & \mathrm{m} / \mathrm{z} & \text { abund. } & \mathrm{m} / \mathrm{z} & \text { abund. } \\ 204.95 & 8547 & 223.00 & 1282 & 235.85 & 159 & 253.05 & 533 \\ 205.95 & 35518 & 223.90 & 17405 & 236.85 & 379 & 254.95 & 71427 \\ 206.95 & 4418 & 224.95 & 4557 & 240.85 & 364 & 255.95 & 10359 \\ 207.95 & 1174 & 225.90 & 116 & 241.90 & 926 & 257.00 & 863 \\ 208.95 & 351 & 226.90 & 6962 & 243.95 & 15145 & 257.90 & 3494 \\ 210.85 & 1626 & 228.00 & 992 & 244.95 & 2002 & 258.80 & 483 \\ 212.10 & 102 & 228.90 & 1407 & 245.90 & 2302 & 264.90 & 1329 \\ 214.95 & 315 & 229.90 & 116 & 246.90 & 424 & 272.95 & 2432 \\ 216.90 & 8039 & 230.90 & 576 & 248.85 & 578 & 274.00 & 6663 \\ 217.90 & 1115 & 233.85 & 381 & 249.95 & 105 & 274.90 & 35219 \\ 220.95 & 10012 & 234.85 & 439 & 251.95 & 112 & 275.90 & 4604\end{array}$


Zverage of 7.596 to $7.613 \mathrm{~min}$.: NQF01TUN.D 50 NG DFTPP (136WS27184)

!'odified:added subtracted scaled

$\begin{array}{rrrrrrrr}\mathrm{m} / z & \text { abund. } & \mathrm{m} / \mathrm{z} & \text { abund. } & \mathrm{m} / \mathrm{z} & \text { abund. } & \mathrm{m} / \mathrm{z} & \text { abund. } \\ 276.90 & 2396 & 315.95 & 431 & 351.90 & 836 & 390.95 & 121 \\ 277.95 & 351 & 320.90 & 182 & 352.90 & 630 & 402.00 & 814 \\ 282.95 & 108 & 323.00 & 3142 & 353.90 & 1183 & 402.95 & 1006 \\ 284.90 & 422 & 323.95 & 585 & 354.90 & 119 & 403.90 & 255 \\ 292.90 & 496 & 326.85 & 476 & 364.90 & 3603 & 420.90 & 829 \\ 295.95 & 7617 & 327.85 & 133 & 365.85 & 512 & 422.95 & 6489 \\ 296.90 & 1061 & 332.95 & 156 & 371.00 & 132 & 423.95 & 1259 \\ 302.90 & 941 & 333.95 & 1932 & 371.95 & 1575 & 441.00 & 4981 \\ 303.90 & 152 & 334.95 & 439 & 372.95 & 363 & 441.95 & 117085 \\ 314.00 & 329 & 340.95 & 469 & 382.95 & 349 & 442.95 & 21966 \\ 314.85 & 853 & 345.90 & 505 & 389.85 & 155 & 443.95 & 2086\end{array}$


$C: \backslash H P C H E M \backslash I \backslash D A T A \backslash 15 J U L 97 N \backslash N R A 01 T U N . D$

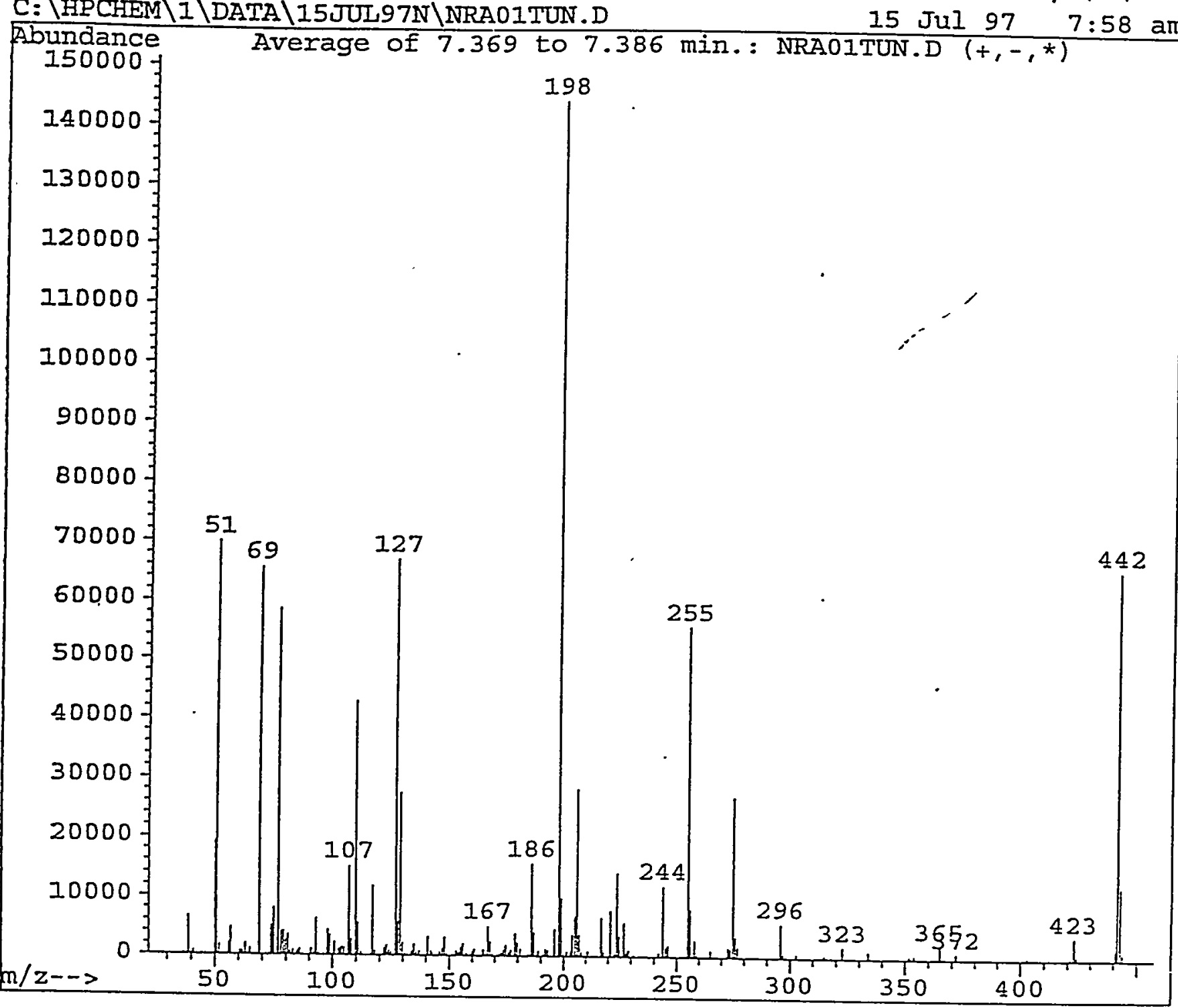

Peak Apex is scan: 256

Average of 3 scans: 255,256,257 minus background scan 251

\begin{tabular}{|c|c|c|c|c|c|}
\hline $\begin{array}{l}\text { Target } \\
\text { Mass }\end{array}$ & $\begin{array}{l}\text { Comparison } \\
\text { Mass }\end{array}$ & $\begin{array}{l}\text { Lower } \\
\text { Iimit, } \%\end{array}$ & $\begin{array}{l}\text { Upper } \\
\text { Limit, } \%\end{array}$ & $\begin{array}{l}\text { Relative } \\
\text { Abundance, } \%\end{array}$ & $\begin{array}{c}\text { Result } \\
\text { Pass/Fail }\end{array}$ \\
\hline $\begin{array}{r}51 \\
68 \\
69 \\
70 \\
127 \\
197 \\
198 \\
199 \\
275 \\
365 \\
441 \\
442 \\
443\end{array}$ & $\begin{array}{r}198 \\
69 \\
198 \\
69 \\
198 \\
198 \\
198 \\
198 \\
198 \\
198 \\
443 \\
198 \\
442\end{array}$ & $\begin{array}{r}30 \\
0 \\
0 \\
0 \\
40 \\
0 \\
100 \\
5 \\
10 \\
1 \\
0 \\
40 \\
17\end{array}$ & $\begin{array}{r}60 \\
2 \\
100 \\
2 \\
60 \\
1 \\
100 \\
9 \\
30 \\
100 \\
100 \\
100 \\
23\end{array}$ & $\begin{array}{r}48.6 \\
0.0 \\
45.5 \\
0.0 \\
46.5 \\
0.0 \\
100.0 \\
6.9 \\
18.8 \\
1.6 \\
14.8 \\
45.7 \\
18.9\end{array}$ & $\begin{array}{l}\text { PASS } \\
\text { PASS } \\
\text { PASS } \\
\text { PASS } \\
\text { PASS } \\
\text { PASS } \\
\text { PASS } \\
\text { PASS } \\
\text { PASS } \\
\text { PASS } \\
\text { PASS } \\
\text { PASS } \\
\text { PASS }\end{array}$ \\
\hline
\end{tabular}


Data File:

Date Acquired:

Operator ID:

Name:

Misc:

Cal Title:

Last Cal Update: Wed Apr 09 20:45:09 1997

EXP. $11 / 07 / 97$
NRAOITUN.D

07/15/97 07:58

TV

50 NG DETPP (143WS28451)

$\operatorname{EXP} .11 / 07 / 97$
Instrument: $5972-\mathrm{N}$

Acq Method: DFTPP625

DA Method: DFTPP625.M

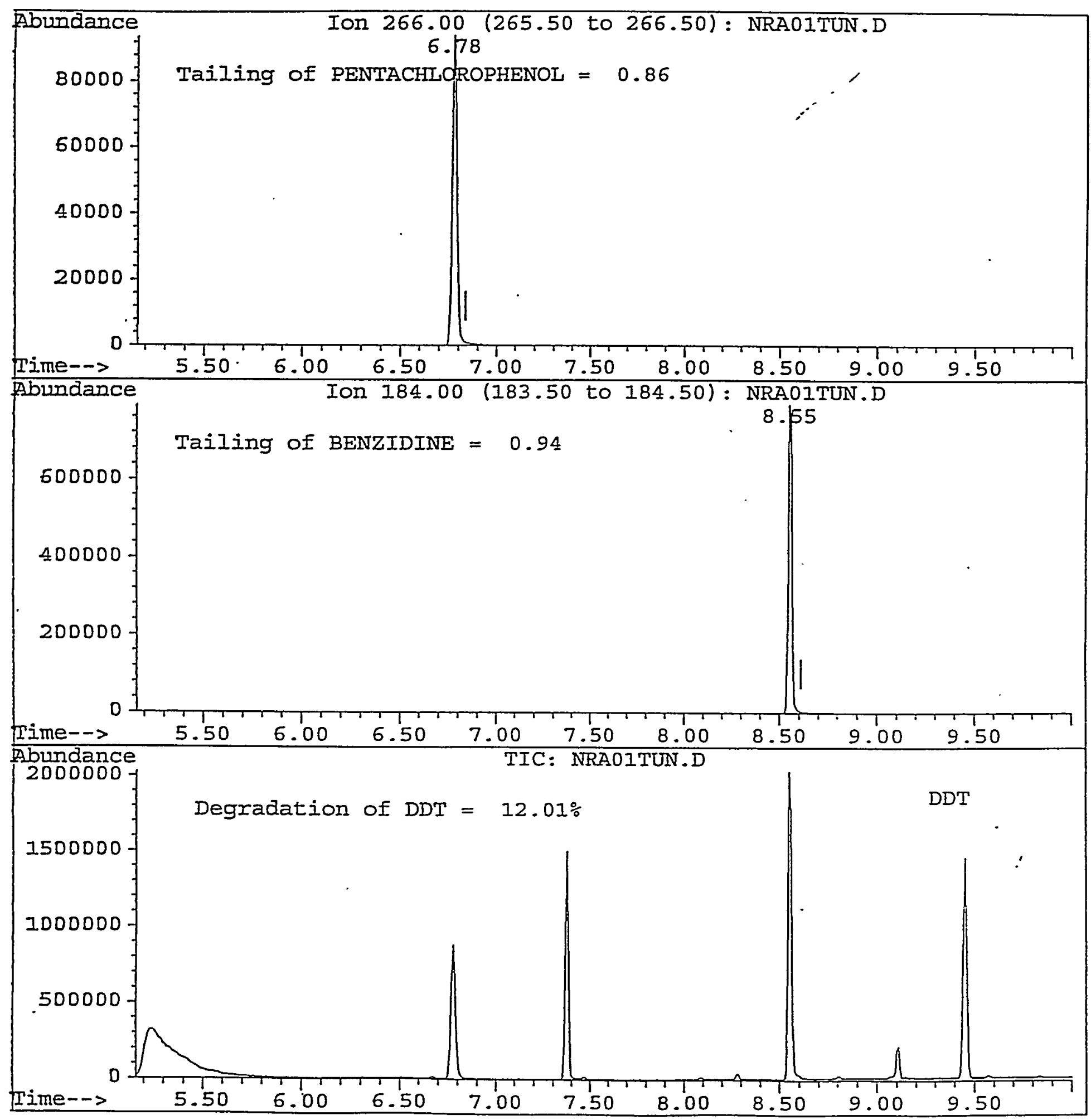

250 
Average of 7.369 to $7.386 \mathrm{~min}$.: NRADITUN.D 50 NG DFTPP (143WS28451)

Modified:added subtracted scaled

$\begin{array}{crrrr}\mathrm{m} / \mathrm{z} & \text { abund. } & \mathrm{m} / \mathrm{z} & \text { abund. } & \mathrm{m} / \mathrm{z} \\ 35.85 & 53 & 57.75 & 102 & 77.90 \\ 36.90 & 314 & 60.85 & 804 & 78.90 \\ 38.90 & 6668 & 61.90 & 831 & 79.90 \\ 39.95 & 341 & 62.90 & 2089 & 80.90 \\ 40.95 & 762 & 63.95 & 295 & 81.90 \\ 44.80 & 147 & 64.85 & 1168 & 82.90 \\ 50.00 & 19351 & 66.75 & 158 & 83.70 \\ 50.85 & 69907 & 68.85 & 65565 & 84.90 \\ 51.85 & 1892 & 73.90 & 5154 & 85.90 \\ 55.85 & 2128 & 74.90 & 8168 & 86.85 \\ 56.85 & 4745 & 76.90 & 58667 & 87.80\end{array}$

Average of 7.369 to $7.386 \mathrm{~min} .:$ NRAOITUN.D

50 NG DFTPP (143WS28451)

Modified:added subtracted scaled

$\begin{array}{crrrrrrr}\mathrm{m} / \mathrm{z} & \text { abund. } & \mathrm{m} / \mathrm{z} & \text { abind. } & \mathrm{m} / \mathrm{z} & \text { abund. } & \mathrm{m} / \mathrm{z} & \text { abund. } \\ 103.85 & 1598 & 119.85 & 262 & 133.90 & 849 & 146.65 & 179 \\ 104.85 & 1434 & 121.80 & 1306 & 134.90 & 1986 & 146.95 & 1352 \\ 106.85 & 15105 & 122.85 & 1801 & 135.90 & 760 & 147.85 & 3194 \\ 107.85 & 2757 & 123.85 & 929 & 136.70 & 155 & 148.85 & 547 \\ 109.85 & 42913 & 124.90 & 675 & 136.95 & 866 & 150.95 & 175 \\ 110.85 & 5688 & 126.90 & 66883 & 139.80 & 136 & 151.25 & 242 \\ 111.90 & 665 & 127.90 & 5913 & 140.80 & 3305 & 152.90 & 931 \\ 112.85 & 105 & 128.90 & 27567 & 141.85 & 1007 & 153.85 & 737 \\ 115.85 & 142 & 129.90 & 2301 & 142.75 & 597 & 154.85 & 1512 \\ 116.85 & 11851 & 130.90 & 456 & 143.75 & 105 & 155.90 & 2200 \\ 117.85 & 932 & 131.90 & 207 & 145.85 & 683 & 157.00 & 612\end{array}$

Average of 7.369 to $7.386 \mathrm{~min}$ : NRAO1TUN.D

50 NG DFTPP (143WS28451)

Modified:added subtracted scaled

$\begin{array}{rrrrrrrr}\mathrm{m} / \mathrm{z} & \text { abund. } & \mathrm{m} / \mathrm{z} & \text { abund. } & \mathrm{m} / \mathrm{z} & \text { abund. } & \mathrm{m} / \mathrm{z} & \text { abund. } \\ 157.55 & 205 & 168.90 & 527 & 180.90 & 1284 & 193.65 & 111 \\ 157.85 & 240 & 169.90 & 102 & 181.90 & 107 & 194.05 & 156 \\ 158.85 & 334 & 170.85 & 228 & 184.00 & 227 & 195.85 & 4696 \\ 159.90 & 894 & 171.80 & 401 & 184.90 & 297 & 197.85 & 143987 \\ 160.85 & 1216 & 172.85 & 621 & 185.85 & 15648 & 198.85 & 9953 \\ 161.85 & 336 & 173.90 & 1090 & 186.85 & 4158 & 199.90 & 767 \\ 163.90 & 136 & 174.90 & 1885 & 187.80 & 393 & 201.35 & 882 \\ 164.80 & 1129 & 175.90 & 766 & 188.80 & 9.02 & 203.00 & 868 \\ 165.90 & 492 & 176.85 & 1066 & 190.85 & 435 & 203.90 & 3720 \\ 165.85 & 5116 & 178.85 & 3793 & 191.85 & 1334 & 205.00 & 6811 \\ 167.80 & 2577 & 179.90 & 2404 & 192.85 & 1157 & 205.95 & 28264\end{array}$

Average of 7.369 to $7.386 \mathrm{~min}$.: NRAOITUN.D 50 NG DFTPP (143WS28451)

Hodified:added subtracted scaled

$\begin{array}{crrrrrrr}\mathrm{m} / z & \text { abund. } & \mathrm{m} / \mathrm{z} & \text { abund. } & \mathrm{m} / \mathrm{z} & \text { abund. } & \mathrm{m} / \mathrm{z} & \text { abund. } \\ 206.95 & 3886 & 223.90 & 14181 & 238.75 & 123 & 255.85 & 8089 \\ 207.85 & 849 & 224.90 & 3588 & 239.85 & 116 & 257.00 & 238 \\ 208.80 & 282 & 226.90 & 5792 & 240.75 & 153 & 257.85 & 2894 \\ 210.20 & 162 & 227.90 & 813 & 241.85 & 765 & 258.85 & 460 \\ 210.75 & 1023 & 228.90 & 1192 & 243.95 & 12004 & 264.80 & 1183 \\ 211.00 & 163 & 229.80 & 122 & 244.95 & 1704 & 272.90 & 1712 \\ 214.80 & 309 & 230.80 & 475 & 245.85 & 2022 & 273.90 & 1537 \\ 216.85 & 6647 & 233.80 & 269 & 246.80 & 331 & 274.90 & 27049 \\ 217.85 & 891 & 234.85 & 314 & 248.85 & 378 & 275.85 & 3571 \\ 220.90 & 7836 & 235.90 & 267 & 252.85 & 169 & 276.85 & 1775 \\ 222.90 & 604 & 236.90 & 348 & 254.90 & 55792 & 277.95 & 157\end{array}$


Average of 7.369 to $7.386 \mathrm{~min}$.: NRAOITUN.D

50 NG DFTPP (I43WS2845I)

Modified:added subtracted scaled

$\begin{array}{crrrrrrr}\mathrm{m} / z & \text { abund. } & \mathrm{m} / \mathrm{z} & \text { abund. } & \mathrm{m} / \mathrm{z} & \text { abund. } & \mathrm{m} / \mathrm{z} & \text { abund. } \\ 282.85 & 110 & 322.85 & 1961 & 364.90 & 2315 & 423.95 & 789 \\ 283.85 & 113 & 323.95 & 246 & 365.85 & 289 & 441.00 & 1837 \\ 284.95 & 141 & 326.85 & 185 & 371.90 & 1044 & 441.90 & 65736 \\ 292.85 & 226 & 332.85 & 110 & 372.95 & 158 & 442.90 & 12442 \\ 295.90 & 5699 & 333.90 & 1247 & 382.95 & 149 & 443.90 & 1138 \\ 296.90 & 782 & 334.85 & 266 & 401.90 & 244 & & \\ 302.90 & 675 & 340.95 & 118 & 402.85 & 566 & \\ 314.00 & 161 & 345.80 & 293 & 404.00 & 129 & \\ 314.90 & 589 & 351.90 & 590 & 420.85 & 379 & \\ 315.85 & 300 & 352.90 & 207 & 421.95 & 190 & \\ 320.95 & 122 & 353.90 & 654 & 422.90 & 3815 & & \end{array}$




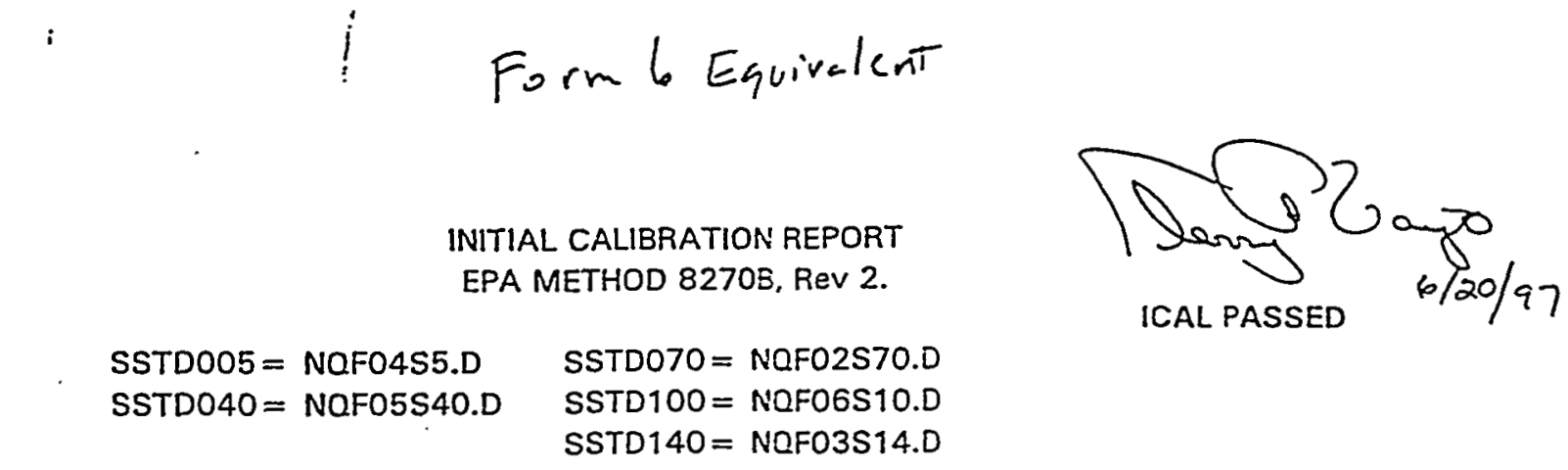

Calibration Check Compounds: \%RSD must be $<30 . \%$

$\begin{array}{rcccccccc} & \text { RFOO5 } & \text { RFO40 } & \text { RF070 } & \text { RF100 } & \text { RF140 } & \text { RF-ave } & \text { RSD } \\ \text { Phenol } & 1.822 & 1.747 & 1.699 & 1.503 & 1.466 & 1.648 & 9.4 & \text { ok } \\ \text { 1,4-dichlorobenzene } & 1.461 & 1.469 & 1.414 & 1.239 & 1.224 & 1.361 & 8.9 & \text { ok } \\ \text { 2-Nitrophenol } & 0.201 & 0.205 & 0.189 & 0.164 & 0.162 & 0.184 & 11.0 & \text { ok } \\ \text { 2,4-Dichlorophenol } & 0.271 & 0.291 & 0.269 & 0.245 & 0.239 & 0.263 & 8.0 & \text { ok } \\ \text { Hexachlorobutadiene } & 0.119 & 0.120 & 0.117 & 0.108 & 0.107 & 0.114 & 5.4 & \text { ok } \\ \text { 4-Chloro-3-Methylphenol } & 0.277 & 0.283 & 0.270 & 0.252 & 0.237 & 0.264 & 7.1 & \text { ok } \\ \text { 2,4,6-Trichlorophenol } & 0.342 & 0.357 & 0.340 & 0.317 & 0.316 & 0.334 & 5.3 & \text { ok } \\ \text { Acenaphthene } & 1.282 & 1.138 & .1 .070 & 0.948 & 0.896 & 1.067 & 14.4 & \text { ok } \\ \text { N-nitrosodiphenylamine } & 0.697 & 0.553 & 0.696 & 0.580 & 0.603 & 0.626 & 10.7 & \text { ok } \\ \text { Pentachlorophenol } & 0.094 & 0.096 & 0.123 & 0.117 & 0.115 & 0.109 & 11.9 & \text { ok } \\ \text { Fluoranthene } & 1.241 & 0.909 & 0.985 & 0.931 & 0.852 & 0.983 & 15.4 & \text { ok } \\ \text { Di-n-octylphthalate } & 2.215 & 2.130 & 2.878 & 2.092 & 3.008 & 2.465 & 17.9 & \text { ok } \\ \text { Benzolalpyrene } & 1.004 & 0.972 & 1.059 & 0.931 & 0.977 & 0.989 & 4.8 & \text { ok }\end{array}$

System Performance Check Compounds: Avg RF must be $>/=.05$

\begin{tabular}{|c|c|c|c|c|c|c|c|}
\hline & RFO05 & RF040 & RFO70 & RF100 & RF140 & RF-ave & RSD \\
\hline N-Nitrosodi-n-propyl amine & 0.889 & $0 . \dot{8} 57$ & 0.828 & 0.726 & 0.650 & 0.790 & 12.6 \\
\hline Hexachlorocyclopentadiene & 0.168 & 0.234 & 0.232 & 0.222 & 0.219 & 0.215 & 12.5 \\
\hline 2,4-Dinitrophenol & 0.133 & 0.205 & 0.209 & 0.223 & 0.214 & 0.197 & 18.5 \\
\hline 4-Nitrophenol & 0.096 & 0.102 & 0.100 & 0.104 & 0.101 & 0.101 & 2.7 \\
\hline Compounds: & RFO05 & RFO40 & RF070 & RFiOO & RF140 & RF-ave & RSD \\
\hline Pyridine & 1.505 & 1.631 & 1.624 & 1.459 & 1.465 & 1.537 & 5.5 \\
\hline $\mathrm{N}$-nitrosodimethylamine & 0.872 & 0.894 & 0.880 & 0.731 & 0.804 & 0.846 & 5.9 \\
\hline 2-Picoline & 1.598 & 1.672 & 1.629 & 1.530 & 1.536 & 1.593 & 3.8 \\
\hline Miethyl methanesulfonate & 0.631 & 0.635 & 0.637 & 0.601 & 0.580 & 0.617 & 4.1 \\
\hline 2-Fluorophenol & 1.346 & 1.351 & 1.342 & 1.211 & 1.210 & 1.292 & 5.8 \\
\hline Ethyl methanesulfonate & 1.100 & 1.064 & 1.067 & 1.021 & 0.964 & 1.043 & 5.0 \\
\hline Phenol-d5 & 1.673 & 1.656 & 1.593 & 1.451 & 1.389 & 1.554 & 7.9 \\
\hline Aniline & 0.885 & 0.987 & 0.940 & 0.873 & 0.848 & 0.907 & 6.2 \\
\hline 2-Chlorophenol-d4 & 1.584 & 1.735 & 1.502 & 1.352 & 1.333 & 1.501 & 11.2 \\
\hline 2-Chlorophenol & 1.508 & 1.698 & 1.445 & 1.314 & 1.277 & 1.448 & 11.6 \\
\hline Decane NOT Present & 0.000 & 0.000 & 0.000 & 0.000 & 0.000 & 0.000 & \\
\hline 1,3-Dichlorobenzene & 1.501 & 1.500 & 1.416 & 1.253 & 1.238 & 1.382 & 9.3 \\
\hline Benzyl alcohol & 0.936 & 0.924 & 0.912 & 0.871 & 0.833 & 0.895 & 4.8 \\
\hline 1.2-Dichlorobenzene-d4 & 0.985 & 0.853 & 0.349 & 0.733 & 0.707 & 0.825 & 13.5 \\
\hline 1,2-dichlorobenzene & 1.416 & 1.240 & $1 . i 67$ & 1.006 & 0.957 & 1.157 & 16.0 \\
\hline 2-Methylphenol & 1.271 & 1.159 & 1.090 & 1.019 & 0.948 & 1.097 & 11.4 \\
\hline Bis(2-chloroisopropyllether & 3.049 & 3.187 & 3.172 & 3.001 & 2.853 & 3.052 & 4.5 \\
\hline 4-methylphenol & 1.5503 & 1.471 & $1 .<48$ & 1.354 & 1.293 & 1.426 & 7.0 \\
\hline Hexachloroethane & 0.508 & 0.523 & 0.519 & 0.457 & 0.461 & 0.496 & 6.0 \\
\hline
\end{tabular}


INITIAL CALIBRATION REPORT

EPA METHOD 8270B, Rev. 2.

$$
\begin{array}{ll}
\text { SSTD005 }=\text { NOFO4S5.D } & \text { SSTD070 }=\text { NOFO2S70.D } \\
\text { SSTDO4O }=\text { NOFO5S40.D } & \text { SSTD100 }=\text { NQFO6S10.D } \\
& \text { SSTD140 }=\text { NOFO3S14.D }
\end{array}
$$

\begin{tabular}{|c|c|c|c|c|c|c|c|}
\hline & & RF040 & RF070 & RF100 & RF140 & RF-ave & RSD \\
\hline Bis (2-chloroethyl)ether & 1.169 & 1.253 & 1.164 & 1.033 & 0.990 & 1.122 & \\
\hline Acetophenone & 0.460 & 0.461 & 0.426 & 0.378 & 0.367 & 0.418 & 10.6 \\
\hline Nitrobenzene-d5 & 0.331 & 0.334 & 0.323 & 0.291 & 0.295 & $.31 .5^{\prime}$ & 65 \\
\hline & .312 & 0.323 & 0.309 & 0.281 & 0.277 & & 8 \\
\hline N-nitrosop & 0.195 & 0.198 & 0.196 & 0.180 & & & \\
\hline & .638 & 0.629 & 0.615 & 0.573 & & & 6.1 \\
\hline 2,4-Dime & 0.455 & 0.335 & 0.317 & 0.280 & 0.282 & & 21.6 \\
\hline & .104 & 0.233 & 0.237 & 0.240 & 0.230 & & \\
\hline Dinla ablarath & 0.424 & 0.421 & 89 & 0.349 & & & - \\
\hline & 0.870 & 1.041 & & 1.012 & & & 7.6 \\
\hline & .280 & 295 & 0.2 & 0.243 & & & \\
\hline & .000 & & & 0.000 & & & \\
\hline & 1.187 & & & 0.937 & & & 10. \\
\hline & 0.430 & 0.410 & & 0.354 & & & 10.9 \\
\hline & 0.278 & 0.266 & & 0.223 & & & \\
\hline & 0.000 & 0.000 & & 0.000 & & & \\
\hline & 0.192 & 97 & & 0.185 & 170 & & 6.1 \\
\hline & 0.609 & & & 0.504 & 0.481 & & 10.2 \\
\hline $1,2,4,5$ & 0.476 & 0.468 & 0.440 & 0.410 & 0.405 & & 7.3 \\
\hline & 0.407 & & & 0.371 & 58 & & 5.3 \\
\hline & 1.341 & & & & & & 10.7 \\
\hline & 0.000 & & & & & 0.000 & \\
\hline & 1.447 & & & 65 & & & 12.9 \\
\hline $1-\mathrm{C}$ & 1.087 & & & & & & 10.3 \\
\hline & 0.314 & & & & & & 3.5 \\
\hline & 1.415 & 08 & & 1.203 & & & 7.9 \\
\hline & 0.317 & 326 & 0.328 & 0.306 & & & 3.6 \\
\hline e & 1.987 & .907. & 831 & 1.628 & 68 & & 10.1 \\
\hline & 0.363 & .367 & .352 & 0.349 & & & 3.2 \\
\hline & 1.708 & 538 & .508 & 1.328 & 262 & & 12.1 \\
\hline & 0.431 & 435 & .417 & 0.396 & 374 & & 6.2 \\
\hline Pent & 0.362 & 22 & 0.335 & 0.314 & 0.302 & & 6.9 \\
\hline & 0.374 & & 0.792 & 888 & 748 & 3 & 26.4 \\
\hline & 1.005 & & & 1.090 & & & 7.5 \\
\hline $2,3,4,6-$ Tetr & 0.221 & 18 & 0.232 & 0.228 & 216 & 223 & 3.0 \\
\hline Dieth & 1.426 & 276 & 1.209 & 1.138 & 880 & & 10.9 \\
\hline 4-Chlorophenyl Phen & 0.536 & 0.428 & 0.452 & 0.397 & 0.375 & & 14.3 \\
\hline & 1.330 & 0.990 & 1.022 & 0.882 & 0.842 & & 18.9 \\
\hline & 0.310 & 0.281 & 0.266 & 0.311 & 0.289 & & 6.7 \\
\hline & 0.127 & 0.136 & & 0.138 & 0.129 & & 3.4 \\
\hline 4,6-Dinitro-2-methylphenol & 0.139 & 0.142 & 0.198 & 0.191 & 0.196 & 0.173 & 17.4 \\
\hline
\end{tabular}


INITIAL CALIBRATION REPORT

EPA METHOD 8270B, Rev. 2.

\begin{tabular}{|c|c|c|c|c|c|c|c|}
\hline $\begin{array}{l}\text { SSTDO05 }=\text { NOFO4S5.D } \\
\text { SSTDO4O }=\text { NOF0 } 540 .\end{array}$ & $\begin{array}{l}\text { SS } \\
\text { SS } \\
\text { SS }\end{array}$ & $\begin{array}{l}\text { STD070 = } \\
\text { STD100 = } \\
\text { STD140 = }\end{array}$ & NOFO6 & $\begin{array}{l}O . D \\
0 . D \\
4 . D\end{array}$ & & & \\
\hline Other Compounds (cont'd): & RF005 & RF040 & RF070 & RF100 & RF140 & RF-ave & RSD \\
\hline 1,2-Diphenylhydrazine & 0.843 & 0.726 & 0.874 & 0.730 & 0.749 & 0.784 & 8.8 \\
\hline Tributylphosphate NOT Present & 0.000 & 0.000 & 0.000 & 0.000 & 0.000 & 0.000 & \\
\hline Phenacetin & 0.431 & 0.322 & 0.426 & 0.415 & 0.400 & & 11.2 \\
\hline 4-Bromophenyl Phenyl Ether & 0.201 & 0.161 & 0.195 & 0.167 & 0.172 & $0 . x 79$ & 9.8 \\
\hline & 0.145 & 0.117 & 0.139 & 0.118 & 0.120 & .128 & 10.4 \\
\hline Hexachloro & 0.211 & 0.166 & 0.201 & 0.178 & 0.181 & 0.187 & 9.7 \\
\hline is(2-CE)phosphate & 0.000 & 0.000 & 0.000 & 0.000 & 0.000 & 0.000 & \\
\hline 4-Amin & 0.824 & 0.713 & 0.789 & 0.668 & & 0.729 & 10.3 \\
\hline & 0.116 & 0.091 & 0.104 & 0.099 & & 0.101 & 9.7 \\
\hline & 0.381 & 0.309 & 0.345 & 0.309 & & 329 & 10.2 \\
\hline & 0.130 & 0.094 & 0.108 & 0.096 & & & 14.3 \\
\hline Pentachloronitr & 0.072 & 0.0 & 0.069 & 0.062 & & & 8.7 \\
\hline Phe & 1.361 & & 1.202 & 1.030 & & 36 & 12.7 \\
\hline & 1.373 & & 1.176 & 0.956 & & & 16.0 \\
\hline & 0.111 & & & 0.073 & & & 20.2 \\
\hline & 0.185 & & & 0.143 & & & 12.3 \\
\hline Butylp & 1.773 & & & 1.357 & & & 13.1 \\
\hline Idrin & 0.158 & & & 0.119 & & 29 & 13.7 \\
\hline dine & 0.370 & & 834 & 0.804 & & 04 & 27.0 \\
\hline & 1.597 & 1.464 & 1.758 & 1.232 & & 97 & 13.1 \\
\hline DDE & 0.248 & 0.235 & 0.299 & 0.216 & & 0.250 & 12.2 \\
\hline Terpher & 1.015 & 78 & 1.087 & 0.785 & & 35 & 12.7 \\
\hline & 0.233 & 0.227 & 0.281 & 0.198 & & & 12.7 \\
\hline p-Dimethylaminoazob & 0.255 & 0.263 & 0.318 & 0.258 & & 75 & 9.5 \\
\hline & 0.442 & 0.410 & 0.502 & 0.387 & 0.440 & & 9.9 \\
\hline Butylbenzylph & 0.906 & & 0.971 & 0.742 & & & 10.2 \\
\hline 4,4 & 0.378 & 0.3 & & 0.357 & & & 8.5 \\
\hline & 0.664 & 0.6 & & 0.676 & 62 & & 4.7 \\
\hline $3,3^{\circ}$-Dichlo & 0.382 & 0.4 & 0.406 & 0.415 & 77 & 96 & 4.0 \\
\hline Benzo[a]an & 1.138 & 1.0 & 1.0 & 0.970 & & 36 & 6.2 \\
\hline & 1.115 & 0.9 & 1.048 & 0.938 & & & 7.8 \\
\hline Bis(2-ethylhexyl)pl & 1.153 & 0.9 & 1.161 & 0.898 & & & 10.8 \\
\hline & 1.248 & 1.1 & 1.273 & 1.138 & 1.2 & 1.204 & 6.5 \\
\hline 2-Dimethylbenz(a)anthracene & 0.221 & 0.4 & 0.519 & 0.429 & & 0.418 & 28.2 \\
\hline Benzo[k]fluoranthene & 1.147 & 1.052 & 1.152 & 0.902 & & 1.053 & 9.9 \\
\hline 3-Methylcholanthrene & 0.468 & 0.499 & 0.534 & 0.486 & & 0.494 & 5.1 \\
\hline Indeno(1,2,3-c, d)pyrene & 0.805 & & 0.870 & 0.748 & & 0.785 & 6.8 \\
\hline Dibenz $[a, h]$ & 0.797 & & 0.867 & 0.768 & .0 .753 & 0.794 & 5.6 \\
\hline Benzo[ghilperylene & 0.801 & 0.704 & 0.813 & 0.688 & 0.679 & 0.737 & 8.8 \\
\hline
\end{tabular}




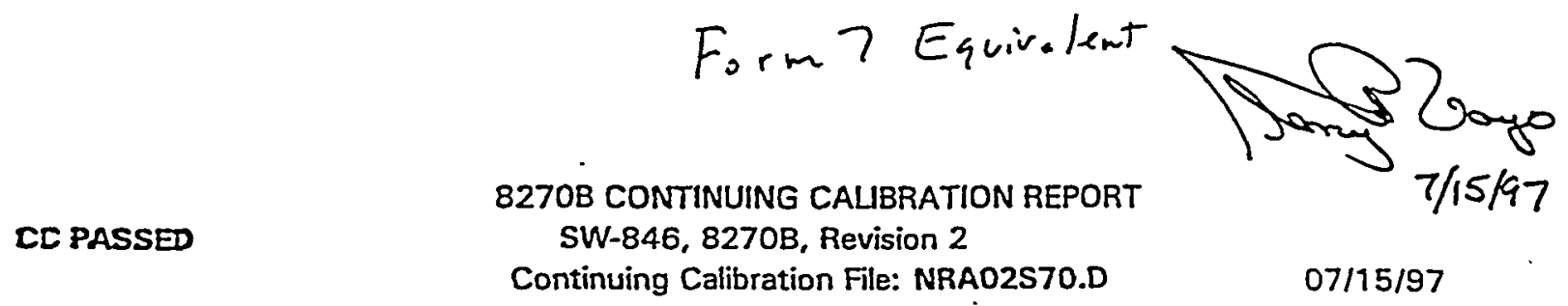

Initial Calibration 5.0 Standard: NOFO4S5.D

Calbration Check Compounds:

$\%$ Drift must be $<20 . \%$

\begin{tabular}{|c|c|c|c|c|c|}
\hline & RT & CC Amnt & CC Target & $\%$ Drift & \\
\hline Phenol & 4.73 & 83.03 & 70.00 & -18.6 & ok \\
\hline 1.4-dichlorobenzene & 5.15 & 75.38 & 70.00 & -7.7 & ok \\
\hline 2-Nitrophenol & $6.14^{\circ}$ & 75.97 & 70.00 & -8.5 & ok \\
\hline 2,4-Dichlorophenol & 6.45 & 63.68 & 70.00 & 9.0 & ok \\
\hline Hexachlorobutadiene & 6.83 & 70.97 & 70.00 & -1.4 & ok \\
\hline 4-Chloro-3-Methylphenol & 7.42 & 76.50 & 70.00 & -9.3 & ok \\
\hline 2,4,6-Trichlorophenol & 8.12 & 68.87 & 70.00 & 1.6 & ok \\
\hline Acenaphthene & 9.47 & 73.68 & 70.00 & -5.3 & ok \\
\hline$N$-nitrosodiphenylamine & 10.58 & 71.05 & 70.00 & -1.5 & ok \\
\hline Pentachlorophenol & 11.74 & 70.95 & 70.00 & -1.4 & ok \\
\hline Fluoranthene & 14.19 & 69.68 & 70.00 & 0.5 & ok \\
\hline Di-s-octylphthalate & 17.45 & 82.62 & 70.00 & -18.0 & ok \\
\hline Benzola]pyrene & 19.16 & 73.68 & 70.00 & -5.3 & ok \\
\hline \multicolumn{2}{|c|}{$\begin{array}{l}\text { Benzolalpyrene } \\
\text { SPCC compounds: }\end{array}$} & \multicolumn{3}{|c|}{$\begin{array}{c}\text { CC RFs must be }>l=0.05 \\
\text { CC RF }\end{array}$} & \\
\hline N-Nitrosodi-n-propyl amine & & \multicolumn{3}{|c|}{0.924} & OK \\
\hline Hexachlorocyclopentadiene & & \multicolumn{3}{|c|}{0.198} & OK. \\
\hline 2,4-Dinitrophenol & & \multicolumn{3}{|c|}{0.167} & OK \\
\hline 4-Nitrophenol & & \multicolumn{3}{|c|}{0.128} & OK \\
\hline & RT & CC Amnt & CC Target & $\%$ Drift & \\
\hline N-Nitrosodi-n-propyl amine & 5.53 & 83.39 & 70.00 & -19.1 & \\
\hline Hexachlorocyclopentadiene & 7.92 & 60.11 & 70.00 & 14.1 & \\
\hline 2,4-Dinitrophenol & 9.52 & 54.74 & 70.00 & 21.8 & warning \\
\hline 4-Nitrophenol & 9.65 & 88.07 & 70.00 & -25.8 & warning \\
\hline Other Compounds: & RT & CC Amnt & CC Target & $\%$ Drift & \\
\hline Pyridine & 2.95 & 88.97 & 70.00 & -27.1 & warning \\
\hline N-nitrosodimethylamine & 2.90 & 84.44 & 70.00 & -20.6 & warning \\
\hline 2-Picoline & 3.49 & 86.87 & 70.00 & -24.1 & warning \\
\hline Methy! methanesulfonate & 3.79 & 84.00 & 70.00 & -20.0 & warning \\
\hline 2-Fluorophenol & 3.93 & 89.21 & 70.00 & -27.4 & warning \\
\hline Ethyl methanesulfonate & 4.36 & 85.44 & 70.00 & -22.1 & warning \\
\hline Phenol-d5 & 4.71 & 85.42 & 70.00 & -22.0 & warning \\
\hline Aniline & 4.79 & 83.25 & 70.00 & -18.9 & \\
\hline 2-Chlorophenol-d4 & 4.90 & 72.90 & 70.00 & -4.1 & \\
\hline 2-Chlorophenol & 4.92 & 74.53 & 70.00 & -6.5 & \\
\hline Decane NOT Present & 0.00 & 0.00 & 70.00 & 100.0 & warning \\
\hline 1,3-Dichlorobenzene & 5.08 & 74.56 & 70.00 & -6.5 & \\
\hline Benzyl alcohol & 5.24 & 81.86 & 70.00 & -16.9 & \\
\hline 1,2-Dichlorobenzene-d4 & 5.29 & 70.76 & 70.00 & -1.1 & \\
\hline 1.2-dichlorobenzene & 5.31 & 85.63 & 70.00 & -22.3 & warning \\
\hline 2-Methylphenol & 5.35 & 87.63 & 70.00 & -25.2 & warning \\
\hline Bis(2-chloroisopropyl)ether & 5.37 & 74.45 & 70.00 & -6.4 & \\
\hline 4-methylphenol & 5.51 & 77.77 & 70.00 & -11.1 & \\
\hline Hexachloroethane & 5.69 & 81.56 & 70.00 & -16.5 & \\
\hline
\end{tabular}

Page 1 
Continuing Calibration File: NRAO2S70.D

\begin{tabular}{|c|c|c|c|c|c|}
\hline $\begin{array}{r}\text { Other Compounds (cont'd): } \\
\text { Bis (2-chloroethyl)ether }\end{array}$ & $\begin{array}{l}\text { RT } \\
4.82\end{array}$ & $\begin{array}{l}\text { CC Amnt } \\
77.68\end{array}$ & $\begin{array}{l}\text { CC Target } \\
70.00\end{array}$ & $\begin{array}{l}\text { \% Drift } \\
-11.0\end{array}$ & \\
\hline Acetophenone & 5.55 & 83.00 & 70.00 & -18.6 & \\
\hline Nitrobenzene-d5 & 5.74 & 82.93 & 70.00 & -18.5 & \\
\hline Nitrobenzene & 5.75 & 80.40 & 70.00 & -14.9 & 1 \\
\hline N-sitrosopiperidine & 5.94 & 73.03 & 70.00 & -4.3 & \\
\hline Isophorone & 6.03 & 80.22 & 70.00 & -14.6 & \\
\hline 2,4-Dimethylphenol & 6.15 & 71.79 & 70.00 & -2.6 & \\
\hline Benzoic acid & 6.25 & 34.55 & 70.00 & 50.6 & warning \\
\hline Bis(2-chloroethoxy)methane & 6.28 & 82.75 & 70.00 & -18.2 & \\
\hline A.-dimethylphenethylamine & 6.53 & 69.69 & 70.00 & 0.4 & \\
\hline 1,2,4-Trichlorobenzene & 6.56 & 61.49 & 70.00 & 12.2 & \\
\hline Dodecane NOT Present & 0.00 & 0.00 & 70.00 & 100.0 & warning \\
\hline Naphthalene & 6.69 & 66.04 & 70.00 & 5.7 & \\
\hline 4-Chloroaniline & 6.73 & 82.17 & 70.00 & -17.4 & \\
\hline 2,6-Dichlorophenol & 6.75 & 64.42 & 70.00 & 8.0 & \\
\hline Benzothiazole NOT Present & 0.00 & 0.00 & 70.00 & 100.0 & warning \\
\hline N-nitroso-di-n-butylamine & 7.19 & 76.66 & 70.00 & -9.5 & \\
\hline 2-Methylnaphthalene & 7.69 & 65.97 & 70.00 & 5.8 & \\
\hline 1,2,4,5-Tetrachlorobenzene & 7.95 & 76.73 & 70.00 & -9.6 & \\
\hline 2,4,5-Trichlorophenol & 8.19 & 69.52 & 70.00 & 0.7 & \\
\hline 2-Fluorobiphenyl & 8.26 & 67.04 & 70.00 & 4.2 & \\
\hline Tetradecane NOT Present & 0.00 & 0.00 & 70.00 & 100.0 & warning \\
\hline 2-Chioronaphthalene & 8.48 & 75.04 & 70.00 & -7.2 & \\
\hline 1-Chloronaphthalene & 8.51 & 66.03 & 70.00 & 5.7 & \\
\hline 2-Nitroaniline & 8.62 & 87.44 & 70.00 & -24.9 & warning \\
\hline Dimethylphthalate & 8.93 & 70.71 & 70.00 & -1.0 & \\
\hline 2,6-Dinitrotoluene & 9.03 & 70.55 & 70.00 & -0.8 & \\
\hline Acenaphthylene & 9.17 & 71.01 & 70.00 & -1.4 & \\
\hline 3-Nitroaniline & 9.33 & 86.50 & 70.00 & -23.6 & warning \\
\hline Dibenzofuran & 9.77 & 69.05 & 70.00 & 1.4 & \\
\hline 2,4-Dinitrotoluene & 9.75 & 70.18 & 70.00 & -0.3 & \\
\hline Pentachlorobenzene & 9.69 & 80.65 & 70.00 & -15.2 & \\
\hline 1-Naphthylamine & 9.90 & 79.94 & 70.00 & -14.2 & \\
\hline 2-Naphthylamine & 10.05 & 75.35 & 70.00 & -7.6 & \\
\hline 2,3,4,6-Tetrachlorophenol & 9.98 & 78.62 & 70.00 & -12.3 & \\
\hline Diethylphthalate & 10.16 & 73.35 & 70.00 & -4.8 & \\
\hline 4-Chlorophenyl Phenyl Ether & 10.37 & 76.57 & 70.00 & -9.4 & \\
\hline Fluorene & 10.38 & 72.99 & 70.00 & -4.3 & \\
\hline 4-Nitroaniline & 10.41 & 85.92 & 70.00 & -22.7 & warning \\
\hline 2,4,6-Tribromophenol & 10.81 & 67.02 & 70.00 & 4.3 & \\
\hline 4.6-Dinitro-2-methylphenol & 10.47 & 67.32 & 70.00 & 3.8 & \\
\hline
\end{tabular}


8270B CONTINUING CALIBRATION REPORT .

Continuing Calibration File: NRAO2S70.D

\begin{tabular}{|c|c|c|c|c|c|}
\hline Other Compounds (cont'd): & RT & CC Amnt & CC Target & $\%$ Drift & \\
\hline 1.2-Diphenylhydrazine & 10.66 & 91.11 & 70.00 & -30.2 & warning \\
\hline Träutyłphosphate NOT Present & 0.00 & 0.00 & 70.00 & 100.0 & warning \\
\hline Phenacetin & 11.16 & 88.95 & 70.00 & -27.1 & warning \\
\hline 4-Bromophenyl Phenyl Ether & 11.27 & 79.70 & 70.00 & -13.9 & $\therefore$ \\
\hline alpha-BHC & 11.29 & 77.72 & 70.00 & -11.0 & \\
\hline Hexachlorobenzene & 11.37 & 74.99 & 70.00 & -7.1 & \\
\hline Tris(2-CE)phosphate NOT Present & 0.00 & 0.00 & 70.00 & 100.0 & warning \\
\hline 4-Aminobiphenyl & 11.75 & 69.18 & 70.00 & 1.2 & \\
\hline beta-BHC & 11.69 & 72.57 & 70.00 & -3.7 & \\
\hline Pronamide & 11.86 & 75.66 & 70.00 & -8.1 & \\
\hline gamma-BHC & 11.83 & 80.93 & 70.00 & -15.6 & \\
\hline Pentachloronitrobenzene & 11.75 & 81.68 & 70.00 & -16.7 & \\
\hline Phenanthrene & 12.13 & 71.71 & 70.00 & -2.4 & \\
\hline Anthracene & 12.22 & 77.98 & 70.00 & -11.4 & \\
\hline delta-BHC & 12.26 & 89.19 & 70.00 & -27.4 & warning \\
\hline Heptachlor & 13.00 & 84.96 & 70.00 & -21.4 & warning \\
\hline Di-n-Butylphthalate & 13.17 & 75.44 & 70.00 & -7.8 & \\
\hline Aldrin & 13.54 & 90.34 & 70.00 & -29.1 & warning \\
\hline Benzidine & 14.40 & 57.82 & 70.00 & 17.4 & \\
\hline Pyrene & 14.54 & 79.99 & 70.00 & -14.3 & \\
\hline $4,4^{\prime}-D D E$ & 14.69 & 87.25 & 70.00 & -24.6 & warning \\
\hline Terphenyl-d14 & 14.76 & 84.30 & 70.00 & -20.4 & warning \\
\hline Dieldrin & 14.83 & 105.18 & 70.00 & -50.3 & warning \\
\hline p-Dimethylaminoazobenzene & 14.96 & 75.97 & 70.00 & -8.5 & \\
\hline $4,4^{\prime}-D D D$ & 15.16 & 85.70 & 70.00 & -22.4 & warning \\
\hline Butylbenzylphthalate & 15.45 & 72.32 & 70.00 & -3.3 & \\
\hline $4,4^{\prime}-\mathrm{DDT}$ & 15.57 & 71.82 & 70.00 & -2.6 & \\
\hline Methoxychlor & 16.16 & 68.21 & 70.00 & 2.6 & \\
\hline 3,3"-Dichlorobenzidine & 16.22 & 67.30 & 70.00 & 3.9 & \\
\hline Benzo[a]anthracene & 16.28 & 73.90 & 70.00 & -5.6 & \\
\hline Chrysene & 16.34 & 73.42 & 70.00 & -4.9 & \\
\hline Bis(2-ethylhexyl)phthalate & 16.29 & 74.57 & 70.00 & -6.5 & \\
\hline Benzo[b]fluoranthene & 18.35 & 77.37 & 70.00 & -10.5 & \\
\hline 7.12-Dimethylbenz(a)anthracene & 18.33 & 82.55 & 70.00 & -17.9 & \\
\hline Benzolklfluoranthene & 18.42 & 76.77 & 70.00 & -9.7 & \\
\hline 3-Methylcholanthrene & 20.13 & 70.77 & 70.00 & -1.1 & \\
\hline Indeno(1,2,3-c,d)pyrene & 22.81 & 67.99 & 70.00 & 2.9 & \\
\hline Dibenz[a,h]anthracene & 22.91 & 68.66 & 70.00 & 1.9 & \\
\hline Benzo[ghi]perylene & 23,90 & 68.96 & 70.00 & 1.5 & \\
\hline
\end{tabular}




\section{INTERNAL STANDARD AREA SUMMARY \\ Form 8 equivalent}

Continuing

Standard

Area Area Area Area Area Area

Filename

NRAOTSTOD

ace-dlo phe-dlo chry-dl2 per-dl2

SSTD070 (143WS28491)
Upper Limit
Lower lintt

$542000 \quad 2174614$

1084000

271000

4349228

943737

1193540

641902

402522

1087307

1887474

2387080

$1283804 \quad .805044$

Area Area

47859

596770

320951

201261

Sample No. Sample No. 1,4-dcb

nap-d8

Area

Area

Area

Area,

\section{BLANK}

LCS I

LCS 2

BL-136532-1 708845

2666679 ace-dio phe-dlo chry-dl2

per-st2

NAP-R2-S2-B

NAP-R2-SA

QC-136532-1

QC-136532-2

591638

$97 C 02897$

$97 C 02898$

681174

623827

2459530

1116204

1420590

971622

660077

2851726

1075581

1360854

897837

625637

2196009

829423

735117

NAPRI-SA

97002901

703030

2619264

1066886

1420013

1317233

1012069

2707533

1089074

1335999

916064

648214

627702

LMITS: Areas should be within 50 to 200 percent of the corresponding area in the calibration standarc. 


\section{INTERNAL STANDARD RETENTION-TIME SUMMARY \\ Form 8 equivalent}

\section{Continuing}

Standard

$\begin{array}{llllll}R T & R T & R T & R T & R T & R T\end{array}$

Filename NRA02S7O.D 1,4-dcb nap-d8 ace-dio phe-dlo chry-dl2 per-d12

$\begin{array}{rllllll}\text { SSTDO70 (143WS28491) } & 5.13 & 6.66 & 9.41 & 12.09 & 16.29 & 19.29 \\ \text { Upper Lmit } & 5.63 & 7.16 & 9.91 & 12.59 & 16.79 & 19.79 \\ \text { Lower Emit } & 4.63 & 0.16 & 8.91 & 11.59 & 15.79 & 18.79\end{array}$

$\begin{array}{llllllll}\text { CLIENT DCL } & \text { RT } & \text { RT } & \text { RT } & \text { RT } & \text { RT } & \text { RT:- }\end{array}$

Sample No. Sample No. 1,4-dcb nap-d8 ace-d10 phe-dlo chry-d12 per-d12

$\begin{array}{lccccccc}\text { BLANK } & \text { BL-136532-1 } & 5.13 & 6.65 & 9.40 & 12.08 & 16.29 & 19.29 \\ \text { LCS 1 } & \text { QC-136532-1 } & 5.13 & 6.65 & 9.41 & 12.08 & 16.29 & 19.29 \\ \text { LCS 2 } & \text { QC-136532-2 } & 5.12 & 6.65 & 9.41 & 12.08 & 16.28 & 19.30 \\ \text { NAP-R2-S2-B } & 97 C 02897 & 5.13 & 6.66 & 9.41 & 12.08 & 16.29 & 19.29 \\ \text { NAP-R2-S4 } & 97 C 02898 & 5.13 & 6.65 & 9.40 & 12.08 & 16.29 & 19.30 \\ \text { NAP-R1-SA } & 97 C 02901 & 5.12 & 6.65 & 9.40 & 12.08 & 16.29 & 19.30\end{array}$

LIMTS: Retention times should be within 30 seconds of the corresponding area in the calibration standarc 


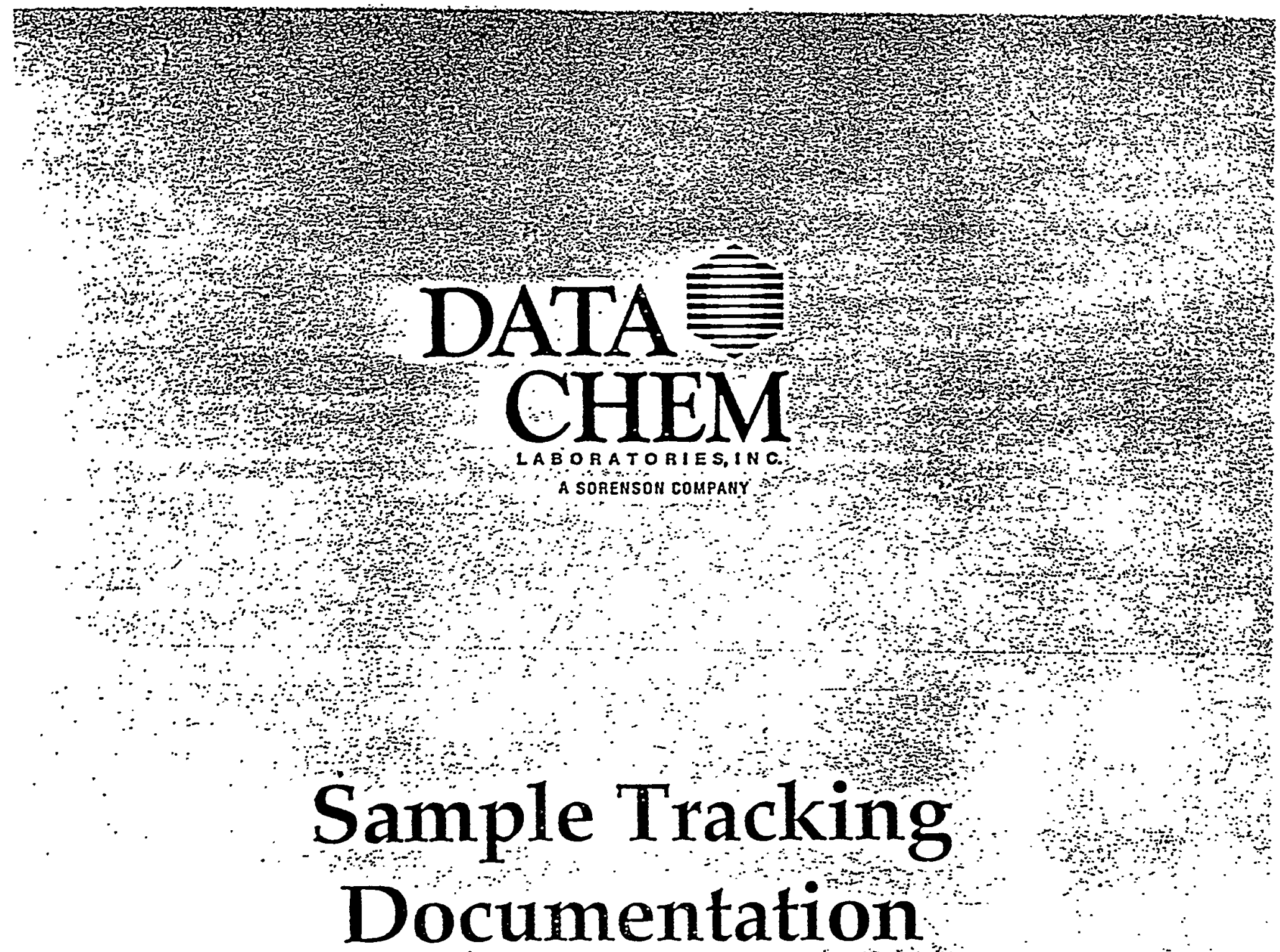

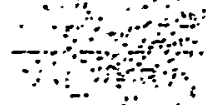

and ond ons

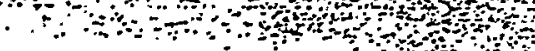

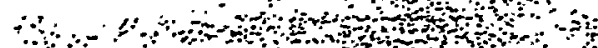

\section{Sample Tracking Documentation}

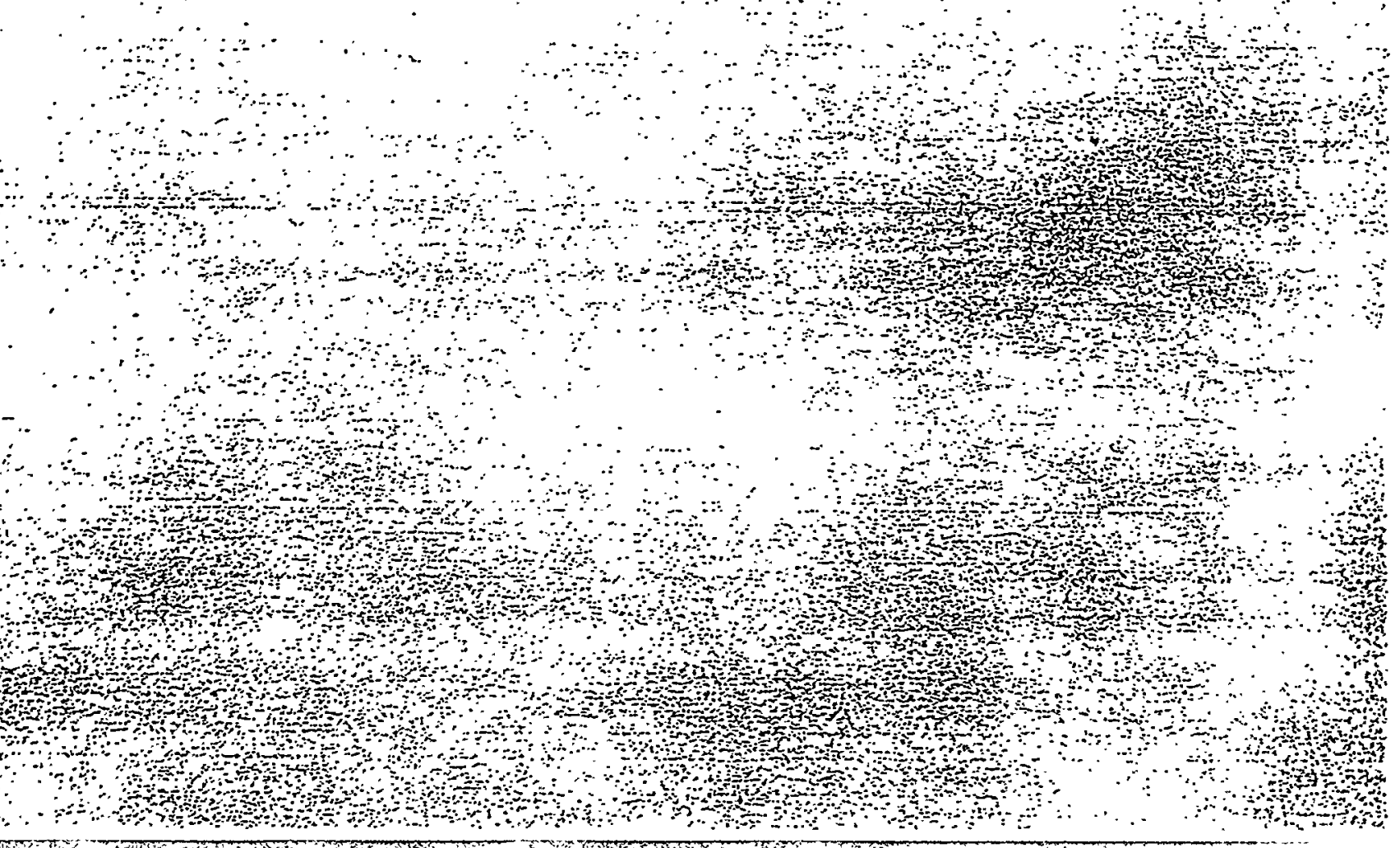


DataChem Laboratories IIMS - Sample Master System

Preparation Group Report

Date: 1-JUL-1997 09:57

User: MOOSMAN

Ireparation Run Name: G9760008
Page :

RIIMS15-

Group ID: G9

Samples: 6

\begin{tabular}{rll} 
& Laboratory & Field Sample \\
Pos & Sample Name & Name I \\
\hline 1 & BL-136532-1 & BL-136532-I \\
2 & QC-136532-1 & QC-136532-1 \\
3 & QC-136532-2 & QC-136532-2 \\
4 & $97 C 02897$. & NAP-R2-S2-B \\
5 & $97 C 02898$ & NAP-R2-S4 \\
6 & $97 C 02901$ & NAP-RI-S4
\end{tabular}

Field Sample Name 2

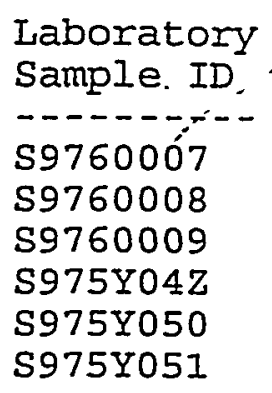

Laboratory Group Name

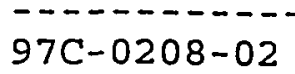

97C-0208-02

97C-0208-02

97C-0208-02

97C-0208-02

97C-0208-02

Bor: $5 w-42$

Due: 7/23/47

Metlad. $8270 b$ 

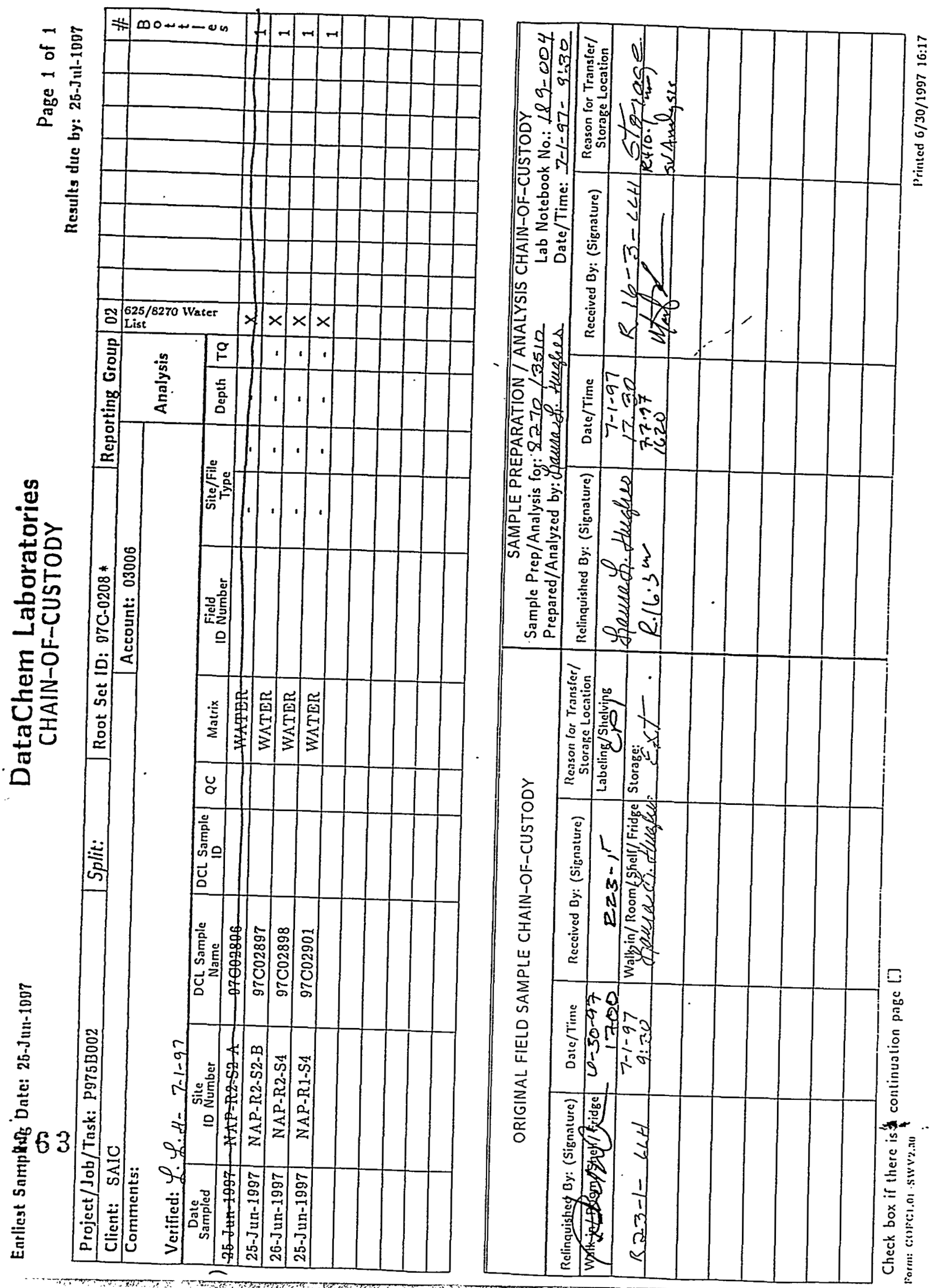


\section{Sample Work Order}

QC Clearance:

DCL Root Set ID: 97C-0208*

Project Manager: Carol M. Bell

DCL Lab. Name: 97C02896-97C02901

Client: SAIC

Total \# Somples: 6

Account: 03006

Sample Entry: Patrick W. Corum

Project/Task: P975B002

Section: FG

Date Received: 27-Jun-1997

Earliest Sampling Date: 24-Jun-1997

Date for Mailing Report: 25-Jul-1997

Preparation Type:

Date for Verbal Report: 25-Jul-1997

\begin{tabular}{|c|c|c|c|c|c|c|c|c|}
\hline $\begin{array}{l}\text { Rep. } \\
\text { Group }\end{array}$ & $\begin{array}{c}\text { FG Section } \\
\text { Analytes Requested }\end{array}$ & $\begin{array}{c}\text { Latest } \\
\text { Prep. Date }\end{array}$ & $\begin{array}{c}\text { Latest } \\
\text { Anal. Date }\end{array}$ & $\begin{array}{l}\text { No. of } \\
\text { Samp. }\end{array}$ & $\begin{array}{l}\text { Storage } \\
\text { Location }\end{array}$ & $\begin{array}{c}\text { Analysis/Prep. } \\
\text { Method }\end{array}$ & Inst. & Matrix \\
\hline 01 & Volatiles by $8260 \mathrm{~A}$ & & 9.Jul-1997c & 6 & & $8260 \mathrm{~A}$ & GC/MS vo & WATER \\
\hline 02 & $625 / 8270$ Water List & 2-Jul-1997c & Prep+40d & 4 & & $8270 / 3510$ & GC/MS SV & WATER \\
\hline
\end{tabular}

${ }^{c}$ Based on date of collection

Special Instructions:

Section Manager: Reed A. Hendricks

$\therefore 264$

Other Sections Receiving Sample Portions: ZI, FC

DataChem Laboratories/ 960 West LeVoy Drive / Salt Lake City, Utah 84123

Form: $1101.04-5 \% 1.12$ 
$\therefore \quad \therefore$ ond

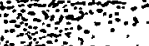

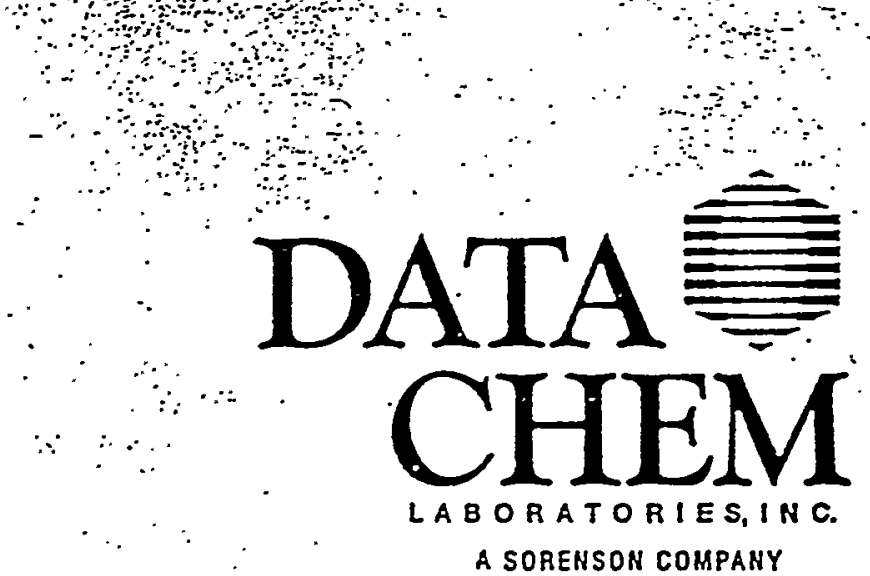

\section{Analytical Documentation}


Date Sct Extracted: 7-1-97

Account No:: 3006

DCL Set No: $97 C-0208-02$

Group ID/Lot: 69760008
DATACHEM

LABORATORIES

EXTRACTION

NOTEBOOK

Book No. EXT 189 Page No. 004

Sample Medium: $\mathrm{H}_{2} \mathrm{O}$

Extraction Method: $8270 / 3510$

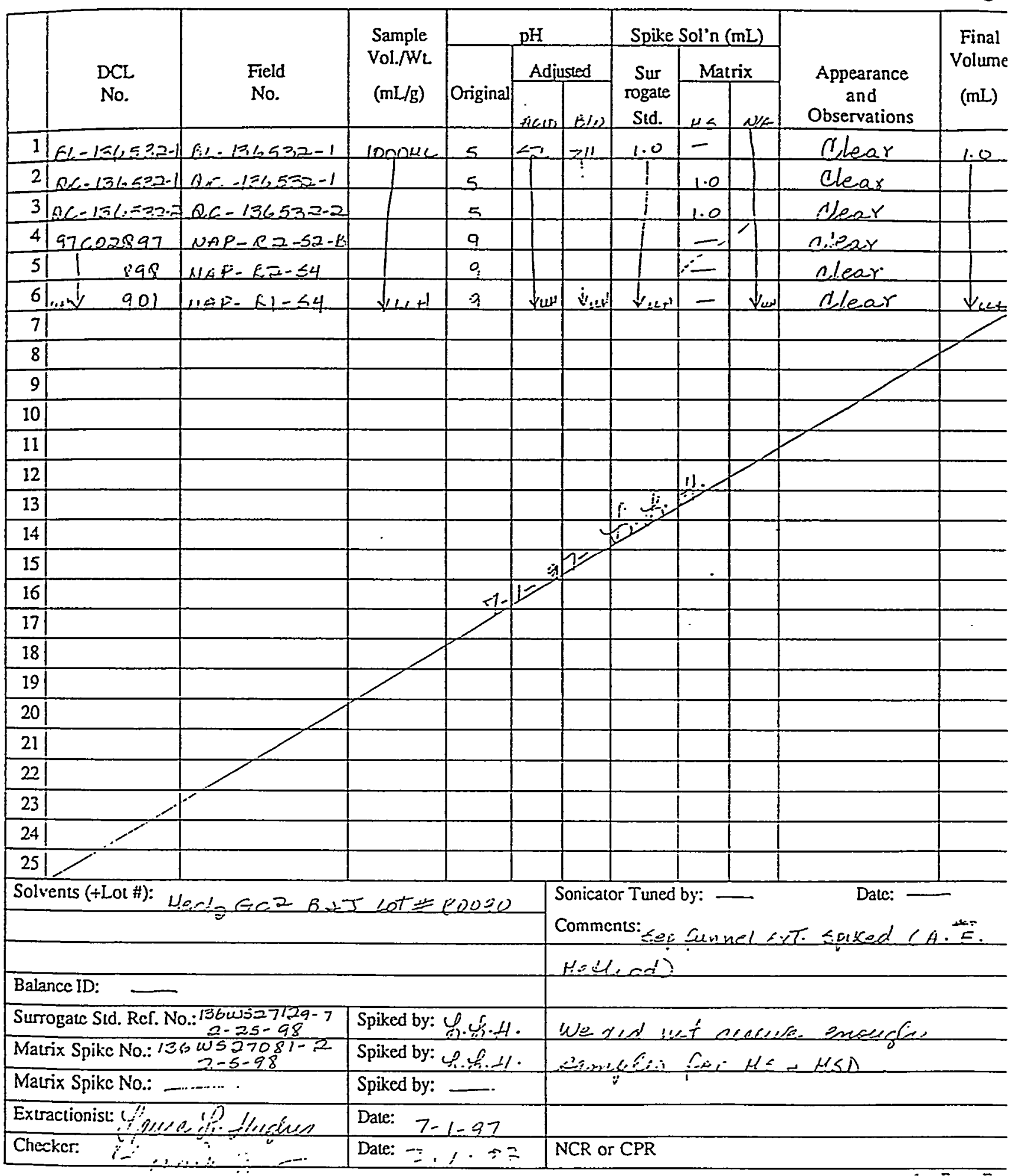


TITLE

$97 C-0208-02$

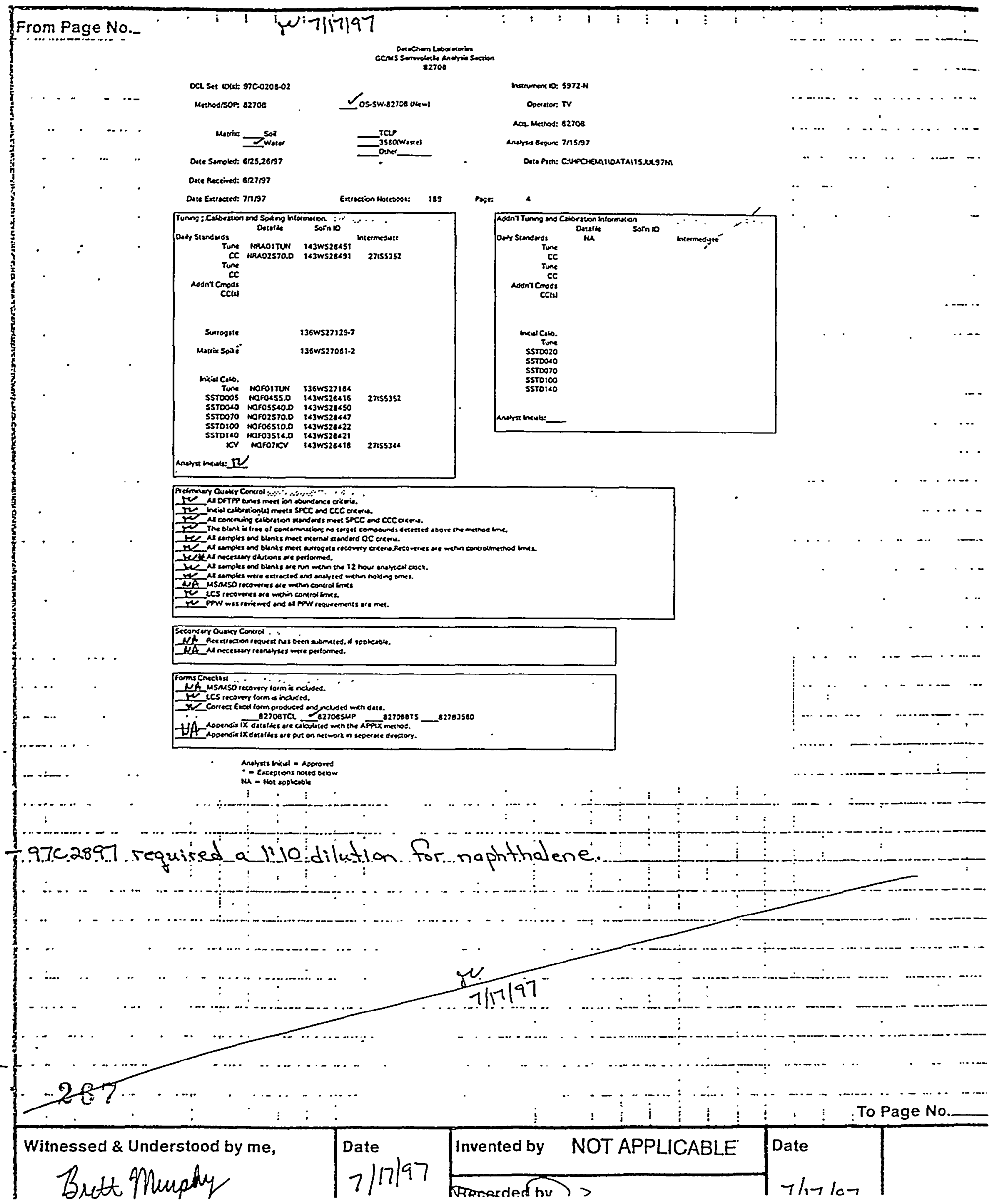




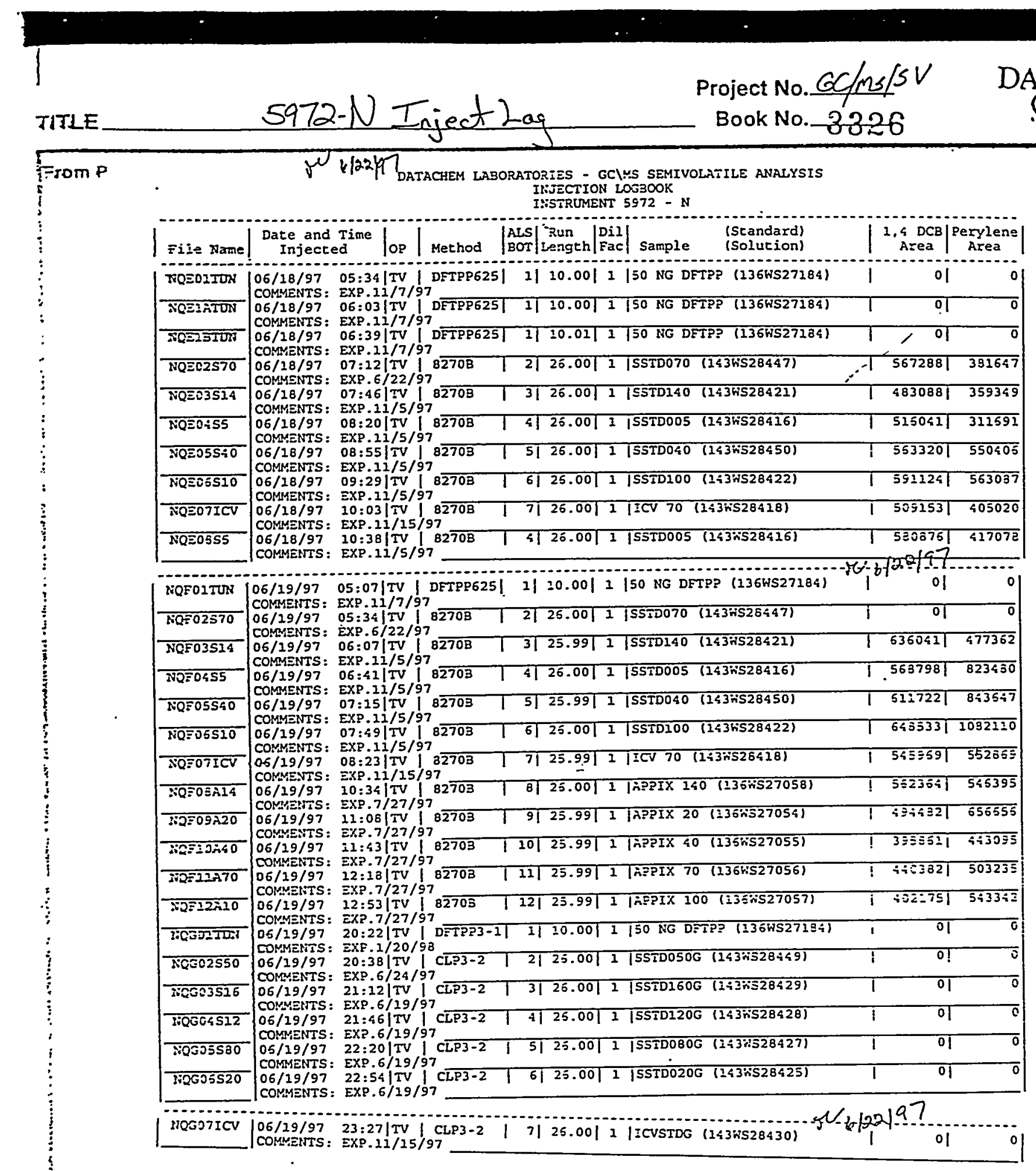


TITLE 5972-N Inject Log.

F $7(17) 97$ DATACHEM LABORATORIES - GClMS SENIVOLATILE ANALYSIS INJECIION LOGBCOK

INSTRUMENT 5972 - N

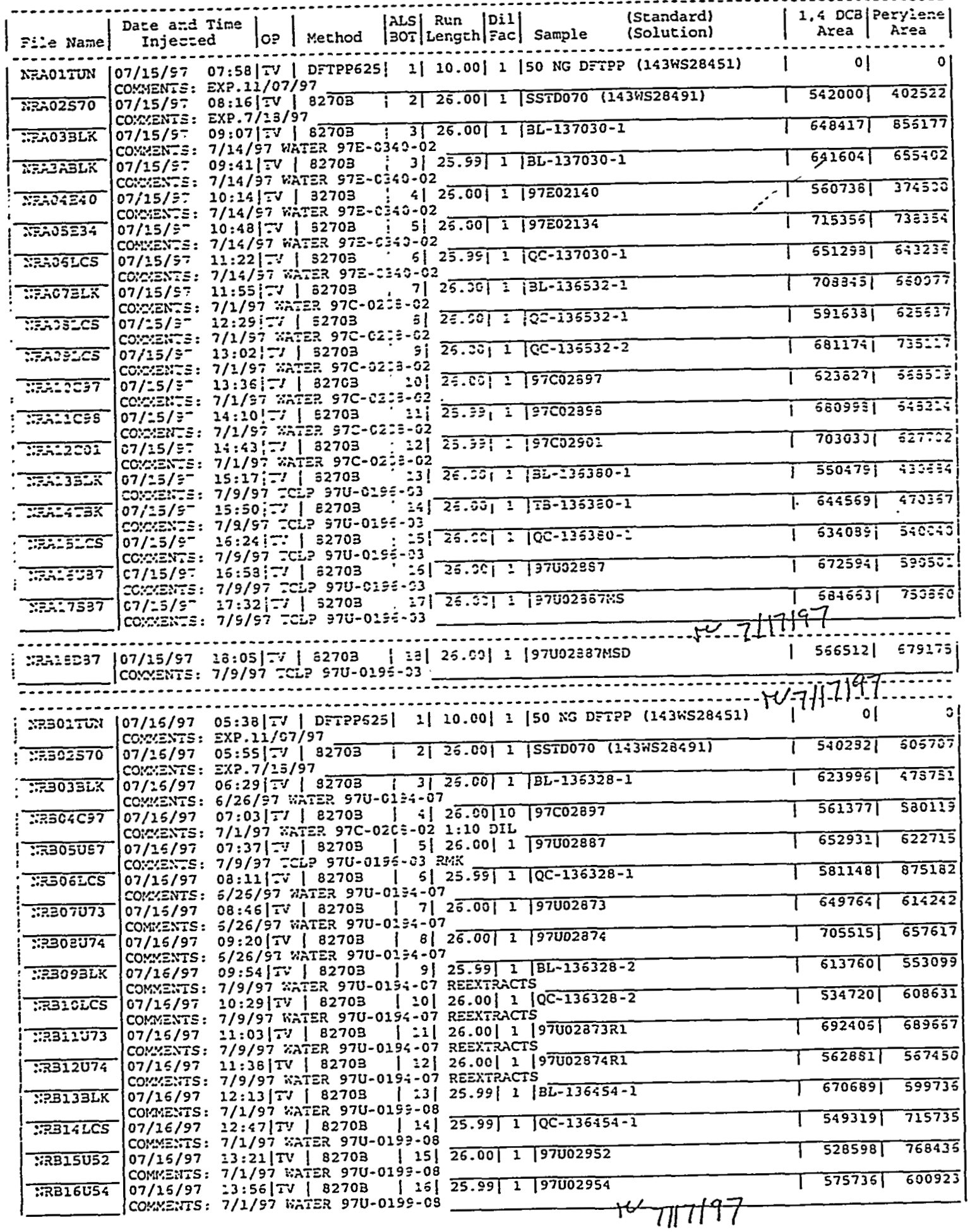

259 $7 / 17 / 17$

To Page No._

Witnessed 2 Understood by me, Beett Pmusply \begin{tabular}{l|l|l} 
Date & Invented by NOT APPLICASiE & Date \\
$7 / 1797$ & $7 / 7 / 97$
\end{tabular} 


\section{Table of Contents}

\section{Standards Traceability Packef}

Part 1: Daily Standards Lee part 5 abo

Part 2: Daily Standards -- additional compounds (if applicable)

Part 3: Surrogate Spiking Solutions

Part 4: Matrix Spiking Solutions (if applicable)

Part 5: Initial Calibration Standards 
Daily standard

WORKING STANDARDS

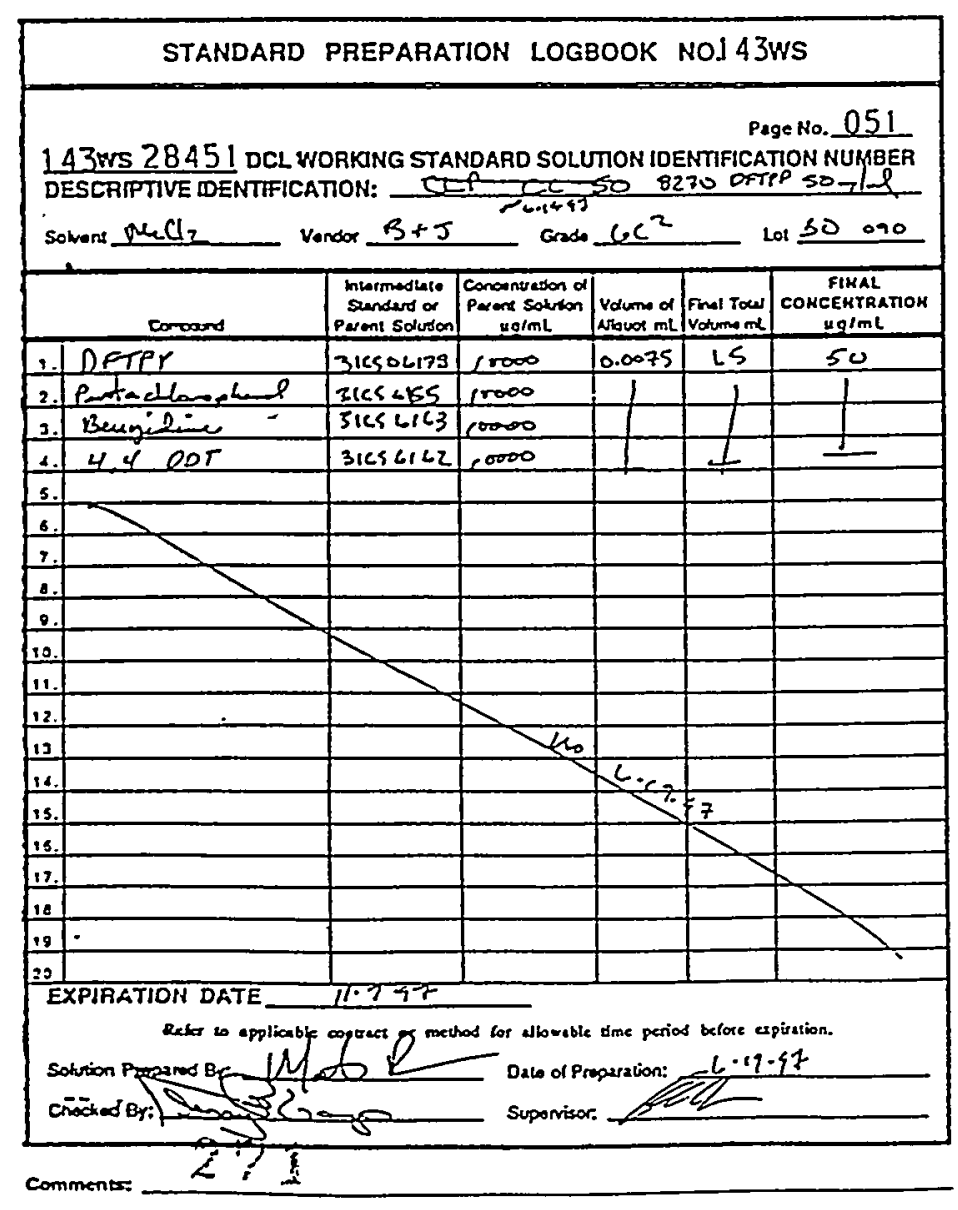

WORKING STANDARDS

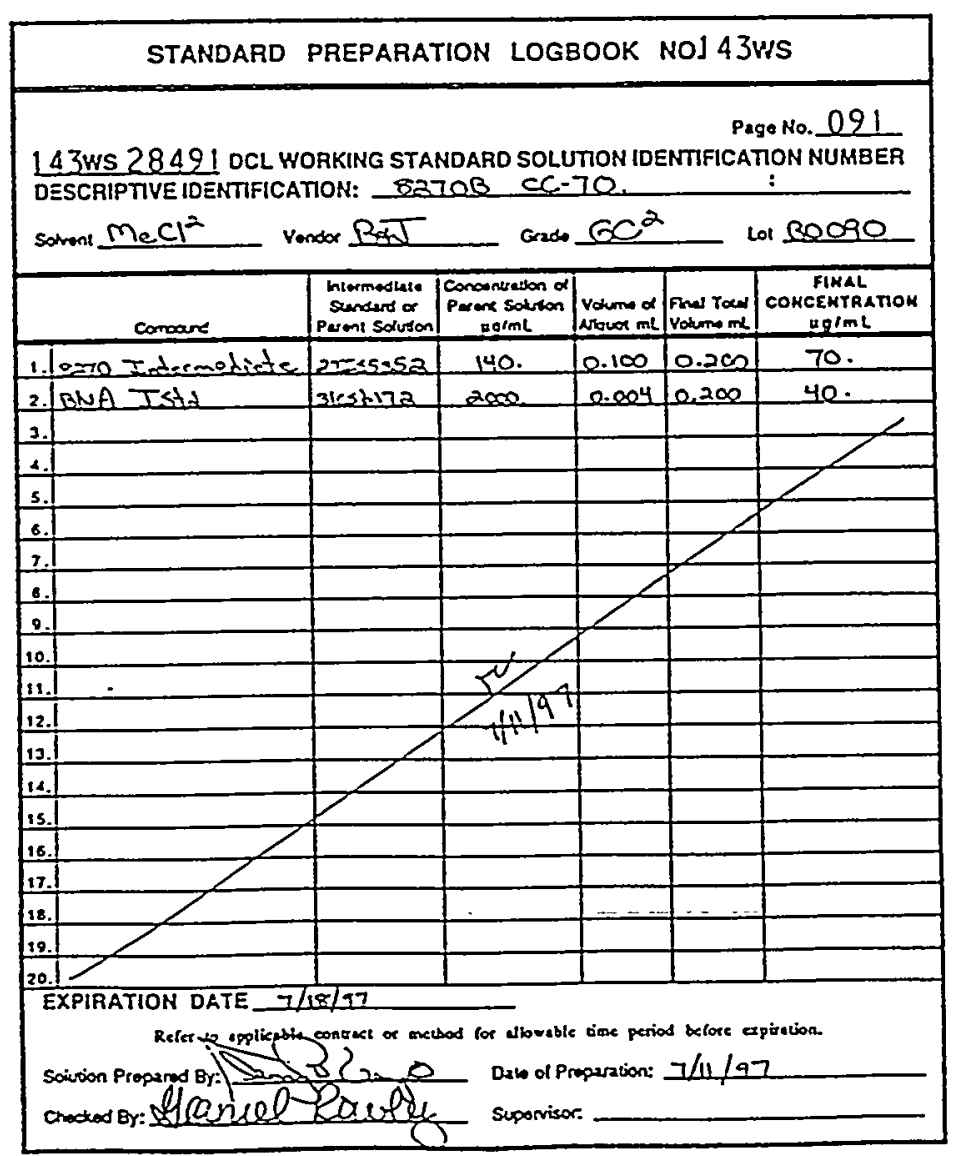

Comments: 
WORKING STANDARDS

PARTY III

\section{SURROGATE SPIKE SOIDTION ;}

\section{-136 WS27129}

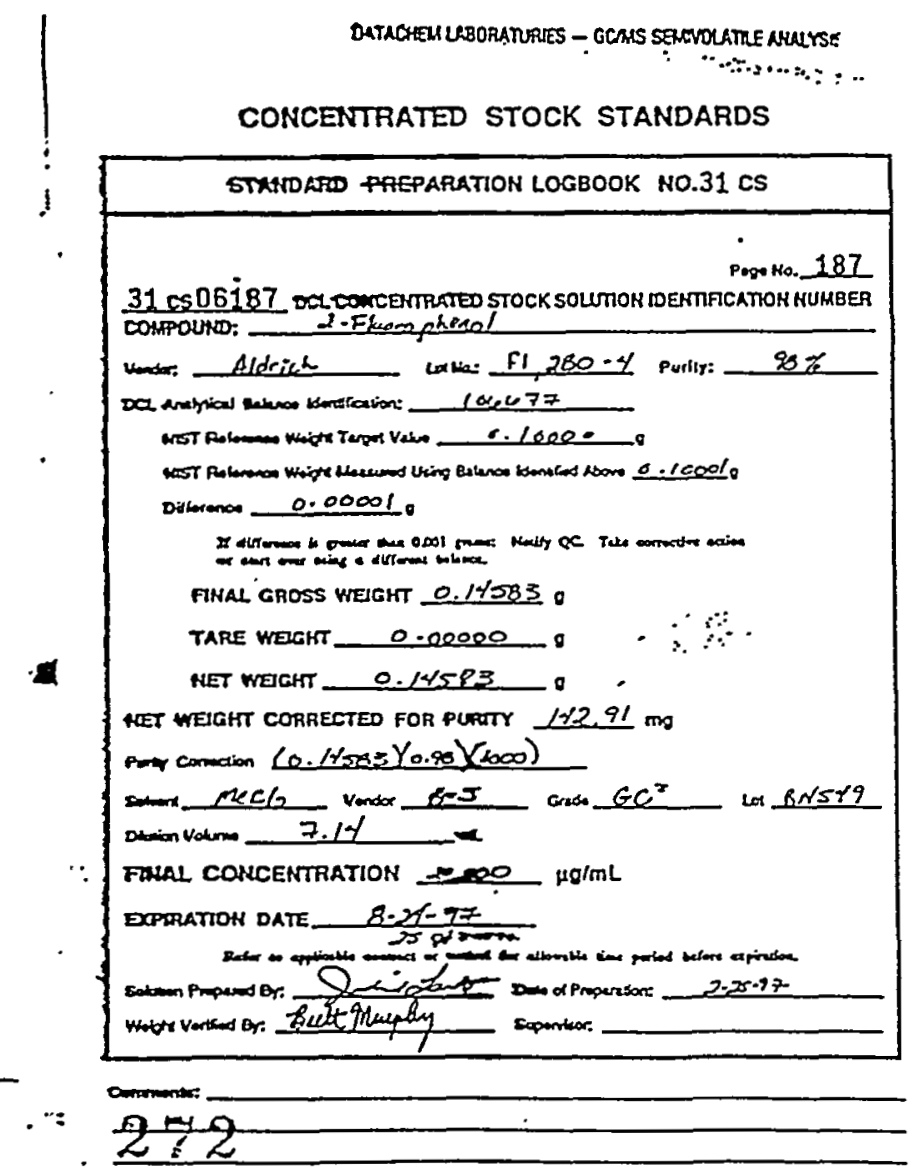

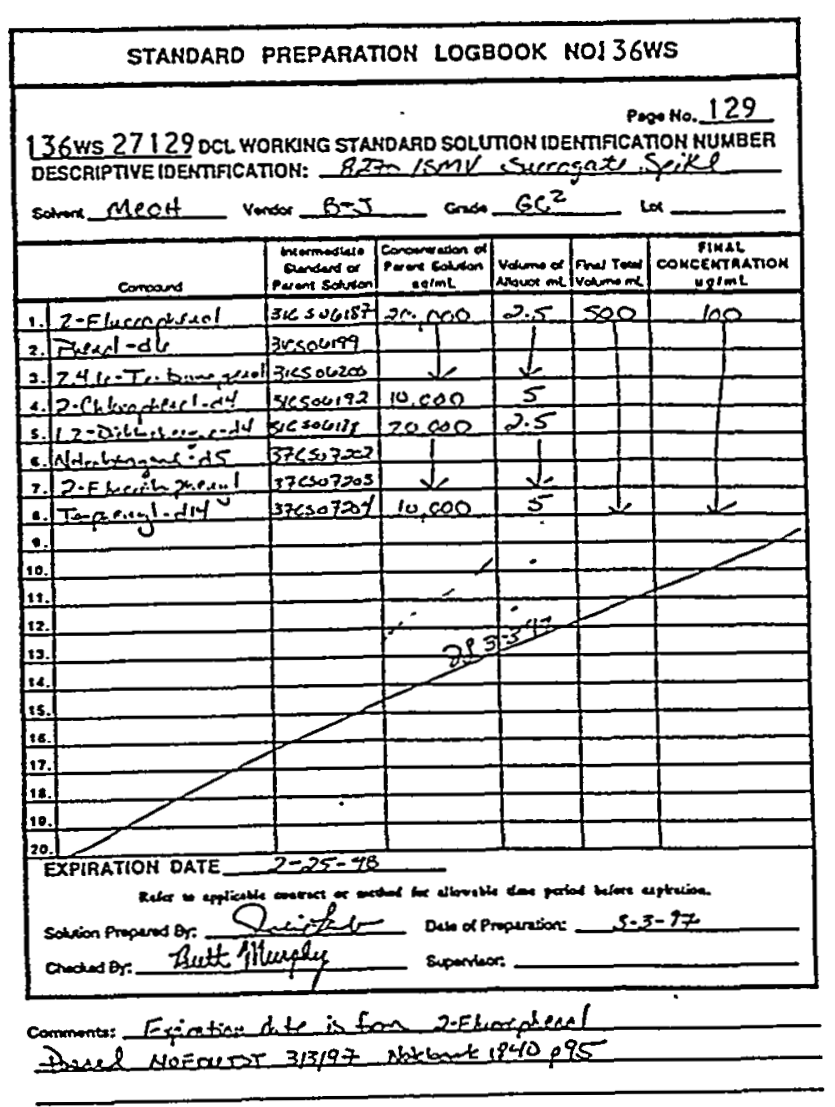

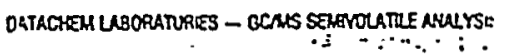

\section{CONCENTRATED STOCK STANDARDS}

STANDARD PREPARATION LOGBOOK NO.31 CS

Page No._199

31 CS 06199 DCL CONCENTRATEO STOCK SOLUTION IDENTFICATON NUHBER

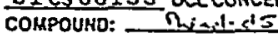

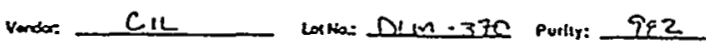

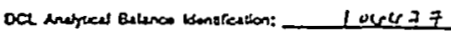

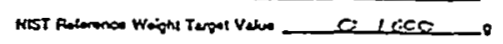

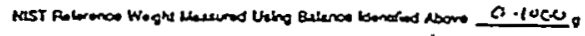

Ditrerencos re.cec00.

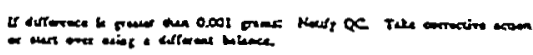

FINAL GROSS WEIGHT 0.12853 。

TARE WEIGHT O.00000."

HET WEIGHT C.12853:

NET WEIGHT CORAECTEO FOR PURTY 17.5.96 mo

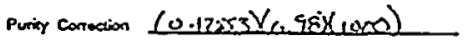

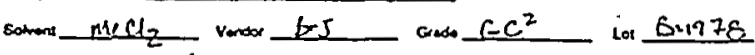

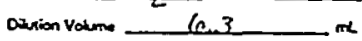

FINAL CONCENTRATION $20,000 \mu \mathrm{g} / \mathrm{mL}$

EXPIRATION DATE_ 3.3.T8

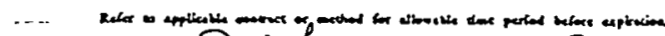

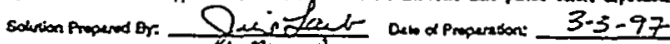

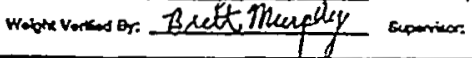

conominas: 


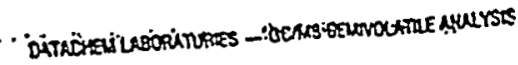

\section{CONCENTRATED STOCK STANDARDS}

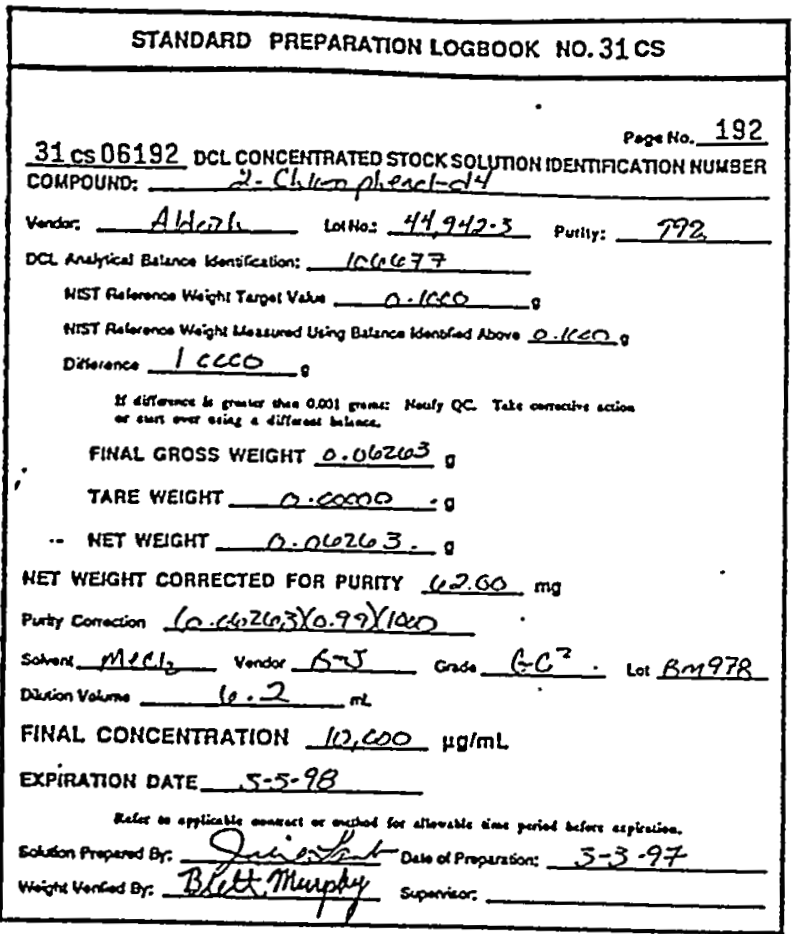

Commorat

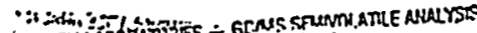

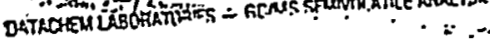

\section{CONCENTRATED STOCK STANDARDS}

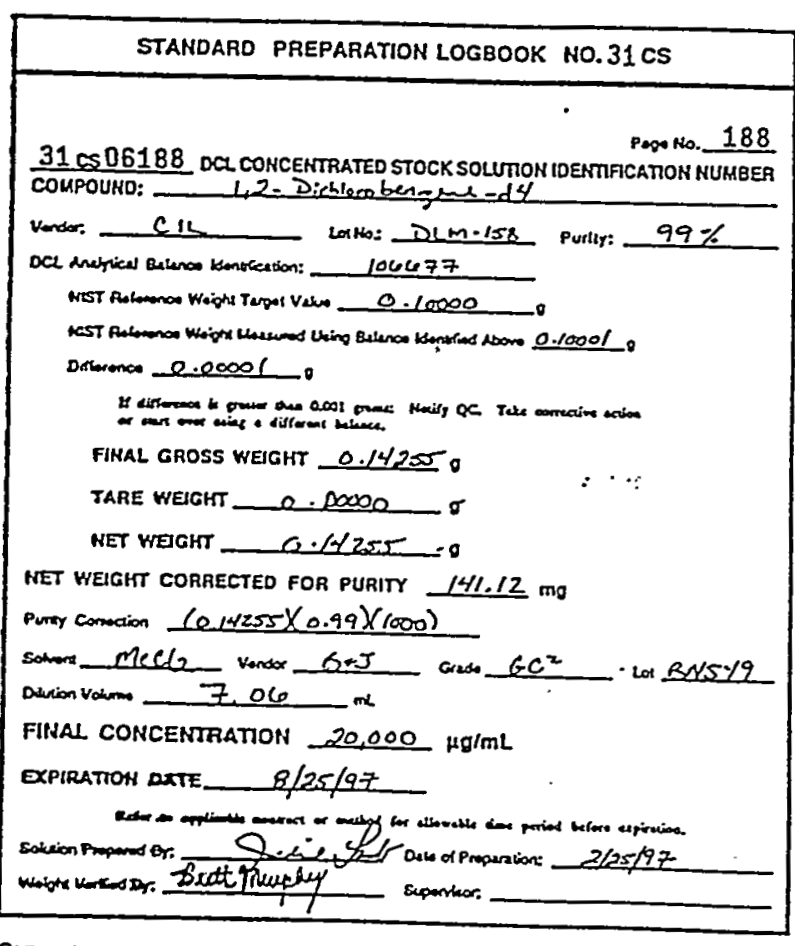

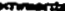

273

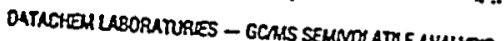

\section{CONCENTRATED STOCK STANDARDS}

STANDARD PREPARATION LOGBOOK NO. $31 \mathrm{cs}$

Pago No. 200

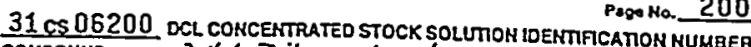

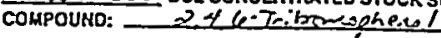

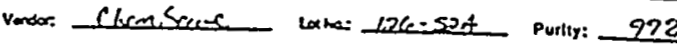

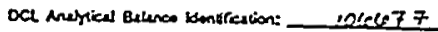

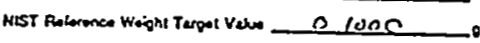

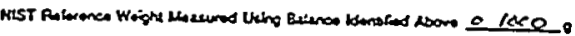

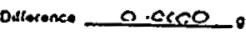

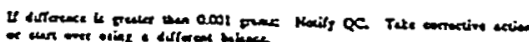

FINAL GROSS WEIGHT $0.1 \%$ COTS.

TARE WEIGHT $0.00000,0$.

NET WEIGHT _ $0.84 \mathrm{~N}$. 15

NET WEIGHT CORRECTED FOA'PURTY $144.99 \mathrm{mg}$

Purity corraction (a 1-6104) $(0.99)$ (1000)

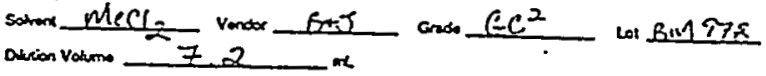

FINAL CONCENTRATION $20, a n O$, $\mu \mathrm{g} / \mathrm{mL}$

EXPIRATION DATE $\quad 3-398$

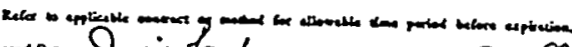

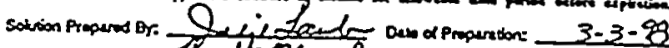

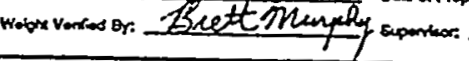

commente

CONCENTRATED STOCK STANDARDS

STANDARD PAEPARATION LOGBOOK NO. $37 \mathrm{CS}$

002

37 CS 07202 OCL CONCENTRATED STOCK SOUTIOH IDENTIFATION NUMBER COMPOUND: Nitribengsos

Vensor: MSD Laxka: 1125K_ Purlir: $97.4 \%$

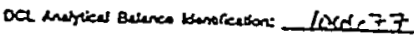

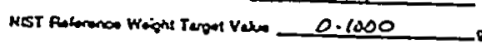

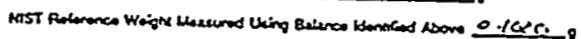

Daterence C.eeco

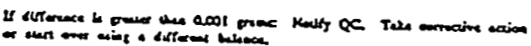

FINAL GROSS WEIGHT Q.l2ya8 a.

TARE WEIGHT Q.00000 -

NET WEIGHT _- $12 \operatorname{seg} 8$

HET HEIGHT CORRECTED FOR PURTं $125.52 \mathrm{mg}$

Puni corretion (0.120.28) $(0.974)(1000)$

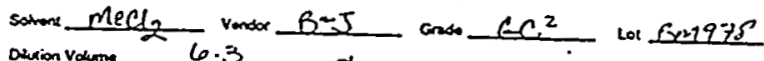

FINAL CONCENTRATION $20,00 \Omega \mu g / m L$

EXPIRATION DATE___ $3-3-28$

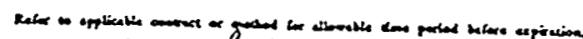

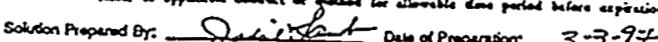

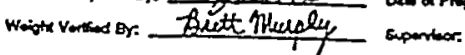

comments: 
comoment:

CONCENTAATED STOCK STANDARDS

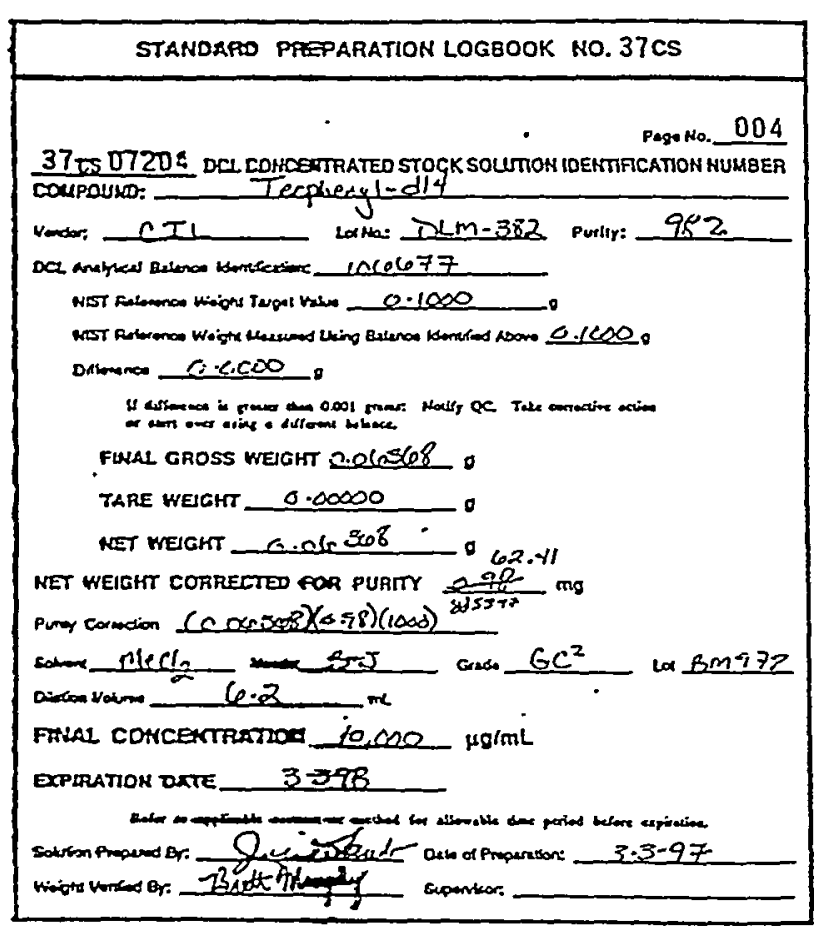

commontr: 
Part TV: Matrix Spike Solution

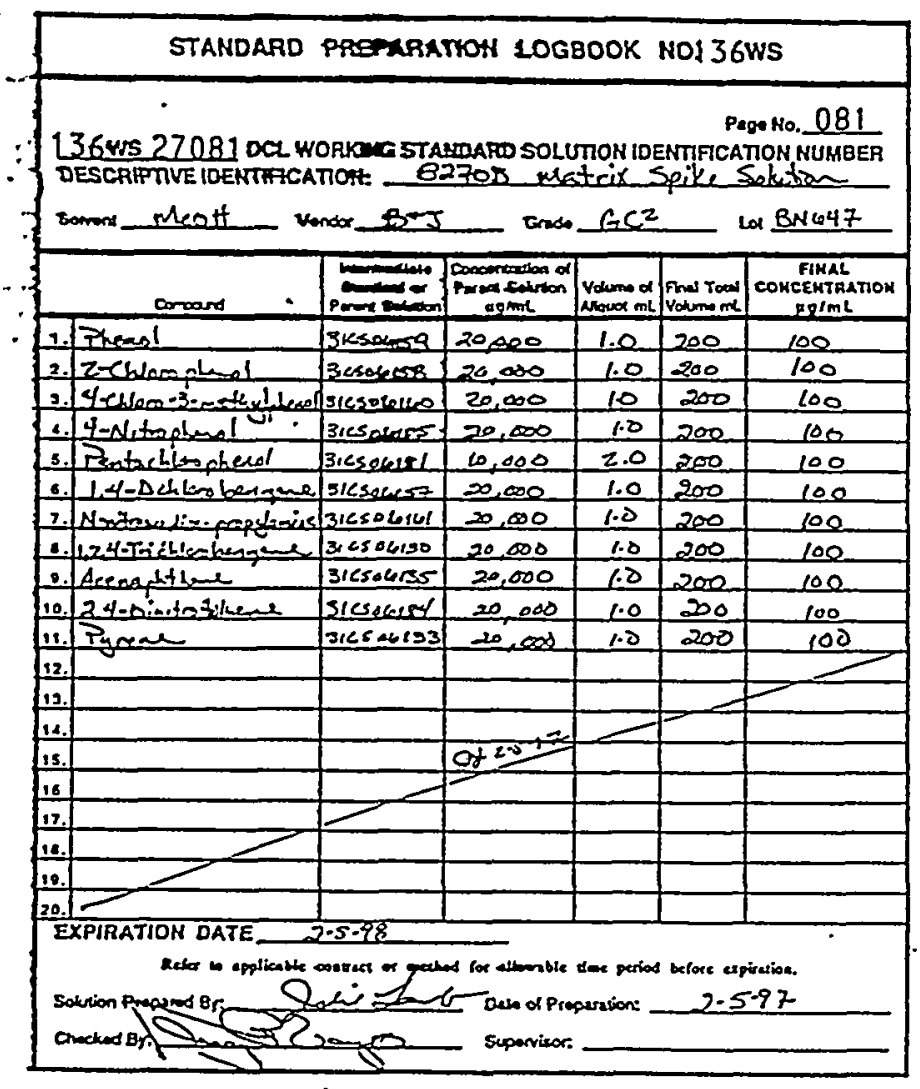

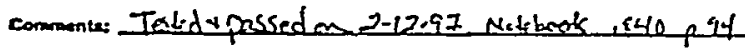

DATACHEHY YASORATURIES - GCMIS SEMNOLATLE ANAL YSTS

$$
\therefore: \therefore
$$

\section{CONCENTRATED STOCK STANDARDS}

STANDARD PREPARATION LOGBOOK NO. $31 \mathrm{CS}$

Page No. 158 31 CS 06158 DCL COHCENTRATEO STOCK SOLUTION IDENTIFICATION NUMBEER COMPOUND: 2-chbropheool. undor, Chemserv Latbo: $96-147$ Purly: $97.0 \%$

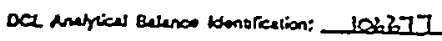

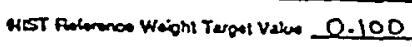

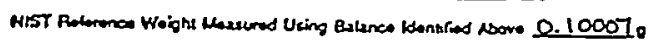

Darman 0.00007 .

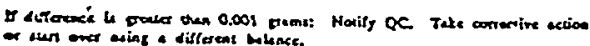

FINAL GROSS WEGHT 0.25597.

TARE WEIGHT 0.00000

NET WEGHT 0.15597

AET WEIGKT CORRECTED FOA FWATY $5 / 3 \mathrm{mg}$

${ }_{0.100069}^{0.000299}$ g Tareffest

0.0302059008

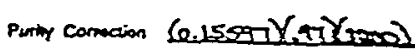

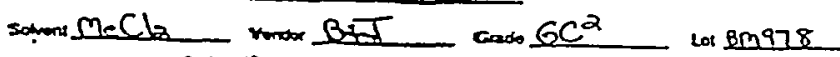

Druion Volum 7.56 - $\alpha$

FINAL CONCENTRATIOH 2DDOR ag/mL

EXPLRATION DATE_b/:209/97

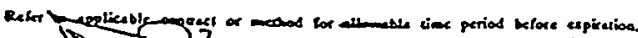

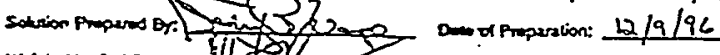

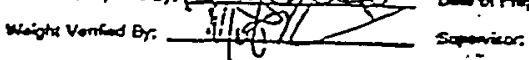

$-$

75
$136 W 527081: \cdots$

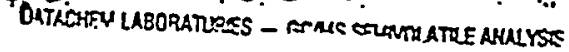
CONCENTRATED STOCK STANDARDS

- standaRd paEparation logBook No.31 CS

Pas NO. 159

31 CS 06159 DCL CONCENTIATED STOCK SOLUTION IDENTIFCATION NUMBER COMPOUND: — Plyethe

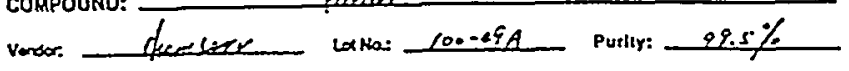

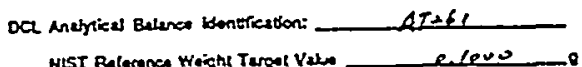

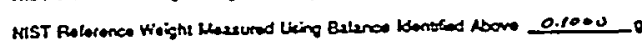

Dillosence 0.0000 -

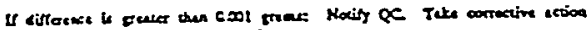

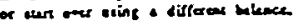

FINAL GROSS WEIGHT Q.PSII:-

TARE WEIGHT 0.0000 0.1521

NET WEIGHT

NET WEIGHT CORRECTED FOR PURTT $15 \% . \mathrm{mg}$

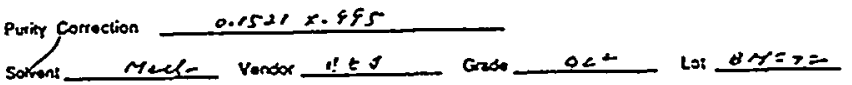

akean volume 7.5 ?

FINAL CONCEMTRATION 20000 $\mu \mathrm{g} / \mathrm{mL}$

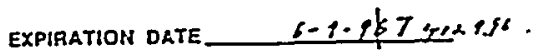

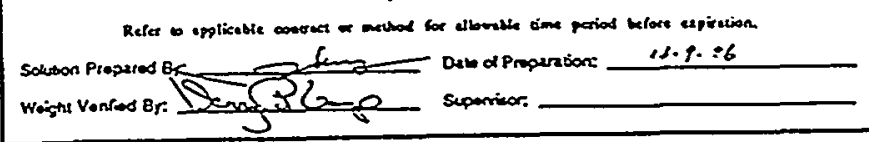

Comments:

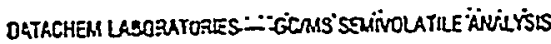

.. $\quad$ ir....

\section{CONCENTRATED STOCK STANDARDS}

STANDARD PREPARATION LOGBOOK NO.31 CS :

Page No. 160

31 CS 06160 DCL CONCENTRATEO STOCK SOUTON IDEMTITCAMON HUMBER

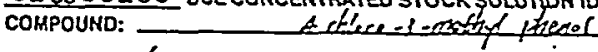

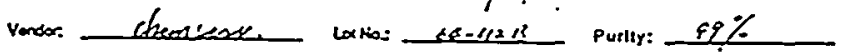

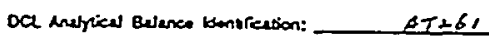

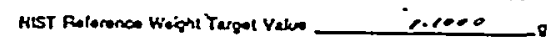

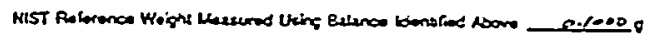

Dillocence 0.0000

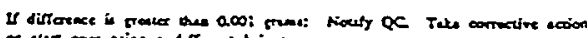

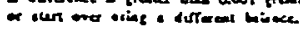

FINAL GROSS WEIGHT C.RSES :

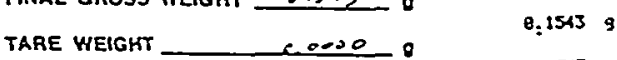

NET WEIGHT _. S.RYS

8.25039

NET WEIGHT CORFECTEO FOR PURTY $15.5 \mathrm{mo}$

Puriog conection 0.1jes C.P9.

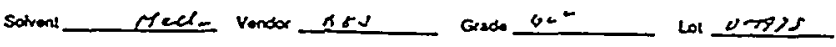

Davion Voume 7.65

FINAL CONCENTRATION ZE TrO $\mu g / m L$

EXPIRATION DATE $\quad 6-8.97$

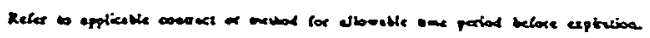

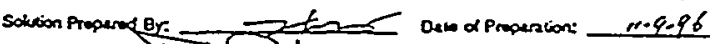

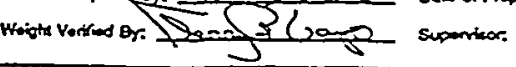




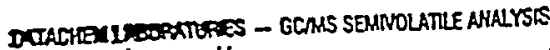

$\because$ -

CONEETTRATED STOCK STANDARDS

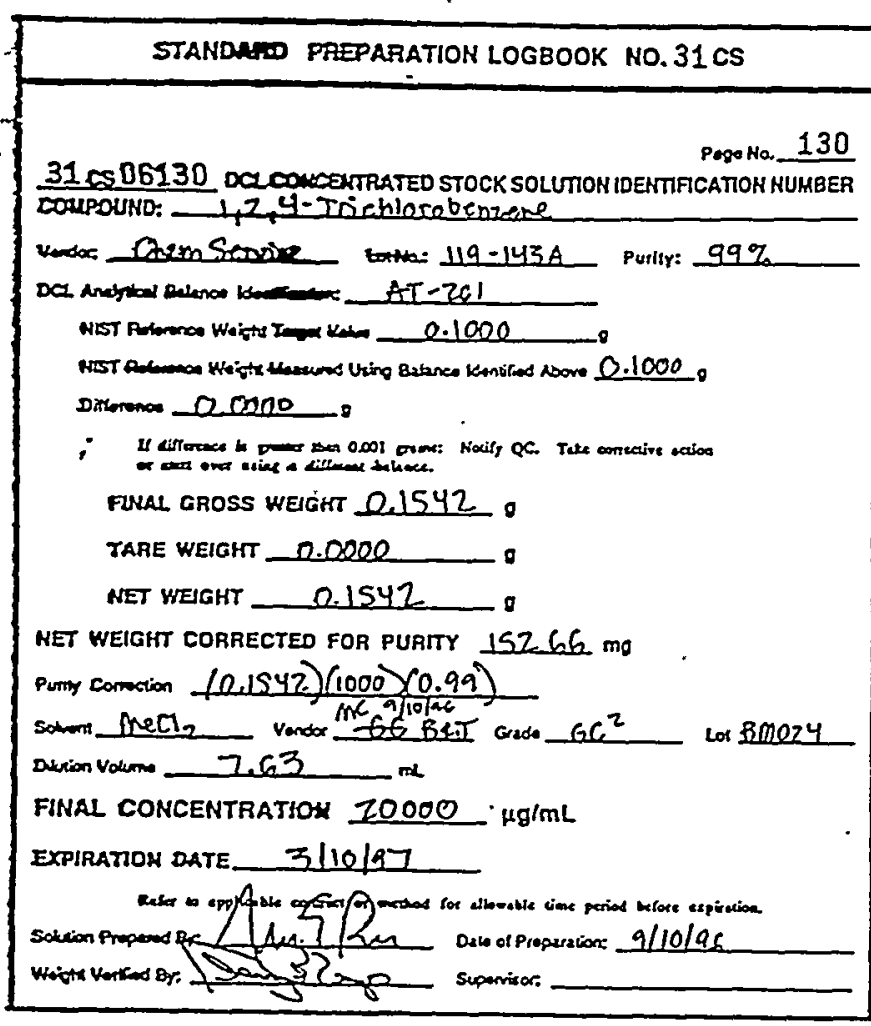

Commontex:

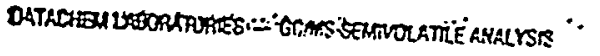
CONCENTRATED STOCK STANDARDS

STANDARD PTEPARATION LOGBOOK NO. $31 \mathrm{CS}$

Page No. 184

31 CS 06184 DCL CONCEMTAXED STOCK SOLUTION IOENTIFICATION HUMBER COKPOUND: 2,4-Dinitotoluene

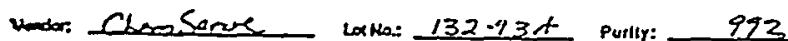

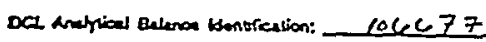

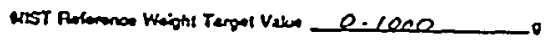

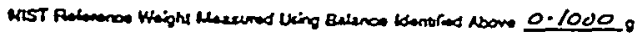

Dirsmanos acea

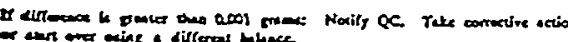

FINAL GROSS WEIGHT 0.13 SYlE 0

TARE WEIGHT 0.00000

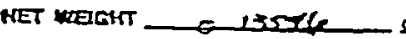

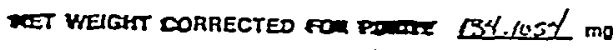

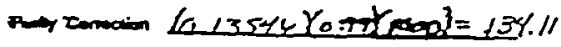

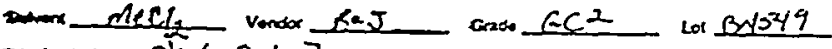

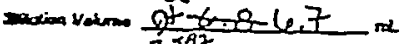

FAMAL CONEENTAATION $20.000 \quad \mu \mathrm{g} / \mathrm{mL}$

EXPLRATLON DATE_ 8-5-97

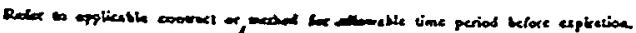

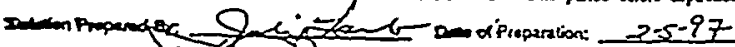

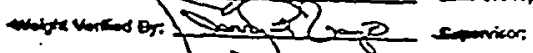

$2 \div 6$

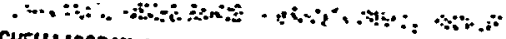

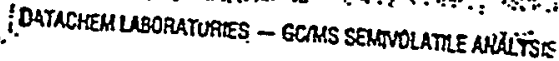

\section{CONCENTRATED STOCK STANDARDS}

STANDARD PREPARATION LOGBOOK NO. $31 \mathrm{CS}$

Page No. 135

31 CS 06135 DCL CONCENTRATED STOCK SOLUTION IDENTIFCATON NUNBER COMPOUND: Acenaghreme

verdor, ChemService Lorta: $127-91 A$ Punty: $99 \%$

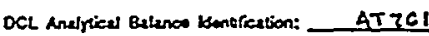

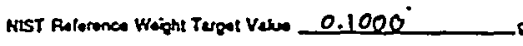

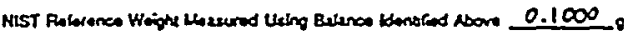
Ditserences 0.0000

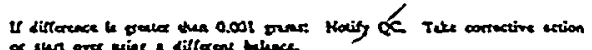

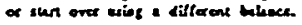

FINAL GROSS WEIGHT $0.146 \overline{3} 0^{\circ}$

TARE WEIGHT 0.0000

MET WEIGHT 0.1453

NET WEIGHT CORRECTED FOR PURITY $146.82 \mathrm{mg}$

Purit Correction $(1000)(0.99)(0.1483)$

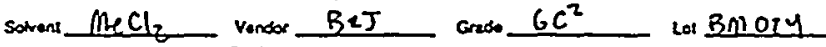

Davion volum 7.34

FINAL CONCENTRATION $20.000 \mu \mathrm{g} / \mathrm{mL}$

EXPIRATION DATE_ 3/24/97

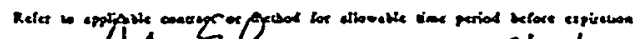

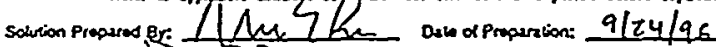

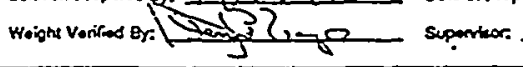

Comments:

DітAсHEM Lisoran

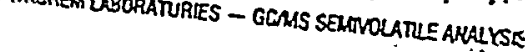

\section{CONCENTRATED STOCK STANDARDS}

STANDARD PREPARATION LOGBOOK NO. $31 \mathrm{cS}$

Pooe No. 133

31 CS 06133 DCL CONCENTRATED STOCK SOLUTIOH IDENTAFCATON NUMBER COMPOUND:

vandor. Chemservice cortas $122-1078$ purlty: $98.0 \%$

OCL Aneyrices Balence bentreation: ___ ATZGI

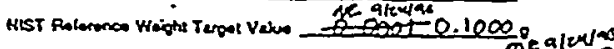

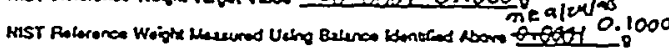

Dillerence 0.0000

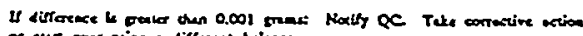

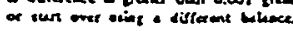

FINAL GROSS WEIGHT 1.1628 .

TARE WEIGHT 0.0000

NET WEIGHT 0.1628

NET WEIGHT CORRECTED FOR PURTY $159.54 \mathrm{mg}$

Puniry Correction $(1000)(0.98)(0.1620)$

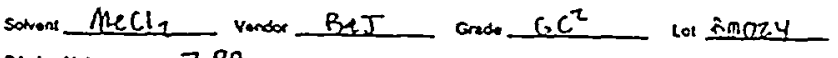

avion valume 7.98 Me

FINAL CONCENTRATION $20,000 \mu \mathrm{g} / \mathrm{mL}$

EXPIRATION DATE $3 / 24 / 97$

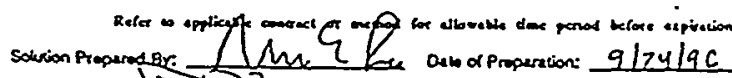

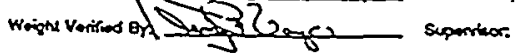




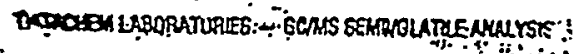 CONCZUTEATED STOCK STANDARDS}

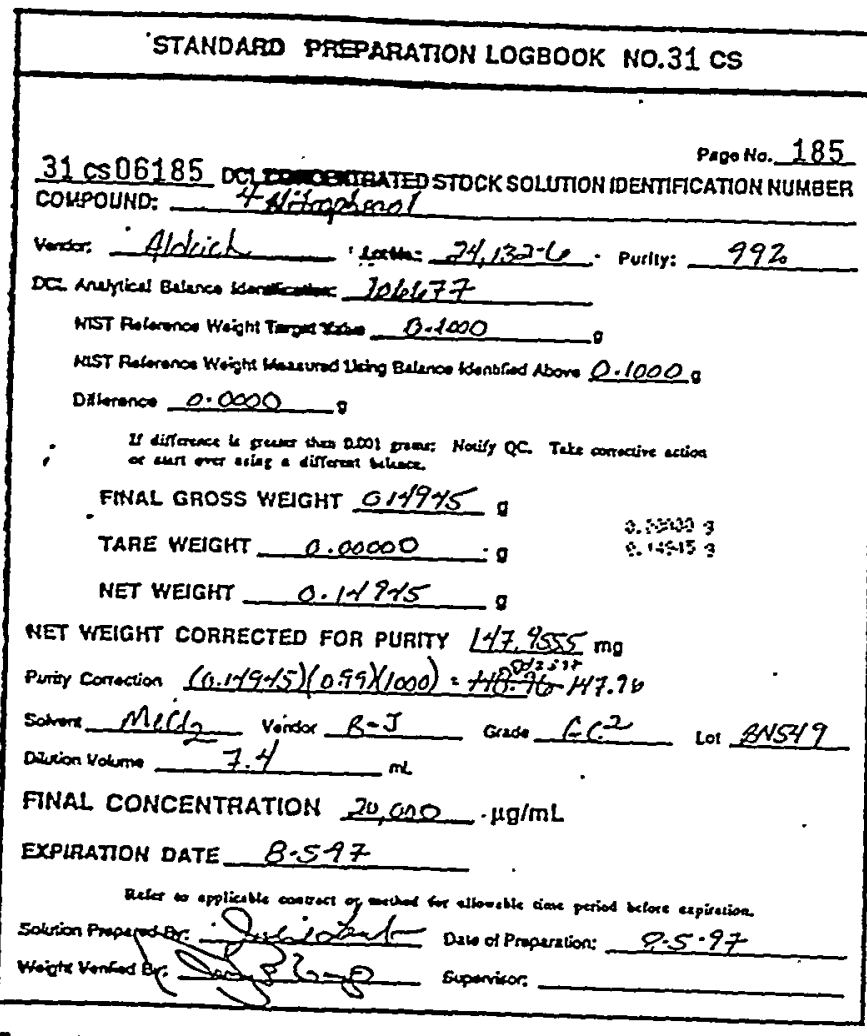

Comonomer:

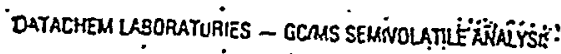

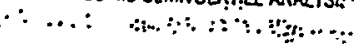

\section{CONCENTRATED STOOK STANDARDS}

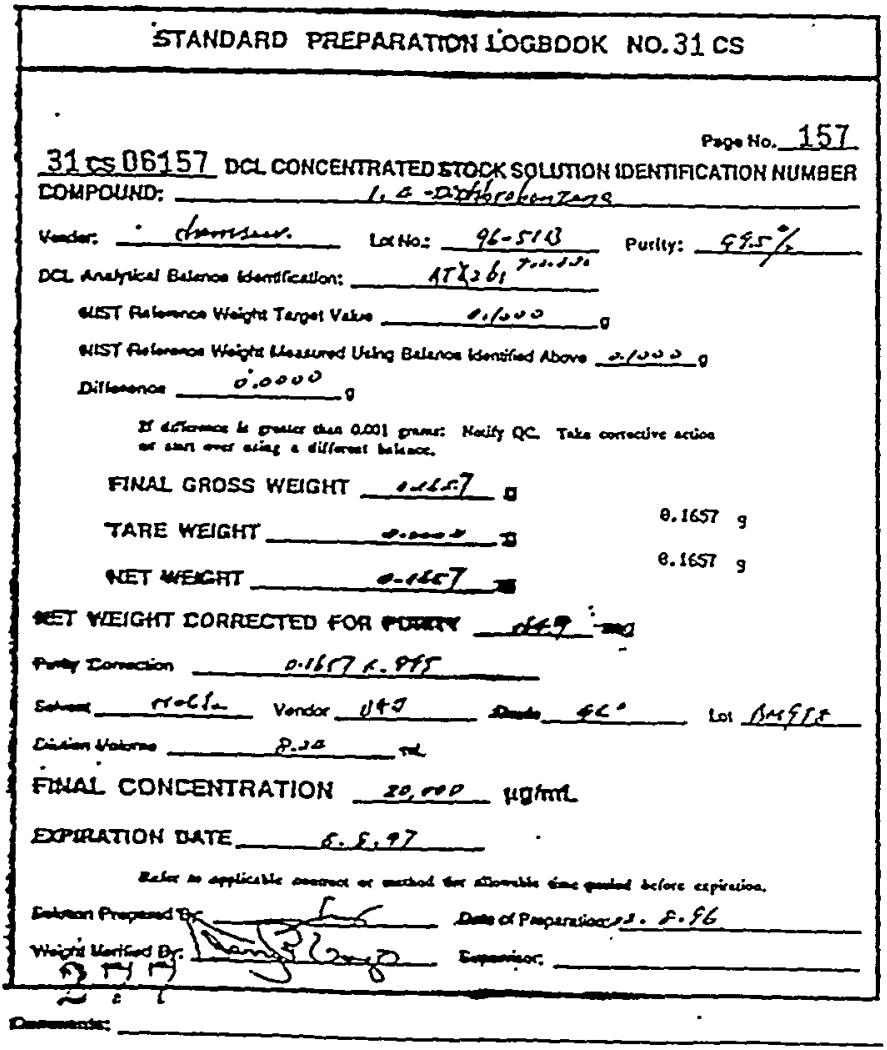

OATACHEM LaboräingIES CONCENTRATED STOCK STANDARDS

STANDARD PREPARATION LOGBOOK NO.31 CS

Pege No. 181

31 CS05181 DCL CONCENTRATEO STOCK SOLUTION IDENTIFCATION NUMEER CoMPOUND: Perdachbrepherel

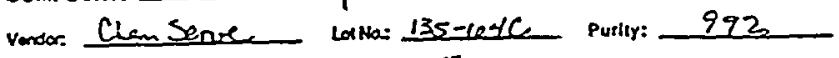

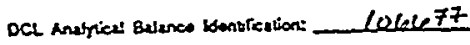

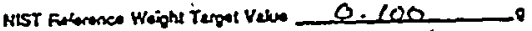

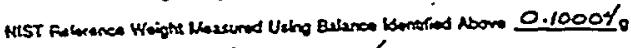

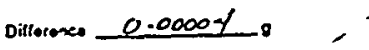

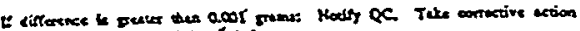

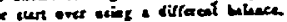

FINAL GROSS WEGHT $0.0833 \mathrm{U} g$

TARE WEIGHT 0.00000

NET WEIGHT -O 202336 - 82.5264

NET WEIGHT CORRECTED FOR PURITY TO

Purity concion $(0.08350)\left(0.79 f^{2000}\right)=82.5204$

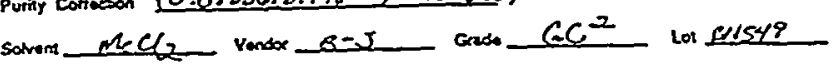
Delvion Votum 8.25

FINAL CONCENTRATION $10,000 \mu \mathrm{g} / \mathrm{mL}$

EXPIRATION DATE_ 8-3-97

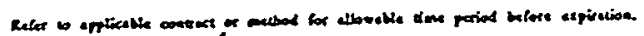

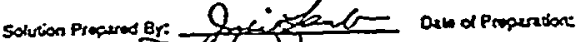

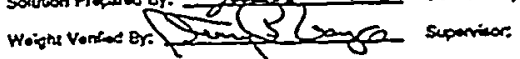

Comments:

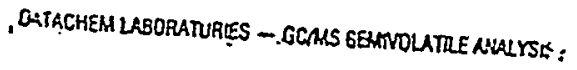

\section{CONCENTRATED STOCK STANDARDS}

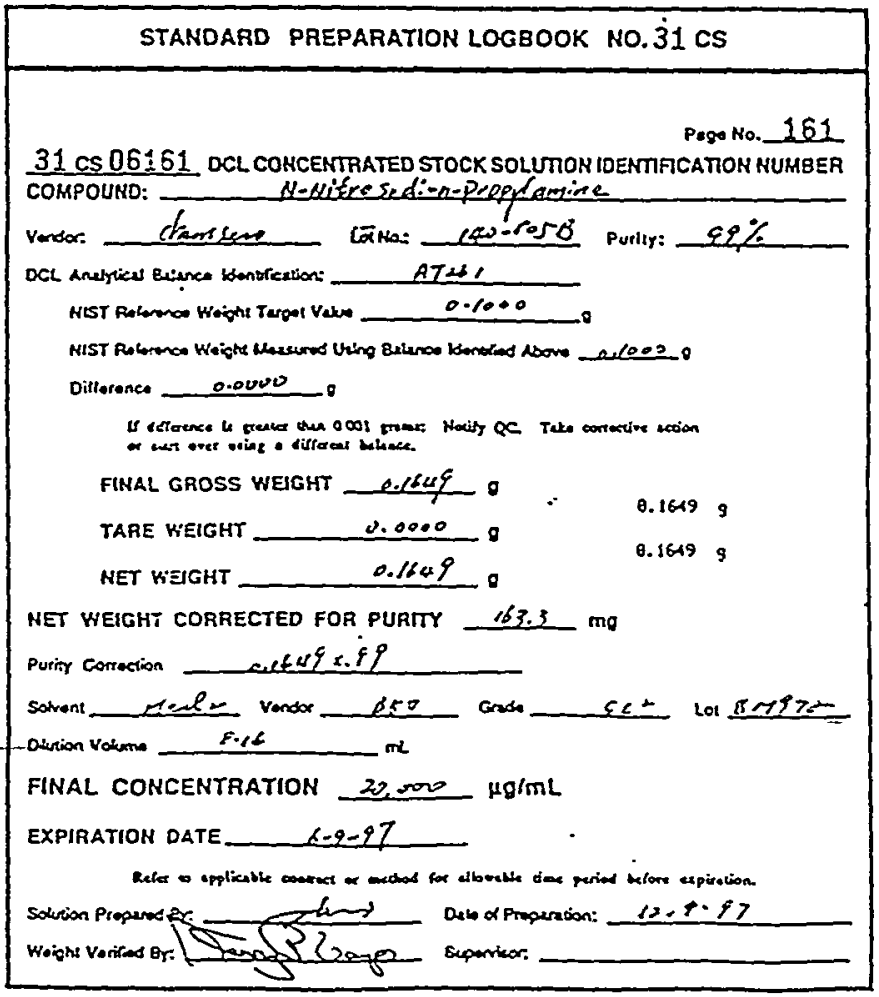

Comments: 


\section{WORKING STANDARDS}

PART V

\section{INTIIAI CALJBRATION STANDARDS}

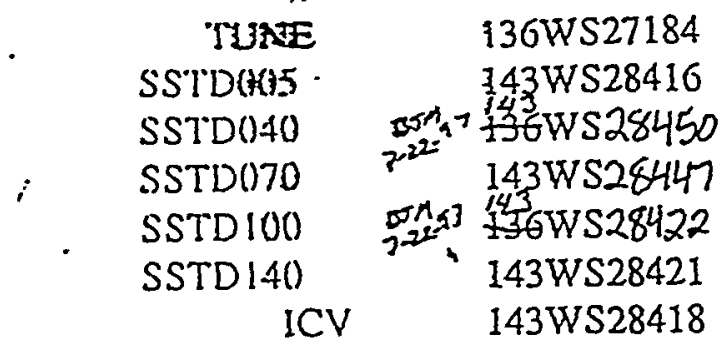

WORKING STANDARDS

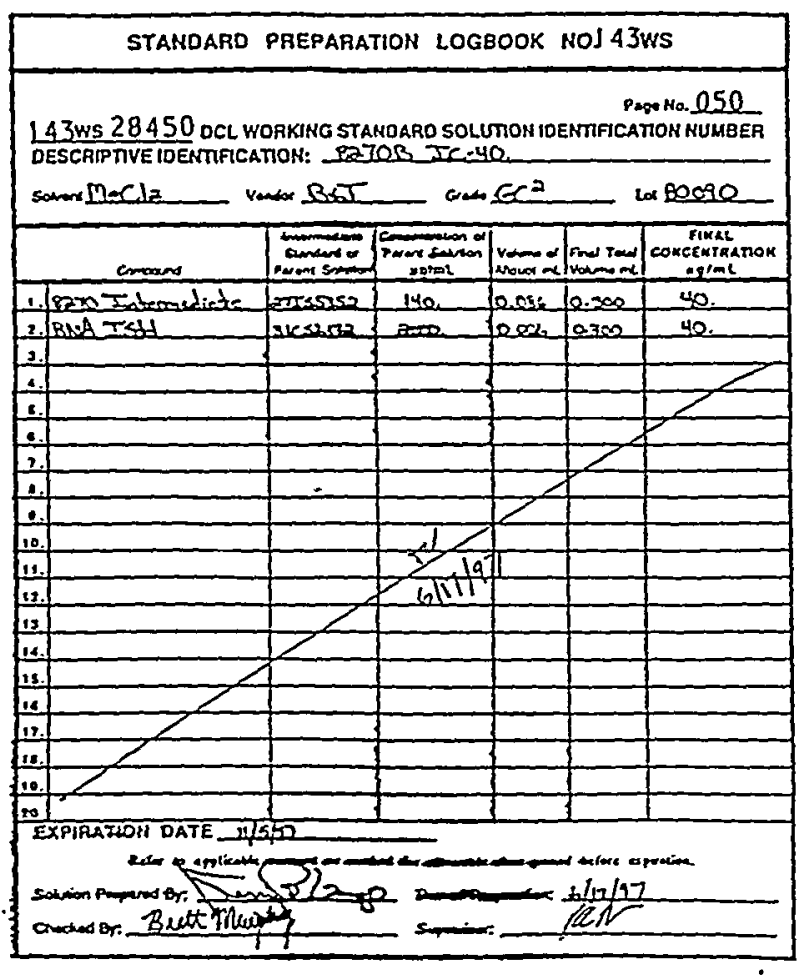

comonerst
STAHDARD PREPARATION LOABOOK NOI 43 WS

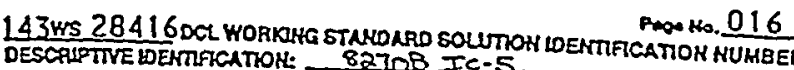

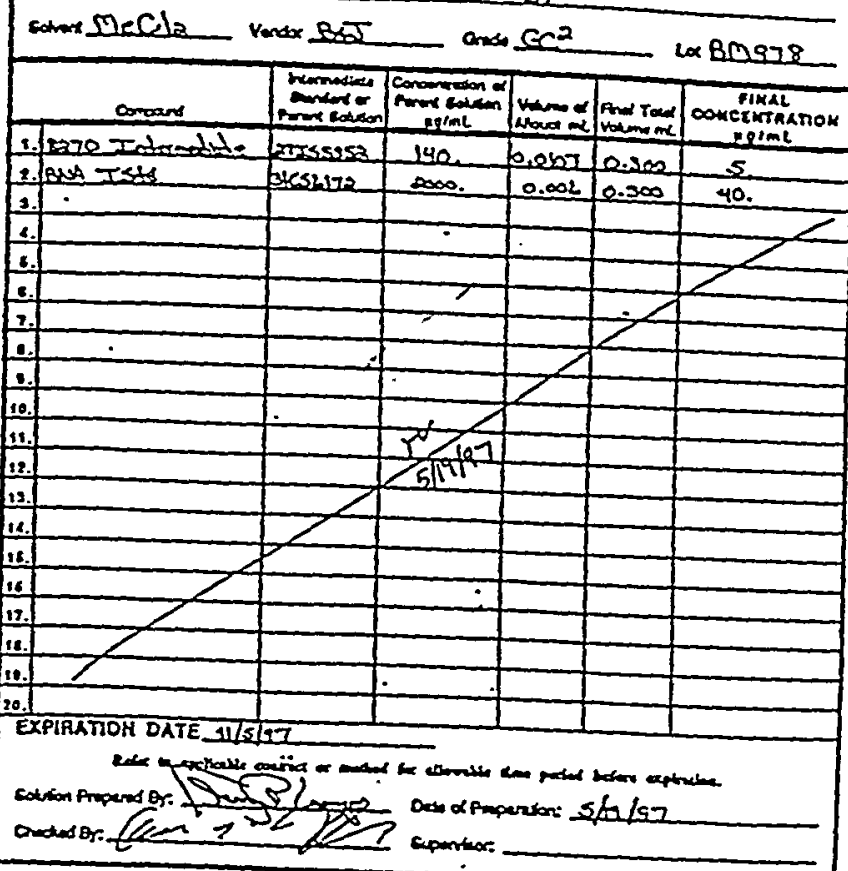

coromente:

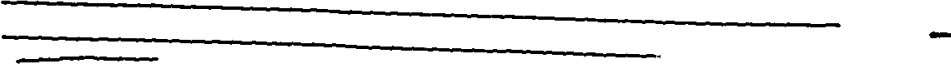

WORKING STANDARDS

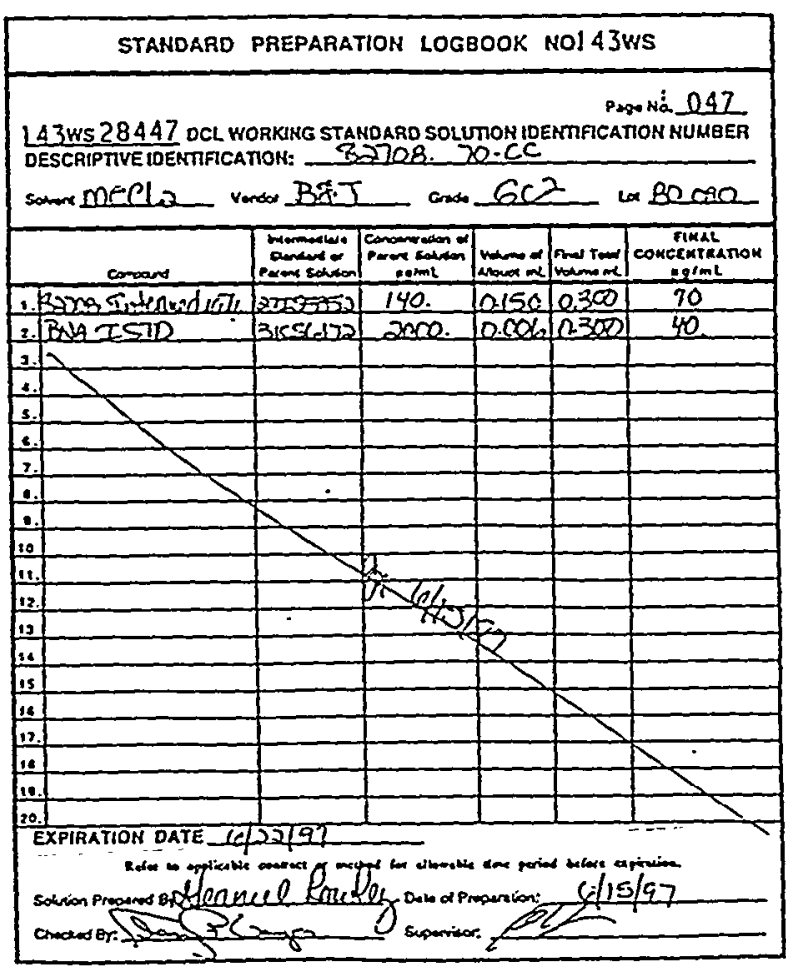

commonts: 


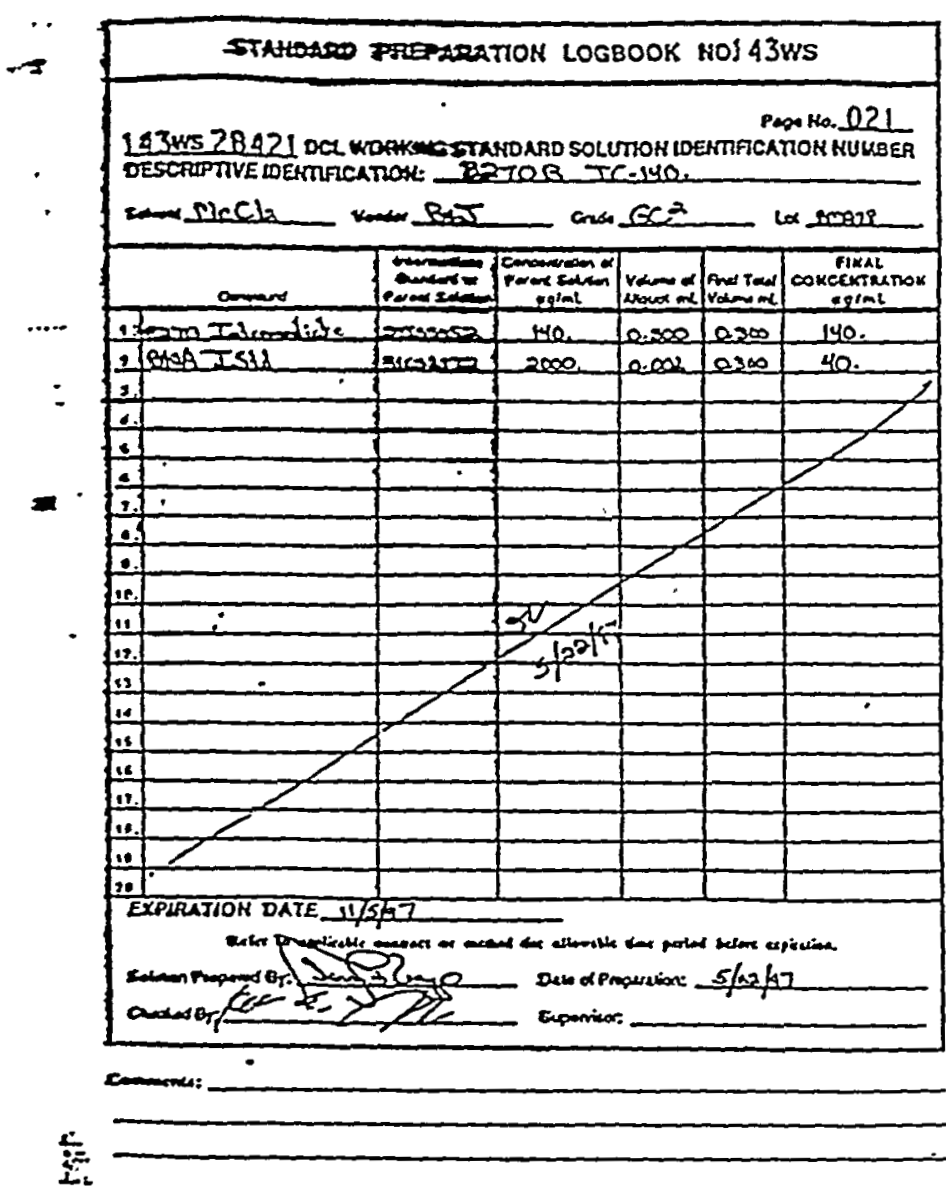

WORKIRG STANDARDS

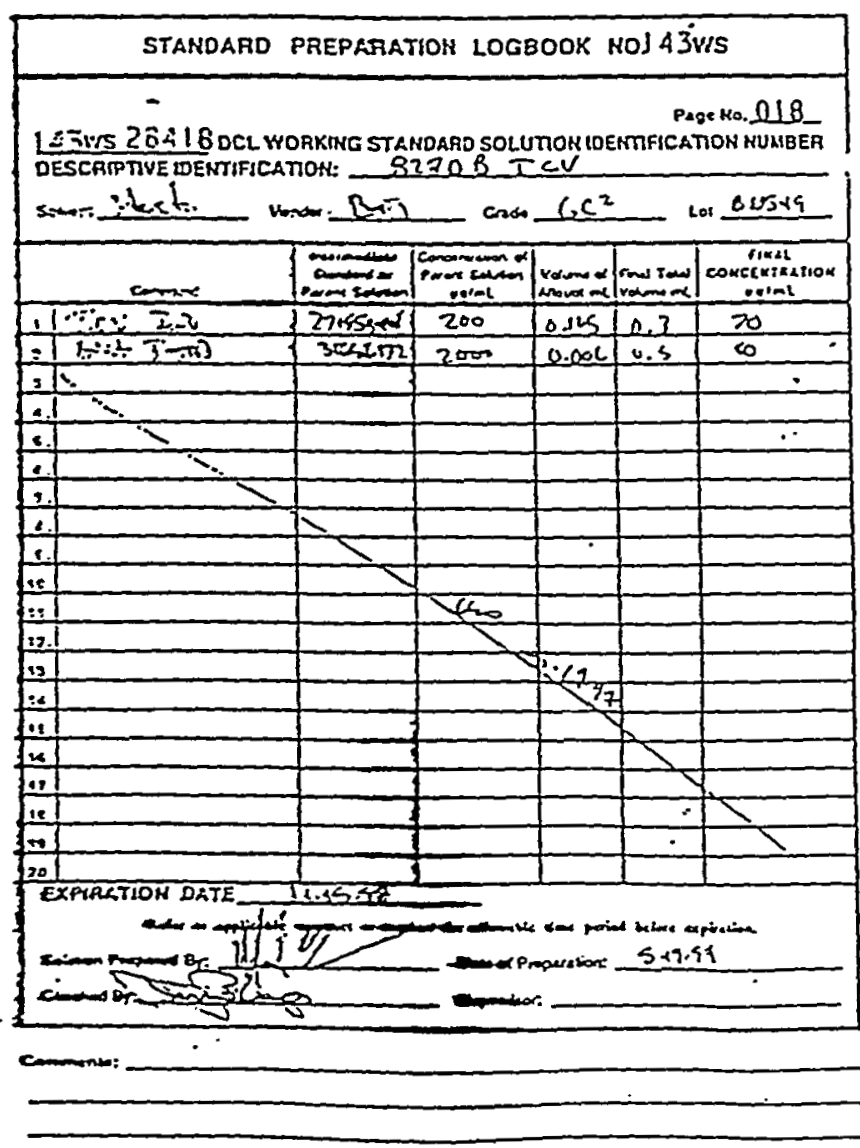

$2 \div 9$
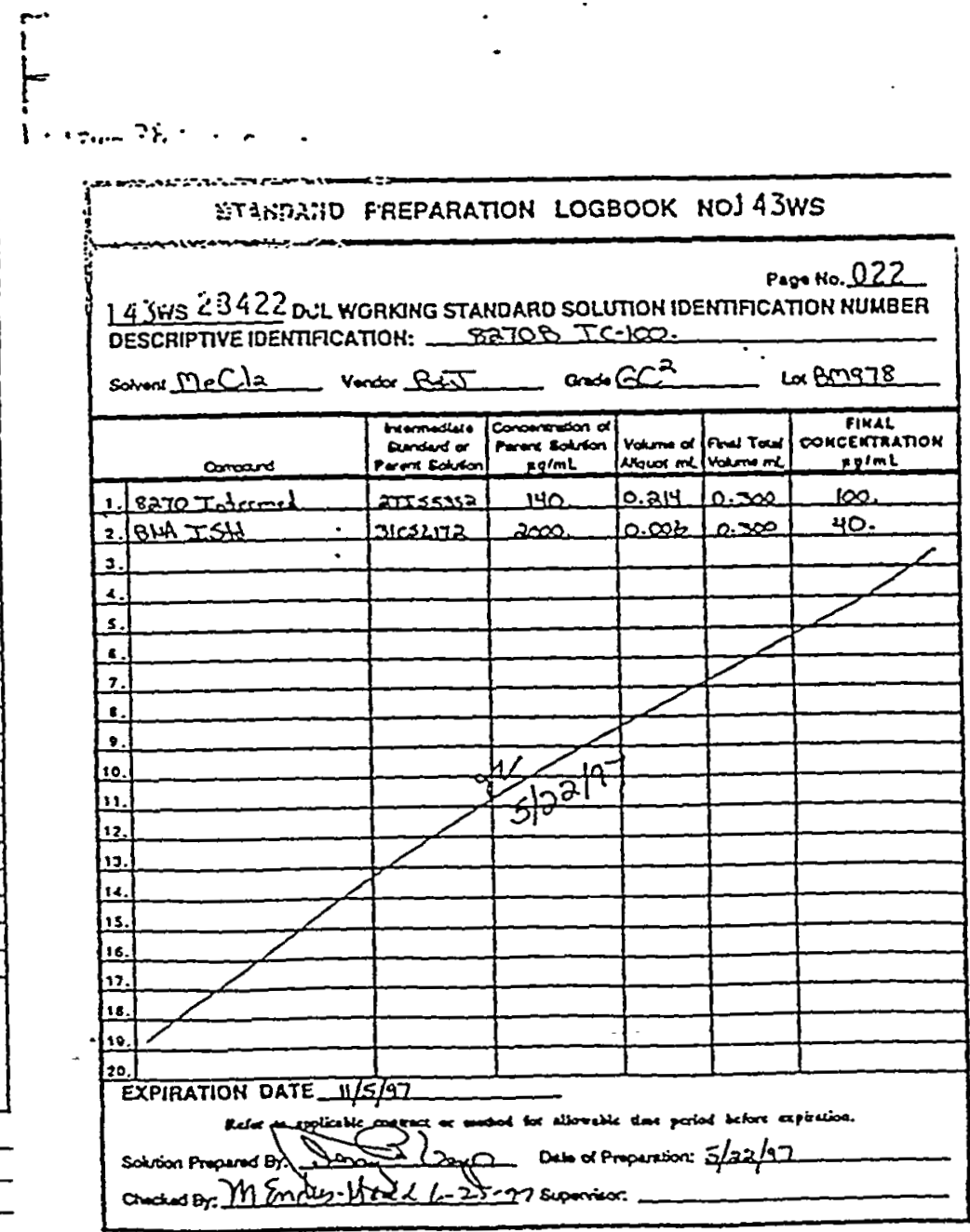

Commonte: 


\section{WORKING STANDARDS}

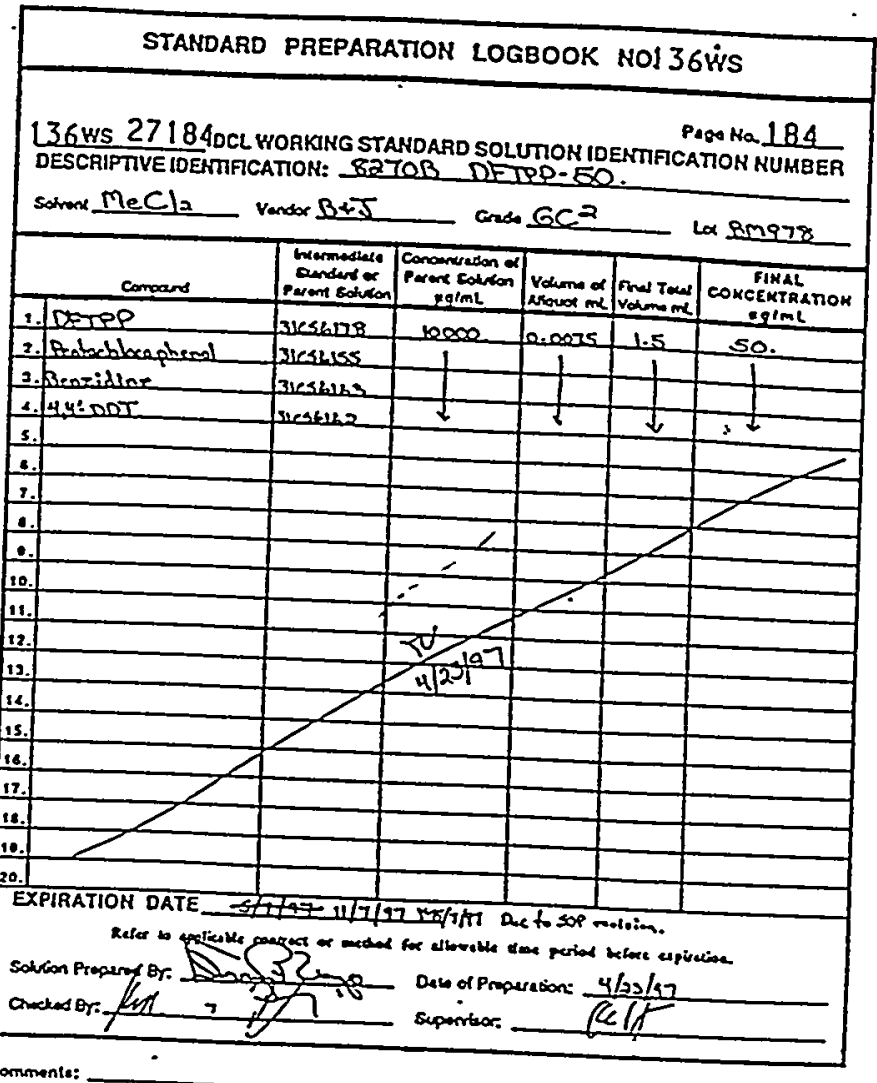

Commente:

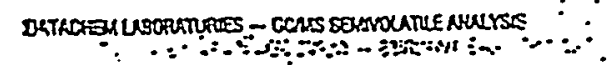

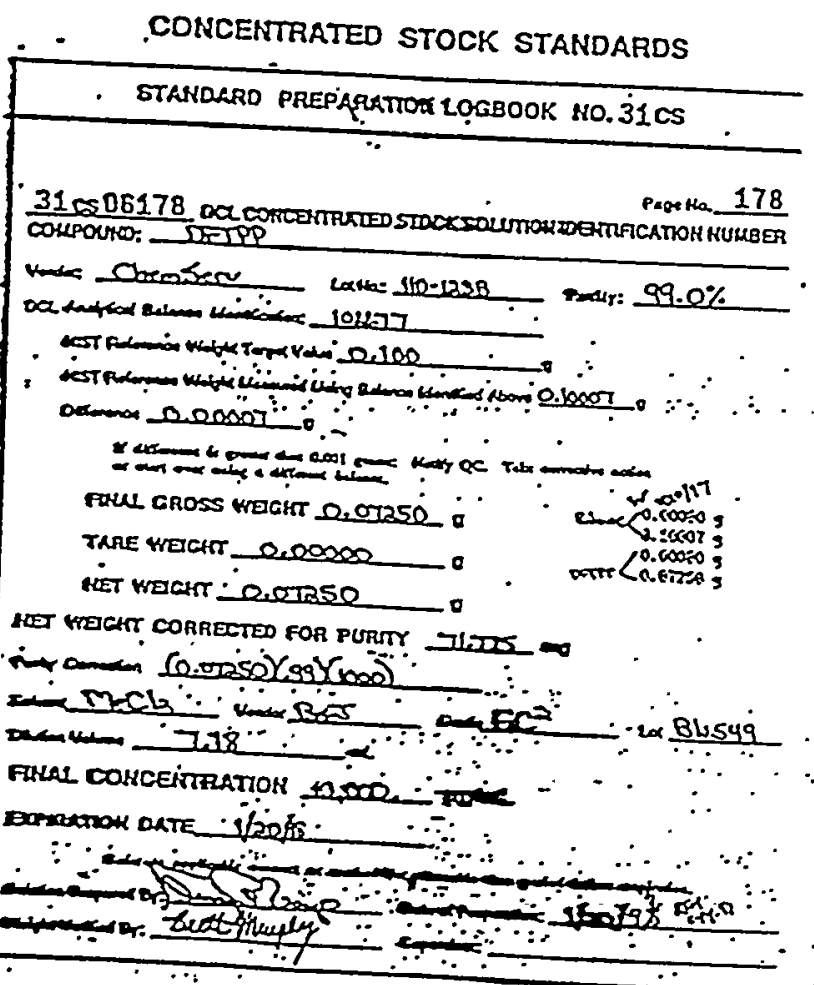

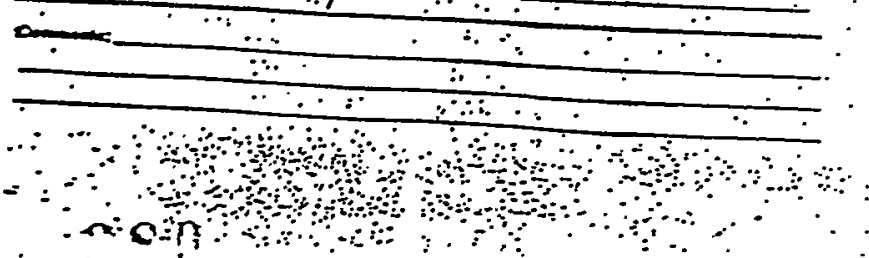

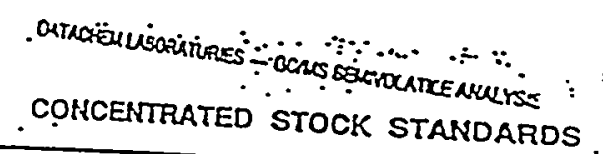
STAMOARD PREPARATION LOGBOOK NO. 31 CS

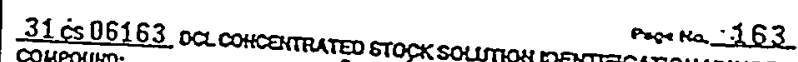

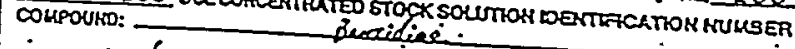

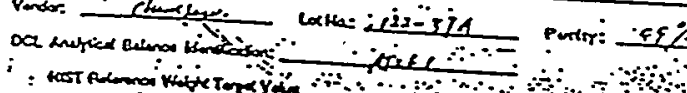

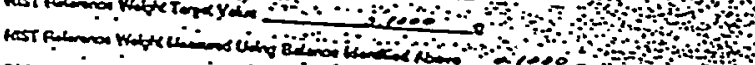

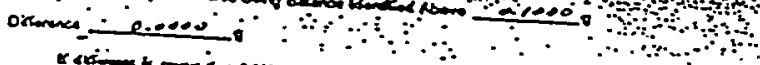

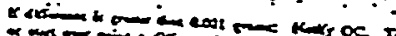
FHUL GROSS Waikt cheitis : TARE KECHT NET weart a.ters: HET HEGHT CORRECTED FOR PURTK :

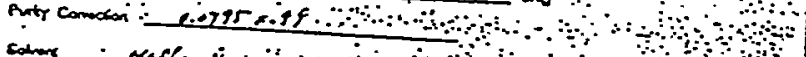

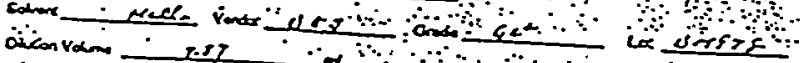

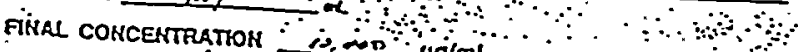

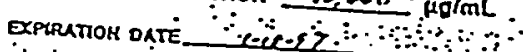

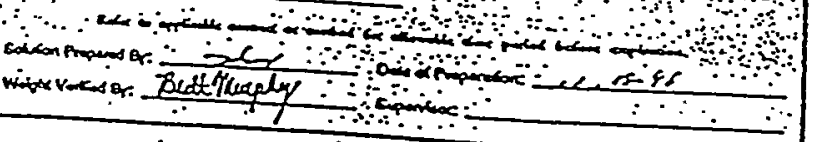




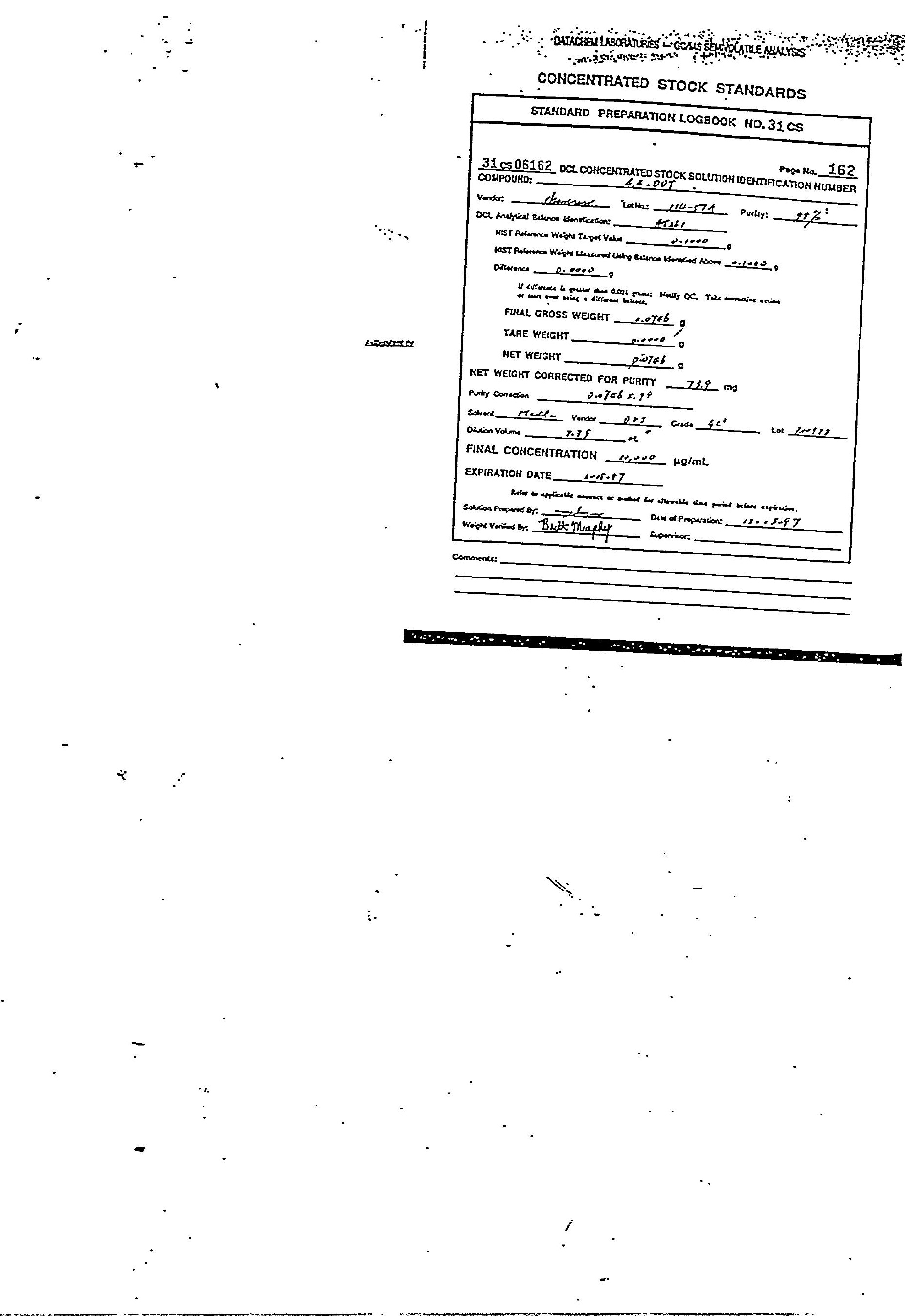




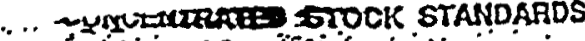

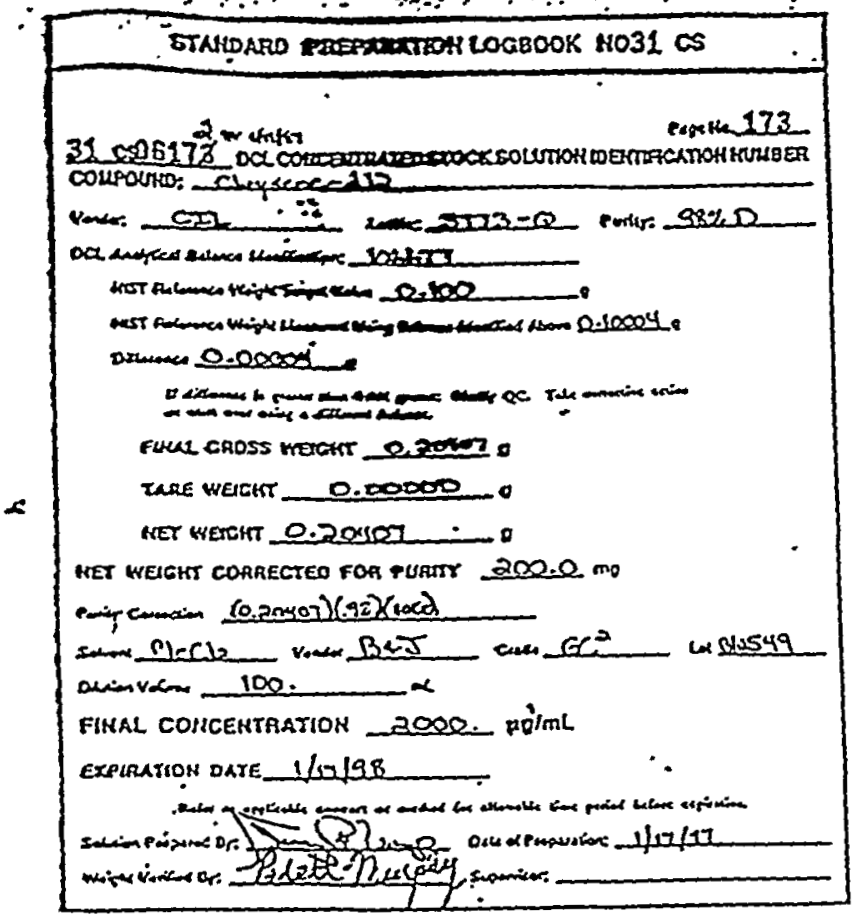

Comomos:

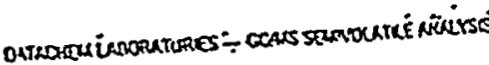

- CONCENTRATED STOCK STANDARDS

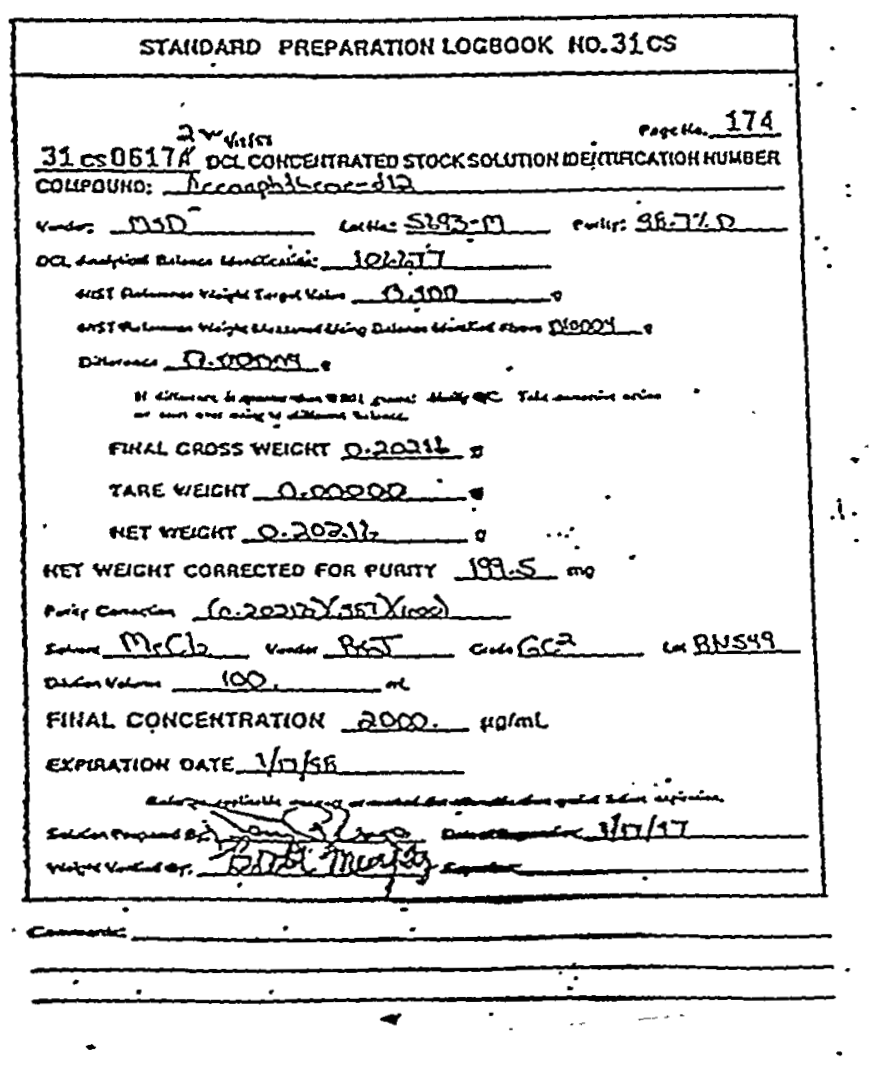

$\because$ - CONCENTRATED STOCK GTAKOARO

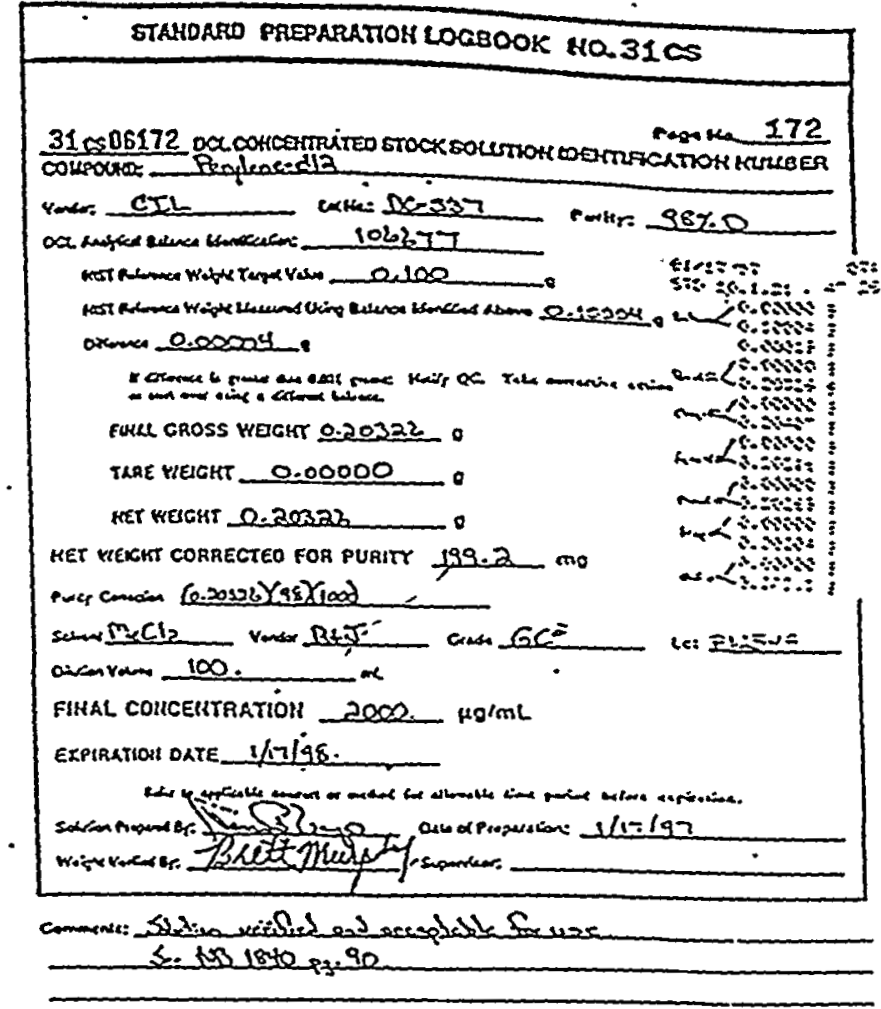

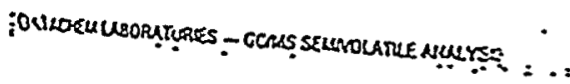

CONCENTRATED STOCK STANDAROS

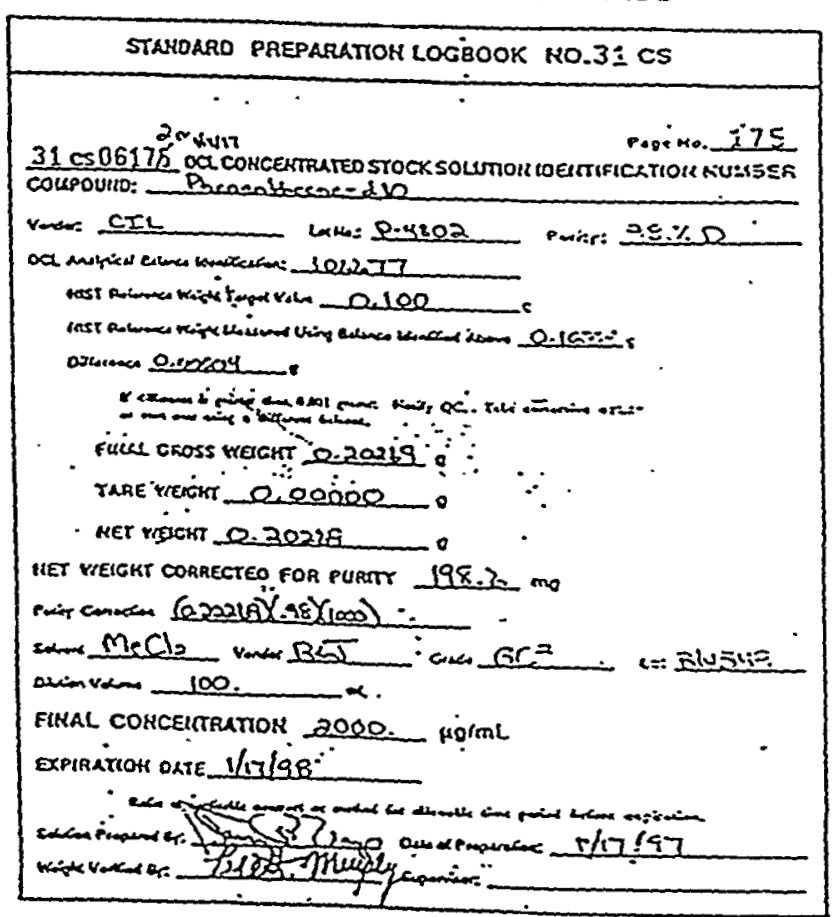

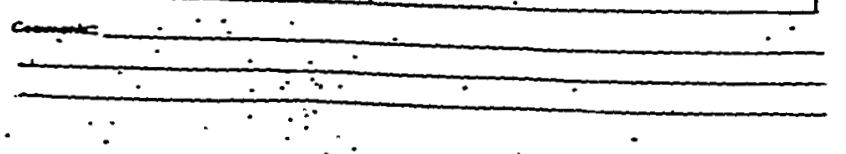

$\cdot \gamma$ 
$\because+$

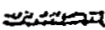

$:$

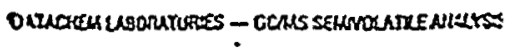

CONCENTRATED STOCK STANDARDS

STANDARD PAEPARATION LOGBOOK NO.31 CS

31 cs06177 Yalkz eagsti. 177

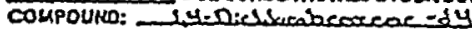

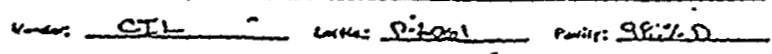

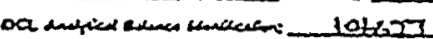

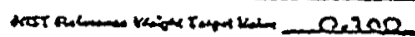

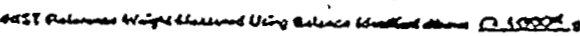

a.coest.

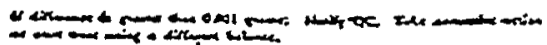

rWML choss weickr $\ddot{0} 203110$.

TARE WEAT _0.00000

NeT WEICKT OL20311

IET MESGK CORRECTEO FOR PURTY $199.0 \mathrm{mo}$

Nir com (0.2031) $(58)(100)$

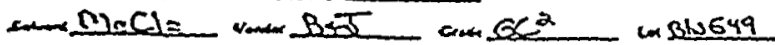

100 .

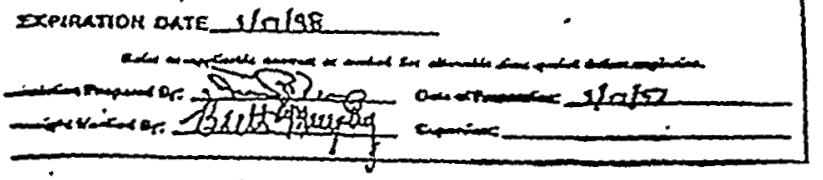

-

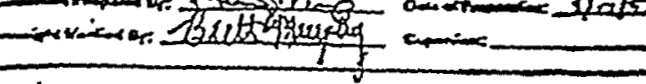

ece

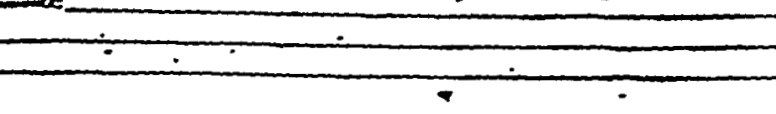

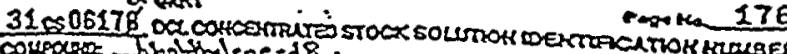
colseser tibativalene-18

von CIL

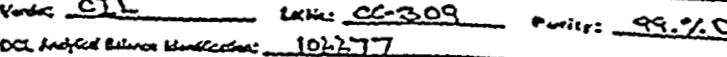

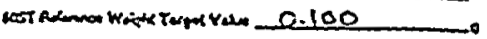

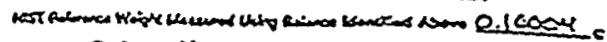

oring 0.00004 .

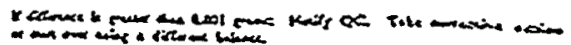

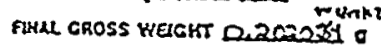

TLRE WESHT 0.00000 0

NET WeIEK 0.30204

HET WESTT CORRECTED FOR PURTK \$0.0 ms

Pvicr commen (0.20201) (so) $(1000)$

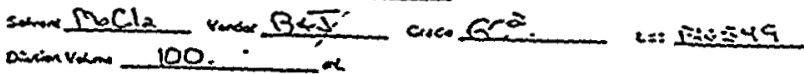

FIHAL COHCENTRATIOH 2000 . $\mu$.

EXPIRATIOH OXTE_//2/96 -

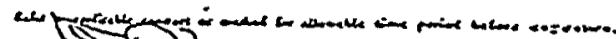

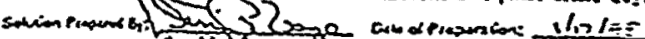

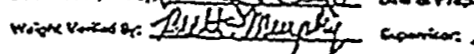

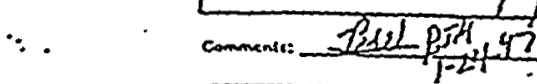

leskes 


\section{STANDARD SOLUTIONS OF INTERMEDIATE CONCENTRATION}

\section{-STANDARD PREPARATION LOGBOOK NO.27 IS}

Page No. 152

27 IS 5352 DCL INTERMEDIATE STANDARD SOLUTIQN IOENTIFICATION NUMBE் DESCRIPTIVE IDENTIFICATION: $8270 \mathrm{~B}$ Indermeliate

Solvent Me $C_{2}$ vendor BFJ Grado ${ }_{2} C^{2}$ Lot'.BN549

\begin{tabular}{|c|c|c|c|c|c|c|}
\hline & Comoound & $\begin{array}{c}\text { Slock Std } \\
\text { or Parent } \\
\text { Solution iD No. } \\
\end{array}$ & $\begin{array}{c}\text { Concenteaton of } \\
\text { Perani Solution } \\
\text { uglmL }\end{array}$ & $\begin{array}{l}\text { Volume of } \\
\text { Allouot } \mathrm{mL}\end{array}$ & $\begin{array}{l}\text { Final Tolal } \\
\text { Volume } \mathrm{mL}\end{array}$ & $\begin{array}{c}\text { FINAL } \\
\text { CONCENTRATION } \\
\text { Hg/mL }\end{array}$ \\
\hline 1. & Abs Mix \# 030696 & 82501 & 2000 & 0.7 & io & 140 \\
\hline 2. & 012297 & 82502 & 1 & 1 & 1 & \\
\hline 3. & 042596 & 91734 & & & & - \\
\hline 4. & 031496 & 10001 & & & & \\
\hline 5. & 041696 & 10002 & & & & \\
\hline 6. & 111396 & 10004 & & & & \\
\hline 7. & 040896 & 10005 & & & & \\
\hline 8. & 102596 & 10007 & & & & \\
\hline 9. & 021696 & 100013 & . & & & \\
\hline 10. & 041696 & 10018 & & & & \\
\hline 11. & 3-Methuldearenthere & $37<507201$ & 10,000 & 0.14 & 10 & 140. \\
\hline 12. & 2Flourophenol & $31<506187$ & 20,050 & 0.07 & & \\
\hline 13. & 2 chloreghenet DU & $31 \operatorname{cs} 06,156$ & $10,000 X_{j}$, 10.78 & $b_{14}\left(x+y_{2}^{2}=2\right.$ & -97 & . \\
\hline 14. & Plenel $05^{\circ}$ & $3)<506168$ & $20,0 \times x^{2}$ & 0.071 & & \\
\hline 15. & 1,2 D, 13 oro benzue-04 & 31050.188 & & \begin{tabular}{|l|l|} 
& $\ddots$ \\
\end{tabular} & & \\
\hline 16. & A trobannen - DS & 310506148 & & & & \\
\hline & 25louns bi phenem & $39 \mathrm{Cs} 06171$ & - & $v_{3}$ & & - \\
\hline 18. & 2,4,6 Tribarainglein: & 310566170 & $d$ & $\therefore .1$ & $\therefore$ & $\because$ \\
\hline 19. & Terduense-D14 & $3 i c s 06169$ & 10,000 & 0.014 & & 1 \\
\hline 20. & & & & & & \\
\hline \multicolumn{7}{|c|}{ EXPIRATION DATE $5 \cdot 5 \cdot 93$} \\
\hline
\end{tabular}

Comments: 


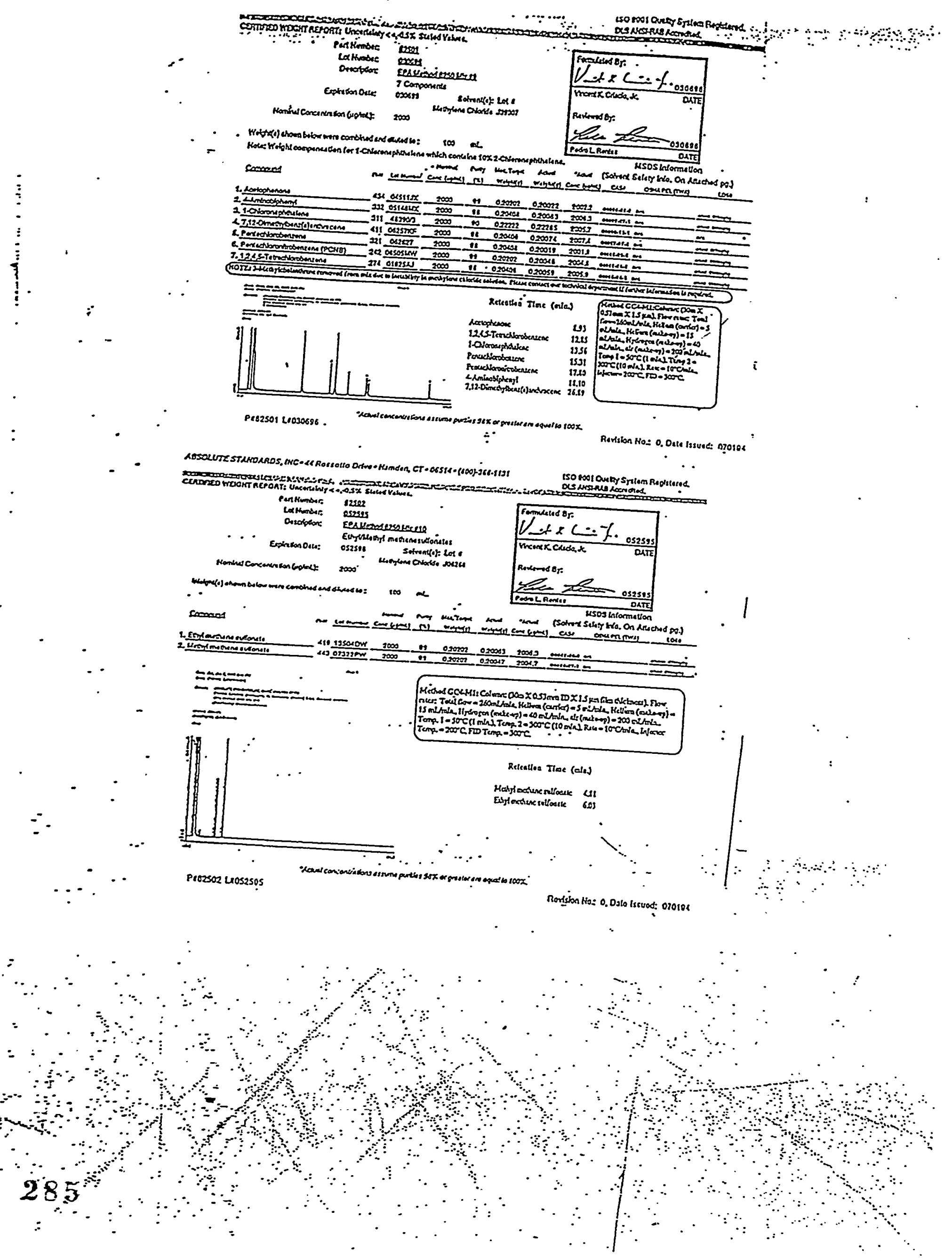




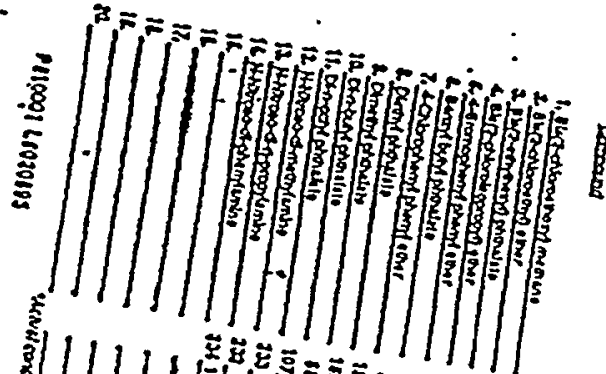

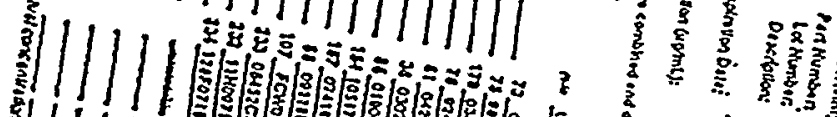
与ิ)

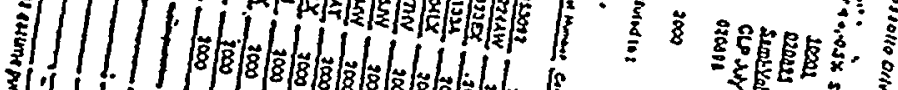

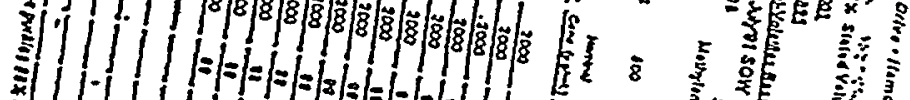

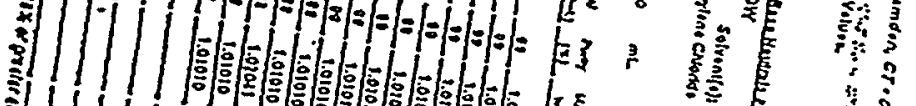

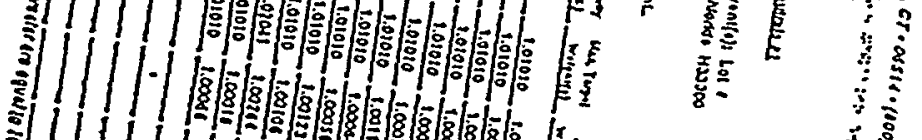

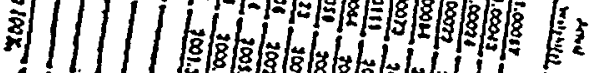
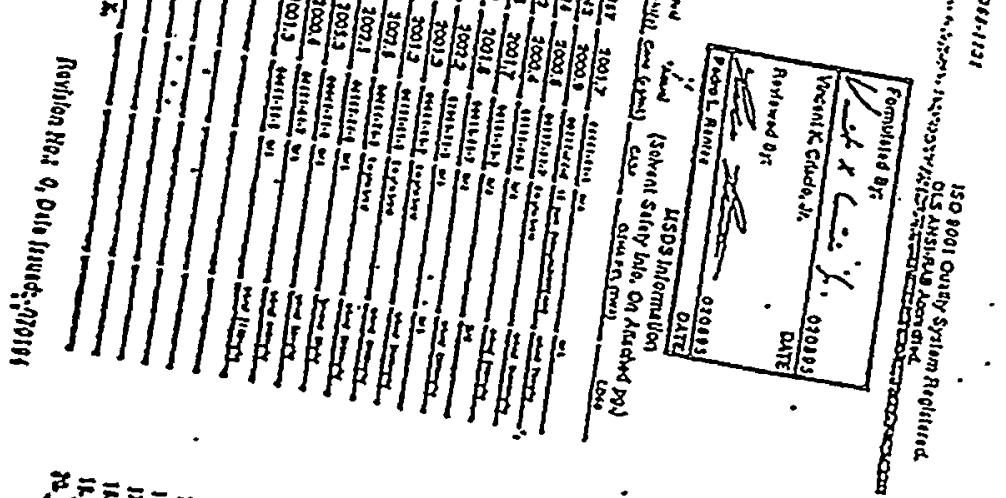
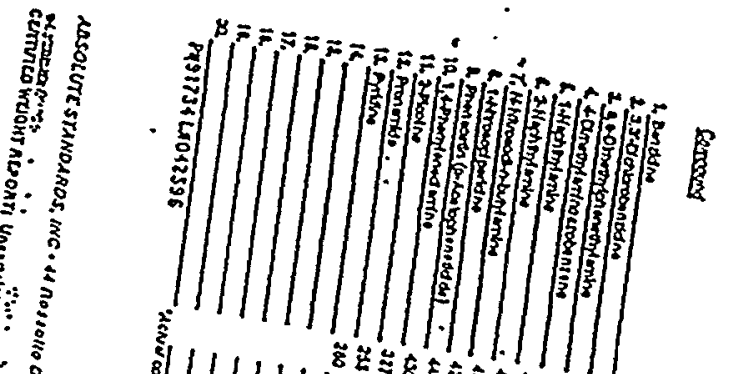

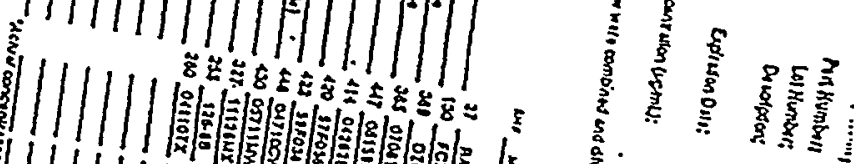

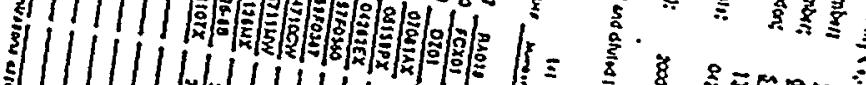

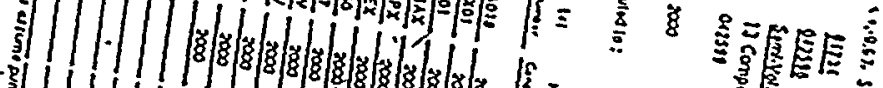

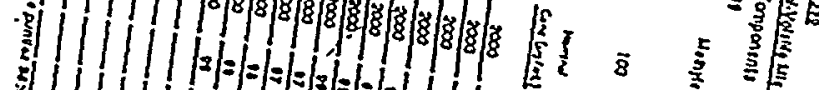
巽 1 (I)

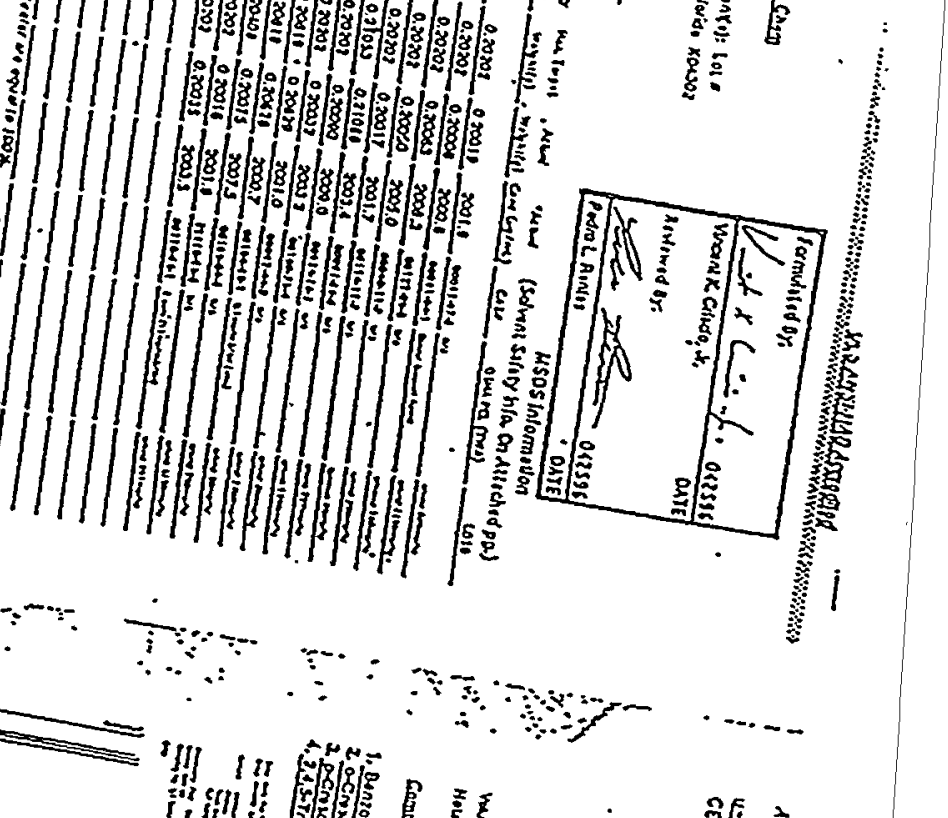

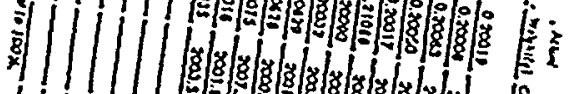
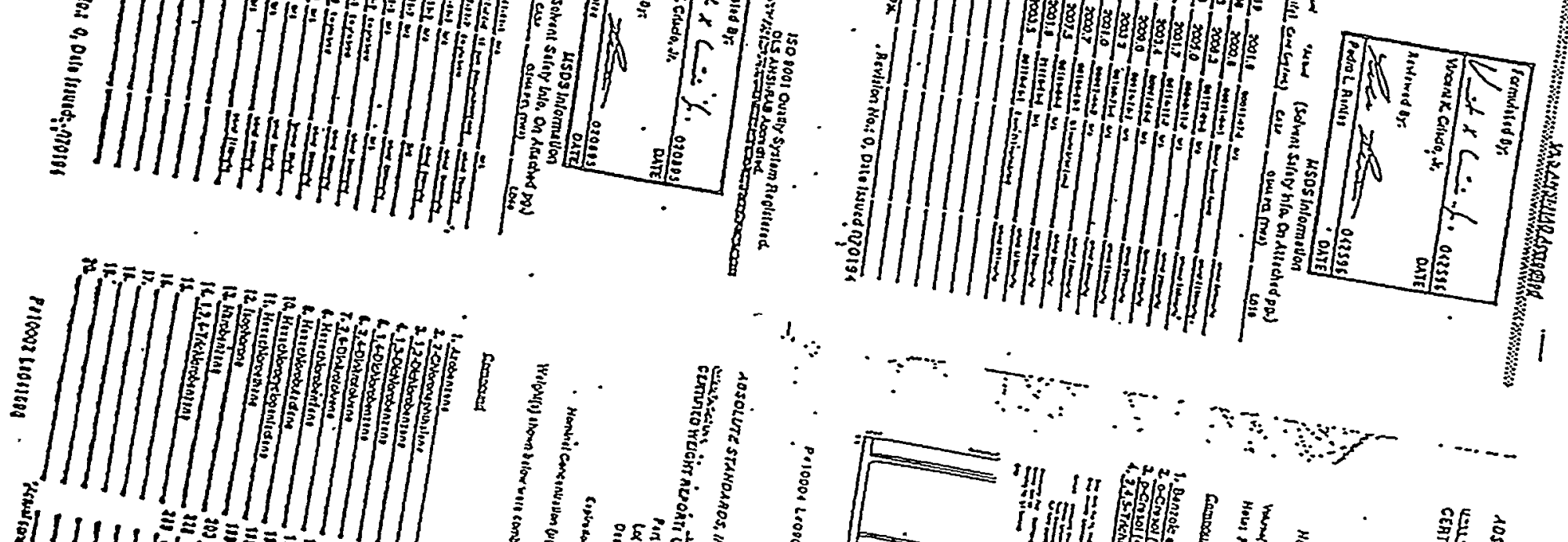

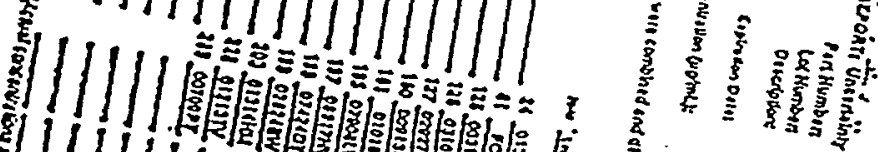

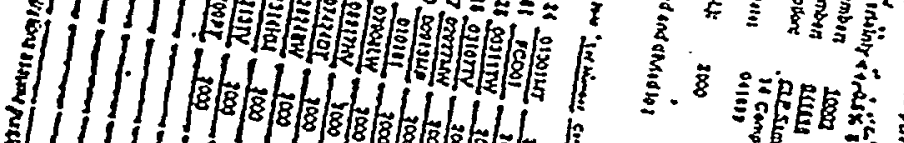

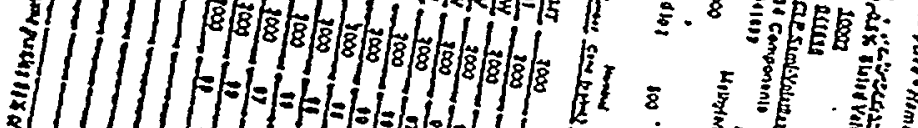

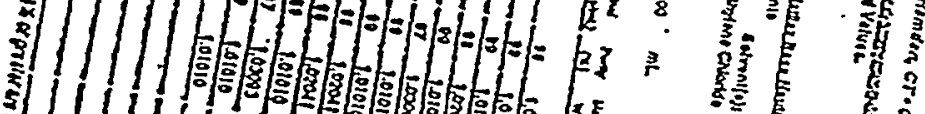

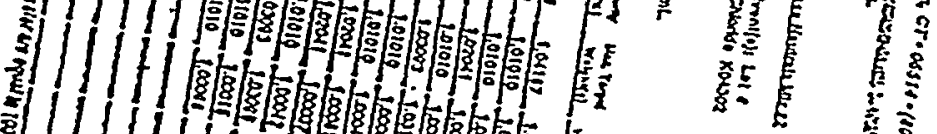

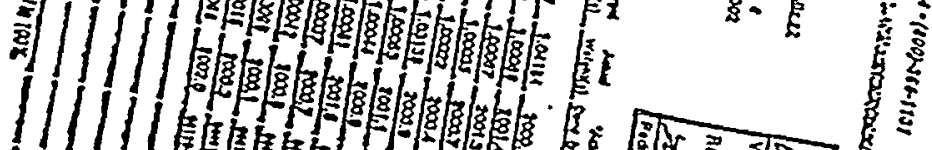

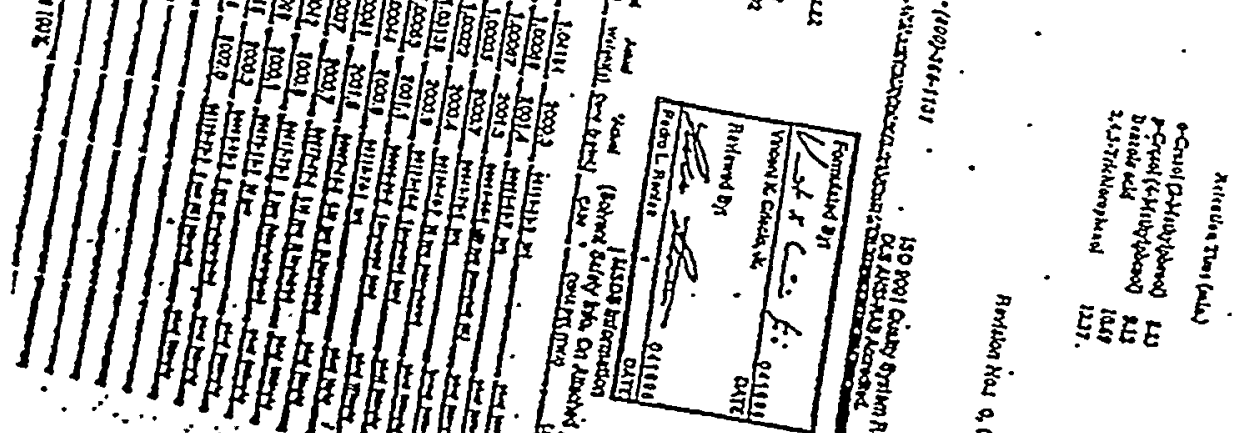

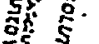

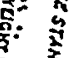

竞

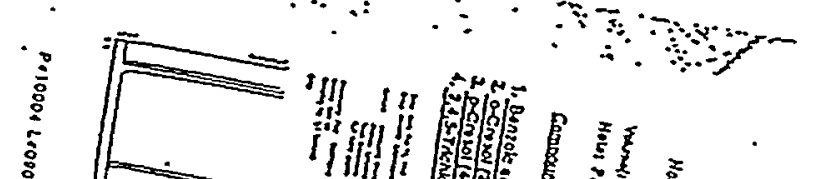



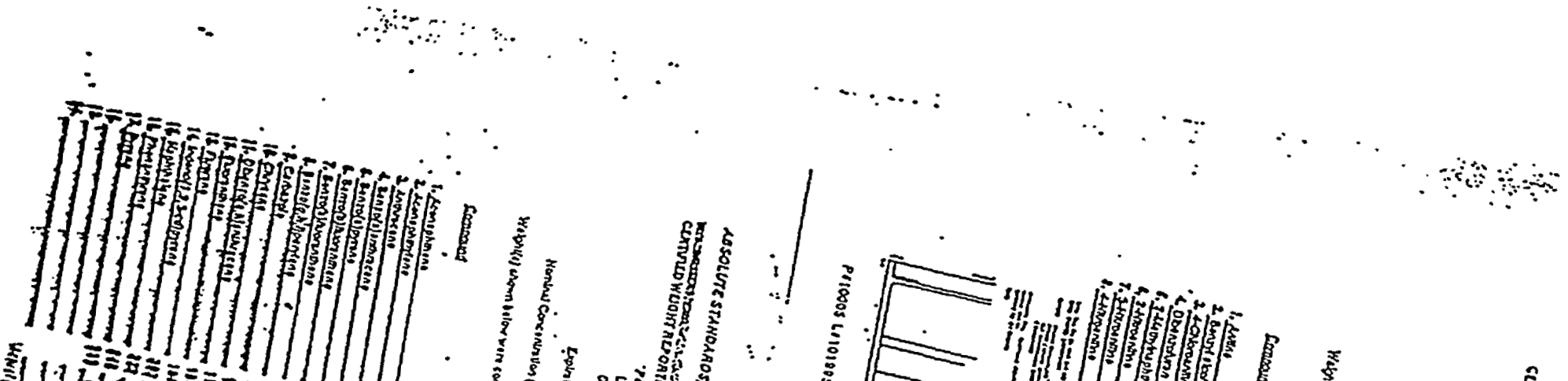

(1)

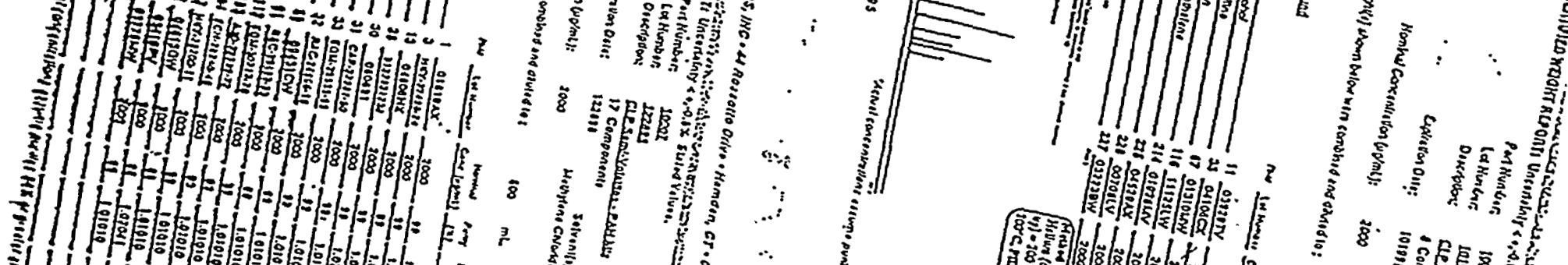

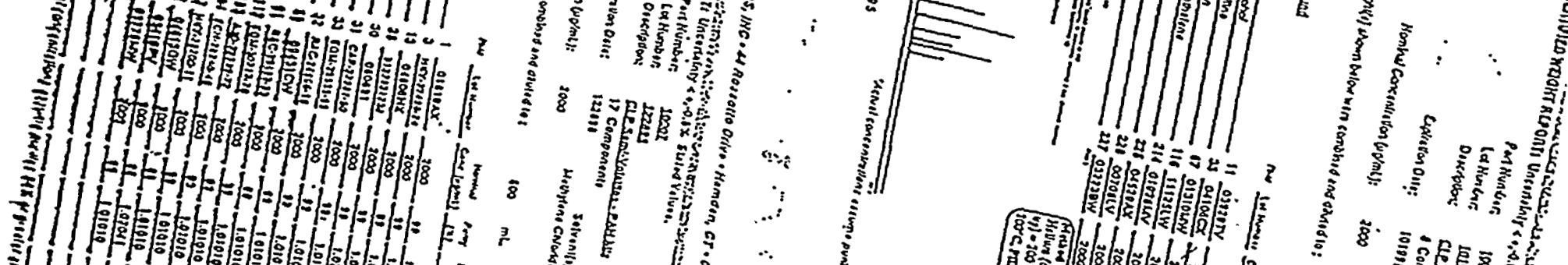

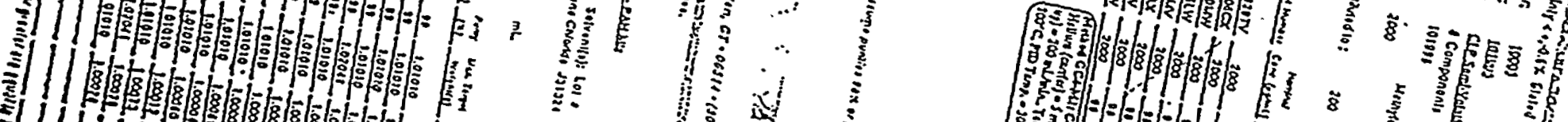
$\therefore$ 政

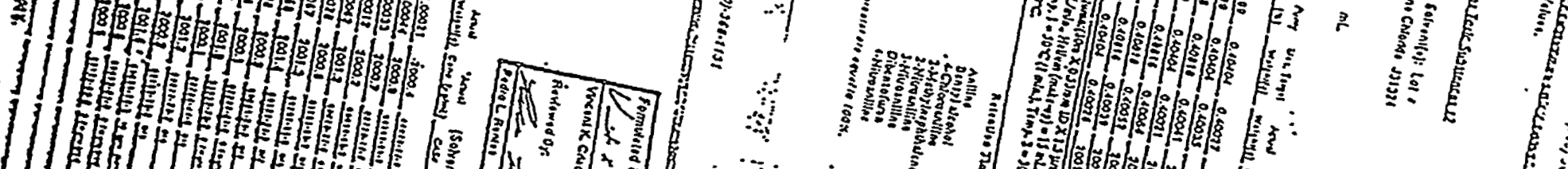

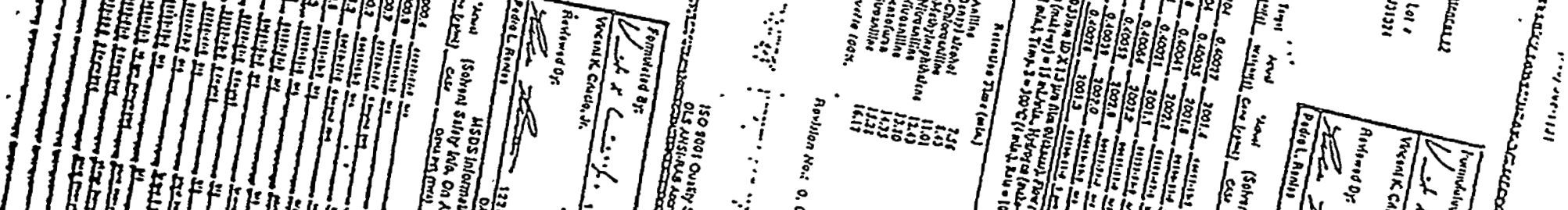

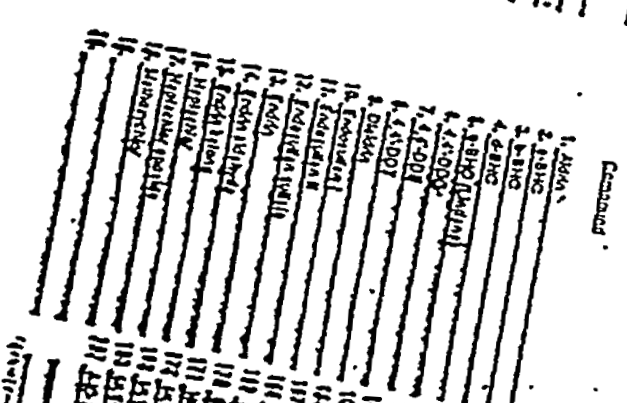

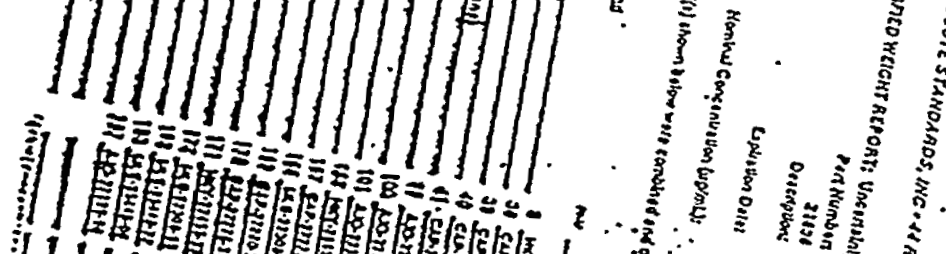

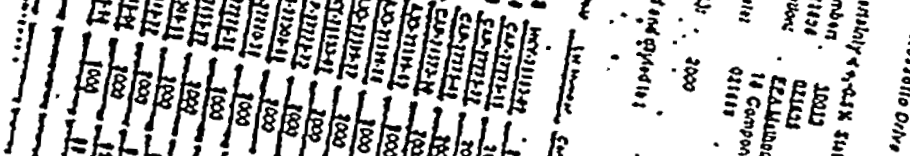

1)

He

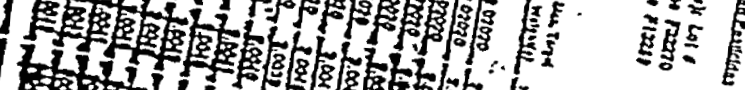

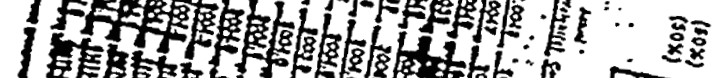

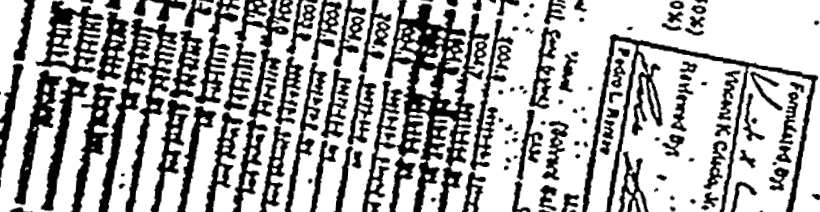

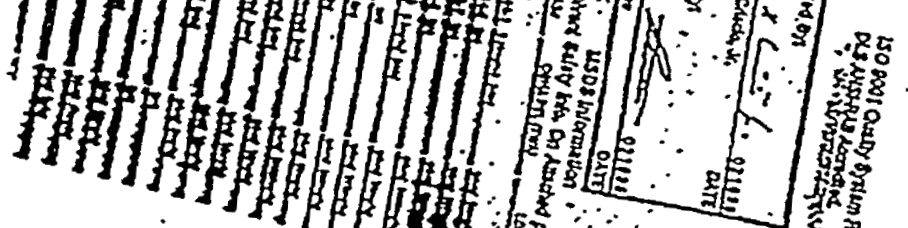

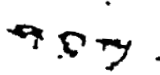
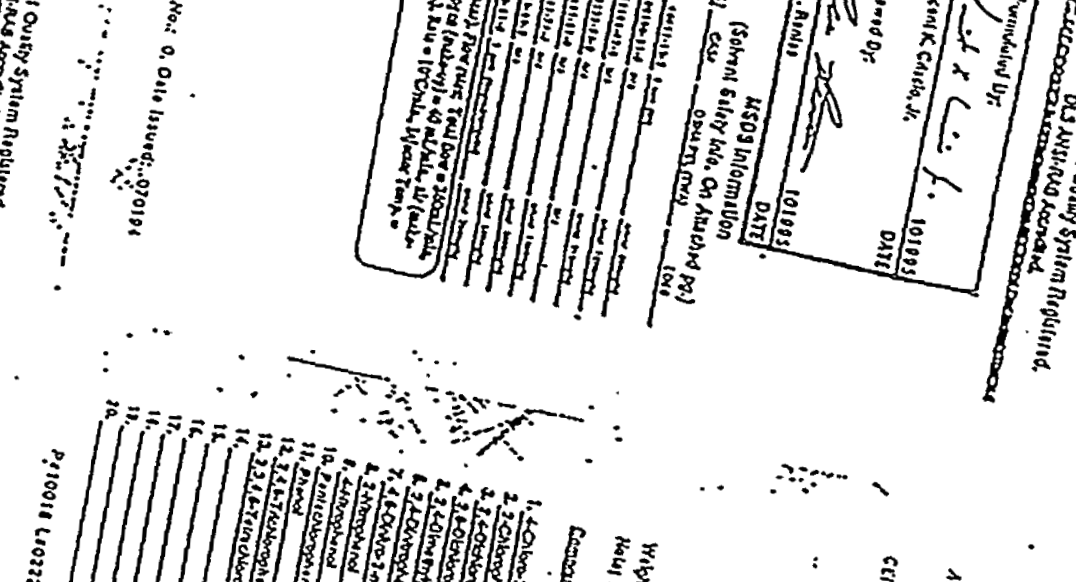

in

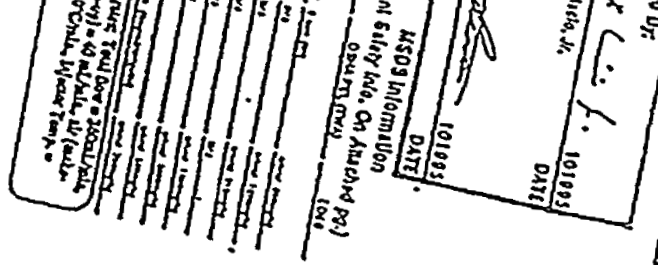

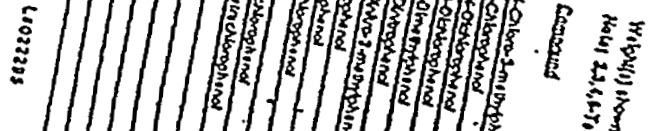

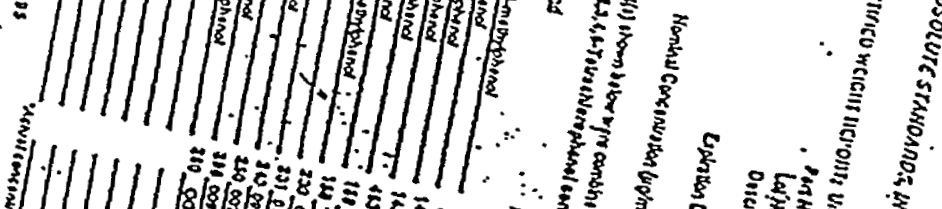

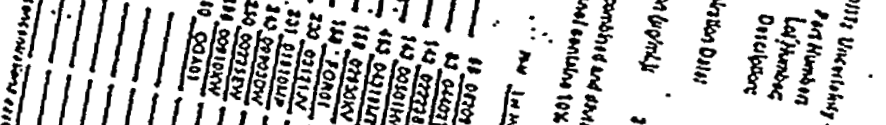

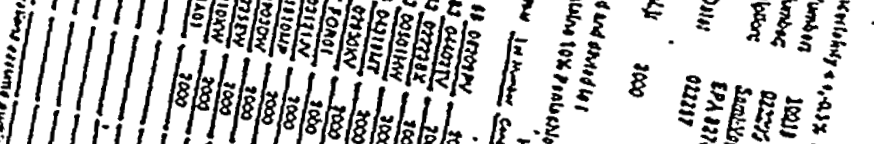

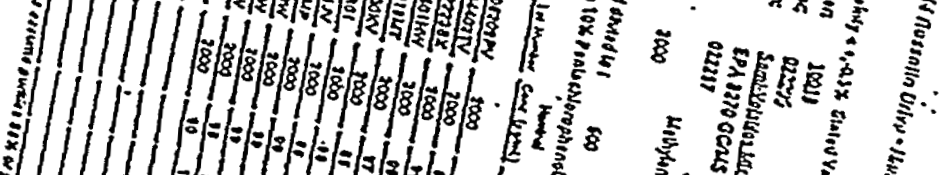

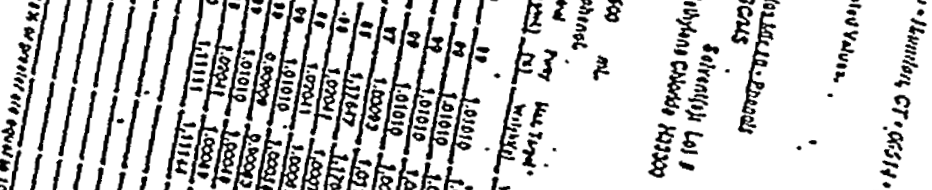

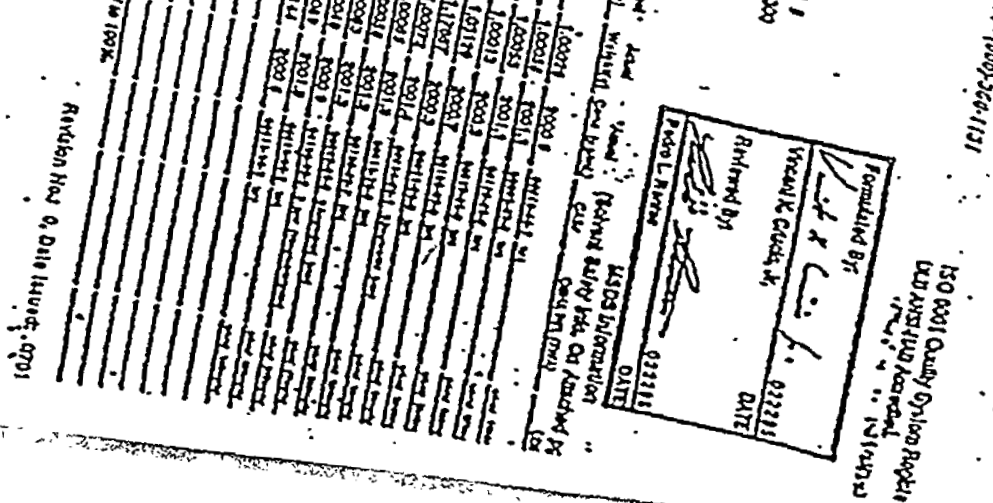


Prostro, 187

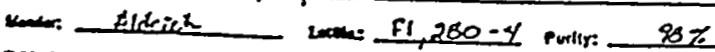

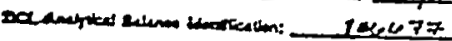

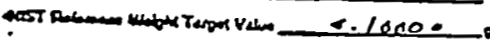

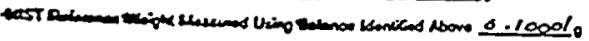

0.000010

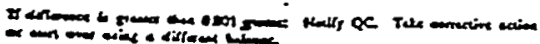

FINM GROSS WELGTT $0.1 \%$ SBS

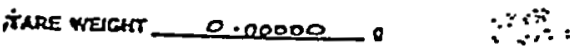

NET waEh 0.14503

HET WEUGH CORRECTED FOR PURTY $112,91 \mathrm{mo}$

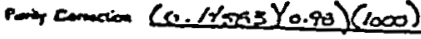

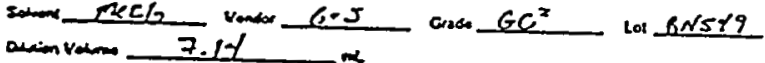

FINAL CONCENTRATION 20,000 $\mu \mathrm{g} / \mathrm{mL}$

EXPLRATION DATE___.27-97

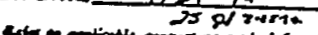

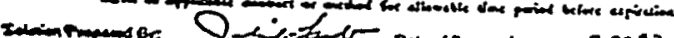

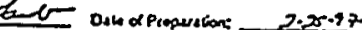

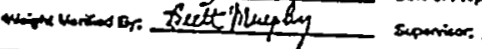

comenes

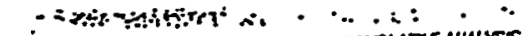

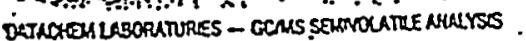

- CONCEENTTRATED STOCK STANDARDS

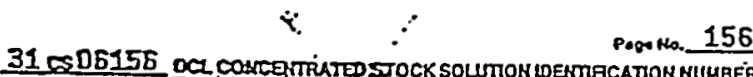

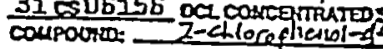

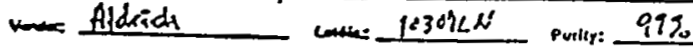

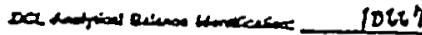

0.10000

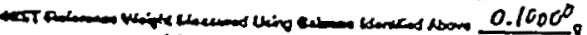

0.00000

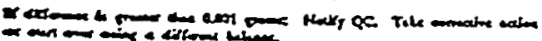

mul cross WECHT 0.0874

TARE WESTT 0.0000 :0.090

Ner waar 0.0974

HET HECATT CORRECTED FOR PURTY 96.53 ing

nitcom $0.0914 \times .91 \times 1000$

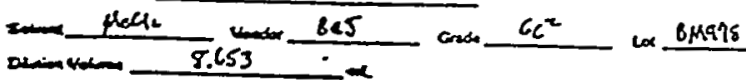

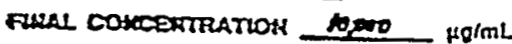

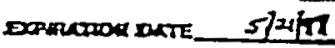

-

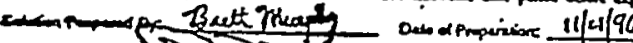

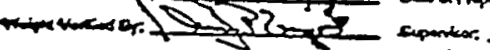

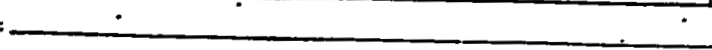

37 CS 07201 OCL CONCEMTRY TED STOCK SOUTON

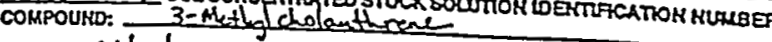

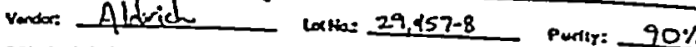

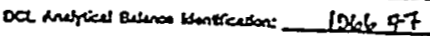

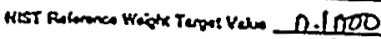
Oatorences 0.0000

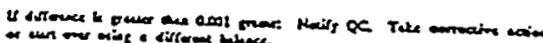

FINAL GROSS WEGHT 0.03835 .

TARE WEGKT _ 0.0000

NET WEGGT 0.03835

HET WEIGHT CORRECTEO FOR PURTY, 34.515 mo

Puiry conosion $0.03535 \times 0.36 ;$

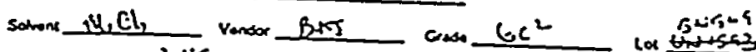

axion Volume 345

FINAL CONCENTRATION $10,050^{\circ} \mu g / m L$

EXPIRATIOH OATE 2.26.1T

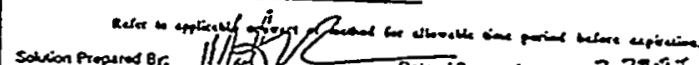

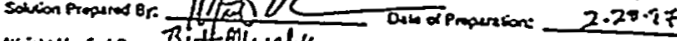

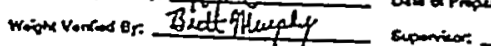

coments: -

ज्smp

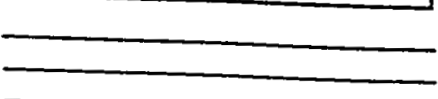

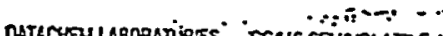

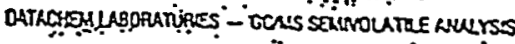

CONCENTRATED STOCK STANDARDS

STANDARD PREPARATION LOGBOOK NO.31CS

31 CS 06168 Pa CONCE 168

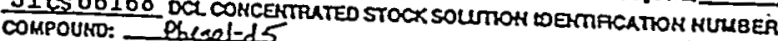

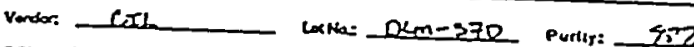

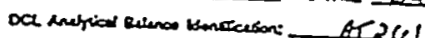

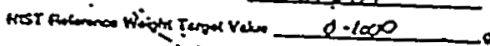

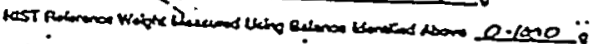

resceos - :

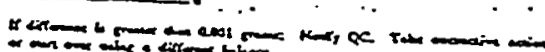

FHAL CROSS WEGHT 0.1442 ?

TARE WEGHT 0.0000

NET WaCKT 0.14k7 :

MET WEIGHT CORRECTEO FOR PURTY 1Y3.8 $\mathrm{mg}$

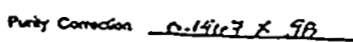

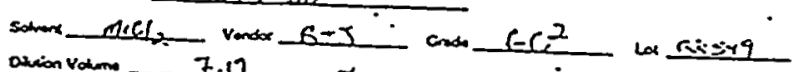

final CONCENTRATION Jo, 300 Ha/mL

EXPIRATION OATE_ $z / z / 97$

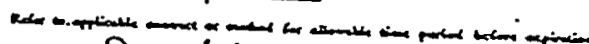

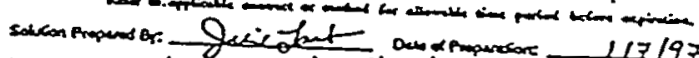

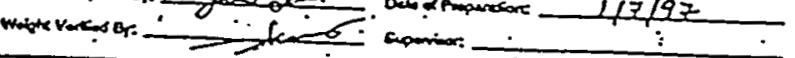

conmente

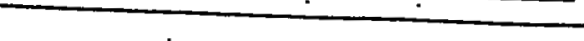

\section{(1)}

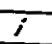




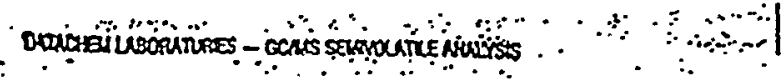
CONCENTIATED STOCK STANDARDS

\begin{tabular}{|c|}
\hline STAKDDARD PREPARATIOH LOGBOOK NO.31CS \\
\hline 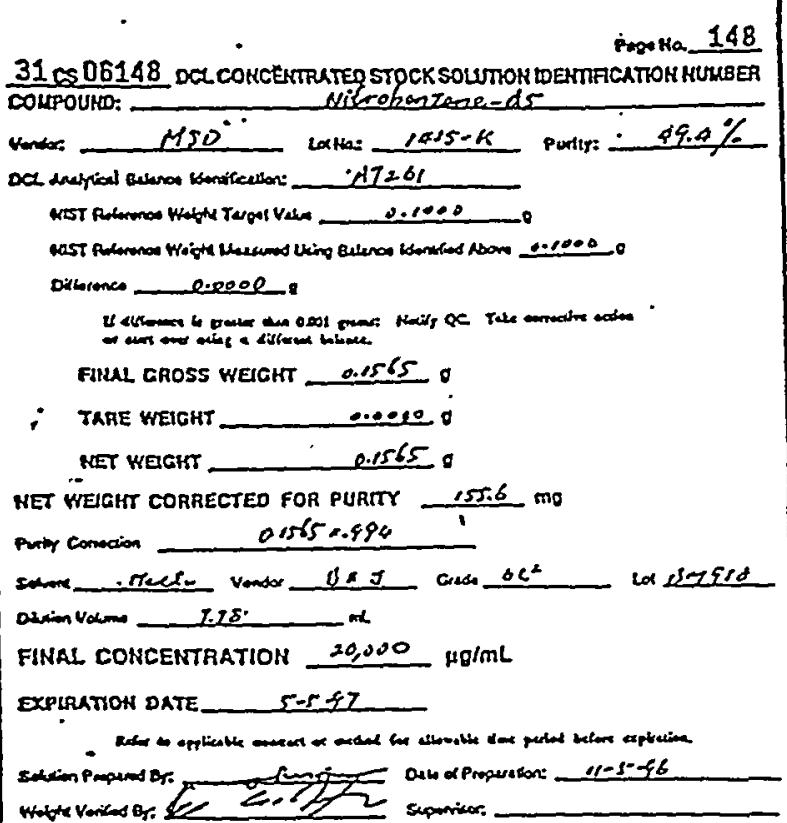 \\
\hline
\end{tabular}

Comments:

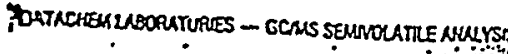

COINCENTRATED STOCK STANDARDS

STAKDARD PREPARATION LOGBOOK NO.31 CS

$\because \quad$.

sect He 172

31 CS 06171 DO CONCEMTATED STOCK SOLUMOH DERTHCATHOK NULBER Coupound: 2-Eluee biphecgs

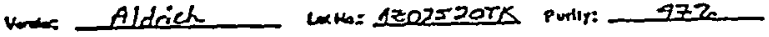

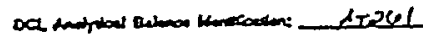

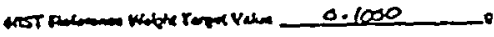

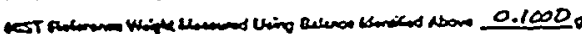

o.0000

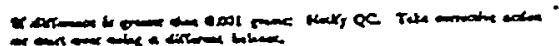

Fall GROSS Wrath $0.1 \% 18$.

TARE WECATI 0.0000

MET WaEkT 0.1418 0

RET WEGAT COARECTED FOR PURTY $133.5 \mathrm{mo}$

riman 0.14R 0.0 .72

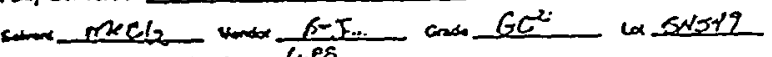

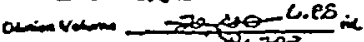

FINAS COUCEMTRATION -20, 500 $\mu \mathrm{g} / \mathrm{mL}$

DPRRATION DATE_ 7/7/97

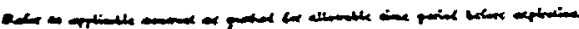

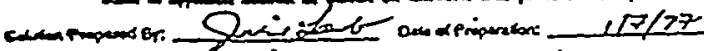

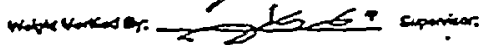

$c 0$

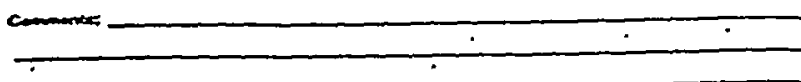

CONCENTRATE STOCK STANDARDS

STAHDARD PREPARATION LOGBOOK HO.31CS

31 CS06188 DCL CONCENTRUTED STOCX SOUTOU PAOKa 188

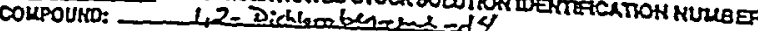

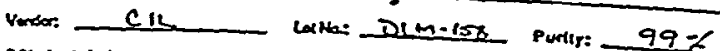

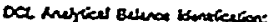

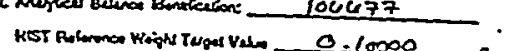

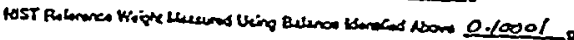
OHotoses e.00001

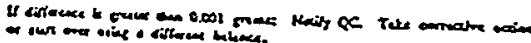

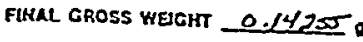

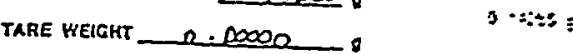

MET WEIGHT C.VPLES 0 ,

KET WEIGHT CORRECTED FOR PURTY, $-141.12 \mathrm{me}$

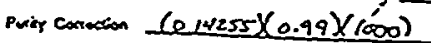

sarom- MeCl2

fIHAL CONCERTRATION $20,000^{-} \mathrm{\mu g} / \mathrm{mL}$

EXPIRATION OATE__ B/25/97

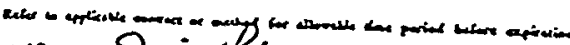

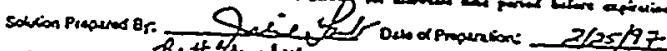

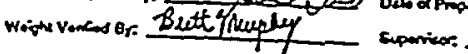

corments:

$\therefore-\cdots$

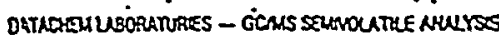

CONCENTRATED STOCK STANDARDS

STANDARD PREPARATION LOGBOOK NO. 31 CS

31006170 Page tea 170

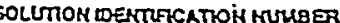

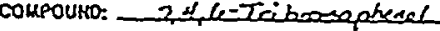

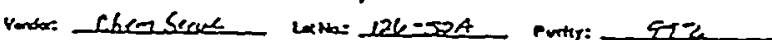

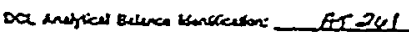

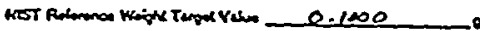

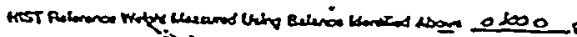

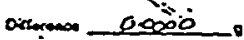

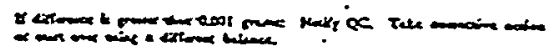

FWAL GROSS MEGGH Q. 1979 :

TARE WEESTT 0.0000

NET KEOKT"

HET HESGHT CORRECTEO FOR PURTT Lite: 1 ' mo

Puity corracion $0.2172<0.99$

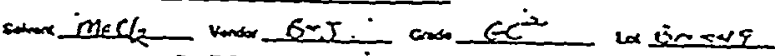
axcion varm 7.52 - $\alpha$.

FIMAL CONCENTRATION $20000 \mathrm{\mu g} / \mathrm{mL}$

EXPIRATION DATE- Z/Z/9T

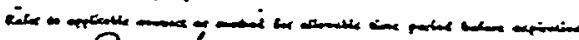

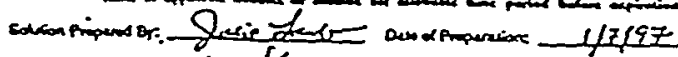

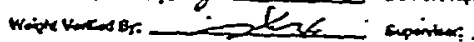

. . . 

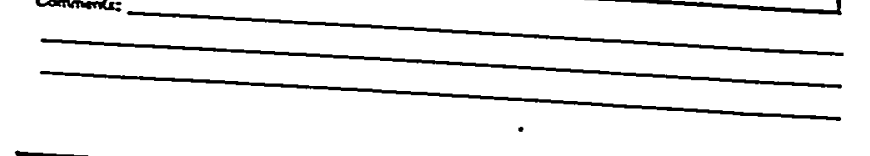

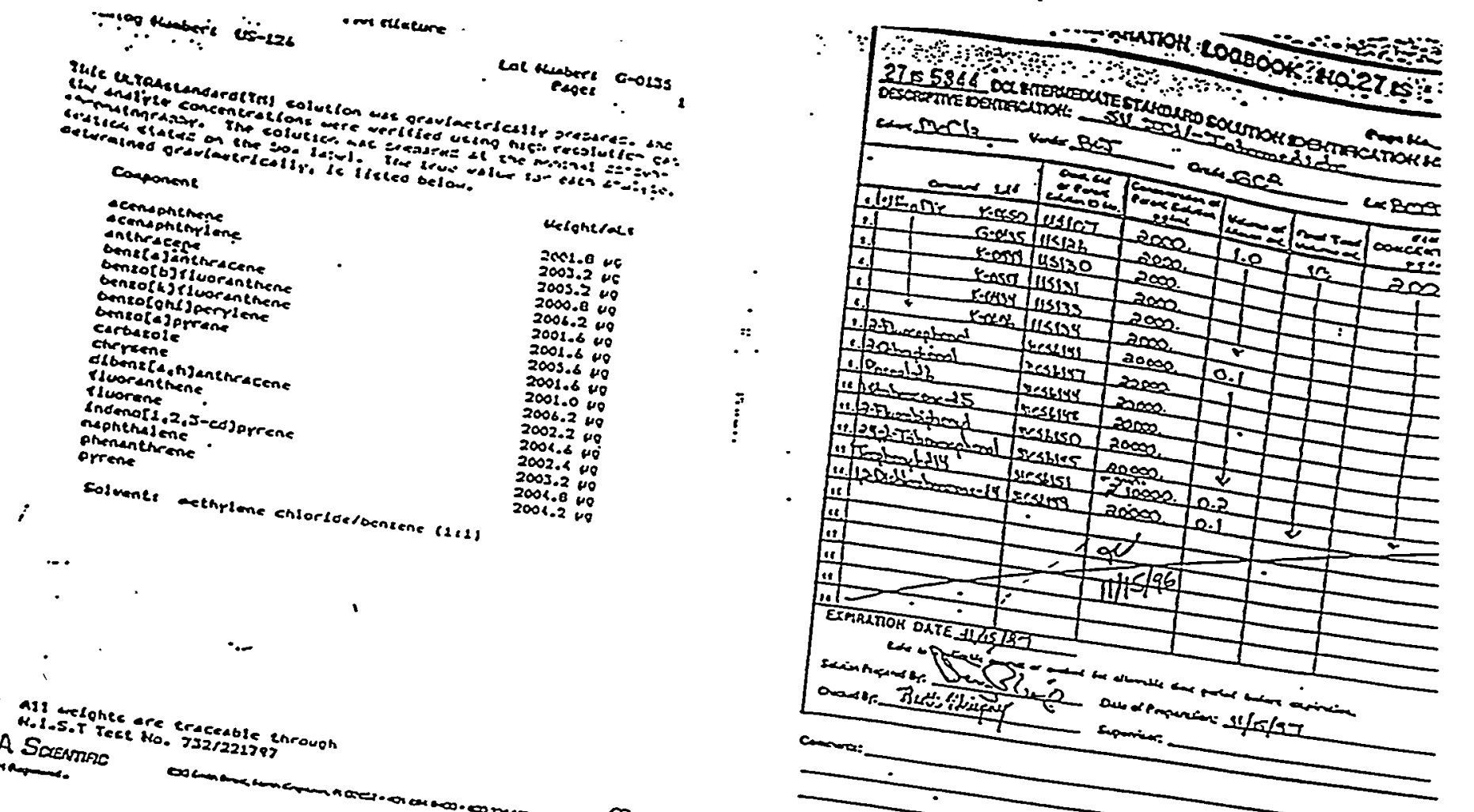

(3)

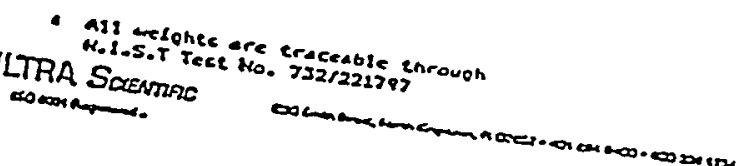

$\rightarrow$ Certificate of Analysis

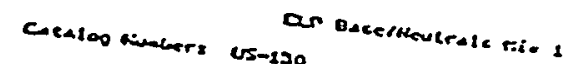

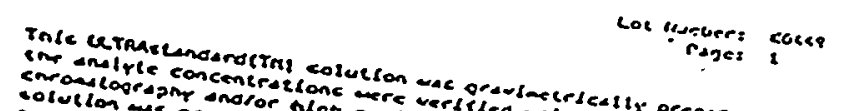

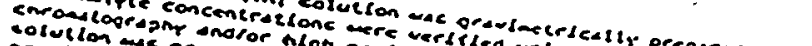

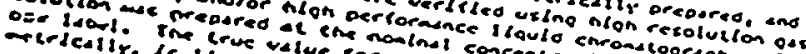

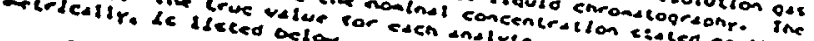

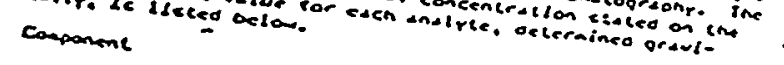

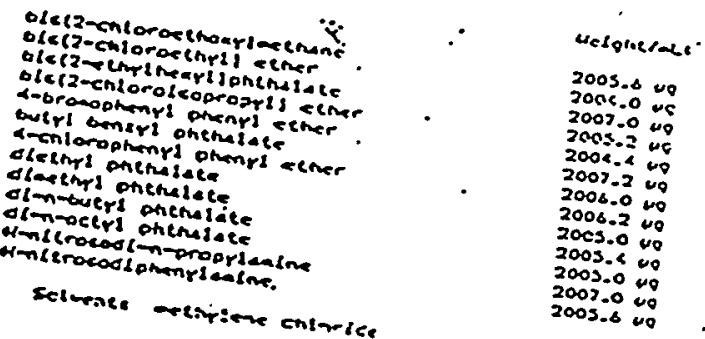

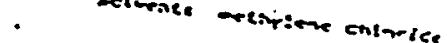

$2003.0 \%$

3005.8 uo
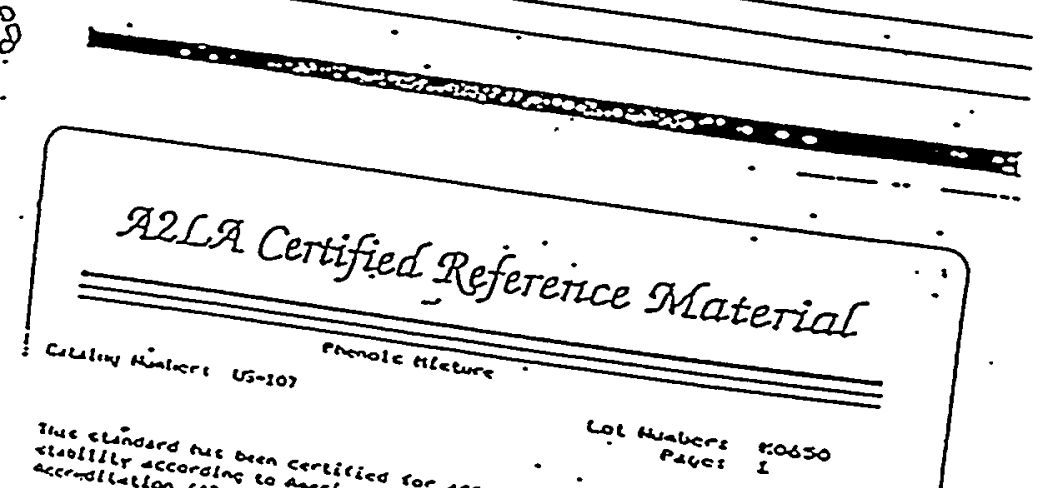

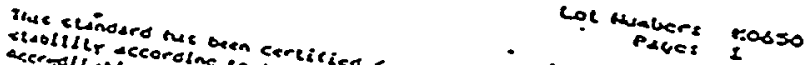

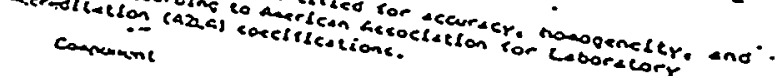

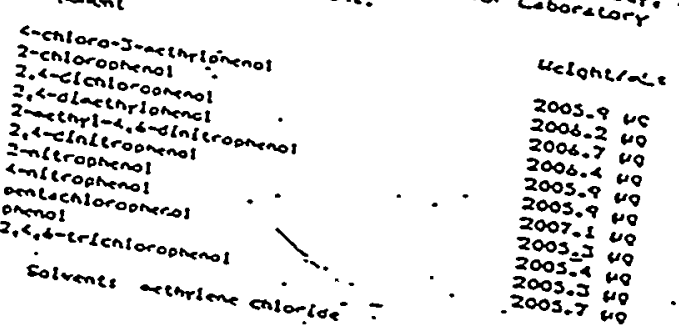

.

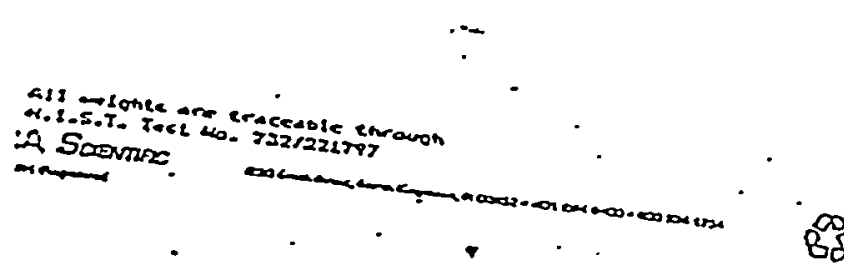

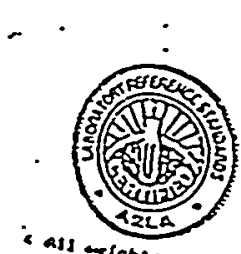

팔

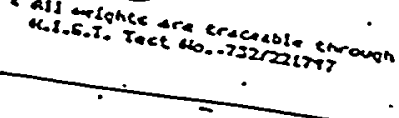




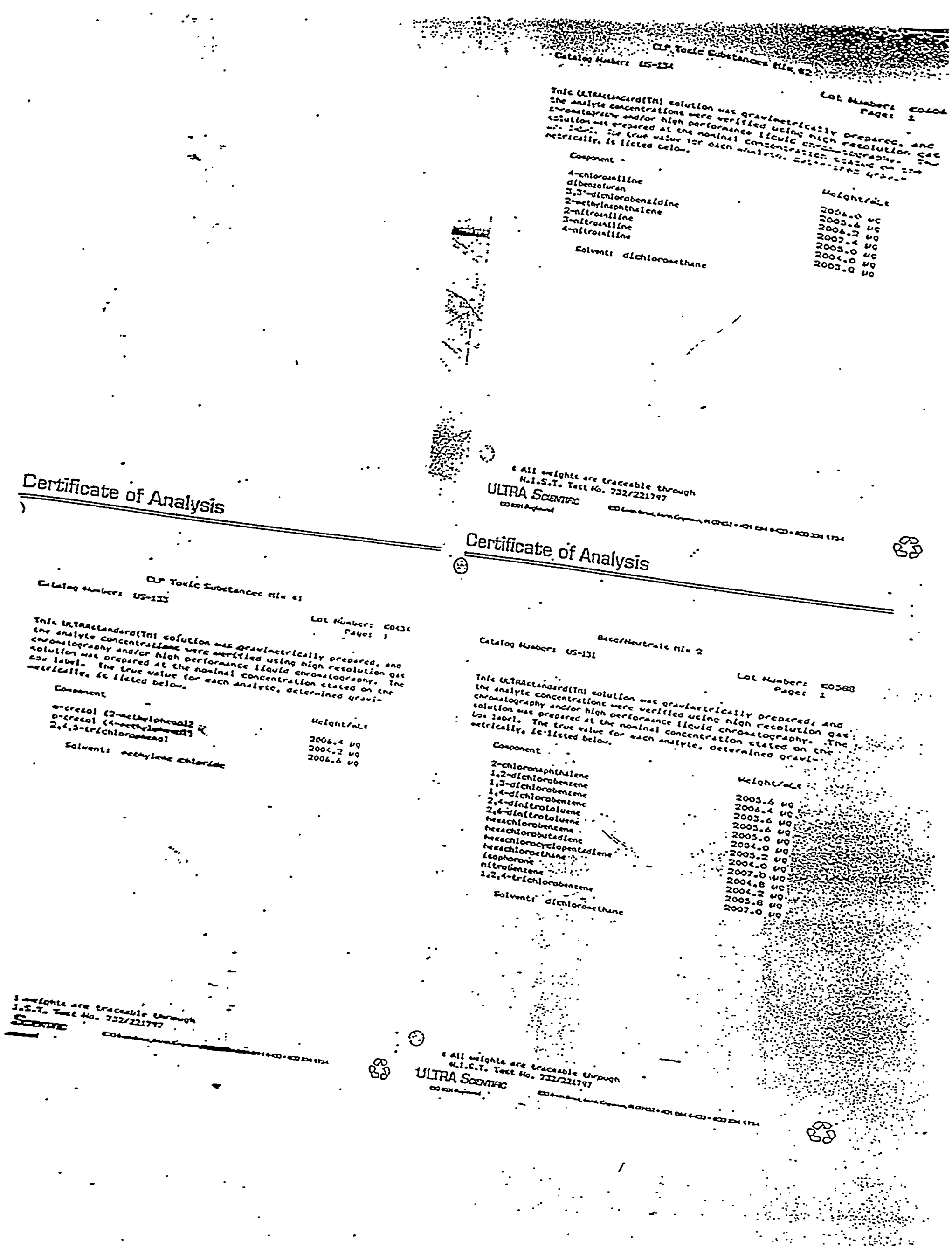




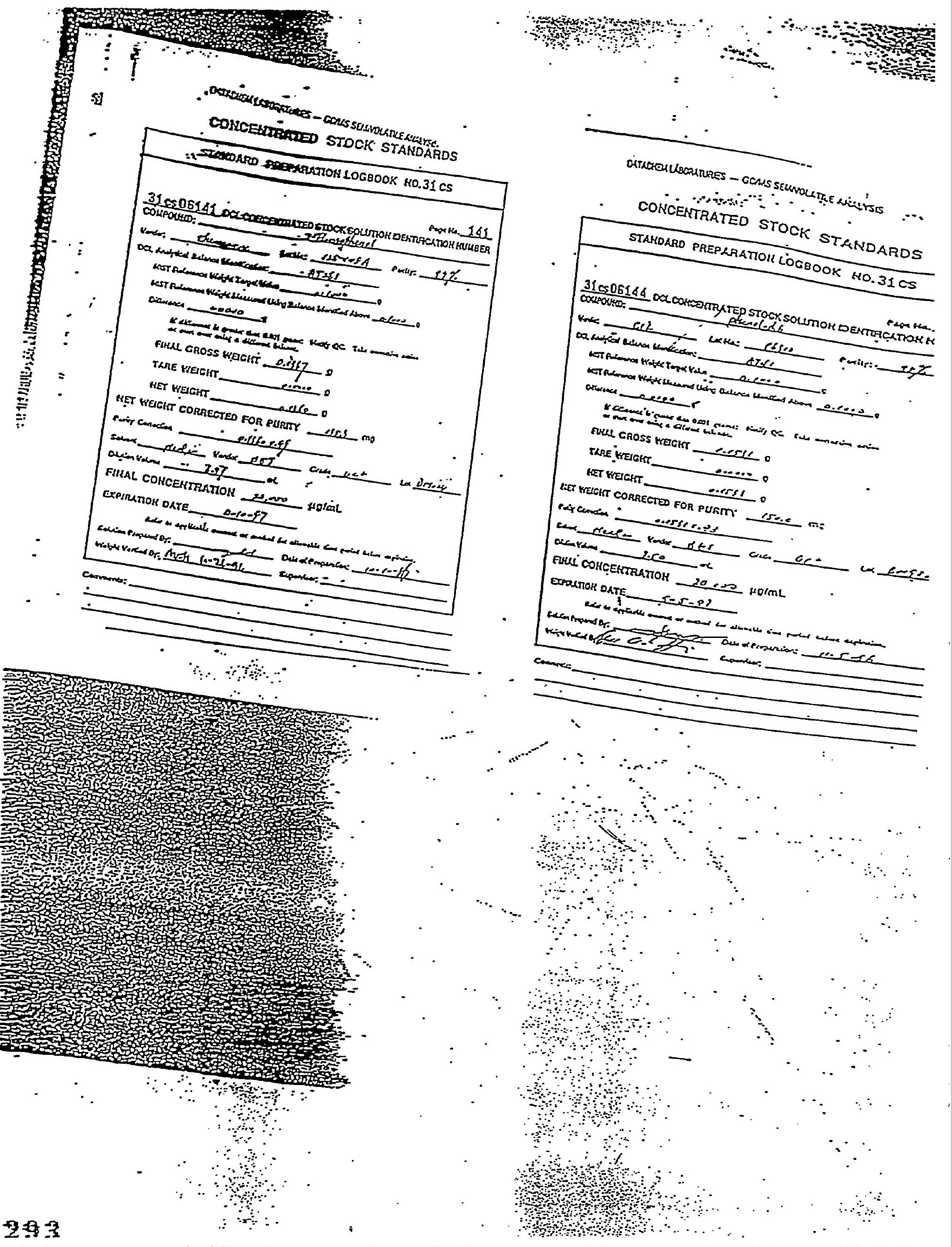




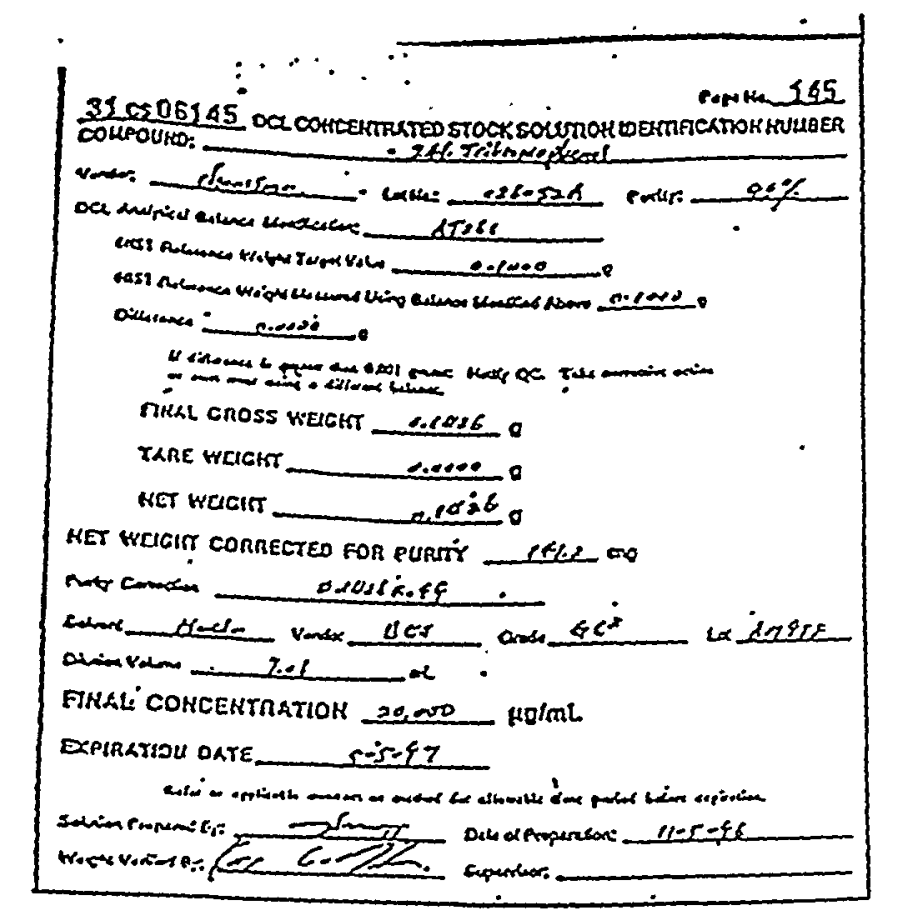

copmon:

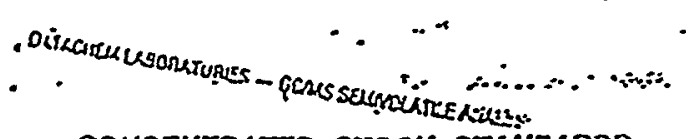
COISCENTAATED STOCK STAKOARDS

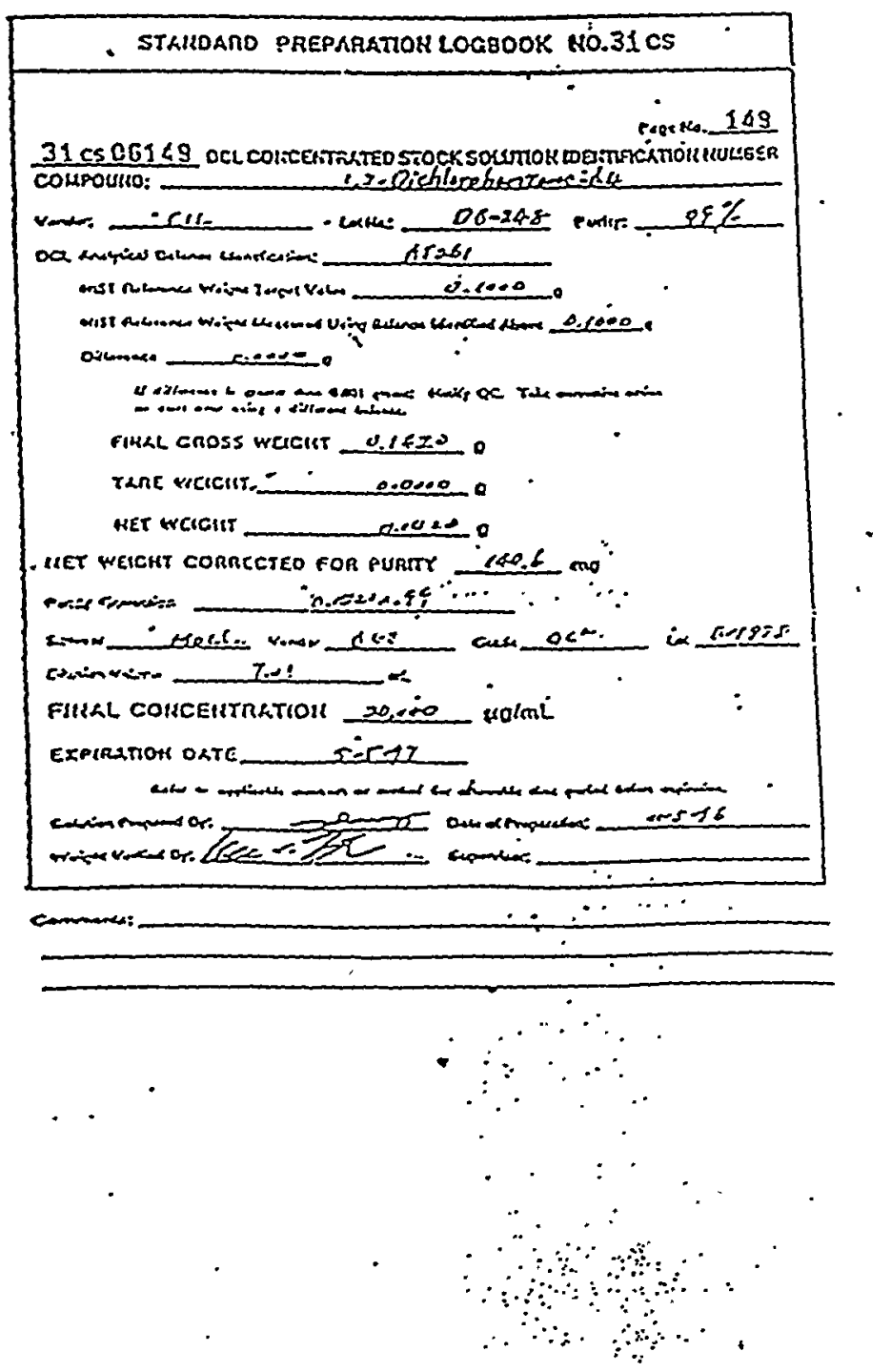

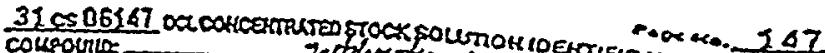

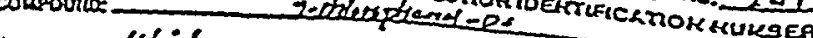

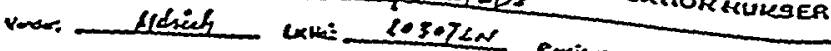

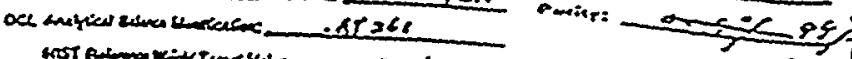

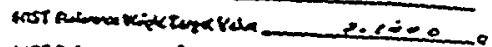

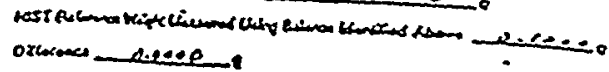

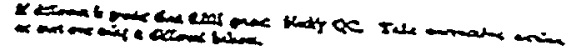

Fithl Cross KackT PC.E.

TARE KaGH

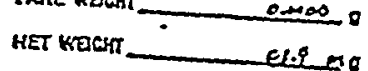

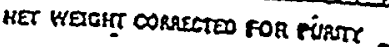

menom flet:

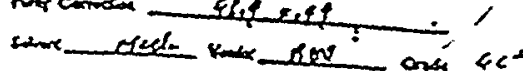

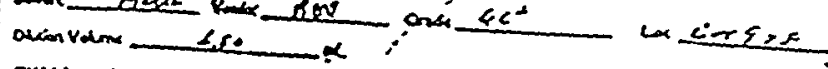

FILAL CONCEMTRTON

ExPIRLTOK OLTE

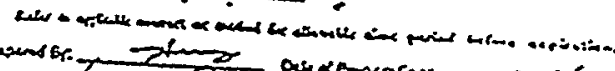

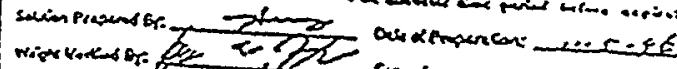

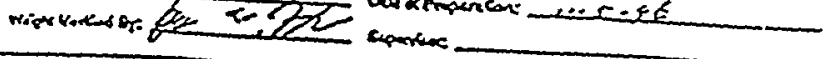

comonese

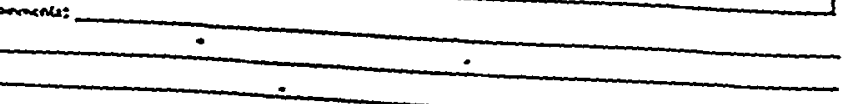

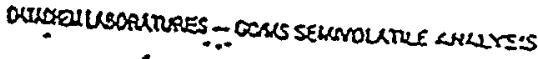

1.

CONCEKTRATED STOCK STANDARDS

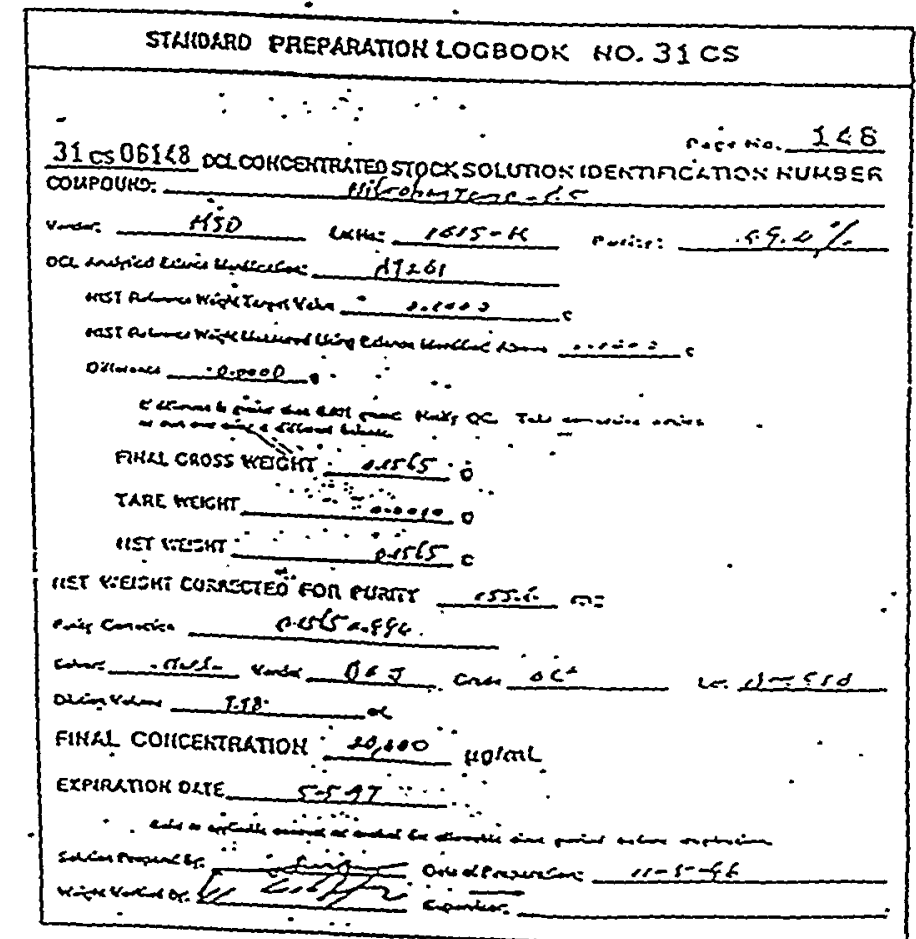

? $\because \because \cdots, \quad \therefore$ :

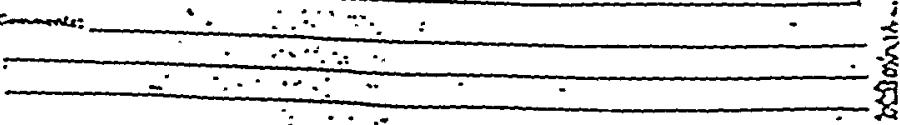



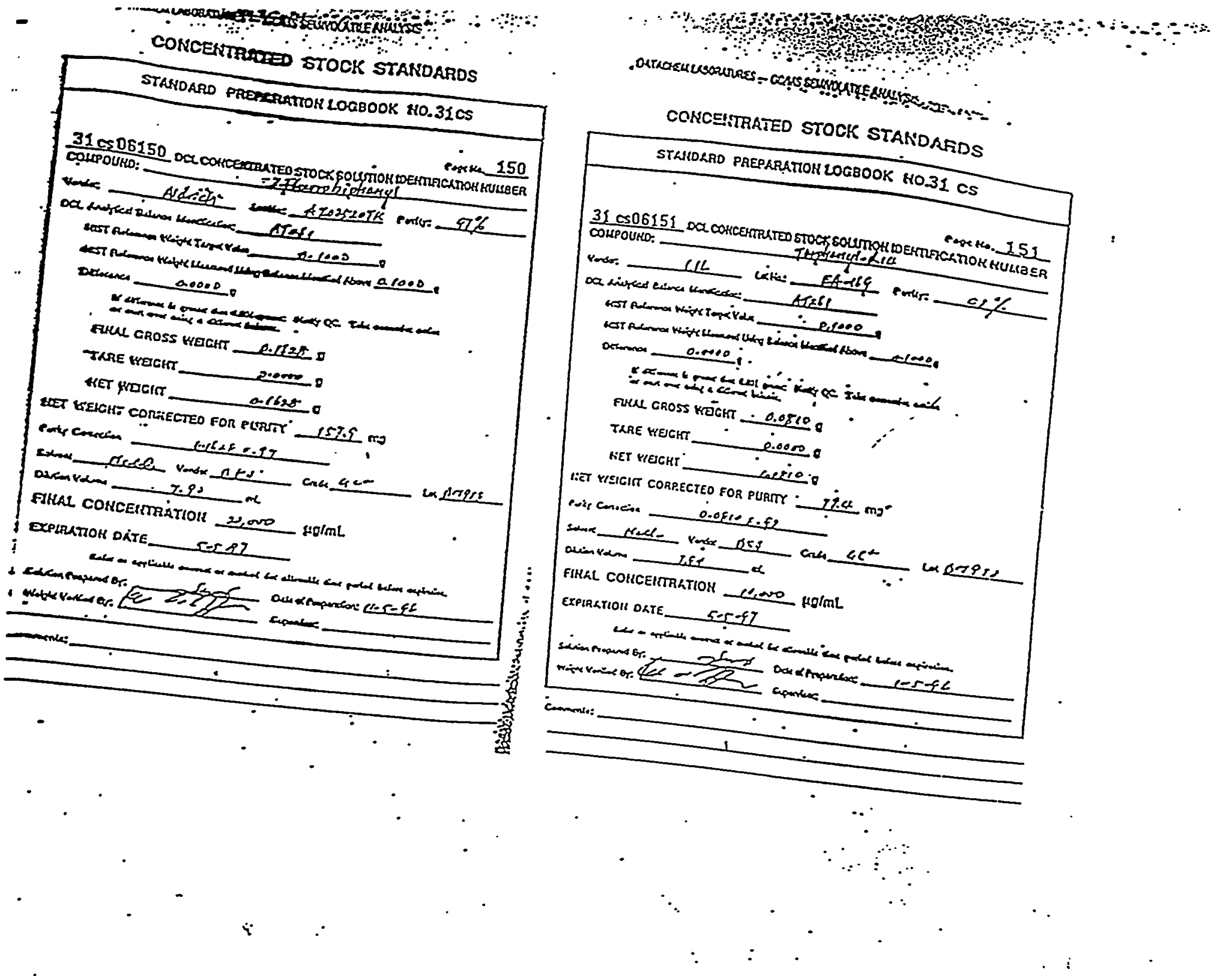
7Pon

(1)

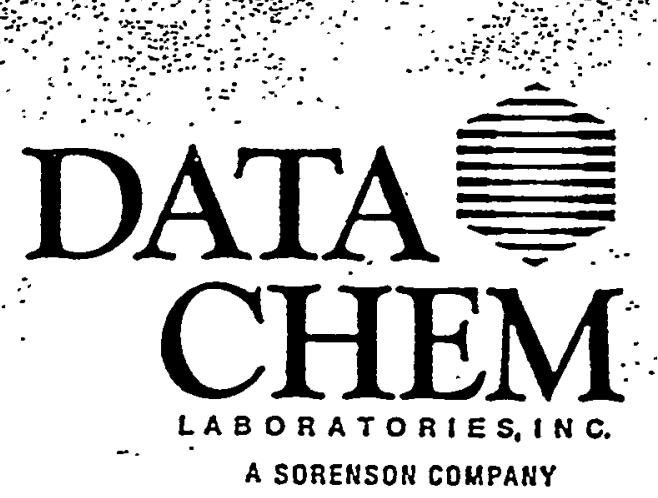

\section{Raw Data}

296 


\title{
PRELIMINARY OC SUMMARY REPORT
}

File: NRAO7BLK.D

\author{
Path: C:IHPCHEMI1IDATAI15JUL97NI 07/15/97 11:55 \\ Sample: $136532-1$ \\ Instrument ID: $5972-N \quad$ Analyst: TV \\ 7/1/97 WATER 97C-0208-02
}

Initial Calibration 5.0 Std: NQF04S5.D

Continuing standard: NRAO2S7O.D

Internal standards:

1,4-Dichlorobenzene-d4 Naphthalene-d8 Acenaphthene-d10

Phenanthrene-d10

Chrysene-d12

Perylene-d12

$\begin{array}{cccc}\text { Area } & \text { Area-70 } & \text { \% area } & \\ 708845 & 542000 & 130.8 & \text { ok } \\ 2666679 & 2174614 & 122.6 & \text { ok } \\ 1116204 & 943737 & 118.3 & \text { ok } \\ 1420590 & 1193540 & 119.0 & \text { ok } \\ 971622 & 641902 & 151.4 & \text { ok } \\ 660077 & 402522 & 164.0 & \text { ok }\end{array}$

CC ISTDs Pasted From: NRAO2S7O.D

Matrix: (Water or Soil): WATER

Multiplier $($ normal water $=1$; soil $=1$ ): $\quad 1$

Surrogates:

$\begin{array}{rccccc} & \text { Quan } & \% \text { Rec } & \text { Method } & \text { Warning } & \text { Control } \\ \text { 2-Fluorophenol } & 47.126 & 47.1 & 21-100 & 23-78 & 10-91 \\ \text { Phenol-d5 } & 34.791 & 34.8 & 10-94 & 9-73 & 0-89 \\ \text { Nitrobenzene-d5 } & 81.103 & 81.1 & 35-114 & 55-102 & 43-114 \\ \text { 2-Fluorobiphenyl } & 73.229 & 73.2 & 43-116 & 63-111 & 51-124 \\ \text { 2,4,6-Tribromophenol } & 60.898 & 60.9 & 10-123 & 36-121 & 14-142 \\ \text { Terphenyl-d14 } & 91.721 & 91.7 & 33-141 & 55-124 & 37-141 \\ \text { 2-Chlorophenol-d4 } & 62.856 & 62.9 & 33-110 & \text { Advisory Use Only } \\ \text { 1,2-Dichlorobenzene-d4 } & 64.445 & 64.4 & 16-110 & & \text { Advisory Use Only }\end{array}$

Limits updated: 12/14/95 


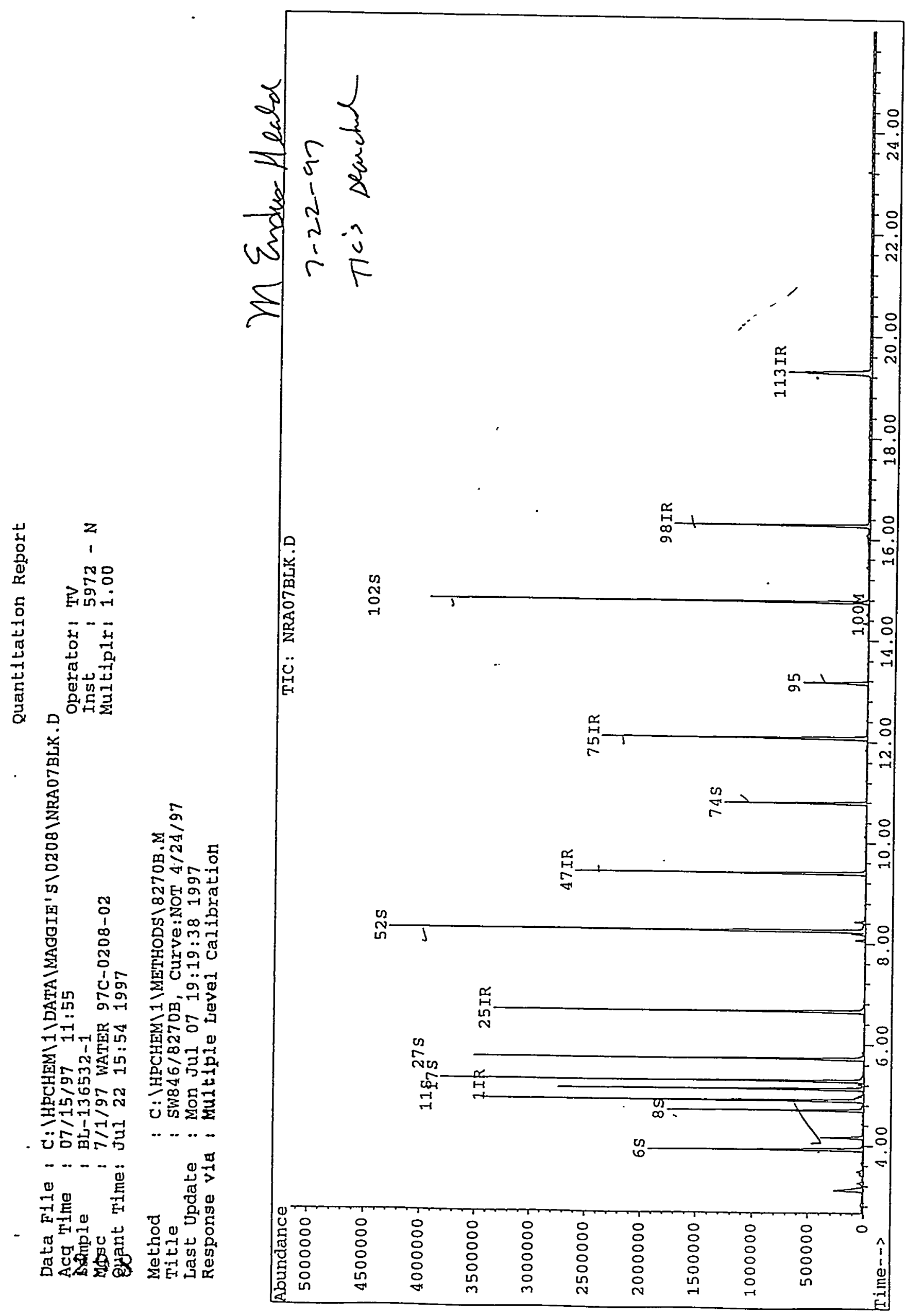

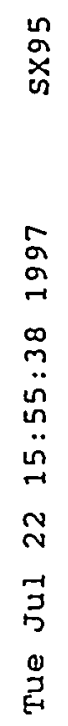




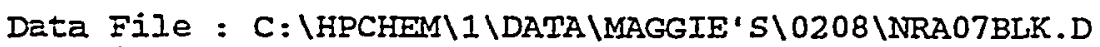

$\begin{array}{lll}\text { Acq Time } & : 07 / 15 / 97 \text { 11:55 } & \text { Operator: TV } \\ \text { Sample } & : \text { BL-136532-1 } & \text { Inst : } 5972-\mathrm{N} \\ \text { Misc } & : 7 / 1 / 97 \text { WATER 97C-0208-02 } & \text { Multiplr: } 1.00\end{array}$

Quant Time: Jul 22 15:54 1997

Method : C: \HPCHEM 1 1METHODS $\backslash 8270 \mathrm{~B} . \mathrm{M}$

Title : SW846/8270B, Curve:NOT 4/24/97

Last Update : Mon. Jul 07 19:19:38 1997

Response via : Multiple Level Calibration

Internal standards

R.T. Scan QIon Response Conc Units Areas

1) 1,4-Dichlorobenzene-d4

25) Naphthalene-d8

47) Acenaphthene- $\mathrm{d} 10$

75) Phenanthrene-d10

98) Chrysene-d12

113) Perylene-d12

$5.13 \quad 285 \quad 152$

$6.65 \quad 460 \quad 136$

$9.40 \quad 776 \quad 164$

$12.08 \quad 1084$

16.291568

19.291914

188

\section{0}

264

708845
2666679
1116204
1420590
971622
660077

$40.00 \mathrm{ug} / \mathrm{mL}$

$40.00 \mathrm{ug} / \mathrm{mL}$

$40.00 \mathrm{ug} / \mathrm{mL}$

$\$ 0.00 \mathrm{ug} / \mathrm{mL}$

40.00 ug tint

$40.00 \mathrm{ug} / \mathrm{mL}$

0.00

0.00

0.00

0.00

0.00

0.00

system Monitoring Compounds

6) 2-Fluorophenol

8) Phenol-a5

11) 2-Chlorophenol-a4

17) 1,2-Dichlorobenzene-d4

27) Nitrobenzene-a5

52) 2-Fluorobiphenyl

74) 2,4,6-Iribromophenol

102) Terphenyl-d14

$\begin{array}{rrrr}3.92 & 146 & 112 & 1108965 \\ 4.70 & 236 & 99 & 1014511 \\ 4.90 & 259 & 132 & 1688949 \\ 5.29 & 304 & 152 & 936368 \\ 5.73 & 354 & 82 & 1676041 \\ 8.25 & 644 & 172 & 2380315 \\ 10.80 & 937 & 330 & 233735 \\ 14.76 & 1393 & 244 & 2026036\end{array}$

\&Recovery

$47.126 \mathrm{ug} / \mathrm{mL} \quad 47.138$

$34.791 \mathrm{ug} / \mathrm{mI} \quad 34.798$

$62.856 \mathrm{ug} / \mathrm{mL} \quad 62.868$

$64.445 \mathrm{ug} / \mathrm{mr} \quad 64.448$

$81.103 \mathrm{ug} / \mathrm{mr} \quad 81.108$

$73.229 \mathrm{ug} / \mathrm{mL} \quad 73.238$

$60.898 \mathrm{ug} / \mathrm{mL} \quad 60.908$

$91.721 \mathrm{ug} / \mathrm{mL}$

$91.72 \%$

Target Compounds

2) Pyridine

0.00

3) N-nitrosodimethylamine

0.00

4) 2-Picoline

0.00

5) Methyl methanesulfonate

0.00

7) Ethyl methanesulfonate

0.00

9) Phenol

0.00

0.00

10) Aniline

0.00
0.00

13) Decane NOT Present

14) 1,3-Dichlorobenzene

0.00

0.00
0.00

16) Benzyl alcohol

0.00
0.00

19) 2-Methylphenol

0.00

0.00

21) 4-methylphenol

22) N-INitrosodi-n-propyl amine

0.00

24) Bis (2-chloroethyl)ether

0.00

0.00

0.00
0.00

28) Nitrobenzene

0.00

0.00

30) Isophorone

0.00

31) 2-Nitrophenol

0.00
0.00

33) Benzoic acia

0.00

0.00

35) 2,4-Dichlorophenol

0.00

0.00

37) $1,2,4-$ Trichlorobenzene

0.00

39) Naphthalene

0.00

0.00

0.00

0.00

42) 2,6-Dichlorophenol

0.00

Not Detected

Qvalue

Not Detected.

Not Detected

Not Detected

Not Detected

Not Detected

Not Detected

Not Detected

Not Detected

Not Detected

Niot Detected

Niot Detected

Niot Detected

Not Detected

Nist Detected

Not Detecied

Nist Detected

Not Detecied

Not Detected

Not Detecied

Not Detected

Not Detected

Nit Detected

Not Detected

Not Detected

Not Detected

Not Detected

Not Detected

Not Detected

Not Detected

Not Detected

Not Detected

Not Detected

Not Detected

Not Detected

Not Detected

Not Detected

44) N-nitroso-di-n-butylamine

(菜) =quajifier out of range $(\mathrm{m})$ = manual integration

(*) IRes at meet EPA spectral criteria (False Hit) sX95 
Deta File : C: IHPCHEM I IDATA IMAGGIE'S\0208\NRA07BLK.D

$\begin{array}{lll}\text { Acg Time } & : 07 / 15 / 97 \quad 11: 55 & \text { Operator: TV } \\ \text { Semple } & : \text { BL-136532-1 } & \text { Inst }: 5972-\mathrm{N} \\ \text { Misc } & : 7 / 1 / 97 \text { WATER 97C-0208-02 } & \text { Multiplr: } 1.00\end{array}$

Quant Time: Jul 22 15:54 1997

Method : C: \HPCHEM $\backslash 1 \backslash M E T H O D S \backslash 8270 B . M$

Ticle : SW846/8270B, Curve:NOT 4/24/97

Last Dpdace : Mon JuI 07 19:19:38 1997

Response via : Multiple Level Calibration

Compound R.T. Scan QIon Response Conc Unit Qvalue

45) 4-Chloro-3-Methylphenol

46) 2-Methylnaphthalene

4B) $1,2,4,5$-Tetrachlorobenzene

49) Hexachlorocyclopentadiene

50) 2,4,6-Irichlorophenol

51) 2,4,5-Irichlorophenol

53) Tetradecane NOT Present

54) 2-Chloronaphthalene

55) 1-Chloronaphthalene

56) 2-Nitroaniline

57) Dimethylphthalate

5B) 2,6-Dinitrotoluene

59) Acenaphthylene

60) 3-Nitroaniline

61) Acenaphthene

62) 2,4-Dinitrophenol

63) 4-Nitrophenol

64) Dibenzofuran

65) 2,4-Dinitrotoluene

66) Pentachlorobenzene

67) 1-Naphthylamine

68) 2-Naphthylamine

69) 2,3,4,6-Tetrachlorophenol

7D) Diethylphthalate

71) 4-Chlorophenyl Phenyl Ethe

72) Fluorene

73) 4-Nitroaniline

76) 4,6-Dinitro-2-methylphenol

77) N-nitrosodiphenylamine

78) 1,2-Diphenylhydrazine

-79) Tributylphosphate NOT Pres

80) Phenacetin

BI) 4-Bromophenyl Phenyl Ether

B2) alpha-3HC

B3) Hexachlorobenzene

B4) Tris (2-CE) phosphate NOT $\mathrm{Pr}$

B5) 4-Aminobiphenyl

B6) beta-Bic

B7) Pentachlorophenol

88) Pronamide

89) gamma-3:C

90) Pentachloronitrobenzene

91) Phenanthrene

92) Antiracene

93) delta-BHC

94) Heptachlor

95) Di-n-Butylphthalate

96) Aldrin

97) Eluoranthene

99) Benzidine

IDD) Pyrene

101) 4,4'-DDE

103) Dielarin

104) D-Dimethylaminoazobenzene

105) $4,4 \cdot-\mathrm{DDD}$ 30
R.T. Scan QIon Response Conc Unit Qvalue

$0.00 \quad$ Not Detected

0.00

0.00

0.00

0.00

0.00

0.00

0.00

0.00

0.00

0.00

0.00

0.00

0.00

0.00

0.00

0.00

0.00

0.00

0.00

0.00

0.00

0.00

0.00

0.00

0.00

0.00

0.00

0.00

0.00

0.00

0.00

0.00

0.00

0.00

0.00

0.00

0.00

0.00

0.00

0.00

0.00

0.00

0.00

0.00

0.00

13.16

120

149

471783

0.00

0.00

0.00

$14.52 \quad 1365 \quad 202 \quad 11999$

0.00

0.00

0.00

0.00

Not Detected

Not Detected

Not Detected-

Not Detectéd

Not Detected

Not Detected

Not Detected

Not Detected

Not Detected

Not Detected

Not Detected

Not Detected

Not Detected

Not Detected

Not Detected

Not Detected

Not Detected

Not Detected

Not Detected.

Not Detected

Not Detected

Not Detected

Not Detected

Not Detected

Not Detected

Not Detected

Not Detected

Not Detected

Not Detected

Not Detected

Not Detected

Not Detected

Not Detected

Not Detected

Not Detected

Not Detected

Not Detected

Not Detected

Not Detected

Not Detected

Not Detected

Not Detected

Not Detected

Not Detected

Not Detected

8.624ug/mL

Not Detected

Not Detected

Not Detected

$0.313 \mathrm{ug} / \mathrm{mL}$

Not Detected

Not Detected

Not Detected

Not Detected 
Data File : C: \HPCHEM \I IDATA \MAGGIE'S\0208\NRA07BLK.D

Acq Time : 07/15/97 11:55

Sample : BL-136532-1

Misc : 7/1/97 WATER 97C-0208-02

Quant Time: Jul 22 15:54 1997

Operator: TV

Inst : $5972-\mathrm{N}$

Multiplr: 1.00

Method : C: IHPCHEM IIMETHODS $\backslash 8270 \mathrm{~B} . \mathrm{M}$

Title : SW846/8270B, Curve:NOT $4 / 24 / 97$

last Update : Mon Jul 07 19:19:38 1997

Response via : Multiple Level Calibration

Compound

106) Butylbenzylphthalate

$107) 4,4^{\prime}-D D T$

108) Methoxychlor

109) 3,3'-Dichlorobenzidine

110) Benzo[a] anthracene

111) Chrysene

112) Bis(2-ethylhexyl) phthalate

114) Di-n-octylphthalate

115) Benzo[b] fluoranthene

116) 7.12-Dimethylbenz (a) anthra

117) Benzo[k] fluoranthene

118) Benzo[a]pyrene

119) 3-Methylcholanthrene

120) Indeno $(1,2,3-c, d)$ pyrene

121) Dibenz $[a, h]$ anthracene

122) Benzo[ghi]perylene

R.T. Scan QIon Response

\subsection{0}

0.00

0.00

0.00

0.00

0.00

0.00

0.00

0.00

0.00

0.00

0.00

0.00

0.00

0.00

0.00
Conc Unit Qvalue

Not Detected

Not Detected

Not Detected

Not Detected-

Not Detectéd

Not Detected

Not Detected

Not Detected

Not Detected

Not Detected

Not Detected

Not Detected

Not Detected

Not Detected.

Not Detected

Not Detected

\section{Data processed вy: Mn Enduc. Hedd 7-22-47}

(H) = qualifier out of range $\langle m\rangle=$ manual integration

(*) Does not meet EPA spectral criteria (False Hit) SX95 


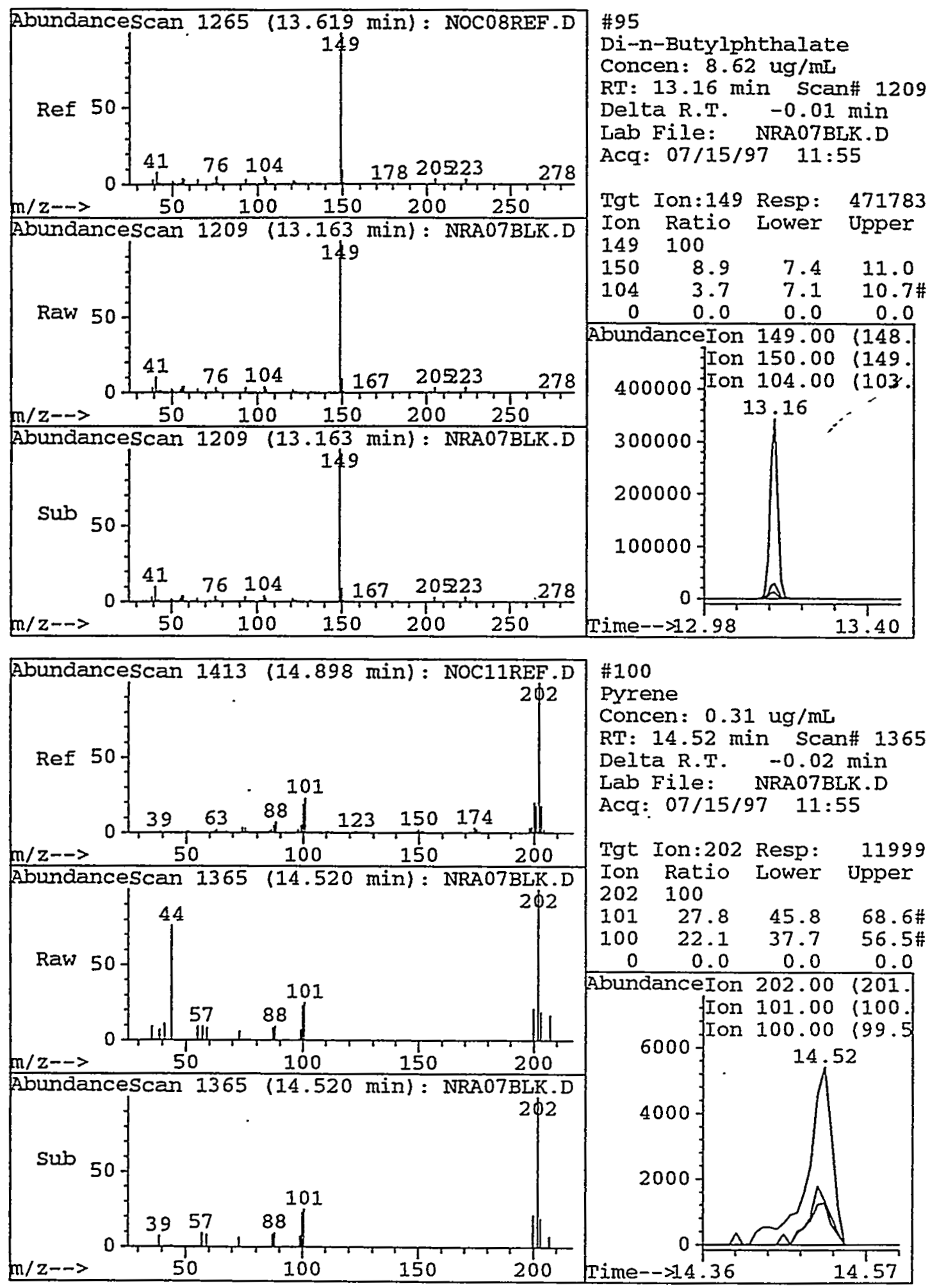

302 


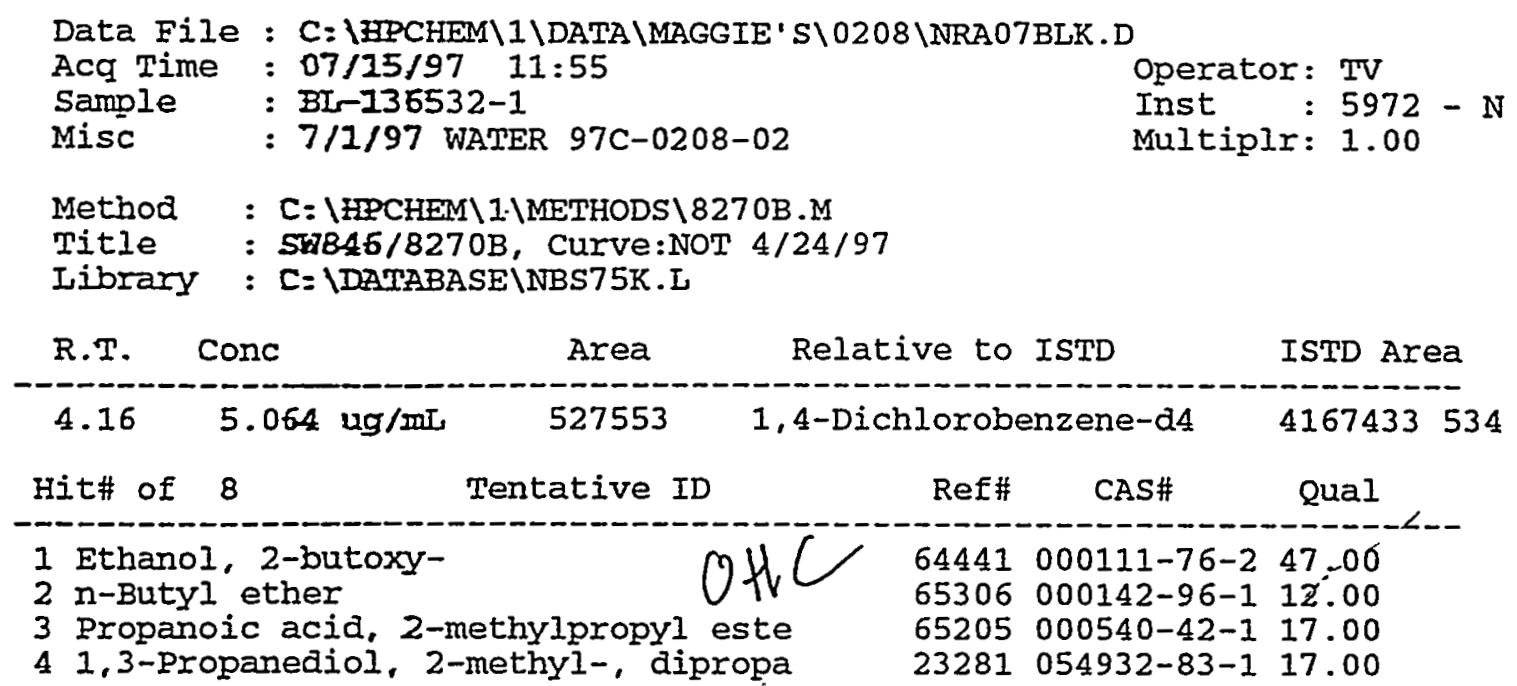

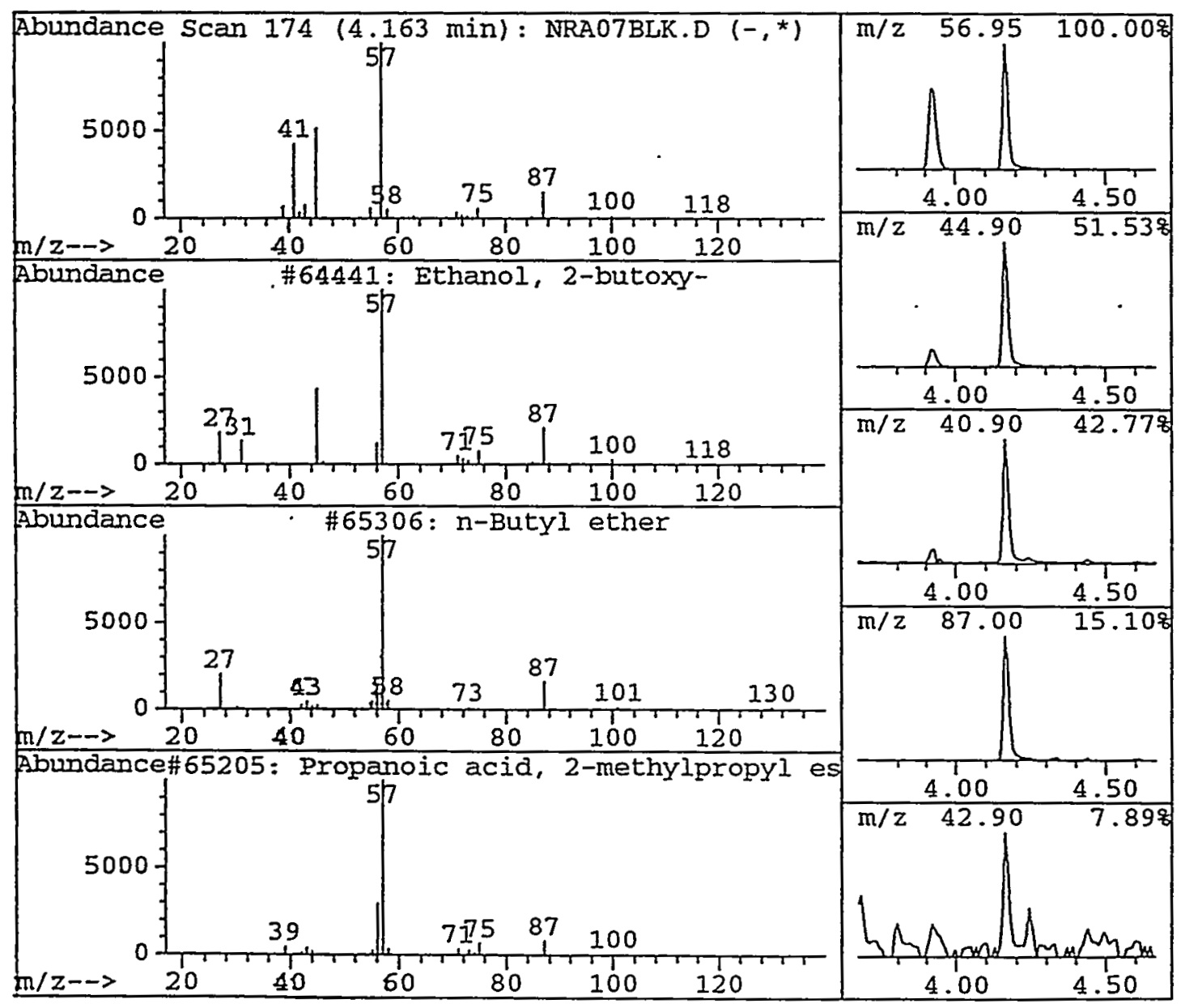



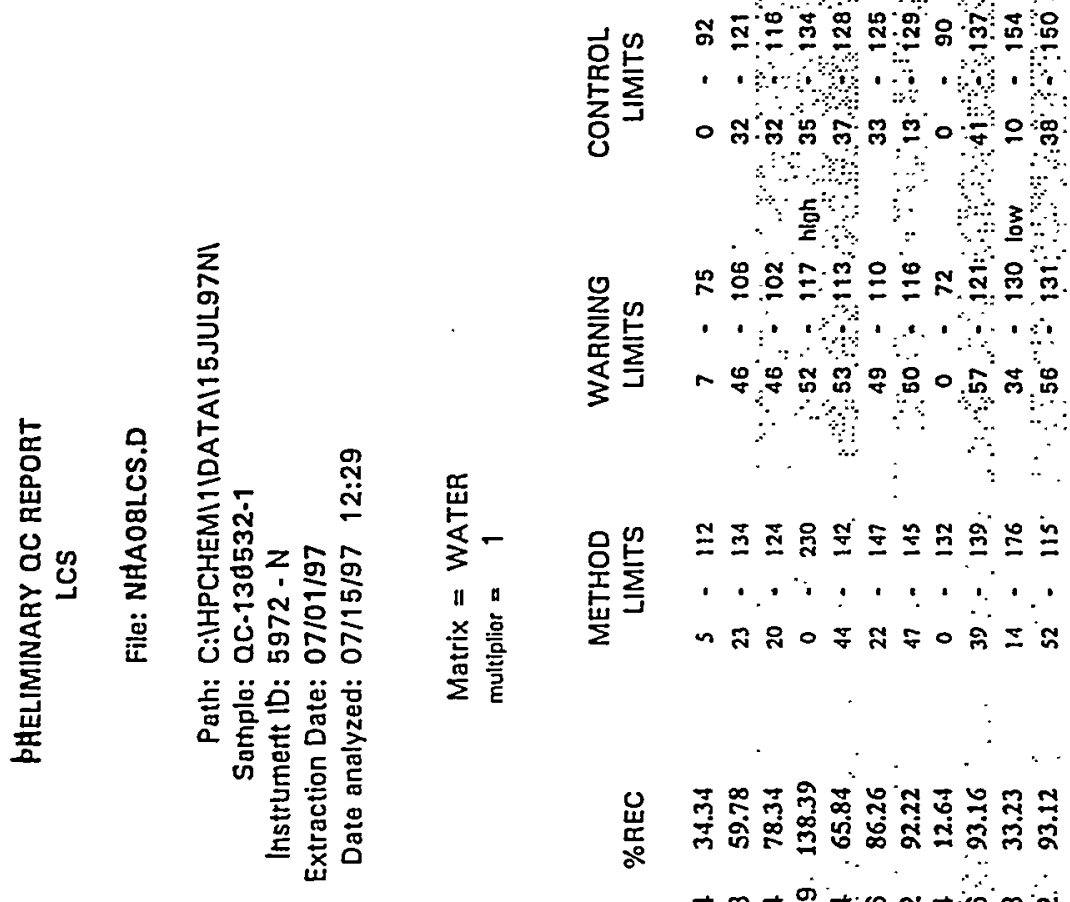

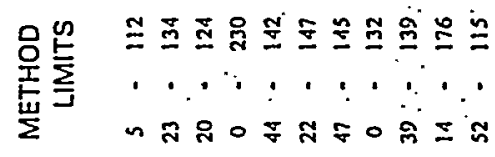

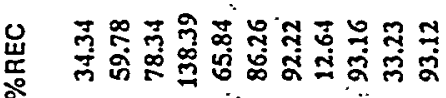

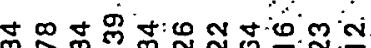

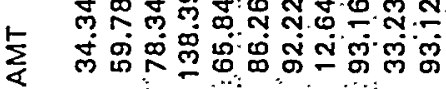

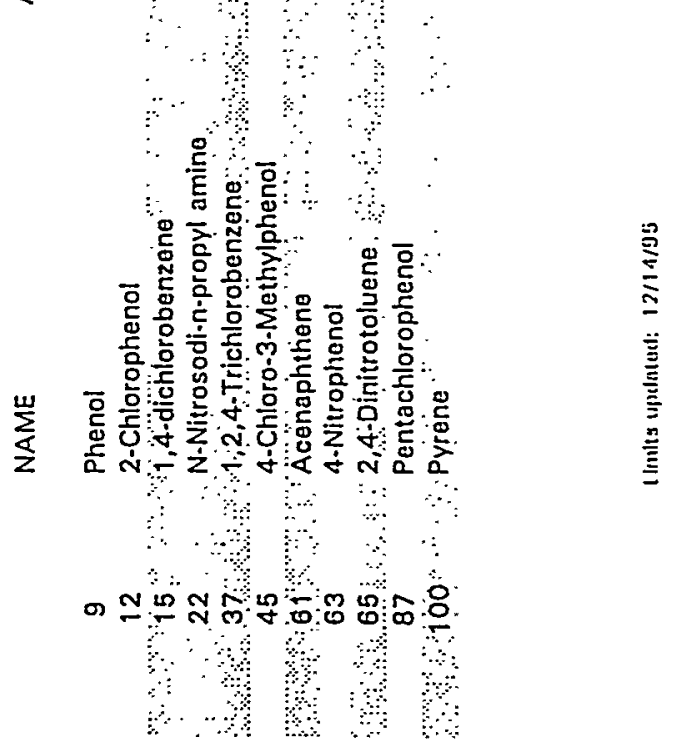




\section{PRELIMINARY OC SUMMARY REPORT}

File: NRAOBLCS.D

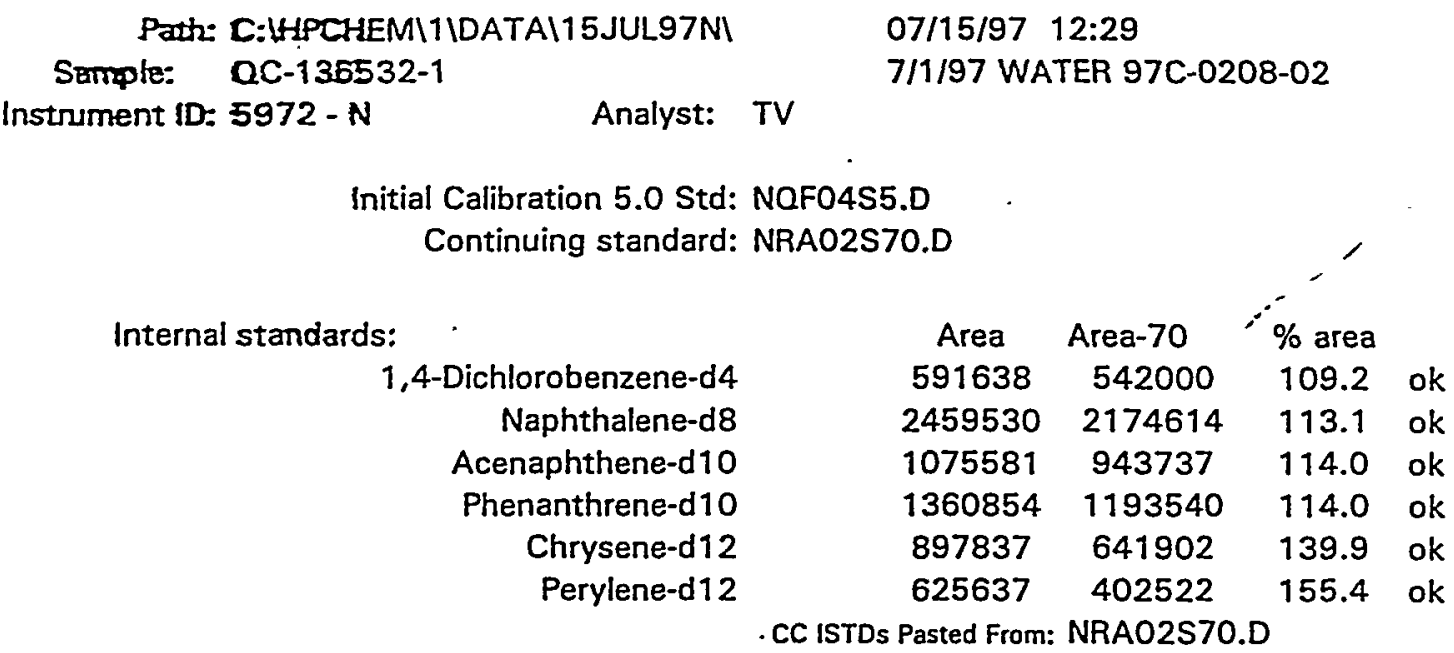

Matrix: (Water or Soill: WATER

Multiplier (normal water $=1$; soil $=1$ ): $\quad 1$

Surrogates:

\begin{tabular}{rccccc} 
& Quan & $\%$ Rec & Method & Warning & Control \\
2-Fluorophenol & 33.773 & 33.8 & $21-100$ & $23-78$ & $10-91$ \\
Phenol-d5 & 32.969 & 33.0 & $10-94$ & $9-73$ & $0-89$ \\
Fitrobenzene-d5 & 89.854 & 89.9 & $35-114$ & $55-102$ & $43-114$ \\
2-Fluorobiphenyl & 81.147 & 81.1 & $43-116$ & $63-111$ & $51-124$ \\
24.6-Jribromophenol & 44.996 & 45.0 & $10-123$ & $36-121$ & $14-142$ \\
Terphenyl-d14 & 98.885 & 98.9 & $33-141$ & $55-124$ & $37-141$ \\
2-Chiorophenol-d4 & 53.874 & 53.9 & $33-110$ & Advisory Use Only \\
1.2-Dichlorobenzene-d4 & 77.201 & 77.2 & $16-110$ & \multicolumn{2}{c}{ Advisory Use Only }
\end{tabular}

Limits updated: 12/14/95 


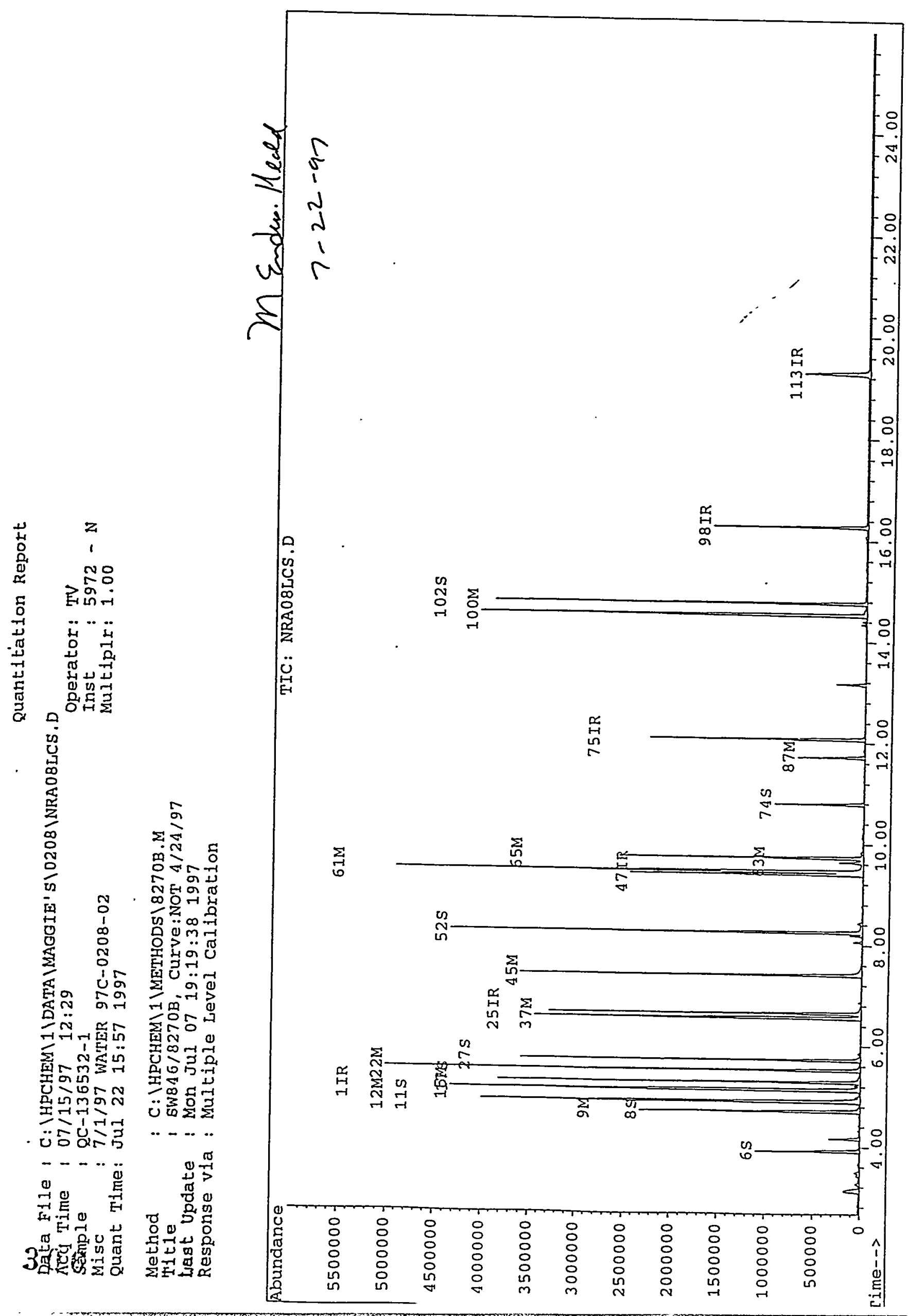

$N$
0
0
$\sigma$
0 
Quantitation Report

Data File : C: \HPCHEM $\backslash 1$ \DATA IMAGGIE'S\0208\NRA08LCS.D

Acq Time : 07/15/97 12:29

Sample : QC-136532-1

Misc : 7/1/97 WATER 97C-0208-02

Operator: TV

Inst : $5972-\mathrm{N}$

Multiplr: 1.00

Quant Time: Jul 22 15:57 1997

Method : C: \HPCHEMIIIMETHODS $18270 B . M$

Title : SW846/8270B, Curve:NOT 4/24/97

Last Update : Mon Jul 07 19:19:38 1997

Response via : Multiple Level Calibration

Internal standards

1) 1,4-Dichlorobenzene-d4
25) Naphthalene-d8
47) Acenaphthene-d10
75) Phenanthrene-d10
98) Chrysene-d12
113) Perylene-d12

System Monitoring Compounds

6) 2-Fluorophenol

B) Phenol-d5

11) 2-Chlorophenol-a4

17) 1,2-Dichlorobenzene-d4

27) Nitrobenzene- $d 5$

52) 2-Eluorobiphenyl

74) 2,4,6-Tribromophenol

102) Terpheny1-d14

Target compounds

9) Phenol

12) 2-Chlorophenol

15) 1,4-dichlorobenzene

22) N-Nitrosodi-n-propyl amine

37) 1,2,4-Trichlorobenzene

45) 4-Chloro-3-Methylphenol

61) Acenaphthene

63) 4-Nitrophenol

65) 2,4-Dinitrotoluene

B7) Pentachlorophenol

100) Pyrene
R.T. Scan QIon Response

Conc Units

Areas

$\begin{array}{rrrrrr}5.13 & 285 & 152 & 591638 & 40.00 \mathrm{ug} / \mathrm{mL} & 0.00 \\ 6.65 & 460 & 136 & 2459530 & 40.00 \mathrm{ug} / \mathrm{mL} & 0.00 \\ 9.41 & 777 & 164 & 1075581 & 40.00 \mathrm{ug} / \mathrm{mL} & 0.00 \\ 12.08 & 1084 & 188 & 1360854 & 40.00 \mathrm{ug} / \mathrm{mL} & 0.00 \\ 16.29 & 1568 & 240 & 897837 & 40.00 \mathrm{ug} / \mathrm{mL} & 0.00 \\ 19.29 & 1914 & 264 & 625637 & 40.00 \mathrm{ug} / \mathrm{mL} & 0.00\end{array}$

\&Recovery

$\begin{array}{rrrr}3.93 & 147 & 112 & 672542 \\ 4.70 & 236 & 99 & 804567 \\ 4.90 & 259 & 132 & 1227094 \\ 5.29 & 304 & 152 & 913124 \\ 5.73 & 354 & 82 & 1697553 \\ 8.25 & 644 & 172 & 2508391 \\ 10.80 & 937 & 330 & 168266 \\ 14.76 & 1393 & 244 & 2010364\end{array}$

$33.773 \mathrm{ug} / \mathrm{mI} \quad 33.778$

$32.969 \mathrm{ug} / \mathrm{mL} \quad 32.978$

$53.874 \mathrm{ug} / \mathrm{mL} \quad 53.878$

$77.201 \mathrm{ug} / \mathrm{mL} \quad 77.208$

$89.854 \mathrm{ug} / \mathrm{mL} \quad 89.85 \%$

$81.147 \mathrm{ug} / \mathrm{mL} \quad 81.15 \%$

$44.996 \mathrm{ug} / \mathrm{mL} \quad 45.008$

$98.885 \mathrm{ug} / \mathrm{mL}$

98.898

$\begin{array}{lllll}4.72 & 238 & 94 & 883655 & 34.343 \mathrm{ug} / \mathrm{mL} \#\end{array}$

$\begin{array}{lllll}4.92 & 261 & 128 & 1309967 \quad 59.780 u g / m L\end{array}$

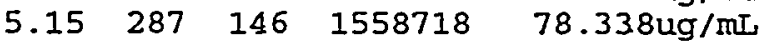

$\begin{array}{llll}5.52 & 330 & 70 & 1333767\end{array}$

$\begin{array}{llll}6.56 & 450 & 180 & 1085551 .\end{array}$

$\begin{array}{llll}7.41 & 547 & 107 & 1381546\end{array}$

$138.387 \mathrm{ug} / \mathrm{mL}$

$65.838 \mathrm{ug} / \mathrm{mL}$

$9.47 \quad 784 \quad 153$

$9.63 \quad 803 \quad 109$

2470368

34853

$9.73 \quad 814 \quad 165$

$11.72 \quad 1043 \quad 266$

1005690

130025

$86.258 \mathrm{ug} / \mathrm{mL}$

$92.223 \mathrm{ug} / \mathrm{mL}$

$12.637 \mathrm{ug} / \mathrm{mL}$

$93.157 \mathrm{ug} / \mathrm{mL} \#$

$33.234 \mathrm{ug} / \mathrm{mL}$

$93.120 \mathrm{ug} / \mathrm{mL} \#$

Qvalue
77
98
95
88
98
90
97
79
90
98
60

Data processed By: M Endu- Heald 7-22-57

(\#) = cualifier out of range $(\mathrm{m})$ = manual integration

( 3 A

Page 1 

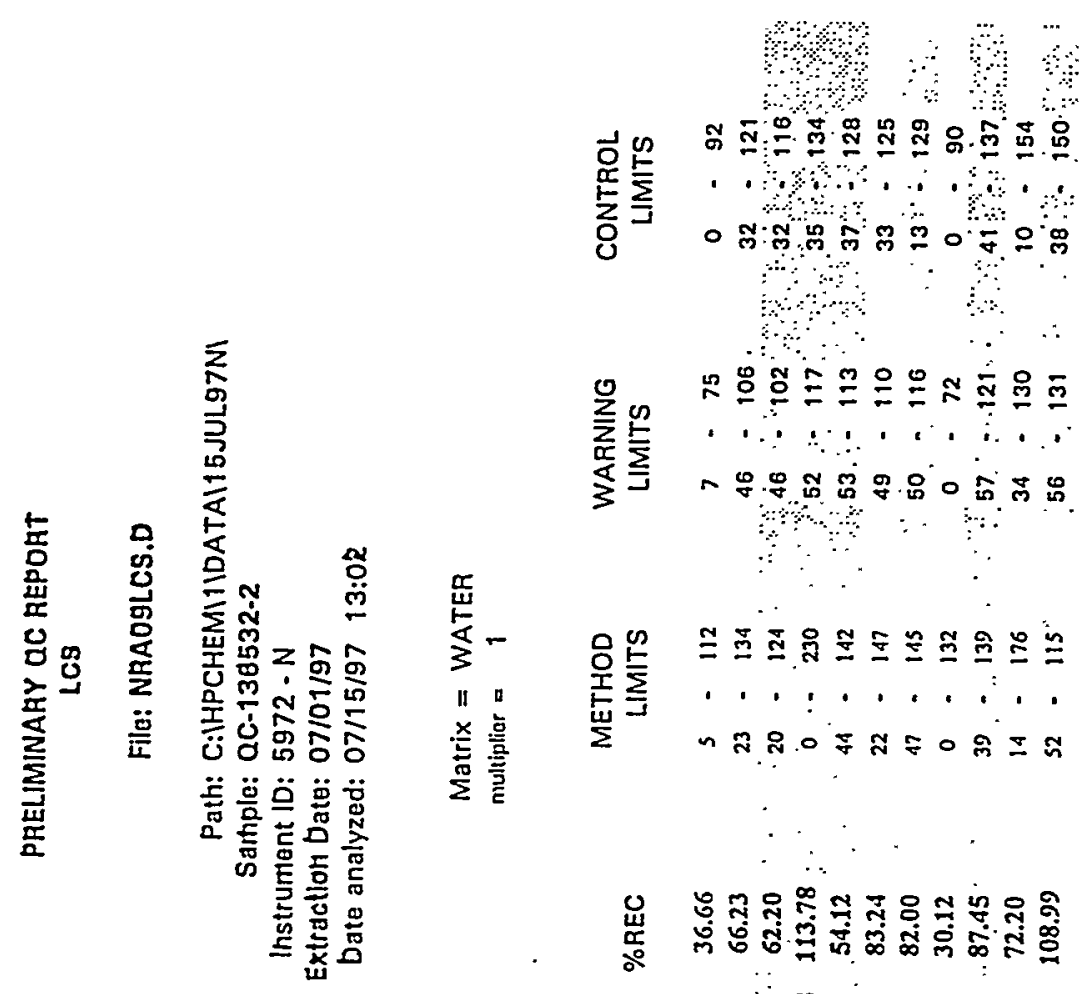

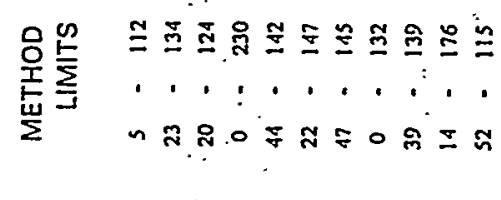

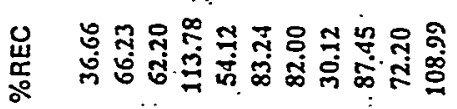
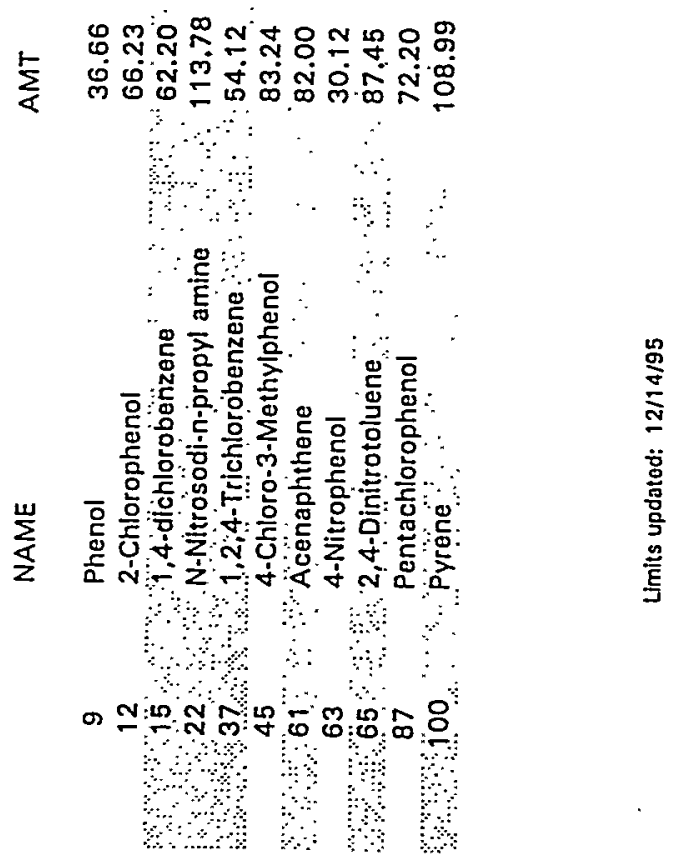


\section{PRELIMINARY QC SUMMARY REPORT}

File: NRAO9LCS.D

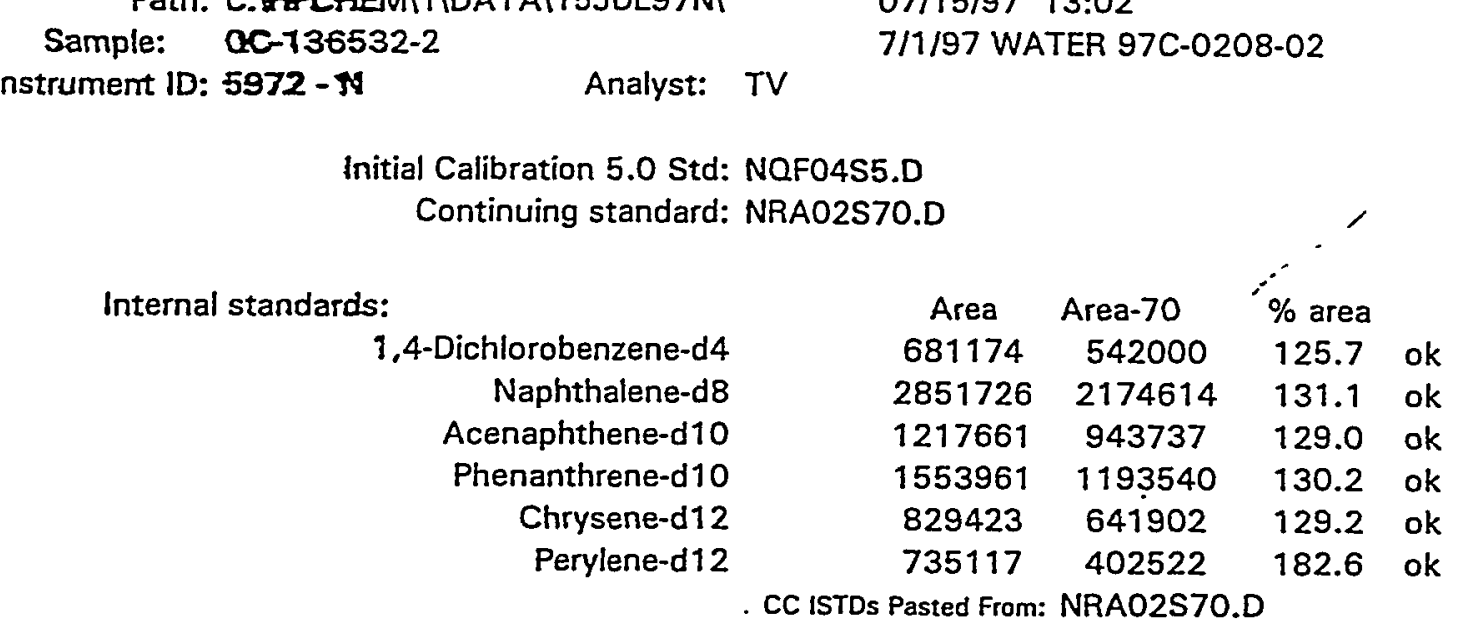

Matrix: (Water or Soil): WATER

Multiplier (normal water $=1$; soil $=1$ : $\quad 1$

Surrogates:

\begin{tabular}{rccccc} 
& Quan & $\%$ Rec & Method & Warning & Control \\
2-Fluorophenol & 43.576 & 43.6 & $21-100$ & $23-78$ & $10-91$ \\
Phenol-d5 & 34.511 & 34.5 & $10-94$ & $9-73$ & $0-89$ \\
Nitrobenzene-d5 & 75.934 & 75.9 & $35-114$ & $55-102$ & $43-114$ \\
2-Fluorobiphenyl & 70.952 & 71.0 & $43-116$ & $63-111$ & $51-124$ \\
2,4,6-Trioromophenol & 65.704 & 65.7 & $10-123$ & $36-121$ & $14-142$ \\
Terphenyl-d14 & 111.950 & 111.9 & $33-141$ & $55-124$ & $37-141$ \\
2-Chlorophenol-d4 & 58.977 & 59.0 & $33-110$ & Advisory Use Only \\
1,2-Dichlorobenzene-d4 & 61.277 & 61.3 & $16-110$ & \multicolumn{2}{c}{ Advisory Use Only }
\end{tabular}

Limits updated: 12/14/95

\section{9}




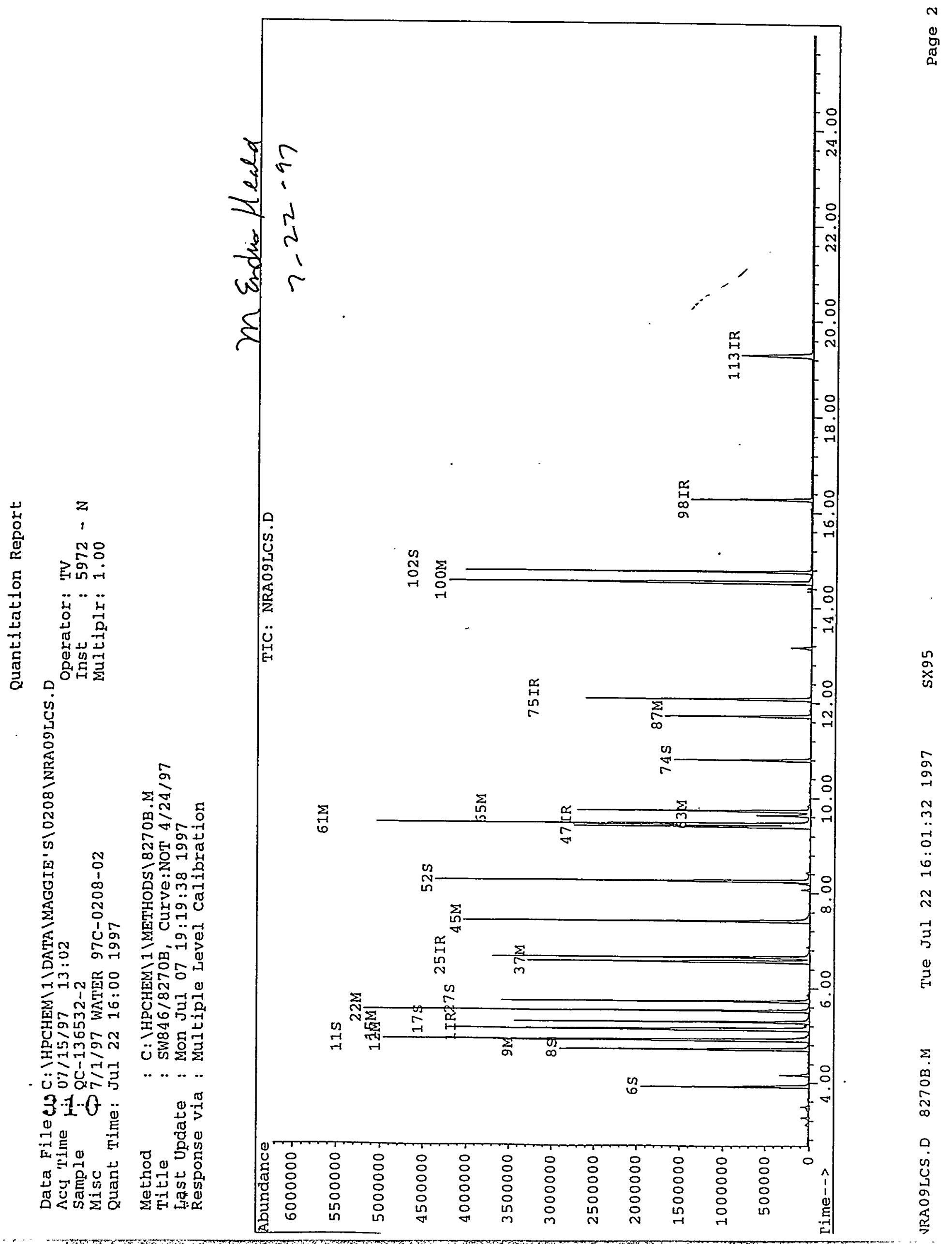


Quantitation Report

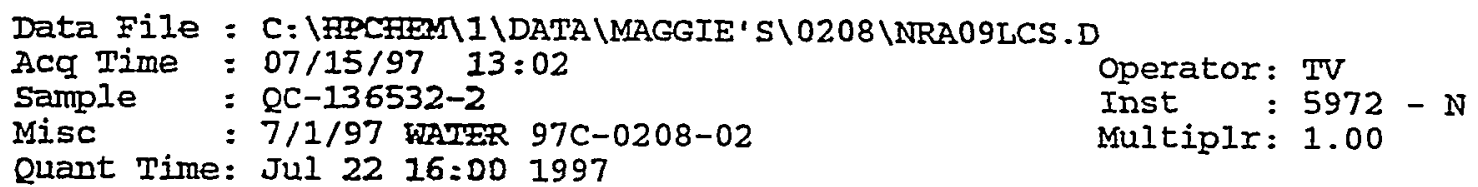

Quant Time: Jul 22 16:00 1997

Method : C: IHPCHEMIIIMETHODS $\backslash 8270 \mathrm{~B} . \mathrm{M}$

Title : S\$B46/8270B, Curve:NOT 4/24/97

- Last Update : Mon JuI D7 19:19:38 1997

Response via : Multiple Level Calibration

Internal standards

R.T. Scan QIon Response Conc Units Area\%

1) 1,4-Dichlorobenzene-d4

$\begin{array}{rrrr}5.12 & 284 & 152 & 681174 \\ 6.65 & 460 & 136 & 2851726 \\ 9.41 & 777 & 164 & 1217661 \\ 12.08 & 1084 & 188 & 1553961 \\ 16.28 & 1567 & 240 & 829423 \\ 19.30 & 1914 & 264 & 735117\end{array}$

25) Naphthalene-dB

47) Acenaphthene-d10

75) Phenanthrene-d10

98) Chrysene-d12

113) Perylene-d12

System Monitoring Compounds
6) 2-Fluorophenol
B) Phenol-a5
11) 2-Chlorophenol-d4
17) 1,2-Dichlorobenzene-d4
27) Nitrobenzene-a5
52) 2-Fluorobiphenyl
74) 2,4,6-Tribromophenol
102) Terpheny $1-d 14$

$\begin{array}{rrrr}3.92 & 146 & 112 & 989028 \\ 4.70 & 236 & 99 & 967441 \\ 4.89 & 258 & 132 & 1533108 \\ 5.29 & 304 & 152 & 860831 \\ 5.73 & 354 & 82 & 1686863 \\ 8.25 & 644 & 172 & 2525429 \\ 10.80 & 937 & 330 & 274176 \\ 14.76 & 1393 & 244 & 2087210\end{array}$

$\begin{array}{lr}40.00 \mathrm{ug} / \mathrm{mL} & 0.00 \\ 40.00 \mathrm{ug} / \mathrm{mL} & 0.00 \\ 40.00 \mathrm{ug} / \mathrm{mL}, & 0.00 \\ 40.00 \mathrm{ug} / \mathrm{mL} & 0.00 \\ 40.00 \mathrm{ug} / \mathrm{mL} & -0.02 \\ 40.00 \mathrm{ug} / \mathrm{mL} & 0.00\end{array}$

Target compounds

9) Phenol

12) 2-Chlorophenol

15) 1,4-dichlorobenzene

22) N-Nitrosodi-n-propyl amine

$\begin{array}{rrrr}4.72 & 238 & 94 & 1082147 \\ 4.91 & 260 & 128 & 1650899 \\ 5.15 & 287 & 146 & 1462505 \\ 5.52 & 330 & 70 & 1370275 \\ 6.56 & 450 & 180 & 1053230 \\ 7.41 & 547 & 107 & 1553794 \\ 9.47 & 784 & 153 & 2544356 \\ 9.63 & 803 & 109 & 93928 \\ 9.73 & 814 & 165 & 1077890 \\ 11.72 & 1043 & 266 & 323812 \\ 14.54 & 1367 & 202 & 3236622\end{array}$

1082147

1462505

37) 1,2,4-Trichlorobenzene

45) 4-Chloro-3-Methylphenol

61) Acenaphthene

63) 4-Nitrophenol

65) 2,4-Dinitrotoluene

B7) Pentachlorophenol

IDD) Pyrene

$36.662 \mathrm{ug} / \mathrm{mLH}$

$66.234 \mathrm{ug} / \mathrm{mL}$

$62.198 \mathrm{ug} / \mathrm{mL}$

$113.781 \mathrm{ug} / \mathrm{mL}$

$54.116 \mathrm{ug} / \mathrm{mr}$

$83.241 \mathrm{ug} / \mathrm{mL}$

$81.999 u g / m r$

$30.122 \mathrm{ug} / \mathrm{mL}$

$87.446 u g / m L$

$72.205 \mathrm{ug} / \mathrm{mL}$

$108.993 \mathrm{ug} / \mathrm{mL}$

\& Recovery

$43.576 \mathrm{ug} / \mathrm{mL} \quad 43.58 \%$

$1 \mathrm{ug} / \mathrm{mL} \quad 34.518$

(27.98 $/ \mathrm{mL} \quad 58.98$

.704ug/mL 65.70 웅

108.993ug/mit

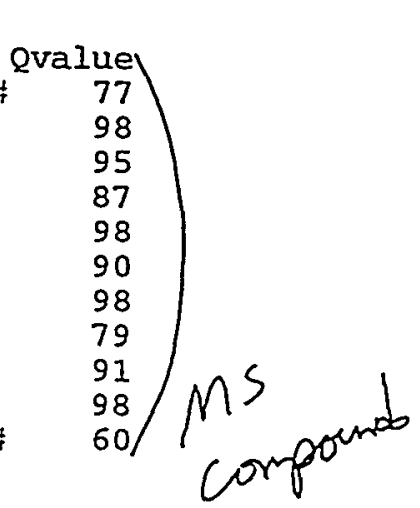

\section{1}

Data Processed By: Wh Sudur-Hesed 7. 22-47

$(\#)$ = qualifier out of range $(m)=$ manual integration

(*) Does not met EPA spectral criteria (False Hit) SX95 
File: NRA 10C97.D

\section{Sample: $97 \mathrm{C02897}$}

Instrument ID: $5972-\mathrm{N}$
DATAI15JUL97NI
07/15/97 13:36

7/1/97 WATER 97C-0208-02

Initial Calibration 5.0 Std: NOF04S5.D

Continuing standard: NRAO2S7O.D

Internal standards:

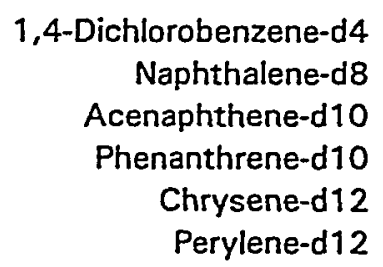

1,4-Dichlorobenzene-d4 Naphthalene-d8 Acenaphthene-d10 Phenanthrene-d10 Chrysene-d12 Perylene-d12

$\begin{array}{cccc} & & & \\ \text { Area } & \text { Area-70 } & \% & \\ 623827 & 542000 & 115.1 & \text { ok } \\ 2196009 & 2174614 & 101.0 & \text { ok } \\ 1077099 & 943737 & 114.1 & \text { ok } \\ 1420013 & 1193540 & 119.0 & \text { ok } \\ 1012069 & 641902 & 157.7 & \text { ok } \\ 668509 & 402522 & 166.1 & \text { ok } \\ \text { Sasted From: } & \text { NRAO2S70.D } & \end{array}$

Matrix: (Water or Soil): WATER

Multiplier (normal water $=1$; soil $=1$ ): $\quad 1$

DILUTION REQUIRED BEYOND CURVE RANGE

Surrogates:

\begin{tabular}{rccccc} 
& Quan & \%Rec & Method & Warning & Control \\
2-Fluorophenol & 40.491 & 40.5 & $21-100$ & $23-78$ & $10-91$ \\
Phenol-d5 & 34.781 & 34.8 & $10-94$ & $9-73$ & $0-89$ \\
Nitrobenzene-d5 & 95.536 & 95.5 & $35-114$ & $55-102$ & $43-114$ \\
2-Fluorobiphenyl & 75.611 & 75.6 & $43-116$ & $63-111$ & $51-124$ \\
2,4,6-Tribromophenol & 62.967 & 63.0 & $10-123$ & $36-121$ & $14-142$ \\
Terphenyl-d14 & 90.183 & 90.2 & $33-141$ & $55-124$ & $37-141$ \\
2-Chlorophenol-d4 & 62.517 & 62.5 & $33-110$ & \multicolumn{2}{c}{ Advisory Use Oniy } \\
1,2-Dichlorobenzene-d4 & 67.750 & 67.8 & $16-110$ & \multicolumn{2}{c}{ Advisory Use Only }
\end{tabular}

Limits updated: $12 / 14 / 95$ 


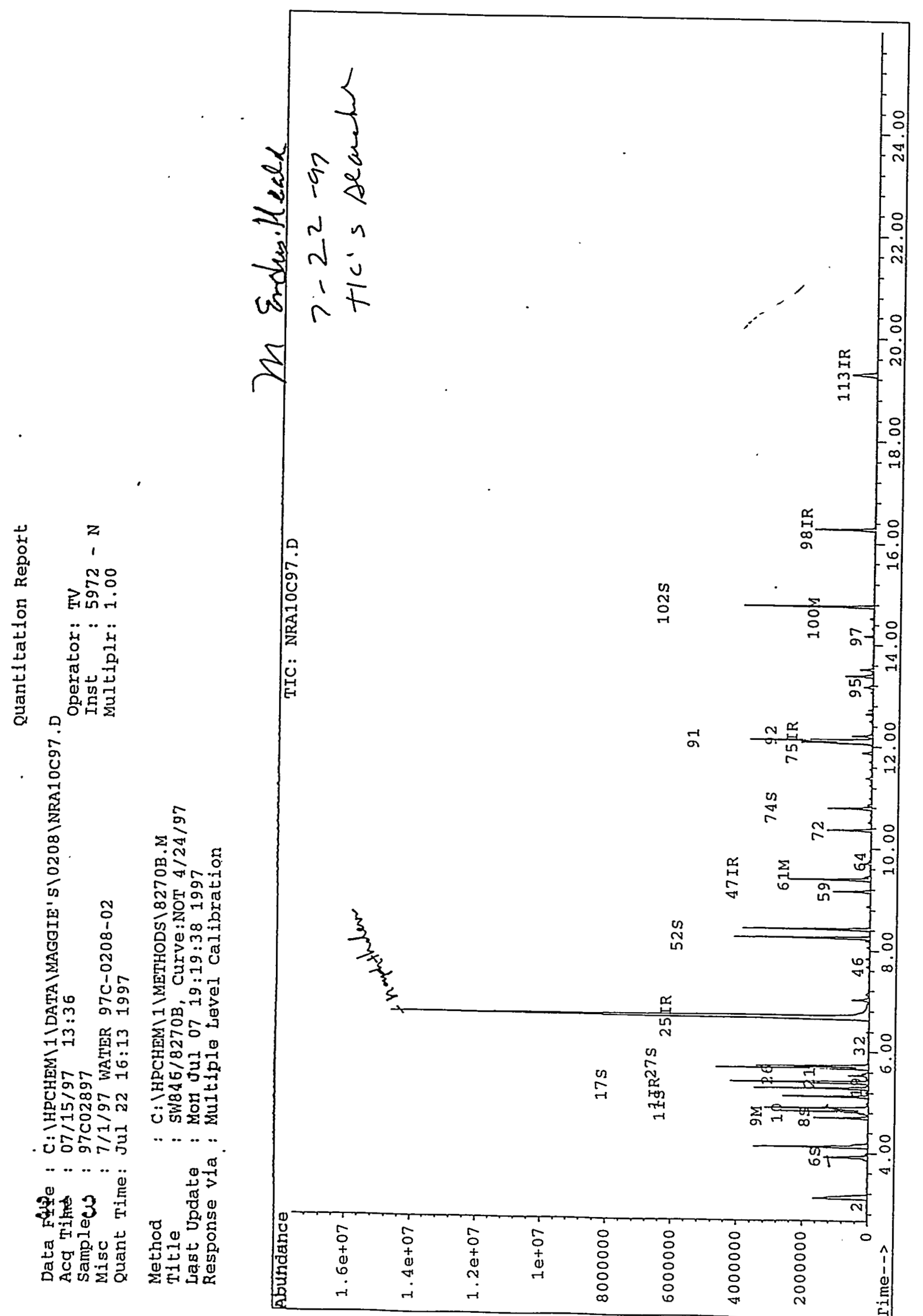


Data File = C: \HPCHEM I IDATA \MAGGIE'S\0208\NRA10C97.D

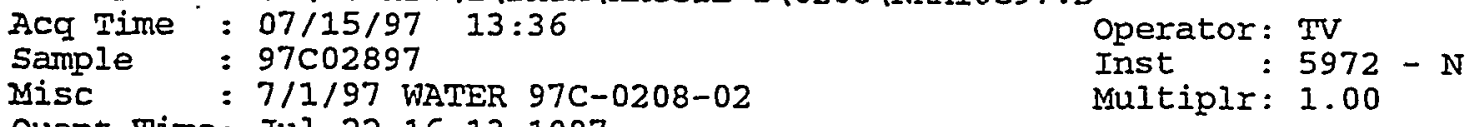

Quant Time: Jul 22 16:13 1997

Method : C: \HPCHEMIIIMETHODS $18270 \mathrm{~B} . M$

Title : SW846/8270B, Curve:NOT 4/24/97

Last Dpdate : Mon Jul 07 19:19:38 1997

Response via : Multiple Level Calibration

Internal standards

R.T. Scan QIon Response Conc Units Area\%

1) 1,4-Dichlorobenzene-d4

25) Naphthalene-d8

47) Acenaphthene-a10

75) Phenanthrene-dio

98) Chrysene-d12

113) Perylene-d12

$\begin{array}{rrrrrrr}5.13 & 285 & 152 & 623827 & 40.00 \mathrm{ug} / \mathrm{mL} & 0.00 \\ 6.66 & 461 & 136 & 2196009 & 40.00 \mathrm{ug} / \mathrm{mL} & 0.00 \\ 9.41 & 777 & 164 & 1077099 & 40.00 \mathrm{ug} / \mathrm{mL} & 0.00 \\ 12.08 & 1084 & 188 & 1420013 & 40.00 \mathrm{ug} / \mathrm{mL} & 0.00 \\ 16.29 & 1568 & 240 & 1012069 & 40.00 \mathrm{ug} / \mathrm{mL} & 0.00 \\ 19.29 & 1914 & 264 & 668509 & 40.00 \mathrm{ug} / \mathrm{mL} & 0.00\end{array}$

System Monitoring Compounds

6) 2-Fluorophenol

B) Phenol-d5

11) 2-Chlorophenol-d4

17) 1,2-Dichlorobenzene-d4

27) Nitrobenzene-d5

52) 2-FIuorobiphenyl

74) 2,4,6-Tribromophenol

102) Terphenyl-d14

$\begin{array}{rrrr}3.93 & 147 & 112 & 844335 \\ 4.71 & 237 & 99 & 892589 \\ 4.90 & 259 & 132 & 1479219 \\ 5.29 & 304 & 152 & 860788 \\ 5.73 & 354 & 82 & 1602208 \\ 8.26 & 645 & 172 & 2362285 \\ 10.80 & 937 & 330 & 232872 \\ 14.76 & 1393 & 244 & 2076769\end{array}$

Target compounds

2) Pyridine

3) N-nitrosodimethylamine

4) 2-Picoline

5) Methyl methanesulfonate

7) Ethyl methanesulfonate

9) Phenol

10) Aniline

12) 2-Chlorophenol

13) Decane NOT Present

14) 1,3-Dichlorobenzene

15) 1,4-dichlorobenzene

16) Benzyl alcohol

18) 1,2-aichlorobenzene

19) 2-Methylphenol

20) Bis (2-chloroisopropyl) ethe

21) 4-methylphenol

22) N-Nitrosodi-n-propyl amine

23) Hexachloroethane

24) Bis (2-chloroethyl) ether

25) Acetophenone

28) Nitrobenzene

29) N-nitrosopiperidine

30) Isophorone

31) 2-Nitrophenol

32) 2,4-Dimethylphenol

33) Benzoic acid

34) Bis (2-chloroethoxy) methane

35) 2,4-Dichlorophenol

36) A. A. -dimethylphenethylami

37) 1,2,4-Trichlorobenzene

38) Dodecane NOT Present

39) Naphthalene

40) 4-Chloroaniline

4i) 2,6-Dichlorophenol

42) Hexachlorobutadiene

43) Benzothiazole NOT Present

3) N4nitroso-di-n-butylamine

$\begin{array}{llll}2.95 & 35 & 79 & 29209 \\ 0.00 & & & \\ 0.00 & & & \\ 0.00 & & & \\ 0.00 & & & \\ 4.72 & 238 & 94 & 50609 \\ 4.79 & 246 & 66 & 67734\end{array}$

0.00

0.00

0.00

0.00

0.00

0.00

5.34

0.00

5.50

0.00

0.00

0.00

5.54

0.00

0.00

0.00

0.00

6.15

0.00

0.00

0.00

0.00

0.00

0.00

0.00

0.00

0.00

0.00

0.00

0.00

$402 \quad 122 \quad 6109$ s Recovery

$40.491 \mathrm{ug} / \mathrm{mL} \quad 40.498$

$34.781 \mathrm{ug} / \mathrm{mL} \quad 34.78 \%$

$62.517 \mathrm{ug} / \mathrm{mL} \quad 62.528$

$67.750 \mathrm{ug} / \mathrm{mL} \quad 67.758$

$95.536 \mathrm{ug} / \mathrm{mL} \quad 95.548$

$.75 .611 \mathrm{ug} / \mathrm{mL} \quad 75.618$

$62.967 \mathrm{ug} / \mathrm{mL} \quad 62.978$

$90.183 \mathrm{ug} / \mathrm{mL} \quad 90.18 \%$

$1.118 u g /$ Qvalue

Not Detected

Not Detected

Not Detected

Not Detected

$1.775 \mathrm{ug} / \mathrm{mL} \#$

$4.312 \mathrm{ug} / \mathrm{mL} \#$

Not Detected

Not Detected

Not Detected

Not Detected

Not Detected

Not Detected

$\begin{array}{llll}309 & 107 & 9866 \quad 0.516 \mathrm{ug} / \mathrm{mL}\end{array}$

Not Detected

$\begin{array}{llll}328 & 108 & 16964 & 0.694 \mathrm{ug} / \mathrm{mL}\end{array}$

Not Detected

Not Detected

Not Detected

$332 \quad 105 \quad 251084$

$9.851 \mathrm{ug} / \mathrm{mL}$

Not Detected

Not Detected

Not Detected

Not Detected

$0.331 \mathrm{ug} / \mathrm{mL} \#$

$70-$

$70 \%$

Not Detected

Not Detected

Not Detected

Not Detected

Not Detected

Not Detected

Nat Dotected

Not Detected

Not Detected

Not Detected

Not Detected

Not Detected 
Data File : C: \HPCHEM \1\DATA \MAGGIE'S\0208\NRA10C97.D
Acc Time
: $07 / 15 / 97 \quad 13: 36$
Sample: 97002897
Misc : 7/1/97 WATER 97C-0208-02
Operator: TV
Inst : $5972-\mathrm{N}$
Multiplr: 1.00

Quant Time: Jul 22 16:13 1997

\begin{abstract}
Method : C:\HPCHEM $\backslash 1 \backslash M E T H O D S \backslash 8270 B . M$
Title : Sw846/8270B, Curve:NOT 4/24/97

Last Update : Mon Jul 07 19:19:38 1997

Response via : Multiple Level Calibration
\end{abstract}

Compound

45) 4-Chloro-3-Methylphenol

46) 2-Methylnaphthalene

48) $1,2,4,5$-Tetrachlorobenzene

49) Hexachlorocyclopentadiene

50) 2,4,6-Trichlorophenol

51) 2,4,5-Irichlorophenol

53) Tetradecane NOT Present

54) 2-Chloronaphthalene

55) 1-Chloronaphthalene

56) 2-Nitroaniline

57) Dimethylphthalate

5B) 2,6-Dinitrotoluene

59) Acenaphthylene

60) 3-Nitroaniline

61) Acenaphthene

62) 2,4-Dinitrophenol

63) 4-Nitrophenol

64) Dibenzofuran

65) 2,4-Dinitrotoluene

65) Pentachlorobenzene

67) 1-Naphthylamine

5B) 2-Naphthylamine

69) 2,3,4,6-Tetrachlorophenol

70) Diethylphthalate

71) 4-Chlorophenyl Phenyl Ethe

72) Fluorene

73) 4-Nitroaniline

76) 4,6-Dinitro-2-methylphenol

77) N-nitrosodiphenylamine

78) 1,2-Diphenylhyarazine

79) Tributylphosphate NOT Pres

BD) Ihenacetin

BI) 4-BIomophenyl phenyl Ether

B2) alpha-BHC

B3) Fexachlorobenzene

B4) Tris (2-CE) phosphate NOT Pr

85) 4-Aminobiphenyl

86) Deta-BHC

B7) Dentachlorophenol

8B) Pronamide

B9) gamma-BHC

9D) Dentaciloronitrobenzene

91) Phenanthrene

92) Inthracene

93) delta-BHC

94) Feptachlor

95) Di-n-Butylphthalate

96) Aldrin

97) Eluoranthene

99) Benzidine

100) Pyrene

101) $4.4^{\prime}-D D E$

103) Dieldrin

104) D-Dimethylaminoazobenzene

$105) \leqslant 4^{\prime}-D D D$ $3+\frac{4}{3}$
R.T. Scan QIon Response Conc Unit Qvalue

0.00 Not Detected

7.69

0.00

579

142

43532

$1.272 \mathrm{ug} / \mathrm{mL}$

Not Detected.

Not Detected

0.00

0.00

0.00

0.00

0.00

0.00

0.00

0.00

9.16

0.00

9.46

0.00

0.00

9.75

0.00

0.00

0.00

0.00

0.00

0.00

0.00

10.37

$749 \quad 152 \quad 887188$

Not Detected

Not Detected

Not Detected

Not Detected

Not Detected

Not Detected

Not Detected

Not Detected

$16.887 \mathrm{ug} / \mathrm{mL}$

Not Detected

$\begin{array}{llll}783 & 153 & 75205 & 2.329 \mathrm{ug} / \mathrm{mL}\end{array}$

Not Detected

$\begin{array}{lllr}817 & 168 & 43297 & \text { Not Detected } \\ 0.970 u g / m I\end{array}$

Not Detected

Not Detected.

Not Detected

Not Detected

Not Detected

Not Detected

Not Detected

0.00

$888 \quad 165 \quad 748568$

$26.374 \mathrm{ug} / \mathrm{mL}$

Not Detected

Not Detected

Not Detected

Not Detected

Not Detected

Not Detected

Not Detected

Not Detected

Not Detected

Not Detected

Not Detected

Not Detected

Not Detected

Not Detected

Not Detected

Not Detected

0.00

0.00

$12.13 \quad 1090 \quad 178 \quad 2820493$

$71.850 \mathrm{ug} / \mathrm{mL}$

$10.687 \mathrm{ug} / \mathrm{mL}$

Not Detected

0.00

0.00

$13.16 \quad 1209 \quad 149 \quad 236555$

0.00

$\begin{array}{llll}14.18 & 1326 & 202 & 179189\end{array}$

0.00

$\begin{array}{llll}14.52 & 1365 & 202 & 64486\end{array}$

0.00

0.00

0.00

0.00
4.305ug/mLt

Not Detected

4.791ug/mL\#

Not Detected

1. $619 \mathrm{ug} / \mathrm{mL}$

Not Detected

Not Detected

Not Detected

Not Detected 99r

97

98

91

$99-$

95

97

$93^{r}$

$72 r$

$66 /$

$(\#)$ = qualifier out of range $(m)=$ manual integration

(*) Does not meet EPA spectral criteria (False Hit) SX95 


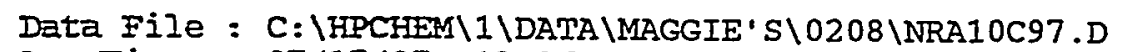

$\begin{array}{lll}\text { Acq Time } & : 07 / 15 / 97 \quad 13: 36 & \text { Operator: } T V \\ \text { Sample } & =97 \text { CO2B97 } & \text { Inst }: 5972-\mathrm{N} \\ \text { Misc } & =7 / 1 / 97 \text { WATER } 97 C-0208-02 & \text { Multiplr: } 1.00 \\ \text { Quant Time: JuI } 22 \text { 16:13 } 1997 & \end{array}$

Quant Time: JuI 22 16:13 1997

Method : C: \HPCHEM I IMETHODS $8270 \mathrm{~B} . \mathrm{M}$

Title : SWB46/827OB, Curve:NOT 4/24/97

$\because$ Iast Update : Mon Jul 07 19:19:38 1997

- Response via : Nultiple Level Calibration

Compound

R.T. Scan QIon Response Conc Unit Qvalue

106) Butylbenzylphthalate

107) $4,4^{\prime}-D D T$

108) Methoxychlor

109) 3,3'-Dichlorobenzidine

110) Benzo[a]anthracene

111) Chrysene

112) Bis(2-ethylhexyl)phthalate

114) Di-n-octylphthalate

115) Benzo[b] fluoranthene

116) 7,12-Dimethylbenz (a) anthra

117) Benzo[k] fluoranthene

118) Benzo[a]pyrene

119) 3-Methylcholanthrene

$120)$ Indeno $(1,2,3-c, d)$ pyrene

121) Dibenz $[a, h]$ anthracene

122) Benzo[ghi]perylene
0.00

0.00

0.00

0.00

0.00

0.00

0.00

0.00

0.00

0.00

0.00

0.00

0.00

0.00

0.00

0.00
Not Detected

Not Detected

Not Detected

Not Detected.

Not Detectéd

Not Detected

Not Detected

Not Detected

Not Detected

Not Detected

Not Detected

Not Detected

Not Detected

Not Detected

Not Detected

Not Detected 

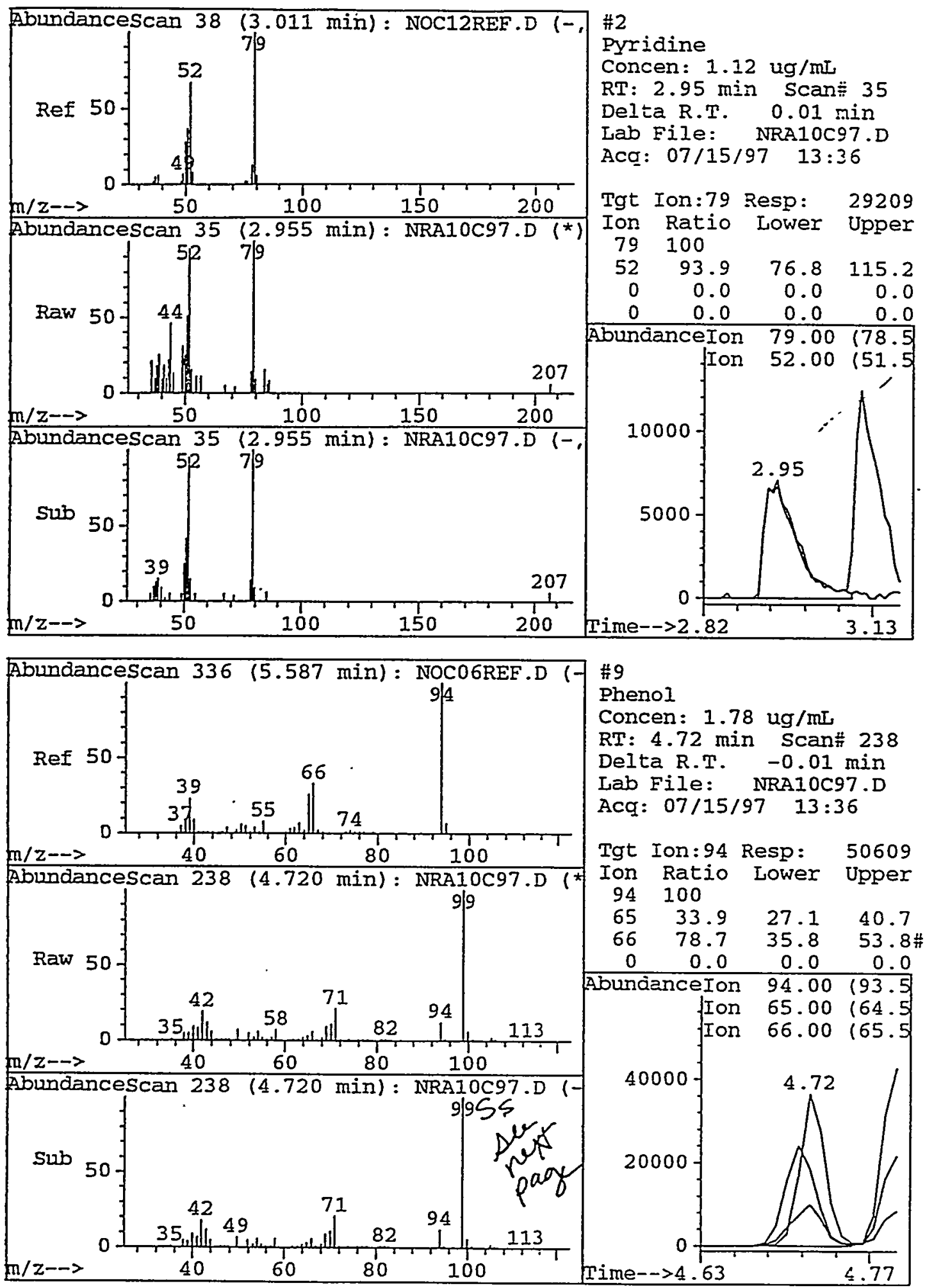
Data File : C: \HPCHEM IIDATA IMAGGIE'S\0208\NRA10C97.D
AcG Time : 07/15/97 13:36
Sarmle : 97C02897
Misc : 7/1/97 WATER 97C-0208-02
Operator: TV
Inst : $5972-\mathrm{N}$

Quent Time: Jul 15 14:03 1997

Method : C: \HPCHEM 11 IMETHODS $\backslash 8270 B . M$

Title : SW846/8270B, Curve:NOT 4/24/97

Iast Update : Mon Jul 07 19:19:38 1997

Response via : Multiple Level Calibration

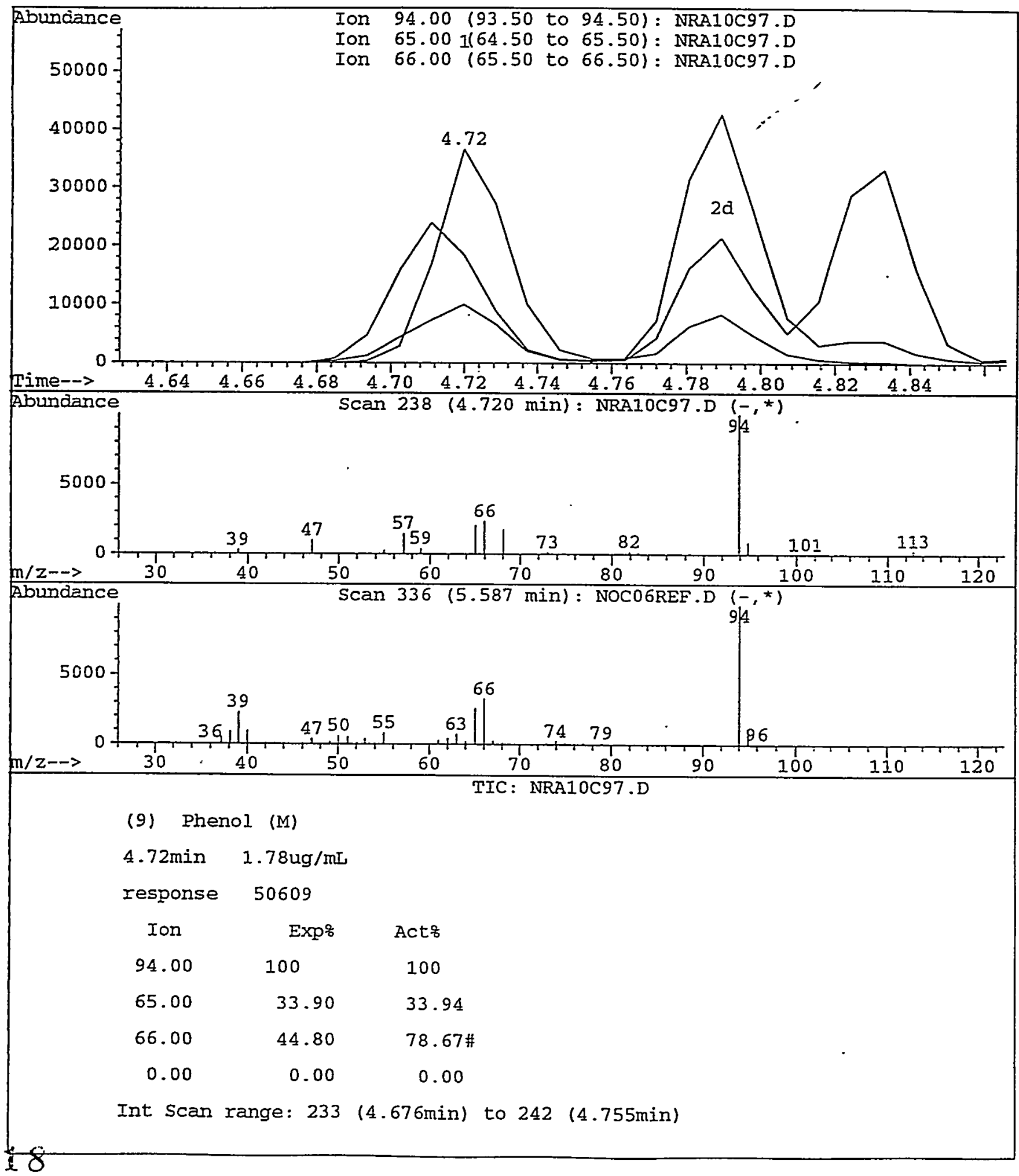



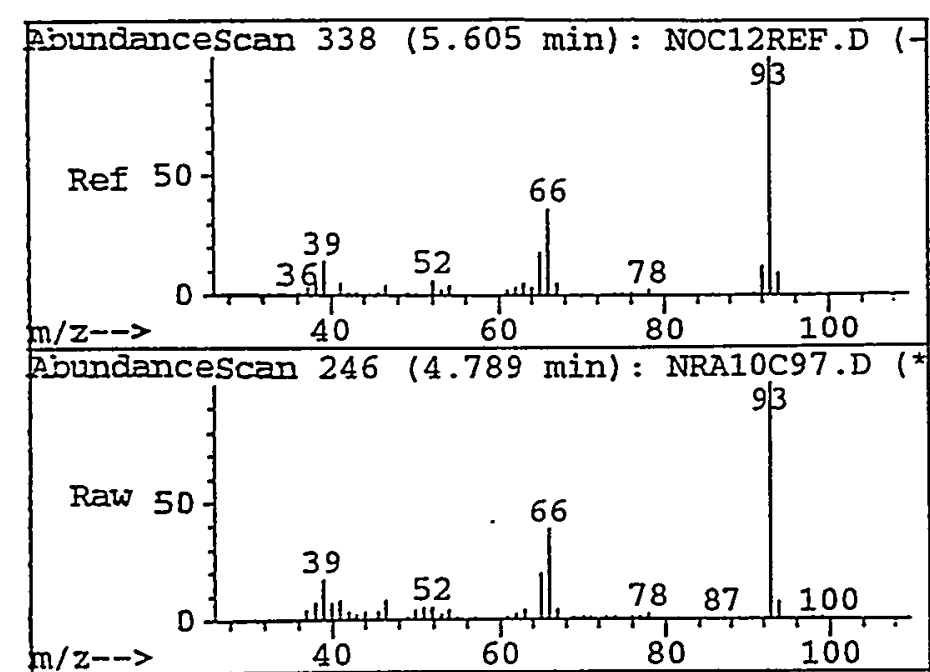

AbundanceScan $246(4.789 \mathrm{~min}):$ NRA10C97.D (-

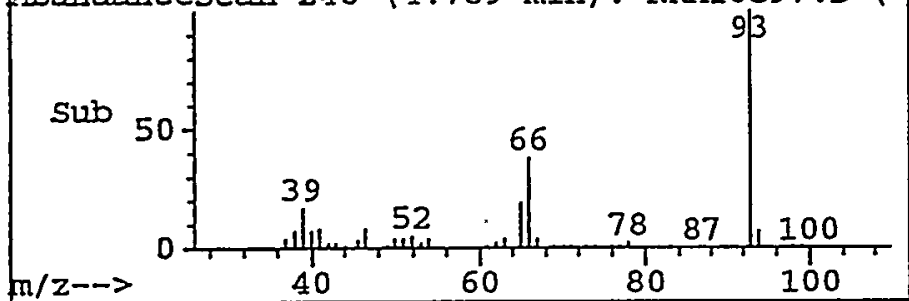

Ajundancescan $246(4.789 \mathrm{~min}):$ NRA10C97.D (*

\#10

Aniline

Concen: $4.31 \mathrm{ug} / \mathrm{mL}$

$\mathrm{RT}: 4.79 \mathrm{~min}$ Scan\# 246

Delta R.T. $0.00 \mathrm{~min}$

Lab File: NRA10C97.D

Acq : $07 / 15 / 97 \quad 13: 36$

Tgt Ion: 66 Resp: 67734

Ion Ratio Lower Upper

$66 \quad 100$

$93 \quad 241.5 \quad 160.0 \quad 240.0 \%$

$\begin{array}{llll}92 & 1.8 & 25.5 & 34.5 \#\end{array}$

$\begin{array}{llll}67 & 12.0 & 21.3 & 28.8 \#\end{array}$

AbundanceIon $66.00 \quad 165.5$

300000 IIon $93.00 \quad(92.5)$

Ion $67.00,166.5$
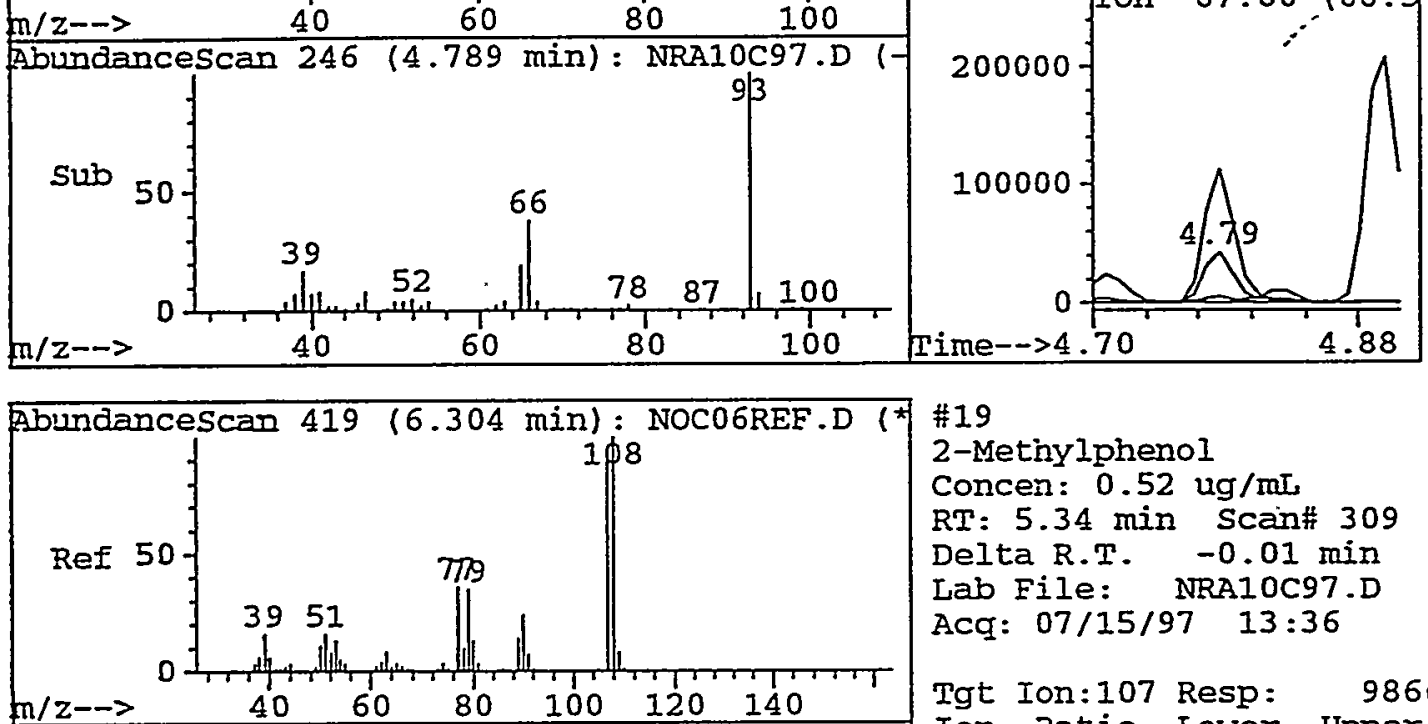

hundancescan $309(5.337 \mathrm{~min}):$ NRA10C97.D (

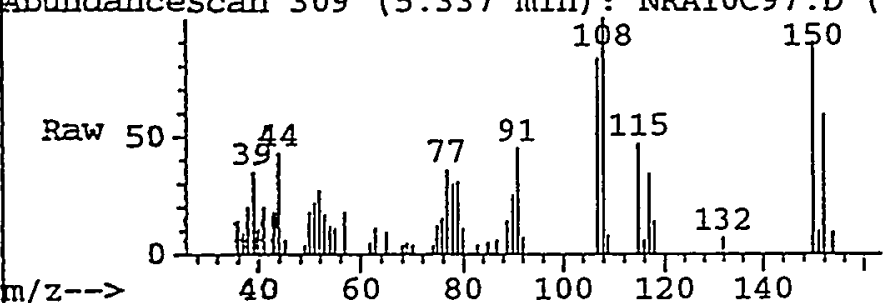

$\mathrm{m} / \mathrm{z} \rightarrow \mathrm{x}$

$\begin{array}{llllll}40 & 60 & 80 & 100 & 120 & 140\end{array}$

Abundancescan 309 (5.337 min): NRA10C97.D (-

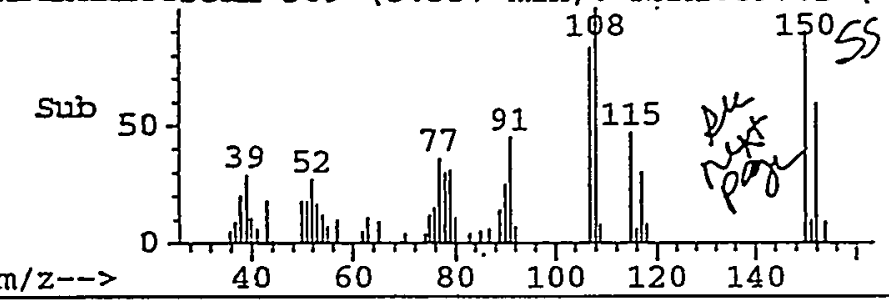

\#19

2-Metinylphenol

Concen: $0.52 \mathrm{ug} / \mathrm{mL}$

RT: 5.34 min Scan\# 309

Delta R.T. $\quad-0.01 \mathrm{~min}$

Lab File: NRA10C97.D

Acq : $07 / 15 / 97 \quad 13: 36$

Tgt Ion:107 Resp: 9866

Ion Ratio Lower Upper

107100

$\begin{array}{llll}108 & 105.5 & 88.6 & 133.0\end{array}$

$\begin{array}{llll}0 & 0.0 & 0.0 & 0.0\end{array}$

$\begin{array}{llll}0 & 0.0 & 0.0 & 0.0\end{array}$

AbundanceIon $107.00 \quad(106$.

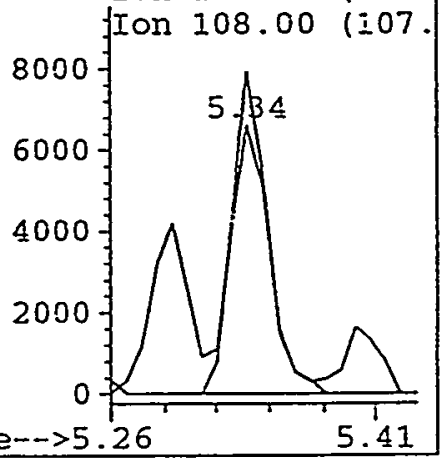



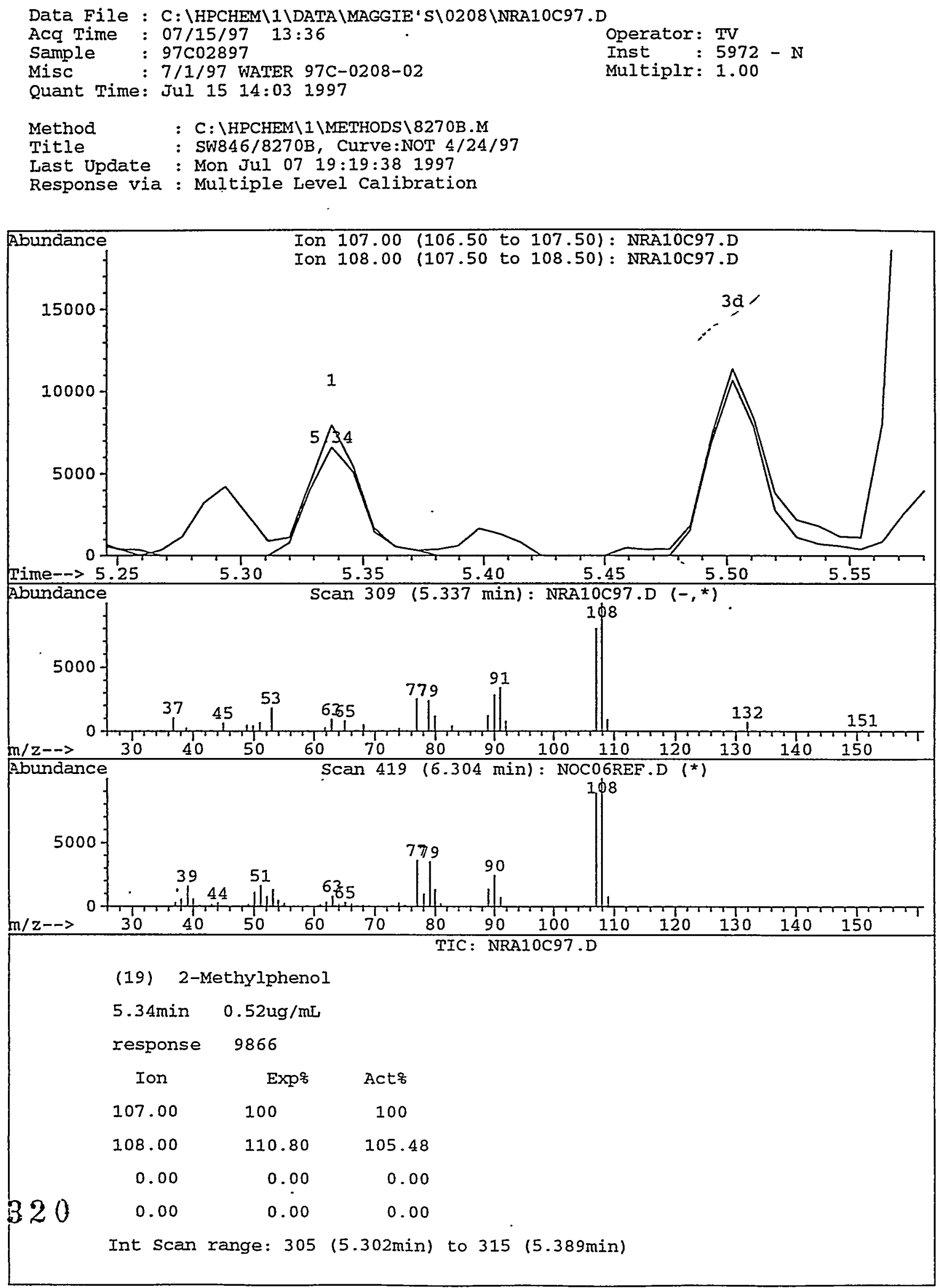


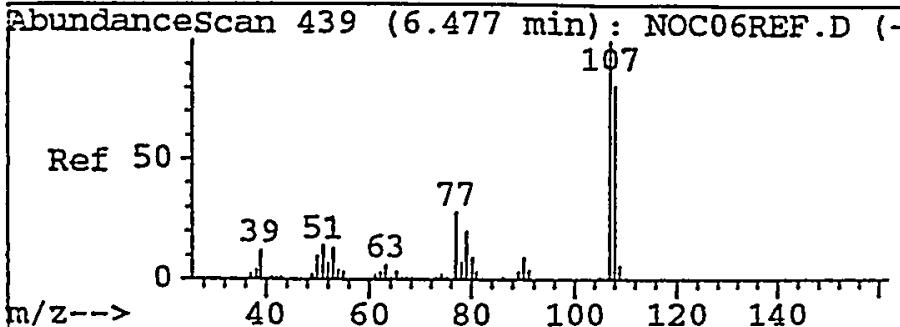

Abundancescan $328(5.502 \mathrm{~min}):$ NRA10C97.D (

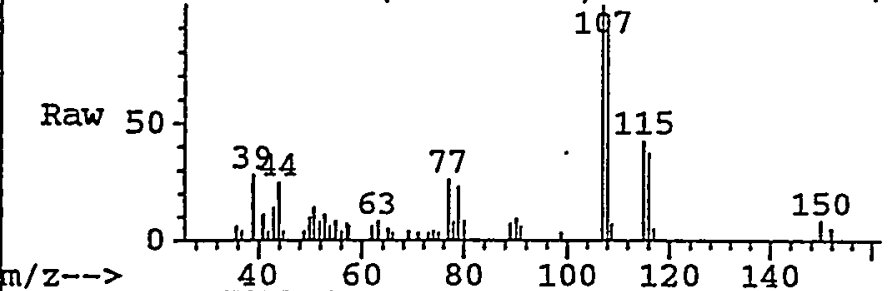

Abundancescan $328(5.502 \mathrm{~min}):$ NRA10C97\% (-
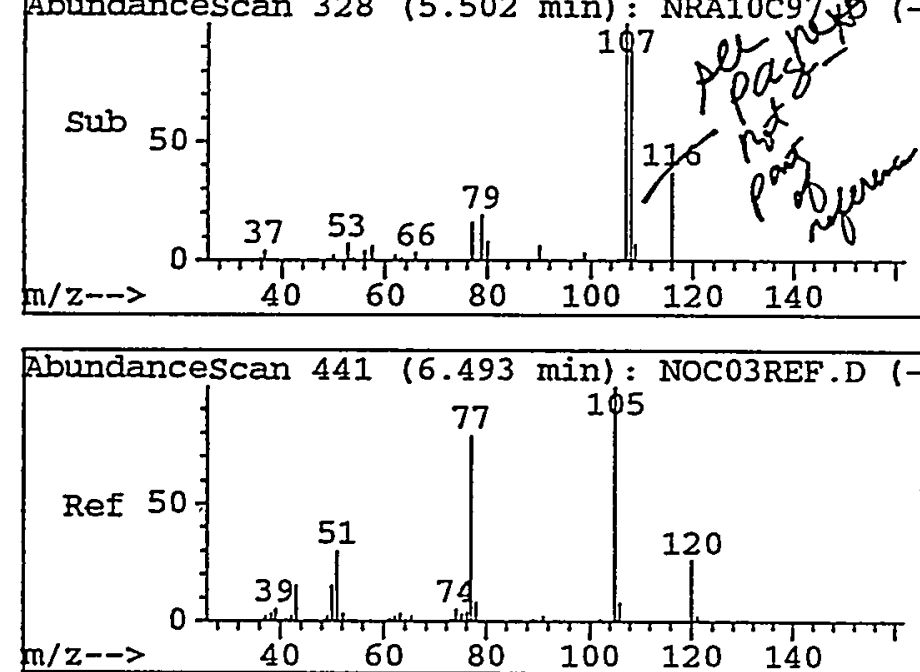

Abundancescan $332(5.537 \mathrm{~min}):$ NRA10C97.D (*

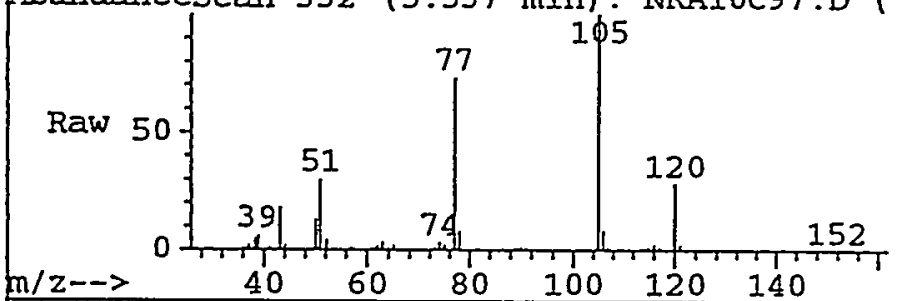

Abundancescan $332(5.537 \mathrm{~min}):$ NRA10C97.D (-

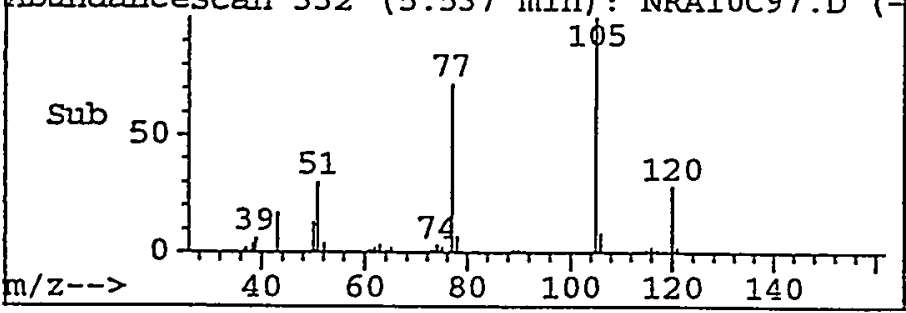

$\# 21$

4-methylphenol

Concen : $0.69 \mathrm{ug} / \mathrm{mL}$

$\mathrm{RT}: 5.50 \mathrm{~min}$ Scan\# 328

Delta R.T. $\quad-0.01 \mathrm{~min}$

Lab File: NRA10C97.D

Acq: $07 / 15 / 97 \quad 13: 36$

Tgt Ion:108 Resp: 16964

Ion Ratio Lower Upper

108100

$\begin{array}{llll}107 & 119.8 & 99.8 & 149.8\end{array}$

$\begin{array}{rrrr}0 & 0.0 & 0.0 & 0.0\end{array}$

abundanceIon 108.00 (107.

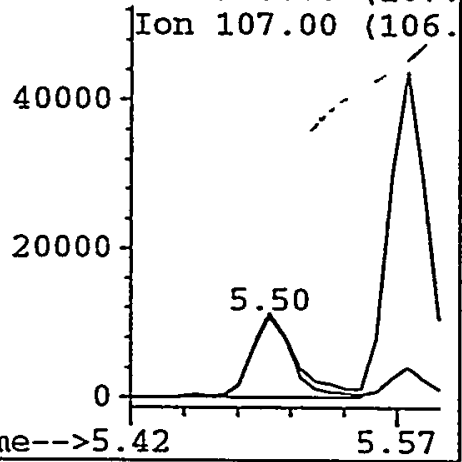

Time-->5.42

5.57

\#26

Acetophenone

Concen: $9.85 \mathrm{ug} / \mathrm{mL}$

RT: 5.54 min Scan\# 332

Delta R.T. $\quad 0.01 \mathrm{~min}$

Lab File: NRA10C97.D

Acq : $07 / 15 / 97 \quad 13: 36$

Tgt Ion:105 Resp: 251084

Ion Ratio Lower Upper

105100

$\begin{array}{llll}71 & 0.0 & 7.0 & 10.4 \#\end{array}$

$\begin{array}{llll}51 & 34.9 & 38.9 & 58.3 \#\end{array}$

$\begin{array}{llll}0 & 0.0 & 0.0 & 0.0\end{array}$

AbundanceIon 105.00 (104.

Ion $71.00 \quad(70.5$

$200000-5.54$

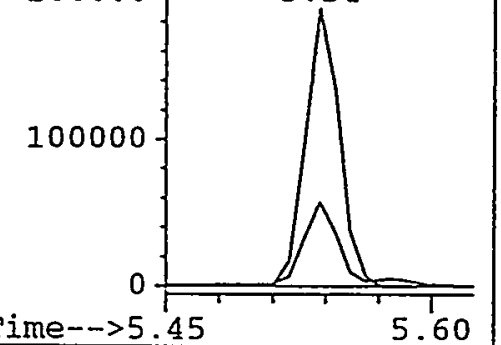




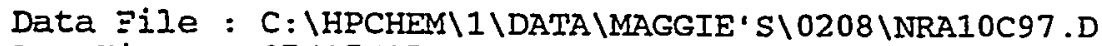

Acq Time : 07/15/97 13:36

Sample : 97002897

Misc : 7/1/97 WATER 97C-0208-02

Quant Time: Jul $1514: 031997$

Operator: TV

Inst : $5972-\mathrm{N}$

Multiplr: 1.00

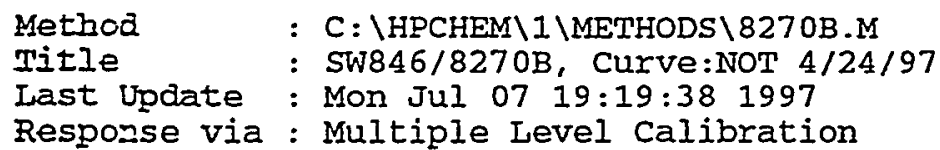

Method : C: \HPCHEM IIIMETHODS $18270 \mathrm{~B} . \mathrm{M}$

Title : SW846/8270B, Curve:NOT 4/24/97

Last Update : Mon Jul 07 19:19:38 1997

Response via : Multiple Level Calibration

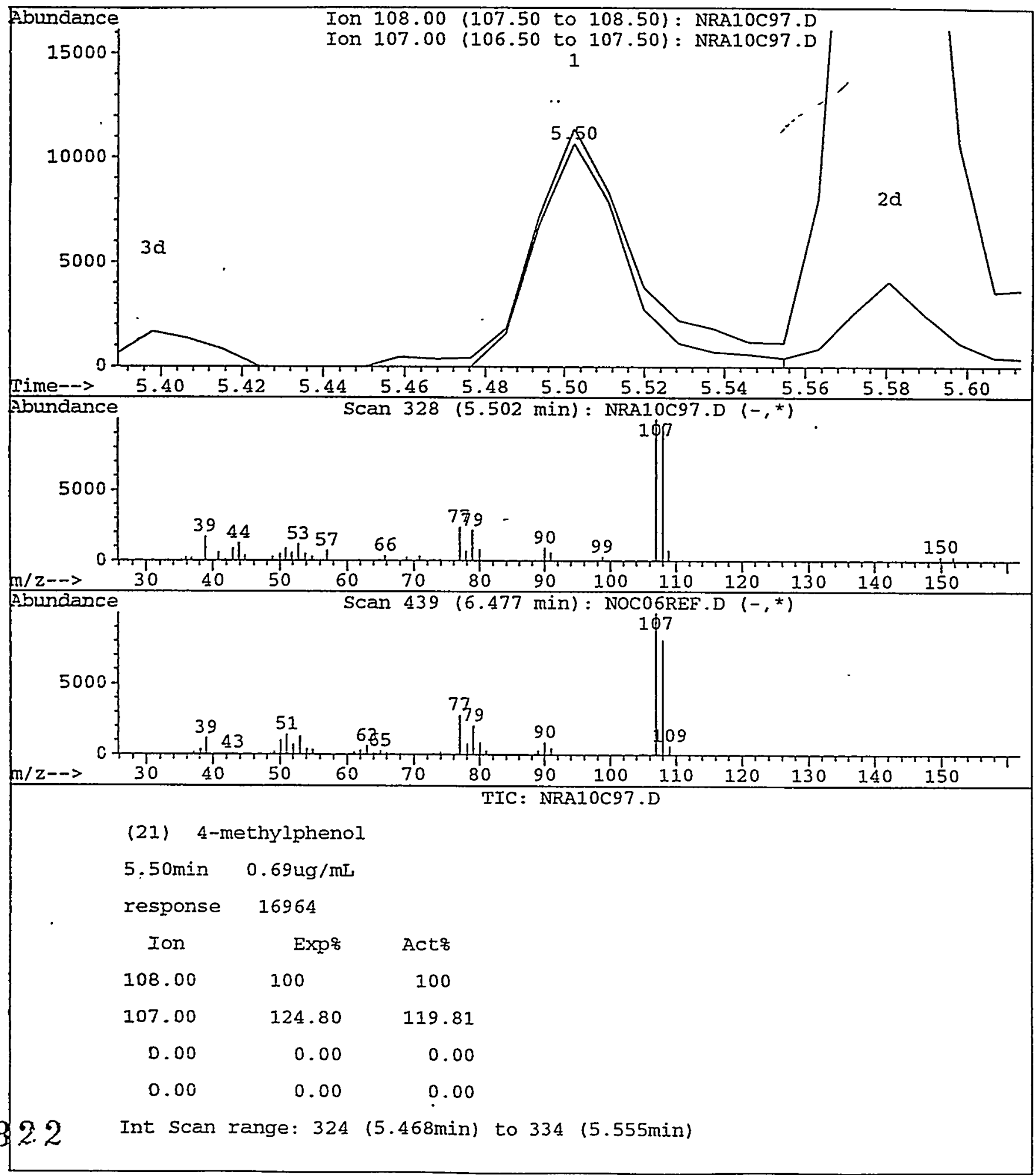




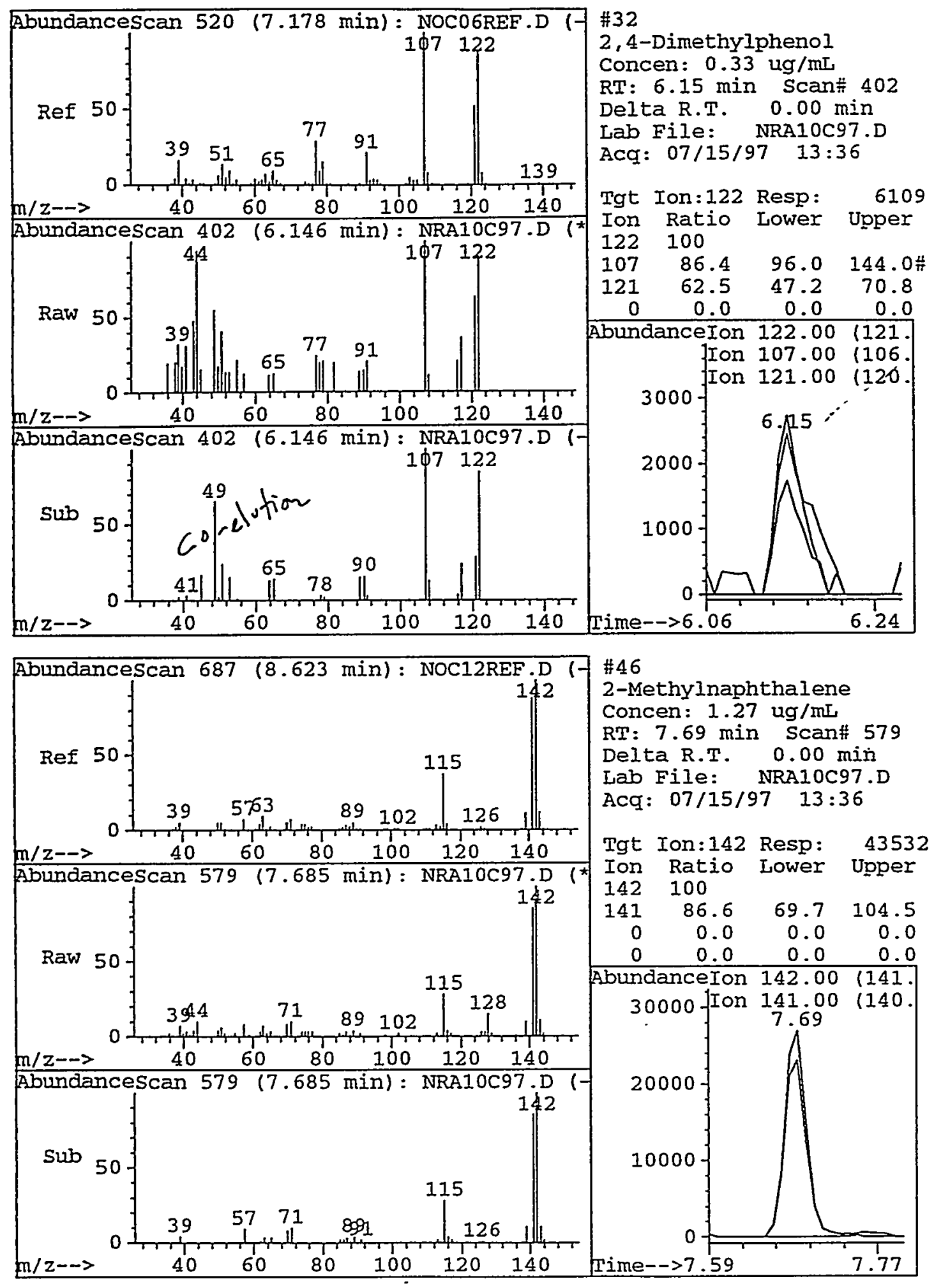

323 

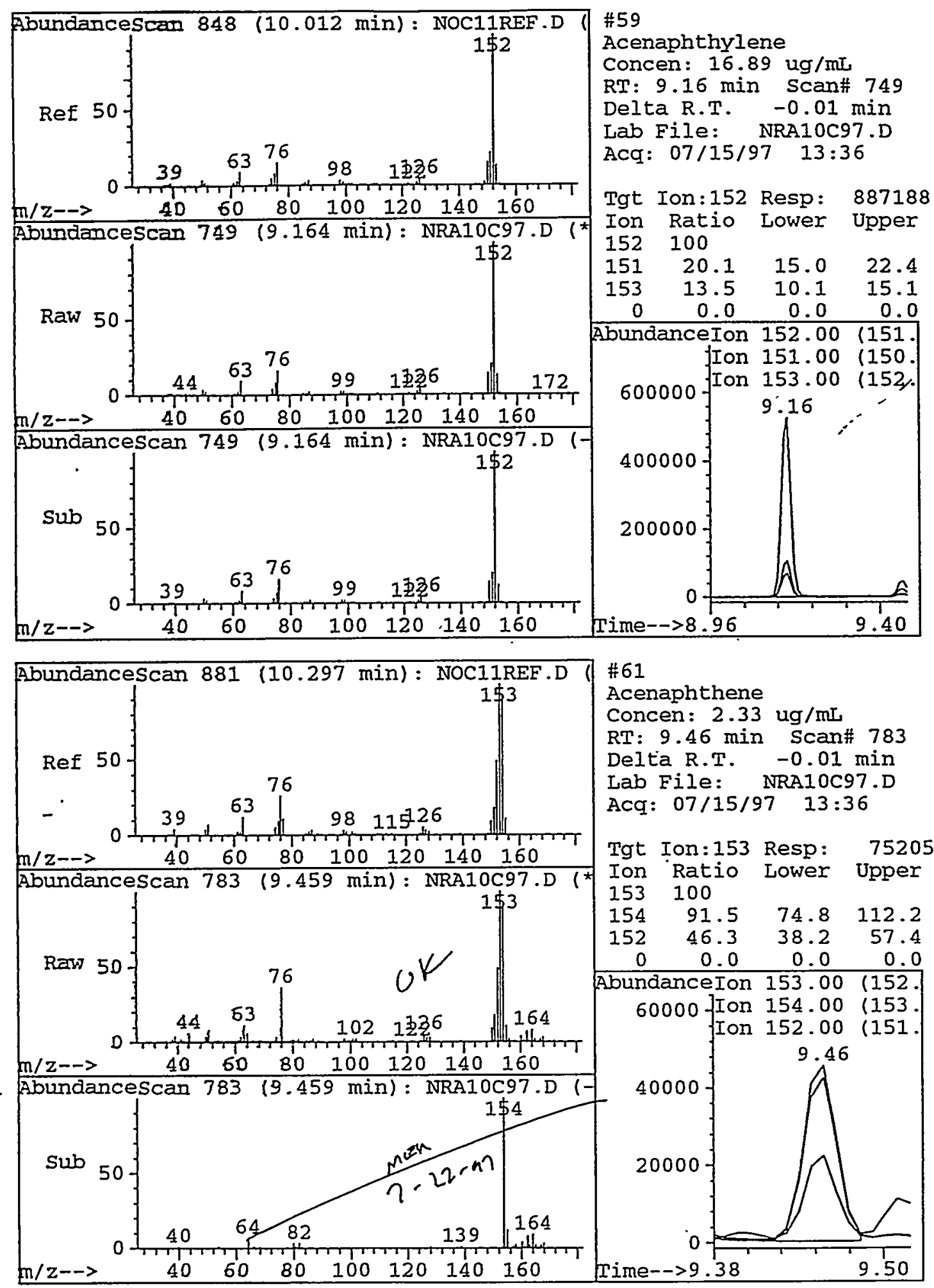

324 


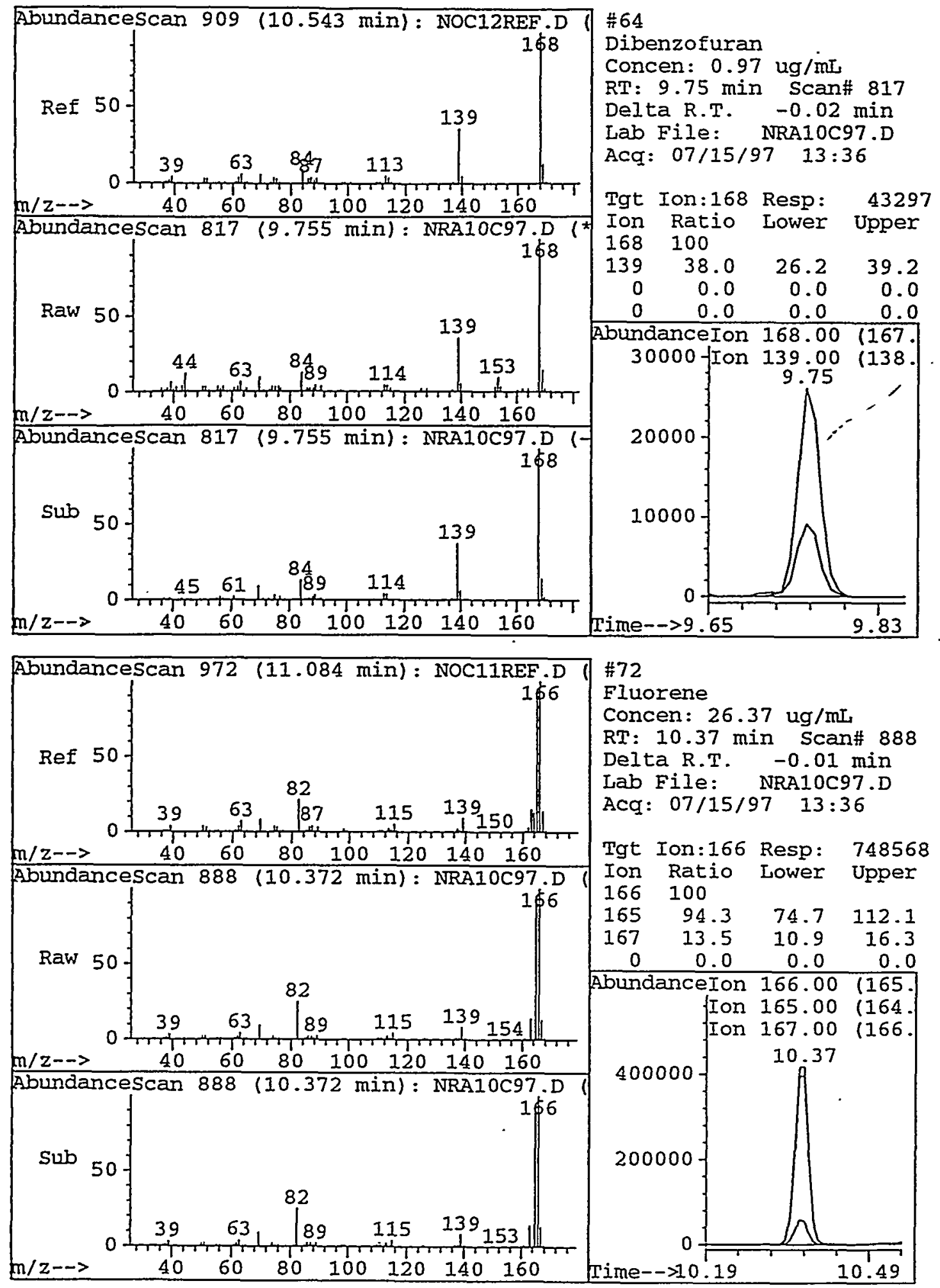




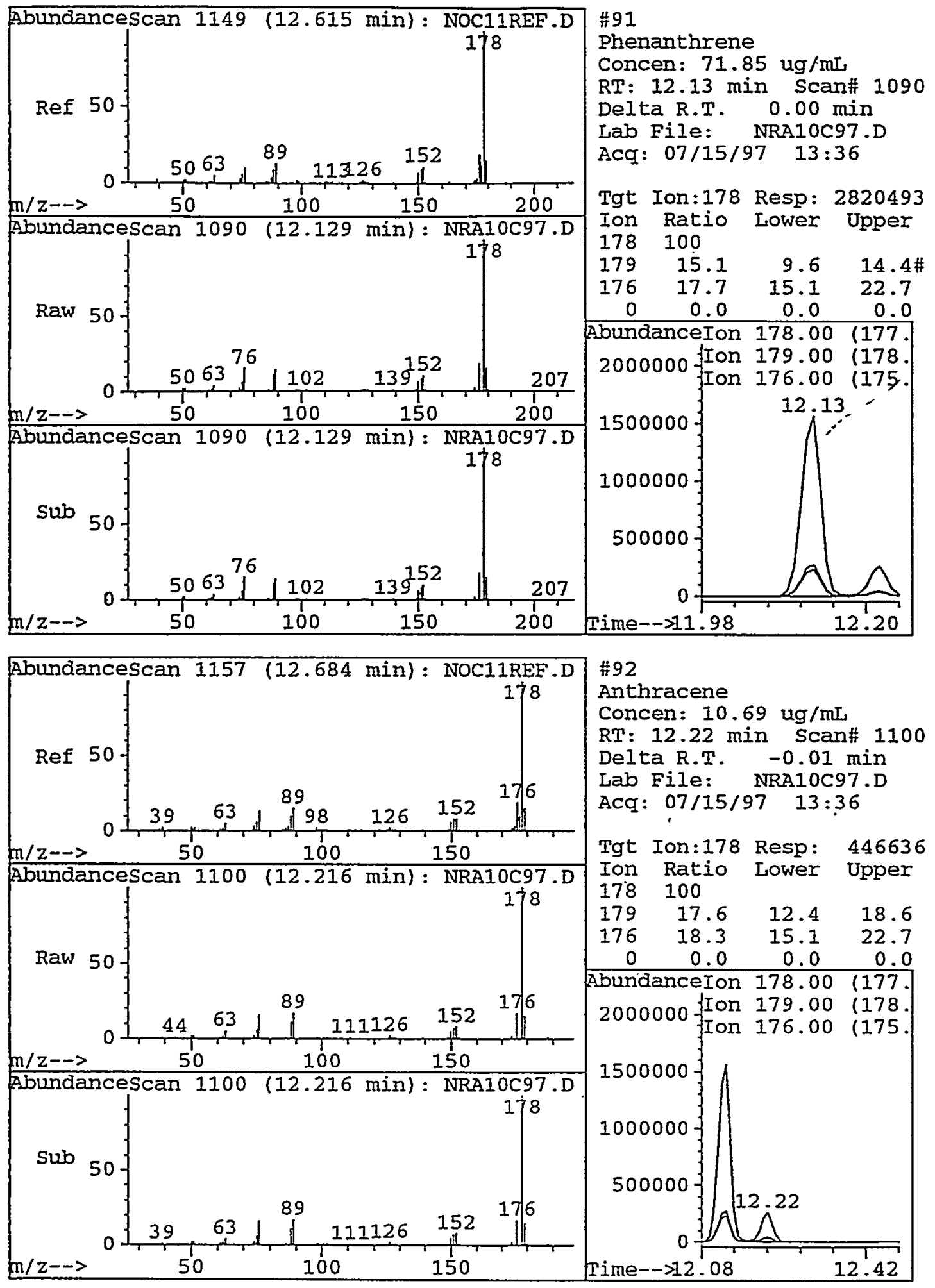

326 


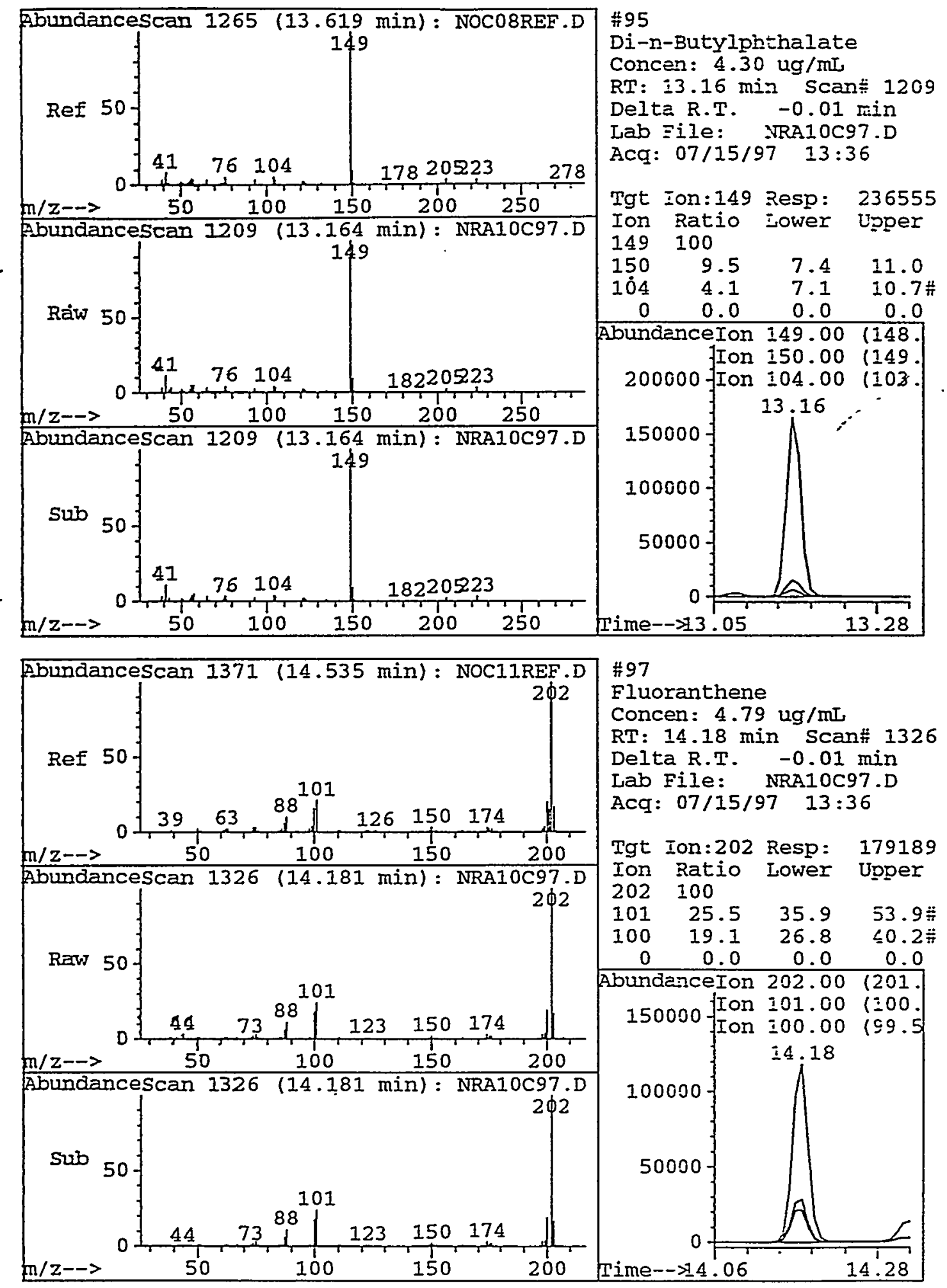




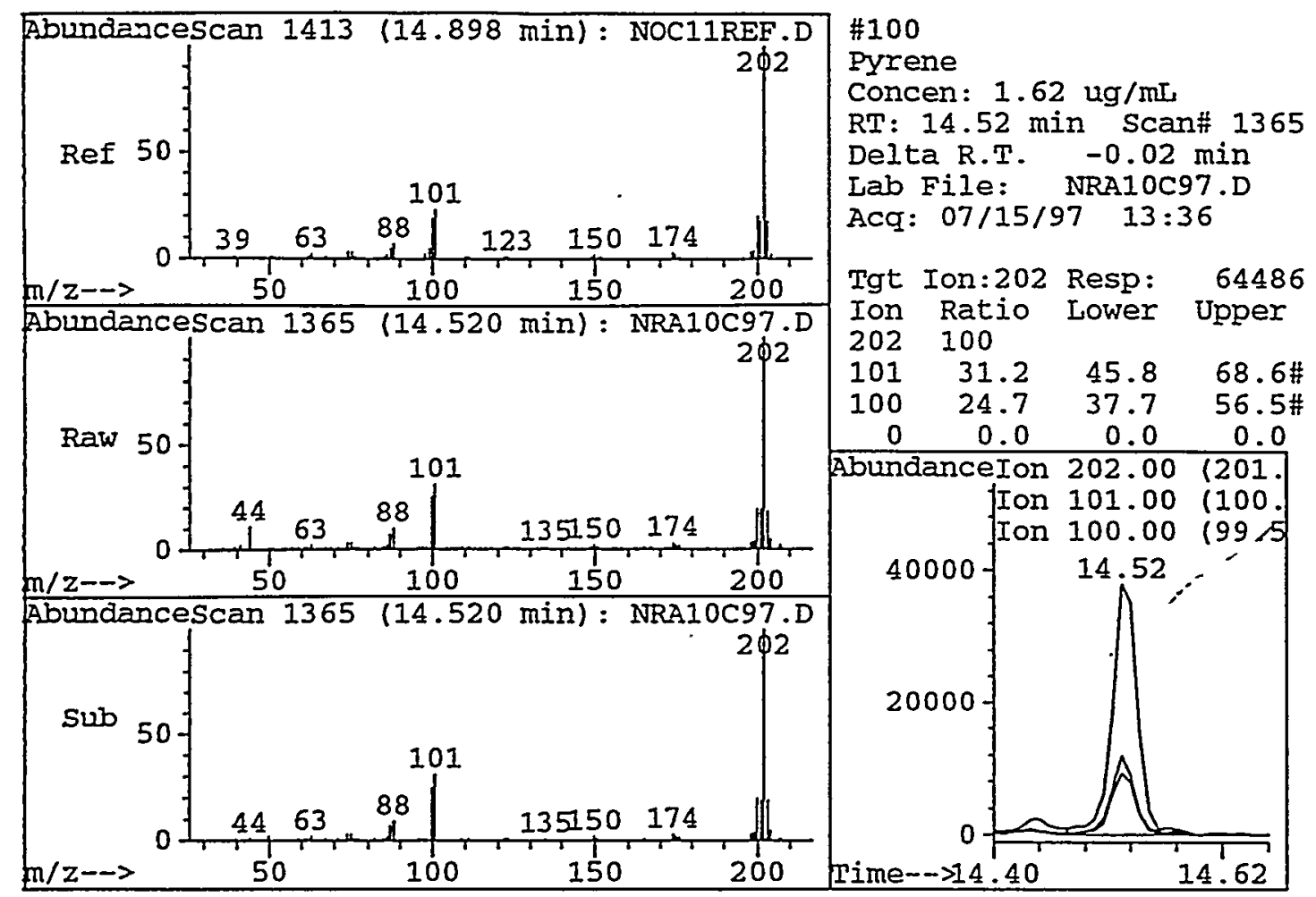




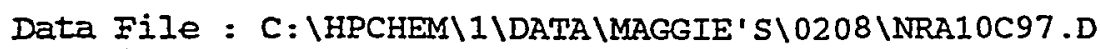

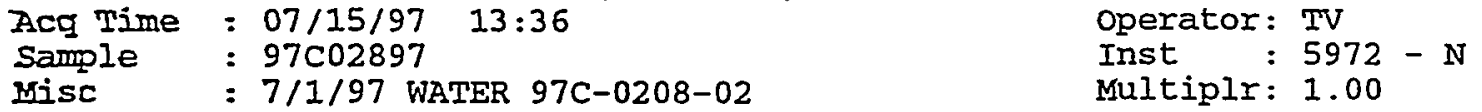

Method : $C: \backslash$ HPCHEM II IMETHODS $\backslash 8270 \mathrm{~B} . \mathrm{M}$

Title : SW846/8270B, Curve:NOT 4/24/97

I.jotary = C: IDATABASE \NBS75K.L

\begin{tabular}{|c|c|c|c|c|c|}
\hline R.T. & Cone & Area & Relative to & ISTD & ISTD Area \\
\hline 4.13 & $68.720 \mathrm{ug} / \mathrm{mL}$ & 6812473 & 1,4-Dichlorob & enzene-d4 & $3965340 \quad 53$ \\
\hline 五it开 of & 20 & Tentative ID & Ref\# & CAS\# & Qual \\
\hline $\begin{array}{ll}1 & \text { Bicycl } \\
2 & \text { Styren } \\
3 & 1,3,5, \\
4 & \text { Butane } \\
5 & 1,3,7\end{array}$ & $\begin{array}{l}\text { lo }[4.2 .0\} \text { octa- } \\
\text { ne } \\
\text { 7-cyclooctate } \\
\text { edioic acid, } \\
\text {-octatrien-5-y }\end{array}$ & $\begin{array}{l}\text {-1,3,5-triene } \\
\text { etraene } \\
\text { ohenyl- } \\
\text { me }\end{array}$ & $\begin{array}{r}1948 \\
1947 \\
1949 \\
21169 \\
1946\end{array}$ & $\begin{array}{l}000694-87-1 \\
000100-42-5 \\
000629-20-9 \\
000635-51-8 \\
016607-77-5\end{array}$ & $\begin{array}{l}90.00 \\
74.00 \\
94.00 \\
16.00 \\
12.00\end{array}$ \\
\hline
\end{tabular}

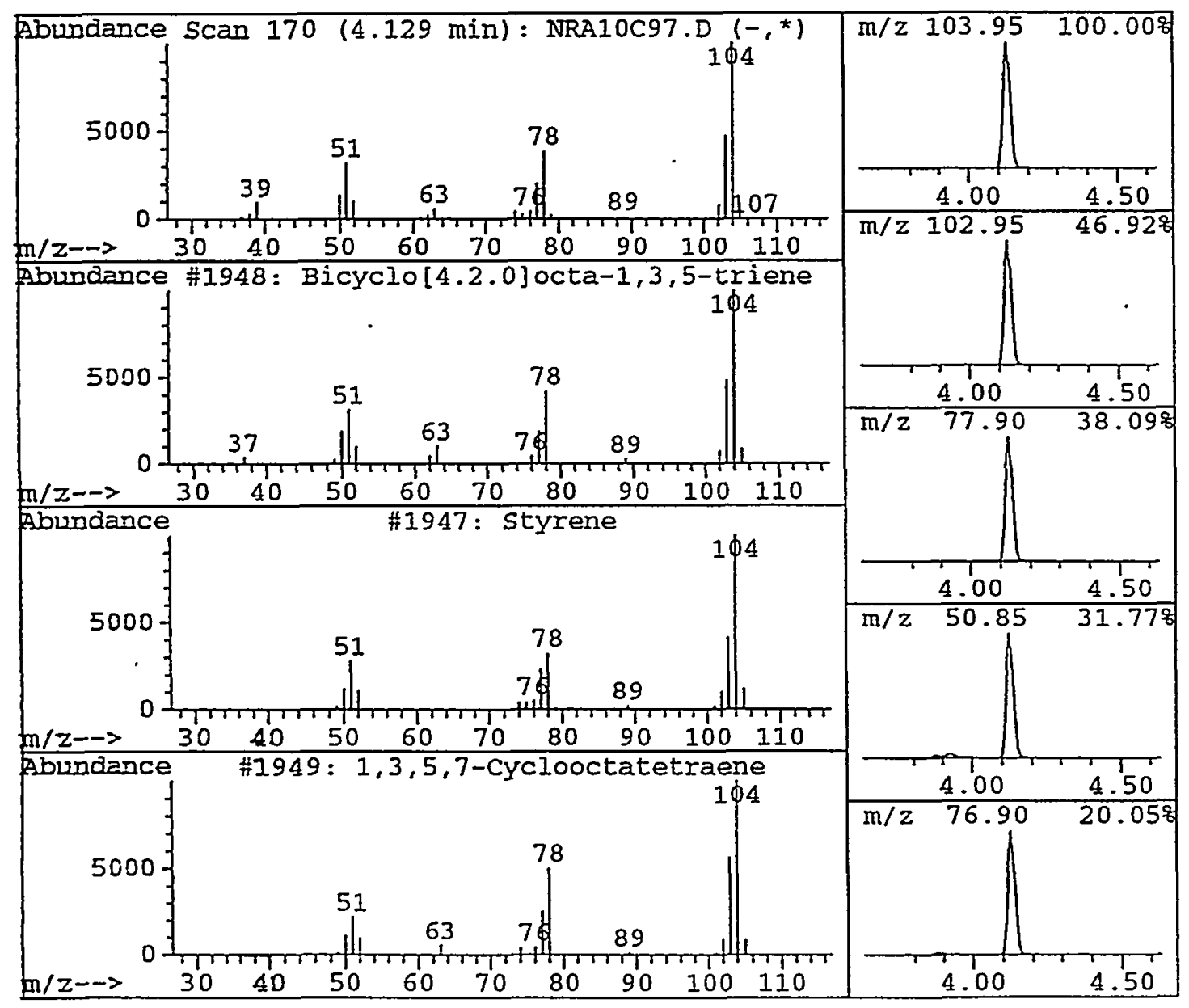

\section{9}



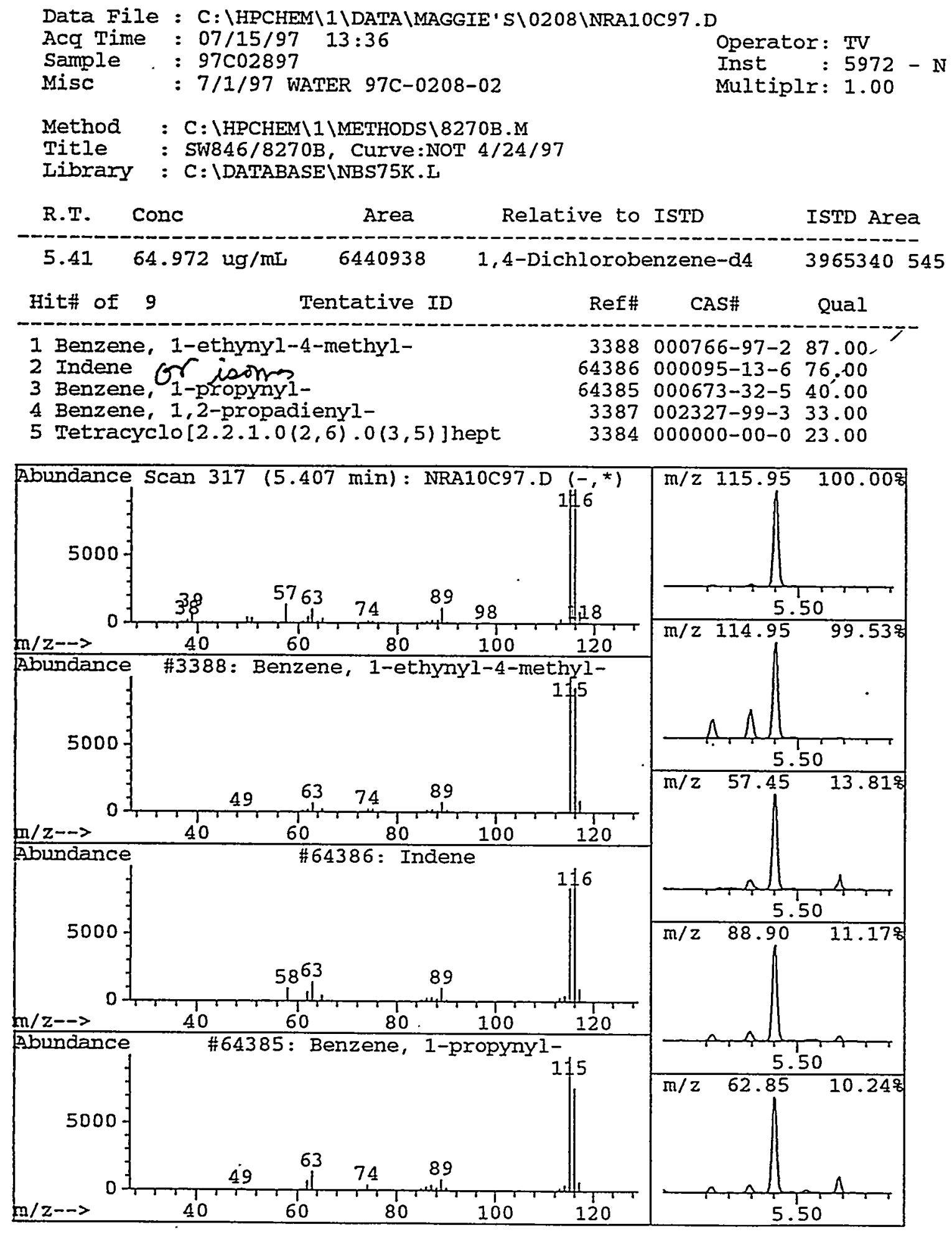

330 


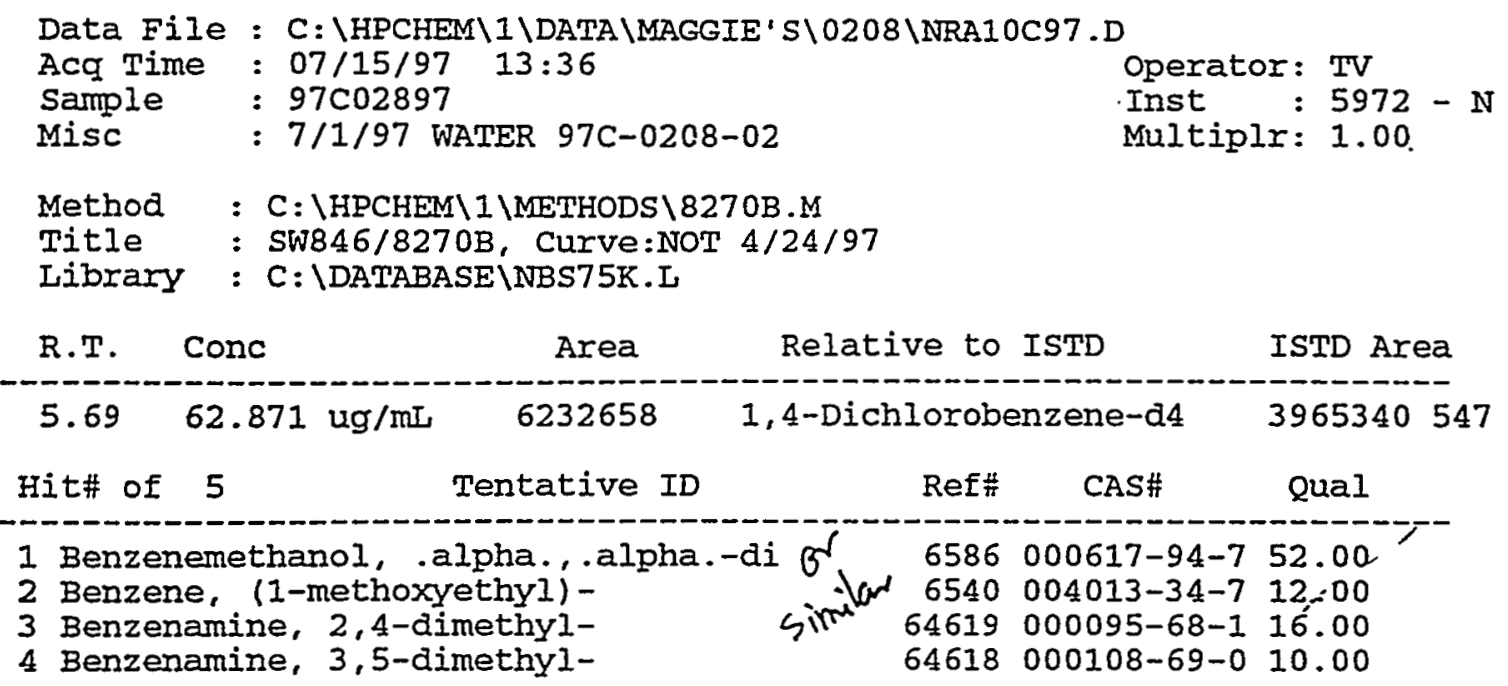

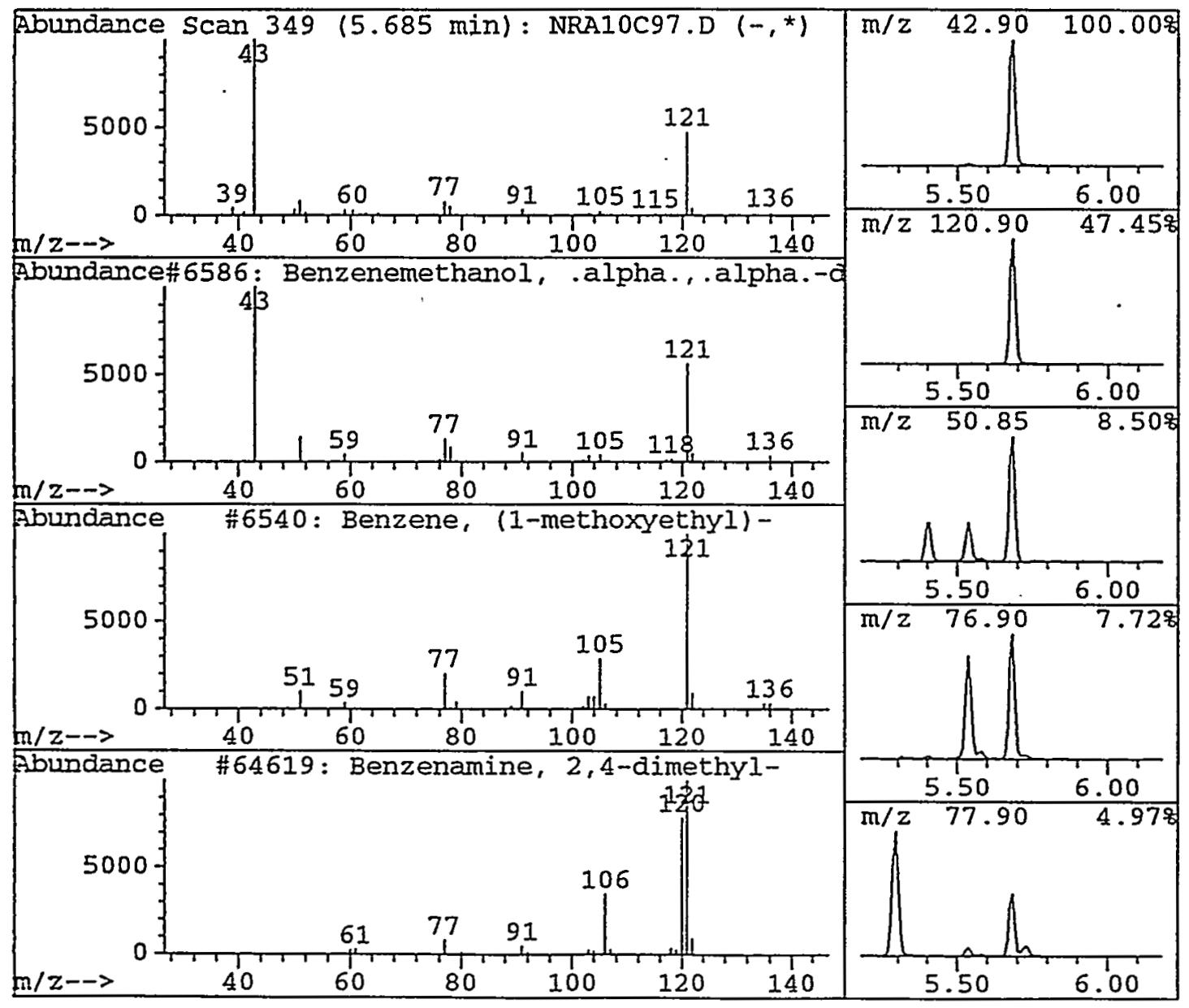




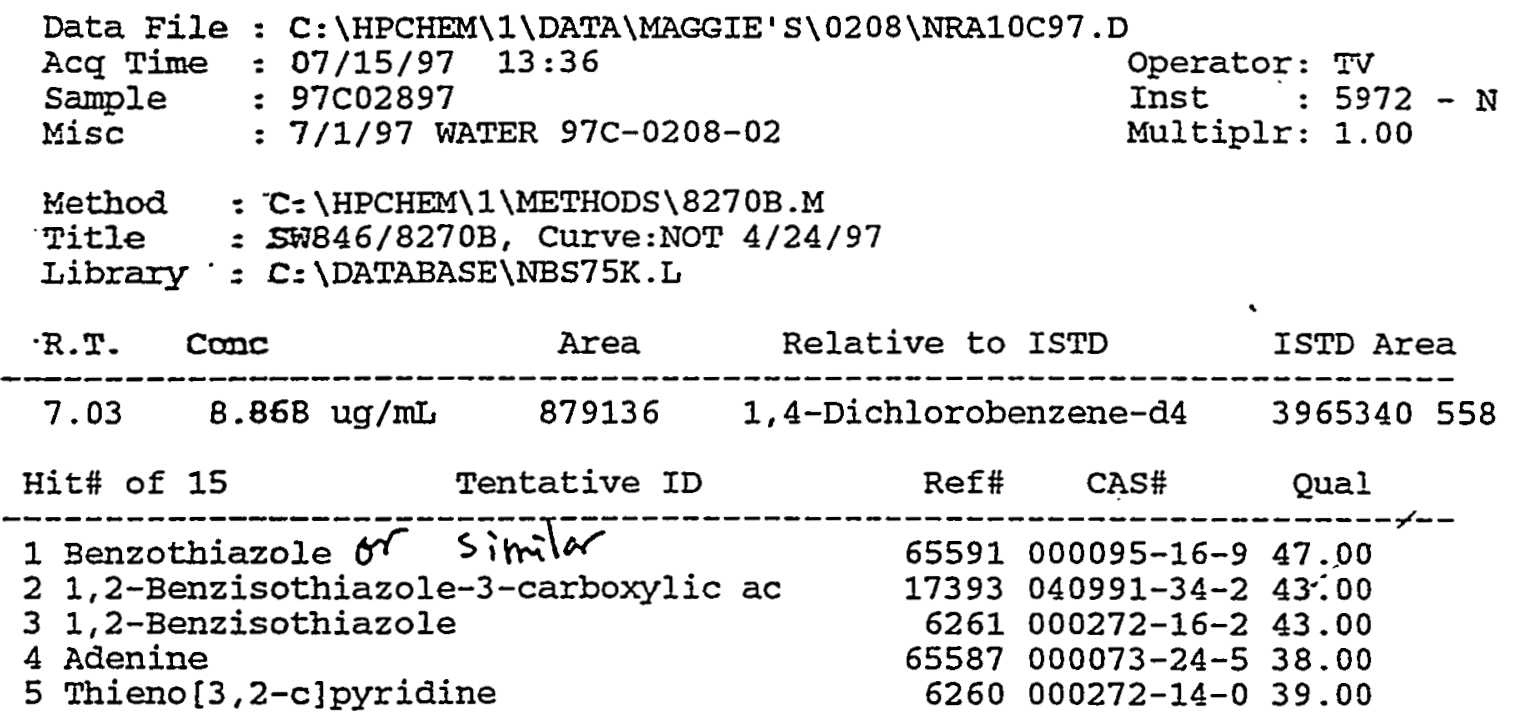

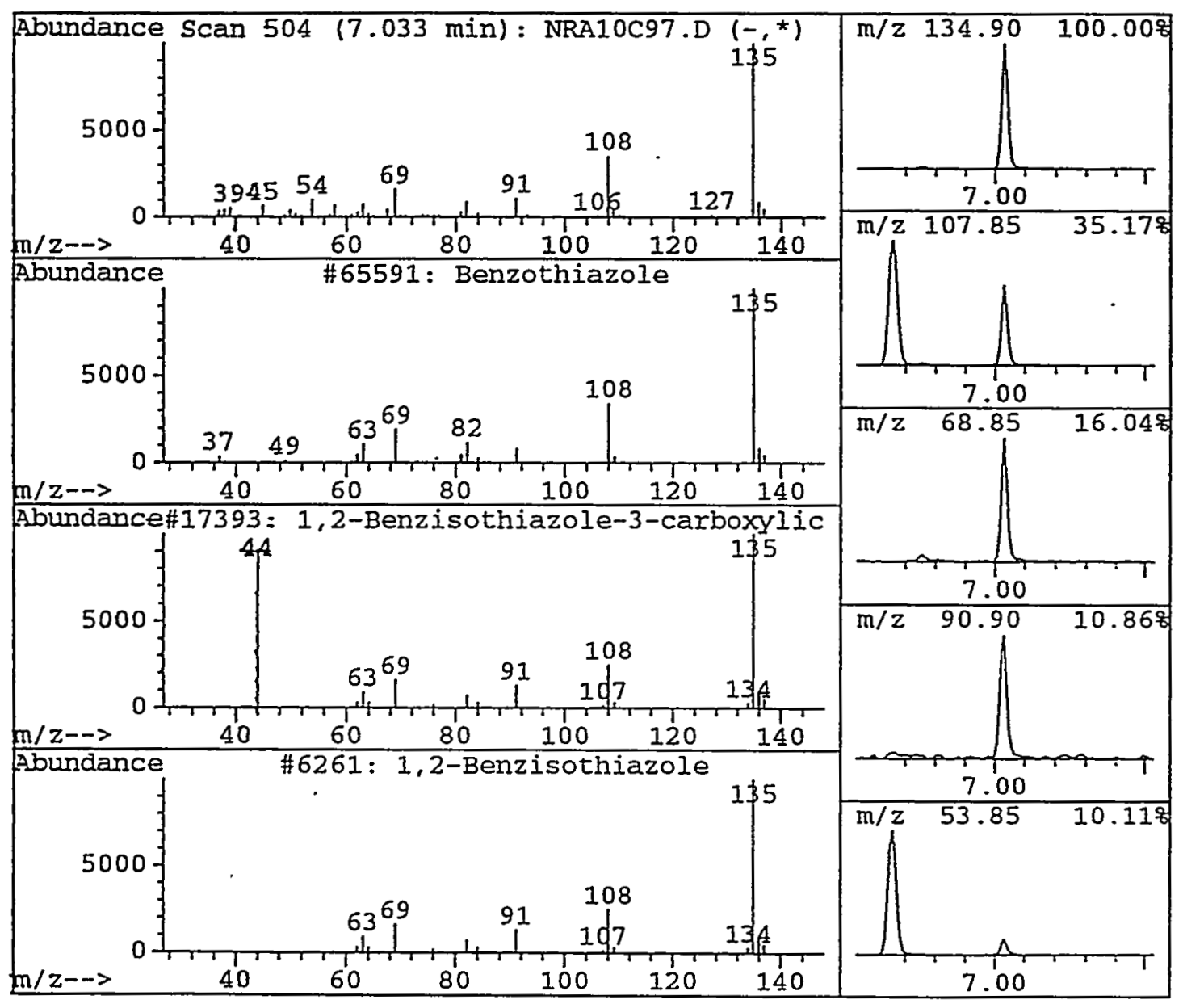




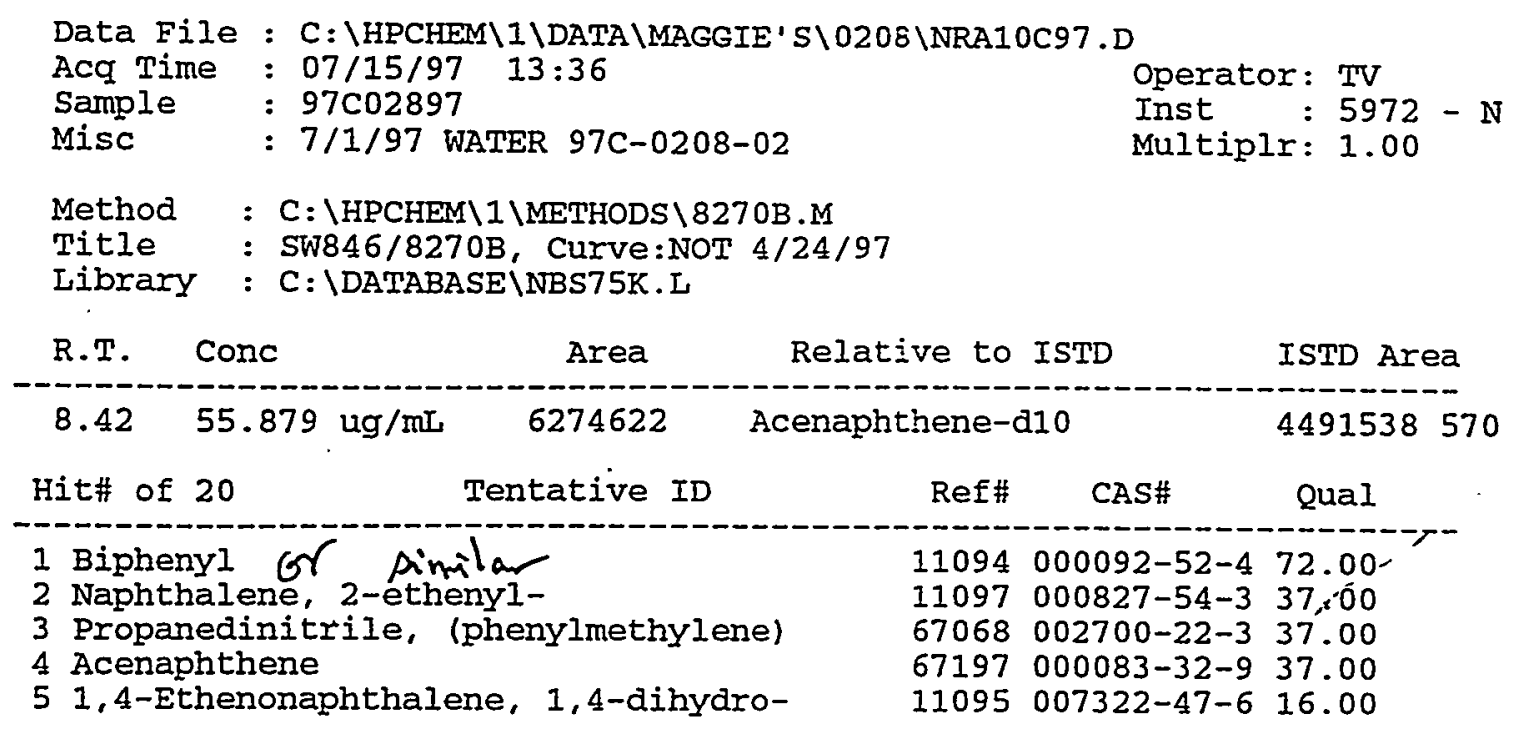

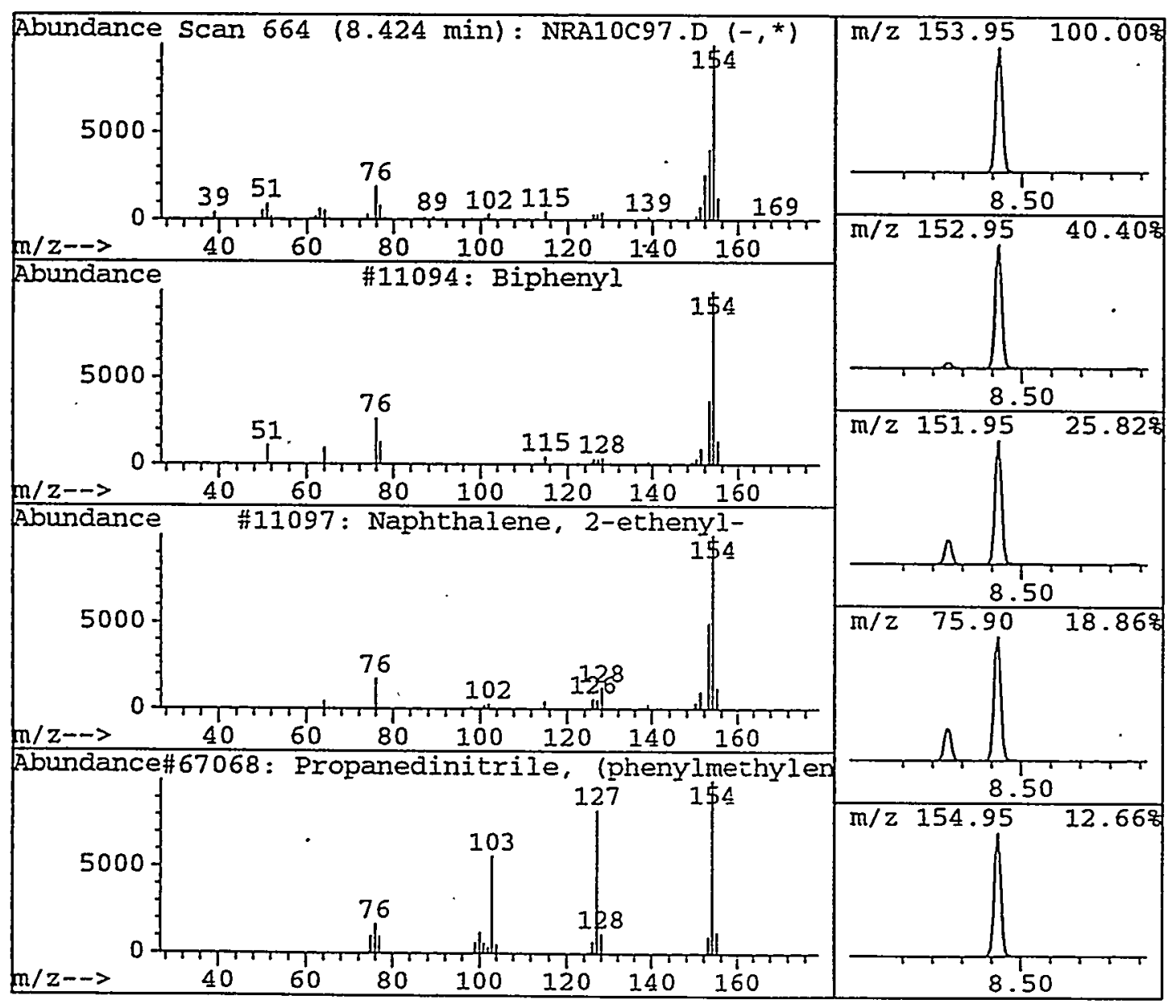




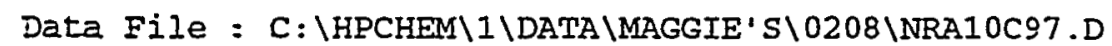

zcq Time : 07/15/97 13:36 Operator: TJ

Sample : 97c02897 Inst : $5972-\mathrm{N}$

Yisc : 7/1/97 WATER 97C-0208-02 Multiplr: 1.00

lethod : $C: \backslash$ HPCHEM 1 \METHODS $18270 \mathrm{~B} . \mathrm{M}$

mitle : SW846/8270B, Curve:NOT 4/24/97

Iibrary : $c:$ IDATABASE \NBS75K.L

\begin{tabular}{|c|c|c|c|c|c|}
\hline B.T. & conc & Area & \multicolumn{2}{|c|}{ Relative to ISTD } & ISTD Area \\
\hline 12.89 & $5.614 \mathrm{ug} / \mathrm{mL}$ & 659028 & \multicolumn{2}{|c|}{ Phenanthrene-d10 } & $4695 \div 30 \quad 59$ \\
\hline Hitt\# of & 20 & Tentative ID & Ref\# & CAS\# & Qual \\
\hline $\begin{array}{ll}1 & \text { Diben } \\
2 & 1,1 \\
3 & \text { Diben } \\
4 & 1,1 \\
5 & 2-\text { Dib }\end{array}$ & $\begin{array}{l}\text { Zothiophene } \\
\text { Biphenyl, 4-m } \\
\text { zothiophene, } \\
\text { Biphenyl, 2-m } \\
\text { enzofuranol }\end{array}$ & $\begin{array}{l}\text { re dinilar } \\
\text { ethoxy- } \\
\text { 5-oxide } \\
\text { ethoxy- }\end{array}$ & $\begin{array}{l}18918 \\
18974 \\
22912 \\
69015 \\
68975\end{array}$ & $\begin{array}{l}000132-65-0 \\
000613-37-6 \\
001013-23-6 \\
000086-26-0 \\
000086-77-1\end{array}$ & $\begin{array}{l}89.00 \\
36: 00 \\
10.00 \\
40.00 \\
38.00\end{array}$ \\
\hline
\end{tabular}

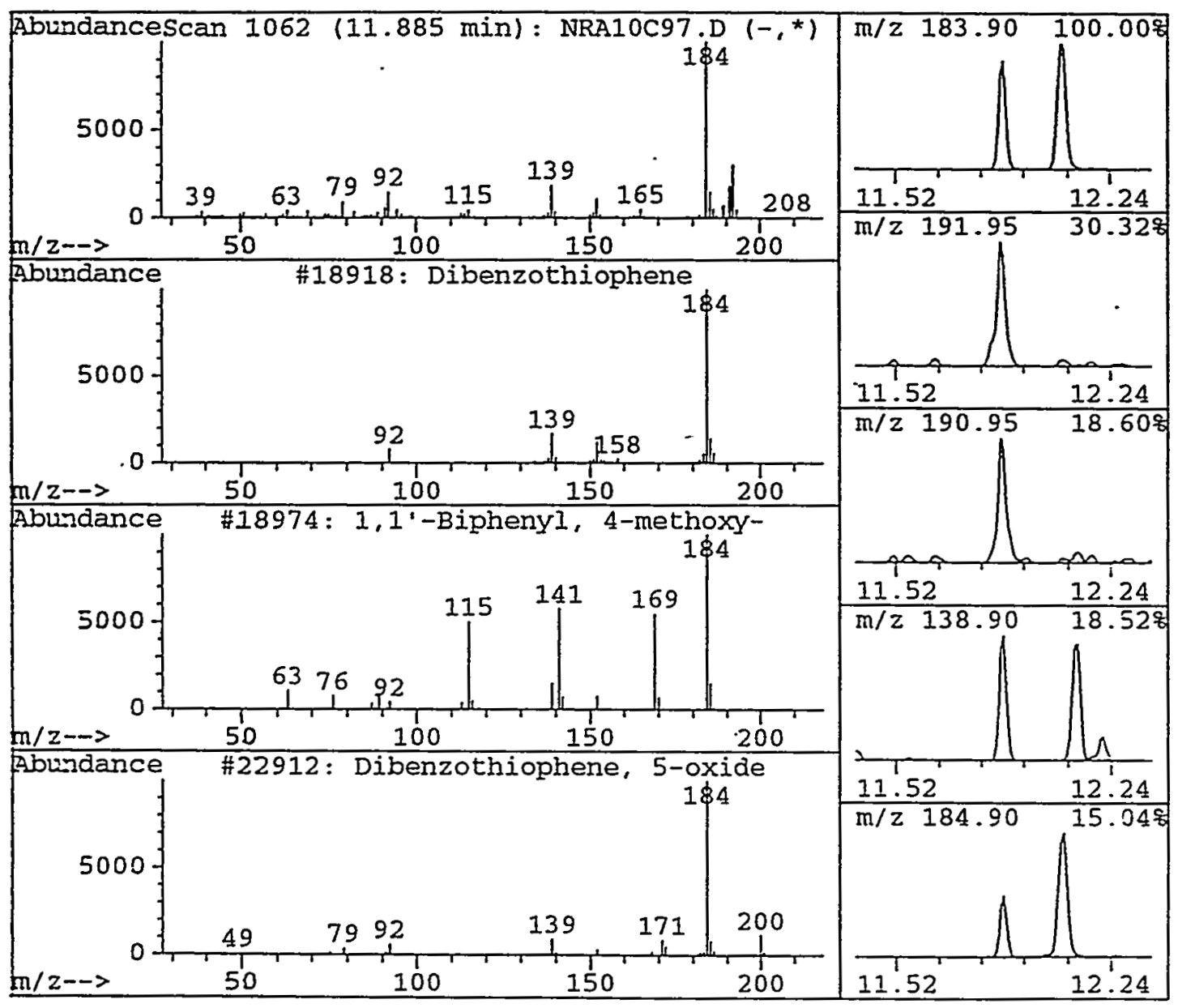



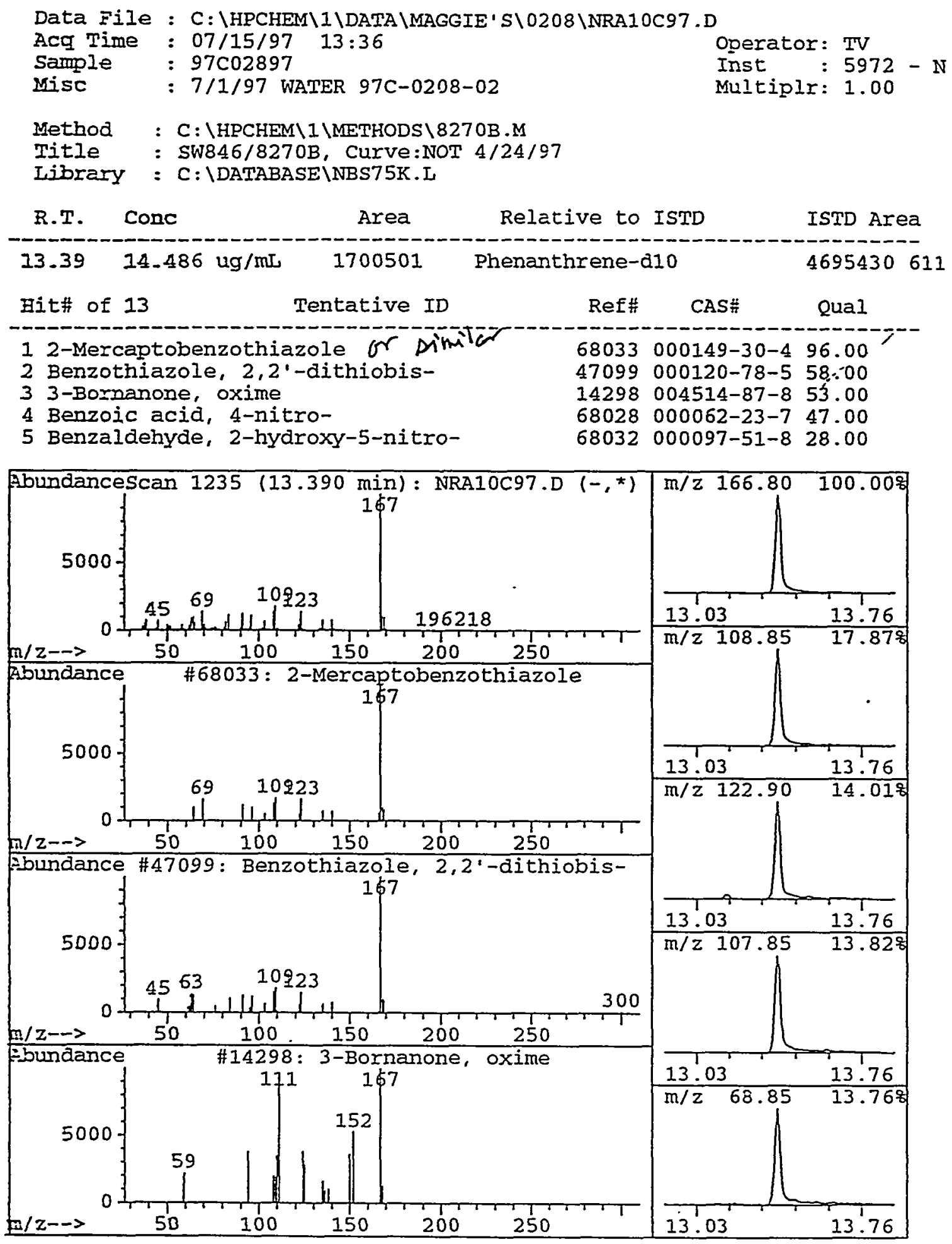

\section{5}




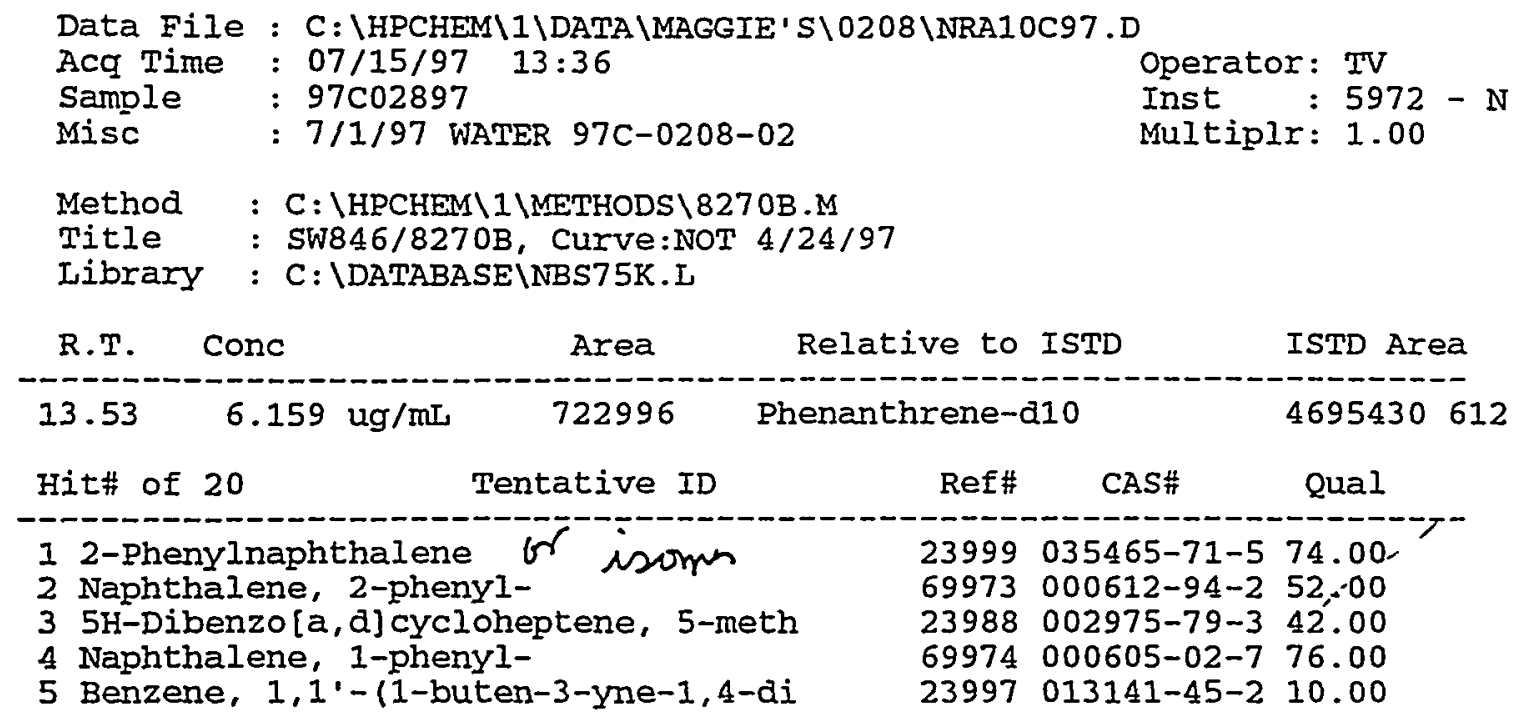

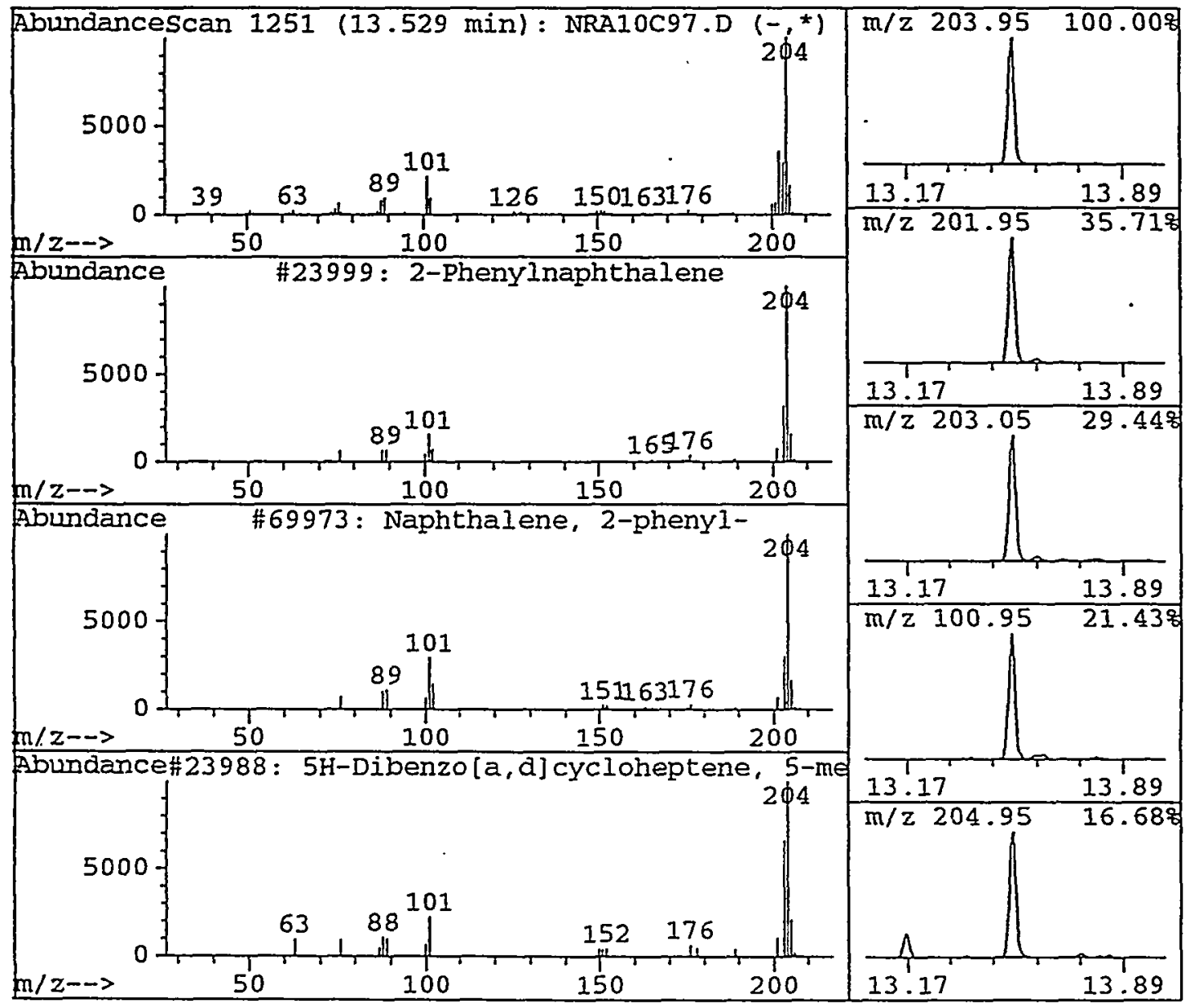




\section{PRELIMINARY QC SUMMARY REPORT}

File: NRB04C97.D

\section{Path: C:IHPCHEMI1IDATAl16JUL97NI 07/16/97 07:03 \\ Sample: 97C02897 \\ 7/1/97 WATER 97C-0208-02 1:10 DIL}

instrument 1D: $5972-\mathrm{N} \quad$ Analyst: TV

Initial Calibration 5.0 Std: NQFO4S5.D

Continuing standard: NRBO2S70.D

Internal standards:

1,4-Dichlorobenzene-d4

Naphthalene-d8

Acenaphthene-d10

Phenanthrene-d10

Chrysene-d12

Perylene-d12

$\begin{array}{lccc}\text { Area } & \text { Area-70 } & \text { \% \% area } & \\ 561377 & 540282 & 103.9 & \text { ok } \\ 2127248 & 2189544 & 97.2 & \text { ok } \\ 969064 & 983363 & 98.5 & \text { ok } \\ 1172298 & 1294075 & 90.6 & \text { ok } \\ 810832 & 819062 & 99.0 & \text { ok } \\ 580119 & 606707 & 95.6 & \text { ok } \\ \text { Pasted From: NRBO2S70.D } & & \end{array}$

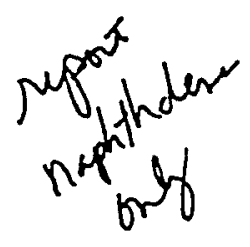

Matrix: (Water or Soil): WATER

Multiplier (normal water $=1$; soil $=1$ ): $\quad$.

Surrogates:

\begin{tabular}{|c|c|c|c|c|c|c|}
\hline & Quan & $\%$ Rec & Method & Warning & Control & \\
\hline 2-Fluorophenol & 3.803 & 3.8 & $21-100$ & $23-78$ & $10-91$ & * Low ** \\
\hline Phenol-d5 & 3.194 & 3.2 & $10-94$ & $9-73$ & $0-89$ & * Low * * \\
\hline Nitrobenzene-d5 & 8.198 & 8.2 & $35-114$ & $55-102$ & $43-114$ & * Low ** \\
\hline 2-Fluorobiphenyl & 7.591 & 7.6 & $43-116$ & $63-111$ & $51-124$ & $*$ Low ** \\
\hline 2,4,6-Tribromophenol & 3.329 & 3.3 & $10-123$ & $36-121$ & $14-142$ & * Low * * \\
\hline Terphenyl-d14 & 10.980 & 11.0 & $33-141$ & $55-124$ & $37-141$ & ** Low ** \\
\hline 2-Chlorophenol-d4 & 5.774 & 5.8 & $33-110$ & \multicolumn{3}{|c|}{ Advisory Use Only } \\
\hline 1,2-Dichlorobenzene-d4 & 6.783 & 6.8 & $16-110$ & \multicolumn{3}{|c|}{ Advisory Use Only } \\
\hline
\end{tabular}

Limits updated: 12/14/95 


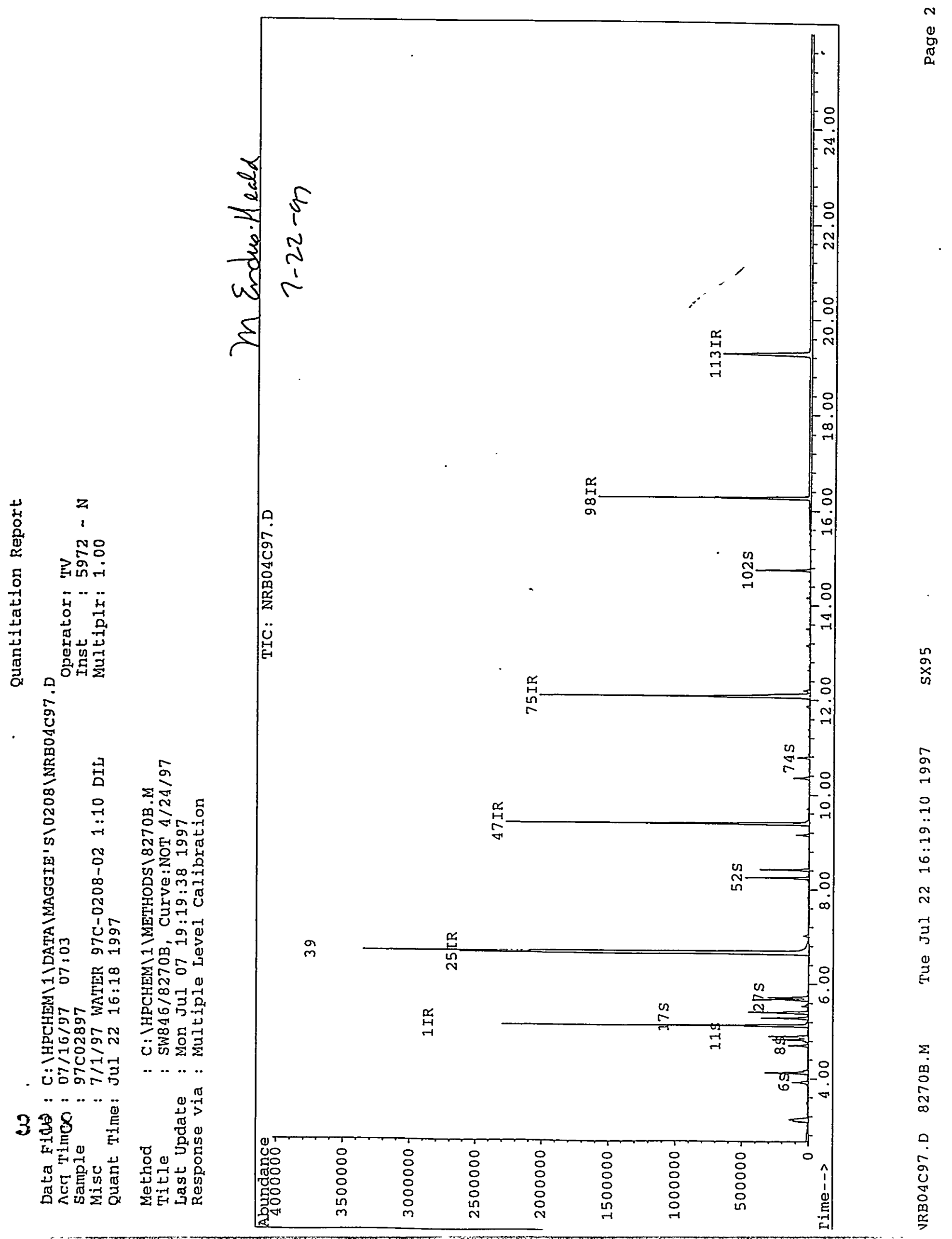


Data File : C: \HPCHEM $\backslash 1 \backslash$ DATA $\backslash$ MAGGIE'S\0208\NRB04C97.D

$\begin{array}{lll}\text { Acq Time } & : 07 / 16 / 97 \text { 07:03 } & \text { Operator: TV } \\ \text { Sample } & : 97 \text { C02897 } & \text { Inst } \\ \text { Misc } & : 7 / 1 / 97 \text { WATER 97C-0208-02 1:10 DIL } & \text { Multiplr: } 1.00\end{array}$

Quant Time: Jul 22 16:18 1997

Method : C: \HPCHEM $\backslash 1 \backslash M E T H O D S \backslash 8270 B . M$

Title : SW846/8270B, Curve:NOT $4 / 24 / 97$

last Update : Mon Jul o7 19:19:38 1997

Response via : Multiple Level Calibration

Internal standards

R.T. Scan QIon Response Conc Units Areas

1) $1,4-\mathrm{Dichlorobenzene-d4}$
47) Naphthalene-d8
75) Phenaphthene-d10
98) Chrysene-d12
113) Perylene-d12

System Monitoring Compounds

6) 2-Fluorophenol

8) Phenol-a5

11) 2-Chlorophenol-d4

17) 1,2-Dichlorobenzene-d4

27) Nitrobenzene-as

52) 2-Fluorobiphenyl

74) 2,4,6-Tribromophenol

102) Terphenyl-d14.

Target Compounds

39) Naphthalene

$\begin{array}{rrrrrr}5.12 & 284 & 152 & 561377 & 40.00 \mathrm{ug} / \mathrm{mL} & 0.00 \\ 6.64 & 459 & 136 & 2127248 & 40.00 \mathrm{ug} / \mathrm{mL} & -0.02 \\ 9.40 & 776 & 164 & 969064 & 40.00 \mathrm{ug} / \mathrm{mL} & -0.02 \\ 12.07 & 1083 & 188 & 1172298 & 40.00 \mathrm{ug} / \mathrm{mL} & -0.03 \\ 16.28 & 1567 & 240 & 810832 & 40.00 \mathrm{ug} / \mathrm{mL} & -0.03 \\ 19.28 & 1912 & 264 & 580119 & 40.00 \mathrm{ug} / \mathrm{mL} & -0.03\end{array}$

$\begin{array}{llll}3.93 & 147 & 112 & 74070\end{array}$

$\begin{array}{llll}4.70 & 236 & 99 & 77191\end{array}$

$4.89 \quad 258 \quad 132 \quad 135059$

$\begin{array}{llll}5.28 & 303 & 152 & 86764\end{array}$

$\begin{array}{llll}5.72 & 353 & 82 & 145073\end{array}$

$8.24 \quad 643 \quad 172 \quad 237501$

$10.79 \quad 936 \cdot 330 \quad 11539$

$14.75 \quad 1392 \quad 244 \quad 211492$

\& Recovery

\section{$3.803 \mathrm{ug} / \mathrm{mL} \quad 3.80 \%$}

$3.194 \mathrm{ug} / \mathrm{mL} \quad 3.198$

$5.774 \mathrm{ug} / \mathrm{m} \quad 5.778$

$6.783 \mathrm{ug} / \mathrm{mL} \quad 6.78 \%$

$8.198 \mathrm{ug} / \mathrm{mL} \quad 8.208$

$7.591 \mathrm{ug} / \mathrm{mL} \quad 7.598 \#$

$3.329 \mathrm{ug} / \mathrm{mL} \quad 3.338$

$10.980 \mathrm{ug} / \mathrm{mL} \quad 10.988$

$\begin{array}{llll}6.68 & 463 & 128 & 3075458\end{array}$

$54.181 \mathrm{ug} / \mathrm{mr}$ Qvalue 


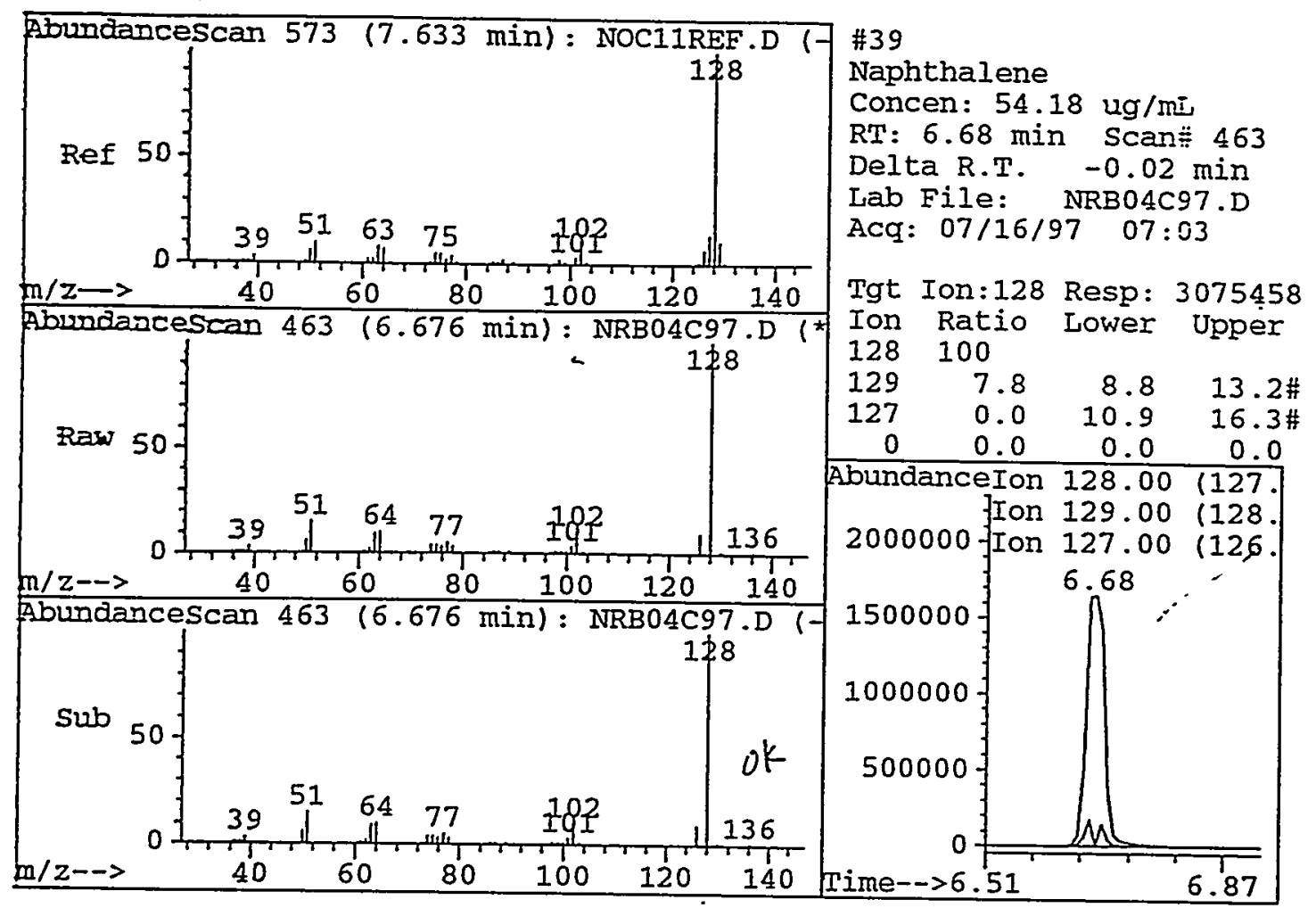


File: NRA11C98.D

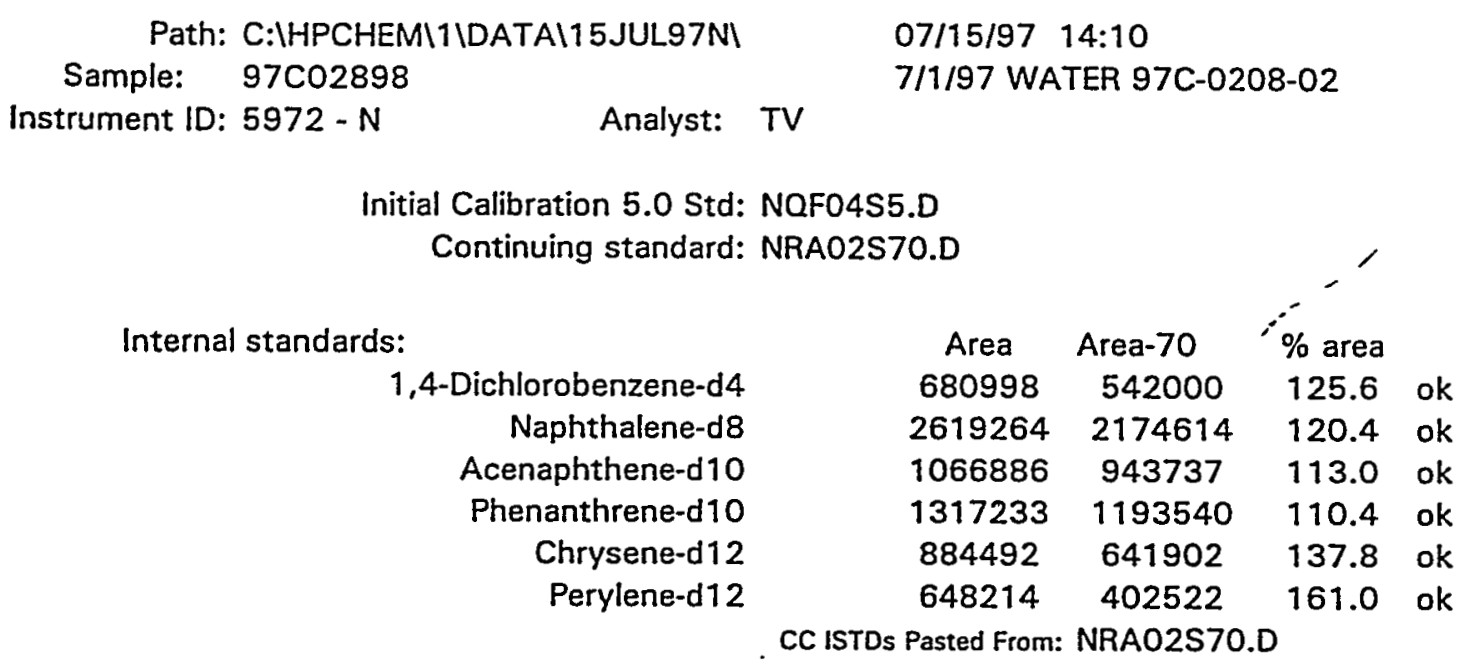

Matrix: (Water or Soill): WATER

Multiplier (normal water $=1$; soil $=1$ ): $\quad$.

$\begin{array}{rccccc}\text { Surrogates: } & & & & & \\ & \text { Quan } & \% \text { Rec } & \text { Method } & \text { Warning } & \text { Control } \\ \text { 2-Fluorophenol } & 34.189 & 34.2 & 21-100 & 23-78 & 10-91 \\ \text { Phenol-d5 } & 36.886 & 36.9 & 10-94 & 9-73 & 0-89 \\ \text { Nitrobenzene-d5 } & 89.095 & 89.1 & 35-114 & 55-102 & 43-114 \\ \text { 2-Fluorobiphenyl } & 87.033 & 87.0 & 43-116 & 63-111 & 51-124 \\ \text { 2,4,6-Tribromophenol } & 39.170 & 39.2 & 10-123 & 36-121 & 14-142 \\ \text { Terphenyl-d14 } & 99.767 & 99.8 & 33-141 & 55-124 & 37-141 \\ \text { 2-Chlorophenol-d4 } & 58.941 & 58.9 & 33-110 & \text { Advisory Use Only } \\ \text { 1,2-Dichlorobenzene-d4 } & 74.283 & 74.3 & 16-110 & & \text { Advisory Use Only }\end{array}$

Limits updated: $12 / 14 / 95$

341 


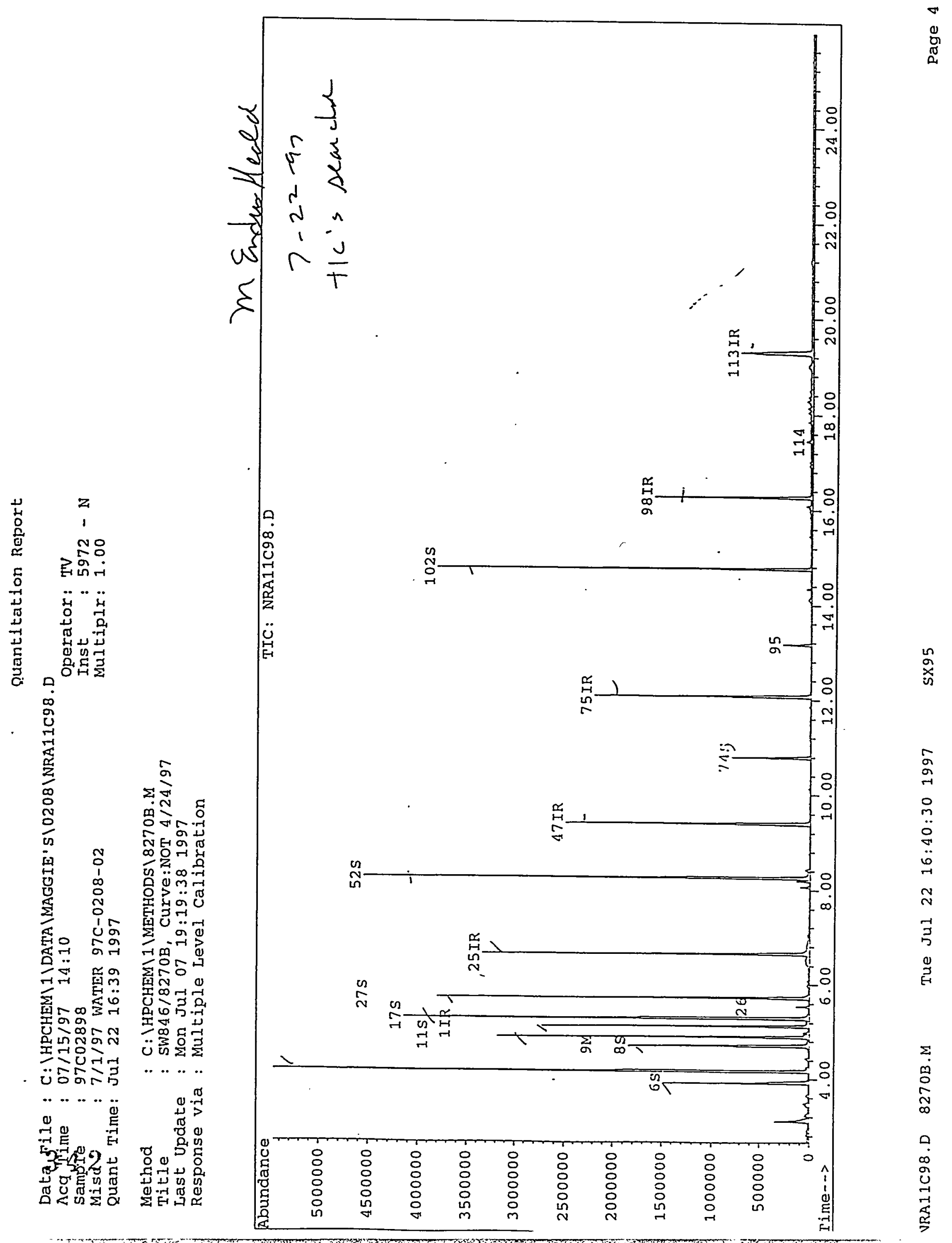




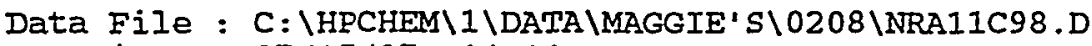

Aca Time : 07/15/97 14:10 Operator: TV

Sample : 97C02898 Inst : $5972-\mathrm{N}$

Misc : 7/1/97 WATER 97C-0208-02 Multiplr: 1.00

Quant Time: Jul 22 16:39 1997

Method : C: \HPCHEM IIMETHODS $\backslash 8270 \mathrm{~B} . \mathrm{M}$

Title : SW846/8270B, Curve:NOT 4/24/97

Last Update : Mon JuI 07 19:19:38 1997

Response via : Multiple Level Calibration

Internal standards

1) 1,4-Dichlorobenzene-d4

25) Naphthalene-d8

47) Acenaphthene-d10

75) Phenanthrene-d10

98) Chrysene-d12

113) Perylene-d12

System Monitoring compounds

6) 2-Fluorophenol

B) Phenol-d5

11) 2-Chlorophenol-a4

17) 1,2-Dichlorobenzene-d4

27) Nitrobenzene-d5

52) 2-Fluorobiphenyl

74) $2,4,6$-Tribromophenol

102) Terphenyl-d14

Target compounds

2) Pyridine

3) N-nitrosodimethylamine

4) 2-Picoline

5) Methyl methanesulfonate

7) Ethyl methanesulfonate

9) Phenol

10) Aniline

12) 2-Chlorophenol

13) Decane NOT Present

14) 1,3-Dichlorobenzene

15) 1,4-dichlorobenzene

16) Benzyl alcohol

18) 1,2-dichlorobenzene

19) 2-Methylphenol

20) Bis (2-chloroisopropyl) ethe

21) 4-methylphenol

22) N-Nitrosodi-n-propyl amine

23) Hexachloroethane

24) Bis (2-chloroethyl)ether

26) Acetophenone

28) Nitrobenzene

29) N-nitrosopiperidine

30) Isophorone

31) 2-Nitrophenol

32) 2,4-Dimethylphenol

$33)$ Benzoic acid

34) Bis (2-chloroethoxy) methane

35) 2,4-Dichlorophenol

36) A. A.-dimethylphenethylami

37) 1,2,4-Trichlorobenzene

38) Dodecane NOT Present

39) Naphthelene

40) 4-Chloroaniline

41) 2,6-Dichlorophenol

42) Hexachlorobutadiene

43) Benzothiazole NOT Present

44) N-nitroso-di-n-butylamine
R.T. Scan QIon Response Conc Units

Areas

$\begin{array}{rrrrrr}5.13 & 285 & 152 & 680998 & 40.00 \mathrm{ug} / \mathrm{mL} & 0.00 \\ 6.65 & 460 & 136 & 2619264 & 40.00 \mathrm{ug} / \mathrm{mL} & 0.00 \\ 9.40 & 776 & 164 & 1066886 & 40.00 \mathrm{ug} / \mathrm{mL} & 0.00 \\ 12.08 & 1084 & 188 & 1317233 & 40.00 \mathrm{ug} / \mathrm{mL}, & 0.00 \\ 16.29 & 1568 & 240 & 884492 & 40.00 \mathrm{ug} / \mathrm{mL} & 0.00 \\ 19.30 & 1914 & 264 & 648214 & 40.00 \mathrm{ug} / \mathrm{mL} & 0.00\end{array}$

$\begin{array}{rrrr}3.92 & 146 & 112 & 783323 \\ 4.70 & 236 & 99 & 1030147 \\ 4.90 & 259 & 132 & 1531874 \\ 5.29 & 304 & 152 & 1017175 \\ 5.73 & 354 & 82 & 1793904 \\ 8.26 & 645 & 172 & 2642244 \\ 10.80 & 937 & 330 & 145882 \\ 14.76 & 1393 & 244 & 1997157\end{array}$

$34.189 \mathrm{ug} / \mathrm{mr}$

\&Recovery

$36.886 \mathrm{ug} / \mathrm{mL} \quad 36.89 \%$

$58.94 \mathrm{lug} / \mathrm{mL} \quad 58.948$

$74.283 \mathrm{ug} / \mathrm{mL} \quad 74.288$

$89.095 \mathrm{ug} / \mathrm{mL} \quad 89.098$

$87.033 \mathrm{ug} / \mathrm{mL} \quad 87.038$

$39.170 \mathrm{ug} / \mathrm{mL} \quad 39.17 \%$

$99.767 \mathrm{ug} / \mathrm{mL}$

$99.77 \%$

Not Detected

Not Detected

Not Detected

Not Detected

Not Detected

0.00

0.00

4.72

$\begin{array}{lll}238 & 94 & 73427\end{array}$

$2.361 \mathrm{ug} / \mathrm{mL}$

Not Detected

Not Detected

Not Detected

Not Detected

Not Detected

Not Detected

Not Detected

Not Detected

Not Detected

Not Detected

Not Detected

Not Detected

Not Detected

0.00

0.00

5.54

$\begin{array}{lll}332 & 105 & 56619\end{array}$

$1.838 \mathrm{ug} / \mathrm{mL} \#$

Not Detected

Not Detected

Not Detected

Not Detected

Not Detected

Not Detected

Not Detected

Not Detected

Not Detected

Not Detected

Not Detected

Not Detected

Not Detected

Not Detected

Not Detected

Not Detected

Not Detected
Qvalue

78

74

$3-3$

(\#) = qualifier out of range $(\mathrm{m})=$ manual integration

(*) Does not meet EPA spectral criteria (False Hit) 
Data File : C: \HPCHEM 1 IDATA \MAGGIE'S\0208\NRA11C98.D

$\begin{array}{lll}\text { Acq Fime } & : 07 / 15 / 97 \quad 14: 10 & \text { Operator: TV } \\ \text { Sampie } & : 97 \text { C2898 } & \text { Inst }: 5972-\mathrm{N} \\ \text { Misc } & : 7 / 1 / 97 \text { WATER 97C-0208-02 } & \text { Multiplr: } 1.00\end{array}$

Quan: Time: Jul 22 16:39 1997

Method : C: \HPCHEM I IMETHODS $\backslash 8270 B . M$

Title : SW846/8270B, Curve:NOT 4/24/97

Iast Upaate : Mon Jul o7 19:19:38 1997

Response via : Multiple Level Calibration

Cormound

R.T. Scan QIon Response Conc Unit Qvalue

45) 4-Chloro-3-Methylphenol

46) 2-Methylnaphthalene

0.00

48) $1,2,4,5$-Tetrachlorobenzene 0.00

49) Hexachlorocyclopentadiene 0.00

50) $2,4,6-$ Trichlorophenol 0.00

51) 2,4.5-Trichlorophenol 0.00

53) Tetradecane NOT Present 0.00

54) 2-Chloronaphthalene 0.00

55) 1-Chloronaphthalene 0.00

56) 2-Nitroaniline 0.00

57) Dimethylphthalate 0.00

5B) 2,6-Dinitrotoluene 0.00

59) Acenaphthylene 0.00

60) 3-Nitroaniline $\quad 0.00$

61) Acenaphthene 0.00

62) 2,4-Dinitrophenol 0.00

63) 4-Nitrophenol 0.00

64) Dibenzofuran 0.00

$65)$ 2,4-Dinitrotoluene $\quad 0.00$

66) Pentachlorobenzene 0.00

67) 1-Naphthylamine $\quad 0.00$

6B) 2-Naphthylamine 0.00

69) 2,3,4,6-Tetrachlorophenol 0.00

70) Diethylphthalate 0.00

71) 4-Chlorophenyl Phenyl Ethe 0.00

72) Fluorene 0.00

73) 4-Nitroaniline 0.00

76) 4,6-Dinitro-2-methylphenol 0.00

77) IV-nitrosodiphenylamine 0.00

78) 1,2-Diphenylhydrazine 0.00

79) Tributylphosphate NOT Pres 0.00

BD) Phonacetin

BI) 4-3romophenyl Phenyl Ether 0.00

B2) alpha-BHC 0.00

B3) Hexachlorobenzene 0.00

B4) Tris(2-CE) phosphate NOT Pr 0.00

B5) 4-iminobiphenyl 0.00

B6) beta-BHC 0.00

87) Pentachlorophenol 0.00

88) Pronamide 0.00

B9) gemma-BHC 0.00

90) Pentachloronitrobenzene 0.00

91) Phenanthrene 0.00

92) Arthracene 0.00

93) de?ta-BHC 0.00

94) Heptachlor 0.00

95) Di-n-Butylphthalate 13.16

96) Alarin

97) Eluoranthene 0.00

99) Benzidine 0.00

-00) Dyzene 0.00

¿01) $4.5^{\prime}-\mathrm{DDE} \quad 0.00$

E03) Dielarin 0.00

-04) p-Dimethylaminoazobenzene 0.00

I05) $4.6^{\prime}-D D D \quad 0.00$

Not Detected

Not Detected

Not Detected

Not Detected.

Not Detectréd

Not Detected

Not Detected

Not Detected

Not Detected

Not Detected

Not Detected

Not Detected

Not Detected

Not Detected

Not Detected

Not Detected

Not Detected

Not Detected

Not Detected

Not Detected.

Not Detected

Not Detected

Not Detected

Not Detected

Not Detected

Not Detected

Not Detected

Not Detected

Not Detected

Not Detected

Not Detected

Not Detected

Not Detected

Not Detected

Not Detected

Not Detected

Not Detected

Not Detected

Not Detected

Not Detected

Not Detected

Not Detected

Not Detected

Not Detected

Not Detected

Not Detected

$4.600 \mathrm{ug} / \mathrm{mL}^{*}$

Not Detected

Not Detected

Not Detected

Not Detected

Not Detected

Not Detected

Not Detected

Not Detected

$-344$

(i) = cualifier out of range $(m)=$ manual integration

(*) Does not meet EPA spectral criteria (False Hit) $5 \times 95$ 
Data File : C: \HPCHEM \1 IDATA \MAGGIE'S\0208\NRA11C98.D

$\begin{array}{lll}\text { Aco Time } & : 07 / 15 / 9714: 10 & \text { Operator: TV } \\ \text { Sanole } & : 97 \text { Co2898 } & \text { Inst }: 5972-N \\ \text { Misc } & : 7 / 1 / 97 \text { WATER 97C-0208-02 } & \text { Multiplr: } 1.00\end{array}$

Quant Time: Jul 22 16:39 1997

Method : C: \HPCHEM $\backslash 1 \backslash M E T H O D S \backslash 8270 B . M$

Title : SW846/8270B, Curve:NOT 4/24/97

Last Update : Mon Jul o7 19:19:38 1997

Response via : Multiple Level Calibration

Compound

106) Butylbenzylphthalate

107) $4.4^{\prime}$-DDT

108) Methoxychlor

109) 3,3'-Dichlorobenzidine

110) Benzo[a]anthracene

111) Chrysene

112) Bis (2-ethylhexyl) phthalate

114) Di-n-octylphthalate

115) Benzo[b] fluoranthene

116) 7,12-Dimethylbenz (a) anthra

117) Benzo[k] fluoranthene

118) Benzola]pyrene

119) 3-Methylcholanthrene

120) Indeno $(1,2,3-c$, d) pyrene

121) Dibenz $[a, h]$ anthracene

122) Benzo[ghi]perylene
R.T. Scan QIon Response
0.00
0.00
0.00
0.00
0.00
0.00

0.00

$\begin{array}{llll}17.46 & 1703 & 149 & 49710\end{array}$

0.00

0.00

0.00

0.00

0.00

0.00

0.00

0.00
Conc Unit Qvalue

Not Detected

Not Detected

Not Detected

Not Detected,

Not Detected

Not Detected

Not Detected

$1.722 \mathrm{ug} / \mathrm{mL}$

Not Detected

Not Detected

Not Detected

Not Detected

Not Detected

Not Detected

Not Detected

Not Detected

345 Data Processed By: Mn Endu-Heald 7-22-97

(it) = cualifier out of range $(\mathrm{m})$ = manual integration

(*) Does not meet EPA spectral criteria (False Hit) sX95 

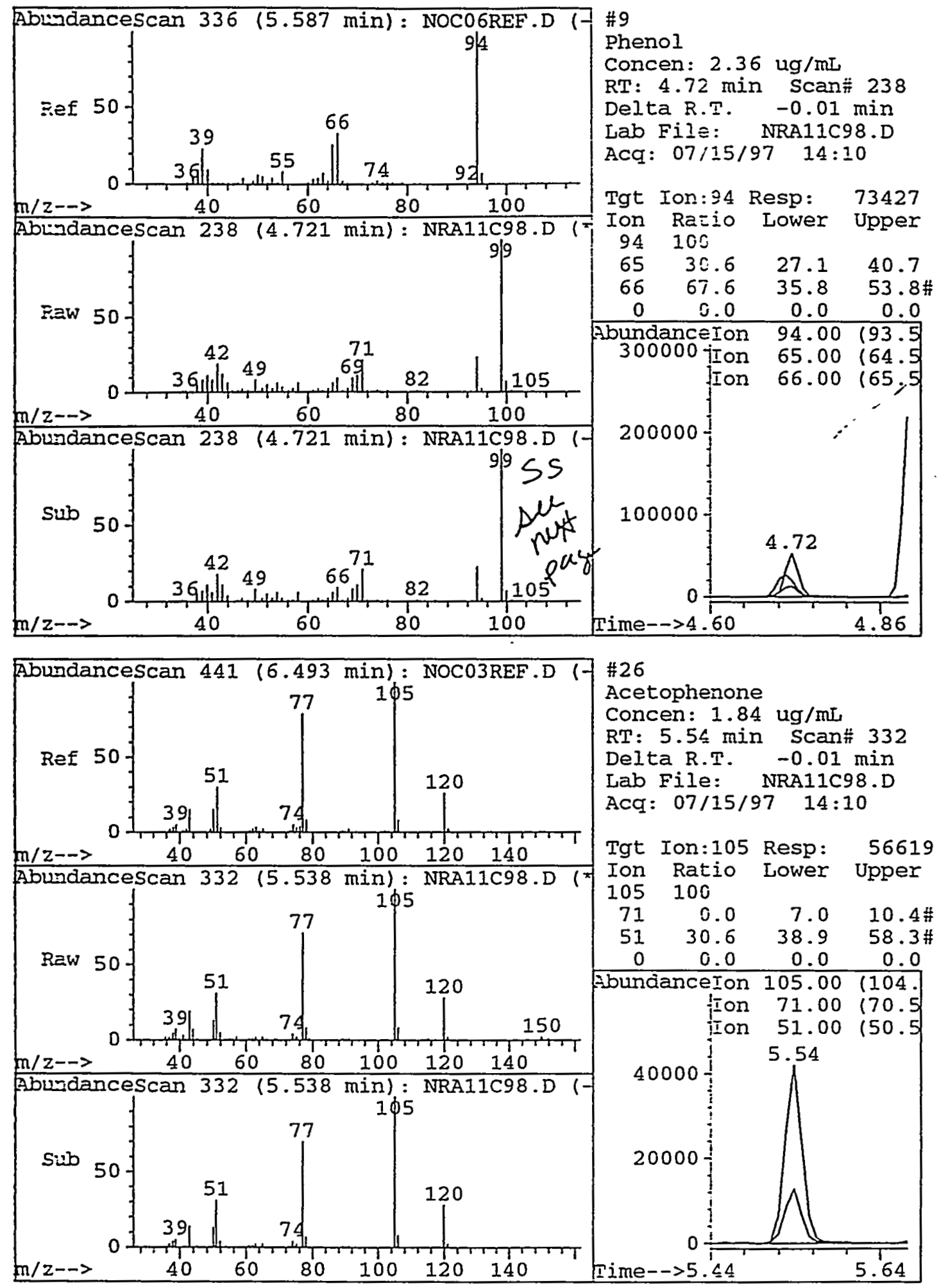
Quantitation Report

Data File : C: \HPCHEM \1 \DATA \MAGGIE'S\0208\NRA11C98.D

Acq Time : 07/15/97 14:10

Sample : 97002898

Misc : 7/1/97 WATER 97C-0208-02

Quant Time: Jul $1514: 361997$

Operator: TV

Inst : $5972-\mathrm{N}$

Multiplr: 1.00

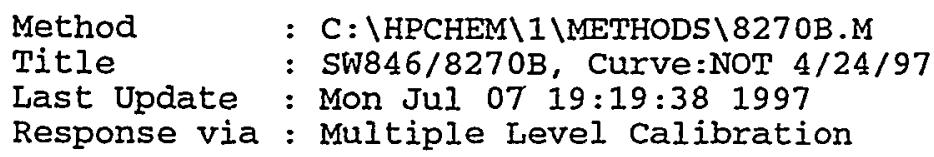

Method : C: \HPCHEM $\backslash 1 \backslash M E T H O D S \backslash 8270 \mathrm{~B} . \mathrm{M}$

Title : SW846/8270B, Curve:NOT 4/24/97

Last Update : Mon Jul 07 19:19:38 1997

Response via : Multiple Level Calibration

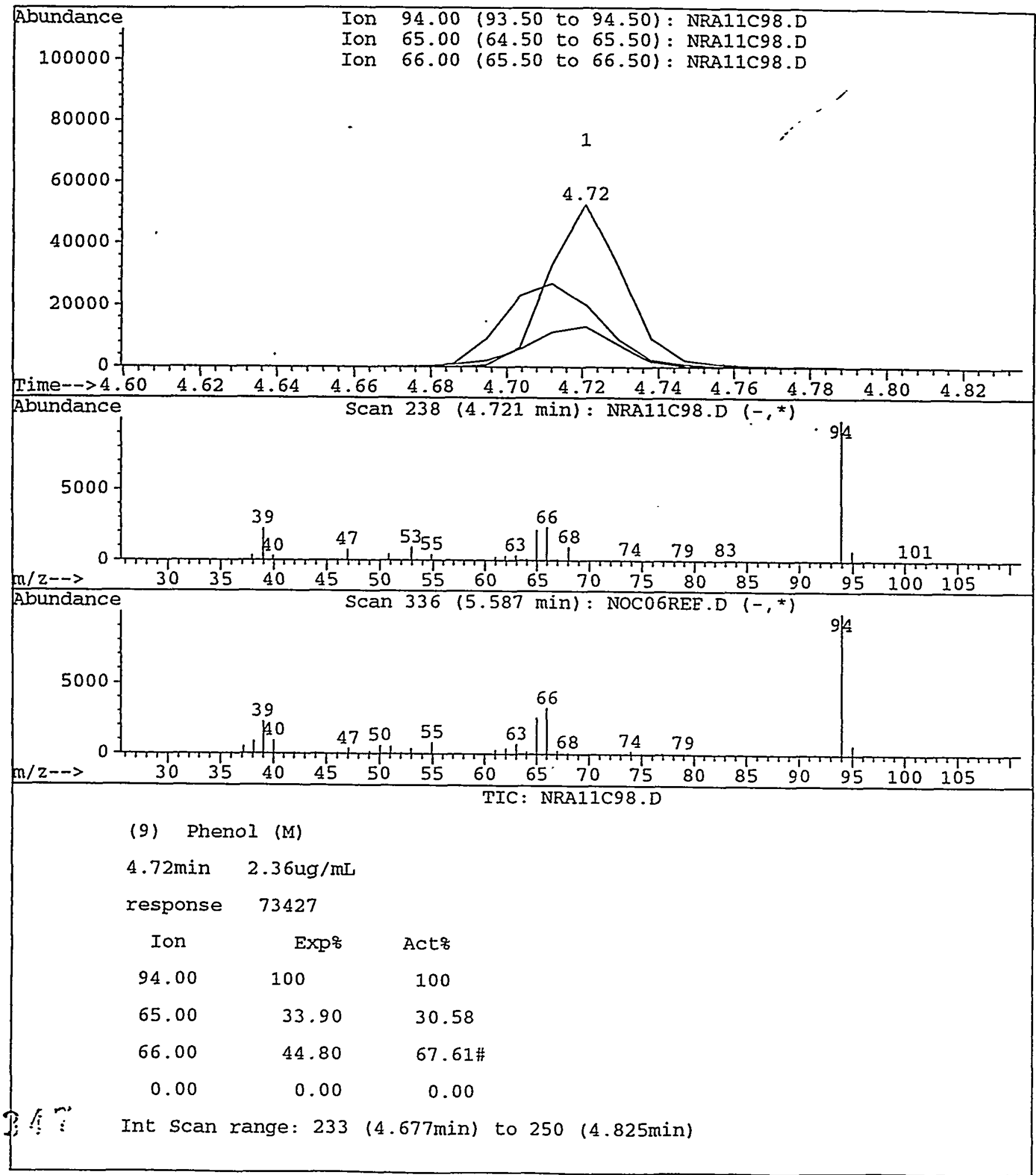




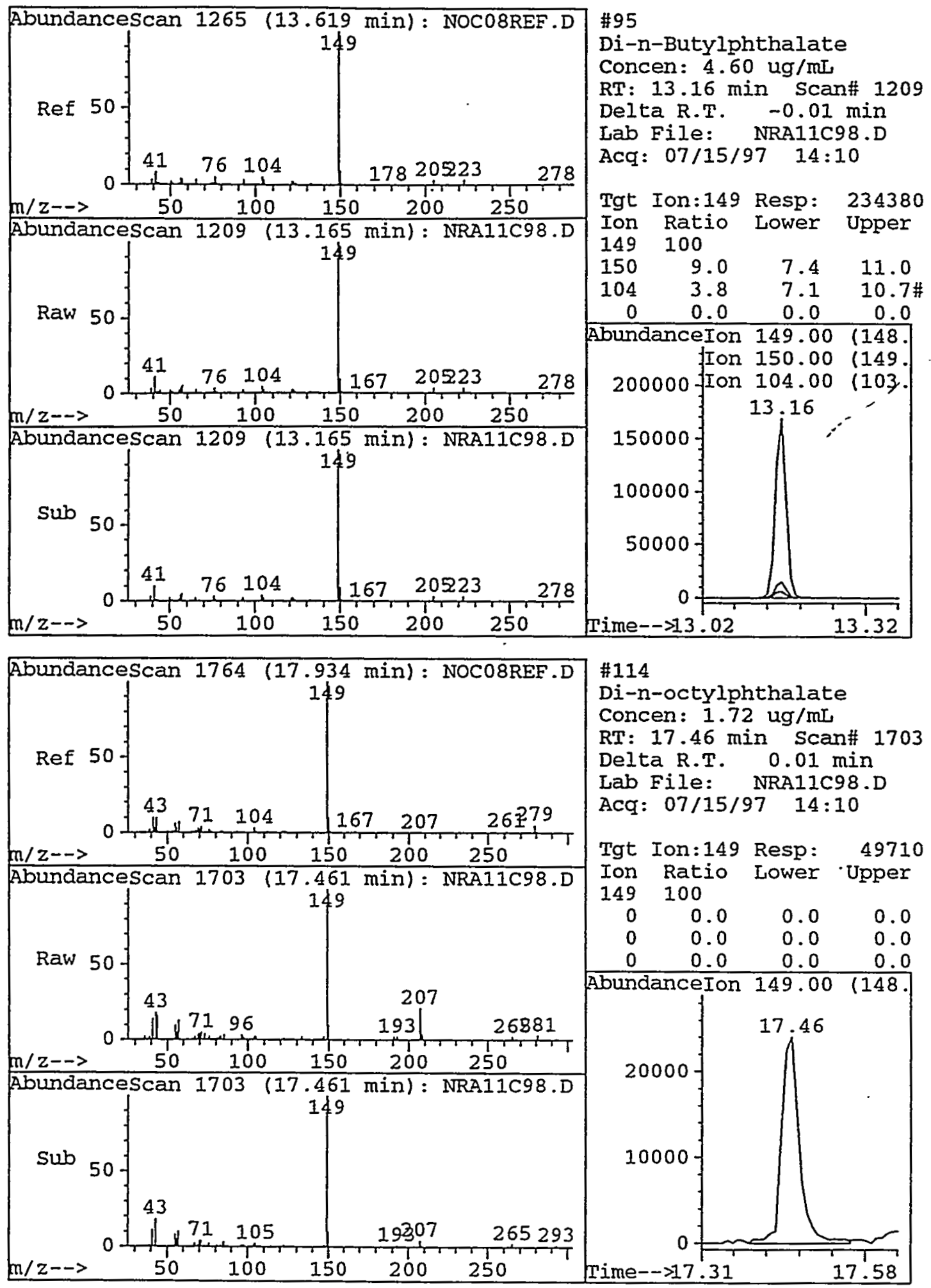

\section{8}




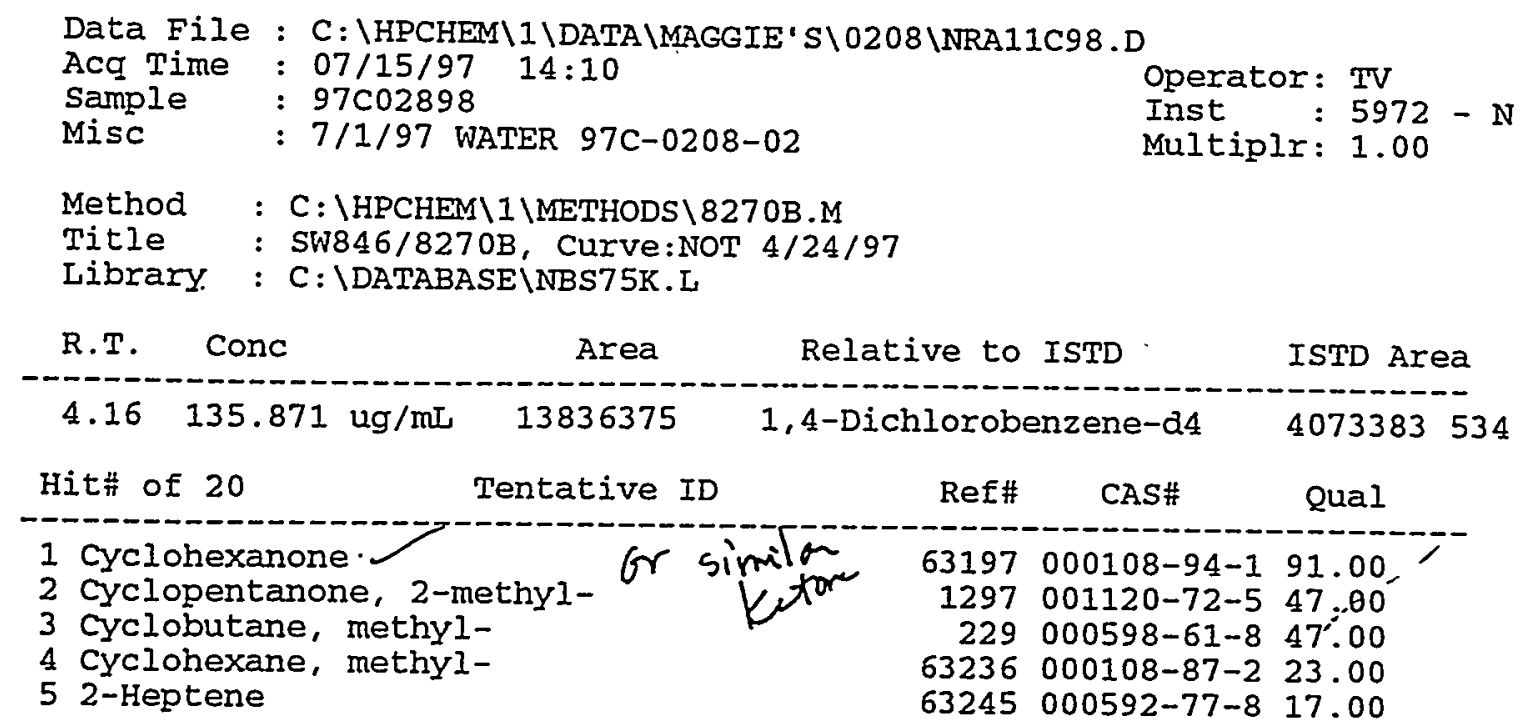

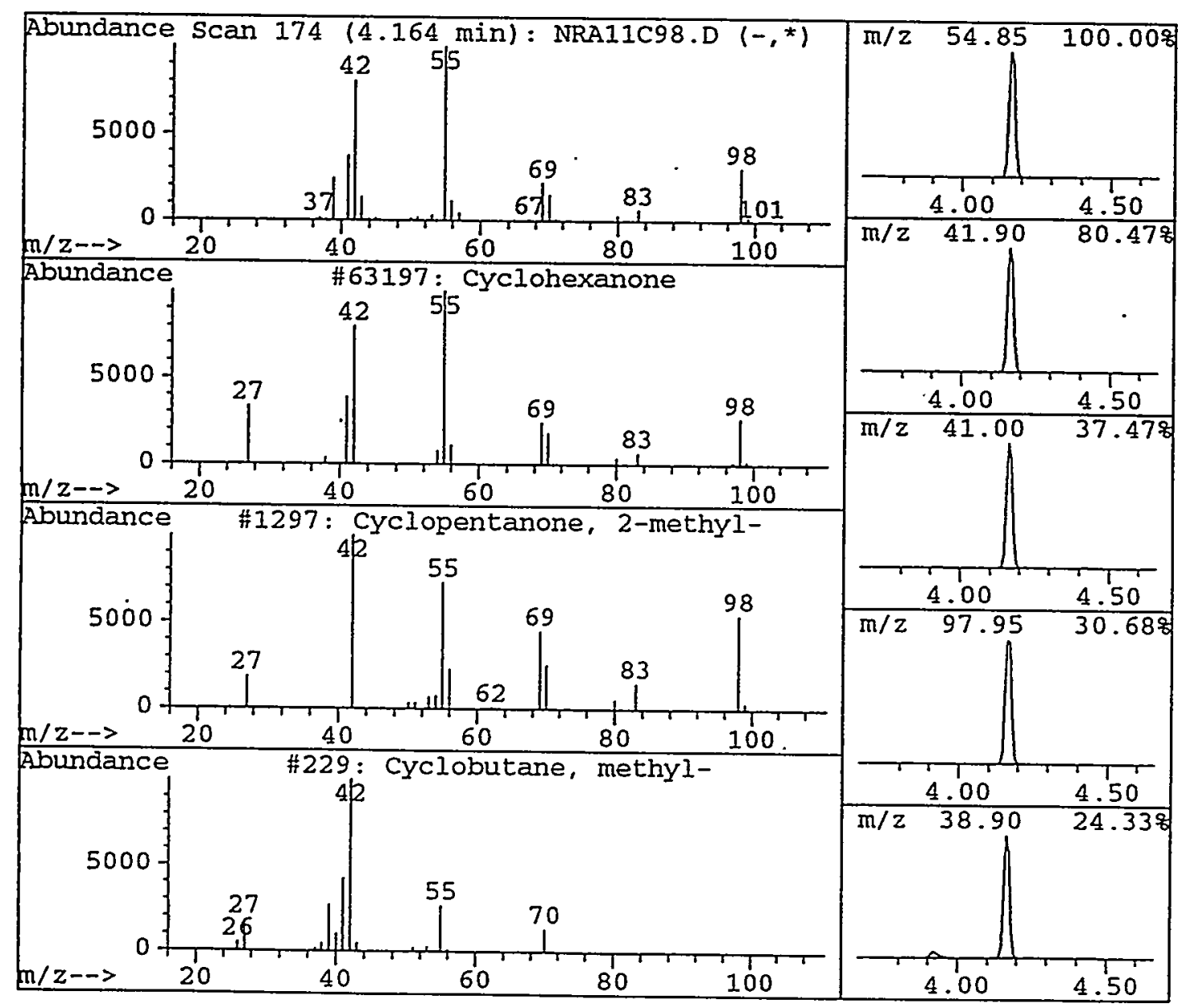

349 


\section{PRELIMINARY QC SUMMARY REPORT}

File: NRA12C01.D

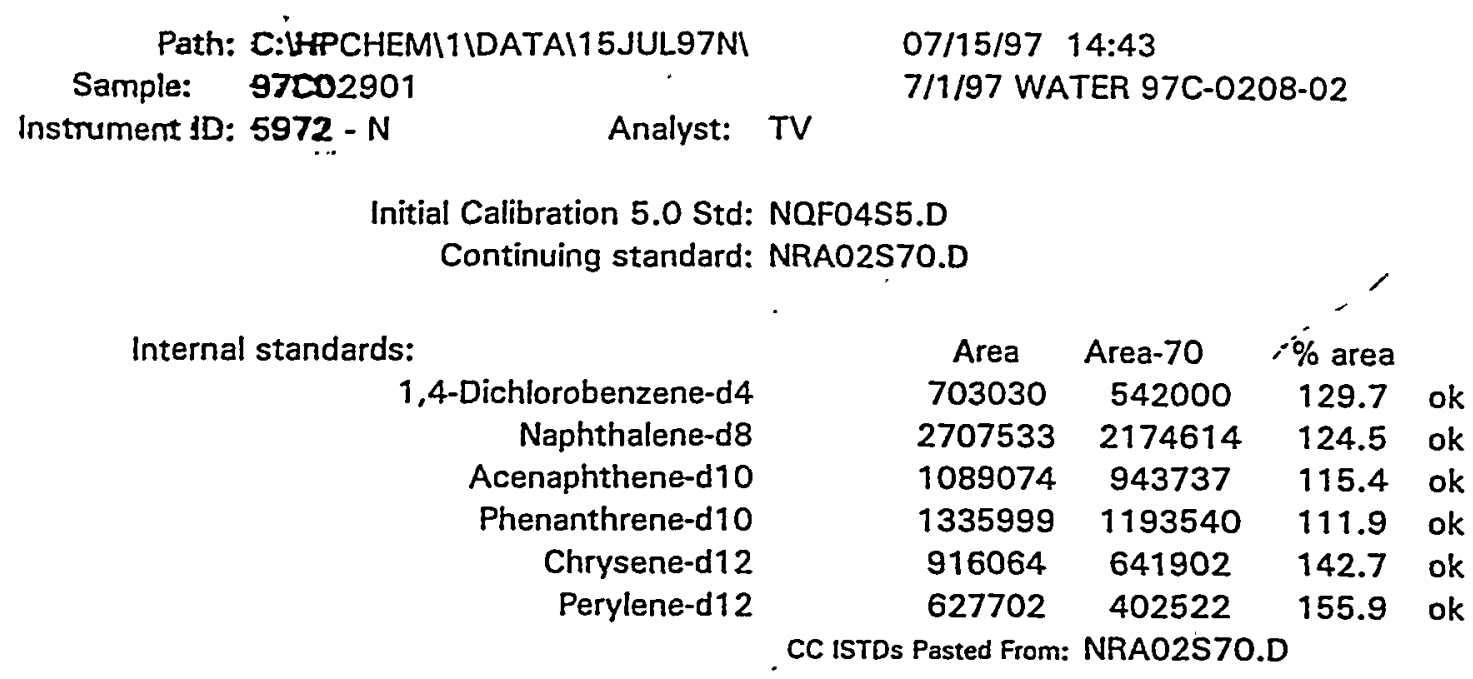

Matrix: (Water or Soil): WATER

Multiplier (normal water $=1$; soil $=1$ ): $\quad$ ?

$\begin{array}{rccccc}\text { Surrogates: } & & & & & \\ & \text { Quan } & \text { \%Rec } & \text { Method } & \text { Warning } & \text { Control } \\ \text { 2-Fluorophenol } & 47.687 & 47.7 & 21-100 & 23-78 & 10-91 \\ \text { Phenol-d5 } & 34.479 & 34.5 & 10-94 & 9-73 & 0-89 \\ \text { Nitrobenzene-d5 } & 73.035 & 73.0 & 35-114 & 55-102 & 43-114 \\ \text { 2-Fluorobiphenyl } & 75.353 & 75.4 & 43-116 & 63-111 & 51-124 \\ \text { 2,4.6-Tribromophenol } & 72.953 & 73.0 & 10-123 & 36-121 & 14-142 \\ \text { Terphenyl-d14 } & 88.104 & 88.1 & 33-141 & 55-124 & 37-141 \\ \text { 2-Chlorophenol-d4 } & 61.972 & 62.0 & 33-110 & \text { Advisory Use Only } \\ \text { 1.2-Dichlorobenzene-d4 } & 59.217 & 59.2 & 16-110 & & \text { Advisory Use Only }\end{array}$

Limits updated: 12/14/95

350 


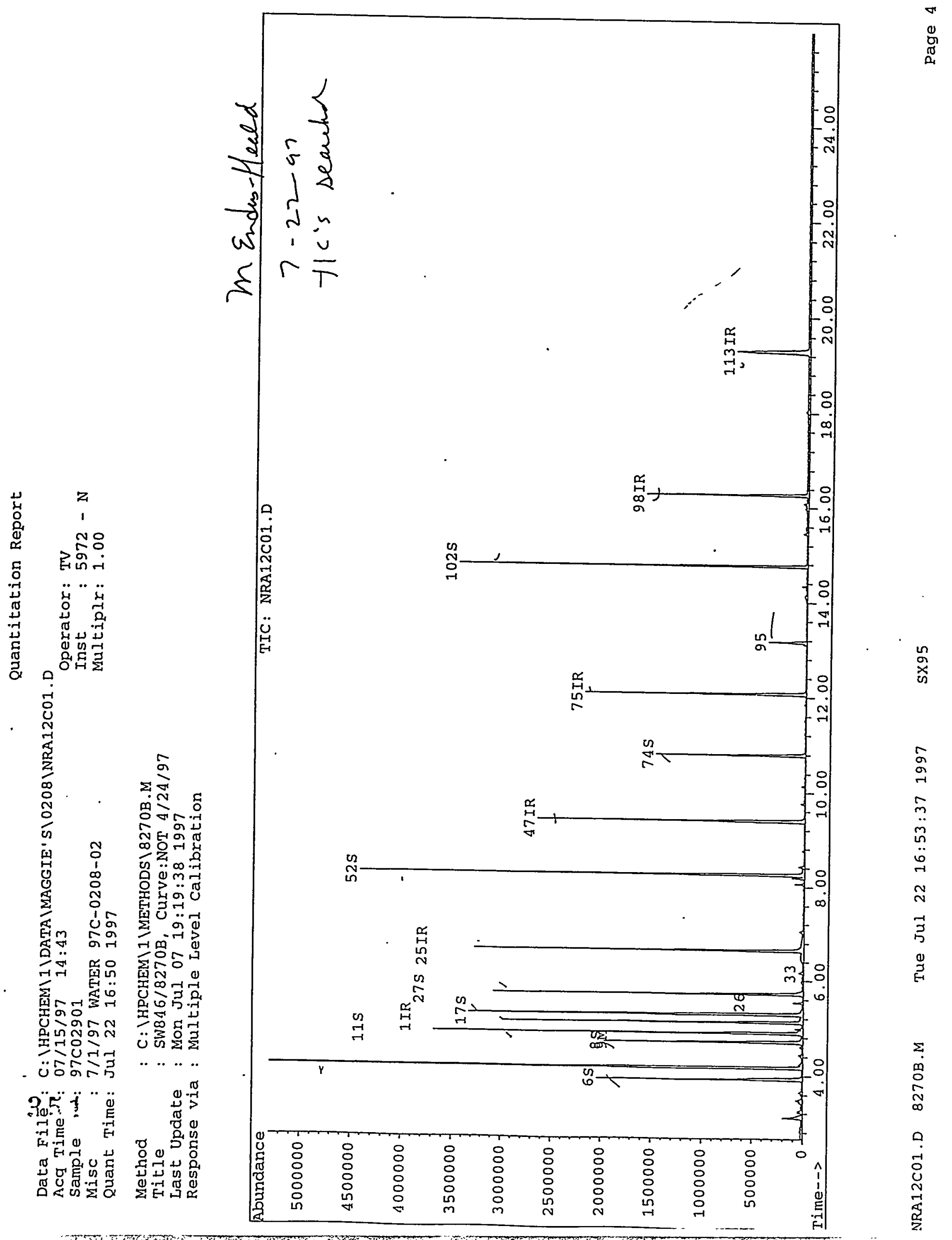


Data File : C: \HPCFEM I IDATA \MAGGIE'S $\backslash 0208 \backslash N R A 12 C 01 . D$

$\begin{array}{lll}\text { Acq Time } & : 07 / 15 / 97 \text { 14:43 } & \text { Operator: TV } \\ \text { Sample } & : 97 \text { C02901 } & \text { Inst } \\ \text { Misc } & : 7 / 1 / 97 \text { WATER 97C-D20B-02 } & \text { Multiplr: } 1.00\end{array}$

Quant Time: Jul 22 16:50 1997

Method : C: IFPCHEMII 1 WETHODS $18270 \mathrm{~B} . \mathrm{M}$

Title : SW846/B270B, curve:NOT 4/24/97

Jast Update : Mon:Jul D7 19:19:38 1997

Response via : Multiple Ievel Calibration

R.T. Scan QIon Response Conc Units Areaz

1) 1,4-Dichlorobenzene- 24

25) Naphthalene-d8

47) Acenaphthene-d10

75) Phenanthrene-d10

98) Chrysene-d12

113) Perylene-d12

System Monitoring Compounds

6) 2-Fluorophenol

8) Phenol-d5

11) 2-Chlorophenol-d4

17) 1,2-Dichlorobenzene-d4

27) Nitrobenzene-d5

52) 2-Fluorobiphenyl

74) 2,4,6-Tribromophenol

102) Terphenyl-d14

Target Compounds

2) Pyridine

3) N-nitrosodimethylamine

4) 2-Picoline

5) Methyl methanesulfonate

7) Ethyl methanesulfonate

9) Phenol

10) Aniline

12) 2-Chlorophenol

13) Decane NOT Present

14) 1,3-Dichlorobenzene

15) 1,4-dichlorobenzene

16) Benzyl alcohol

18) 1,2-dichlorobenzene

19) 2-Methylphenol

20) Bis (2-chloroisopropyl) ethe

21) 4-methylphenol

22) N-Nitrosodi-n-propyl amine

23) Hexachloroethane

24) Bis (2-chloroethyl)ether

26) Acetophenone

28) Nitrobenzene

29) N-nitrosopiperidine

30) Isophorone

31) 2-Nitrophenol

32) 2,4-Dimethylphenol

33) Benzoic acid

34) Bis (2-chloroethoxy) methane

35) 2,4-Dichlorophenol

36) A., A.-dimethylphenethylami

37) 1,2,4-Trichlorobenzene

38) Dodecane NOT Present

39) Naphthalene

40) 4-Chloroaniline

41) 2,6-Dichlorophenol

42) Hexachlorobutadiene

$\begin{array}{rrrr}5.12 & 284 & 152 & 703030 \\ 6.65 & 460 & 136 & 2707533 \\ 9.40 & 776 & 164 & 1089074 \\ 12.08 & 1084 & 188 & 1335999 \\ 16.29 & 1568 & 240 & 916064 \\ 19.30 & 1914 & 264 & 627702\end{array}$

$19.30 \quad 1914 \quad 264 \quad 627702$

$\begin{array}{rrrr}3.92 & 146 & 112 & 1112310 \\ 4.71 & 236 & 99 & 997603 \\ 4.90 & 258 & 132 & 1654068 \\ 5.30 & 304 & 152 & 861978 \\ 5.72 & 353 & 82 & 1544908 \\ 8.25 & 644 & 172 & 2381435 \\ 10.80 & 937 & 330 & 270891 \\ 14.77 & 1393 & 244 & 1838556\end{array}$

0.00

0.00

0.00

0.00

4.72

0.00

0.00

0.00

0.00

D. DO

0.00

0.00

0.00

0.00

0.00

0.00

0.00

0.00

5.54

0.00

$332 \quad 105 \quad 42010$

0.00

0.00

0.00

0.00

6.18

$\begin{array}{lll}238 & 94 \quad 90177\end{array}$

0.00

0.00

0.00

D.DD

D. DD

0.00

0.00

0.00

0.00

0.00

44. ${ }^{N}=n j$ troso-di-n-butylamine
$40.00 \mathrm{ug} / \mathrm{mL} \quad 0.00$

$40.00 \mathrm{ug} / \mathrm{mL} \quad 0.00$

$40.00 \mathrm{ug} / \mathrm{mL}, 0.00$

$40.00 \mathrm{ug} / \mathrm{mL}$. 0.00

$40.00 \mathrm{ug} / \mathrm{mL} \quad 0.00$

$40.00 \mathrm{ug} / \mathrm{mL} \quad 0.00$

\& Recovery

$47.687 \mathrm{ug} / \mathrm{mL} \quad 47.69 \%$

$34.479 \mathrm{ug} / \mathrm{mL} \quad 34.48 \%$

$61.972 \mathrm{ug} / \mathrm{mL} \quad 61.97 \%$

$59.217 \mathrm{ug} / \mathrm{mL} \quad 59.228$

$73.035 \mathrm{ug} / \mathrm{mL} \quad 73.048$

$75.353 \mathrm{ug} / \mathrm{mL} \quad 75.35 \%$

$72.953 \mathrm{ug} / \mathrm{mL} \quad 72.95 \%$

$88.104 \mathrm{ug} / \mathrm{mL} \quad 88.10 \%$

Qvalue

Not Detected

Not Detected

Not Detected

Not Detected

Not Detected

$2.811 \mathrm{ug} / \mathrm{mL}$

Not Detected

Not Detected

Not Detected

Not Detected

Not Detected

Not Detected

Not Detected

Not Detected

Not Detected

Not Detected

Not Detected

Not Detected

Not Detected

$1.318 \mathrm{ug} / \mathrm{mL}$

Not Detected

Not Detected

Not Detected

Not Detected

Not Detected

$0.721 \mathrm{ug} / \mathrm{mL}$

Not Detected

Not Detected

Not Detected

Not Detected

Not Detected

Not Detected

Not Detected

Not Detected

Not Detected

Not Detected

Not Detected
84

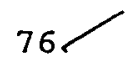

96 352

(\#) = qualifier out of range $(m)=$ manual integration

(*) Does not meet EPA spectral criteria (False Hit) SX95 
Data File : C:IHPCHEM IIDATA IMAGGIE'S\0208\NRA12C01.D

$\begin{array}{lll}\text { Acq Time } & : 07 / 15 / 97 \quad 14: 43 & \text { Operator: TV } \\ \text { Sample } & : 97 C 02901 & \text { Inst }: 5972-\mathrm{N} \\ \text { Misc } & : 7 / 1 / 97 \text { WATER 97C-0208-02 } & \text { Multiplr: } 1.00\end{array}$

Quant Time: Jul 22 16:50 1997

Method : C: IHPCHEM IIMETHODS $\backslash 8270 \mathrm{~B} . \mathrm{M}$

ritle : SWB46/8270B, Curve:NOT 4/24/97

- Iast Update : Mon Jul o7 19:19:38 1997

- Response via : Nultiple Level Calibration

Compouna

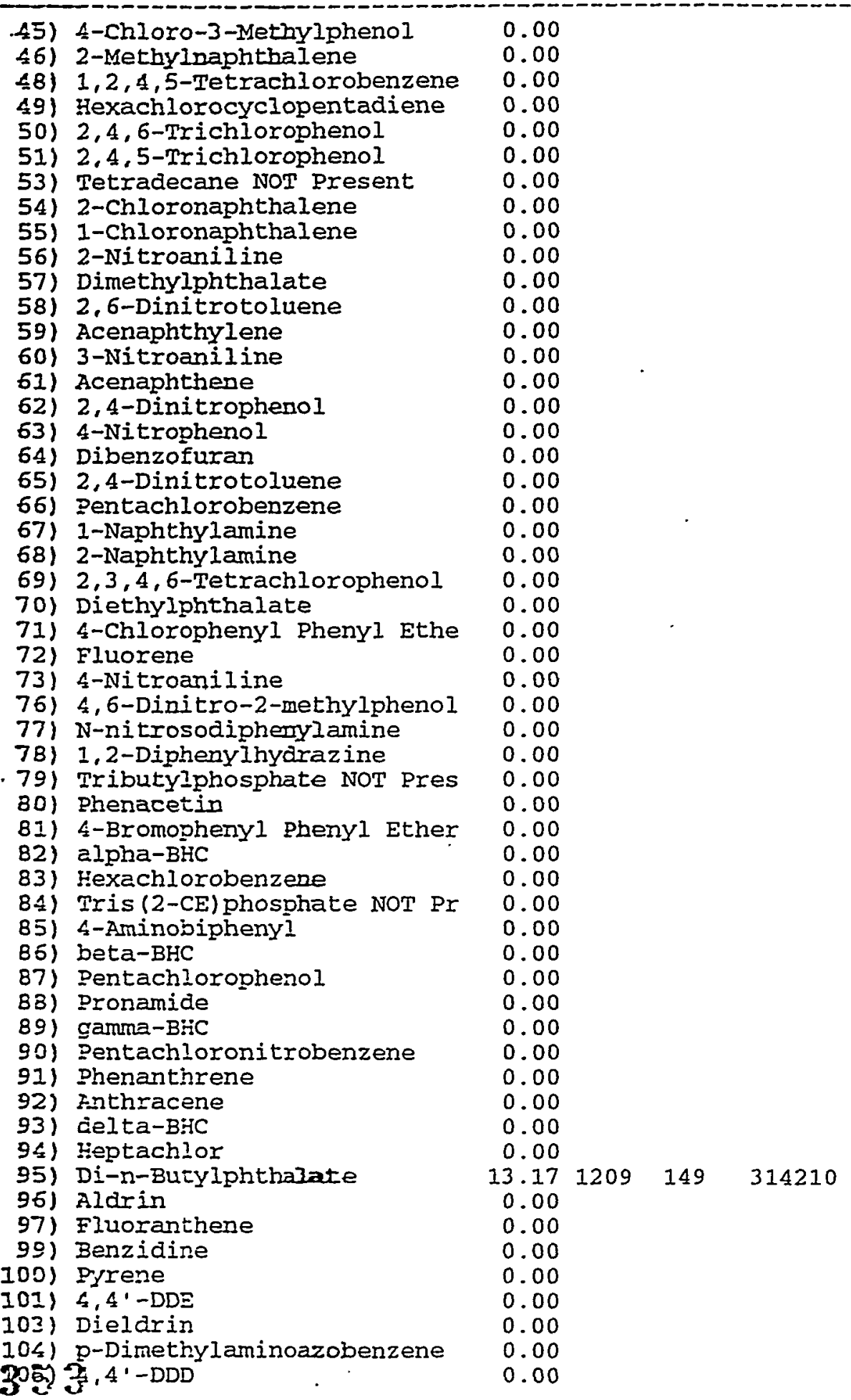

Conc Unit Qvalue

Not Detected

Not Detected

Not Detected

Not Detected-

Not Detectéd

Not Detected

Not Detected

Not Detected

Not Detected

Not Detected

Not Detected

Not Detected

Not Detected

Not Detected

Not Detected

Not Detected

Not Detected

Not Detected

Not Detected

Not Detected

Not Detected

Not Detected

Not Detected

Not Detected

Not Detected

Not Detected

Not Detected

Not Detected

Not Detected

Not Detected

Not Detected

Not Detected

Not Detected

Not Detected

Not Detected

Not Detected

Not Detected

Not Detected

Not Detected

Not Detected

Not Detected

Not Detected

Not Detected

Not Detected

Not Detected

Not Detected

6. $090 \mathrm{ug} / \mathrm{mL}$

Not Detected

Not Detected

Not Detected

Not Detected

Not Detected

Not Detected

Not Detected

Not Detected 


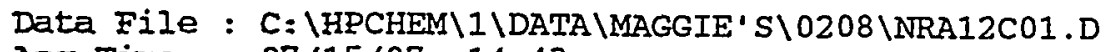

Acq Time : 07/15/97 14:43

Sample : 97c02901

HisC : 7/1/97 WATER 97C-0208-02

quant Time: Ju1 22 16:50 1997

Operator: TV

Inst : $5972-\mathrm{N}$

Multiplr: 1.00

$\begin{array}{ll}\text { Hethod } & : \text { C: \HPCHEM I IMETHODS } \backslash 8270 B . M \\ \text { Title } & : \text { SW846/8270B, Curve:NOT 4/24/97 } \\ \text { Iast Update : Mon Jul 07 19:19:38 1997 }\end{array}$

Response via : Multiple Level Calibration

Compouna

1D5) Butylbenzylphthalate

107) 4,4'-DDT

108) Methoxychlor

ID9) 3,3'-Dichlorobenzidine

110) Benzo[a] anthracene

111) Chrysene

112) Bis(2-ethylhexyl)phthalate

114) Di-n-octylphthalate

115) Benzo[b] fluoranthene

116) 7,12-Dimethylbenz (a) anthra

117) Benzo[k] fluoranthene

118) Benzola]pyrene

119) 3-Methylcholanthrene

$120)$ Indeno $(1,2,3-c, d)$ pyrene

121) Dibenz $[a, h]$ anthracene

122) Benzo[ghi]perylene
R.T. Scan QIon Response Conc Unit Qvalue

$0.00 \quad$ Not Detected

0.00

0.00

0.00

0.00

0.00

0.00

0.00

0.00

0.00

0.00

0.00

0.00

0.00

0.00

0.00
Not Detected

Not Detected

Not Detected.

Not Detected

Not Detected

Not Detected

Not Detected

Not Detected

Not Detected

Not Detected

Not Detected

Not Detected

Not Detected

Not Detected

Not Detected 


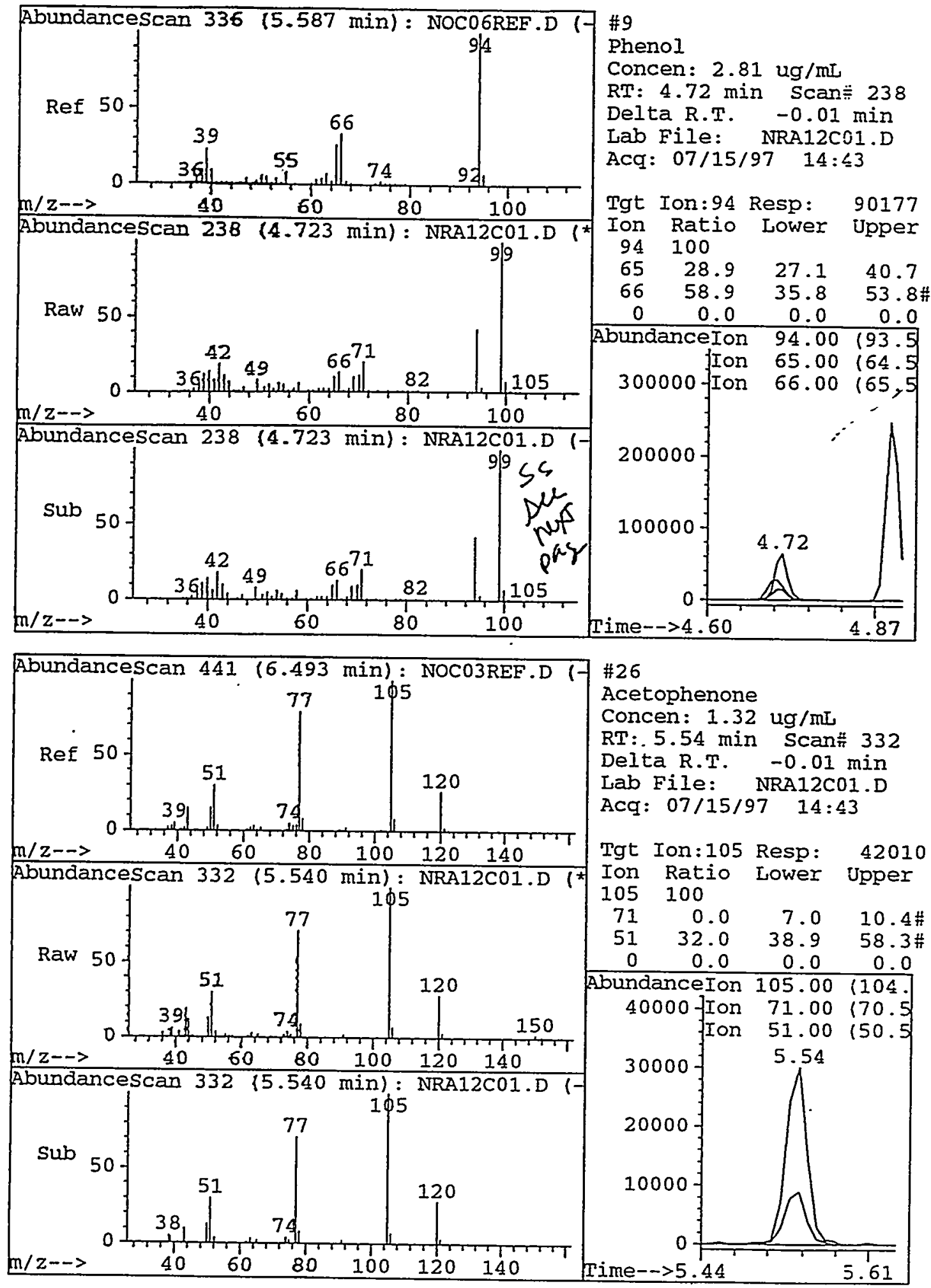

$? \div$ 
Data File : C: \HPCHEM I IDATA \MAGGIE'S\0208\NRA12C01.D

Acq Time : 07/15/97 $14: 43$

Sample : 97C02901

Misc : 7/1/97 WATER 97C-0208-02

Operator: TV

Inst : $5972-\mathrm{N}$

Quant Time: Jul 15 15:10 1997

Multiplr: 1.00
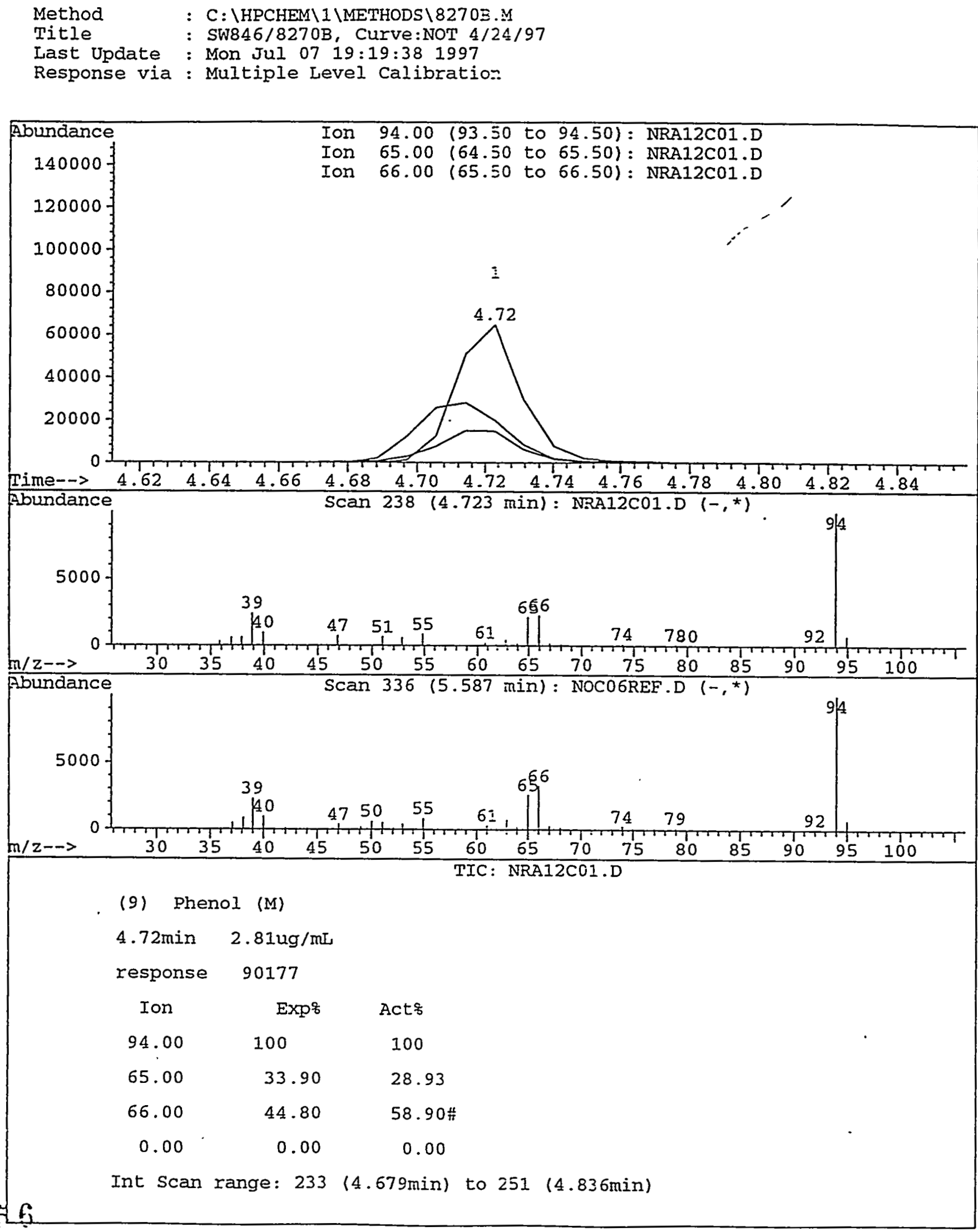


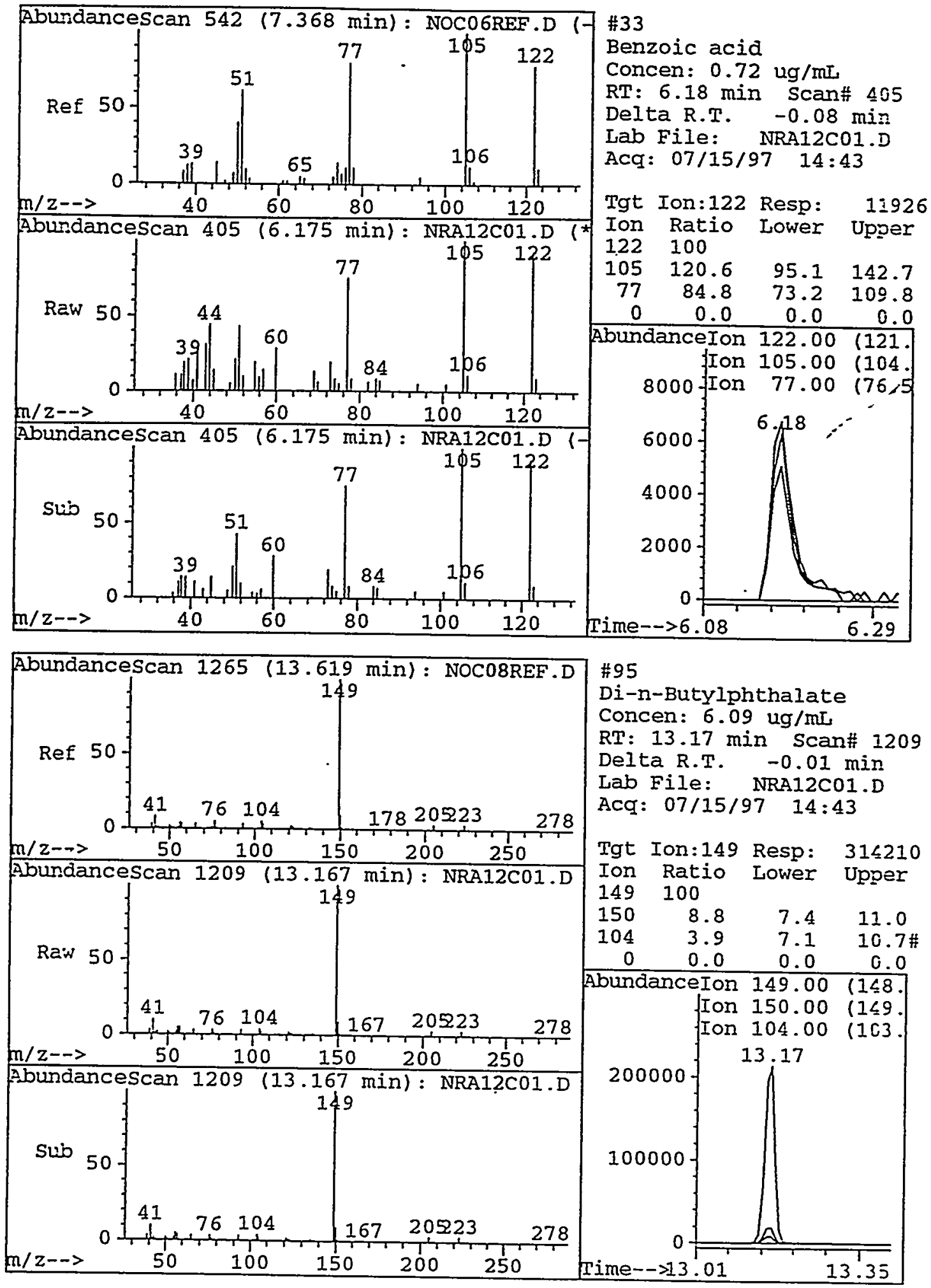




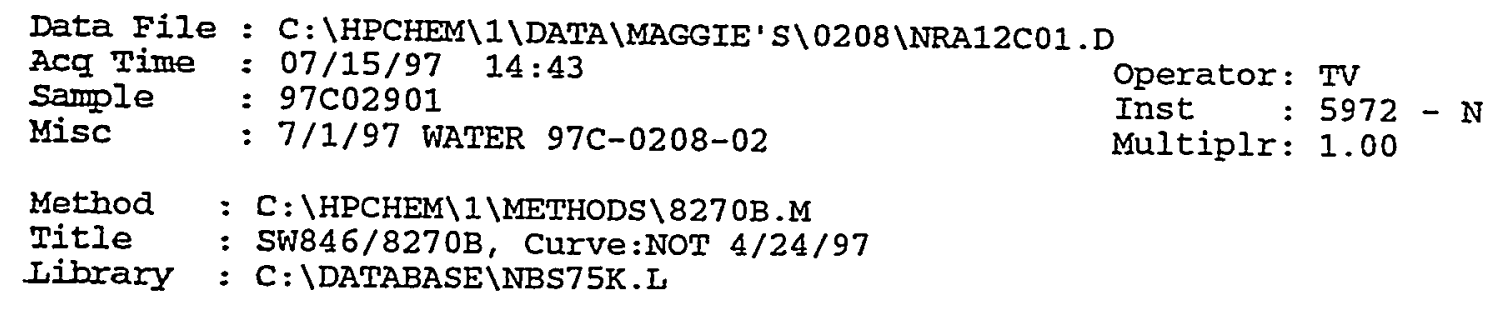

R.T. Conc Area Relative to ISTD ISTD Area

$4.17147 .102 \mathrm{ug} / \mathrm{mL} \quad 15365625 \quad 1,4-D i c h l o r o b e n z e n e-d 4 \quad 4178217534$

\begin{tabular}{|c|c|c|c|c|}
\hline Hit\# of & ID & Ref\# & CAS\# & Qual \\
\hline $\begin{array}{l}\text { I Cyclohexanone } \\
2 \text { cyclopentanone, 2-methyl- } \\
3 \text { cyclobutanone, 2-ethyl- } \\
4 \text { cyclobutane, methyl- } \\
5 \text { cyclohexane, methyl- }\end{array}$ & $\begin{array}{l}\text { sintor } \\
\text { Setree }\end{array}$ & $\begin{array}{r}63197 \\
1297 \\
1238 \\
229 \\
63236\end{array}$ & $\begin{array}{l}000108-94-1 \\
001120-72-5 \\
010374-14-8 \\
000598-61-8 \\
000108-87-2\end{array}$ & $\begin{array}{l}91.00 \\
47.00 \\
72.00 \\
47.00 \\
23.00\end{array}$ \\
\hline
\end{tabular}

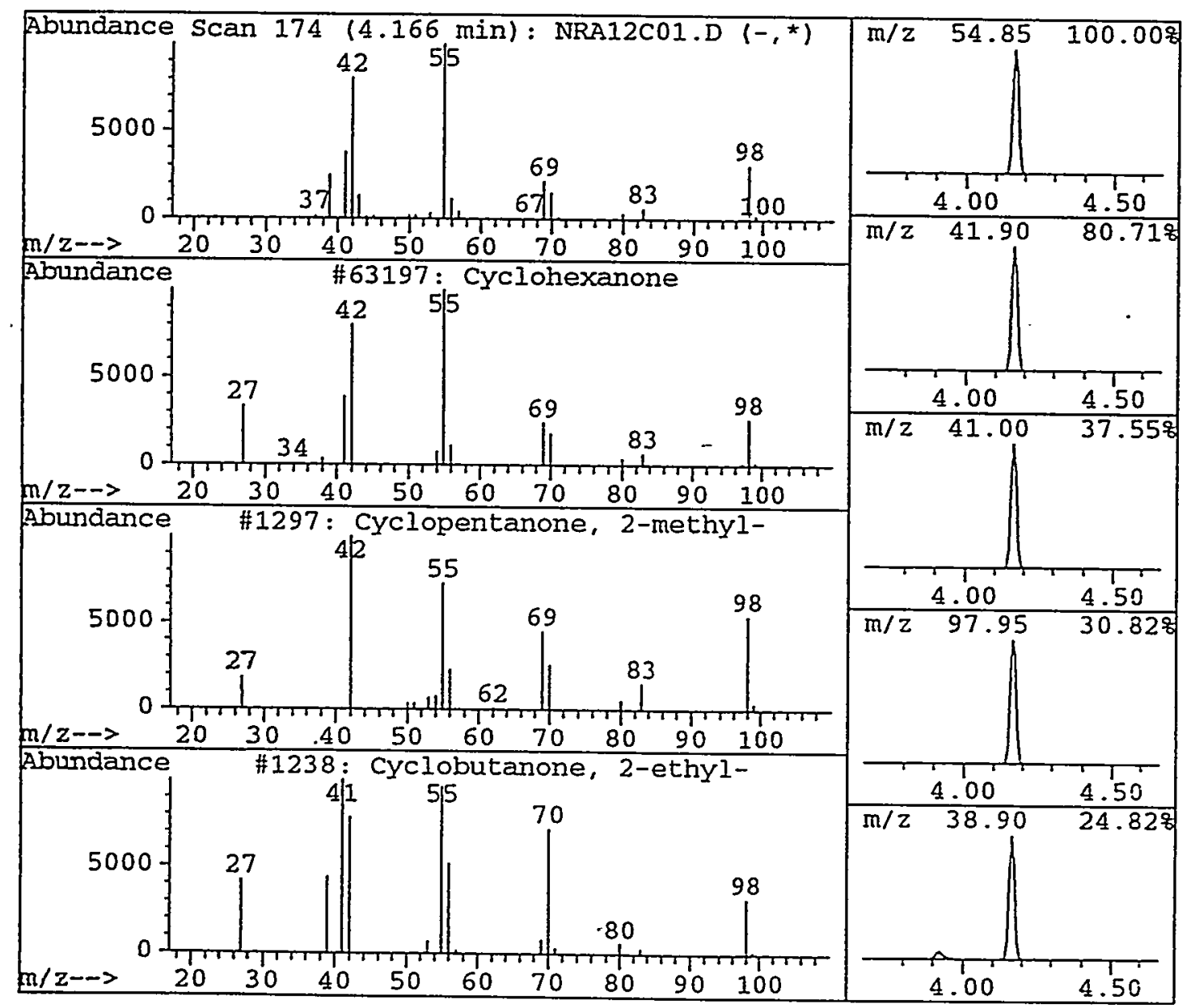




\section{PASSED}

\section{B CONTINUING CALIBRATION REPORT}

SW-846, 8270B, Revision 2

Continuing Calibration File: NRA02S70.D

Initial Calibration 5.0 Standard: NQFO4S5.D

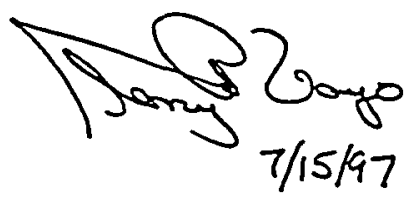

$07 / 15 / 97$

Calibration Check Compounds: $\quad \%$ Drift must be $<20 . \%$

$\begin{array}{rccccc} & \text { RT } & \text { CC Amnt } & \text { CC Target } & \% \text { Drift } & \\ \text { Phenol } & 4.73 & 83.03 & 70.00 & -18.6 & \text { ok } \\ \text { 1.4-dichlorobenzene } & 5.15 & 75.38 & 70.00 & -7.7 & \text { ok } \\ \text { 2-Nitrophenol } & 6.14 & 75.97 & 70.00 & -8.5 & \text { ok } \\ \text { 2,4-Dichlorophenol } & 6.45 & 63.68 & 70.00 & 9.0 & \text { ok } \\ \text { Hexachlorobutadiene } & 6.83 & 70.97 & 70.00 & -1.4 & \text {. - } \bar{k} \\ \text { 4-Chloro-3-Methylphenol } & 7.42 & 76.50 & 70.00 & -9.3 & \text { ok } \\ \text { 2.4,6-Trichlorophenol } & 8.12 & 68.87 & 70.00 & 1.6 & \text { ok } \\ \text { Acenaphthene } & 9.47 & 73.68 & 70.00 & -5.3 & \text { ok } \\ \text { N-nitrosodiphenylamine } & 10.58 & 71.05 & 70.00 & -1.5 & \text { ok } \\ \text { Pentachlorophenol } & 11.74 & 70.95 & 70.00 & -1.4 & \text { ok } \\ \text { Fluoranthene } & 14.19 & 69.68 & 70.00 & 0.5 & \text { ok } \\ \text { Di-n-octylphthalate } & 17.45 & 82.62 & 70.00 & -18.0 & \text { ok } \\ \text { Benzolalpyrene } & 19.16 & 73.68 & 70.00 & -5.3 & \text { ok }\end{array}$

SPCC compounds:

N-Nitrosodi-n-propyl amine Hexachlorocyclopentadiene

2,4-Dinitrophenol

4-Nitrophenol

N-Nitrosodi-n-propyl amine Hexachlorocyclopentadiene

2,4-Dinitrophenol

4-Nitrophenol

Other Compounds:

Pyridine

$\mathrm{N}$-nitrosodimethylamine 2-Picoline

Methyl methanesulfonate

2-Fluorophenol

Ethyl methanesulfonate

Phenol-d5

Aniline

2-Chlorophenol-d4

2-Chlorophenol

Decane NOT Present

1,3-Dichlorobenzene

Benzyl alcohol

1,2-Dichlorobenzene-d4

1.2-dichlorobenzene

2-Methylphenol

Bis(2-chloroisopropyllether

4-methylphenol

Hexachloroethane
CC RFs must be $>l=0.05$

CC RF

0.924

0.198

0.167

0.128

\section{RT}

5.53

7.92

9.52

9.65

RT

2.95

2.90

3.49

3.79

3.93

4.36

4.71

4.79

4.90

4.92

0.00

5.08

5.24

5.29

5.31

5.35

5.37

5.51

5.69
CC Amnt

83.39

60.11

54.74

88.07

CC Amnt

88.97

84.44

$86: 87$

84.00

89.21

85.44

85.42

83.25

72.90

74.53

0.00

74.56

81.86

70.76

85.63

87.63

74.45

77.77

81.56
CC Target

$$
70.00
$$

70.00

70.00

70.00

CC Target

70.00

70.00

70.00

70.00

70.00

70.00

70.00

70.00

70.00

70.00

70.00

70.00

70.00

70.00

70.00

70.00

70.00

70.00

70.00
$-11.1$
OK

OK

OK

OK

\% Drift

$-19.1$

14.1

21.8 warning

-25.8 warning

$\%$ Drift

-27.1 warning

-20.6 warning

-24.1 warning

-20.0 warning

-27.4 warning

-22.1 warning

-22.0 warning

$-18.9$

$-4.1$

$-6.5$

100.0 warning

$-6.5$

$-16.9$

$-1.1$

-22.3 warning

-25.2 warning

$-16.5$ 
Continuing Calibration File: NRA02S70.D

\begin{tabular}{|c|c|c|c|c|c|}
\hline $\begin{array}{l}\text { Other Compounds (cont'd): } \\
\text { Bis (2-chloroethyl)ether }\end{array}$ & $\begin{array}{l}\mathrm{RT} \\
4.82\end{array}$ & $\begin{array}{l}\text { CC Amnt } \\
77.68\end{array}$ & $\begin{array}{l}\text { CC Target } \\
70.00\end{array}$ & $\begin{array}{l}\text { \% Drift } \\
-11.0\end{array}$ & \\
\hline Acetophenone & 5.55 & 83.00 & 70.00 & -18.6 & \\
\hline Nitrobenzene-d5 & 5.74 & 82.93 & 70.00 & -18.5 & \\
\hline Nitrobenzerze & 5.75 & 80.40 & 70.00 & -14.9 & - \\
\hline N-nitrosopiperidine & 5.94 & 73.03 & 70.00 & -4.3 & $\therefore$ \\
\hline Isophorone & 6.03 & 80.22 & 70.00 & -14.6 & \\
\hline 2,4-Dimethylphenol & 6.15 & 71.79 & 70.00 & -2.6 & \\
\hline Benzoic acid & 6.25 & 34.55 & 70.00 & 50.6 & warning \\
\hline Bis(2-chloroethoxy)methane & 6.28 & 82.75 & 70.00 & -18.2 & \\
\hline A.-dimethylphenethylamine & 6.53 & 69.69 & 70.00 & 0.4 & \\
\hline 1,2,4-Trichlorobenzene & 6.56 & 61.49 & 70.00 & 12.2 & \\
\hline Dodecane NOT Present & 0.00 & 0.00 & 70.00 & 100.0 & warning \\
\hline Naphthalene & 6.69 & 66.04 & 70.00 & 5.7 & \\
\hline 4-Chloroaniline & 6.73 & 82.17 & 70.00 & -17.4 & \\
\hline 2,6-Dichlorophend] & 6.75 & 64.42 & 70.00 & 8.0 & \\
\hline Benzothiazole NOT Present & 0.00 & 0.00 & 70.00 & 100.0 & warning \\
\hline N-nitroso-di-n-butylamise & 7.19 & 76.66 & 70.00 & -9.5 & . \\
\hline 2-Methylnaphthalene & 7.69 & 65.97 & 70.00 & 5.8 & \\
\hline 1.2,4,5-Tetrachlorobenzene & 7.95 & 76.73 & 70.00 & -9.6 & \\
\hline 2,4,5-Trichlorophenol & 8.19 & 69.52 & 70.00 & 0.7 & \\
\hline 2-Fluorobiphenyl & 8.26 & 67.04 & 70.00 & 4.2 & \\
\hline Tetradecane NOT Present & 0.00 & 0.00 & 70.00 & 100.0 & warning \\
\hline 2-Chloronaphthalene & 8.48 & 75.04 & 70.00 & -7.2 & \\
\hline 1-Chloronaphthalene & 8.51 & 66.03 & 70.00 & 5.7 & \\
\hline 2-Nirtomiline & 8.62 & 87.44 & 70.00 & -24.9 & warning \\
\hline Dimetriplotinalate & 8.93 & 70.71 & 70.00 & -1.0 & \\
\hline 2,6-Dinitrototuene & 9.03 & 70.55 & 70.00 & -0.8 & \\
\hline Acenaphthylese & 9.17 & 71.01 & 70.00 & -1.4 & \\
\hline 3-Nitroanilize & 9.33 & 86.50 & 70.00 & -23.6 & warning \\
\hline Dibenzofures & 9.77 & 69.05 & 70.00 & 1.4 & \\
\hline 2,4-Dinitrotoluene & 9.75 & 70.18 & 70.00 & -0.3 & \\
\hline Pentachlorobenzene & 9.69 & 80.65 & 70.00 & -15.2 & \\
\hline 1-Naphthylamine & 9.90 & 79.94 & 70.00 & -14.2 & \\
\hline 2-Naphthylamine & 10.05 & 75.35 & 70.00 & -7.6 & \\
\hline 2,3,4,6-Tetrachlorophenol & 9.98 & 78.62 & 70.00 & -12.3 & \\
\hline Diethylphthalate & 10.16 & 73.35 & 70.00 & -4.8 & \\
\hline 4-Chlorophenyl Phenyl Ether & 10.37 & 76.57 & 70.00 & -9.4 & \\
\hline Futorere & 10.38 & 72.99 & 70.00 & -4.3 & \\
\hline : 4ntiutromilize & 10.41 & 85.92 & 70.00 & -22.7 & warning \\
\hline 2,4;6-Tribromopherea & 10.81 & 67.02 & 70.00 & 4.3 & \\
\hline 4,6-Dinitro-2-medrytuera & 10.47 & 67.32 & 70.00 & 3.8 & \\
\hline
\end{tabular}


Continuing Calibration File: NRA02S70.D

\begin{tabular}{|c|c|c|c|c|c|}
\hline Dther Compounds (cont'd): & RT & CC Amnt & CC Target & $\%$ Drift & \\
\hline 1,2-Diphenylhydrazine & 10.66 & 91.11 & 70.00 & -30.2 & warning \\
\hline Tributylphosphate NOT Present & 0.00 & 0.00 & 70.00 & 100.0 & warning \\
\hline Phenacetin & 11.16 & 88.95 & 70.00 & -27.1 & warning \\
\hline 4-Bromophenyl Phenyl Ether & 11.27 & 79.70 & 70.00 & -13.9 & $\therefore$ \\
\hline alpha-BHC & 11.29 & 77.72 & 70.00 & -11.0 & \\
\hline Hexachlorobenzene & 11.37 & 74.99 & 70.00 & -7.1 & \\
\hline Tris(2-CE)phosphate NOT Present & 0.00 & 0.00 & 70.00 & 100.0 & warning \\
\hline 4-Aminobiphenyl & 11.75 & 69.18 & 70.00 & 1.2 & \\
\hline beta-BHC & 11.69 & 72.57 & 70.00 & -3.7 & \\
\hline Pronamide & 11.86 & 75.66 & 70.00 & -8.1 & \\
\hline gamma-BHC & 11.83 & 80.93 & 70.00 & -15.6 & \\
\hline Pentachloronitrobenzene & 11.75 & 81.68 & 70.00 & -16.7 & \\
\hline Phenanthrene & 12.13 & 71.71 & 70.00 & -2.4 & \\
\hline Anthracene & 12.22 & 77.98 & 70.00 & -11.4 & \\
\hline delta-BHC & 12.26 & 89.19 & 70.00 & -27.4 & warning \\
\hline Heptachlor & 13.00 & 84.96 & 70.00 & -21.4 & warning \\
\hline Di-n-Butylphthalate & 13.17 & 75.44 & 70.00 & -7.8 & \\
\hline Aldrin & 13.54 & 90.34 & 70.00 & -29.1 & warning \\
\hline Benzidine & 14.40 & 57.82 & 70.00 & 17.4 & \\
\hline Pyrene & 14.54 & 79.99 & 70.00 & -14.3 & \\
\hline $4,4^{\circ}-D D E$ & 14.69 & 87.25 & 70.00 & -24.6 & warning \\
\hline Terphenyl-d14 & 14.76 & 84.30 & 70.00 & -20.4 & warning \\
\hline Dieldrin & 14.83 & 105.18 & 70.00 & -50.3 & warning \\
\hline p-Dimethylaminoazobenzene & 14.96 & 75.97 & 70.00 & -8.5 & \\
\hline $4,4^{\prime}-D D D$ & 15.16 & 85.70 & 70.00 & -22.4 & warning \\
\hline Butylbenzylphthalate & 15.45 & 72.32 & 70.00 & -3.3 & \\
\hline $4,4^{\circ}-\mathrm{DDT}$ & 15.57 & 71.82 & 70.00 & -2.6 & \\
\hline Methoxychlor & 16.16 & 68.21 & 70.00 & 2.6 & \\
\hline 3,3'-Dichlorobenzidine & 16.22 & 67.30 & 70.00 & 3.9 & \\
\hline Benzo[a]anthracene & 16.28 & 73.90 & 70.00 & -5.6 & \\
\hline $\begin{array}{l}\text { Chrysene } \\
\text { Che }\end{array}$ & 16.34 & 73.42 & 70.00 & -4.9 & \\
\hline Bis(2-ethylhexyl)phthalate & 16.29 & 74.57 & 70.00 & -6.5 & \\
\hline Benzo[b]fluoranthene & 18.35 & 77.37 & 70.00 & -10.5 & \\
\hline 7.12-Dimethylbenz(a)anthracene & 18.33 & 82.55 & 70.00 & -17.9 & \\
\hline Benzo[k]fluoranthene & 18.42 & 76.77 & 70.00 & -9.7 & \\
\hline 3-Methylcholanthrene & 20.13 & 70.77 & 70.00 & -1.1 & \\
\hline Indeno(1,2,3-c, d)pyrene & 22.81 & 67.99 & 70.00 & 2.9 & \\
\hline Dibenz $[a, h] a n t h r a c e n e$ & 22.91 & 68.66 . & 70.00 & 1.9 & \\
\hline Benzo[ghilperylene & 23.90 & 68.96 & 70.00 & 1.5 & \\
\hline
\end{tabular}




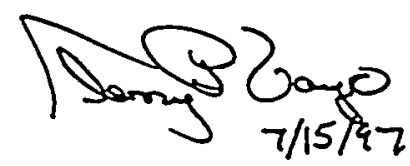

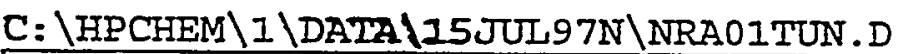

15 Jul $97 \quad 7: 58$ am

$\therefore \quad$ Abundance

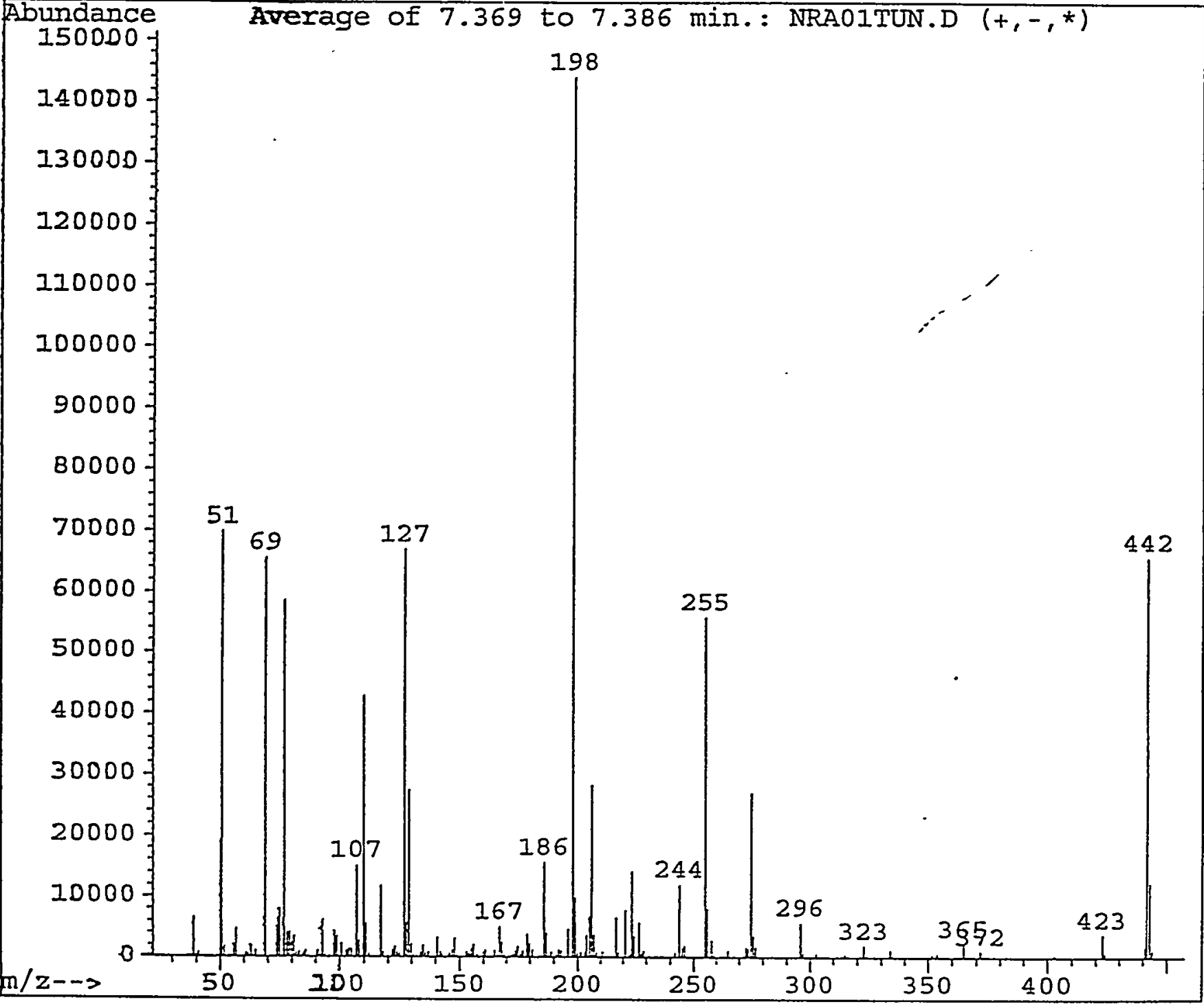

Peak Apex is scan: 256

Average of 3 scans: $255,256,257$ minus background scan 251

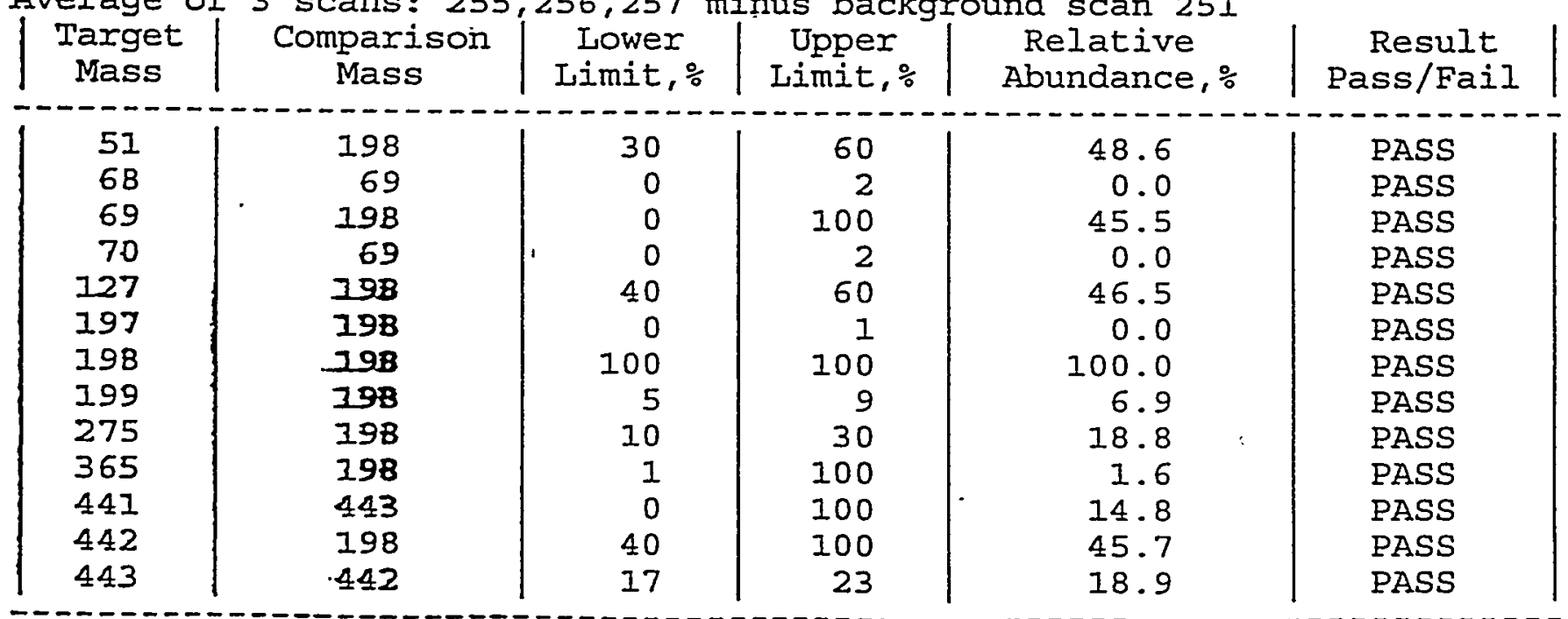


Data File:

Date-Acquired:

Operator ID:

Name:

Misc:
NRA01TUN.D

$07 / 15 / 97 \quad 07: 58$

TV

50 NG DFTPP (143WS2845I)

EXP.11/07/97
Instrument: $5972-\mathrm{N}$

Acq Method: DETPP625

DA Method: DETPP625.M

Cal Title:

Method 8270 Tuning Standard

last Cal Dpdate= Wed Apr 09 20:45:09 1997

EXP_-27/07/97

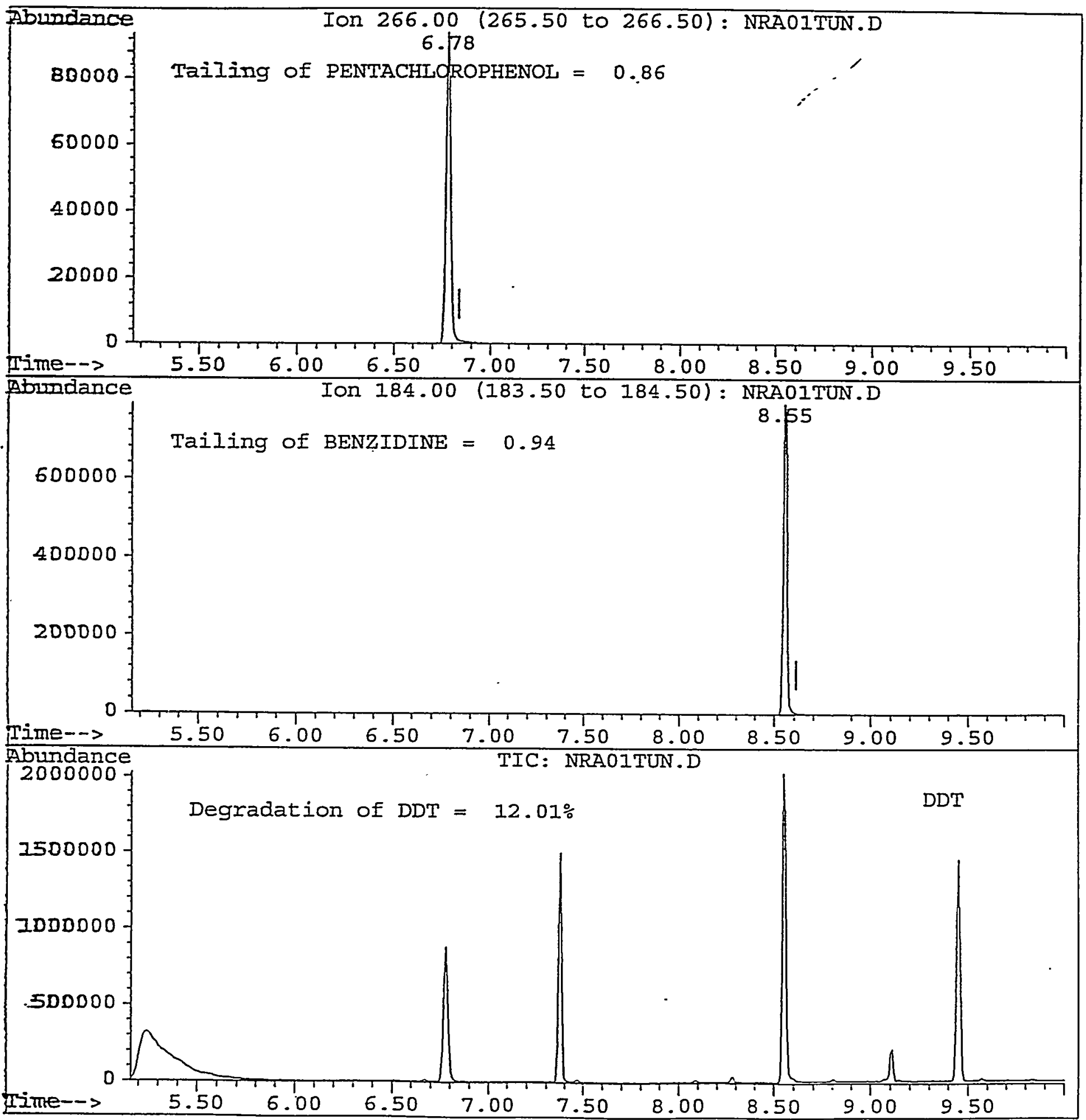

363 
I.369 to $7.386 \mathrm{~min}$ : NRAOITUN.D 50 NG DETPP (143WS28451)

Thäfied:added subtracted scaled

$\begin{array}{lrrrr}\mathrm{m} / \mathrm{z} & \text { abund. } & \mathrm{m} / \mathrm{z} & \text { abund. } & \mathrm{m} / \mathrm{z} \\ 35.85 & 53 & 57.75 & 102 & 77.90 \\ 36.90 & 314 & 60.85 & 804 & 78.90 \\ 38.90 & 6668 & 61.90 & 831 & 79.90 \\ 39.95 & 341 & 62.90 & 2089 & 80.90 \\ 40.95 & 752 & 63.95 & 295 & 81.90 \\ \text { 44.BD } & 147 & 64.85 & 1168 & 82.90 \\ 50.0 D & 19351 & 66.75 & 158 & 83.70 \\ 50.85 & 69907 & 68.85 & 65565 & 84.90 \\ 51.85 & 1892 & 73.90 & 5154 & 85.90 \\ 55.85 & 2128 & 74.90 & 8168 & 86.85 \\ 55.85 & 4745 & 76.90 & 58667 & 87.80\end{array}$

Average of 7.369 to $7.386 \mathrm{~min}$ : NRAOITUN.D

50 NG DFTPP (143WS28451)

MoBified:adied subtracted scaled

$\begin{array}{crcrc}\mathrm{m} / \mathrm{z} & \text { abund. } & \mathrm{m} / \mathrm{z} & \text { abund. } & \mathrm{m} / \mathrm{z} \\ 103.85 & 1598 & 119.85 & 262 & 133.90 \\ 104.85 & 1434 & 121.80 & 1306 & 134.90 \\ 106.85 & 15105 & 122.85 & 1801 & 135.90 \\ 107.85 & 2757 & 123.85 & 929 & 136.70 \\ 109.85 & 42913 & 124.90 & 675 & 136.95 \\ 110.85 & 5688 & 126.90 & 66883 & 139.80 \\ 117.90 & 665 & 127.90 & 5913 & 140.80 \\ 112.85 & 105 & 128.90 & 27567 & 141.85 \\ 115.85 & 142 & 129.90 & 2301 & 142.75 \\ 116.85 & 11851 & 130.90 & 456 & 143.75 \\ 117.85 & 932 & 131.90 & 207 & 145.85\end{array}$

Average of 7.369 to $7.386 \mathrm{~min}$.: NRAOITUN.D

50 NG DFTPP (143WS28451)

Modified:added subtracted scaled

$\begin{array}{rrrrrrrr}\mathrm{m} / z & \text { abund. } & \mathrm{m} / \mathrm{z} & \text { abund. } & \mathrm{m} / \mathrm{z} & \text { abund. } & \mathrm{m} / \mathrm{z} & \text { abund. } \\ 157.55 & 205 & 168.90 & 527 & 180.90 & 1284 & 193.65 & 111 \\ 157.85 & 240 & 169.90 & 102 & 181.90 & 107 & 194.05 & 156 \\ 158.85 & 334 & 170.85 & 228 & 184.00 & 227 & 195.85 & 4696 \\ 159.90 & 894 & 171.80 & 401 & 184.90 & 297 & 197.85 & 143987 \\ 160.85 & 1216 & 172.85 & 621 & 185.85 & 15648 & 198.85 & 9953 \\ 161.85 & 336 & 173.90 & 1090 & 186.85 & 4158 & 199.90 & 767 \\ 153-90 & 136 & 174.90 & 1885 & 187.80 & 393 & 201.35 & 882 \\ 154-80 & 1129 & 175.90 & 766 & 188.80 & 902 & 203.00 & 868 \\ 165.90 & 492 & 176.85 & 1066 & 190.85 & 435 & 203.90 & 3720 \\ 165.85 & 5116 & 178.85 & 3793 & 191.85 & 1334 & 205.00 & 6811 \\ 167.80 & 2577 & 179.90 & 2404 & 192.85 & 1157 & 205.95 & 28264\end{array}$

Average of 7.369 to $7.386 \mathrm{~min}$.: NRAO1TUN.D

50 NG DFTPP (I43WS2845I)

Nodified:added subtracted scaled

\begin{tabular}{|c|c|c|c|c|c|c|c|}
\hline$m / 2$ & abound. & $\mathrm{m} / \mathrm{z}$ & abund. & $\mathrm{m} / \mathrm{z}$ & abund. & $\mathrm{m} / \mathrm{z}$ & abund. \\
\hline 206.95 & 3886 & 223.90 & 14181 & 238.75 & 123 & 255.85 & 8089 \\
\hline 207-85 & $B 49$ & 224.90 & 3588 & 239.85 & 116 & 257.00 & 238 \\
\hline $2 B B-B D$ & 282 & 226.90 & 5792 & 240.75 & 153 & 257.85 & 2894 \\
\hline 230.20 & 152 & 227.90 & 813 & 241.85 & 765 & 258.85 & 460 \\
\hline 270.75 & 1023 & 228.90 & 1192 & 243.95 & 12004 & 264.80 & 1183 \\
\hline 271_.00 & 163 & 229.80 & 122 & 244.95 & 1704 & 272.90 & 1712 \\
\hline $21 \leq-80$ & 309 & 230.80 & 475 & 245.85 & 2022 & 273.90 & 1537 \\
\hline 216.85 & 6547 & 233.80 & 269 & 246.80 & 331 & 274.90 & 27049 \\
\hline 217.85 & B91 & 234.85 & 314 & 248.85 & 378 & 275.85 & 3571 \\
\hline 220.90 & 7836 & 235.90 & 267 & 252.85 & 169 & 276.85 & 1775 \\
\hline 222.90 & 604 & 236.90 & 348 & 254.90 & 55792 & 277.95 & 157 \\
\hline
\end{tabular}


zwerage of 7.369 to 7.386 min.: NRAOITUN.D

50 NG DFTPP (143WS2845I)

Modified:added subtracted scaled

$$
\mathrm{m} / \mathrm{z}
$$

282.85

283.85

284.95

292.85

295.90

296.90

302.90

374.00

374.90

315.85

320.95

\begin{abstract}
abuna.
$\mathrm{m} / \mathrm{z}$
\end{abstract}

110

113

141

226

5699

782

675

261

589

300

122
322.85

323.95

326.85

332.85

333.90

334.85

340.95

345.80

351.90

352.90

353.90 abund. 1961

246

185

110

1247

266

118

293

590

207

654 $\mathrm{m} / \mathrm{z}$

364.90

365.85

371.90

372.95

382.95

401.90

402.85

404.00

420.85

421.95

422.90 abund.

2315

289

1044

158

149

244

566

129

379

190

3815 

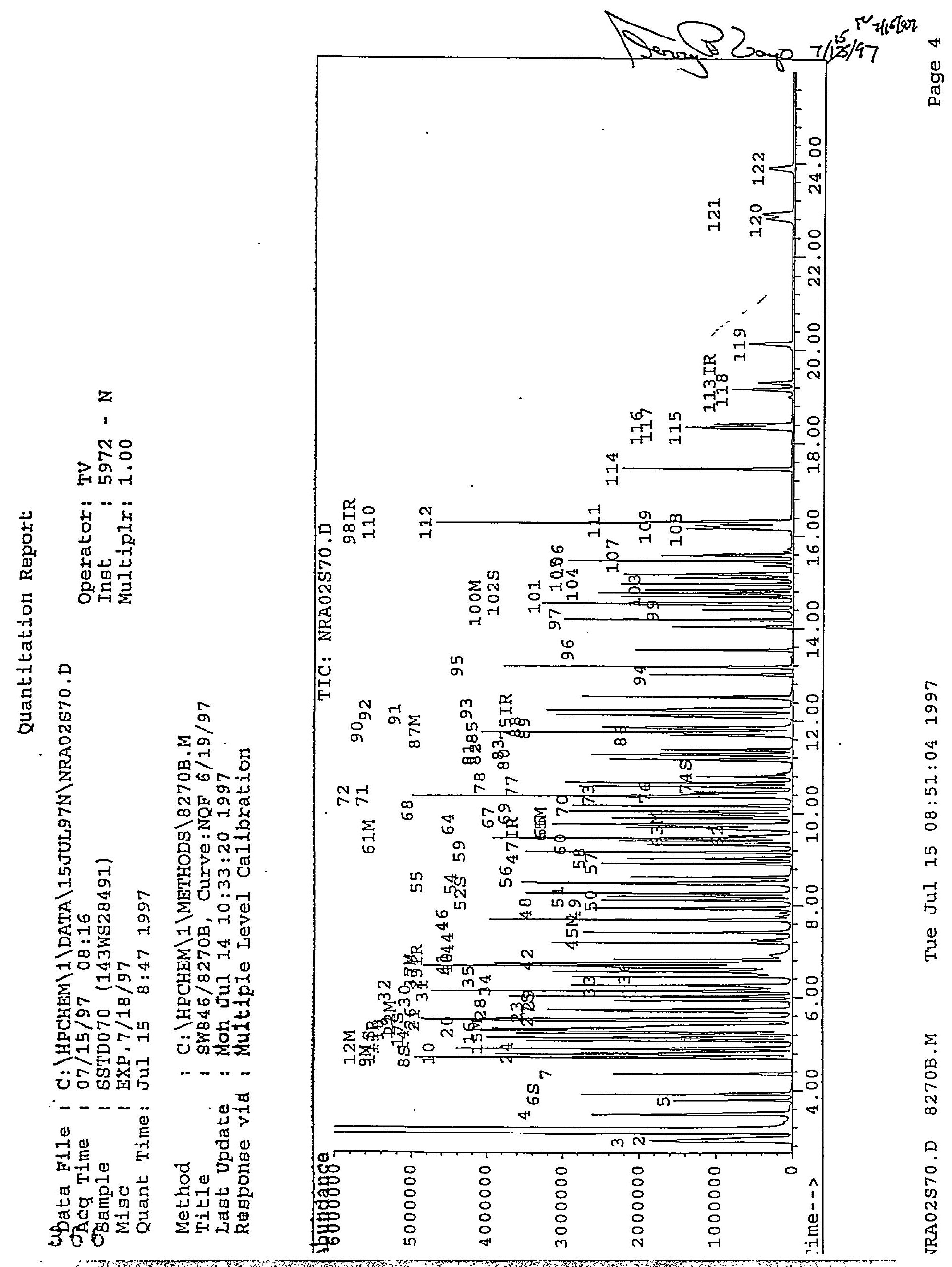


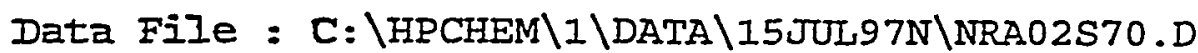

Acq Time $=07 / 15 / 97 \quad 08: 16$

Sample = SSTD070 (143WS28491)

Misc : EXP.7/18/97

Quant Time: Jul $15 \quad 8: 47 \quad 1997$
Operator: TV

Inst : $5972-\mathrm{N}$

Multiplr: 1.00

Method : C: $\backslash$ HPCHEM $\backslash I \backslash M E T H O D S \backslash 8270 B . M$

Title : SW846/8270B, Curve:NQF 6/19/97

Iast Ipdate = Mon JuI 14 10:33:20 1997

Tesponse via : Multiple Level Calibration

Internal standards

1) 1,4-Dichlorobenzene- $\mathrm{d4}$

25) Naphthalene-d8

47) Acenaphthene-d10

75) Phenanthrene-dio

98) Chrysene-d12

113) Perylene-d12

System Monitoring Compounds

6) 2-FIuorophenol

B) Phenol-d5

11) 2-Chlorophenol-d4

17) 1.2-Dichlorobenzene-d4

27) Nitrobenzene-a5

52) 2-Eluorobipheny 1

74) 2,4,6-Tribromophenol

1D2) Terphenyl-d14

Target Compounds

2) Pyridine

3) N-nitrosodimethylamine

4) 2-Picoline

5) Methyl methanesulfonate

7) Ethyl mettanesulfonate

9) Phenol

ID) Aniline

12) 2-ChIorophenol

13) Decane NOT Present

14) 1,3-Dichlorobenzene

15) 1,4-dichlorobenzene

16) Benzyl alcohol

IB) 1,2-dichlorobenzene

I9) 2-Methylphenol

20) Bis (2-chloroisopropyl) ethe

21) 4-methylphenol

22) N-Nitrosodi-n-propyl amine

23) Hexachloroethane

24) Bis (2-chloroethyl) ether

26) Acetophenome

2B) Nitrobenzene

29) N-nitrosopiperidine

30) Isopiorone

31) 2-Nitrophenol

32) 2.4-Dimethylphenol

R.T. Scan QIon Response Conc Units Area\%

$\begin{array}{rrrrrr}5.13 & 285 & 152 & 542000 & 40.00 \mathrm{ug} / \mathrm{mL} & 0.02 \\ 6.66 & 461 & 136 & 2174614 & -40.00 \mathrm{ug} / \mathrm{mL} & 0.02 \\ 9.41 & 777 & 164 & 943737 & 40.00 \mathrm{ug} / \mathrm{mL} & 0.02 \\ 12.09 & 1085 & 188 & 1193540 & 40.00 \mathrm{ug} / \mathrm{mL} & 0.02 \\ 16.29 & 1569 & 240 & 641902 & 40.00 \mathrm{ug} / \mathrm{mL} & 0.00 \\ 19.29 & 1914 & 264 & 402522 & 40.00 \mathrm{ug} / \mathrm{mL} & 0.02\end{array}$

$\because$ Recovery

$\begin{array}{rrrr}3.93 & 147 & 112 & 1535056 \\ 4.71 & 237 & 99 & 1762500 \\ 4.90 & 259 & 132 & 1471707 \\ 5.29 & 304 & 152 & 776530 \\ 5.74 & 355 & 82 & 1395006 \\ 8.26 & 645 & 172 & 1861292 \\ 10.81 & 938 & 330 & 216554 \\ 14.76 & 1393 & 244 & 1235243\end{array}$

2.95

2.90

3.49

3.79

4.36

4.73

4.79

4.92

0.00

5.08

5.15

5.24

5.31

5.35

5.37

5.51

5.53

5.69

4.82

5.55

5.75

5.94

6.03

6.14

6.15
34

29

97

131

197

239

246

261

279

287

298

306

310

313

329

331

350

250

333

357

378

389

401

402

79
42
93
8
7
94
6
128
146
146
108
146
107
45
108
70
11
63
105
77
11
8
139
12

79

42

93

80

79

94

66

128

146

146

108

146

107

45

108

70

117

63

105

77

114

82

139

122
1849165

954103

1862936

701929

1199748

1808221

1028856

1454929

1379994

1380741

989242

1263579

1248597

3138842

1491341

876670

546644

1180891

1837059

1303119

755452

2602727

750254

1196176
$89.214 \mathrm{ug} / \mathrm{mL}$ $85.419 u \mathrm{~g} / \mathrm{mL}$

$72.905 \mathrm{ug} / \mathrm{mL}$

$70.761 \mathrm{ug} / \mathrm{mL}$

$82.931 \mathrm{ug} / \mathrm{mL}$

$67.039 \mathrm{ug} / \mathrm{mL}$

$67.020 \mathrm{ug} / \mathrm{mL}$

$84.297 \mathrm{ug} / \mathrm{mL}$

$89.21 \%$

$85.42 \%$

$72.90 \%$

$70.76 \%$

$82.93 \%$

$67.04 \%$

$67.02 \%$

$84.30 \%$

Qvalue

$84.97 \mathrm{ug} / \mathrm{mL}$

$86.865 \mathrm{ug} / \mathrm{mL} \quad 95$

$84.001 \mathrm{ug} / \mathrm{mL} \# \quad 76$

$85.436 \mathrm{ug} / \mathrm{mL} \quad 94$

$83.027 \mathrm{ug} / \mathrm{mL} \# \quad 78$

$83.252 \mathrm{ug} / \mathrm{mL} \# \quad 52$

$74.528 \mathrm{ug} / \mathrm{mL} \quad 98$

Not Detected

$74.559 \mathrm{ug} / \mathrm{mL} \quad 96$

$75.384 \mathrm{ug} / \mathrm{mL} \quad 95$

$81.858 \mathrm{ug} / \mathrm{mL} \quad 79$

$85.627 \mathrm{ug} / \mathrm{mLm}^{\mathrm{N}} \mathrm{s} / \mathrm{s7} 97$

$87.626 \mathrm{ug} / \mathrm{mL}$ 1/15/ 94

$74.447 \mathrm{ug} / \mathrm{mL} \# \quad 90$

$77.774 \mathrm{ug} / \mathrm{mL} \quad 96$

$83.391 \mathrm{ug} / \mathrm{mL} \quad 90$

$81.559 u \mathrm{~g} / \mathrm{mL} \# \quad 75$

$77.683 \mathrm{ug} / \mathrm{mL} \# \quad 87$

$82.997 \mathrm{ug} / \mathrm{mL} \# \quad 74$

$80.397 \mathrm{ug} / \mathrm{mL} \# \quad 100$

$73.033 \mathrm{ug} / \mathrm{mL} \# \quad 1$

$80.221 \mathrm{ug} / \mathrm{mL} \# \quad 87$

$75.968 \mathrm{ug} / \mathrm{mL} \# \quad 79$

$71.788 \mathrm{ug} / \mathrm{mL}$

89

$(\#)=$ qualifier art of range $(\mathrm{m})$ = manual integration

(*) Dopes not meet spectral criteria (False Hit) 
Quantitation Report

Data File = C: $\backslash$ HPCHEM \I\DATA \15JUL97N\NRA02570.D

Acq Time : 07/15/97 08:16

Sample : SSTD070 (143WS28491)

Mise $=\operatorname{EXP} .7 / 18 / 97$

Quant Time: Jul 15 8:47 1997

Operator: TV

Inst : $5972-\mathrm{N}$

Multiplr: 1.00

Method $=\mathrm{C}: \backslash$ HPCHEM $\backslash 1 \backslash$ METHODS $\backslash 8270 \mathrm{~B} . \mathrm{M}$

Title : Sw846/8270B, Curve:NQF 6/19/97

Iast Jpdate : Mon Jul 14 10:33:20 1997

Response via : wltiple Level Calibration

Compound

33) Benzoic acid

34) Bis (2-chloroethoxy) methane

35) 2,4-Dichlorophenol

36) A., A.-dimethylphenethylami

37) 1,2,4-Trichlorobenzene

3B) Dodecane NOT Present

39) Naphthalene

40) 4-Chloroaniline

4I) 2,6-Dichlorophenol

42) Hexachlorobutadiene

43) Benzothiazole NOT Present

44) N-nitroso-di-n-butylamine

45) 4-Chloro-3-Methylphenol

46) 2-Methylnaphthalene

48) $1,2,4,5$-Tetrachlorobenzene

49) Hexachlorocyclopentadiene

50) 2,4,6-Trichlorophenol

51) 2,4,5-Trichlorophenol

53) Tetradecane NOT Present

54) 2-Chloronaphthalene

55) 1-Chloronaphthalene

56) 2-Nitroaniline

57) Dimethylphthalate

5B) 2,6-Dinitrotoluene

59) Acenaphthylene

50) 3-Nitroaniline

6I) Acenaphthene

62) 2,4-Dinitrophenol

63) 4-Nitrophenol

64) Dibenzofuran

65) 2,4-Dinitrotoluene

66) Pentachlorobenzene

67) 1-Naphthylamine

6B) 2-Naphthylamine

59) 2,3,4,6-Tetrachlorophenol

7D) Diethylphthalate

71) 4-chloropheryl Phenyl Ethe

T2) Fluorene

73) 4-Nitroaniline

76) 4,6-Dinitro-2-methylphenol

77) N-nitrosodiphenylamine

7B) 1,2-Diphenylizydrazine

79) Tributylphosphate NOT Pres
R.T. Scan QIon Response Conc Unit Qvalue

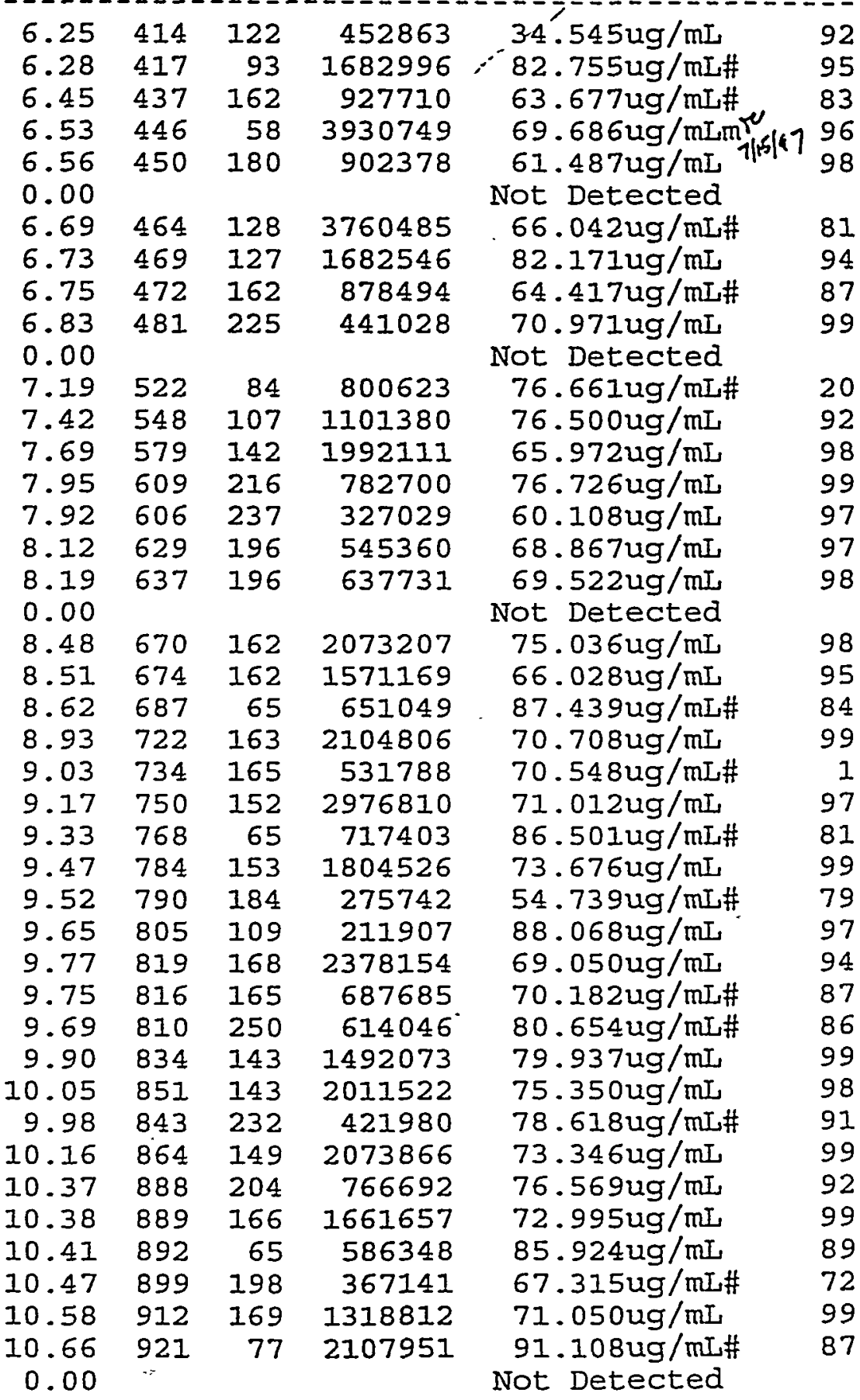

(\#) = qualifier out of range $\cdot(\mathrm{m})=$ manual integration

(*) Does not meet EPA spectral criteria (False Hit) 


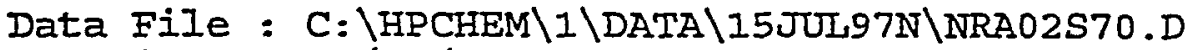

Acq Time : 07/15/97 08:16

Sample : SSTD070 (143WS28491)

Misc : EXP.7/18/97

Operator: TV

Inst : $5972-\mathrm{N}$

Multiplr: 1.00

Quant Time: Jul 15 8:47 1997

Method : C: $\backslash$ HPCHEM $\backslash 1 \backslash M E T H O D S \backslash 8270 B . M$

Title : SW846/8270B, Curve:NQF 6/19/97

Last Update : Mon JuI 14 10:33:20 1997

Response via : Multiple Level Calibration

Compound

BD) Phenacetin

BI) 4-Bromophenyl Phenyl Ether

B2) alpha-BHC

B3) Hexachlorobenzene

B4) Tris (2-CE) phosphate NOT Pr

B5) 4-Aminobiphenyl

B6) beta-BHC

B7) Pentachlorophenol

BB) Pronamide

B9) gamma-BHC

90) Pentachloronitrobenzene

91) Phenanthrene

92) Anthracene

93) delta-BHC

94) Heptachlor

95) Di-n-Butylphthalate

96) Aldrin

97) Fluoranthene

99) Benzidine

100) Pyrene

101) 4,4'-DDE

103) Dieldrin

104) p-Dimethylaminoazobenzene

105) 4.4'-DDD

106) Butylbenzylphthalate

107) 4,4'-DDT

IDB) Methoxychlor

109) 3.3'-Dichlorobenzidine

110) Benzo [a] anthracene

1II) Chrysene

112) Bis(2-ethylhexyl) phthalate

114) Di-n-octylphthalate

115) Benzo [b] fluoranthene

116) 7,12-Dimethylbenz (a) anthra

117) Benzo[k] fluoranthene

118) Benzo [a] pyrene

119) 3-Methylcholanthrene

120) Indeno $(1,2,3-c, d)$ pyrene

121) Dibenz $[a, h]$ anthracene

122) Benzo [ghi] perylene
R.T. Scan QIon Response Conc Unit Qvalue

$\begin{array}{rrr}11.16 & 979 & 108 \\ 11.27 & 991 & 248 \\ 11.29 & 993 & 183 \\ 11.37 & 1003 & 284 \\ 0.00 & & \end{array}$

11.751046

11.691039

$11.74 \cdot 1045$

11.861059

11.831056

$11.75 \quad 1047$

12.131090

$12.22 \quad 1101$

$12.26 \quad 1105$

$13.00 \quad 1190$

$13.17 \quad 1210$

$13.54 \quad 1252$

$14.19 \quad 1327$

$14.40 \quad 1351$

$14.54 \quad 1367$

$14.69 \quad 1384$

$14.83 \quad 1401$

14.961416

$15.16 \quad 1439$

$15.45 \quad 1472$

$15.57 \quad 1486$

$16.16 \quad 1553$

16.221561

$16.28 \quad 1567$

$16.34 \quad 1574$

$16.29 \quad 1568$

$17.45 \quad 1702$

18.351805

169

181

266

173

183

237

178

178

183

100

149

66

202

184

202

246

79

225

235

149

235

227

252

228

228

149

149

252

$\begin{array}{lll}18.33 & 1803 \quad 256\end{array}$

18.421813

19.161898

252

252

$20.13 \quad 2010 \quad 268$

$22.812318 \quad 276$

$22.91 \quad 2330 \quad 278$

23.902444
1069970

420173

291539

413296

1512094

216021

244348

730959

242145

155897

2366265

2458865

202797

383688

3201718

330287

1995587

753058

1887553

350087

387612

343106

596895

981696

454614

768342

446304

1198792

1165929

1218330

2025618

921339

384827

791441

732806

362663

551492

564083

518642
$88.946 \mathrm{ug} / \mathrm{mL \#}$

$79.698 \mathrm{ug} / \mathrm{mL} \#$

$77.724 \mathrm{ug} / \mathrm{mL} \#$

$74.992 \mathrm{ug} / \mathrm{mL} \#$

Not Detected

$69.184 \mathrm{ug} / \mathrm{mL}$

$72.565 \mathrm{ug} / \mathrm{mL} \#$

$70.948 \mathrm{ug} / \mathrm{mL}$

$75.664 \mathrm{ug} / \mathrm{mL} \#$

$80.929 \mathrm{ug} / \mathrm{mL} \#$

$81.676 \mathrm{ug} / \mathrm{mL} \#$

$71.707 \mathrm{ug} / \mathrm{mL} \#$

$77.985 \mathrm{ug} / \mathrm{mL}$

$89.193 \mathrm{ug} / \mathrm{mL} \#$

$84.957 \mathrm{ug} / \mathrm{mL} \#$

$75.441 \mathrm{ug} / \mathrm{mL}$ \#

$90.343 \mathrm{ug} / \mathrm{mr}$

$69.676 \mathrm{ug} / \mathrm{mL} \#$

$57.819 \mathrm{ug} / \mathrm{mL}$ \#

$79.990 \mathrm{ug} / \mathrm{mL} \#$

$87.250 \mathrm{ug} / \mathrm{mL} \#$

$105.184 \mathrm{ug} / \mathrm{mL}$

$75.970 \mathrm{ug} / \mathrm{mL}^{\#}$

$85.700 \mathrm{ug} / \mathrm{mL} \#$

$72.319 u \mathrm{~g} / \mathrm{mL}$

$71.822 \mathrm{ug} / \mathrm{mL} \#$

$68.214 \mathrm{ug} / \mathrm{mL}$

$67.302 \mathrm{ug} / \mathrm{mL}$

$73.898 \mathrm{ug} / \mathrm{mL}$

$73.417 \mathrm{ug} / \mathrm{mL}$

$74.574 \mathrm{ug} / \mathrm{mL}$

$82.621 \mathrm{ug} / \mathrm{mL}$

$77.375 \mathrm{ug} / \mathrm{mL} \#$

$82.547 \mathrm{ug} / \mathrm{mL}$

$76.766 \mathrm{ug} / \mathrm{mL} \#$

$73.683 \mathrm{ug} / \mathrm{mL}$

$70.767 \mathrm{ug} / \mathrm{mL}$

$67.985 \mathrm{ug} / \mathrm{mL}$

$68.656 \mathrm{ug} / \mathrm{mL}$

$68.959 \mathrm{ug} / \mathrm{mL}$
65

86

74

83

$$
96
$$

63

98

94

$$
77
$$

68

95

$$
98
$$

91

78

93

96

69

89

60

100

90

62

86

100

84

98

\section{5}

99

99

98

100

\section{8}

97

88

88

99

98

87

90

(\#) = qualifier out of range $(\mathrm{m})$ = manual integration

(*) Does not meet EPA spectral criteria (False Hit) 
MANUAI I IEGRATION FOR 1,2-dichlorobenzene

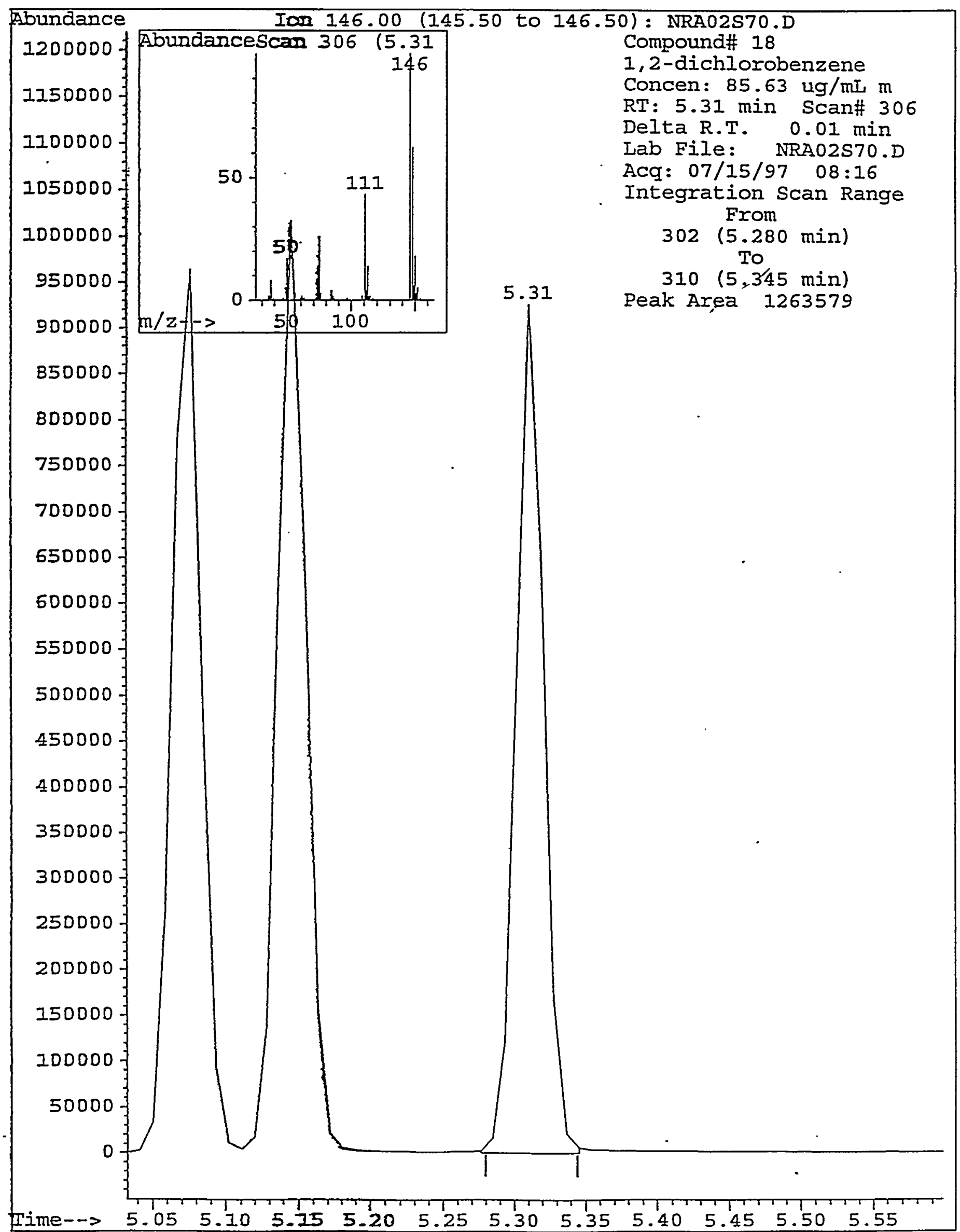


$M A N$ U I IN TEGRATION FOR A.,A.-dimethylphenethylamine

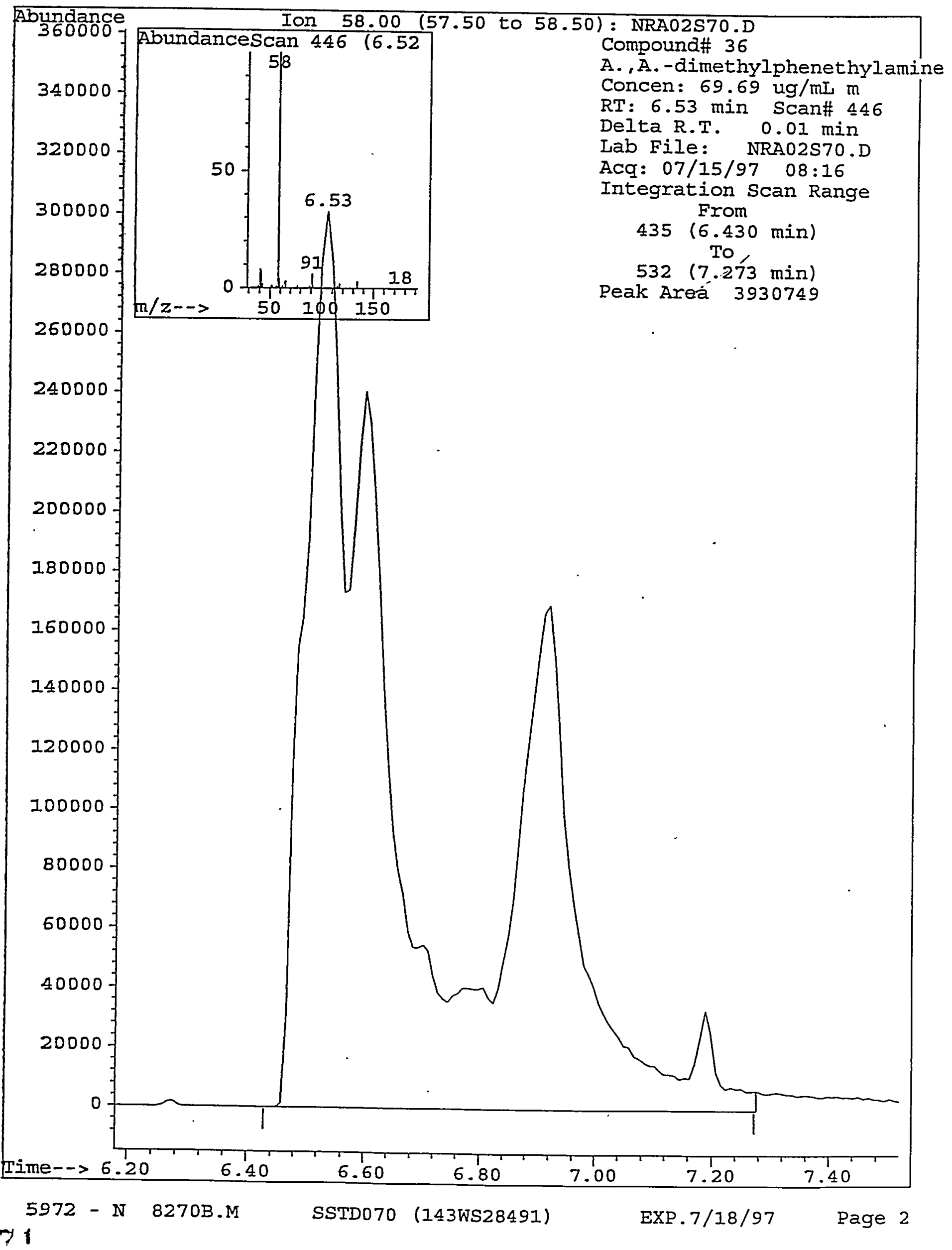


INITIAL CALIBRATION REPORT

EPA METHOD 8270B, Rev 2.

$$
\begin{array}{ll}
\text { SSTDO05 }=\text { NOFO4S5.D } & \text { SSTD070 }=\text { NOFO2S70.D } \\
\text { SSTDO4O }=\text { NOFO5S4O.D } & \text { SSTD100 }=\text { NOFO6S10.D } \\
& \text { SSTD140 }=\text { NOF03S14.D }
\end{array}
$$

Calibration Check Compounds: \%RSD must be $<30 . \%$

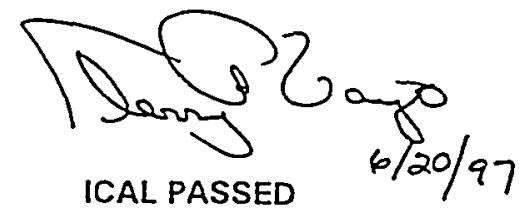

ICAL PASSED
RF005 RF040 RF070 RF100 RF140 RF-ave RSD

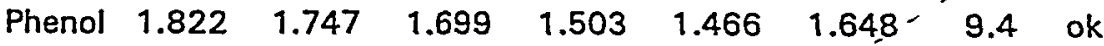

$\begin{array}{lllllllll}\text { 1.4-dichlorobenzene } & 1.461 & 1.469 & 1.414 & 1.239 & 1.224 & 1.361 & 8.9 & \text { ok }\end{array}$

$\begin{array}{lllllllll}\text { 2-Nitrophenol } & 0.201 & 0.205 & 0.189 & 0.164 & 0.162 & 0.184 & 11.0 & \text { ok }\end{array}$

$\begin{array}{lllllllll}\text { 2,4-Dichlorophenol } & 0.271 & 0.291 & 0.269 & 0.245 & 0.239 & 0.263 & 8.0 & \text { ok }\end{array}$

$\begin{array}{llllllllll}\text { Hexachlorobutadiene } & 0.119 & 0.120 & 0.117 & 0.108 & 0.107 & 0.114 & 5.4 & \text { ok }\end{array}$

$\begin{array}{llllllllll}\text { 4-Chloro-3-Methylphenol } & 0.277 & 0.283 & 0.270 & 0.252 & 0.237 & 0.264 & 7.1 & \text { ok }\end{array}$

$\begin{array}{lllllllllll}2,4,6-\text { Trichlorophenol } & 0.342 & 0.357 & 0.340 & 0.317 & 0.316 & 0.334 & 5.3 & \text { ok }\end{array}$

$\begin{array}{llllllllll}\text { Acenaphthene } & 1.282 & 1.138 & 1.070 & 0.948 & 0.896 & 1.067 & 14.4 & \text { ok }\end{array}$

$\begin{array}{llllllllll}\mathrm{N} \text {-nitrosodiphenylamine } & 0.697 & 0.553 & 0.696 & 0.580 & 0.603 & 0.626 & 10.7 & \text { ok }\end{array}$

$\begin{array}{llllllllll}\text { Pentachlorophenol } & 0.094 & 0.096 & 0.123 & 0.117 & 0.115 & 0.109 & 11.9 & \text { ok }\end{array}$

$\begin{array}{llllllllll}\text { Fluoranthene } & 1.241 & 0.909 & 0.985 & 0.931 & 0.852 & 0.983 & 15.4 & \text { ok }\end{array}$

$\begin{array}{lllllllll}\text { Di-n-octylphthalate } & 2.215 & 2.130 & 2.878 & 2.092 & 3.008 & 2.465 & 17.9 & \text { ok }\end{array}$

$\begin{array}{lllllllll}\text { Benzo[a]pyrene } & 1.004 & 0.972 & 1.059 & 0.931 & 0.977 & 0.989 & 4.8 & \text { ok }\end{array}$

System Performance Check Compounds: Avg RF must be $>/=.05$

RF005 RF040 RF070 RF100 RF140 RF-ave RSD

$\begin{array}{llllllll}\text { N-Nitrosodi-n-propyl amine } & 0.889 & 0.857 & 0.828 & 0.726 & 0.650 & 0.790 & 12.6\end{array}$

Hexachlorocyclopentadiene 0.168

2,4-Dinitrophenol 0.133

4-Nitrophenol 0.096

$0.2340 .232 \cdot 0.222$

$\begin{array}{lll}0.205 & 0.209 & 0.223\end{array}$

$0.102 \quad 0.100 \quad 0.104$

0.219

0.214

0.101

$0.215 \cdot 12.5$

$0.197 \quad 18.5$

$0.101 \quad 2.7$

Other Compounds:

RF005

RF040 RF070 RF100

RF140

RF-ave RSD

$\mathrm{N}$-nitrosodimethylamine 0.872

2-Picoline 1.598

Methyl methanesulfonate 0.631

2-Fluorophenol 1.346

Ethyl methanesulfonate 1.100

Phenol-d5 1.673

Aniline 0.885

2-Chlorophenol-d4 1.584

2-Chlorophenol 1.508

1.631

0.894

1.624

1.459

1.465

1.672

0.880

0.781

0.804

1.537

0.846

5.5

1.536

1.593

1.351

0.637

0.601

0.580

0.617

$\begin{array}{lll}1.064 & 1.067 & 1.021\end{array}$

1.210

1.292

0.964

1.043

1.656

1.593

1.451

1.399

1.554

0.987

0.940

0.873

0.848

0.907

1.735

1.502

1.352

1.333

1.501

1.448

Decane NOT Present 0.000

1.698

1.445

1.314

1.277

0.000

1,3-Dichlorobenzene 1.501

Benzyl alcohol 0.936

1,2-Dichlorobenzene-d4 0.985

1.500

0.000

0.000

.000

1.382

$0.924 \quad 0.912$

1.253

0.853

0.733

0.833

0.895

0.825

$0.957 \quad 1.157$

2-Methylphenol 1.271

Bis\{2-chloroisopropyl)ether 3.049

$1.159 \cdot 1.090 \quad 1.019$

0.948

1.097

2.853

3.052

1.471

3.172

3.001

1.293

1.426

0.496

5.9

3.8

4.1

5.8

5.0

7.9

6.2

11.2

11.6

9.3

4.8

13.5

16.0

11.4

4.5

7.0

4:methylphenol 1.553
Hexachloroethane 0.508

0.523

$\begin{array}{ll}0.519 & 0.467\end{array}$

0.461

6.0 
INITIAL CALIBRATION REPORT

EPA METHOD 8270B, Rev. 2.

\begin{tabular}{|c|c|c|c|c|c|c|c|}
\hline $\begin{array}{l}\text { SSTD005 }=\text { NQF04S5.D } \\
\text { SSTD040 }=\text { NOF05S40. }\end{array}$ & 5 & $\begin{array}{l}\text { TD100 }= \\
\text { TD140 }=\end{array}$ & NOFO6 & $\begin{array}{l}\text { O.D } \\
\text { 4.D }\end{array}$ & & & \\
\hline Other Compounds (cont'd): & RF005 & RF040 & RF070 & RF100 & RF140 & RF-ave & RSD \\
\hline Bis (2-chloroethyl)ether & 1.169 & 1.253 & 1.164 & 1.033 & 0.990 & 1.122 & 9.6 \\
\hline Acetophenone & 0.460 & 0.461 & 0.426 & 0.378 & 0.367 & 0.418 & 10.6 \\
\hline Nitrobenzene-d5 & 0.331 & 0.334 & 0.323 & 0.291 & 0.295 & 0.315 & 6.5 \\
\hline Nitrobenzene & 0.312 & 0.323 & 0.309 & 0.281 & 0.277 & 0.301 & 6.8 \\
\hline $\mathrm{N}$-nitrosopiperidine & 0.195 & 0.198 & 0.196 & 0.180 & 0.180 & 0.190 & 4.8 \\
\hline Isop & 0.638 & 0.629 & 0.615 & 0.573 & 0.553 & 0.601 & 6.1 \\
\hline 2,4-Dimethylphenol & 0.455 & 0.335 & 0.317 & 0.280 & 0.282 & 0.334 & 21.6 \\
\hline Benzoic acid & 0.104 & 0.233 & 0.237 & 0.240 & 0.230 & 0.209 & 28.1 \\
\hline Bis(2-chloroethoxy)m & 0.424 & 0.421 & 0.389 & 0.349 & 0.341 & 0.385 & 10.1 \\
\hline .A.-dimethylphenet & 0.870 & 41 & 1.039 & 1.012 & 0.939 & 0.980 & 7.6 \\
\hline 1,2,4-Trichloro & 0.280 & 0.295 & 0.271 & 0.243 & 0.240 & 0.266 & 8.9 \\
\hline Dodecane NOT & 0.000 & $0.000^{\circ}$ & 0.000 & 0.000 & 0.000 & 0.000 & \\
\hline Nap & 1.187 & & 1.083 & 0.937 & 0.936 & 1.055 & 10.8 \\
\hline 4-Chio & 0.430 & 0.410 & 0.398 & 0.354 & 0.328 & 0.384 & 10.9 \\
\hline 2,6-Dichlo & 0.278 & 0.266 & 0.257 & 0.223 & 0.210 & 0.247 & 11.7 \\
\hline Benzothiazole $N$ & 0.000 & 000 & 000 & 0.000 & 0.000 & 0.000 & \\
\hline$N$-nitrosc & 0.192 & & & 0.185 & 0.170 & 0.188 & 6.1 \\
\hline & 0.609 & & & 0.504 & 0.481 & & 10.2 \\
\hline $1,2,4,5-\mathrm{T}$ & 0.476 & & 440 & 0.410 & 0.405 & & 7.3 \\
\hline $2,4,5-7$ & 0.407 & & & 0.371 & & & 5.3 \\
\hline 2-Flu & 1.341 & & 207 & 68 & & & 10.7 \\
\hline Tetradecane & 0.000 & & 000 & 0.000 & & & \\
\hline & 1.447 & & & 65 & & & 12.9 \\
\hline 1-Chloror & 1.087 & & & 24 & & & 10.3 \\
\hline & 0.31 & & & & & & 3.5 \\
\hline & 1.415 & & & & & & 7.9 \\
\hline & 0.317 & & & 26 & & & 3.6 \\
\hline Acer & 1.987 & & & 1.628 & & & 10.1 \\
\hline & 0.363 & 367 & 352 & 0.3 & & & 3.2 \\
\hline Dibe & 1.708 & 38 & 508 & 28 & 262 & & 12.1 \\
\hline 1-Din & 0.431 & .435 & $\$ 17$ & 96 & & & 6.2 \\
\hline Pent & 0.362 & & & 14 & & & 6.9 \\
\hline & 0.374 & & 792 & .788 & & & 26.4 \\
\hline & 1.005 & & & .090 & & & 7.5 \\
\hline $2,3,4,6-\mathrm{Te}$ & 0.22 & & & 0.228 & & & 3.0 \\
\hline $\mathrm{Di}$ & 1.426 & & & 1.138 & & & 10.9 \\
\hline H-Chlorophenyl Phen & 0.53 & & & 0.397 & & & 14.3 \\
\hline & 1.330 & & & 0.882 & & & 18.9 \\
\hline & 0.310 & 0.2 & & & & & 6.7 \\
\hline & 0.127 & & & 0.138 & & & 3.4 \\
\hline 4,6-Dinitro-2-methylphenol & 0.139 & 0.142 & 0.198 & 0.191 & 0.196 & 0.173 & 17.4 \\
\hline
\end{tabular}


INITIAL CALIBRATION REPORT

EPA METHOD 8270B, Rev. 2.

\begin{tabular}{|c|c|c|c|c|c|c|c|}
\hline $\begin{array}{l}\text { SSTD005 }=\text { NQF04S5.D } \\
\text { SSTDO40 }=\text { NOF05S40. }\end{array}$ & $\begin{array}{l}\text { SS } \\
\text { SS } \\
\text { SS }\end{array}$ & $\begin{array}{l}\text {TD070 = } \\
T \text { 100 }= \\
T D 140=\end{array}$ & $\begin{array}{l}\text { NOF02S } \\
\text { NaF06S } \\
\text { NaF03s }\end{array}$ & $\begin{array}{l}70 . D \\
10 . D \\
14 . D\end{array}$ & & & \\
\hline Other Compounds (cont'd): & RF005 & RFO40 & RF070 & RF100 & RF140 & RF-ave & RSD \\
\hline 1,2-Diphenylhydrazine & 0.843 & 0.726 & 0.874 & 0.730 & 0.749 & 0.784 & 8.8 \\
\hline Tributylphosphate NOT Present & 0.000 & 0.000 & 0.000 & 0.000 & 0.000 & 0.000 & \\
\hline Phenacetin & 0.431 & 0.322 & 0.426 & 0.415 & 0.400 & & 11.2 \\
\hline 4-Bromophenyl Phenyl Ether & 0.201 & 0.161 & 0.195 & 0.167 & 0.172 & & 9.8 \\
\hline alpha-BHC & 0.145 & 0.117 & 0.139 & 0.118 & 0.120 & & 10.4 \\
\hline Hexachlorobenzene & 0.211 & 0.166 & 0.201 & 0.178 & 0.181 & & 9.7 \\
\hline is(2-CE)phosphate NOT Present & 0.000 & 0.000 & 0.000 & 0.000 & 0.000 & 0.000 & \\
\hline 4-Aminobiphenyl & 0.824 & 0.713 & 0.789 & 0.668 & & & 10.3 \\
\hline beta-BHC & 0.116 & 0.091 & 0.104 & 0.099 & & & 9.7 \\
\hline Pronamide & 0.381 & 0.3 & 0.345 & 0.309 & & & 10.2 \\
\hline gamma-BHC & 0.130 & 0.094 & 0.108 & 0.096 & & & 14.3 \\
\hline Pentachloronitrobenzene & 0.072 & 0.0 & 0.069 & 0.062 & & & 8.7 \\
\hline Phenanthrene & 1.361 & 1.0 & 1.202 & 1.030 & & & 12.7 \\
\hline cene & 1.373 & 1.0 & 1.176 & 0.956 & & & 6.0 \\
\hline & 0.111 & 0.0 & 0.083 & 0.073 & & & 0.2 \\
\hline Heptachlor & 0.185 & 0.148 & 0.166 & 0.143 & & & 12.3 \\
\hline Di-n-Butylphthalate & 1.773 & 1.3 & 1.519 & 1.357 & & & 13.1 \\
\hline Aldrin & 0.158 & 0.119 & 0.133 & 0.119 & & & 13.7 \\
\hline Benzidine & 0.370 & 0.743 & 0.834 & 0.804 & & & 7.0 \\
\hline Pyrene & 1.597 & 1.464 & 1.758 & 1.232 & & & 13.1 \\
\hline $4,4^{\circ}-\mathrm{DDE}$ & 0.248 & 0.235 & 0.299 & 0.216 & & & 12.2 \\
\hline Terphenyl-d14 & 1.015 & 0.878 & 1.087 & 0.785 & & & 12.7 \\
\hline Dieldrin & 0.233 & 0.227 & 0.281 & 0.198 & & & 12.7 \\
\hline p-Dimethylaminoazobenzene & 0.255 & 0.263 & 0.318 & 0.258 & 0.281 & & 9.5 \\
\hline $4,4^{\prime}-D D D$ & 0.442 & 0.410 & 0.502 & 0.387 & 0.440 & & 9.9 \\
\hline Butylbenzylphthalate & 0.906 & 0.823 & 0.971 & 0.742 & 0.836 & & 10.2 \\
\hline $4,4^{\prime}-$ DDT & 0.378 & 0.373 & 0.442 & 0.357 & 0.373 & & 8.5 \\
\hline Methoxychlor & 0.664 & 0.663 & 0.737 & 0.676 & 0.662 & 80 & 4.7 \\
\hline 3,3'-Dichloro & 0.382 & 0.400 & 0.406 & 0.415 & 0.377 & 396 & 4.0 \\
\hline Benzo[a]anthracene & 1.138 & 1.011 & 1.055 & 0.970 & 1.007 & 36 & 6.2 \\
\hline Chrysene & 1.115 & 0.970 & 1.048 & 0.938 & 0.937 & 1.002 & 7.8 \\
\hline Bis(2-ethylhexyl)p & 1.153 & 0.998 & 1.161 & 0.898 & 0.998 & 1.042 & 10.8 \\
\hline Benzo[b]fluo & 1.248 & 1.104 & 1.273 & 1.138 & 1.259 & 1.204 & 6.5 \\
\hline 7.12-Dimethylbenz(a)a & 0.221 & 0.423 & 0.519 & 0.429 & 0.497 & 0.418 & 28.2 \\
\hline Benzo[k]fluo & 1.147 & 1.052 & 1.152 & 0.902 & 10 & & 9.9 \\
\hline 3-Methylcholanthrene & 0.468 & 0.499 & 0.534 & 0.486 & 0.481 & & 5.1 \\
\hline Indeno(1,2,3-c,d)pyrene & 0.805 & 0.762 & 0.870 & 0.748 & 0.741 & 85 & 6.8 \\
\hline Dibenz $[a, h]$ anthracene & 0.797 & 0.782 & 0.867 & 0.768 & & & 5.6 \\
\hline Benzo[ghilperylene & 0.801 & 0.704 & 0.813 & 0.688 & 0.679 & 0.737 & 8.8 \\
\hline
\end{tabular}




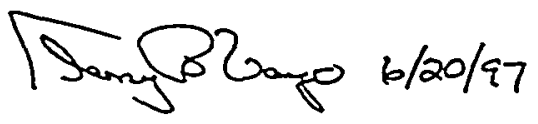

C: \HPCHEM \I\DATA \19JUN97N\NQF01TUN.D

19 Jun 97 5:07 am

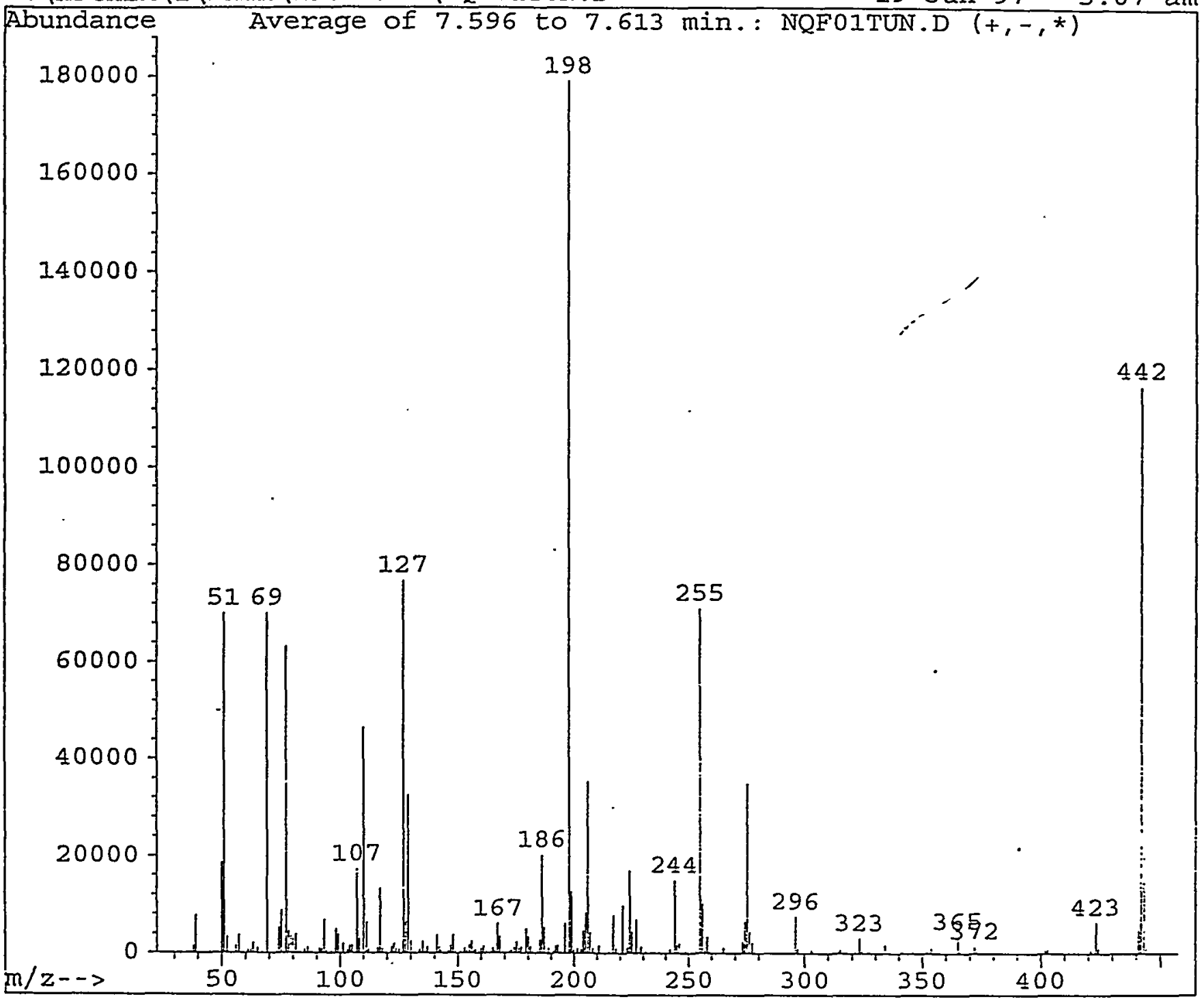

Peak Apex is scan: 296

Average of 3 scans: 295,296,297 minus background scan 290

\begin{tabular}{|c|c|c|c|c|c|}
\hline $\begin{array}{l}\text { Target } \\
\text { Mass }\end{array}$ & $\begin{array}{c}\text { Comparison } \\
\text { Mass }\end{array}$ & $\begin{array}{l}\text { Lower } \\
\text { Limit, } \%\end{array}$ & $\begin{array}{l}\text { Upper } \\
\text { Iimit, \% }\end{array}$ & $\begin{array}{l}\text { Relative } \\
\text { Abundance, } \%\end{array}$ & $\begin{array}{c}\text { Result } \\
\text { Pass/Fail }\end{array}$ \\
\hline 51 & 198 & 30 & 60 & 392 & PASS \\
\hline 68 & 69 & 0 & 2 & $\begin{array}{r}0.2 \\
0.0\end{array}$ & PASS \\
\hline 69 & 198 & 0 & 100 & 39.3 & PASS \\
\hline 70 & 69 & 0 & 2 & 0.0 & PASS \\
\hline 127 & 198 & 40 & 60 & 43.0 & PASS \\
\hline 197 & 198 & 0 & 1 & 0.0 & PASS \\
\hline 198 & 198 & 100 & 100 & 100.0 & PASS \\
\hline 199 & 198 & 5 & 9 & 7.2 & PASS \\
\hline 275 & 198 & 10 & 30 & 19.6 & PASS \\
\hline 365 & 198 & $I$ & 100 & 2.0 & PASS \\
\hline 441 & 443 & 0 & 100 & 22.7 & PASS \\
\hline 442 & 198 & 40 & 100 & 65.3 & PASS \\
\hline 443 & 442 & 17 & 23 & 18.8 & PASS \\
\hline
\end{tabular}


Data File: NQFOITUN.D

Date Acquired: 06/19/97 05:07

Operator ID: TV

Name:

Misc:
50 NG DFTPP (136WS27184)

EXP.11/7/97
Instrument: $5972-\mathrm{N}$

Acq Method: DFTPP625

DA Method: DFTPP625.M

Cal Title: Method 8270 Tuning Standard

Last Cal Update: Wed Apr 09 20:45:09 1997

EXP.11/7/97

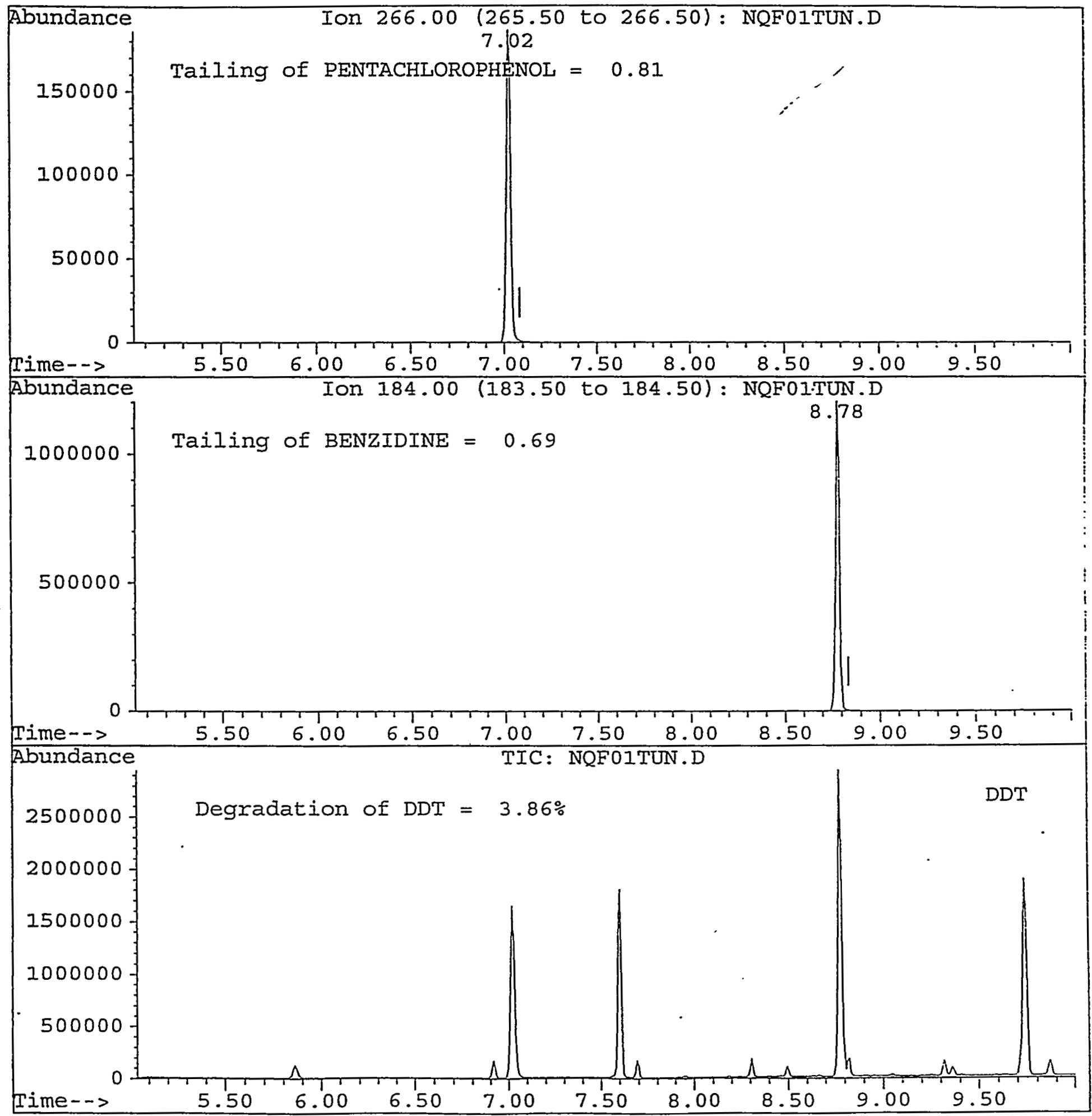

376 
Average of 7.596 to $7.613 \mathrm{~min} .:$ NQFO1TUN.D 50 NG DFTPP (136WS27184)

Modified:added subtracted scaled

$\begin{array}{rrrrr}\mathrm{m} / z & \text { abund. } & \mathrm{m} / \mathbf{z} & \text { abund. } & \mathrm{m} / \mathrm{z} \\ 36.10 & 225 & 55.90 & 1678 & 74.90 \\ 36.70 & 186 & 56.95 & 3888 & 77.00 \\ 37.10 & 273 & 60.95 & 696 & 77.95 \\ 37.95 & 1661 & 62.00 & 771 & 78.90 \\ 39.00 & 7774 & 63.00 & 2208 & 79.90 \\ 39.85 & 172 & 64.00 & 337 & 80.90 \\ 44.90 & 350 & 64.95 & 1242 & 81.95 \\ 50.00 & 18753 & 66.95 & 151 & 83.00 \\ 51.00 & 70257 & 68.95 & 70378 & 84.95 \\ 51.90 & 3470 & 73.05 & 475 & 85.95 \\ 52.85 & 101 & 74.00 & 5447 & 86.90\end{array}$

Average of 7.596 to $7.613 \mathrm{~min}$.: NQF01TUN.D

50 NG DFTPP (136WS27184)

Modified:added subtracted scaled

$\begin{array}{rrrrrrrr}\mathrm{m} / \mathrm{z} & \text { abund. } & \mathrm{m} / \mathrm{z} & \text { abund. } & \mathrm{m} / \mathrm{z} & \text { abund. } & \mathrm{m} / \mathrm{z} & \text { abund. } \\ 102.95 & 981 & 117.95 & 1136 & 130.90 & 481 & 144.95 & 156 \\ 103.90 & 1669 & 118.85 & 101 & 131.95 & 351 & 145.90 & 652 \\ 104.95 & 1744 & 119.80 & 108 & 133.90 & 846 & 146.95 & 1747 \\ 106.95 & 17421 & 121.90 & 1474 & 134.95 & 2481 & 147.90 & 3923 \\ 107.95 & 3076 & 122.90 & 2062 & 135.95 & 868 & 148.95 & 805 \\ 109.95 & 46613 & 123.95 & 1116 & 137.00 & 1367 & 150.15 & 127 \\ 110.95 & 6525 & 124.95 & 939 & 139.70 & 125 & 151.00 & 458 \\ 111.95 & 806 & 126.90 & 77115 & 140.00 & 151 & 151.60 & 325 \\ 112.95 & 118 & 128.00 & 6777 & 141.00 & 3896 & 152.90 & 1166 \\ 116.05 & 1295 & 128.90 & 32704 & 142.00 & 1368 & 153.95 & 756 \\ 116.95 & 13525 & 129.90 & 2845 & 142.80 & 748 & 154.95 & 1994\end{array}$

Average of 7.596 to $7.613 \mathrm{~min}$ : NQFO1TUN.D

50 NG DETPP (136WS27184)

Modified:added subtracted scaled

$\begin{array}{crcrc}\mathrm{m} / z & \text { abund. } & \mathrm{m} / \mathrm{z} & \text { abund. } & \mathrm{m} / \mathrm{z} \\ 155.95 & 2699 & 167.90 & 3612 & 179.90 \\ 157.00 & 784 & 168.90 & 464 & 180.90 \\ 157.85 & 833 & 169.90 & 396 & 182.00 \\ 158.95 & 506 & 170.70 & 272 & 184.00 \\ 159.95 & 1264 & 171.90 & 572 & 185.00 \\ 160.95 & 1672 & 172.95 & 870 & 186.00 \\ 161.90 & 460 & 173.90 & 1450 & 187.00 \\ 163.90 & 258 & 174.95 & 2488 & 188.00 \\ 164.95 & 1272 & 176.00 & 1237 & 188.85 \\ 166.00 & 723 & 176.90 & 1414 & 189.85 \\ 166.90 & 6217 & 178.90 & 5173 & 190.90\end{array}$

$\begin{array}{rc}\text { abund. } & \mathrm{m} / \mathrm{z} \\ 3501 & 191.95 \\ 1710 & 192.95 \\ 385 & 193.95 \\ 501 & 195.95 \\ 2901 & 197.85 \\ 20380 & 198.85 \\ 5525 & 199.85 \\ 542 & 201.45 \\ 1176 & 202.05 \\ 152 & 202.90 \\ 711 & 203.95\end{array}$

abund.

1675

1852

419

6209

179256

12905

840

1064

157

1000

4825

Average of 7.596 to $7.613 \mathrm{~min}$.: NQF01TUN.D

50 NG DFTPP (136WS27184)

Modified:added subtracted scaled

$\begin{array}{crrrrrrr}\mathrm{m} / \mathrm{z} & \text { abund. } & \mathrm{m} / \mathrm{z} & \text { abund. } & \mathrm{m} / \mathrm{z} & \text { abund. } & \mathrm{m} / \mathrm{z} & \text { abund. } \\ 204.95 & 8547 & 223.00 & 1282 & 235.85 & 159 & 253.05 & 533 \\ 205.95 & 35518 & 223.90 & 17405 & 236.85 & 379 & 254.95 & 71427 \\ 206.95 & 4418 & 224.95 & 4557 & 240.85 & 364 & 255.95 & 10359 \\ 207.95 & 1174 & 225.90 & 116 & 241.90 & 926 & 257.00 & 863 \\ 208.95 & 351 & 226.90 & 6962 & 243.95 & 15145 & 257.90 & 3494 \\ 210.85 & 1626 & 228.00 & 992 & 244.95 & 2002 & 258.80 & 483 \\ 212.10 & 102 & 228.90 & 1407 & 245.90 & 2302 & 264.90 & 1329 \\ 214.95 & 315 & 229.90 & 116 & 246.90 & 424 & 272.95 & 2432 \\ 216.90 & 8039 & 230.90 & 576 & 248.85 & 578 & 274.00 & 6663 \\ 217.90 & 1115 & 233.85 & 381 & 249.95 & 105 & 274.90 & 35219 \\ 220.95 & 10012 & 234.85 & 439 & 251.95 & 112 & 275.90 & 4604\end{array}$


Average of 7.596 to $7.613 \mathrm{~min}$ : NQF01TUN.D 50 NG DFTPP (136WS27184)

Modified:added subtracted scaled

$\begin{array}{rrrrrrrr}\mathrm{m} / z & \text { abund. } & \mathrm{m} / \mathrm{z} & \text { abund. } & \mathrm{m} / \mathrm{z} & \text { abund. } & \mathrm{m} / \mathrm{z} & \text { abund. } \\ 76.90 & 2396 & 315.95 & 431 & 351.90 & 836 & 390.95 & 121 \\ 77.95 & 351 & 320.90 & 182 & 352.90 & 630 & 402.00 & 814 \\ 82.95 & 108 & 323.00 & 3142 & 353.90 & 1183 & 402.95 & 1006 \\ 84.90 & 422 & 323.95 & 585 & 354.90 & 119 & 403.90 & 255 \\ 92.90 & 496 & 326.85 & 476 & 364.90 & 3603 & 420.90 & 829 \\ 95.95 & 7617 & 327.85 & 133 & 365.85 & 512 & 422.95 & 6489 \\ 96.90 & 1061 & 332.95 & 156 & 371.00 & 132 & 423.95 & 1259 \\ 32.90 & 941 & 333.95 & 1932 & 371.95 & 1575 & 441.00 & 4981 \\ 303.90 & 152 & 334.95 & 439 & 372.95 & 363 & 441.95 & 117085 \\ 14.00 & 329 & 340.95 & 469 & 382.95 & 349 & 442.95 & 21966 \\ 14.85 & 853 & 345.90 & 505 & 389.85 & 155 & 443.95 & 2086\end{array}$




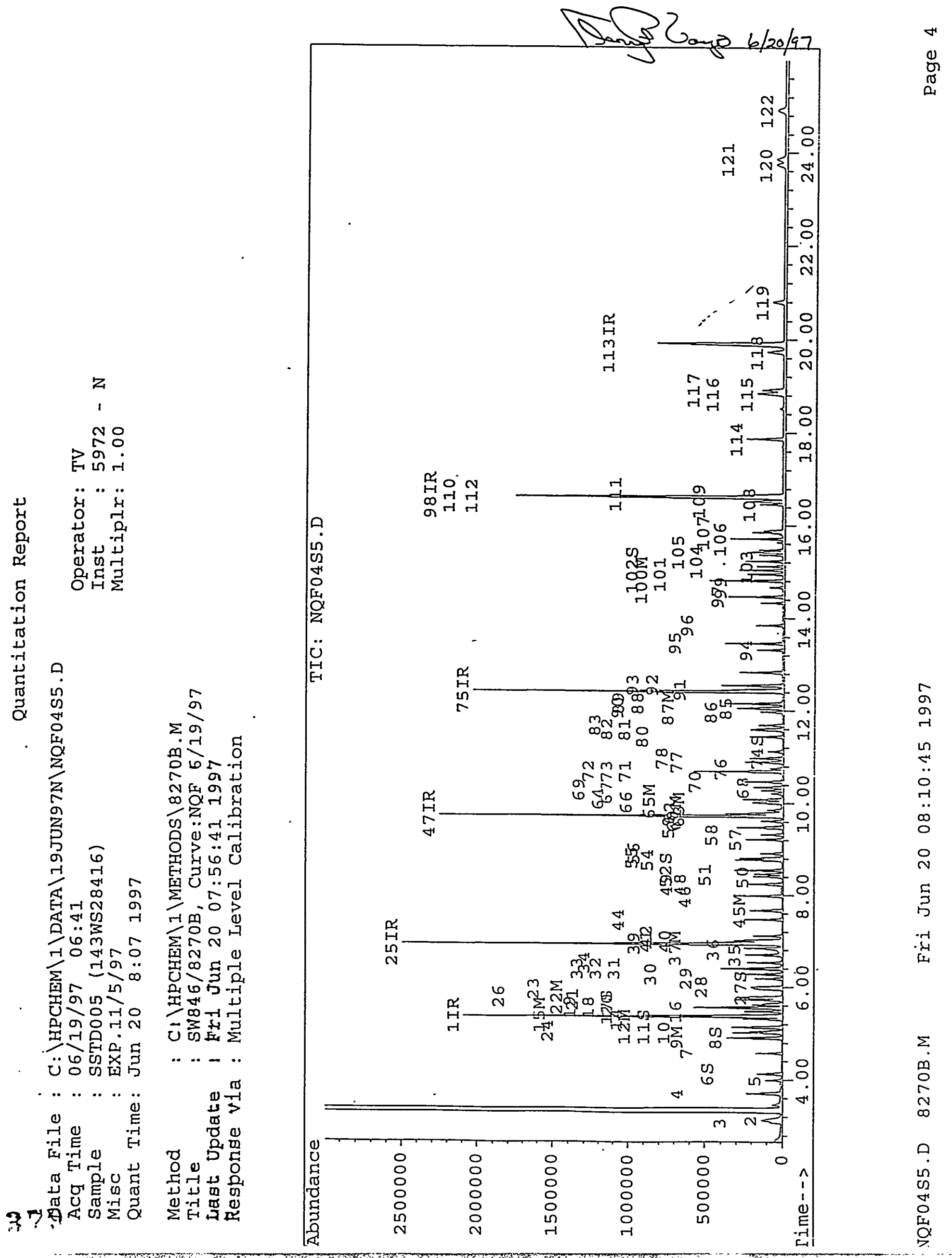




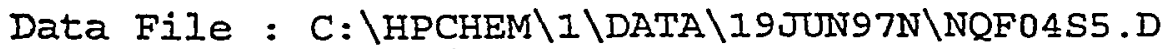

$\begin{array}{ll}\text { Acq Time } & =06 / 19 / 9706: 41 \\ \text { Sample } & : \text { SSTD005 (143WS28416) } \\ \text { Misc } & : \text { EXP.11/5/97 }\end{array}$

Quant Time: Jun 20 8:07 1997

Operator: TV

Inst : $5972-\mathrm{N}$

Multiplr: 1.00

Method : C: $\backslash$ HPCHEM $\backslash I \backslash M E T H O D S \backslash 8270 \mathrm{~B} . \mathrm{M}$

Title : SW846/8270B, Curve:NQF 6/19/97

Iast Update : Fri Jun 20 07:56:41 1997

Response via : Multiple Level Calibration

Internal standards
1) 1,4-Dichlorobenzene-d4

25) Naphthalene-d8

47) Acenaphthene- $\mathrm{d} 10$

75) Phenanthrene-d10

98) Chrysene-d12

113) Perylene-d12

System Monitoring Compounds

6) 2-FIuorophenol

B) Phenol-d5

11) 2-Chlorophenol-d4

17) 1,2-Dichlorobenzene-d4

27) Nitrobenzene-d5

52) 2-Fluorobiphenyl

74) 2,4,6-Tribromophenol

102) Terphenyl-d14

Target Compounds

2) Pyridine

3) N-nitrosodimethylamine

4) 2-Picoline

5) Methyl methanesulfonate

7) Ethyl methanesulfonate

9) Phenol

10) Aniline

12) 2-Chlorophenol

13) Decane NOT Present

14.) 1,3-Dichlorobenzene

15) 1,4-dichlorobenzene

16) Benzyl alcohol

18) 1,2-dichlorobenzene

19) 2-Methylphenol

20) Bis (2-chloroisopropyl) ethe

21) 4-methylphenol

22) N-Nitrosodi-n-propyl amine

23) Hexachloroethane

24) Bis (2-chloroethyl) ether

25) Acetophenone

28.). Nitrobenzene

29) N-nitrosopiperidine

30) Isophorone

3I) 2-Nitroptenol

32) 2,4-Dimethylphenol
R.T. Scan QIon Response Conc Units

Area:

$\begin{array}{rrrrrr}5.37 & 313 & 152 & 568798 & -40.00 \mathrm{ug} / \mathrm{mL} & 0.00 \\ 6.94 & 493 & 136 & 2171482, & 40.00 \mathrm{ug} / \mathrm{mL} & -0.01 \\ 9.73 & 814 & 164 & 1018582 & 40.00 \mathrm{ug} / \mathrm{mL} & -0.01 \\ 12.42 & 1123 & 188 & 1370982 & 40.00 \mathrm{ug} / \mathrm{mL} & -0.01 \\ 16.63 & 1607 & 240 & 1094116 & 40.00 \mathrm{ug} / \mathrm{mL} & -0.01 \\ 19.90 & 1984 & 264 & 823480 & 40.00 \mathrm{ug} / \mathrm{mL} & 0.00\end{array}$

$\div$ Recovery

$\begin{array}{rrrr}4.12 & 169 & 112 & 95703 \\ 4.90 & 259 & 99 & 118985 \\ 5.13 & 285 & 132 & 112655 \\ 5.55 & 333 & 152 & 70043 \\ 5.98 & 383 & 82 & 89971 \\ 8.56 & 679 & 172 & 170728 \\ 11.12 & 974 & 330 & 16213 \\ 15.03 & 1424 & 244 & 138829\end{array}$

\subsection{3}

55

3.09

3.70

3.99

4.56

4.92

5.02

5.15

0.00

5.31

5.39

5.47

5.56

5.56

5.62

5.73

5.76

5.96

5.05

5.79

6.01 .

6.19

6.29

6.40

6.40

50
120
154
220
261
272
287
306
315
324
334
335
341
354
358
380
276
361
386
407
418
431
431

\section{9}

42

93

80

79

94

66

128

146

146

108

146

107

45

108

70

117

63

105

77

114

82

139

122
106993

61982

113592

44882

78245

129541

62952

107238

106707

103901

66578

100709

90376

216796

110433

63199

36130

83085

124761

84792

52960

173240

54652

123538

\begin{tabular}{ll}
\multicolumn{3}{c}{$\%$ Recovery } \\
$4.959 u g / m L$ & $4.96 \%$ \\
$5.386 u g / m L$ & $5.39 \%$ \\
$5.392 u g / m L$ & $5.39 \%$ \\
$5.852 u g / m L$ & $5.85 \% \ddagger$ \\
$5.532 u g / m L$ & $5.53 \%$ \\
$5.497 u g / m L$ & $5.50 \% \ddagger$ \\
$5.047 u g / m L$ & $5.05 \% \ddagger$ \\
$4.867 u g / m L$ & $4.87 \% \ddagger$
\end{tabular}

Qvalue

$5.471 \mathrm{ug} / \mathrm{mL} \quad 97$

$5.508 \mathrm{ug} / \mathrm{mL} \# \quad 71$

$4.777 \mathrm{ug} / \mathrm{mL} \quad 96$

$4.859 \mathrm{ug} / \mathrm{mL} \# \quad 76$

$5.224 \mathrm{ug} / \mathrm{mL} \quad 90$

$5.635 \mathrm{ug} / \mathrm{mL} \# \quad 75$

$4.773 \mathrm{ug} / \mathrm{mL} \# \quad 69$

$5.339 \mathrm{ug} / \mathrm{mL} \quad 98$

Not Detected

$5.437 \mathrm{ug} / \mathrm{mL} \quad 97$

$5.305 \mathrm{ug} / \mathrm{mLm} / \mathrm{m} / 97$

$5.419 \mathrm{ug} / \mathrm{mL} \# \mathrm{p} / 20 / \mathrm{kT} 72$

$5.616 \mathrm{ug} / \mathrm{mL}$

$5.251 \mathrm{ug} / \mathrm{mI}$

$4.795 \mathrm{ug} / \mathrm{mL} \#$

$5.724 \mathrm{ug} / \mathrm{mL}$

$5.498 u g i / m L$

$4.940 \mathrm{ug} / \mathrm{mL} \#$

$5.032 \mathrm{ug} / \mathrm{mL \#}$

$5.402 \mathrm{ug} / \mathrm{mL} \#$

$5.127 \mathrm{ug} / \mathrm{mL \#}$

$5.119 u g / m L \#$

$5.101 \mathrm{ug} / \mathrm{mL} \#$

$5.182 \mathrm{ug} / \mathrm{mL} \#$

$7.379 \mathrm{ug} / \mathrm{mL} \#$
98

99

91

i00

94

80

90

75

100

83

83
81

64

(\#) = qualifier out of range $(m)$ = manual integration

(*) Does not meet EPA spectral criteria (False Hit)

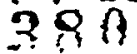




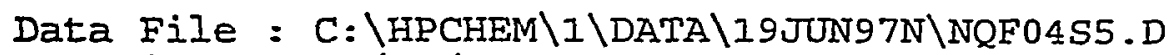

Acq Time : 06/19/97 06:41

Sample = SSTD005 (143WS28416)

Misc : EXP.11/5/97

Quant Time: Jun 20 8:07 1997

Operator: TV

Inst : $5972-\mathrm{N}$

Multiplr: 1.00

$\begin{array}{ll}\text { Method } & : C: \backslash H P C H E M \backslash I \backslash M E T H O D S \backslash 8270 B . M \\ \text { Title } & =S W 846 / 8270 B, \text { Curve:NQF } 6 / 19 / 97 \\ \text { Irast Update } & =\text { Fri Jun 20 07:56:4I 1997 } \\ \text { Response via } & \text { Multiple Level Calibration }\end{array}$

Compound

R.T. Scan QIon Response Conc Unit Qvalue

33) Benzoic acid

34) Bis (2-chloroethoxy) methane

35) 2,4-Dichlorophenol

36) A.,A.-dimethylphenethylami

37) 1,2,4-Trichlorobenzene

38) Dodecane NOT Present

39) Naphthalene

40) 4-Chloroaniline

41) 2,6-Dichlorophenol

42) Hexachlorobutadiene

43) Benzothiazole NOT Present

44) N-nitroso-di-n-butylamine

45) 4-Chloro-3-Methylphenol

46) 2-Methylnaphthalene

48) $1,2,4,5$-Tetrachlorobenzene

49) Hexachlorocyclopentadiene

50) 2,4,6-Trichlorophenol

51) 2,4,5-Trichlorophenol

53) Tetradecane NOT Present

54) 2-Chloronaphthalene

55) I-Chloronaphthalene

56) 2-Nitroaniline

57) Dimethylphthalate

5B) 2,6-Dinitrotoluene

59) Acenaphthylene

60) 3-Nitroaniline

6I) Acenaphthene

62) 2,4-Dinitrophenol

63) 4-Nitrophenol

64) Dibenzofuran

65) 2,4-Dinitrotoluene

66) Pentachlorobenzene

67) 1-Naphthylamine

68) 2-Naphthylamine

69) 2,3,4,6-Tetrachlorophenol

70) Diethylphthalate

71) 4-Chlorophenyl Phenyl Ethe

72) Fluorene

73) 4-Nitroaniline

76) 4,6-Dinitro-2-methylphenol

7.7) N-nitrosodiphenylamine

78) 1,2-Diphenyihyorazine

79) Tributylphosphate NOT Pres $\begin{array}{lll}6.41 & 432 & 122\end{array}$

$\begin{array}{lll}6.53 & 446 \quad 93\end{array}$

$6.70 \quad 466 \quad 162$

$\begin{array}{lll}6.82 & 479 \quad 58\end{array}$

$6.84 \quad 482 \quad 180$

0.00

6.96

7.00

7.02

$496 \quad 128$

$500 \quad 127$

7.11

502

162

0.00

7.47

7.67

7.98

8.24

8.23

8.41

8.46

0.00

8.77

8.82

8.91

9.22

9.33

9.49

9.62

9.78

9.80

9.86

10.09

10.03

10.01

10.22

10.35

10.28

10.47

10.69

10.70

10.69

10.76

10.90

10.97

0.00
$513 \quad 225$

$554 \quad 84$

$577 \quad 107$

$613 \quad 142$

$643 \quad 216$

$641 \quad 237$

$662 \quad 196$

$668 \quad 196$

$704 \quad 162$

$709 \quad 162$

$720 \quad 65$

$\begin{array}{ll}755 & 163\end{array}$

$\begin{array}{ll}768 & 165\end{array}$

$\begin{array}{lll}786 & 152\end{array}$

$801 \quad 65$

$820 \quad 153$

$822 \quad 184$

829109

855168

$849 \quad 165$

$846 \quad 250$

$870 \quad 143$

$885 \quad 143$

$877 \quad 232$

$899 \quad 149$

$924 \quad 204$

925166

$924 \quad 65$

$932 \quad 198$

$948 \quad 169$

$957 \quad 77$
28204

115009

73665

236105

76045

322278

116666

75472

32176

52208

75216

165313

60608

21436

43487

51869

184263

138450

39972

180100

40343

253048

46200

163215

16914

12268

217463

54834

46062

47675

127902

28197

181517

68263

169369

39522

23809

119467

144401
$-4.940 \mathrm{ug} / \mathrm{mLm}_{6 / 2019727}^{\mathrm{r}}$

$5.382 \mathrm{ug} / \mathrm{mL} \# \quad 97$

$5.265 \mathrm{ug} / \mathrm{mL}$ \# 79

$\begin{gathered}14.289 \mathrm{ug} / \mathrm{mLm} \\ 5.255 \mathrm{ug} / \mathrm{mL}\end{gathered} / \mathrm{k} 7_{99}^{88}$

Not Detected

$5.971 \mathrm{ug} / \mathrm{mL} \# \quad 81$

$5.438 \mathrm{ug} / \mathrm{mLm} / 20 \mathrm{ki} 81$

$5.752 \mathrm{ug} / \mathrm{mL} \# \quad 84$

$4.982 \mathrm{ug} / \mathrm{mL}$

99

Not Detected

$4.942 \mathrm{ug} / \mathrm{mL} \quad I I$

$5.219 \mathrm{ug} / \mathrm{mL} \quad 100$

$.5 .624 \mathrm{ug} / \mathrm{mL} \quad 99$

$5.061 \mathrm{ug} / \mathrm{mL} \quad 98$

4.390ug/mL $\quad 94$

$5.193 \mathrm{ug} / \mathrm{mL} \quad 97$

$5.261 \mathrm{ug} / \mathrm{mL}$

93

Not Detected

$5.806 \mathrm{ug} / \mathrm{mL}$

94

$5.475 \mathrm{ug} / \mathrm{mI}$

$4.852 \mathrm{ug} / \mathrm{mL}$

$5.467 \mathrm{ug} / \mathrm{mL}$

$4.988 \mathrm{ug} / \mathrm{mL}=$

$5.534 \mathrm{ug} / \mathrm{mL}$

$5.301 \mathrm{ug} / \mathrm{mL}$

$5.944 \mathrm{ug} / \mathrm{mL}$

$4.923 \mathrm{ug} / \mathrm{mL}$

$5.204 \mathrm{ug} / \mathrm{mL}$

$6.194 \mathrm{ug} / \mathrm{mL}$

$6.102 \mathrm{ug} / \mathrm{mL}$

$5.274 \mathrm{ug} / \mathrm{mL} \#$

$2.604 \mathrm{ug} / \mathrm{mL}$

$4.624 \mathrm{ug} / \mathrm{mL}$

$5.304 \mathrm{ug} / \mathrm{mL}$ \#

$5.693 \mathrm{ug} / \mathrm{mL}$

$5.995 \mathrm{ug} / \mathrm{mL}$

$6.586 \mathrm{ug} / \mathrm{mL}$

$5.727 \mathrm{ug} / \mathrm{mL}$

$4.825 \mathrm{ug} / \mathrm{mL} \#$

$5.498 \mathrm{ug} / \mathrm{mL}$

$5.029 u g / m L \#$

Not Detected

(\#) = qualifier out of range $(\mathrm{m})=$ manual integration

(*) Does not meet EPA spectral criteria (False Hit) 


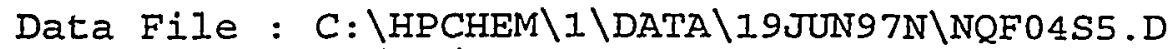

$\begin{array}{ll}\text { Acq Time } & \text { : } 06 / 19 / 9706: 41 \\ \text { Sample } & : \text { SSTD005 (143WS28416) } \\ \text { Misc } & : \text { EXP.11/5/97 }\end{array}$

Quant Time: Jun 20 8:07 1997

Operator: TV

Inst : $5972-\mathrm{N}$

Multiplr: 1.00

Method : C: $\backslash$ HPCHEM $\backslash I \backslash M E T H O D S \backslash 8270 B . M$

Title : Sw846/8270B, Curve:NQF 6/19/97

Last Update : Fri Jun 20 07:56:41 1997

Response via : Multiple Level Calibration

Compound

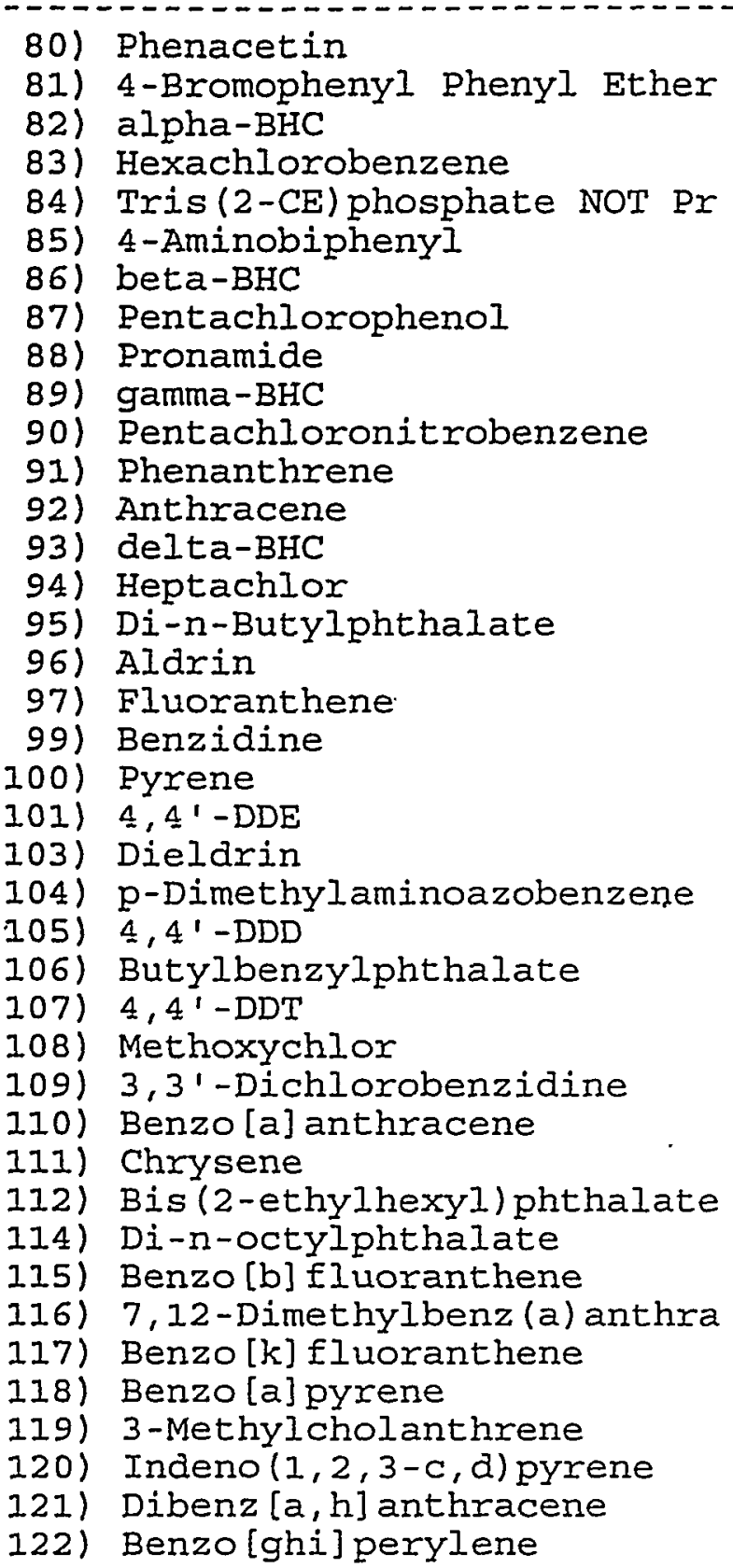

R.T. Scan QIon Response Conc Unit

Qvalue

$\begin{array}{rrr}11.43 & 1010 & 10 \\ 11.58 & 1027 & 24 \\ 11.60 & 1029 & 18 \\ 11.69 & 1039 & 28 \\ 0.00 & & \end{array}$

12.051081

11.971072

12.041080

12.161093

$12.16 \quad 1093$

12.071083

$12.46 \quad 1128$

$12.55 \quad 1138$

$12.57 \quad 1140$

$13.32 \quad 1227$

$13.46 \quad 1243$

$13.84 \quad 1287$

$14.47 \quad 1359$

$14.66 \quad 1381$

$14.81 \quad 1398$

$14.95 \quad 1414$

$15.11 \quad 1433$

$15.23 \quad 1446$

$15.43 \quad 1469$

$15.71 \quad 1502$

$15.85 \quad 1518$

$16.47 \quad 1589$

$16.54 \quad 1597$

16.611605

$16.67 \quad 1612$

$16.60 \quad 1604$

$17.87 \quad 1750$

$18.84 \quad 1862$

$18.82 \quad 1859$

$18.91 \quad 1870$

$19.73 \quad 1964$

20.812088

$23.73 \quad 2424$

$23.84 \quad 2437$

$24.92 \quad 2561$

108
248
183
284
169
181
186
173
183
237
178
178
183
100
149
66
202
184
202
246
79
225
235
149
235
227
252
228
228
149
149
252
256
252
252
268
276
278
27

108

248

84

169

181

266

173

237

178

178

183

100

149

66

202

184

202

246

79

225

235

149

235

227

252

228

228

149

149

252

256

252

252

268

276

278

276
73945

34442

24895

36080

141208

19891

16193

65270

22204

12274

233202

235329

18963

31759

303877

27122

212648

50639

218416

33901

31906

34832

60427

123865

51730

90870

52253

155691

152527

157648

227951

128493

22734

118113

103371

48133

82853

82085

82434
'5.558ug/mL\#

$6.734 \mathrm{ug} / \mathrm{mL} \#$

$5.410 \mathrm{ug} / \mathrm{mL} \#$

$5.414 \mathrm{ug} / \mathrm{mL} \#$

Not Detected

$5.917 \mathrm{ug} / \mathrm{mL}$

$5.495 \mathrm{ug} / \mathrm{mL} \#$

$6.777 \mathrm{ug} / \mathrm{mL}$

$5.349 \mathrm{ug} / \mathrm{mL \#}$

$5.713 \mathrm{ug} / \mathrm{mL} \#$

$5.424 \mathrm{ug} / \mathrm{mL} \#$

$6.080 \mathrm{ug} / \mathrm{mL} \#$

$5.972 \mathrm{ug} / \mathrm{mL}$

$5.805 \mathrm{ug} / \mathrm{mL}$

$5.554 \mathrm{ug} / \mathrm{mL} \#$

$5.897 \mathrm{ug} / \mathrm{mL \#}$

$5.633 \mathrm{ug} / \mathrm{mL}$

$6.740 \mathrm{ug} / \mathrm{mL} \#$

$2.369 u \mathrm{~g} / \mathrm{mL \#}$

$4.594 \mathrm{ug} / \mathrm{mL} \#$

$4.182 \mathrm{ug} / \mathrm{mL}$

$3.952 \mathrm{ug} / \mathrm{mL} \#$

$4.456 \mathrm{ug} / \mathrm{mL \#}$

$4.304 \mathrm{ug} / \mathrm{mL}$

$4.805 \mathrm{ug} / \mathrm{mL}$

$5.713 \mathrm{ug} / \mathrm{mL} \#$

$4.840 \mathrm{ug} / \mathrm{mL}$

$4.625 \mathrm{ug} / \mathrm{mL} \#$

$5.246 \mathrm{ug} / \mathrm{mL}$

$5.332 \mathrm{ug} / \mathrm{mL}$

$4.874 \mathrm{ug} / \mathrm{mL} \#$

$4.118 \mathrm{ug} / \mathrm{mL}$

$5.191 \mathrm{ug} / \mathrm{mL} \#$

$2.429 \mathrm{ug} / \mathrm{mL}$

$5.097 \mathrm{ug} / \mathrm{mL} \#$

$5.015 \mathrm{ug} / \mathrm{mL} \#$

$5.086 \mathrm{ug} / \mathrm{mL}$

$4.802 \mathrm{ug} / \mathrm{mI}$

$5.425 \mathrm{ug} / \mathrm{mL}$

$5.912 \mathrm{ug} / \mathrm{mL} \#$
42

85

71

86

98

57

99

95

88

66

94

97

93

81

92

93

66

85

58

100

87

51

91

89

85

99

94

98

99

98

100

87

93

87

87

100

93

83

84

Data Processed By: Desp omp 6/20/97

(\#) = qualifier out of range (m) = manual integration

(*) Does not meet EPA spectral criteria (False Hit)

Page 3 $3 \& 2$ 
Path: L:IHPCHEMIIIDATAI19JUN97NI

$06 / 19 / 97$

Sample: S5TDO05 (143WS28416)

instrument 1D: $5972-\mathrm{N}$

Analyst: TV

No.

25

47

75

98

113

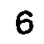

$$
\begin{array}{r}
\text { 1,4ame } \\
\text { Nichlorobemzene-d4 } \\
\text { Naphthalene-d8 } \\
\text { Pcenaphthene-d30 } \\
\text { Phenanthrene-d10 } \\
\text { Chrysene-d12 } \\
\text { Perylene-d12 } \\
\text { 2-Fluorophenol } \\
\text { Phenol-d5 } \\
\text { 2-Chlorophenol-d4 } \\
\text { 1,2-Dichlorobenzene-d4 } \\
\text { Nitrobenzene-d5 } \\
\text { 2-Fluorobipheny1 } \\
\text { 2,4,6-Tribromophenol } \\
\text { Terphenyl-d14 }
\end{array}
$$

3.13

3.09

3.70

3.99

4.56

4.92

5.02

5.15

0.00

5.31

5.39

5.47

5.56

5.56

5.62

5.73

5.76

5.96

5.05

5.79

6.01

6.19

6.29

640

540

5.41

553

6.70

scan
313
493
814
1123
1607
1984
169
259
285
333
383
679
974
1424

$\mathrm{m} / \mathrm{z}$

152.00

136.00

164.00

188.00

240.00

264.00

112.00

99.00

132.00

152.00

82.00

172.00

330.00

244.00

55

50

120

154

220

261

272

287

1

306

315

324

334

335

341

354

358

380

276

361

386

407

418

431

431

432

446

466
79.00

42.00

93.00

80.00

79.00

94.00

66.00

128.00

57.00

146.00

146.00

108.00

146.00

107.00

45.00

108.00

70.00

117.00

63.00

105.00

77.00

114.00

82.00

139.00

122.00

122.00

93.00

162.00

area
568798
2171482
1018582
1370982
1094116
823480

RF

40.000

$1.000 \quad 40.000$

$1.000 \quad 40.000$

$1.000 \quad 46.000$

$1.000 \quad 40.000$

$1.000 \quad 40.000$

$\begin{array}{ccc}95703 & 1.346 & 5.00 \\ 118985 & 1.673 & 5.00 \\ 112655 & 1.584 & 5.00 \\ 70043 & 0.985 & 5.00 \\ 89971 & 0.331 & 5.00 \\ 170728 & 1.341 & 5.00 \\ 16213 & 0.127 & 5.00 \\ 138829 & 1.015 & 5.00\end{array}$

106993

$1.505 \cdot 5.00$

$\begin{array}{lll}61982 & 0.872 \quad 5.00\end{array}$

$\begin{array}{lll}113592 & 1.598 & 5.00\end{array}$

$\begin{array}{lll}44882 & 0.631 & 5.00\end{array}$

$\begin{array}{lll}78245 & 1.100 \quad 5.00\end{array}$

$\begin{array}{lll}129541 & 1.822 & 5.00\end{array}$

$\begin{array}{lll}62952 & 0.885 & 5.00\end{array}$

$\begin{array}{lll}107238 & 1.508 \quad 5.00\end{array}$

$\begin{array}{lll}0 & 0.000 \quad 5.00\end{array}$

$\begin{array}{lll}106707 & 1.501 & 5.00\end{array}$

$103901 \quad 1.461 \quad 5.00$

$\begin{array}{lll}66578 & 0.936 \quad 5.00\end{array}$

$100709 \quad 1.416 \quad 5.00$

$90376 \quad 1.271 \quad 5.00$

$\begin{array}{lll}216796 & 3.049 \quad 5.00\end{array}$

$\begin{array}{lll}110433 & 1.553 \quad 5.00\end{array}$

$\begin{array}{lll}63199 & 0.889 & 5.00\end{array}$

$\begin{array}{lll}36130 & 0.508 & 5.00\end{array}$

$83085 \quad 1.169 \quad 5.00$

$\begin{array}{lll}124761 & 0.460 \quad 5.00\end{array}$

$84792 \quad 0.312$

5.00

52960

0.195

5.00

$\begin{array}{lll}173240 & 0.638 & 5.00\end{array}$

54652

0.201

5.00

123538

28204

0.455

5.00

115009

0.424

5.00

73665

0.271

5.00 
Quantitation Report File: NQF04S5.D

Path: C:IHPCHEMI1IDATAI19JUN97NI

$06 / 19 / 97$

Sample: SSTD005 (143WS28416)

instrument ID: $5972-\mathrm{N}$

Analyst: TV

No.

36

37

38

39

40

41

42

43

44

45

46

48

49

50

51

53

54

55

56

57

58

59

60

61

62

63

64

65

66

67

68

69

70

71

72

73

76

77

78

79

80

81

\begin{tabular}{|c|c|c|c|c|c|c|}
\hline Name & RT & scan & $\mathrm{m} / \mathrm{z}$ & area & RF & amt \\
\hline A.,A.-dimethylphenethylamine & 6.82 & 479 & 58.00 & 236105 & 0.870 & 5.00 \\
\hline 1,2,4-Trichlorobenzene & 6.84 & 482 & 180.00 & 76045 & 0.280 & 5.00 \\
\hline Dodecane NOT Present & 0.00 & 1 & 57.00 & 0 & 0.000 & \\
\hline$\cdot$ Naphthalene & 6.96 & 496 & 128.00 & 322278 & 1.187 & \\
\hline 4-Chloroaniline & 7.00 & 500 & 127.00 & 116666 & 0.430 & \\
\hline 2,6-Dichlorophenol & 7.02 & 502 & 162.00 & 75472 & 0.278 & \\
\hline Hexachlorobutadiene & 7.11 & 513 & 225.00 & 32176 & 0.119 & \\
\hline Benzothiazole NOT Present & 0.00 & 1 & 135.00 & 0 & 0.000 & \\
\hline N-nitroso-di-n-butylamine & 7.47 & 554 & 84.00 & 52208 & 0.192 & .0 \\
\hline 4-Chloro-3-Methylphenol & 7.67 & 577 & 107.00 & 75216 & 0.277 & .0 \\
\hline 2-Methyinaphthalene & 7.98 & 613 & 142.00 & 165313 & 0.609 & .0 \\
\hline $1,2,4,5$-Tetrachlorobenzene & 8.24 & 643 & 216.00 & 60608 & 0.476 & .0 \\
\hline Hexachlorocyclop & 8.23 & 641 & 237.00 & 21436 & 0.168 & .00 \\
\hline 2,4,6-Trichlorophenol & 8.41 & 662 & 196.00 & 43487 & 0.342 & .0 \\
\hline 2,4,5-Trichlo & 8.46 & 668 & 196.00 & 51869 & 0.407 & .08 \\
\hline Tetradecane NOT Present & 0.00 & 1 & 57.00 & 0 & 0.000 & .00 \\
\hline 2-Chioronaphthalene & 8.77 & 704 & 162.00 & 184263 & 1.447 & 5.00 \\
\hline 1-Chlorona & 8.82 & 709 & 162.00 & 138450 & 1.087 & 5.00 \\
\hline $2-\mathrm{Ni}$ & 8.91 & 720 & 65.00 & 39972 & 0.314 & 5.0 \\
\hline Dimethylp & 9.22 & 755 & 163.00 & 180100 & 1.415 & 5.0 \\
\hline 2,6-Dini & 9.33 & 768 & 165.00 & 40343 & 0.317 & 5.0 \\
\hline Acena & 9.49 & 786 & 152.00 & 253048 & 1.987 & 5.00 \\
\hline iline & 9.62 & 801 & 65.00 & 46200 & 0.363 & 5.00 \\
\hline Acen & 9.78 & 820 & 153.00 & 163215 & 1.282 & 5.00 \\
\hline 2,4-Dini & 9.80 & 822 & 184.00 & 16914 & 0.133 & 5.00 \\
\hline $4-N$ & 9.86 & 829 & 109.00 & 12268 & 0.096 & 5.0 \\
\hline Dibe & 10.09 & 855 & 168.00 & 2174 & 1.708 & 5.00 \\
\hline 2,4-Dinits & 10.03 & 849 & 165.00 & & 0.431 & 5.0 \\
\hline Pentachlor & 10.01 & 846 & 250.00 & 46062 & 0.362 & 5.0 \\
\hline 1-Napt & 10.22 & 870 & 143.00 & 4767 & 0.374 & 5.0 \\
\hline 2- $\mathrm{Napl}$ & 10.35 & 885 & 43.00 & 127902 & 1.005 & 5.0 \\
\hline $2,3,4,6$-Tetrach & 10.28 & 877 & 232.00 & 281 & 0.221 & .0 \\
\hline Diethylf & 10.47 & 899 & 149.00 & 181517 & 1.426 & 5.0 \\
\hline 4-Chlorophenyl Phenyl & 10.69 & 924 & 204.00 & 68263 & 0.536 & 5.0 \\
\hline & 10.70 & 925 & 166.00 & 169369 & 1.330 & 5.0 \\
\hline & 10.69 & 924 & 65.00 & 39522 & 0.310 & 5.0 \\
\hline 4,6-Dinitro-2-met & 10.76 & 932 & 198.00 & 23809 & 0.139 & 5.0 \\
\hline N-nitrosodiph & 10.90 & 948 & 169.00 & 119467 & 0.697 & 5.0 \\
\hline 1,2-Diphenyl & 10.97 & 957 & 77.00 & 144401 & 0.843 & \\
\hline ibutylphosphate NOT & 0.00 & 1 & 99.00 & 0 & 0.000 & .0 \\
\hline Phenacetin & 11.43 & 1010 & 108.00 & 73945 & 0.431 & .0 \\
\hline 4-Bromophenyl Phenyl Ether & 11.58 & 1027 & 248.00 & 34442 & 0.201 & 5.0 \\
\hline
\end{tabular}


Quantitation Report

File: NOF04S5.D

Path: C:IHPCHEMI7IDATAI19JUN97NI

$06 / 19 / 97$

Sample: SSTDO05 (143WS28416)

Instrument ID: $5972-\mathrm{N}$

Analyst: TV

\begin{tabular}{|c|c|c|c|c|c|c|c|}
\hline No. & Name & RT & scan & $\mathrm{m} / \mathrm{z}$ & area & RF & amt \\
\hline 82 & alpha-BHC & 11.60 & 1029 & 183.00 & 24895 & 0.145 & \\
\hline 83 & Hexachlorobenzene & 11.69 & 1039 & 284.00 & 36080 & 0.211 & \\
\hline 84 & Tris(2-CE)phosphate NOT Present & 0.00 & 1 & 249.00 & 0 & 0.000 & \\
\hline 85 & 4-Aminobiphenyl & 12.05 & 1081 & 169.00 & 141208 & 0.824 & 5.00 \\
\hline 86 & beta-BHC & 11.97 & 1072 & 181.00 & 19891 & 0.1 .16 & \\
\hline 87 & Pentachlorophenol & 12.04 & 1080 & 266.00 & 16193 & 0.094 & \\
\hline 88 & Pronamide & 12.16 & 1093 & 173.00 & 65270 & 0.381 & \\
\hline 89 & gamma-BHC & 12.16 & 1093 & 183.00 & 22204 & 0.130 & \\
\hline 90 & Pentachloronitrobenzene & 12.07 & 1083 & 237.00 & 12274 & 0.072 & \\
\hline 91 & Phenanthrene & 12.46 & 1128 & 178.00 & 233202 & 1.361 & \\
\hline 92 & Anthracene & 12.55 & 1138 & 178.00 & 235329 & 1.373 & \\
\hline 93 & delta-BHC & 12.57 & 1140 & 183.00 & 18963 & 0.111 & \\
\hline 94 & Heptachlor & 13.32 & 1227 & 100.00 & 31759 & 0.185 & \\
\hline 95 & Di-n-Butylphthalate & 13.46 & 1243 & 149.00 & 303877 & 1.773 & \\
\hline 96 & Aldrin & 13.84 & 1287 & 66.00 & 27122 & 0.158 & \\
\hline 97 & Fluoranthene & 14.47 & 1359 & 202.00 & 212648 & 1.241 & \\
\hline 99 & Benzidine & 14.66 & 1381 & 184.00 & 50639 & 0.370 . & \\
\hline 100 & Pyrene & 14.81 & 1398 & 202.00 & 218416 & 1.597 & \\
\hline 101 & $4,4^{\circ}-D D E$ & 14.95 & 1414 & 246.00 & 33901 & 0.248 & \\
\hline 103 & Dieldrin & 15.11 & 1433 & 79.00 & 31906 & 0.233 & \\
\hline 104 & p-Dimethylaminoazobenzene & 15.23 & 1446 & 225.00 & 34832 & 0.255 & \\
\hline 105 & $4,4^{\prime}-D D D$ & 15.43 & 1469 & 235.00 & 60427 & 0.442 & \\
\hline 106 & Butylbenzylphthalate & 15.71 & 1502 & 149.00 & 123865 & 0.906 & \\
\hline 107 & $4,4^{\prime}-$ DDT & 15.85 & 1518 & 235.00 & 51730 & 0.378 & \\
\hline 108 & Methoxychlor & 16.47 & 1589 & 227.00 & 90870 & 0.664 & \\
\hline 109 & 3,3'-Dichlorobenzidine & 16.54 & 1597 & 252.00 & 52253 & 0.382 & \\
\hline 110 & Benzo[a]anthracene & 16.61 & 1605 & 228.00 & 155691 & 1.138 & \\
\hline 111 & Chrysene & 16.67 & 1612 & 228.00 & 152527 & 1.115 & \\
\hline 112 & Bis(2-ethylhexyl)phthalate & 16.60 & 1604 & 149 & 157648 & 1.153 & \\
\hline 114 & Di-n-octylphthalate & 17.87 & 1750 & 149.00 & 227951 & 2.215 & \\
\hline 115 & Benzolblfluorar & 18.84 & 1862 & 252.00 & 128493 & 1.248 & \\
\hline 116 & 12-Dimethylbenz(a) anthracene & 18.82 & 1859 & 256.00 & 22734 & 0.221 & \\
\hline 117 & Benzo[k]fluoranthene & 18.91 & 1870 & 252.00 & 118113 & 1.147 & \\
\hline 118 & Benzolajpyrene & 19.73 & 1964 & 252.00 & 103371 & 1.004 & \\
\hline 119 & 3-Methylcholanthrene & 20.81 & 2088 & 268.00 & 48133 & 0.468 & \\
\hline 120 & ndeno $(1,2,3-c, d)$ pyrene & 23.73 & 2424 & 276.00 & 82853 & 0.805 & \\
\hline 121 & Dibenz $[a, h] a n t h r a c e n e$ & 23.84 & 2437 & 278.00 & 82085 & 0.797 & \\
\hline 122 & Benzolghi]perylene & 24.92 & 2561 & 276.00 & 82434 & 0.801 & \\
\hline
\end{tabular}




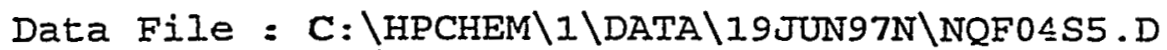

Acq Time $=06 / 19 / 97 \quad 06: 41$

Sample = SSTD005 (143WS28416)

Misc $=$ EXP.11/5/97

Quant Time: Jun 19 7:07 1997

Operator: TV

Inst : $5972-\mathrm{N}$

Multiplr: 1.00
Method
C $: \backslash$ HPCHEM $\backslash I \backslash M E T H O D S \backslash 8270 B . M$
Title
: SW846/8270B, Curve:NQF 6/19/97
Last Update : Fri Jun 20 07:56:41 1997
Response via : Multiple Level Calibration

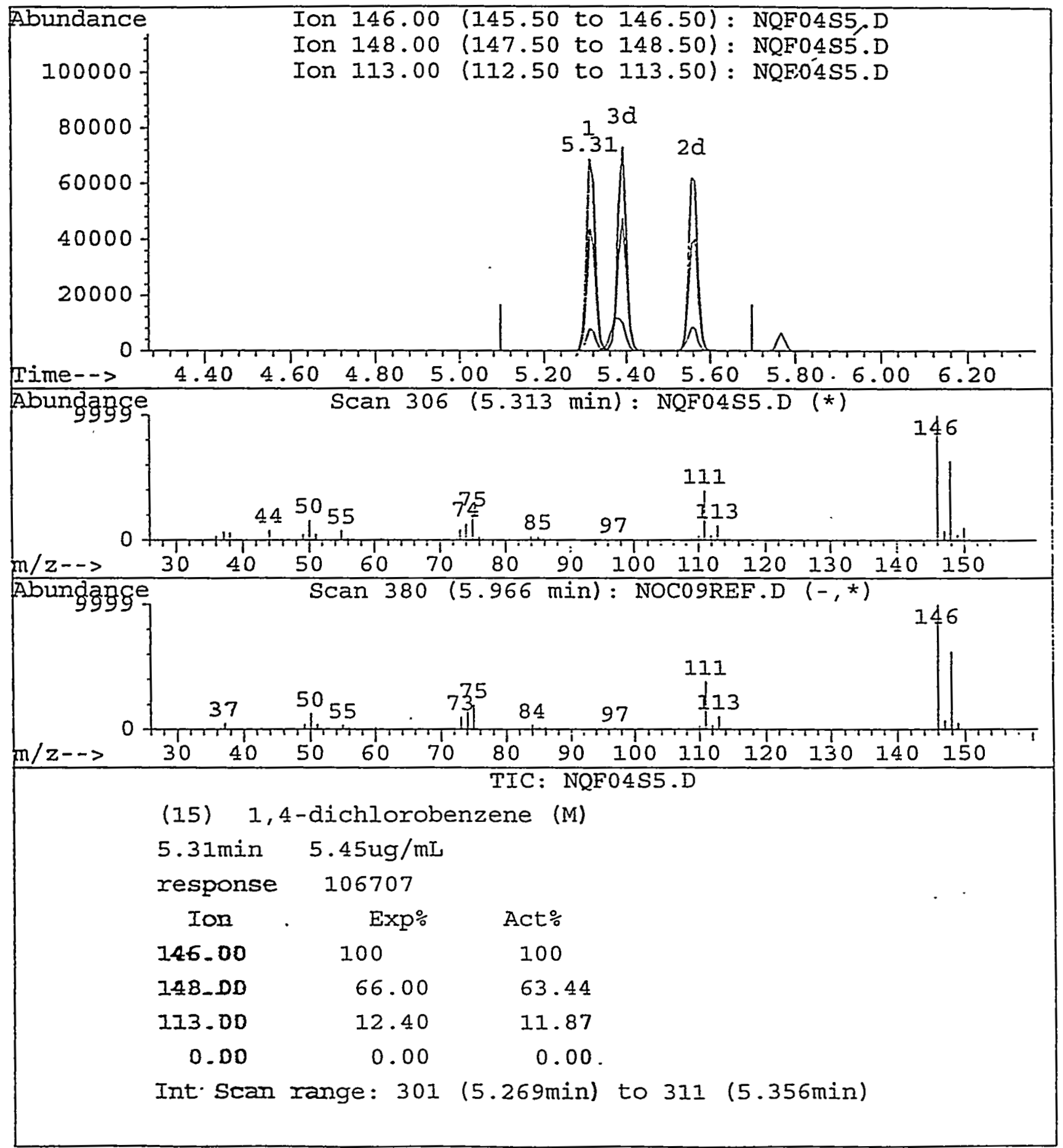


Quantitation Report

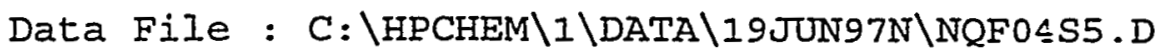

$\begin{array}{ll}\text { Acq Time } & : 06 / 19 / 9706: 41 \\ \text { Sample } & : \text { SSTD005 (143WS28416) } \\ \text { Misc } & : \text { EXP.11/5/97 }\end{array}$

Operator: TV

Inst : $5972-\mathrm{N}$

Quant Time: Jun 20 8:05 1997

Multiplr: 1.00

Method : C: \HPCHEM $\backslash I \backslash M E T H O D S \backslash 8270 \mathrm{~B} . \mathrm{M}$

Title : SW846/8270B, Curve:NQF 6/19/97

Last Update : Fri Jun 20 07:56:41 1997

Response via : Multiple Level Calibration

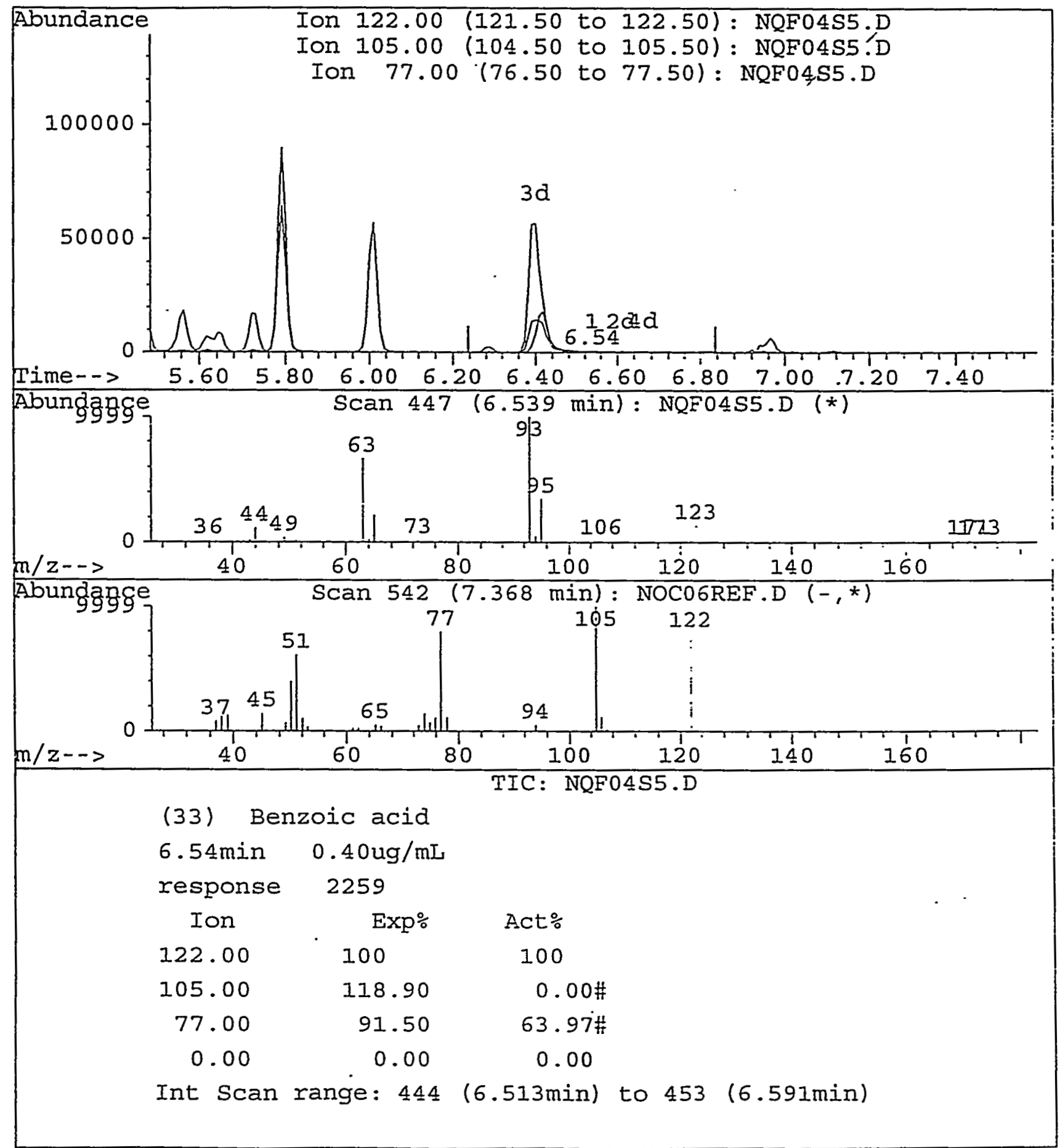

\footnotetext{
NQFO4S5.D 8270B.M Fri Jun 20 08:06:24 1997
} 


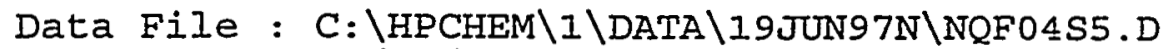

Acq Time : 06/19/97 06:41

Sample : SSTD005 (143WS28416)

Misc : EXP.11/5/97

Quant Time: Jun 20 8:06 1997

Operator: TV

Inst : $5972-\mathrm{N}$

Multiplr: 1.00

Method : C: \HPCHEM $\backslash 1 \backslash$ METHODS $\backslash 8270 \mathrm{~B} . \mathrm{M}$

Title : SW846/8270B, Curve:NQF 6/19/97

Last Update : Fri Jun 20 07:56:41 1997

Response via : Multiple Level Calibration

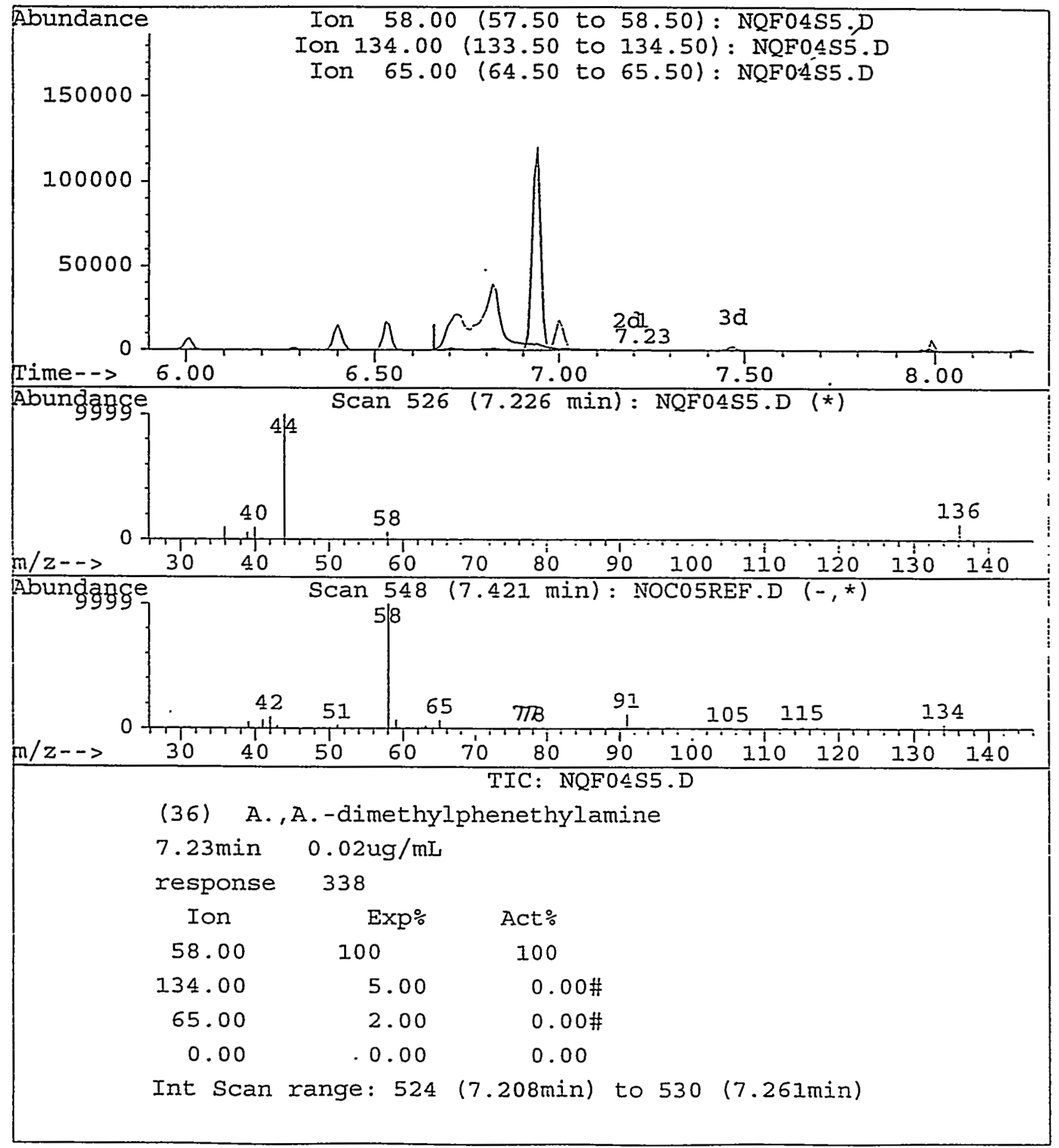


Quantitation Report

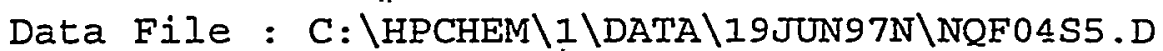

Acq Time : 06/19/97 06:41

Sample : SSTD005 (143WS28416)

Misc : EXP.11/5/97

Quant Time: Jun 20 8:07 1997

Operator: TV

Inst : $5972-\mathrm{N}$

Multiplr: 1.00

Method : C: \HPCHEM \I\METHODS $\backslash 8270 B . M$

Title : Sw846/8270B, Curve:NQF 6/19/97

Last Update : Fri Jun 20 07:56:41 1997

Response via : Multiple Level Calibration

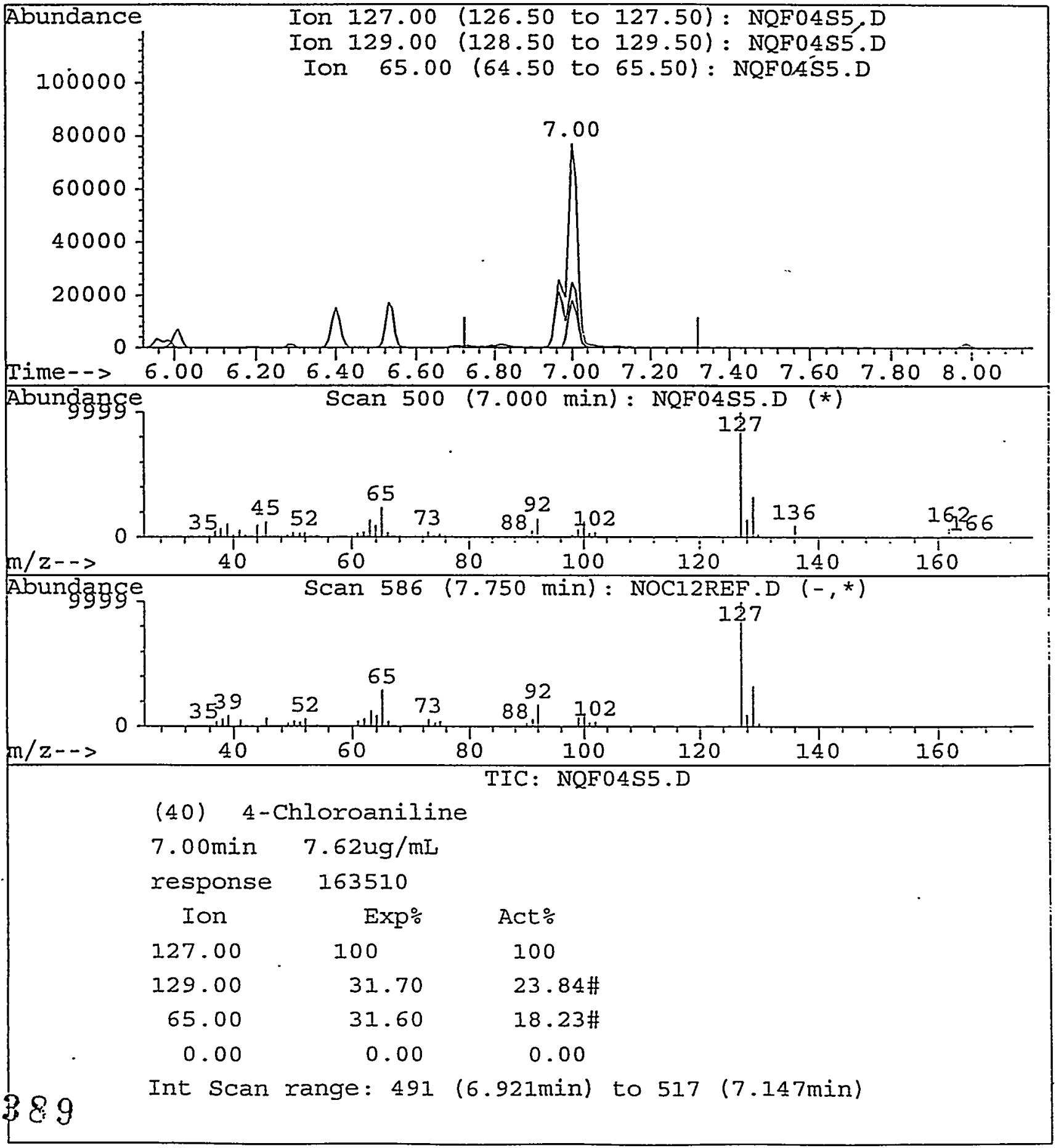


MA N U A L IN TEGRA T I ON FOR 1,4-dichlorobenzene

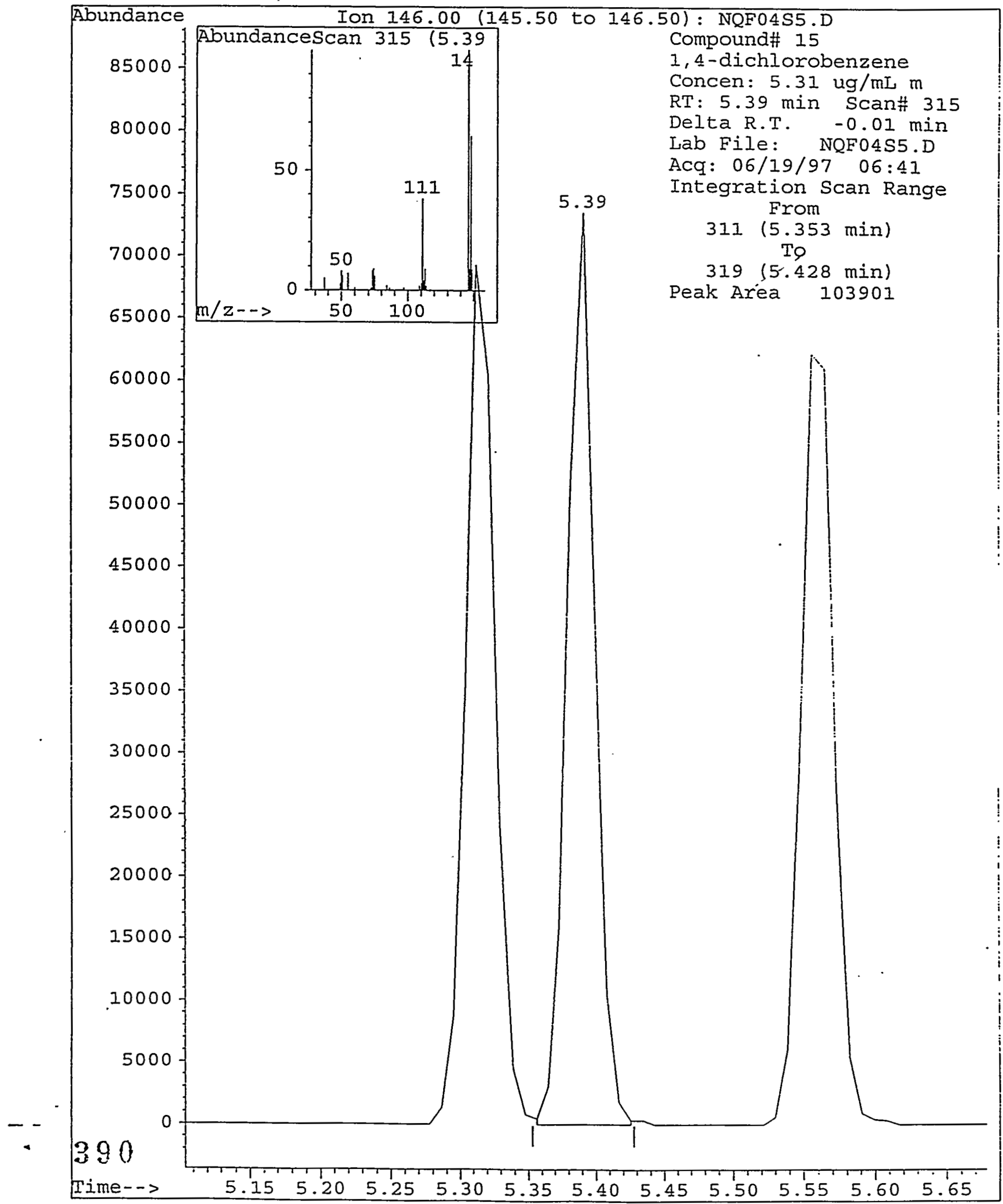

5972 - N 8270B.M SSTD005 (143WS28416)

$\operatorname{EXP} .11 / 5 / 97$

Page 1 
$M A N U A L$ I N T E G R A I ON FOR Benzoic acid

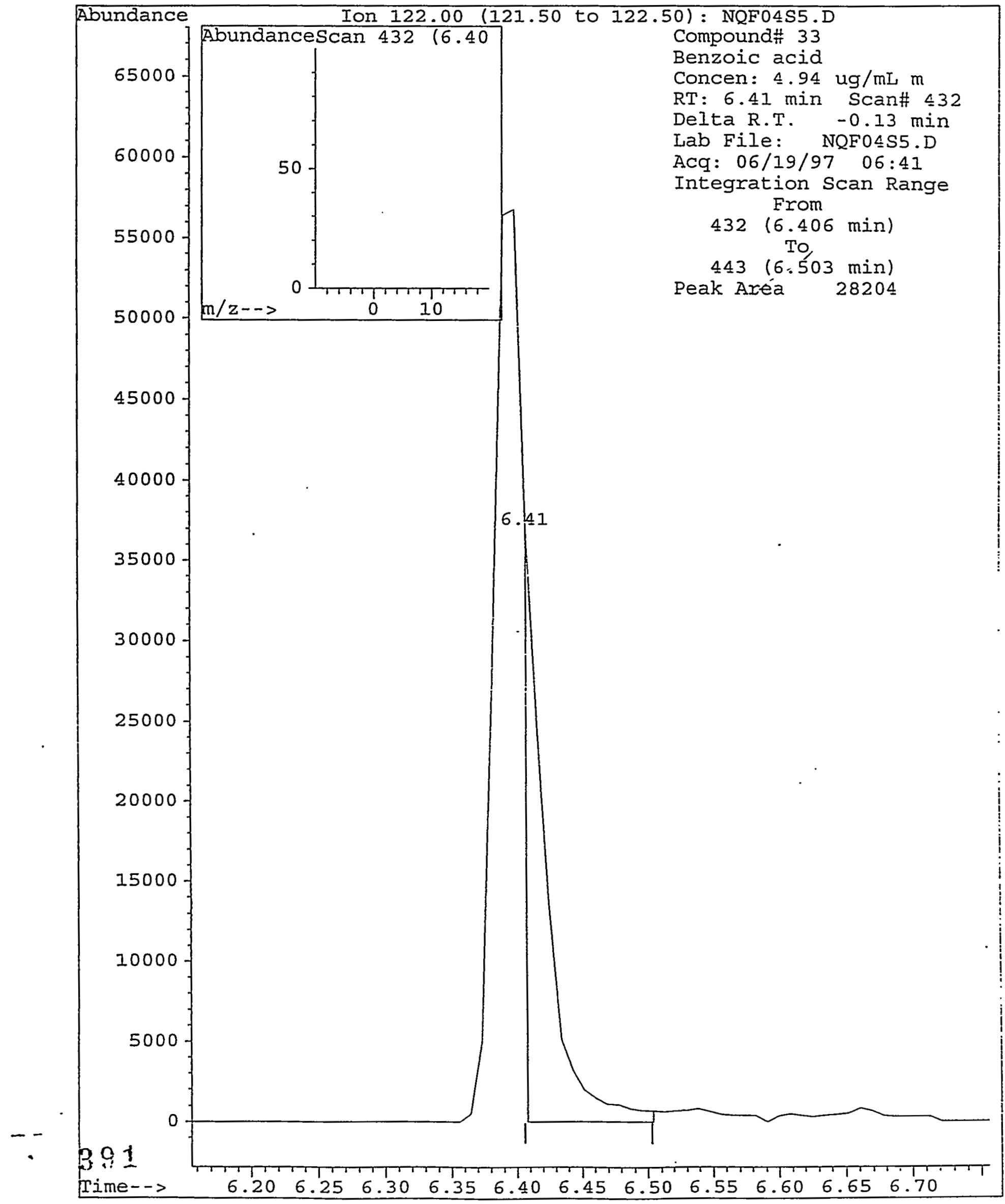

5972 - N 8270B.M SSTD005 (143WS28416)

EXP.11/5/97 Page 2 
M A N U A I IN TE G R A I O N FOR A.,A.-dimethylphenethylamine

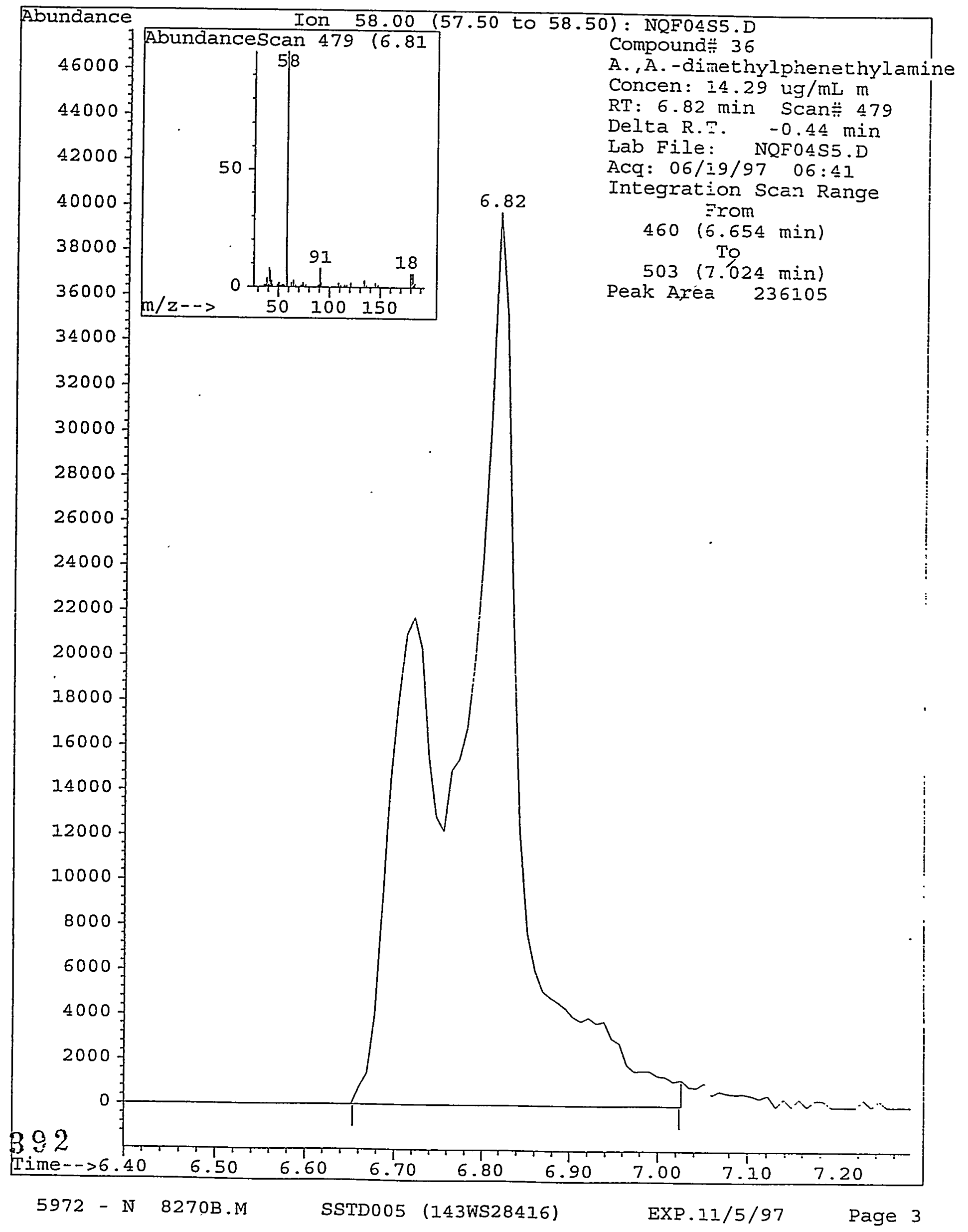


$M A N U A L$ I N TEGRA I I ON FOR 4-Chloroaniline

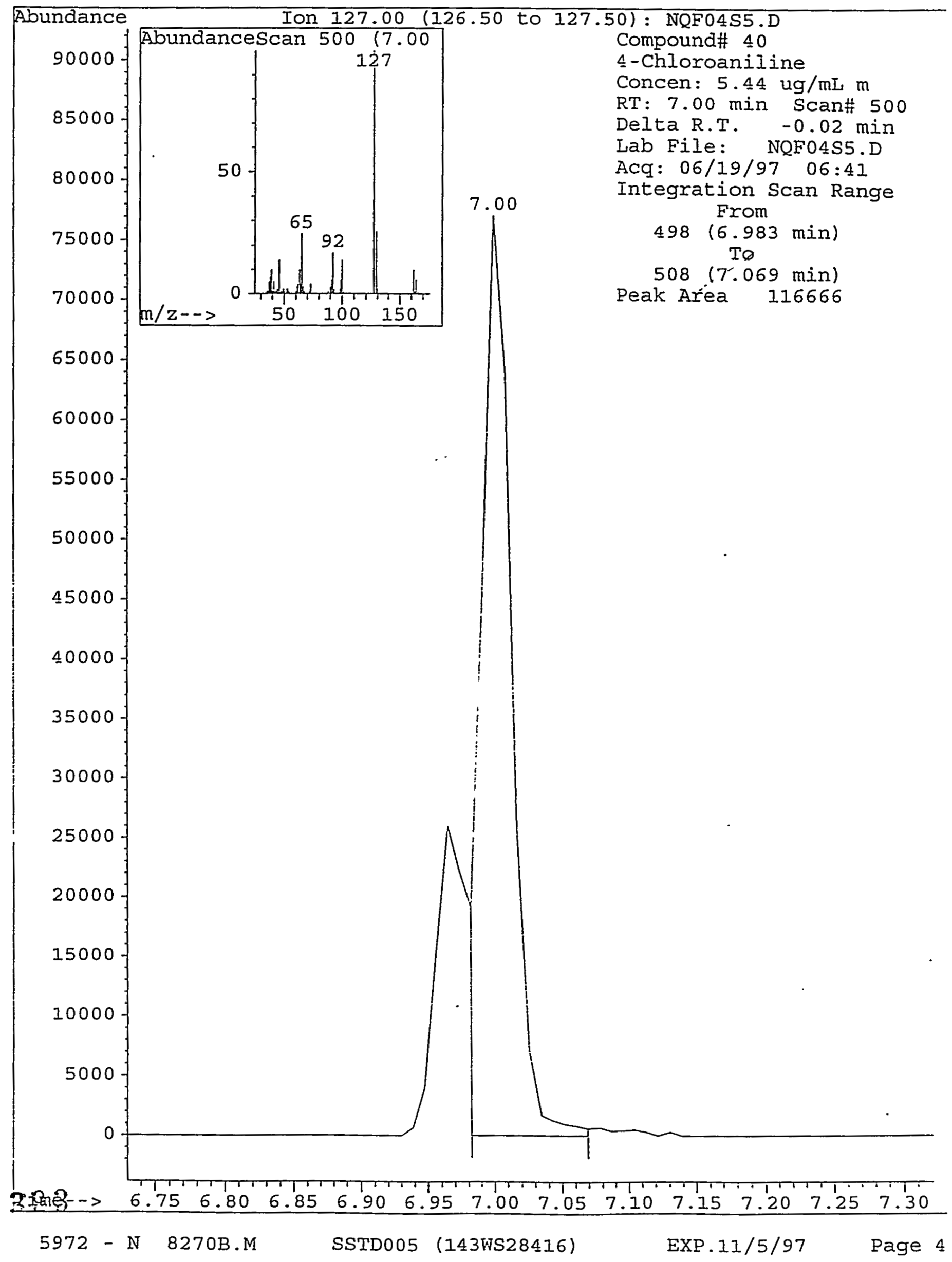




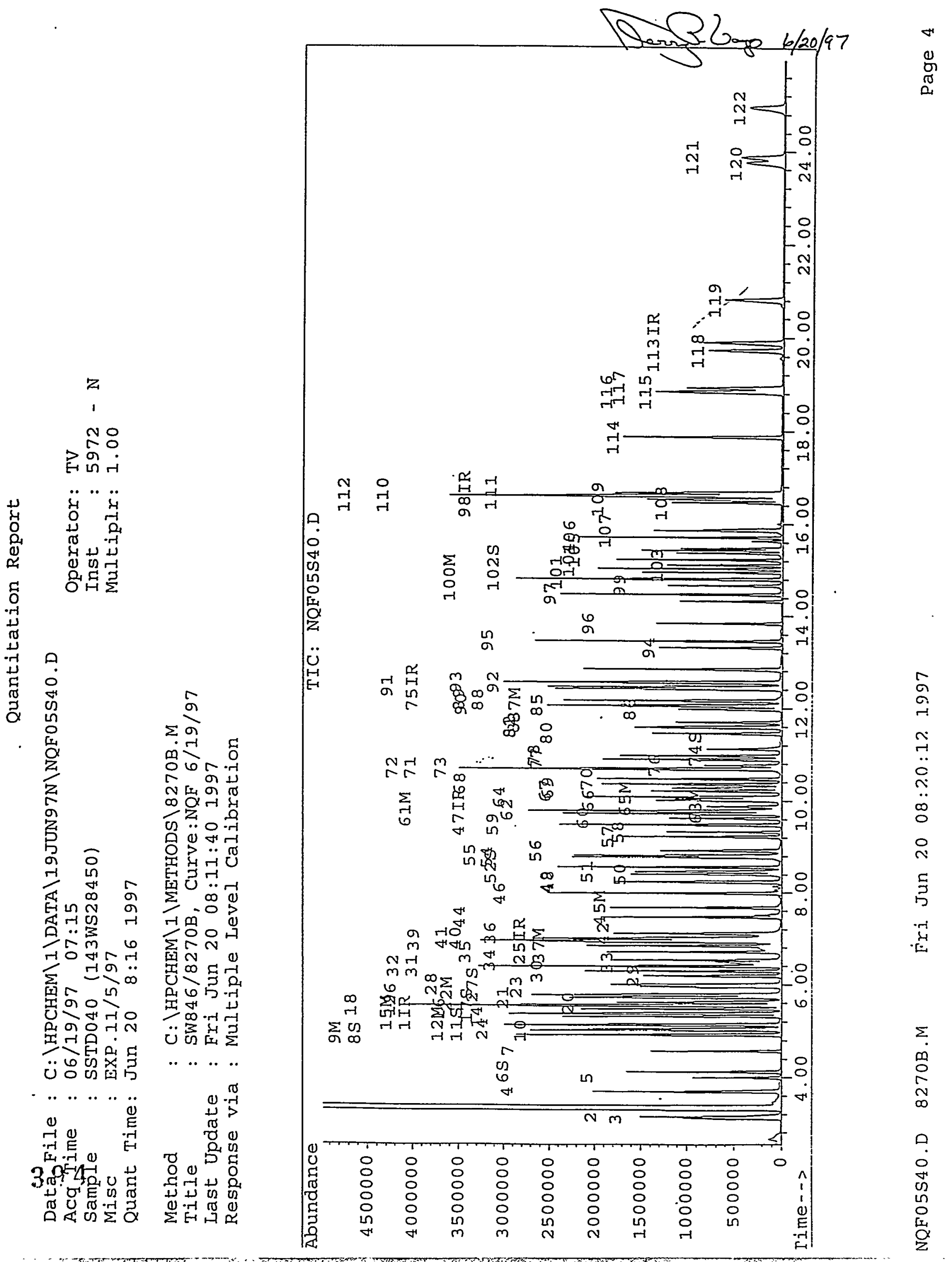




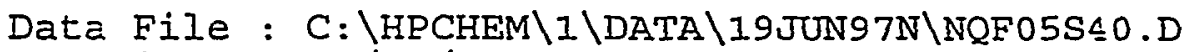

Acq Time : 06/19/97 07:15

Sample : SSTD040 (143WS28450)

Misc : EXP.11/5/97

Quant Time: Jun 20 8:16 1997
Operator: TV

Inst : $5972-\mathrm{N}$

Multiplr: 1.00

Method : C: $\backslash H P C H E M \backslash I \backslash M E T H O D S \backslash 8270 B . M$

Title : SW846/8270B, Curve:NQF 6/19/97

Last Update : Fri Jun 20 08:11:40 1997

Response via : Multiple Level Calibration

Internal standards

1) 1,4-Dichlorobenzene- $d 4$

25) Naphthalene-d8

47) Acenaphthene-d10

75) Phenanthrene-d10

98) Chrysene-d12

113) Perylene-di2

System Monitoring Compounds

6) 2-Fluorophenol

8) Phenol-d5

11) 2-Chlorophenol-d4

17) 1,2-Dichlorobenzene- 24

27) Nitrobenzene-d5

52) 2-Fluorobiphenyl

74) 2,4,6-Tribromophenol

102) Terphenyl-d14

Target Compounds

2) Pyridine

3) N-nitrosodimethylamine

4) 2-Picoline

5) Methyl methanesulfonate

7) Ethyl methanesulfonate

9) Phenol

10) Aniline

12) 2-Chlorophenol

13) Decane NOT Present

14) 1,3-Dichlorobenzene

15) 1,4-dichlorobenzene

16) Benzyl alcohol

18) 1,2-dichlorobenzene

19) 2-Methylphenol

20) Bis (2-chloroisopropyl) ethe

21) 4-methylphenol

22) N-Nitrosodi-n-propyl amine

23) Hexachloroethane

24) Bis (2-chloroethyl)ether

26) Acetophenone

28) Nitrobenzene

29) N-nitrosopiperidine

30) Isophorone

31) 2-Nitrophenol

32) 2,4-Dimethylphenol
R.T. Scan QIon Response Conc Units

Area\%

$\begin{array}{rrrr}5.37 & 326 & 152 & 611722 \\ 6.94 & 507 & 136 & 2299996 \\ 9.73 & 828 & 164 & 1106610 \\ 12.42 & 1137 & 188 & 1681225 \\ 16.63 & 1621 & 240 & 1032899 \\ 19.90 & 1997 & 264 & 843647\end{array}$

$\begin{array}{ll}40.00 \mathrm{ug} / \mathrm{mL} & -0.01 \\ 40.00 \mathrm{ug} / \mathrm{mL} & -0.01 \\ 40.00 \mathrm{ug} / \mathrm{mL} & -0.01 \\ 40.00 \mathrm{ug} / \mathrm{mL} & -0.01 \\ 40.00 \mathrm{ug} / \mathrm{mL} & -0.01 \\ 40.00 \mathrm{ug} / \mathrm{mL} & -0.01\end{array}$

$\div$ Recovery

$\begin{array}{rrrr}4.12 & 183 & 112 & 826717 \\ 4.91 & 274 & 99 & 1013045 \\ 5.13 & 299 & 132 & 1061442 \\ 5.54 & 346 & 152 & 522044 \\ 5.98 & 397 & 82 & 768023 \\ 8.56 & 693 & 172 & 1399222 \\ 11.12 & 988 & 330 & 150032 \\ 15.04 & 1438 & 244 & 906910\end{array}$

$39.831 \mathrm{ug} / \mathrm{mL}$

$39.83 \%$

$42.642 \mathrm{ug} / \mathrm{mL}$

$47.239 \mathrm{ug} / \mathrm{mL}$

$40.552 \mathrm{ug} / \mathrm{mL}$

$44.582 \mathrm{ug} / \mathrm{mL}$

$.41 .471 \mathrm{ug} / \mathrm{mL}$

$42.990 \mathrm{ug} / \mathrm{mL}$

$33.678 \mathrm{ug} / \mathrm{mL}$

$42.64 \%$

$47.24 \%$

$40.55 \%$

$44.58 \%$

$41.47 \%$

$42.99 \%$

$33.68 \%$

$\begin{array}{rrrr}3.14 & 70 & 79 & 997547 \\ 3.10 & 66 & 42 & 546621 \\ 3.70 & 134 & 93 & 1022968 \\ 3.99 & 168 & 80 & 388401 \\ 4.57 & 235 & 79 & 650696 \\ 4.93 & 276 & 94 & 1068898 \\ 5.02 & 286 & 66 & 603654 \\ 5.15 & 301 & 128 & 1038480\end{array}$

Qvalue

$47.429 \mathrm{ug} / \mathrm{mL} \quad 100$

$45.163 \mathrm{ug} / \mathrm{mL} \# \quad 66$

$39.998 \mathrm{ug} / \mathrm{mL} \quad 96$

$39.098 \mathrm{ug} / \mathrm{mL} \# \quad 78$

$40.394 \mathrm{ug} / \mathrm{mL} \quad 90$

$43.231 \mathrm{ug} / \mathrm{mL} \# \quad 77$

$42.555 \mathrm{ug} / \mathrm{mL} \# \quad 50$

$48.077 \mathrm{ug} / \mathrm{mL} \quad 97$

0.00

$5.31 \quad 320 \quad 146$

$5.38 \quad 328 \quad 146$

$5.48 \quad 339 \quad 108$

$5.56 \quad 348 \quad 146$

$5.57 \quad 349 \quad 107$

$5.62 \quad 355 \quad 45$

$5.74 \quad 359$

108

$5.77 \quad 373$

70

$\begin{array}{lll}5.96 & 394 & 117\end{array}$

$5.05 \quad 290 \quad 63$

$5.79 \quad 375 \quad 105$

$6.01 \quad 400$

105
77

$6.19 \leq 21$

$6.30 \quad 433$

114

82

$6.40 \quad 445 \quad 139$

917452

898429

565050

758749

708847

1949402

899889

524463

320147

766205

1061229

743482

455785

1446067

$6.40 \quad 445 \quad 122$

Not Detected

$43.470 \mathrm{ug} / \mathrm{mL} \quad 97$

$42.655 \mathrm{ug} / \mathrm{mL} \quad 97$

$42.763 \mathrm{ug} / \mathrm{mL} \# \quad 72$

$39.344 \mathrm{ug} / \mathrm{mL} \quad 98$

$38.295 \mathrm{ug} / \mathrm{mL} \quad 99$

$40.094 \mathrm{ug} / \mathrm{mL} \# \quad .91$

$43.369 \mathrm{ug} / \mathrm{mL} \quad 99$

$42.424 \mathrm{ug} / \mathrm{mL} \quad 96$

$40.704 \mathrm{ug} / \mathrm{mL} \quad 83$

$43.152 \mathrm{ug} / \mathrm{mL} \# \quad 77$

$43.384 \mathrm{ug} / \mathrm{mL} \# \quad 74$

$42.44 \mathrm{Iug} / \mathrm{mL} \# \quad 100$

$41.597 \mathrm{ug} / \mathrm{mI \#} \quad 6$

$40.198 \mathrm{ug} / \mathrm{mI} \# \quad 82$

$42.273 \mathrm{ug} / \mathrm{mL \#} \quad 81$

472248

769882

(\#) 3 Qualifier out of range $(m)=$ manual integration

(*) Does not meet EPA spectral criteria (Ealse Hit) 
Quantitation Report

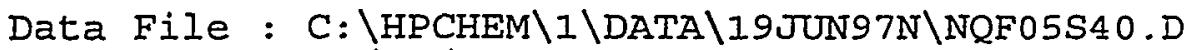

Acq Time : 06/19/97 07:15

Sample : SSTD040 (143WS28450)

Misc : EXP.11/5/97

Quant Time: Jun 20 8:16 1997

Operator: TV

Inst : $5972-\mathrm{N}$

Multiplr: 1.00

Method : C: \HPCHEM $\backslash I \backslash M E T H O D S \backslash 8270 \mathrm{~B} . \mathrm{M}$

Title : SW846/8270B, Curve:NQF 6/19/97

Last Update : Fri Jun 20 08:11:40 1997.

Response via : Multiple Level Calibration

Compound

R.T. Scan QIon Response Conc Unit Qvalue

33) Benzoic acid

34) Bis (2-chloroethoxy) methane

35) 2,4-Dichlorophenol

36) A., A. -dimethylphenethylami

37) 1,2,4-Trichlorobenzene

38) Dodecane NOT Present

39) Naphthalene

40) 4-Chloroaniline

41) 2,6-Dichlorophenol

42) Hexachlorobutadiene

43) Benzothiazole NOT Present

44) N-nitroso-di-n-butylamine

45) 4-Chloro-3-Methylphenol

46) 2-Methylnaphthalene

48) 1,2,4,5-Tetrachlorobenzene

49) Hexachlorocyclopentadiene

50) 2,4,6-Trichlorophenol

51) 2,4,5-Trichlorophenol

53) Tetradecane NOT Present

54) 2-Chloronaphthalene

55) 1-Chloronaphthalene

56) 2-Nitroaniline

57) Dimethylphthalate

58) 2,6-Dinitrotoluene

59) Acenaphthylene

60) 3-Nitroaniline

61) Acenaphthene

62) 2,4-Dinitrophenol

63) 4-Nitrophenol

64) Dibenzofuran

65) 2,4-Dinitrotoluene

66) Pentachlorobenzene

67) 1-Naphthylamine

68) 2-Naphthylamine.

69) $2,3,4,6$-Tetrachlorophenol

70) Diethylphthalate

71) 4-Chlorophenyl Phenyl Ethe

72) Fluorene

73) 4-Nitroaniline

76) 4,6-Dinitro-2-methylphenol

77) N-nitrosodiphenylamine

78) 1,2-Diphenyl hydrazine

79) Tributylphosphate NOT Pres

\begin{tabular}{|c|c|c|c|c|c|}
\hline 6.50 & 456 & 122 & 536795 & \multicolumn{2}{|c|}{${ }^{\prime} 88.774 \mathrm{ug} / \mathrm{mLm}_{\mathrm{rpp}} \mid \mathrm{i1} 1$} \\
\hline 5.54 & 461 & 93 & $969057^{\circ}$ & $42.813 \mathrm{ug} / \mathrm{mL} \#$ & 96 \\
\hline & 480 & 162 & 669395 & $45.167 \mathrm{ug} / \mathrm{mL} \#$ & 79 \\
\hline 7.11 & 527 & 58 & 2395 & $136.865 \mathrm{ug} / \mathrm{mLm}$ & \\
\hline 6.84 & 496 & 180 & 678198 & $44.244 \mathrm{ug} / \mathrm{mL}$ & 98 \\
\hline 0.00 & & & & Not Detected & \\
\hline 5.97 & 510 & 128 & 2605235 & $\cdot 45.569 \mathrm{ug} / \mathrm{mL} \#$ & 8 \\
\hline 7.01 & 515 & 127 & 942284 & $41.470 \mathrm{ug} / \mathrm{mLm}$ & \\
\hline 7.03 & 517 & 162 & 612159 & $44.045 u g / m L \#$ & \\
\hline 7.11 & 527 & 225 & 275116 & $40.218 \mathrm{ug} / \mathrm{mL}$ & \\
\hline 0.00 & & & & Not Detected & \\
\hline 7.47 & 568 & 84 & 453024 & $40.489 \mathrm{ug} / \mathrm{mL} \#$ & 1 \\
\hline 7.67 & 591 & 107 & 649920 & $42.580 \mathrm{ug} / \mathrm{mL}$ & \\
\hline 7.98 & 627 & 142 & 1370454 & $015 u g / m L$ & 10 \\
\hline 8.24 & 657 & 216 & 1690 & $\mathrm{ug} / \mathrm{mL}$ & \\
\hline 8.23 & 655 & 237 & 233 & $\mathrm{~g} / \mathrm{mL}$ & \\
\hline 8.41 & 676 & 196 & 395354 & 43.45 & 96 \\
\hline 8.46 & 682 & 196 & 436980 & $40.795 \mathrm{ug} / \mathrm{mL}$ & \\
\hline 0.00 & & & & Not Detected & \\
\hline 8.78 & 719 & 162 & 1443213 & $41.859 \mathrm{ug} / \mathrm{mL}$ & 94 \\
\hline 8.82 & 723 & 162 & 1158285 & $42.162 \mathrm{ug} / \mathrm{mL}$ & \\
\hline 8.92 & 735 & 65 & 366884 & $40.995 \mathrm{ug} / \mathrm{mL} \#$ & \\
\hline 9.23 & 770 & 163 & 1447060 & $40.429 \mathrm{ug} / \mathrm{mL}$ & \\
\hline 9.33 & 782 & 165 & 361197 & $41.109 \mathrm{ug} / \mathrm{mL} \#$ & \\
\hline 9.49 & 800 & 152 & 211 & $3 \mathrm{ug} / \mathrm{mL}$ & $y$ \\
\hline 9.63 & 816 & 65 & 742 & 19/mL\# & \\
\hline 9.78 & 834 & 153 & 125 & 4 & \\
\hline 81 & 837 & 184 & 983 & L\# & \\
\hline .88 & 845 & 109 & 112496 & 43.927 & 78 \\
\hline .09 & 869 & 168 & 1701762 & $44.617 \mathrm{ug} / \mathrm{mL}$ & 95 \\
\hline .04 & 864 & 165 & 480959 & $49.268 \mathrm{ug} / \mathrm{mL} \#$ & 79 \\
\hline 01 & 860 & 250 & 355892 & $37.507 \mathrm{ug} / \mathrm{mL} \#$ & 85 \\
\hline 22 & 884 & 143 & 902545 & $45.370 \mathrm{ug} / \mathrm{mL}$ & .94 \\
\hline 36 & 900 & 143 & 1328913 & $44.223 \mathrm{ug} / \mathrm{mL}$ & $92-x-2 \cdot x$ \\
\hline .29 & 892 & 232 & 241753 & $41.858 \mathrm{ug} / \mathrm{mL} \#$ & 8 \\
\hline .47 & 913 & 149 & 1411992 & $40.761 \mathrm{ug} / \mathrm{mL}$ & $y$ \\
\hline 69 & 938 & 204 & 473564 & $.283 \mathrm{ug} / \mathrm{mL}$ & 9 \\
\hline & 939 & 166 & 1095647 & .216 & 10 \\
\hline & 941 & 65 & 311282 & $41.522 \mathrm{ug} / \mathrm{mL}$ & 90 \\
\hline .77 & 947 & 198 & 237904 & $39.313 \mathrm{ug} / \mathrm{mL} \#$ & 6 \\
\hline .90 & 962 & 169 & 930063 & $34.906 \mathrm{ug} / \mathrm{mL}$ & 9 \\
\hline 97 & 971 & 77 & 1220958 & $34.676 \mathrm{ug} / \mathrm{mL} \#$ & 84 \\
\hline & & & & & \\
\hline
\end{tabular}

$6.54 \quad 461 \quad 93 \quad 969057^{\circ} \quad 42.813 \mathrm{ug} / \mathrm{mL} \# 96$

$\begin{array}{lllll}6.70 & 480 & 162 & 669395 \quad 45.167 u g / m L \# & \end{array}$

$\begin{array}{rrrrr}7.11 & 527 & 58 & 2395304 & 136.865 \mathrm{ug} / \mathrm{mLm} / 2 \mathrm{df} 7 \\ 6.84 & 496 & 180 & 678198 & 44.244 \mathrm{ug} / \mathrm{mL}\end{array}$

Not Detected

$\begin{array}{llll}6.97 & 510 & 128 & 2605235\end{array}$

$7.71-527-225 \quad 275116$

0.00

7.47

7.67

7.98

8.24

8.23

8.41

8.46

8.78

8.82

8.92

9.23

9.49

9.63

9.78

9.81

9.88

10.09

10.04

10.22

10.36

10.29

10.47

10.69

10.70

10.71

10.90

0.00

Not Detected

\section{(9.98}

98

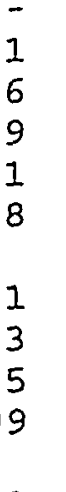




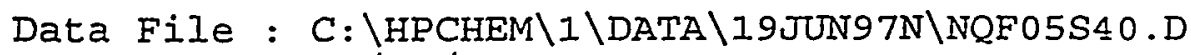

AcG Time : 06/19/97 07:15

Sample : SSTD040 (143WS28450)

Misc : EXP.11/5/97

Operator: TV

Quant Time: Jun 20 8:16 1997

Inst : $5972-\mathrm{N}$

Multiplr: 1.00

Method : C: \HPCHEM $\backslash I \backslash M E T H O D S \backslash 8270 \mathrm{~B} . \mathrm{M}$

Title : SW846/8270B, Curve:NQF 6/19/97

Iast Update : Fri Jun 20 08:11:40 1997

Response via : Multiple Level Calibration

Compound

80) Phenacetin

81) 4-Bromophenyl Phenyl Ether

82) alpha-BHC

83) Hexachlorobenzene

84) Tris (2-CE) phosphate NOT Pr

85) 4-Aminobiphenyl

86) beta-BHC

B7) Pentachlorophenol

88) Pronamide

89) gamma-BHC

90) Pentachloronitrobenzene

91) Phenanthrene

92) Anthracene

93) delta-BHC

94) Heptachlor

95) Di-n-Butylphthalate

96) Aldrin

97) Fluoranthene

99) Benzidine

100) Pyrene

101) $\leq .4 '-D D E$

103) Dieldrin

$10 \leq)$ p-Dimethylaminoazobenzene

105) $4,4 \cdot-D D D$

106) Butylbenzylphthalate

107) $4,41-D D T$

108) Nethoxychlor

109) 3,3'-Dichlorobenzidine

110) Benzo[a] anthracene

111) Chrysene

112) Bis (2-ethylhexyl) phthalate

11s) Di-n-octylphthalate

115) Benzo[b] fluoranthene

116) 7,12-Dimethylbenz (a) anthra

117) Benzo[k] fluoranthene

118) Benzo[a] pyrene

119) 3-Methylcholanthrene

120) Indeno $(1,2,3-c, d)$ pyrene

121) Dibenz $[a, h]$ anthracene

122) Benzo[ghi] perylene
R.T. Scan QIon Response Conc Unit Qvalue

\begin{tabular}{|c|c|c|c|c|}
\hline 11.46 & 1027 & 108 & 540810 & $-33.147 \mathrm{ug} / \mathrm{mL} \#$ \\
\hline 11.58 & 1041 & 248 & $2713730^{\circ}$ & $43.269 \mathrm{ug} / \mathrm{mL} \#$ \\
\hline 11.61 & 1044 & 183 & 196241 & $3.4 .774 \mathrm{ug} / \mathrm{mL} \#$ \\
\hline $\begin{array}{r}11.70 \\
0.00\end{array}$ & 1054 & 284 & 278401 & $\begin{array}{l}34.066 \mathrm{ug} / \mathrm{mL} \# \\
\text { Not Detected }\end{array}$ \\
\hline 12.06 & 1096 & 169 & 1198861 & $40.963 \mathrm{ug} / \mathrm{mL}$ \\
\hline 11.98 & 1087 & 181 & 152500 & $34.352 \mathrm{ug} / \mathrm{mL}$ \\
\hline 12.04 & 1094 & 266 & 162197 & $55.357 \mathrm{ug} / \mathrm{mL}$ \\
\hline 12.17 & 1108 & 173 & 520230 & $34.764 \mathrm{ug} / \mathrm{mL}$ \\
\hline 12.16 & 1107 & 183 & 158483 & $.255 \mathrm{ug} / \mathrm{mL} \#$ \\
\hline 12.08 & 1098 & 237 & 98763 & $35.587 \mathrm{ug} / \mathrm{mL} \#$ \\
\hline 12.46 & 1142 & 178 & 1760462 & $37.430 \mathrm{ug} / \mathrm{mL} \#$ \\
\hline 12.56 & 1153 & 178 & 1764815 & $36.522 \mathrm{ug} / \mathrm{mL}$ \\
\hline 12.57 & 1154 & 183 & 132662 & $33.117 \mathrm{ug} / \mathrm{mL}$ \\
\hline 13.32 & 1241 & 100 & 248488 & $35.435 \mathrm{ug} / \mathrm{mL} \#$ \\
\hline 13.46 & 1257 & 149 & 2268020 & $35.891 \mathrm{ug} / \mathrm{mL} \#$ \\
\hline 13.84 & 1301 & 66 & 200544 & $33.967 \mathrm{ug} / \mathrm{mL}$ \\
\hline $1 \leq .47$ & 1373 & 202 & 1527493 & $39.482 \mathrm{ug} / \mathrm{mL}$ \\
\hline $1 \leq .67$ & 1396 & 184 & 767188 & $.011 \mathrm{ug} / \mathrm{mL}$ \\
\hline 14.82 & 1413 & 202 & 1512258 & $.691 \mathrm{ug} / \mathrm{mL} \ddot{\pi}$ \\
\hline 14.95 & 1428 & 246 & 242994 & $.754 \mathrm{ug} / \mathrm{mL}$ \\
\hline 15.11 & $1 \leq 47$ & 79 & 234958 & $30.827 \mathrm{ug} / \mathrm{mL} \#$ \\
\hline 15.23 & 1460 & 225 & 271811 & $36.835 \mathrm{ug} / \mathrm{mL} \#$ \\
\hline 15.43 & 1483 & 235 & 423775 & $31.973 \mathrm{ug} / \mathrm{mL} \#$ \\
\hline 15.71 & 1516 & 149 & 850135 & $34.935 \mathrm{ug} / \mathrm{mL}$ \\
\hline 15.85 & 1532 & 235 & 385181 & $45.057 \mathrm{ug} / \mathrm{mL} \#$ \\
\hline 16.46 & 1602 & 227 & 685006 & $38.647 \mathrm{ug} / \mathrm{mL}$ \\
\hline 16.54 & 1611 & 252 & 413374 & $38.760 \mathrm{ug} / \mathrm{mL} \#$ \\
\hline 16.61 & 1619 & 228 & 1044744 & $37.290 u g / m L$ \\
\hline 16.67 & 1626 & 228 & 1001608 & $37.092 \mathrm{ug} / \mathrm{mL}$ \\
\hline 16.60 & 1618 & 149 & & $33.750 u g / m L \#$ \\
\hline 17.87 & 1764 & 149 & 1796952 & $31.685 \mathrm{ug} / \mathrm{mL}$ \\
\hline 18.85 & 1877 & 252 & 931182 & $36.722 \mathrm{ug} / \mathrm{mL \#}$ \\
\hline 18.84 & 1875 & 256 & 357043 & $37.232 \mathrm{ug} / \mathrm{mL}$ \\
\hline 18.93 & 1886 & 252 & 887466 & $37.385 \mathrm{ug} / \mathrm{mL} \#$ \\
\hline 19.74 & 1979 & 252 & 819852 & $38.823 \mathrm{ug} / \mathrm{mL} \#$ \\
\hline 20.82 & 2103 & 268 & 420716 & $43.395 \mathrm{ug} / \mathrm{mL}$ \\
\hline 23.75 & 2440 & 276 & 642616 & $36.353 \mathrm{ug} / \mathrm{mL}$ \\
\hline 23.87 & 2454 & 278 & 659975 & $42.576 \mathrm{ug} / \mathrm{mL} \#$ \\
\hline 24.95 & & 276 & 593781 & $41.563 \mathrm{ug} / \mathrm{mL} \#$ \\
\hline
\end{tabular}

$--$

71

86

97

57

95

96

85

62

95

94

75

92

98

55

84

56

80

55

90

88

87

99

95

00

99

99

00

95

88

88

98

98

85

85

Data Processed By: Sens (2aye b/20/97

Ho $\Rightarrow$ qualifier out of range $(\mathrm{m})=$ manual integration

(*) Does not meet EPA spectral criteria (False Hit) 
$\begin{array}{lll}\text { Quantitation Report } \quad \text { File: NQF05S40.D standard amount: } \quad 40 & 40\end{array}$

Path: C:IHPCHEMI1IDATAI19JUN97NI

$06 / 19 / 97$

Sample: SSTD040 (143WS28450)

Instrument ID: $5972-\mathrm{N}$

Analyst: TV

No.

scan
326
507
828
1137
1621
1997
183
274
299
346
397
693
988
1438

$$
\begin{array}{r}
\text { Pyridine } \\
\text { N-nitrosodimethylamine } \\
\text { 2-Picoline }
\end{array}
$$

$3.14 \quad 70$

3.10

3.70

3.99

4.57

4.93

5.02

5.15

0.00

5.31

5.38

5.48

5.56

5.57

5.62

5.74

5.77

5.96

5.05

5.79

6.01

6.19

6.30

6.40

6.40

6.50

6.54

6.70$$
\mathrm{m} / \mathrm{z}
$$$$
152.00
$$$$
136.00
$$$$
164.00
$$

188.00

240.00

264.00

112.00

99.00

132.00

152.00

82.00

172.00

330.00

244.00

79.00

42.00

93.00

80.00

79.00

94.00

66.00

128.00

57.00

146.00

146.00

108.00

146.00

107.00

45.00

108.00

70.00

117.00

63.00

105.00

77.00

114.00

82.00

139.00

122.00

122.00

93.00

162.00 611722 2299996 1106610 1681225 1032899 843647

826717 1013045

1061442

522044

768023

1399222

150032

906910

997547

546621

1022968

388401

650696

1068898

603654

1038480

$$
0
$$

917452

898429

565050

758749

708847

1949402

899889

524463

320147

766205

1061229

743482

455785

1446067

472248

769882

536795

969057

669395
RF amt

$1.000 \quad 40.000$

$1.000 \quad 40.000$

$1.000 \quad 40.000$

$1.000-40.000$

$1.000 \quad 40.000$

$1.000 \quad 40.000$

$1.351 \quad 40.00$

$1.656 \quad 40.00$

$1.735 \quad 40.00$

$0.853 \quad 40.00$

$0.334 \quad 40.00$

$1.264 \quad 40.00$

$0.136 \quad 40.00$

$0.878 \quad 40.00$

$1.631 \cdot 40.00$

$0.894 \quad 40.00$

1.67240 .00
0.635

0.63540 .00

$1.064 \quad 40.00$

$1.747 \quad 40.00$

0.98740 .00

$\begin{array}{ll}1.698 & 40.00\end{array}$

$0.000 \quad 40.00$

$1.500 \quad 40.00$

$1.469 \quad 40.00$

$0.924 \quad 40.00$

$\begin{array}{lll}1.240 & 40.00\end{array}$

$1.159 \quad 40.00$

$3.187 \quad 40.00$

1.47140 .00

$0.857 \cdot 40.00$

0.52340 .00

$1.253 \quad 40.00$

$0.461 \quad 40.00$

0.32340 .00

$0.198 \quad 40.00$

0.62940 .00

$0.205 \quad 40.00$

$0.335 \quad 40.00$

$0.233 \quad 40.00$

$0.421 \quad 40.00$

$0.291 \quad 40.00$ 
Patl: C:IHPCHEMI1IDATAI19JUN97NI

06/19/97

Sample: SSTD040 (143WS28450)

Instrument ID: $5972-\mathrm{N}$

\begin{tabular}{|c|c|c|c|c|c|c|c|}
\hline $\begin{array}{l}\text { No. } \\
36\end{array}$ & $\begin{array}{c}\text { Name } \\
\text { A.,A.-dimethylphenethylamine }\end{array}$ & $\begin{array}{l}\text { RT } \\
7.11\end{array}$ & $\begin{array}{l}\text { scan } \\
527\end{array}$ & $\begin{array}{l}\mathrm{m} / \mathrm{z} \\
58.00\end{array}$ & $\begin{array}{c}\text { area } \\
2395304\end{array}$ & $\begin{array}{c}\mathrm{RF} \\
1.041\end{array}$ & $\begin{array}{c}\text { amt } \\
40.00\end{array}$ \\
\hline 37 & 1.2,4-Trichlorobenzene & 6.84 & 496 & 180.00 & 678198 & 0.295 & 40.00 \\
\hline 38 & Dodecane NOT Present & 0.00 & 1 & 57.00 & 0 & 0.000 & 49.00 \\
\hline 39 & Naphthalene & 6.97 & 510 & 128.00 & 2605235 & 1.133 & -40.00 \\
\hline 40 & 4-Chloroaniline & 7.01 & 515 & 127.00 & 942284 & $0.410^{\circ}$ & 40.00 \\
\hline 41 & 2,6-Dichlorophenol & 7.03 & 517 & 162.00 & 612159 & 0.266 & 40.00 \\
\hline 42 & Hexachlorobutadiene & 7.11 & 527 & 225.00 & 275116 & 0.120 & 40.00 \\
\hline 43 & Benzothiazole NOT Present & 0.00 & 1 & 135.00 & 0 & 0.000 & 40.00 \\
\hline 44 & N-nitroso-di-n-butylamine & 7.47 & 568 & $84.00^{\circ}$ & 453024 & 0.197 & 40.00 \\
\hline 45 & 4-Chloro-3-Methylphenol & 7.67 & 591 & 107.00 & 649920 & & 40.00 \\
\hline 46 & 2-Methylnaphthalene & 7.98 & 627 & 142.00 & 1370454 & & 40.00 \\
\hline 48 & $1,2,4,5$-Tetrachlorobenzene & 8.24 & 657 & 216.00 & 517690 & & 40.00 \\
\hline 49 & Hexachlorocyclopentadiene & 8.23 & 655 & 237.00 & 259233 & & 40.00 \\
\hline 50 & 2,4,6-Trichlorophenol & 8.41 & 676 & 196.00 & 395354 & & 40.00 \\
\hline 51 & $2,4,5$ & 8.46 & 682 & 196.00 & 436980 & & .00 \\
\hline 53 & Tetradecane NOT & 0.00 & 1 & 57.00 & 0 & & .00 \\
\hline 54 & 2-Chloronapl & 8.78 & 719 & 162.00 & 1443213 & 04 & .00 \\
\hline 55 & 1-Chlorona: & 8.82 & 723 & 162.00 & 1158285 & 47 & 40.00 \\
\hline 56 & niline & 8.92 & 735 & 65.00 & 366884 & 0.332 & 40.00 \\
\hline 57 & Dimethy & 9.23 & 770 & 163.00 & 1447060 & 08 & 40.00 \\
\hline 58 & 2,6-Dinit & 9.33 & 782 & 165.00 & 361197 & 0.326 & 40.00 \\
\hline 59 & Acena & 9.49 & 800 & 152.00 & 2110037 & 1.907 & 40.00 \\
\hline 60 & line & 9.63 & 816 & $65.00^{\circ}$ & 405742 & 367 & 40.00 \\
\hline 61 & Acen & 9.78 & 834 & 153.00 & 1259182 & 38 & 40.00 \\
\hline 62 & 2,4-Dini & 9.81 & 837 & 184.00 & 226983 & 05 & 40.00 \\
\hline 63 & $4-\mathrm{Ni}$ & 9.88 & 845 & 109.00 & 112496 & 02 & 40.00 \\
\hline 64 & furan & 10.09 & 869 & 168.00 & 1701762 & 1.538 & 40.00 \\
\hline 65 & 2,4-Dinit & 10.04 & 864 & 165.00 & 480959 & 0.435 & 40.00 \\
\hline 66 & Pentachloro & 10.01 & 860 & 250.00 & 355892 & 0.322 & 40.00 \\
\hline 67 & 1-Napht & 10.22 & 884 & 143.00 & 902545 & 0.816 & 40.00 \\
\hline 68 & 2-Napht & 10.36 & 900 & 143.00 & 1328913 & 1.201 & 40.00 \\
\hline 69 & 2,3,4,6-Tetrachlor & 10.29 & 892 & 232.00 & 241753 & 0.218 & 40.00 \\
\hline 70 & Diethyl & 10.47 & 913 & 149.00 & 1411992 & 1.276 & 40.00 \\
\hline 71 & 4-Chlorophenyl Phenyl Ether & 10.69 & 938 & 204.00 & 473564 & 0.428 & 40.00 \\
\hline 72 & & 10.70 & 939 & 166.00 & 1095647 & 0.990 & 40.00 \\
\hline 73 & & 10.71 & 941 & 65.00 & 311282 & 0.281 & 40.00 \\
\hline 76 & 4,6-Dinitro-2-methylphenol & 10.77 & 947 & 198.00 & 237904 & 0.142 & 40.00 \\
\hline 77 & $\mathrm{~N}$-nitrosodiphenylamine & 10.90 & 962 & 169.00 & 930063 & 0.553 & 40.00 \\
\hline 78 & 1,2-Diphenylhydrazine & 10.97 & 971 & 77.00 & 1220958 & 0.726 & 40.00 \\
\hline 79 & Tributylphosphate NOT Present & 0.00 & 1 & 99.00 & 0 & 0.000 & 40.00 \\
\hline 80 & Phenacetin & 11.46 & 1027 & 108.00 & 540810 & 0.322 & 40.00 \\
\hline 81 & 4-Bromophenyl Phenyl Ether & 11.58 & 1041 & 248.00 & 271373 & 0.161 & 40.00 \\
\hline
\end{tabular}


Path: C:IHPCHEMITIDATAI19JUN97NI

06/19/97

Sample: SSTD040 (143WS28450)

Instrument ID: $5972-\mathrm{N}$

\begin{tabular}{|c|c|c|c|c|c|c|c|}
\hline $\begin{array}{l}\text { No. } \\
82\end{array}$ & $\begin{array}{l}\text { Name } \\
\text { alpha-BHC }\end{array}$ & $\begin{array}{c}\text { RT } \\
11.61\end{array}$ & $\begin{array}{l}\text { scan } \\
1044\end{array}$ & $\begin{array}{c}\mathrm{m} / \mathrm{z} \\
183.00\end{array}$ & $\begin{array}{c}\text { area } \\
196241\end{array}$ & $\begin{array}{c}\text { RF } \\
0.117\end{array}$ & $\begin{array}{l}\text { amt } \\
40.00\end{array}$ \\
\hline 83 & Hexachlorobenzene & 11.70 & 1054 & 284.00 & 278401 & 0.166 & 40.00 \\
\hline 84 & ris (2-CE)phosphate NOT Present & 0.00 & 1 & 249.00 & 0 & 0.000 & 40.00 \\
\hline 85 & 4-Aminobiphenyl & 12.06 & 1096 & 169.00 & 1198861 & 0.713 & 40.00 \\
\hline 86 & beta-BHC & 11.98 & 1087 & 181.00 & 152500 & 0.091 & 40.00 \\
\hline 87 & Pentachlorophenol & 12.04 & 1094 & 266.00 & 162197 & .096 & 40.00 \\
\hline 88 & Pronamide & 12.17 & 1108 & 173.00 & 520230 & .309 & 40.00 \\
\hline 89 & gamma-BHC & 12.16 & 1107 & 183.00 & 158483 & .094 & 40.00 \\
\hline 90 & Pentachloronitrobenzene & 12.08 & 1098 & 237.00 & 98763 & & 40.00 \\
\hline 91 & Phenanthrene & 12.46 & 1142 & 178.00 & 1760462 & & 40.00 \\
\hline 92 & acene & 12.56 & & & 1764815 & & 40.00 \\
\hline 93 & -BHC & 12.57 & 11 & & & & 40.00 \\
\hline 94 & achlor & 13.32 & 12 & & 248488 & & 40.00 \\
\hline 95 & Di-n-Butylph & 13.46 & 12 & & 2268 & & 40.00 \\
\hline 96 & Aldrin & 13.8 & 13 & & & & 40.00 \\
\hline 97 & Fluoranthene & 14.47 & 13 & & 1527 & & 40.00 \\
\hline 99 & Benzidine & 14.67 & 13 & & & 43. & 40.00 \\
\hline 100 & Pyrene & 14.82 & 14 & 20 & 1512258 & & 40.00 \\
\hline 101 & $4,4^{\prime}-D D E$ & 14.95 & 14 & 246 & 242994 & & 40.00 \\
\hline 103 & Dieldrin & 15.11 & 14 & 79. & 234 & 27 & 40.00 \\
\hline 104 & p-Dimethylaminoazobenzene & 15.23 & 1460 & 225.00 & 271811 & .263 & 40.00 \\
\hline 105 & $4,4^{\prime}-D D D$ & 15.43 & 1483 & 235.00 & 423775 & 0.410 & 40.00 \\
\hline 106 & Butylbenzylphthalate & 15.71 & 1516 & 149.00 & 850135 & 0.823 & 40.00 \\
\hline 107 & $4,4^{\prime}-\mathrm{DDT}$ & 15.85 & 1532 & 235.00 & 385181 & 0.373 & 40.00 \\
\hline 108 & Methoxychlor & 16.46 & 1602 & 227.00 & 685006 & 0.663 & 40.00 \\
\hline 109 & 3,3'-Dichlorober & 16.54 & 1611 & 252.00 & 413374 & 0.400 & 40.00 \\
\hline 110 & Benzo[a]anth & 16.61 & 161 & 228.00 & 1044744 & 1.011 & 40.00 \\
\hline 111 & sene & 16.67 & 162 & 228.00 & 1001608 & 0.970 & 40.00 \\
\hline 112 & Bis(2-ethylhexyl)p & 16.60 & 16 & 149.00 & 1030518 & 0.998 & 40.00 \\
\hline 114 & Di-n-octylpl & 17.87 & 17 & 149.00 & 1796952 & 30 & 40.00 \\
\hline 115 & enzo[b]fluor & 18.85 & 187 & 252.00 & 931182 & .1 .104 & 40.00 \\
\hline 116 & 12-Dimethylben & 18.84 & 18 & 256.00 & 357043 & 0.423 & 40.00 \\
\hline 117 & Benzolklfluor & 18.93 & 188 & 252.00 & 887466 & 1.052 & 40.00 \\
\hline 118 & Benzola & 19.74 & 19 & 252.00 & 819852 & 0.972 & 40.00 \\
\hline 119 & 3-Methylcholanthrene & 20.82 & 2103 & 268.00 & 420716 & 0.499 & 40.00 \\
\hline 120 & Indeno( $1,2,3-c, d)$ pyrene & 23.7 & 24 & 276.00 & 642616 & 0.762 & 40.00 \\
\hline 121 & ibenz[a, h]anthracene & 23.87 & $24 !$ & 278.00 & 659975 & 0.782 & 40.00 \\
\hline 122 & Benzo[ghi]perylene & 24.95 & 2578 & 276.00 & 593781 & 0.704 & 40.00 \\
\hline
\end{tabular}


Quantitation Report

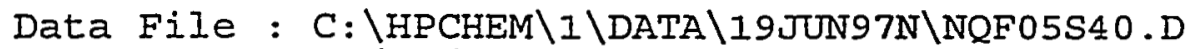

$\begin{array}{ll}\text { Acq Time } & : 06 / 19 / 9707: 15 \\ \text { Sample } & : \text { SSTD040 (143WS28450) } \\ \text { Misc } & : \text { EXP.11/5/97 }\end{array}$

Misc : EXP.11/5/97

Quant Time: Jun 19 7:41 1997

Operator: TV

Inst : $5972-\mathrm{N}$

Multiplr: 1.00

Method : C: \HPCHEM \I\METHODS $\backslash 8270 \mathrm{~B} . \mathrm{M}$

Title : Sw8 $46 / 8270 B$, Curve:NQF 6/19/97

Last Update : Fri Jun 20 08:11:40 1997

Response via : Multiple Level Calibration

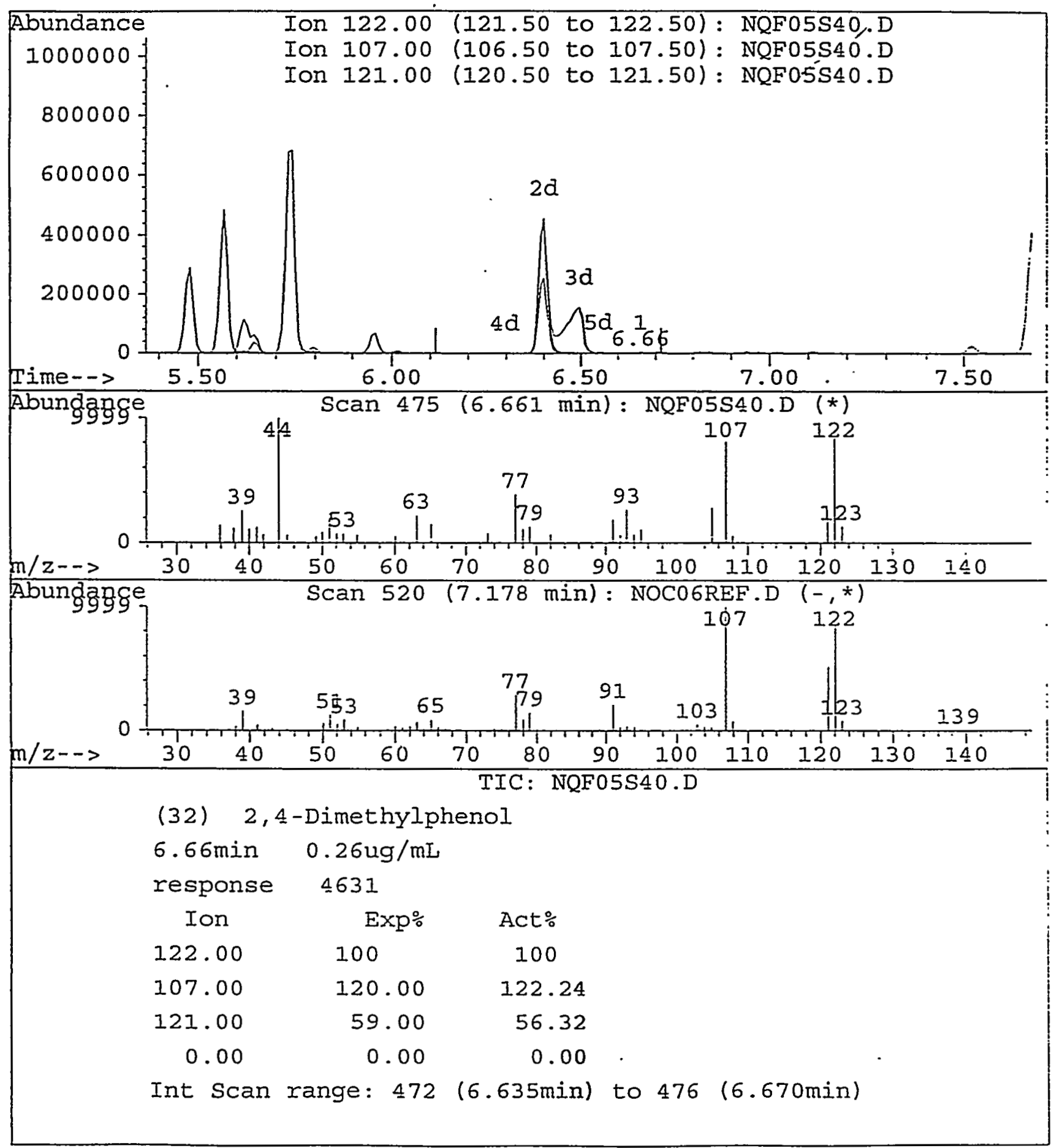




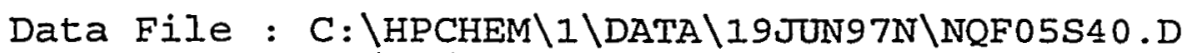

Acq Time : 06/19/97 07:15

Sample : SSTD040 (143WS28450)

Misc : EXP.11/5/97

Quant Time: Jun 20 8:15 1997

Operator: TV

Inst : $5972-\mathrm{N}$

Multiplr: 1.00 .

Method : C: $\backslash$ HPCHEM $\backslash I \backslash M E T H O D S \backslash 8270 B . M$

Title : SW846/8270B, Curve:NQF 6/19/97

Last Update : Fri Jun 20 08:11:40 1997

Response via : Multiple Level Calibration

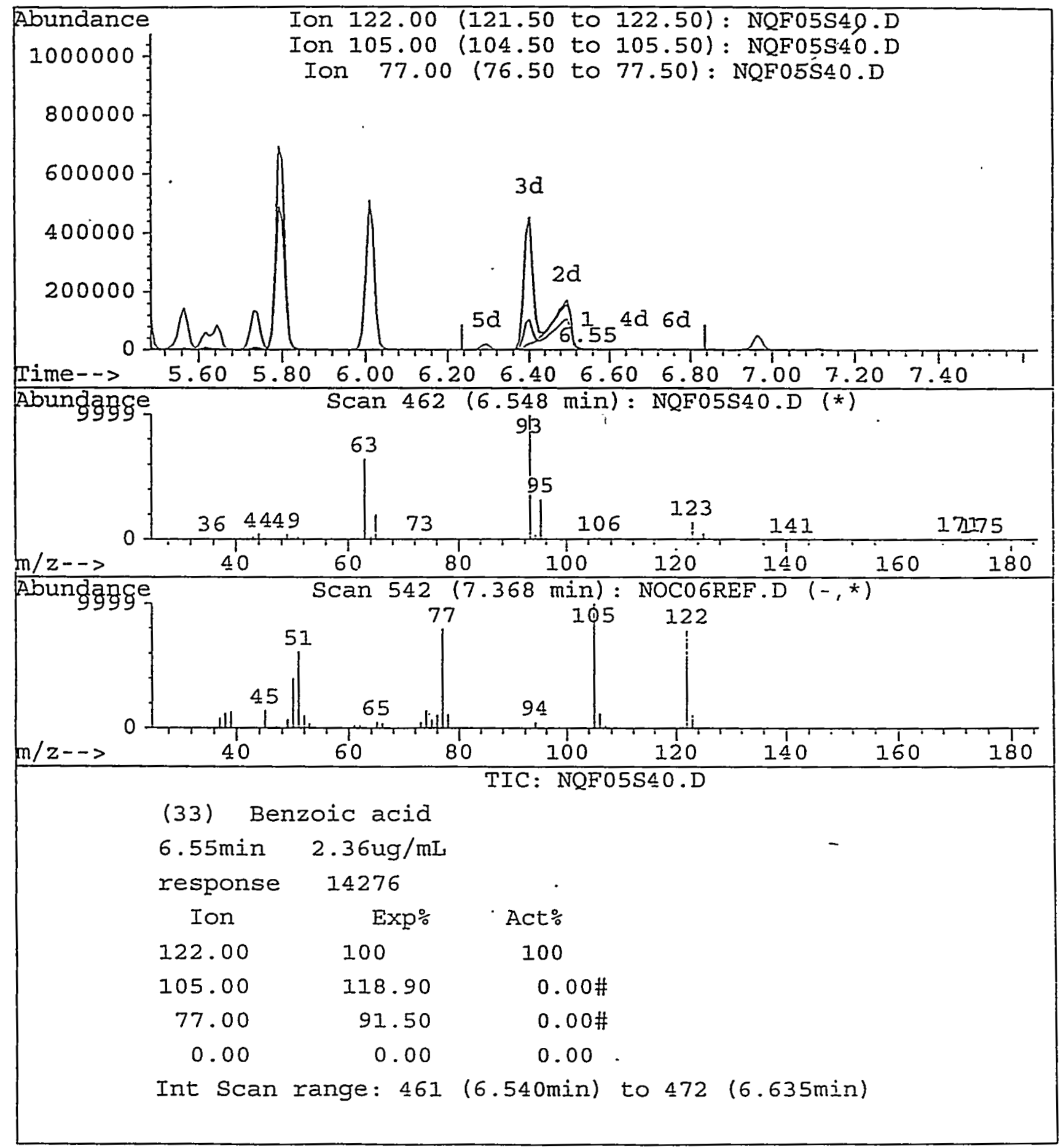




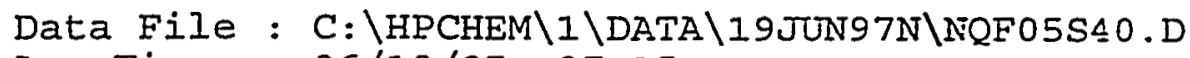

Acq Time : 06/19/97 07:15

Sample : SSTD040. (I43WS28450)

Misc : EXP.11/5/97

Quant Time: Jun 20 8:15 1997

Operator: TV

Inst : $5972-\mathrm{N}$

Multiplr: 1.00

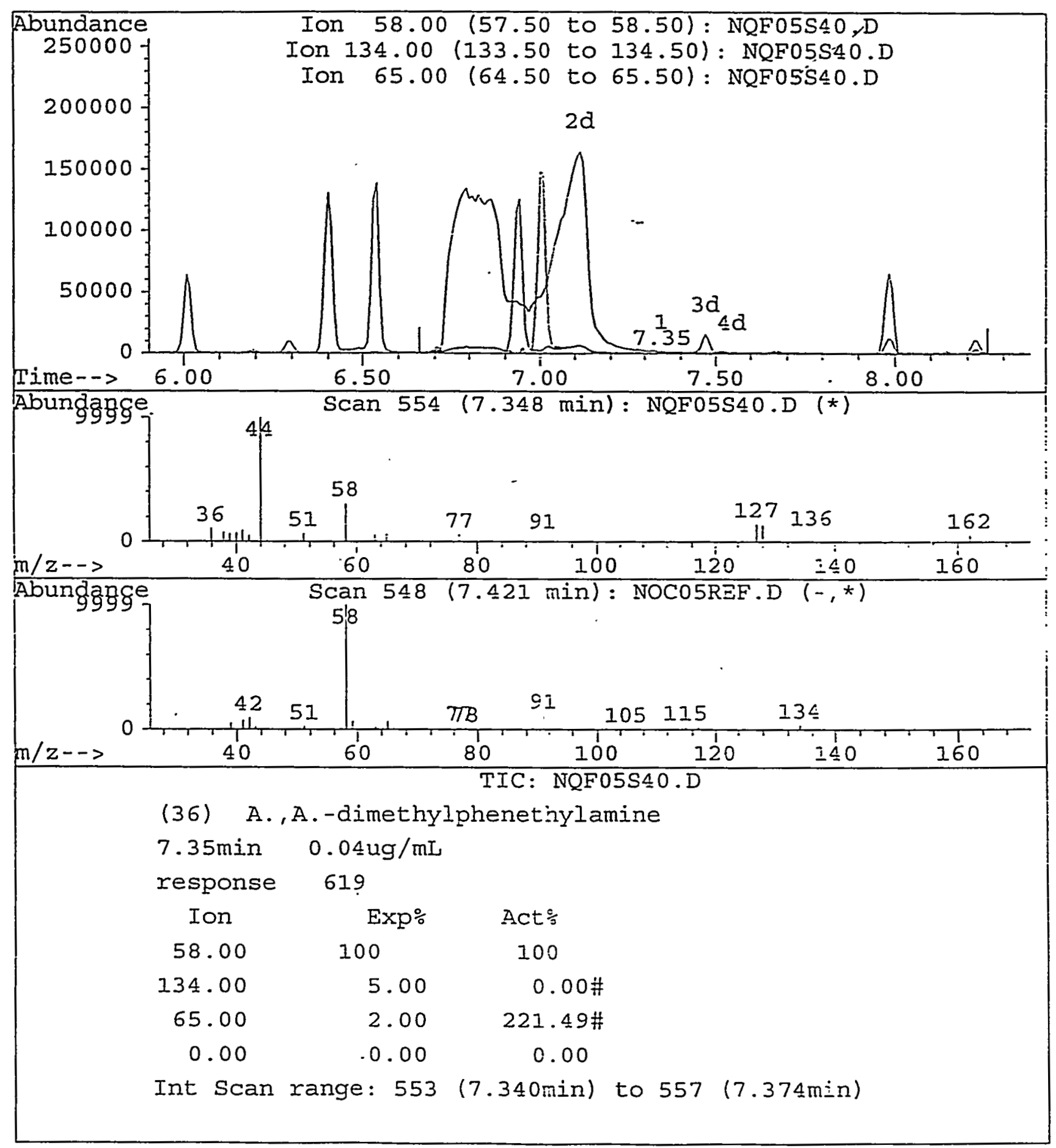




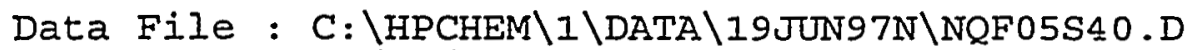
Acq Time
$06 / 19 / 97 \quad 07: 15$
Sample
: SSTD040 (143W528450)
Misc : EXP.11/5/97

Quant Time: Jun 20 8:16 1997

Operator: TV

Inst : $5972-\mathrm{N}$

Multiplr: 1.00

Method : C: \HPCHEM $\backslash 1 \backslash$ METHODS $\backslash 8270 \mathrm{~B} . \mathrm{M}$

Title

: SW846/8270B, Curve:NQF 6/19/97

Last Update : Fri Jun 20 08:11:40 1997

Response via : Multiple Level Calibration

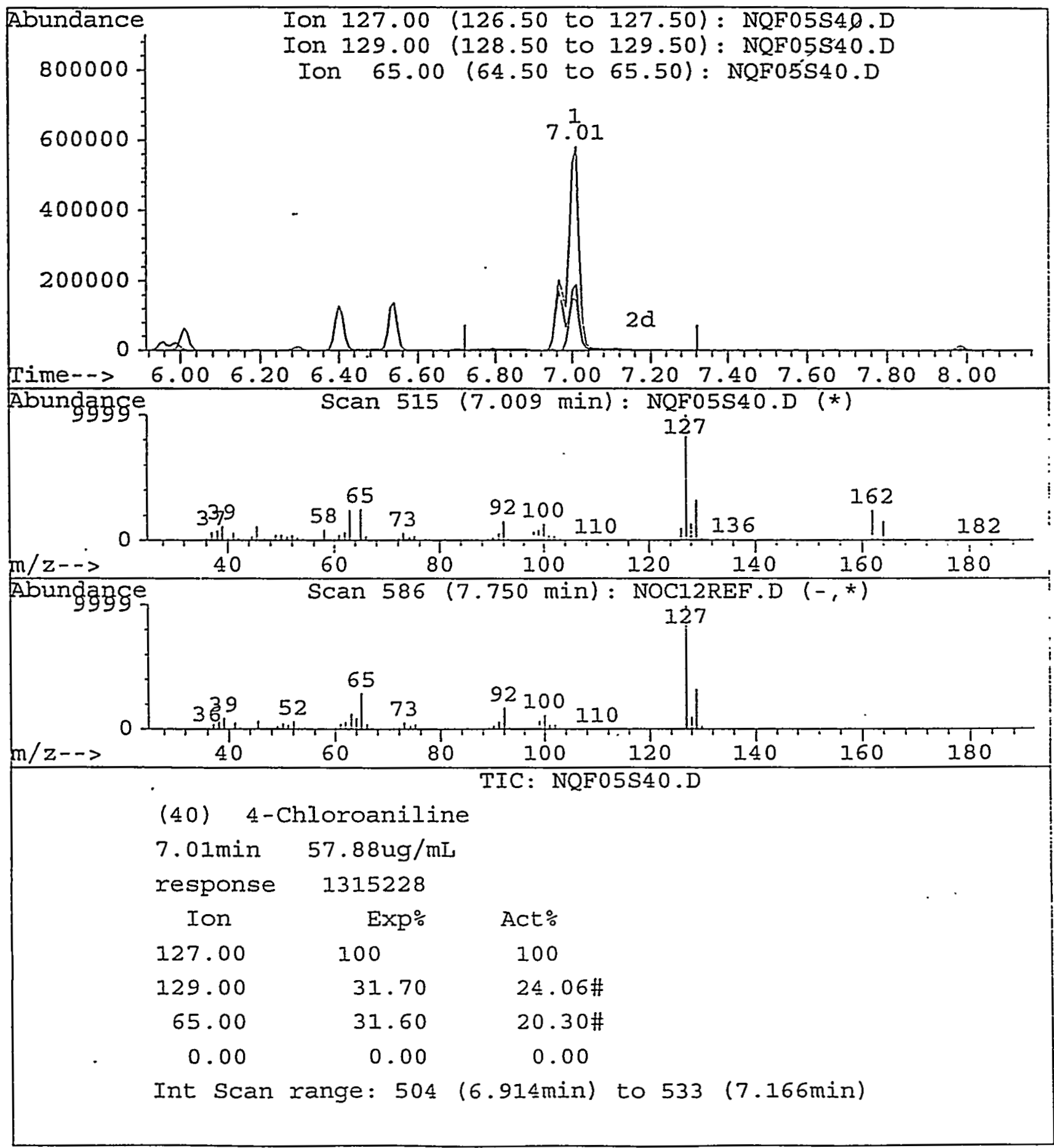


$M A N U A I \quad I N T E G R A T I O N$ FOR 2,4-Dimethylphenol

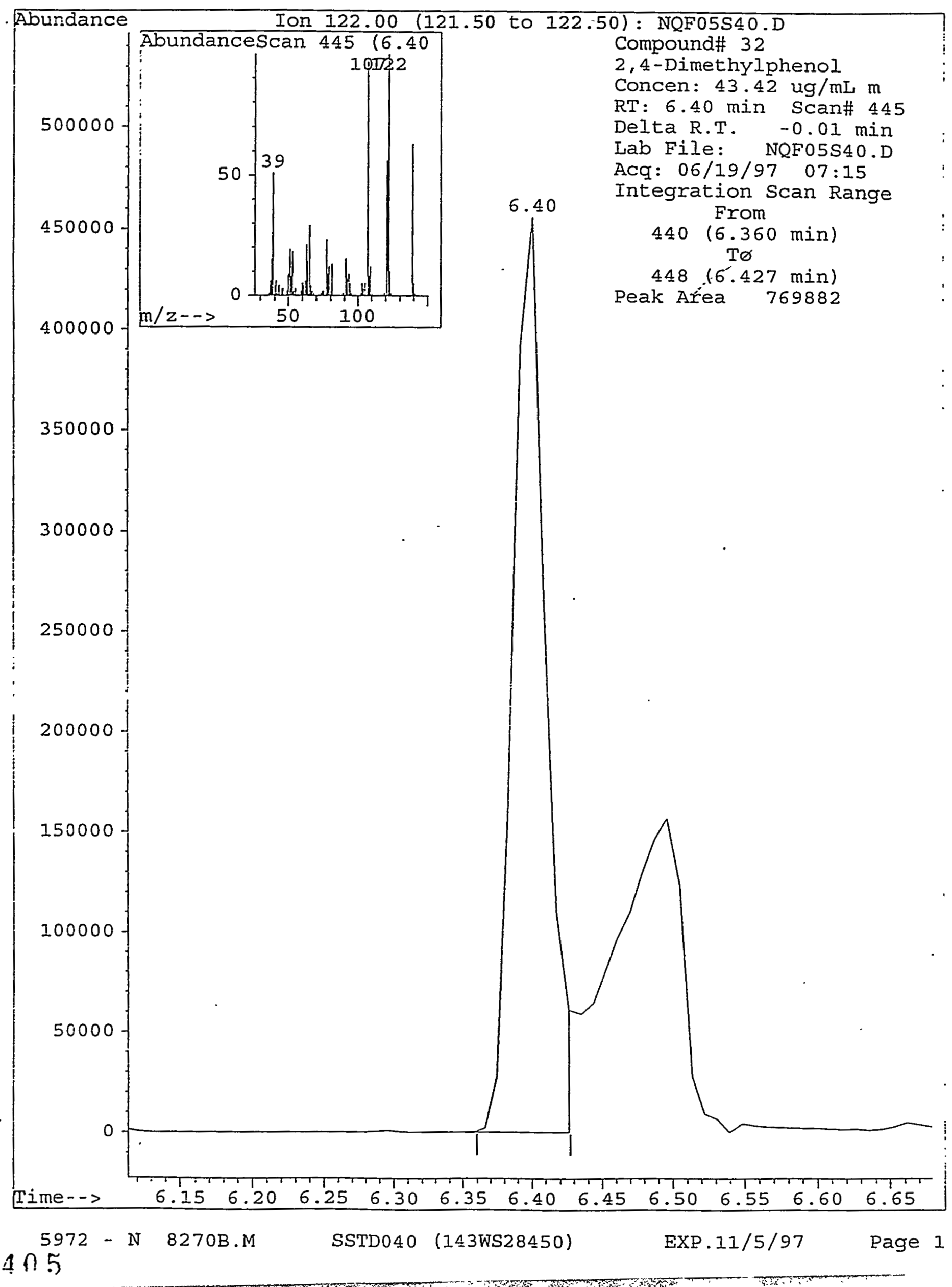


$M A N U A L$ I N T E G A T I O N FOR Benzoic acid

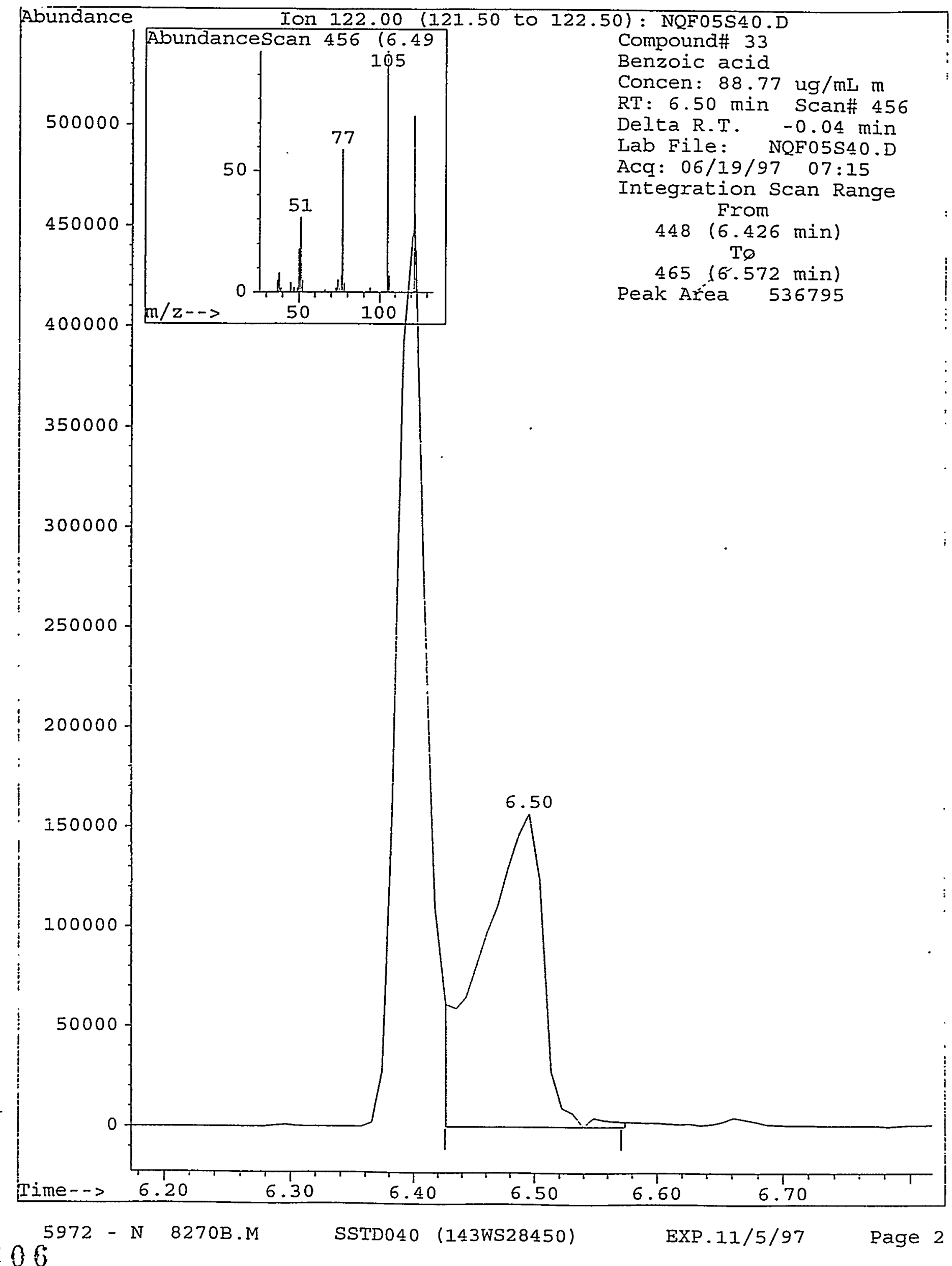


$M A N U A I \quad I N T E G R A T I O N$ FOR A.,A.-dimethylphenethylamine

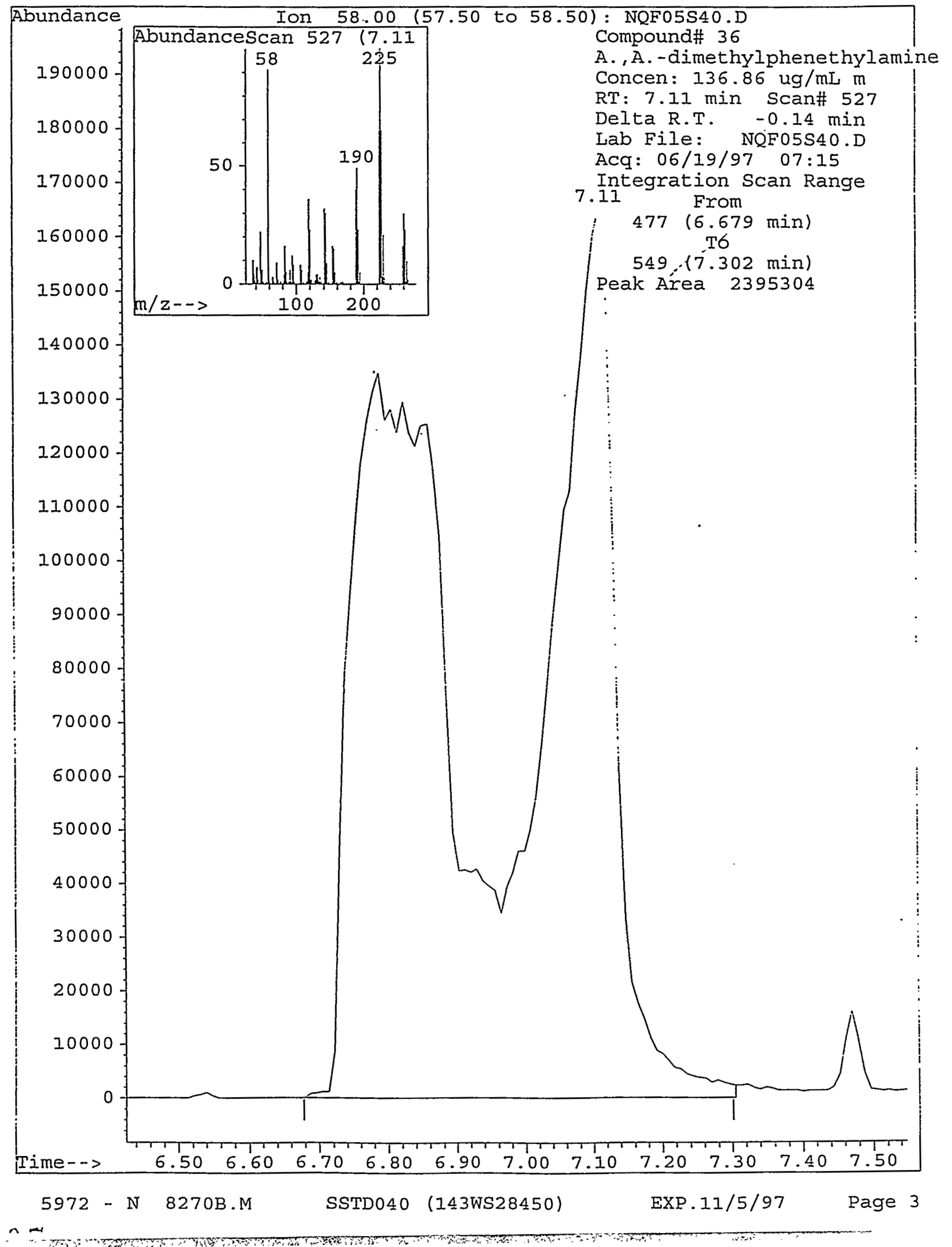


MAN UA L IN TE G R T I ON FOR 4 -Chloroaniline

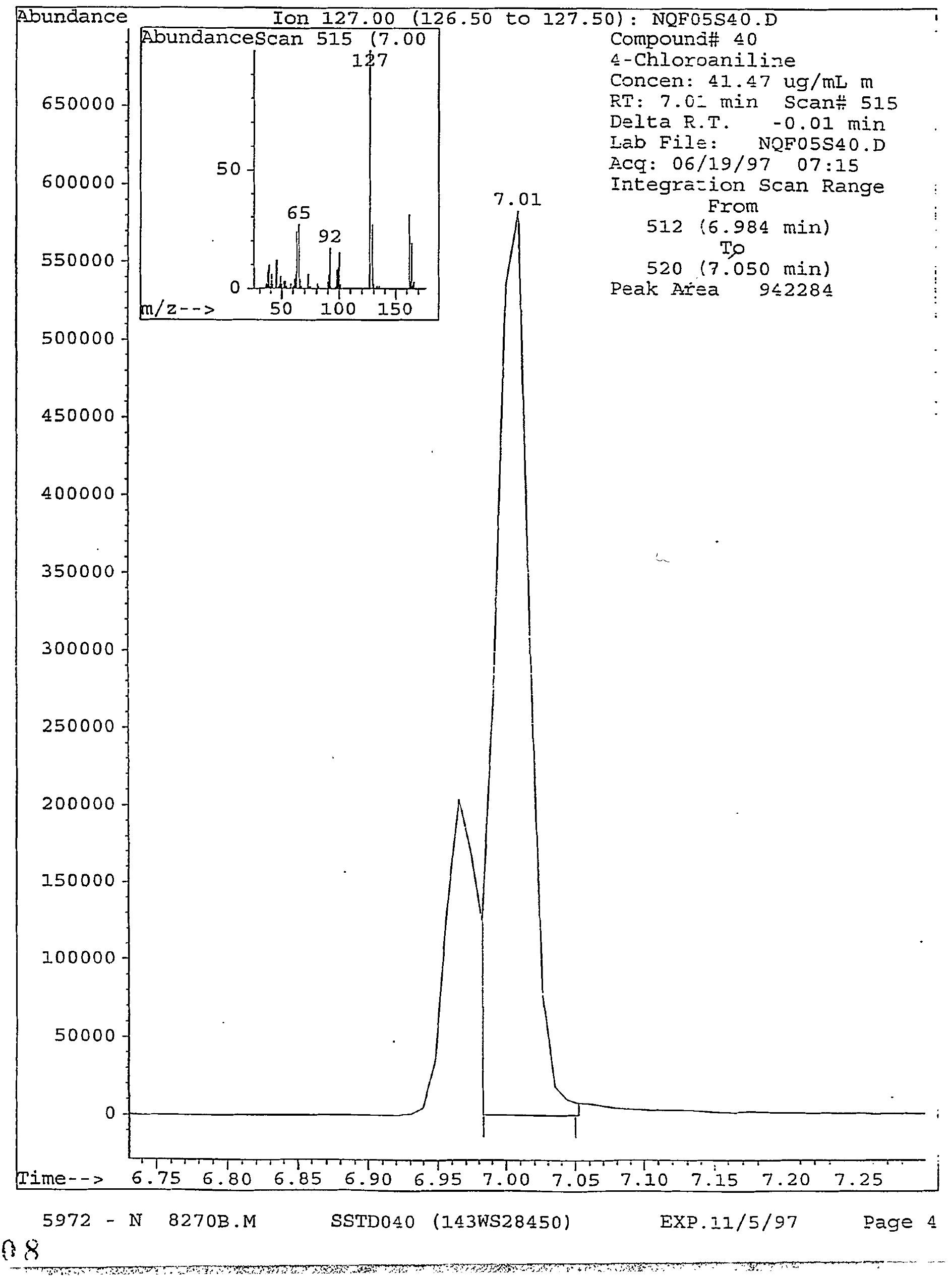




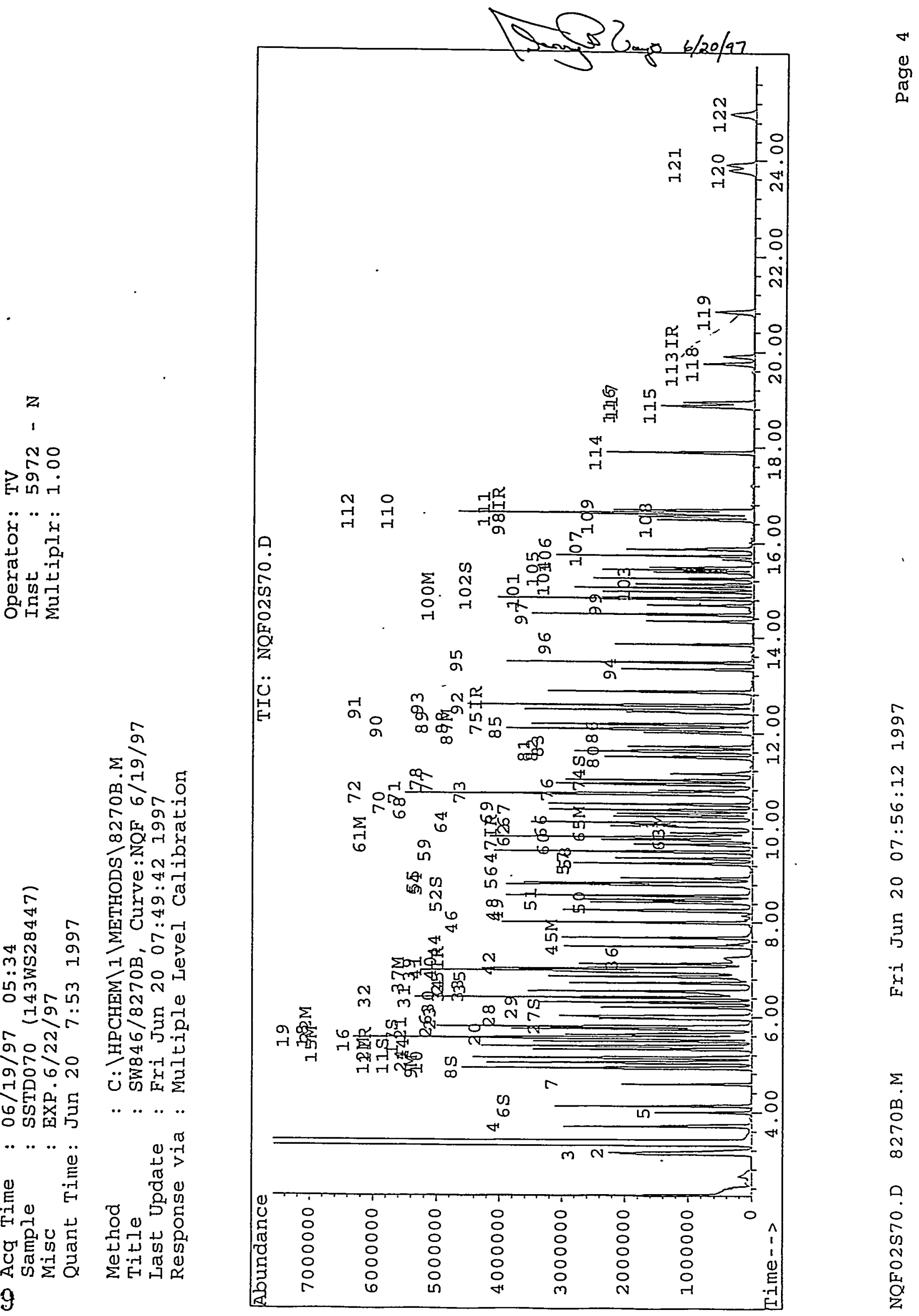




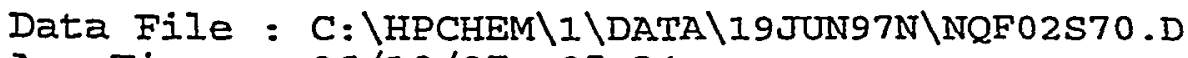

Acq Time : 06/19/97 05:34

Sample : SSTD070 (143WS28447)

Misc : EXP.6/22/97

Quant Time: Jun 20 7:53 1997

Operator: TV

Inst : $5972-\mathrm{N}$

Multiplr: 1.00

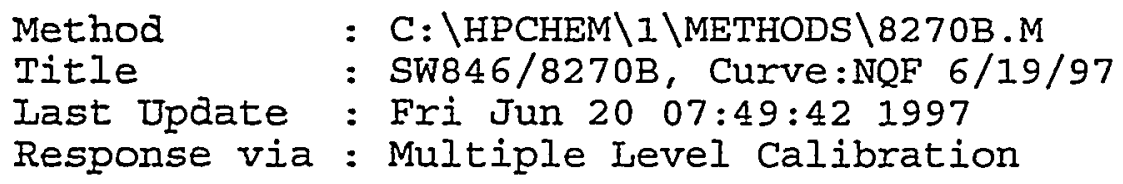

Internal standards

R.T. Scan QIon Response Conc Units Area\%

1).1,4-Dichlorobenzene-d4

25) Naphthalene-d8

47) Acenaphthene-d10

75) Phenanthrene-d10

98) Chrysene- $d 12$

113) Perylene-d12

System Monitoring Compounds

5) 2-Fluorophenol

B) Phenol-d5

1I) 2-Chlorophenol- $d 4$

I7) I,2-Dichlorobenzene-d4

27) Nitrobenzene-d5

52) 2-Fluorobiphenyl

74) 2,4,6-Tribromophenol

102) TerphenyI-d14

Target Compounds

2) Pyridine

3) N-nitrosodimethylamine

4) 2-Picoline

5) Methyl methanesulfonate

7) Ethyl methanesulfonate

.9) Phenol

10) Aniline

12) 2-Chlorophenol

13) Decane NOT Present

14) 1,3-Dichlorobenzene

15) 1,4-dichlorobenzene

16) Benzyl alcohol

18) 1,2-dichlorobenzene

19) 2-Methylphenol

20) Bis(2-chloroisopropyl) ethe

21) $₫$-methylphenol

22) N-Nitrosodi-n-propyl amine

23) Hexachloroethane

24) Bis (2-chioroethyl) ether

26) Acetophenone

28) Nitrobenzene

29) N-nitrosopiperidine

30) Isophorone

3I) 2-Nitrophenol

32) 2,4-Dimethylphenol

$\begin{array}{rrr}5.38 & 362 & 152 \\ 6.95 & 543 & 136 \\ 9.74 & 864 & 164 \\ 12.43 & 1173 & 188 \\ 16.64 & 1657 & 240 \\ 19.91 & 2033 & 264\end{array}$

4.13

218

4.93

310

5.14

335

5.55

382

6.00

433

$8.57 \quad 729$

11.141025

$15.05 \quad 1474$

112

99

132

152

82

172

330

244

1423988

1690500

1594339

901110

1341912

2428289

269261

1398123

3.15

106

3.13

103

3.70

169

4.01

204

4.59

271

4.94

312

5.03

322

5.16

0.00

5.33

5.40

5.49

5.57

5.58

5.63

5.75

5.80

5.96

5.07

5.81

6.02

6.21

6.31

6.41

6.41
337

356

364

375

384

385

391

405

410

429

326

412

436

458

469

481

481
79

42

93

80

79

94

66

128

146

146

108

146

107

45

108

70

117

63

105

77

114

82

139

122
1723754

934410

1728891

676352

1132640

1803596

998165

1503184

1500793.

968467

1239075

1157481

3366718

1536692

879365

551378

1235537

1769291

1281631

812600

2550097

784951

1313641
1533396
Area\%

$\begin{array}{rrr}40.00 \mathrm{ug} / \mathrm{mL} & 0.00 \\ -40.00 \mathrm{ug} / \mathrm{mL} & 0.00 \\ 40.00 \mathrm{ug} / \mathrm{mL} & 0.00 \\ 40.00 \mathrm{ug} / \mathrm{mL} & 0.00 \\ 40.00 \mathrm{ug} / \mathrm{mL} & 0.00 \\ 40.00 \mathrm{ug} / \mathrm{mL} & 0.00\end{array}$

$\div$ Recovery

$69.191 \mathrm{ug} / \mathrm{mL} \quad 69.19 \%$

$71.763 \mathrm{ug} / \mathrm{mL} \quad 71.76 \%$

$71.558 \mathrm{ug} / \mathrm{mL} \quad 71.56 \%$

$70.593 \mathrm{ug} / \mathrm{mL}$

$75.572 \mathrm{ug} / \mathrm{mL}$

$69.295 \mathrm{ug} / \mathrm{mL}$

$70.59 \%$

$75.57 \%$

$74.285 \mathrm{ug} / \mathrm{mL}$

$72.959 \mathrm{ug} / \mathrm{mL}$

$69.30 \%$

$74.29 \%$

$72.96 \%$

Qvalue

68. $174 \mathrm{ug} / \mathrm{mL}$ \#

$68.663 \mathrm{ug} / \mathrm{mL} \# \quad 80$

$70.911 \mathrm{ug} / \mathrm{mL} \quad 90$

$73.566 \mathrm{ug} / \mathrm{mL} \# \quad 77$

$70.964 \mathrm{ug} / \mathrm{mL} \# \quad 1$

$71.592 \mathrm{ug} / \mathrm{mL} \quad 98$

Not Detected

$71.828 \mathrm{ug} / \mathrm{mL} \quad 97$

$71.859 \mathrm{ug} / \mathrm{mL} \quad 96$

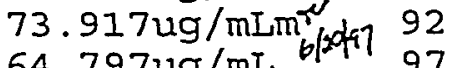

$64.797 \mathrm{ug} / \mathrm{mL}$ b/xt) 97

$63.064 \mathrm{ug} / \mathrm{mI} \quad 99$

$69.834 \mathrm{ug} / \mathrm{mL} \quad 91$

$74.688 \mathrm{ug} / \mathrm{mL} \quad 100$

$71.738 \mathrm{ug} / \mathrm{mL} \quad 96$

$70.699 \mathrm{ug} / \mathrm{mL} \quad 83$

$70.176 \mathrm{ug} / \mathrm{mL} \# \quad 22$

$70.174 \mathrm{ug} / \mathrm{mL} \# \quad 76$

$70.979 \mathrm{ug} / \mathrm{mL} \# \quad 100$

$71.951 \mathrm{ug} / \mathrm{mL} \# \quad 7$

$68.775 \mathrm{ug} / \mathrm{mL} \# \quad 82$

$68.170 \mathrm{ug} / \mathrm{mL} \# \quad 82$

$71.870 \mathrm{ug} / \mathrm{mL} \#$

$(\#)=$ qualifier out of range $(\mathrm{m})=$ manual integration

(*) Does not meet EPA spectral criteria (False Hit) 


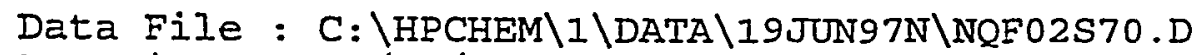

Acq Time : 06/19/97 05:34

Sample : SSTD070 (143WS28447)

Misc : EXP.6/22/97

Operator: TV

Inst : $5972-\mathrm{N}$

Multiplr: 1.00

Quant Time: Jun 20 7:53 1997

Method : C: \HPCHEM $\backslash \backslash \backslash M E T H O D S \backslash 8270 \mathrm{~B} . \mathrm{M}$

Title : Sw846/8270B, Curve:NQF 6/19/97

Last Update : Fri Jun 20 07:49:42 1997

Response via : Multiple Ievel Calibration

Compound

33) Benzoic acid

34) Bis (2-chloroethoxy) methane

35) 2,4-Dichlorophenol

36) A.,A.-dimethylphenethylami

37) 1,2,4-Trichlorobenzene

38) Dodecane NOT Present

39) Naphthalene

10) 4-Chloroaniline

AI) 2,6-Dichlorophenol

12) Hexachlorobutadiene

43) Benzothiazole NOT Present

44) N-nitroso-di-n-butylamine

45) 4-Chloro-3-Methylphenol

46) 2-Methylnaphthalene

A8) $1,2,4,5$-Tetrachlorobenzene

49) Hexachlorocyclopentadiene

50) 2,4,6-Trichlorophenol

51) 2,4,5-Trichlorophenol

53) Tetradecane NOT Present

54) 2-Chloronaphthalene

55) 1-Chloronaphthalene

56) 2-Nitroaniline

57) Dimethylphthalate

58) 2,5-Dinitrotoluene

59) Acenaphthylene

50) 3-Nitroaniline

51) Acenaphthene

52) 2, 4-Dinitrophenol

53) 4-Nitrophenol

54) Dibenzofuran

55) 2,4-Dinitrotoluene

55) Pentachlorobenzene

57) 1-Naphthylamine

58) 2-Naphthylamine

59) 2,3,4,6-Tetrachlorophenol

70) Diethylphthalate

71) 4-Chlorophenyl Phenyl Ethe

72) Fluorene

73) 4-Nitroaniline

75) 4,6-Dinitro-2-methylphenol

77) N-nitrosodiphenylamine

78) 1,2-Diphenyl hydrazine

T9) Tributylphosphate NOT Pres 41
R.T. Scan QIon Response Conc Unit Qvalue

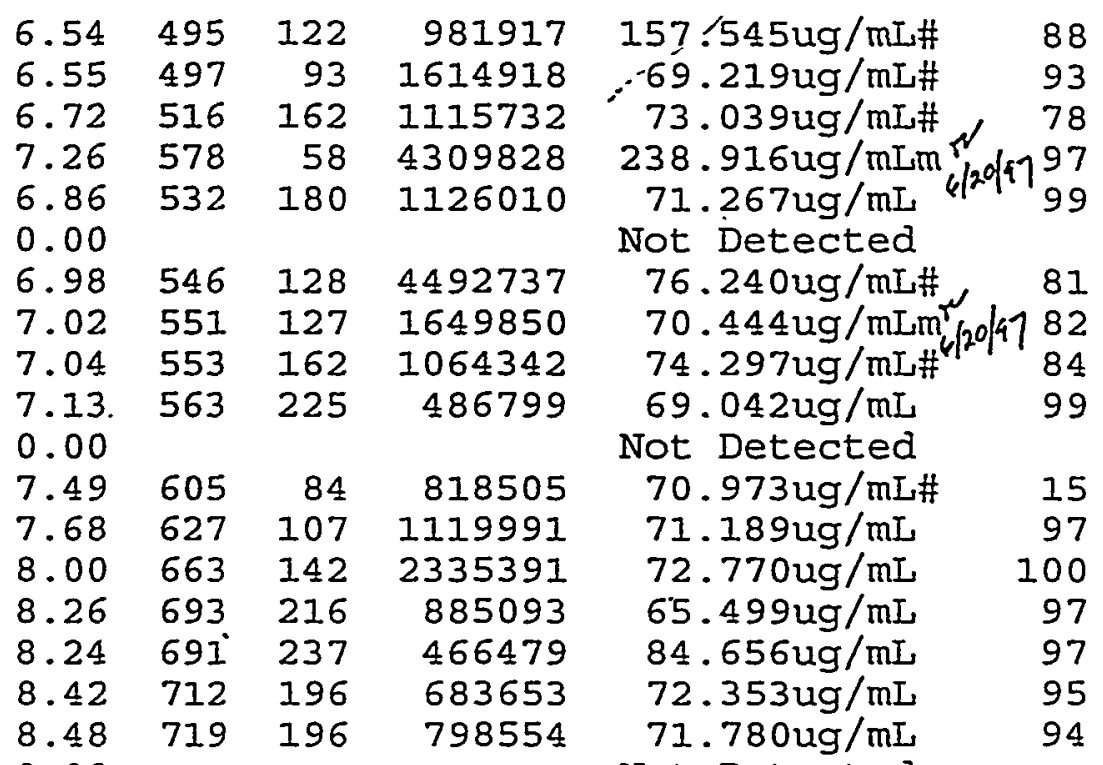

0.00

8.80

$\begin{array}{ll}755 & 162\end{array}$

2452005

Not Detected

$68.473 \mathrm{ug} / \mathrm{mL}$

94

$\begin{array}{llll}8.84 & 760 & 162 & 2101996\end{array}$

$73.669 \mathrm{ug} / \mathrm{mL}$

$69.601 \mathrm{ug} / \mathrm{mL \#}$

$69.108 \mathrm{ug} / \mathrm{mL}$

$72.250 \mathrm{ug} / \mathrm{mL \#}$

$71.375 \mathrm{ug} / \mathrm{mL}$

$72.076 \mathrm{ug} / \mathrm{mL} \#$

$69.493 \mathrm{ug} / \mathrm{mL}$

$108.598 \mathrm{ug} / \mathrm{mL} \#$

$75.867 \mathrm{ug} / \mathrm{mL}$

$76.554 \mathrm{ug} / \mathrm{mL}$

$82.627 \mathrm{ug} / \mathrm{mL} \#$

$68.391 \mathrm{ug} / \mathrm{mL} \#$

$77.102 \mathrm{ug} / \mathrm{mL}$

$73.330 \mathrm{ug} / \mathrm{mL}$

$77.690 \mathrm{ug} / \mathrm{mL \#}$

$67.591 \mathrm{ug} / \mathrm{mL}$

$70.688 \mathrm{ug} / \mathrm{mL}$

$70.826 \mathrm{ug} / \mathrm{mL}$

$68.638 \mathrm{ug} / \mathrm{mL}$

$96.246 \mathrm{ug} / \mathrm{mL}$

$76.860 \mathrm{ug} / \mathrm{mL}$

$73.007 \mathrm{ug} / \mathrm{mL} \#$

Not Detected

(弚) = qualifier out of range $(\mathrm{m})=$ manual integration

(*) Does not meet EPA spectral criteria (False Hit) 
Quantitation Report

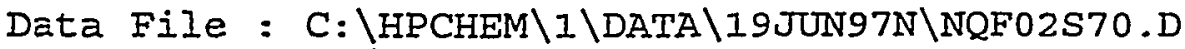

Acq Time : 06/19/97 05:34

Sample : SSTD070 (143WS28447)

Misc : EXP.6/22/97

Operator: TV

Quant Time: Jun 20 7:53 1997

Inst : $5972-\mathrm{N}$

Multiplr: 1.00

Method : C: $\backslash$ HPCHEM $\backslash 1 \backslash$ METHODS $\backslash 8270 \mathrm{~B} . \mathrm{M}$

Title : SW846/8270B, Curve:NQF 6/19/97

Last Update : Fri Jun 20 07:49:42 1997

Response via : Multiple Level Calibration

Compound

R.T. Scan QIon Response Conc Unit Qvalue

80) Phenacetin

81) 4-Bromophenyl Phenyl Ether

82) alpha-BHC

83) Hexachlorobenzene

84) Tris (2-CE) phosphate NOT $\mathrm{Pr}$

B5) 4-Aminobiphenyl

B6) beta-BHC

B7) Pentachlorophenol

B8) Pronamide

B9) gamma-BHC

90) Pentachloronitrobenzene

91) Phenanthrene

92) Anthracene

93) delta-BHC

94) Heptachlox

95) Di-n-Butylphthalate

96) Aldrin

97) Eluoranthene

99) Benzidine

100) Pyrene

101) $4,4 \cdot-D D E$

103) Dieldrin

104) p-Dimethylaminoazobenzene

i05) $4,41-D D D$

106) Butylbenzylphthalate

107) $4,4 i-D D T$

IOB) Methoxychlor

109) 3,3'-Dichlorobenzidine

110) Benzo[a] anthracene

111) Chrysene

112) Bis (2-ethylhexyl) phthalate

114) Di-n-octylphthalate

115) Benzo[b] fluoranthene

116) 7,12-Dimethylbenz (a) anthra

117) Benzo[k] fluoranthene

1IB) Benzo[a] pyrene

119) 3-Methylcholanthrene

120) Indeno $(1,2,3-c, d)$ pyrene

121) Dibenz $[a, h]$ anthracene

122) Benzo[ghi] perylene
R.

$11.48 \quad 1064 \quad 108 \quad 1033456 \quad-76.745 \mathrm{ug} / \mathrm{mL \#} \quad 42$

$11.61 \quad 1078 \quad 248 \quad 473667^{\circ} 91.505 \mathrm{ug} / \mathrm{mL} \# \quad 84$

$\begin{array}{lllll}11.62 & 1080 & 183 & 336569 & 72.261 \mathrm{ug} / \mathrm{mL} \# \quad 70\end{array}$

$11.711090 \cdot 284 \quad 487086 \cdot 72.215 \mathrm{ug} / \mathrm{mL} \# \quad 87$

0.00

$\begin{array}{llll}12.08 & 1133 & 169 & 1915646\end{array}$

$\begin{array}{llll}12.01 & 1124 & 181 & 253661\end{array}$

$\begin{array}{llll}12.07 & 1131 & 266 & 299558\end{array}$

$\begin{array}{llll}12.19 & 1145 & 173 & 837578\end{array}$

$\begin{array}{llll}12.17 & 1143 & 183 & 262844\end{array}$

$\begin{array}{llll}12.09 & 1134 & 237 & 168077\end{array}$

$12.48 \quad 1179 \quad 178 \quad 2919859$

$\begin{array}{llll}12.57 & 1189 & 178 & 2855131\end{array}$

$\begin{array}{llll}12.58 & 1190 & 183 & 202732\end{array}$

$13.33 \quad 1276 \quad 100 \quad 404096$

$\begin{array}{llll}13.48 & 1293 & 149 & 3687985\end{array}$

$13.86 \quad 1337$

$14.48 \quad 1409$

$14.68 \quad 1432$

$14.83 \quad 1449$

$14.96 \quad 1464$

$15.12 \quad 1482$

15.241496

$15.43 \quad 1518$

$15.73 \quad 1552$

15.871568

16.481638

$16.55 \quad 1647$

$16.62 \quad 1655$

16.681662

16.611654

$17.88 \quad 1800$

$18.88 \quad 1914$

18.861912

$18.95 \quad 1922$

19.752015

20.832139

$23.78 \quad 2478$

$23.90 \quad 2492$

24.992617
66

202

184

202

246

79

225

235

149

235

227

252

228

228

149

149

252

256

252

252

268

276

278

276
Not Detected

$79.306 \mathrm{ug} / \mathrm{mL}$

98

$69.231 \mathrm{ug} / \mathrm{mL} \#$

55

$123.872 \mathrm{ug} / \mathrm{mL}$

98

$67.814 \mathrm{ug} / \mathrm{mL}$

$66.825 \mathrm{ug} / \mathrm{mLm} / \mathrm{F}^{\prime} \mid 1792$

$73.380 \mathrm{ug} / \mathrm{mL} \# \quad 6 \leqslant$

$75.218 \mathrm{ug} / \mathrm{mL} \# \quad 95$

$71.589 \mathrm{ug} / \mathrm{mI} \quad 98$

$61.318 \mathrm{ug} / \mathrm{mL}$

$9 \leq$

69.819ug/mL\#

72

323338

2390798

1073254

2261019

384072

360884

408589

645155

1249382

568399

947462

521884

1357049

1347723

1493094

2371431

1049425

427716

949094

872674

440244

716827

$70.712 \mathrm{ug} / \mathrm{mL} \#$

$66.354 \mathrm{ug} / \mathrm{mL}$

$74.874 \mathrm{ug} / \mathrm{mL} \#$

$74.724 \mathrm{ug} / \mathrm{mL}$

$70.785 \mathrm{ug} / \mathrm{mL}$

$70.528 \mathrm{ug} / \mathrm{mI}$

$66.537 \mathrm{ug} / \mathrm{mL}$

$77.808 \mathrm{ug} / \mathrm{mL}$

$68.400 \mathrm{ug} / \mathrm{mL}_{\pi}$

$72.147 \mathrm{ug} / \mathrm{mL}$

$93.432 \mathrm{ug} / \mathrm{mL}$

$75.115 \mathrm{ug} / \mathrm{mL}$

$68.763 \mathrm{ug} / \mathrm{mL} \#$

$68.065 \mathrm{ug} / \mathrm{mL}$

$70.135 \mathrm{ug} / \mathrm{mL}$

$68.715 \mathrm{ug} / \mathrm{mL}$

$74.912 \mathrm{ug} / \mathrm{mL}$

$74.143 \mathrm{ug} / \mathrm{mL} \#$

$79.905 \mathrm{ug} / \mathrm{mL}$

$71.628 \mathrm{ug} / \mathrm{mL} \#$

$74.034 \mathrm{ug} / \mathrm{mL \#}$

$81.353 \mathrm{ug} / \mathrm{mL}$

$72.648 \mathrm{ug} / \mathrm{mL}$

714760

$82.607 \mathrm{ug} / \mathrm{mL} \#$

$83.974 \mathrm{ug} / \mathrm{mL \#}$

92

95

$6 \leqslant$

85

55

$10 C$

$8 \leqslant$

56

89

87

86

98

95

99

100

99

100

.88

95

89

88

98

97

669634

\section{2}

Data Processed By: Dure Gay $6 / 20 / 47$

$\left(\frac{H}{\pi}\right)=$ qualifier out of range $(m)=$ manual integration

(*) Does not meet EPA spectral criteria (False Hit) 
Quantitation Report File: NQFO2S70.D $\quad$ standard amount: 70

Path: C:IHPCHEMI1IDATAI19JUN97NI

06/19/97

Sample: SSTD070 (143WS28447)

Instrument ID: $5972-\mathrm{N} \quad$ Analyst: TV

\begin{tabular}{|c|c|c|c|c|c|c|c|}
\hline $\begin{array}{r}\text { No. } \\
1\end{array}$ & $\begin{array}{c}\text { Name } \\
\text { 1,4-Dichlorobenzene-d4 }\end{array}$ & $\begin{array}{l}\text { RT } \\
5.38\end{array}$ & $\begin{array}{l}\text { scan } \\
362\end{array}$ & $\begin{array}{c}\mathrm{m} / \mathrm{z} \\
152.00\end{array}$ & $\begin{array}{c}\text { area } \\
606564\end{array}$ & $\begin{array}{c}\mathrm{RF} \\
1.000\end{array}$ & $\begin{array}{c}\text { amt } \\
40.000\end{array}$ \\
\hline 25 & Naphthalene-d8 & 6.95 & 543 & 136.00 & 2370684 & 1.000 & 40.000 \\
\hline 47 & Acenaphthene-d10 & 9.74 & 864 & 164.00 & 1149337 & 1.000 & 40.000 \\
\hline 75 & Phenanthrene-d10 & 12.43 & 1173 & 188.00 & 1387588 & 1.000 & 40.000 \\
\hline 98 & Chrysene-d12 & 16.64 & 1657 & 240.00 & 735038 & 1.000 & 40.000 \\
\hline 113 & Perylene-d12 & 19.91 & 2033 & 264.00 & 470908 & 1.000 & 40.000 \\
\hline 6 & 2-Fluorophenol & 4.13 & 218 & 112.00 & 1423988 & 1.342 & 70.00 \\
\hline 8 & Phenol-d5 & 4.93 & 310 & 99.00 & 1690500 & 1.593 & 70.00 \\
\hline 11 & 2-Chlorophenol-d4 & 5.14 & 335 & 132.00 & 1594339 & 1.502 & 70.00 \\
\hline 17 & 1,2-Dichlorobenzene-d4 & 5.55 & 382 & 152.00 & 901110 & 0.849 & 70.00 \\
\hline 27 & Nitrobenzene-d5 & 6.00 & 433 & 82.00 & 1341912 & 0.323 & 70.00 \\
\hline 52 & 2-Fluorobiphenyl & 8.57 & 729 & 172.00 & 2428289 & 1.207 & 70.00 \\
\hline 74 & 2,4,6-Tribromophenol & 11.14 & 1025 & 330.00 & 269261 & 0.134 & 70.00 \\
\hline 102 & Terphenyl-d14 & 15.05 & 1474 & 244.00 & 1398123 & 1.087 & 70.00 \\
\hline 2 & Pyridine & 3.15 & 106 & 79.00 & 1723754 & $1.624^{\circ}$ & 70.00 \\
\hline 3 & $\mathrm{~N}$-nitrosodimethylamine & 3.13 & 103 & 42.00 & 934410 & 0.880 & 70.00 \\
\hline 4 & 2-Picoline & 3.70 & 169 & 93.00 & 1728891 & 1.629 & 70.00 \\
\hline 5 & Methyl methanesulfonate & 4.01 & 204 & 80.00 & 676352 & 0.637 & 70.00 \\
\hline 7 & Ethyl methanesulfonate & 4.59 & 271 & 79.00 & 1132640 & 1.067 & 70.00 \\
\hline 9 & Phenol & 4.94 & 312 & 94.00 & 1803596 & 1.699 & 70.00 \\
\hline 10 & Aniline & 5.03 & 322 & 66.00 & 998165 & 0.940 & 70.00 \\
\hline 12 & 2-Chlorophenol & 5.16 & 337 & 128.00 & 1533396 & 1.445 & 70.00 \\
\hline 13 & Decane NOT Present & 0.00 & 1 & 57.00 & 0 & 0.000 & 70.00 \\
\hline 14 & 1,3-Dichlorobenzene & 5.33 & 356 & 146.00 & 1503184 & 1.416 & 70.00 \\
\hline 15 & 1.4-dichlorobenzene & 5.40 & 364 & 146.00 & 1500793 & 1.414 & 70.00 \\
\hline 16 & Benzyl alcohol & 5.49 & 375 & 108.00 & 968467 & 0.912 & 70.00 \\
\hline 18 & 1,2-dichlorobenzene & 5.57 & 384 & 146.00 & 1239075 & 1.167 & 70.00 \\
\hline 19 & 2-Methylphenol & 5.58 & 385 & 107.00 & 1157481 & 1.090 & 70.00 \\
\hline 20 & Bis(2-chloroisopropyl)ether & 5.63 & 391 & 45.00 & 3366718 & 3.172 & 70.00 \\
\hline 21 & 4-methylphenol & 5.75 & 405 & 108.00 & 1536692 & 1.448 & 70.00 \\
\hline 22 & N-Nitrosodi-n-propyl amine & 5.80 & 410 & 70.00 & 879365 & 0.828 & 70.00 \\
\hline 23 & Hexachloroethane & 5.96 & 429 & 117.00 & 551378 & 0.519 & 70.00 \\
\hline 24 & Bis (2-chloroethyl)ether & 5.07 & 326 & 63.00 & 1235537 & 1.164 & 70.00 \\
\hline 26 & Acetophenone & 5.81 & 412 & 105.00 & 1769291 & 0.426 & 70.00 \\
\hline 28 & Nitrobenzene & 6.02 & 436 & 77.00 & 1281631 & 0.309 & 70.00 \\
\hline 29 & $\mathrm{~N}$-nitrosopiperidine & 6.21 & 458 & 114.00 & 812600 & 0.196 & 70.00 \\
\hline 30 & Isophorone & 6.31 & 469 & 82.00 & 2550097 & 0.615 & 70.00 \\
\hline 31 & 2-Nitrophenol & 6.41 & 481 & 139.00 & 784951 & 0.189 & 70.00 \\
\hline 32 & 2,4-Dimethylphenol & 6.41 & 481 & 122.00 & 1313641 & 0.317 & 70.00 \\
\hline 33 & Benzoic acid & 6.54 & 495 & 122.00 & 981917 & 0.237 & 70.00 \\
\hline 34 & Bis(2-chloroethoxy)methane & 6.55 & 497 & 93.00 & 1614918 & 0.389 & 70.00 \\
\hline 35 & 2,4-Dichlorophenol & 6.72 & 516 & 162.00 & 1115732 & 0.269 & 70.00 \\
\hline
\end{tabular}


Path: C:IHPCHEMI1LATAI19JUN97NI

$06 / 19 / 97$

Sample: SSTDO7O (143WS28447)

Instrument ID: 5972 - N

Analyst: TV

\begin{tabular}{|c|c|c|c|c|c|c|c|}
\hline $\begin{array}{l}\text { No. } \\
36\end{array}$ & A.,A.-dimethylphenethylamine & $\begin{array}{l}R T \\
7.26\end{array}$ & $\begin{array}{l}\text { scan } \\
578\end{array}$ & $\begin{array}{l}\mathrm{m} / \mathrm{z} \\
58.00\end{array}$ & $\begin{array}{c}\text { area } \\
4309828\end{array}$ & $\begin{array}{l}\mathrm{RF} \\
1.039\end{array}$ & $\begin{array}{l}\text { amt } \\
70.00\end{array}$ \\
\hline 37 & $1,2,4$-Trichlorobenzene & 6.86 & 532 & 180.00 & 1126010 & 0.271 & 70.00 \\
\hline 38 & Dodecane NOT Present & 0.00 & 1 & 57.00 & 0 & 0.000 & 70.00 \\
\hline 39 & Naphthalene & 6.98 & 546 & 128.00 & 4492737 & 1.083 & -70.00 \\
\hline 40 & 4-Chloroaniline & 7.02 & 551 & 127.00 & 1649850 & 0.398 & 70.00 \\
\hline 41 & 2,6-Dichlorophenol & 7.04 & 553 & 162.00 & 1064342 & 0.257 & 70.00 \\
\hline 42 & Hexachlorobutadiene & 7.13 & 563 & 225.00 & 486799 & 0.117 & 70.00 \\
\hline 43 & Benzothiazole NOT Present & 0.00 & 1 & 135.00 & 0 & 0.000 & 70.00 \\
\hline 44 & N-nitroso-di-n-butylamine & 7.49 & 605 & 84.00 & 818505 & 0.197 & 70.00 \\
\hline 45 & 4-Chloro-3-Methylphenol & 7.68 & 627 & 107.00 & 1119991 & 0.270 & 70.00 \\
\hline 46 & 2-Methylnaphthalene & 8.00 & 663 & 142.00 & 2335391 & 0.563 & 70.00 \\
\hline 48 & 1,2,4,5-Tetrachlorobenzene & 8.26 & 693 & 216.00 & 885093 & 0.440 & 70.00 \\
\hline 49 & Hexachlorocyclopentadiene & 8.24 & 691 & 237.00 & 466479 & 0.232 & 70.00 \\
\hline 50 & 2,4,6-Trichlorophenol & 8.42 & 712 & 196.00 & 683653 & 0.340 & 70.00 \\
\hline 51 & 2,4,5-Trichlorophenol & 8.48 & 719 & 196.00 & 798554 & 0.397 & 70.00 \\
\hline 53 & Tetradecane NOT Present & 0.00 & 1 & 57.00 & 0 & 0.000 & 70.00 \\
\hline 54 & 2-Chloronaphthalene & 8.80 & 755 & 162.00 & 2452005 & 1.219 & 70.00 \\
\hline 55 & 1-Chloronaphthalene & 8.84 & 760 & 162.00 & 2101996 & 1.045 & 70.00 \\
\hline 56 & 2-Nitroaniline & 8.94 & 771 & 65.00 & 646947 & 0.322 & 70.00 \\
\hline 57 & Dimethylphthalate & 9.24 & 806 & 163.00 & 2569057 & 1.277 & 70.00 \\
\hline 58 & 2,6-Dinitrotoluene & 9.35 & 819 & 165.00 & 659318 & 0.328 & 70.00 \\
\hline 59 & Acenaphthylene & 9.50 & 836 & 152.00 & 3682746 & 1.831 & 70.00 \\
\hline 60 & 3-Nitroaniline & 9.65 & 853 & 65.00 & 708875 & 0.352 & 70.00 \\
\hline 61 & Acenaphthene & 9.81 & 871 & 153.00 & 2153104 & 1.070 & 70.00 \\
\hline 62 & 2,4-Dinitrophenol & 9.83 & 874 & 184.00 & 421039 & 0.209 & 70.00 \\
\hline 63 & 4-Nitrophenol & 9.90 & 882 & 109.00 & 201795 & 0.100 & 70.00 \\
\hline 64 & Dibenzofuran & 10.10 & 905 & 168.00 & 3032655 & 1.508 & 70.00 \\
\hline 65 & 2,4-Dinitrotoluene & 10.07 & 901 & 165.00 & 837759 & 0.417 & 70.00 \\
\hline 66 & Pentachlor & 10.03 & 897 & 250.00 & 674002 & 0.335 & 70.00 \\
\hline 67 & 1-Naphthylamine & 10.24 & 921 & 143.00 & 1593021 & 0.792 & 70.00 \\
\hline 68 & 2-Naphthylamine & 10.38 & 937 & 143.00 & 2288667 & 1.138 & 70.00 \\
\hline 69 & $2,3,4,6$-Tetrachloro & 10.30 & 928 & 232.00 & 466025 & 0.232 & 70.00 \\
\hline 70 & Diethyl & 10.49 & 950 & 149.00 & 2431777 & 1.209 & 70.00 \\
\hline 71 & 4-Chlorophenyl Phenyl Ether & 10.71 & 975 & 204.00 & 908179 & 0.452 & 70.00 \\
\hline 72 & $\begin{array}{l}\text { Fluorene } \\
\end{array}$ & 10.72 & 976 & 166.00 & 2055188 & 1.022 & 70.00 \\
\hline 73 & 4-Nitroaniline & 10.74 & 978 & 65.00 & 534432 & 0.266 & 70.00 \\
\hline 76 & 4,6-Dinitro-2-methylphenol & 10.79 & 984 & 198.00 & 480705 & 0.198 & 70.00 \\
\hline 77 & $N$-nitrosodiphenylamine & 10.92 & 999 & 169.00 & 1690230 & 0.696 & 70.00 \\
\hline 78 & 1,2-Diphenylhydrazine & 11.00 & 1008 & 77.00 & 2121616 & 0.874 & 70.00 \\
\hline 79 & Tributylphosphate NOT Present & 0.00 & 1 & 99.00 & 0 & 0.000 & 70.00 \\
\hline 80 & & 11.48 & 1064 & 108.00 & 1033456 & 0.426 & 70.00 \\
\hline 81 & 4-Bromophenyl Phenyl Ether & 11.61 & 1078 & 248.00 & 473667 & 0.195 & 70.00 \\
\hline
\end{tabular}


Quantitation Report File: NQF02S70.D

Path: C:IHPCHEMI1IDATAI19JUN97NI

$06 / 19 / 97$

Sample: SSTD070 (143WS28447)

Instrument ID: $5972-\mathrm{N} \quad$ Analyst: TV

\begin{tabular}{|c|c|c|c|c|c|c|c|}
\hline No. & Name & RT & scan & $\mathrm{m} / \mathrm{z}$ & area & $\mathrm{RF}$ & amt \\
\hline 82 & alpha-BHC & 11.62 & 1080 & 183.00 & 336569 & 0.139 & 70.00 \\
\hline 83 & Hexachlorobenzene & 11.71 & 1090 & 284.00 & 487086 & 0.201 & 70.00 \\
\hline 84 & ris(2-CE)phosphate NOT Present & 0.00 & 1 & 249.00 & 0 & 0.000 & 70.00 \\
\hline 85 & 4-Aminobiphenyl & 12.08 & 1133 & 169.00 & 1915646 & 0.789 & 70.00 \\
\hline 86 & beta-BHC & 12.01 & 1124 & 181.00 & 253661 & $0.10^{\circ}$ & 70.00 \\
\hline 87 & Pentachlorophenol & 12.07 & 1131 & 266.00 & 299558 & 0.123 & 70.00 \\
\hline 88 & Pronamide & 12.19 & 1145 & 173.00 & 837578 & 0.345 & 70.00 \\
\hline 89 & gamma-BHC & 12.17 & 1143 & 183.00 & 262844 & 0.108 & 70.00 \\
\hline 90 & Pentachloronitrobenzene & 12.09 & 1134 & 237.00 & 168077 & 0.069 & 70.00 \\
\hline 91 & Phenanthrene & 12.48 & 1179 & 178.00 & 2919859 & 1.202 & 70.00 \\
\hline 92 & Anthracene & $\cdot 12.57$ & 1189 & 178.00 & 2855131 & 1.176 & 70.00 \\
\hline 93 & delta-BHC & 12.58 & 1190 & 183.00 & 202732 & 0.083 & 70.00 \\
\hline 94 & Heptachlor & 13.33 & 1276 & 100.00 & 404096 & 0.166 & 70.00 \\
\hline 95 & Di-n-Butylphthalate & 13.48 & 1293 & 149.00 & 3687985 & 1.519 & 70.00 \\
\hline 96 & Aldrin & 13.86 & 1337 & 66.00 & 323338 & 0.133 & 70.00 \\
\hline 97 & Fluoranthene & 14.48 & 1409 & 202.00 & 2390798 & 0.985 & 70.00 \\
\hline 99 & -Benzidine & 14.68 & 1432 & 184.00 & 1073254 & $0.834^{\circ}$ & 70.00 \\
\hline 100 & Pyrene & 14.83 & 1449 & 202.00 & 2261019 & 1.758 & 70.00 \\
\hline 101 & $4,4^{\prime}-\mathrm{DDE}$ & 14.96 & 1464 & 246.00 & 384072 & 0.299 & 70.00 \\
\hline 103 & Dieldrin & 15.12 & 1482 & 79.00 & 360884 & 0.281 & 70.00 \\
\hline 104 & p-Dimethylaminoazobenzene & 15.24 & 1496 & 225.00 & 408589 & 0.318 & 70.00 \\
\hline 105 & $4,4^{\prime}-D D D$ & 15.43 & 1518 & 235.00 & 645155 & 0.502 & 70.00 \\
\hline 106 & Butylbenzylphthalate & 15.73 & 1552 & 149.00 & 1249382 & 0.971 & 70.00 \\
\hline 107 & $4,4^{\prime}-$ DDT & 15.87 & 1568 & 235.00 & 568399 & 0.442 & 70.00 \\
\hline 108 & Methoxychlor & 16.48 & 1638 & 227.00 & 947462 & 0.737 & 70.00 \\
\hline 109 & 3,3'-Dichlorobenzidine & 16.55 & 1647 & 252.00 & 521884 & 0.406 & 70.00 \\
\hline 110 & Benzo[a]anthracene & 16.62 & 1655 & 228.00 & 1357049 & 1.055 & 70.00 \\
\hline 111 & Chrysene & 16.68 & 1662 & 228.00 & 1347723 & 1.048 & 70.00 \\
\hline 112 & Bis(2-ethylhexyl)phthalate & 16.61 & 1654 & 149.00 & 1493094 & $1.16 \underline{1}$ & 70.00 \\
\hline 114 & Di-n-octylphthalate & 17.88 & 1800 & 149.00 & 2371431 & 2.878 & 70.00 \\
\hline 115 & Benzo[b]fluoranthene & 18.88 & 1914 & $252.00^{\circ}$ & 1049425 & 1.273 & 70.00 \\
\hline 116 & 12-Dimethylbenz(a)anthracene & 18.86 & 1912 & 256.00 & 427716 & 0.519 & 70.00 \\
\hline 117 & Benzo[k]fluoranthene & 18.95 & 1922 & 252.00 & 949094 & 1.152 & 70.00 \\
\hline 118 & Benzo[a]pyrene & 19.75 & 2015 & 252.00 & 872674 & 1.059 & 70.00 \\
\hline 119 & 3-Methylcholanthrene & 20.83 & 2139 & 268.00 & 440244 & 0.534 & 70.00 \\
\hline 120 & deno $(1,2,3-c, d)$ pyrene & 23.78 & 2478 & 276.00 & 716827 & 0.870 & 70.00 \\
\hline 121 & ibenz[a,h]anthracene & 23.90 & 2492 & 278.00 & 714760 & 0.867 & 70.00 \\
\hline 122 & Benzo[ghi]perylene & 24.99 & 2617 & 276.00 & 669634 & 0.813 & 70.00 \\
\hline
\end{tabular}




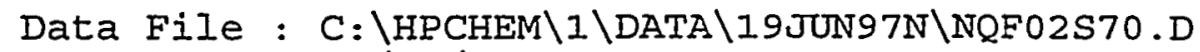

Acq Time : 06/19/97 05:34

Sample : SSTD070 (143WS28447)

Misc : EXP.6/22/97

Quant Time: Jun 19 6:15 1997

Operator: TV

Inst : $5972-\mathrm{N}$

Multiplr: 1.00

Method : C: \HPCHEM $\backslash I \backslash M E T H O D S \backslash 8270 B . M$

Title : Sw846/8270B, Curve:NQF 6/19/97

Last Update : Fri Jun 20 07:49:42 1997

Response via : Multiple Level Calibration

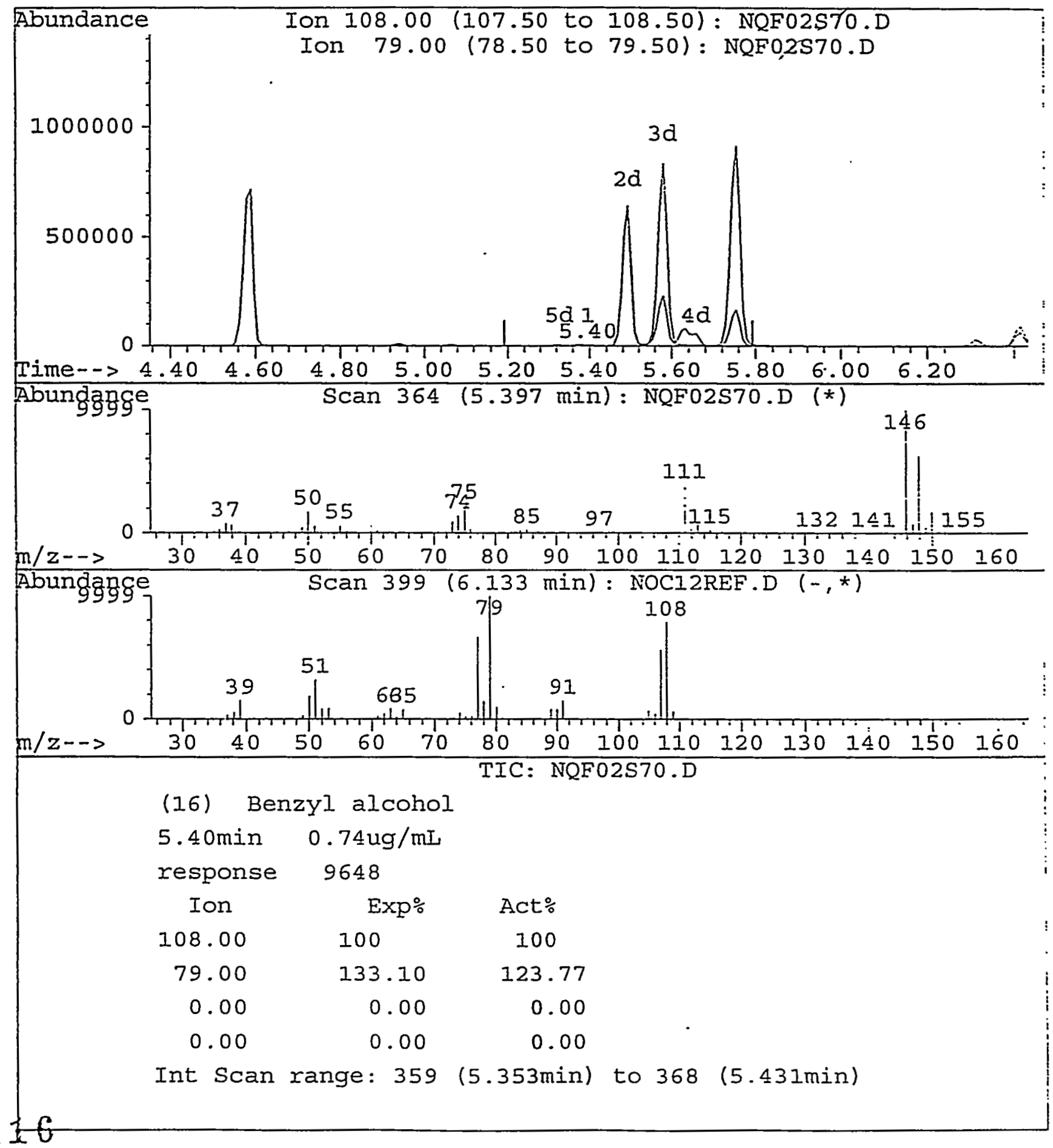




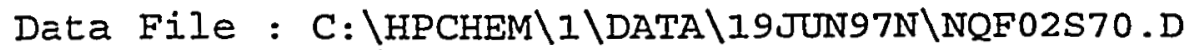

$\begin{array}{ll}\text { Acq Time } & : 06 / 19 / 9705: 34 \\ \text { Sample } & : \text { SSTD070 (143WS28447) } \\ \text { Misc } & \text { : EXP.6/22/97 }\end{array}$

Quant Time: Jun $20 \quad 7: 501997$

Operator: TV

Inst : $5972-\mathrm{N}$

Multiplr: 1.00

Method : C: $\backslash$ HPCHEM $\backslash \backslash \backslash M E T H O D S \backslash 8270 B . M$

Title : SW846/8270B, Curve:NQF 6/19/97

Last Update : Fri Jun 20 07:49:42 1997

Response via : Multiple Level Calibration

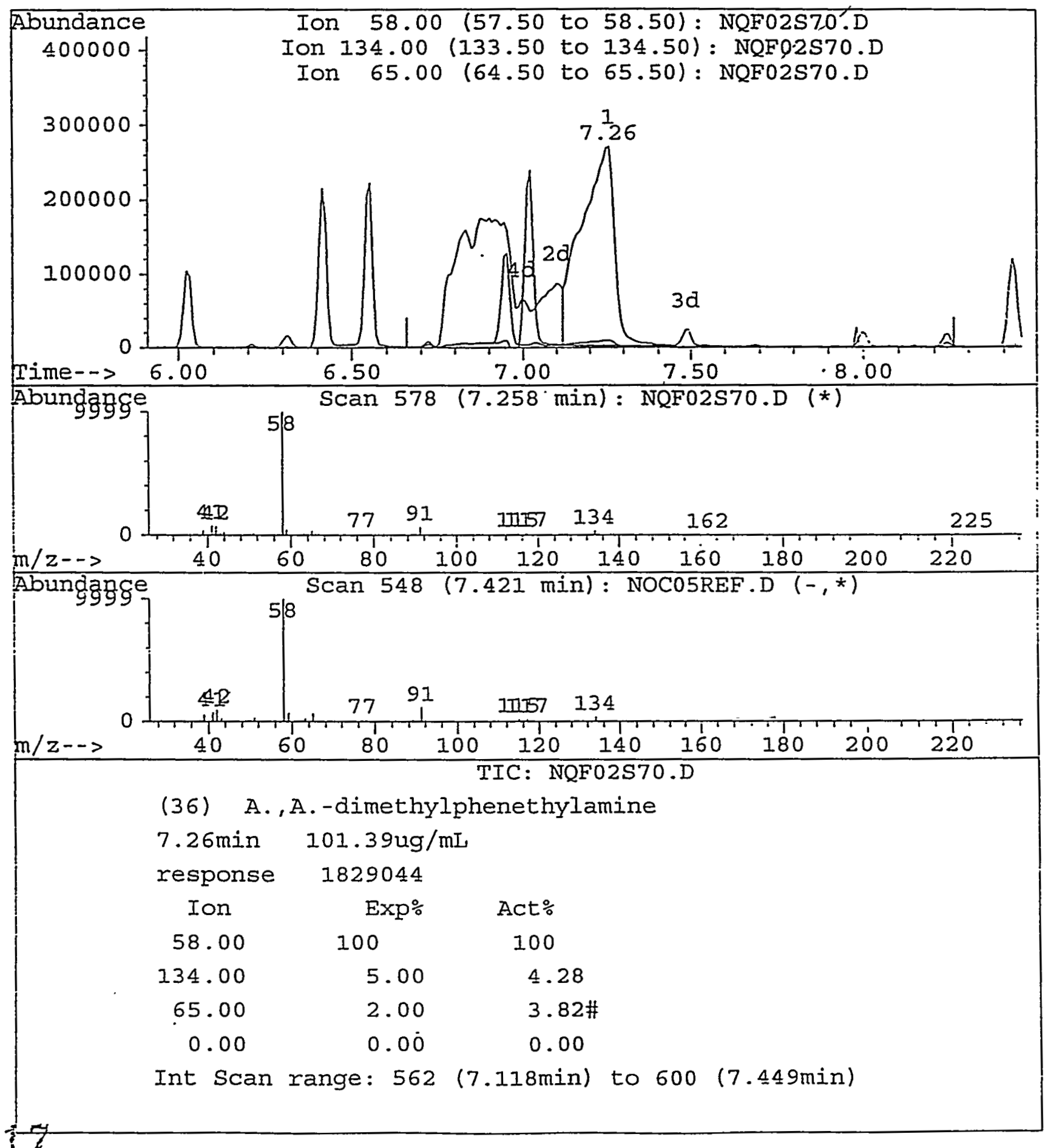


Quantitation Report

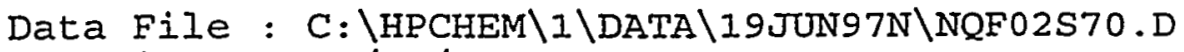

Acq Time : 06/19/97 05:34

Sample : SSTD070 (143WS28447)

MisC : EXP.6/22/97

Operator: TV

Quant Time: Jun 20 7:51 1997

Inst : $5972-\mathrm{N}$

Multiplr: 1.00

Method : C: $\backslash$ HPCHEM $\backslash I \backslash M E T H O D S \backslash 8270 \mathrm{~B} . \mathrm{M}$

Title : SW846/8270B, Curve:NQF 6/19/97

Last Update : Fri Jun 20 07:49:42 1997

Response via : Multiple Level Calibration

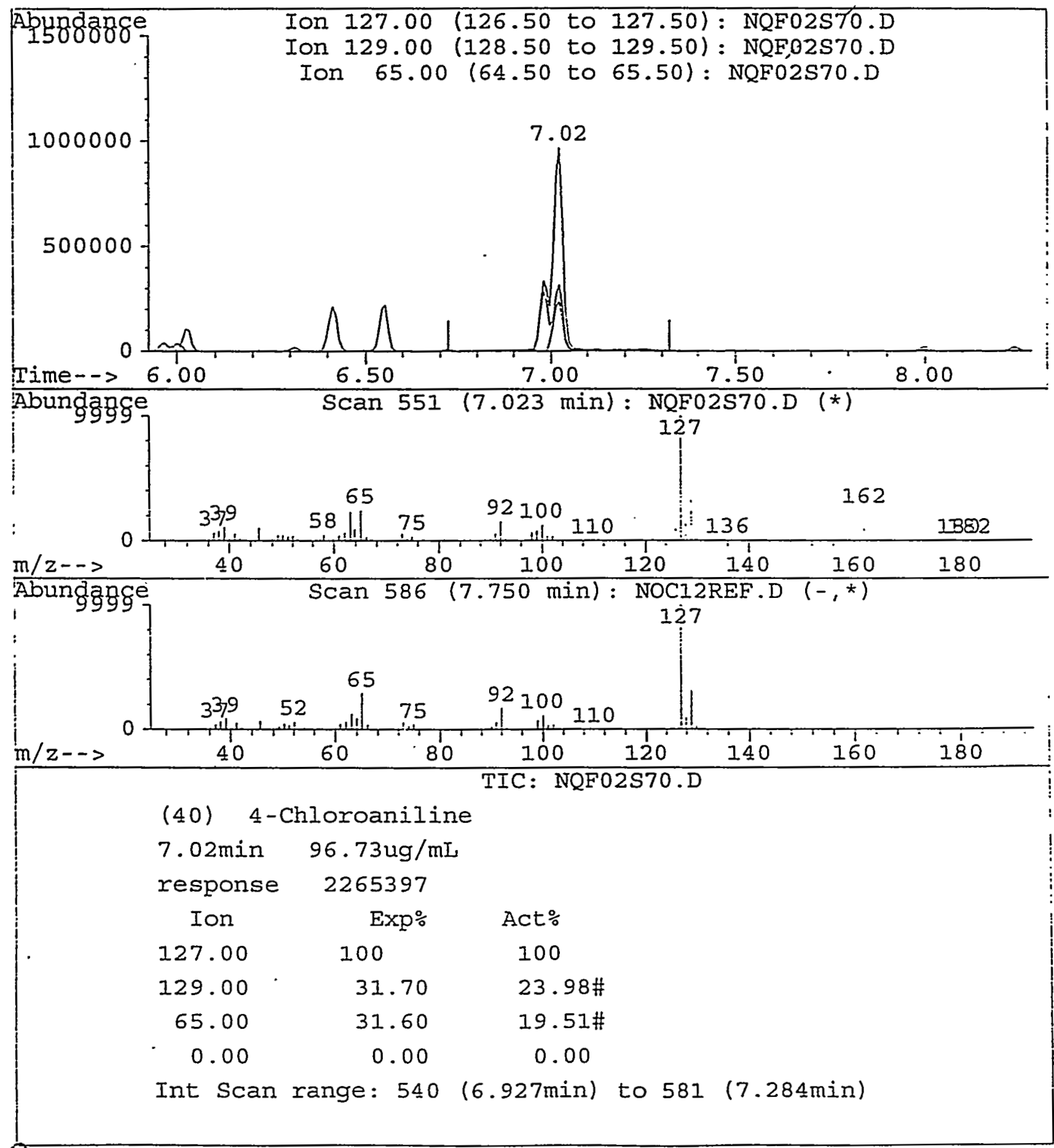




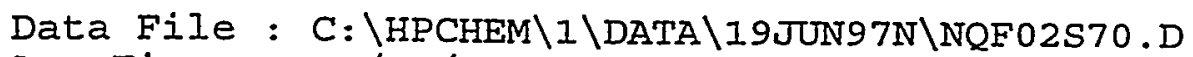

Acq Time : 06/19/97 05:34

Sample : SSTD070 (143WS28447)

Misc : EXP.6/22/97

Quant Time: Jun 20 7:52 1997

Operator: TV

Inst : $5972-\mathrm{N}$

Multiplr: 1.00

$\begin{array}{ll}\text { Method } & : \text { C: } \backslash \text { HPCHEM } \backslash I \backslash M E T H O D S \backslash 8270 B . M \\ \text { Title } & : \text { SW846/8270B, Curve:NQF 6/19/97 } \\ \text { Last Update : Fri Jun 20 07:49:42 1997 } \\ \text { Response via : Multiple Level Calibration }\end{array}$

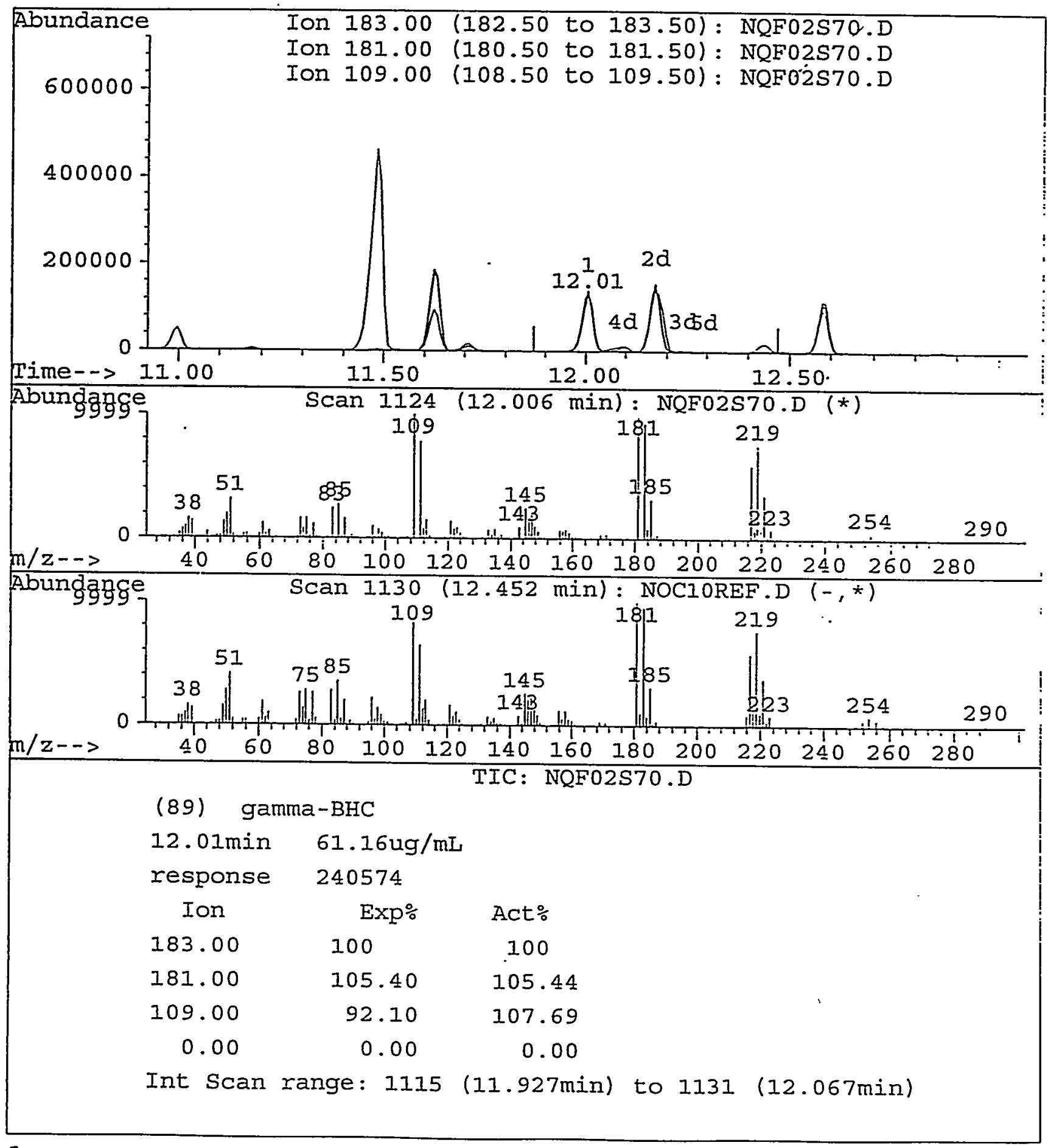

419

NQF02S70.D 8270B.M Fri Jun 20 07:53:34 1997 
MANUAL IN TEG R A T I O N FOR Benzyl alcohol

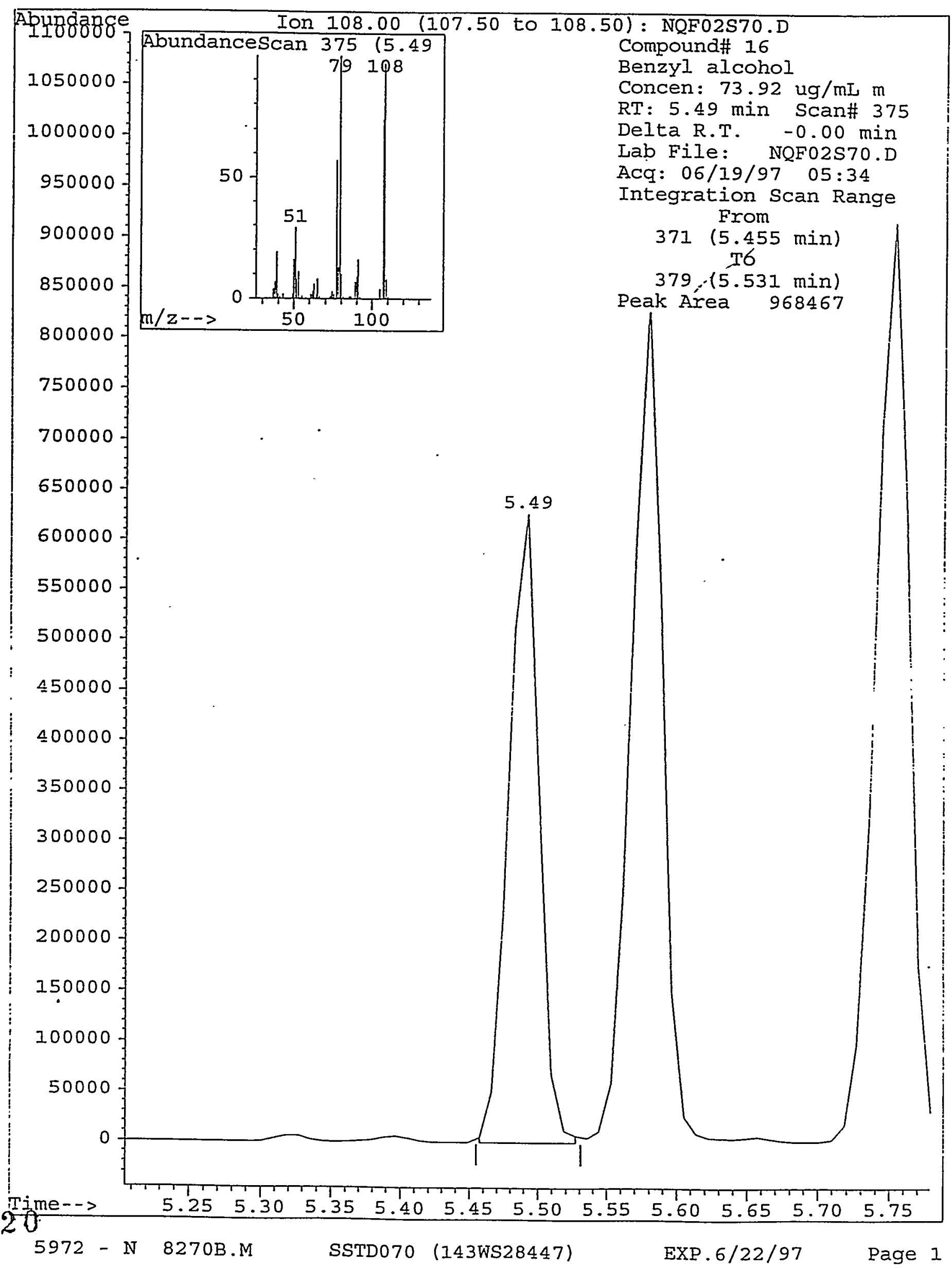


MANUAI IN TEGRATION FOR A.,A.-dimethylphenethylamine

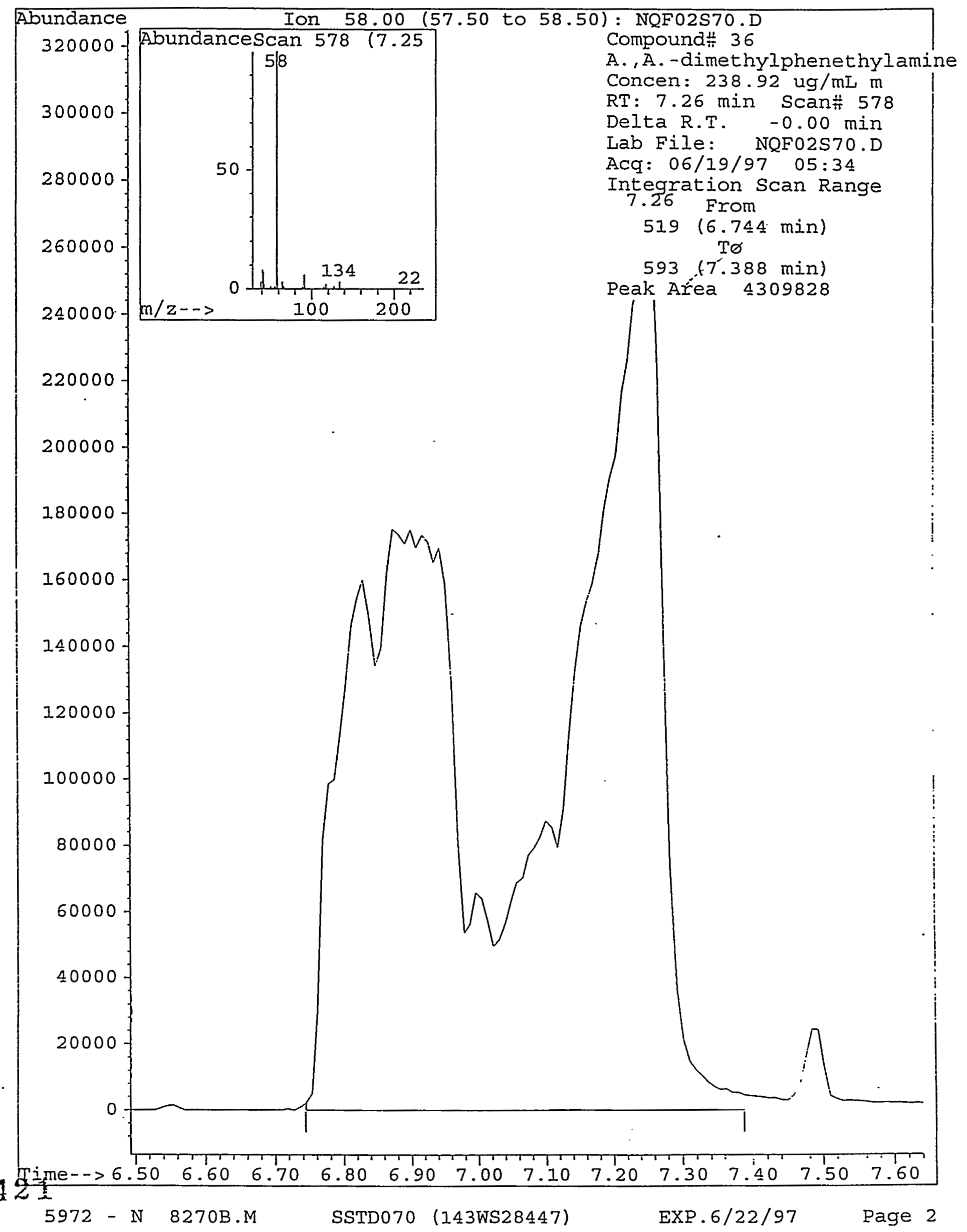


$M$ A N U A I I N T E G R A T I O N FOR 4-Chloroaniline

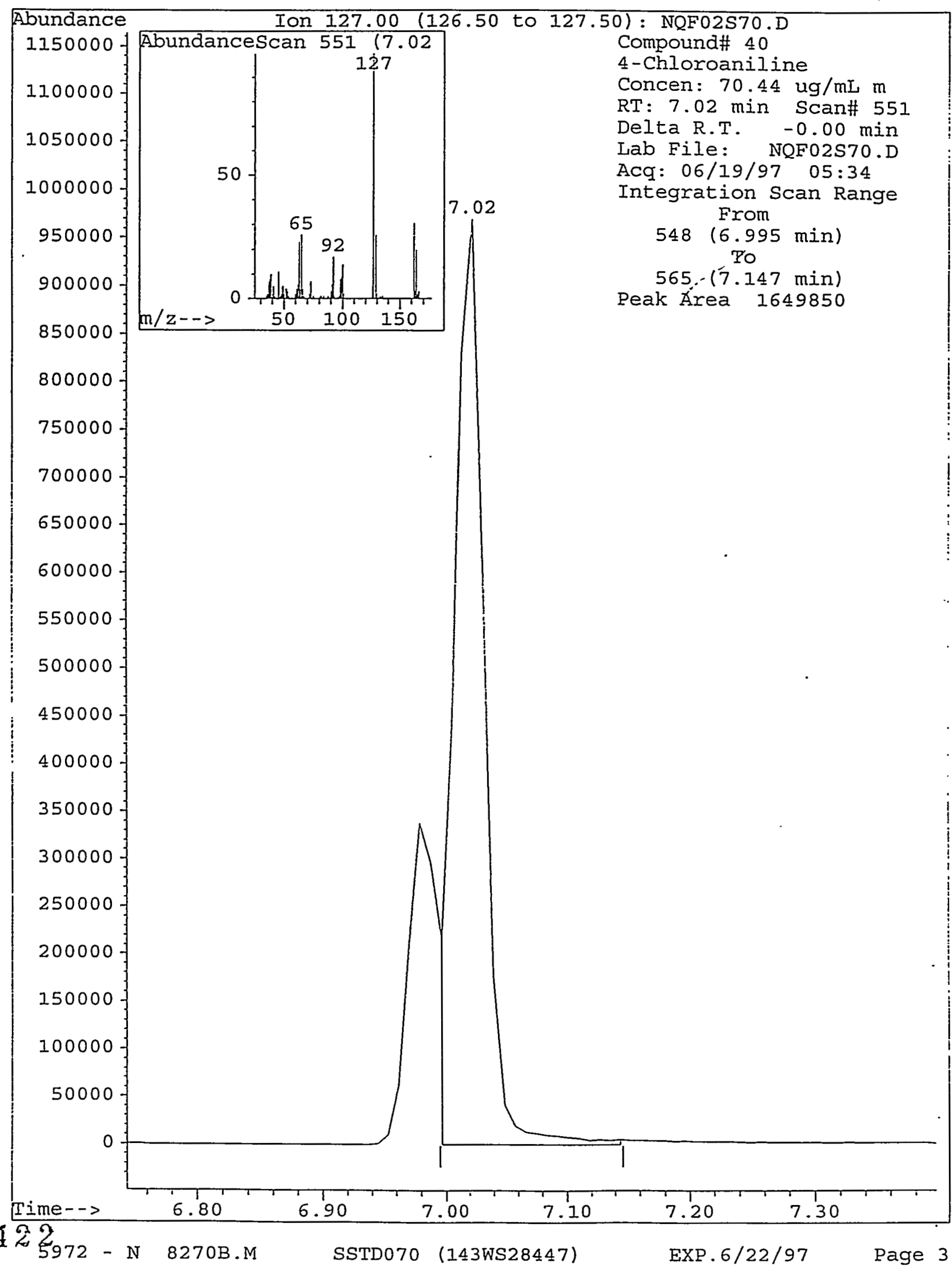


$M A N U A I$ I N T E R A T I ON FOR gamma-BHC

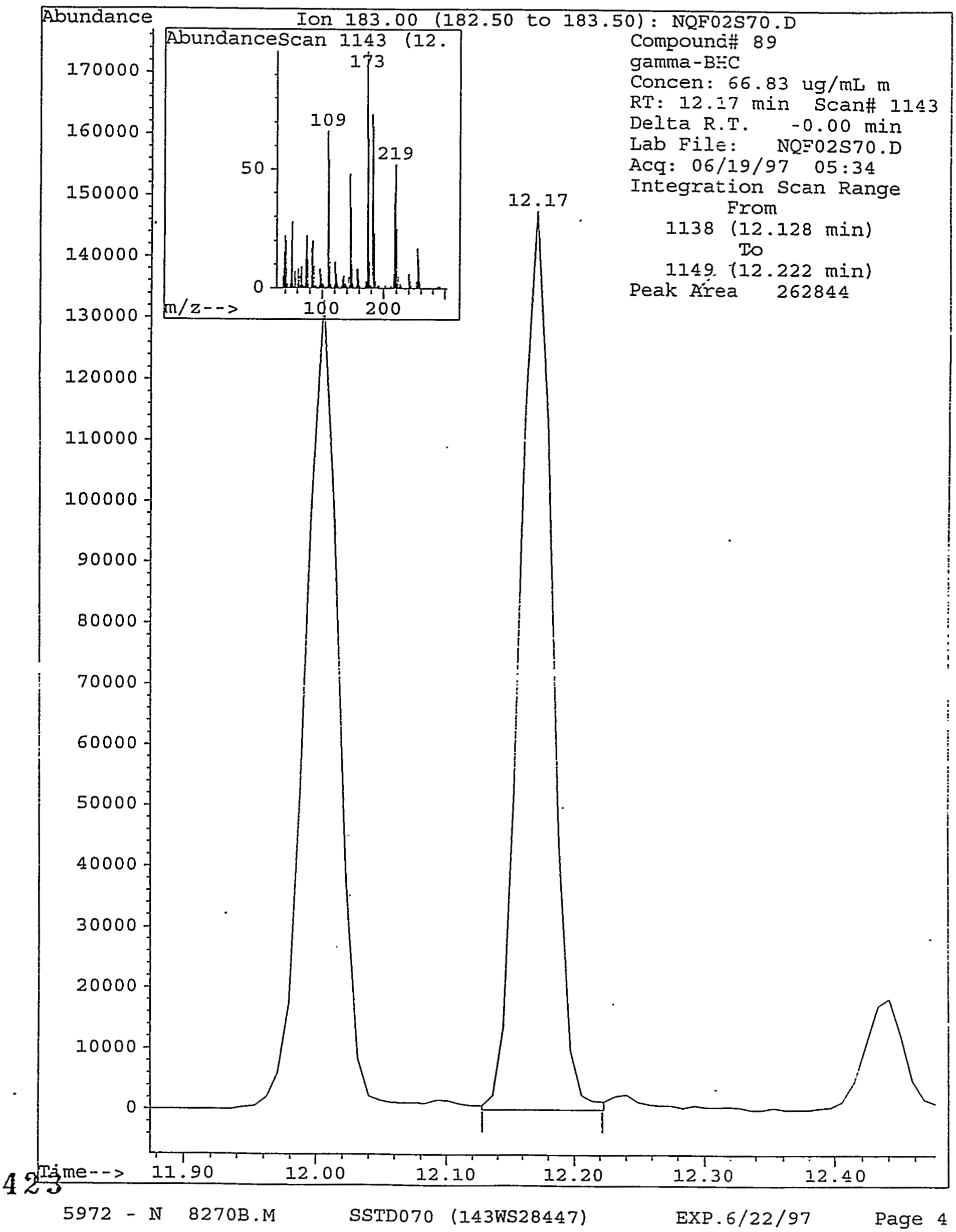




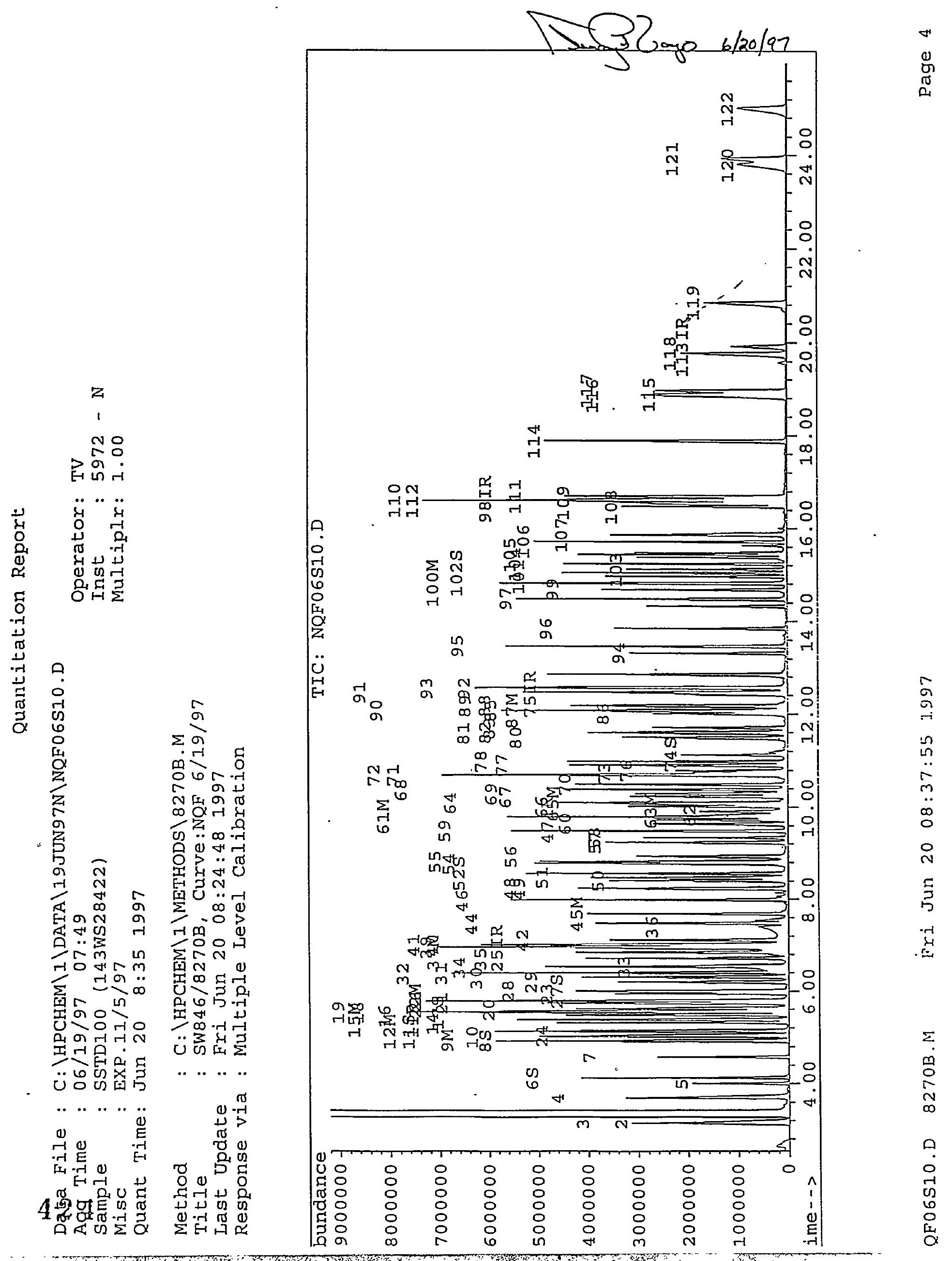


Quantitation Report

$\begin{array}{ll}\text { Data File } & : C: \backslash H P C H E M \backslash I \backslash D A T A \backslash 19 J U N 97 N \backslash N Q F 06 S 10 . D \\ \text { Acq Time } & : 06 / 19 / 9707: 49 \\ \text { Sample } & : \text { SSTD100 (143WS28422) } \\ \text { MisC } & : \text { EXP.11/5/97 }\end{array}$

Quant Time: Jun 20 8:35 1997

Method : C: $\backslash$ HPCHEM $\backslash I \backslash M E T H O D S \backslash 8270 \mathrm{~B} . \mathrm{M}$

Title : SW846/8270B, Curve:NQF 6/19/97

Last Update : Fri Jun 20 08:24:48 1997

Response via : Multiple Level Calibration
Operator: TV

Inst : $5972-\mathrm{N}$

Multiplr: 1.00

Internal Standards

R.T. Scan QIon Response Conc Units

Area $\%$

1) 1,4-Dichlorobenzene- $\mathrm{d} 4$

25) Naphthalene-d8

47) Acenaphthene-d10

75) Phenanthrene-dio

98) Chrysene-d12

113) Perylene-d12

System Monitoring Compounds

6) 2-Fluorophenol

8) Phenol-d5

11) 2-Chlorophenol-d4

17) 1,2-Dichlorobenzene-d4

27) Nitrobenzene-d5

52) 2-Fluorobiphenyl

74) 2,4,6-Tribromophenol

102) Terphenyl-d14

Target Compounds

2) Pyridine

3) N-nitrosodimethylamine

4) 2-Picoline

5) Methyl methanesulfonate

7) Ethyl methanesulfonate

9) Phenol

10) Aniline

12) 2-Chlorophenol

13) Decarie NOT Present

14) 1,3-Dichlorobenzene

15) 1,4-dichlorobenzene

16) Benzyl alcohol

18) 1,2-dichlorobenzene

19) 2-Methylphenol

20) Bis (2-chloroisopropyl) et he

21) 4-methylphenol

22) N-Nitrosodi-n-propyl amine

23) Hexachloroethane

24) Bis (2-chloroethyl)ether

26) Acetophenone

28) Nitrobenzene

29) N-nitrosopiperidine

30) Isophorone

31) 2-Nitrophenol

32) 2,4-Dimethylphenol

$\begin{array}{rrrr}5.37 & 326 & 152 & 648533 \\ 6.93 & 506 & 136 & 2632726 \\ 9.73 & 827 & 164 & 1293340 \\ 12.42 & 1137 & 188 & 1730929 \\ 16.64 & 1622 & 240 & 1248821 \\ 19.92 & 1999 & 264 & 1082110 \\ & & & \\ 4.13 & 183 & 112 & 1963588 \\ 4.93 & 275 & 99 & 2352116 \\ 5.13 & 299 & 132 & 2191651 \\ 5.54 & 346 & 152 & 1187781 \\ 5.99 & 398 & 82 & 1915863 \\ 8.55 & 693 & 172 & 3451887 \\ 11.13 & 989 & 330 & 445868 \\ 15.04 & 1438 & 244 & 2450300\end{array}$

3.16

3.14

3.70

4.00

4.58

4.93

5.03

5.15

0.00

5.32

5.39

5.48

5.56

5.57

5.62

5.75

5.79

5.95

5.06

5.80

6.02

6.20

6.31

6.40

6.40

72
70
134
169
235
276
287
301

320
328
339
348
349
355
370
375
393
290
376
401
422
434
445
445

72
70

79

42

93

80

79

94

66

128

146

146

108

146

107

45

108

70

117

63

105

77

114

82

139

122
2365732

1266375

2480107

974773

1655754

2437668

1415854

2130768

2031247

2009196

1411770

1631054

1651988

4866364

2211610

1177615

757678

1674866

2486438

1850342

1187289

3769296

1080839

1840177

$\begin{array}{lr}40.00 \mathrm{ug} / \mathrm{mL} & -0.01 \\ 40.00 \mathrm{ug} / \mathrm{mL} & -0.02 \\ 40.00 \mathrm{ug} / \mathrm{mL} & -0.02 \\ 40.00 \mathrm{ug} / \mathrm{mL} & -0.01 \\ 40.00 \mathrm{ug} / \mathrm{mL} & 0.00 \\ 40.00 \mathrm{ug} / \mathrm{mL} & 0.00\end{array}$

$\because$ Recovery $89.236 \mathrm{ug} / \mathrm{mL} \quad 89.24 \%$ $93.388 \mathrm{ug} / \mathrm{mL} \quad 93.39 \%$ $92.002 \mathrm{ug} / \mathrm{mL} \quad 92.00 \%$ $87.029 u \mathrm{~g} / \mathrm{mL} \quad 87.03 \%$ $97.156 \mathrm{ug} / \mathrm{mL} \quad 97.16 \%$ $.87 .538 \mathrm{ug} / \mathrm{mL} \quad 87.54 \%$ $109.312 \mathrm{ug} / \mathrm{mL} 109.31 \%$ $75.259 u \mathrm{~g} / \mathrm{mL} \quad 75.26 \%$

$\begin{array}{cr} & \text { Qvalue } \\ 106.096 \mathrm{ug} / \mathrm{mL} & 99 \\ 98.691 \mathrm{ug} / \mathrm{mL} \# & 71 \\ 91.467 \mathrm{ug} / \mathrm{mL} & 97 \\ 92.554 \mathrm{ug} / \mathrm{mL} & 78 \\ 96.953 \mathrm{ug} / \mathrm{mL} & 91 \\ 92.994 \mathrm{ug} / \mathrm{mL} & 79 \\ 94.145 \mathrm{ug} / \mathrm{mL} & 1 \\ 93.045 \mathrm{ug} / \mathrm{mL} & 97\end{array}$

Not Detected

$90.779 \mathrm{ug} / \mathrm{mL} \quad 96$

$89.976 \mathrm{ug} / \mathrm{mL} \quad 96$

$100.779 \mathrm{ug} / \mathrm{mL} \# \quad 74$

$79.776 \mathrm{ug} / \mathrm{mL} \quad 96$

$84.183 \mathrm{ug} / \mathrm{mL} \quad 100$

$94.408 \mathrm{ug} / \mathrm{mL \#} \quad .91$

$100.535 \mathrm{ug} / \mathrm{mL} \quad 99$

$89.852 \mathrm{ug} / \mathrm{mL} \quad 97$

$90.864 \mathrm{ug} / \mathrm{mL} \cdot 86$

$88.972 \mathrm{ug} / \mathrm{mL} \#$

$88.802 \mathrm{ug} / \mathrm{mL} \#$

$92.276 \mathrm{ug} / \mathrm{mL} \#$

$94.663 \mathrm{ug} / \mathrm{mL} \#$

$91.538 \mathrm{ug} / \mathrm{mL} \#$

$84.523 \mathrm{ug} / \mathrm{mL} \#$

$90.657 \mathrm{ug} / \mathrm{mL} \#$
75
100
6
83
82
83 


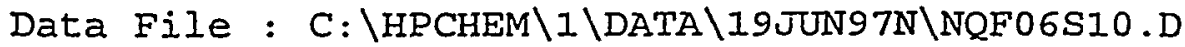

$\begin{array}{ll}\text { Acq Time } & : 06 / 19 / 9707: 49 \\ \text { Sample } & : \text { SSTD100 (143WS28422) } \\ \text { Misc } & : \text { EXP.11/5/97 }\end{array}$

Operator: TV

Quant Time: Jun 20 8:35 1997

Inst : $5972-\mathrm{N}$

Multiplr: 1.00

Method : C: \HPCHEM $\backslash 1 \backslash$ METHODS $\backslash 8270 \mathrm{~B} . \mathrm{M}$

Title : SW846/8270B, Curve:NQF 6/19/97

Last Update : Fri Jun 20 08:24:48 1997

Response via : Multiple Level Calibration

Compound

R.T. Scan QIon Response Conc Unit

Qvalue

33) Benzoic acid

34) Bis (2-chloroethoxy) methane

35) 2,4-Dichlorophenol

36) A. A. -dimethylphenethylami

37) 1,2,4-Trichlorobenzene

38) Dodecane NOT Present

39) Naphthalene

40) 4-Chloroaniline

41) 2,6-Dichlorophenol

42) Hexachlorobutadiene

43) Benzothiazole NOT Present

44) N-nitroso-di-n-butylamine

45) 4-Chloro-3-Methylphenol

46) 2-Methylnaphthalene

48) $1,2,4,5$-Tetrachlorobenzene

49) Hexachlorocyclopentadiene

50) 2,4,6-Trichlorophenol

51) 2,4,5-Trichlorophenol

53) Tetradecane NOT Present

54) 2-Chloronaphthalene

55) 1-Chloronaphthalene

56) 2-Nitroaniline

57) Dimethylphthalate

58) 2,6-Dinitrotoluene

59) Acenaphthylene

60) 3-Nitroaniline

61) Acenaphthene

62) 2,4-Dinitrophenol

63) 4-Nitrophenol

64) Dibenzofuran

65) 2,4-Dinitrotoluene

66) Pentachlorobenzene

67) 1-Naphthylamine

68) 2-Naphthylamine

69) 2,3,4,6-Tetrachlorophenol

70) Diethylphthalate

71) 4-Chlorophenyl Phenyl Ethe

72) Fluorene

73) 4-Nitroaniline

75) $4,6-$ Dinitro-2-methylphenol

77). N-nitrosodiphenylamine

78) 1,2-Diphenylhydrazine

79) Tributylphosphate NOT Pres

\section{$6.57 \quad 464$}

$6.54 \quad 461$

$6.72 \quad 481$

7.40

6.85

0.00

6.97

7.01

7.03

7.12

0.00

7.48

7.68

7.99

8.25

8.23

8.41

8.47

0.00

8.79

8.83

8.93

9.24

9.35

9.49

9.65

9.80

9.83

9.91

10.10

10.06

10.01

10.23

10.38

10.29

10.49

10.70

10.71

10.74

10.80

10.91

10.99

0.00
122

93

162

58

496

180

510

515

517

527

569

592

627

657

655

676

683

128

127

162

225

\section{4}

107

142

216

237

196

196

71

72

736

771

784

800

818

835

839

848

870

866

860

885

902

892

915

939

940

944

950

963

972
162

162

65

163

165

152

65

153

184

109

168

165

250

143

143

232

149

204

166

65

198

169

77
1579809

$2298268^{\circ}$

1612234

6660582

1602438

6167815

2328538

1466370

709061

1216898

1658598

3320145

1326344

719331

1025278

1198912

3444198

2922345

1001007

3890319

987918

5262802

1129662

3064492

720744

335508

4294465

1280932

1016616

2546422

3524588

736915

3680936

1282783

2853172

1004935

828322

2511982

3159681 $\begin{array}{lr}228.246 \mathrm{ug} / \mathrm{mL} \# & 87 \\ 88.704 \mathrm{ug} / \mathrm{mL} & 93 \\ 95.037 \mathrm{ug} / \mathrm{mL} & 79 \\ 332.479 \mathrm{ug} / \mathrm{mL} / \mathrm{m} / 20 / 17 & 94 \\ 91.327 \mathrm{ug} / \mathrm{mL} & 99 \\ \text { Not Detected } & \end{array}$

$94.248 \mathrm{ug} / \mathrm{mL}$

$89.527 \mathrm{ug} / \mathrm{mL}$

$92.172 \mathrm{ug} / \mathrm{mL \#}$

$90.555 \mathrm{ug} / \mathrm{mL}$

Not Detected

$95.015 \mathrm{ug} / \mathrm{mL} \#$

$94.931 \mathrm{ug} / \mathrm{mL}$

$.93 .157 \mathrm{ug} / \mathrm{mL}$

$87.224 \mathrm{ug} / \mathrm{mL}$

$116.009 \mathrm{ug} / \mathrm{mL}$

$96.427 \mathrm{ug} / \mathrm{mL}$

$95.768 \mathrm{ug} / \mathrm{mL}$

Not Detected

$85.472 \mathrm{ug} / \mathrm{mL}$

$91.016 \mathrm{ug} / \mathrm{mL}$

$95.702 \mathrm{ug} / \mathrm{mL} \#$

$92.998 \mathrm{ug} / \mathrm{mL}$

$96.206 \mathrm{ug} / \mathrm{mL}$ \#

$90.641 \mathrm{ug} / \mathrm{mL}$

$102.072 \mathrm{ug} / \mathrm{mL} \#$

$87.896 \mathrm{ug} / \mathrm{mL}$

$165.201 \mathrm{ug} / \mathrm{mL} \#$

$112.093 \mathrm{ug} / \mathrm{mL \#}$

$96.336 \mathrm{ug} / \mathrm{mL}$

$112.270 \mathrm{ug} / \mathrm{mL}$

$91.670 \mathrm{ug} / \mathrm{mL \#}$

$109.524 \mathrm{ug} / \mathrm{mL}$

$100.356 \mathrm{ug} / \mathrm{mL}$

$109.171 \mathrm{ug} / \mathrm{mL} \#$

$90.919 \mathrm{ug} / \mathrm{mL}$

$88.728 \mathrm{ug} / \mathrm{mL}$

$87.378 \mathrm{ug} / \mathrm{mL}$

$114.694 \mathrm{ug} / \mathrm{mL} \#$

$132.949 \mathrm{ug} / \mathrm{mL} \#$

$91.570 \mathrm{ug} / \mathrm{mL}$

$87.161 \mathrm{ug} / \mathrm{mL} \#$

Not Detected

98

95

84

99

15

98

100

99

9.7

95

94

95

52

72

59

17

98

76

93

55

72

95

97

85

.93

93

so

97

95

100 
Quantitation Report

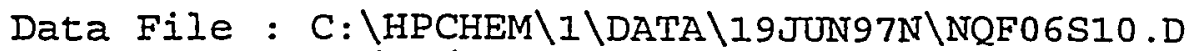

Acq Time : 06/19/97 07:49

Sample : SSTD100 (143WS28422)

Misc : EXP.11/5/97

Quant Time: Jun 20 8:35 1997

Operator: TV

Inst : $5972-\mathrm{N}$

Multiplr: 1.00

Method : C: \HPCHEM \I\METHODS $\backslash 8270 B . M$

Title : SWB46/8270B, Curve:NQF 6/19/97

Last Update : Fri Jun 20 08:24:48 1997

Response via : Multiple Level Calibration

Compound

\begin{tabular}{|c|c|}
\hline 80) & Phenacetin \\
\hline B1) & 4-Bromophenyl Phenyl Ether \\
\hline 82) & alpha-BHC \\
\hline B3) & Hexachlorobenzene \\
\hline B4) & Tris (2-CE) phosphate NOT Pr \\
\hline 85) & 4-AminobiphenyI \\
\hline B6) & beta-BHC \\
\hline B7. & Pentachlorophenol \\
\hline 88) & Pronamide \\
\hline B9) & gamma-BHC \\
\hline 90) & Pentachloronitrobenzene \\
\hline 91) & Phenanthrene \\
\hline 92) & Anthracene \\
\hline 93) & delta-BHC \\
\hline 94 & HeptachIor \\
\hline 95 & Di-n-Butylphthalate \\
\hline 96) & Aldrin \\
\hline 97) & Fluoranthene \\
\hline 99) & Benzidine \\
\hline 0) & Pyrene \\
\hline 01) & $4,4^{\prime}-\mathrm{DDE}$ \\
\hline 03) & Dieldrin \\
\hline 04$)$ & p-Dimethylaminoazobenzene \\
\hline .05 & $4,4^{\prime}-\mathrm{DDD}$ \\
\hline 06) & Butylbenzylphthalate \\
\hline 07) & $4,4 i-\mathrm{DDT}$ \\
\hline (8) & Methoxychlor \\
\hline & 3,3'-Dichlorobenzidine \\
\hline & Benzo [a] anthracene \\
\hline 11) & Chrysene \\
\hline 12 & Bis (2-ethylhexyl) phthalate \\
\hline & Di-n-octylphthalate \\
\hline 15 & Benzo [b] fluoranthene \\
\hline 16 & 7,12-Dimethylbenz (a) anthra \\
\hline & Benzo [k] fluoranthene \\
\hline & Benzo[a] pyrene \\
\hline & 3-Methylcholanthrene \\
\hline & Indeno $(1,2,3-c, d)$ pyrene \\
\hline & Dibenz $[a, h]$ anthracene \\
\hline & Benzo[ghi] perylene \\
\hline
\end{tabular}

R.T. Scan QIon Response Conc Unit Qvalue

$11.50 \quad 1031$

$11.60 \quad 1042$

11.621045

11.711055

0.00

$12.08 \quad 1098$

12.011090

$12.06 \quad 1095$

12.191110

12.161107

12.091099

$12.47 \quad 1143$

$12.57 \quad 1154$

$12.58 \quad 1155$

$13.32 \quad 1240$

$13.47 \quad 1258$

13.851301

$14.48 \quad 1374$

$14.68 \quad 1397$

$14.83 \quad 1414$

$14.96 \quad 1429$

$15.12 \quad 1447$

$15.24 \quad 1461$

$15.43 \quad 1483$

$15.73 \quad 1517$

$15.86 \quad 1532$

$16.47 \quad 1603$

$16.56 \quad 1613$

16.621620

16.691628

16.601618

$17.88 \quad 1765$

$18.90 \quad 1882$

$18.87 \quad 1878$

$18.96 \quad 1889$

$19.77 \quad 1982$

20.852106

$23.80 \quad 2445$

23.932460

25.012585
108

248

183

284

169

181

266

173

183

237

178

178

183

100

149

66

202

184

202

246

79

225

235

149

235

227

252

228

228

149

149

252

256

252

252

268

276

278

276
1796482

723728

510988

770908

2890879

426268

504320

1337915

416346

268054

$\triangle 455585$

4136198

315809

620179

5871924

512975

4026969

2510103

3847787

675257

619166

804411

1207517

2315739

1116086

2110574

1295621

3029195

2929454

2804330

5659829

3078543

1159552

2440621

2519608

1314190

2024562

2077023

1862160
$.106 .946 \mathrm{ug} / \mathrm{mL} \#$

$112.080 \mathrm{ug} / \mathrm{mL \#}$

$87.948 \mathrm{ug} / \mathrm{mL} \#$

$91.623 \mathrm{ug} / \mathrm{mL} \#$

Not Detected

$95.941 \mathrm{ug} / \mathrm{mL}$

93.264ug/mL\#

$167.178 \mathrm{ug} / \mathrm{mL}$

$86.837 \mathrm{ug} / \mathrm{mL}$

96

$84.855 \mathrm{ug} / \mathrm{mLm} / \mathrm{mok} 792$

$93.815 \mathrm{ug} / \mathrm{mL}{ }^{k / 20} 67$

$92.013 \mathrm{ug} / \mathrm{mL}$

$83.138 \mathrm{ug} / \mathrm{mL}$

$.76 .572 \mathrm{ug} / \mathrm{mL}$

$85.898 \mathrm{ug} / \mathrm{mL} \#$

$90.254 \mathrm{ug} / \mathrm{mLH}$

$84.390 \mathrm{ug} / \mathrm{mL}$

$101.099 \mathrm{ug} / \mathrm{mL \#}$

$102.863 \mathrm{ug} / \mathrm{mL \#}$

$70.902 \mathrm{ug} / \mathrm{mL} \#$

$72.984 \mathrm{ug} / \mathrm{mL}$

$67.191 \mathrm{ug} / \mathrm{mL} \#$

$90.163 \mathrm{ug} / \mathrm{mL}$

$75.352 \mathrm{ug} / \mathrm{mL}$

$78.709 \mathrm{ug} / \mathrm{mL}$

$107.981 \mathrm{ug} / \mathrm{mL} \#$

$98.486 \mathrm{ug} / \mathrm{mL}$

$100.478 \mathrm{ug} / \mathrm{mL \#}$

$89.426 \mathrm{ug} / \mathrm{mL}$

$89.729 \mathrm{ug} / \mathrm{mL}$

$75.964 \mathrm{ug} / \mathrm{mL}$

$77.806 \mathrm{ug} / \mathrm{mL}$

$94.652 \mathrm{ug} / \mathrm{mL} \#$

$94.269 \mathrm{ug} / \mathrm{mL}$

$80.156 \mathrm{ug} / \mathrm{mL} \#$

$93.020 \mathrm{ug} / \mathrm{mL} \#$

$105.682 \mathrm{ug} / \mathrm{mL}$

$89.291 \mathrm{ug} / \mathrm{mL}$

$104.464 \mathrm{ug} / \mathrm{mL} \#$

$101.623 \mathrm{ug} / \mathrm{mL \#}$
43

87

71

88

98

55

98

95

98

93

65

92

97

62

84

53

100

83

51

87

90

85

99

95

98

99

97

100

89

95

90

89

98

98

87

86

Data Processed By:

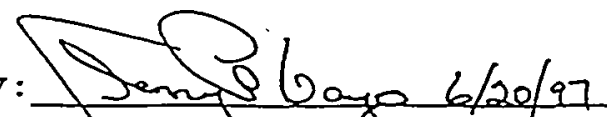

$\left(\frac{H}{\pi}\right)=$ qualifier out of range $(\mathrm{m})=$ manual integration

(*) Does not meet EPA spectral criteria (False Hit) 
Quantitation Report File: NQFO6S10.D $\quad$ standard amount: 100

Path: C:IHPCHEMI1IDATAI19JUN97NI

Sample: SSTD100 (143WS28422)

Instrument ID: 5972 - N

No.

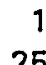

47

75

98

113

8

11

17

27

52

74

102

2

3

$$
\begin{array}{r}
\text { Name } \\
\text { 1,4-Dichlorobenzene-d4 } \\
\text { Naphthalene-d8 } \\
\text { Acenaphthene-d10 } \\
\text { Phenanthrene-d10 } \\
\text { Chrysene-d12 } \\
\text { Perylene-d12 } \\
\text { 2-Fluorophenol } \\
\text { Phenol-d5 } \\
\text { 2-Chlorophenol-d4 } \\
\text { 1,2-Dichlorobenzene-d4 } \\
\text { Nitrobenzene-d5 } \\
\text { 2-Fluorobiphenyl } \\
\text { 2,4,6-Tribromophenol } \\
\text { Terphenyl-d14 }
\end{array}
$$

Pyridine

$\mathrm{N}$-nitrosodimethylamine 2-Picoline

Methyl methanesulfonate

Ethyl methanesulfonate Phenol Aniline

2-Chlorophenol

Decane NOT Present

1,3-Dichlorobenzene

1,4-dichlorobenzene

Benzyl alcohol

1,2-dichlorobenzene

2-Methylphenol

Bis(2-chloroisopropyl)ether 4-methylphenol

$\mathrm{N}$-Nitrosodi-n-propyl amine

Hexachloroethane

Bis (2-chloroethyl)ether Acetophenone Nitrobenzene

$\mathrm{N}$-nitrosopiperidine Isophorone 2-Nitrophenol

2,4-Dimethylphenol Benzoic acid

Bis(2-chloroethoxy)methane 2,4-Dichlorophenol

$R T$
5.37
6.93
9.73
12.42
16.64
19.92

4.13

4.93

5.13

5.54

5.99

8.56

11.13

15.04

3.14

5.15

0.00

5.32

5.39

5.48

5.56

5.57

5.62

5.75

5.79

5.95

5.06

5.80

6.02

6.20

6.31

6.40

6.40

6.57

6.54

6.72
Analyst: TV

$\begin{array}{ccc}\text { scan } & \mathrm{m} / \mathrm{z} & \text { area } \\ 326 & 152.00 & 648533 \\ 506 & 136.00 & 2632726 \\ 827 & 164.00 & 1293340 \\ 1137 & 188.00 & 1730929 \\ 1622 & 240.00 & 1248821 \\ 1999 & 264.00 & 1082110 \\ & & \\ 183 & 112.00 & 1963588 \\ 275 & 99.00 & 2352116 \\ 299 & 132.00 & 2191651 \\ 346 & 152.00 & 1187781 \\ 398 & 82.00 & 1915863 \\ 693 & 172.00 & 3451887 \\ 989 & 330.00 & 445868 \\ 1438 & 244.00 & 2450300\end{array}$

RF amt $1.000 \quad 40.000$

$1.000 \quad 40.000$

$1.000 \quad 40.000$

$1.000-40.000$

$1.000 \quad 40.000$

$1.000 \quad 40.000$

1438

$3.16 \quad 72$

70

$3.70 \quad 134$

$4.00 \quad 169$

$4.58 \quad 235$

$4.93 \quad 276$

$5.03 \quad 287$

\subsection{0}

42.00

93.00

80.00

79.00

94.00

66.00

128.00

57.00

146.00

146.00

108.00

146.00

107.00

45.00

108.00

70.00

117.00

63.00

105.00

77.00

114.00

82.00

139.00

122.00

122.00

93.00

162.00
2365732 1266375 2480107

974773

1655754

2437668

1415854 2130768

$$
0
$$

2031247

2009196

1411770

1631054

1651988

4866364

2211610

1177615

757678

1674866

2486438

1850342

1187289

3769296

1080839

1840177

1579809

2298268

1612234
1.211100 .00

$1.451 \quad 100.00$

1.352100 .00

0.733100 .00

0.291100 .00

$\begin{array}{ll}1.068 & 100.00\end{array}$

0.138100 .00

0.785100 .00

$1.459 \cdot 100.00$

$0.781 \quad 100.00$

1.530100 .00

0.601100 .00

$1.021 \quad 100.00$

1.503100 .00

0.873100 .00

$\begin{array}{ll}1.314 & 100.00\end{array}$

$0.000 \quad 100.00$

1.253100 .00

1.239100 .00

1.006100 .00

1.019100 .00

3.001100 .00

$\begin{array}{ll}1.364 & 100.00\end{array}$

0.726100 .00

0.467100 .00

1.033100 .00

0.378100 .00

0.281100 .00

0.180100 .00

0.573100 .00

0.164100 .00

0.280100 .00

0.240100 .00

0.349100 .00

0.245100 .00
0.871100 .00 
Path: C:IHPCHEMI1IDATAI19JUN97NI

06/19/97

Sample: SSTD100 (143WS28422)

Instrument ID: 5972 - N

Analyst: TV

\begin{tabular}{|c|c|c|c|c|c|c|c|}
\hline $\begin{array}{l}10 . \\
36\end{array}$ & $\begin{array}{c}\text { Name } \\
\text { A.,A.-dimethylphenethylamine }\end{array}$ & $\begin{array}{l}\text { RT } \\
7.40\end{array}$ & $\begin{array}{l}\text { scan } \\
560\end{array}$ & $\begin{array}{l}\mathrm{m} / \mathrm{z} \\
58.00\end{array}$ & $\begin{array}{c}\text { area } \\
6660582\end{array}$ & $\begin{array}{c}R F \\
1.012\end{array}$ & $\begin{array}{c}\text { amt } \\
100.00\end{array}$ \\
\hline 37 & 1,2,4-Trichlorobenzene & 6.85 & 496 & 180.00 & 1602438 & 0.243 & 100.00 \\
\hline 38 & Dodecane NOT Present & 0.00 & 1 & 57.00 & 0 & 0.000 & 100.00 \\
\hline 39 & Naphthalene & 6.97 & 510 & 128.00 & 6167815 & 0.937 & 100.00 \\
\hline 40 & 4-Chloroaniline & 7.01 & 515 & 127.00 & 2328538 & 0.354 & 100.00 \\
\hline 41 & 2,6-Dichlorophenol & 7.03 & 517 & 162.00 & 1466370 & 0.223 & 100.00 \\
\hline 42 & Hexachlorobutadiene & 7.12 & 527 & 225.00 & 709061 & 0.108 & 100.00 \\
\hline 43 & Benzothiazole NOT Present & 0.00 & 1 & 135.00 & 0 & 0.000 & 100.00 \\
\hline 44 & N-nitroso-di-n-butylamine & 7.48 & 569 & 84.00 & 1216898 & 0.185 & 100.00 \\
\hline 45 & 4-Chloro-3-Methylphenol & 7.68 & 592 & 107.00 & 1658598 & 0.252 & 100.00 \\
\hline 46 & 2-Methylnap & 7.99 & 627 & 42.00 & 3320145 & 0.504 & 100.00 \\
\hline 48 & 1,2,4,5-Tetrachlorc & 8.25 & 657 & 216.00 & 1326344 & 0.410 & 100.00 \\
\hline 49 & Hexachlorocyclop & 8.23 & 655 & 237.00 & 719331 & 0.222 & 100.00 \\
\hline 50 & $2,4,6$ & 8.41 & 676 & 196.00 & 1025278 & 0.317 & \\
\hline 51 & $2,4,5$ & 8.47 & 683 & 196.00 & 1198912 & 0.371 & \\
\hline 53 & Tetradecane NO & 0.00 & 1 & & 0 & 0.000 & \\
\hline 54 & & 8.79 & 719 & & 3444198 & & \\
\hline 55 & ne & 8.83 & 724 & 162.00 & 29223 & 04 & \\
\hline 56 & & 8.93 & 736 & 65.00 & 10010 & 310 & \\
\hline 57 & Dimethy & 9.24 & 771 & & 3890 & 203 & \\
\hline 58 & 2,6-Din & 9.35 & 784 & $65.00^{\circ}$ & 987918 & 306 & \\
\hline 59 & Acena & 9.49 & 800 & 152.00 & $5262 \varepsilon$ & 1.628 & \\
\hline 60 & & 9.65 & 818 & 65.00 & 11296 & & \\
\hline 61 & Ace & 9.80 & 835 & 153.00 & 30644 & 0.948 & \\
\hline 62 & 2,4-Din & 9.83 & 839 & 84.00 & 720744 & 0.223 & .00 \\
\hline 63 & & 9.91 & 848 & 09.00 & 335508 & & \\
\hline 64 & & 10.10 & 870 & 68.00 & 4294 & 1.328 & \\
\hline 65 & 2,4-Dinit & 10.06 & 866 & 165.00 & 1280932 & 0.396 & \\
\hline 66 & Pentachlor & 10.01 & 860 & 250.00 & 1016616 & & \\
\hline 67 & & 10.23 & 885 & 143.00 & 2546422 & & 100.00 \\
\hline 68 & Imine & 10.38 & 902 & 143.00 & 3524588 & 1.090 & 100.00 \\
\hline 69 & $2,3,4,6$-Tetrachl & 10.29 & 892 & 232.00 & 736915 & 0.228 & 100.00 \\
\hline 70 & Diethylphthalate & 10.49 & 915 & 149.00 & 3680936 & 1.138 & 100.00 \\
\hline 71 & 4-Chlorophenyl Phenyl Ether & 10.70 & 939 & 204.00 & 1282783 & 0.397 & 100.00 \\
\hline 72 & Jorene & 10.71 & 940 & 166.00 & 2853172 & 0.882 & 100.00 \\
\hline 73 & 4-Nitroaniline & 10.74 & 944 & 65.00 & 1004935 & 0.311 & 100.00 \\
\hline 76 & 4,6-Dinitro-2-methyl & 10.80 & 950 & 198.00 & 828322 & 0.191 & 100.00 \\
\hline 77 & N-nitrosodiphen & 10.91 & 963 & 169.00 & 2511982 & 0.580 & 100.00 \\
\hline 78 & 1,2-Diphenylhydrazine & 10.99 & 972 & 77.00 & 3159681 & 0.730 & 100.00 \\
\hline 79 & ributylphosphate NOT Present & 0.00 & 1 & 99.00 & 0 & 0.000 & 100.00 \\
\hline 80 & Phenacetin & 11.50 & 1031 & 108.00 & 1796482 & 0.415 & 100.00 \\
\hline 81 & 4-Bromophenyl Phenyl Ether & 11.60 & 1042 & 248.00 & 723728 & 0.167 & 100.00 \\
\hline
\end{tabular}


Path: C:IHPCHEMI1\DATAI19JUN97NI Sample: SSTD100 (143WS28422) Instrument ID: $5972-\mathrm{N}$

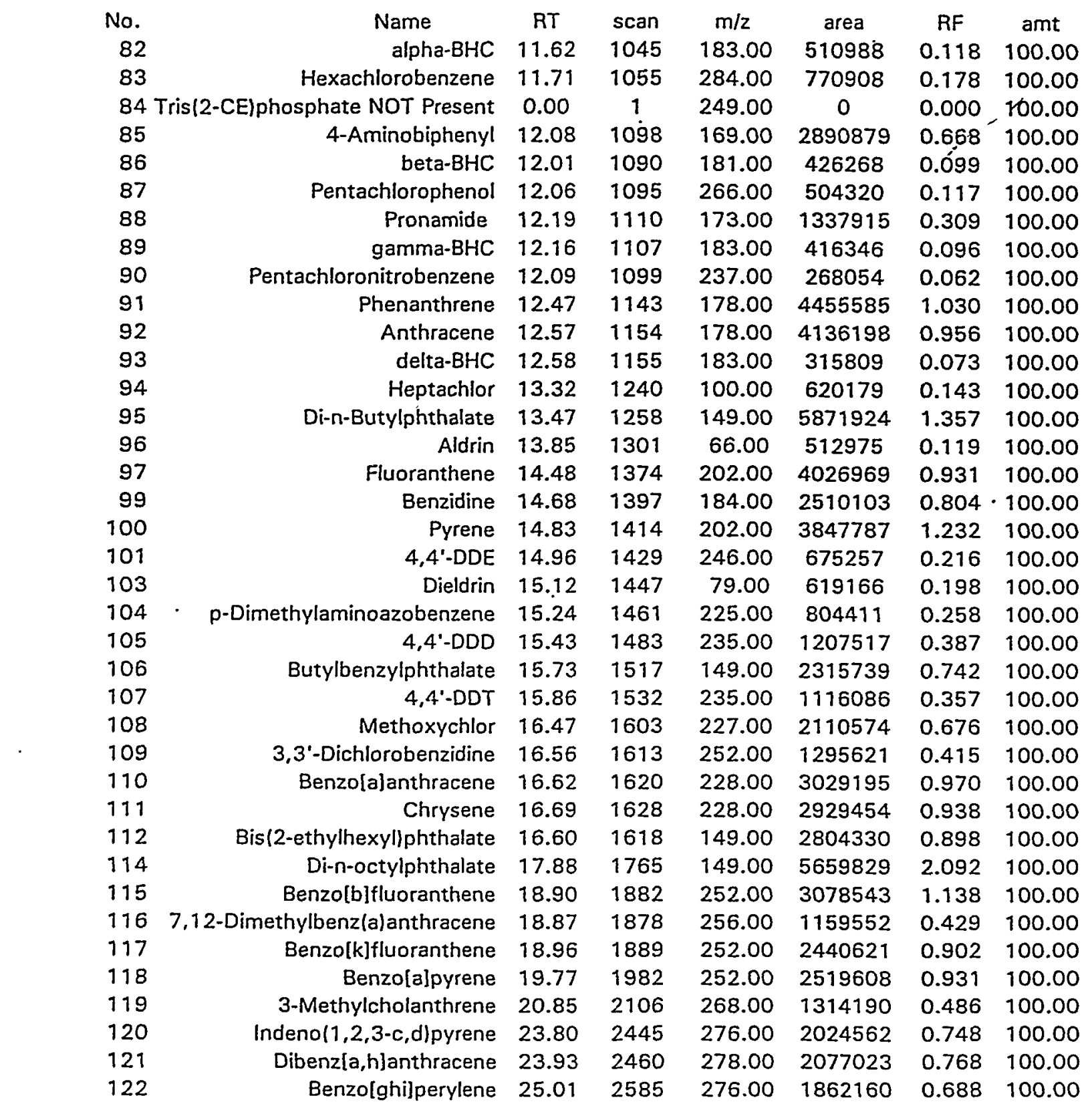

$06 / 19 / 97$

Analyst: TV 


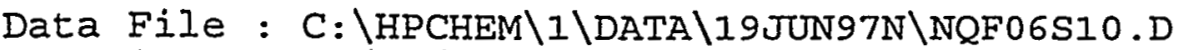

Acq Time : 06/19/97 07:49

Sample : SSTDI00 (143WS28422)

MisC : EXP.11/5/97

Quant Time: Jun 19 8:15 1997

Operator: TV Inst : $5972-\mathrm{N}$ Multiplr: 1.00

Method : C: $\backslash$ HPCHEM $\backslash I \backslash$ METHODS $\backslash 8270 \mathrm{~B} . \mathrm{M}$

Title : SW846/8270B, Curve:NQF 6/19/97

Last Update : Fri Jun 20 08:24:48 1997

Response via : Multiple Level Calibration

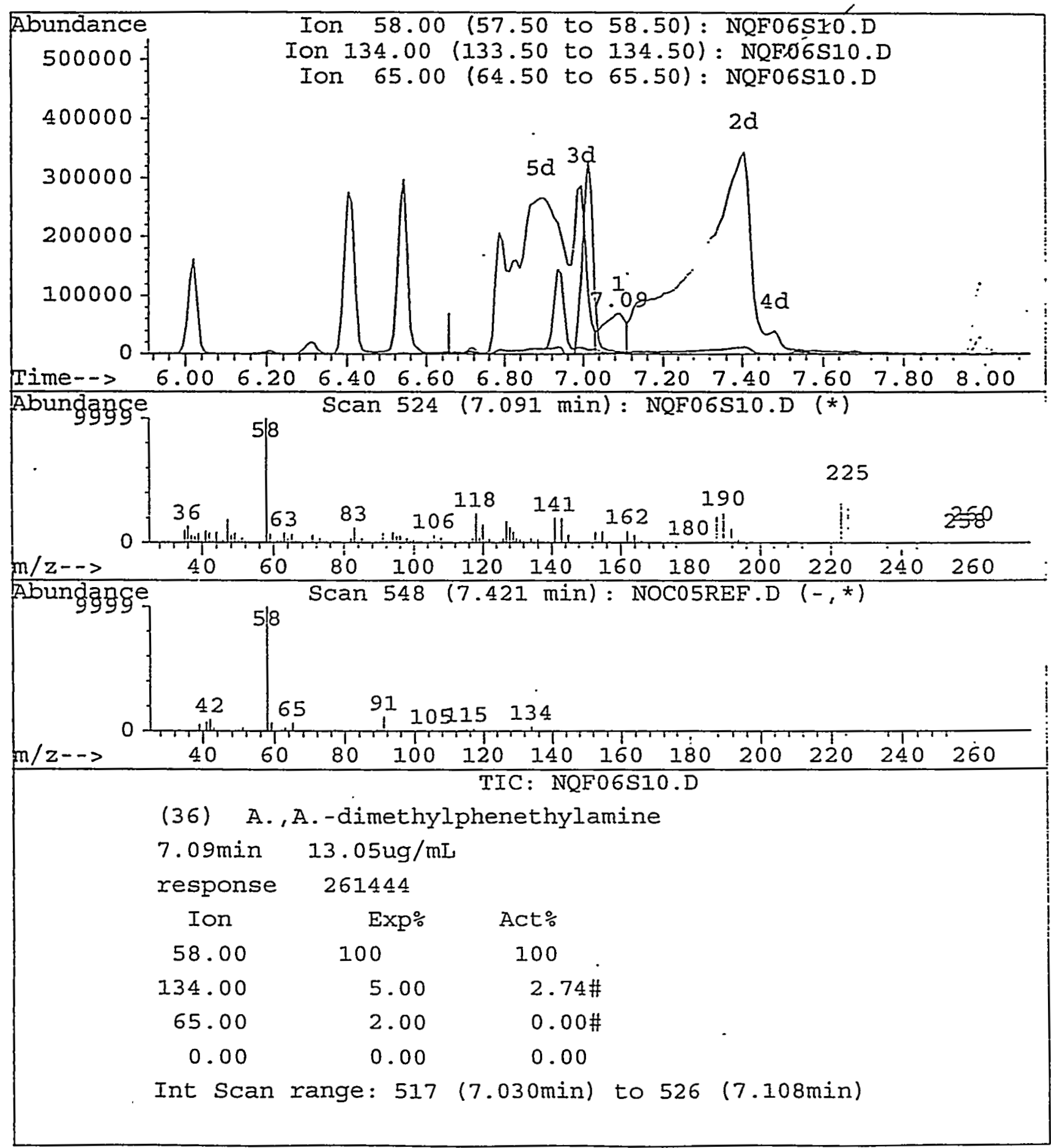




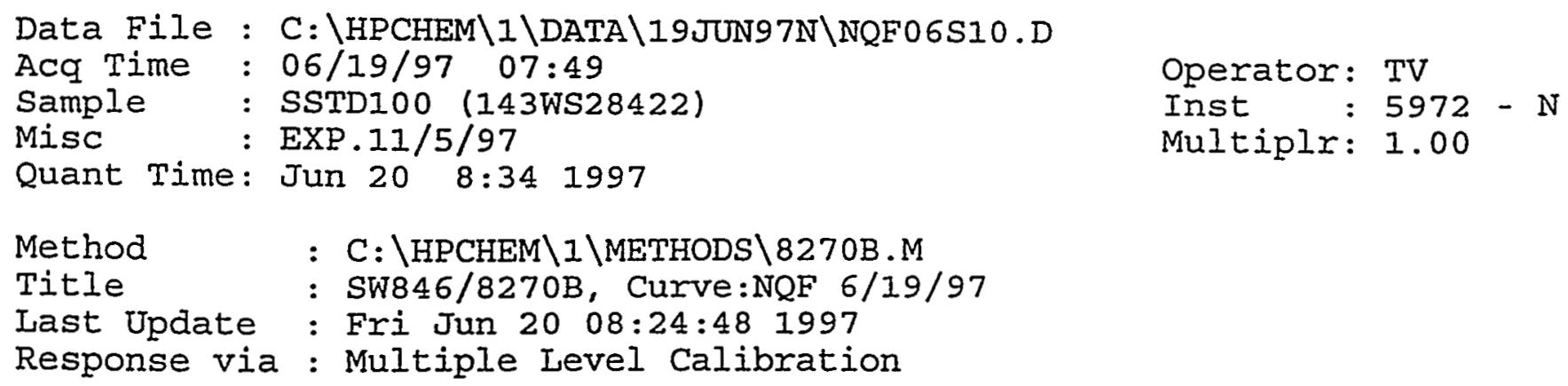

Operator: TV Inst : $5972-\mathrm{N}$ Multiplr: 1.00

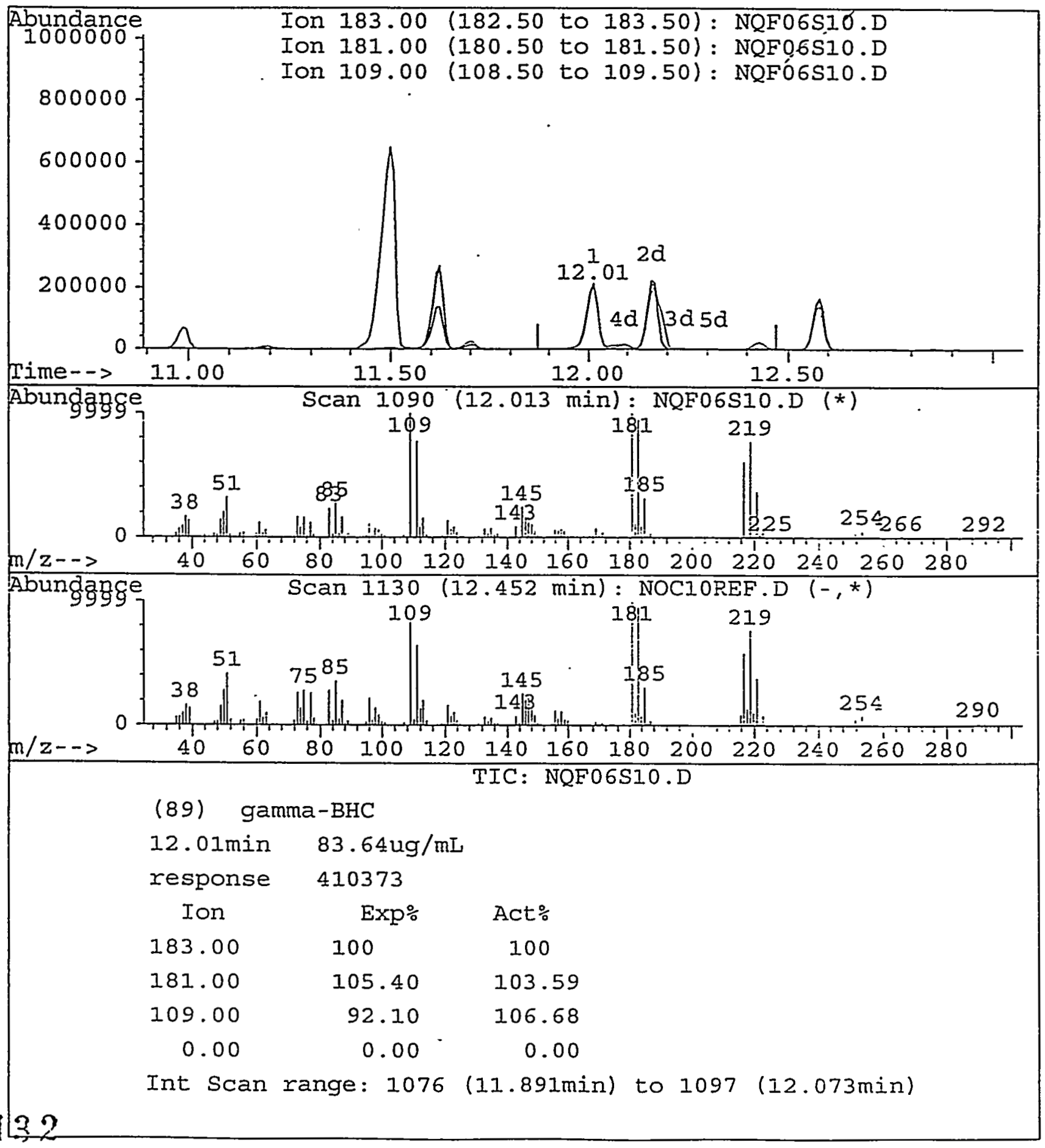


$M A N U A I \quad I N T E G R A T I O N$ FOR A.,A.-dimethylphenethylamine

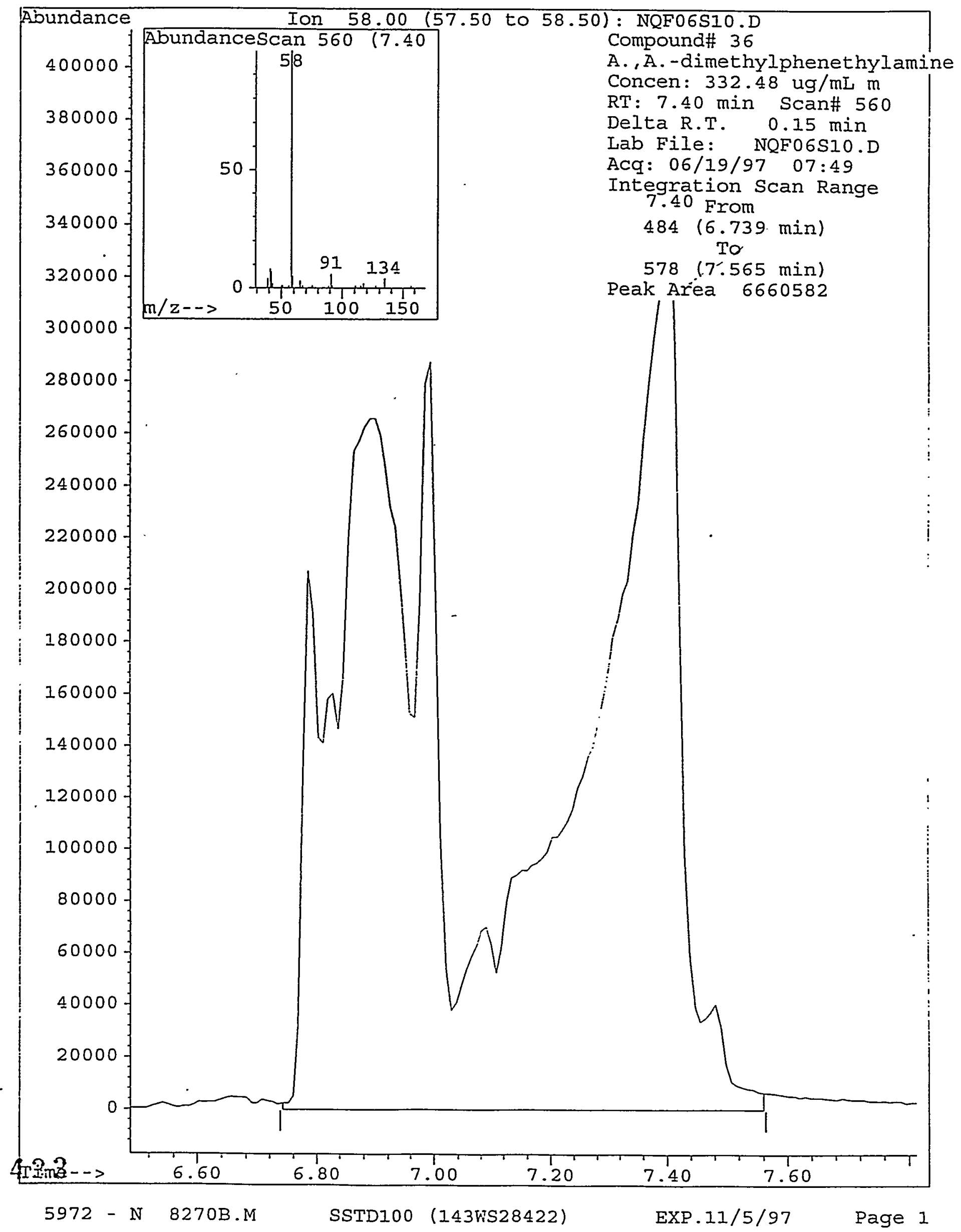


$M A N U A L$ I $N$ T E R A T I O N FOR gamma-BHC

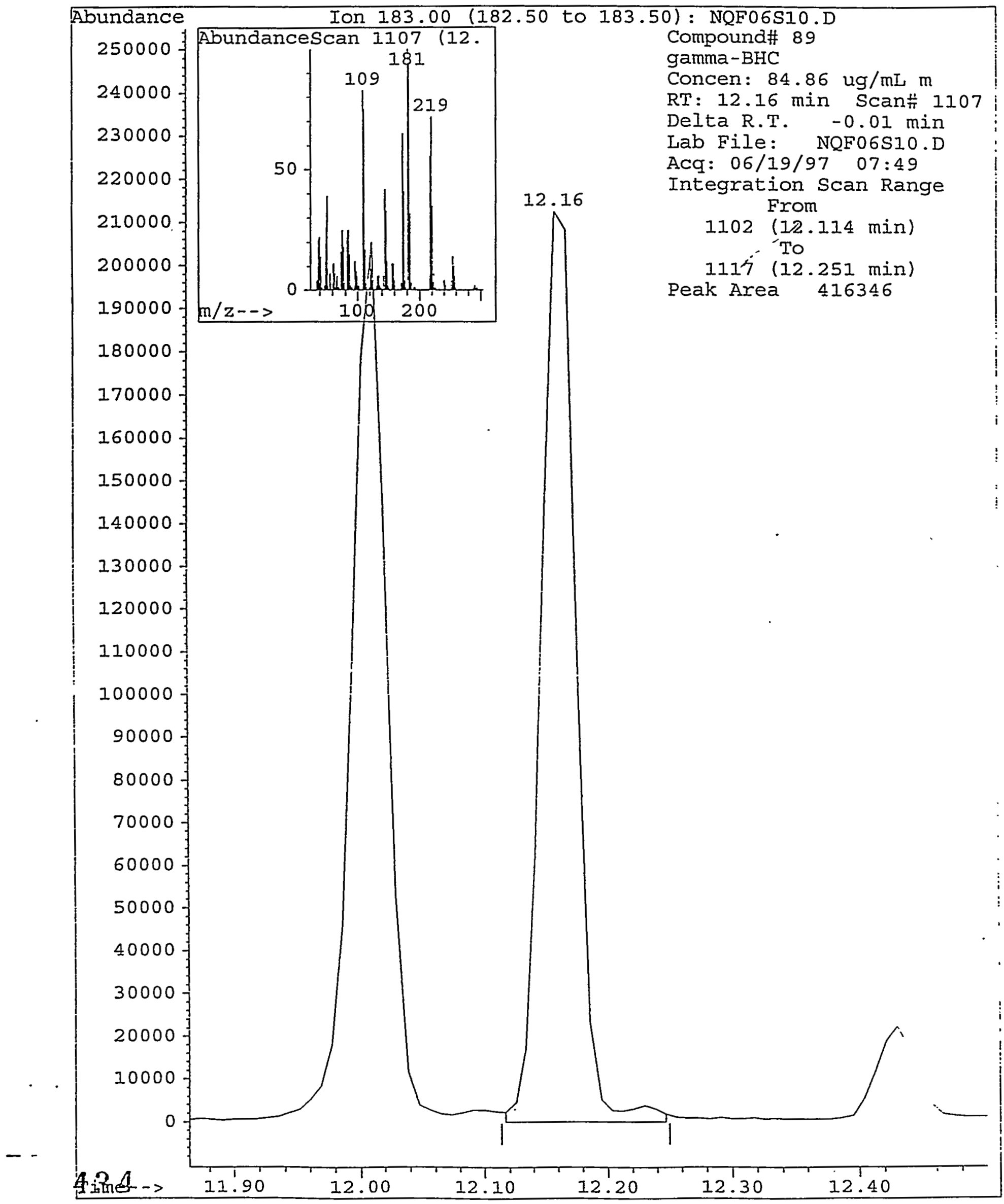




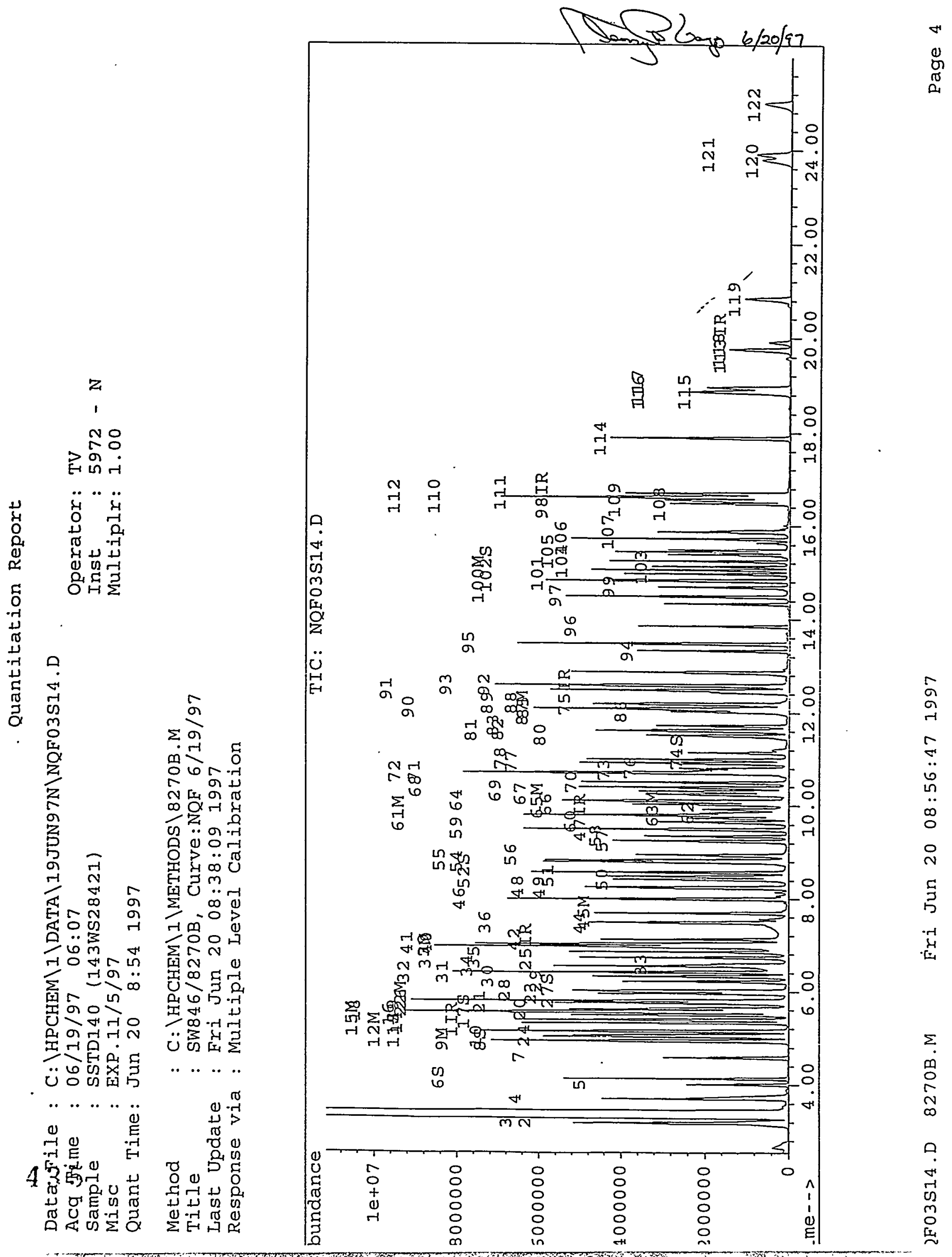




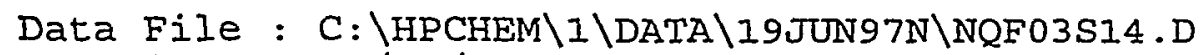

Acq Time : 06/19/97 06:07

Sample : SSTD140 (143WS28421)

Misc : EXP.11/5/97

Quant Time: Jun 20 8:54 1997

Method : C: $\backslash$ HPCHEM $\backslash I \backslash M E T H O D S \backslash 8270 B . M$

Title : Sw846/8270B, Curve:NQF 6/19/97

Last Update : Fri Jun 20 08:38:09 1997

Response via : Multiple Level Calibration
Operator: TV

Inst $\quad: 5972-\mathrm{N}$

Multiplr: 1.00

Internal standards

R.T. Scan QIon Response Conc Units

Area

\begin{tabular}{|c|c|c|c|c|c|c|c|c|}
\hline 1) & 1,4-Dichlorobenzene-d4 & 5.37 & 327 & 152 & 636041 & 40.00 & $\mathrm{ug} / \mathrm{mL}$ & 0.00 \\
\hline 25) & Naphthalene-d8 & 6.95 & 508 & 136 & 2437149 & 40.00 & $\mathrm{ug} / \mathrm{mL}$ & 0.00 \\
\hline 47) & Acenaphthene-d10 & 9.74 & 829 & 164 & 1149398 & 40.00 & $\mathrm{ug} / \mathrm{mL}$ & 0.00 \\
\hline 751 & Phenanthrene-d10 & 12.44 & 1139 & .188 & 1416091 & 40.00 & $\mathrm{ug} / \mathrm{mL}$ & 0.00 \\
\hline 981 & Chrysene-d12 & 16.64 & 1623 & 240 & 787909 & 40.00 & $\mathrm{ug} / \mathrm{mL}$ & 0.00 \\
\hline 113) & Perylene-d12 & 19.91 & 1998 & 264 & 477362 & 40.00 & $\mathrm{ug} / \mathrm{mL}$ & 0.0 \\
\hline
\end{tabular}

System Monitoring Compounds

6) 2-Fluorophenol

8) Phenol-d5

11) 2-Chlorophenol-d4

17) 1,2-Dichlorobenzene-d4

27) Nitrobenzene-d5

52) 2-FIuorobiphenyl

74) 2,4,6-Tribromophenol

102) Terphenyl-d14

Target Compounds

2) Dyridine

3) N-nitrosodimethylamine

4) 2-Picoline

5) Methyl methanesulfonate

7) Ethyl methanesulfonate

9) Phenol

10) Aniline

12) 2-Chlorophenol

13) Decane NOT Present

14) 1,3-Dichlorobenzene

15) 1,4 -dichlorobenzene

16) Benzyl alcohol

18) 1,2-dichlorobenzene

19) 2-Methylphenol

20) Bis (2-chloroisopropyl) ethe

21) 4-methylphenol

22) N-Nitrosodi-n-propyl amine

23) Hexachloroethane

24) Bis (2-chloroethyl)ether

26) Acetophenone

2B) Nitrobenzene

29) N-nitrosopiperidine

30) Isophorone

31) 2-Nitrophenol

32) 2,4-Dimethylphenol
$4.14 \quad 185 \quad 112$

4.94

5.15

5.55

277

6.01

301

347

8.57

400

11.15

15.05

695

991

1440

\subsection{9}

3.19

3.70

4.01

4.59

4.96

5.04

5.17

0.00

5.32

5.40

5.50

5.57

5.58

5.63

5.77

5.81

5.97

5.07

5.82

6.04

6.22

6.33

6.42

6.43
99

132

152

82

172

330

244
2693205

3115001

2967617

1573212

2514749

4213624

518437

2507627 $\because$ Recovery $124.798 \mathrm{ug} / \mathrm{mL} 124.80 \%$ $126.106 \mathrm{ug} / \mathrm{mL} 126.11 \%$ $127.022 \mathrm{ug} / \mathrm{mL} 127.02 \%$ $117.534 \mathrm{ug} / \mathrm{mL} 117.53 \%$ $137.760 \mathrm{ug} / \mathrm{mL} 137.76 \%$ $120.236 \mathrm{ug} / \mathrm{mL} 120.24 \%$ $143.021 \mathrm{ug} / \mathrm{mL} 143.02 \%$ $122.076 \mathrm{ug} / \mathrm{mL} 122.08 \%$ 


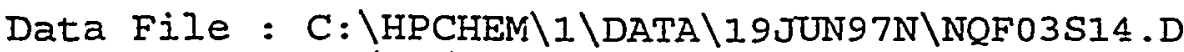

Acq Time : 06/19/97 06:07

Sample : SSTD140 (I43WS2842I)

Misc : EXP.11/5/97

Quant Time: Jun 20 8:54 1997
Operator: TV

Inst : $5972-\mathrm{N}$

Multiplr: 1.00

Method : C: $\backslash$ HPCHEM $\backslash I \backslash M E T H O D S \backslash 8270 B . M$

Title : SW846/8270B, Curve:NQF 6/19/97

Last Update : Fri Jun 20 08:38:09 1997

Response via : Multiple Level Calibration

Compound

33) Benzoic acid

34) Bis (2-chloroethoxy) methane

35) 2,4-Dichlorophenol

36) A. A. -dimethylphenethylami

37) 2,2,4-Trichlorobenzene

38) Dodecane NOT Present.

39) Naphthalene

40) 4-ChIoroaniline

41) 2,6-Dichlorophenol

42) Hexachlorobutadiene

43) Benzothiazole NOT Present

44) N-nitroso-di-n-butylamine

45) 4-Chloro-3-Methylphenol

46) 2-Methylnaphthalene

48) $1,2,4,5$-Tetrachlorobenzene

49) Hexachlorocyclopentadiene

50) 2,4,6-Trichlorophenol

51) 2,4,5-Trichlorophenol

53) Tetradecane NOT Present

54) 2-ChIoronaphthalene

55) 1-Chloronaphthalene

56) 2-Nitroaniline

57) Dimethylphthalate

5B) 2,6-Dinitrotoluene

59) Acenaphthylene

60) 3-Nitroaniline

61) Acenaphthene

62) 2,4-Dinitrophenol

63) 4-Nitrophenol

64) Dibenzofuran

65) 2,4-Dinitrotoluene

66) Pentachlorobenzene

57) 1-Naphthylamine

5B) 2-Naphthylamine

69) 2,3,4,6-Tetrachlorophenol

70) Diethylphthalate

71) 4-Chlorophenyl Phenyl Ethe

72) Fluorene

73) 4-Nitroaniline

76) 4,6 -Dinitro-2-methylphenol

77) iv-nitrosodiphenylamine

78) 1,2-Diphenylhydrazine

79) Tributylphosphate NOT Pres
R.T. Scan QIon Response Conc Unit Qvalue

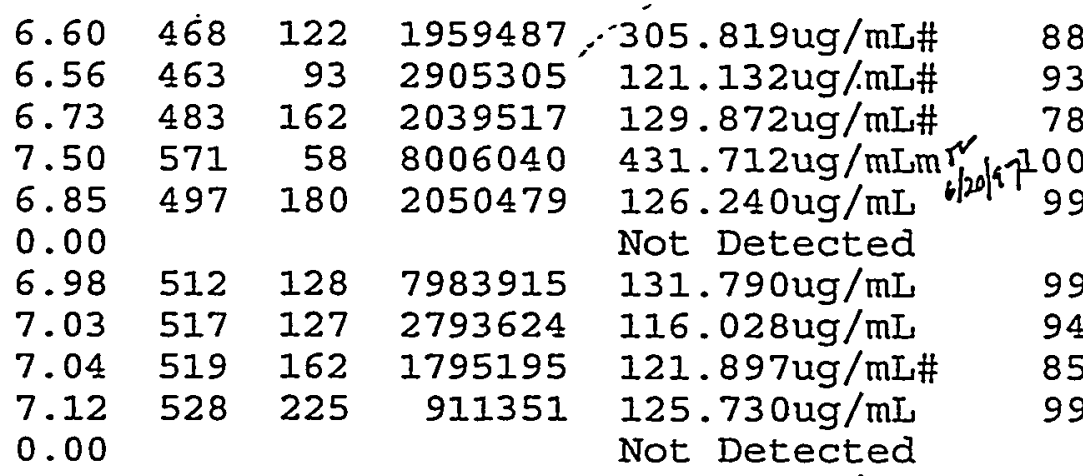

7.50

7.70

571

84

1448196

8.00

594

107

2022623

4105843

1631150

880443

8.24

659

216

8.43

656

237

8.49

678

196

0.00

8.80

685196

1271055

14389.19

8.84

$\begin{array}{ll}721 & 162\end{array}$

4388671

$122.149 \mathrm{ug} / \mathrm{mL} \#$

$125.056 \mathrm{ug} / \mathrm{mL}$

$1 \cdot 24.447 \mathrm{ug} / \mathrm{mL}$

$120.702 \mathrm{ug} / \mathrm{mL}$

$159.774 \mathrm{ug} / \mathrm{mL}$

$134.513 \mathrm{ug} / \mathrm{mL}$

$129.333 \mathrm{ug} / \mathrm{mL}$

Not Detected

1219091

$738 \quad 65$

$9.25 \quad 773 \quad 163$

$\triangle 637795$

1220218

6307234

1361915

3603940

862271

406885

5076475

1502781

1216836

3007777

4099711

868236

4345921

1508929

3388553

1163914

$\begin{array}{llll}952 & 198 \quad 970246 \quad 190.351 \mathrm{ug} / \mathrm{mL} \# \quad 66\end{array}$

10.81

10.92

11.00

0.00

$122.549 \mathrm{ug} / \mathrm{mL}$

$120.360 \mathrm{ug} / \mathrm{mL}$

$131.148 \mathrm{ug} / \mathrm{mL} \#$

$124.750 u g / m L$

$133.708 \mathrm{ug} / \mathrm{mL} \# \quad 18$

$122.233 \mathrm{ug} / \mathrm{mL} \quad 98$

$138.468 \mathrm{ug} / \mathrm{mL} \# \quad 74$

$116.314 \mathrm{ug} / \mathrm{mL} \quad 93$

$222.392 \mathrm{ug} / \mathrm{mL} \#, 62$

$152.965 \mathrm{ug} / \mathrm{mLm} / \mathrm{m} / 20 / 787$

$128.140 \mathrm{ug} / \mathrm{mL}$ b/20/17 97

$148.209 \mathrm{ug} / \mathrm{mL} \quad 98$

$123.466 \mathrm{ug} / \mathrm{mL} \# \quad 85$

$145.568 \mathrm{ug} / \mathrm{mL} \quad .93$

$131.350 \mathrm{ug} / \mathrm{mL} \quad 93$

$144.733 \mathrm{ug} / \mathrm{mL} \# \quad 89$

$120.787 \mathrm{ug} / \mathrm{mL} \quad 97$

$117.441 \mathrm{ug} / \mathrm{mL} \quad 94$

$116.770 \mathrm{ug} / \mathrm{mL} \quad 100$

$149.475 \mathrm{ug} / \mathrm{mL} \# \quad 83$

$\begin{array}{lllll}965 & 169 & 2989983 & 133.227 \mathrm{ug} / \mathrm{mL} & 97\end{array}$

$\begin{array}{llll}974 & 77 & 3712251 & 125.171 \mathrm{ug} / \mathrm{mL} \# \quad 82\end{array}$

Not Detected

(\#) A.qualifier out of range $(\mathrm{m})=$ manual integration

(*) Does not meet EPA spectral criteria (False Hit) 


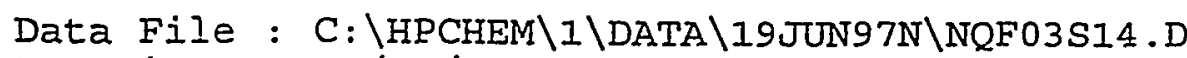

Acq Time : 06/19/97 06:07

Sample : SSTD140 (143WS28421)

Misc : EXP.11/5/97

Quant Time: Jun 20 8:54 1997

Method : C: \HPCHEM \IMETHODS $\backslash 8270 \mathrm{~B} . M$

Title : SW846/8270B, Curve:NQF 6/19/97

Last Update : Fri Jun 20 08:38:09 1997

Response via : Multiple Level Calibration
Operator: TV

Inst : $5972-\mathrm{N}$

Multiplr: 1.00

Compound

R.T. Scan QIon Response Conc Unit Qvalue

Bo) Phenacetin

BI) 4-Bromophenyl Phenyl Ether

82) alpha-BHC

B3) Hexachlorobenzene

B4) Tris (2-CE) phosphate NOT $\operatorname{Pr}$

85) 4-Aminobiphenyl

B6) beta-BHC

B7) Pentachlorophenol

B8) Pronamide

B9) gamma-BHC

90) Pentachloronitrobenzene

91) Phenanthrene

92) Anthracene

93) delta-BHC

94) Heptachlor

95) Di-n-Butylphthalate

96) Aldrin

97) Fluoranthene

99) Benzidine

100) Pyrene

101) 4,41-DDE

103) Dieldrin

104) p-Dimethylaminoazobenzene

105) 4, 4'-DDD

106) Butylidenzylphthalate

107) 4,4:-DDT

108) Methoxychlor

109) 3,3'-Dichlorobenzidine

110) Benzo[a] anthracene

111) Chrysene

112) Bis (2-ethyl hexyl) phthalate

114) Di-n-octylphthalate

115) Benzo[b] Iluoranthene

116) 7,12-Dimethylbenz (a) anthra

117) Benzo[k] fluoranthene

11.8) Benzo[a] pyrene

119) 3-Metinylcholanthrene

120) Indeno $(1,2,3-c, d)$ pyrene

121) Dibenz $[a, h]$ anthracene

122) Benzo[ghi]perylene $\begin{array}{lll}11.51 & 1033 \quad 108\end{array}$

$\begin{array}{lll}11.61 & 1044 & 248\end{array}$

$11.64 \quad 1047 \quad 183$

$11.71 \quad 1056 \quad 284$

0.00

12.101100

12.031092

12.071097

12.201112

12.171109

12.111101

12.491145

12.581156

$12.59 \quad 1157$

$13.33 \quad 1242$

13.481259

13.851302

$14.49 \quad 1375$

14.691398

$14.84 \quad 1415$

$14.97 \quad 1430$

$15.12 \quad 1448$

$15.24 \quad 1462$

$15.44 \quad 1484$

$15.73 \quad 1518$

15.871534

16.481604

16.571614

16.631621

$16.70 \quad 1629$

16.611619

$17.89 \quad 1766$

18.891881

$18.86 \quad 1878$

$18.96 \quad \dot{1889}$

$19.77 \quad 1982$

20.842106

23.792445

23.912458

$25.00 \quad 2584$

\section{5 \\ 853632 \\ 592863 \\ 898789}

169

181

266

173

183

237

178

178

183

100

149

66

202

184

202

246

79

225

235

149

235

227

252

228

228

149

149

252

256

252

252

268

276

278

276
3232190

471359

571712

1489960

473651

302521

5143968

4741195

335734

690929

6486171

572805

4223282

2125381

3958216

694208

633554

775153

1212088

2304363

1029499

1825229

1040991

2777362

2583519

2752668

5026396

2102710

830760

1687249

1633015

804363

1238625

1257700

1133878 $\begin{array}{ll}-144.305 \mathrm{ug} / \mathrm{mL} \# & 42 \\ 161.589 u \mathrm{~g} / \mathrm{mL} \# & 85 \\ 124.726 \mathrm{ug} / \mathrm{mL} \# & 71 \\ 130.571 \mathrm{ug} / \mathrm{mL} \# & 88 \\ \text { Not Detected } & \end{array}$

$131.117 \mathrm{ug} / \mathrm{mL}$

$126.058 \mathrm{ug} / \mathrm{mL} \#$

$231.654 \mathrm{ug} / \mathrm{mL}$

$118.206 \mathrm{ug} / \mathrm{mL}$

$117.997 \mathrm{ug} / \mathrm{mLm} / 20 / 1793$

$129.417 \mathrm{ug} / \mathrm{mL} \#$

$129.846 \mathrm{ug} / \mathrm{mI} \#$

$116.486 \mathrm{ug} / \mathrm{mL}$

$.99 .501 \mathrm{ug} / \mathrm{mL}$

$116.974 \mathrm{ug} / \mathrm{mL}$

$121.861 \mathrm{ug} / \mathrm{mL} \#$

$115.183 \mathrm{ug} / \mathrm{mL}$

$129.600 \mathrm{ug} / \mathrm{mL}$

$138.047 \mathrm{ug} / \mathrm{mI} \#$

$115.604 \mathrm{ug} / \mathrm{mIt}$

$118.924 \mathrm{ug} / \mathrm{mL} \#$

$108.972 \mathrm{ug} / \mathrm{mE}$

$137.709 \mathrm{ug} / \mathrm{mL}$

$119.883 \mathrm{ug} / \mathrm{mL}$ \#

$124.139 \mathrm{ug} / \mathrm{mL}$

$157.870 \mathrm{ug} / \mathrm{mL \#}$

$134.994 \mathrm{ug} / \mathrm{mI}$

$127.957 \mathrm{ug} / \mathrm{mL} \#$

$129.955 \mathrm{ug} / \mathrm{mL}$

$125.424 \mathrm{ug} / \mathrm{mL}$

$118.183 \mathrm{ug} / \mathrm{mL}$

$156.635 \mathrm{ug} / \mathrm{mL}$

$146.550 u g / m L \#$

$153.102 \mathrm{ug} / \mathrm{mL}$

$125.614 \mathrm{ug} / \mathrm{mL} \#$

$136.665 \mathrm{ug} / \mathrm{mL} \#$

$146.628 \mathrm{ug} / \mathrm{mL}$

$123.834 \mathrm{ug} / \mathrm{mL}$

$143.392 \mathrm{ug} / \mathrm{mL} \#$

$140.270 \mathrm{ug} / \mathrm{mL \#}$
98

55

97

66

95

98

93

55

92

98

63

85

53

100

82

52

88

90

86

99

\section{5}

99

99

98

100

.90

95

88

88

98

98

86

85 
Quantitation Report File: NOFO3S14.D $\quad$ standard amount: 140

Path: C:IHPCHEMI1IDATAl19JUN97NI

$06 / 19 / 97$

Sample: SSTD140 (143WS28421)

Instrument ID: 5972 - N

Analyst: TV

\begin{tabular}{|c|c|c|c|c|c|c|c|}
\hline No. & $\begin{array}{c}\text { Name } \\
\text { 1,4-Dichlorobenzene-d4 }\end{array}$ & $\begin{array}{l}\text { RT } \\
5.37\end{array}$ & $\begin{array}{l}\text { scan } \\
327\end{array}$ & $\begin{array}{c}\mathrm{m} / \mathrm{z} \\
152.00\end{array}$ & $\begin{array}{c}\text { area } \\
636041\end{array}$ & $\begin{array}{c}\mathrm{RF} \\
1.000\end{array}$ & $\begin{array}{c}\text { amt } \\
40.000\end{array}$ \\
\hline 25 & Naphthalene-d8 & 6.95 & 508 & 136.00 & 2437149 & 1.000 & 40.000 \\
\hline 47 & Acenaphthene-d10 & 9.74 & 829 & 164.00 & 1149398 & 1.000 & 49.000 \\
\hline 75 & Phenanthrene-d10 & 12.44 & 1139 & 188.00 & 1416091 & 1.000 & -40.000 \\
\hline 98 & Chrysene-d12 & 16.64 & 1623 & 240.00 & 787909 & 1.000 & 40.000 \\
\hline 113 & Perylene-d12 & 19.91 & 1998 & 264.00 & 477362 & 1.000 & 40.000 \\
\hline 6 & 2-Fluorophenol & 4.14 & 185 & 112.00 & 2693205 & 1.210 & 140.00 \\
\hline 8 & Phenol-d5 & 4.94 & 277 & 99.00 & 3115001 & 1.399 & 140.00 \\
\hline 11 & 2-Chlorophenol-d4 & 5.15 & 301 & 132.00 & 2967617 & 1.333 & 140.00 \\
\hline 17 & 1,2-Dichlorobenzene-d4 & 5.55 & 347 & 152.00 & 1573212 & 0.707 & 140.00 \\
\hline 27 & Nitrobenzene-d5 & 6.01 & 400 & 82.00 & 2514749 & 0.295 & 140.00 \\
\hline 52 & 2-Fluorobiphenyl & 8.57 & 695 & 172.00 & 4213624 & 1.047 & 140.00 \\
\hline 74 & 2,4,6-Tribromophenol & 11.15 & 991 & 330.00 & 518437 & 0.129 & 140.00 \\
\hline 102 & Terphenyl-d14 & 15.05 & 1440 & 244.00 & 2507627 & 0.909 & 140.00 \\
\hline 2 & $\begin{array}{l}\text { Pyridine }\end{array}$ & 3.19 & 76 & 79.00 & 3260721 & $1.465^{\circ}$ & 140.00 \\
\hline 3 & $\mathrm{~N}$-nitrosodimethylamine & 3.19 & 76 & 42.00 & 1788925 & 0.804 & 140.00 \\
\hline 4 & 2-Picoline & 3.70 & 135 & 93.00 & 3418269 & 1.536 & 140.00 \\
\hline 5 & Methyl methanesulfonate & 4.01 & 170 & 80.00 & 1291226 & 0.580 & 140.00 \\
\hline 7 & Ethyl methanesulfonate & 4.59 & 237 & 79.00 & 2145690 & 0.964 & 140.00 \\
\hline 9 & Phenol & 4.96 & 279 & 94.00 & 3263185 & 1.466 & 140.00 \\
\hline 10 & Aniline & 5.04 & 288 & 66.00 & 1888780 & 0.848 & 140.00 \\
\hline 12 & 2-Chlorophenol & 5.17 & 303 & 128.00 & 2843637 & 1.277 & 140.00 \\
\hline 13 & Decane NOT Present & 0.00 & 1 & 57.00 & 0 & 0.000 & 140.00 \\
\hline 14 & 1,3-Dichlorobenzene & 5.32 & 321 & 146.00 & 2757070 & 1.238 & 140.00 \\
\hline 15 & 1,4-dichlorobenzene & 5.40 & 330 & 146.00 & 2723814 & 1.224 & 140.00 \\
\hline 16 & Benzyl alcohol & 5.50 & 341 & 108.00 & 1854433 & 0.833 & 140.00 \\
\hline 18 & 1.2-dichlorobenzene & 5.57 & 350 & 146.00 & 2130333 & 0.957 & 140.00 \\
\hline 19 & 2-Methylphenol & 5.58 & 351 & 107.00 & 2110805 & 0.948 & 140.00 \\
\hline 20 & Bis (2-chloroisopropyl)ether & 5.63 & 356 & 45.00 & 6351538 & 2.853 & 140.00 \\
\hline 21 & 4-methylphenol & 5.77 & 372 & 108.00 & 2878700 & 1.293 & 140.00 \\
\hline 22 & N-Nitrosodi-n-propyl amine & 5.81 & 377 & 70.00 & 1446853 & 0.650 & 140.00 \\
\hline 23 & Hexachloroethane & 5.97 & 395 & 117.00 & 1026491 & 0.461 & 140.00 \\
\hline 24 & Bis (2-chloroethyl)ether & 5.07 & 292 & 63.00 & 2202892 & 0.990 & 140.00 \\
\hline 26 & Acetophenone & 5.82 & 378 & 105.00 & 3129340 & 0.367 & 140.00 \\
\hline 28 & Nitrobenzene & 6.04 & 403 & 77.00 & 2363242 & 0.277 & 140.00 \\
\hline 29 & $N$-nitrosopiperidine & 6.22 & 424 & 114.00 & 1531870 & 0.180 & 140.00 \\
\hline 30 & Isophorone & 6.33 & 437 & 82.00 & 4717867 & 0.553 & 140.00 \\
\hline 31 & 2-Nitrophenol & 6.42 & 447 & 139.00 & 1384069 & 0.162 & 140.00 \\
\hline 32 & 2,4-Dimethylphenol & 6.43 & 448 & 122.00 & 2402445 & 0.282 & 140.00 \\
\hline 33 & Benzoic acid & 6.60 & 468 & 122.00 & 1959487 & 0.230 & 140.00 \\
\hline 34 & Bis(2-chloroethoxy)methane & 6.56 & 463 & 93.00 & 2905305 & 0.341 & 140.00 \\
\hline 35 & 2,4-Dichlorophenol & 6.73 & 483 & 162.00 & 2039517 & 0.239 & 140.00 \\
\hline
\end{tabular}


Quantitation Report File: NQF03S14.D

Path: C:IHPCHEMI1IDATAI19JUN97NI

$06 / 19 / 97$

Sample: SSTD140 (143WS28421)

Instrument ID: 5972 - N

Analyst: TV

\begin{tabular}{|c|c|c|c|c|c|c|c|}
\hline $\begin{array}{l}\text { No. } \\
36\end{array}$ & $\begin{array}{c}\text { Name } \\
\text { A.,A.-dimethylphenethylamine }\end{array}$ & $\begin{array}{l}\text { RT } \\
7.50\end{array}$ & $\begin{array}{l}\text { scan } \\
571\end{array}$ & $\begin{array}{l}\mathrm{m} / \mathrm{z} \\
58.00\end{array}$ & $\begin{array}{c}\text { area } \\
8006040\end{array}$ & $\begin{array}{l}\text { RF } \\
0.939\end{array}$ & $\begin{array}{c}\text { amt } \\
140.00\end{array}$ \\
\hline 37 & 1,2,4-Trichlorobenzene & 6.85 & 497 & 180.00 & 2050479 & 0.240 & 140.00 \\
\hline 38 & Dodecane NOT Present & 0.00 & 1 & 57.00 & 0 & 0.000 & 140.00 \\
\hline 39 & Naphthalene & 6.98 & 512 & 128.00 & 7983915 & 0.936 & 140.00 \\
\hline 40 & 4-Chloroaniline & 7.03 & 517 & 127.00 & 2793624 & 0.328 & 140.00 \\
\hline 41 & 2,6-Dichlorophenol & 7.04 & 519 & 162.00 & 1795195 & 0.210 & 140.00 \\
\hline 42 & Hexachlorobutadiene & 7.12 & 528 & 225.00 & 911351 & 0.107 & 140.00 \\
\hline 43 & Benzothiazole NOT Present & 0.00 & 1 & 135.00 & 0 & 0.000 & 140.00 \\
\hline 44 & $\mathrm{~N}$-nitroso-di-n-butylamine & 7.50 & 571 & 84.00 & 1448196 & 0.170 & 140.00 \\
\hline 45 & 4-Chloro-3-Methyiphenol & 7.70 & 594 & 107.00 & 2022623 & 0.237 & 140.00 \\
\hline 46 & 2-Methyinaphthalene & 8.00 & 629 & 142.00 & 4105843 & 0.481 & 140.00 \\
\hline 48 & $1,2,4,5$-Tetrachlorobenzene & 8.26 & 659 & 216.00 & 1631150 & 0.405 & 140.00 \\
\hline 49 & Hexachlorocyclopentadiene & 8.24 & 656 & 237.00 & 880443 & 0.219 & 140.00 \\
\hline 50 & 2,4,6-Trichlorophenol & 8.43 & 678 & 196.00 & 1271055 & 0.316 & 140.00 \\
\hline 51 & 2,4,5-Trichlorophenol & 8.49 & 685 & 196.00 & 1438919 & 0.358 & 140.00 \\
\hline 53 & Tetradecane NOT Present & 0.00 & 1 & 57.00 & 0 & 0.000 & 140.00 \\
\hline 54 & 2-Chloronaphthalene & 8.80 & 721 & 162.00 & 4388671 & 1.091 . & 140.00 \\
\hline 55 & 1-Chloronaphthalene & 8.84 & 726 & 162.00 & 3434433 & 0.854 & 140.00 \\
\hline 56 & 2-Nitroaniline & 8.95 & 738 & 65.00 & 1219091 & 0.303 & 140.00 \\
\hline 57 & Dimethylphthalate & 9.25 & 773 & 163.00 & 4637795 & 1.153 & 140.00 \\
\hline 58 & 2,6-Dinitrotoluene & 9.37 & 786 & 165.00 & 1220218 & 0.303 & 140.00 \\
\hline 59 & Acenaphthylene & 9.50 & 802 & 152.00 & 6307234 & 1.568 & 140.00 \\
\hline 60 & 3-Nitroaniline & 9.66 & 820 & 65.00 & 1361915 & 0.339 & 140.00 \\
\hline 61 & Acenaphthene & 9.80 & 836 & 153.00 & 3603940 & 0.896 & 140.00 \\
\hline 62 & 2,4-Dinitrophenol & 9.84 & 841 & 184.00 & 862271 & 0.214 & 140.00 \\
\hline 63 & 4-Nitrophenol & 9.92 & 850 & 109.00 & 406885 & 0.101 & 140.00 \\
\hline 64 & Dibenzofuran & 10.11 & 872 & 168.00 & 5076475 & 1.262 & 140.00 \\
\hline 65 & 2,4-Dinitrotoluene & 10.08 & 868 & 165.00 & 1502781 & 0.374 & 140.00 \\
\hline 66 & Pentachlorobenzene & 10.03 & 862 & 250.00 & 1216836 & 0.302 & 140.00 \\
\hline 67 & 1-Naphthylamine & 10.24 & 887 & 143.00 & 3007777 & 0.748 & 140.00 \\
\hline 68 & 2-Naphthylamine & 10.39 & 904 & 143.00 & 4099711 & 1.019 & 140.00 \\
\hline 69 & $2,3,4,6$-Tetrachlorophenol & 10.30 & 894 & 232.00 & 868236 & 0.216 & 140.00 \\
\hline 70 & Diethylphthalate & 10.50 & 917 & 149.00 & 4345921 & 1.080 & 140.00 \\
\hline 71 & 4-Chlorophenyl Phenyl Ether & 10.70 & 940 & 204.00 & 1508929 & 0.375 & 140.00 \\
\hline 72 & Fluorene & 10.72 & 942 & 166.00 & 3388553 & 0.842 & 140.00 \\
\hline 73 & 4-Nitroaniline & 10.77 & 947 & 65.00 & 1163914 & 0.289 & 140.00 \\
\hline 76 & 4,6-Dinitro-2-methylphenol & 10.81 & 952 & 198.00 & 970246 & 0.196 & 140.00 \\
\hline 77 & N-nitrosodiphenylamine & 10.92 & 965 & 169.00 & 2989983 & 0.603 & 140.00 \\
\hline 78 & 1,2-Diphenylhydrazine & 11.00 & 974 & 77.00 & 3712251 & 0.749 & 140.00 \\
\hline 79 & Tributylphosphate NOT Present & 0.00 & 1 & 99.00 & 0 & 0.000 & 140.00 \\
\hline 80 & Phenacetin & 11.51 & 1033 & 108.00 & 1983135 & 0.400 & 140.00 \\
\hline 81 & 4-Bromophenyl Phenyl Ether & 11.61 & 1044 & 248.00 & 853632 & 0.172 & 140.00 \\
\hline
\end{tabular}


Path: C:IHPCHEMI1IDATAI19JUN97NI

$06 / 19 / 97$

Sample: SSTD140 (143WS28421)

Instrument ID: $5972-\mathrm{N}$

Analyst: TV

\begin{tabular}{|c|c|c|c|c|c|c|c|}
\hline $\begin{array}{l}\text { No. } \\
82\end{array}$ & $\begin{array}{l}\text { Name } \\
\text { alpha-BHC }\end{array}$ & $\begin{array}{c}\mathrm{RT} \\
11.64\end{array}$ & $\begin{array}{l}\text { scan } \\
1047\end{array}$ & $\begin{array}{c}\mathrm{m} / \mathrm{z} \\
183.00\end{array}$ & $\begin{array}{c}\text { area } \\
592863\end{array}$ & $\begin{array}{c}R F \\
0.120\end{array}$ & $\begin{array}{c}\text { amt } \\
140.00\end{array}$ \\
\hline 83 & Hexachlorobenzene & 11.71 & 1056 & 284.00 & 898789 & 0.181 & 140.00 \\
\hline $84^{-}$ & ris(2-CE)phosphate NOT Present & 0.00 & 1 & 249.00 & 0 & .000 & 140.00 \\
\hline 85 & 4-Aminobiphenyl & 12.10 & 1100 & 169.00 & 3232190 & .652 & 140.00 \\
\hline 86 & beta-BHC & 12.03 & 092 & 181.00 & 471359 & 0.095 & 140.00 \\
\hline 87 & Pentachlorophenol & 12.07 & 097 & 266.00 & 571712 & 115 & 140.00 \\
\hline 88 & ide & 12.20 & 112 & 173.00 & 1489960 & 301 & 140.00 \\
\hline 89 & gamn & 12.17 & 109 & 183.00 & 473651 & .096 & 140.00 \\
\hline 90 & achloronitrobe & 12.11 & 101 & 237.00 & 302521 & .061 & 140.00 \\
\hline 91 & Phenar & 12.49 & 145 & 178.00 & 51435 & 038 & 140.00 \\
\hline 92 & Ant & 12.58 & 156 & 178.00 & 4741 & 957 & 140.00 \\
\hline 93 & & 12.59 & .157 & 183 & 335 & 068 & 140 \\
\hline 94 & Hep & 13.33 & 242 & 100 & 690 & 139 & \\
\hline 95 & Di-n-Butylpht & 13.48 & 259 & 145 & 648 & 309 & 00 \\
\hline 96 & drin & 13.85 & 302 & & & & \\
\hline 97 & hene & 14.49 & 1375 & .00 & 32 & 52 & \\
\hline 99 & dine & 14.69 & 1398 & .00 & 31 & 771 & $\cdot 140$ \\
\hline 100 & rene & 14.84 & 1415 & 202.00 & 3958 & 435 & \\
\hline 101 & $4,4^{\prime}-\mathrm{DDE}$ & 14.97 & 1430 & 246.00 & 694208 & .252 & \\
\hline 103 & eldrin & 15.12 & 1448 & 79.00 & 54 & 230 & 140.00 \\
\hline 104 & p-Dimethylaminoazobenzene & 15.24 & 1462 & 225.00 & 775 & 281 & 140.00 \\
\hline 105 & ODD & 15.44 & 1484 & 235.00 & 88 & .440 & 140.00 \\
\hline 106 & Butylbenzylpht & 15.73 & 1518 & 149.00 & 63 & .836 & 140.00 \\
\hline 107 & DDT & 15.87 & 1534 & 235.00 & 99 & 373 & 140.00 \\
\hline 108 & hlor & 16.48 & 1604 & 227.00 & 29 & 662 & 140.00 \\
\hline 109 & 3,3'-Dichlorobenzidine & 16.57 & 1614 & 252.00 & 91 & .377 & 140.00 \\
\hline 110 & Benzo[a]anthracene & 16.63 & 1621 & 228.00 & 362 & .007 & 140.00 \\
\hline 111 & sene & 16.70 & 1629 & 228.00 & 19 & 937 & 140.00 \\
\hline 112 & Bis(2-ethylhexyl)pht & 16.61 & 1619 & 149.00 & 2752668 & 998 & .140 .00 \\
\hline 114 & alate & 17.89 & 1766 & 149.00 & 5026396 & 008 & 140.00 \\
\hline 115 & & 18.89 & 1881 & 252.00 & 2102710 & 259 & 140.00 \\
\hline 116 & 12-Dimethylbenz(a)anthracene & 18.86 & 1878 & 256.00 & 830760 & 0.497 & 140.00 \\
\hline 117 & thene & 18.96 & 1889 & 252.00 & 1687249 & 1.010 & 140.00 \\
\hline 118 & & 19.77 & 1982 & 252.00 & 1633015 & .977 & 140.00 \\
\hline 119 & & 20.84 & 2106 & 268.00 & 8043 & .481 & 140.00 \\
\hline 120 & & 23.79 & 2445 & & 1238625 & .741 & 140.00 \\
\hline 121 & & 23.91 & & & & 0.753 & 140.00 \\
\hline 122 & Benzo[ghilperylene & 25.00 & 2584 & 276.00 & 1133878 & 0.679 & 140.00 \\
\hline
\end{tabular}




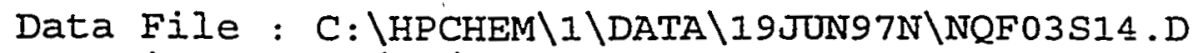

Acq Time : 06/19/97 06:07

Sample : SSTD140 (143WS28421)

Misc : EXP.11/5/97

Quant Time: Jun 19 6:34 1997

Operator: TV

Inst : $5972-\mathrm{N}$

Multiplr: 1.00

Method : C: $\backslash$ HPCHEM $\backslash$ I $\backslash M E T H O D S \backslash 8270 B . M$

Title : SW846/8270B, Curve:NQF 6/19/97

Last Update : Fri Jun 20 08:38:09 1997

Response via : Multiple Level Calibration
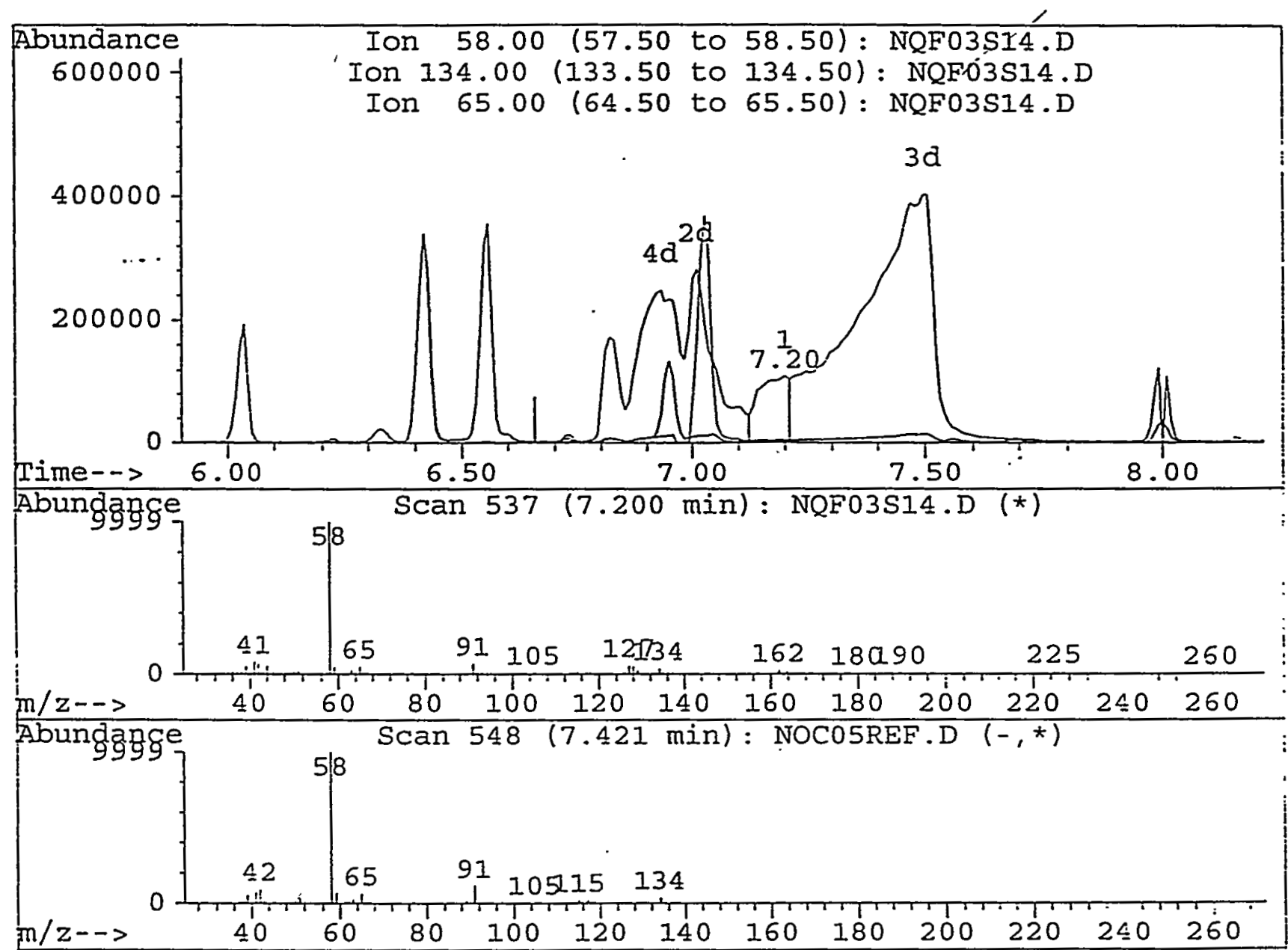

(36) A.,A.-dimethylphenethylamine

$7.20 \mathrm{~min} \quad 26.54 \mathrm{ug} / \mathrm{mL}$

response 492206

\begin{tabular}{rcc}
\multicolumn{1}{c}{ Ion } & Exp\% & Act\% \\
58.00 & 100 & 100 \\
134.00 & 5.00 & 4.98 \\
65.00 & 2.00 & 1.72 \\
0.00 & 0.00 & 0.00
\end{tabular}

Int Scan range: $528(7.122 \mathrm{~min})$ to $538(7.209 \mathrm{~min})$ 
Quantitation Report

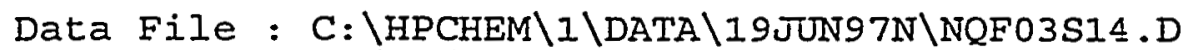

Acq Time : 06/19/97 06:07

Sample : SSTD140 (I43WS28421)

Misc : EXP.11/5/97

Quant Time: Jun 20 8:53 1997

Operator: TV

Inst : $5972-\mathrm{N}$

Multiplr: 1.00

Method $\quad$ C: $\backslash$ HPCHEM $\backslash I \backslash$ METHODS $\backslash 8270 B . M$

Title

: SW846/8270B, Curve:NQF 6/19/97

Last Update : Fri Jun 20 08:38:09 1997

Response via : Multiple Level Calibration

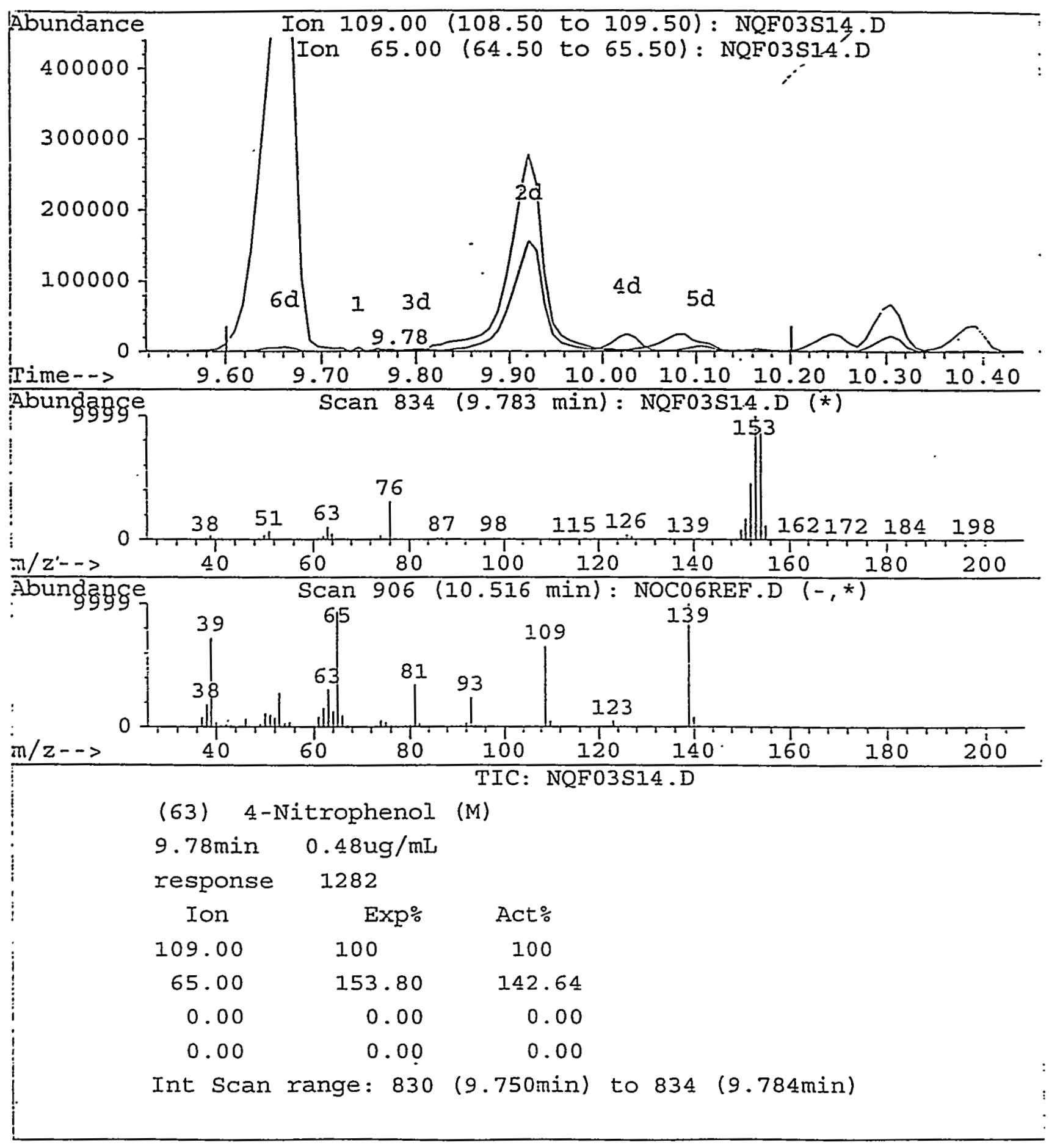




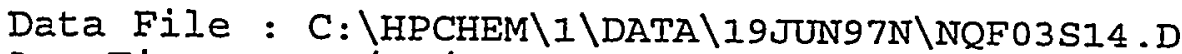

Acq Time : 06/19/97 06:07

Sample : SSTD140 (143WS28421)

MisC : EXP.11/5/97

Quant Time: Jun 20 8:53 1997

Operator: TV

Inst : $5972-\mathrm{N}$

Multiplr: 1.00

Method : C: \HPCHEM $\backslash I \backslash M E T H O D S \backslash 8270 \mathrm{~B} . \mathrm{M}$

Title : SW846/8270B, Curve:NQF 6/19/97

Last Update : Fri Jun 20 08:38:09 1997

Response via : Multiple Level Calibration

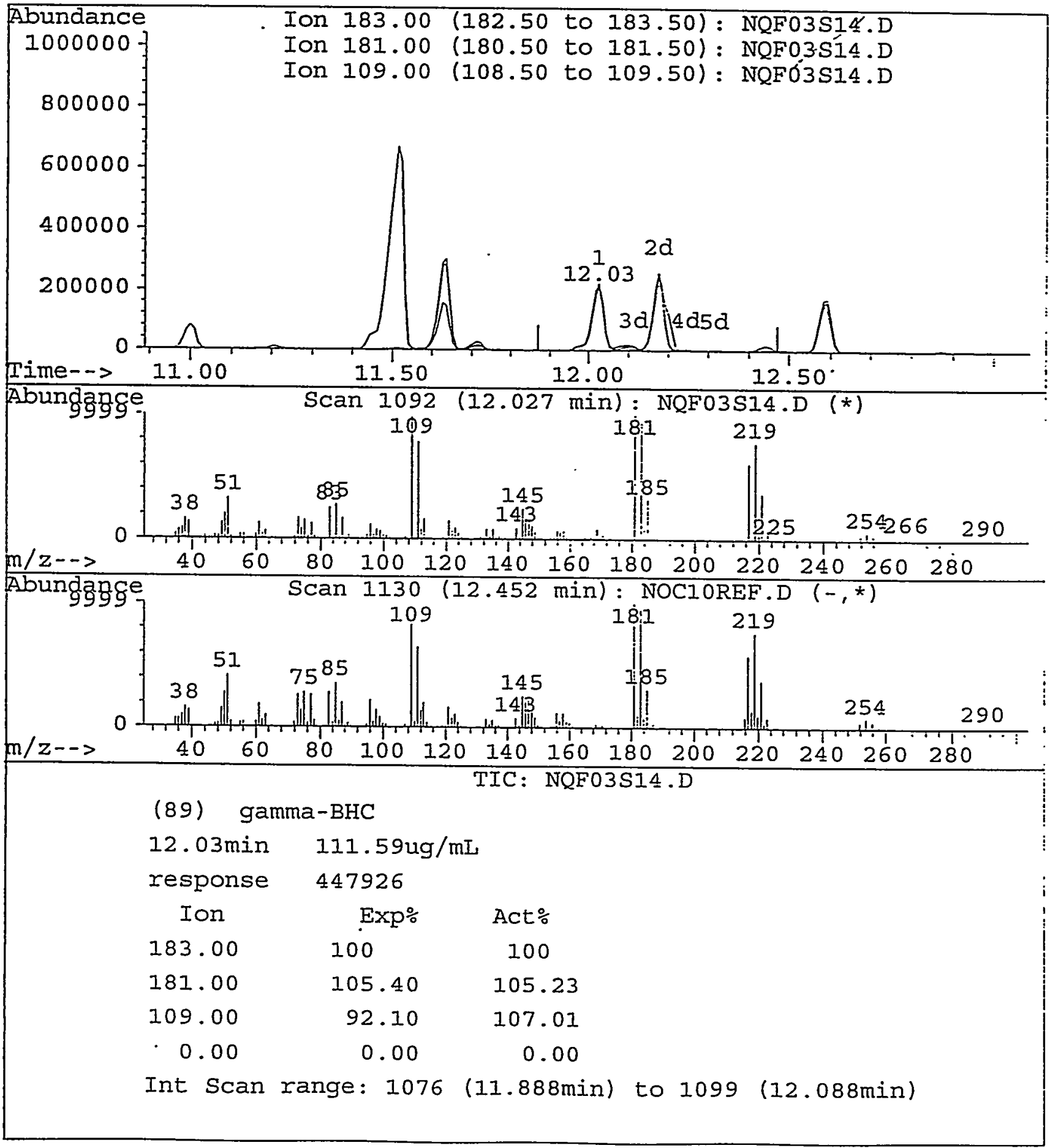


$M A N U A I$ I $N$ T E R A I O N FOR A.,A.-dimethyIphenethylamine

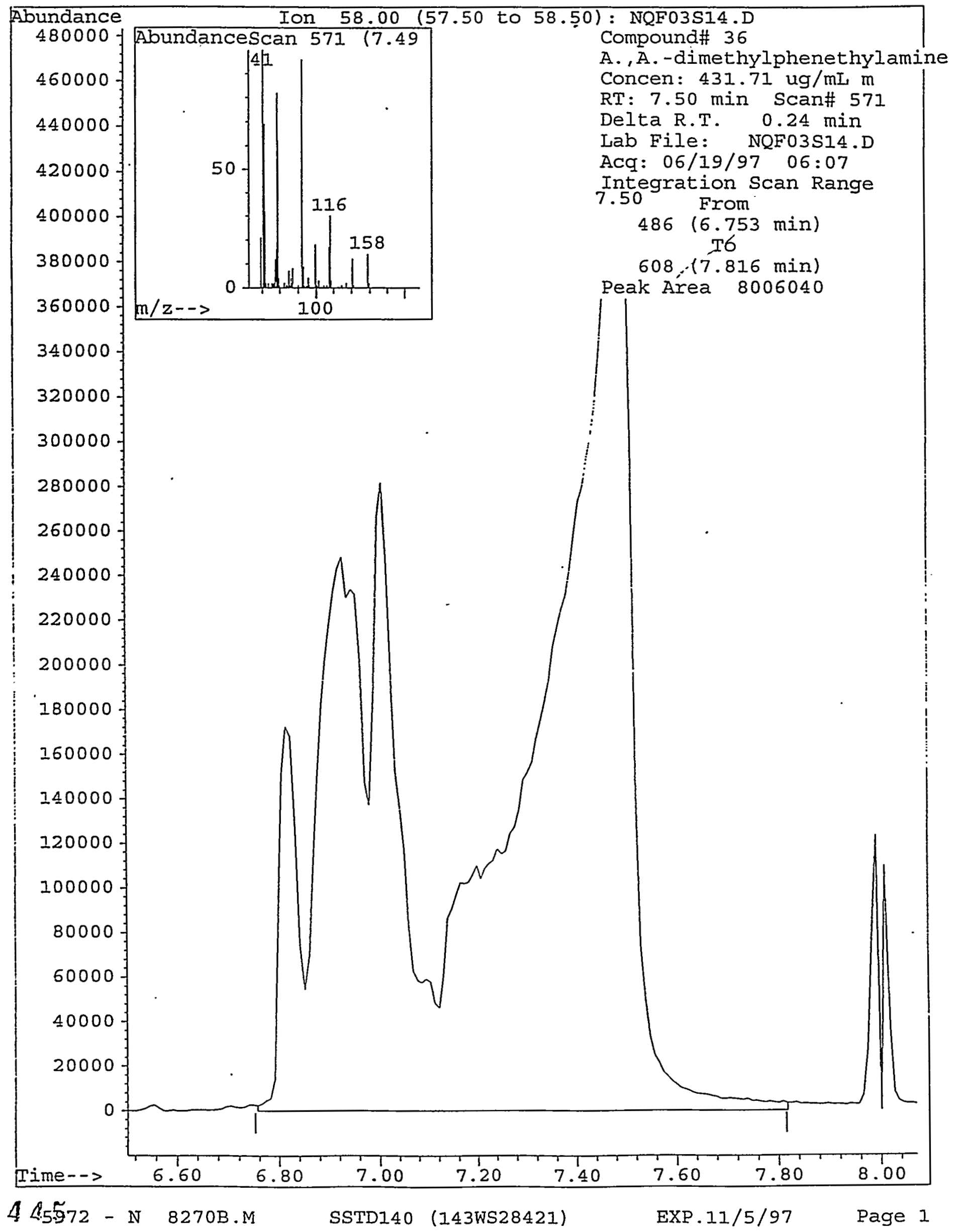


$M A N U A I \quad I N T E G R A T I O N$ FOR 4-Nitrophenol

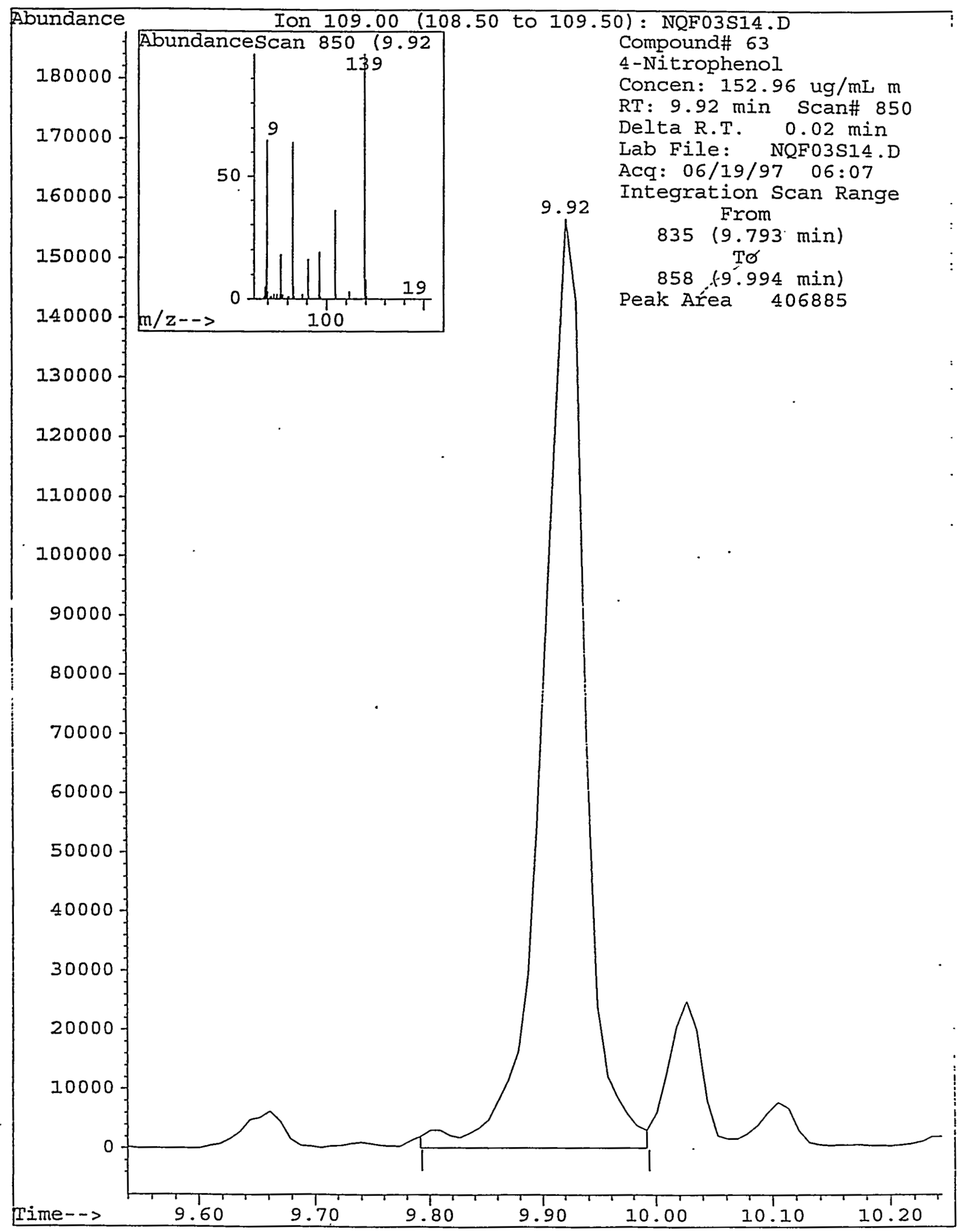


$M A N \cup A I \quad I N T E G R A T I O N$ FOR gamma-BHC

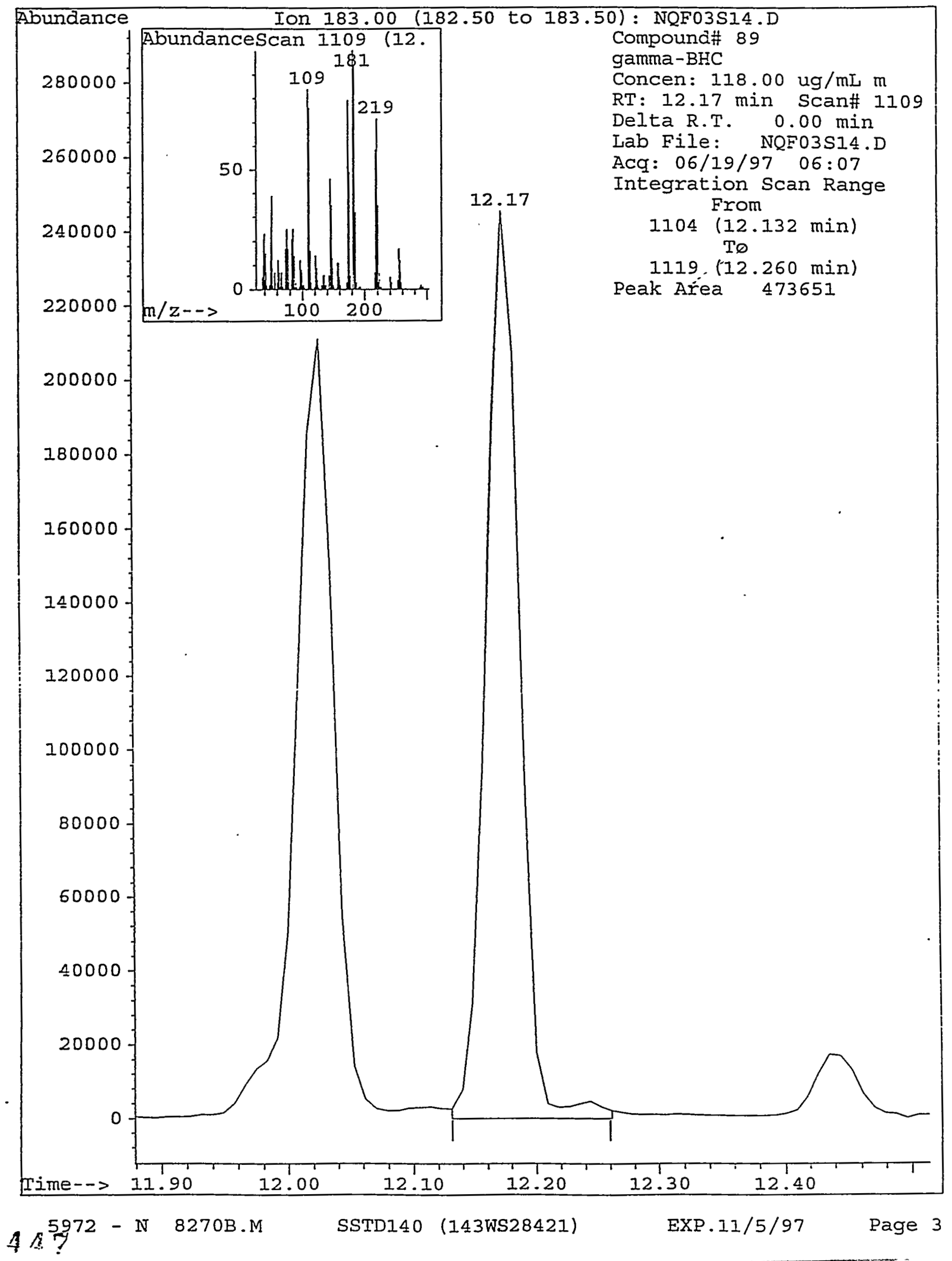


Initial Calibration 5.0 Standard: NQF04S5.D

Solution Monitoring Compounds: $\quad \%$ Drift must be $<I=25 . \%$

\begin{tabular}{|c|c|c|c|c|c|}
\hline & RT & CC Amnt & CC Target & $\%$ Drift & \\
\hline 2-Fluorophenol & 4.11 & 77.67 & 70.00 & -10.96 & \\
\hline Phenol-d5 & 4.91 & 76.24 & 70.00 & -8.91 & \\
\hline Nitrobenzene-d5 & 5.98 & 69.63 & 70.00 & 0.53 & \\
\hline 2-Fluorobiphenyl & 8.56 & 71.35 & 70.00 & -1.93 & \\
\hline 2,4,6-Tribromophenol & 11.11 & 70.85 & 70.00 & -1.21 & \\
\hline Terphenyl-d14 & 15.03 & 74.38 & 70.00 & -6.25 & \\
\hline Phenol & 4.92 & 73.92 & 70.00 & -5.61 & \\
\hline 2-Chlorophenol & 5.14 & 71.98 & 70.00 & -2.83 & \\
\hline 1,3-Dichlorobenzene & 5.31 & 72.14 & 70.00 & -3.06 & \\
\hline 1,4-dichlorobenzene & 5.38 & 72.19 & 70.00 & -3.13 & \\
\hline 1,2-dichlorobenzene & 5.55 & 71.99 & 70.00 & -2.85 & \\
\hline 2-Methylphenol & 5.56 & 65.60 & 70.00 & 6.29 & \\
\hline 4-methylphenol & 5.73 & 67.56 & 70.00 & 3.49 & \\
\hline N-Nitrosodi-n-propyl amine & 5.78 & 82.97 & 70.00 & -18.52 & \\
\hline Hexachloroethane & 5.95 & 72.20 & 70.00 & -3.15 & \\
\hline Bis (2-chloroethyl)ether & 5.04 & 63.71 & 70.00 & 8.98 & \\
\hline Nitrobenzene & 6.01 & 70.53 & 70.00 & -0.76 & \\
\hline Isophorone & 6.30 & 65.16 & 70.00 & 6.91 & \\
\hline 2-Nitrophenol & 6.40 & 72.14 & 70.00 & -3.05 & \\
\hline 2,4-Dimethylphenol & 6.39 & 63.15 & 70.00 & 9.78 & 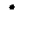 \\
\hline Bis(2-chloroethoxy)methane & 6.53 & 68.12 & 70.00 & 2.68 & \\
\hline 2,4-Dichlorophenol & 6.71 & 69.27 & 70.00 & 1.04 & \\
\hline 1,2,4-Trichlorobenzene & 6.84 & 69.46 & 70.00 & 0.77 & . \\
\hline Naphthalene & 6.97 & 64.47 & 70.00 & 7.90 & \\
\hline 4-Chloro-3-Methylphenol & 7.66 & 71.82 & 70.00 & -2.60 & \\
\hline 2-Methylnaphthalene & 7.98 & 70.52 & 70.00 & -0.75 & \\
\hline 2,4,6-Trichlorophenol & 8.40 & 69.06 & 70.00 & 1.35 & \\
\hline 2,4,5-Trichlorophenol & 8.46 & 66.62 & 70.00 & 4.83 & \\
\hline 2-Chloronaphthalene & 8.78 & 61.49 & 70.00 & 12.16 & \\
\hline 2,6-Dinitrotoluene & 9.33 & 72.14 & 70.00 & -3.06 & \\
\hline Acenaphthylene & 9.48 & 65.41 & 70.00 & 6.56 & \\
\hline Acenaphthene & 9.78 & 69.76 & 70.00 & 0.34 & \\
\hline Dibenzofuran & 10.08 & 70.82 & 70.00 & -1.17 & \\
\hline 2,4-Dinitrotoluene & 10.04 & 72.75 & 70.00 & -3.93 & \\
\hline 4-Chlorophenyl Phenyl Ether & 10.69 & 71.84 & 70.00 & -2.63 & \\
\hline Fluorene & 10.70 & 71.63 & 70.00 & -2.32 & \\
\hline 4-Bromophenyl Phenyl Ether & 11.58 & 71.41 & 70.00 & -2.01 & \\
\hline Hexachlorobenzene & 11.69 & 71.89 & 70.00 & -2.71 & \\
\hline Pentachlorophenol & 12.04 & 82.20 & 70.00 & -17.42 & \\
\hline Phenanthrene & 12.45 & 71.48 & 70.00 & -2.12 & \\
\hline Anthracene & 12.55 & 76.45 & 70.00 & -9.21 & \\
\hline - Fluoranthene & 14.47 & 74.72 & 70.090 & -6.75 & \\
\hline $\begin{array}{l}\text { Pyrene } \\
\end{array}$ & 14.81 & 72.97 & 70.00 & -4.24 & \\
\hline Benzola]anthracene & 16.61 & 68.49 & 70.00 & 2.15 & \\
\hline Chrysene & 16.67 & 65.62 & 70.00 & 6.26 & \\
\hline Benzolb]fluoranthene & 18.85 & 72.43 & 70.00 & -3.48 & \\
\hline Benzo[k]fluoranthene & 18.92 & 80.40 & 70.00 & -14.85 & \\
\hline Benzolalpyrene & 19.73 & 70.78 & 70.00 & -1.11 & \\
\hline Indeno(1,2,3-c,d)pyrene & 23.73 & 50.28 & 70.00 & 28.17 & $\#$ \\
\hline Dibenz[a,h]anthracene & 23.85 & 51.07 & 70.00 & 27.05 & $\#$ \\
\hline Benzolghi]perylene & 24.93 & 49.82 & 70.00 & 28.83 & $\#$ \\
\hline
\end{tabular}




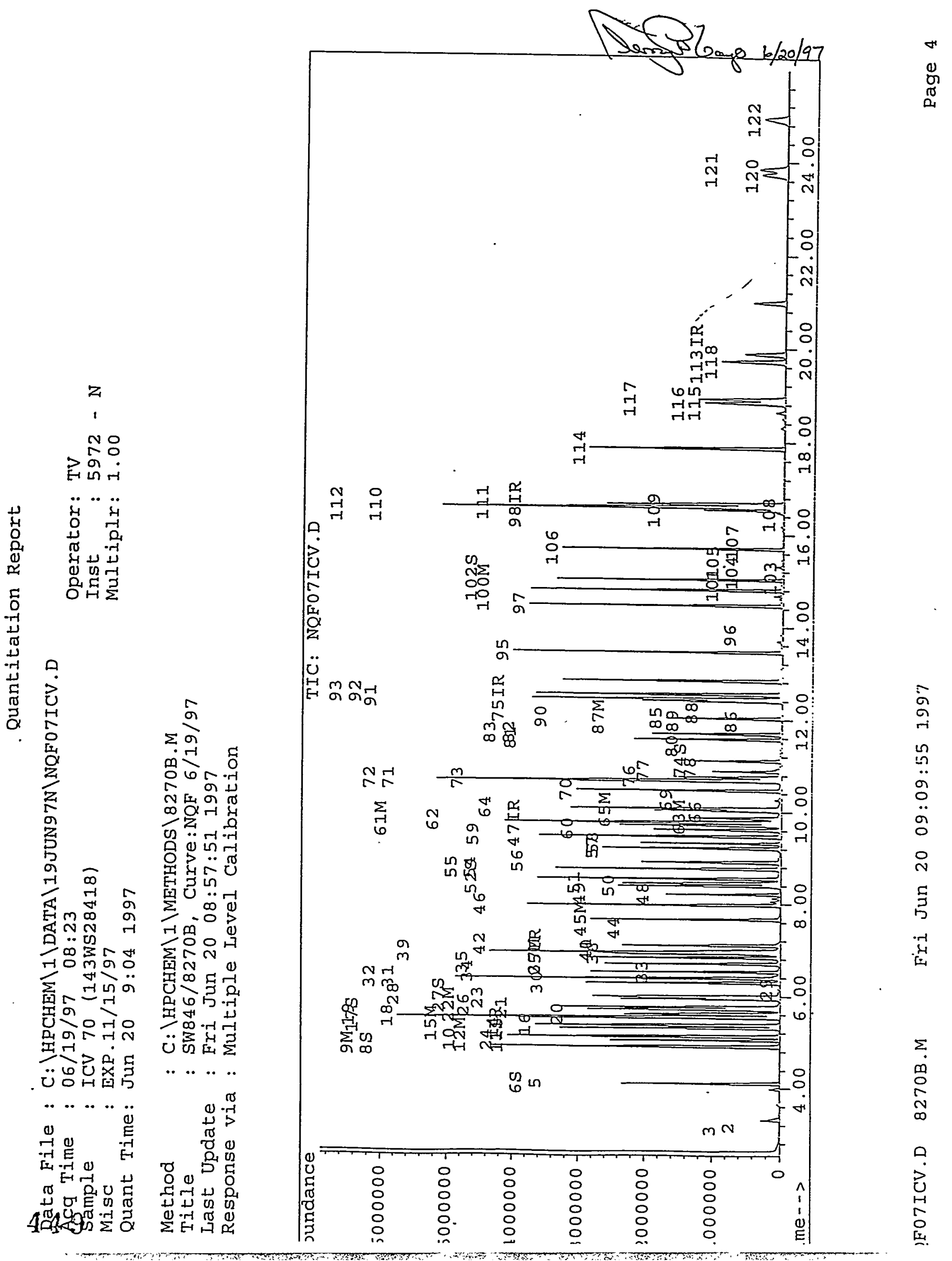


Quantitation Report

Data File : C: $\backslash$ HPCHEM \I\DATA $\backslash 19 \pi U N 97 N \backslash N Q F 07 I C V . D$

Acq Time : 06/19/97 08:23

Sample : ICV 70 (143WS28418)

MisC : EXP.11/15/97

Quant Time: Jun 20 9:04 1997

Operator: TV

Inst : $5972-\mathrm{N}$

Multiplr: 1.00

Method : C: $\backslash$ HPCHEM $\backslash I \backslash M E T H O D S \backslash 8270 B . M$

Title : SW846/8270B, Curve:NQF 6/19/97

Last Update : Fri Jun 20 08:57:51 1997

Response via : Multiple Level Calibration

Internal standards

I) 1,4-Dichlorobenzene-d4

25) Naphthalene-d8

47) Acenaphthene-d10

75) Phenanthrene-dI0

98) Chrysene-d12

113) Perylene-d12

System Monitoring Compounds

6) 2-Fluorophenol

B) Phenol-d5

11) 2-Chlorophenol-d4

17) 1,2-Dichlorobenzene-d4

27) Nitrobenzene-d5

52) 2-Fluorobipheny 1

74) 2,4,6-Tribromophenol

102) Terphenyl-d14

Target Compounds

2) Pyridine

3) N-nitrosodimethylamine

4) 2-Picoline

5) Methyl methanesulfonate

7) Ethyl methanesulfonate

9) Phenol

1D) Aniline

12) 2-Chlorophenol

13) Decane NOT Present

14) 1,3-Dichlorobenzene

15) I,4-dichlorobenzene

15) Benzyl alcohol

IB) 1,2-dichlorobenzene

19) 2-Methylphenol

2D) Bis (2-chloroisopropyl) ethe

21) 4-methylphenol

22) N-Nitrosodi-n-propyl amine

23) Hexachloroethane

24) Bis (2-chloroethyl) ether

26) Acetophenone

28) Nitrobenzene

29) N-nitrosopiperidine

30) Isophorone

31) 2-Nitrophenol

32) 2,4-Dimethylphenol
R.T. Scan QIon Response

Conc Units

Area:

$\begin{array}{rrr}5.36 & 325 & 152 \\ 6.93 & 506 & 136 \\ 9.72 & 827 & 164 \\ 12.41 & 1136 & 188 \\ 16.63 & 1621 & 240 \\ 19.89 & 1996 & 264\end{array}$

545969 .

2240763

1088016

1454698

951171

552869

$\begin{array}{rrrr}4.11 & 181 & 112 & 1363133 \\ 4.91 & 273 & 99 & 1607763 \\ 5.12 & 298 & 132 & 1428189 \\ 5.53 & 345 & 152 & 769055 \\ 5.98 & 397 & 82 & 1223144 \\ 8.56 & 693 & 172 & 2267660 \\ 11.11 & 987 & 330 & 263206 \\ 15.03 & 1437 & 244 & 1623829\end{array}$

\subsection{7}

73

3.10

0.00

4.11

0.00

4.92

5.04

5.14

0.00

5.31

5.38

5.38

5.55

5.56

5.61

5.73

5.78

5.95

5.04

5.79

6.01

6.18

6.30

6.40

6.39
65

181

275

289

300

319

328

328

347

348

354

368

373

393

289

375

400

420

433

445

444

\section{9}

42

80

94

66

128

146

146

108

146

107

45

108

70

117

63

105

77

114

82

139

122
1744

2304

$7 \leq 62$

1646741

10025

1422455

1350205

1338903

8574

1105434

981327

2855888

1323554

879675

493309

1003317

1478

1193267

607

2220522

739376

1097174

$\begin{array}{ll}40.00 \mathrm{ug} / \mathrm{mL} & -0.02 \\ 40.00 \mathrm{ug} / \mathrm{mL} & -0.02 \\ 40.00 \mathrm{ug} / \mathrm{mL} & -0.02 \\ 40.00 \mathrm{ug} / \mathrm{mL} & -0.02 \\ 40.00 \mathrm{ug} / \mathrm{mL} & -0.01 \\ 40.00 \mathrm{ug} / \mathrm{mL} & -0.02\end{array}$

$\because$ Recovery

$77.673 \mathrm{ug} / \mathrm{mL}$ $76.239 \mathrm{ug} / \mathrm{mL}$ $69.858 \mathrm{ug} / \mathrm{mL}$ $69.383 \mathrm{ug} / \mathrm{mL}$ $69.632 \mathrm{ug} / \mathrm{mL}$ $.71 .349 \mathrm{ug} / \mathrm{mL}$ $70.847 \mathrm{ug} / \mathrm{mL}$ $74.376 \mathrm{ug} / \mathrm{mL}$

$77.67 \%$

$76.24 \%$

$69.86 \%$

$69.38 \%$

$69.63 \%$

$71.35 \%$

$70.85 \%$

$74.38 \%$

$0.076 u g /$ Qvalue

$0.189 \mathrm{ug} / \mathrm{mL} \ddot{\pi} \quad .23$

Not Detected

$0.813 \mathrm{ug} / \mathrm{mL}$ \#

1

Not Detected

$73.925 \mathrm{ug} / \mathrm{mL}$ \#

$0.726 \mathrm{ug} / \mathrm{mL}$

78

$71.983 \mathrm{ug} / \mathrm{mL}$

Not Detected

$72.142 \mathrm{ug} / \mathrm{mL}$

$72.193 \mathrm{ug} / \mathrm{mL}$

$0.645 \mathrm{ug} / \mathrm{mL}$

$71.992 \mathrm{ug} / \mathrm{mL}$

$65.599 \mathrm{ug} / \mathrm{mL}$

$66.581 \mathrm{ug} / \mathrm{mL} \#$

$67.558 \mathrm{ug} / \mathrm{mL}$

$82.966 \mathrm{ug} / \mathrm{mL}$

$72.202 \mathrm{ug} / \mathrm{mL}$

$63.714 \mathrm{ug} / \mathrm{mL} \#$

$0.056 \mathrm{ug} / \mathrm{mL}$

$70.529 u g / m L \#$

$0.053 \mathrm{ug} / \mathrm{mL} \#$

$65.164 \mathrm{ug} / \mathrm{mL \#}$

$72.138 \mathrm{ug} / \mathrm{mL} \#$

$63.152 \mathrm{ug} / \mathrm{mL}$
1

99

97

96

97

98

100

.91

99

93

84

94

1

100

1

83

82

87 
Quantitation Report

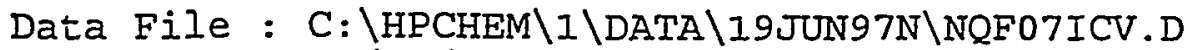

Acq Time : 06/19/97 08:23

Sample : ICV 70 (143WS28418)

Misc : EXP.11/15/97

Quant Time: Jun 20 9:04 1997

Operator: TV

Inst : $5972-\mathrm{N}$

Multiplx: 1.00

Method : C: \HPCHEM $\backslash I \backslash$ METHODS $\backslash 8270 \mathrm{~B} . \mathrm{M}$

Title : SW846/8270B, Curve:NQF 6/19/97

Last Update : Fri Jun 20 08:57:51 1997

Response via : Multiple Level Calibration

Compound

33) Benzoic acid

34) Bis (2-chloroethoxy) methane

35) 2,4-Dichlorophenol

36) A.,A.-dimethylphenethylami

37) 1,2,4-Trichlorobenzene

38) Dodecane NOT Present

39) Naphthalene

40) 4-Chloroaniline

4I) 2,6-Dichlorophenol

42) Hexachlorobutadiene

43) Benzothiazole NOT Present

44) N-nitroso-di-n-butylamine

45) 4-Chloro-3-Methylphenol

46) 2-Methylnaphthalene

48) $1,2,4,5$-Tetrachlorobenzene

49) Hexachlorocyclopentadiene

50) 2,4,6-Trichlorophenol

51) 2,4,5-Trichlorophenol

53) Tetradecane NOT Present

54) 2-Chloronaphthalene

55) I-Chloronaphthalene

56) 2-Nitroaniline

57) Dimethylphthalate

58) 2,6-Dinitrotoluene

59) Acenaphthylene

60) 3-Nitroaniline

51) Acenaphthene

52) 2,4-Dinitrophenol

53) 4-Nitrophenol

64) Dibenzofuran

65) 2,4-Dinitrotoluene

66) Pentachlorobenzene

67) 1-Naphthylamine

68) 2-Naphthylamine

69) $2,3,4,6$-Tetrachlorophenol

70) Diethylphthalate

71) 4-Chlorophenyl Phenyl Ethe

72) FIuorene

73) 4-Nitroaniline

7.6) 4 ,6-Dinitro-2-methylphenol

77) N-nitrosodiphenylamine

78) 1,2-Diphenylhydrazine

79) Tributylphosphate NOT Pres
R.T. Scan QIon Response

Conc Unit

Qvalue

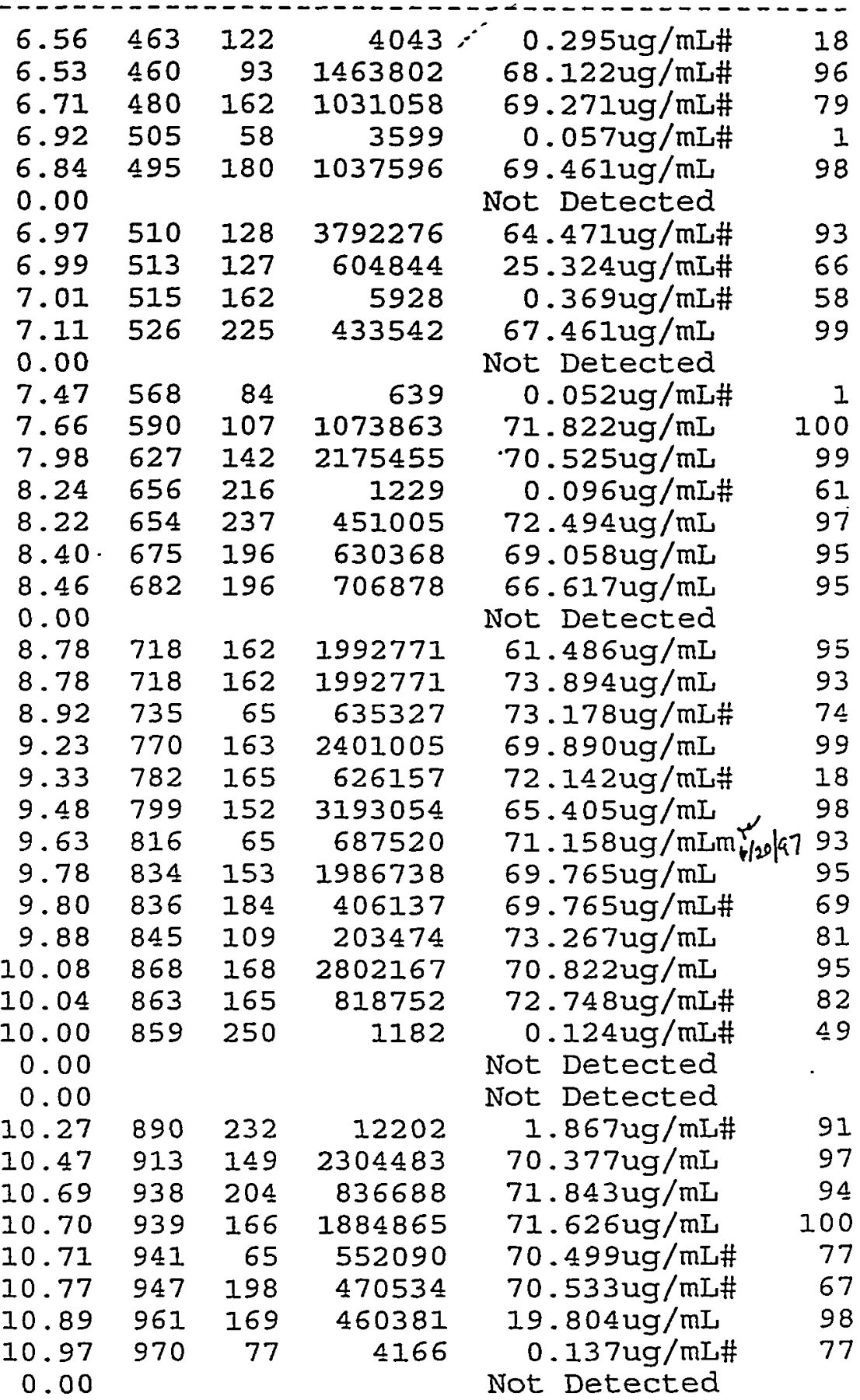

$($ \#) = qualifier out of range $(\mathrm{m})=$ manual integration

(*) ${ }^{*}$ Dpes not meet EPA spectral criteria (False Hit) 
Quantitation Report

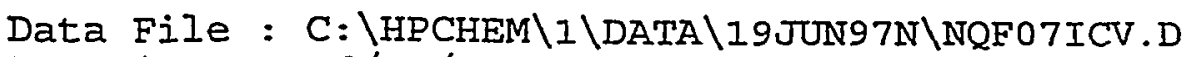

Acq Time : 06/19/97 08:23

Sample : ICV 70 (143WS28418)

Misc : EXP.11/15/97

Quant Time: Jun 20 9:04 1997

Operator: TV

Inst : $5972-\mathrm{N}$

Multiplr: 1.00

Method : C: $\backslash$ HPCHEM $\backslash 1 \backslash$ METHODS $\backslash 8270 \mathrm{~B} . \mathrm{M}$

Title : SW846/8270B, Curve:NQF 6/19/97

Last Update : Fri Jun 20 08:57:51 1997

Response via : Multiple Level Calibration

Compound

Bo) Phenacetin

BI) 4-Bromophenyl Phenyl Ether

82) alpha-BHC

83) Hexachlorobenzene

84) Tris (2-CE) phosphate NOT Pr

B5) 4-Aminobiphenyl

B6) beta-BHC

B7) Pentachlorophenol

B8) Pronamide

B9) gamma-BHC

90) Pentachloronitrobenzene

91) Phenanthrene

92) Anthracene

93) delta-BHC

94) Heptachlor

95) Di-n-Butylphthalate

96) Aldrin

97) Fluoranthene

99) Benzidine

100) Pyrene

101) 4,41-DDE

103) Dieldrin

104) p-Dimethylaminoazobenzene

105) $4,41-D D D$

106) Butylbenzylphthalate

107) 4,4 -DDT

10B) Methoxychlor

109) 3,3'-Dichlorobenzidine

110) Benzo[a] anthracene

111) Chrysene

112) Bis(2-ethylhexyl) phthalate

114) Di-n-octylphthalate

115) Benzo[b] fluoranthene

116) 7,12-Dimethylbenz (a) anthra

117) Benzo[k] fluoranthene

.118) Benzo[a] pyrene

119) 3-Methylcholanthrene

120) Indeno $(I, 2,3-c, d)$ pyrene

121) Dibenz $[a, h]$ anthracene

122) Benzo[ghi]perylene
R.T. Scan QIon Response Conc Unit Qvalue

\begin{tabular}{|c|c|c|c|c|}
\hline 11.43 & 1023 & 108 & 3394 & $0.234 \mathrm{ug} / \mathrm{mL} \#$ \\
\hline 11.58 & 1041 & 248 & 460788 & $71.406 \mathrm{ug} / \mathrm{mL} \#$ \\
\hline 11.69 & 1053 & 183 & 1638 & $0.336 \mathrm{ug} / \mathrm{mL} \#$ \\
\hline $\begin{array}{r}11.69 \\
0.00\end{array}$ & 1053 & 284 & 483396 & $\begin{array}{l}71.895 \mathrm{ug} / \mathrm{mI} \# \\
\text { Not Detected }\end{array}$ \\
\hline 12.04 & 1093 & 169 & 57035 & $1.930 \mathrm{ug} / \mathrm{mI}$ \\
\hline 11.98 & 1086 & 181 & 374 & $0.099 \mathrm{ug} / \mathrm{mL} \#$ \\
\hline 04 & 1093 & 266 & 345410 & $82.197 \mathrm{ug} / \mathrm{mI}$ \\
\hline 15 & 1106 & 173 & 1444 & $0.113 \mathrm{ug} / \mathrm{mL} \#$ \\
\hline 97 & 1085 & 183 & 327 & $0.084 \mathrm{ug} / \mathrm{mL} \#$ \\
\hline 2.04 & 1093 & 237 & 10785 & $4.371 \mathrm{ug} / \mathrm{mI} \#$ \\
\hline .45 & 1141 & 178 & 2875624 & $71.482 \mathrm{ug} / \mathrm{mL} \#$ \\
\hline .55 & 1152 & 178 & 2945533 & $76.449 u \mathrm{~g} / \mathrm{mL}$ \\
\hline .56 & 1153 & 183 & 338 & $0.102 \mathrm{ug} / \mathrm{mL} \#$ \\
\hline 0.00 & & & & Not Detected \\
\hline 3.46 & 1257 & 149 & 3756569 & $72.346 \mathrm{ug} / \mathrm{mL} \#$ \\
\hline .84 & 1300 & 66 & 580 & $0.118 \mathrm{ug} / \mathrm{mL} \#$ \\
\hline 4.47 & 1373 & 202 & 2588623 & $74.723 \mathrm{ug} / \mathrm{mL} \#$ \\
\hline .00 & & & & Not Detected \\
\hline 81 & 1412 & 202 & 2567566 . & $72.969 \mathrm{ug} / \mathrm{mL}_{\pi}^{\#}$ \\
\hline 94 & 1427 & 246 & 1298 & $0.212 \mathrm{ug} / \mathrm{mL} \#$ \\
\hline 5.10 & 1445 & 79 & 1056 & $0.178 \mathrm{ug} / \mathrm{mL}$ \\
\hline 5.22 & 1459 & 225 & 856 & $0.126 \mathrm{ug} / \mathrm{mL} \#$ \\
\hline 5.42 & 1482 & 235 & 1816 & $0.174 \mathrm{ug} / \mathrm{mL}$ \\
\hline 5.71 & 1515 & 149 & 1400446 & $69.524 \mathrm{ug} / \mathrm{mL}$ \\
\hline 5.85 & 1531 & 235 & 1154 & $0.115 \mathrm{ug} / \mathrm{mL} \#$ \\
\hline .46 & 1602 & 227 & 2667 & $0.152 \mathrm{ug} / \mathrm{mL} \#$ \\
\hline .54 & 1611 & 252 & $40 \leq 220$ & $39.997 \mathrm{ug} / \mathrm{mL \#}$ \\
\hline .61 & 1619 & 228 & 1647984 & $68.493 \mathrm{ug} / \mathrm{mL}$ \\
\hline .67 & 1626 & 228 & 1554661 & $65.620 u g / m L$ \\
\hline .59 & 1617 & 149 & 1662780 & $68.425 \mathrm{ug} / \mathrm{mL}$ \\
\hline .86 & 1763 & 149 & 3108611 & $90.082 \mathrm{ug} / \mathrm{mL}$ \\
\hline .85 & 1876 & 252 & 1179786 & $72.434 \mathrm{ug} / \mathrm{mL} \#$ \\
\hline 8.82 & 1873 & 256 & 1058 & $0.179 \mathrm{ug} / \mathrm{mL} \#$ \\
\hline 3.92 & 1885 & 252 & 1136002 & $80.396 \mathrm{ug} / \mathrm{mL} \#$ \\
\hline 9.73 & 1978 & 252 & 967720 & $70.778 \mathrm{ug} / \mathrm{mL} \#$ \\
\hline .00 & & & & Not Detected \\
\hline .73 & 2438 & 276 & 571889 & $50.282 \mathrm{ug} / \mathrm{mL}$ \\
\hline & 2452 & 278 & 587745 & $51.067 \mathrm{ug} / \mathrm{mL} \#$ \\
\hline & 2576 & 276 & 528008 & $49.816 u g / n$ \\
\hline
\end{tabular}

Data Processed By: Saros Gap b/20/97

(H) = qualifier out of range $(m)=$ manual integration

(4) Bpes not meet EPA spectral criteria (False Hit) 
Quantitation Report

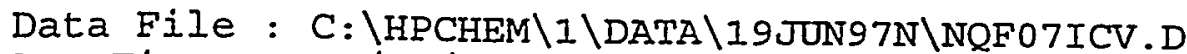

Acq Time : 06/19/97 08:23

Sample : ICV 70 (143WS28418)

Misc' : EXP.11/15/97

Quant Time: Jun 20 9:01 1997

Operator: TV

Inst : $5972-\mathrm{N}$ Multiplr: 1.00

Method

: C: $\backslash$ HPCHEM $\backslash 1 \backslash$ METHODS $\backslash 8270 B . M$

Title

: SW846/8270B, Curve:NQF 6/19/97

Last Update : Fri Jun 20 08:57:51 1997

Response via : Multiple Level Calibration

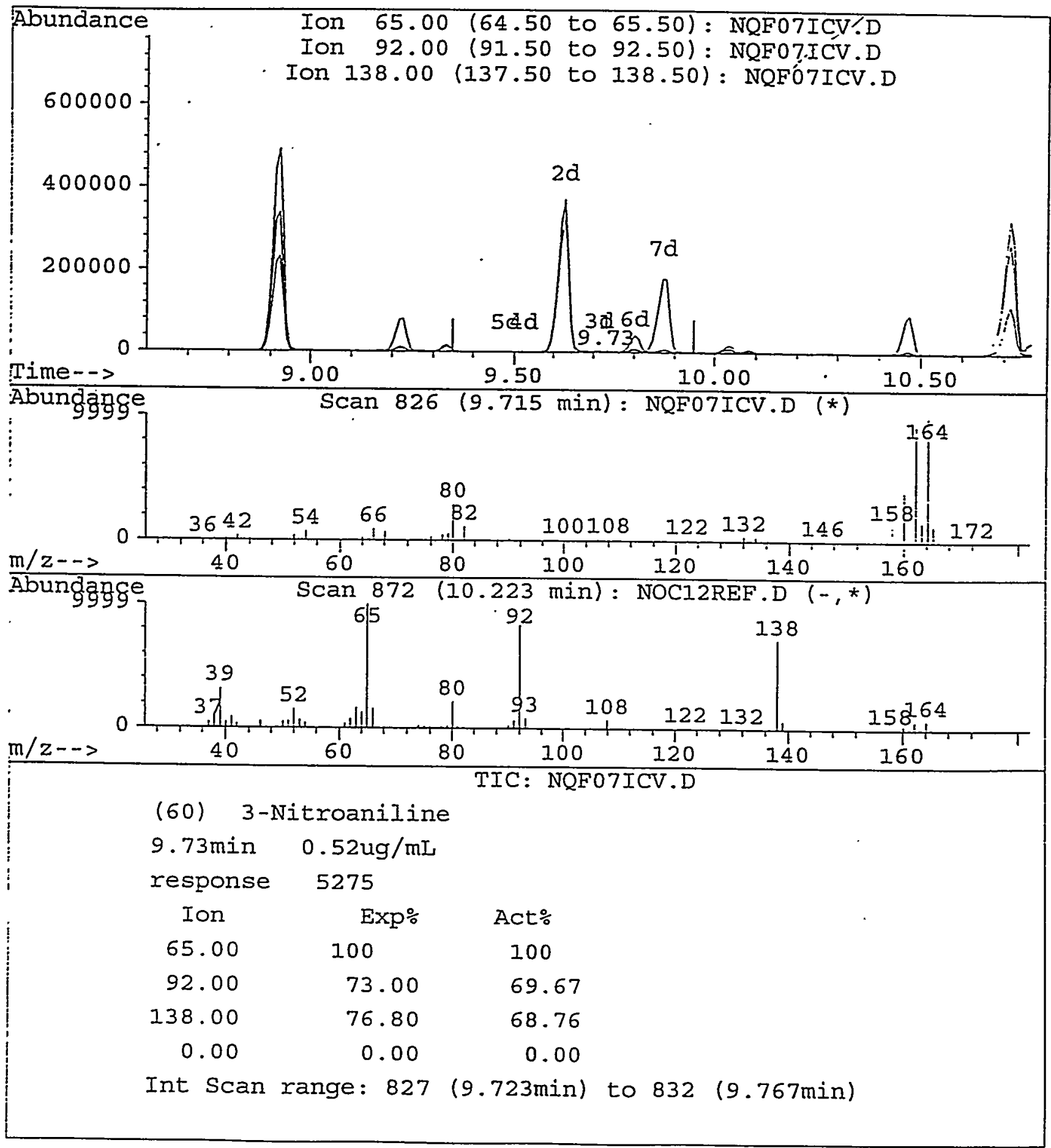


$M A N U A L \quad I N T E G R A T I O N$ FOR 3-Nitroaniline

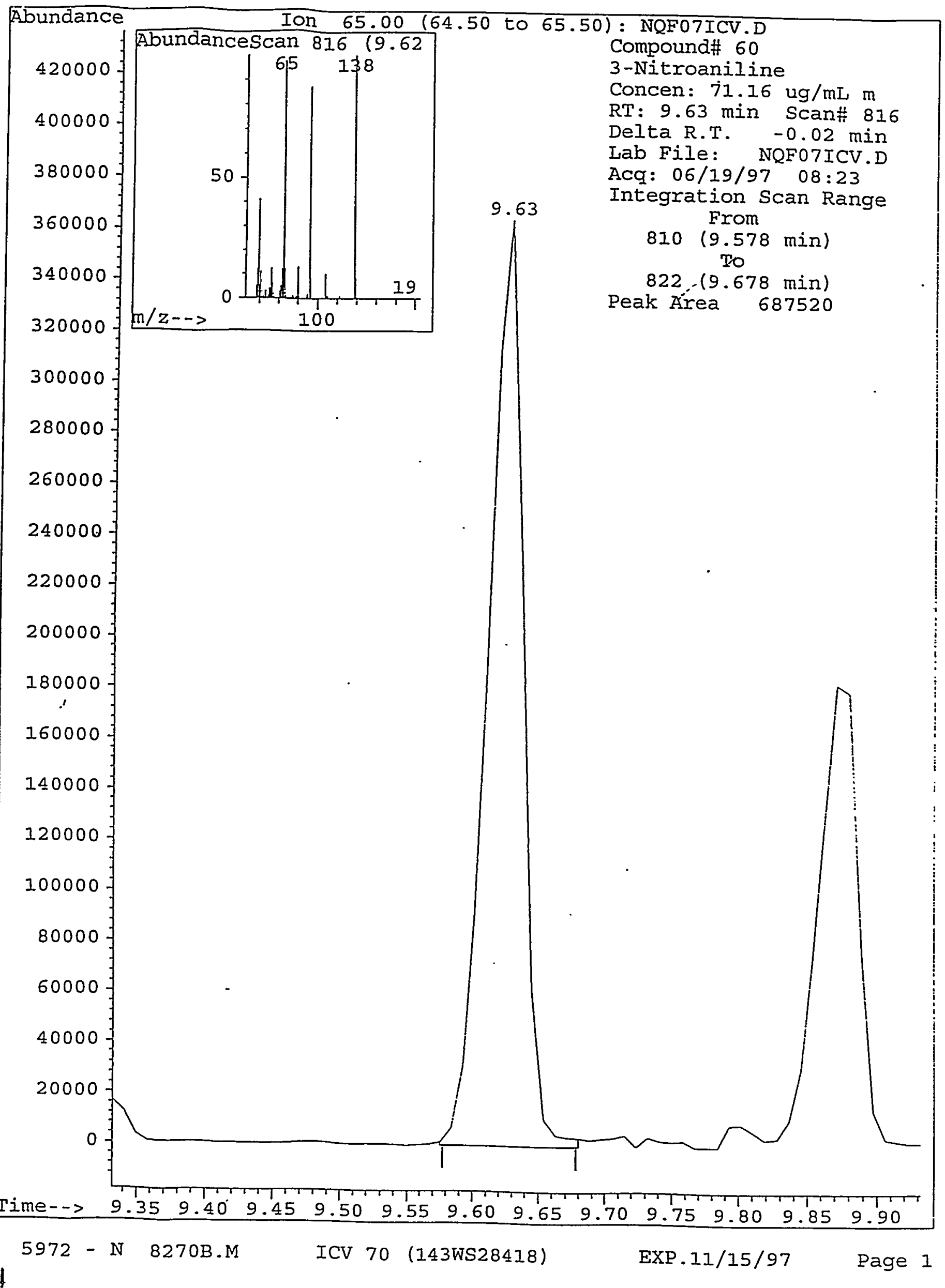




\section{DATA \\ CHEM \\ a SOREKSON SOMPAKY}

Method: EPA 418.1

Analysis: Total Petroleum Hydrocarbons

Preparation SOP : IW-EP-418.1

Analysis SOP : IW-EP-418.1

DCL Set D: 97C-0208-03
Client: SAIC

Account: 3006

Matrix: Water

Group ID: G9769006

:jeneral Set Information: There were eight field samples including the MS/MSD samples in this set which were analyzed for total petroleum hydrocarbons. This set was analyzed with 97C-0208-04.

Method Summary: Each sample bottle is marked at the meniscus with a piece of tape and an ink marker for initial volume. $\mathrm{pH}$ is taken from each sample to verify that sample is preserved. Pour sample into 2-L separatory funnel. Rinse each sample bottle with 30 $\mathrm{mL}$ of freon 113 and transfer the solvent into the funnel. Shake the funnel for two minutes and allow the two layers to separate. Drain the freon 113 layer through a Whatman \#40 filter cone containing about $10 \mathrm{~g}$ of anhydrous $\mathrm{Na}_{2} \mathrm{SO}_{4}$. Collect the filtrate in a $100 \mathrm{~mL}$ volumetric flask. Repeat shaking the funnel two more times with $30 \mathrm{~mL}$ portions of freon 113, combining each successive filtrate in the flask. Bring the extracted sample to volume in a $100 \mathrm{~mL}$ volumetric flask. Discard about 5-10 $\mathrm{mL}$ of solution from the volumetric flask. Add about $3 \mathrm{~g}$ silica gel and a stirring bar into the flask. Stir the solution for seven minutes on a magnetic stirrer. Wait for the silica gel to settle in the sample extract, then analyze sample using the FTIR-1000C, by direct comparison with standards using a linear curve.

Sample Preparation: All samples were extracted and prepared in accordance with the published DCL SOF.

Holding Times: The holding times were met for both preparation and analysis.

Dilution(s): NA

QC Data: All QC data were within method limits.

MS/MSD Analysis: The MS/MSD recoveries were all within limits.

455 
Instrument QC: Instrument initial and continuing calibrations were performed in accordance with published procedures.

NC/CAR: N/A

Flagging Codes: N/A.

Sample Calculation: The final results are calculated in $\mu \mathrm{g} / \mathrm{L}$ by the equation:

$$
\mu g / L T P H=\frac{R \times D \times 1000 \mu g / \mathrm{mg}}{V}
$$

where:

$\mathrm{R}=$ Mass of TPH determined from the FTR (mg).

$D=$ Extract dilution factor, if used.

$V=$ Volume of water sample (L).

Example: Calculation of TPH concentration ( $\mu \mathrm{g} / \mathrm{L}$ ) in sample

97C02897MS:

$$
\frac{(1.65054 \mathrm{mg}) \times(1) \times(1000 \mu \mathrm{g} / \mathrm{mg})}{1.06 \mathrm{~L}}=1557.11 \mu \mathrm{g} / \mathrm{L}
$$

Miscellaneous Comments: All samples except $97 \mathrm{C} 02899$ were preserved to a $\mathrm{pH}<2$.

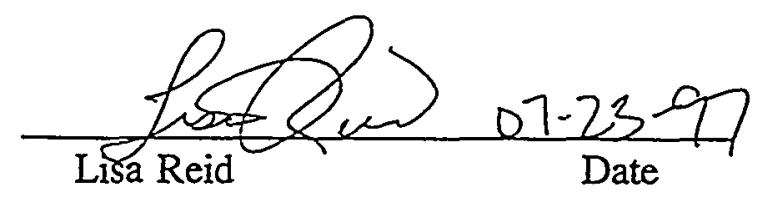

- 456 


\section{DATA 産 CHEM}

\section{Datapackage Table of Contents}

Information pertaining to this datapackage is divided into the four categories listed below. A Case Narrative immediately precedes this Table of Contents and contains pertinent information about this datapackage.

Aralytical Results ............... Yellow

Sample Tracking Documentation ............ Pink.

Analytical Documentation ............. Blue

Raw Data

: Green

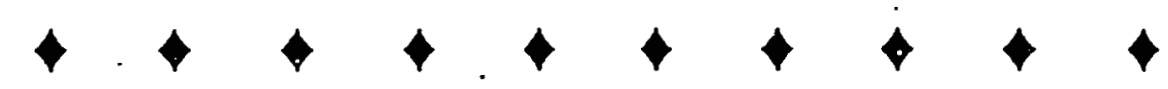

$\because 3$

\section{Analytical Results}




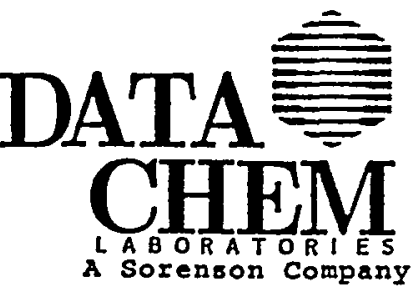

SAIC

Attention: Art Shattuck 411 Hackensack Avenue Hackensack, NJ 07601

\section{COVER PAGE}

ANALYTICAL REPORT FOR SAIC

Phone(201) 498-8496

$\operatorname{Fax}(201) \quad 489-1592$
Form COVER-V1.3

Page 1

07239709051984

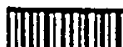

G9757027

DCI Report Group..: 97C-0208-03

Date Printed...... 23-JUL-9709:06

Project Protocol *: P975B002

client Ref Nugber.: Not Provided

Release Number.... Not Provided $\therefore$

Analysis Hethod(s): 418.1

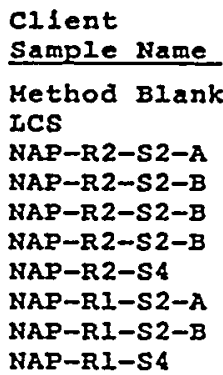

Sample Name

Iaboratory

Sample Name

BI-136533-1

QC-136533-1

97002896

$97 \mathrm{C02897}$

$97 \mathrm{C02897 \textrm {HS }}$

97C02897KSD

97002898

97002899

97002900

97002901
Date

Samplea

NA

MA

25-JUस-97

25-JUN-97

25-JUN-97

25-JUN-97

26-JUस-97

24-JUस-97

24-JUस-97

25-JUR-97
Date

Recelved

*ล

NR

27-JUN-97

27-JUस-97

27-JUN-97

27-JUR-97

27-JUN-97

27-J Uस -97

27-JUस-97

27-JUस-97

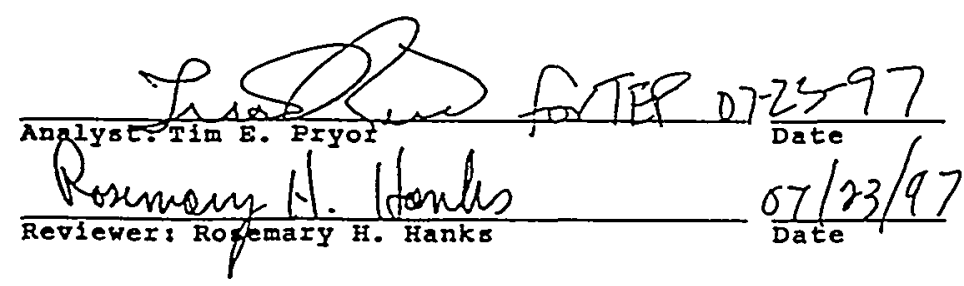




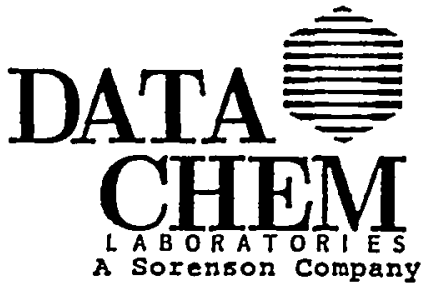

CIleat Name... SAIC
EORM H (TYPE I)

SINGLE METHOD ANALYSES

SAMPLE GROUP COMMENTS
Form RLIMS63H-V1.3

Page 2

07239709051984

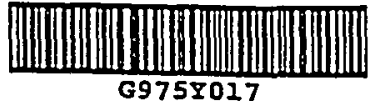

DCI Report Group... : 97C-0208-03

Date Printed.....: 23-JUI-97 09:06

Release Number.... Not Provided

\section{Generzl Information}

The DCI QC Database maintalns all numerical flgures which are input from the pertinent data source. These data have not been rounded to signiflcant figures nor have they been molsture corrected. Reports generated from the system, hovever, llst data which have been rounded to the number of signiflcant flgures requested by the client or deemed appropriate for the method. Thls may create minor discrepancles between data which appear on the QC Summary Forms (Porms B-G) and those that would be calculated from rounded analytical results. Additionally, if a molsture correction is performed, differences will be observed between the QC data and the surrogate data reported on Form $A$ (or other report forms) and corresponding data reported on QC sumary Forms. In these cases, the Form $A$ will indicate the "Report Basis" as well as the molsture value used for making the correction. Report generation options: MX

\section{Result Syabol Definitions}

2ND - Not Detected above the KDL or IDL (LID or HDC for radiochemistry).

** - No result could be reported, see sample comments for detalls.

\section{Qualiflex Symbol Deflaitions}

0 - Not Detected above the KDI or IDI (IID or HDC fOr radiochemistIY). For radiochemistry the nuclide was not ldentified by the camberra Nuclear NID program, activity values reported are calculated using the Canberra Nuclear mINACr program.

$B$ - For organic analysis the qualifier indicates that this analgte was found in the method blank. For inorganic analysis the qualifler signifles the value is between the IDI and PQI.

$J$ - The qualifier indlcates that the value is between the HDI and the PQL. It is also used for Indlating an estimated value for tentatively ldentified compounds in mass spectrometry where a $1: 1$ response is assumed. 


\section{DATA \\ FORY A (TYPE I) \\ SINGLE METHOD ANATYSES} CHEM A Soremson Company

Date Pxinted........: 23-JuL-97 09:06

client rame.......... saIC

cllent Ref Number.... Not Provided

sampling slte........ Not Applicable

Release Number....... Hot Rrovided

Date Received........ Not Applicable

DCL Preparation Group: G9761010

Date Prepared.......: 02-JUI-97 00:00

Preparation Hethod...: 418.1

Aliquot Weight/Volume: 1.00

Net Weight/VoIume.... Not Required
Form RLIMS63A-V1.3 07239709051984

\section{Page 3}

SAMPLE ANALYSIS DATA SHEET
s976102L
Client Sample Name: BL-136533-1 DCI Sample Name...: Br-136533-I DCI Report Group... 97C-0208-03

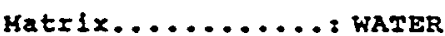
Date Sampled....... Not Applicable Reporting Units...: ug/I $\therefore$

DCL Analy:1: Group: G9769006 Analysis Method...: 418.1 Instrument Type...: IR Instrument ID....: FTIR-1000C colum Type...... Not Applicable

Analytical Results

\begin{tabular}{|c|c|c|c|c|c|c|c|}
\hline Aralyte & $\begin{array}{c}\text { Date } \\
\text { Analyzed } \\
\end{array}$ & KDL & Result & Comment & Qual. & Dilution & CRDL \\
\hline TRPH & 09-JUI-97 00:00 & 100. & ND & & & & 100. \\
\hline
\end{tabular}




\section{$\stackrel{\text { FORH A (TYPE I) }}{=}$ \\ DATA} CHEM

LABORATORIES

A Sorenson Company

\section{SAMPLE ANALYSIS DATA SHEET}

Form RLIMS63A-V1.3

Page 4
Date Printed.......: 23-JUL-97 09:06

Client Name.......... SAIC

Client Ref Number..... Not Provided

Sampling site........ Not Applicable

Release Number........ Not Provided

Date Recelved....... I Not Appllcable

DCI Preparation Group: G9761010

Date Prepared.......: 02-JUL-97 00:00

Preparation Method...: 418.1

Aliquot Welght/Volume: 1.00

Net Welght/Volume.:. . : Not Required
Cllent Sample Name: QC-136533-1

DCI Sample Name...: QC-136533-1

DCI Report Group..: 97C-0208-03

Matrix.......... HATER

Date Sampled...... Yot Applicable

Reporting Units.... ug/I

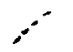

DCI Analysis Group: G9769006

Analysis Method...: 418.1

Instrument Type...: IR

Instrument ID....: FTIR-1000C

Column Type...... Not Applicable

Analytical Results

\begin{tabular}{|c|c|c|c|c|c|c|c|}
\hline Analyte & \begin{tabular}{|c|} 
Date \\
Analyzed \\
\end{tabular} & MDL & Result & Comment & Qual. & Dilution & CRDI \\
\hline TRPH & $09-3 U L-97 \quad 00: 00$ & 100. & 1200 & & & & 100 \\
\hline
\end{tabular}




\section{DATA CHEM \\ A Sorenson Company}

Date Printed.......: 23-JUI-97 09:06

cilent Name......... SAIC

Client Ref Number..... Not Provided

Sampling site........ Not Provided

Release Number....... Not Provided

Date Recelved......: 27-JUN-97 00:00

DCL Preparation Group: G9761010

Date Prepared.......: 02-JUL-97 00:00

Preparation Method...: 418.1

Allquot welght/Volume: 1.06

Net Welght/Volume..... Not Required
FORM A (TYPE I)

SINGLE MEIHOD ANALYSES

SAMPLE ANALYSIS DATA SHEET

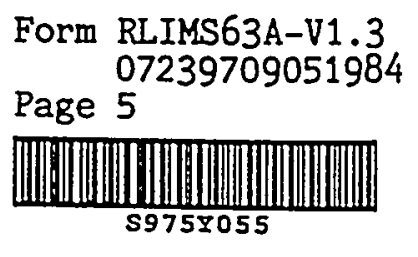

Client Sample Name: HNP-R2-82-X

DCL Sample Name...: 97602896

DCL Report Group..: 97C-0208-03

Matrix......... : WATER

Date Sampled......:25-JUN-97 00:00

Reporting Units....: ug/I

Report Basls.,:... 团As Recelved $\square$ Dried

DCL Analysis Group: 69769006

Analysis Hethod...: 418.1

Instrument Type...: IR

Instrument ID.....: ETIR-100OC

Column Type....... Not Applicable

Analytical Results

\begin{tabular}{|c|c|c|c|c|c|c|c|}
\hline Analyte & $\begin{array}{c}\text { Date } \\
\text { Aralyzed } \\
\end{array}$ & MDL & Result & Comment & Qual. & Dizution & CRDL \\
\hline IRPH & $09-30 L-97 \quad 00: 00$ & 100. & ND & & & & 100. \\
\hline
\end{tabular}




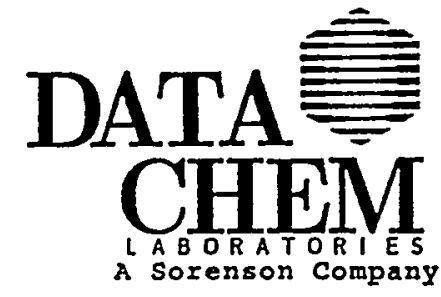

Date Printed.......: 23-JUL-97 09:06

Client Name.......... SAIC

cllent Ref Number..... Not Provided Sampling site......... Not provided Release Number....... Not Provided

Date Recelyed.......: 27-JUN-97 00:00

DCL Preparation Group: G9761010

Date Prepared......: 02-JUL-97 00:00

Preparation Method...: 418.1

Allquot Welght/Volume: 1.06

Net Weight/Volume.... : Not Requlred
FORY A (TYPE I)

SINGLE METHOD ANATYSES

SAMPLE ANALYSIS DATA SHEXET
Form RLIMS63A-V1.3 07239709051984

\section{Page 6}

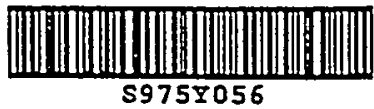

Client Sample Name: ExP-R2-82-B

DCI Sample Name...: 97002897

DCL Report Group..: 97C-0208-03

Matrix......... WATER

pate Sampled......2 25-JUN-97 00:00

Reporting Uaits..., ug/I

Report Basls....... 因As Recelved $\square$ Drled

DCL Analysis Group: 69769006

Analysis Hethod...: 418.1

Instrument Type...: IR

Instrument ID..... FTIR-1000C

Column Type....... Not Applicable

Analytical Results

\begin{tabular}{|c|c|c|c|c|c|c|c|}
\hline Analgte & $\begin{array}{c}\text { Date } \\
\text { Analyzed }\end{array}$ & KDL & Result & Comment & Qual. & Dilution & CRDL \\
\hline TRPH & 09-JUL-97 $00: 00$ & 100. & ND & & & & 100. \\
\hline
\end{tabular}




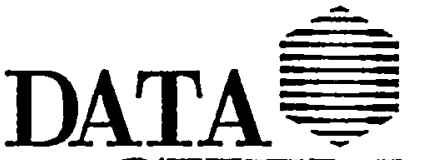

\section{FORM A (TYPE I) \\ SINGLE KEIHOD ANALYSES}

SAMPLE ANALYSIS DATA SHEET
Form RLIMS63A-V1.3 Page 7 07239709051984

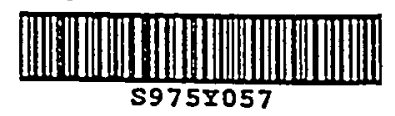

Date Printed.......: 23-JUL-97 09:06

Client Name.......... SAIC

Cllent Ref Number....: Not Provided

Sampling site........ Not Provided

Release Number....... Not Provided

Date Received.......: 27-JUN-97 00:00

DCI Preparation Group: G9761010

Date Prepared.......: 02-JUL-97 00:00

Preparation Method...: 418.1

Allquot Welght/Volume: 1.06

Net Welght/Volume.... : Not Required
Client Sample' Name: HAP-R2-82-B

DCI Sample Name...: 97C02897Ks

DCI Report Group..: 97C-0208-03

Matrix......... WATER

Date Sampled......: 25-JUN-97 00:00

Reporting Units...: ug/L

Report Basis...... 因As Recelved $\square$ Dried

DCL Analysis Group: G9769006

Analysis Method...: 428.1

Instrument Type...: IR

Instrument ID....: ETIR-1000C

Column Type....... Not Appllcable

Analytical Results

\begin{tabular}{|c|c|c|c|c|c|c|c|}
\hline Analyte & $\begin{array}{c}\text { Date } \\
\text { Analyzed } \\
\end{array}$ & HDI & Result & Comment & Qual. & Dilution & CRDI \\
\hline TRPH & $09-J U L-97 \quad 00: 00$ & 100. & 1600 & & & & 100. \\
\hline
\end{tabular}



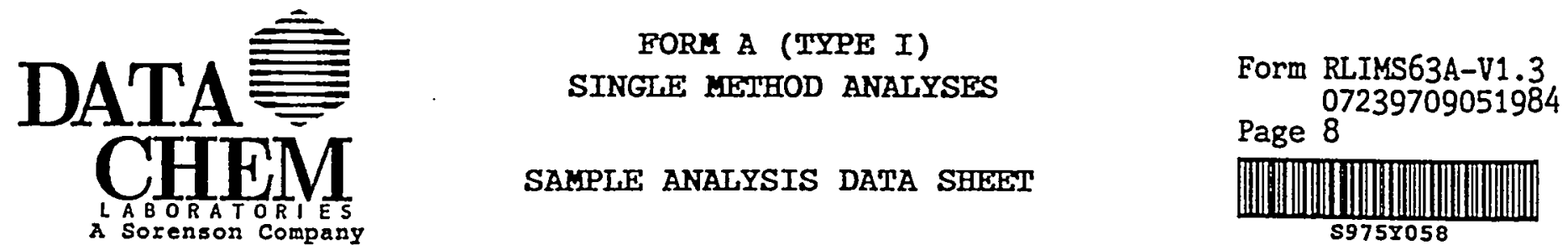

Date Printed.......: 23-JUL-97 09:06

Client sample Name: MAP-R2-82-B

c1lent Name......... SAIC

cllent Ref Number.... Not Provided

sampling site........ Not Provided

Release Number....... Not Provided

DCI Sample Name... 9 97C02897ksD

DCI Report Group... 97C-0208-03

HatrIx........... HATER

Date Sampled...... 25-Jర1-97 00:00

Reporting Units.... ug/I

Date Recelved.......27-JUN-97 00:00

Report Basis....... [ 8 Xs Recelved $\square$ Drled

DCI Preparation Group: G9761010

Date Prepared....... 02-JuL-97 00:00

DCI Analys is Group: G9769006

Preparation Hethod...:418.1

Aliquot Welght/Volume: 1.06

Analysis Hethod...: 418.1

Instrument Type...: IR

set Welght/Volume.... Not Required

Instrument ID..... FTIR-1000C

Column Type....... Not Appllable

Analytical Results

\begin{tabular}{|c|c|c|c|c|c|c|c|}
\hline Analyto & $\begin{array}{c}\text { Date } \\
\text { Analyzed }\end{array}$ & MDI & Result & Comment & Qual. & Dilution & CRDI \\
\hline TRPH & $09-J U L-97 \quad 00: 00$ & 100 . & 1500 & & & & 100. \\
\hline
\end{tabular}



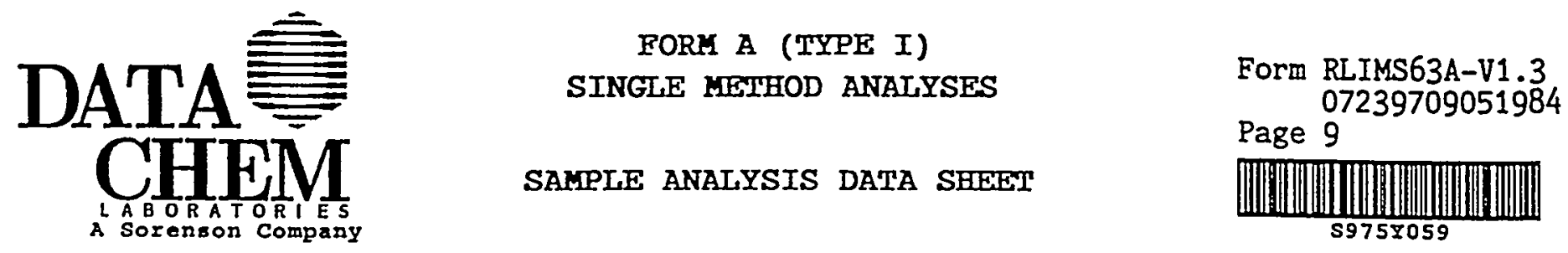

Date Printed........23-JUI-9709:06

Client Sample Name: MNP-R2-84

client Name.......... SAIC

client Ref Number.... Hot Provided

Sampling site........ Not Provided

Release Number....... Not Provided

DCI Sample Name...: 97602898

DCI Report Group..: 97C-0208-03

Matrix.......... WATER

Date Sampled...... 26-JUN-97 00:00

Reporting Units.... ug/I

Date Received......2 27-JUN-97 00:00

Report Bas1s.;:... 目As Recelved $\square$ Dried

DCL Preparation Group: G9761010

Date Prepared........ 02-JUL-97 00:00

DCL Analysis Group: G9769006

Preparation Method...: 418.1

Allquot Welght/Volume: 1.06

Analysis Hethod...: 418.1

Instrument Type...: IR

Net Welght/Volume.... Not Required

Instrument ID ....: FTIR-1000C

Colum Type....... Not Applicable

Analytical Results

\begin{tabular}{|c|c|c|c|c|c|c|c|}
\hline Analyte & $\begin{array}{c}\text { Date } \\
\text { Analyzed }\end{array}$ & MDI & Result & Comment & Qual. & Dilution & CRDL \\
\hline TRPH & $09-J U I-97 \quad 00=00$ & 100. & ND & & & & 100. \\
\hline
\end{tabular}




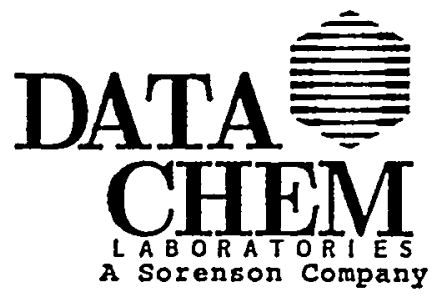

Date Printed.......: 23-JUL-97 09:06

Cllent Name......... SAIC

client Ref Number.... Hot Provided

Sampiling site........ Not Provided

Release Number....... Not Provided

Date Recelved....... 27-JUR-97 00:00

DCI Preparation Group: G9761010

Date Prepared........ 02-JuL-97 00:00

Preparation Hethod...:418.1

Aliquot Welght/Volume: 1.06

Net Weight/Volume.... Not Required
FORM A (TYPE I)

SINGLE METHOD ANALYSES

\title{
SAMPLE ANALYSIS DATA SHEET
}

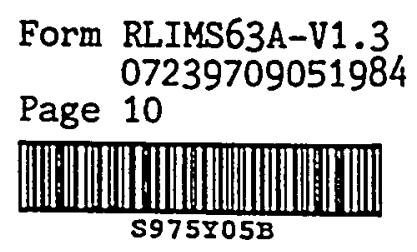

\author{
Client Sample Name: MAP-RI-82-A \\ DCI Sample Name...: $97 C 02899$ \\ DCL Report Group..: 97C-0208-03 \\ Hatrix.......... WATER \\ Date Sampled.....: 3A-JUN-97 00:00 \\ Reporting Unlts...t ug/I \\ Report Basis..., $\because$. [ [X] Received $\square$ Dried \\ DCI Analysis Group: G9769006 \\ Analysis Hethod.... 418.1 \\ Instrument TYPe... IR \\ Instrument ID....: FTIR-I000C \\ Column Tqpe....... Not Applicable
}

Analytical Results

\begin{tabular}{|c|c|c|c|c|c|c|c|}
\hline Analyte & $\begin{array}{c}\text { Date } \\
\text { Mnalyzed } \\
\end{array}$ & KDI & Result & Comment & Qual. & Dilution & CRDL \\
\hline IRPH & 09-JUL-9700:00 & 100. & RD & & & & 100. \\
\hline
\end{tabular}




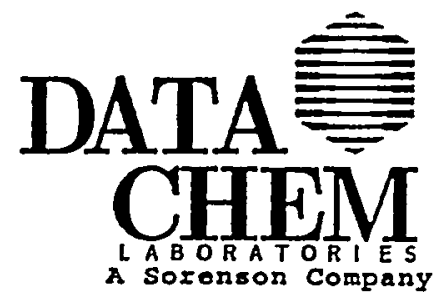

Date Printed.......: 23-JUI-97 09:06

Client Name......... SAIC

client Ref Number.... Not Provided

Sampling site........ Not Provided

Release Number........ Not Provided

Date Received......: 27-JUN-97 00:00

DCL Preparation Group: G9761010

Date Prepared.......:02-JUI-97 00:00

Preparation Hethod...: 418.1

Allquot Weight/Volume: 1.06

Net Welght/Volume.... Hot Requlred
FORY A (TYPE I)

SINGLE MEIHOD ANALYSES

\section{SAMPLE ANALYSIS DATA SHEET}

Form RLIMS63A-V1.3
07239709051984

\section{Page 11}
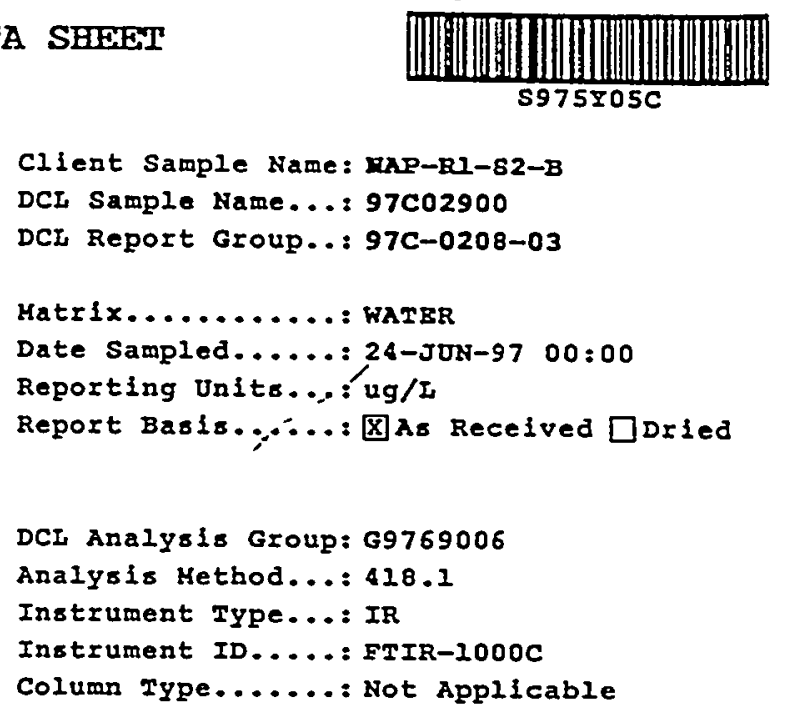

Analytical Results

\begin{tabular}{|c|c|c|c|c|c|c|c|}
\hline Analyte & $\begin{array}{c}\text { Date } \\
\text { Analyzed } \\
\end{array}$ & KDI & Result & Comment & Qual. & Dilution & CRDL \\
\hline TRPH & $09-50 I-97 \quad 00: 00$ & 100 & ND & & & & 100 \\
\hline
\end{tabular}


DATA
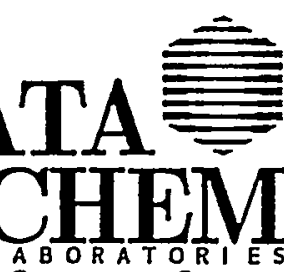

A Sorenson Company

Date P=inted........23-JUL-97 09:06

Cllent Name.......... SAIC

Cl lent Ref Number....: Not Provided

Sampling site......... Not Provided

Release vimber....... : Not Brovided

Date Received.......27-JUN-97 00:00

DCI Preparation Group: G9761010

Date Prepared.......: 02-JUI-97 00:00

Preparation Method...: 428.1

Al lquot Welght/Volume: 1.06

Net Weight/Volume....: Not Reguired
FORM A (TYPE I)

SINGLE METHOD ANALYSES

SAMPLE ANALYSIS DATA SHEET
Form RLIMS63A-V1.3 07239709051984 Page 12

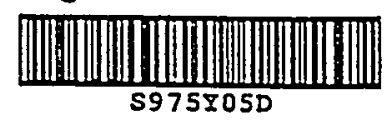

Cllent Sample Name: wnP-Rl-se

DCI Sample Name...: 97C02901

DCI Report Group..: 97C-0208-03

Hatz1x.......... HATER

Date Sampled......25-JON-97 00:00

Reporting Units.... ug/L

Report Basis...... $\$ As Recelved $\square$ Drled

DCL Analysis Group: G9769006

Analysis Hethod...: 418.1

Instrument Type...: IR

Instrument ID.....: FIIR-1000C

Column Type....... Not Applicable

Analytical Results

\begin{tabular}{|c|c|c|c|c|c|c|c|}
\hline Analgte & $\begin{array}{c}\text { Date } \\
\text { Analyzed } \\
\end{array}$ & MDL & Result & Comment & Qual. & Dilution & CRDL \\
\hline TRPH & $09-5 U L-97 \quad 00: 00$ & 100. & ND & & & & 100. \\
\hline
\end{tabular}




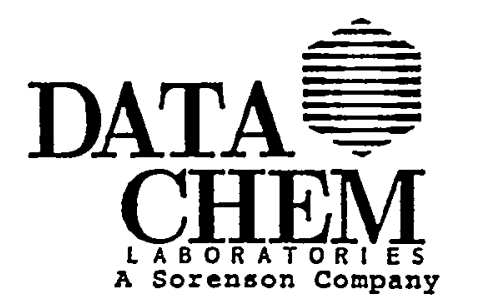

Client Name.......... SAIC

Release Number....... Not Provided

Matrix............ : HATER

Reporting onits....... ug/L
FORM B (TYPE I)

\section{SINGLE METHOD ANATYSES}

QUALITY CONTROL DATA SHEET

ILABORATORY CONTROI SAKPLE (ICS)

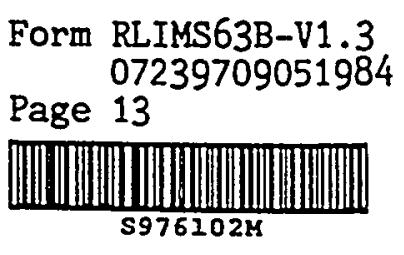

DCL Sample Name...: Q QC-136533-1

Date Printed.....: 23-J0L-97 09:06

DCL Analysis Group: G9769006

Analgsis Hethod... $\$ 18.1$

Instrument Type...: IR

Instrument ID.....: ETIR-1000C

Column Type......: Not Applicable

DCI Preparation Group: G9761010

Date Prepared......: 02-JUL-97 00:00

Preparation Method...: 418.1

QC Iimit Type....: Hethod

Analytical Results

\begin{tabular}{|c|c|c|c|c|c|c|}
\hline Analyte & $\begin{array}{c}\text { Date } \\
\text { Aralyzed }\end{array}$ & Target & Result & $\begin{array}{l}\text { Percent } \\
\text { Recovery }\end{array}$ & $\begin{array}{c}Q C \\
\text { Limits }\end{array}$ & $\begin{array}{l}\text { QC } \\
\text { Fiag }\end{array}$ \\
\hline TRPH & |09-JUL-97 $00: 00$ & 1200 & 1100 & 91.9 & $75.0 / 125$ & \\
\hline
\end{tabular}




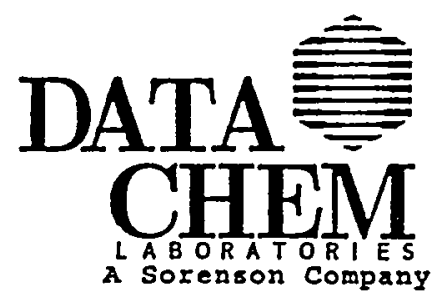

Client Name.......... saIC

Release 2iumber....... Not Provided

Matr1x............. WATER

Reportlng Unlts...... ug/L

DCI Preparation Group: G9761010

Date Prepared.......: 02-JUI-97 00:00

Preparation Method... 418.1

Analytical Results

\begin{tabular}{|c|c|c|c|c|c|c|c|}
\hline Analyte & $\begin{array}{c}\text { Date } \\
\text { Analyzed } \\
\end{array}$ & $\begin{array}{l}\text { Sample } \\
\text { Result }\end{array}$ & $\begin{array}{l}\text { Splked } \\
\text { Result }\end{array}$ & $\begin{array}{l}\text { Splke } \\
\text { Added }\end{array}$ & $\begin{array}{c}\text { Percent } \\
\text { Recovery }\end{array}$ & $\begin{array}{c}\text { QC } \\
\text { Iimits }\end{array}$ & $\begin{array}{c}\text { QC } \\
\text { Elag }\end{array}$ \\
\hline TRPH & $109-J U L-97 \quad 00: 00$ & 60.6 & 1560 & 1670 & 93.2 & $75.0 / 125$ & \\
\hline
\end{tabular}

QUALITY CONTROL DATA SHEET

MATRIX SPIKE SAMPLE

MATRIX SPIKE DUPLICATE SAMPLE
Form RLIMS63F-V1.3 07239709051984

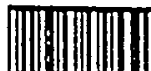

59759057 Page 14

DCI Sample Name...: 97C02897xs

Date Printed..... 23-JUL-97 09:06

DCI Analysis Group: G9769006

Analysis Hethod.... 418.1

Instrument Type... IR

Instrument ID..... ETIR-1000C

Colum Type........ Not Applicable

QC IImit Type..... Method

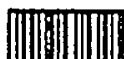

DCI Sample Name. $\therefore$ : 97C02897k8D

Analytical Results

\begin{tabular}{|c|c|c|c|c|c|c|c|c|c|}
\hline Analyte & $\begin{array}{r}\text { Date } \\
\text { Aralyz }\end{array}$ & $\operatorname{sed}$ & $\begin{array}{c}\text { Duplicate } \\
\text { Result }\end{array}$ & $\begin{array}{c}\text { Percent } \\
\text { Recovery }\end{array}$ & Hean. & Range & RPD & $\begin{array}{c}\text { QC } \\
\text { Inits }\end{array}$ & $\begin{array}{l}\text { QC } \\
\text { PIag }\end{array}$ \\
\hline TRPF & 109-JUI-97 & $00: 00$ & 1540 & 92.1 & 1550 & 18.3 & 1.2 & $0.00 / 20.0$ & \\
\hline
\end{tabular}




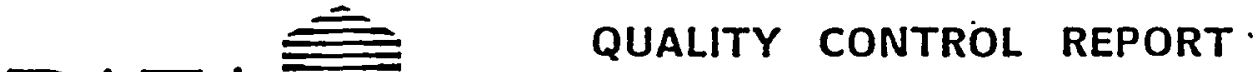

Form RLIMS65-V1.0

Page 1

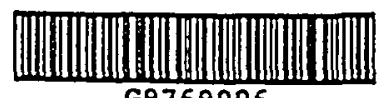

Method... . : 18.1
Analyte... : TRPH
Hatrix... . : WATER

Blank Results

Sample ID Sample Name S976102I BL-136533-1
QC Date 09-JUL-1997 $\frac{\begin{array}{c}\text { Blank } \\ \text { Result }\end{array}}{27.8}$ Lower $\frac{\operatorname{limlt}}{-100}$

\section{LCS Results}

-

Sample ID Sample Name $\overline{\text { S976102M }} \overline{\text { QC-136533-1 }}$
QC Date 09-JUL-1997

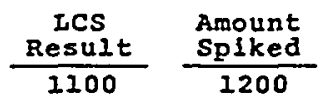

Percent

$\frac{\text { Recovery }}{91.9}$

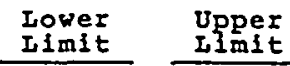
Iimst 125 .

Matrix Spike Results

Sample ID Sample Name QC Date

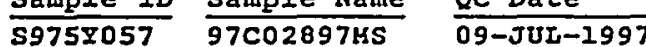

Sample Sample $\frac{\text { Result }}{60.6}$

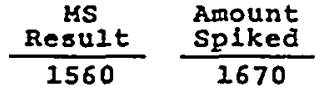

QC Iimit Type... : Hethod

\author{
Date Printed...: 23-JUL-1997 09:04 \\ Group Name........ G9769006 \\ Analyst........ PRTOR \\ Jnits.......... ug $/ \mathrm{I}$
}

Matrix Spike Duplicate Results

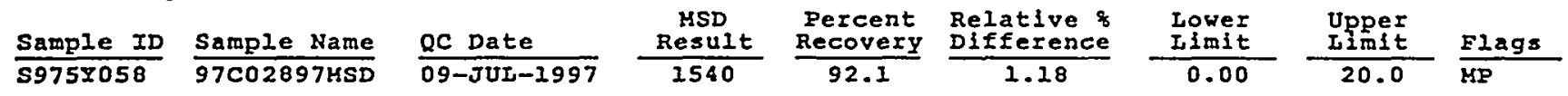

Flag code definitlons (An * before the sample name means the sample failed a limit check.)

m - No Kethod (Absolute) limits found. M - Passed Method (Absolute) 11mits check.

$p$ - No Performance limits found.

5 - No client limits found.

a - Below lower Hethod (Absolute) 1 imit.

$P$ - Passed Performance limits check.

S - Passed client Iimits check.

c - Below lower Control (Performance) limit.

A - Above upper Method (Absolute) 1imit.

C - Above upper Control (Performance) limit.

w - Below lower Warning (Performance) Iimit.

I - Below lower Client Ilmit.

$?$ - Inconsistent Method (Absolute) IImits.

W - Above upper harning (Performance) limit.

$R$ - Above upper Client limit.

1 - Inconsistent Performance I1mits.

- Inconsistent cilent limits.

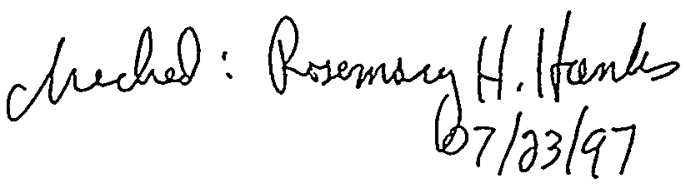




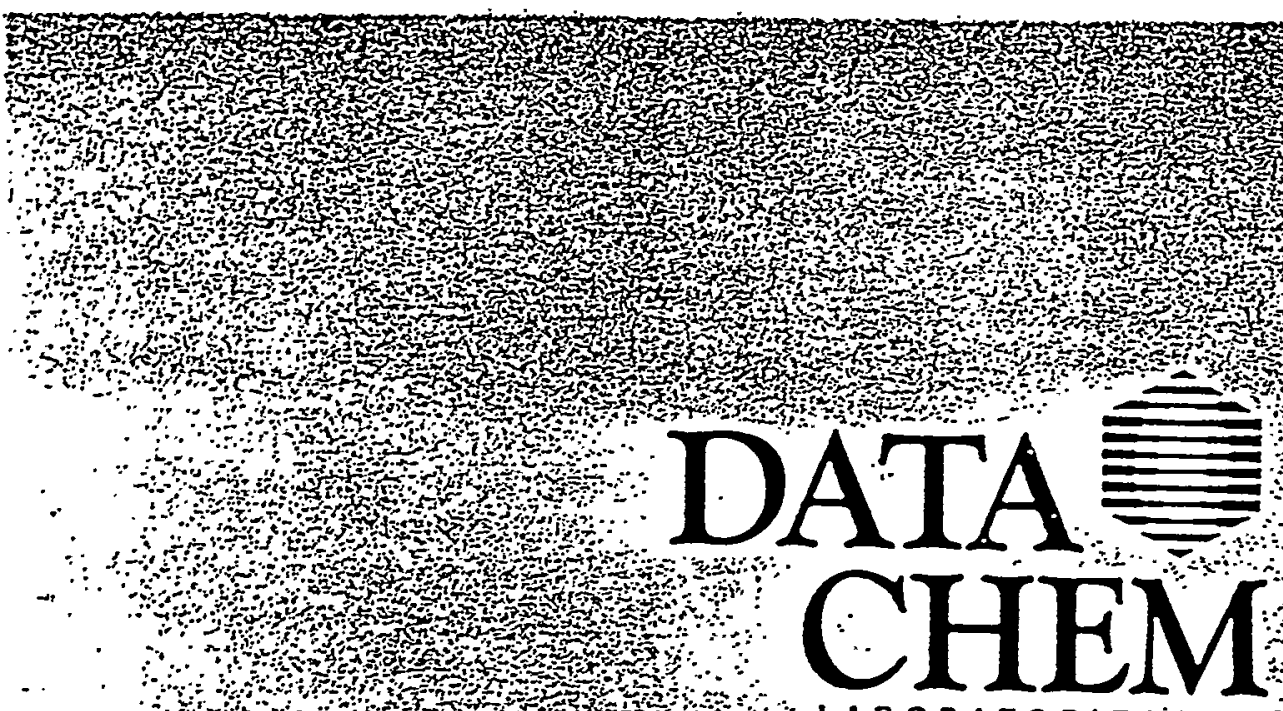

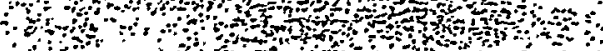

की

of
A SORENSON COMPANY
LABORATORIESIINC.

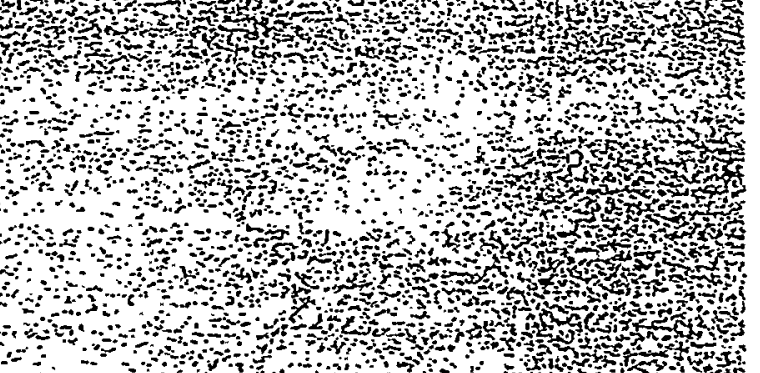

(n)

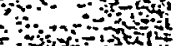
110

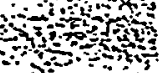
$3 x$ 40 and

\title{
o \\ Sample Tracking Documentation
}

\author{
? \\ and
}

and

$\therefore$ a

40

(n)

(n)

on

why

(a)

$8-1$

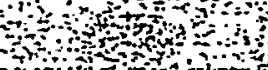

and

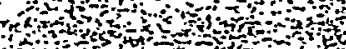

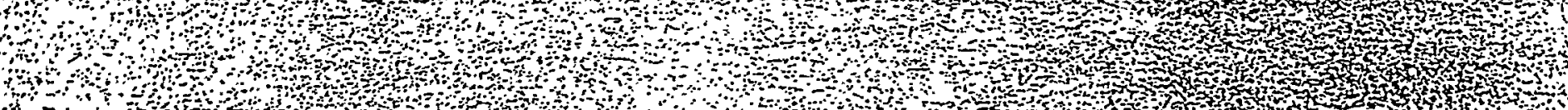

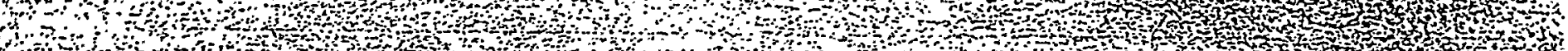

and

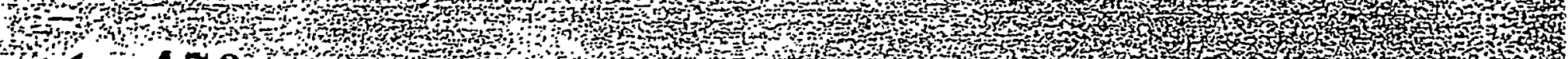

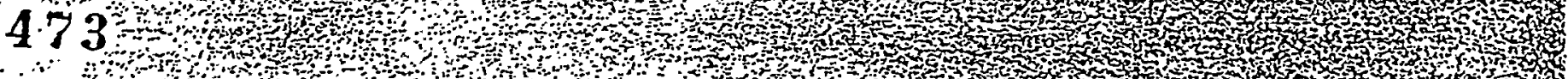

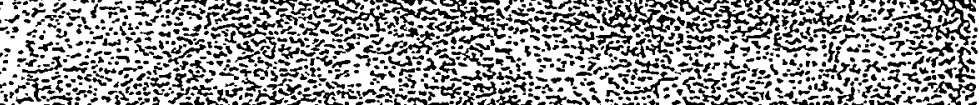

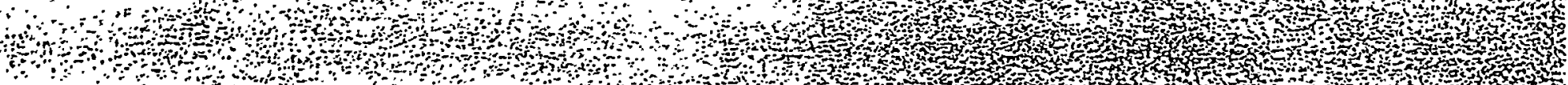

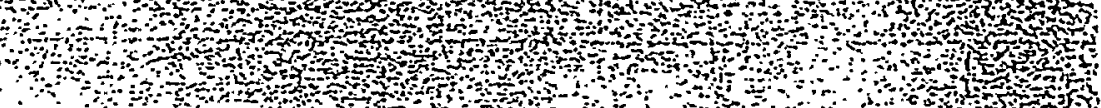


DataChem Laboratories

LIMS - Sample Master System

Analysis Group Report
Date: 10-JUL-1997 09:25

User: PRYOR

Analysis Run Name: G9769006
Page: 1

RLIMS15-V1.2

Group ID: G9769006

Samples: 17

\begin{tabular}{rlll} 
Pos & $\begin{array}{l}\text { Laboratory } \\
\text { Sample Name }\end{array}$ & $\begin{array}{l}\text { Field Sample } \\
\text { Name 1 }\end{array}$ & $\begin{array}{l}\text { Field Sample } \\
\text { Name 2 }\end{array}$ \\
\hline 1 & BL-136533-1 & BL-136533-1 & \\
2 & QC-136533-1 & QC-136533-1 & \\
3 & $97 C 02896$ & NAP-R2-S2-A & \\
4 & $97 C 02897$ & NAP-R2-S2-B & \\
5 & $97 C 02897 M S$ & NAP-R2-S2-B & \\
6 & $97 C 02897 M S D$ & NAP-R2-S2-B & \\
7 & $97 C 02898$ & NAP-R2-S4 & \\
8 & $97 C 02899$ & NAP-R1-S2-A \\
9 & $97 C 02900$ & NAP-R1-S2-B \\
10 & $97 C 02901$ & NAP-R1-S4 & \\
11 & BL-136534-1 & BL-136534-1 \\
12 & QC-136534-1 & QC-136534-1 \\
13 & $97 C 02902$ & NAP-R1-S6 & \\
14 & $97 C 02903$ & NAP-R2-S6 & \\
15 & $97 C 02908$ & NAPR2S6BLANK & \\
16 & $97 C 02908 M S$ & NAPR2S6LCS & \\
17 & $97 C 02908 M S D$ & NAPR2S6LCSD
\end{tabular}

Laboratory
Sample ID

-

S976102L

S976102M

S975Y055

S975Y056

S975Y057

S975Y058

S975Y059

S975Y05B

S975Y05C

S975Y05D

S976102T

S976102V

S975Y05L

S975Y05M

S975Y05N

S975Y05P

S975Y05Q
Laboratory.

Group Name

97C-0208-03

97C-0208-03

97C-0208-03

97C-0208-03

97C-0208-03

97C-0208-03

97C-0208-03

97C-0208-03

97C-0208-03

97C-0208-03

97C-0208-04

97C-0208-04

97C-0208-04

97C-0208-04

97C-0208-04

97C-0208-04

97C-0208-04
Accnt. Number

03006

03006

03006

03006

03006

03006

03006

03006

03006

03006

03006

03006

03006

03006

03006

03006

03006

END OE LISTING 
DataChem Laboratories

LIMS - Sample Master System

Preparation Group Report
Date: 2-JUL-1997 15:42

User: PRYOR

Preparation Run Name: G9761010
Page: 1

RLIMS15-V1.2

Group ID: G9761010

Samples: 10

\begin{tabular}{rl} 
Pos & $\begin{array}{l}\text { Laboratory } \\
\text { Sample Name }\end{array}$ \\
\hline 1 & BL-136533-1 \\
2 & QC-136533-1 \\
3 & 97 C02896 \\
4 & $97 \mathrm{CO2897}$ \\
5 & $97 \mathrm{CO2897 \textrm {MS }}$ \\
6 & $97 \mathrm{C02897 \textrm {MSD }}$ \\
7 & $97 \mathrm{C02898}$ \\
8 & $97 \mathrm{C02899}$ \\
9 & $97 \mathrm{C} 02900$ \\
10 & $97 \mathrm{C} 02901$
\end{tabular}

END OF LISTING
Field Sample

Name 1

BL-136533-1

QC-136533-1

NAP-R2-S2-A

NAP-R2-S2-B

NAP-R2-S2-B

NAP-R2-S2-B

NAP-R2-S4

NAP-R1-S2-A

NAP-R1-S2-B

NAP-R1-S4
Field Sample Name 2

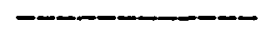

Laboratory Sample ID

S976102L

S976102M

S975Y055

S975Y056

S975Y057

S975Y058

S975Y059

S975Y05B

S975Y05C

S975Y05D
Laboratory Group Name

97C $\div 00208-03$

97C-0208-03

97C-0208-03

97C-0208-03

97C-0208-03

97C-0208-03

97C-0208-03

97C-0208-03

97C-0208-03

97C-0208-03
Accnt.

Number

03006

03006

03006

03006

03006

03006

03006

03006

03006

03006 


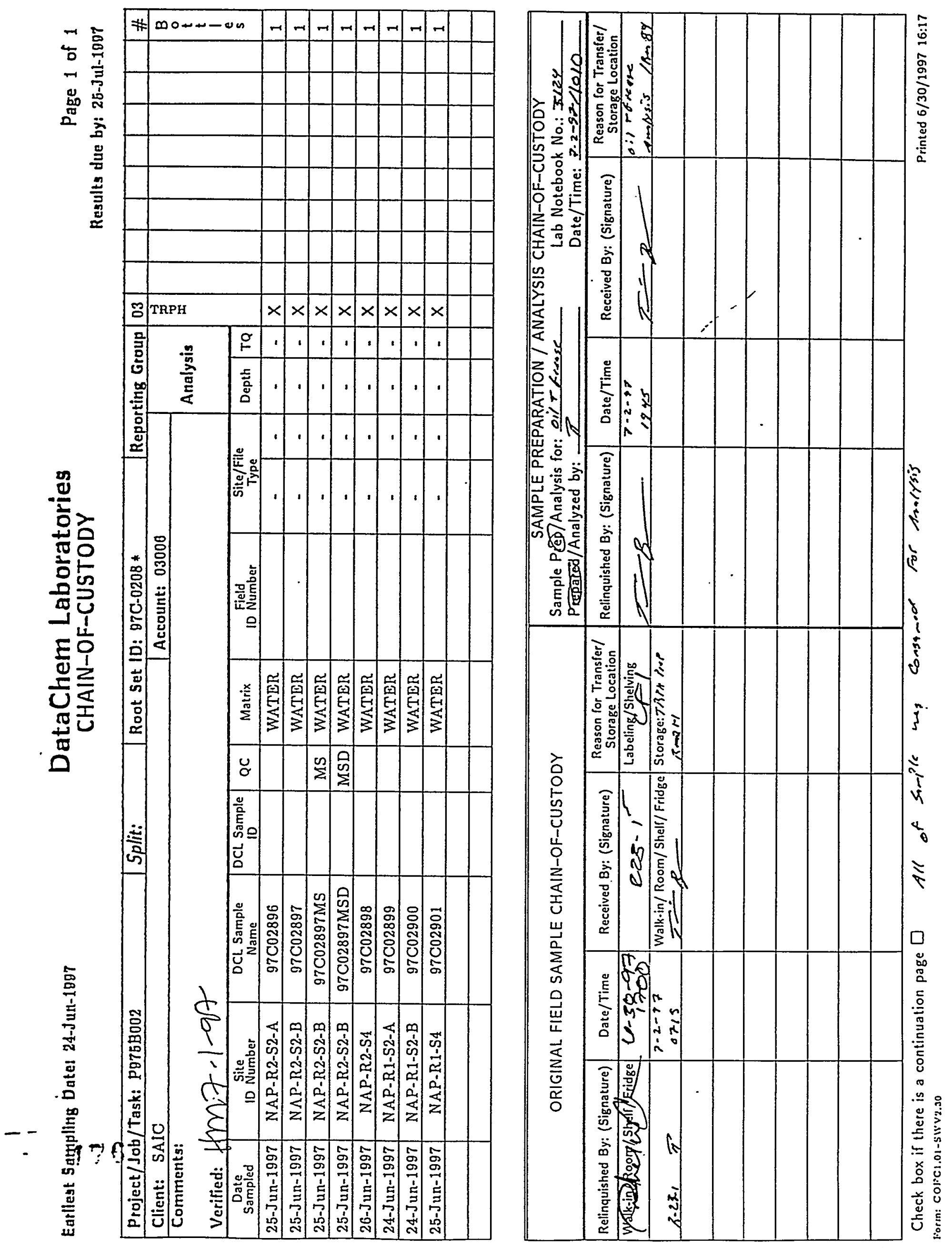


QC Clearance:

Project Manager: Carol M. Bell

Client: SAIC

Account: 03006

Project/Task: P975B002

Date Received: 27-Jun-1997

Date for Mailing Report: 25-Jul-1897

Date for Verbal Report: 25-Jul-1897

\begin{tabular}{|c|c|c|c|c|c|c|c|c|}
\hline $\begin{array}{l}\text { Rep. } \\
\text { Group }\end{array}$ & $\begin{array}{l}\text { Zl Section } \\
\text { Analytes Requested }\end{array}$ & $\begin{array}{c}\text { Latest } \\
\text { Prep. Date }\end{array}$ & $\begin{array}{c}\text { Latest } \\
\text { Anal. Date }\end{array}$ & $\begin{array}{l}\text { No. of } \\
\text { Samp. }\end{array}$ & $\begin{array}{l}\text { Storage } \\
\text { Location }\end{array}$ & $\begin{array}{l}\text { Analysis/Prep. } \\
\text { - Method }\end{array}$ & Inst. & Matrix \\
\hline 03 & TRPH & & 23-Jut-1997c & 8 & & 418.1 & IR & WATER \\
\hline 04 & TRPH & & 23-Jut-1997 & 5 & & 418.1 & IR & WIPE \\
\hline
\end{tabular}

${ }^{\text {Based on date of collection }}$

\section{Special Instructions:}

Section Manager: Brett G. Lee

\section{Sample Work Order}

DCL Root Set ID: 97C-0208*

DCL Lab. Name: 97C02896-97C02908MSD

Total \# Samples: 13

Somple Entry: Patrick W. Corum

Section: ZI

Earliest Sampling Date: 24-Jun-1997

Preparation Type: 
$\because \because$
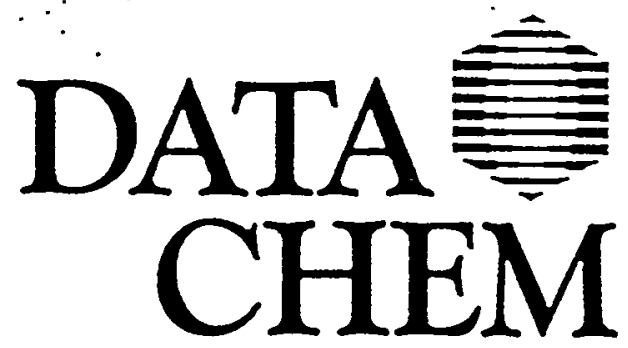

LABORATORIESI INC.

A SORENSON COMPANY

\section{Analytical Documentation}




\section{Analysis Record Page}

Group \#: 69769006

LOT \#: in

Preparation Date: $\quad>-q-9 z$

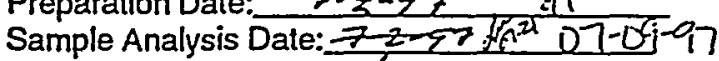

Method\#: 418.1 Mod

Matrix: wipes / warr

DCL I.D.

\begin{tabular}{|c|}
\hline $976-02+8-04$ \\
\hline $97 c-0205-0 s$ \\
\hline
\end{tabular}

DCL SAMPLE \#

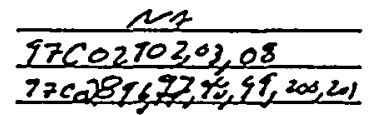

MDL: $\frac{100 \text { rege } / L}{100^{2}}$

Instrument iD: FTIR-1000C

- Samples were analyzed by method 418.0 Analysis procedure can be found on

page 31 of logbook $2084 \quad 2-07.22-974$

\section{ACCOUNT}

$\frac{\frac{N}{3006}}{206}$

- Sample results can be found in the datapack.

Reagents:

Freon 113 B.ker Lot $T 22217$

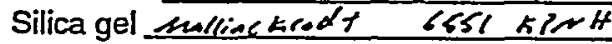

- Primary stock solution can be found on page 13 of logbook 2017.

$$
\text { IR-QS-PAF 6-16-97 IR-S-TP 6-16-97 }
$$

- Intermediate stock solution can be found on page 26 of logbook 2019.
IR-QI-LMR 6-24-97
IR-I-TP $6-24-97$
IR-QC-LMR 6-24-97
IR-ISP-TP 6-24-97

-Working standard solution's can be found on page 2 and $y, 5$ of logbook 3131.

IR-WS-TP $\quad$-2-24-97

$$
4.4265 \text { (2) } 2.2133 \mathrm{mg} \quad 0.8853 \mathrm{mg} \quad 0.4427 \mathrm{mg}
$$

$\begin{array}{lll}\text { (1) } \mathrm{CCB} & \text { (2) } \mathrm{CCV}\end{array}$

IR-ICV- $2 \sin \quad 1-24-47 \quad 1.2003 \mathrm{mg}$ (ICV)

- QC preparation can be found on page 50 and $s \leqslant$ of logbook 3131.

Comments: $\frac{\text { Qc }-136533-1}{0 .-13634-1}$

. $\frac{\text { ac-136533-1 }}{\text { se-136534-1 }}$

Comments: $\frac{2 c-136533-1}{Q_{2}-13654-1}$

$-$

\section{9}

To Page No.

Witnessed \& Understood by me,

Posemaygh Hento

07122197

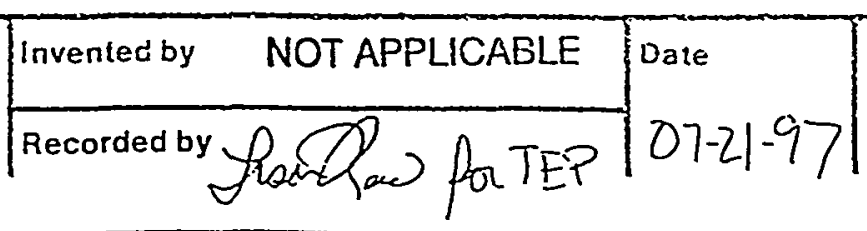


TITLE_ZRPH . Wipes

Project No.

Book No3124
DATA

CHEAM

From Page No.=

Setip: 9zc-0208-04

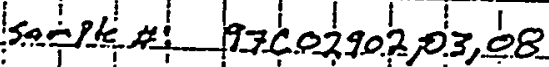

ifrokP 2P: G5ZE1011
Prep pate: $7-2=97$

Noteix: zipes

Acecurt 3006

Mrizdi ! 4to: mod

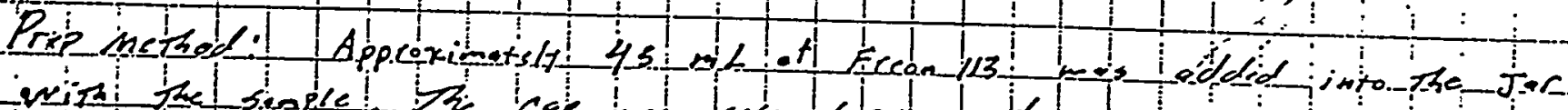

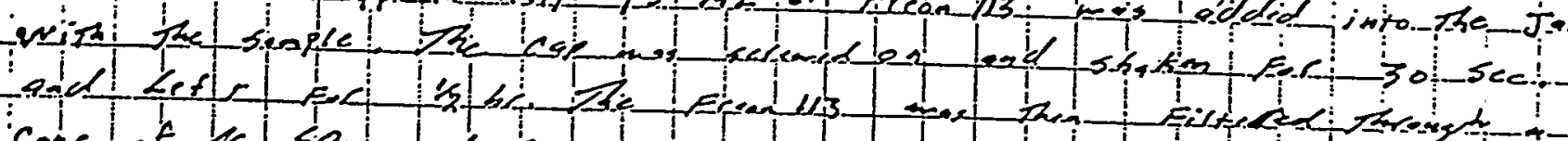

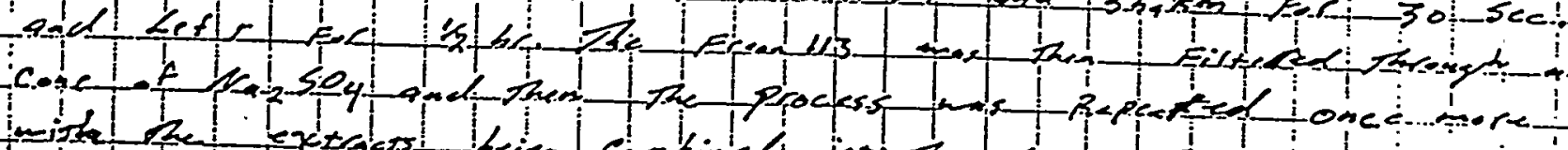

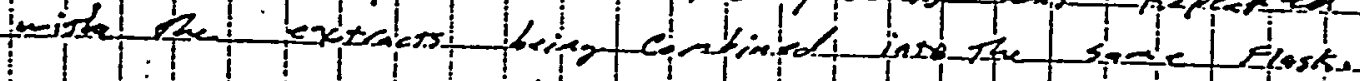

\section{$1+\frac{1}{1} 1+1$

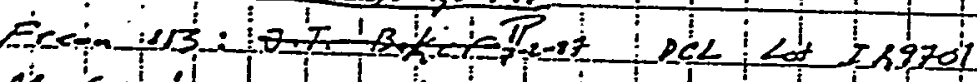

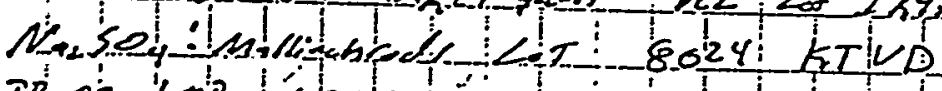

IR-a.c-2nR $6=24-87$

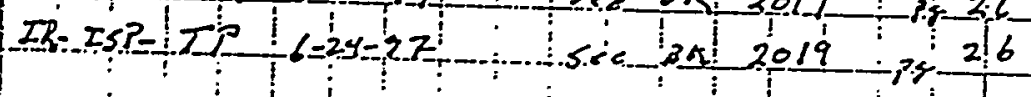

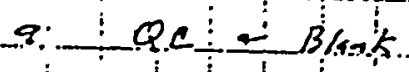

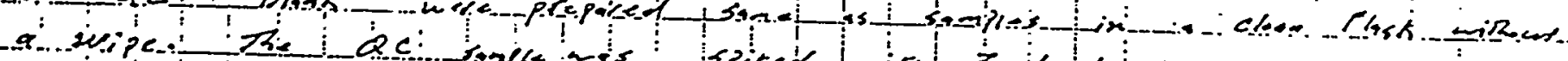

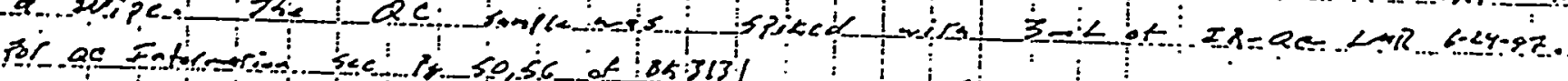

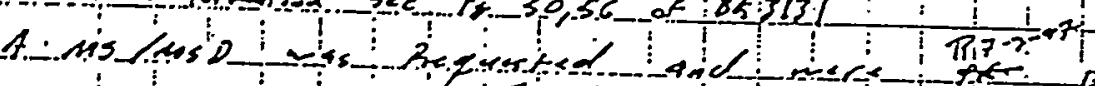

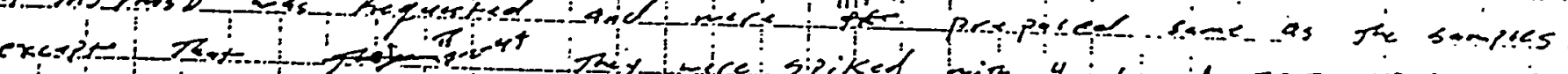

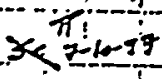

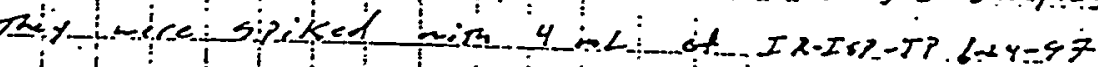

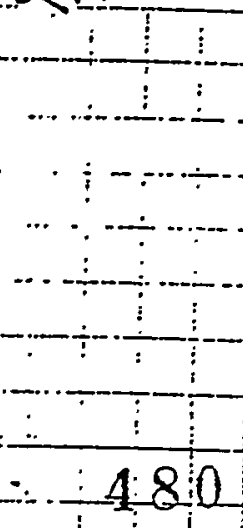

Witnessed \& Understoog byne,

fropere

Date
$07-2$

$+$ 
From Page No.:! : $\quad$ TP $7-2-97$

Water prep

Set ID: $9 \dot{7} c-0208-03$

Sample \#'s: $97602856-701$

Lot/Group ID: $\$ 9761010$

i Method: 418.1

- Matrix:2water

Account \#: 3006

Prep. Date: $z-2-97$

STANDARDS AND REAGENTS

$\mathrm{Na}_{2} \mathrm{SO}_{4}$ :Medlinckreds Lr 8024 KTVD

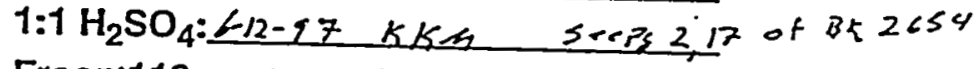

Freon'113:DCL Let $\bar{Z} 27701$

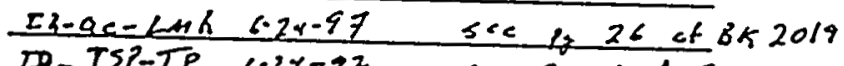

IR-IST-TP $6.21-97$ Sore $P_{2} 26$ of B +2017

see is so,56 of $B A 3131$ for aC Infurustion

\begin{tabular}{|c|c|c|}
\hline Sample ID & $\mathrm{pH}$ & Vol. (L) \\
\hline$\therefore P L-13 \leq 533-1$ & $x / 4$ & 1,00 \\
\hline$\therefore Q_{C=136533-1}$ & $M$ & 1.00 \\
\hline$\frac{9.7602896}{97 \cos 89 j}$ & $<2$ & l.o6. \\
\hline 9.7602897 & $\leqslant 2$ & 1.06 \\
\hline . $.7 \mathrm{Co} 2897^{\mathrm{m}} \mathrm{ss}$ & $<2$ & 1.06 \\
\hline - I7Co2897 ASD. & $<2$ & 1.0 .6 \\
\hline S7cole98 & $<2$ & 1.06 \\
\hline$\frac{77 \cos 2899}{.77 \cos 300}$ & -12 & 1.06 \\
\hline 97002901 & $\begin{array}{l}<2 \\
<2\end{array}$ & $\begin{array}{l}1.06 \\
1.06\end{array}$ \\
\hline
\end{tabular}

481

$$
\text { - }
$$

.. $\quad \cdots+\cdots,-\cdots$

Witnessed \& Understood by one,

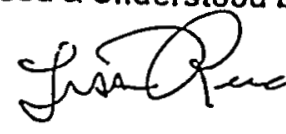


TITLE TPH \& Oil and Gneose in $\mathrm{H}_{2} \mathrm{O}$ by IR

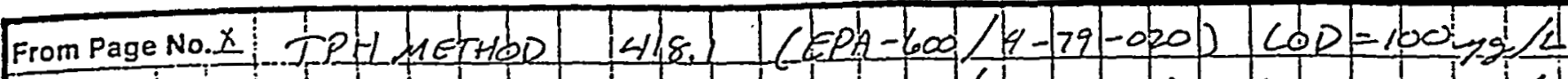

\section{WATER EXTRACTIan}

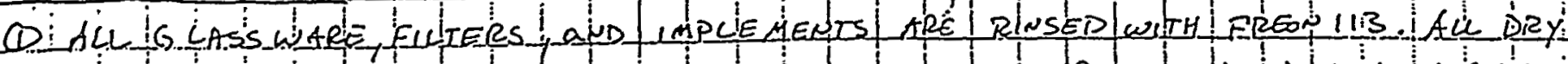

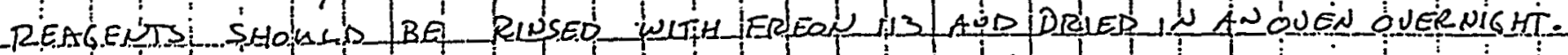

(2) USE A PIECE OA IAPE AND AN WUK MARKE TO MARK EASH SAMBL BOTLEATI

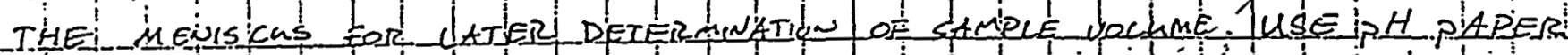
TO ENSURE THAT SAMPLES WERE PRESERED TO PH 2 ar LESS

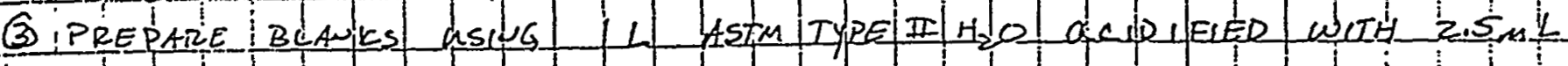

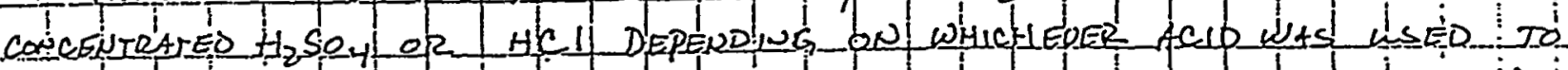

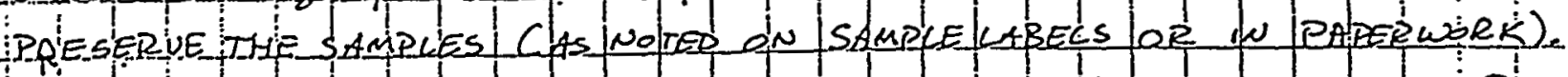

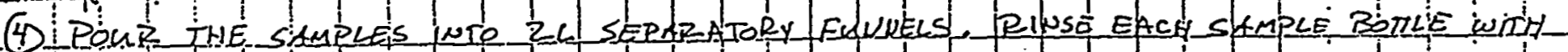

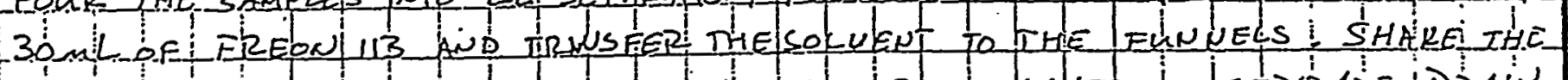

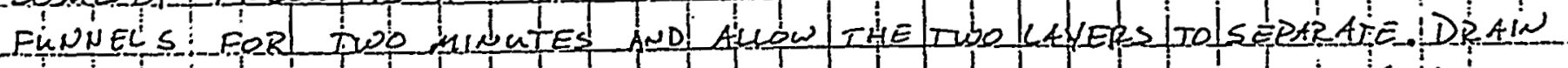

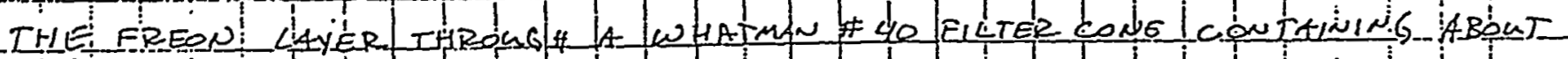

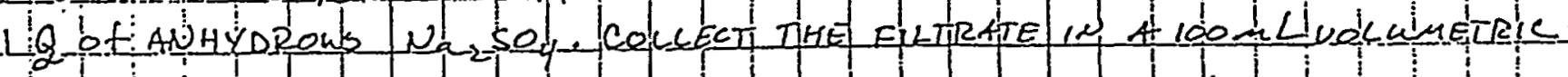
ints

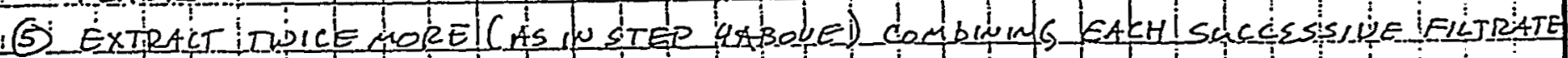
NTHE EEASK

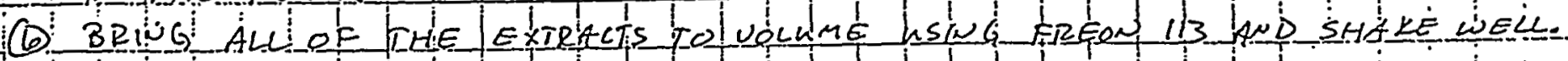

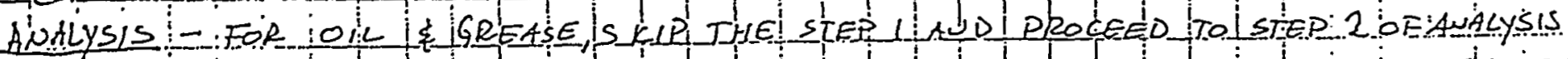

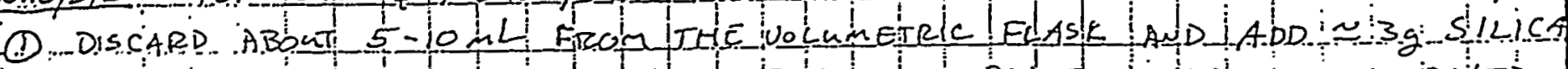
GEL AND A STIRIBAR STOPPER IHE FLASK AND PLACEON MAGUETIC STR PLTTE AND STR EOR SEUEN MNUTES ALLOW TO SETLE BEEORE AUAYYSIS

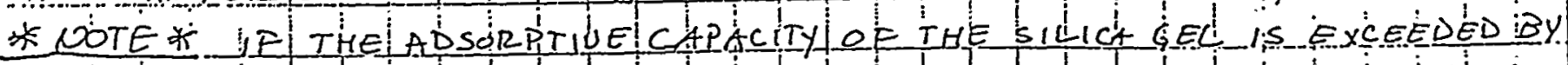

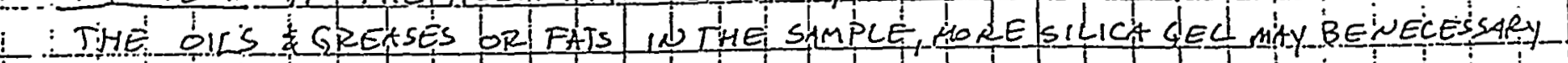

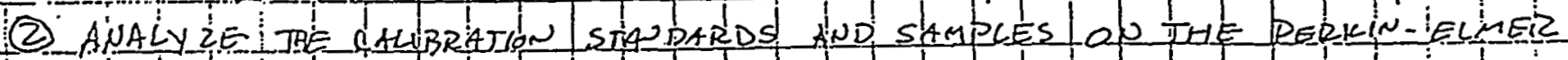

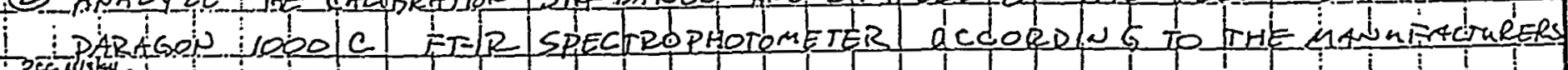

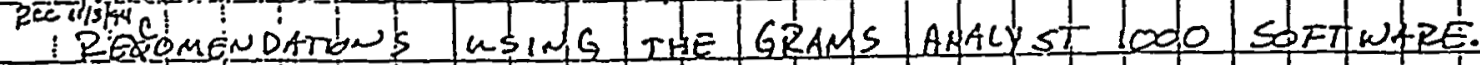

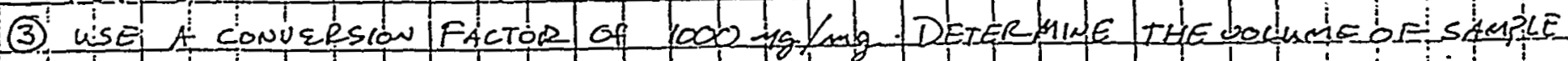
AND USE, IN N THELEINAL CALCL ULATON.

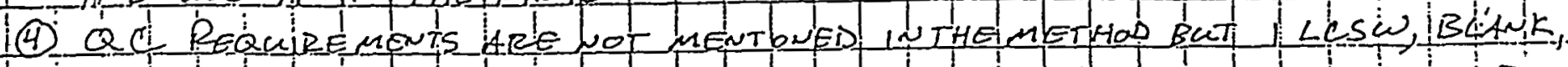
DUPLICATE AUD SPIKE IPER zO SAMPLES OR BIKTCH IS RECOMAEDED HF

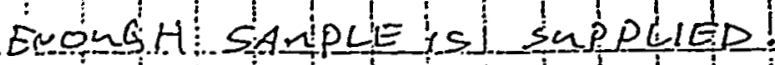

482

Witnessed \& yzdeygtood by me,

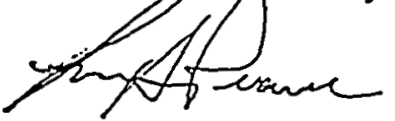

Date

$11-03-q 4$ Recorded by

fadd ell 
Book No.2579

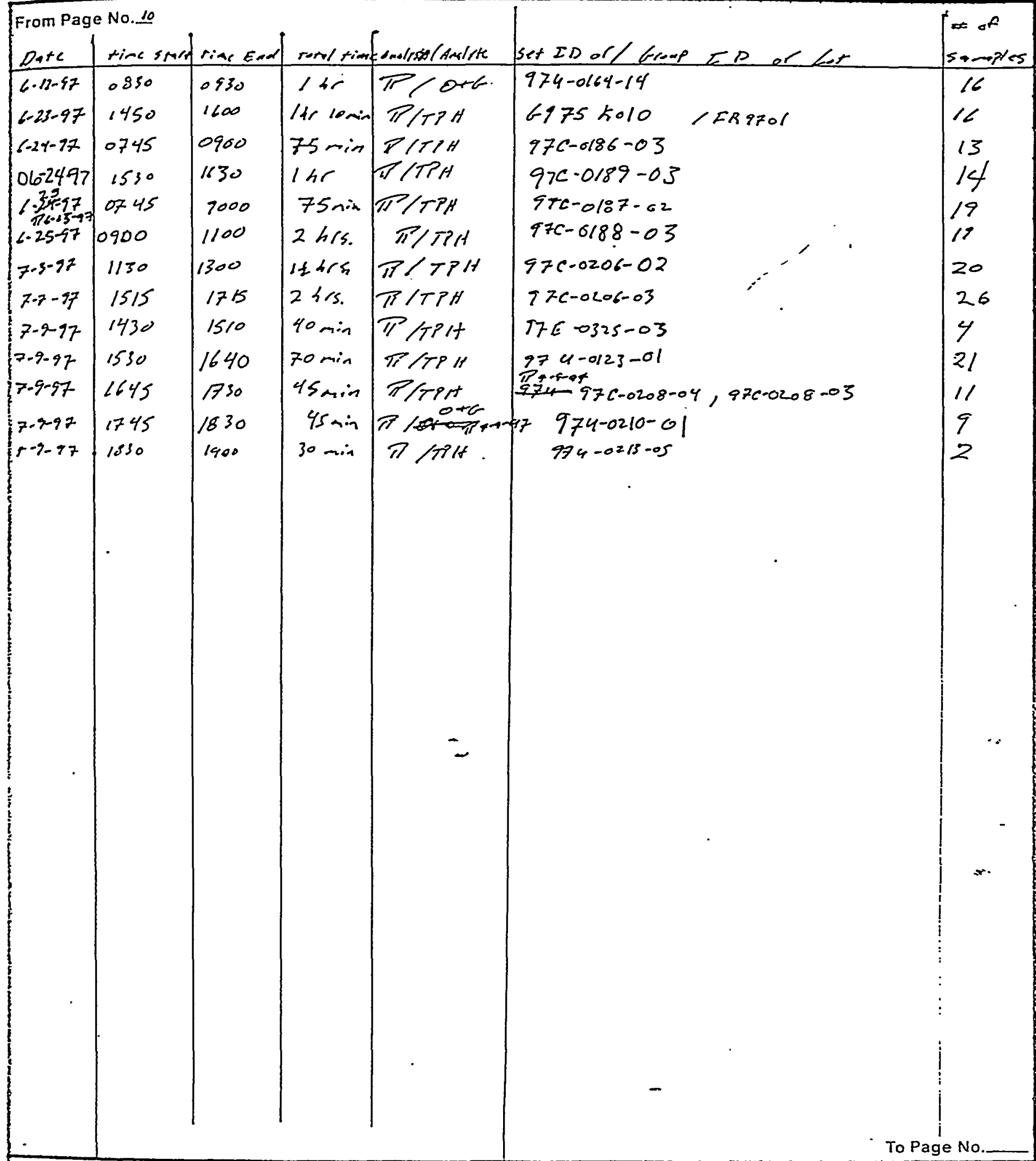

Witnessed \& Understood by me, Date

\begin{tabular}{|l|l|}
\hline Invehted by & Date \\
\hline Recorded by &
\end{tabular}



TITLE

From Page No. $=$

Working standards are prepared by rinsing seven $100 \mathrm{~mL}$ volumetric flasks with Ereon 113. The first 3 standards are made Irom the intermediate spiking solution. Use glass, class A, pipets and transfer $10 \mathrm{~mL}, 5 \mathrm{~mL}$, and $2 \mathrm{~mL}$ to 3 different volumetric flask and dilute to volume with freon 113 . The'se are $10 x, 20 x$, and a $50 x$ dilution of the intermediate spiking solution.

The next three standards are made from the $10 \mathrm{x}$ dilution just made. Transfer $10 \mathrm{~mL}, 5 \mathrm{~mL}$, and $2 \mathrm{~mL}$ to 3 different volumetric flask and dilute to volume with freon 113. These are 10x, 20x, and a $50 x$ dilution of the $10 x$ dilution. The last standard is a blank which is $100 \mathrm{~mL}$ of freon 113 .

The ICV is prepared by adding $3 \mathrm{~mL}$ intermediate QC spiking solution to a $100 \mathrm{~mL}$ volumetric flask and diluted to volume with freon 113 .

Labeling of solutions will be as follows:

$10 \mathrm{x}$

$20 \mathrm{x}$

$50 \mathrm{x}$

$100 \mathrm{x}$

$200 \mathrm{x}$

$500 \mathrm{x}$

Blank

ICV
IR-WS-(INITIAIS), Date, Concentration

..IR-WS-(INITIAIS), Date, Concentration

IR-WS-(INITIAIS), Date, Concentration

IR-WS-(INITIALS), Date, Concentration

IR-WS-(INITIAIS), Date, Concentration

IR-WS-(INITIALS), Date, Concentration

IR-WS-(INITIALS), Date, Concentration

IR-ICV-(INITIAIS), Date, Concentration

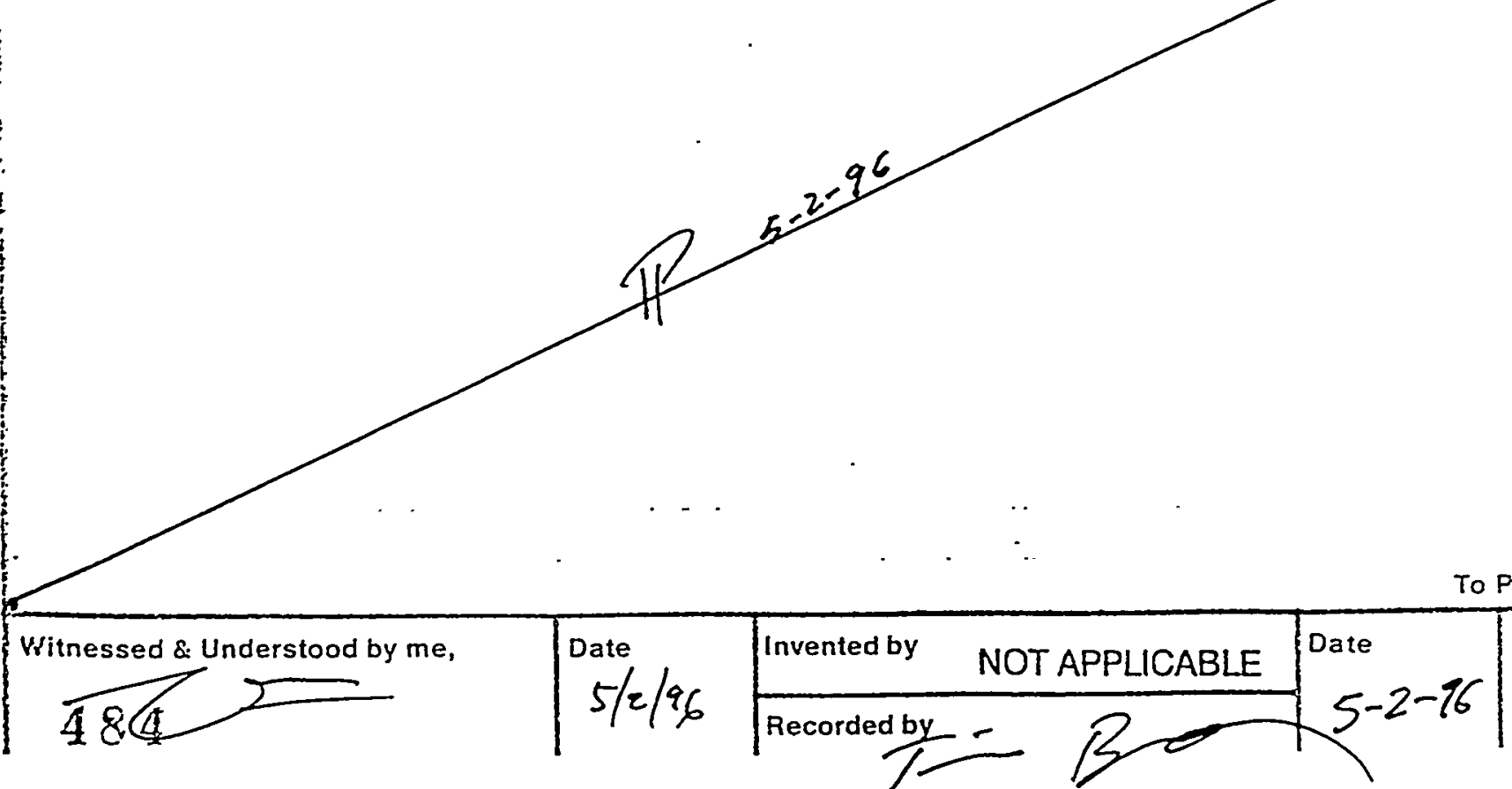




\begin{tabular}{|c|c|c|c|}
\hline $\begin{array}{l}\text { From Page N } \\
\text { Date } \\
\text { Enitials }\end{array}$ & Freon $1 / 3$ Lot & Entrernediate standyed & rencentratioes \\
\hline Po 7 & $\begin{array}{l}\text { 34xter Lot } \\
532267\end{array}$ & $\begin{array}{l}I A . \cdots Q C-K L H \\
8-21-96\end{array}$ & $1.3395 \mathbb{3}^{2+17+46} \mathrm{mg}$ \\
\hline $10.26-96$ & $\begin{array}{l}\text { Brxter Lot } \\
522267\end{array}$ & $\begin{array}{r}I R-I S P-T P \\
10-25-96 \\
\end{array}$ & 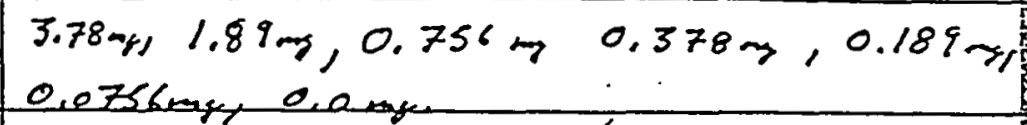 \\
\hline Ma-26-76 & $\begin{array}{l}5 x+20+\angle 2 \\
52267\end{array}$ & 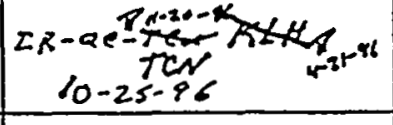 & 1.2136 .5 mg $\left(C^{\prime} C D\right)$ \\
\hline $\begin{array}{c}12-5-52 \\
\pi P\end{array}$ & 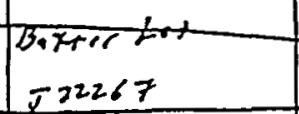 & $\begin{array}{c}5-02-x c x \\
10-25-96\end{array}$ & $1.21365 \pi 12-5-91$ \\
\hline $12-89-96$ & $\begin{array}{l}\text { J.T. B.x4\% Lat } \\
J_{22267}\end{array}$ & 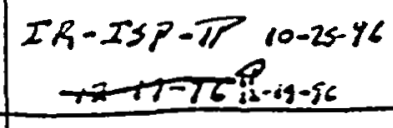 & $\begin{array}{l}3.78 \mathrm{mp} \quad 1.89 \mathrm{mg} 0.756 \mathrm{mg} 0.378 \mathrm{r} \\
0.189 \mathrm{mo} \quad 0.0756 \mathrm{~m} 0.0 \mathrm{mt}\end{array}$ \\
\hline $\begin{array}{c}12-19-96 \\
C A\end{array}$ & $\begin{array}{l}\text { J.T. Baxter Lt } \\
\text { T.22Z6Z }\end{array}$ & $\begin{array}{l}I R-a c-T C N \\
D-25-46 \\
\end{array}$ & $1.21365 \mathrm{mg}$ \\
\hline $\mathbb{P}^{22-2 x-46}$ & $\begin{array}{l}\text { J. } 5.8 \text { stier Lt } \\
532267\end{array}$ & 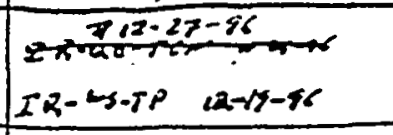 & $0.375 \mathrm{~ms} \quad-0.185 \mathrm{mg}$ \\
\hline $\begin{array}{c}2.28-97 \\
0\end{array}$ & $\begin{array}{l}J_{1} T_{1} \text { Baker bot } \\
J 2226 z\end{array}$ & $\begin{array}{r}I R \cdot Q C-T C N \\
16-25-96 \\
\end{array}$ & $-1.21365 \mathrm{mg}$ (ICV) \\
\hline $\begin{array}{l}2-23-47 \\
2\end{array}$ & $\begin{array}{l}\text { J. T. B.ker Lot } \\
\text { J3ग267 }\end{array}$ & $\begin{array}{l}I X-レ S-T P \\
10-45-96 \\
\end{array}$ & $\begin{array}{ccc}3.78 \mathrm{r} & 1.89 \mathrm{rg} \quad 0.756 \gamma & 0.378 \mathrm{~m} \\
0.189 \mathrm{rg} & 0.0756 \% & 0.074\end{array}$ \\
\hline$\stackrel{5-13-87}{17}$ & $\begin{array}{l}\text { J. T. B,tor Lot } \\
\text { J22267 }\end{array}$ & $\begin{array}{l}28 \cdot Q C-T C N \\
1025-96\end{array}$ & $1.21365 \mathrm{mg} \quad(I C N)$ \\
\hline $\begin{array}{c}5-13-17 \\
\sin B\end{array}$ & $\begin{array}{l}J .7 .8,50 r \text { Lot } \\
I_{22263}\end{array}$ & $\begin{array}{l}I R-\sum^{T S}-77^{5-22-47} \\
5-5-97\end{array}$ & 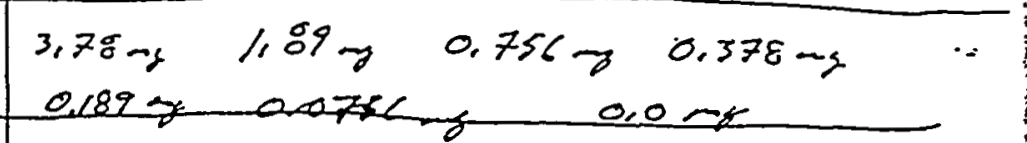 \\
\hline$\frac{5-13-57}{28}$ & $\begin{array}{l}\text { T. T. B.her Let } \\
522267\end{array}$ & $\begin{array}{l}{[B-Q C-T P} \\
5-1 S-I Z\end{array}$ & $1.21365 \mathrm{mg}$ Ic \\
\hline $\begin{array}{l}6-10-17 \\
\end{array}$ & 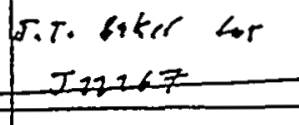 & Th-ac- & $T_{6-12-17}$ \\
\hline$\frac{6.33 .97}{11}$ & 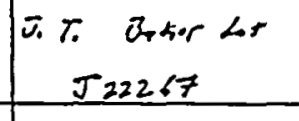 & $\begin{array}{l}2 x-2 \leq p^{-17} \\
6-16-97\end{array}$ & 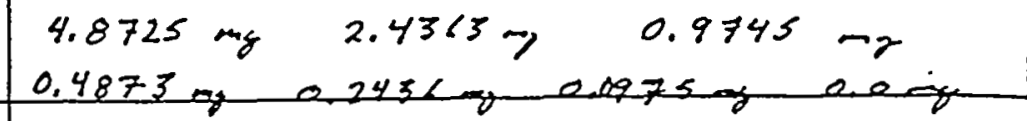 \\
\hline $\begin{array}{l}6-23-57 \\
4.25858\end{array}$ & $\begin{array}{l}\text { J. T. Sather Lot } \\
\text { J } 222<7\end{array}$ & $\begin{array}{l}I h-Q C-C A \\
6 \cdot 16-97 \\
\end{array}$ & $1.1785 \mathrm{mg}$ \\
\hline $\begin{array}{l}06-24-97 \\
\quad \text { hen } \\
\end{array}$ & $\begin{array}{l}\text { J.T.Baker Lot } \\
\text { J22267 }\end{array}$ & $\begin{array}{r}I R-Q C-C A \\
06-11=-97\end{array}$ & $1.1985 \mathrm{mg}$ (ICD) \\
\hline$\prod_{I P}^{4-24-97}$ & $\begin{array}{l}\text { DCL } \angle A T \\
\text { ZRT7OI }\end{array}$ & $\begin{array}{c}I R-I S P-T P \\
6.24-17\end{array}$ & 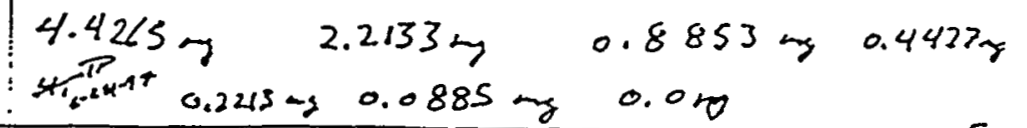 \\
\hline & & & $i \quad$ lo Pageno. 5 \\
\hline \multicolumn{2}{|c|}{ Witnessed \& Understood by me, } & $06 / 24 / 97$ & vented by NOT APPLUCABIE \\
\hline
\end{tabular}


1

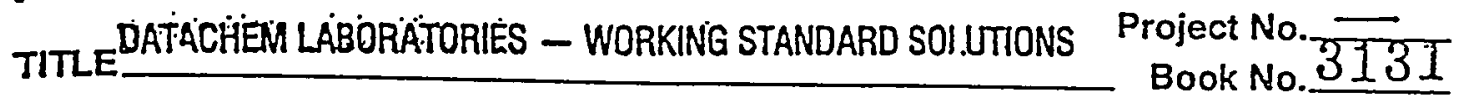

5

From Page No. $f$

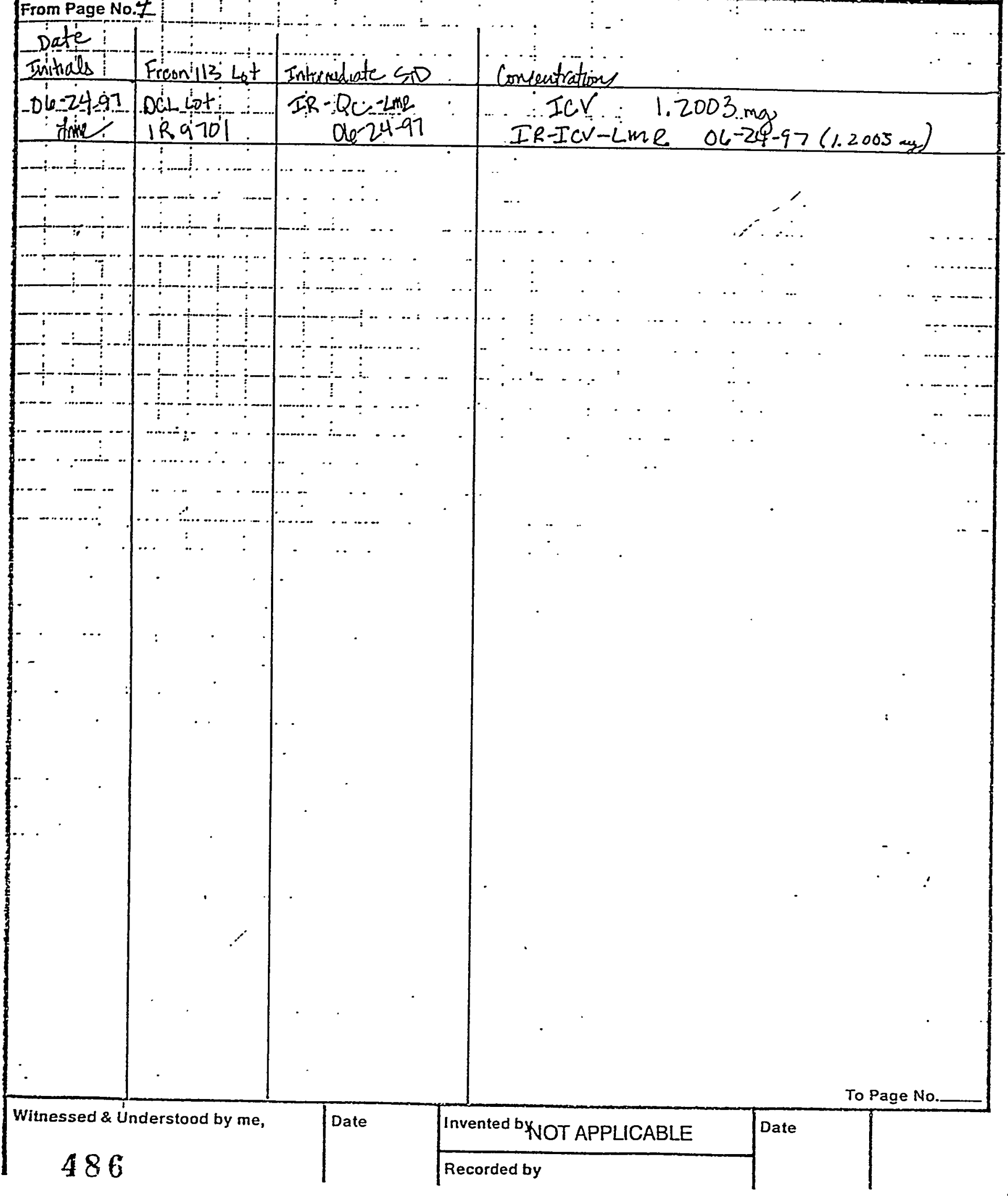

Wm 


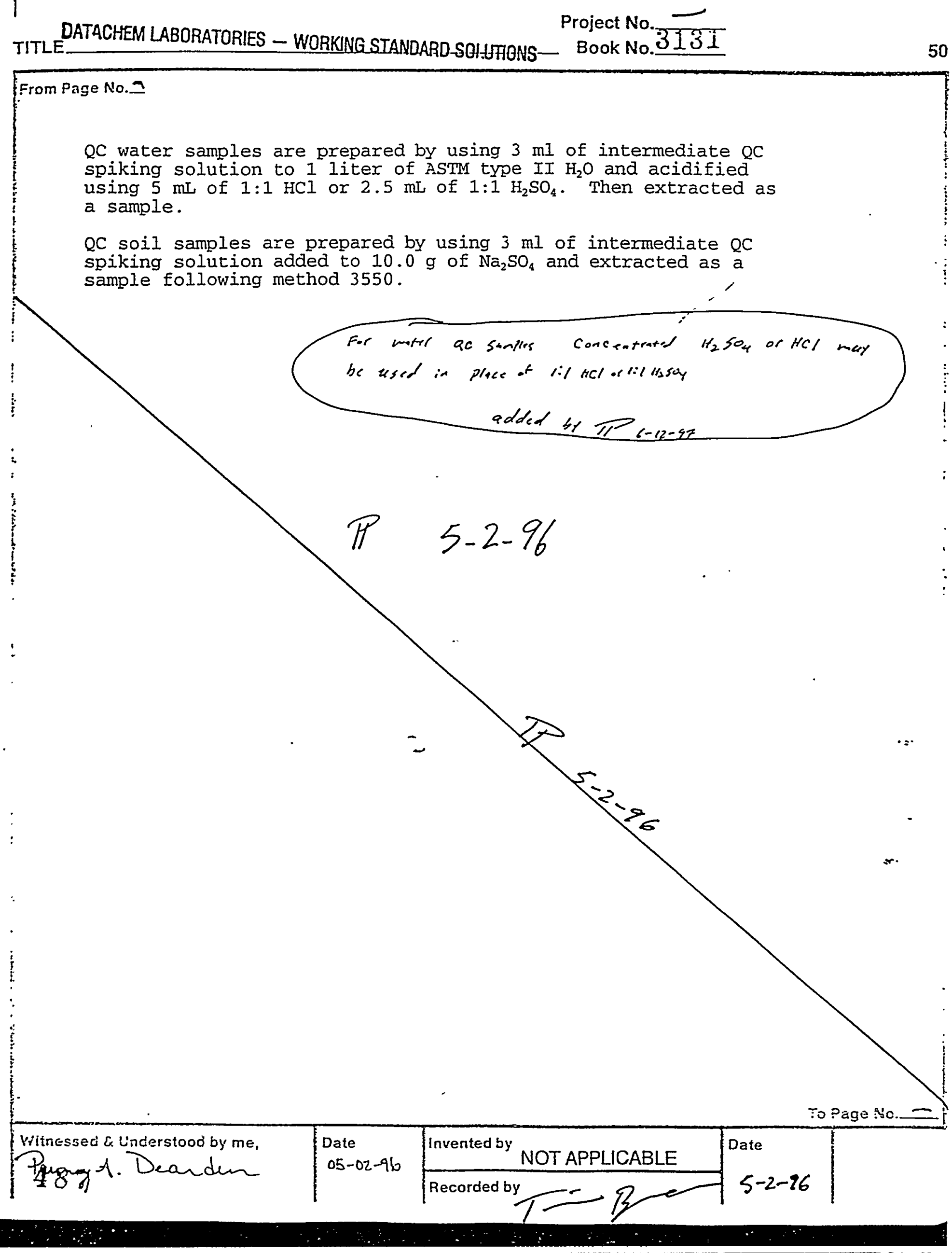


TITLE-DATACHEM LABORATORIES - WORKNG STANDARDSOIUIIONS

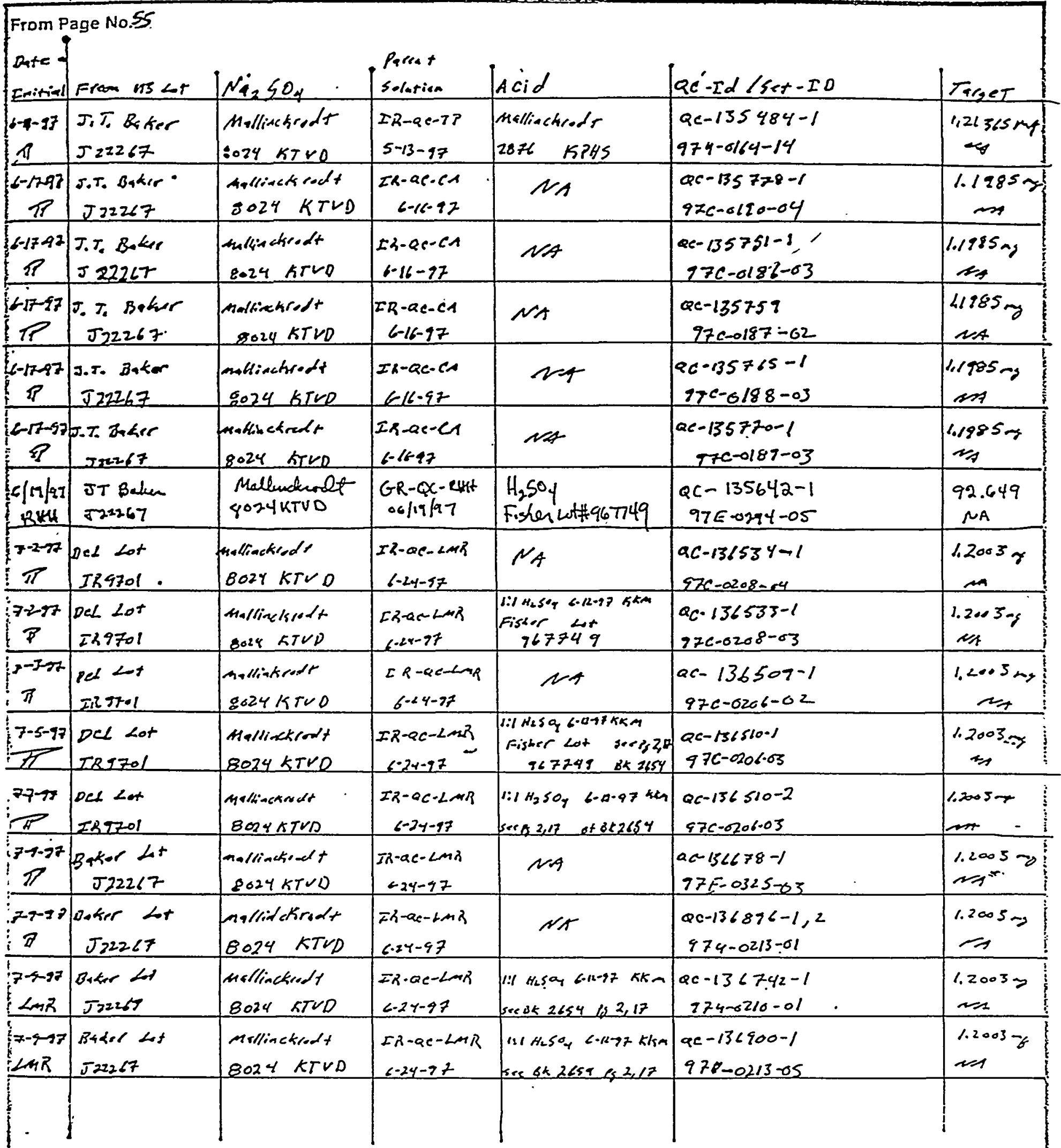

To Page No.

\begin{tabular}{|l|l|l|l|}
\hline $\begin{array}{l}\text { witnessed \& Understood by me, } \\
488\end{array}$ & Date & Invented by NOT PFilibisigiE & Date \\
\hline Recorded by
\end{tabular}


Analysis Record Page

Group \#: 69769006

LOT H: m

Preparation Date: $\quad z-g-q 7$

Sample Analysis Date: $7 z-Z_{7}$ d

Methodt: 418.1 mod

- Matrix: Wipes / warer
DCL I.D.

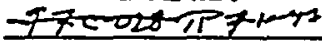

97C-0208-04

97c-0200-03

\section{SAMPLE \#

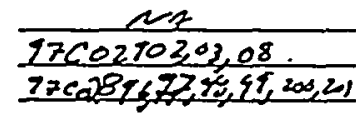

MOD: $\frac{100 \text { erg/h }}{100 \text { ex/L }}$

instrument P.D: FIR-1000C

- Samples were analyzed by method $418 . f$

page 3/ of logbook 2084

- Sample Preparation Record Page can be four

Analysis procedure can be found on

- Sample results can be found in the datapack.

\section{Reagents:}

\section{ACCOUNT}

$\frac{\text { wA }}{3006}$

Freon 113 Bofir LT J T2217

Silica gel funlianetrodt CSSl $\mathrm{kPNH}$

- Primary stock solution can be found on page 13 of logbook 2017. IR-QS-PAF 6-16-97 IR-S-TP 6-16-97

- Intermediate stock solution can be found on page 26 of logbook 2019.
IR-QI-LMR 6-24-97
IR-I-TP 6-24-97
IR-QC-LMA 6-24-97
IR-ISP-TP 6-24-97

- Working standard solution's can be found on page 2 and y,5 of logbook 3131 .

IR-WS-TP $\quad$-2:24-4z

$$
\begin{array}{cccc}
4.4265 & \text { (2) } 2.2133 \mathrm{mg} & 0.8853 \mathrm{mg} & 0.4427 \mathrm{mg} \\
0.2213 \mathrm{mg} & 0.0885 \mathrm{mg} & \text { (1) } 0.0 \mathrm{mg}
\end{array}
$$

IR-ICV-LMB (-24-3 7 (1) $12003 \mathrm{mg}$ (ICV)

- QC preparation can be found on page 50 and _ $5 \leqslant$ of logbook 3131 .

- $\quad \frac{Q C-136533-1}{0.136534-1}$

Comments;

To Page ito.

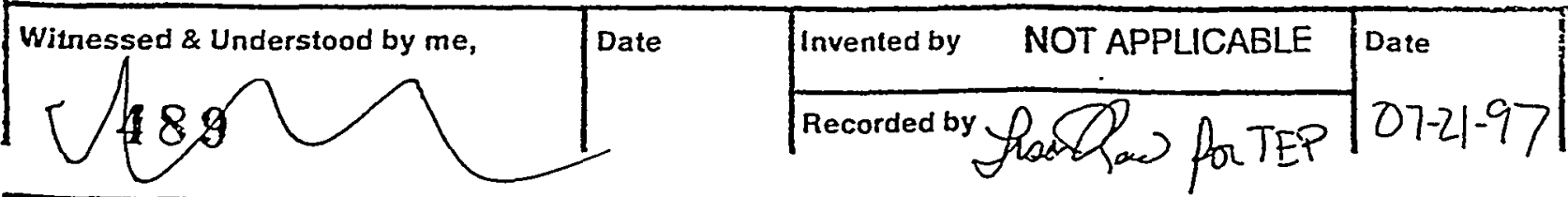


DATACHEM LABORATORIES - SECONDARY STOCK STANDARD SOLUTONS Project No. $\cong A$ TITLE

From Page No.35

$T R=T P=T P=\angle C=9.7$

10_-

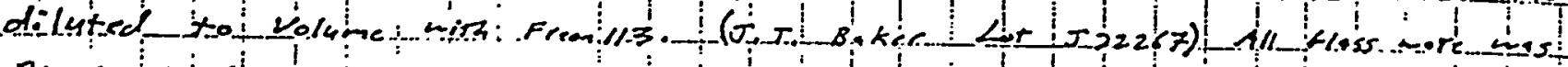

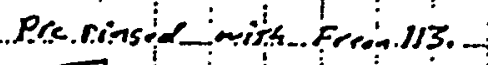

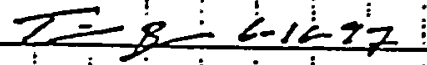

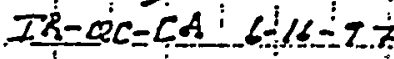

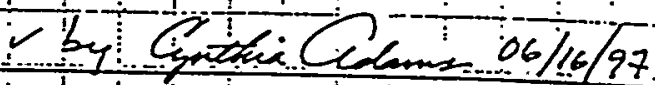

(

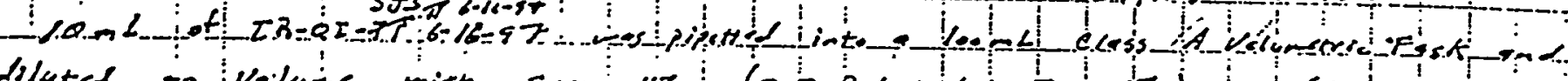

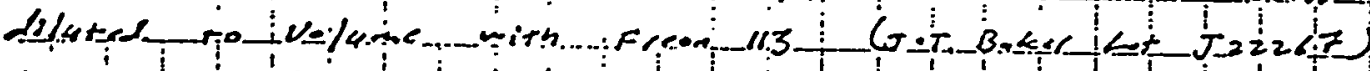

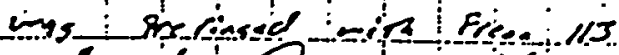

Cynthed Chene: $06 / 16 / 97$

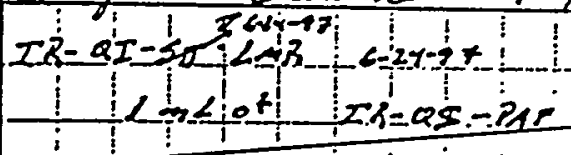

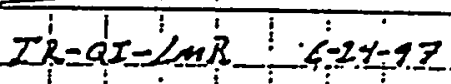

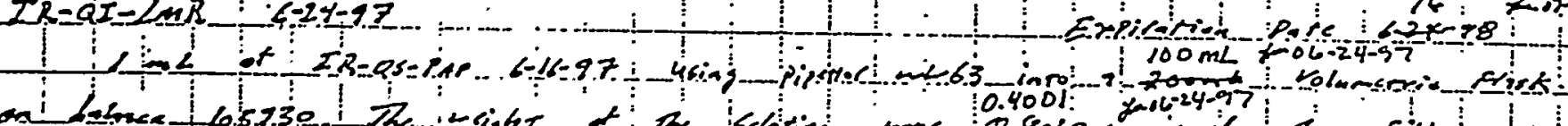

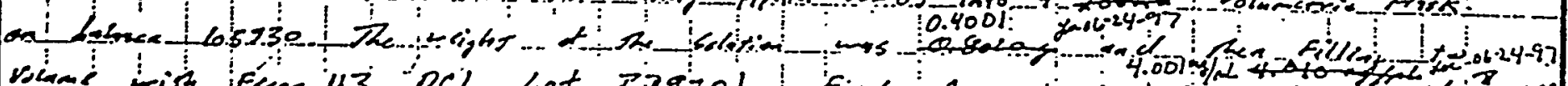

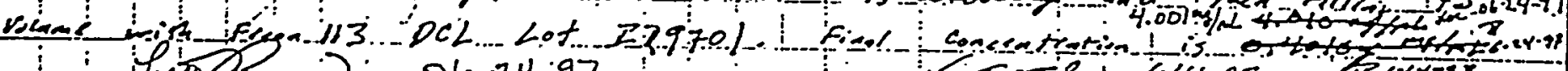
Thos $06-24-97$

$2 B=a c=\sin -24-27$

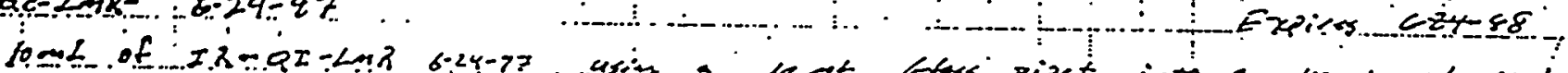
$16-2<07+21.92$

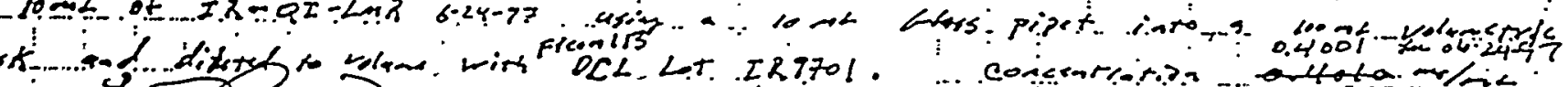

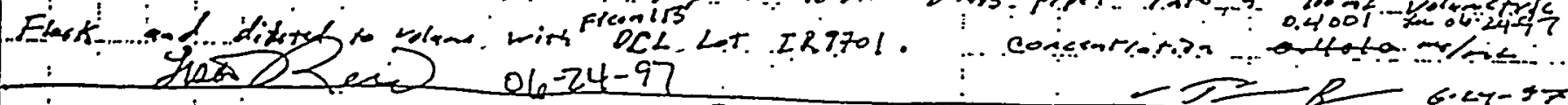

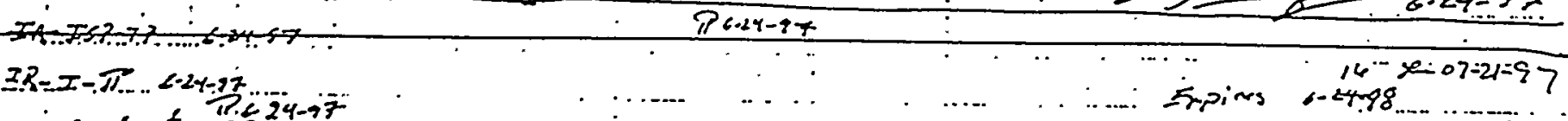

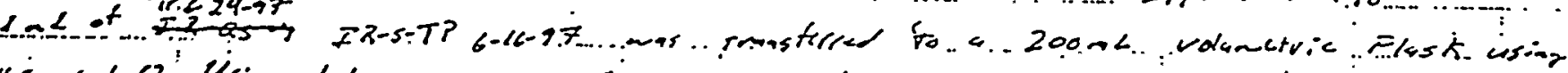

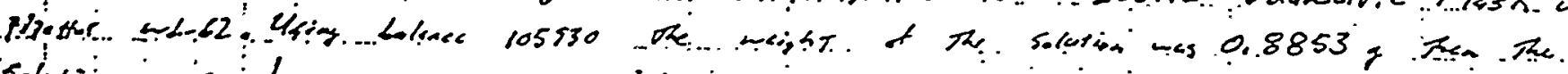

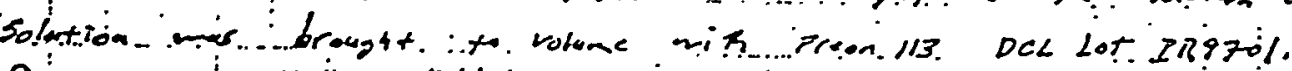

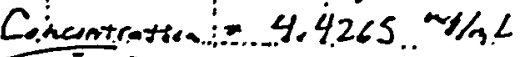

$=2-24-97$

IR-TSP-TP $<-24-97$

1e-4 of $=R-T-T 3$

$+$.

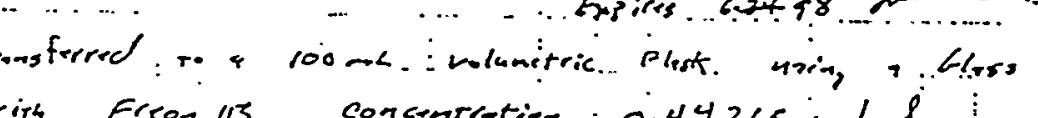

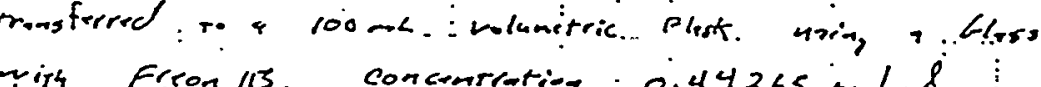

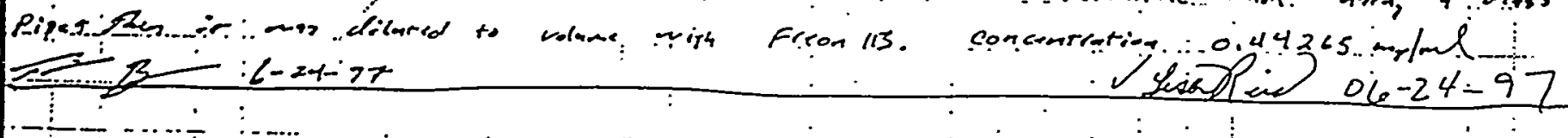

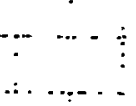

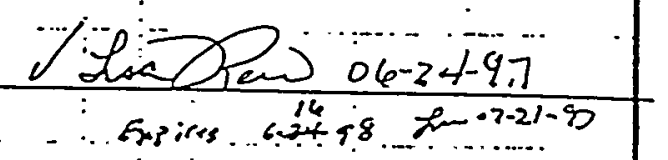


TITLE DATACHEM LABORATORIES - PRIMARY STOCK STANDARDS

Project No.

Book No. 2017

13

From Page No.12

GB-QC-TP $\quad 10.7-96$

Concentration? $33.149 \mathrm{mSSh}$

$33.149 \mathrm{mgsc}$

Expiration Date: $10-7-97$

$3.3149 \mathrm{~g}$ of 0.1 (Valvoline Tull Formula. SAE $10 \mathrm{w} 30$ High Pertomeace Motor Oil) into . loo ord class A Volumetric .. Flask. . Then.. diluted to volume wish Flem 113 (Baker Lot J22267). This is to be used. only for oil and Case amlysis by brevametlic:..

$=10-7-96$

Jedi:Dand Daniel $10-796$

GR -S DA $10-14-96$

$3.3299 \mathrm{~g}$ of Mazola corn oil (Freshnesdate Nov 1896 ) into a $100 \mathrm{ml}$ class. A volumetric flask which was diluted to volume with Freon 113 (Baker Lot J2226.7). Volumentic was rinsed with Freon 113 . This is to be wed for 0 il and Grease analysis. by granitic method. Expiration date: 10-14-97. Concentration: $33.299 \mathrm{mg} / \mathrm{ke}$

Pun ce A. Tote $10-14-96$

$2 n-5-T>2-16-97$ $2=10-14-5<-14-96$ P 17

Expires $6-16.98$

$15 \mathrm{~mL}$ of Isooctane $(2,2,9$, Trimbarf pentane) J. T. Bat ier Lot J12285

$15 \mathrm{~nL}$ of Hexudthe Aldrich Lot $01218 \mathrm{~J} X$

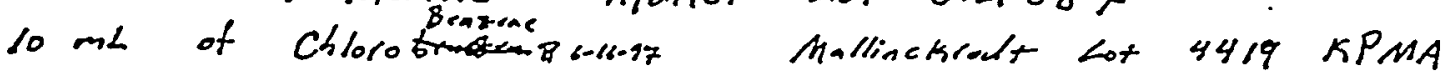

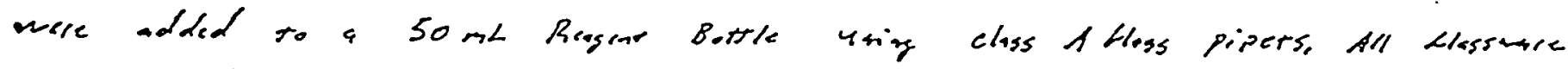
was gre rinsed wish Freed 113 . $=$

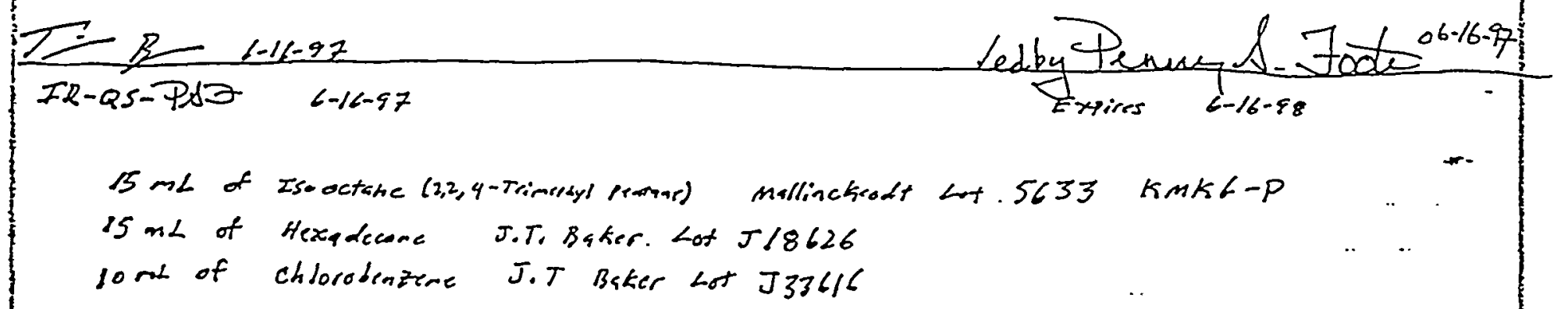

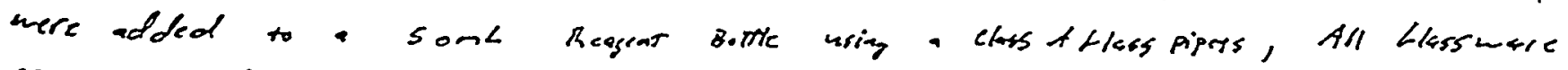
ines prefigured with freon 115 .

Penny. Grote $\infty$ - $16-97$

$$
-06-16-9.7 P A>
$$

$6.16 \div .97$

To Page No. 14

$$
\begin{array}{|l|l|l|l|}
\hline \text { Witnessed \& Understood by me, } & \text { Date } & \text { Invented by NAte } & \text { Nate } \\
\hline
\end{array}
$$




\section{TPH in Water}

Group \# : G9769006

Preparation Date : 7/2/97

Analysis Date : 7/9/97

Instrument 1.D: FTIR-1000C

Set I.D's: $97 C-0208-03 \& 04$

\author{
WATER LOD : $100 \mu \mathrm{g} / \mathrm{L}$ \\ Method: 418.1 \\ Account \#: 3006 \\ - Analyst : TP
}

Results are calculated according to the following formula: $\mu \mathrm{g} /$ wipe Petroleum Hydrocarbons $=R^{*} D *(1000 \mu \mathrm{g} / \mathrm{mg}) /$ \# of wipes $\mathrm{\mu g} / \mathrm{L}$. Petroleum Hydrocarbons $=R * D *(1000 \mu \mathrm{g} / \mathrm{mg}) / \mathrm{V}$

$R=m g$ of Petroleum Hydrocarbons as determined from the calibration plot. $D=$ extract dilution factor, if used.

$V=$ Volume of water sample, in liters.

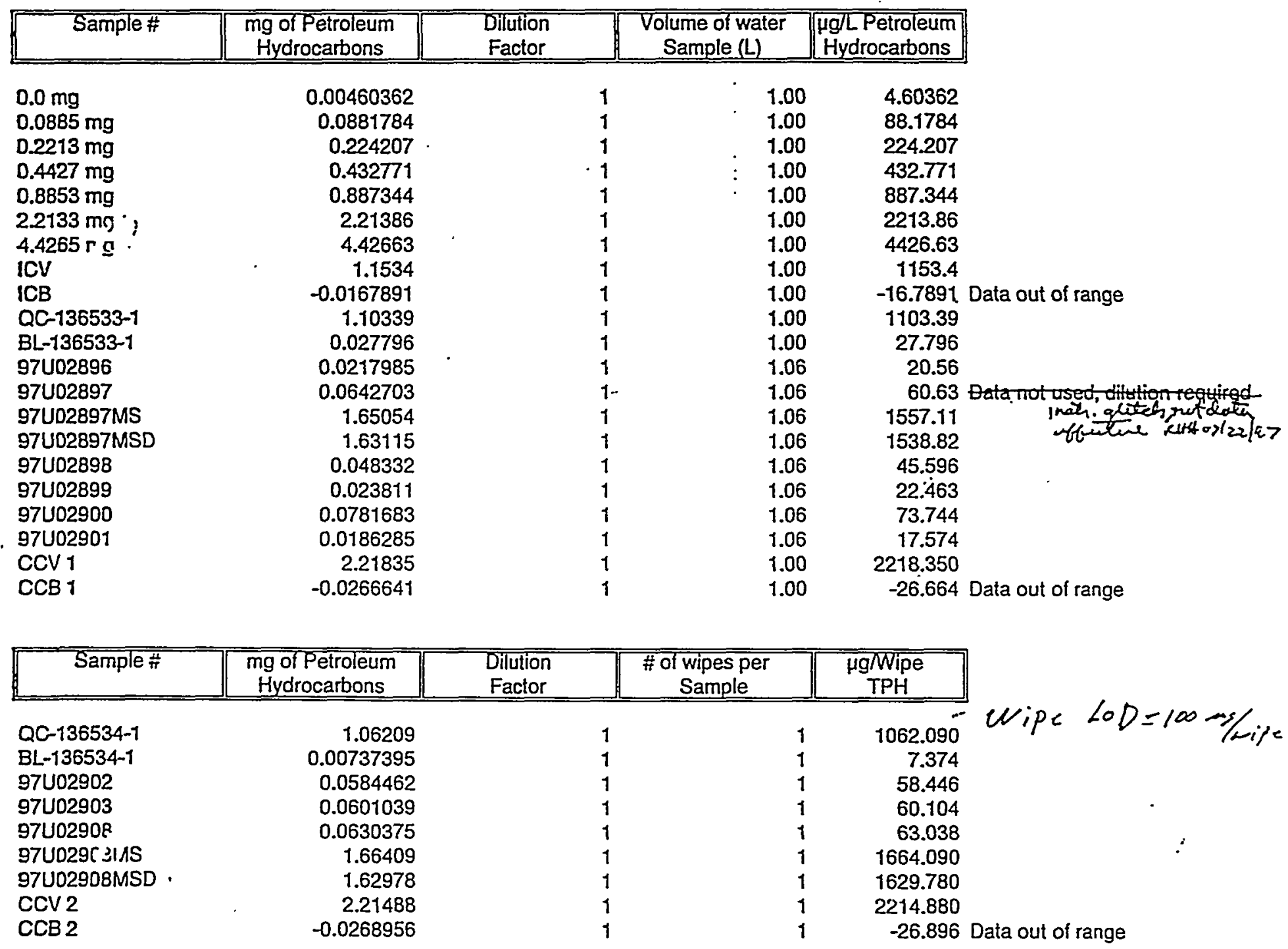

\section{3}




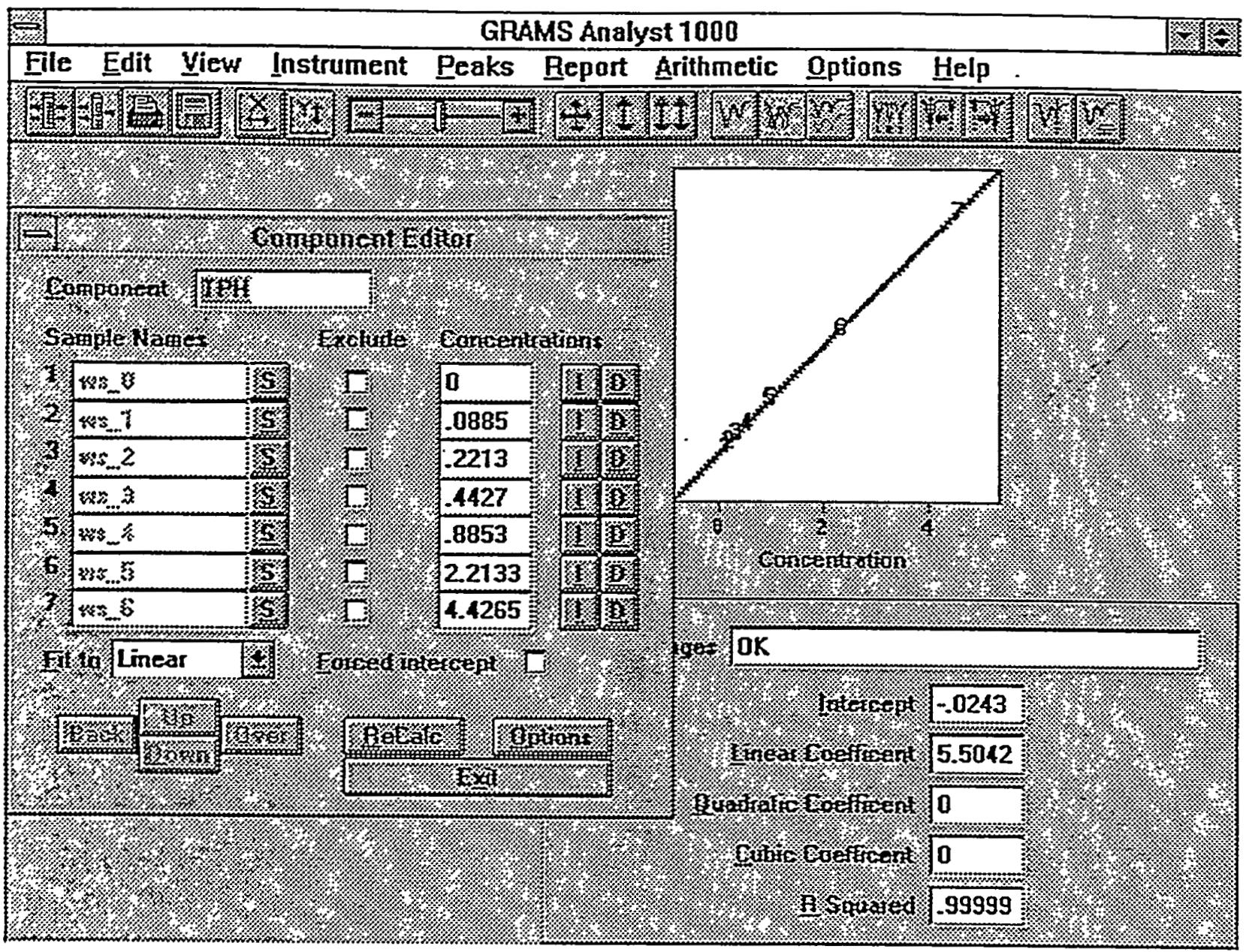

Calibration data for set 97C-0208-04 and 97C-0208-03

Instrument ID: FTIR-1000C 
QuantBasic Report

Quant Type: Multlpolnt $\quad$ 7/15/97 5:01 pm

Method: C:IPE1000GSIDATAL703GTPH.Q

Sample: c:lpe1000gsidatal97036lcalcurve.spc

Analyst: TP

Sample ID: 0.0

Component Concen R-Squared

TPH

$.00460362 \quad .999991$

Instrument 1.D.: FTIR-1000C

Analyzed on: 09-JUL-1997

Date and time on printout is the day and time copy was printed.

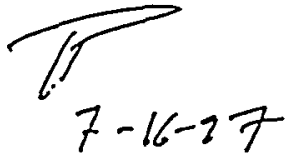

$\therefore 495$
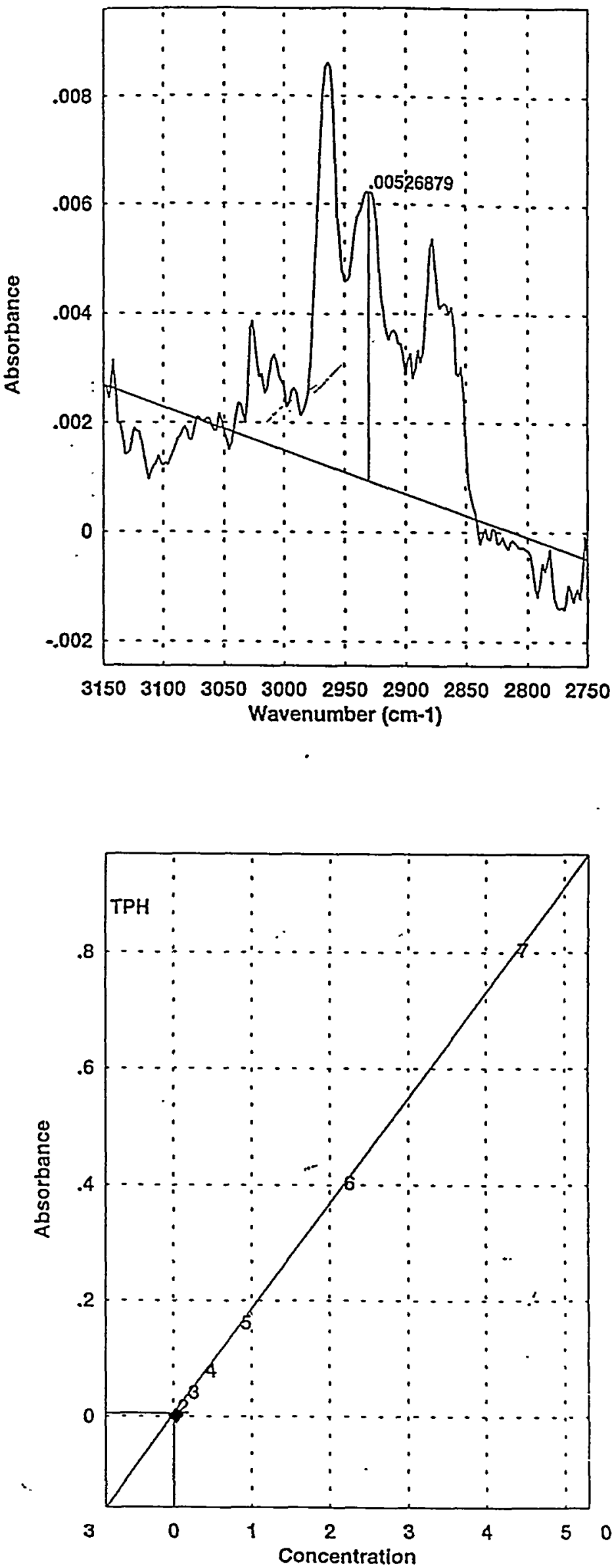
QuantBaslc Report

Quant Type: Multipolnt $\quad 7 / 15 / 975: 01 \mathrm{pm}$

Method: C:lPE1000GSIDATAL703GTPH.Q

Sample: c:lpe1000gsldatal97036lcalcurve.spc

Analyst: TP

Sample ID: 0.0885

Component Concen R-Squared

$\begin{array}{lll}\text { TPH } & .0881784 \quad .999991\end{array}$

Instrument I.D.: FTIR-1000C

Analyzed on: 09-JUL-1997

Date and time on printout is the day and time copy was printed.

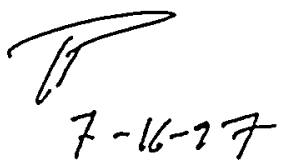

- 496
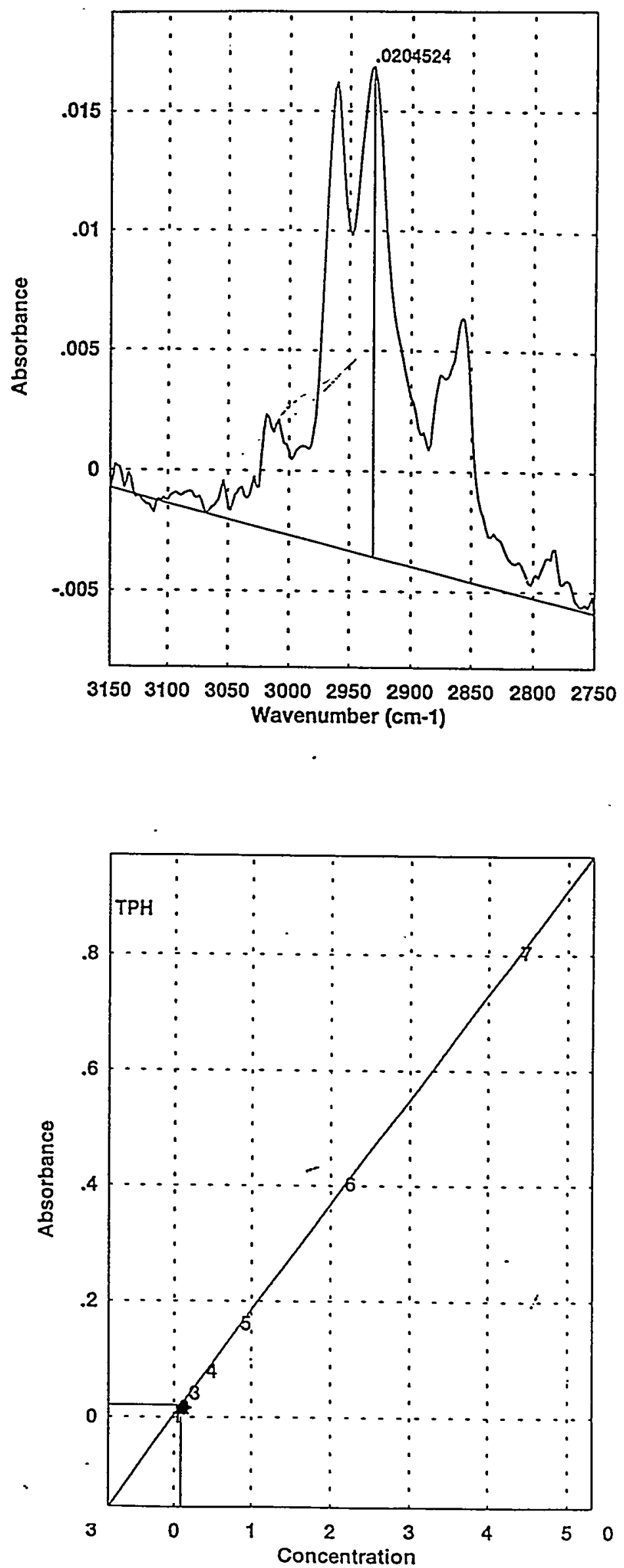
QuantBasic Report

Quant Type: Multipolnt $\quad 7 / 15 / 975: 00 \mathrm{pm}$

Method: C:IPEYO00GSIDATAL7036TTH.Q

Sample: c:lpe1000gsidatal97036lcalcurve.spe

Analyst: TP

Sample ID: 0.2213

Component Concen R-Squared

$\begin{array}{lll}\text { TPH } & .224207 \quad .999991\end{array}$

Instrument I.D.: FTIR-1000C

Analyzed on: 09-JUL-1997

Date and time on printout is the day and time copy was printed.

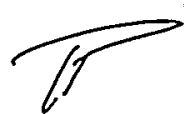

$$
7-16-27
$$
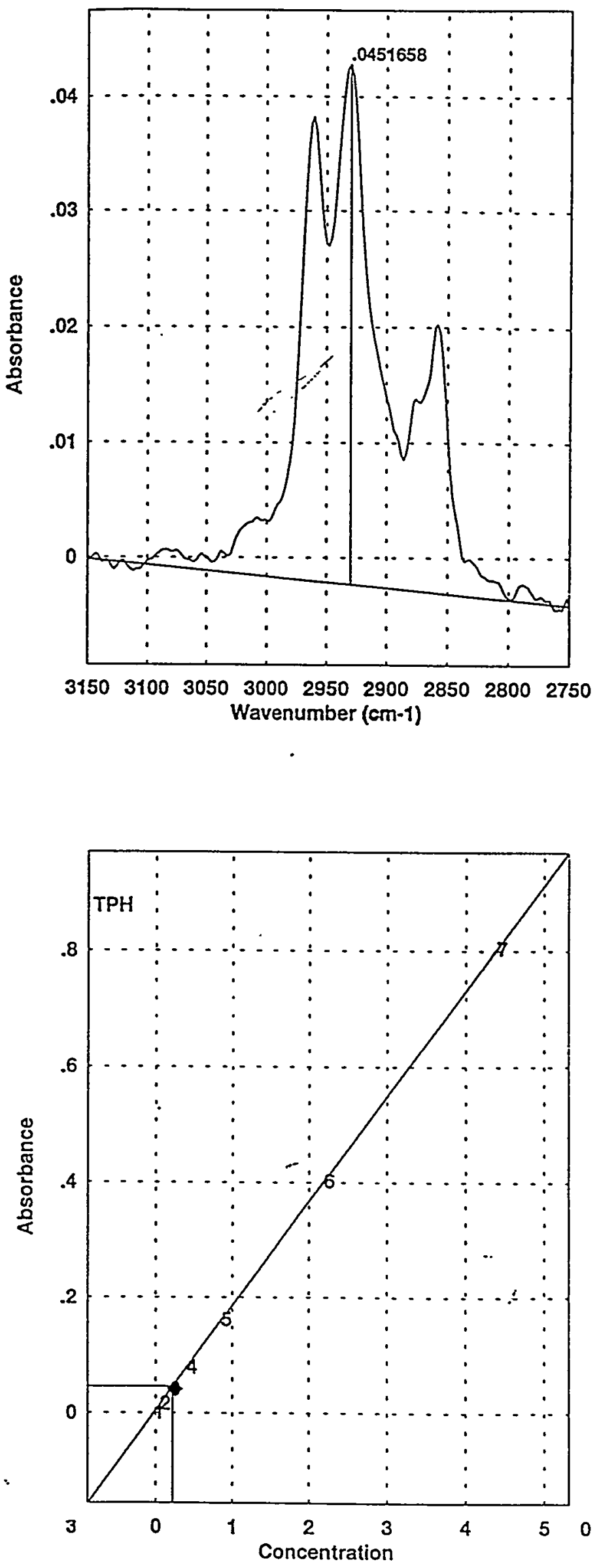
QuantBasic Report

Quant Type: Multlpolnt 7/15/97 5:00 pm

Method: C:IPE1000GSIDATAL703GITPH.Q

Sample: cilpe1000gsidatal97036lcalcurve.spe

Analyst: TP

Sample ID: 0.4427

Component Concen R-Squared

$\begin{array}{lll}\text { TPH } & .432771 & .999991\end{array}$

Instrument I.D.: FTIR-1000C

Analyzed on: 09-JUL-1997

Date and time on printout is the day and time copy was printed.

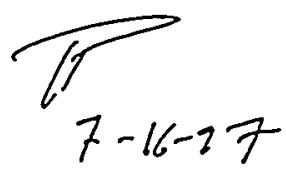

- 498
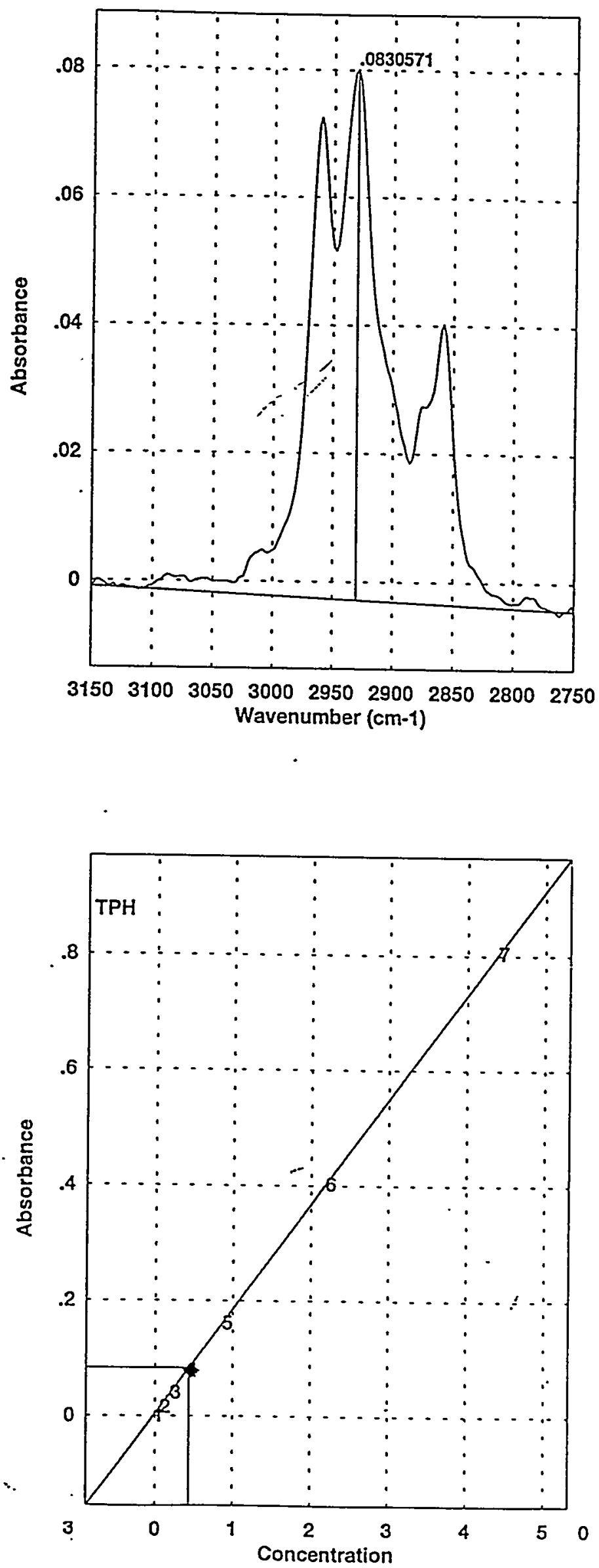
QuantBasic Report

Quant Type: Multlpoint $\quad 7 / 15 / 97$ 5:00 pm

Method: C:UPE1000GSDATA197036TPH.Q

Sample: c:lpe1000gsidatal97036lcalcurve.spc

Analyst: TP

Sample 1D: 0.8853

Componẹnt Concen R-Squared

$\begin{array}{lll}\text { TPH } & .887344 & .999991\end{array}$

;

Instrument 1.D.: FTIR-1000C

Analyzed on: 09-JUL-1997

Date and time on printout is the day and time copy was printed.

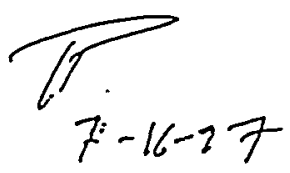

$\because 499$
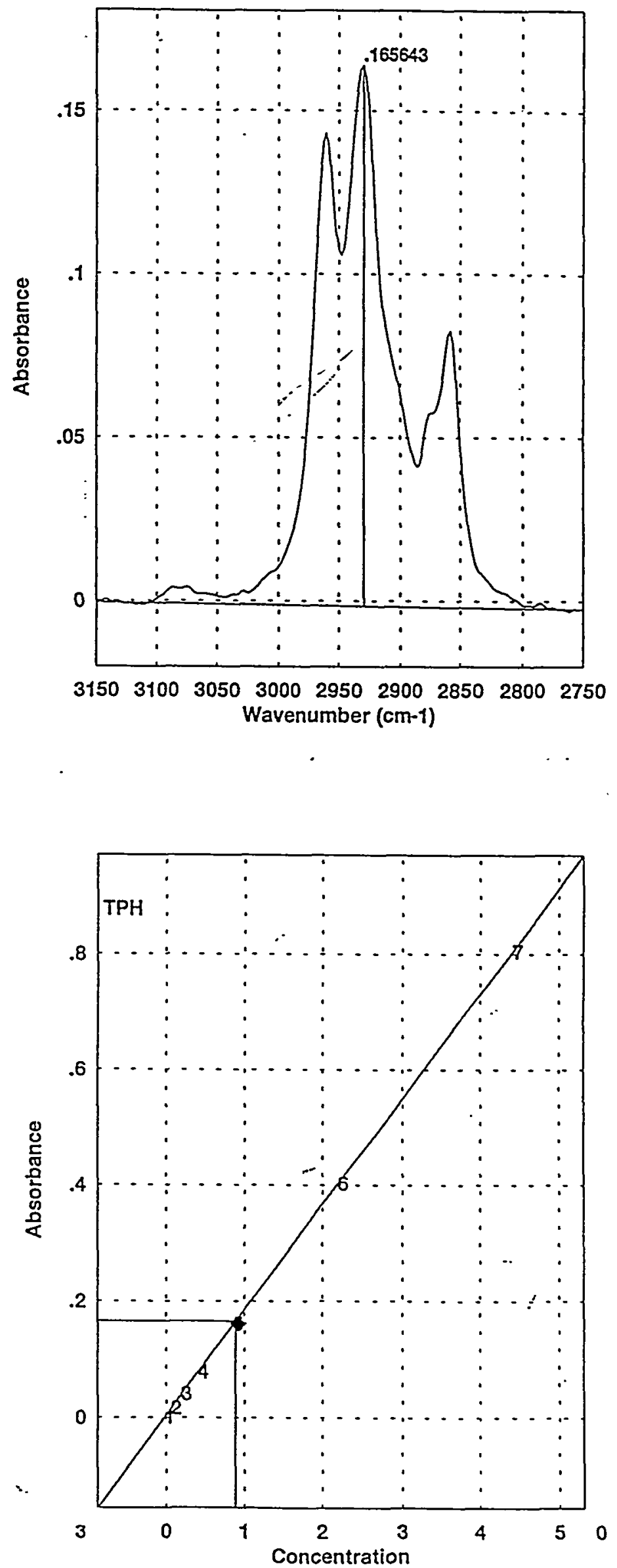
QuantBasic Report

Quant Type: Multipoint $\quad 7 / 15 / 97$ 4:59 pm

Method: C:LE1000GSLDATALIO7036ITPH.Q

Sample: c:lpe1000gsidatal97036lcalcurve.spc

Analyst: TP

Sample ID: 2.2133

Component Concen R-Squared

TPH $\quad 2.21386 \quad .999991$

Instrument I.D.: FTIR-1000C

Analyzed on: 09-JUL-1997

Date and time on printout is the day and time copy was printed.

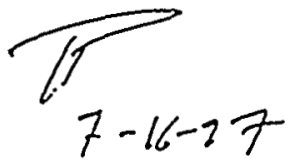

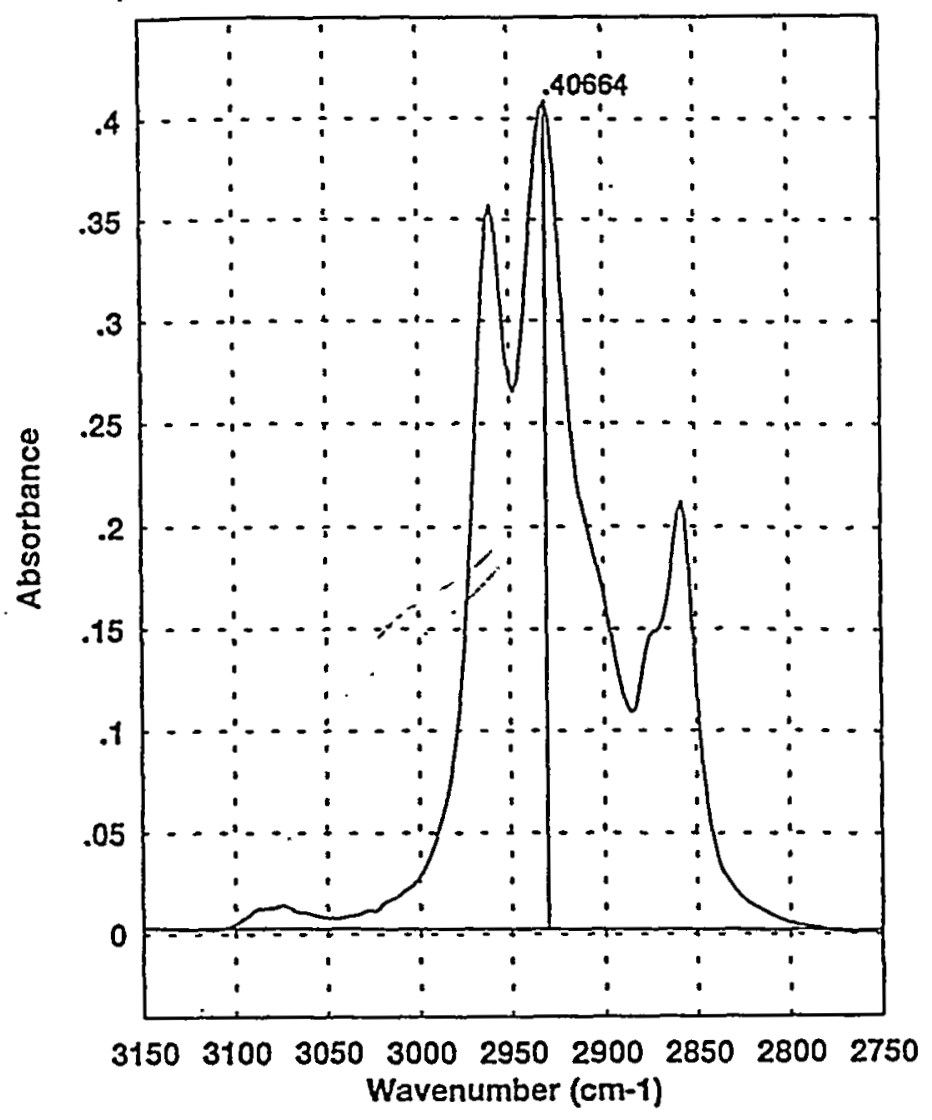

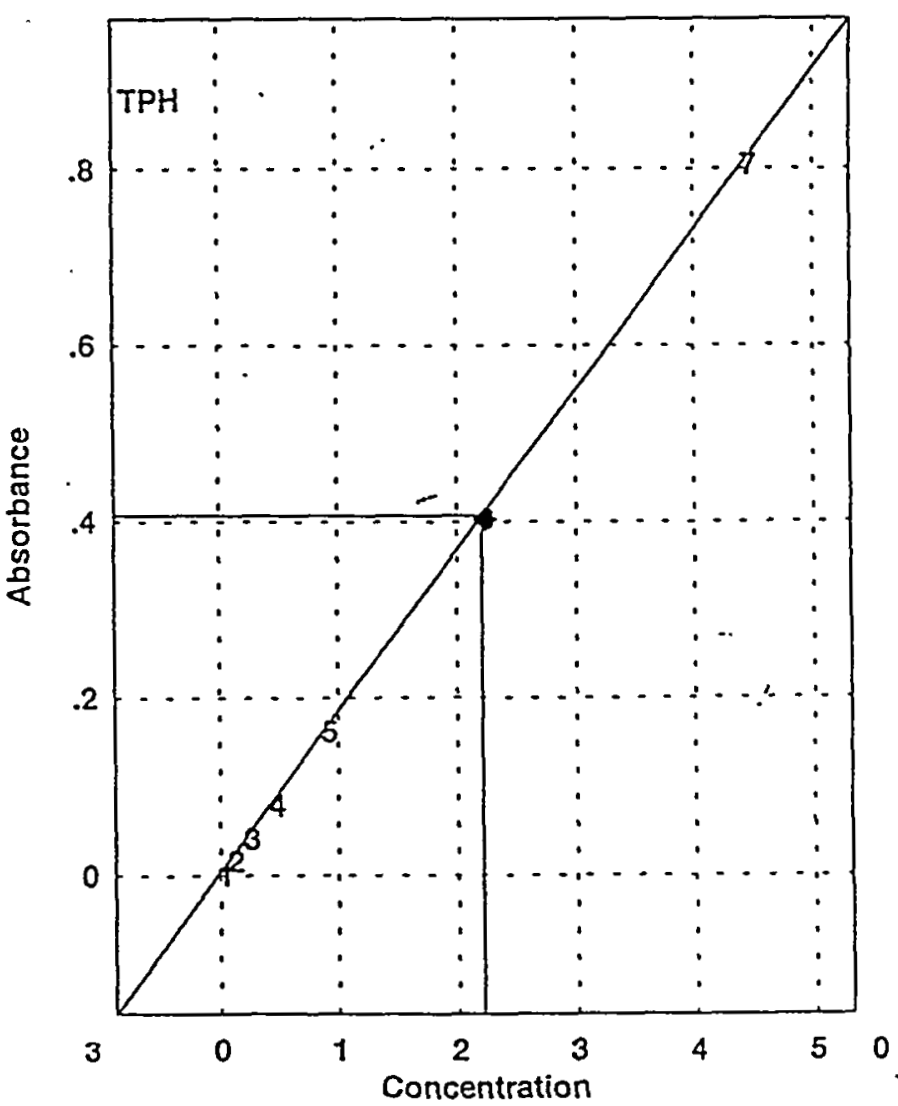




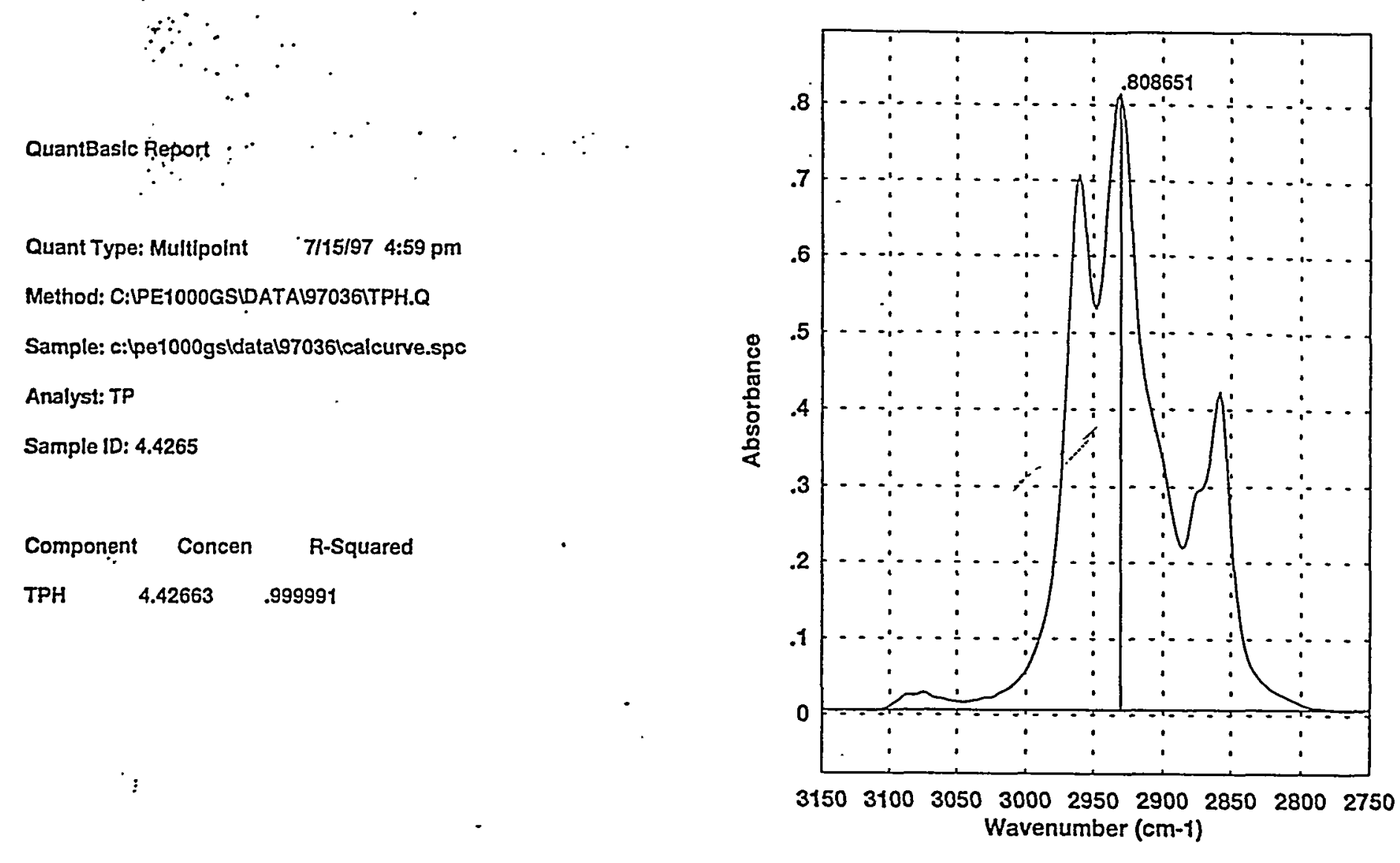

Instrument I.D.: FTIR-1000C

Analyzed on: 09-JUL-1997

Date and time on printout is the day and time copy was printed.
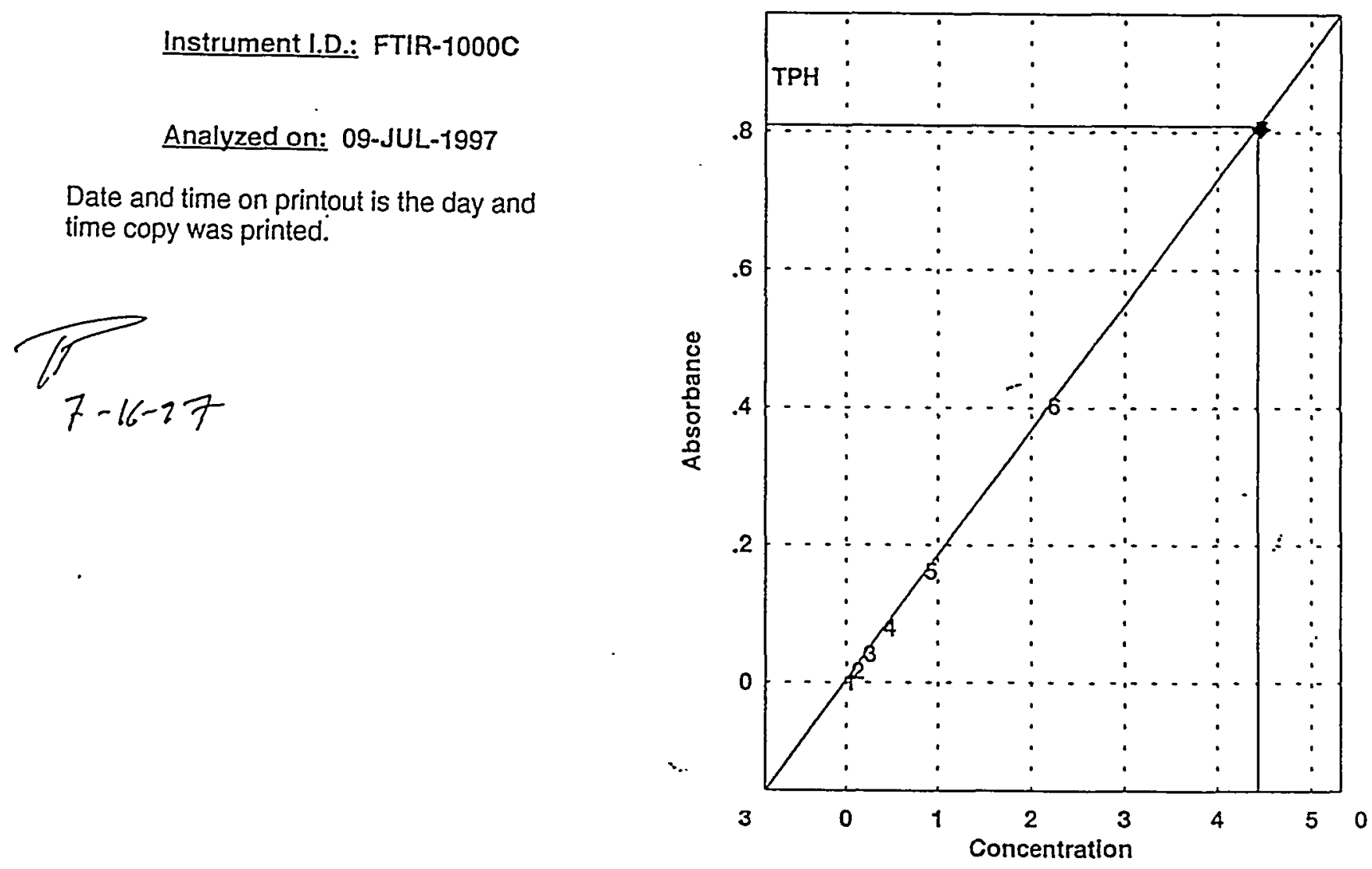
QuantBaslc Report

Quant Type: Multlpolnt $\quad$ 7/15/97 5:02 pm

Melhod: C:LPE1000GSLDATAL703GTPH.Q

Sample: c:lpe1000gsidatal97036lcalcurve.spc

Analyst: TP

Sample 1D: ICV

Component Concen R-Squared

$\begin{array}{lll}\text { TPH } & 1.1534 & .999991\end{array}$

Instrument 1.D.: FTIR-1000C

Analyzed on: 09-JUL-1997

Date and time on printout is the day and time copy was printed.

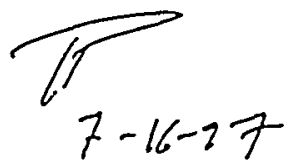

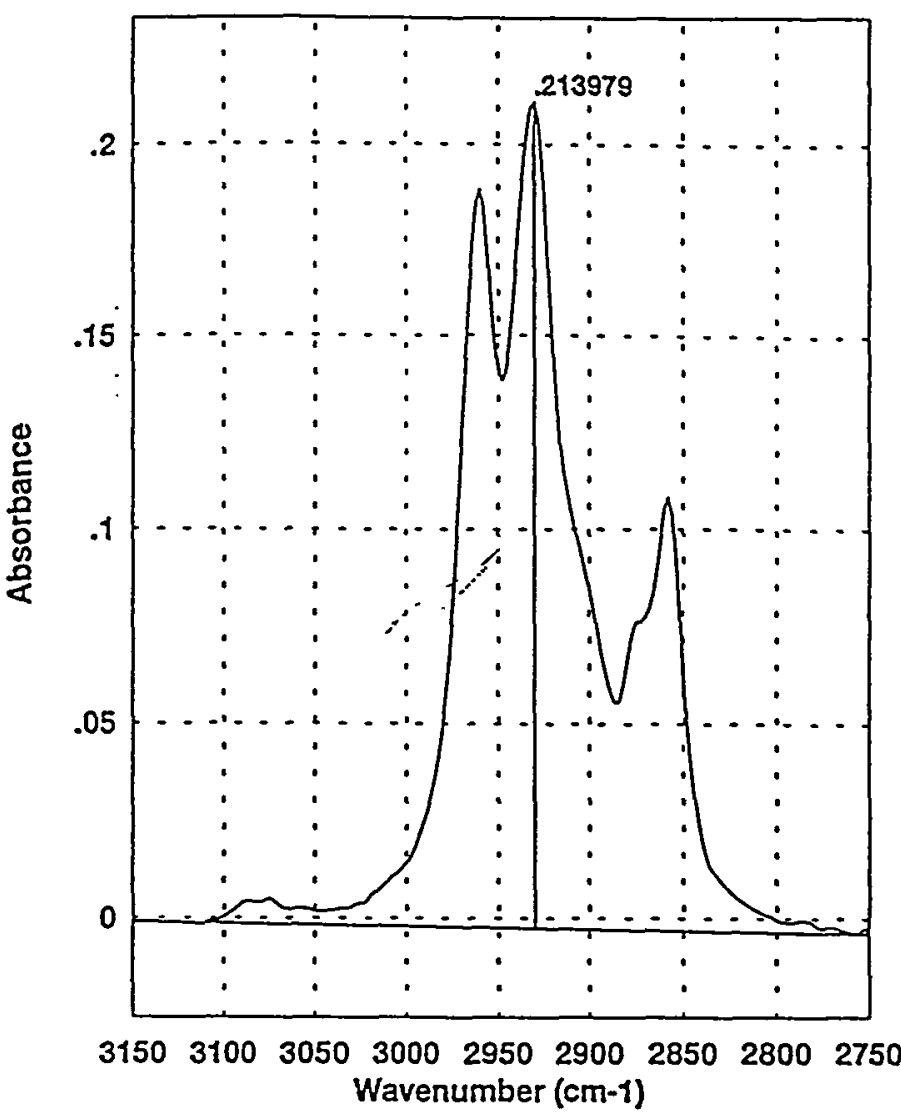

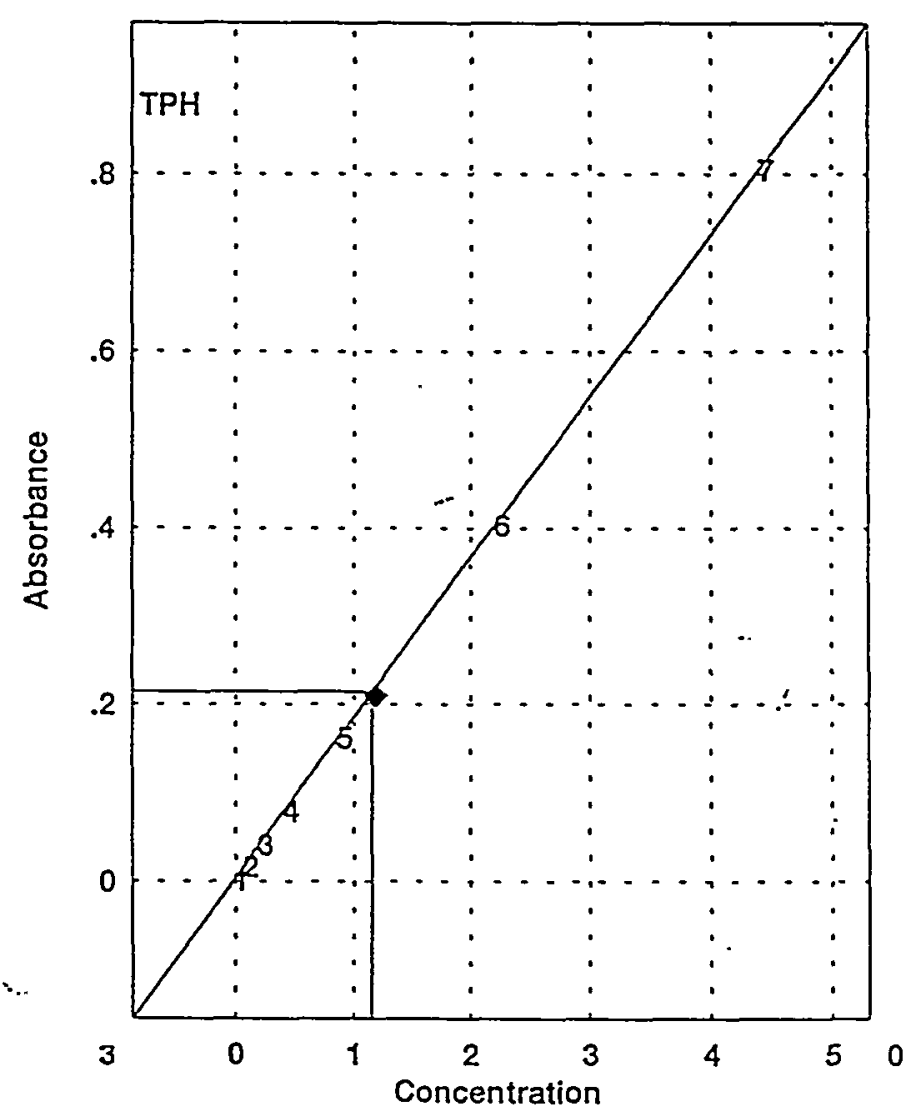


QuantBasic Report

Quant Type: Multipoint $\quad$ 7/15/97 5:02 pm

Method: C:IPE1000GSLATALTO3GTPH.Q

Sample: c:lpe1000gsidatalo7036lcalcurve.spc

Analyst: TP

Sample ID: ICB

Component Concen R-Squared

TPH $\quad-.0167891 \quad .999991$

Instrument I.D.: FTIR-1000C

Analyzed on: 09-JUL-1997

Date and time on printout is the day and time copy was printed.

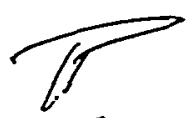

$7-16-37$
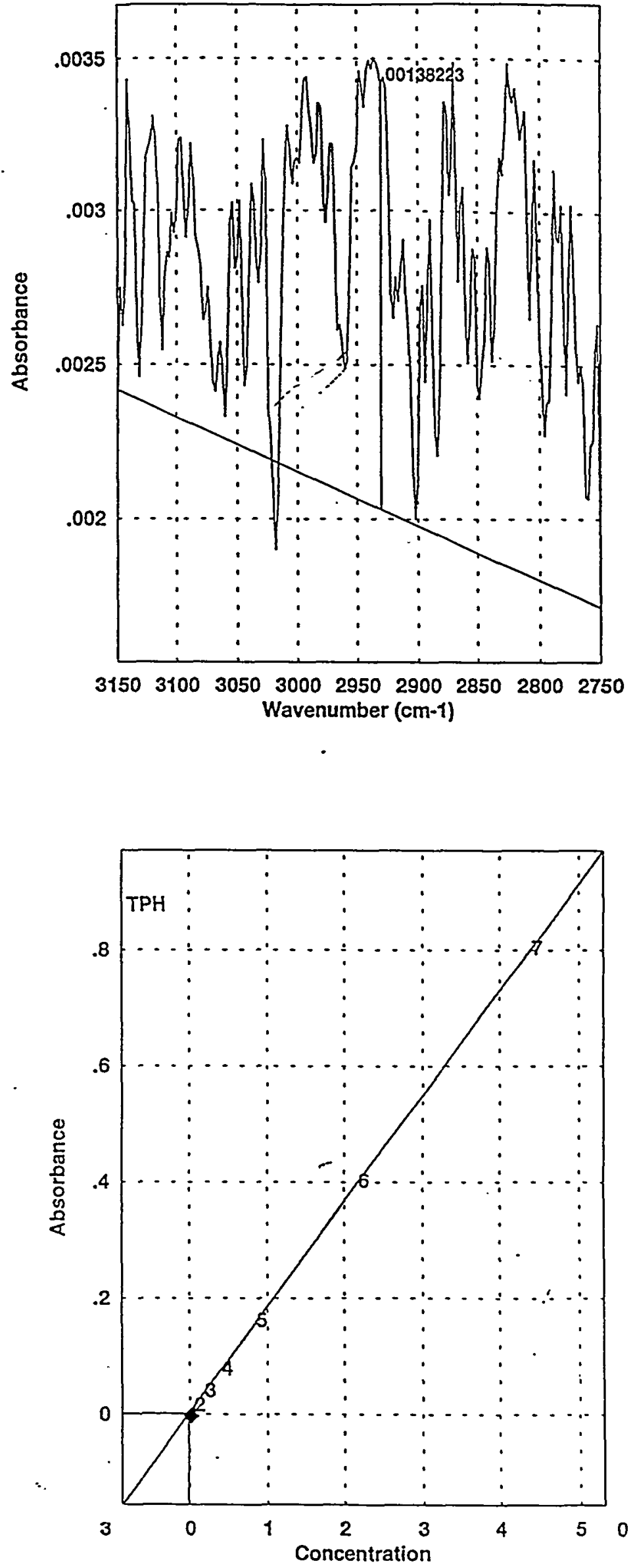
QuantBasic Report

Quant Type: Multlpolnt $\quad 7 / 15 / 97$ 4:52 pm

Method: C:IPE1000GSIDATAL7036ITPH.Q

Sample: c:pe1000gsidatal97036lcalcurve.spc

Analyst: TP

Sample ID: QC-136533-1

Component Concen R-Squared

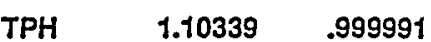

Instrument I.D.: FTIR-1000C

Analyzed on: 09-JUL-1997

Date and time on printout is the day and time copy was printed.

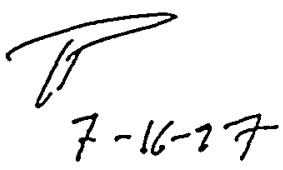

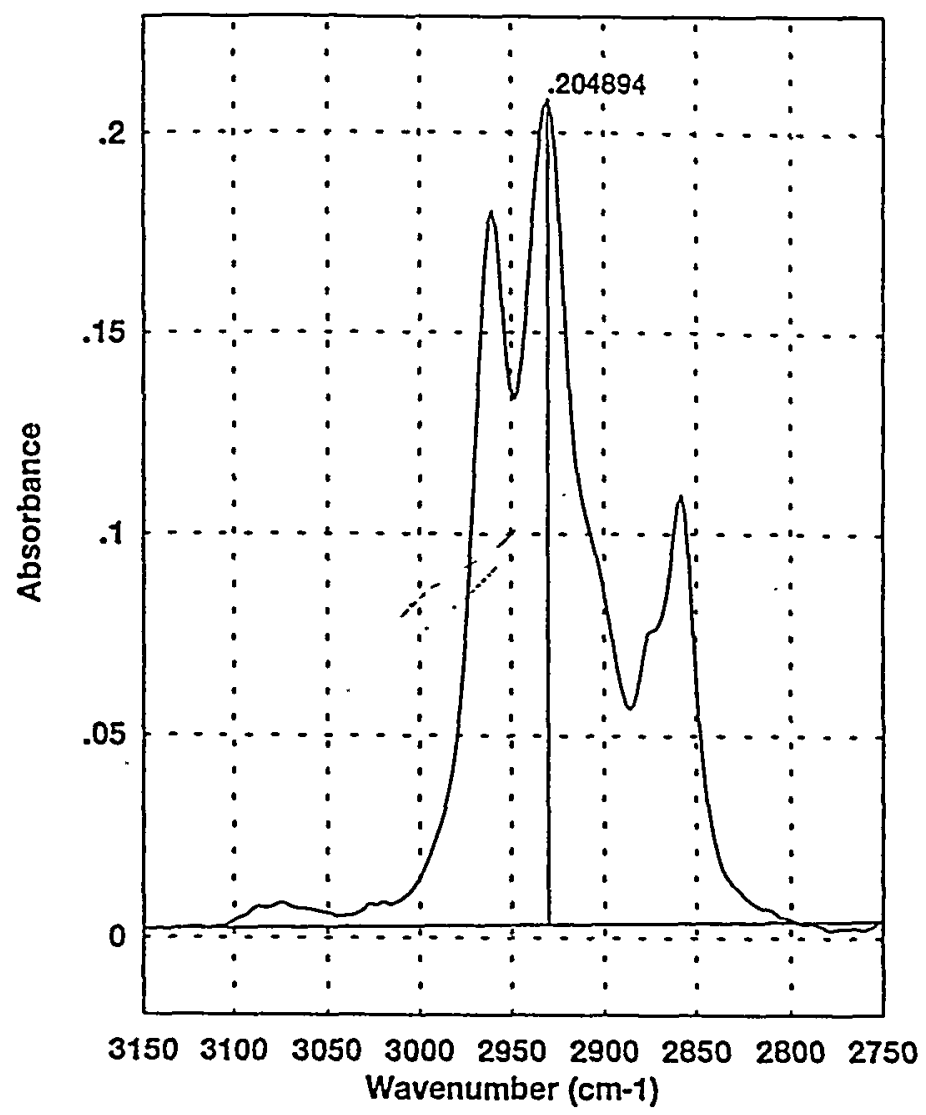

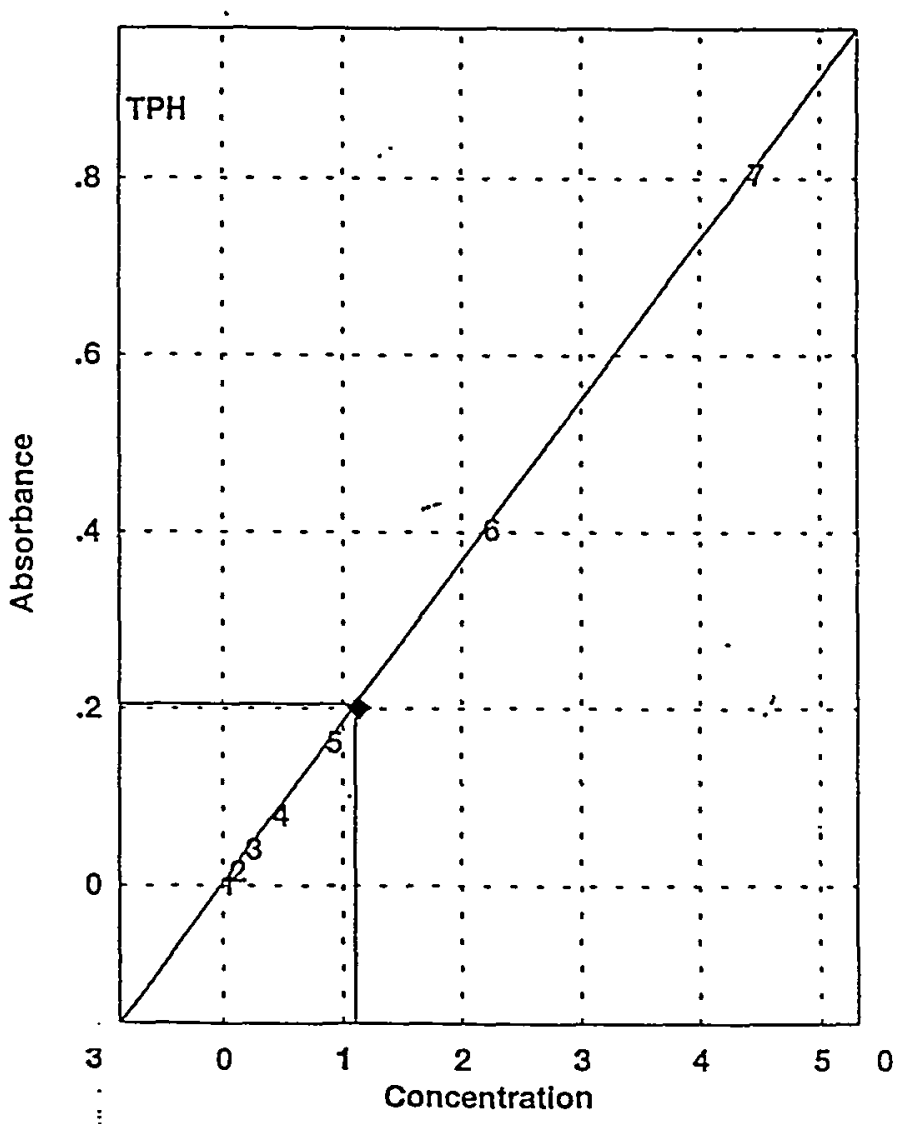


QuantBasic Report

Quant Type: Multipolnt $\quad$ 7/15/97 4:53 pm

Method: C:IPE1000GSIDATAL7036ITPH.Q

Sample: c:lpe1000gsldatal97036lcalcurve.spc

Analyst: TP

Sample ID: BL-136533-1

Component Concen R-Squared

TPH $.027796 \quad .999991$

Instrument I.D.: FTIR-1000C

Analyzed on: 09-JUL-1997

Date and time on printout is the day and time copy was printed.

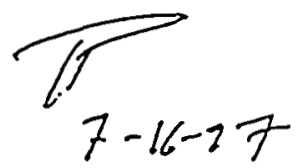

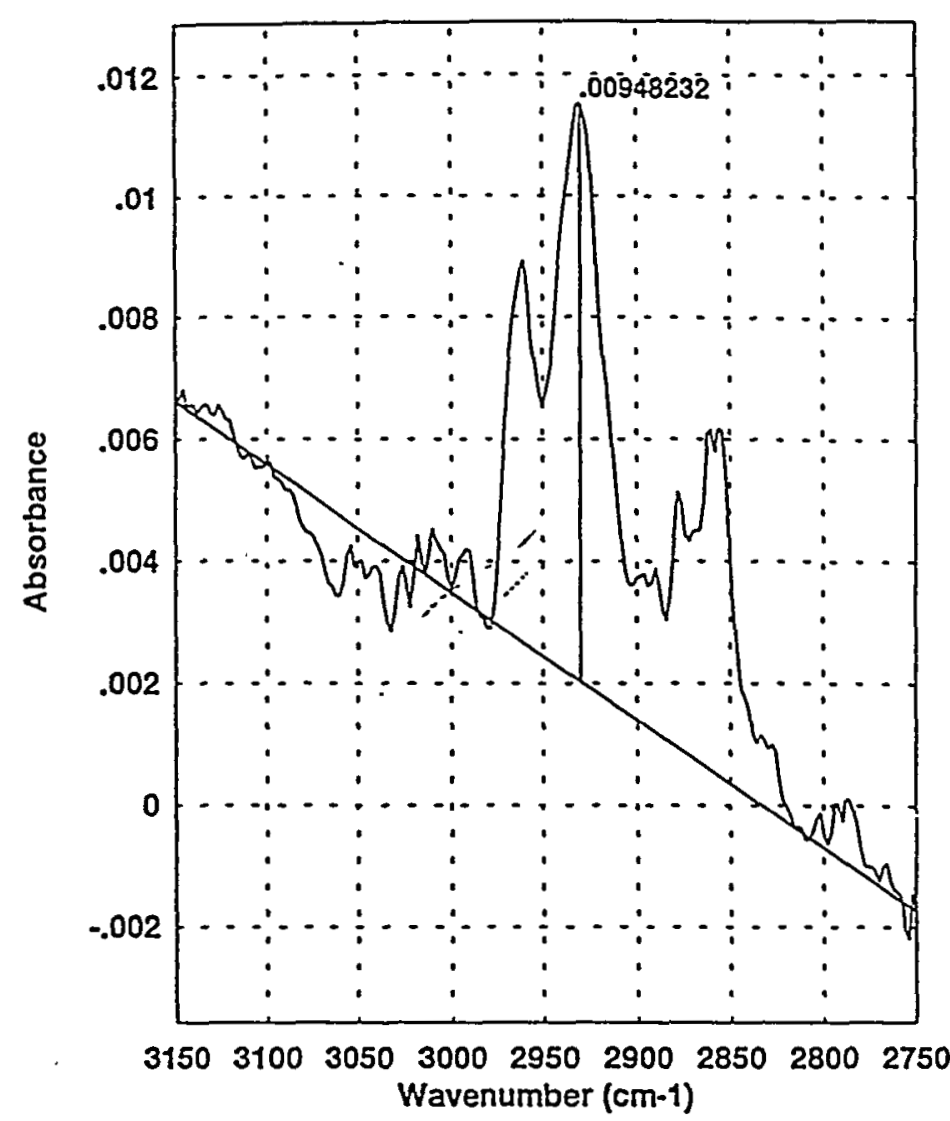

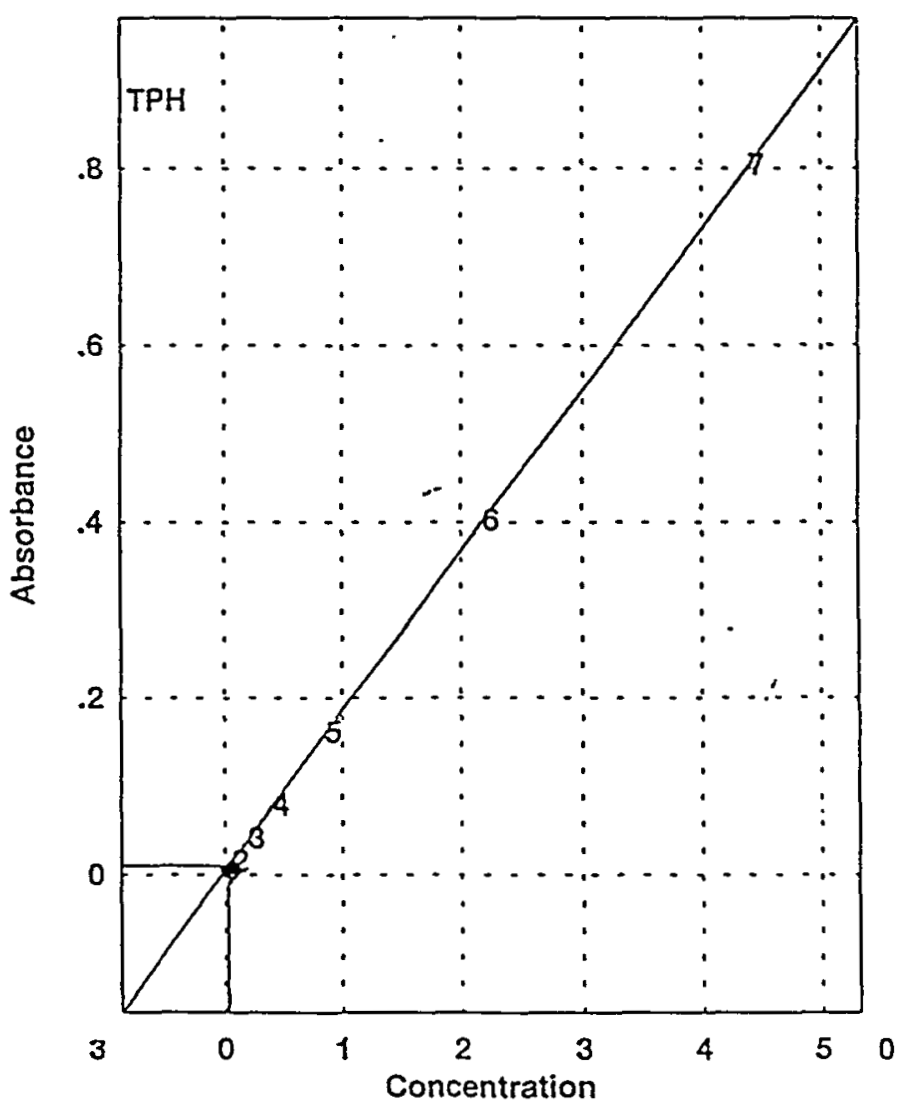


QuantBasic Report

Quant Type: Multlpoint $\quad$ 7/15/97 4:57 pm

Method: C:IPE1000GSIDATALT036ITPH.Q

Sample: c:lpe1000gsidatal97036lcalcurve.spc

Analyst: TP

Sample ID: $97 \mathrm{C02896}$

Component Concen R-Squared

TPH $\quad .0217985 \quad .999991$

Instrument I.D.: FTIR-1000C

Analyzed on: 09-JUL-1997

Date and time on printout is the day and time copy was printed.

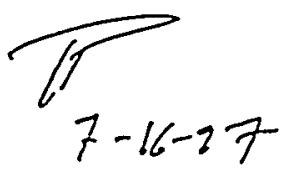

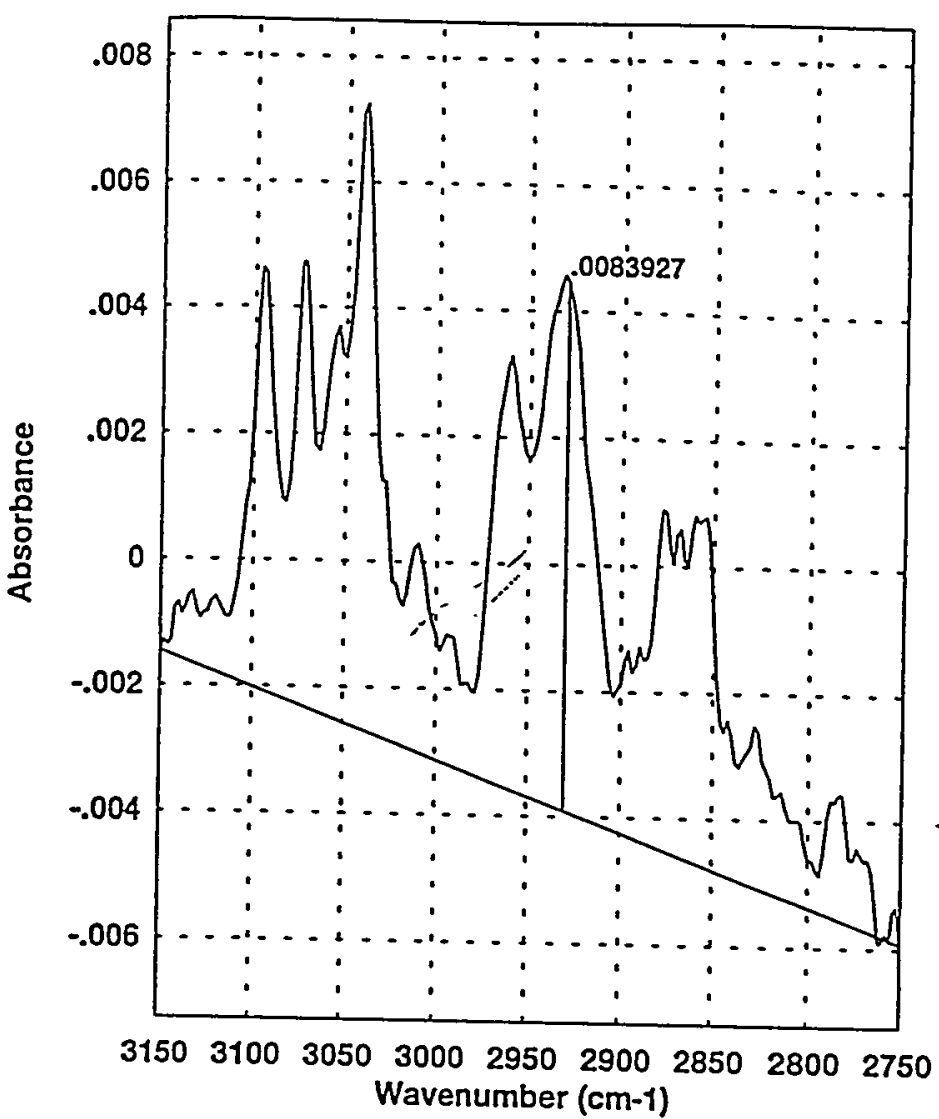

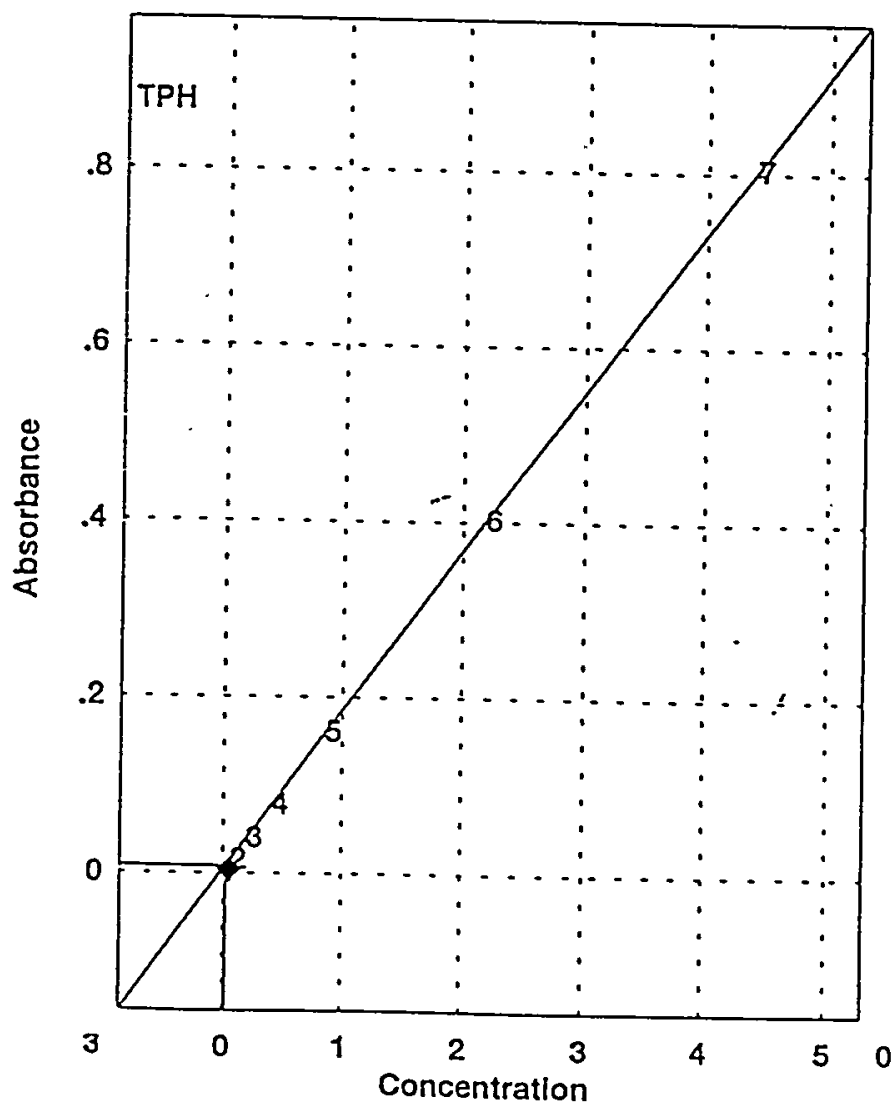


QuantBasic Report

Quant Type: Multlpoint $\quad$ 7/15/97 4:57 pm

Method: C:IPE1000GSLATAL703GTPH.Q

Sample: c:lpe1000gsldatal97036lcalcurve.spc

Analyst: TP

Sample ID: 97002897

Component Concen R.Squared

$\begin{array}{lll}\text { TPH } & .0642703 \quad .999991\end{array}$

Instrument l.D.: FTIR-1000C

Analyzed on: 09-JUL-1997

Date and time on printout is the day and time copy was printed.

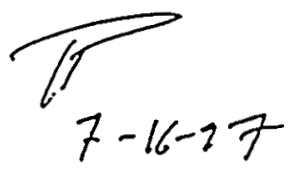

507
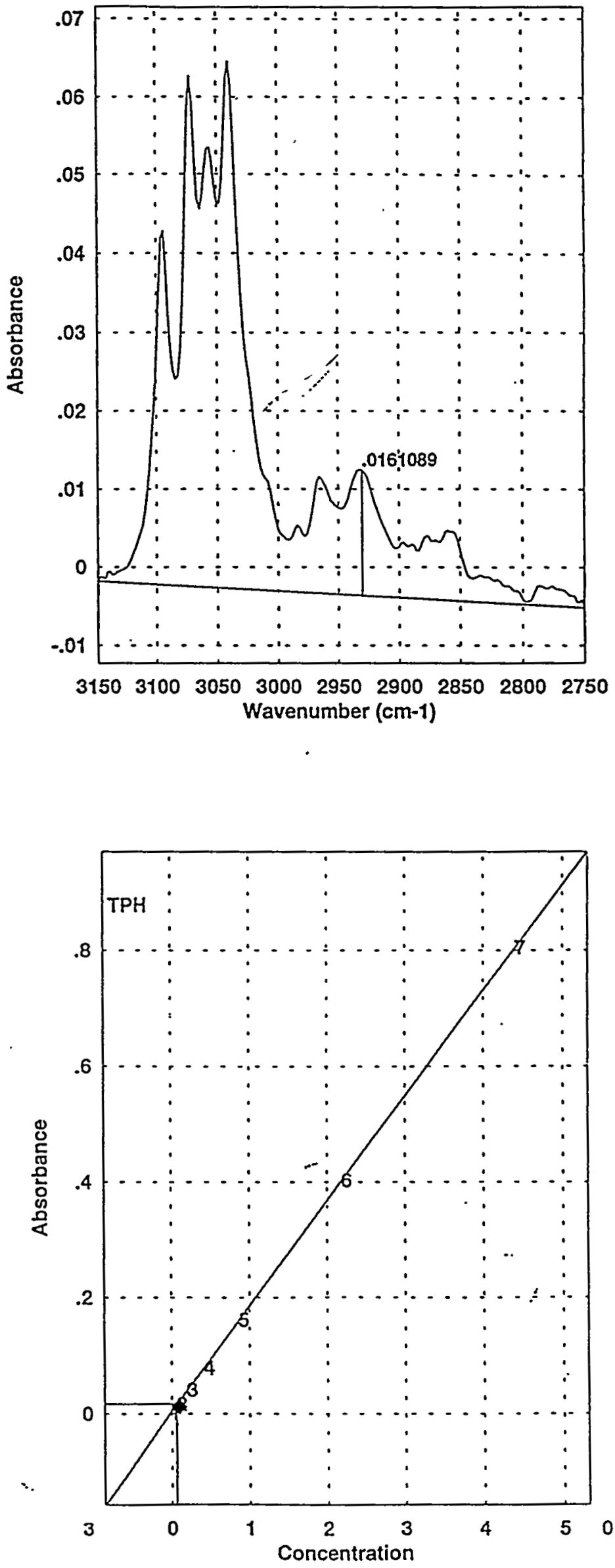
QuantBaslc Report

Quant Type: Multipoint $\quad 7 / 15 / 97 \quad 4: 57 \mathrm{pm}$

Method: C:IPE1000GSIDATALT036ITPH.Q

Sample: c:lpe1000gsidatal97036lcalcurve.spc

Analyst: TP

Sample ID: 97C02897MS

Componẹnt Concen R-Squared

$\begin{array}{lll}\text { TPH } & 1.65054 \quad .999991\end{array}$

;

Instrument 1.D.: FTIR-1000C

Analyzed on: 09-JUL-1997

Date and time on printout is the day and time copy was printed.

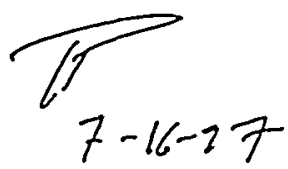

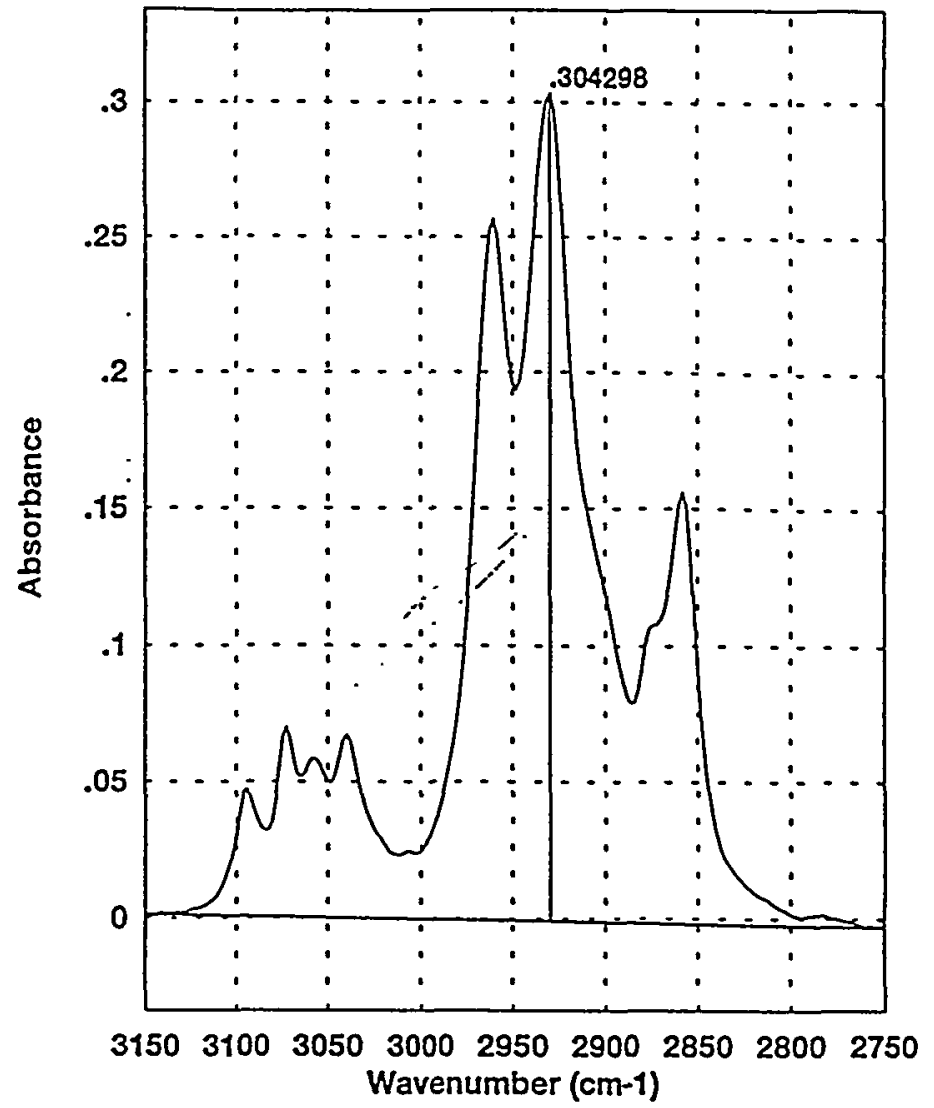

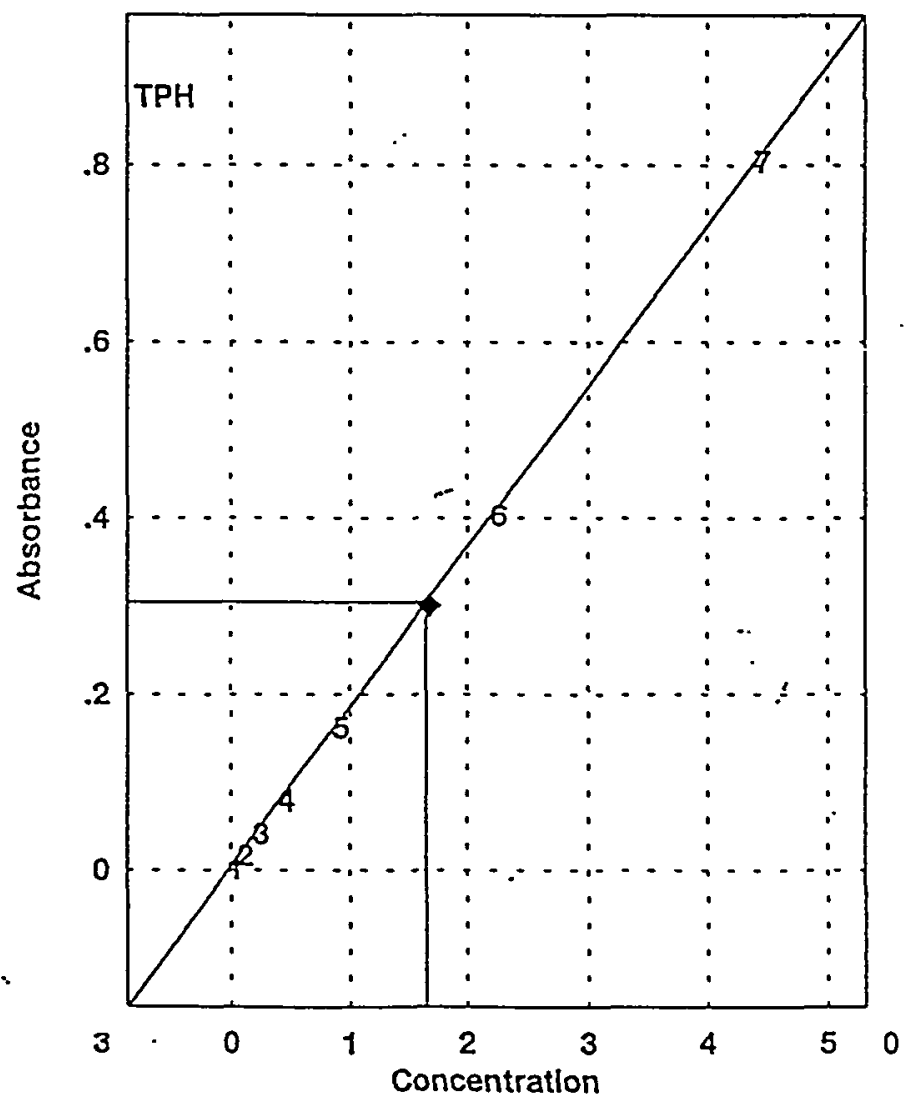


QuantBasic Report

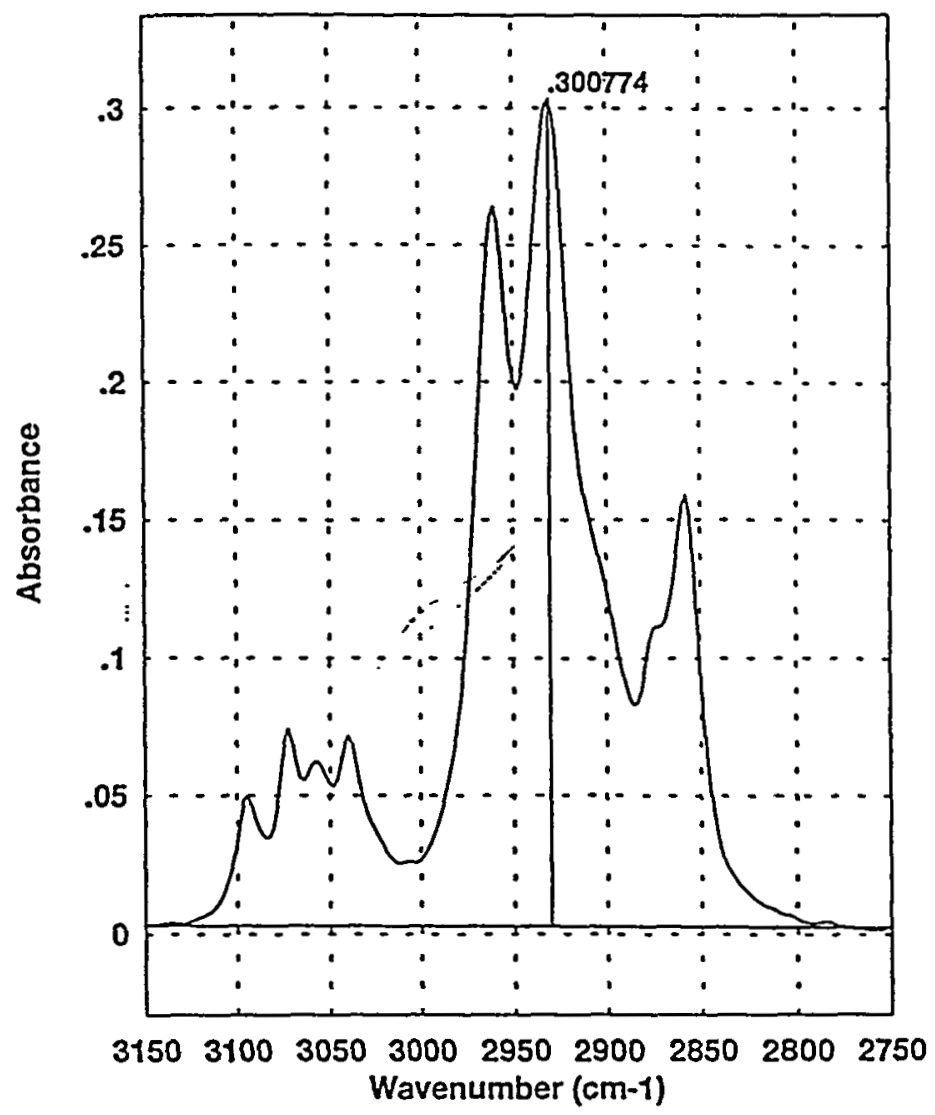

Instrument 1.D.: FTIR-1000C

Analyzed on: 09-JUL-1997

Date and time on printout is the day and time copy was printed.
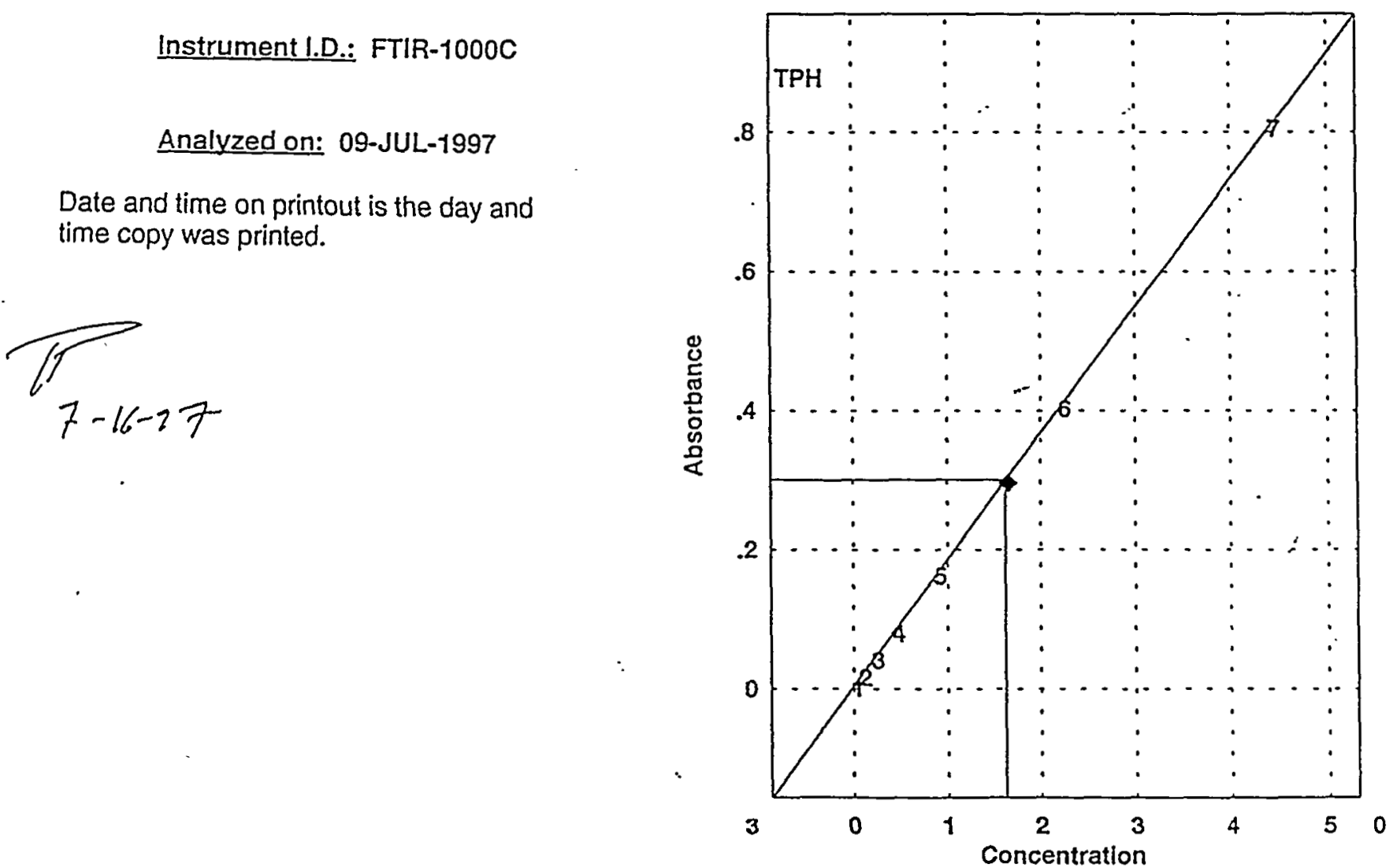
QuantBaslc Report

Quant Type: Multipoint $\quad$ 7/15/97 4:56 pm

Method: C:LPE1000GSIDATAL7036TTPH.Q

Sample: c:lpe1000gsidatal97036lcalcurve.spc

Analyst: TP

Sample ID: $97 \overline{502898}$

Componẹnt Concen R-Squared

$\begin{array}{lll}\text { TPH } & \cdot 048332 \quad .999991\end{array}$

Instrument I.D.: FTIR-1000C

Analyzed on: 09-JUL-1997

Date and time on printout is the day and time copy was printed.

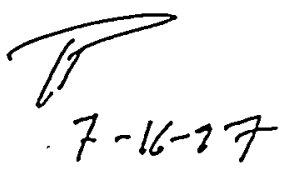

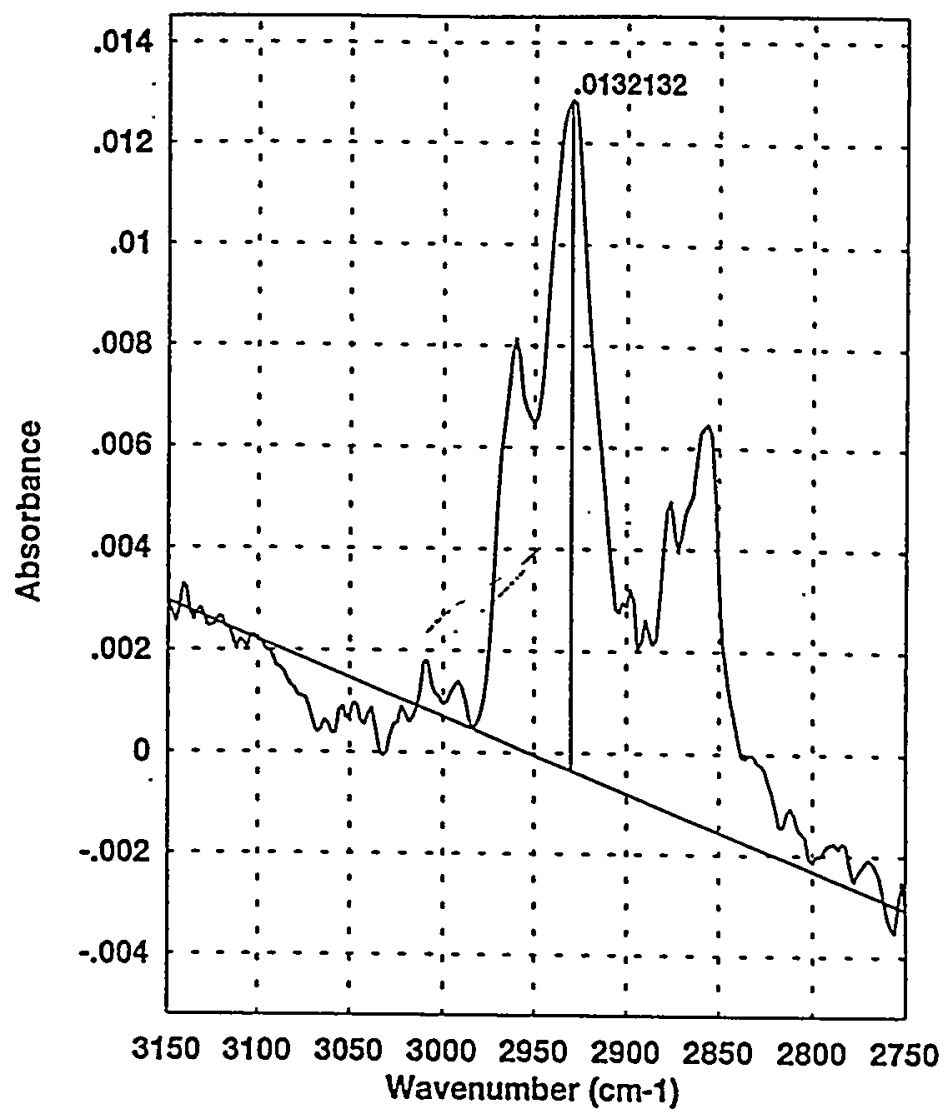

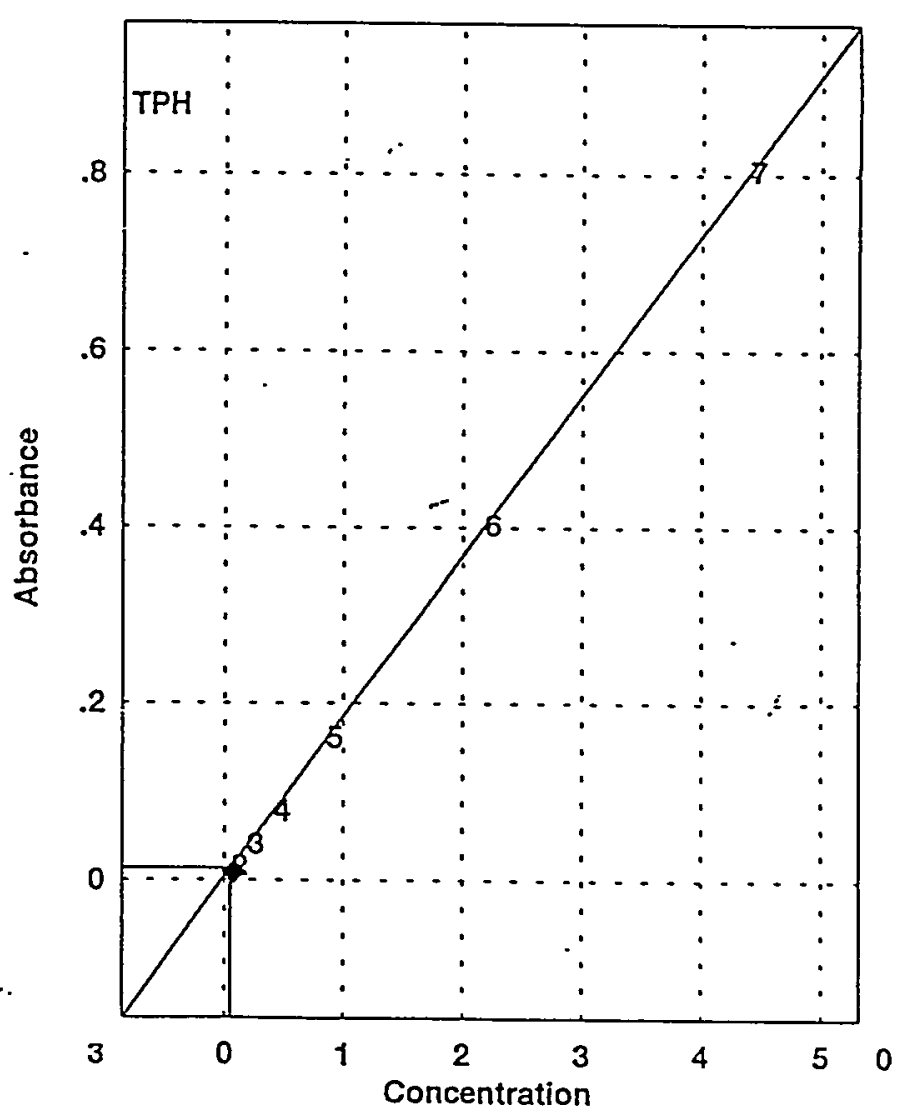


QuantBasic Report

Quant Type: Multlpoint $\quad 7 / 15 / 974: 55$ pm

Method: C:IPE1000GSDATAL7036TPH.Q

Sample: c:lpe1000gsldatal97036lcalcurve.spc

Analyst: TP

Sample 1D: $97 \hat{4} 02899$

$7-2-91$

Componeint Concen R-Squared

TPH $\quad .023811 \quad .999991$

Instrument I.D.: FTIR-1000C

Analyzed on: 09-JUL-1997

Date and time on printout is the day and time copy was printed.

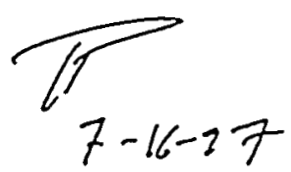

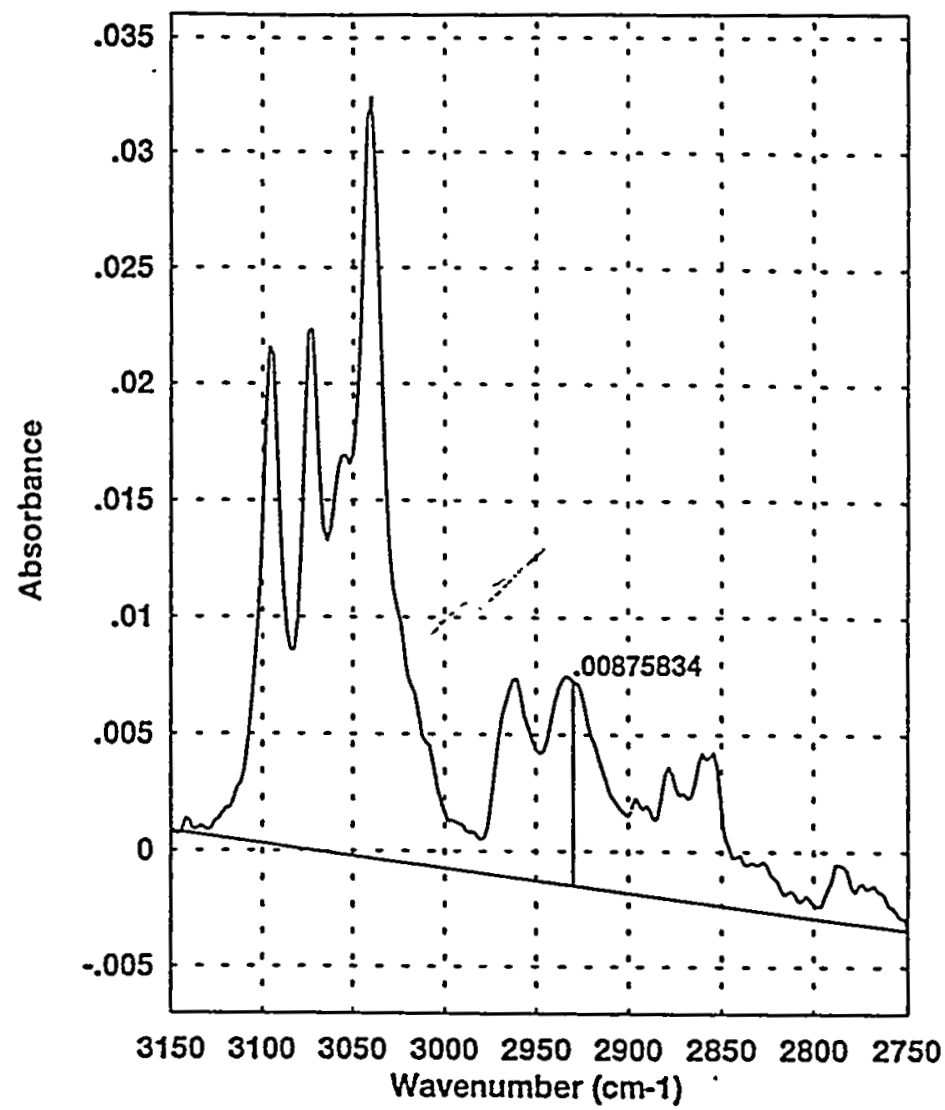

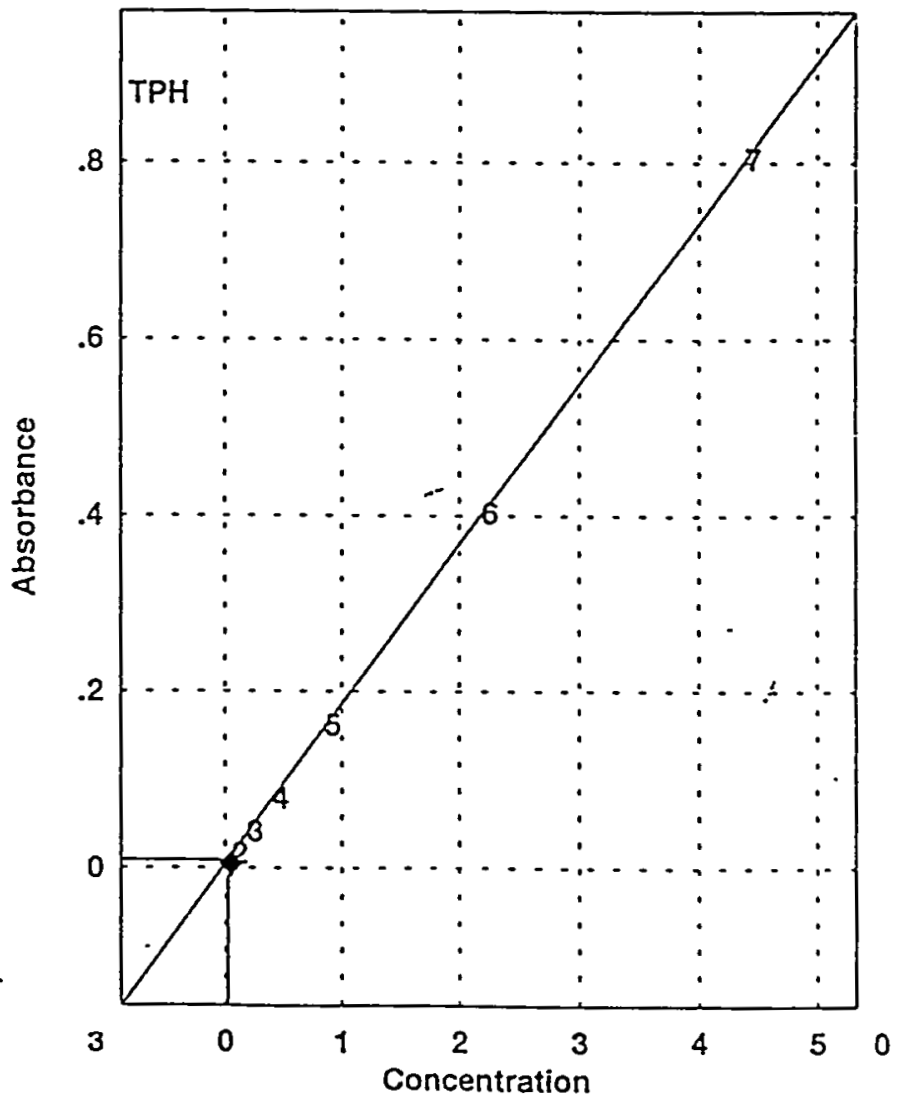




\section{QuantBasic Report}

\section{Quant Type: Multlpolnt $\quad$ 7/15/97 4:55 pm}

\section{Method: C:LPE1000GSLATAL7036TPH.Q}

Sample: c:lpe1000gsidatal97036lcalcurve.spc

Analyst: TP

Sample ID: 97402900

Fient

Componẹnt Concen R-Squared

TPH

$.0781683 \quad .999991$

Instrument I.D.: FTIR-1000C

Analyzed on: 09-JUL-1997

Date and time on printout is the day and time copy was printed.

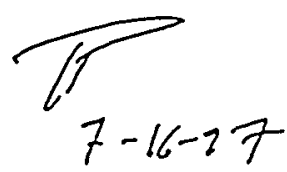

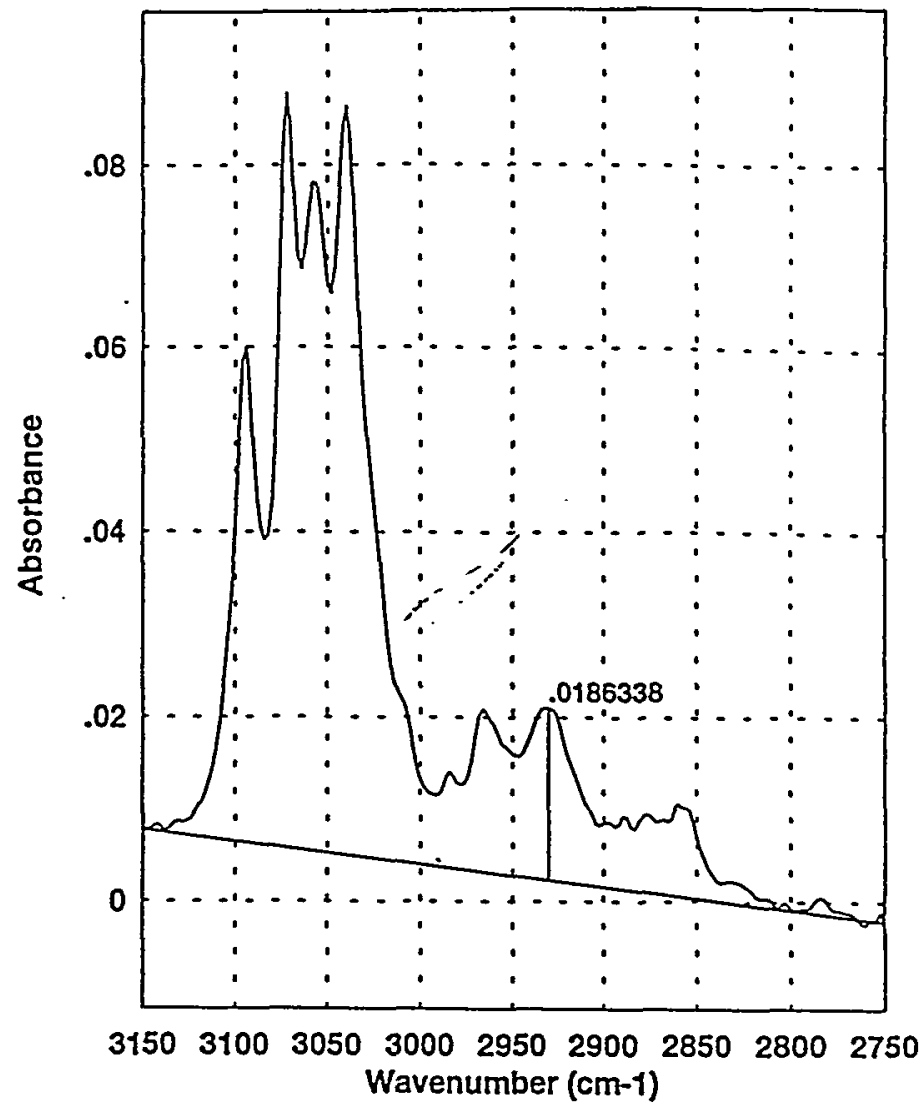

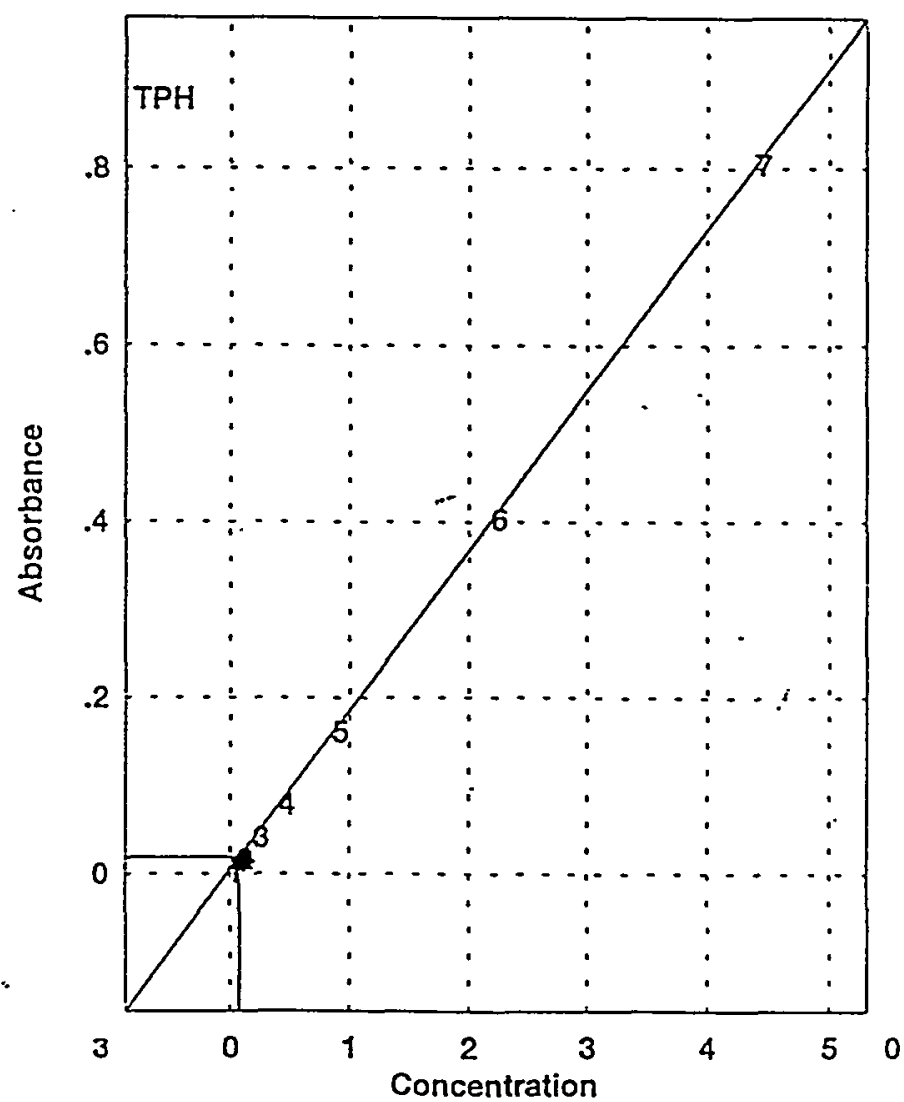




\section{QuantBasic Report}

Quạnt Type: Multlpoint $\quad$ 7/15/97 4:55 pm

Method: C:LPE10DOGSIDATAL7036ITPH.Q

Sample: c:lpe1000gsidatal97036lcalcurve.spc

Analyst: TP

Sample 1D: 97 CO02901

Componẹnt Concen R-Squared

$\begin{array}{lll}\text { TPH } & .0186285 & .999991\end{array}$

Instrument I.D.: FTIR-1000C

Analyzed on: 09-JUL-1997

Date and time on printout is the day and time copy was printed.

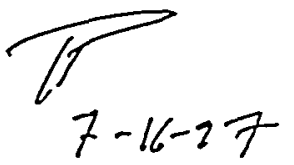

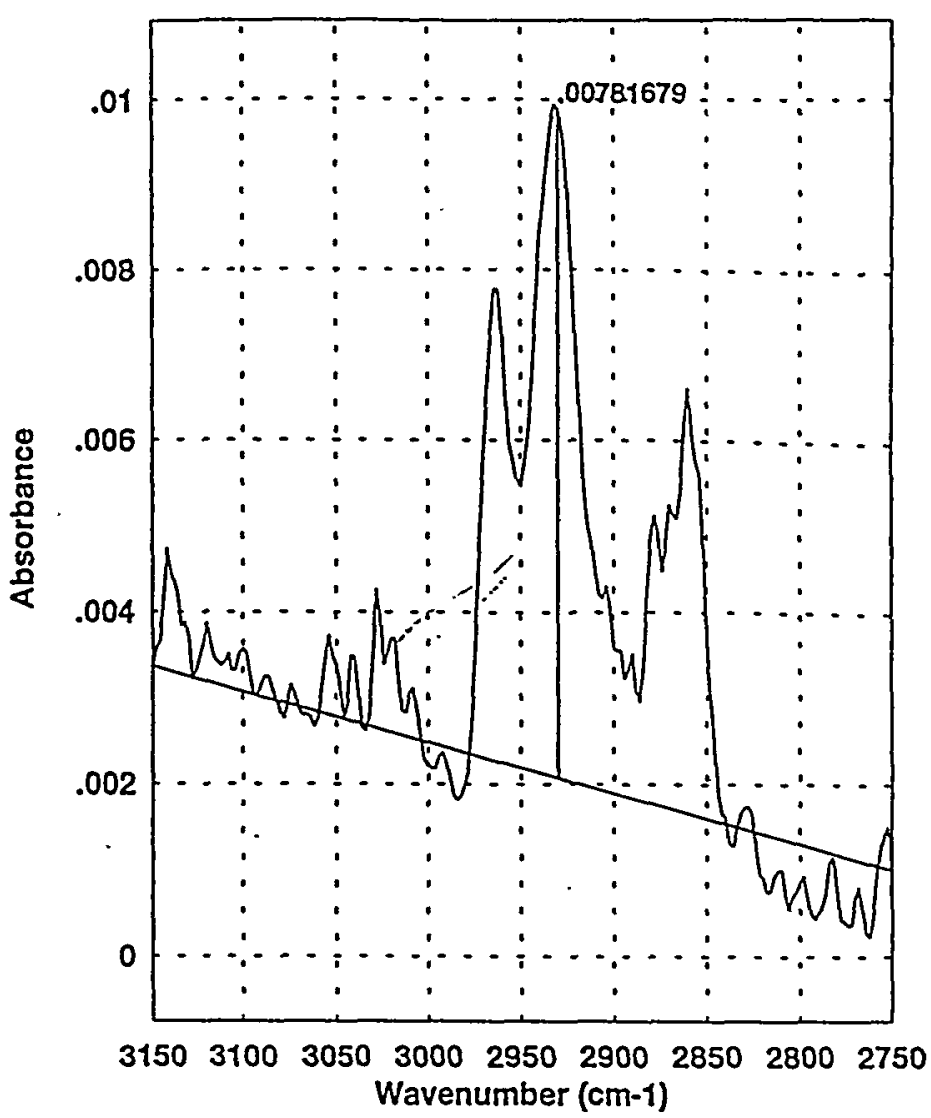

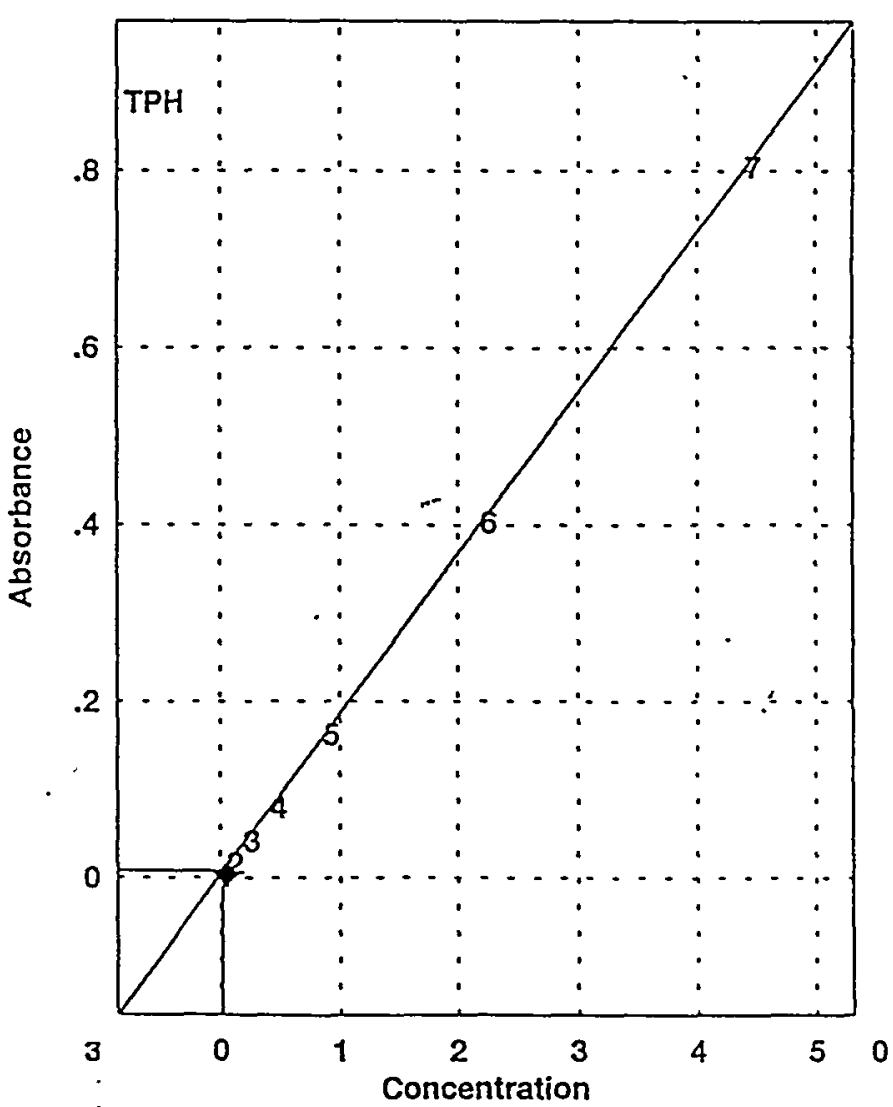


QuantBaslc Report

Quant Type: Multipoint $\quad 7 / 15 / 975: 03 \mathrm{pm}$

Method: C:IPE1000GSIDATAL703GITPH.Q

Sample: c:lpe1000gsldatalo7036lcalcurve.spc

Analyst: TP

Sample ID: CCV

Componẹnt Concen R-Squared

$\begin{array}{lll}\text { TPH } & 221835 \quad .999991\end{array}$

Instrument I.D.: FTIR-1000C

Analyzed on: 09-JUL-1997

Date and time on printout is the day and time copy was printed.

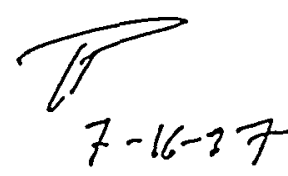

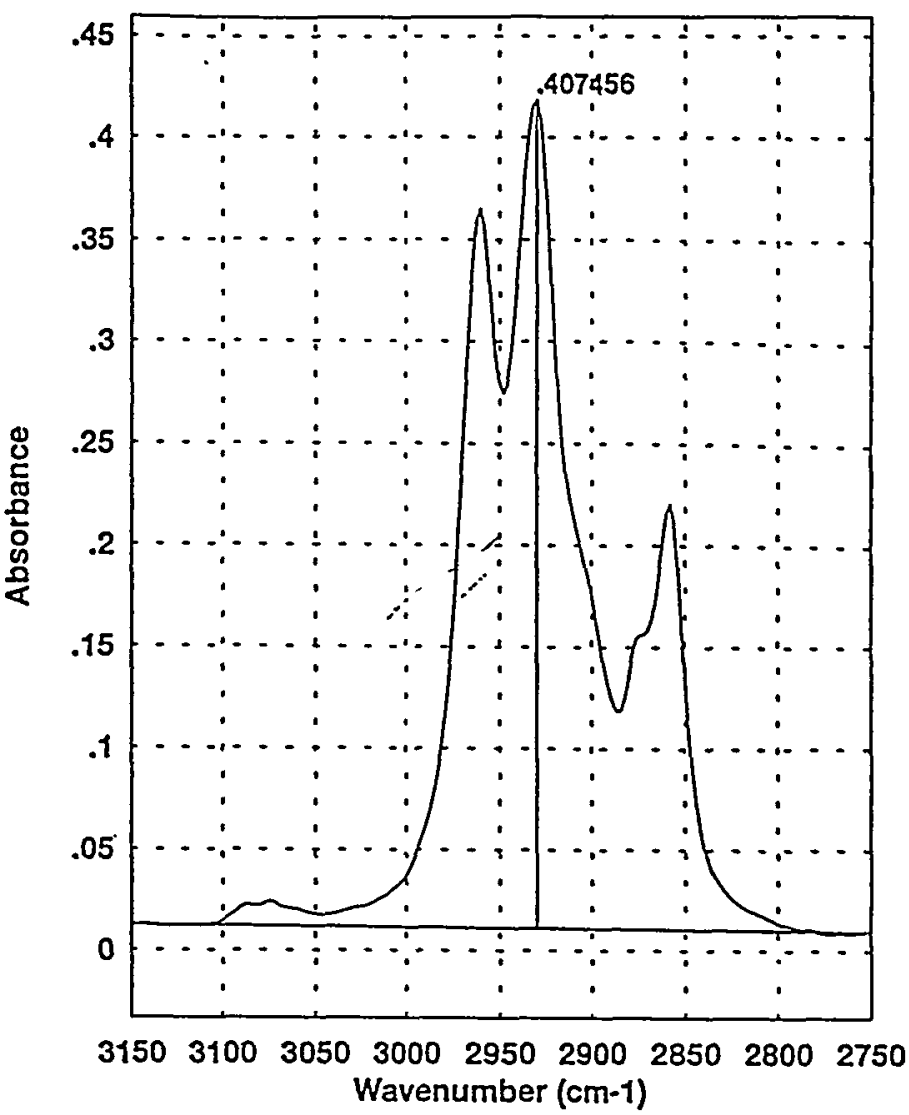

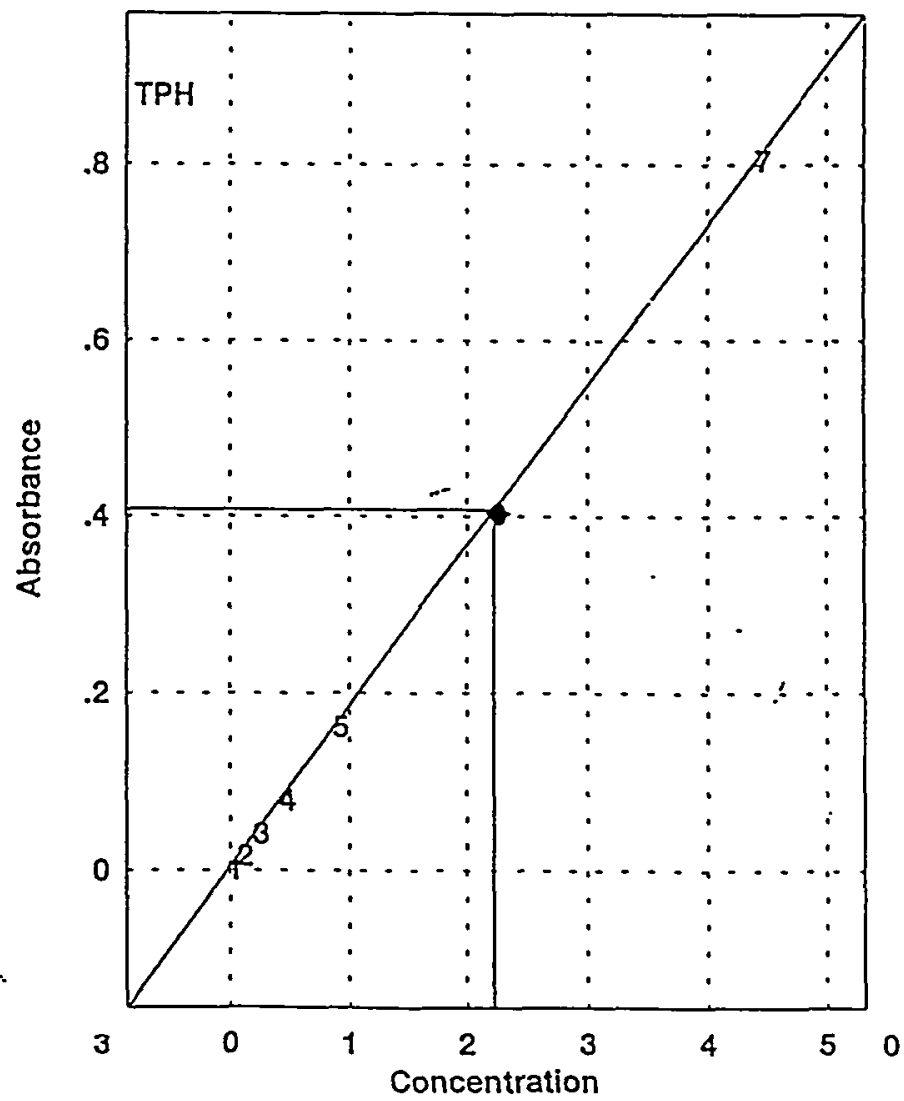


QuantBasic Report

Quant Type: Multlpoint $\quad$ 7/15/97 5:04 pm

Method: CilPE1000GSIDATAL7036TTPH.Q

Sample: c:lpe1000gsidatal97036lcalcurve.spc

Analyst: TP

Sample ID: CCB

Component Concen R-Squared

TPH $\quad .0266641 \quad .999991$

Instrument 1.D.: FTIR-1000C

Analyzed on: 09-JUL-1997

Date and time on printout is the day and time copy was printed.

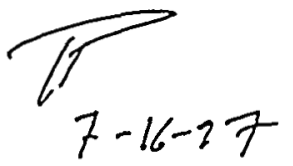

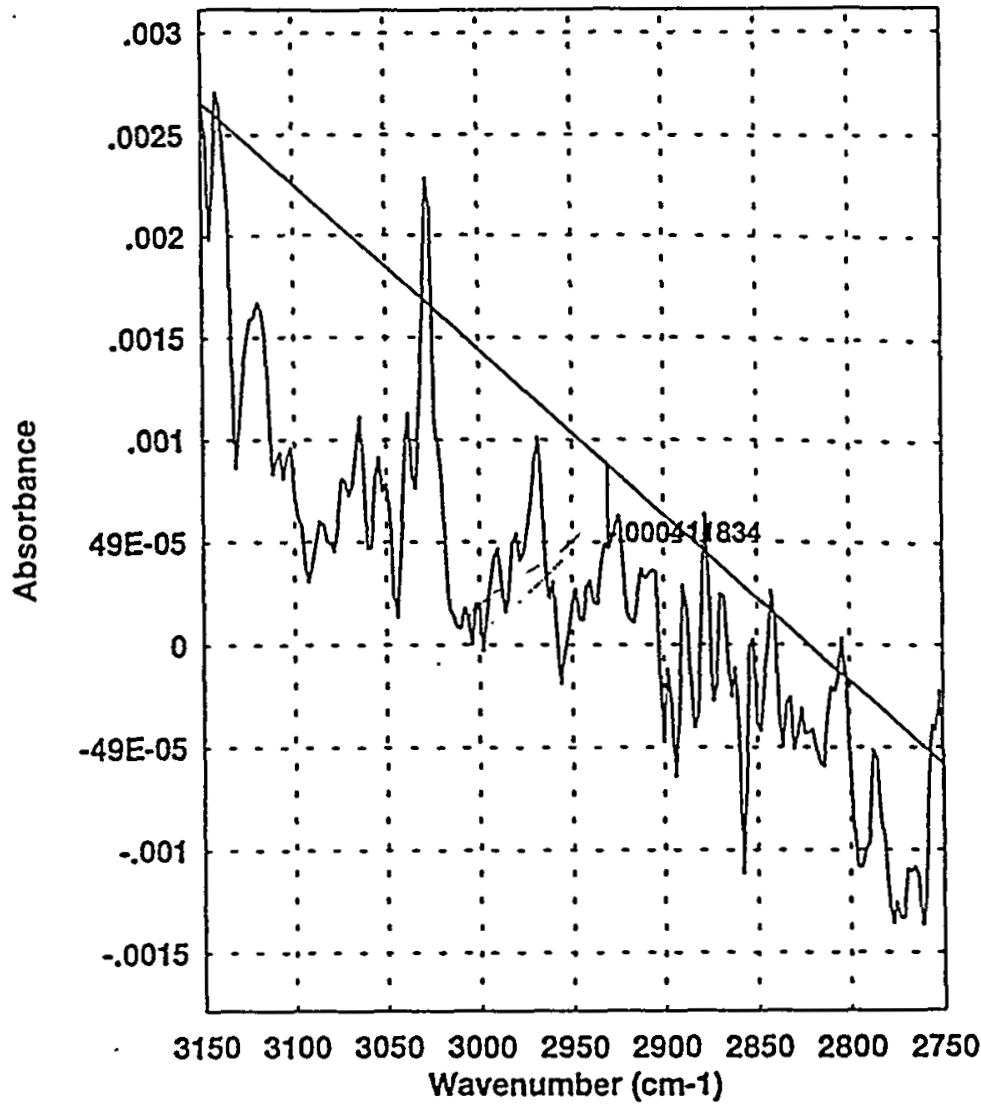

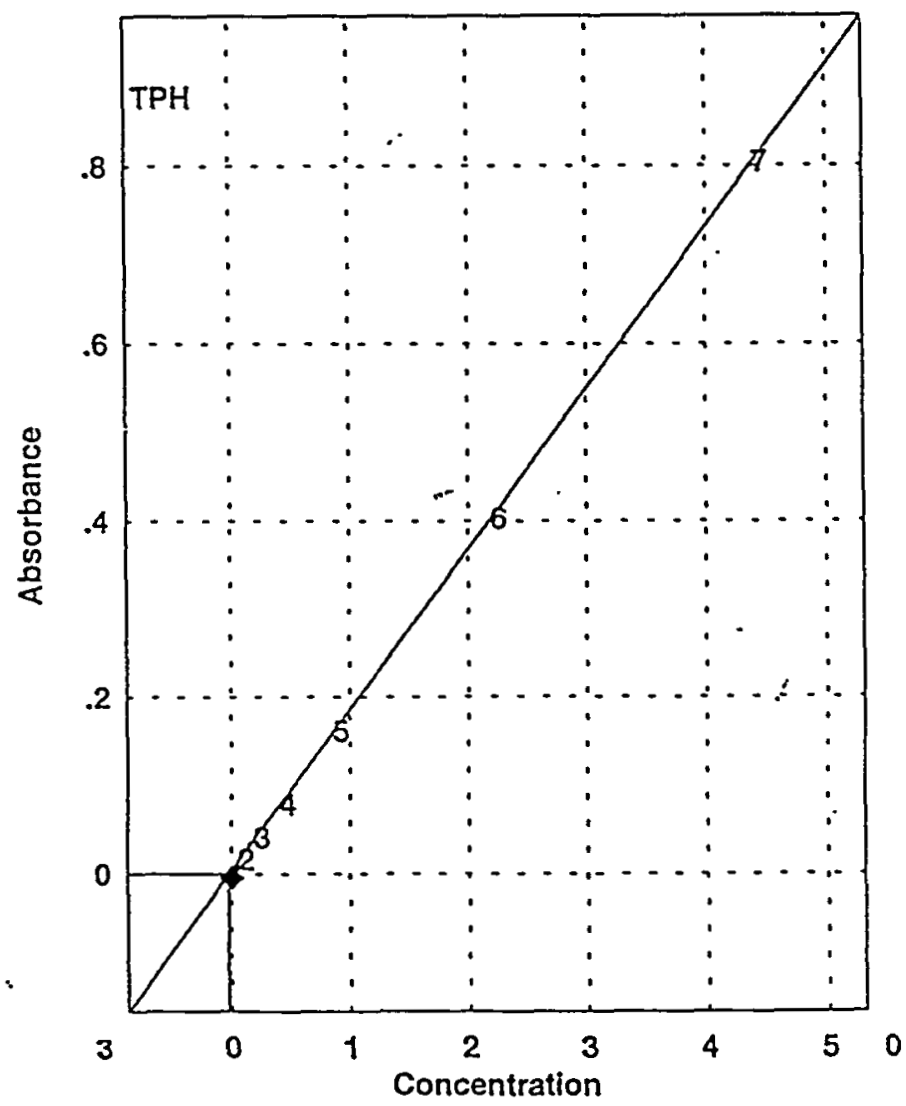


QuántBasic Report

Quant Type: Multipolnt $\quad 7 / 15 / 97$ 4:51 pm

Method: C:LPE1000GSIDATAL703GITPH.Q

Sample: c:lpe1000gsidatal07036lcalcurve.spc

Analyst: TP

Sampie ID: QC-1365341

Componẹt Concen R-Squared

$\begin{array}{lll}\text { TPH } & 1.06209 & .999991\end{array}$

Instrument I.D.: FTIR-1000C

Analyzed on: 09-JUL-1997

Date and time on printout is the day and time copy was printed.

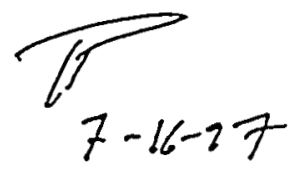

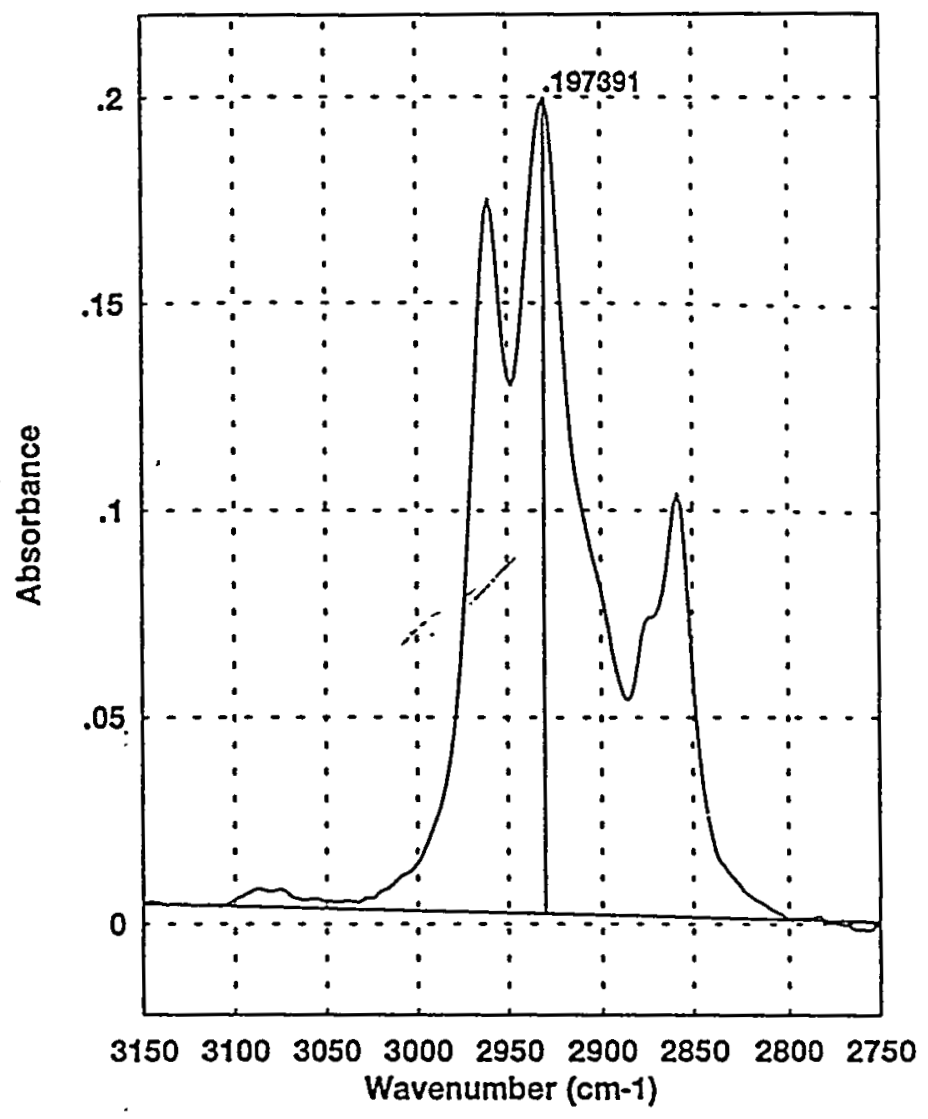

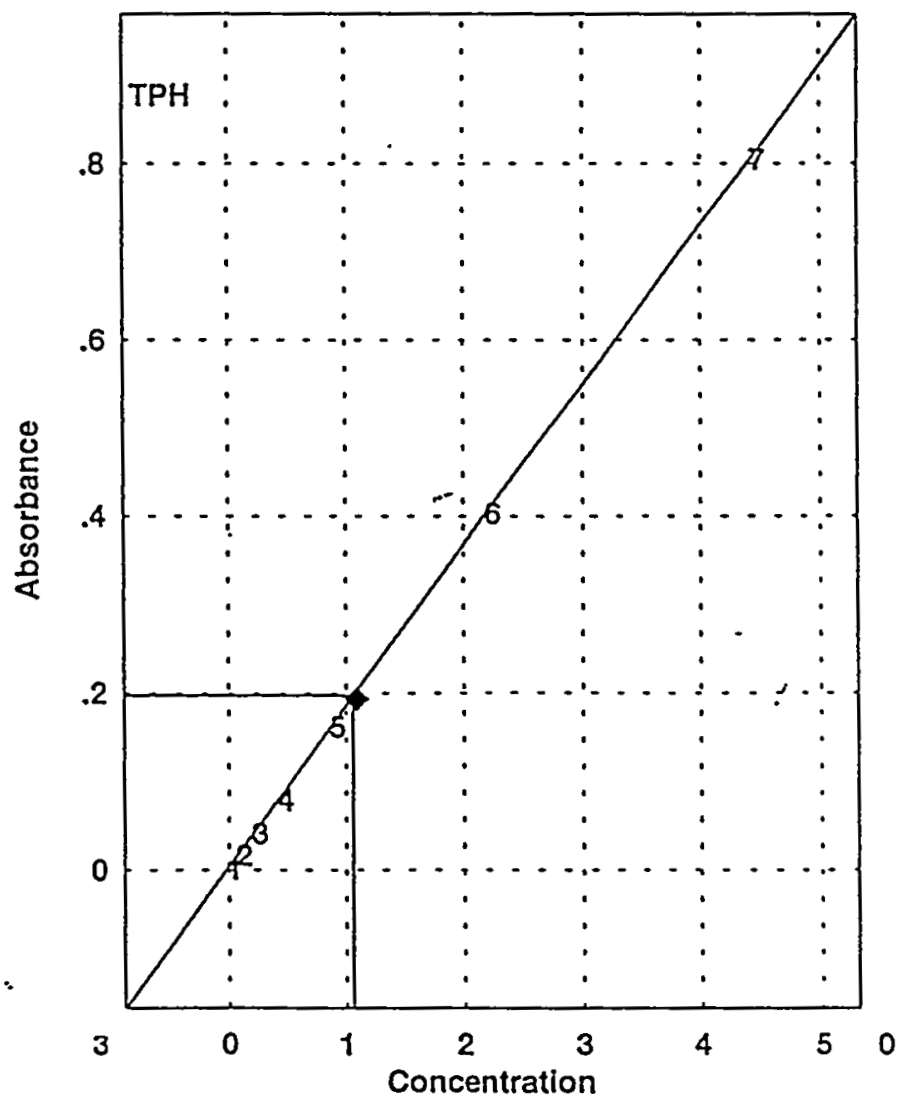

516 
QuantBaslc Report

Quant Type: Multlpolnt $\quad 7 / 15 / 974: 52 \mathrm{pm}$

Method: C:IPE1000GSIDATAL7036TPH.Q

Sample: c:lpe1000gsidatal97036lcalcurve.spc

Analyst: TP

Sample ID: BL-1136534-1

Component Concen R-Squared

TPH $\quad .00737395 \quad .999991$

Instrument 1.D.: FTIR-1000C

Analyzed on: 09-JUL-1997

Date and time on printout is the day and time copy was printed.

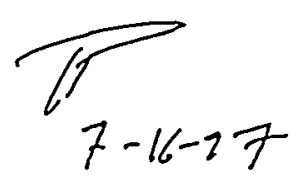

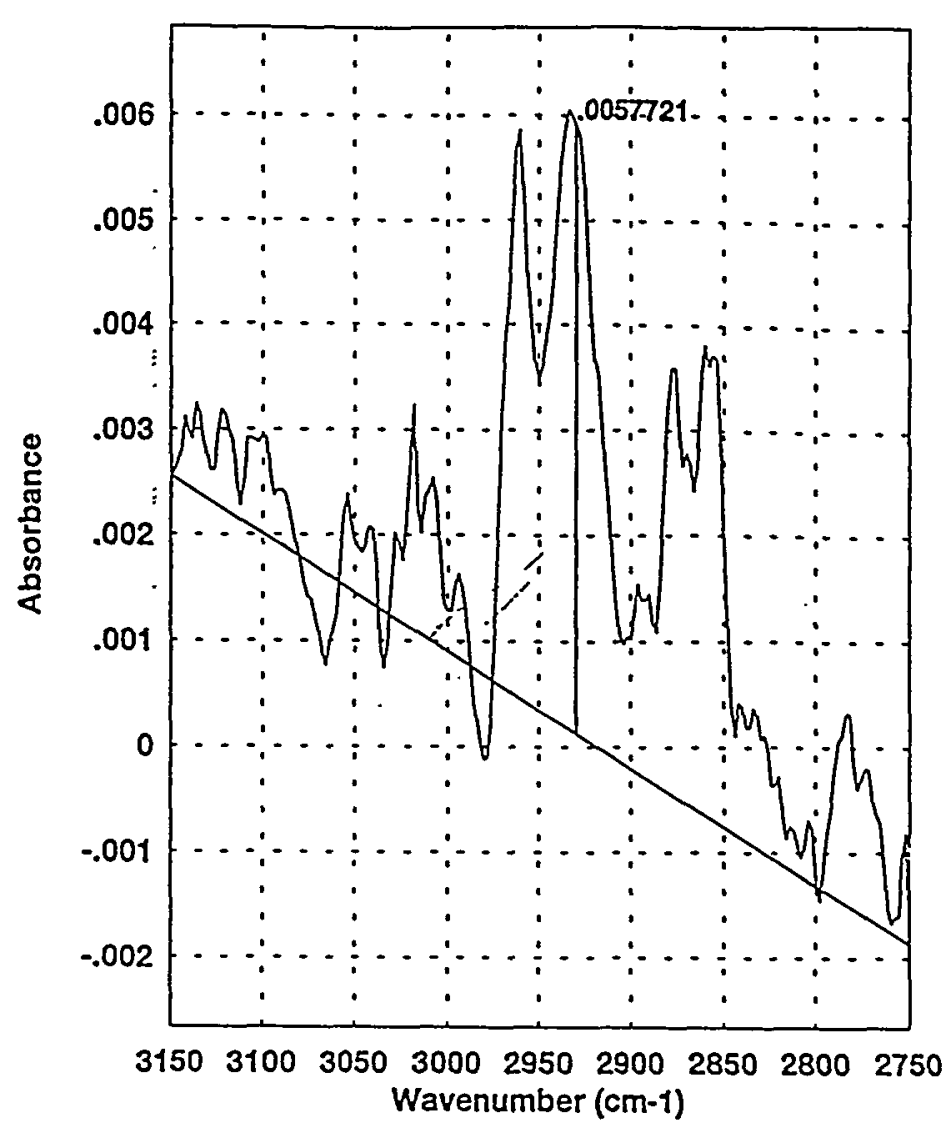

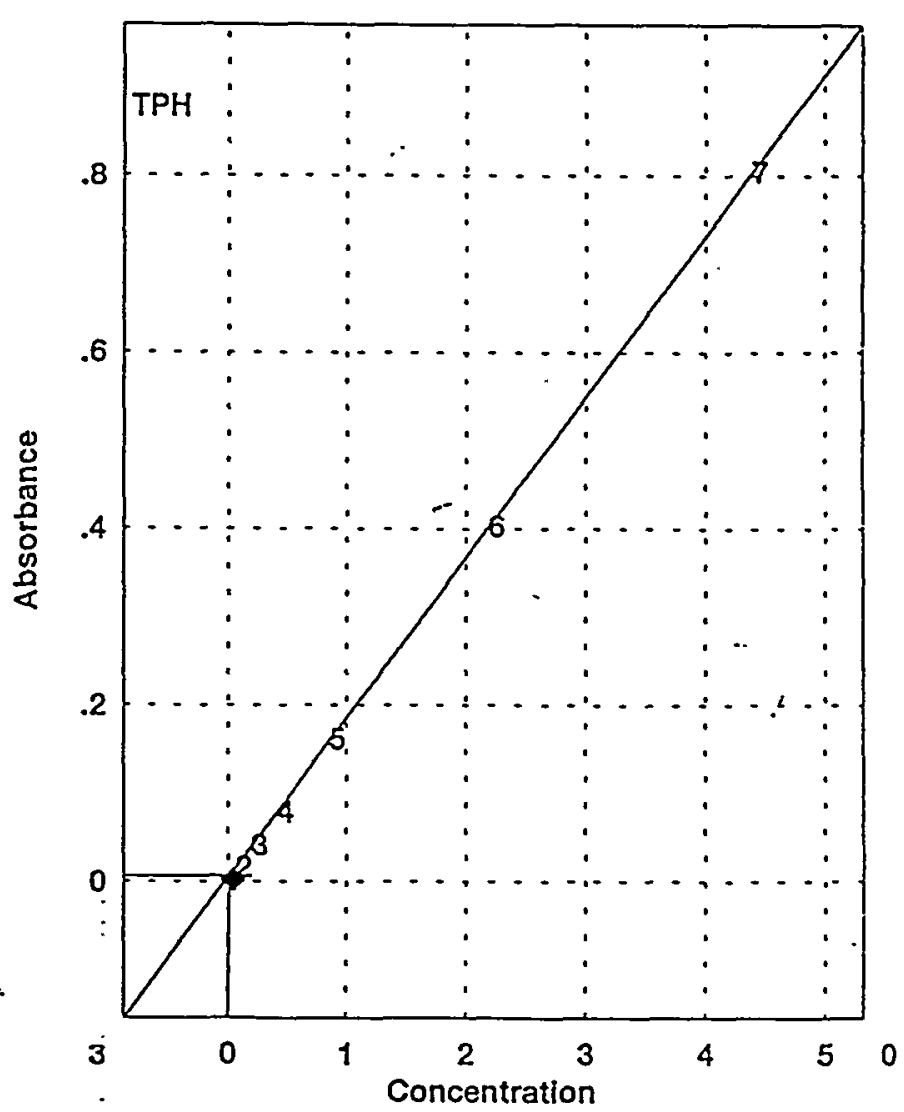




\section{QuantBaslc Report}

Quant Type: Multlpolnt $\quad$ 7/15/97 4:54 pm

Method: C:IPE1000GSLDATALTO3GTPH.Q

Sample: c: $p e 1000$ gsldatalo7036lcalcurve.spc

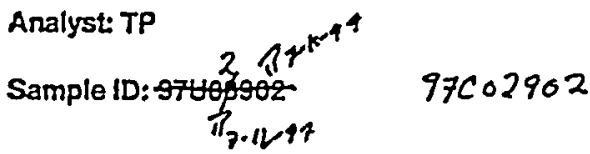

Componẹint Concen. R-Squared

TPH

$.0584462 \quad .999991$

Instrument I.D.: FTIR-1000C

Analyzed on: 09-JUL-1997

Date and time on printout is the day and time copy was printed.

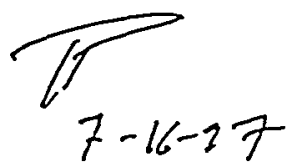

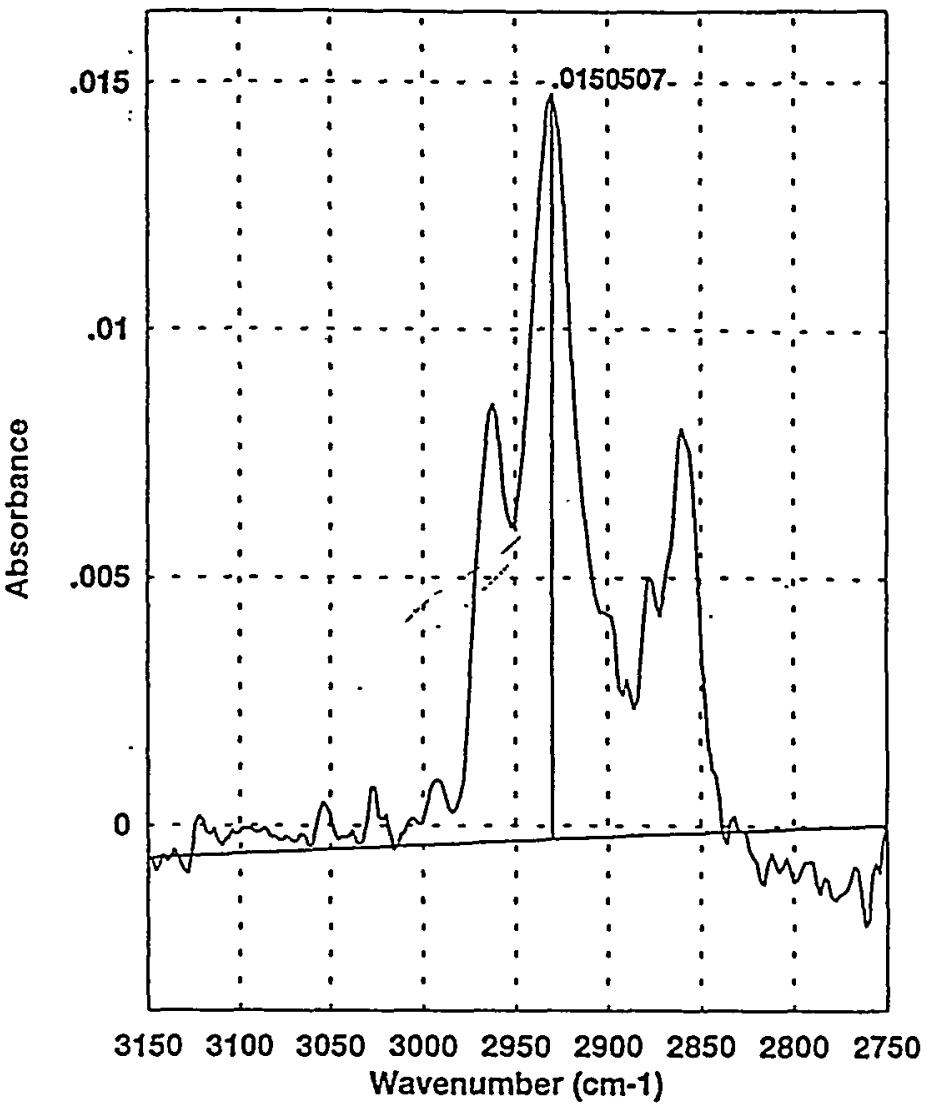

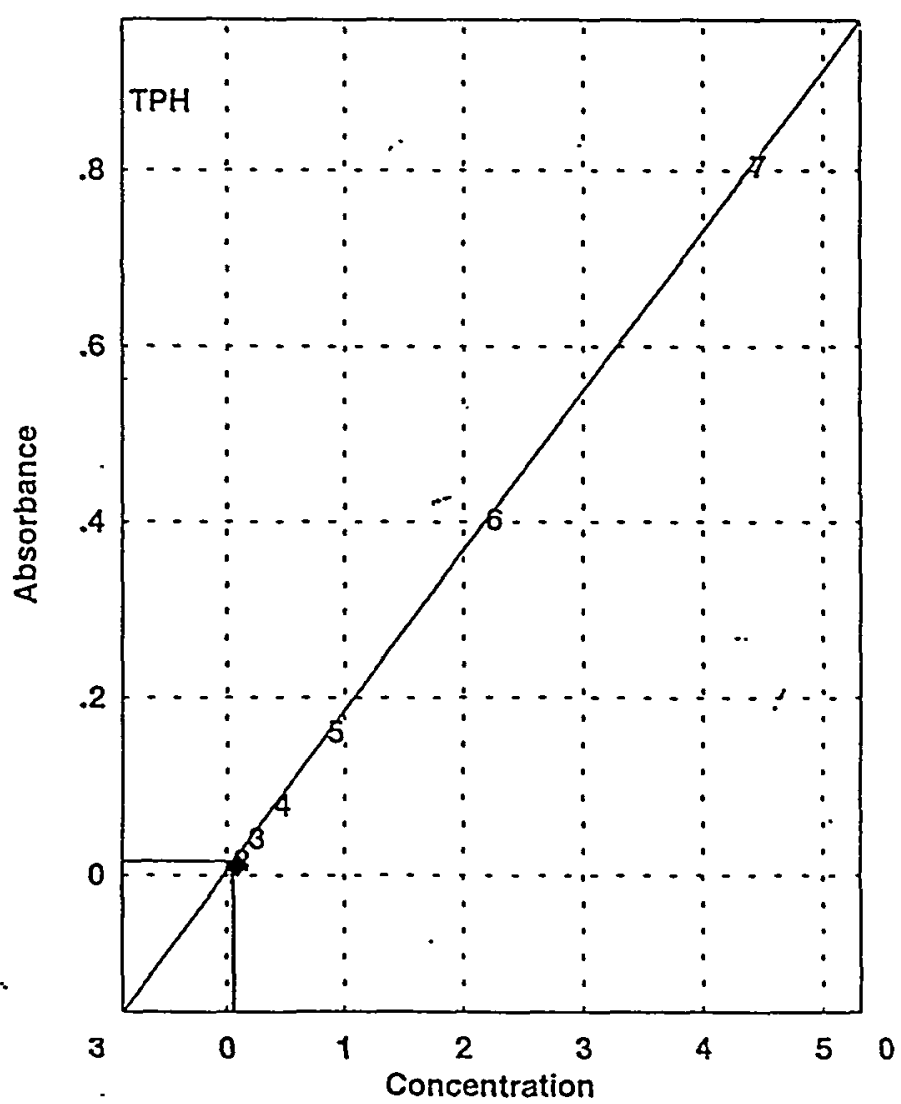


QuantBasie Report

Quant Type: Multipoint $\quad 7 / 15 / 974: 54 \mathrm{pm}$

Method: C:IPE1000GSLATAL703GTPH.Q

Sample: c:lpet00ogsldatalo7036lcalcurve.spc

Analyst: TP

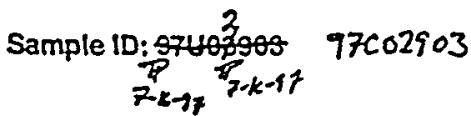

Component Concen R-Squared

TPH

$.0601039 \quad .999991$

Instrument 1.D.: FTIR-1000C

Analyzed on: 09-JUL-1997

Date and time on printout is the day and time copy was printed.

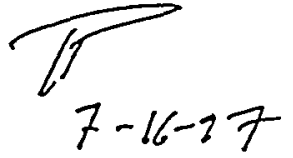

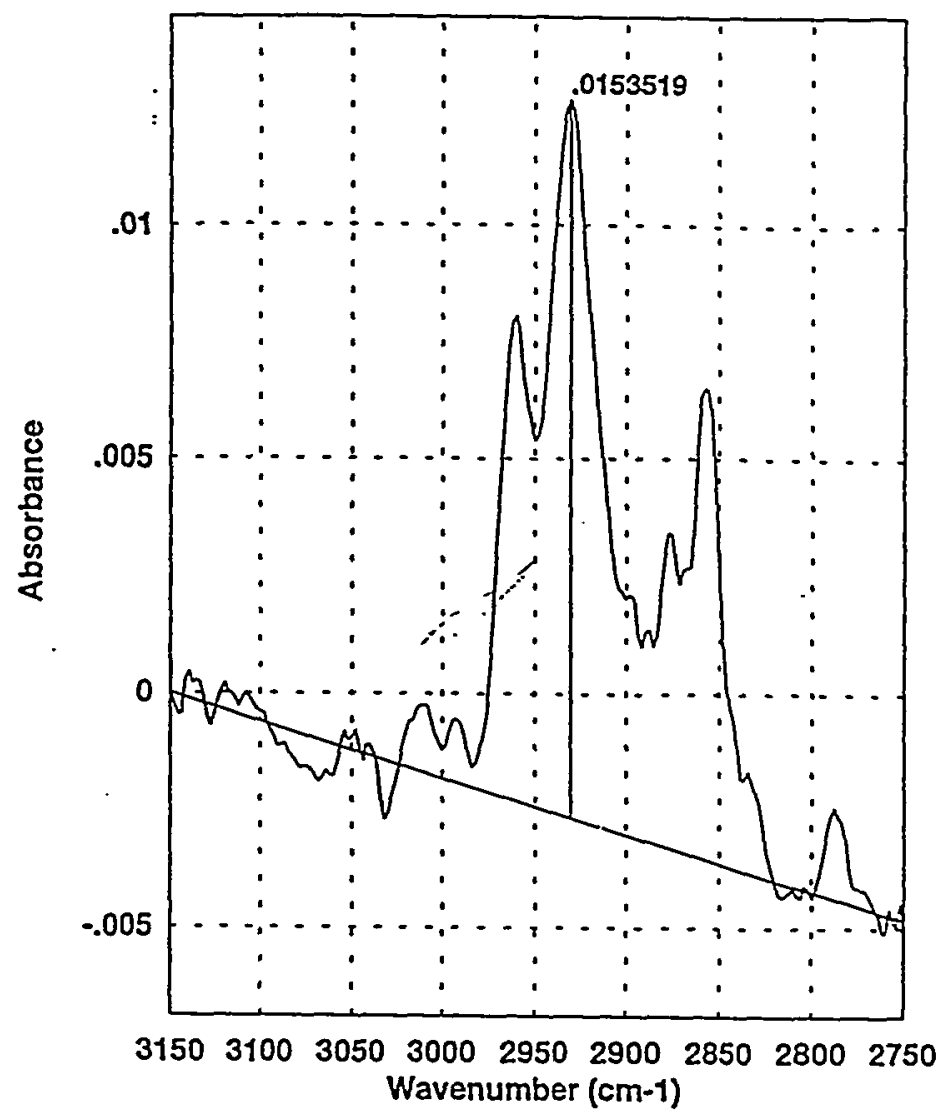

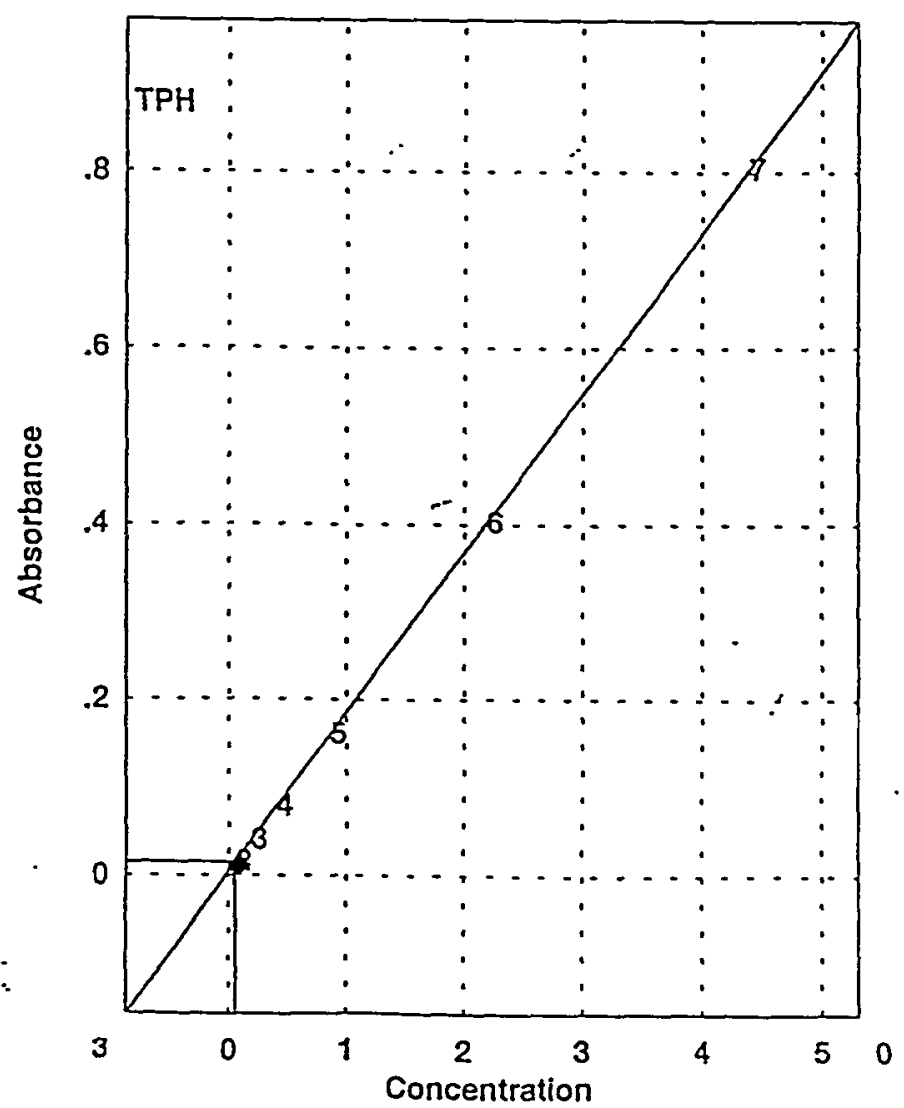




\section{QuantBasic Report}

Quant Type: Multipolnt $\quad 7 / 15 / 974: 54 \mathrm{pm}$

Method: C:IPE1000GSIDATAL70703GITPH.Q

Sample: c:lpe1000gsldatal97036lcalcurve.spc

Analyst: TP

Sample 10:-97492308 77002968

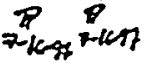

Componẹt Concen R-Squared

$\begin{array}{lll}\text { TPH } & .0630375 \quad .999991\end{array}$

Instrument I.D.: FTIR-1000C

Analyzed on: 09-JUL-1997

Date and time on printout is the day and time copy was printed.

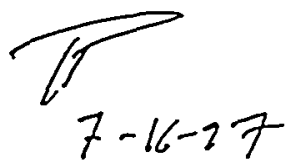

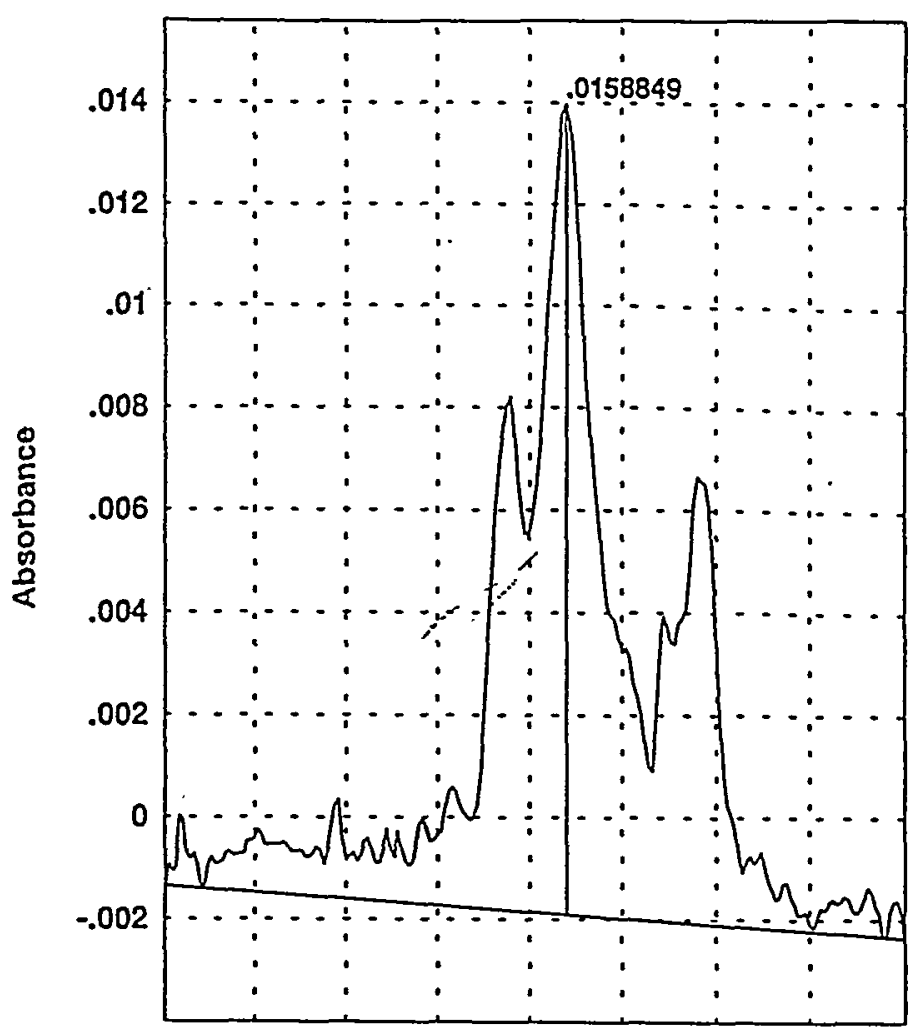

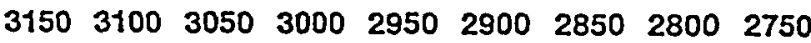
Wavenumber $(\mathrm{cm}-1)$

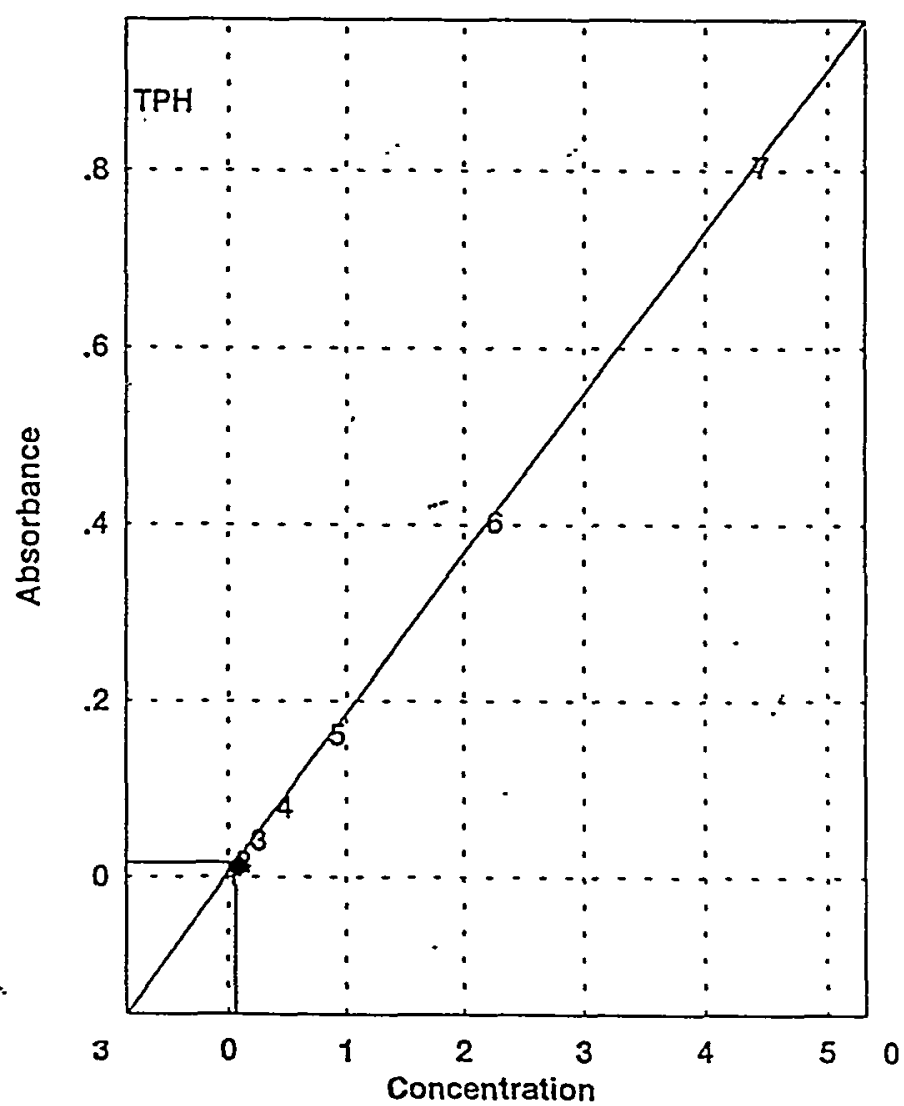


QuantBasic Report

Quant Type: Multlpolnt $\quad 7 / 15 / 97 \quad 4: 53 \mathrm{pm}$

Method: C:IPE1000GSDATAL703GTPH.Q

Sample: c:lpe1000gsidatalo7036lcalcurve.spc

Analyst: TP

Sample ID: $9700^{2} 900 \mathrm{~ms} 97 \mathrm{~m} 02908 \mathrm{~ms}$

Component Concen R-Squared

$\begin{array}{lll} & \text { TPH } \quad 1.66409 \quad .999991\end{array}$

Instrument I.D.: FTIR-1000C

Analyzed on: 09-JUL-1997

Date and time on printout is the day and time copy was printed.

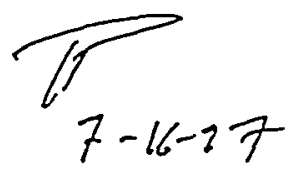

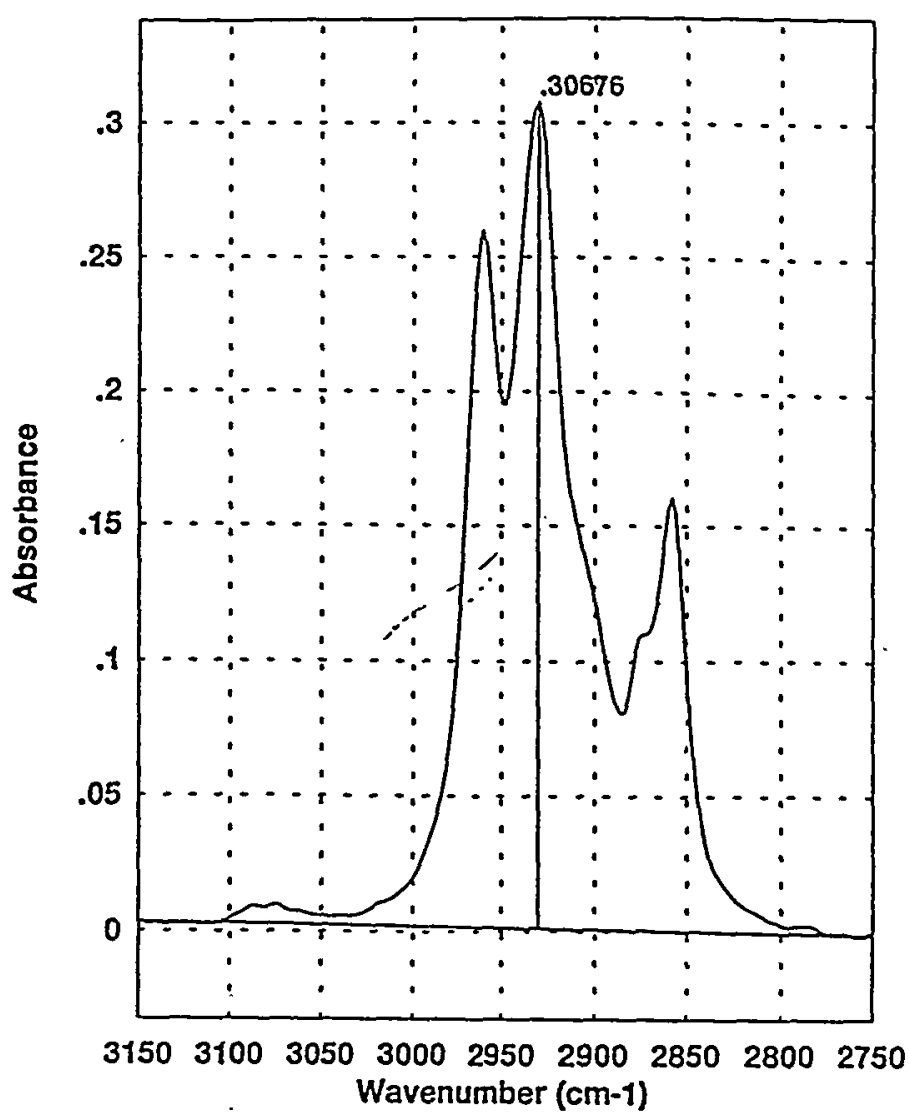

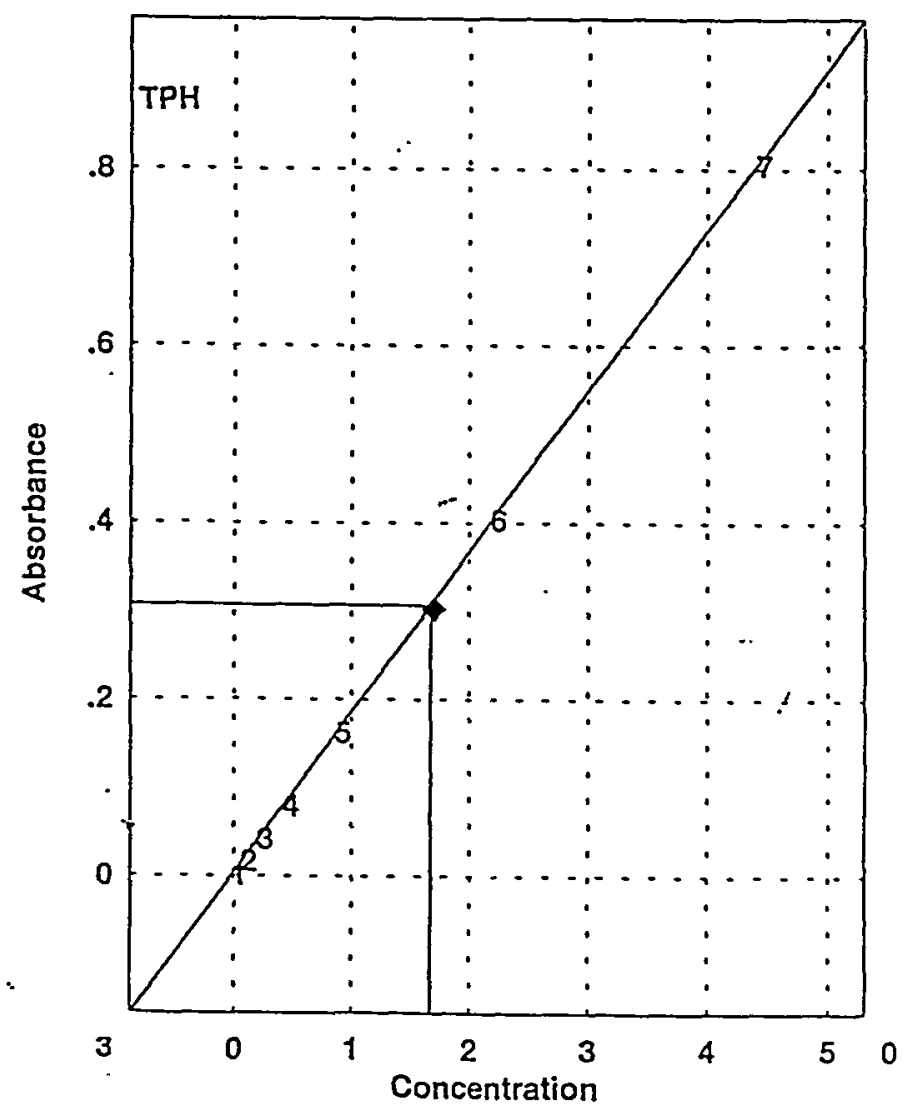


QuantBasic Report

Quant Type: Multlpoint $\quad$ 7/15/97 4:53 pm

Method: C:IPE1000GSIDATAL703GITPH.Q

Sample: c:lpe100ogsidatal97036lcalcurve.spc

Analyst: TP

Sample 1D:07ugionsusD 77602708450

$$
\left.\operatorname{Tip}_{\rightarrow-47}\right)^{3}+6-19
$$

Component Concen R-Squared

$\begin{array}{lll}\text { TPH } & 1.62978 \quad .999991\end{array}$

Instrument 1.D.: FTIR-1000C

Analyzed on: 09-JUL-1997

Date and time on printout is the day and time copy was printed.

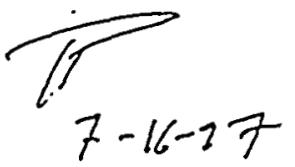

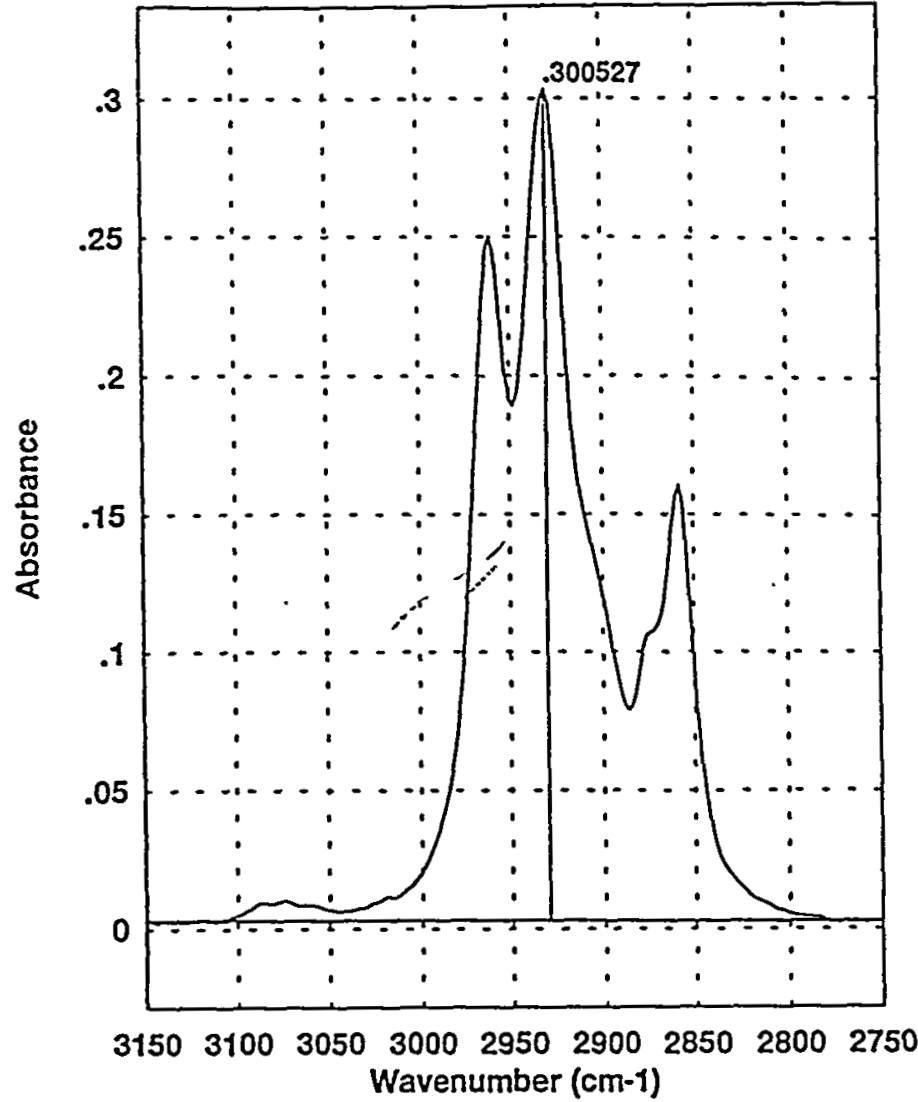

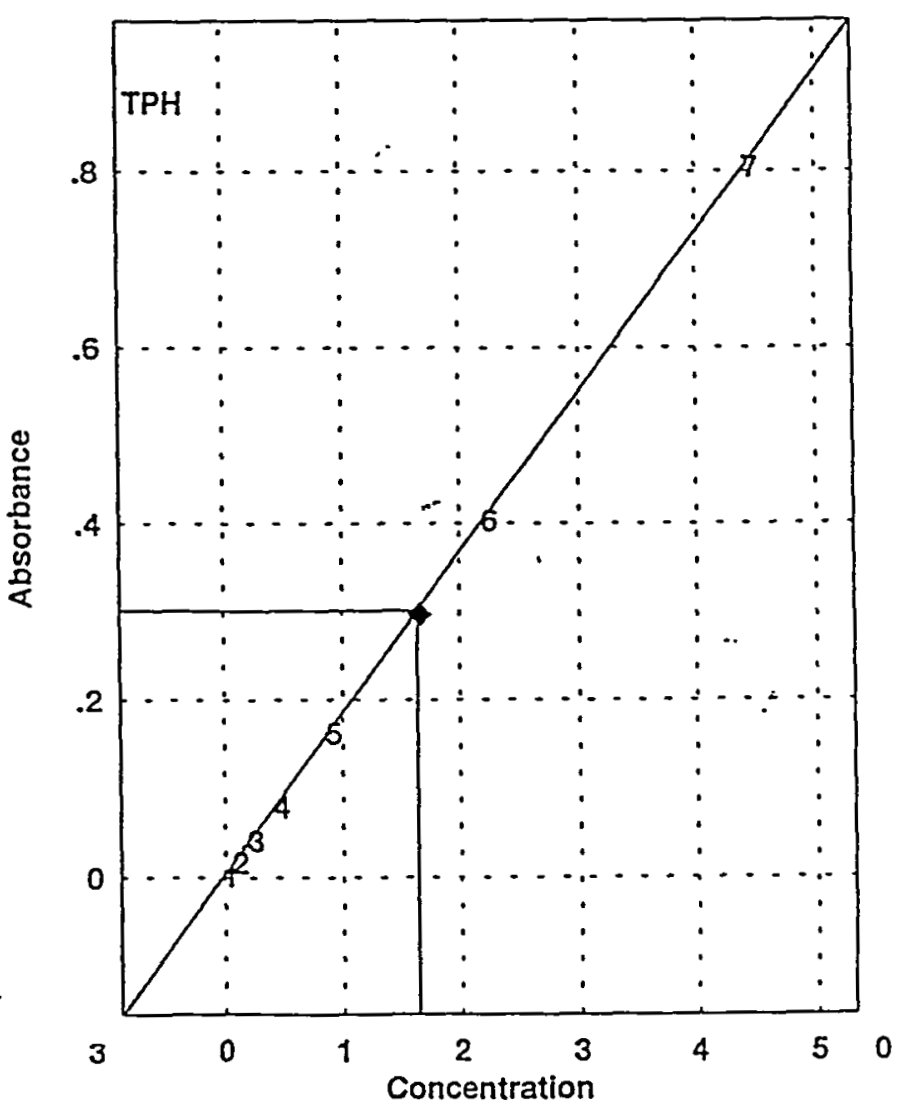


QuantBasic Report

Quant Tyype: Multlpoint $\quad 7 / 15 / 975: 03 \mathrm{pm}$

Method: C:PPE1000GSIDATAL703GTPH.Q

Sample: c:lpe1000gsidatal97036lcalcurve.spc

Analyst: TP

Sample ID: CCV2

Component Concen R-Squared

$\begin{array}{lll}\text { TPH } & 2.21488 \quad .999991\end{array}$

Instrument I.D.: FTIR-1000C

Analyzed on: 09-JUL-1997

Date and time on printout is the day and time copy was printed.

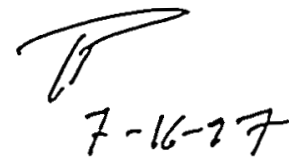

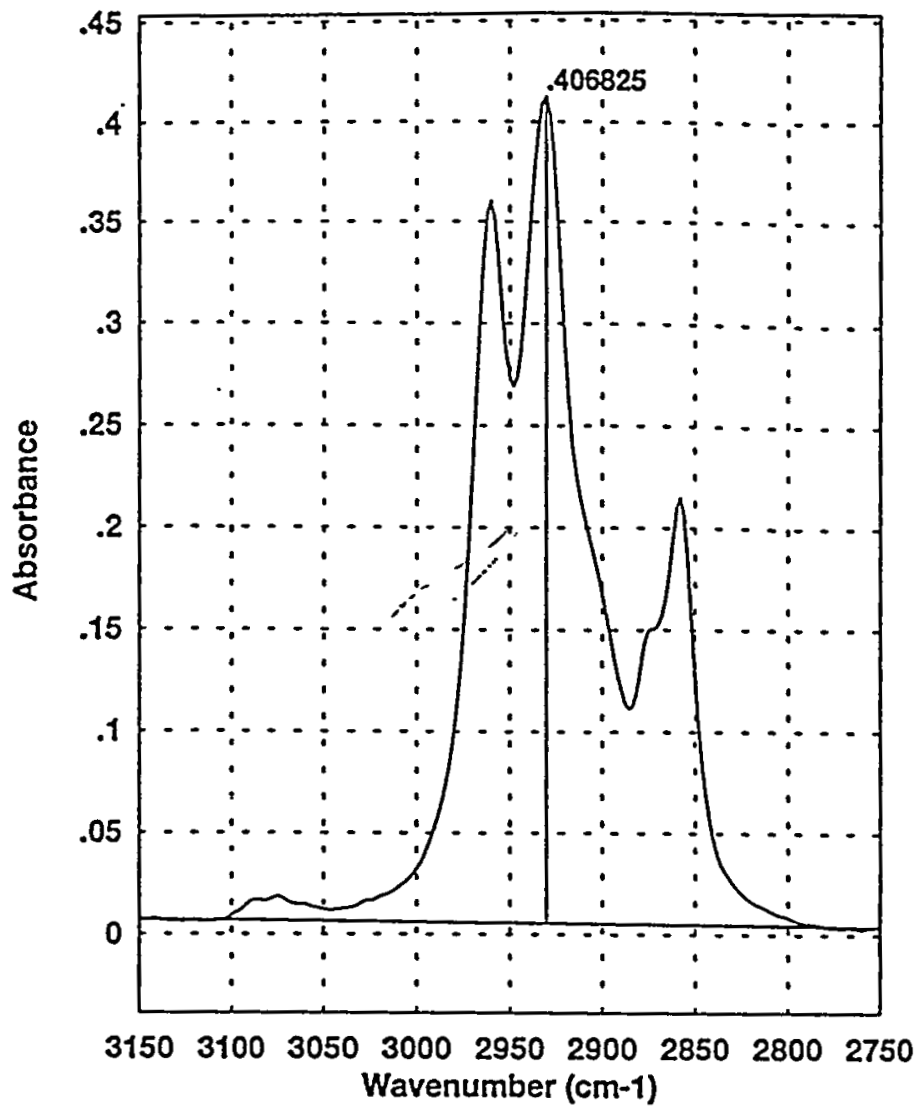

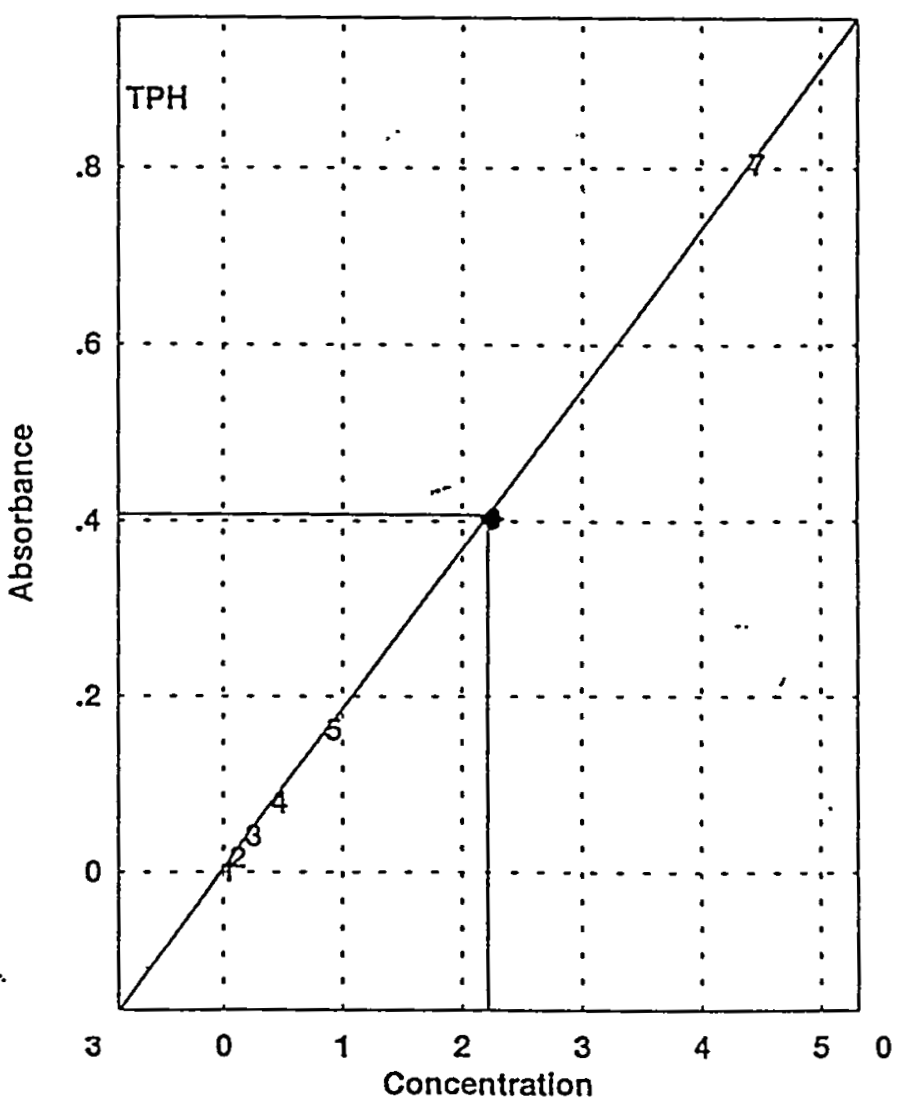


QuantBasic Report

Quant Type: Multipoint $\quad$ 7/15/97 5:03 pm

Method: C:LE1000GSIDATAL703GTPH.Q

Sample: c:1po1000gsldatal97036lcalcurve.spc

Analyst: TP

Sample ID: CCB2

Componẹnt Concen R-Squared

$\begin{array}{lll}\text { TPH } & -.0268956 & .999991\end{array}$

;

Instrument I.D.: FTIR-1000C

Analyzed on: 09-JUL-1997

Date and time on printout is the day and time copy was printed.

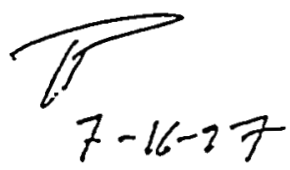

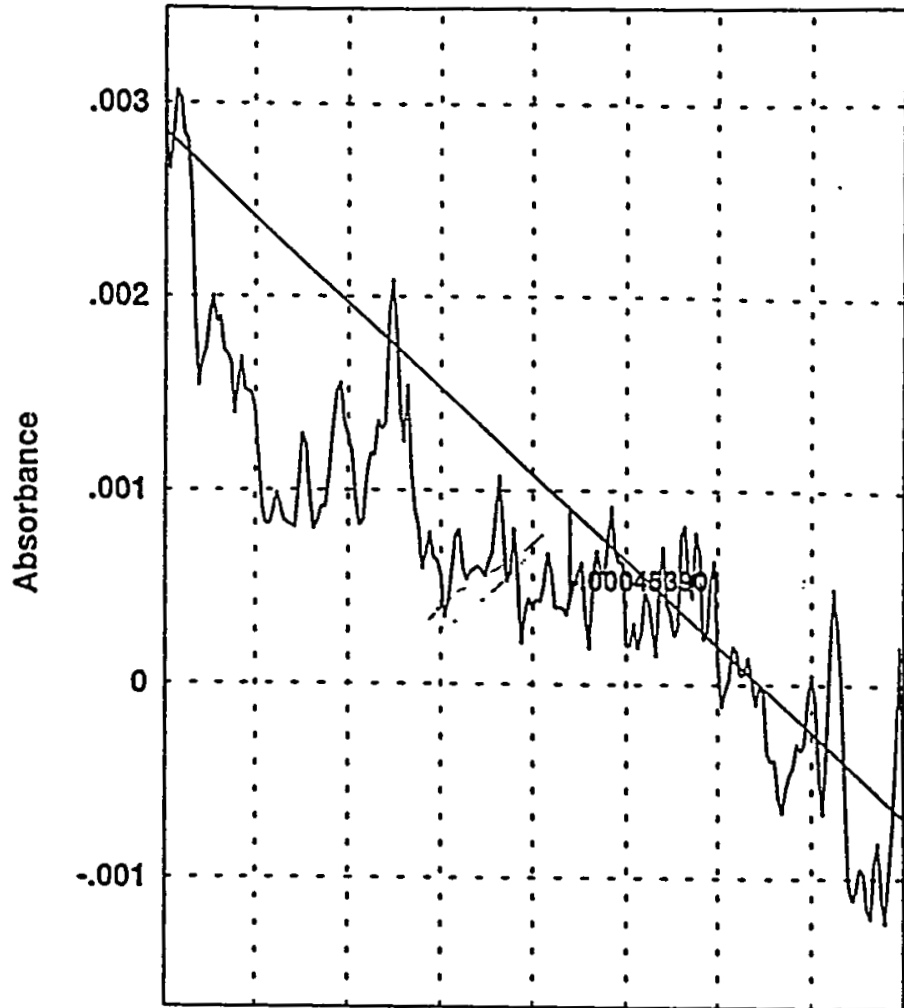

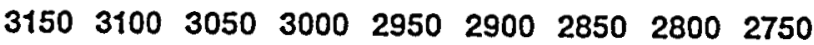
Wavenumber $(\mathrm{cm}-1)$

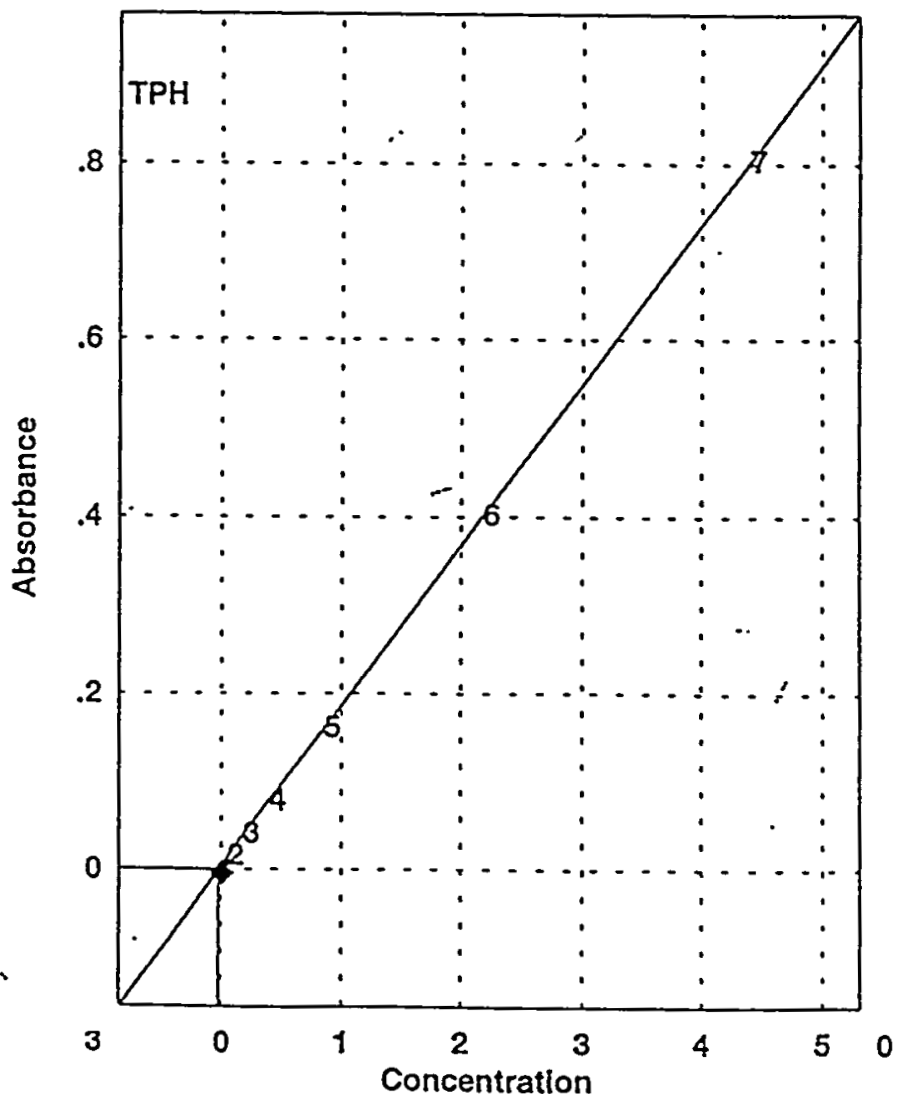




\section{DATA 産 \\ CHEM \\ a SOREKSOH COMPAKY}

\section{Case Narrative}

Method: EPA 418.1

Analysis: Total Petroleum Hydrocarbons

Preparation SOP : IW-EP-418.1

Analysis SOP : IW-EP-418.1

DCL Set ID: 97C-0208-04
Client: SAIC

Account: 3006

Matrix: Wipe

Group D: G9769006

General Set Information: There were five field samples including the MS/MSD samples in this set which were analyzed for total petroleum hydrocarbons. This set was also analyzed with 97C-0208-03.

Method Summary: Approximately $45 \mathrm{~mL}$ of Freon 113 was added into the jar with the sample. The cap was screwed on and shaken for 30 seconds and left for $0.5 \mathrm{hr}$. The Freon was then filtered through a cone of $\mathrm{Na}_{2} \mathrm{SO}_{4}$ and then the process was repeated once more with the extracts being combinmed into the same flask. Add about $3 \mathrm{~g}$ silica gel and a stiring bar into the flask. Stir the solution for seven minutes on a magnetic stirrer. Wait for the silica gel to settle in the sample extract, then analyze sample using the FTIR-1000C, by direct comparison with standards using a linear curve.

Sample Preparation: All samples were extracted and prepared in accordance with the published DCL SOP.

Holding Times: The holding times were met for both preparation and analysis.

Dilution(s): NA

QC Data: All QC data were within method limits.

NC/CAR: N/A

Flagging Codes: N/A.

MS/MSD Analysis: The MS/MSD recoveries for sample 97002908 were within limits.

Instrument QC: Instrument initial and continuing calibrations were performed in accordance with published procedures.

525 
Sample Calculation: The final results are calculated in $\mu \mathrm{g} /$ wipe by the equation:

$$
\mu g / \text { wipe } T P H=\frac{R \times D \times 1000 \mu g / m g}{W}
$$

where:

$\mathrm{R}=$ Mass of TPH determined from the FTR (mg).

$D=$ Extract dilution factor, if used.

$\mathrm{W}=\#$ of wipes.

Example: Calculation of TPH concentration ( $\mu \mathrm{g} / \mathrm{wipe})$ in sample 97C02908MS:

$$
\frac{(1.66409 \mathrm{mg}) \times(1) \times(1000 \mu \mathrm{g} / \mathrm{mg})}{1 \text { wipe }}=1664.09 \mu \mathrm{g} / \text { wipe }
$$

Miscellaneous Comments: None.

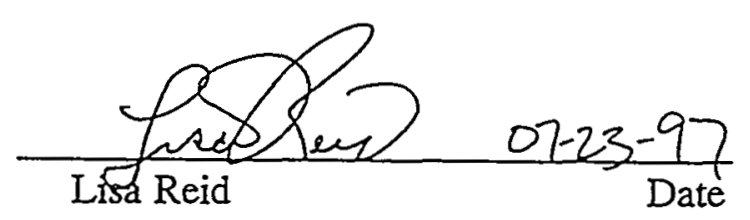


Information pertaining to this datapackage is divided into the four categories listed below. A Case Narrative immediately precedes this Table of Contents and contains pertinent information about this datapackage.

Analytical Results ................ Yellow

Sample Tracking Documentation ......... Pink

Analytical Documentation ............ Blue

Raw Data .................... Green

\section{$+1+1+1+1$}

\section{Analytical Results}

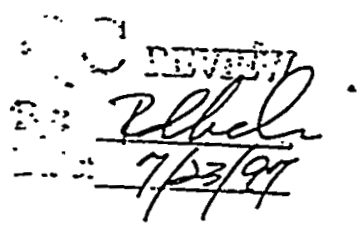




\section{DATA CHEM \\ A Sorenson Company}

\section{COVER PAGE}

ANALYTICAL REPORT FOR SAIC

Phone(201) 498-8496
Form COVER-V1.3 07239709280286 Page 1

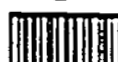

DCI Report Group..: 97C-0208-04

Date Printed.....: 23-JUL-97 09:27

Project Protocol *: P975B002

Cllent Ref Number.s Not Propided

Release Number..... Not Proplded $\therefore$

Analysis Method (s): 418.1
Attention: Art Shattuck 411 Hackensack Avenue Hackensack, NJ 07601

Client
Sample Name
Method Blank
LCS
NAP-R1-S6
NAP-R2-S6
NAPR2S6BLANR
NAPR2S6LCS
NAPR2S6ICSD
BL-136533-1
QC- $136533-1$

\begin{tabular}{l} 
Laboratory \\
Sample Name \\
\hline BL-136534-1 \\
QC-136534-1 \\
$97 C 02902$ \\
$97 C 02903$ \\
$97 C 02908$ \\
$97 C 0290845$ \\
$97 C 0290845 D$ \\
BL-136533-1 \\
$Q C-136533-1$
\end{tabular}

Date

Sampled

NA

NA

25-JUN-97

26-JUK-97

26-JUN-97

26-JUN-97

26-JUN-97
Date

Recelved

NA

NA

27-JUN-97

27-JUR-97

27-JUN-97

27-JUN-97

27-JUस-97

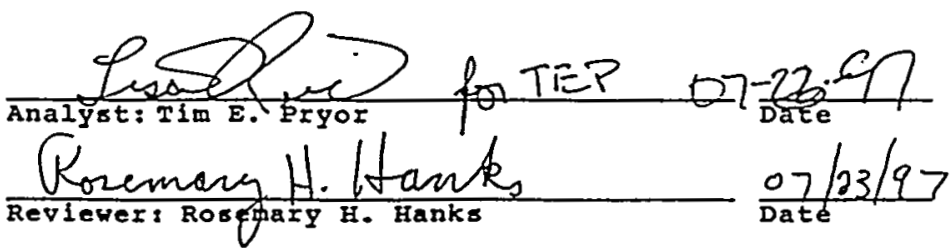




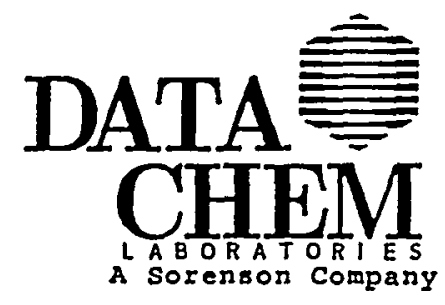

client Name... SAIC
FORM E (TYPE I)

SINGLE YEIHOD ANALYSES

SAMPLE GROUP COMMENTS
Form RLIMS63H-V1.3

\section{Page 2}

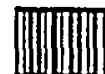

G9759018

DCI Report Group... 97C-0208-04

Date Printed......: 23-JUL-97 09:27

Release Number.... not Provided

General Information

The DCI QC Database maintains all numerfcal figures which are input from the pertinent data source. These data have not been rounded to slgnificant flgures nor have they been molsture corrected. Reports generated from the system, hovever, list data which have been rounded to the number of signiflcant figures requested by the client or deemed approprlate for the method. This may create minor discrepancles between data whlch appear on the QC summary Forms (Forms B-G) and those that would be calculated from rounded analgtical results. Additionally, if a molsture correction is performed,

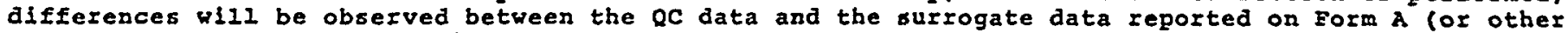
report forms) and corresponding data reported on QC Sumary Forms. In these cases, the Form $A$ will indicate the "Report Basis" as well as the molsture value used for making the correctlon.

report generation options: $M X$

\section{Resalt Symbol Definitions}

ND - Not Detected above the MDI or IDI (IID or MDC for radiochemistry).

** - No result could be reported, see sample comments for details.

\section{Daalifier Sybol Definitions}

$U$ - Not Detected above the HDL or IDL (ILD or MDC for radiochemistry). For Iadochemistry the nuclide was not ldentifled by the Canberra Nuclear NID program, activity values reported are calculated using the Canberra Nuclear mINACT program.

B - For organic analysis the qualifier indicates that this analyte was found in the method blank. For Inorganic analysis the gualifier signifies the value is between the IDI and PQL.

$J$ - The qualifier indicates that the value is between the MDI and the PQL. It 15 also used for indicating an estlmated value for tentatively identified compounds in mass spectrometry where a $1: 1$ response is assumed. 


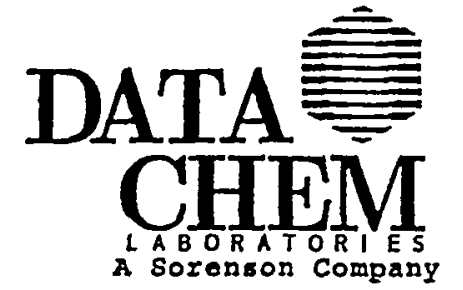

Date Printed........ 23-JUL-97 09:27

Client wame........... SAIC

client Ref Number.... Not Provided

samling site........ Not Appllcable

Release number....... Not Rrovided

Date Received........ Not Applicable

DCI Preparation Group: G9761011

Date Prepared........ 02-JUL-97 00:00

Preparation Hethod....

AIIquot Weight/Polumes Not Appllcable

Net Welght/Volume.... Not Requlred
FORM A (TYPE I)

SINGLE METHOD ANALYSES

SAMPLE ANALYSIS DATA SHEET

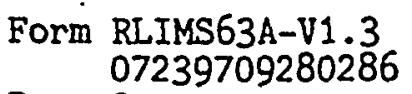

Page 3

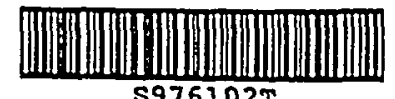

s9761022

Cllent Sample Hame: BL-136534-1

DCL Sample Name... B BI-136534-I

DCI Report Group..: 97C-0208-04

Hatrix........... HIPE

Date Sampled....... Not Appl1cable

Reporting Ualts... ug/wipe

$\therefore$

DCI Analysis Group: 69769006

Analysis Method... 418.1

Instrument Type...: IR

Instrument ID....: ETIR-1000C

Column Type....... Not Applicable

Analytical Results

\begin{tabular}{|c|c|c|c|c|c|c|c|}
\hline Analgte & $\begin{array}{c}\text { Date } \\
\text { Anajyzed }\end{array}$ & YDL & Result & Comment & Qual. & Dilution & CRDI \\
\hline ThPR & $09-J U L-97 \quad 00: 00$ & 100. & ND & & & & 100. \\
\hline
\end{tabular}

590 


\section{DATA

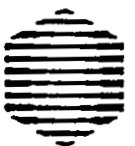 \\ FORM A (TYPE I) \\ SINGLE METHOD ANALYSES

Date Priated......... 23-JUL-97 09:27

client rame.......... SAIC

Cllent Ref Number....: Not Provided

Sampling slte........ Not Applicable

Release Number....... Not Rrovided

Date Received....... Not Applicable

DCL Preparation Group: G9761011

Date Frepared........ 02-JUL-97 00:00

Preparation Method...:

Allquot Welght/Volume: Not Applicable

Net Welght/Volume.... . Not Required
Cllent Sample Name: QC-136534-1 DCI Sample Name... I QC-136534-1 DCI Report Group... 97C-0208-04

Mat $=1 \times \ldots \ldots \ldots \ldots$ WIRE Date Sampled...... Not Applicable Reporting Units.... ug/wipe $\therefore$

DCI Analysis Group: G9769006 Analysis Method...: 418.1 Instrument Type...: IR Instrument ID....: FTIR-1000C column Type....... Not Applicable

Analytical Results

\begin{tabular}{|c|c|c|c|c|c|c|c|}
\hline Aralyte & $\begin{array}{c}\text { Date } \\
\text { Analyzed }\end{array}$ & MDI & Result & Coment & Qual. & Dilution & CRDI \\
\hline TRPR & $09-5 U L-97 \quad 00: 00$ & 100. & 1100 & & & & 100 \\
\hline
\end{tabular}




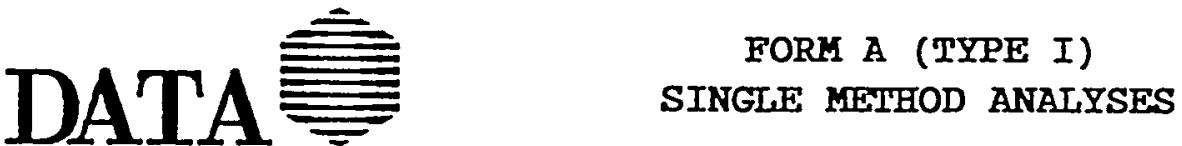

SAMPLE ANALYSIS DATA SHEET
Form RLIMS63A-V1.3 07239709280286 Page 5

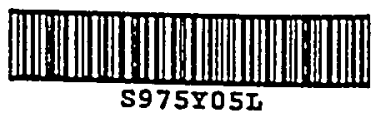

Date Printed........: 23-JUL-97 09:27

client Name.......... SAIC

Cllent Ref Number..... Not Provided

Sampilng Site......... Not Provided

Release Number........ Not Proyided

Date Received.......: 27-JUמ-97 00:00

DCr Preparation Group: G9761011

Date Prepared........: 02-JUI-97 00:00

Ireparation Method...:

Allquot Welght/Volume: Not Applleable

Net Welght/volume.... : Not Roquired
Client Sample Name: MAP-RI-S6 DCL Sample Name...: 97C02902

DCL Report Group..: 97C-0208-04

Matrix.......... พIPE

Date Sampled......:25-JuN-97 00:00

Reporting Units...: ug/wipe

Report Basis...... : $\mathrm{XAs}$ Recelved $\square$ Dried

DCL Analysis Group: G9769006

Analysis Method...: 418.1

Instrument Type.... IR

Instrument ID.....: FTIR-1000C

Column Type...... Not Applicable

Analytical Results

\begin{tabular}{|c|c|c|c|c|c|c|c|}
\hline Analyte & $\begin{array}{c}\text { Date } \\
\text { Analyzed } \\
\end{array}$ & MDI & Result & Comment & Qual. & Dilution & CRDI \\
\hline TREF & 09-JUL-97 00:00 & 100. & ND & & & & 100. \\
\hline
\end{tabular}




\section{DATA

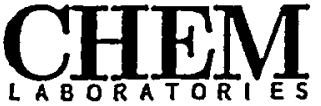 \\ A Sorenson Company}

Date Printed........: 23-JuL-97 09:27

Client vame........... SAIC

cllent Ref Number.... Not Provided

Sampling site........ Not Provided

Release Number....... Hot Provided

Date Recelved.......:27-JUN-97 00:00

DCI Preparation Group: G9761011

Date Prepared........ 02-JUL-97 00:00

Preparation Hethod...:

Allquot Welght/Volume: Not Applicable

set Welght/Volume.... Hot Requlred
FORM A (TYPE I)

SINGLE WEIHOD ANALYSES

SAMPLE ANALYSIS DATA SHEET
Form RLIMS63A-V1.3

Page 6

07239709280286

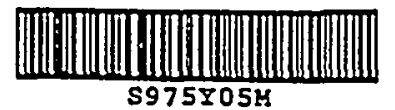

Client Sample Name: axp-R2-86

DCI Sample Name...: 97C02903

DCL Report Group..: 97C-0208-04

Matr1x.......... HIPE

Date Sampled.....3,26-JUN-97 00:00

Reporting Unito... ug/wipe

Report Basis,..... $X$ As Recelved $\square$ Drled

DCI Analysis Group: G9769006

Analysis Method...: 418.1

Instrument Type...: IR

Instrument ID....: RTIR-1000C

Column Type....... Not Applicable

Analytical Results

\begin{tabular}{|c|c|c|c|c|c|c|c|}
\hline Analyte & $\begin{array}{c}\text { Date } \\
\text { Analyzed } \\
\end{array}$ & MDI & Result & Comment & Qual. & Dilution & CRDL \\
\hline TRPH & $09-J \cup I-97 \quad 00=00$ & 100. & ND & & & & 100. \\
\hline
\end{tabular}


DATA
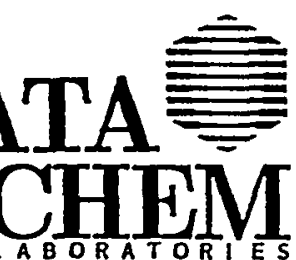

A Sorenzon Company

Date Printed........ 23-JUI-97 $09: 27$

Cl1ent Mame.......... SAIC

cllent Ref Number.... Not Rrovided

Sampling site........ Not Rrovided

Release Number....... Not Provided

Date Recelved......: 27-JUN-97 00:00

DCI Prepaxation Group: G9761011

Date Prepared........ 02-JUL-97 00:00

Preparation Method...

Allquot Welght/Volume: Not Applicable

liet Weight/Volume.... Not Required
FORH A (TYPE I)

SINGLE MIEIHOD ANALYSES

\section{SAMPLE ANALYSIS DATA SHEET}

Form RLIMS63A-V1.3 07239709280286

Page 7

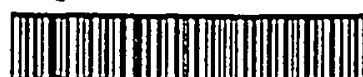

S975Y05K
Cllent Sample Name: HAPR2s6Brinta

DCy Sample kame... 97C02908

DCI Report Group.. : 97C-0208-04

Hatr $1 \times \ldots \ldots \ldots \ldots$...... WIPE

Date Sampled......; 26-Jus-97 00:00

Reporting Units...: ug/wipe

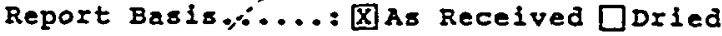

DCI Analysis Group: G9769006

Analysis Hethod...:418.1

Instrument Type...: IR

Instrument ID....: ETIR-1000C

Column Type....... Not Applicable

Analytical Results

\begin{tabular}{|c|c|c|c|c|c|c|c|}
\hline Analyte & $\begin{array}{c}\text { Date } \\
\text { Analyzed } \\
\end{array}$ & HDI & Result & Comment & Qual. & Dilution & CRDI \\
\hline TRPH & $09-J 0 I_{1}-97 \quad 00=00$ & 100. & ND & & & & 100. \\
\hline
\end{tabular}




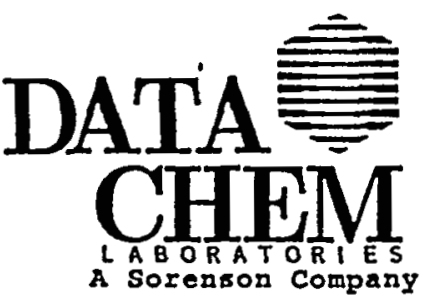

Date Printed.......: 23-JUL-97 09:27

Cllent Name.......... SAIC

Client Ref Number....: Not Provided Sampling site......... Not Provided

Relense Number........ Not Provided

Date Recelved........27-JUN-97 00:00

DCI Preparation Group: G9761011

Date Prepared....... 02-JUL-97 00:00

Preparation Method...:

Aliquot Weight/Volume: Not Applicable

Net Welght/Volume.... . Not Required
FORM A (TYPE I)

SINGLE MEIHOD ANATYSES

SAMPLE ANALYSIS DATA SHEET

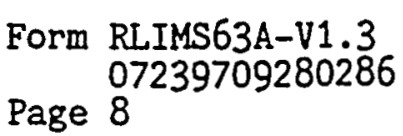

Page 8

S975Y05P
Cllent Sample Name: BApE2s6LCs

DCL Sample Name...: 97C02908ks

DCI Report Group... 97C-0208-04

Matrix........... HIPE

Date Sampled......2/26-J0k-97 00:00

Reporting Units...s ug/wipe

Report Basis.a... 囚As Received $\square$ Dried

DCI. Analysis Group: G9769006

Analysis Hethod...8418.1

Instrument Type...? IR

Instrument ID...... FTIR-1000C

Colum Type....... Not Applicable

Analytical Results

\begin{tabular}{|c|c|c|c|c|c|c|c|}
\hline Analyte & $\begin{array}{c}\text { Dato } \\
\text { Analyzed } \\
\end{array}$ & MDI & Result & Comment & Qual. & Dilution & CRDI \\
\hline TRPH & $09-J U L-97 \quad 00200$ & 100. & 2700 & & & & 200. \\
\hline
\end{tabular}

Sample Comments

Splke added $=1770.6 \mu \mathrm{g} /$ wipe. 


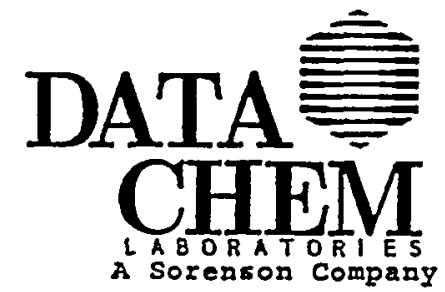

Date PIinted........ 23-JUL-97 09:27

cllent Hame.......... SAIC

client Ref Number.... Hot provided

Sampling Site........ Not Provided

Release Number....... Not Provided

Date Received.......: 27-JUH-9700:00

DCI Preparation Group: G9761011

Date Prepared........: 02-JUI-97 00:00

Preparation Kethod....

Al1quot Weight/Volume: Not Applicable

Net Welght/Volume..... Not Required
FORH A (TYPE I)

SINGLE METHOD - ANALYSES

\section{SAMPLE ANALYSIS DATA SHEET}

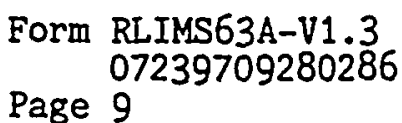

Page 9
Client Sample Name: unpa286LCSD

DCL Sample Name... 97c02908KsD

DCI Report Group.. : 97C-0208-04

Matr1x.......... WIPE

Date sampled......2,26-JUN-97 00:00

Reporting Units... i ug/ulpe

Report Basis...... $囚$ As Recelved $\square$ Dried

DCI Analysis Group: G9769006

Analysis Hethod...: 418.1

Instrument Type.... IR

Instrumerit ID..... PTIR-1000C

Column Type....... Not Applicable

Analytical Results

\begin{tabular}{|c|c|c|c|c|c|c|c|}
\hline Analgte & $\begin{array}{c}\text { Date } \\
\text { Analyzed } \\
\end{array}$ & MDI & Result & Comment & Qual. & Dilution & CRDI \\
\hline TRPH & 09-JUL-97 $00: 00$ & 100 & 1600 & & & & 100. \\
\hline
\end{tabular}

Sample Comraents

Splke added $=1770.6 \mu \mathrm{g} / \mathrm{wl}$ pe. 


\section{DATA

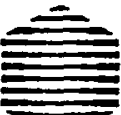 \\ FORY A (TYPE I) SINGLE MEITHOD ANALYSES}

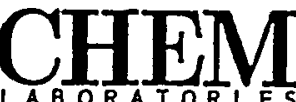

A Sorenson Company

\section{SAMMLE ANALYSIS DATA SHEET}

Form RLIMS63A-V1.3 07239709280286

Page 10

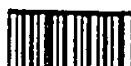

$\$ 9761025$
Date Printed........ 23-JUI-97 09:27

client rame........... SAIC

Cllent Ref Number.... Not Provided

Sampling site........ Not Applicable

Releare Number....... Not Provided

Date Recelved....... Not Applicable

DCI Preparation Group: G9761010

Date Prepared........: 02-JUI,-97 00:00

Preparation Method...: 418.1

Al Iquot Welght/Volume: 1.00

Net Welght/Volume.... Not Requlred
Client Sample Name: BL-136533-1

DCI Sample Name...: BL-I36533-1

DCI Report Group.. : 97C-0208-03

Hatrix........... HATER

Date Sampled...... Not Applicable

Reporting Unlts... : ug/L<smiles>C[Hg]</smiles>

DCI Analysis Group: G9769006

Analysis Hethod...: 418.1

Instrument rype...: IR

Instrument ID.....: ETIR-1000C

Column Type....... Not Applicable

\section{Analytical Results}

\begin{tabular}{|c|c|c|c|c|c|c|c|}
\hline Analyte & $\begin{array}{c}\text { Date } \\
\text { Analyzed } \\
\end{array}$ & MDI & Result & Comment & Qual. & Dilution & CRDL \\
\hline TRPH & $09-J U 5-97 \quad 00=00$ & 100. & ND & & & & 100. \\
\hline
\end{tabular}




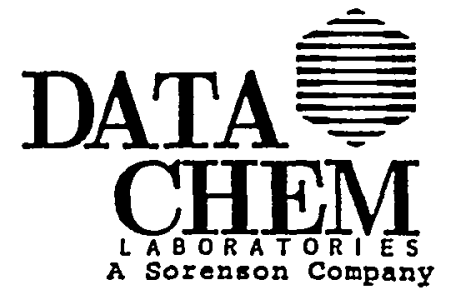

Date Printed........: 23-JUI-97 09:27

Client Name........... SAIC

client Ref Number.... Not Providea

sampling site........ Not Applicable

Release Number....... Not Provided

Date Recelved........ Not Applicable

DCI Preparation Group: G9761010

Date Prepared........ 02-JuI-97 00:00

Preparation Method...: 418.1

Allquot Weight/volume: 1.00

Net Welght/Volume..... Not Required
FORM A (TYPE I)

SINGLE METHOD ANALYSES

\section{SAMPLE ANALYSIS DATA SHEET}

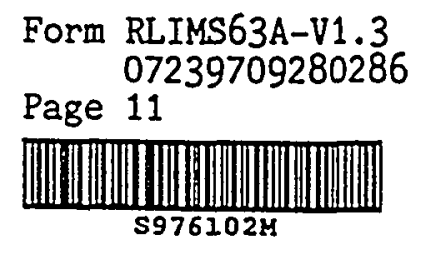

DCE Analysis Group: G9769006

Analysis Method...: 418.1

Instrument Type...: IR

Instrument ID..... ETIR-1000C

Column rype....... Not Applicable

Analytical Results

\begin{tabular}{|c|c|c|c|c|c|c|c|}
\hline Analyte & $\begin{array}{c}\text { Date } \\
\text { Analyzed }\end{array}$ & HDI & Result & Comment & Qual. & D11ution & CRDI \\
\hline TREF & $09-J U I-97 \quad 00=00$ & 100. & 1100 & & & & 100. \\
\hline
\end{tabular}



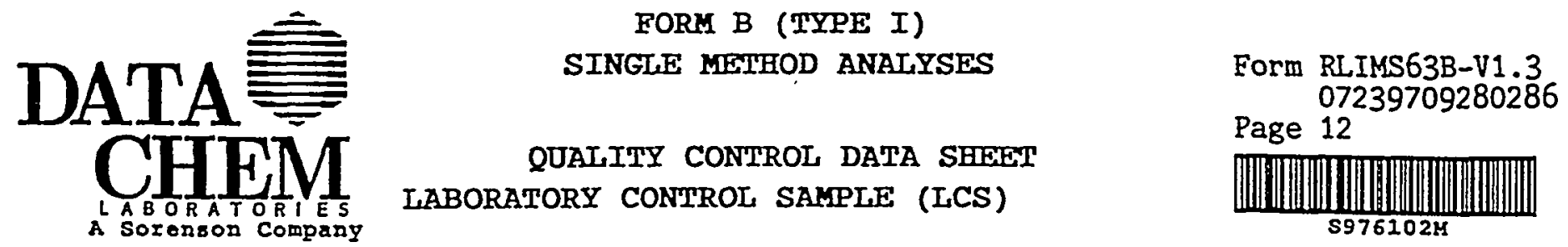

Client Hame.......... SAIC

Release Number....... Not Provided

DCI Sample Name...: QC-136533-1

Date Printed.....: 23-JUI-97 09:27

Matr1x............ : WATER

Reporting Units....... ug/I

DCL Analys 18 Group: 99769006

Analysis Hethod...: 418.1

Instrument Type...: IR

Instrument ID..., : FTIR-1000C

Column Type........ Not Applicable

DCI Preparation Group: G9761010

Date Prepared.......:02-JUL-97 00:00

Preparation Method...: 418.1

QC Iimit Type..... : Hethod

Analytical Results

\begin{tabular}{|c|c|c|c|c|c|c|}
\hline Ane 1gte & $\begin{array}{c}\text { Date } \\
\text { Analyzed } \\
\end{array}$ & Target & Result & $\begin{array}{l}\text { Porcent } \\
\text { Recovery }\end{array}$ & $\begin{array}{c}Q C \\
\text { Iimits } \\
\end{array}$ & $\begin{array}{l}Q C \\
\text { Flag }\end{array}$ \\
\hline TRPH & $109-J U L-97 \quad 00: 00$ & $=1200$ & 1100 & 91.9 & $75.0 / 125$ & \\
\hline
\end{tabular}




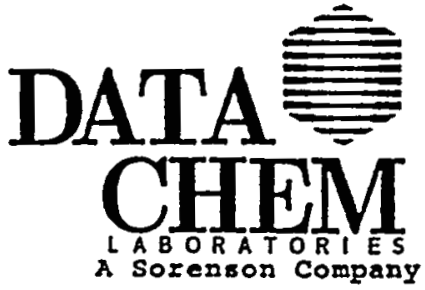

Cllent Name.......... SAIC

Release Number....... Not Provlded

Matrix............. WATER

Reporting UnIta....... ug/L

DCI Preparation Group: G9761010

Date Prepared........ 02-JuI-97 00:00

Preparation Method...: 418.1

Analytical Results
FORM F (TYPE I)

SINGLE METHOD ANALYSES

QUALITY CONTROL DATA SHEET HATRIX SPIKE SAMPLE MATRIX SPIKE DUPLICATE SAMPLE
Form RLIMS63F-V1.3

07239709280286

Page 13

S975\%057

DCL Sample Name... 97c02897ks

Date Printed.....: 23-JUI-97 09:27

DCL Analys : Group: G9769006

Analysis Hetbod...:418.1

Instrument Iype... IR

Instrument ID..... ETIR-2000C

Column Type....... Not Appllcable

QC Iimlt Type..... Hethod

\begin{tabular}{|c|c|c|c|c|c|c|c|}
\hline Analgte & \begin{tabular}{|c|} 
Date \\
Analyzed \\
\end{tabular} & $\begin{array}{l}\text { Sample } \\
\text { Result }\end{array}$ & $\begin{array}{l}\text { Spiked } \\
\text { Result }\end{array}$ & $\begin{array}{l}\text { Spike } \\
\text { Added } \\
\end{array}$ & $\begin{array}{l}\text { Percent } \\
\text { Recovery }\end{array}$ & Iimits & $\begin{array}{l}\text { QC } \\
\text { Elag }\end{array}$ \\
\hline TRPH & $09-J 0 x_{-97} 00: 00$ & 60.6 & 1560 & 1670 & 93.2 & $75.0 / 125$ & \\
\hline
\end{tabular}

59757058

DCL Sample Name.:. 97C02897k8D

Analytical Results

\begin{tabular}{|c|c|c|c|c|c|c|c|c|}
\hline Analgte & $\begin{array}{c}\text { Date } \\
\text { Analyzed }\end{array}$ & $\begin{array}{c}\text { Duplicate } \\
\text { Result }\end{array}$ & $\begin{array}{c}\text { Percent } \\
\text { Recovery }\end{array}$ & Hean & Range & RPD & $\begin{array}{l}Q C \\
\text { Inits }\end{array}$ & $\begin{array}{l}\text { QC } \\
\text { Flag }\end{array}$ \\
\hline TRPH & 109-JUL-97 $00: 00$ & 1540 & 92.1 & 1550 & 18.3 & 1.2 & $0.00 / 20.0$ & \\
\hline
\end{tabular}




\section{DataChem Laboratories Quality Control Data Sheet INORGANIC - ENVIRONMENTAL CHEMISTRY}

Mothod No: $418.1 \mathrm{Mod}$

Battelle Code (if eppiceble) N//

Analyst Initials: II

Matrix: SOIL WATER OThO (chath ons)
Place Group Sticker Hore.

Dato of Analysis: $z-q-97$

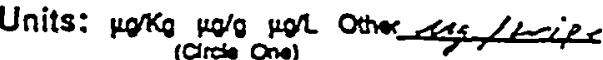

Sot ID(s) Included in This Group: $9 z 1-6208-04$

Analyto $\triangle P H$

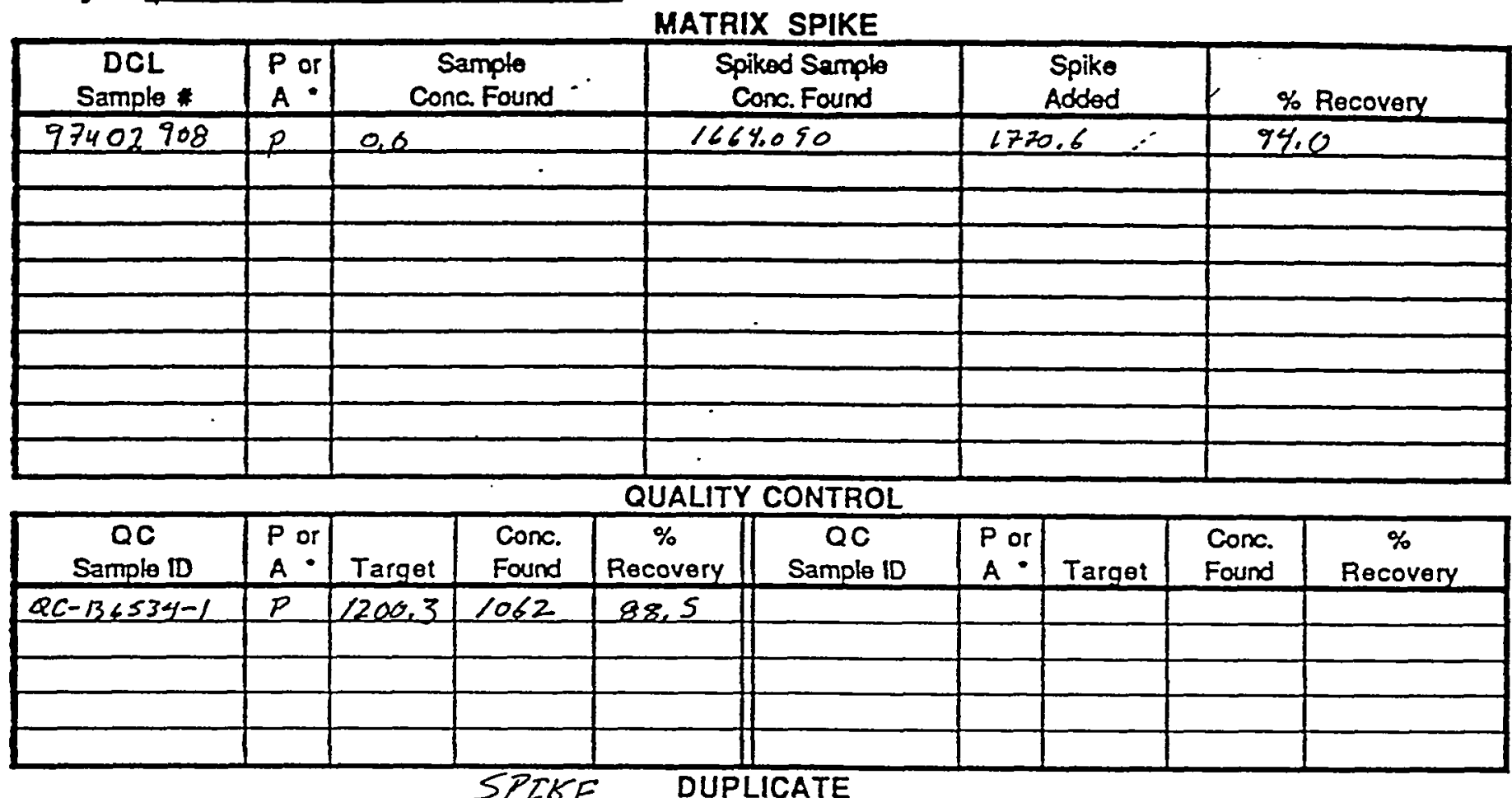

\begin{tabular}{|c|c|c|c|c|c|c|}
\hline \multirow[b]{2}{*}{ DCL Sample \# } & \multirow{2}{*}{$\begin{array}{l}P \text { or } \\
A\end{array}$} & \multicolumn{2}{|c|}{ Eencentration } & \multirow[b]{2}{*}{ Mean } & \multirow[b]{2}{*}{ Range } & \multirow[b]{2}{*}{ RPD } \\
\hline & & spike semole ${ }^{2}$ z-16-99 & Duplicate & & & \\
\hline 97402408 & $p$ & 1664,090 & 1629.790 & 1646.935 & 34.31 & 2.08 \\
\hline MMD & & & & & & \\
\hline & & & & & & \\
\hline & & & & & & \\
\hline & & & & & & \\
\hline & & & & & & \\
\hline & & & & & & \\
\hline & & & & & & \\
\hline & & & & & & \\
\hline & & & & & & \\
\hline
\end{tabular}

chocked os cheched hy! Rosemang 61.1 tand $07 / 23 / 97$

If data required for this form are submitted to customers in report format, simply attach copy to QC data sheet.

Report concentrations found to three significant figures.

- Indicate with the letter. samoles which were soiked orior to oredaration (P) or soiked prior to analvsis (A).

$\simeq \pi$ 

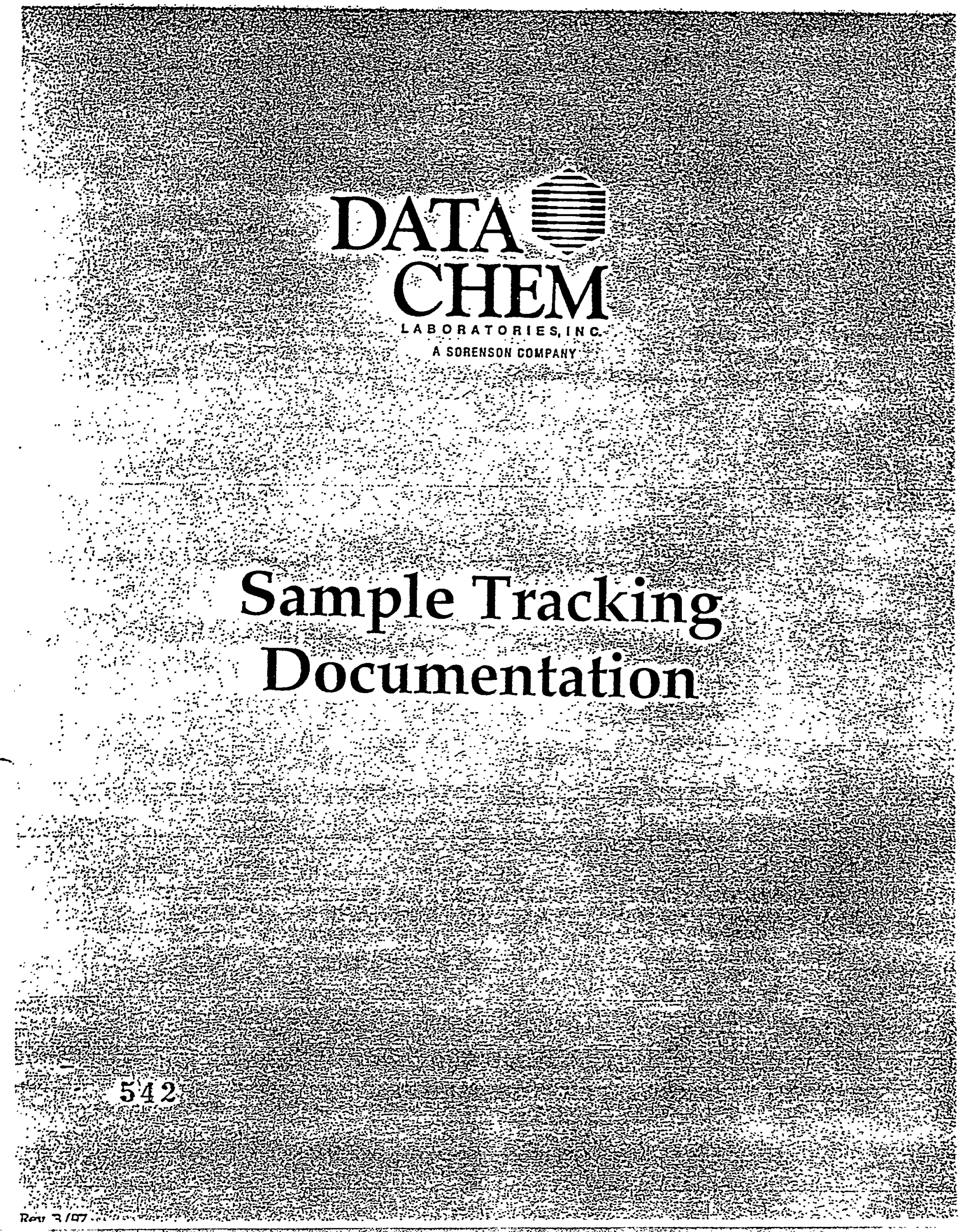
DataChem Laboratories

LIMS - Sample Master System

Analysis Group Report
Date: 10-JUL-1997 09:25

User: PRYOR

Analysis Run Name: G9769006

Samples: 17
Page: 1

RLIMS15-V1.2

Group ID: G9769006

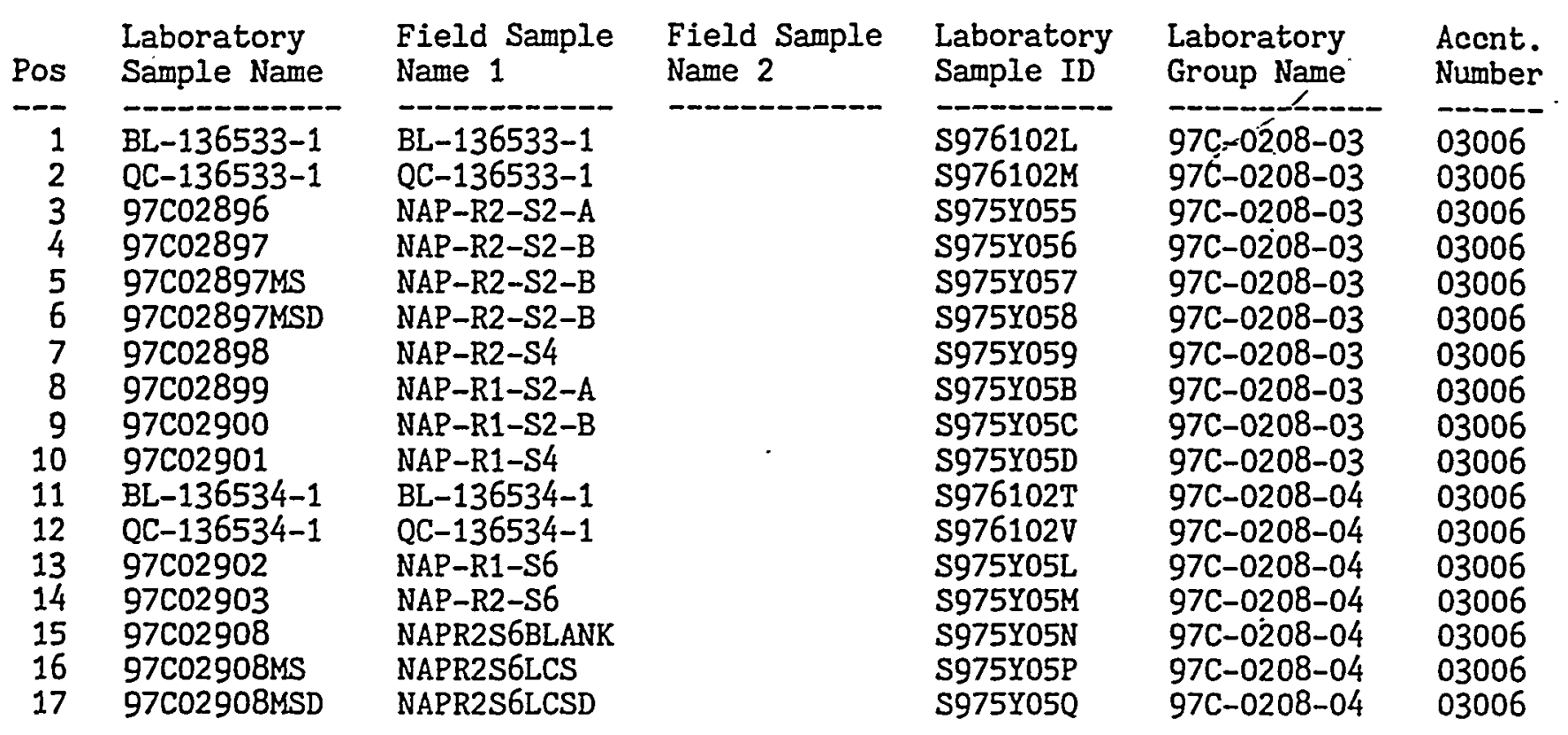

543 


\section{DataChem Laboratories \\ LIMS - Sample Master System \\ Preparation Group Report}

Date: 2-JUL-1997 15:42

User: PRYOR

Preparation Run Name: G9761011

Samples: 7

\begin{tabular}{cll} 
Pos & $\begin{array}{l}\text { Laboratory } \\
\text { Sample Name }\end{array}$ & $\begin{array}{l}\text { Field Sample } \\
\text { Name 1 }\end{array}$ \\
\cline { 3 - 3 } 1 & BL-136534-1 & BL-136534-1 \\
2 & 0C-136534-1 & QC-136534-1 \\
3 & $97 C 02902$ & NAP-R1-S6 \\
4 & $97 C 02903$ & NAP-R2-S6 \\
5 & $97 C 02908$ & NAPR2S6BLANK \\
6 & 97 C02908MS & NAPR2S6LCS \\
7 & $97 C 02908 M S D$ & NAPR2S6LCSD
\end{tabular}

Field Sample Laboratory Name 2

END OF IISTING

S975Y050
Page: 1

RLIMS15-V1.2

Group ID: G9761011
Group Name Number

S976102T

S976102V

S975Y05L

S975Y05M

S975Y05N

S975Y05P
97C-0208-04

97Ć-0208-04

97C-0208-04

97C-0208-04

97C-0208-04

97C-0208-04

97C-0208-04
03006

03006

03006

03006

03006

03006

03006

染 

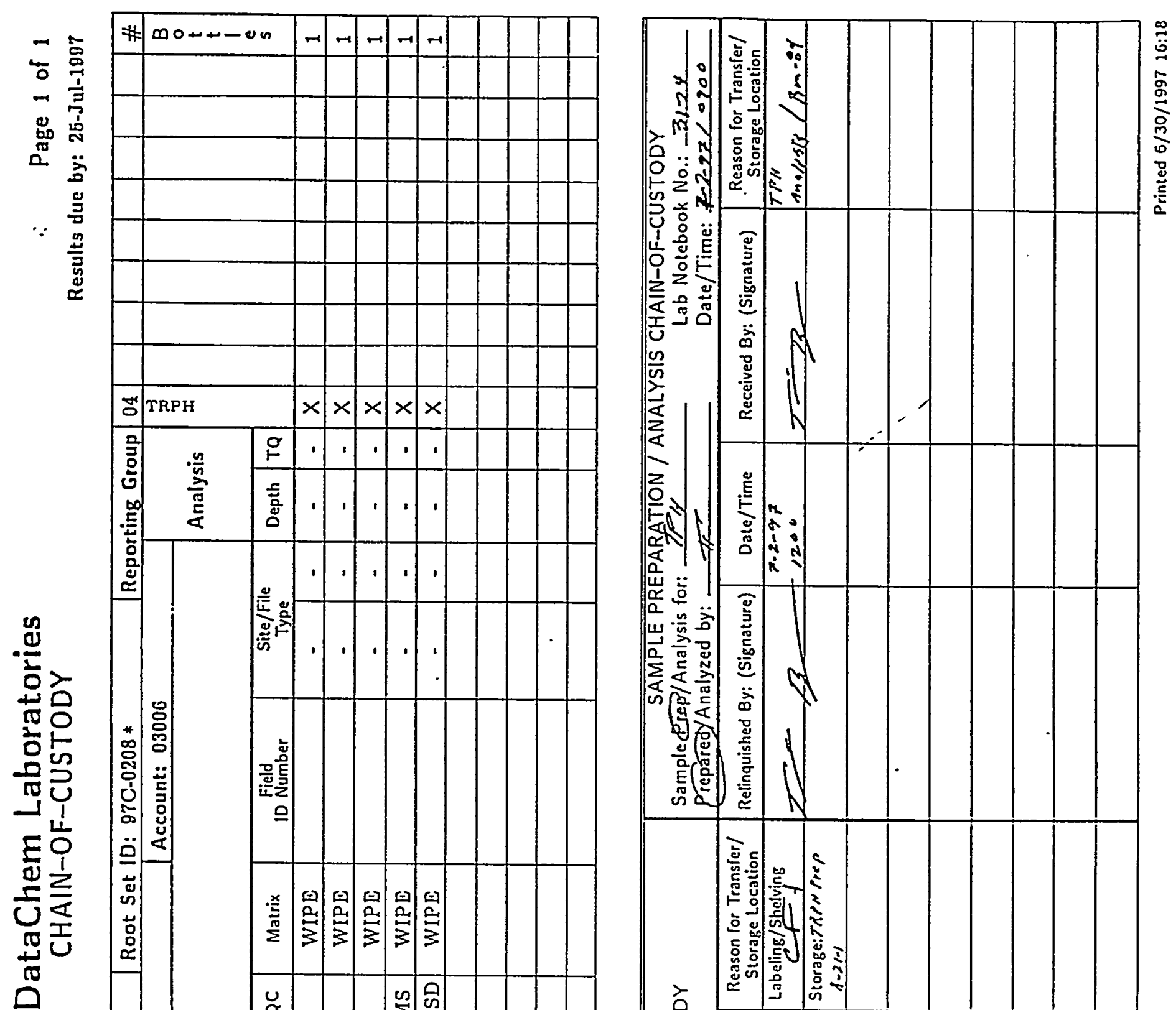

运哭 


\section{Sample Work Order}

QC Clearance:

Project Manager: Carol M. Bell

Client: SAIC

Account: 03006

Project/Task: P975B002

Date Received: 27-Jun-1997

Date for Mailing Report: 25-Jul-1997

Date for Verbal Report: 25-Jul-1987

\begin{tabular}{|c|c|c|c|c|c|c|c|c|}
\hline $\begin{array}{l}\text { Rep. } \\
\text { Group }\end{array}$ & $\begin{array}{l}\text { Zl Section } \\
\text { Analytes Requested }\end{array}$ & $\begin{array}{c}\text { Latest } \\
\text { Prep. Date }\end{array}$ & $\begin{array}{c}\text { Latest } \\
\text { Anal. Date }\end{array}$ & No. of & $\begin{array}{l}\text { Storage } \\
\text { Location }\end{array}$ & $\begin{array}{l}\text { Analysis/Prep. } \\
\therefore \text {-Method }\end{array}$ & Inst. & Matrix \\
\hline 03 & TRPH & & 23-Jul-1997 & 8 & & 418.1 & IR & WATER \\
\hline 04 & TRPE & & $23-$ Jul-1997c & 5 & & 418.1 & IR & WIPE \\
\hline
\end{tabular}

${ }^{c}$ Based on date of collection

Special Instructions:

Section Manager: Brett G. Lee
DCL Root Set ID: 97C-0208*

DCL Lab. Name: 97C02896-97C02908MSD

Total \# Samples: 13

Sample Entry: Patrick W. Corum

Section: ZI

Earliest Sampling Date: 24-Jun-1997

Preparation Type:

Other Sections Receiving Sample Portions: FG, FC

- 5 DataChem Laboratories/ 860 West LeVoy Drive / Salt Lake City, Utah 84123

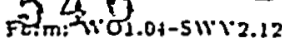




\section{DATA CHEM \\ LABORATORIES, INC. A SORENSON COMPANY}

$\stackrel{5}{3}$

$\because 547$

\section{Analytical Documentation}




\section{Analysis Record Page}

Group \#: $\angle 7769006$

LOT \#: $\mathrm{m}$

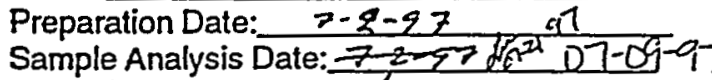

Method\#: 418.1 mod

Matrix: wipes / ware

DCL I.D.

\begin{tabular}{|c|}
\hline $97 c-02+8-04$ \\
\hline $570-0203-03$ \\
\hline
\end{tabular}

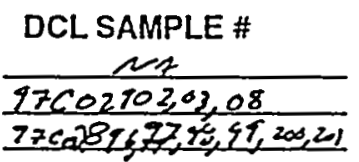

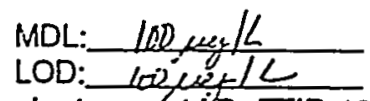

Instrument i. D: FTIR-1000C

- Samples were analyzed by method 418.

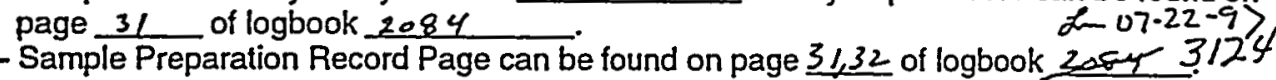
Analysis procedure can be found on

- Sample results can be found in the datapack.

Reagents:

Freon 113 Baker LoT J22217

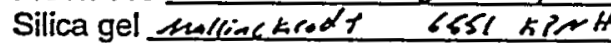

- Primary stock solution can be found on page 13 of logbook 2017.

$$
\text { IR-QS-PAF 6-16-97 IR-S-TP 6-16-97 }
$$

- Intermediate stock solution can be found on page 26 of logbook 2019.
IR-Q!-LMR 6-24-97
IR-I-TP $6-24-97$
IR-QC-LMR 6-24-97
IR-ISP-TP $\quad 6-24-97$

- Working standard solution's can be found on page 2 and 4,5 of logbook 3131 .
IR-WS-TP $6 \cdot 2+1-97$
4.4265
(2) $2.2133 \mathrm{mg} 0.8853 \mathrm{mg}$
$0.4427 \mathrm{mg}$

(1) $\mathrm{CCB}$

(2) $\mathrm{CCV}$

DCLACCOUNT

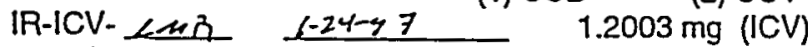

- QC preparation can be found on page 50 and $S \delta$ of logbook 3131. ac $-136533-1$ Qc $-13+534-1$

\section{Comments:}

\section{8}

To Fage No.

Witnessed \& Understood by me,

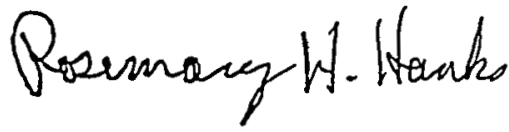
$\left\{\begin{array}{l}\text { Date } \\ 09 / 22197\end{array}\right.$

\begin{tabular}{|l|l|l|} 
invented by NOT APPLICABLE & Date \\
\hline Recorded by fosthas for TEP & $07-21-97$
\end{tabular}


TITLE TBPH

stripes
Project No. $=$

Book No 3124
DATA

CHEM

From Page No.=

- Set top: 97c-0208-04

prep Dote: 7-2-97!

15012 $97002902,03,08$

Mate ix zexipes

from IP: |6jz<1011

Accost: 3006

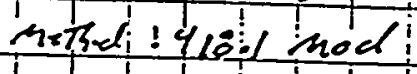

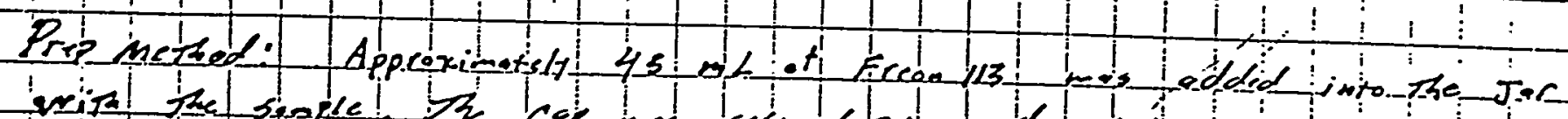

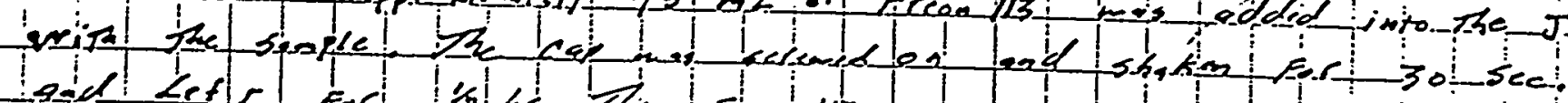

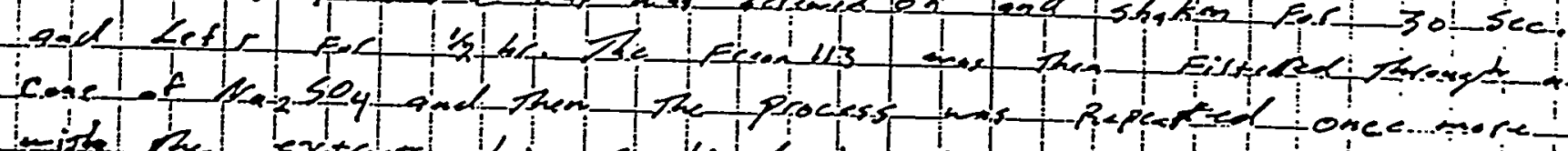

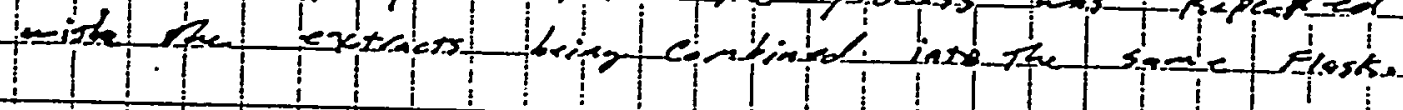

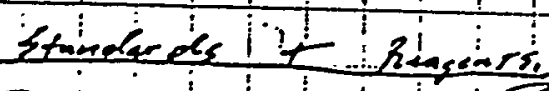

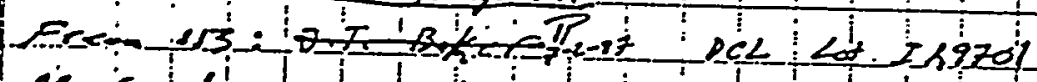

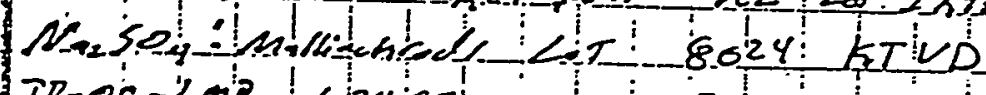

$I R-2 C-2 \operatorname{Rn} \mid 6=24-97$

Se BA 20lj! 2,6

IRIS? IS: $6=24=97$ sec 3019 is $206 !$

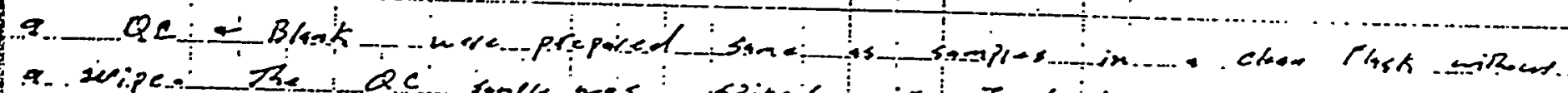

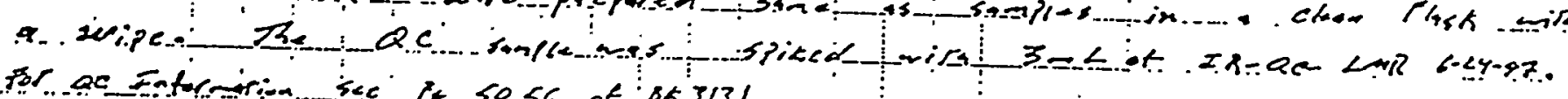

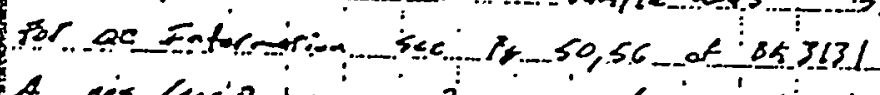

A. nishens'D

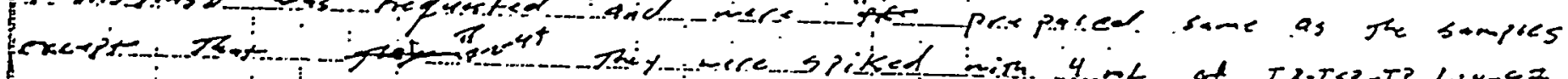

$x<\frac{\pi}{20-15}$

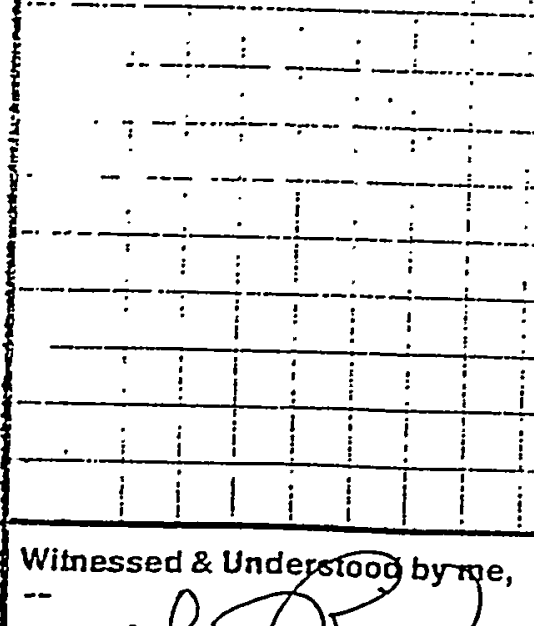

Witnessed 2 Underengoog by

\begin{tabular}{l|l|l}
\hline Date & Invented by \\
$07-21-9$ & Recorded by \\
\hline
\end{tabular}

To Page No. $=$ 
Water prep

Set ID: $97 c-0208-03$

Sample \#'s: $97602856-701$

Lot/Group ID: 69761010

Method:418.1

Matrix:water

Account \#: 3006

Prep. Date: $z-2-97$

STANDARDS AND REAGENTS

$\mathrm{Na}_{2} \mathrm{SO}_{4}: \mu$ ellinckred $\angle r \quad 8024$ KTVD

$1: 1 \mathrm{H}_{2} \mathrm{SO}_{4}:-12-57 \quad \mathrm{KK} \quad \mathrm{Serpz} 2,17$ of $8 \mathrm{~K} 2 \mathrm{CS4}$

Freon 113: $D C L$ Lot $I Z 7701$

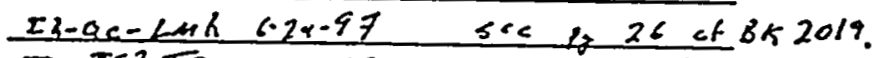

IR-ISP-TP $6.29-77$ Sere $P_{2} 26$ of $8 \times 2017$

sec if 50,56 of $8 A 3131$ For aC Inturuation

\begin{tabular}{|c|c|c|}
\hline Sample ID & $\mathrm{pH}$ & Vol. (L) \\
\hline$B L-13 \leqslant 533-1$ & $1 / 4$ & 1.00 \\
\hline$\vdots Q R C=13553.3-1$ & $\mathscr{B}$ & 1.00 \\
\hline 97602896 & $<2$ & 1.06 \\
\hline 97002897 & $<^{2}$ & 1.06 \\
\hline $77 \mathrm{Co} 28.9 \mathrm{~ms}$ & $<2^{2}$ & 1.06 \\
\hline .7700 .2897 ASD & $<2$ & 1.06 \\
\hline $97.002898 \ldots$ & $<2$ & 1.06 \\
\hline .77002899 & -12 & 1.06 \\
\hline $\begin{array}{r}97602700 \\
97602901\end{array}$ & $\begin{array}{l}<2 \\
<2\end{array}$ & $\begin{array}{l}1.06 \\
1.06\end{array}$ \\
\hline
\end{tabular}

Witnessed \& Understood by me,

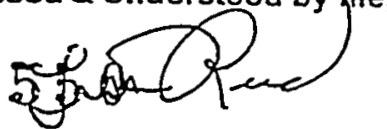

To Page No.

$\begin{aligned} & \text { Date } \\ & 1 \times 2-7 \\ & x=17 \\ & 7-2-87\end{aligned} \mid$


TITLE DATACHEKLABORATORIES DINSTRUMENTLOGIO NOF.2579

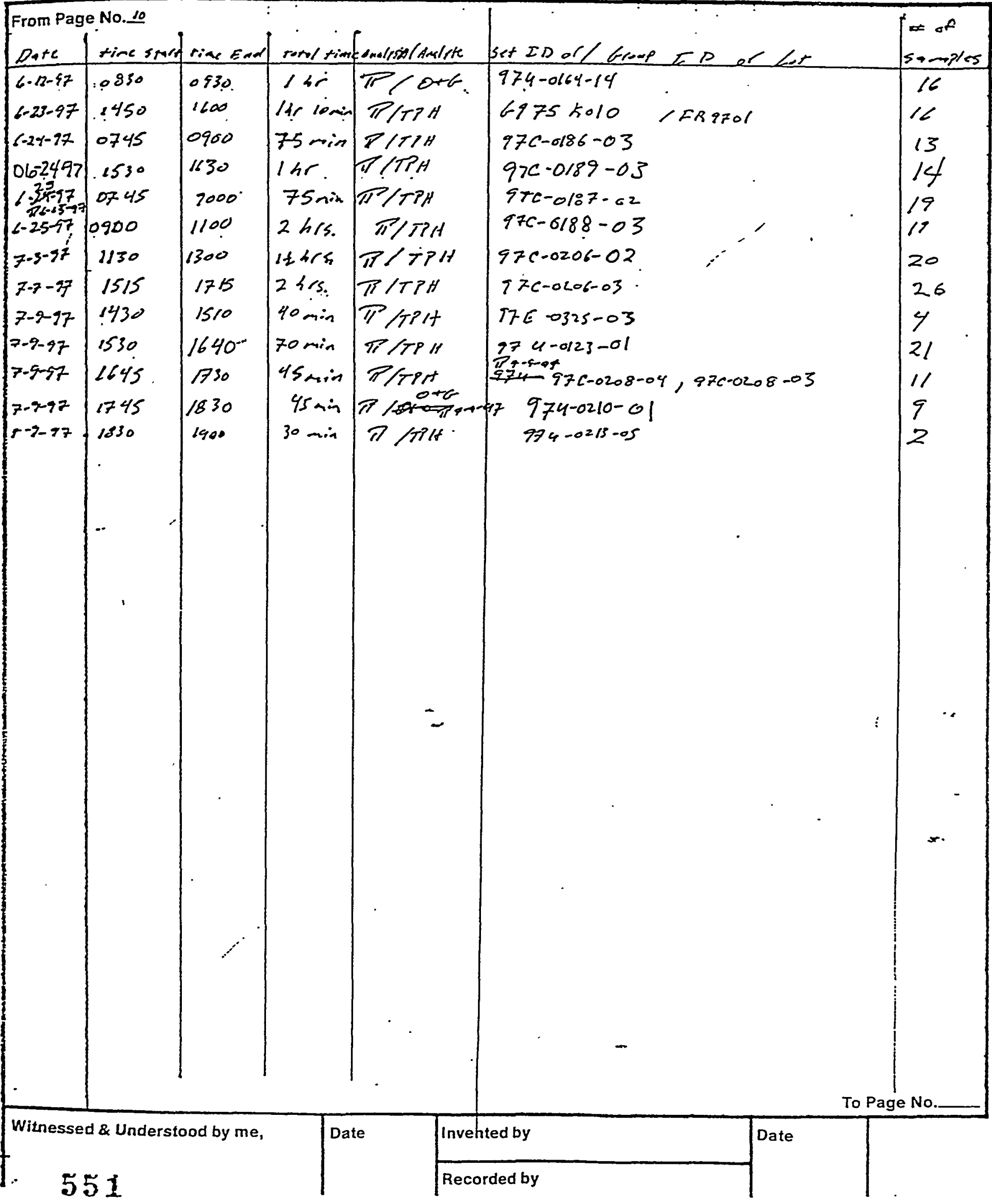


TITLE IPHE Oil Gaose in $\mathrm{H}_{2} \mathrm{O}$ by IR Book No.2089 LABORATORIES

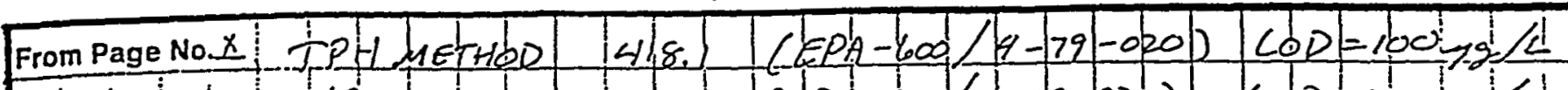
O\$S SETHOD 413.2 $(G P A-600)(4-29-020)$ LOP $=100-15 \%$

WATER SXTRACTLOU

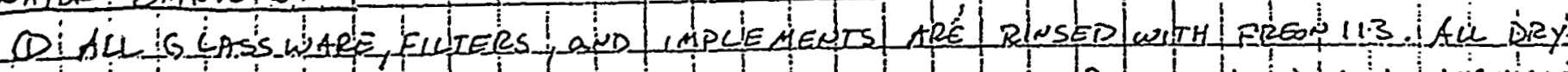

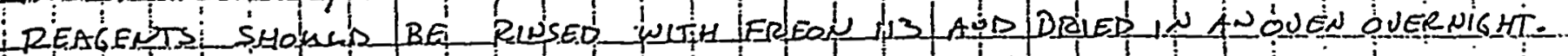

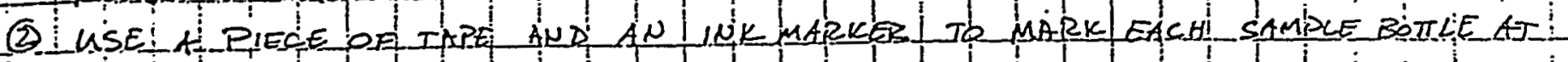

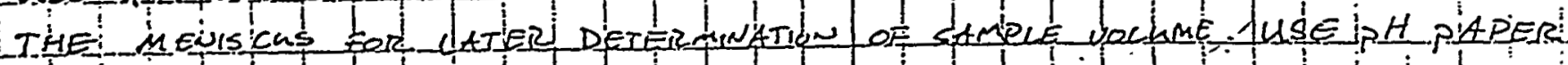

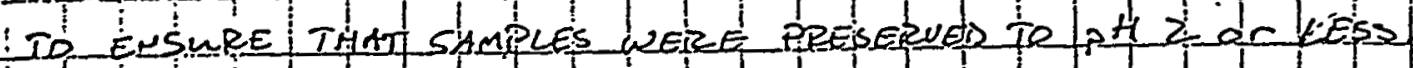

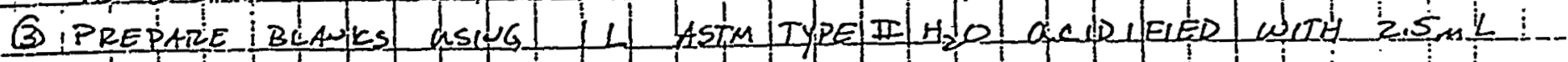

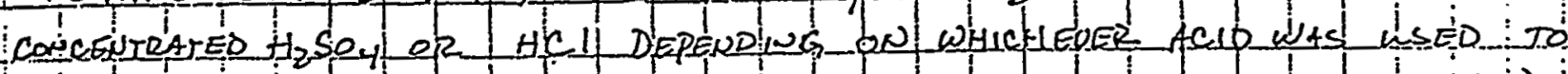

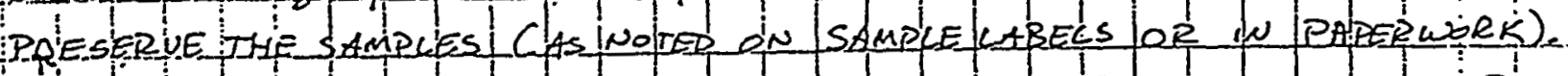

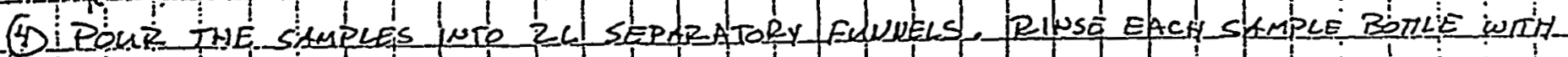

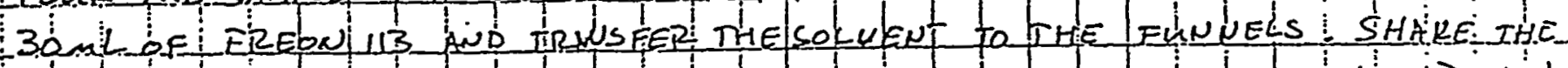

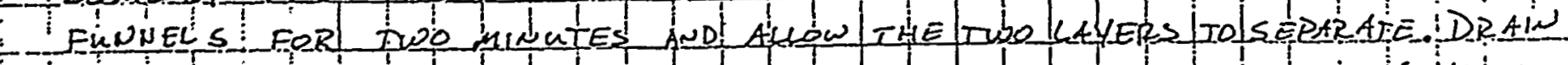

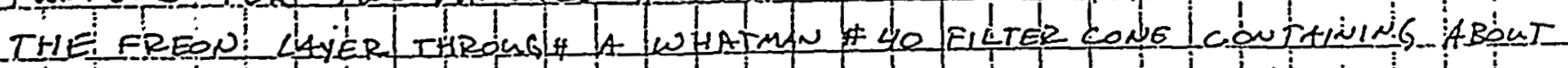

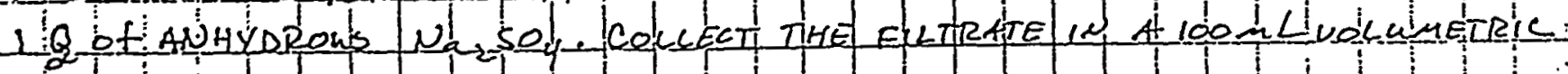
$\because$ FLASIS:

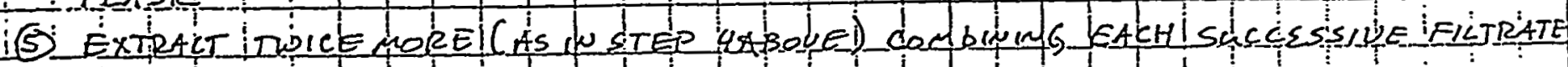
STHE ELASK

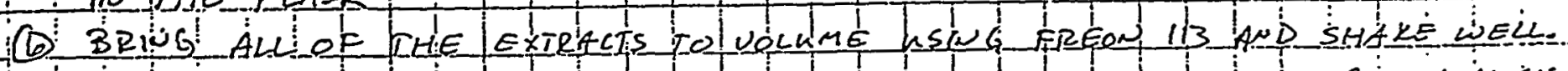

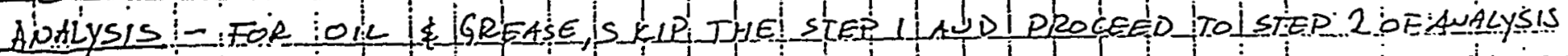

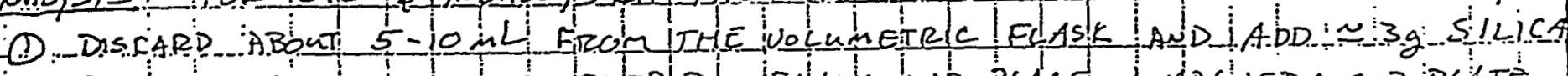
- SEL AND A STIRIBAR STOPPER IHE FLASK TND PLACEON MAGNETIC SRR OPLTTR AND STR EOR SEVEN MNNTES ALLOW TO SEILEREEORE AUALYSIS

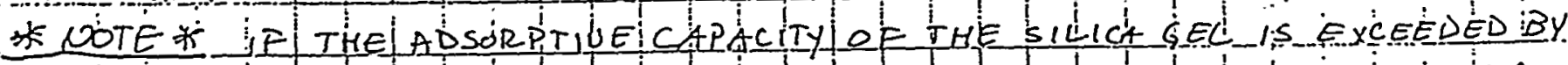

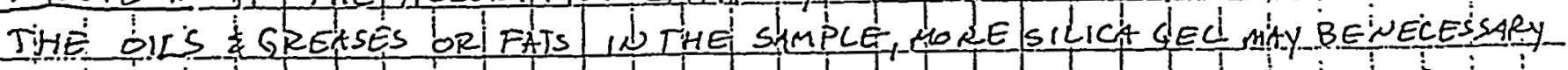

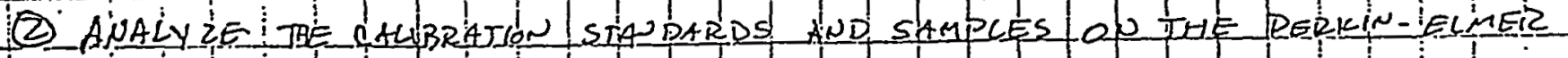

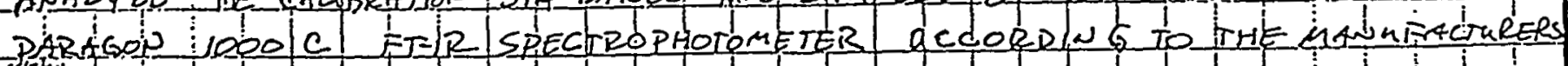
IRQDOMENDATOUS USING THE GRAMS ANACY ST IOOO SOFTWHZE.

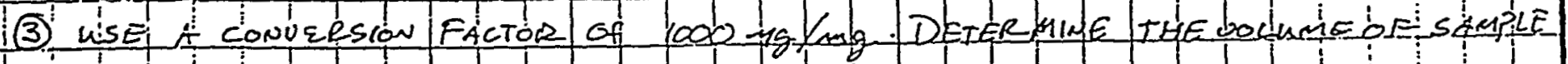

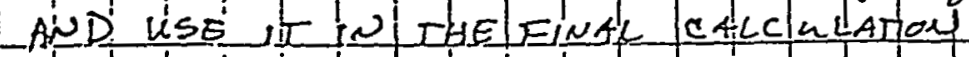

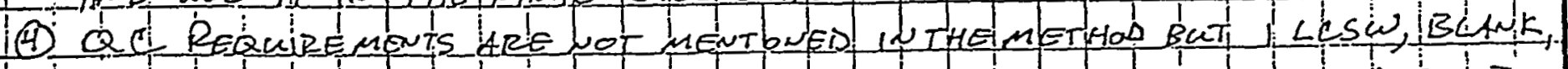
DUPLICAIE A ID SPIKE I PER 2O SAMPLES OR BATCHIS PEGOMMEODED IF EROOULH SANPLE SI SUPPLIER
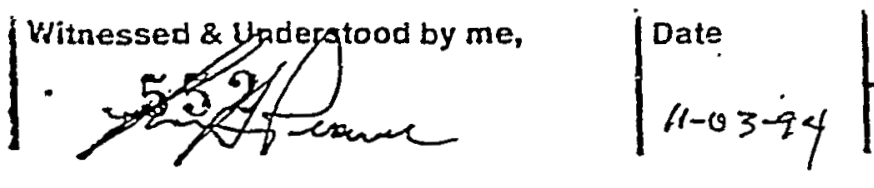

Inyented by

Date

Faddely

$11-3-94$ 
$\overline{-1}$

TITLE DATACHEM LABORATORIES - REAGENT PREPARATION Project No. $\frac{2}{2645}$

2

From Page No. 2

Ciponide Dotillations

Reagents Preparation.

$1.25 \mathrm{~N} N \mathrm{NaOH}$

Water dissolve $100 \mathrm{~g}$ pellet. $\mathrm{NaOH}$ in ASTM Type II Water. Dilute to $2000 \mathrm{~mL}$. Mix thouncughey.

$0.25 \mathrm{~N}$ NaH.

Water absolute $20 \mathrm{~g}$ pellet $\mathrm{NaOH}$ in AsTi Type II water. Dilute to $2000 \mathrm{~mL}$. mix thouroughly:

$\mathrm{MgCl}_{2}$

ASTM dissolve $1020 \mathrm{~g}$ magnesium chloride in flask. Mix thouroughly-

1: $\mathrm{H}_{2} \mathrm{SO}_{4}$

ASTM add $500 \mathrm{~mL}$ concentrated $\mathrm{H}_{2} \mathrm{SO}_{4}$ to $500 \mathrm{ml}$ ASTM Type II Water. mix thouroughly-Cool before ling:-

Sulfuric Acid Solution

Dissolve $40 \mathrm{~g}$ o $8 \mathrm{NH}_{2} \mathrm{SO}_{3} \mathrm{H}$ in AsTr Type It water.
Dilute to $1000 \mathrm{~mL}$ and mix thourghly. Pi ad ted o5-14-94

${\mathrm{Ca}(O C 1)_{2}}_{2}$ Solution: Dissolve 59 \& calcium hypochlorite in $160 \mathrm{~mL}$ \& ASTiR Type II water. (Added os-22-45PAD)

Bismuth nitrate solution:. Dissolve $30.0 \mathrm{~g}$ of $\mathrm{Bi}\left(\mathrm{NC}_{3}\right)_{3}$ in $100 \mathrm{~mL}$ of ASTM type II water... While stirring, add $250 \mathrm{~mL}$ of glacial acetic.. acid. Stir until dissolved. Dillecte...to I... with ASTM type II water. . (Added o1/2/96 KKM) 10 N NaOs: Dissolve 400 g. NoaH and dilute to IL with. Astrol. Type II water. Mix

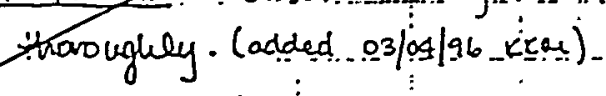

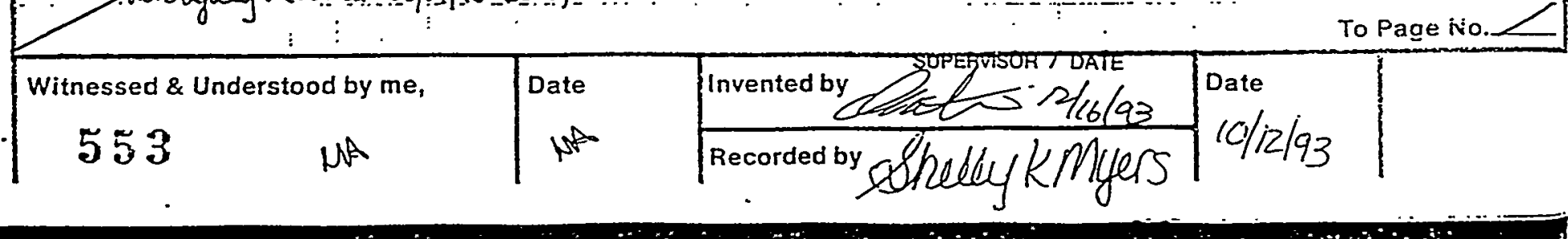




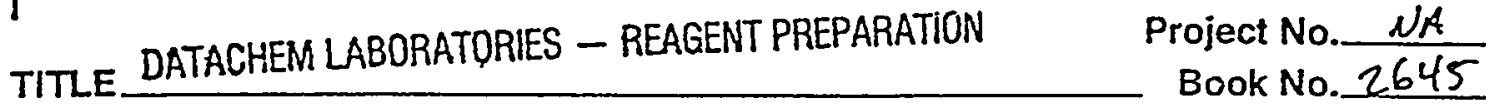

From Page No.16

\begin{tabular}{|c|c|c|c|c|c|}
\hline REAGENT & DATE & LNALYST & CHEMICAL USED & VENSOR & LOT \\
\hline $.0 .25 \mathrm{~N} \mathrm{NaOlt}$ & $03 / 03 / 97$ & $C A$ & NaOH & Mallinckrodt & 7708 KTKE \\
\hline Sulfamic Acid & $03 / 03 / 97$ & $C A$ & $\mathrm{NH}_{2} \mathrm{SO}_{3} \mathrm{H}$ & Baker & 637712 \\
\hline .. MgCl $C_{2}:$ soln. & $03 / 03 / 97$ & $C A$ & $\mathrm{MgCl}_{2} \cdot 6 \mathrm{H}_{2} \mathrm{O}$ & Mallinckrodt & $5956 \mathrm{KPTL}$ \\
\hline $1: 1 \mathrm{H}_{2} \mathrm{SO}_{4}$ & $03-03-97$ & PAJ & conc. $\mathrm{H}_{2} \mathrm{SO}_{4}$ & Fishen & 964339 \\
\hline $0.25 \ldots \mathrm{NaOH}$ & $03.03-97$ & PSJ & $\mathrm{NaOH}$ & Mallinckrodt & $7708 \mathrm{KTKS}$ \\
\hline $0.25 \mathrm{~N} N \mathrm{NOOH}$ & $03 / 16 / 97$ & $\mathrm{keM}$ & $\mathrm{NaOH}$ & Mallineḱrodt. & 7708 KTKE \\
\hline $0.25 \mathrm{~N} N \mathrm{~N}$.0H & $03 / 23 / 97$ & KKM & $\mathrm{NaOH}$ & Mallinckrodt & $7708 \mathrm{KTKE}$ \\
\hline .. Sulfaneic. Acid & $03 / 23 / 9$ & KKM & $\mathrm{NH}_{2} \mathrm{SO}_{3} \mathrm{H}$ & Baker & $G 37712$ \\
\hline $0.25 N . N S a O H \quad \times 2$ & $3 / 28 / 97$ & KEM & $\mathrm{NaOH}$ & Mallinckrodt & 7708 KTRE \\
\hline $1: 1 \mathrm{H}_{2} \mathrm{SO}_{4}$ & $3 / 28.97$ & $\mathrm{KKH}$ & $\mathrm{H}_{2} \mathrm{SO}_{4}$ & Fisher & 967749 \\
\hline $0.25 \mathrm{~N} N \mathrm{NaOH}$ & $3 / 29 / 97$ & $\mathrm{KCH}$ & $\mathrm{NaOH}$ & Mallinctrodt & T708 KTKE \\
\hline $0.25 N \mathrm{NaOH}$ & $4 / 10 / 97$ & $\mathrm{ECH}$ & $\mathrm{NaOH}$ & Baker & $k 03931$ \\
\hline 0.25 in NaOHt & $4-17-97$ & CASt & $\mathrm{NaOH}$ & Baker & 103931 \\
\hline $0.25 \mathrm{~N}$ NaOH $\times 2$ & 4120197 & inst & $\mathrm{NaOH}$ & Baker & $\operatorname{ko3931}$ \\
\hline$n=1 \mathrm{H}_{2} \mathrm{SO}_{4} \ldots$ & 4120197 & ustert & $\mathrm{H}_{2} \mathrm{SO}_{4}$ & Fisher & 967749 \\
\hline .. Sulftamic Acid & 4120197 & vesst. & $\mathrm{NH}_{2} \mathrm{SO}_{3} \mathrm{H}$ & Baker & 637712 \\
\hline$-0.25 N N .00 \mathrm{~A} \quad \times 2$ & $5 / 3 / 97$ & $\varepsilon \subset \mu$ & NaOH & Baker & $K 03931$ \\
\hline Sulfapre Acid & $5 / 11197$ & KKH & $\mathrm{NH}_{2} \mathrm{SO}_{3} \mathrm{H}$ & Baker & $G 37712$ \\
\hline $0.25 \mathrm{~N} . \mathrm{NaOH}$ & $5 / 11 / 97$ & Kera & $\mathrm{NaOH}$ & Balcer & $k 03931$ \\
\hline 0.2LNN VuaCt & Eilojit & Kะ & $\sin \div$ & 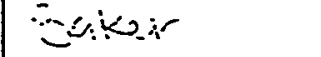 & KLラシジ \\
\hline D. $25 \mathrm{~N}$. NaOlt & $05 / 21 / 97$ & $C A$ & $\mathrm{NaOH}$ & Baker & K03931 \\
\hline $.0 .25 \mathrm{~N} \mathrm{NaOH}$ & $5 / 28 / 97$ & кen & $\mathrm{NaOH}$ & Baker & $k 03931$ \\
\hline $0.25 \mathrm{~N}$ NaOH & $6-12-y 7$ & verst & $\mathrm{NaOH}$ & Buker & Ko333i \\
\hline Sultamic Acid & $6-12-97$ & $10+21$ & $\mathrm{NH}_{2} \mathrm{SO}_{3} \mathrm{H}$ & Boikr & 348727 \\
\hline$\therefore \mathrm{H}_{2} \mathrm{SO}_{<1}$ & $6 \cdot 12 \cdot 97$ & lusant & $\mathrm{H}_{2} \mathrm{SO}_{4}$ & Fislier & 967745 \\
\hline $\operatorname{Magh}_{2} \mathrm{Sith}$ & $(=-12-5)$ & cister & $\operatorname{WhaCl}{ }_{2} \cdot 6 \mathrm{H}_{2} \mathrm{O}$ & Wallinckrodt & 964335 \\
\hline $0.25 N \mathrm{NaOH}$ & $6121 / 97$ & $\mathrm{KKM}$ & NaOH & Baker & $K 03931$ \\
\hline$\ldots .25 \mathrm{~N}_{-} \mathrm{NaOH}$ & $6 / 29 / 97$ & $\mathrm{kech}$ & $\mathrm{NaOH}$ & Baker & $K 03931$ \\
\hline - & & & & & \\
\hline$\div-\bar{T}$ & & $\cdots$ & & & \\
\hline$\frac{\vdots}{\cdot}-\cdots \cdot \cdots \quad \cdot$ & & $\cdots$ & & & \\
\hline$+\frac{\vdots}{i}-\cdots$ & .* & & $\cdots$ & & \\
\hline$\vdots$ & & & & & \\
\hline
\end{tabular}

Witnessed \& Understood by me, Date

554

\begin{tabular}{|l|l|}
\hline Invented by & Date \\
\hline Recorded by
\end{tabular}


From Page No. $=$

Working standards are prepared by rinsing seven $100 \mathrm{~mL}$ volumetric flasks with freon 113. The first 3 standards are made from the intermediate spiking solution. Use glass, class A, ; pipets and transfer $10 \mathrm{~mL}, 5 \mathrm{~mL}$, and $2 \mathrm{~mL}$ to 3 different volumetric flask and dilute to volume with freon 113 . These are $1.0 x, 20 x$, and a $50 x$ dilution of the intermediate spiking solution.

The next three standards are made from the $10 \mathrm{x}$ dilution just made. Transfer $10 \mathrm{~mL}, 5 \mathrm{~mL}$, and $2 \mathrm{~mL}$ to 3 different volumetric flask and dilüte to volume with freon 113. These are 10x, 20x, and a 50x dilution of the $10 x$ dilution. The last standard is a blank which is $100 \mathrm{~mL}$ of Ereon 113.

The ICV is prepared by adding $3 \mathrm{~mL}$ intermediate QC spiking solution to a $100 \mathrm{~mL}$ volumetric flask and diluted to volume with freon 113 .

Labeling of solutions will be as follows:

$10 \mathrm{x}$ IR-WS-(INITIALS), Date, Concentration

$20 x$ IR-WS-(INITIALS), Date, Concentration

$50 \mathrm{x}$ IR-WS-(INITIALS), Date, Concentration

100x IR-WS-(INITIALS), Date, Concentration

200x IR-WS-(INITIALS), Date, Concentration

500x IR-WS-(INITIALS), Date, Concentration

Blank IR-WS-(INITIALS), Date, Concentration

ICV IR-ICV-(INITIALS), Date, Concentration
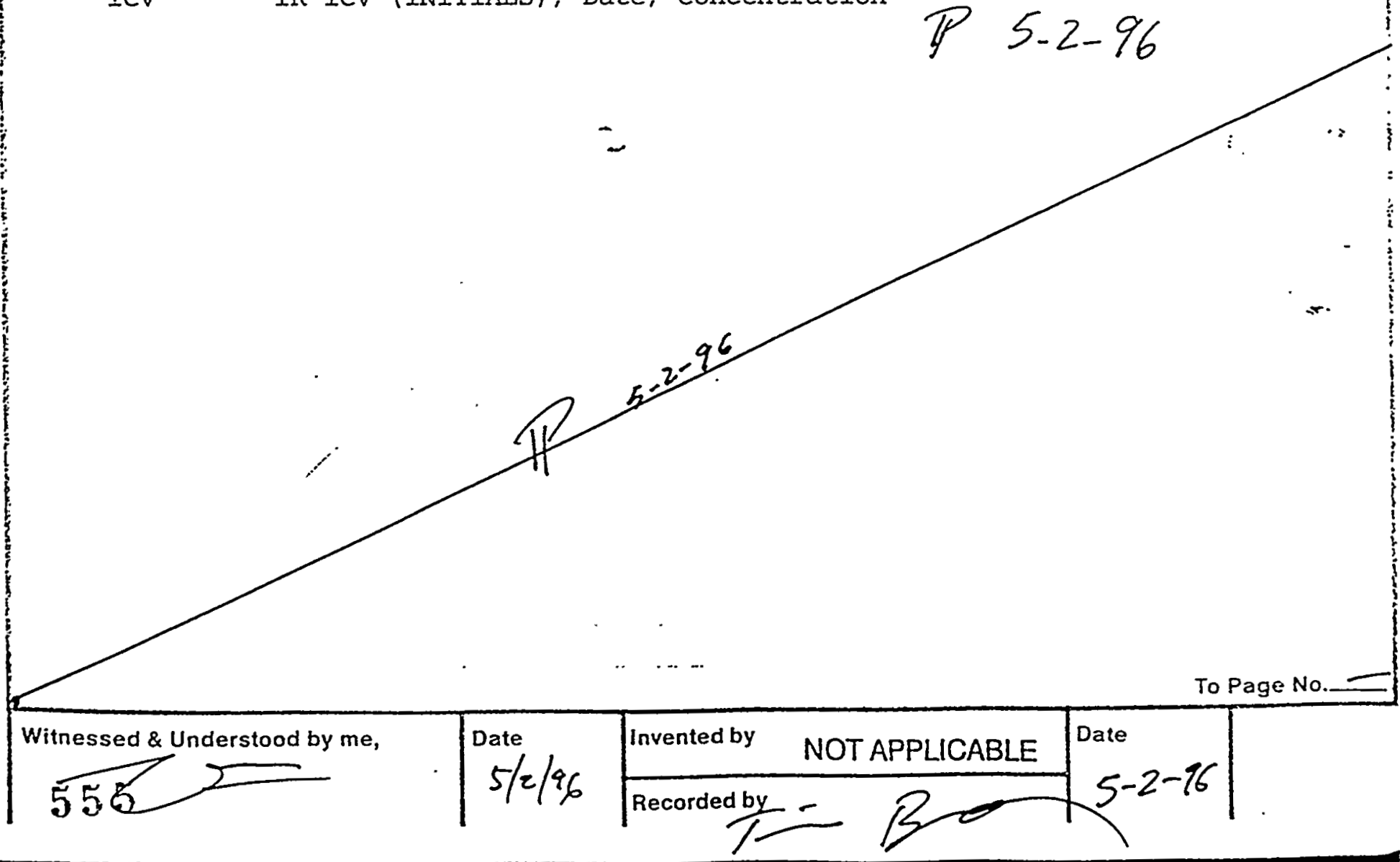


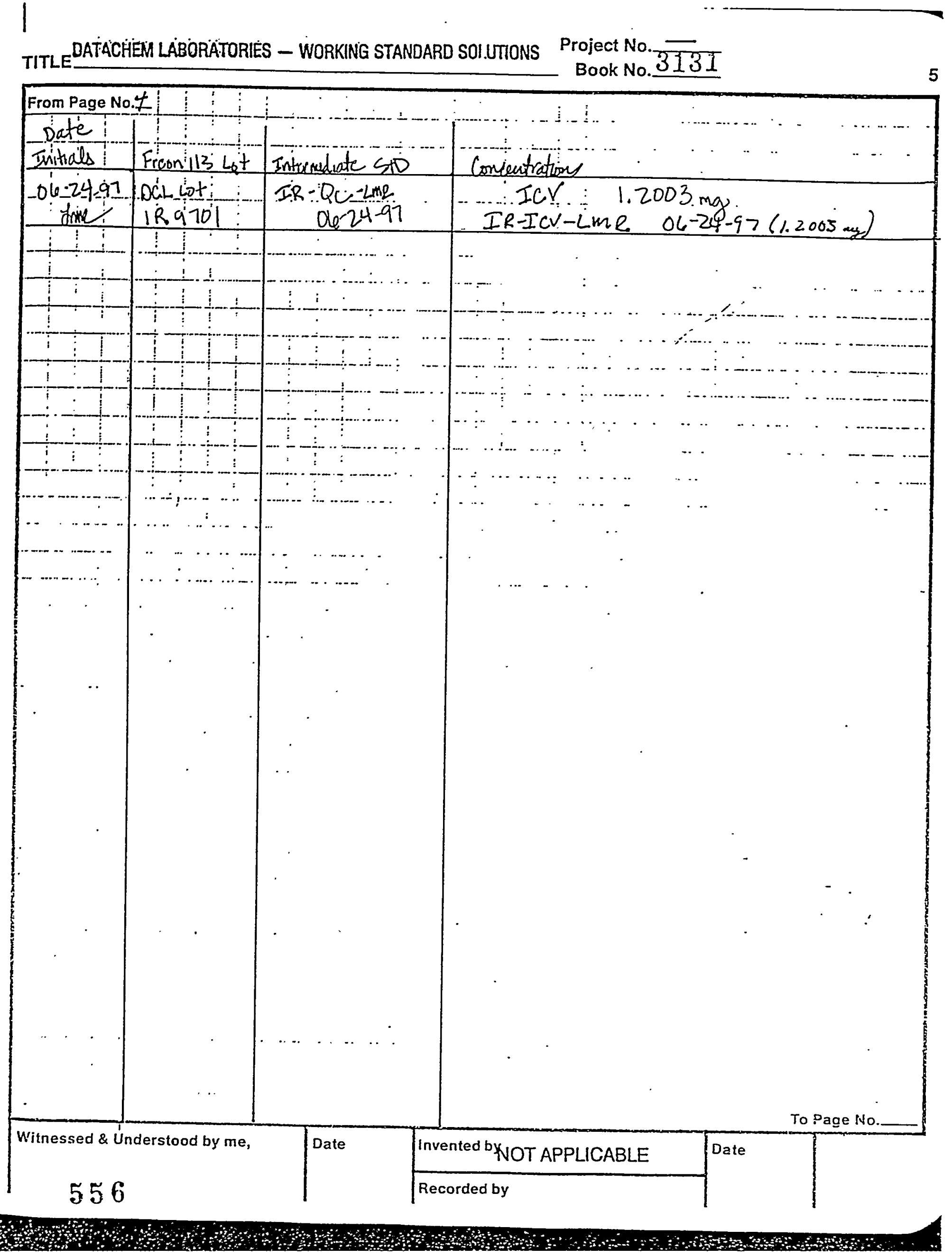




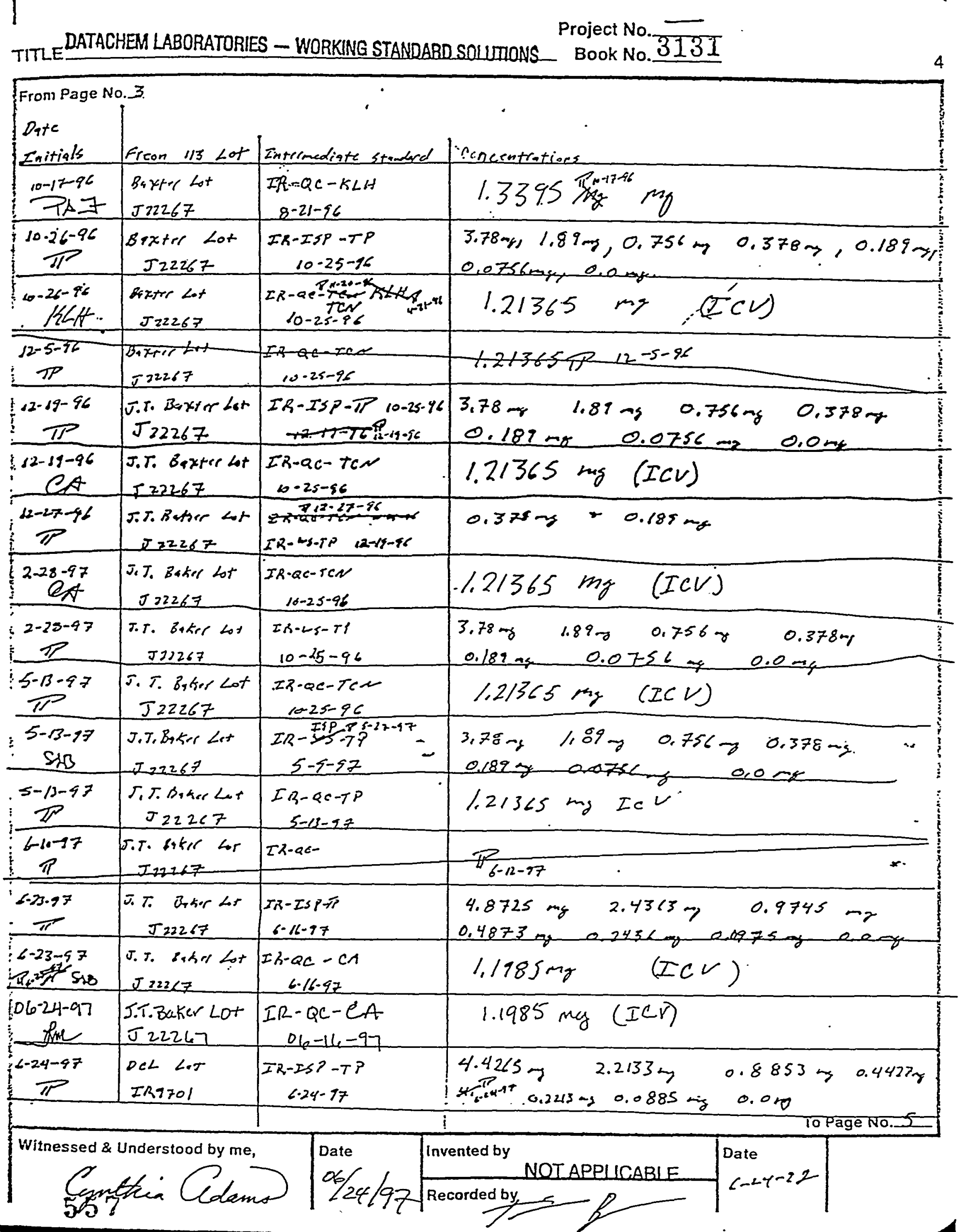


DATACHEM LABORATORIES - WORKING STANDARDSOHUHONS- BOok No. B

From Page No.I

QC water samples are prepared by using $3 \mathrm{ml}$ of intermediate QC spiking solution to 1 liter of ASTM type II $\mathrm{H}_{2} \mathrm{O}$ and acidified using $5 \mathrm{~mL}$ of $1: 1 \mathrm{HCI}$ or $2.5 \mathrm{~mL}$ of $1: 1 \mathrm{H}_{2} \mathrm{SO}_{4}$. Then extracted as a sample.

QC soil samples are prepared by using $3 \mathrm{ml}$ of intermediate QC spiking solution added to $10.0 \mathrm{~g}$ of $\mathrm{Na}_{2} \mathrm{SO}_{4}$ and extracted as a ; sample following method 3550 .

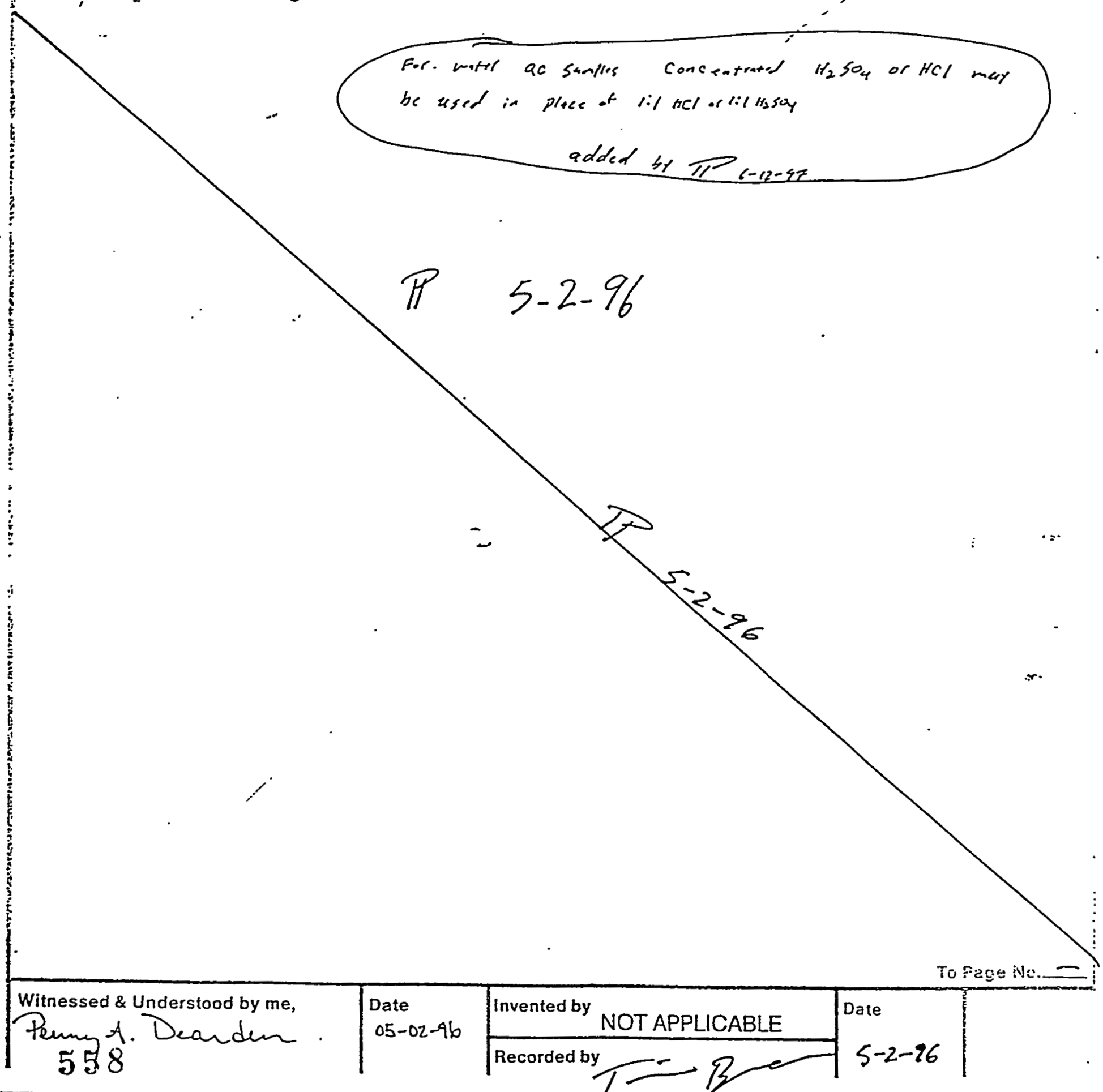




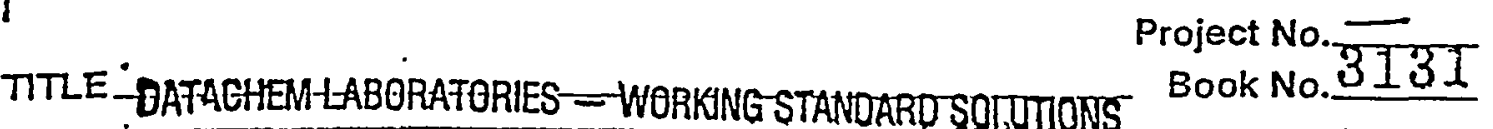

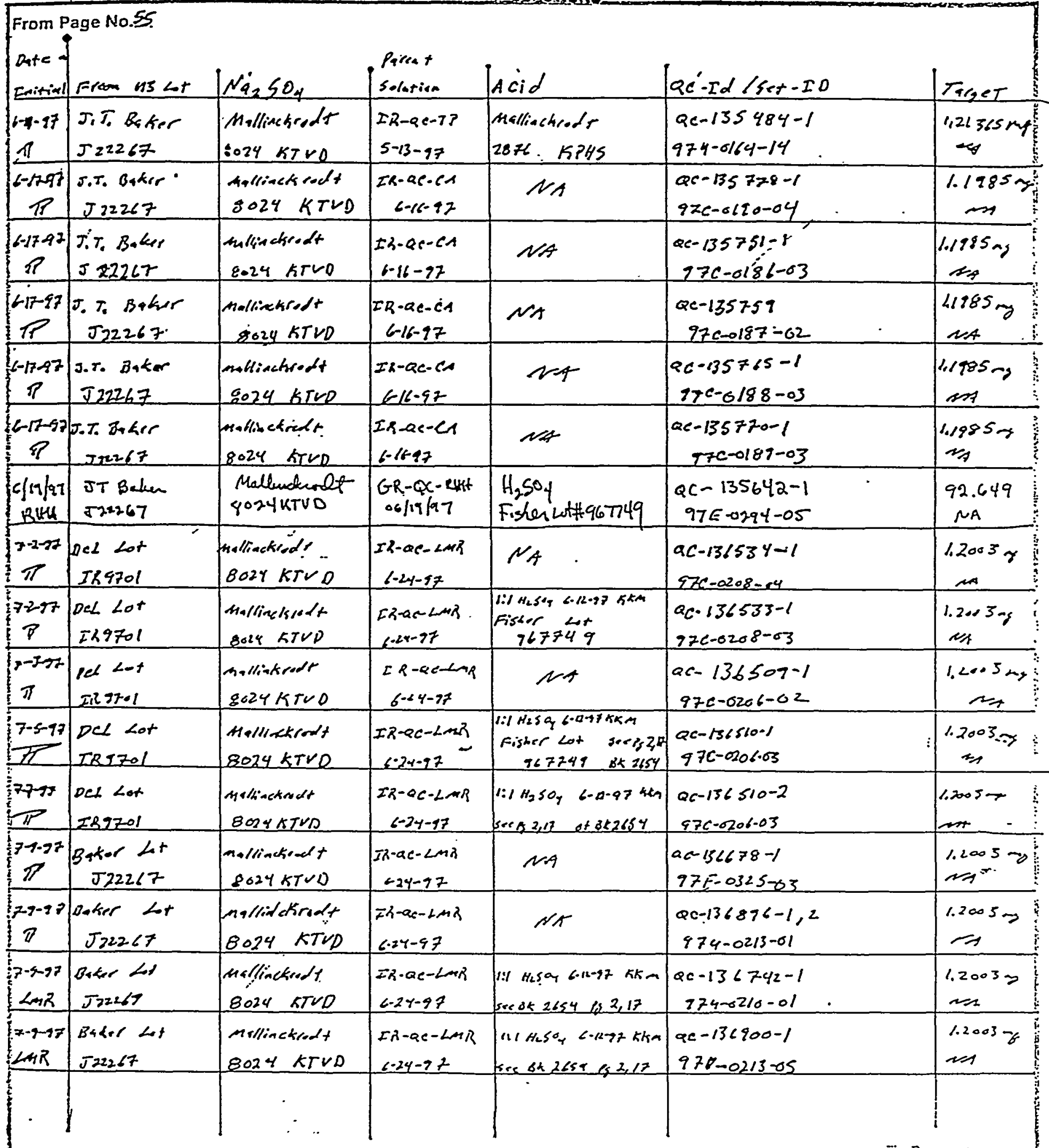

TO Fag : :

\begin{tabular}{|l|l|l|l|l}
\hline Witnessed \& Understood by me, & Date & Invented by NOT AFiLLGABLE & Date
\end{tabular}

559

Recorded by 
DATACHEM LABORATORIES - SECONDARY STOCK STANDARO SOUTONS Project No.NA TITLE

From Page No.35

$I R-I S \dot{P}-T P \quad 6=\dot{T}=9=$

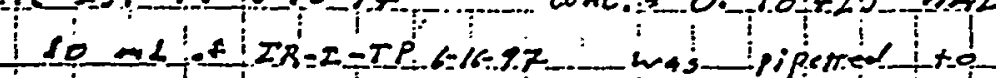

:

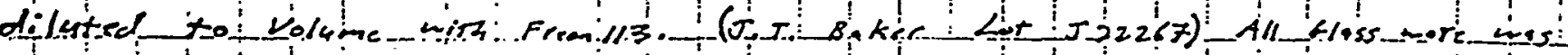

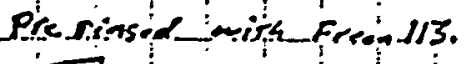

$Z=8 \dot{2}<-1<z z$

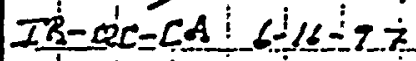

$\checkmark b y$

Cymai Celoms $06 / 16 / 97$

$\operatorname{conc}=0,32552 \pi / 4$

Expires ! 6:16-\$?

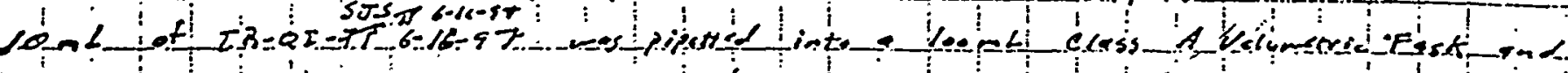

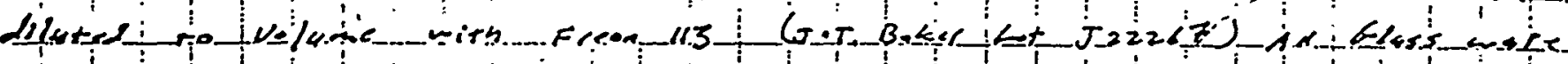

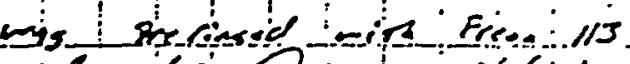

i Conthed Clemw: $06 / 16 / 97$

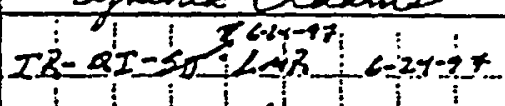

i l

$I X=R I=\ln \dot{R}+\dot{i}+24-9 z$

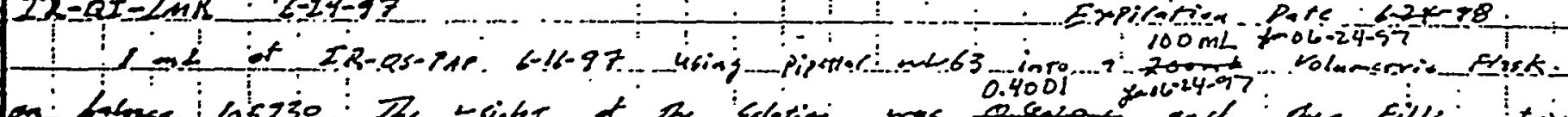

$16-16-97$

$\dot{x}-6=23=9.7$

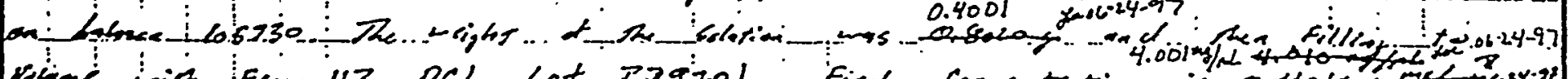

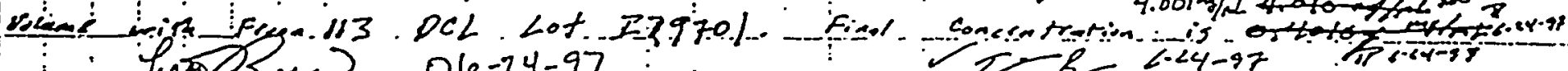

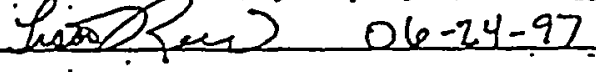

$23=0.4-4 \pi R-2-24-27$ $2=\not \beta-24-97$ $16-2-07-21.9$

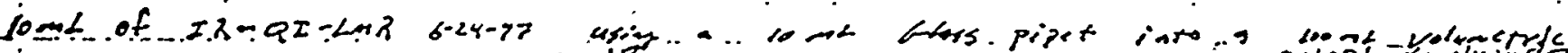

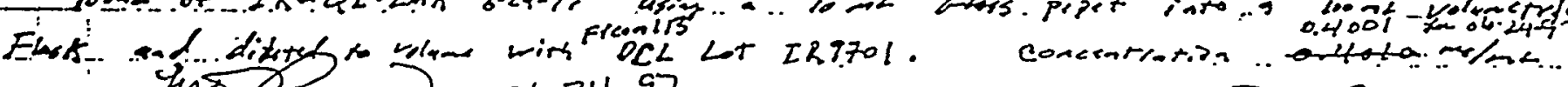

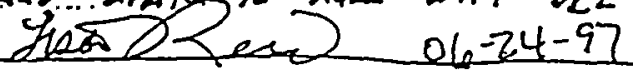

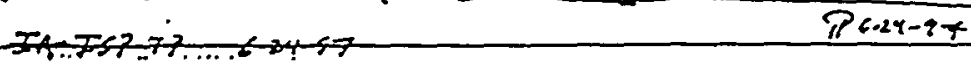

$2 B-2=\pi-2-2 x-97$

$1-20$ of $\frac{1}{2}+24-97$

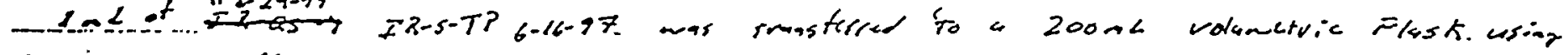

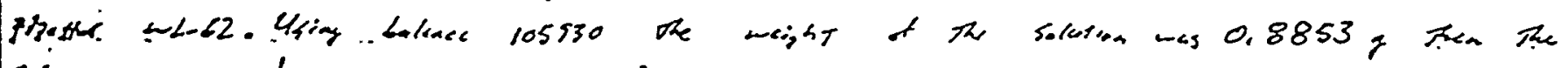

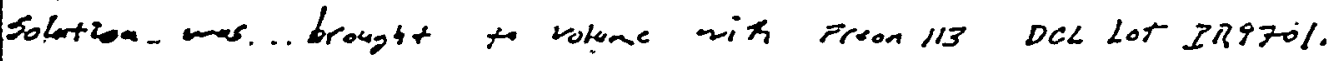

Concontration......4.4265.. M//sL

$=2.24-97$

$T R=T S P-. T P \ldots .6-24-47$

$12 \operatorname{los} 06-24-9.7$

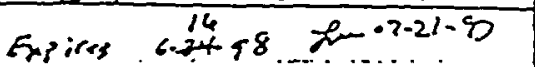

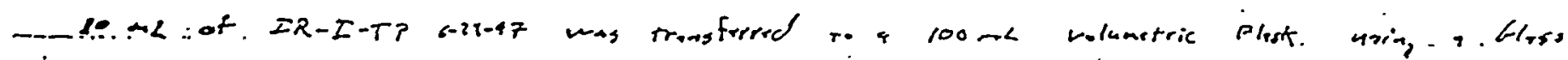

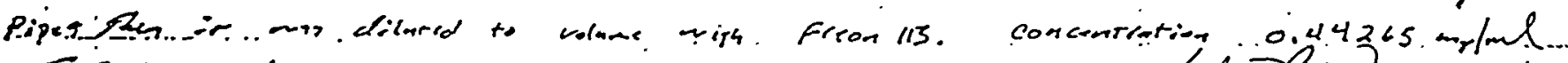

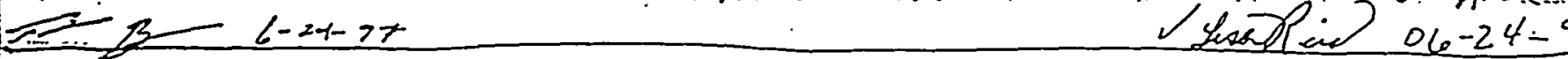
$-2=2-6 \cdot c y-37$ Spiors $16 \cdot x-2498 \ldots \ldots+21-97$ 


$$
\begin{array}{lr}
\text { TITLE DATACHEM LABORATORIES - PRIMARY STOCK STANDARDS } & \text { Project No. } \overline{-} \\
\text { Book No. } 2017
\end{array}
$$

13

From Page No.L2.

$$
\text { GR-QC-TP 10:7-96. }
$$

Concentration: 33.

$33.149 \mathrm{mg} / \mathrm{s}$

Expiration Date: 10-7-97

$3.3149 \mathrm{~g}$. of Oil. (Valvoline Tubb Formula. SAE $10 \mathrm{w} 30$ High Pertomence Motor Dil) into loomL ..class. A Volumetriz.. Flask.o. Then . diluted to volume wirt Freon 113 (Baker Lot. J.22267)...This is to. be wred only for oil and

Grase andysis by Giavametic..

$$
\text { GR-5 } 13 A 710-14-96
$$

Sed: Daud Danued - 10-796

$3.3299 \mathrm{~g}$ of Mazjola.corn Dil (Freshresodati Nov.1896) into a $100 \mathrm{~mL}$ class. . voluinetrie flaok which was dibet to volume with Frcon 113 (.Bater Lot. J22267). Volumitic was rinsed with Frcon 113. Thio is to bu be used. for oil and Grease analyeis by gramithic method.

Expination date: $10-14.97 \ldots$ Concentration: $33.299 \mathrm{mg} / \mathrm{nd}$

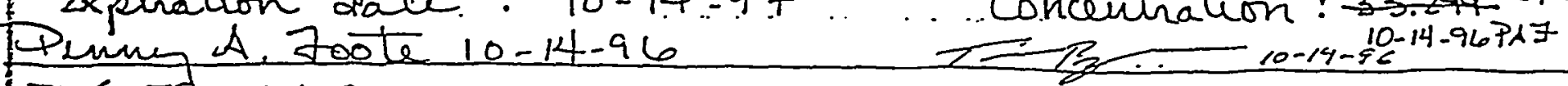
$2 x-5-T>2-16-1 z$

Expires. 6:16:98

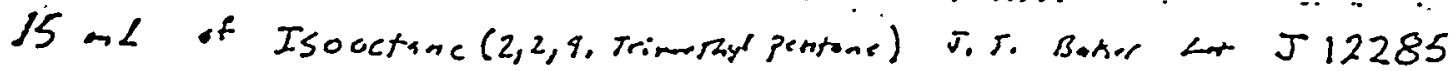
$15 \mathrm{aL}$ of Hexadane Aldrich Lot $01218 \mathrm{~J} X$

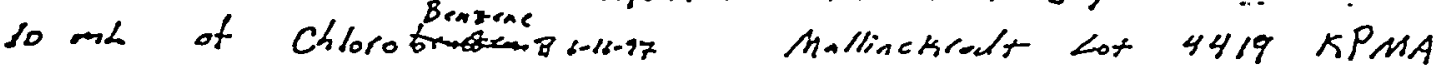

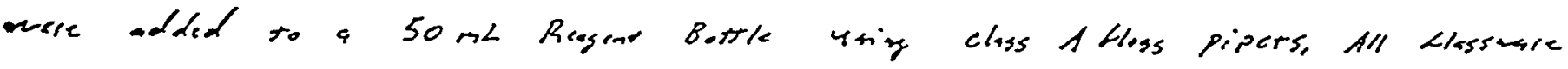
thas pre riused with Freed 113 . -

$72-Q 5-903$

$1-16-97$

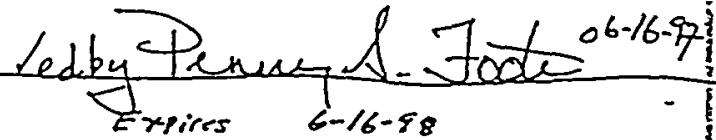

$6-16-97$

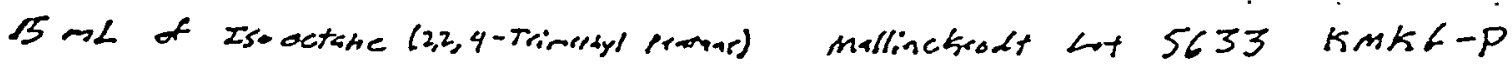

$15 \mathrm{~nL}$ of Hexadecane. J.T. Bater Lof J/8626

loont of chlorobenzene J:T Baker Lot J33l/6

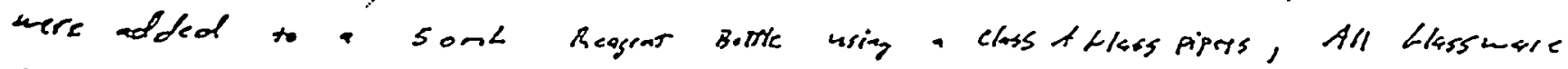
such presigsed with freon 113 .

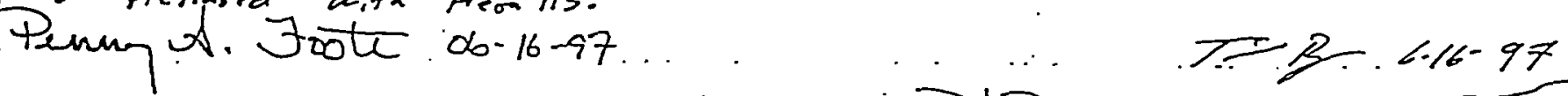

$$
06-16-97 \text { PAP. }
$$

To Page No. 14

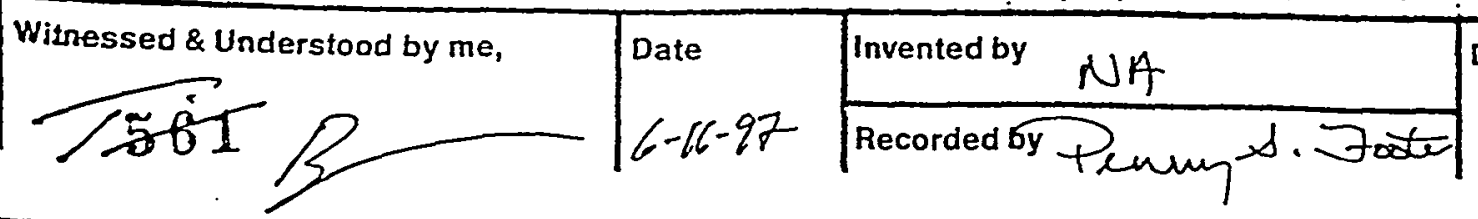

Date

$06-16-97$ 


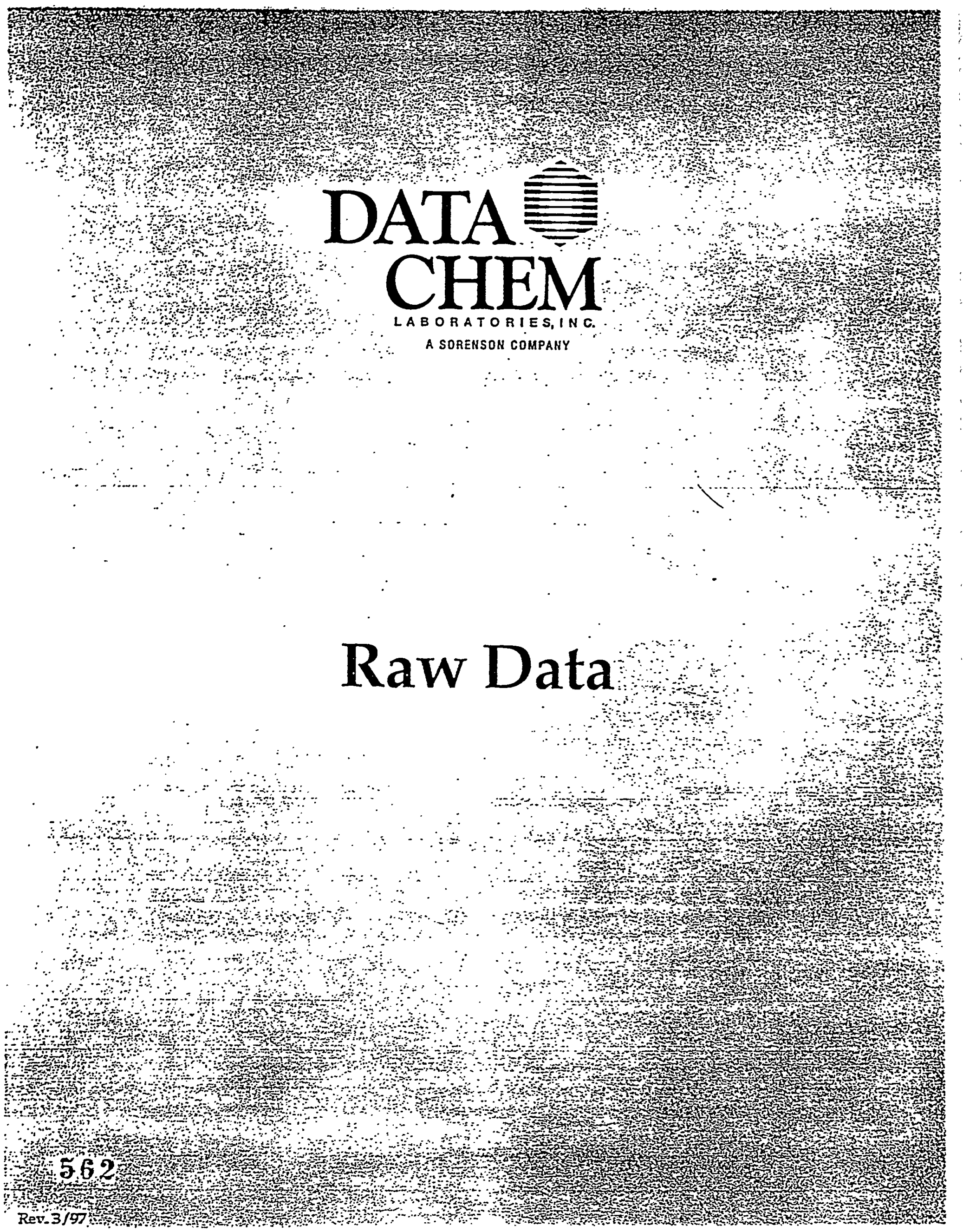




\section{TPH in Water}

Group \# : G9769006

Preparation Date : 7/2/97

Analysis Date : 7/9/97

Instrument I.D: FTIR-1000C

Set I.D's : 97C-0208-03 \& 04
WATER LOD : $100 \mu \mathrm{g} / \mathrm{L}$

Method: 418.1

Account \#: 3006

Analyst: TP .

Results are calculated according to the following formula:

$\mu \mathrm{g} /$ wipe Petroleum Hydrocarbons $=R^{*} D^{*}(1000 \mu \mathrm{g} / \mathrm{mg}) /$ \# of wipes

$\mathrm{Hg} / \mathrm{L}$ Petroleum Hydrocarbons $=R^{*} D^{*}(1000 \mu \mathrm{g} / \mathrm{mg}) / \mathrm{V}$

$\mathrm{F}=\mathrm{mg}$ of Petroleum Hydrocarbons as determined from the calibration plot.

$D=$ extract dilution factor, if used.

$\mathrm{V}=$ Volume of water sample, in liters.

\begin{tabular}{|c|c|c|c|c|c|}
\hline Sample\# & $\begin{array}{l}\mathrm{mg} \text { of Petroleum } \\
\text { Hydrocarbons }\end{array}$ & $\begin{array}{l}\text { Dilution } \\
\text { Factor }\end{array}$ & $\begin{array}{c}\text { Volume of water } \\
\text { Sample (L) }\end{array}$ & $\begin{array}{l}\mu \mathrm{p} / \mathrm{L} \text { Petroleum } \\
\text { Hydrocarbons }\end{array}$ & \\
\hline $0.0 \mathrm{mg}$ & 0.00460362 & 1 & 1.00 & 4.60362 & \\
\hline D.08B5 mg & 0.0881784 & 1 & 1.00 & 88.1784 & \\
\hline $0.2213 \mathrm{mg}$ & 0.224207 & 1 & 1.00 & 224.207 & \\
\hline $0.4427 \mathrm{mg}$ & 0.432771 & 1 & 1.00 & 432.771 & \\
\hline D.8B53 mg & 0.887344 & 1 & 1.00 & 887.344 & \\
\hline $2.2133 \mathrm{mr}$ & 2.21386 & 1 & 1.00 & 2213.86 & \\
\hline $4.4265 \mathrm{rg}$ & $4.426 € 3$ & 1 & 1.00 & 4426.63 & \\
\hline ICV - & 1.1534 & 1 & 1.00 & 1153.4 & \\
\hline ICB & -0.0167891 & 1 & 1.00 & -16.7891 & Data out of range \\
\hline QC-136533-1 & 1.10339 & 1 & 1.00 & 1103.39 & \\
\hline BL-136533-1 & 0.027796 & 1 & 1.00 & 27.796 & \\
\hline 97UD2896 & 0.0217985 & 1 & 1.06 & 20.56 & \\
\hline $97 \cup 02897$ & 0.0642703 & 1 & 1.06 & 60.63 & Data not used, dilution required \\
\hline 97UD2B97MS & 1.65054 & 1 & 1.06 & 1557.11 & \\
\hline 97UD2897MSD & 1.63115 & 1 & 1.06 & 1538.82 & \\
\hline 97UD2B9B & 0.048332 & 1 & 1.06 & 45.596 & \\
\hline 97U02B99 & 0.023811 & 1 & 1.06 & 22.463 & \\
\hline $97 \cup 02900$ & 0.0781683 & 1 & 1.06 & 73.744 & \\
\hline $97 \cup 02901$ & 0.0186285 & 1 & 1.06 & 17.574 & \\
\hline $\operatorname{ccV} 1$ & 2.21835 & 1 & 1.00 & 2218.350 & \\
\hline ССВ 1 & -0.0266641 & 1 & 1.00 & -26.664 & Data out of range \\
\hline
\end{tabular}

\begin{tabular}{|c|c|c|c|c|c|c|}
\hline Sample \# & $\begin{array}{l}\mathrm{mg} \text { of Petroleum } \\
\text { Hydrocarbons }\end{array}$ & $\begin{array}{l}\text { Dilution } \\
\text { Factor }\end{array}$ & $\begin{array}{c}\text { \# of wipes per } \\
\text { Sample }\end{array}$ & & $\begin{array}{l}\mu \mathrm{g} / \mathrm{Wipe} \\
\mathrm{TPH} \\
\end{array}$ & \\
\hline QC-136534-1 & 1.06209 & & & 1 & 1062.090 & ipe \\
\hline BL-136534-1 & 0.00737395 & & & 1 & 7.374 & \\
\hline 97U02902 & 0.0584462 & & & 1 & 58.446 & \\
\hline $97 \cup 02903$ & 0.0601039 & & & 1 & 60.104 & \\
\hline $97 \cup 0290 R$ & 0.0630375 & & & 1 & 63.038 & \\
\hline 97UD29[ 31/S & 1.66409 & & & 1 & 1664.090 & \\
\hline 97UD290BMSD & 1.62978 & & & 1 & 1629.780 & \\
\hline $\operatorname{ccv} 2$ & 2.21488 & & & 1 & 2214.880 & \\
\hline $\mathrm{CCB} 2$ & -0.0268956 & & & 1 & -26.896 & Data out of range \\
\hline
\end{tabular}

$-$

- 563 


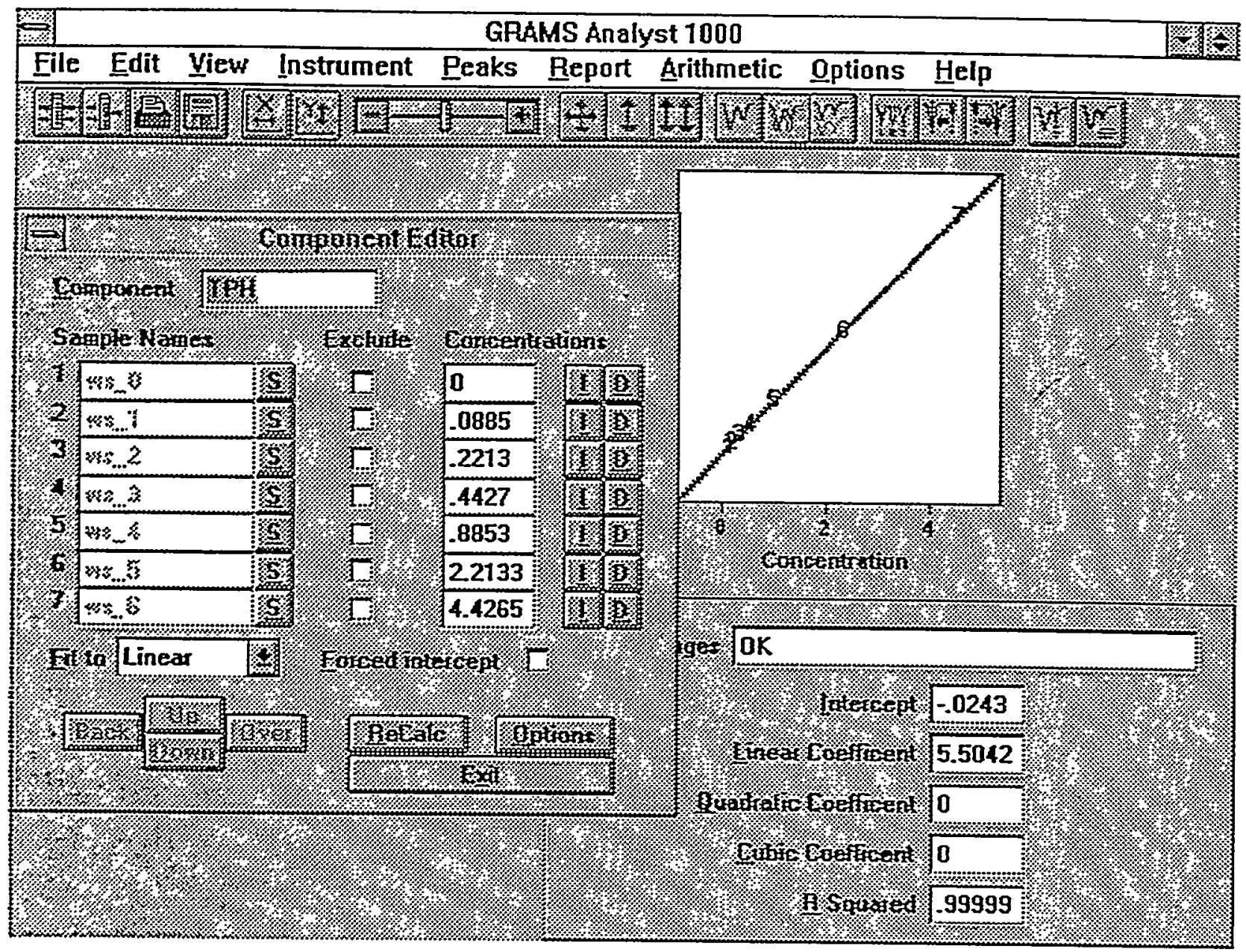

Calibration data for set 97C-0208-04 and 97C-0208-03 Instrument ID: FTIR-1000C 
QuantBasic Report

Quant Type: Multipoint $\quad$ 7/15/97 5:01 pm

Method: C:LE1000GSIDATAL703GTPH.Q

Sample: c:pe1000gsldata197036lcalcurve.spc

Analyst: TP

Sample ID: 0.0

Component Concen R-Squared

$\begin{array}{lll}\text { TPH } & .00460362 & .999991\end{array}$

Instrument 1.D.: FTIR-1000C

Analyzed on: 09-JUL-1997

Date and time on printout is the day and time copy was printed.

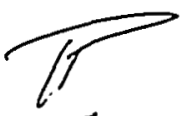

$$
7-16-37
$$
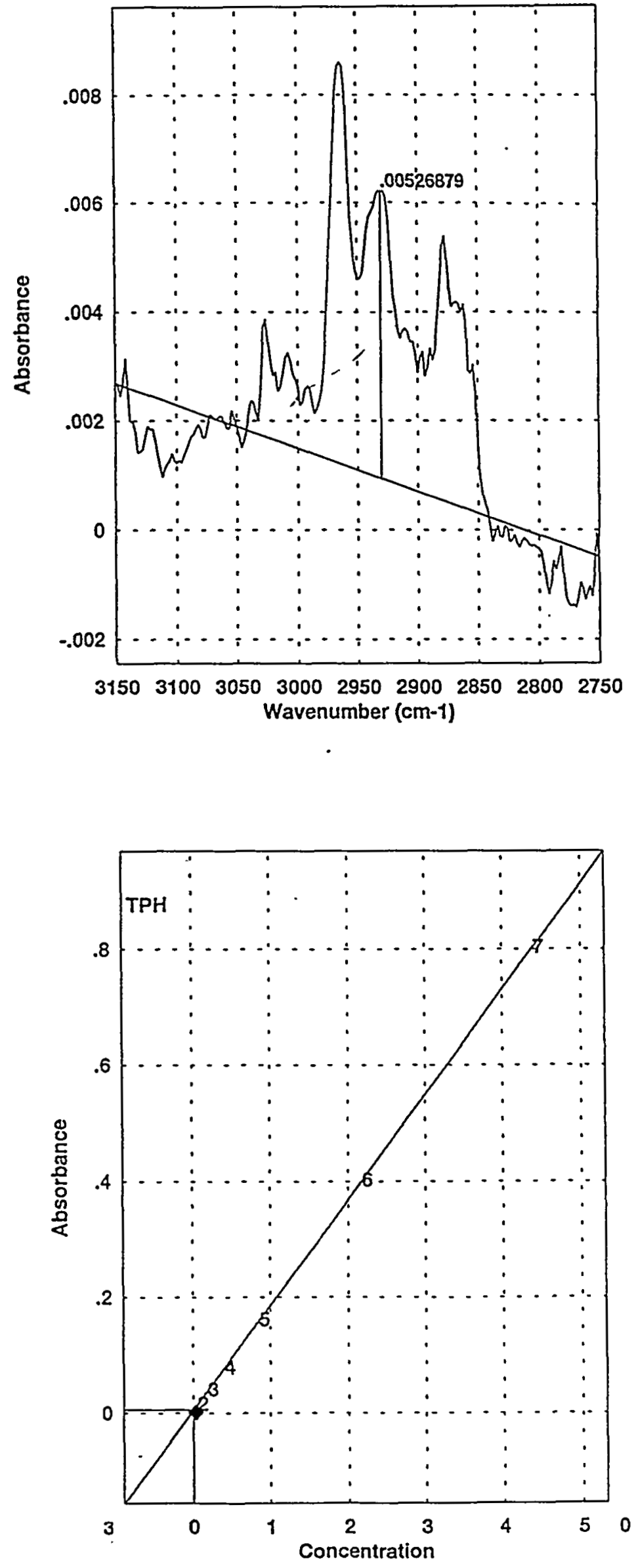
QuantBasic Report

Quant Type: Multipoint $\quad 7 / 15 / 97$ 5:01 pm

Method: C:PE1000GSDATAL703GTPH.Q

Sample: c:lpe1000gsldatal97036lcalcurve.spc

Analyst: TP

Sample ID: 0.0885

Component Concen R-Squared

$\begin{array}{lll}\text { TPH } & .0881784 & .999991\end{array}$

Instrument 1.D.: FTIR-1000C

Analyzed on: 09-JUL-1997

Date and time on printout is the day and time copy was printed.

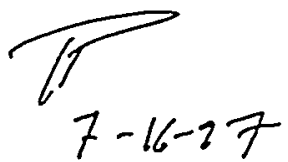

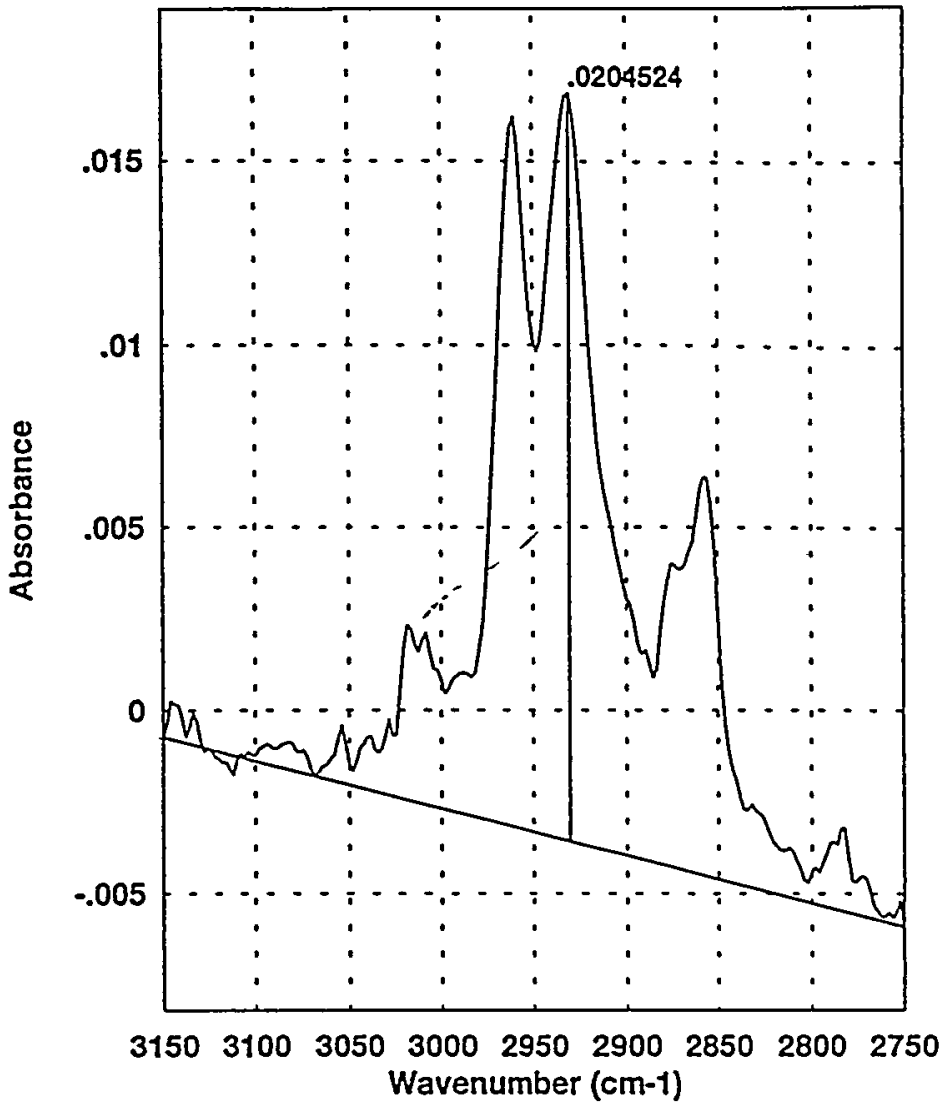

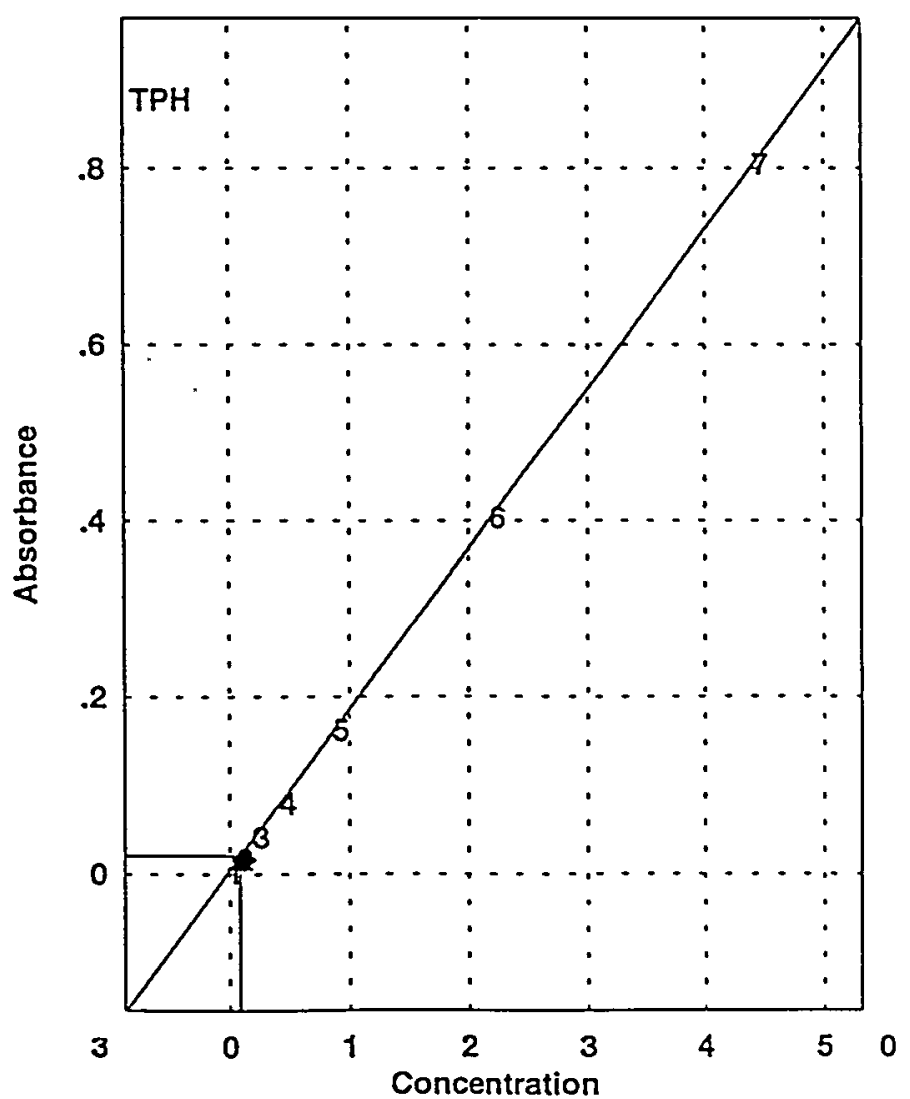


QuantBasic Report

Quant Type: Multipoint $\quad 7 / 15 / 97$ 5:00 pm

Method: C:IPE1000GSIDATAL7036TPH.Q

Sample: c:lpe1000gsldatal07036lcalcurve.spe

Analyst: TP

Sample ID: 0.2213

Component Concen R-Squared

TPH $\quad .224207 \quad .999991$

Instrument l.D.: FTIR-1000C

Analyzed on: 09-JUL-1997

Date and time on printout is the day and time copy was printed.

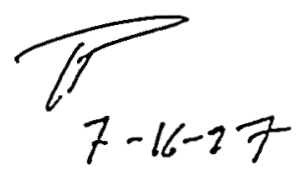

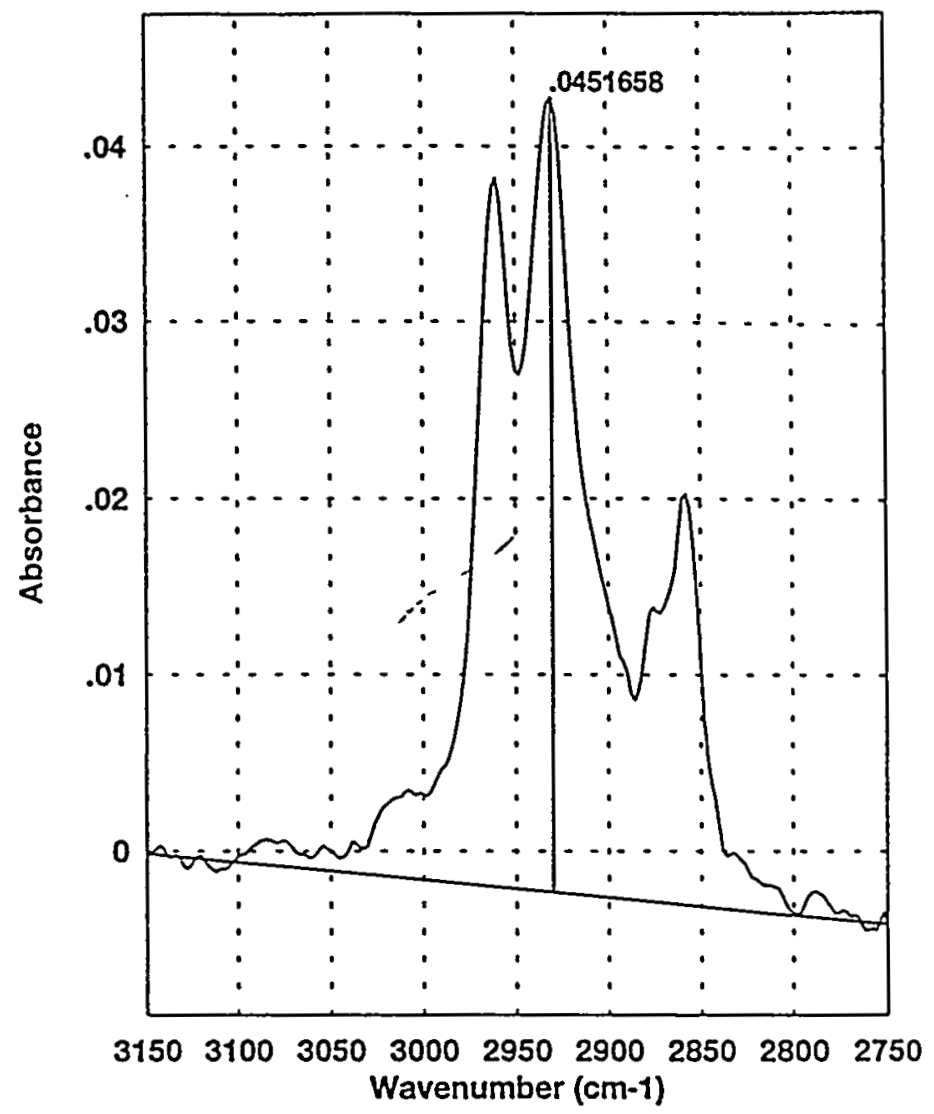

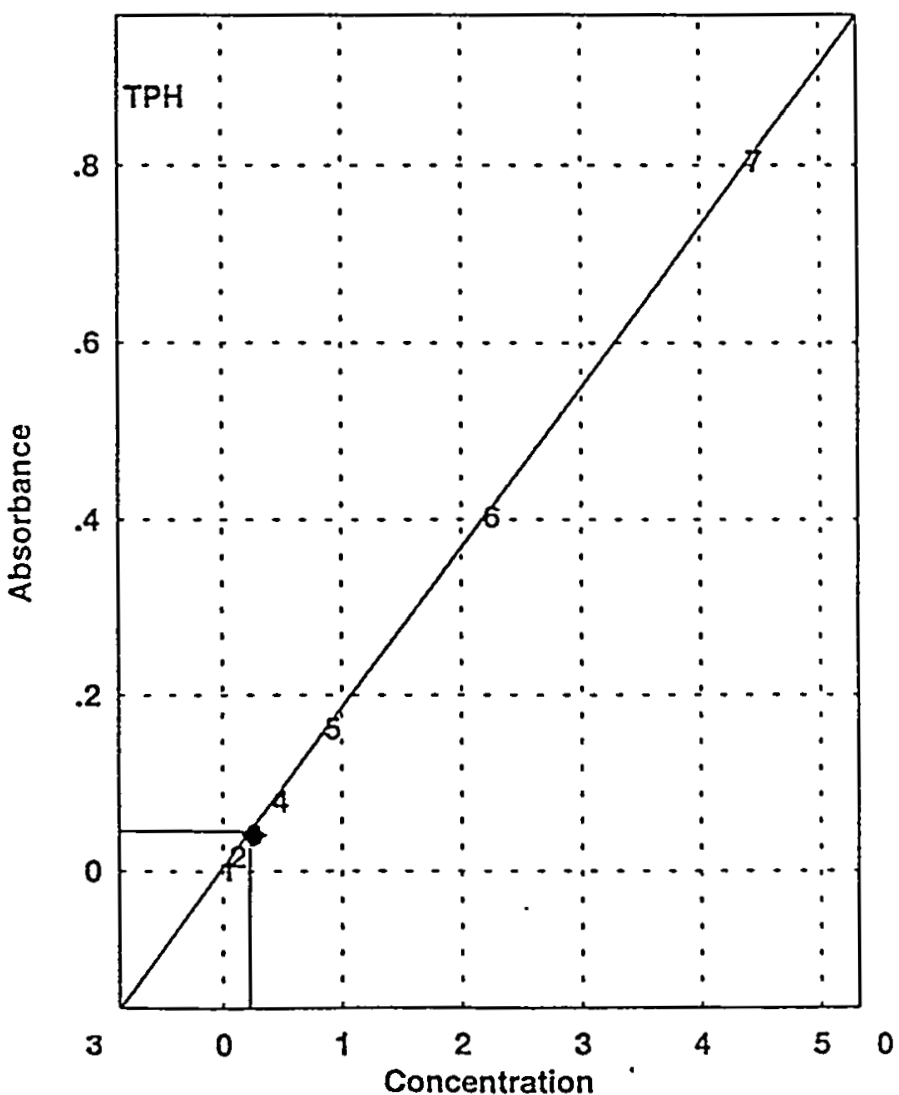


QuantBasic Report

Quant Type: Multipoint $\quad 7 / 15 / 97$ 5:00 pm

Method: C:IPE1000GSUATAL703GTPH.Q

Sample: c:lpe1000gsldatalo7036lcalcurve.spc

Analyst: TP

Sample ID: 0.4427

Component Concen R-Squared

$\begin{array}{lll}\text { TPH } & .432771 \quad .999991\end{array}$

Instrument 1.D.: FTIR-1000C

Analyzed on: 09-JUL-1997

Date and time on printout is the day and time copy was printed.

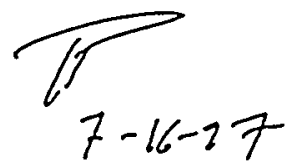

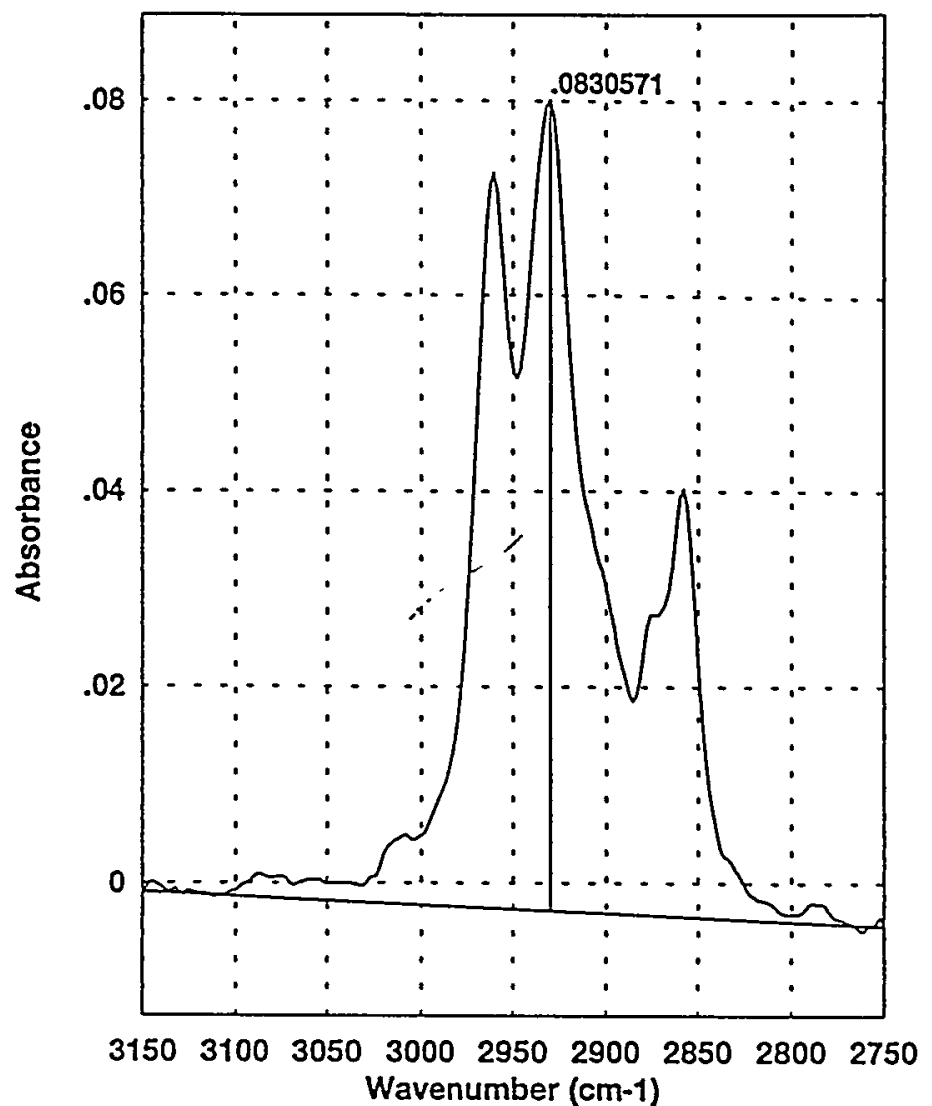

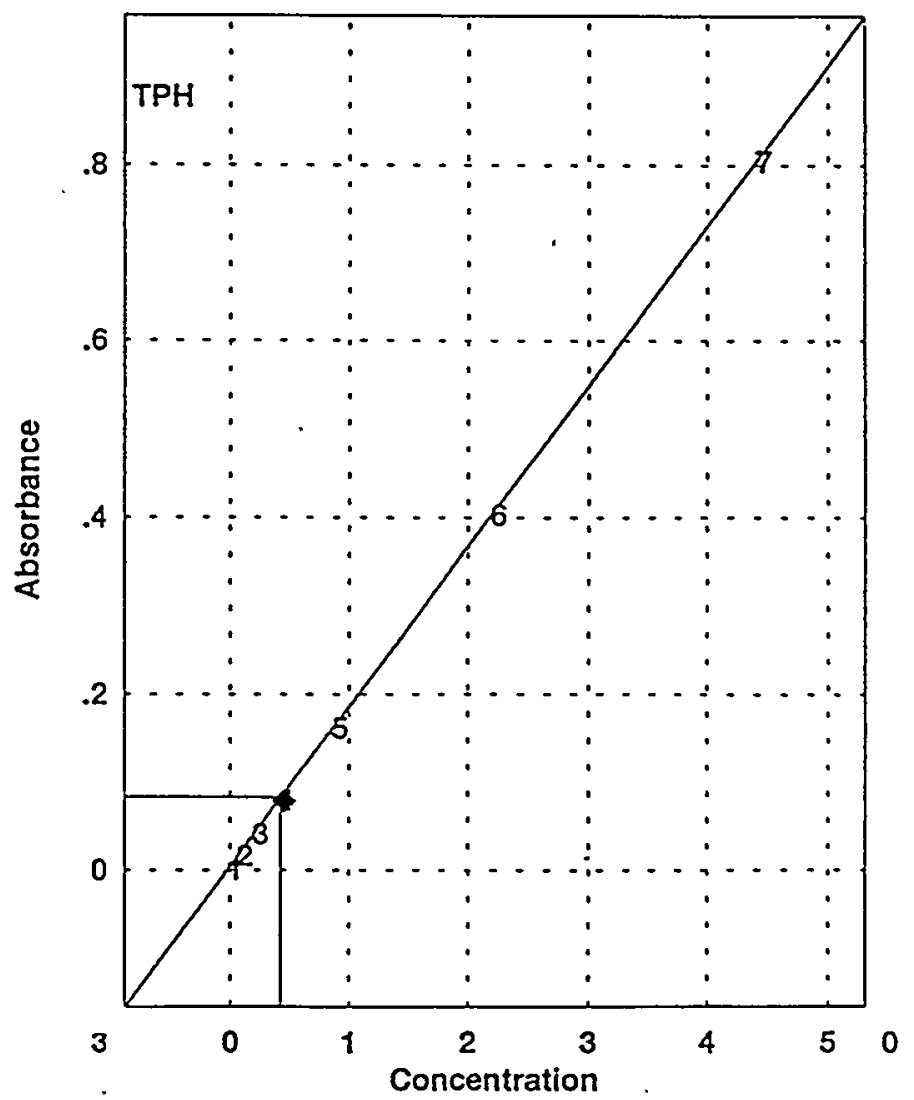


QuantBasic Report

Quant Type: Multipoint $\quad 7 / 15 / 97$ 5:00 pm

Method: C:IPE1000GSLATAL703GTPH.Q

Sample: c:lpe1000gsldata197036lcalcurve.spc

Analyst: TP

Sample ID: 0.8853

Componẹnt Concen R-Squared

$\begin{array}{lll}\text { TPH } & .887344 & .999991\end{array}$

Instrument I.D.: FTIR-1000C

Analyzed on: 09-JUL-1997

Date and time on printout is the day and time copy was printed.

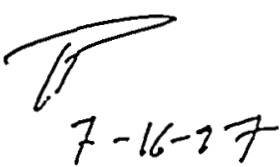

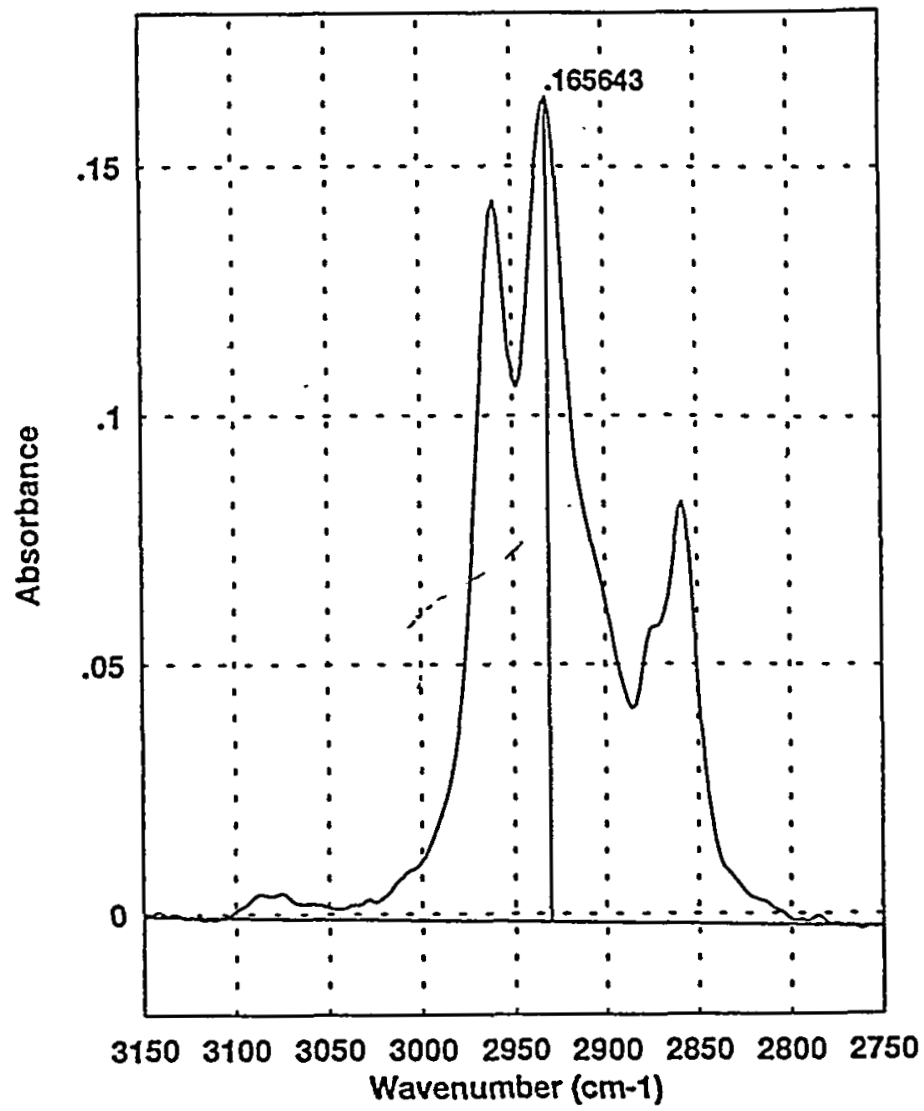

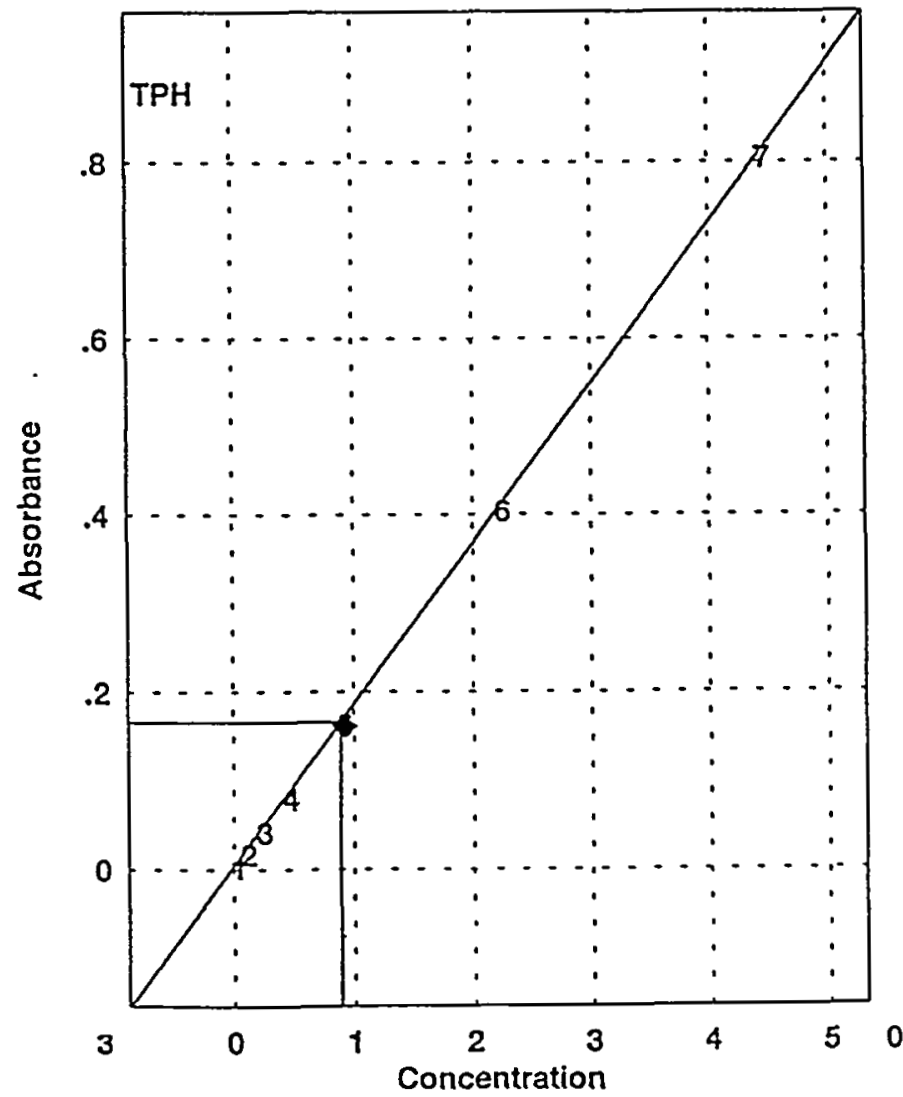


QuantBasic Report

Quant Type: Muttipolnt $\quad 7 / 15 / 97$ 4:59 pm

Method: C:IPE1000GSLATAL7036ITPH.Q

Sample: c:lpe1000gsldatal97036lcalcurve.spo

Analyst: TP

Sample ID: 22133

Component Concen R-Squared

$\begin{array}{lll}\text { TPH } & 221386 & .999991\end{array}$

Instrument I.D.: FTIR-1000C

Analyzed on: 09-JUL-1997

Date and time on printout is the day and time copy was printed.

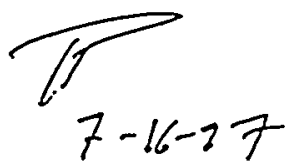

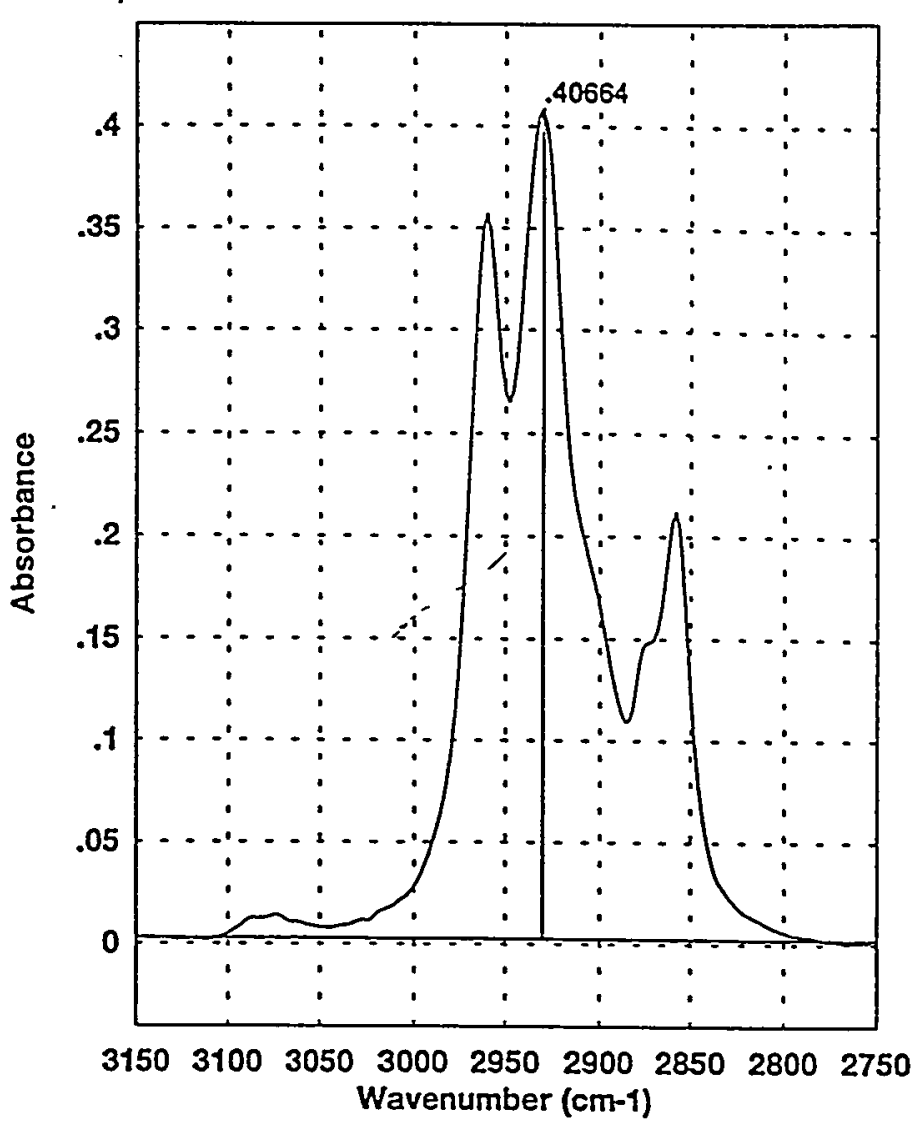

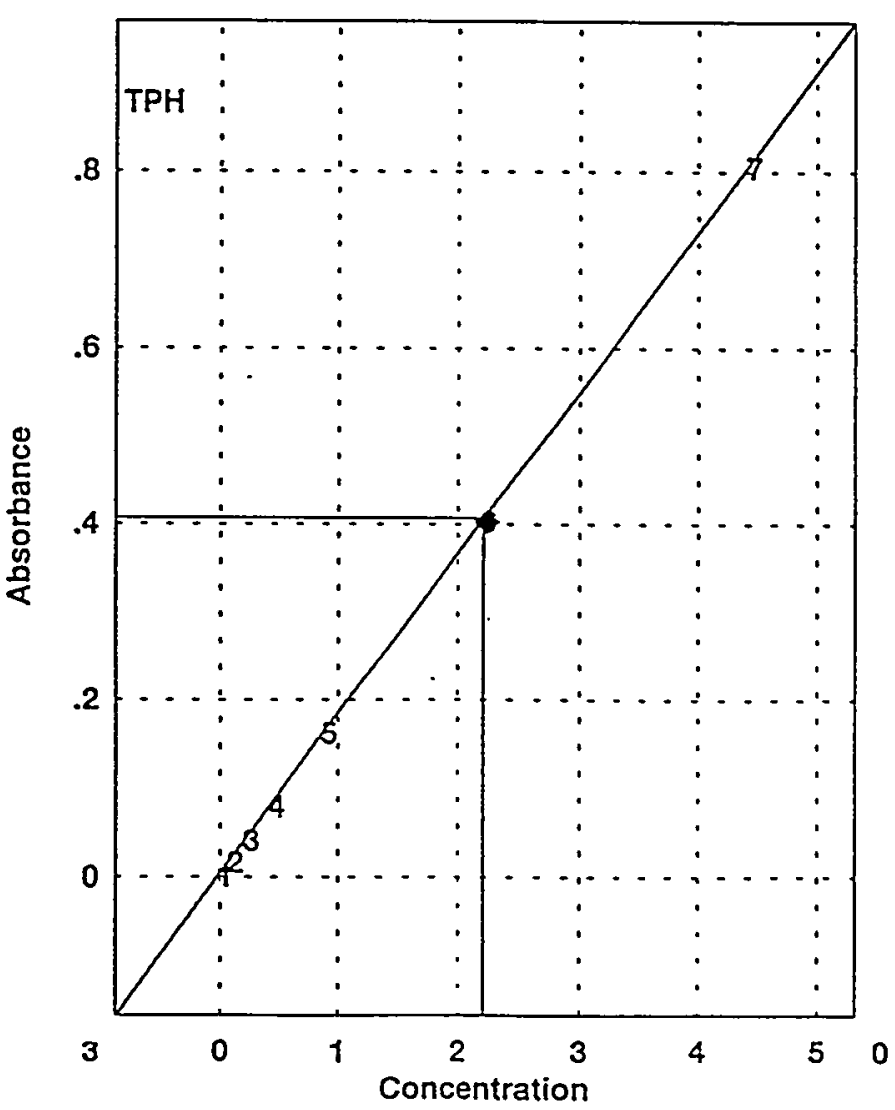

590 


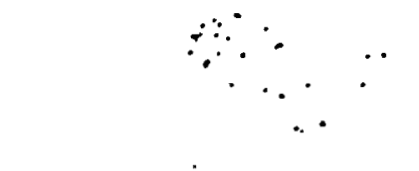

QuantBasic Report

Quant Type: Multipolnt $\quad 7 / 15 / 97$ 4:59 pm

Method: C:PEE1000GSLATAL703GTPH.Q

Analyst: TP

Sample ID: 4.4265

Component Concen R-Squared

$\begin{array}{lll}\text { TPH } & 4.42663 \quad .999991\end{array}$

(1)

\section{Sample: c:lpe 1000gsidatal97036lcalcurve.spc}
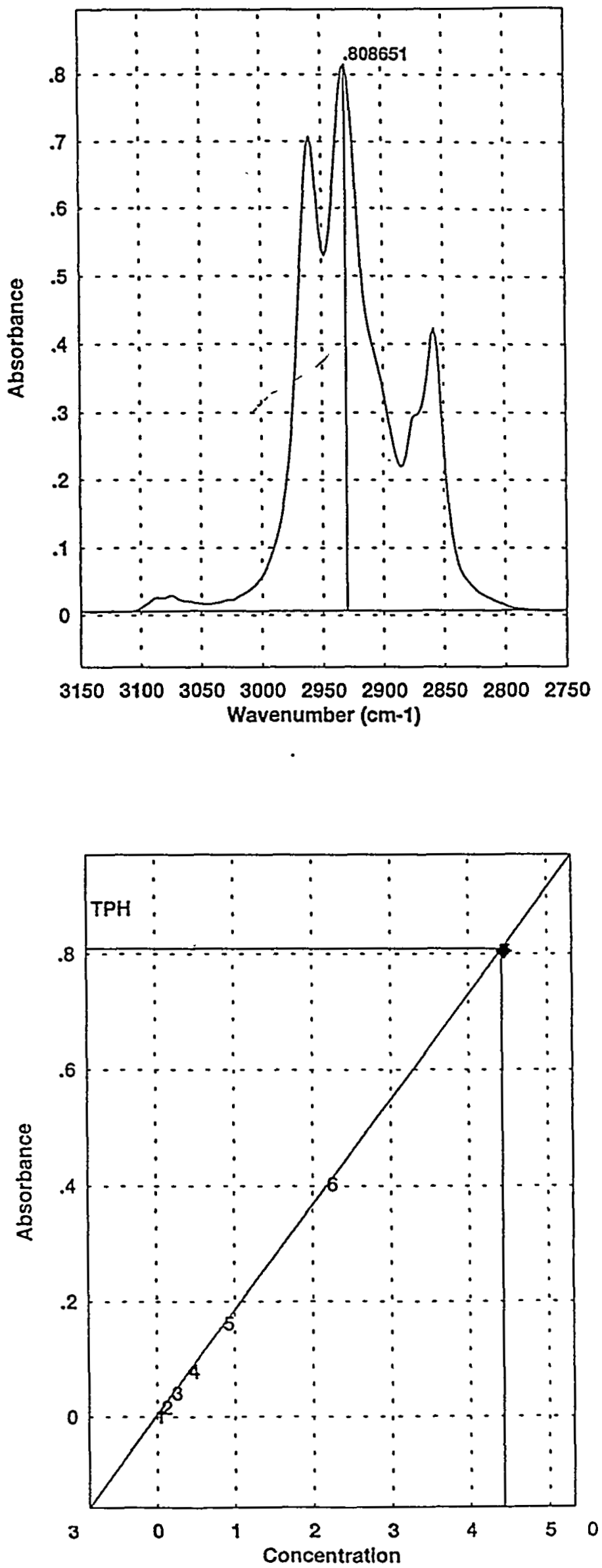

Date and time on printout is the day and time copy was printed.

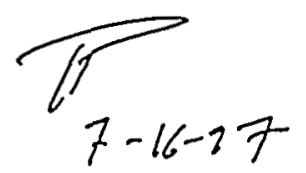

$$
7-16-27
$$

Instrument I.D.: FTIR-1000C

Analyzed on: 09-JUL-1997 
QuantBasic Report

Quant Type: Multipoint $\quad$ 7/15/97 5:02 pm

Method: C:LPE1000GSIDATAL7036ITPH.Q

Sample: c:\$pe1000gsidatal97036lcalcurve.spc

Analyst: TP

Sample ID: ICV

Component Concen R-Squared

TPH $1.1534 \quad .999991$

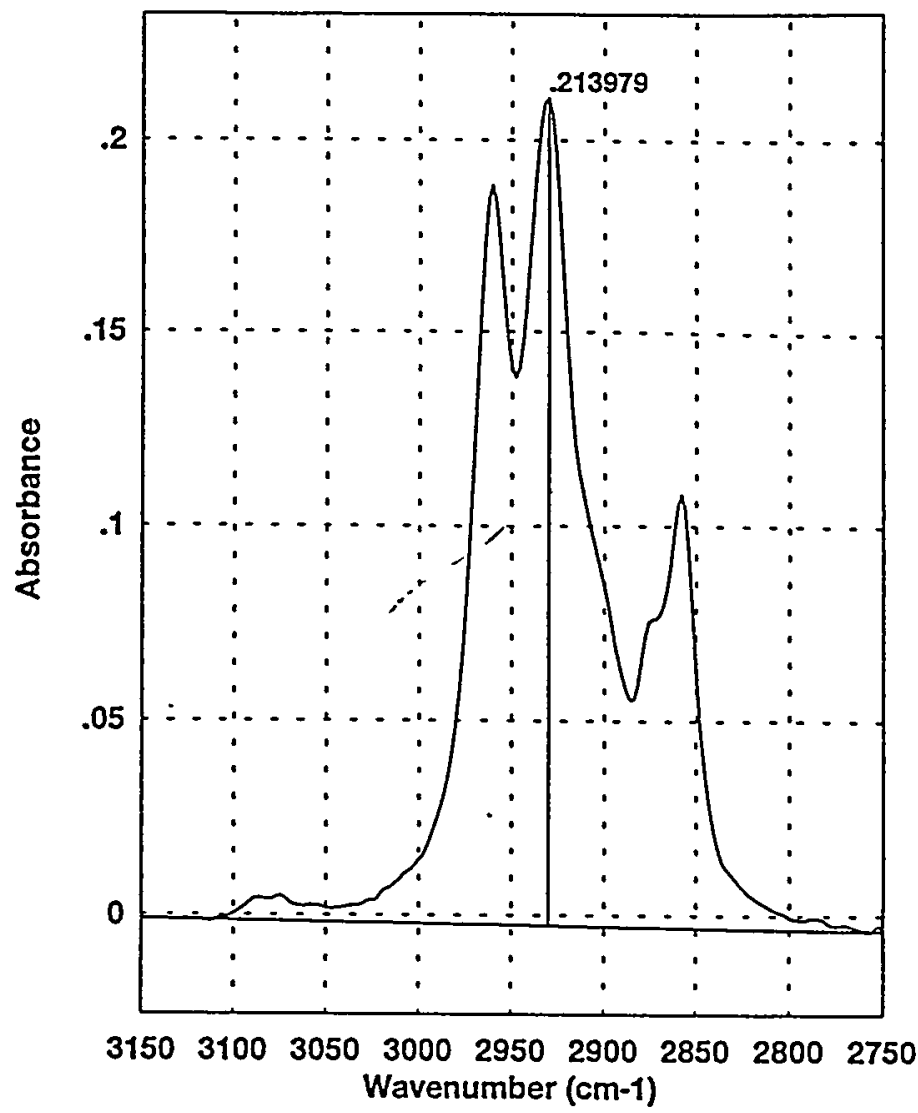

Instrument I.D.: FTIR-1000C

Analyzed on: 09-JUL-1997

Date and time on printout is the day and time copy was printed.
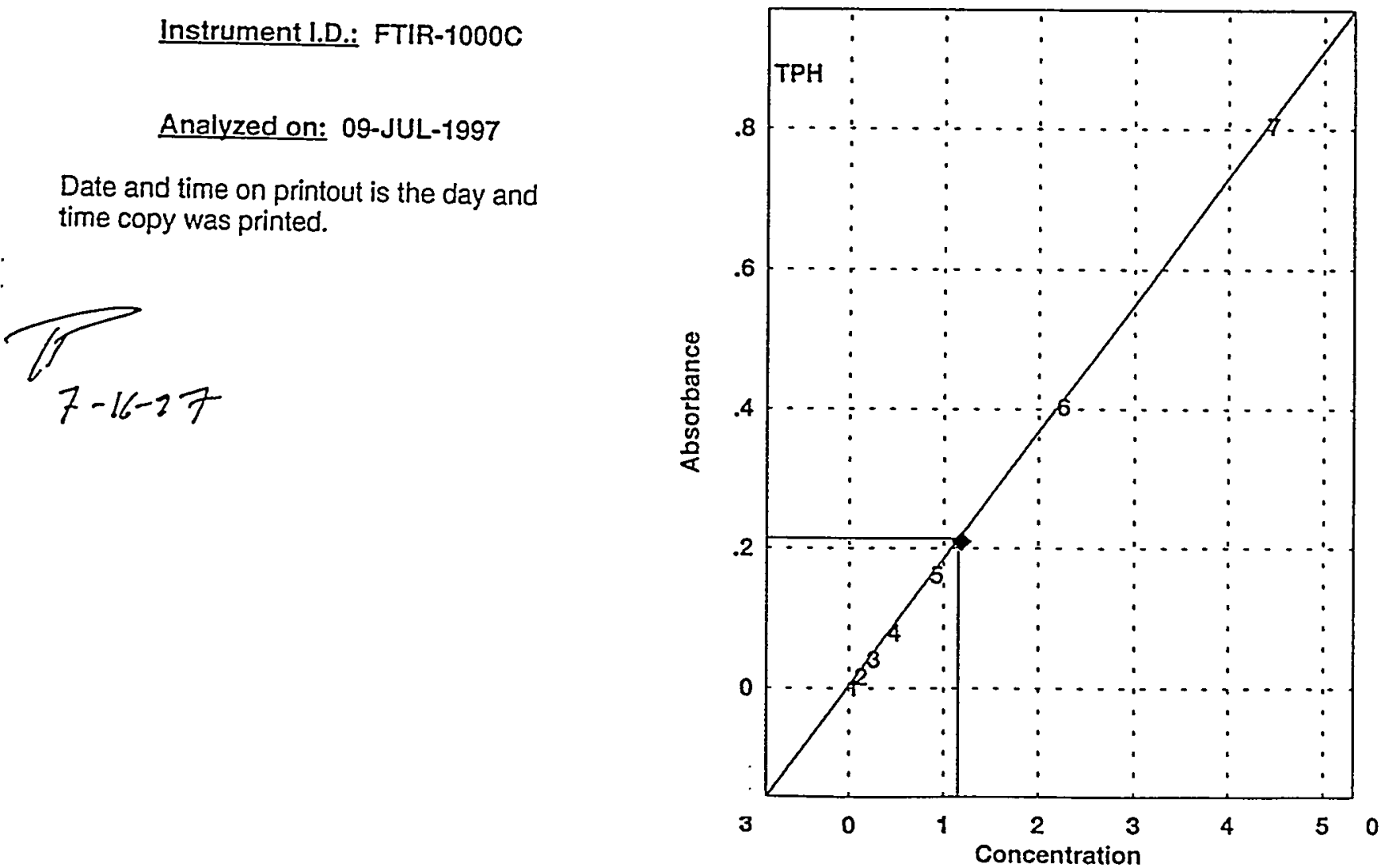
QuantBasic Report

Quant Type: Multipoint $\quad$ 7/15/97 5:02 pm

Method: C:IPE1000GSIDATAL707036ITPH.Q

Sample: c:lpe1000gsldatala7036lcalcurve.spc

Analyst: TP

Sample ID: ICB

Component Concen R-Squared

TPH $\quad-.0167891 \quad .999991$

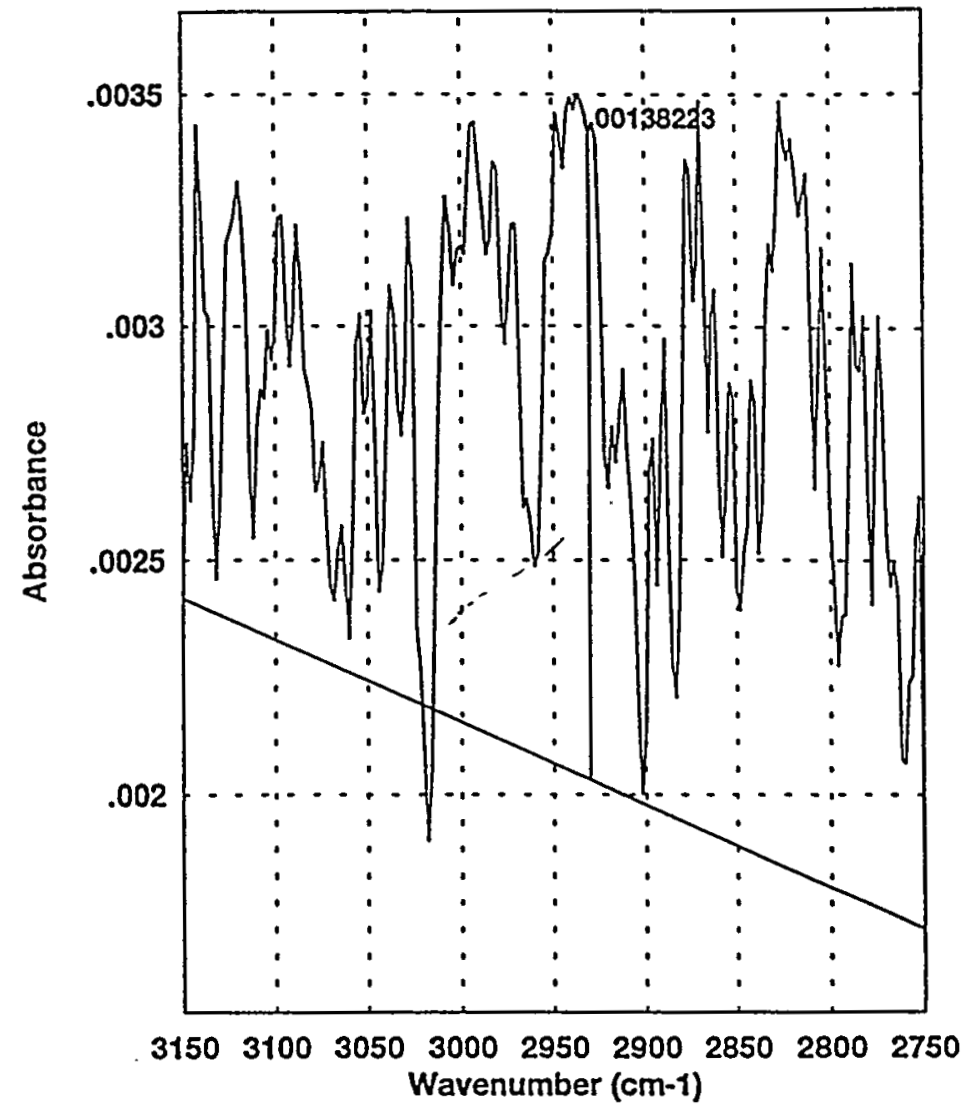

Instrument 1.D.: FTIR-1000C

Analyzed on: 09-JUL-1997

Date and time on printout is the day and time copy was printed.

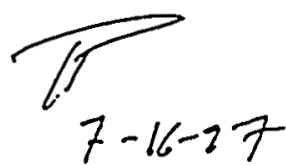

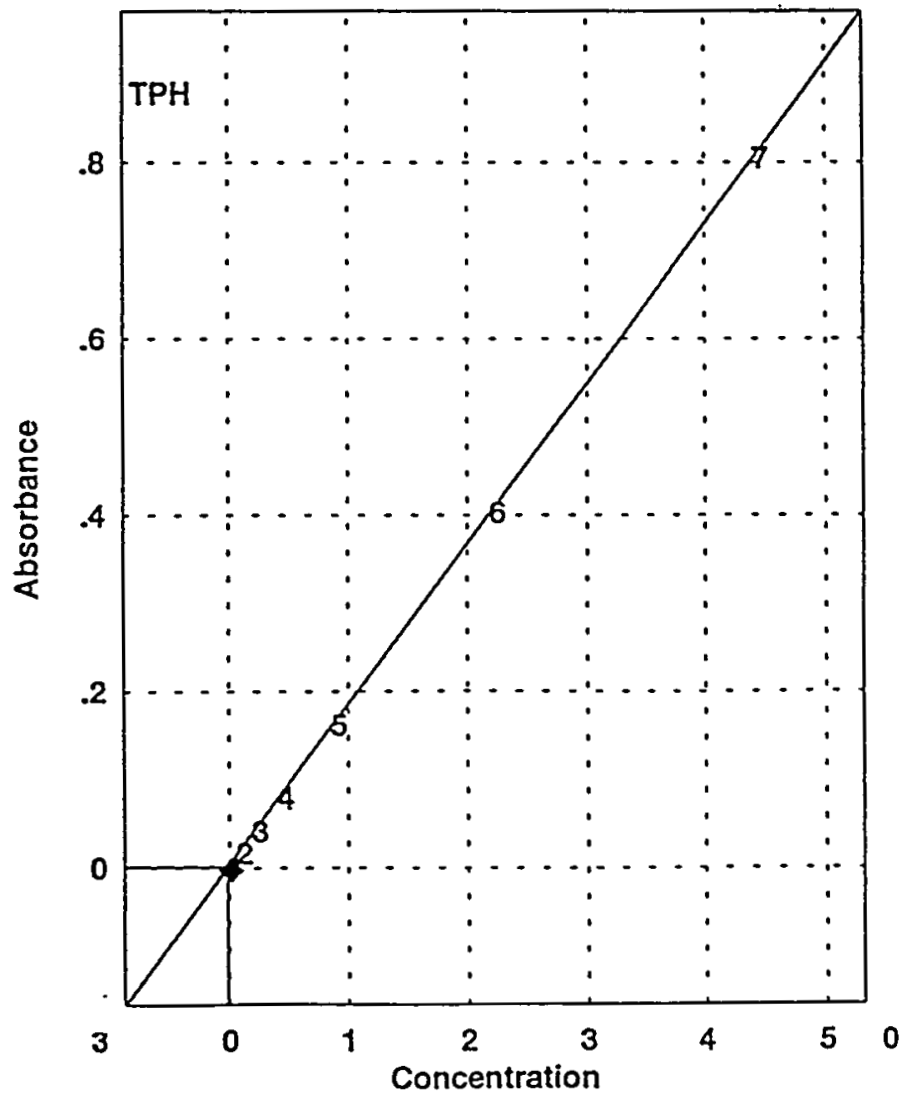


QuantBasic Report

Quant Type: Multipoint $\quad 7 / 15 / 97$ 4:52 pm

Method: C:IPE1000GSIDATAL7036TPH.Q

Sample: c:lpe1000gsldatal97036lcalcurve.spc

Analyst: TP

Sample ID: QC-136533-1

Component Concen R-Squared

$\begin{array}{lll}\text { TPH } & 1.10339 & .999991\end{array}$

Instrument I.D.: FTIR-1000C

Analyzed on: 09-JUL-1997

Date and time on printout is the day and time copy was printed.

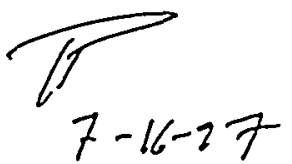

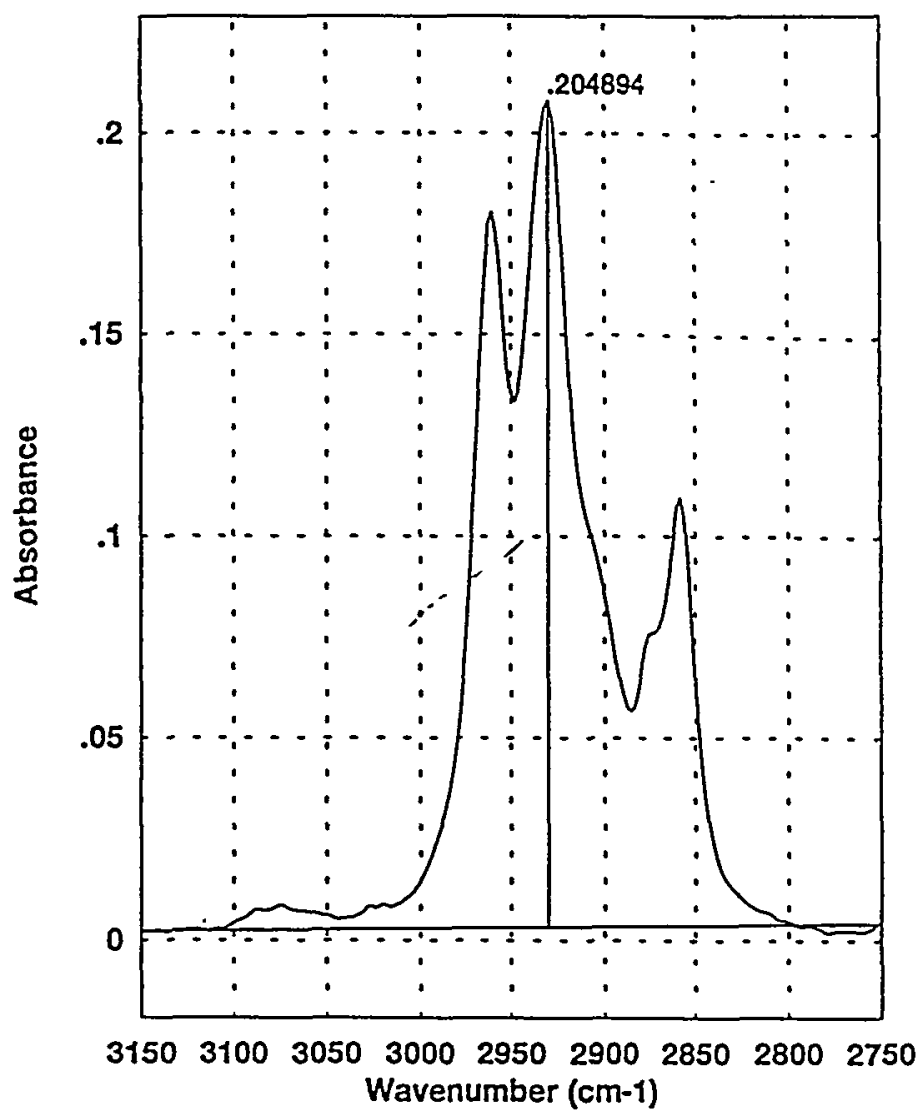

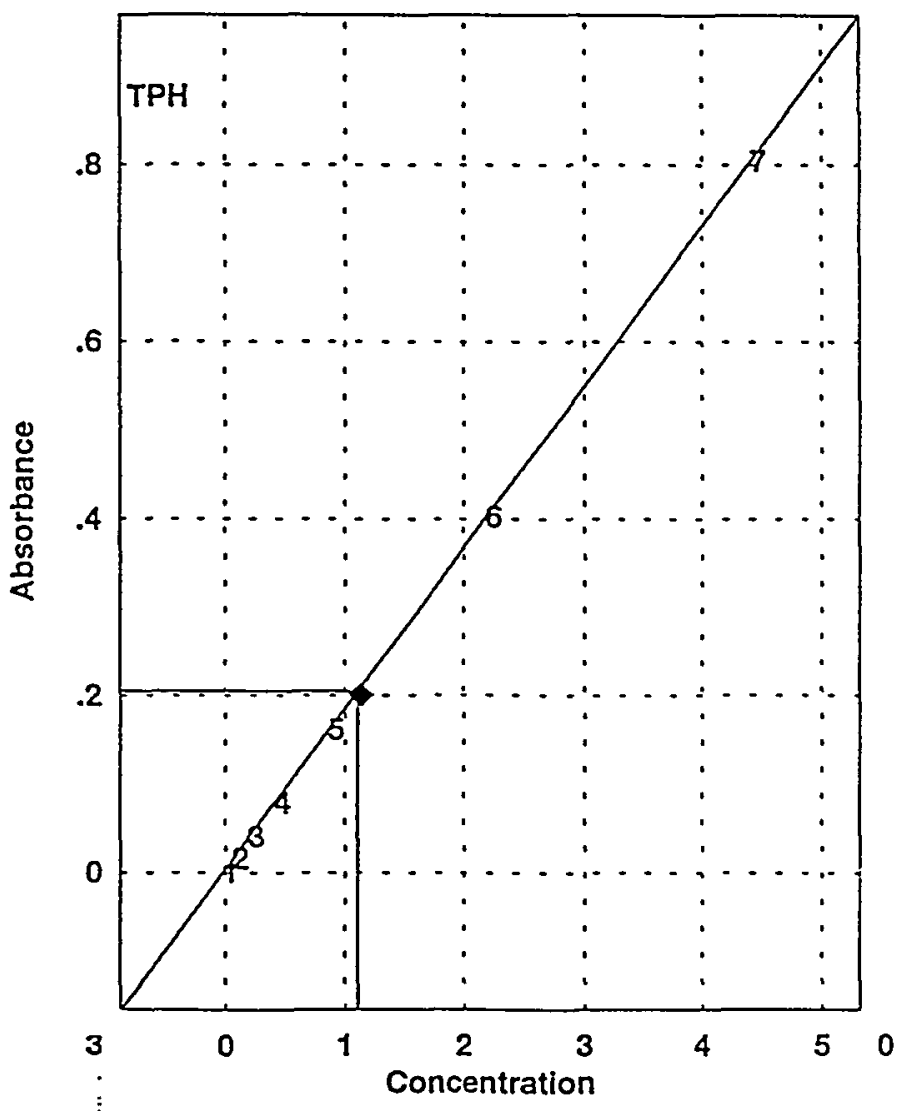


QuantBasic Report

Quant Type: Multipolnt $\quad 7 / 15 / 97$ 4:53 pm

Method: C:IPE1000GSIDATAL703GTPH.Q

Sample: c:lpe1000gsldatal97036lcalcurve.spc

Analyst: TP

Sample 1D: BL-136533-1

Component Concen R-Squared

$\begin{array}{lll}\text { TPH } & .027796 \quad .999991\end{array}$

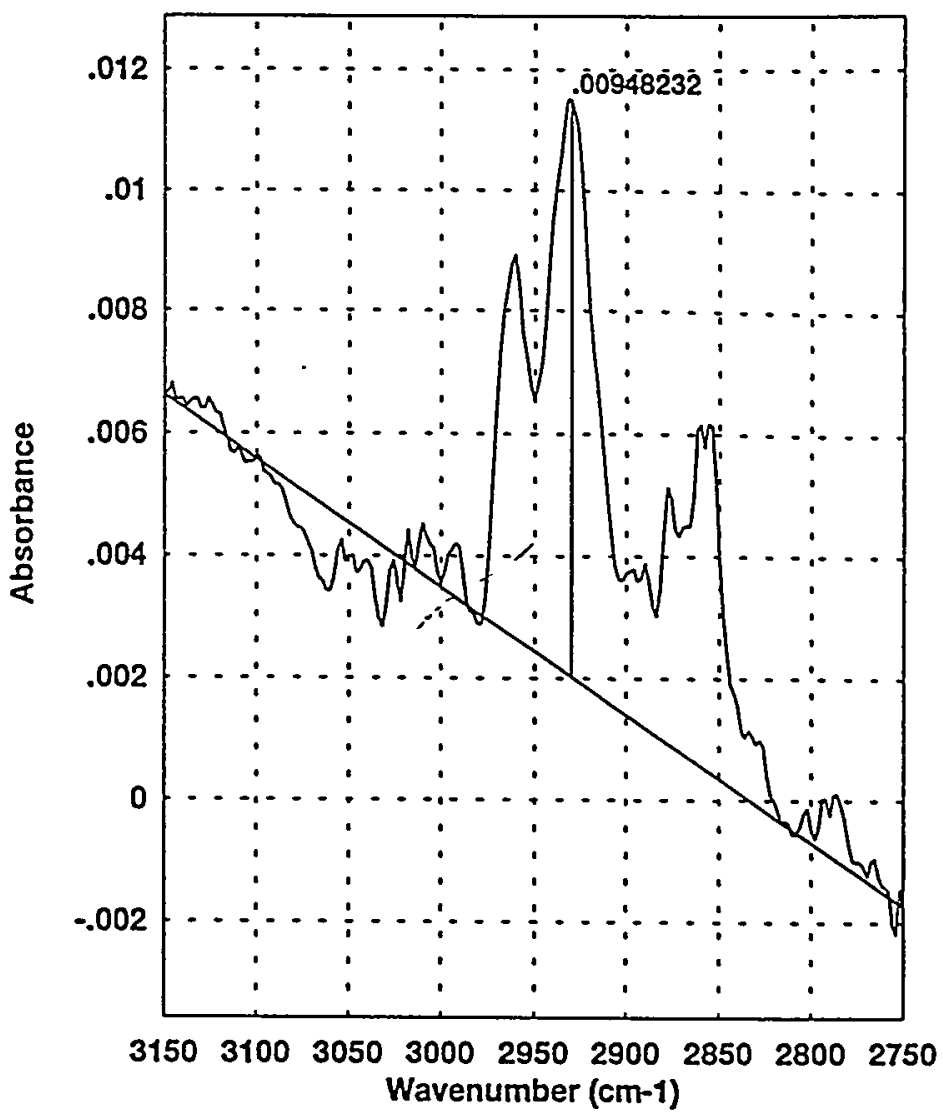

Instrument 1.D.: FTIR-1000C

Analyzed on: 09-JUL-1997

Date and time on printout is the day and time copy was printed.
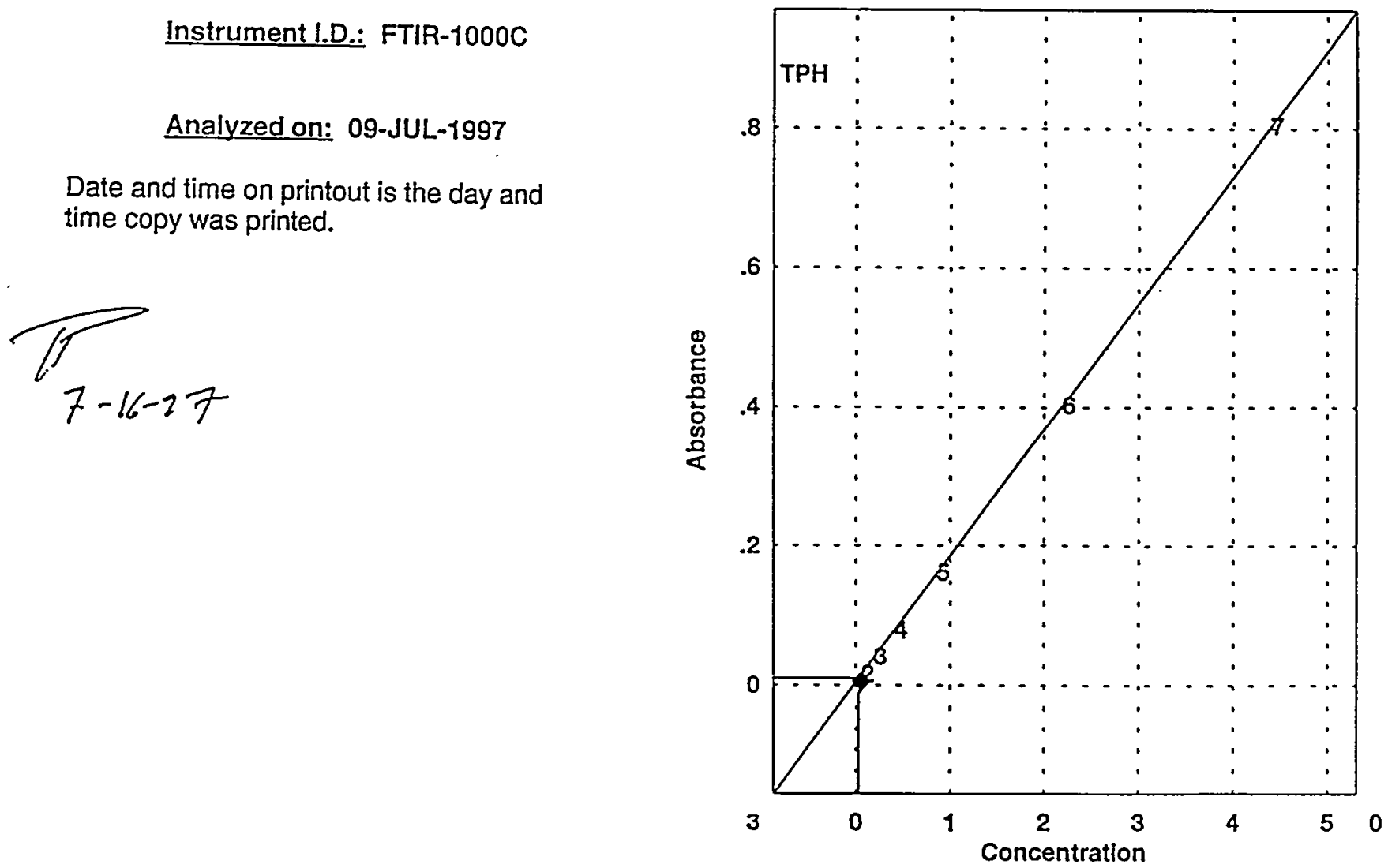
QuantBasic Report

Quant Type: Multlpoint $\quad$ 7/15/97 4:57 pm

Method: C:IPE1000GSLATAL7036TPH.Q

Sample: c:lpe1000gsidatal97036lcalcurve.spc

Analyst: TP

Sample ID: 97C02896

Component Concen R-Squared

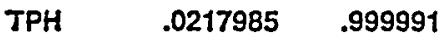

Instrument I.D.: FTIR-1000C

Analyzed on: 09-JUL-1997

Date and time on printout is the day and time copy was printed.

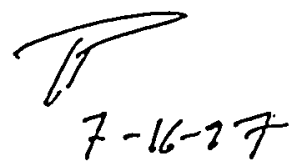

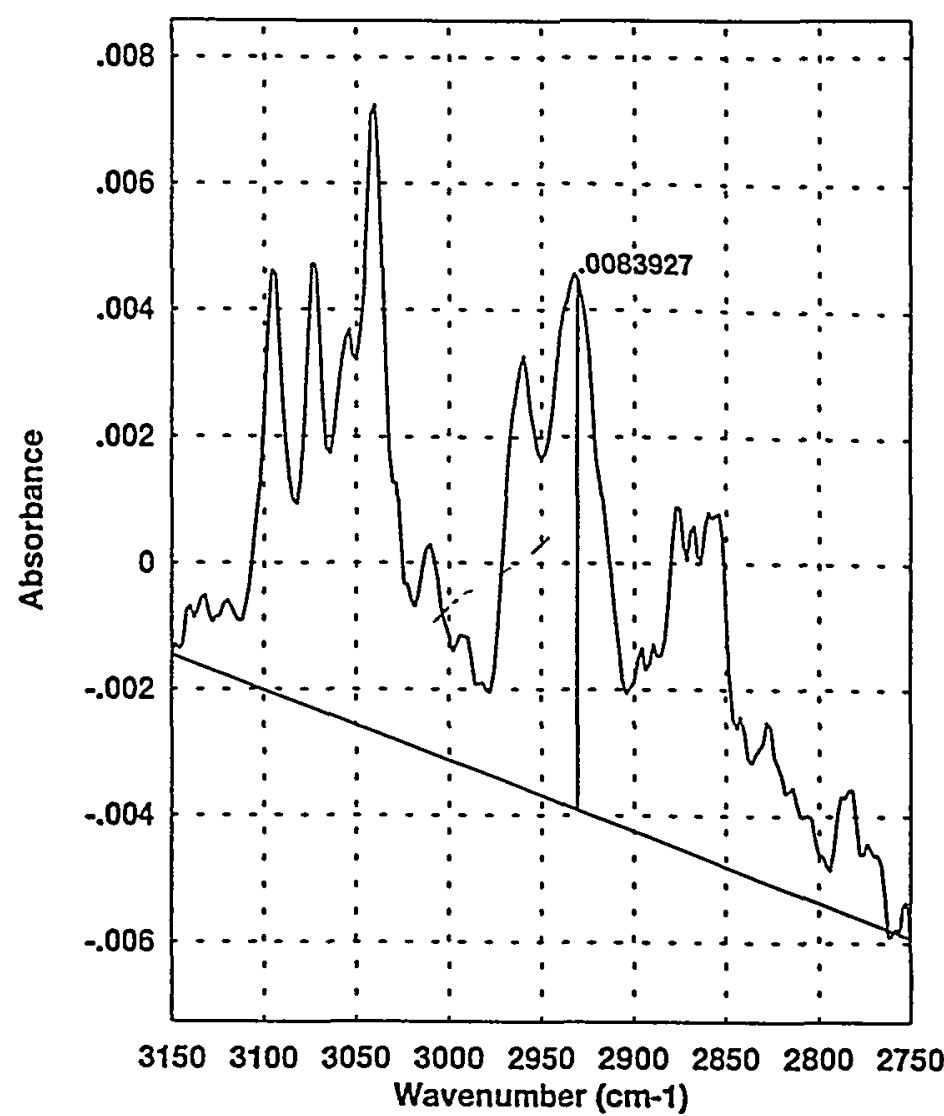

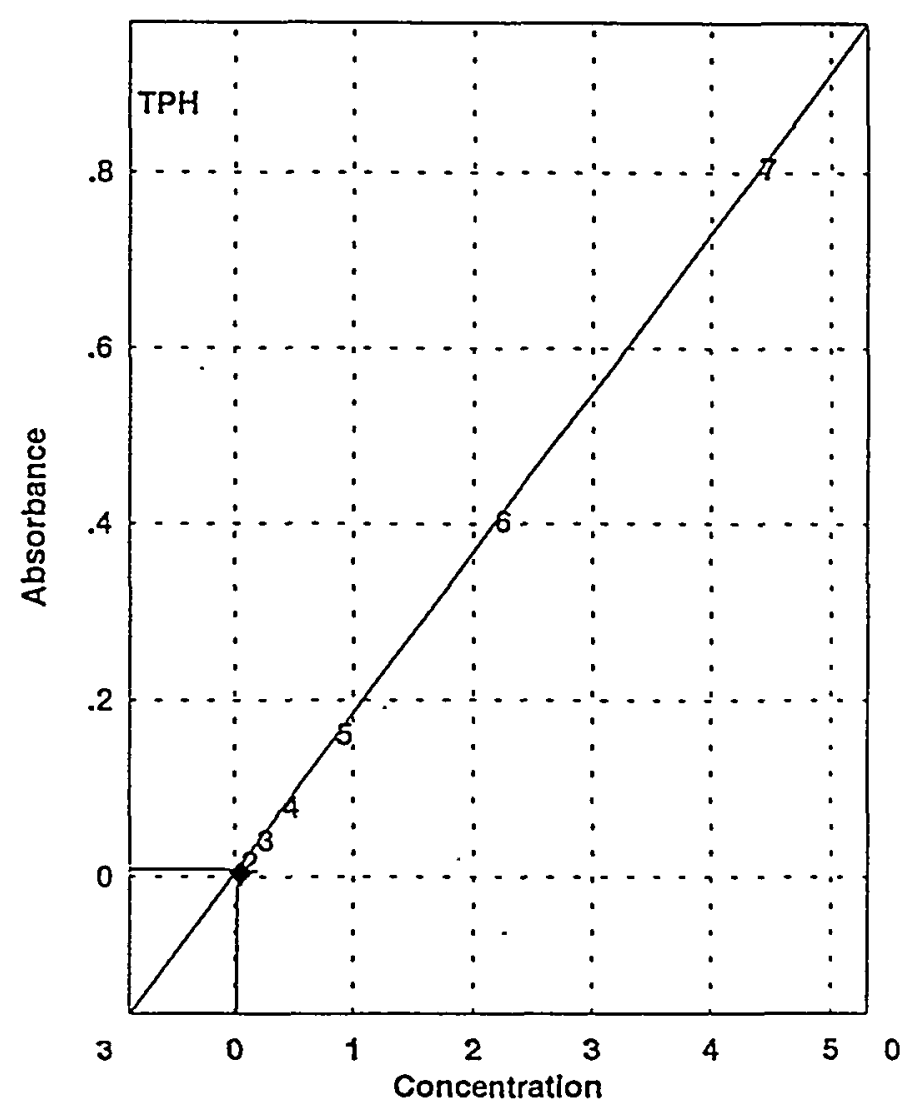




\section{QuantBasic Report}

Quant Type: Multipoint $\quad 7 / 15 / 974: 57 \mathrm{pm}$

Method: C:PPE1000GSIDATAL703GTPH.Q

Sample: c:lpe1000gsidatal97036lcalcurve.spc

Aralyst: TP

Sample ID: 97002897

Component Concen R-Squared

$\begin{array}{lll}\text { TPH } & .0642703 \quad .999991\end{array}$

Instrument I.D.: FTIR-1000C

Analyzed on: 09-JUL-1997

Date and time on printout is the day and time copy was printed.

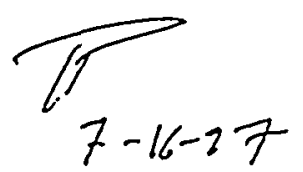

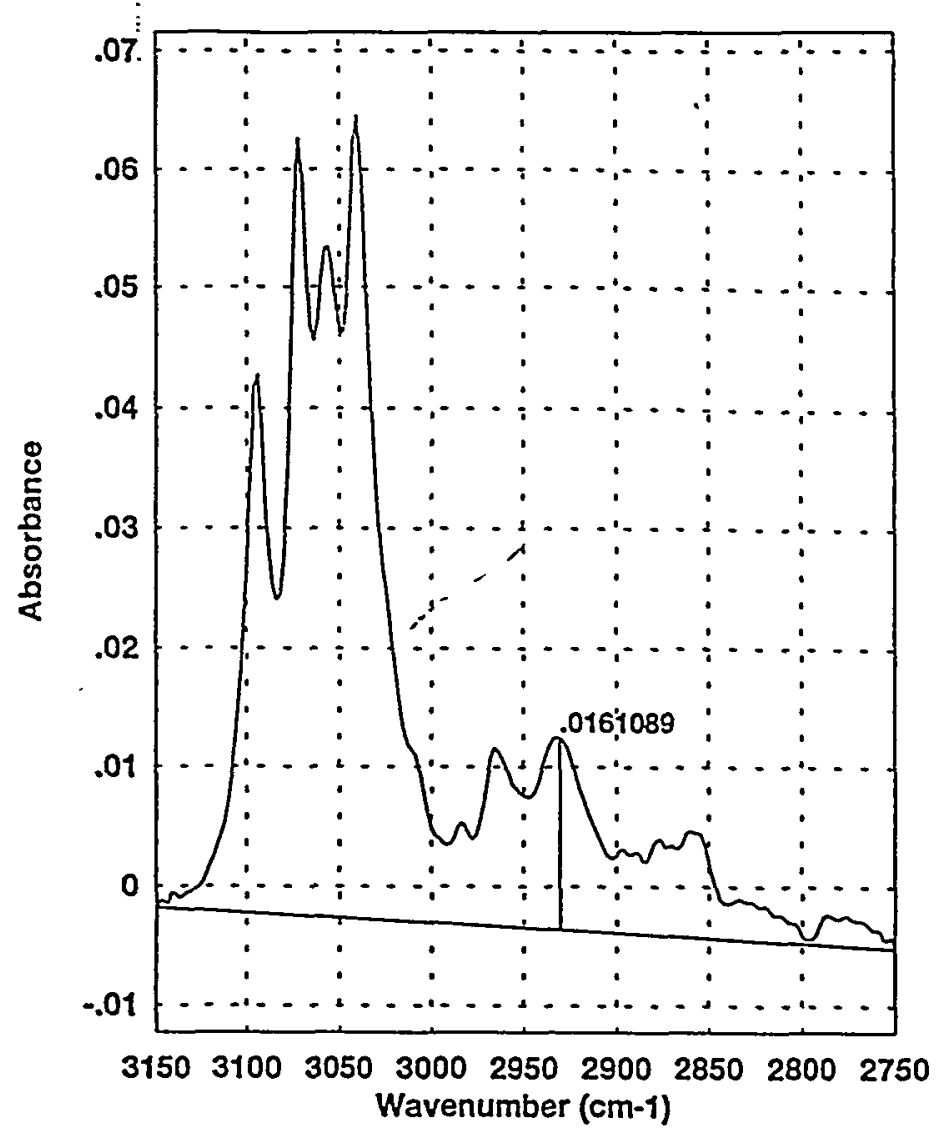

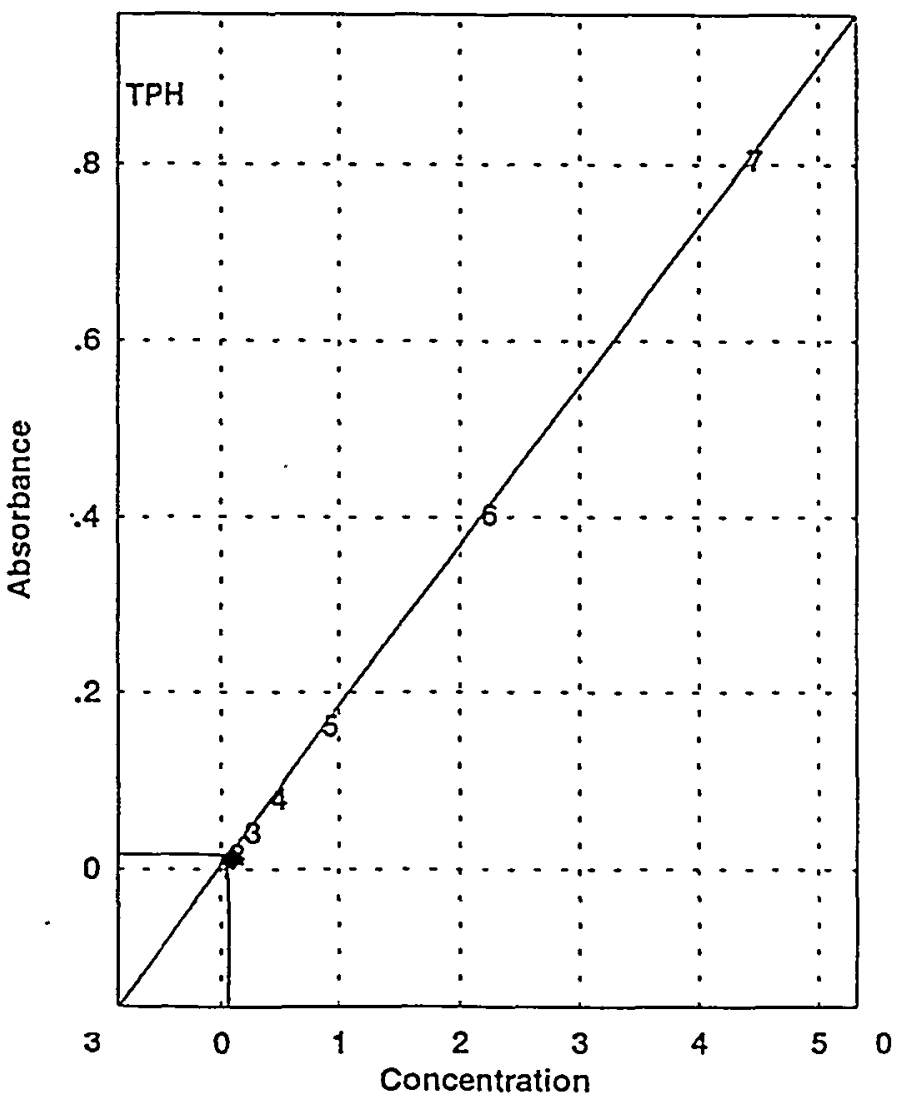


QuantBasle Report

Quant Type: Multipoint $\quad$ 7/15/97 4:57 pm

Method: C:IPE1000GSLDATAL703GTPH.Q

Sample: c:lpe1000gsidatal97036lcalcurve.spc

Analyst: TP

Sample D: 97C02897MS

Component Concen R-Squared

$\begin{array}{lll}\text { TPH } & 1.65054 \quad .999991\end{array}$

Instrument 1.D.: FTIR-1000C

Analyzed on: 09-JUL-1997

Date and time on printout is the day and time copy was printed.

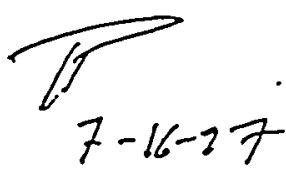

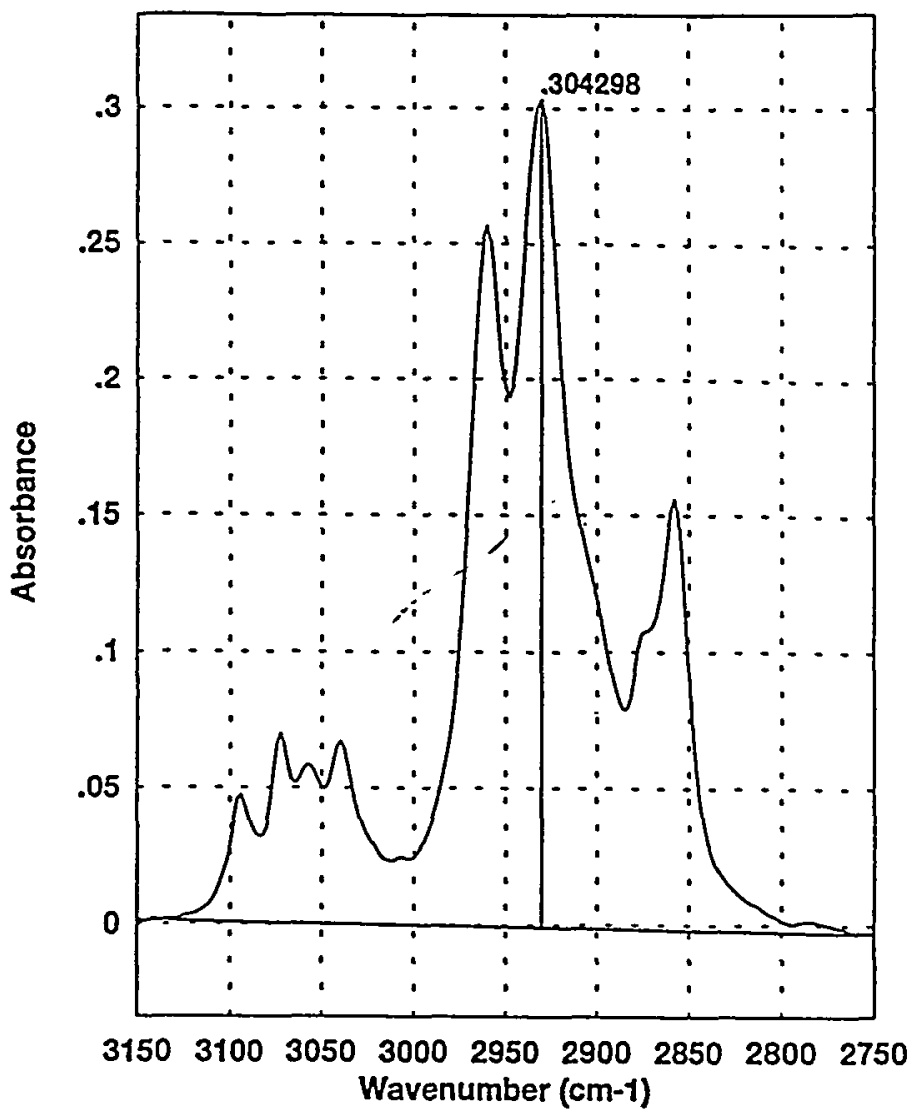

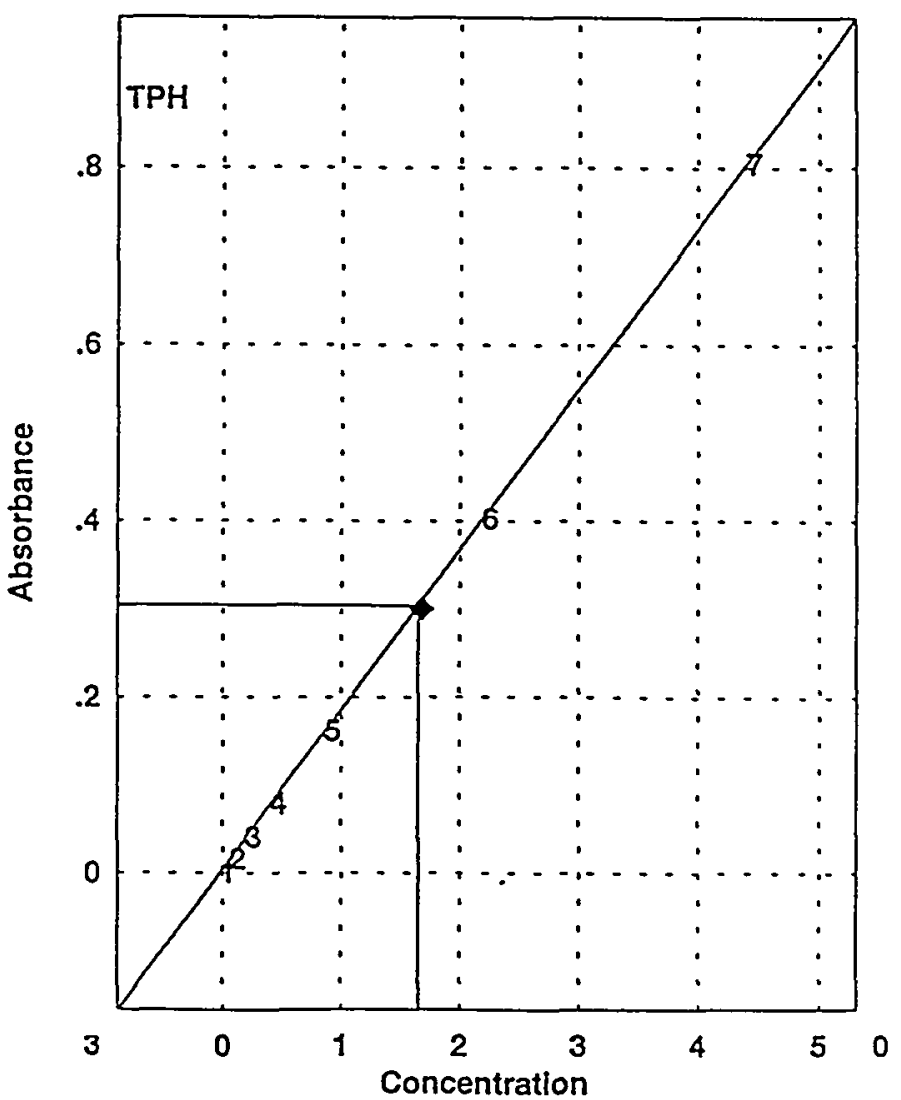


QuantBasic Report

Quant Type: Muttlpoint $\quad$ 7/15/97 4:56 pm

Method: C:IPE1000GSIDATAlo7036TTPH.Q

Sample: c:lpe1000gsldatal97036lcalcurve.spc

Analyst: TP

Sample ID: 97C02897MSD

Component Concen R-Squared

$\begin{array}{lll}\text { TPH } & 1.63115 \quad .999991\end{array}$

Instrument 1.D.: FTIR-1000C

Analyzed on: 09-JUL-1997

Date and time on printout is the day and time copy was printed.

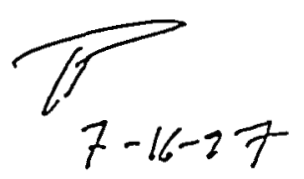

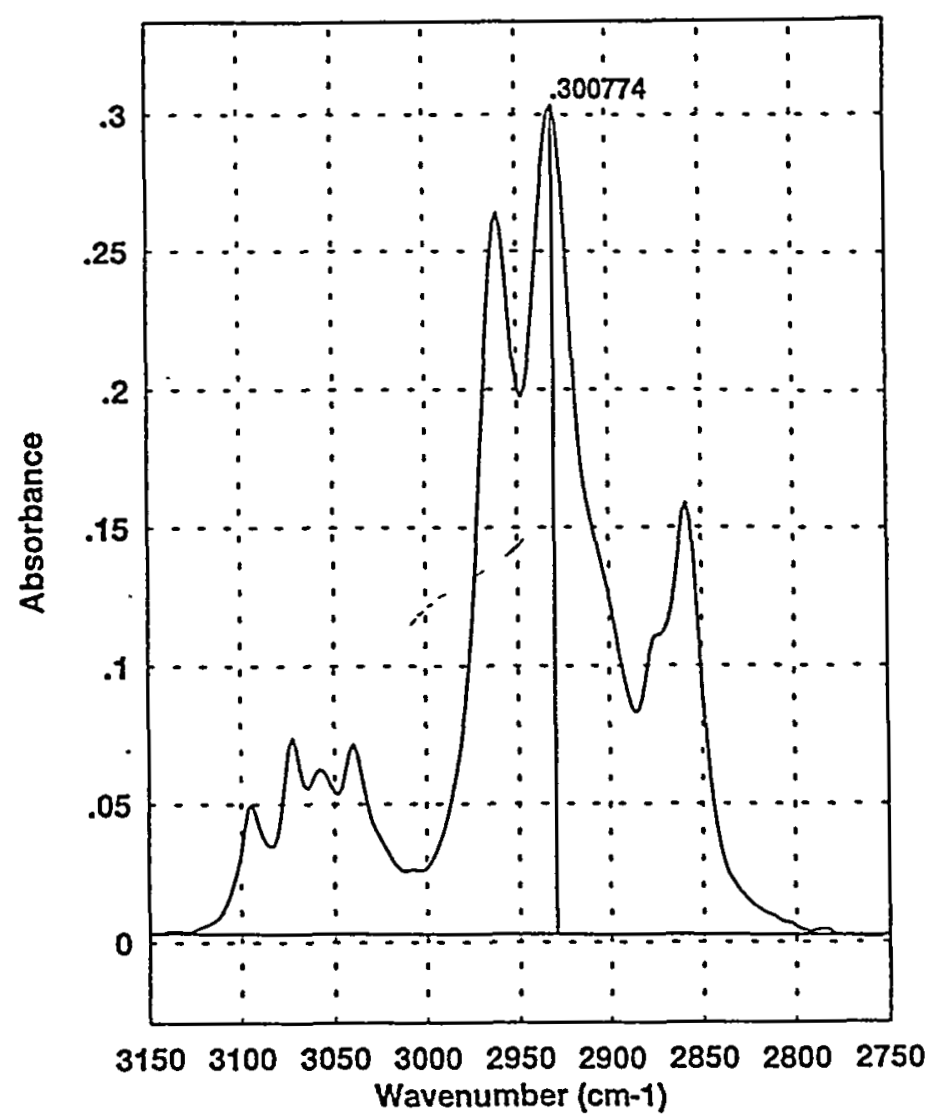

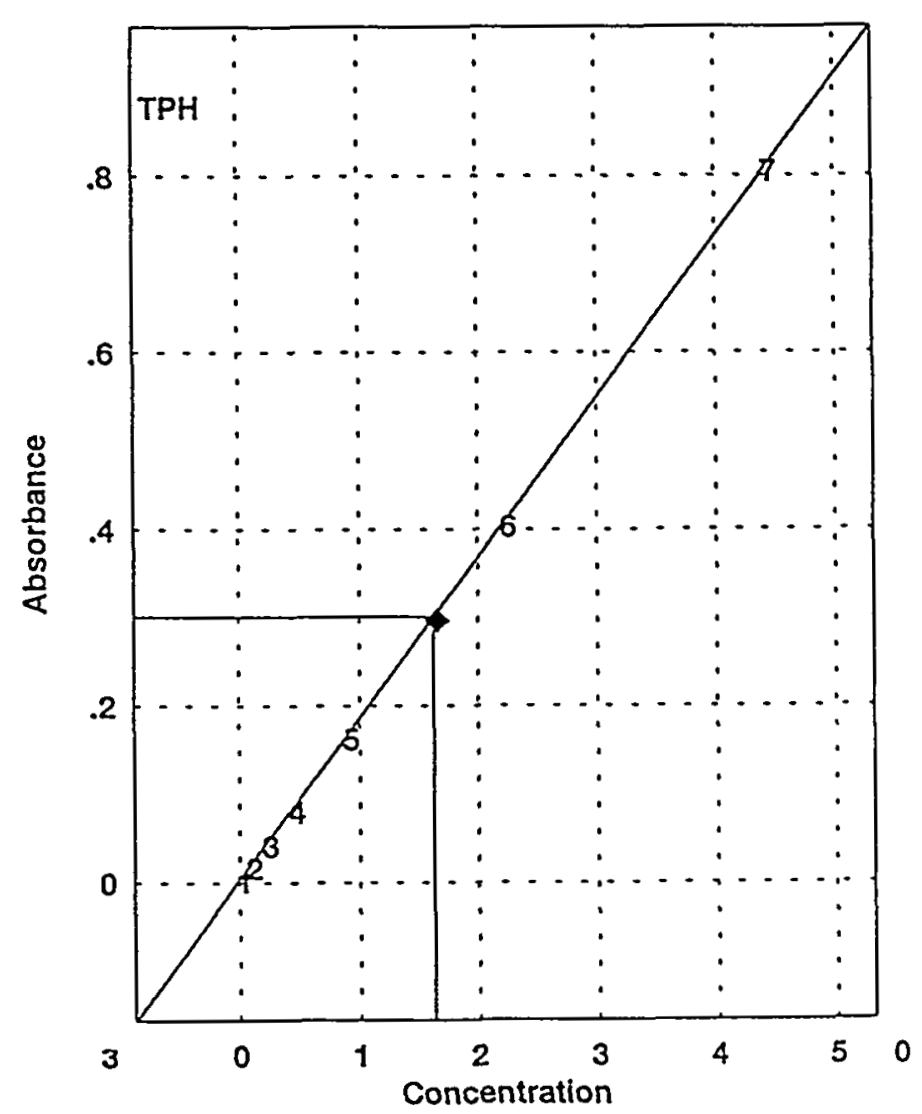


QuantBasic Report

Quant Type: Multipoint $\quad$ 7/15/97 4:56 pm

Method: C:LPE1000GSIDATAL7036ITPH.Q

Sample: c:lpe1000gsidatal97036lcalcurve.spc

Analyst: TP

Sample 1D: 97ð̌02898

Component Concen R-Squared

$\begin{array}{lll}\text { TPH } & .048332 & .999991\end{array}$
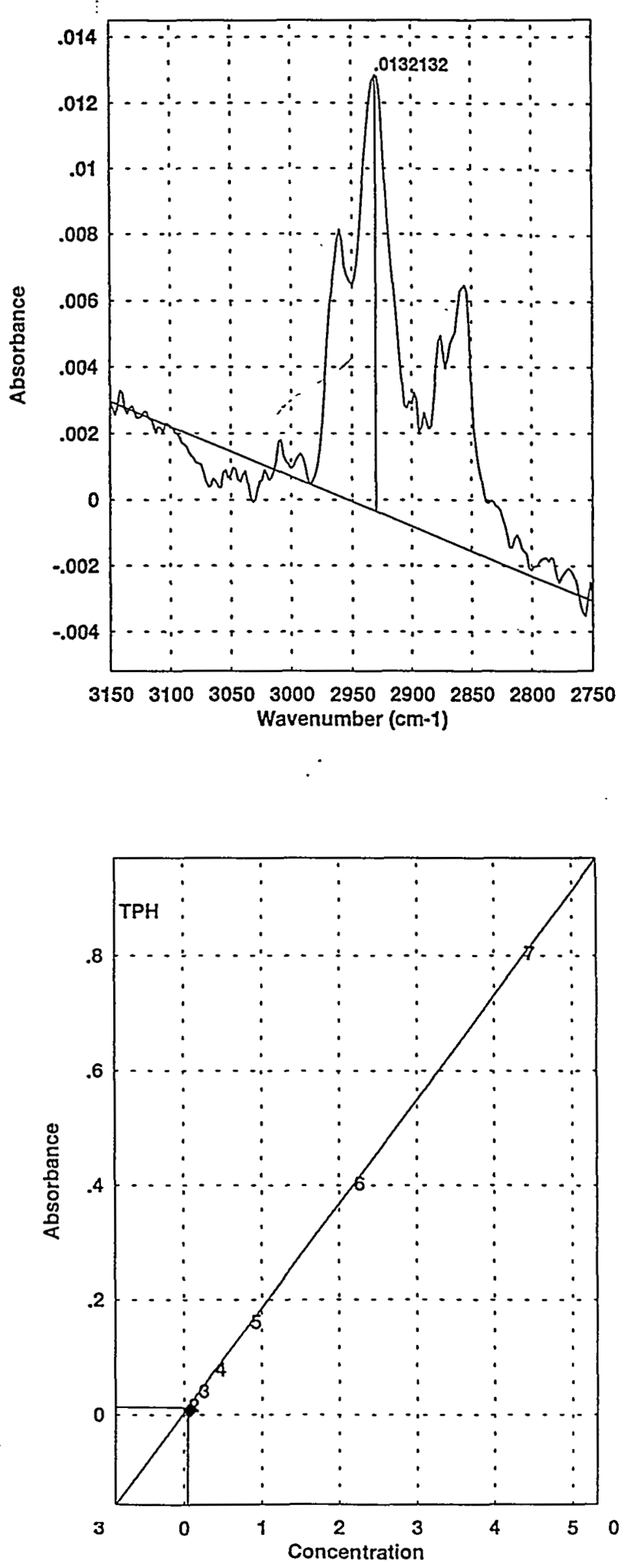

Instrument 1.D.: FTIR-1000C

Analyzed on: 09-JUL-1997

Date and time on printout is the day and time copy was printed.

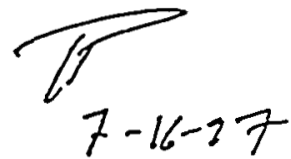


QuantBasic Report

Quant Type: Multipolnt $\quad$ 7/15/97 4:55 pm

Method: C:LPE1000GSIDATA19703GTPH.Q

Sample: c:lpe1000gsidatal97036lcalcurve.spc

Analyst: TP

Sample 1D: $97 \hat{6} 02899$

$2-21$

Componeint Concen R-Squared

$\begin{array}{lll}\text { TPH } & .023811 & .999991\end{array}$

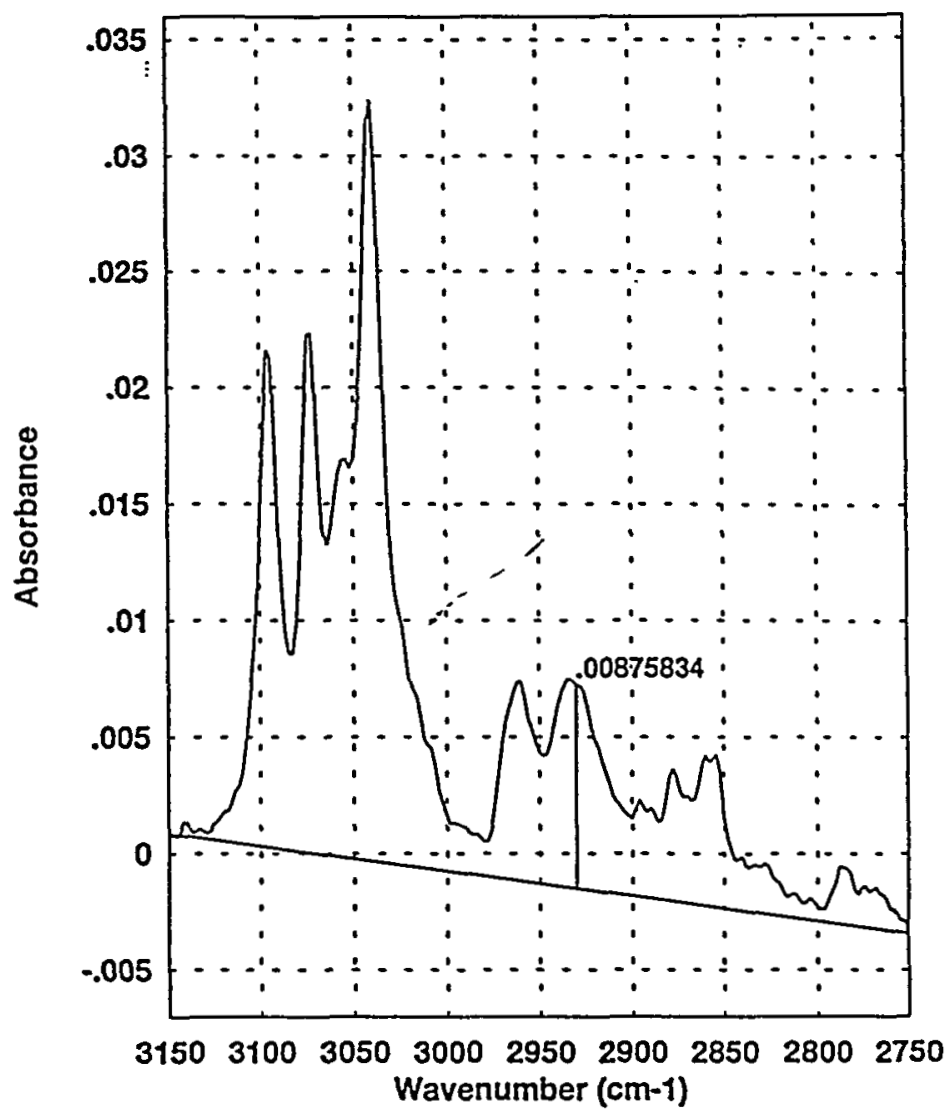

Instrument 1.D.: FTIR-1000C

Analyzed on: 09-JUL-1997

Date and time on printout is the day and time copy was printed.
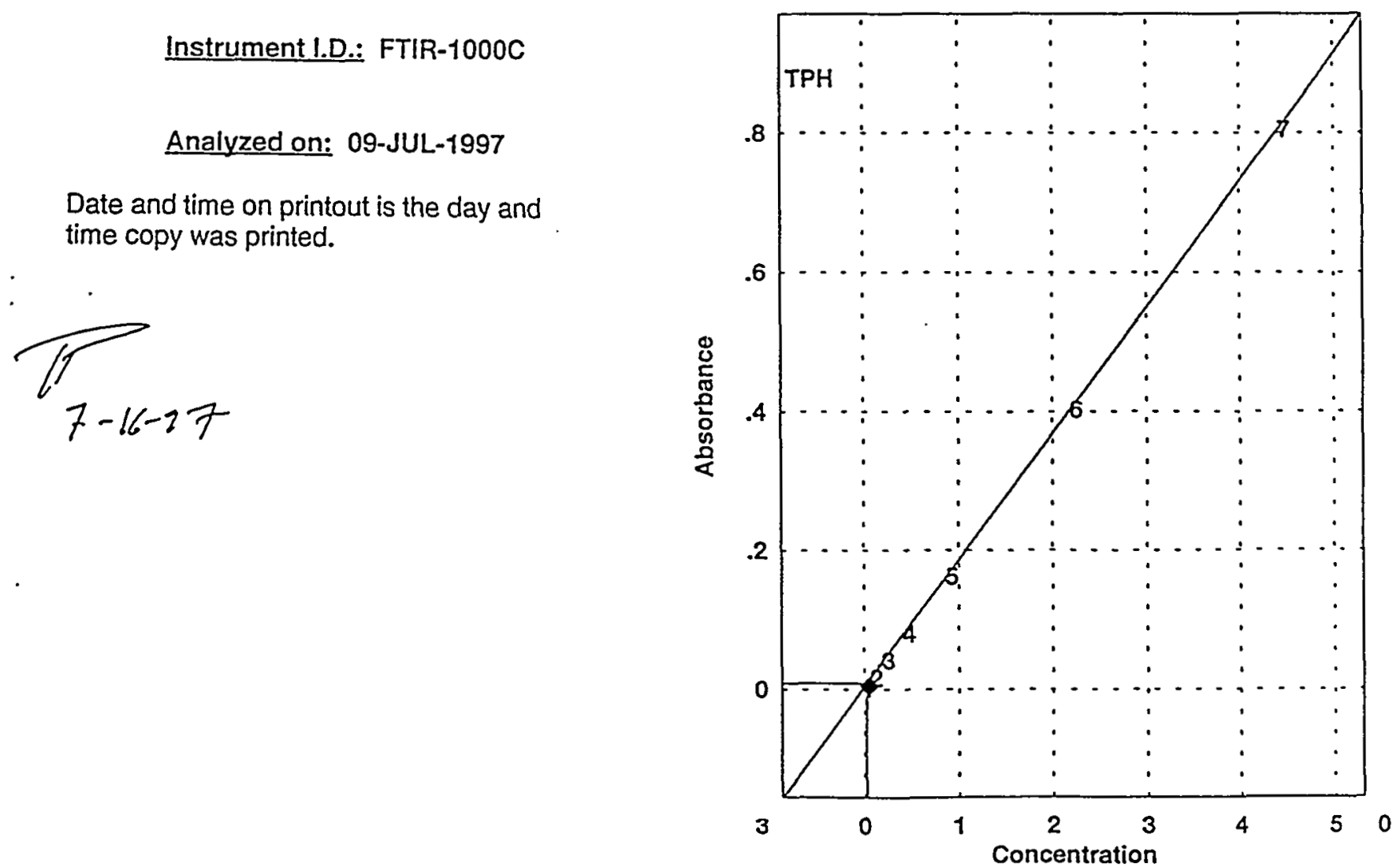
QuantBaslc Report

Quant Type: Multipoint $\quad$ 7/15/97 4:55 pm

Method: C:IPE1000GSIDATAL7036ITPH.Q

Sample: c:lpe1000gsldatal97036lcalcurve.spc

Analyst: TP

Sample ID: 97ఝुo2900

Component Concen R-Squared

$\begin{array}{lll}\text { TPH } & .0781683 & .999991\end{array}$

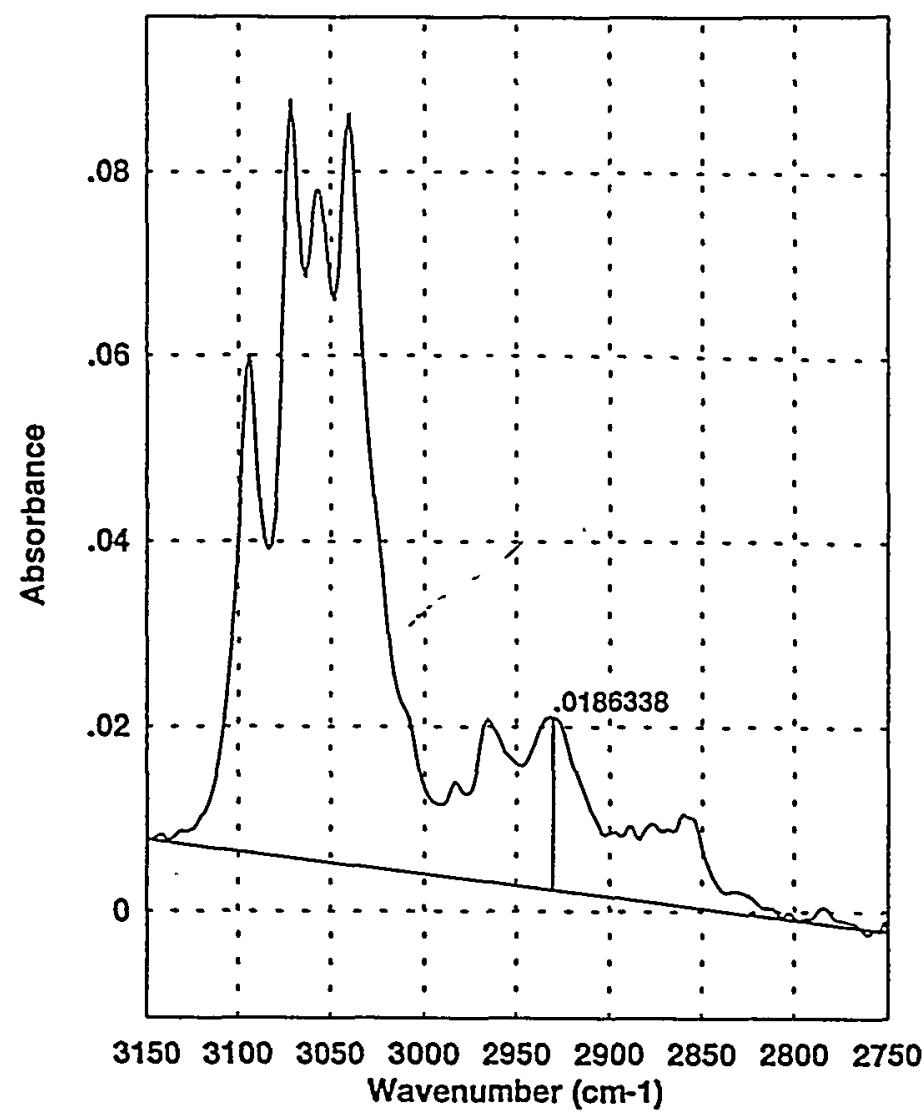

Instrument l.D.: FTIR-1000C

Analyzed on: 09-JUL-1997

Date and time on printout is the day and time copy was printed.

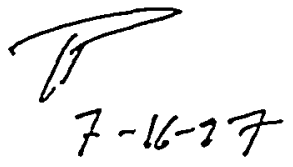

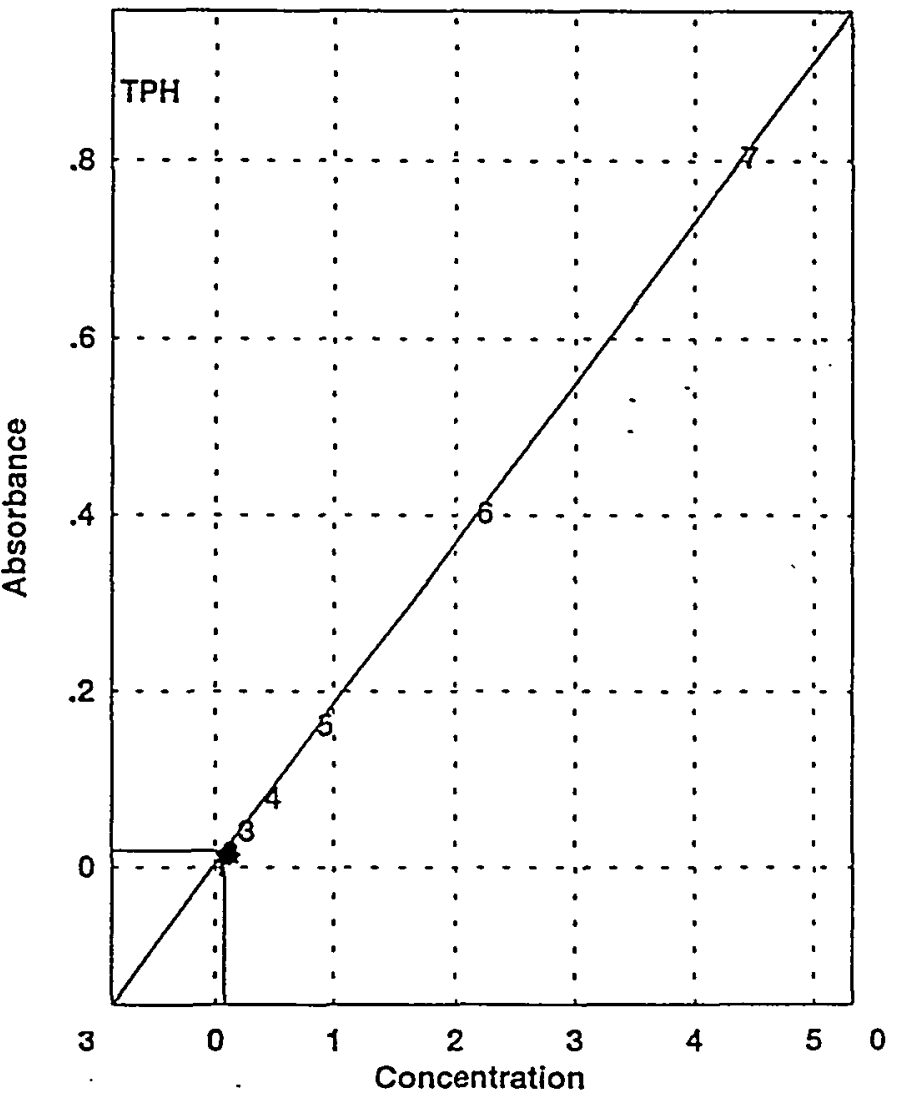


QuantBasic Report

Quant Type: Multipolnt $\quad$ 7/15/97 4:55 pm

Method: C:LPE1000GSIDATAL7036ITPH.Q

Sample: c:lpe1000gsidatal97036lcalcurve.spc

Analyst: TP

Sample 10: 97ழु02901

Component Concen R-Squared

$\begin{array}{lll}\text { TPH } & .0186285 & .999991\end{array}$

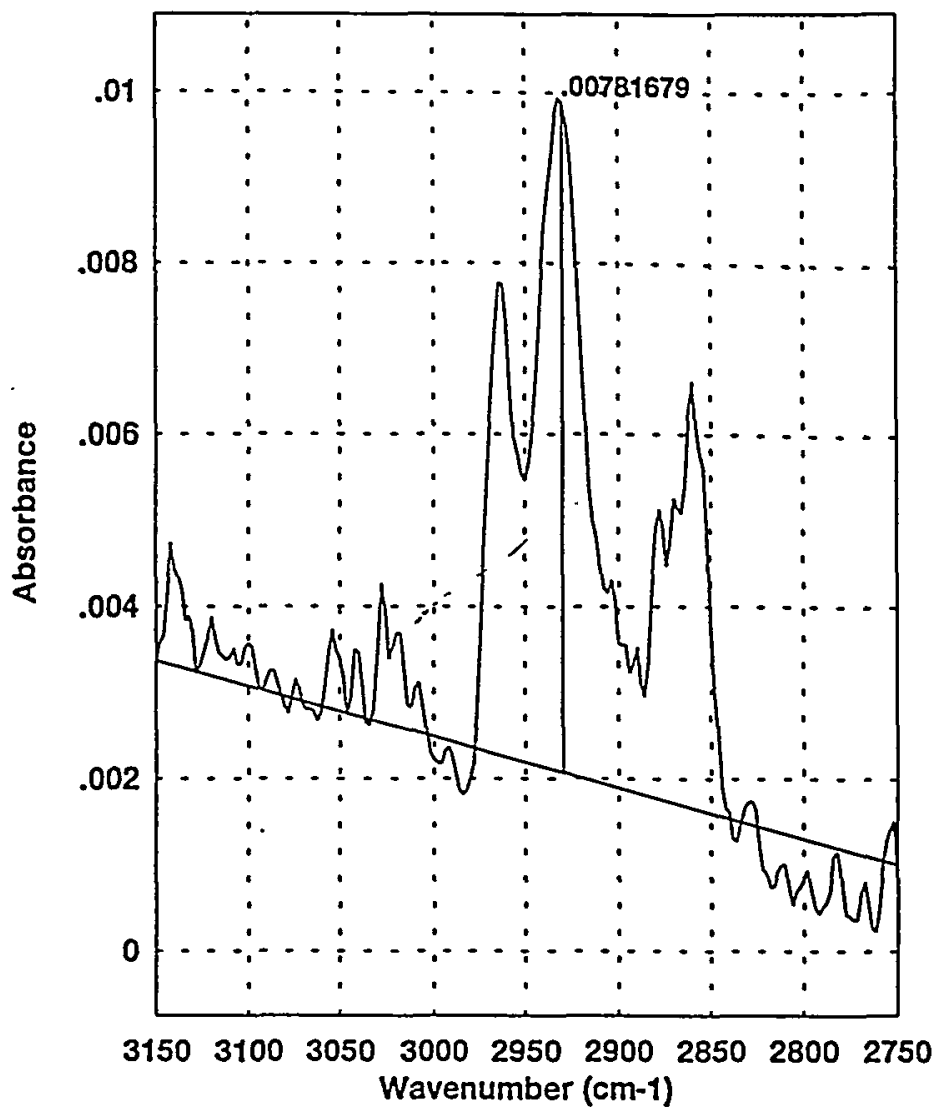

Instrument 1.D.: FTIR-1000C

Analyzed on: 09-JUL-1997

Date and time on printout is the day and time copy was printed.
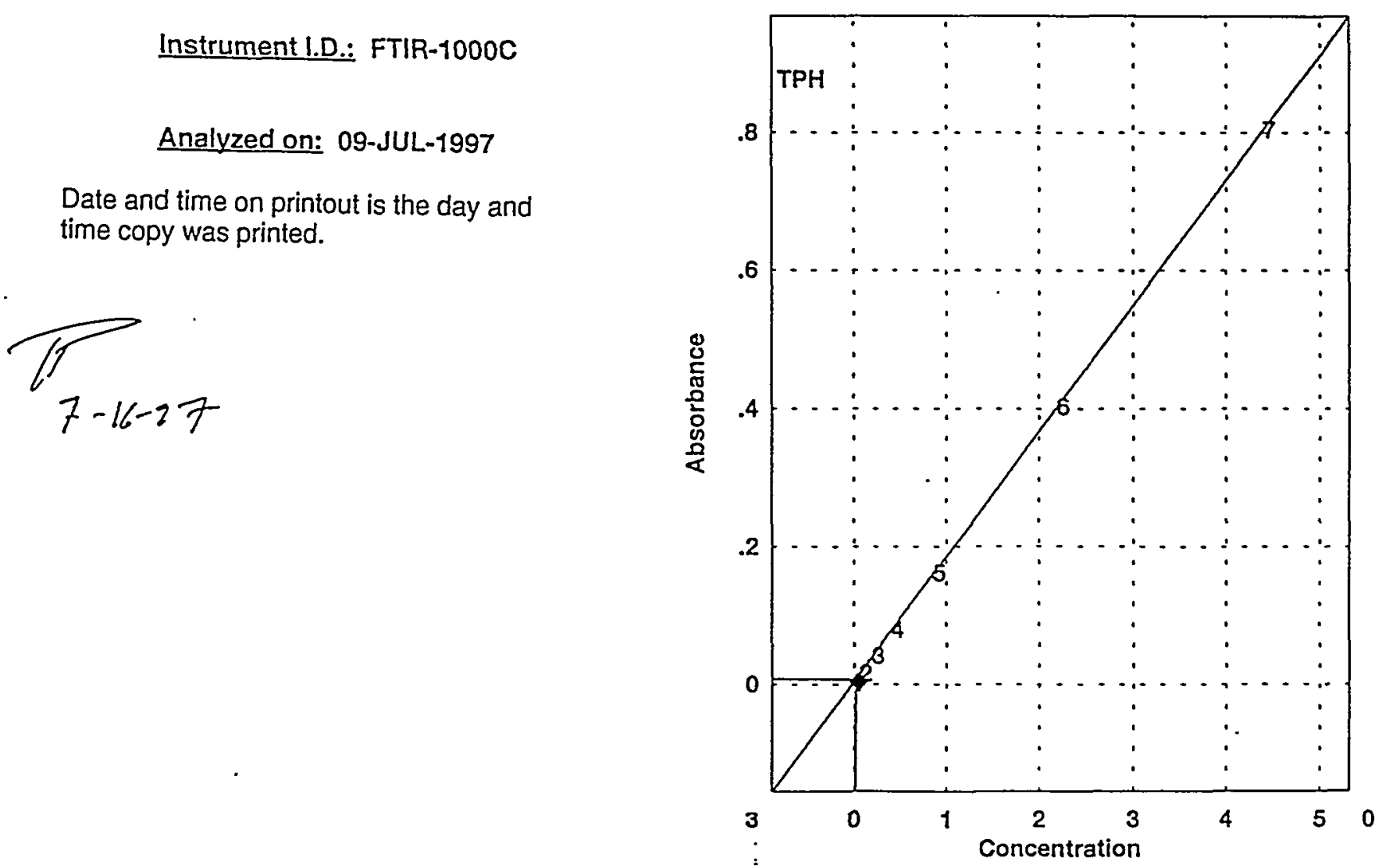
QuantBasic Report

Quant Type: Multipolnt $\quad$ 7/15/97 5:03 pm

Method: C:IPE1000GSIDATAL703GTPH.Q

Sample: c:lpe1000gsidatalo7036lcalcurve.spo

Analyst: TP

Sample ID: CCV

Component Concen R-Squared

$\begin{array}{lll}\text { TPH } & 2.21835 \quad .999991\end{array}$

Instrument I.D.: FTIR-1000C

Analyzed on: 09-JUL-1997

Date and time on printout is the day and time copy was printed.

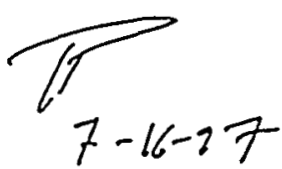

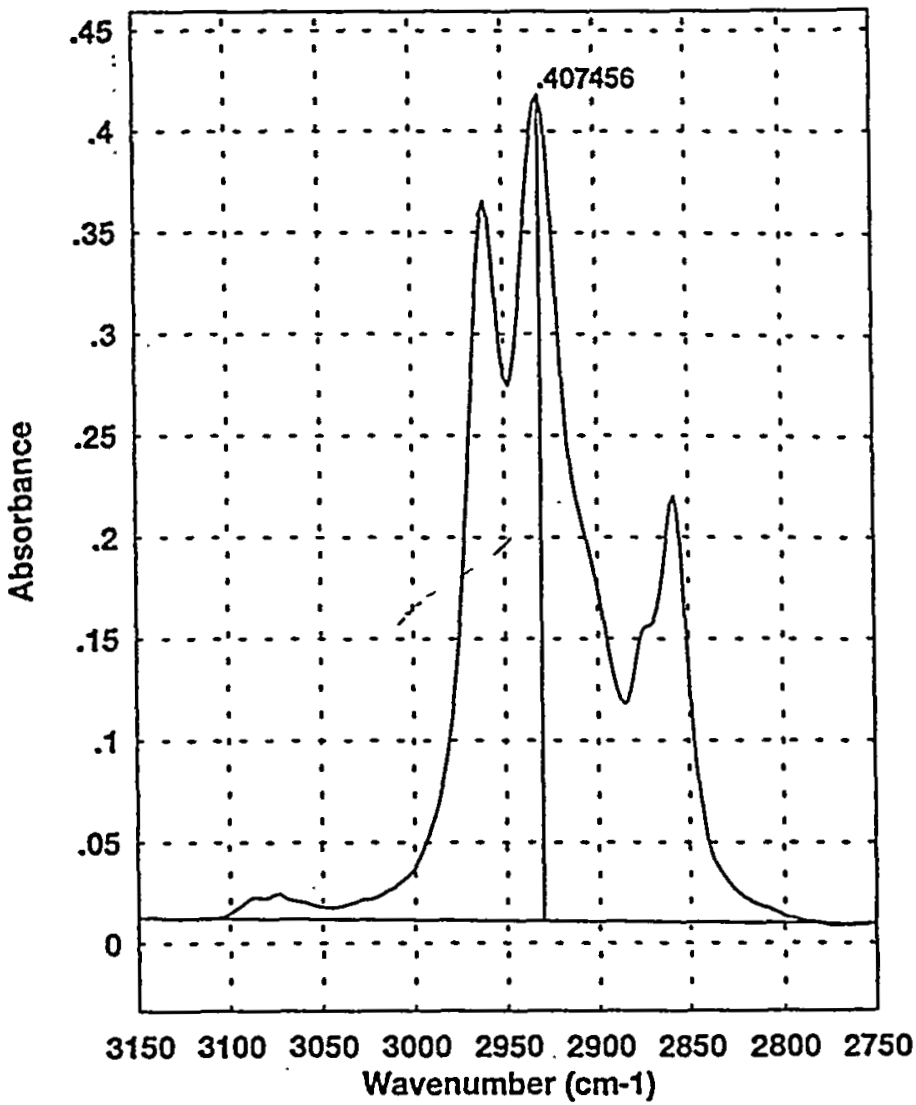

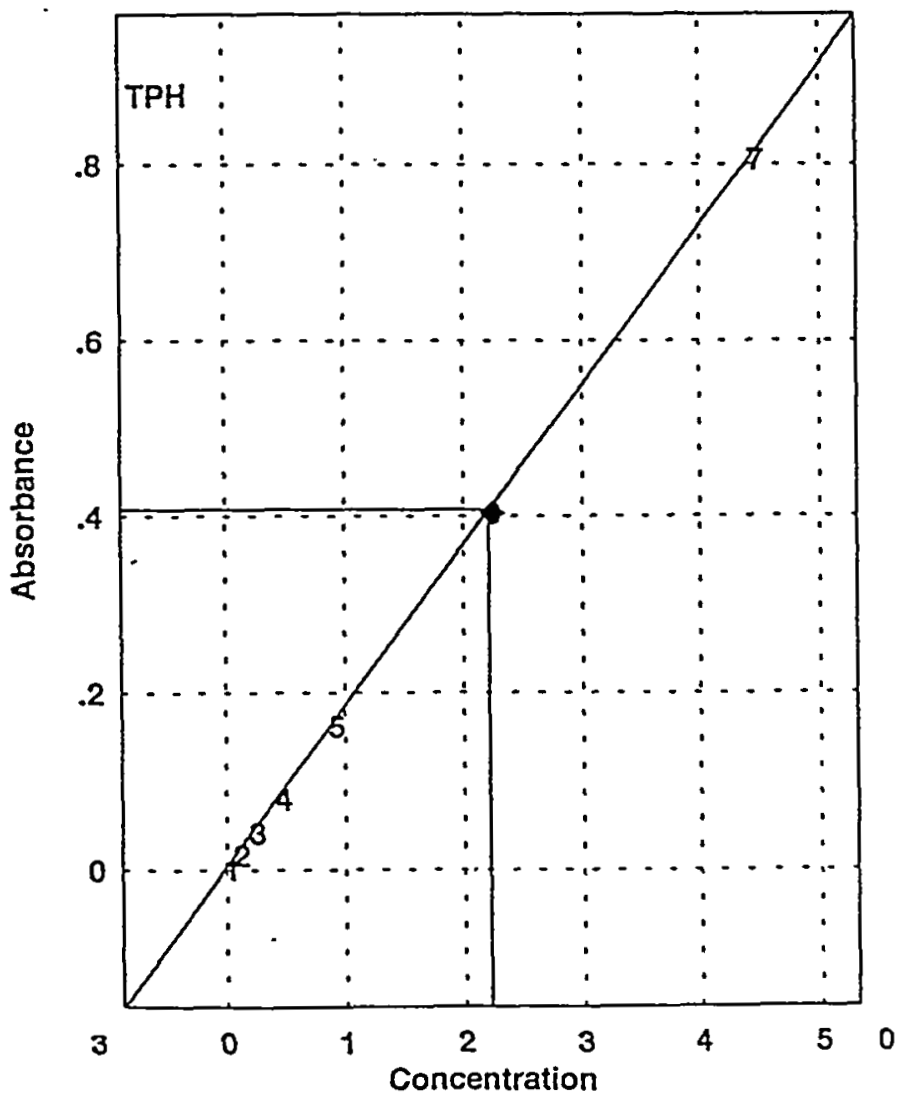

584 
QuantBasic Report

Quant Type: Multipolnt $\quad$ 7/15/97 5:04 pm

Method: C:IPE1000GSDATAL7036ITPH.Q

Sample: c:lpe1000gsldatalo7036lcalcurve.spc

Analyst: TP

Sample 1D: CCB

Component Concen R-Squared

TPH $\quad-.0266641 \quad .999991$

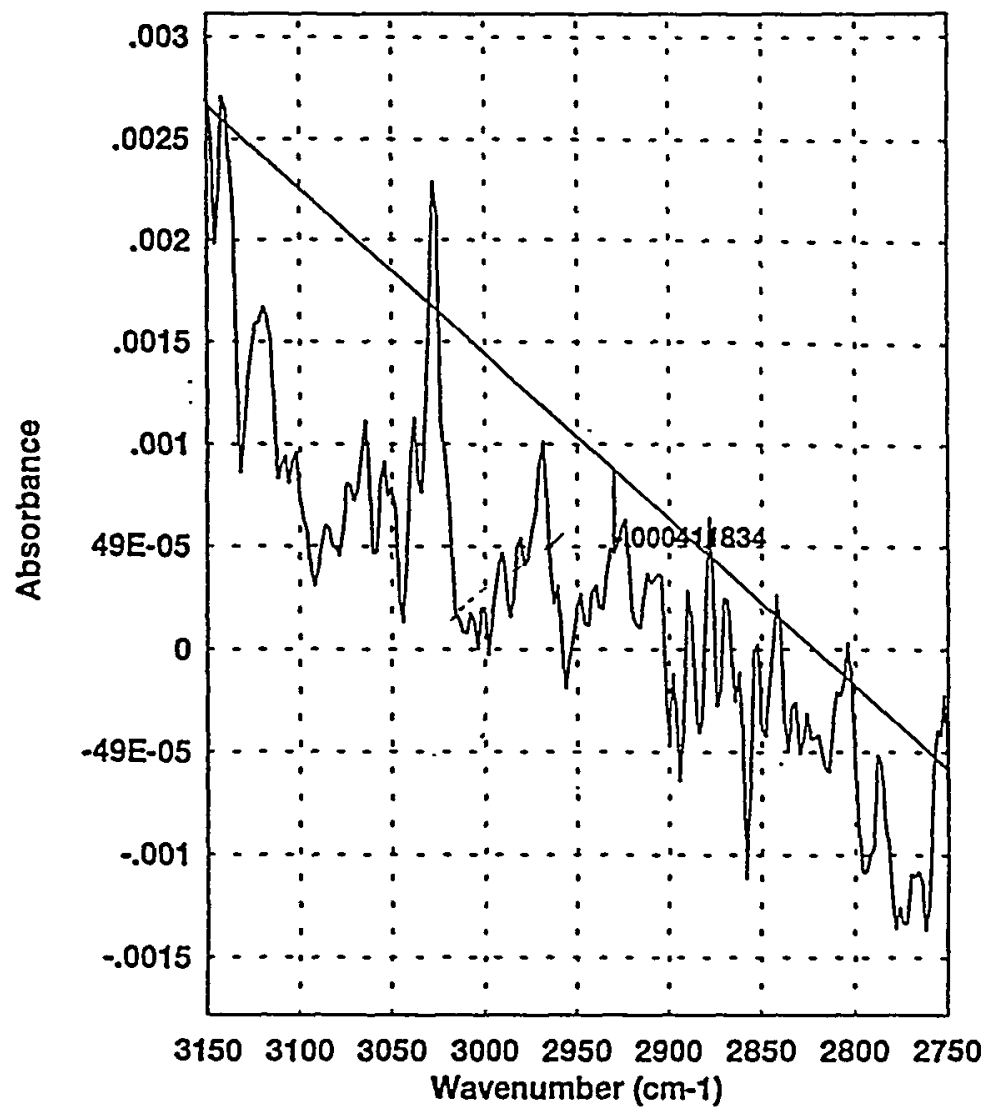

Instrument I.D.: FTIR-1000C

Analyzed on: 09-JUL-1997

Date and time on printout is the day and time copy was printed.

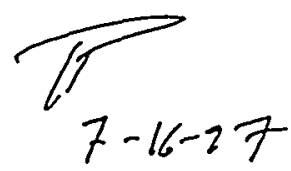

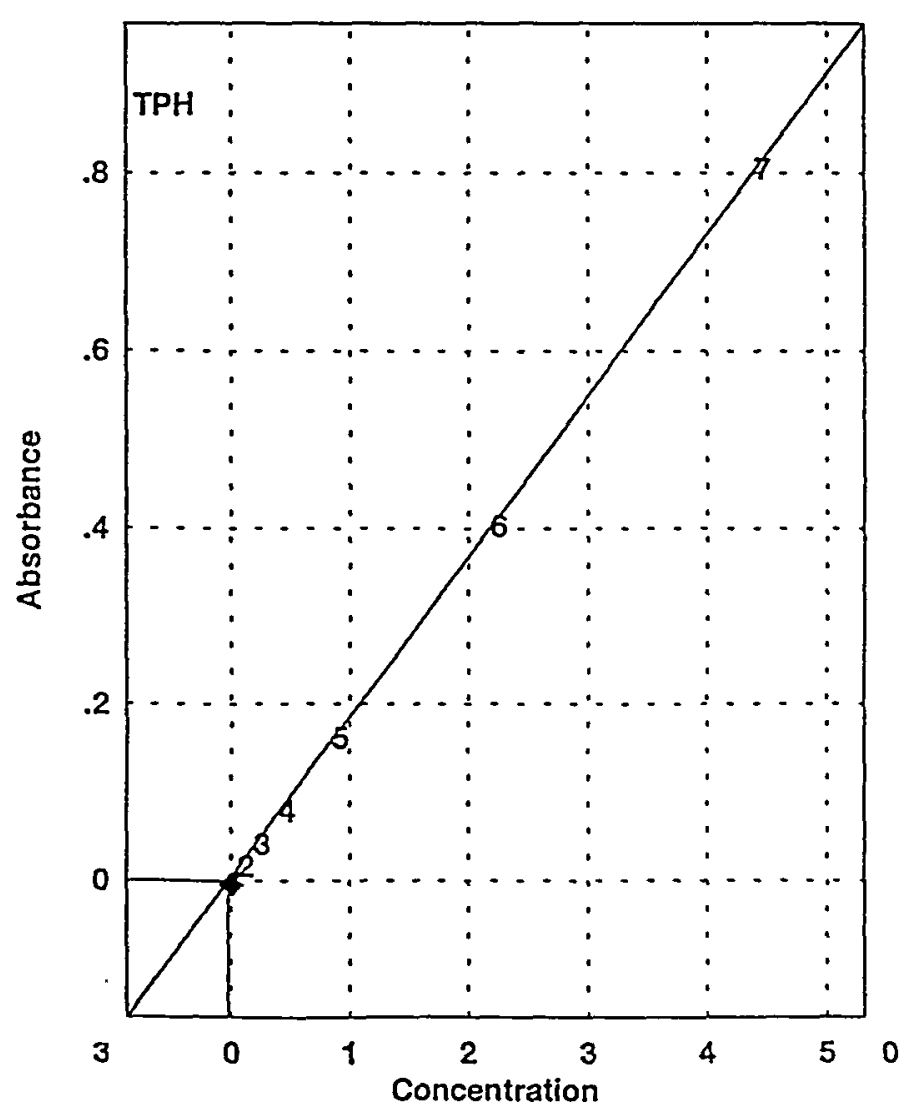

585 
QuantBaslc Report

Quant Type: Multlpoint $\quad 7 / 15 / 974: 51 \mathrm{pm}$

Method: C:IPE1000GSIDATALT036TPH.Q

Sample: c:Le1000gsldatal07036lcalcurve.spc

Analyst: TP

Sample ID: QC-1365341

Component Concen R-Squared

TPH $\quad 1.06209 \quad .999991$

Instrument I.D.: FTIR-1000C

Analyzed on: 09-JUL-1997

Date and time on printout is the day and time copy was printed.

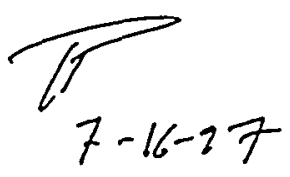

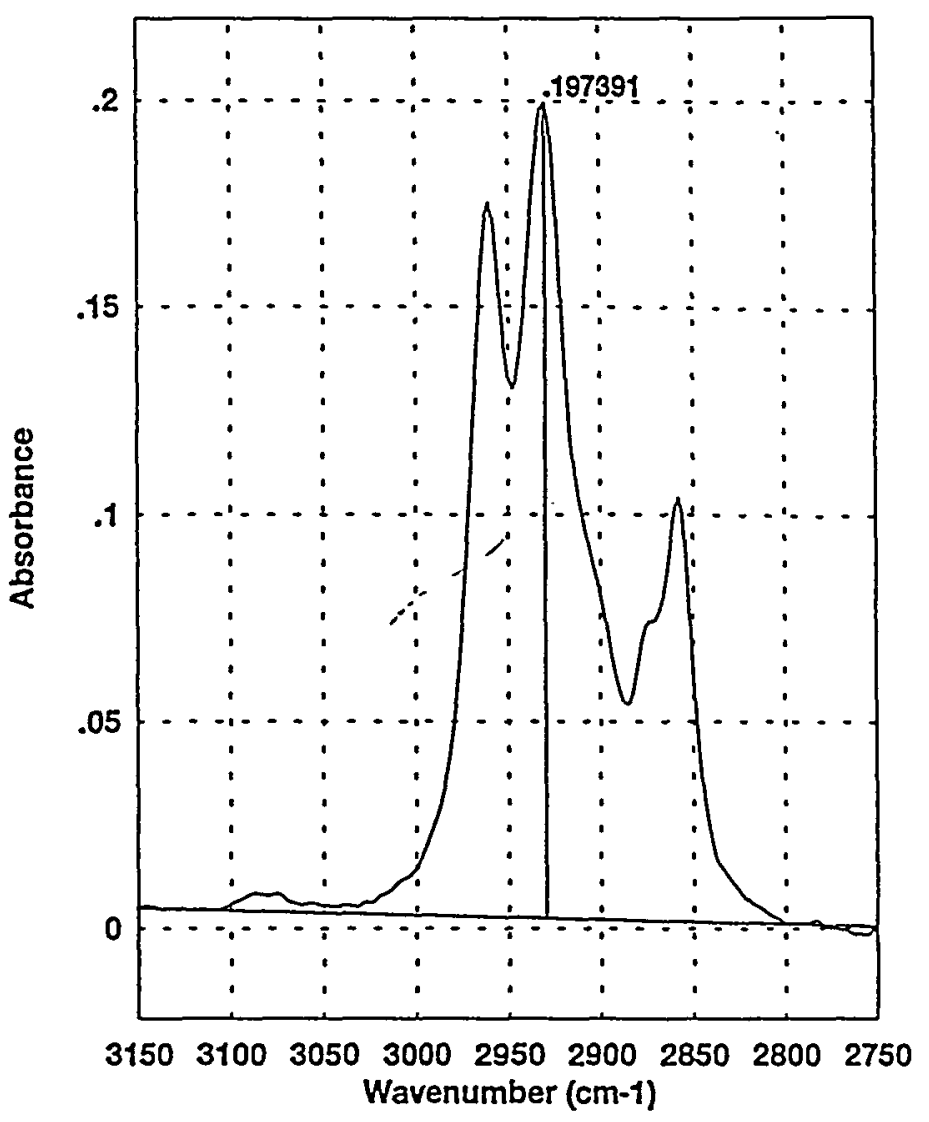

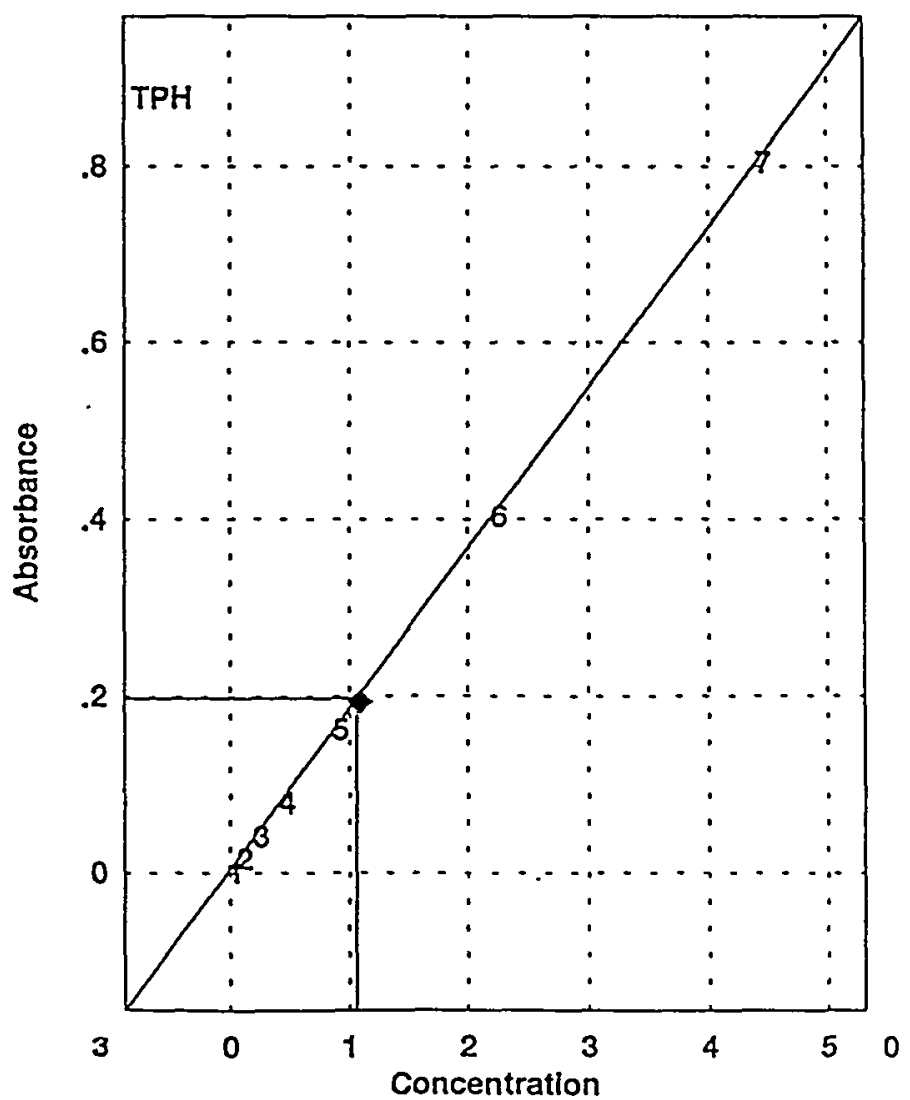


QuantBasic Report

Quant Type: Multlpoint $\quad 7 / 15 / 97$ 4:52 pm

Method: CilPE1000GSLATALT03GTPH.Q

Sample: c:lpe1000gsidatal97036lcaicurve.spc

Analyst: TP

Sample ID: BL-1136534-1

$\begin{array}{llll}\text { Component } & \text { Concen } & \text { R-Squared } \\ \text { TPH } & .00737395 & .999991\end{array}$

Instrument I.D.: FTIR-1000C

Analyzed on: 09-JUL-1997

Date and time on printout is the day and time copy was printed.

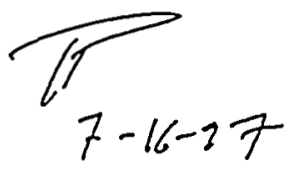

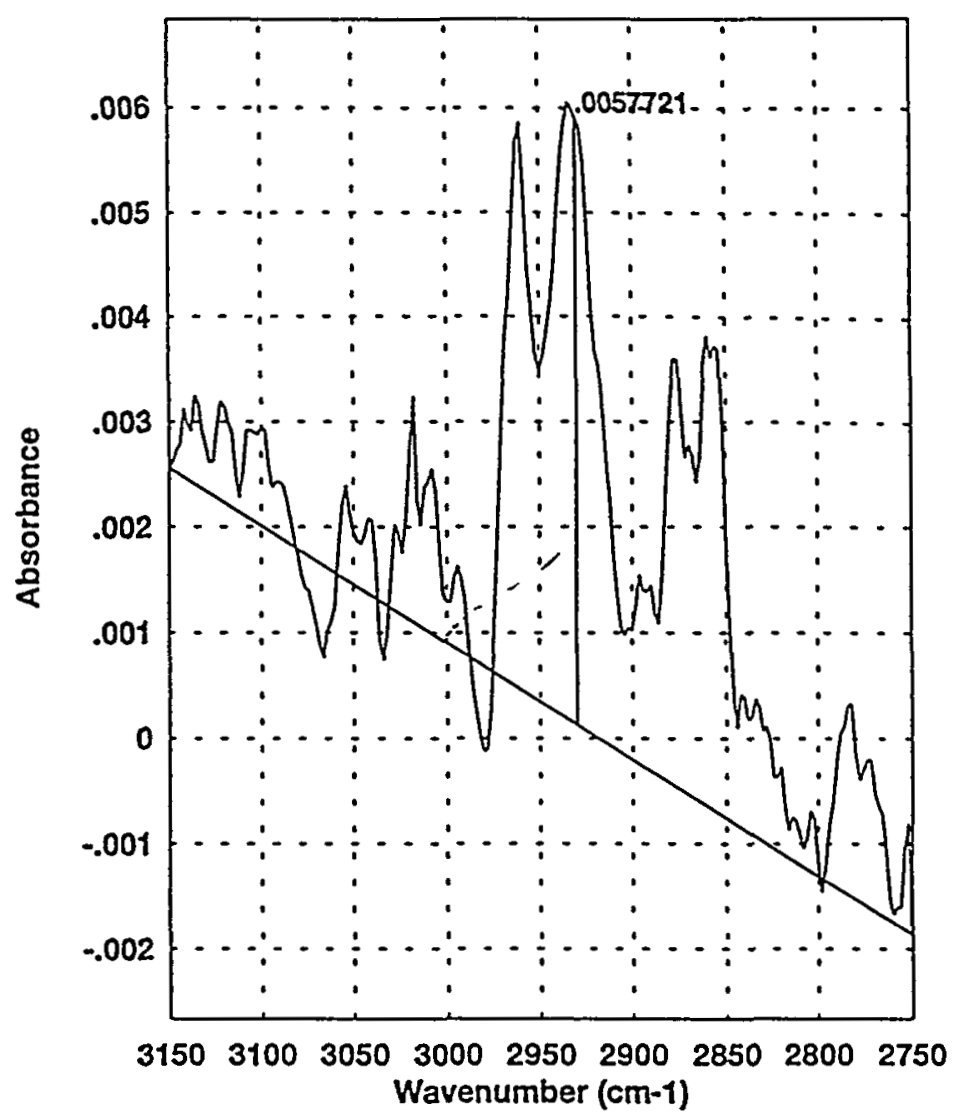

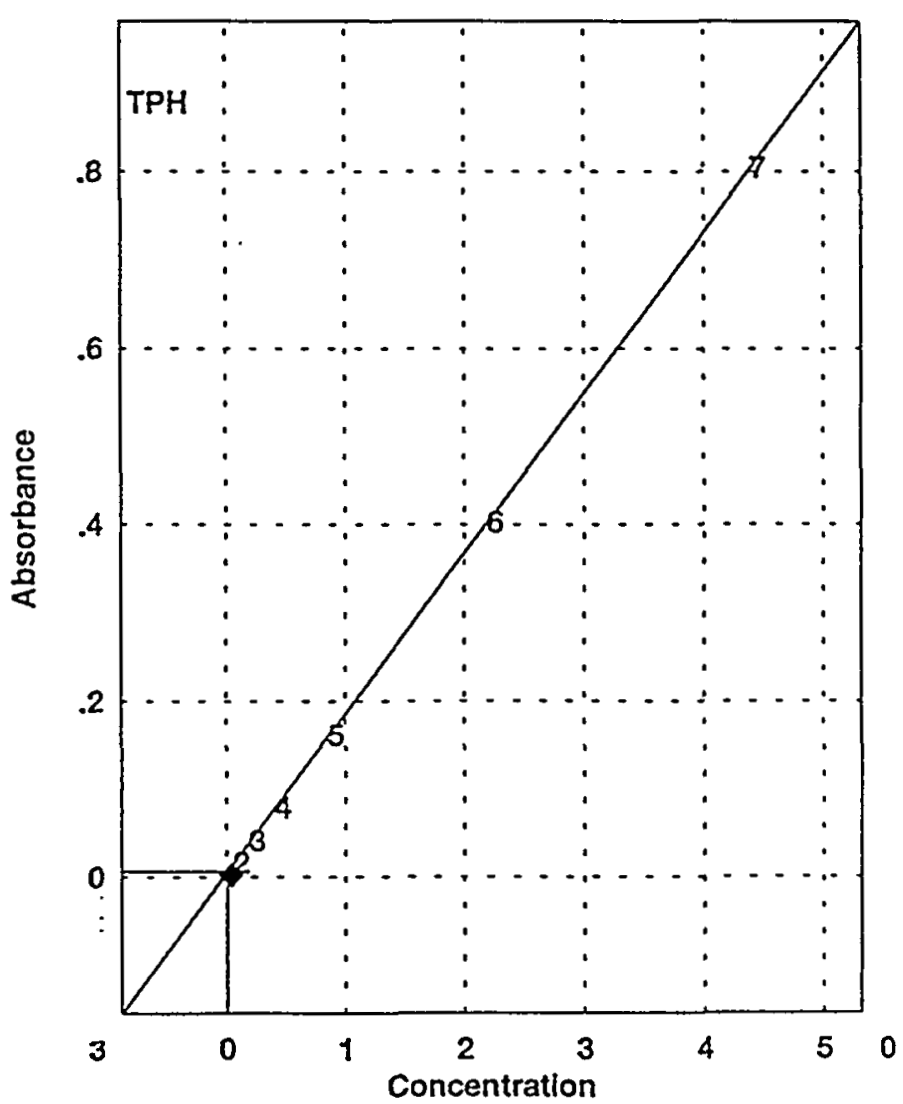

วั 87 
QuantBasic Report

Quant Type: Multipolnt $\quad$ 7/15/97 4:54 pm

Method: C:LE1000GSUATAL703GITPH.Q

Sample: c:lpe1000gsidatala7036lcalcurve.spc

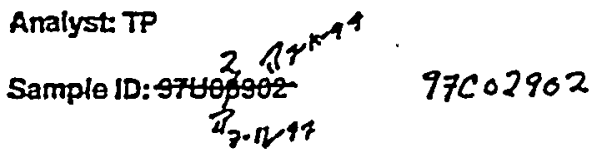

Componeint Concen R-Squared

TPH $\quad 0584462 \quad .999991$

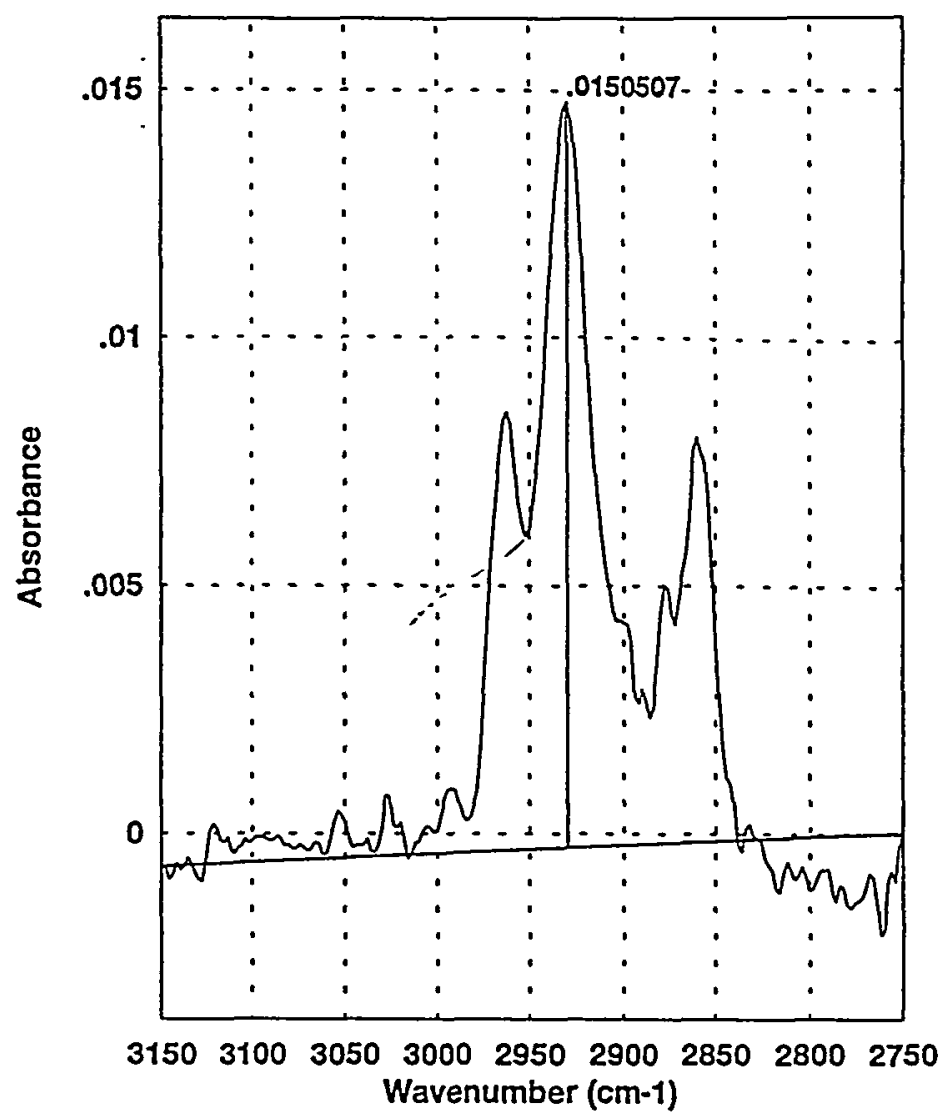

Instrument I.D.: FTIR-1000C

Analyzed on: 09-JUL-1997

Date and time on printout is the day and time copy was printed.

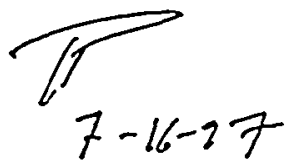

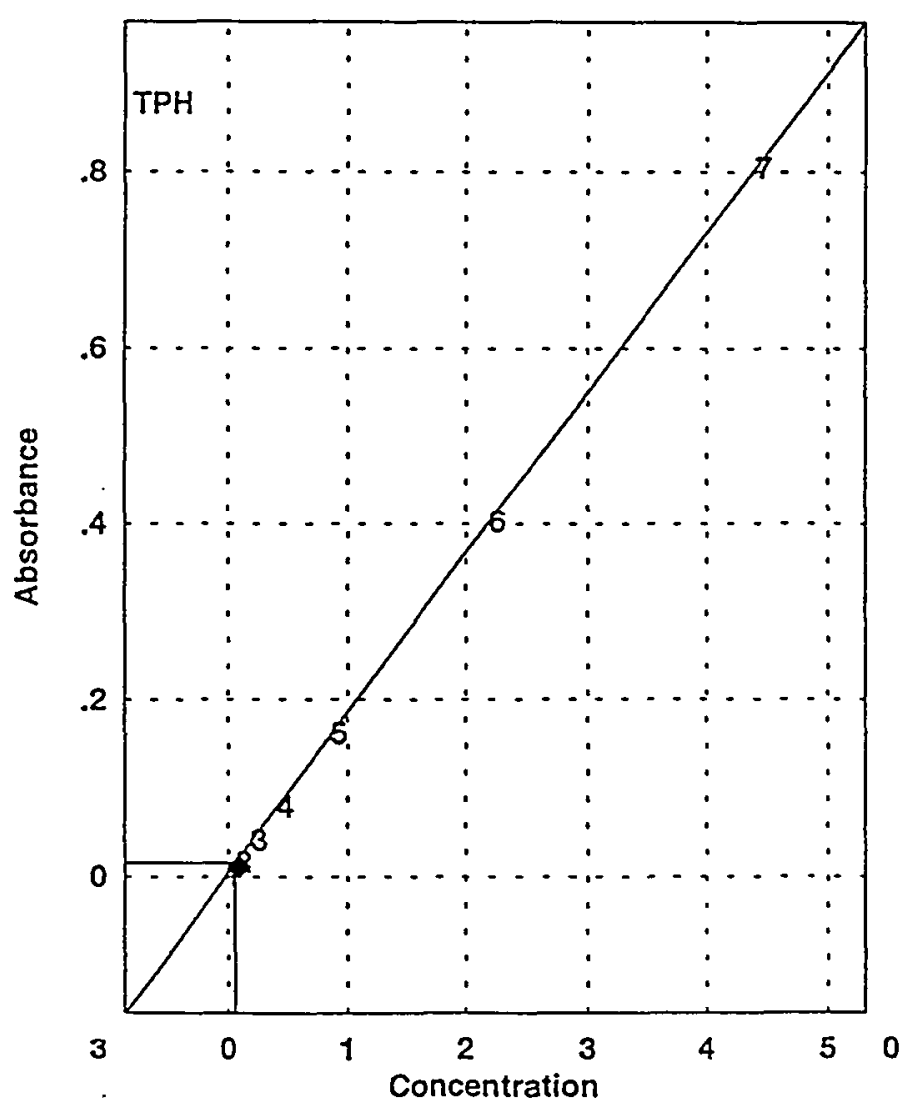


QuantBasic Report

Quant Type: Multipoint $\quad 7 / 15 / 974: 54$ pm

Wethod: C:IPE1000GSIDATAL7036TTPH.Q

Sample: c:lpe1000gsldatal07036lcalcurve.spc

Analyst TP

Sample ID:

Component Concen R-Squared

$\begin{array}{lll}\text { TPH } & \text {.0601039 } & \text { 99999 }\end{array}$
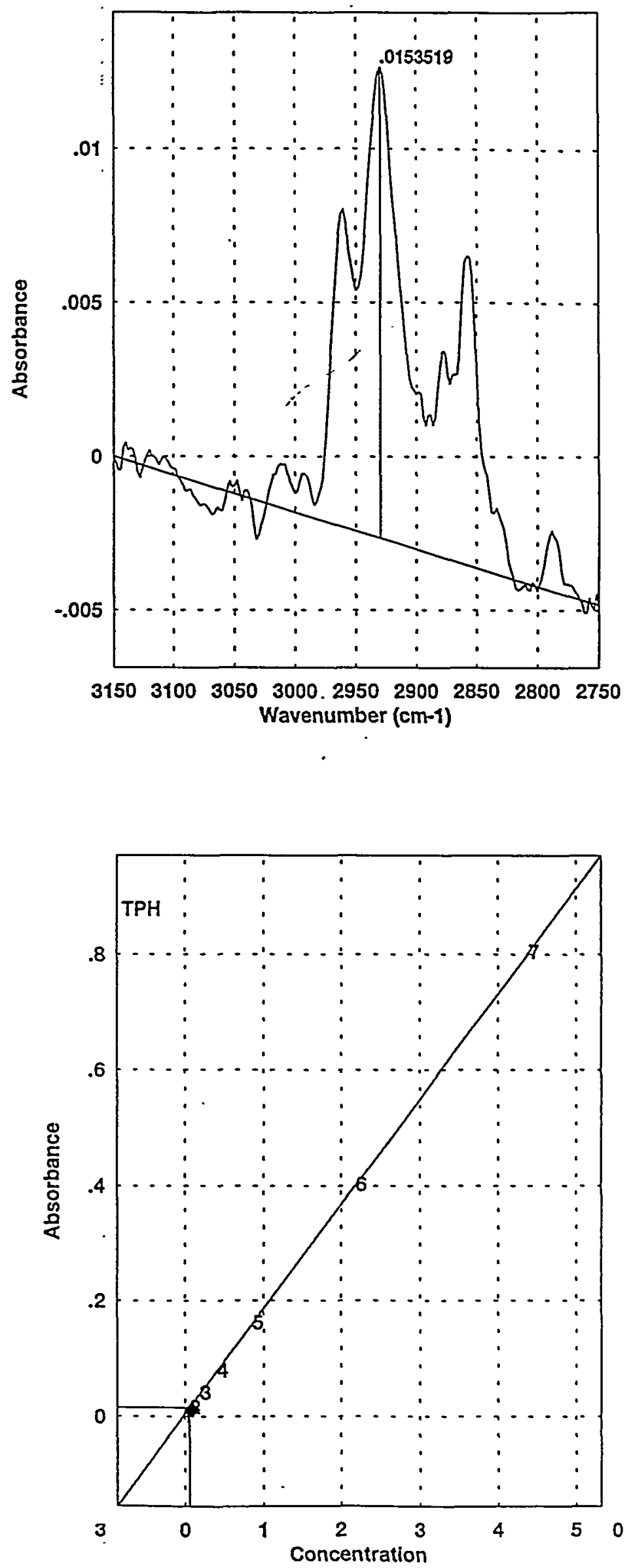

Wavenumber (cm-1)
Instrument I.D.: FTIR-1000C

Analyzed on: 09-JUL-1997

Date and time on printout is the day and time copy was printed.

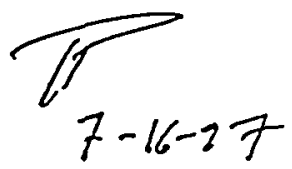

a


QuantBasic Report

Quant Type: Muttlpoint $\quad 7 / 15 / 974: 54$ pm

Method: C:IPE10ODGSUDATALTO3GTPH.Q

Sample: c:lpe1000gsldatal97036lcalcurve.spe

Analyst: TP

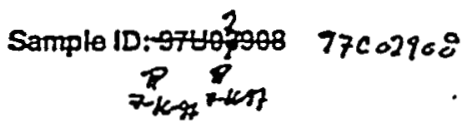

Component Concen R-Squared

$\begin{array}{lll}\text { TPH } & .0630375 & .999991\end{array}$

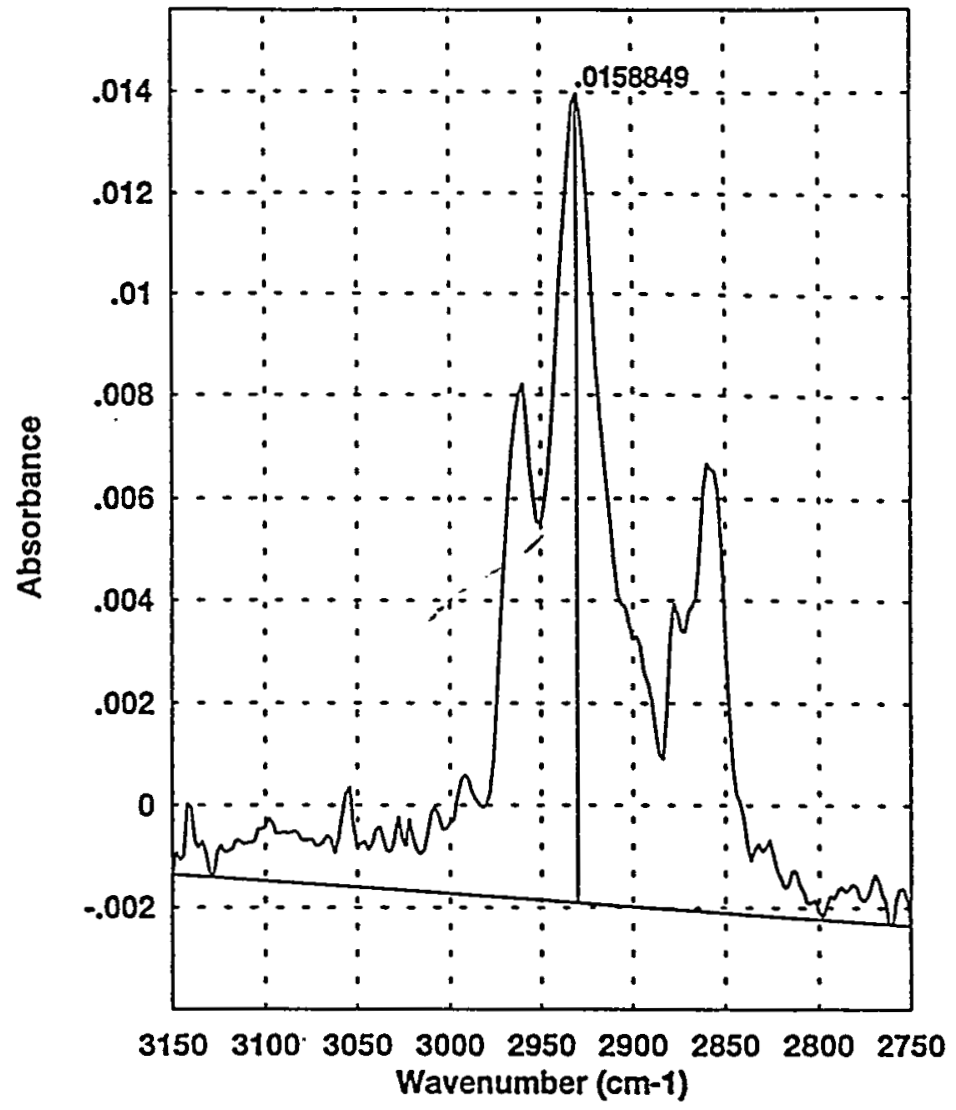

Instrument I.D.: FTIR-1000C

Analyzed on: 09-JUL-1997

Date and time on printout is the day and time copy was printed.

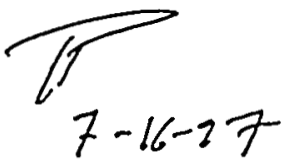

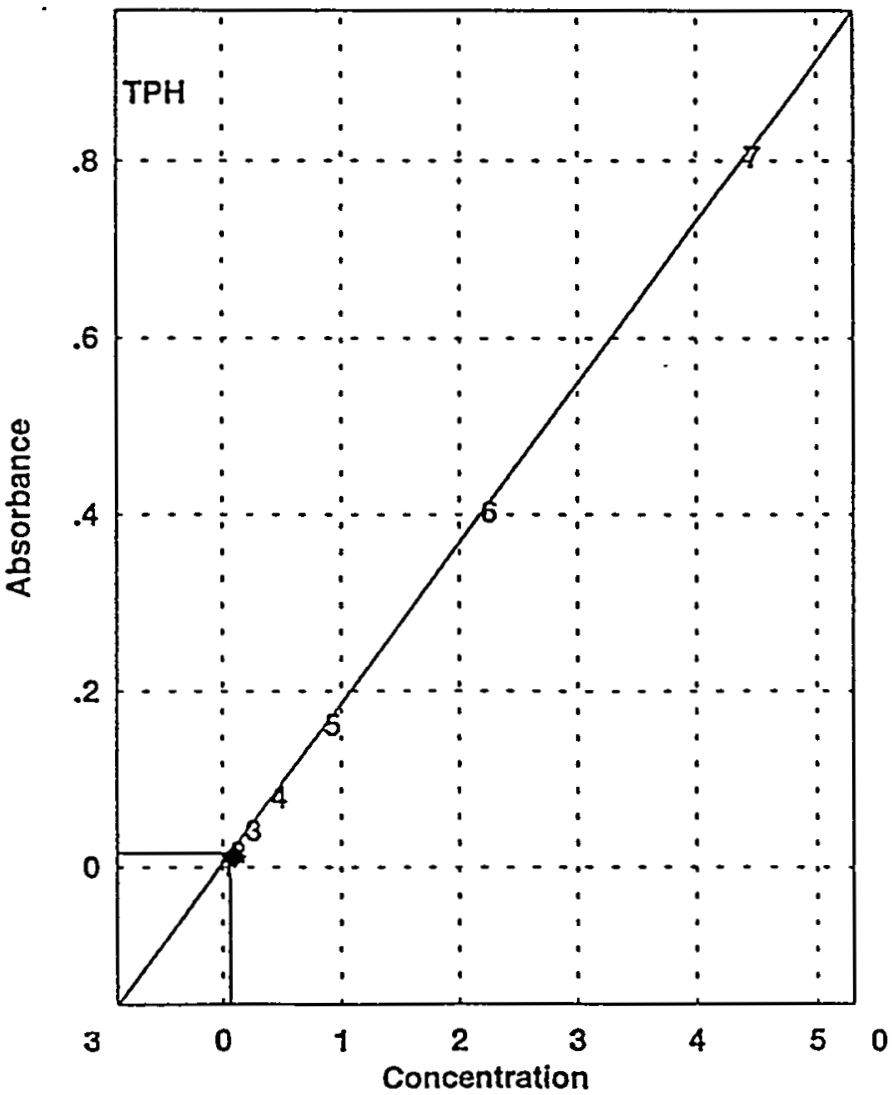


QuantBasic Report

Quant Type: Multipoint $\quad 7 / 15 / 97$ 4:53 pm

Method: C:LPE1D00GSIDATAL703GTPH.Q

Sample: c:lpe1000gsldatal97036lcalcurve.spe

Analyst TP

Sample 1D: $97400^{2} 908 m s \quad 97002908 \mathrm{MS}$

$$
2 / 6,7 x+1
$$

Component Concen R-Squared

TPH

$1.66409 \quad .999991$

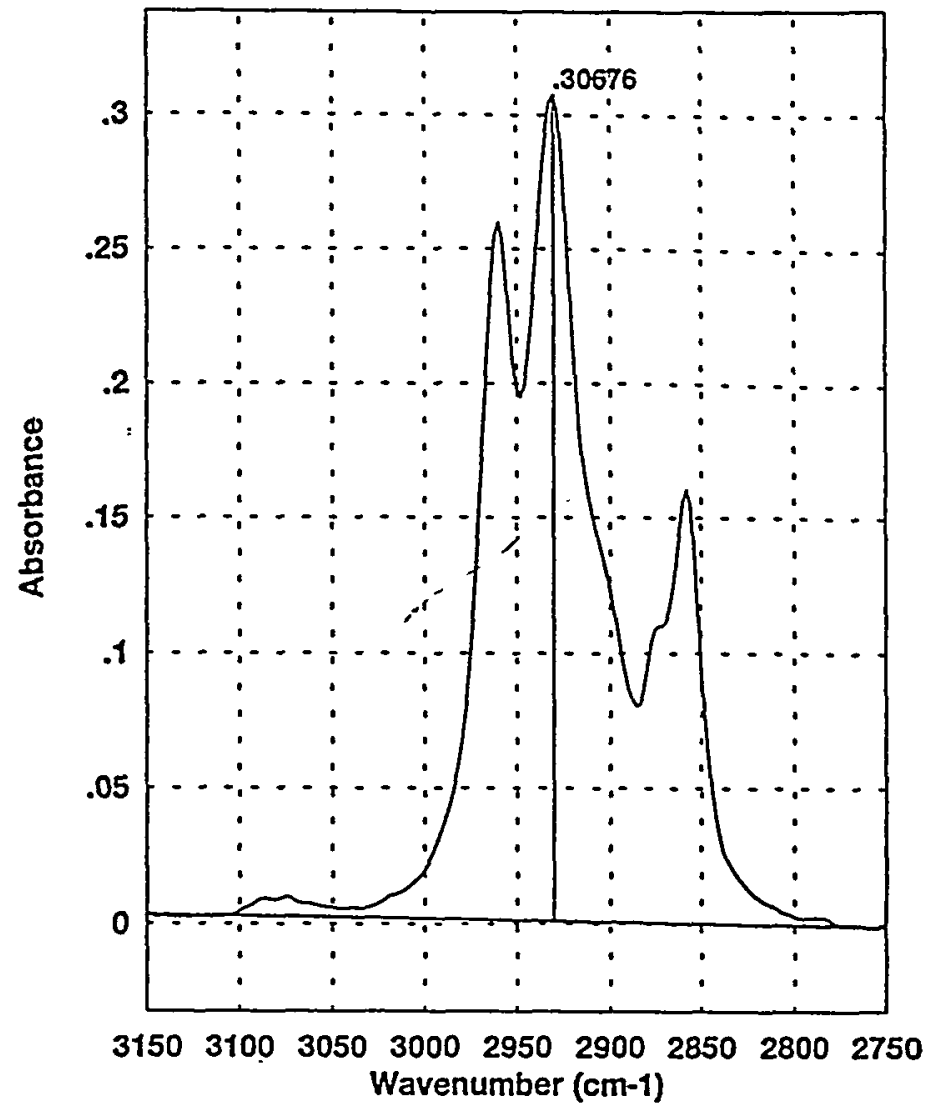

Instrument 1.D.: FTIR-1000C

Analyzed on: 09-JUL-1997

Date and time on printout is the day and time copy was printed.

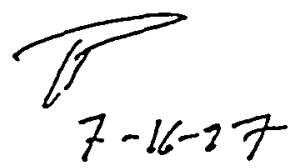

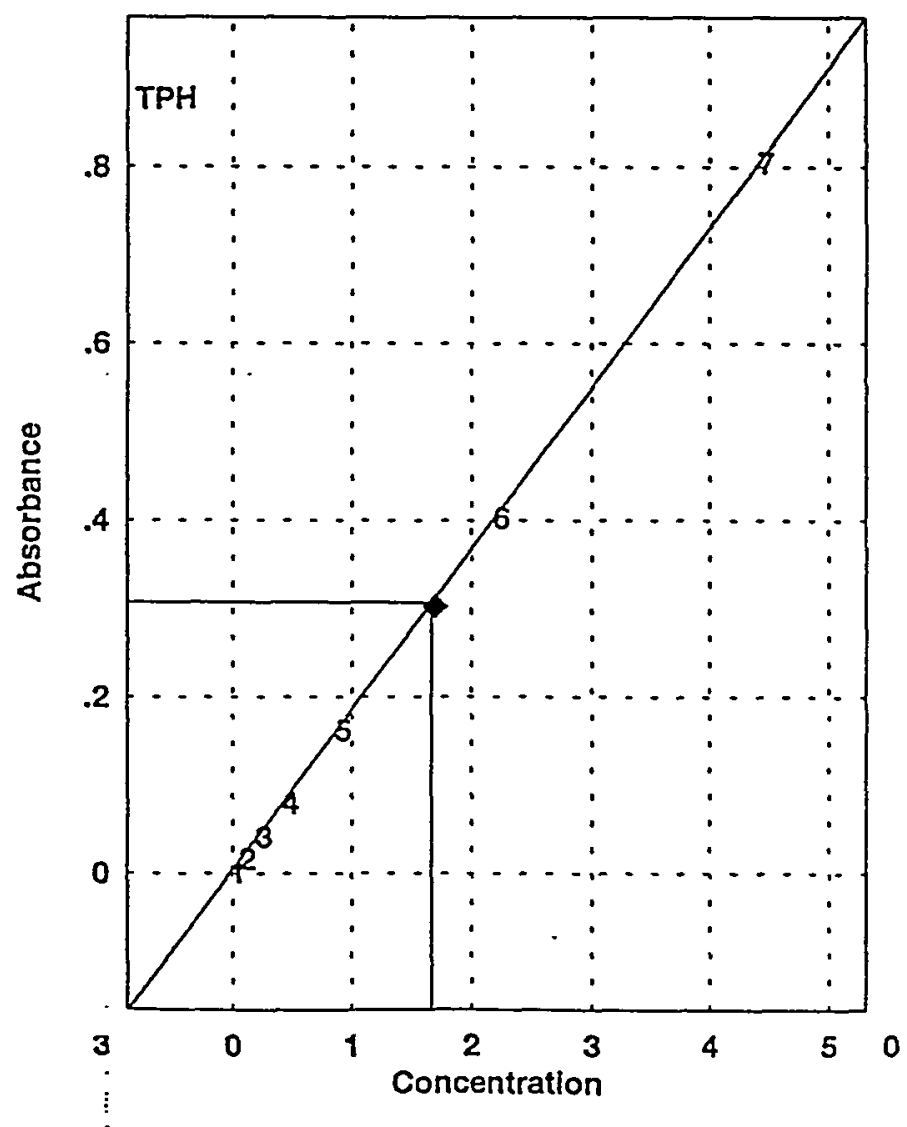


QuantBaslc Report

Quant Type: Muttipoint $\quad$ 7/15/97 4:53 pm

Method: C:IPE1000GSIDATAL707036ITPH.Q

Sample: c:lpe1000gsidatal97036lcalcurve.spc

Analyst: TP

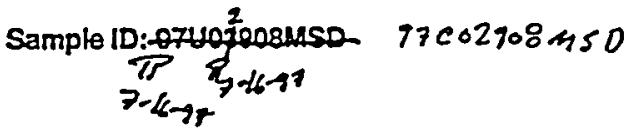

Component Concen R-Squared

$\begin{array}{lll}\text { TPH } & 1.62978 \quad .999991\end{array}$

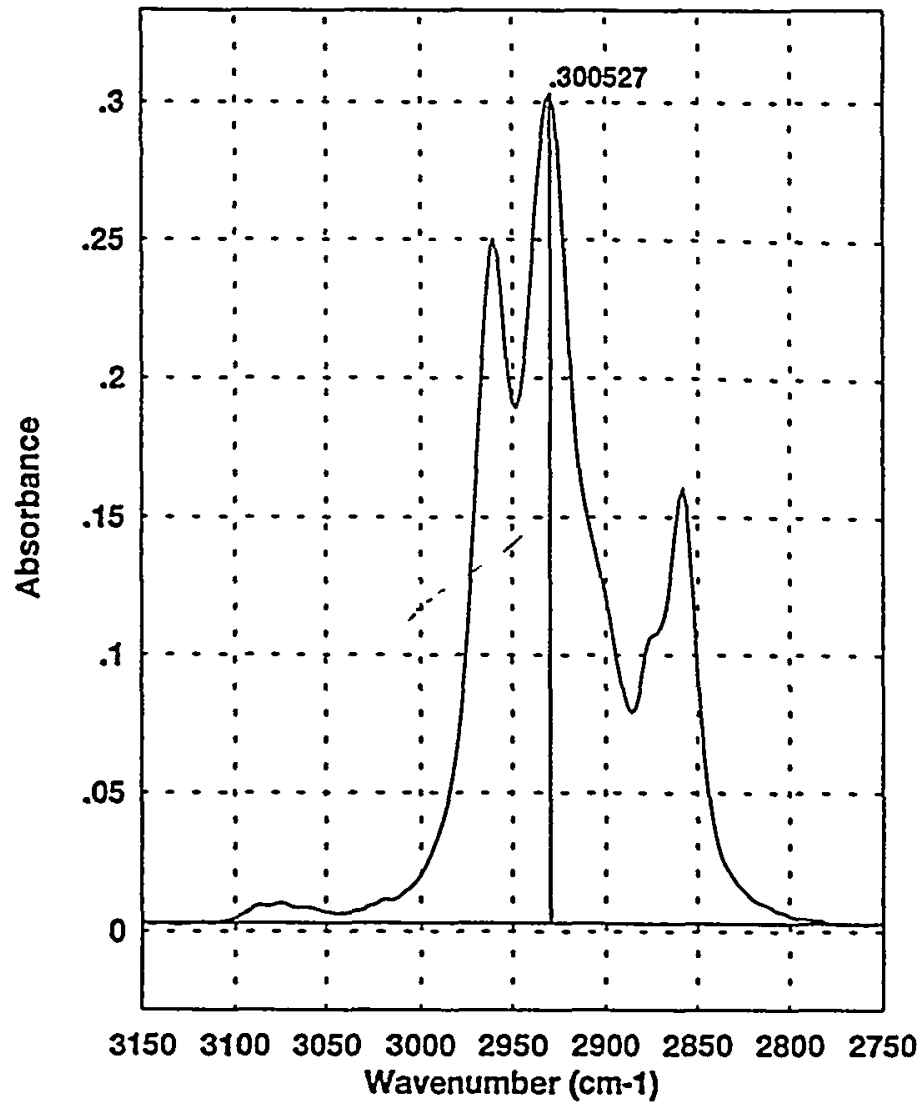

Instrument I.D.: FTIR-1000C

Analyzed on: 09-JUL-1997

Date and time on printout is the day and time copy was printed.

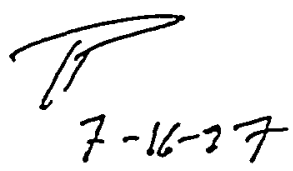

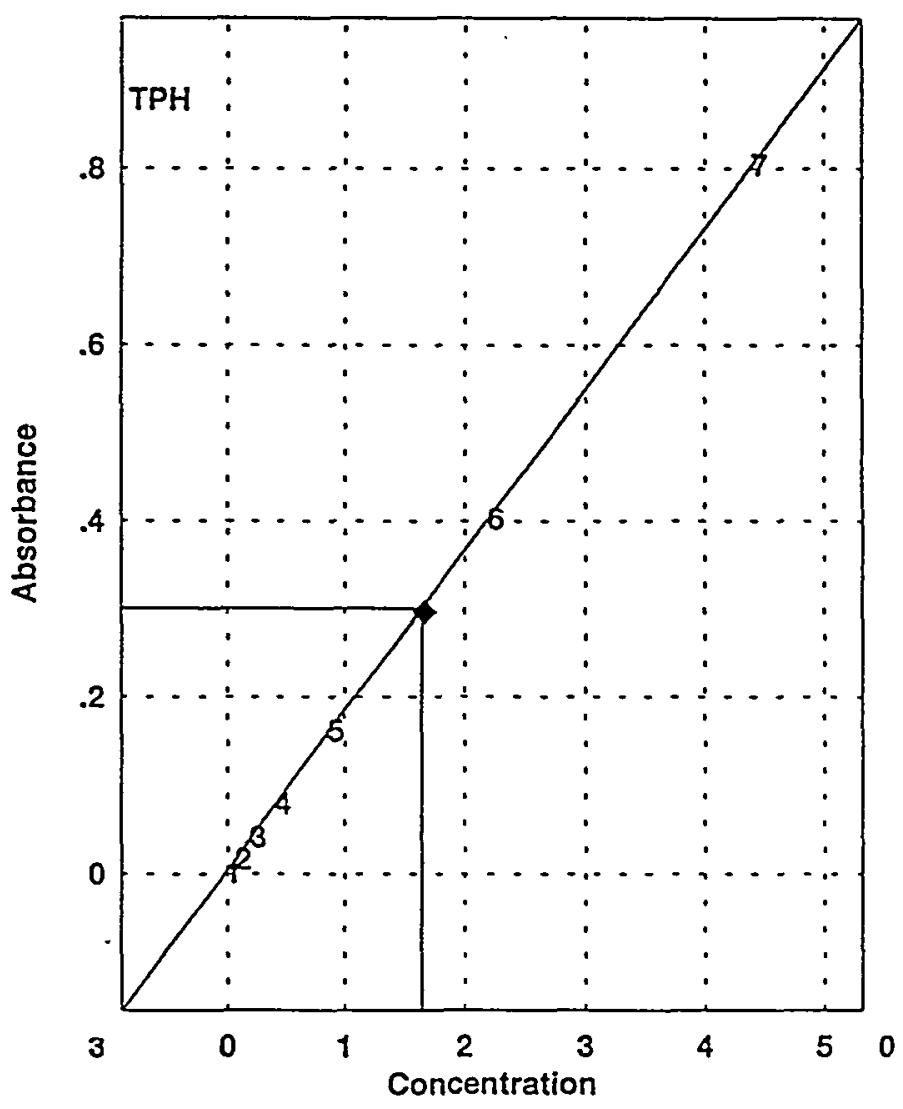


QuantBasic Report

Quant Type: Multipoint $\quad$ 7/15/97 5:03 pm

Method: C:IPE1000GSDATAL703GITPH.Q

Sample: c:lpe1000gsidatal97036lcalcurve.spc

Analyst: TP

Sample ID: CCV2

Component Concen R-Squared

$\begin{array}{lll}\text { TPH } & 2.21488 \quad .999991\end{array}$
Instrument I.D.: FTIR-1000C

Analyzed on: 09-JUL-1997

Date and time on printout is the day and time copy was printed.

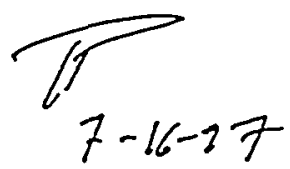

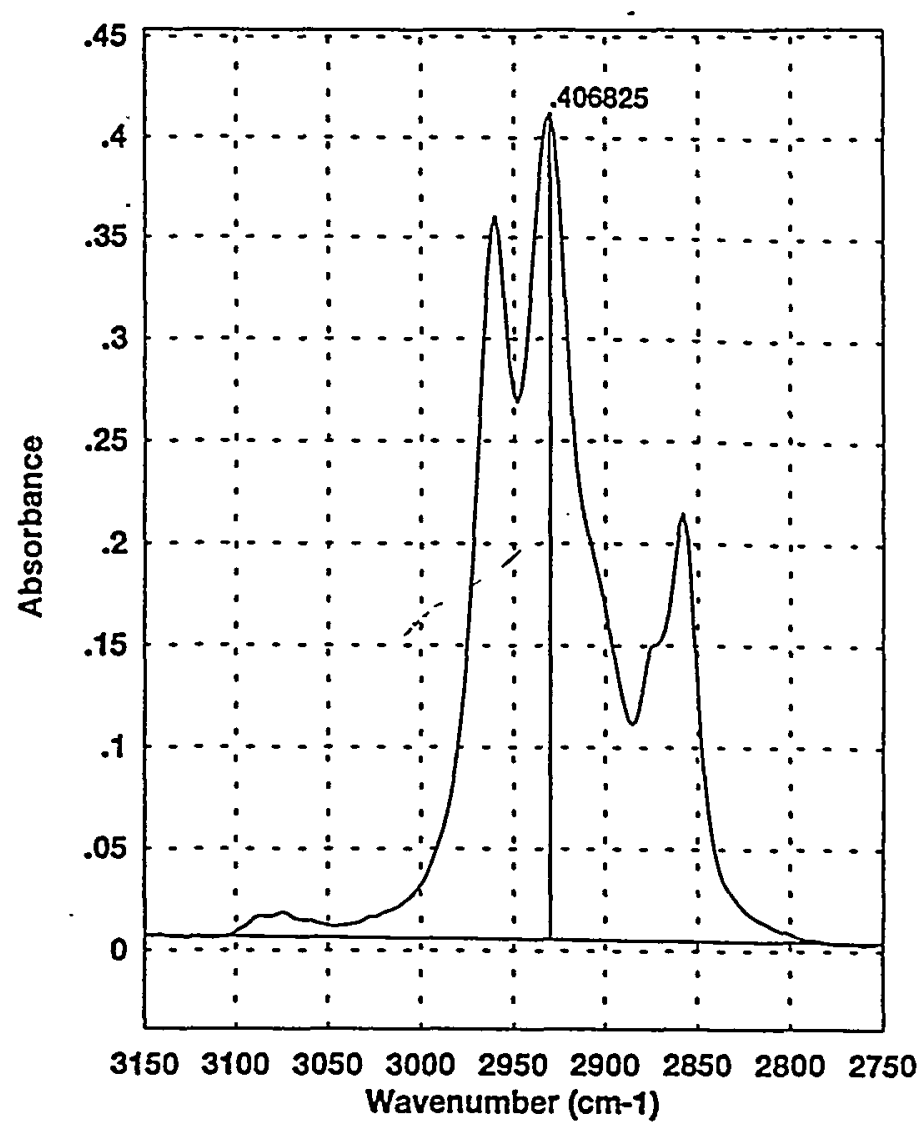

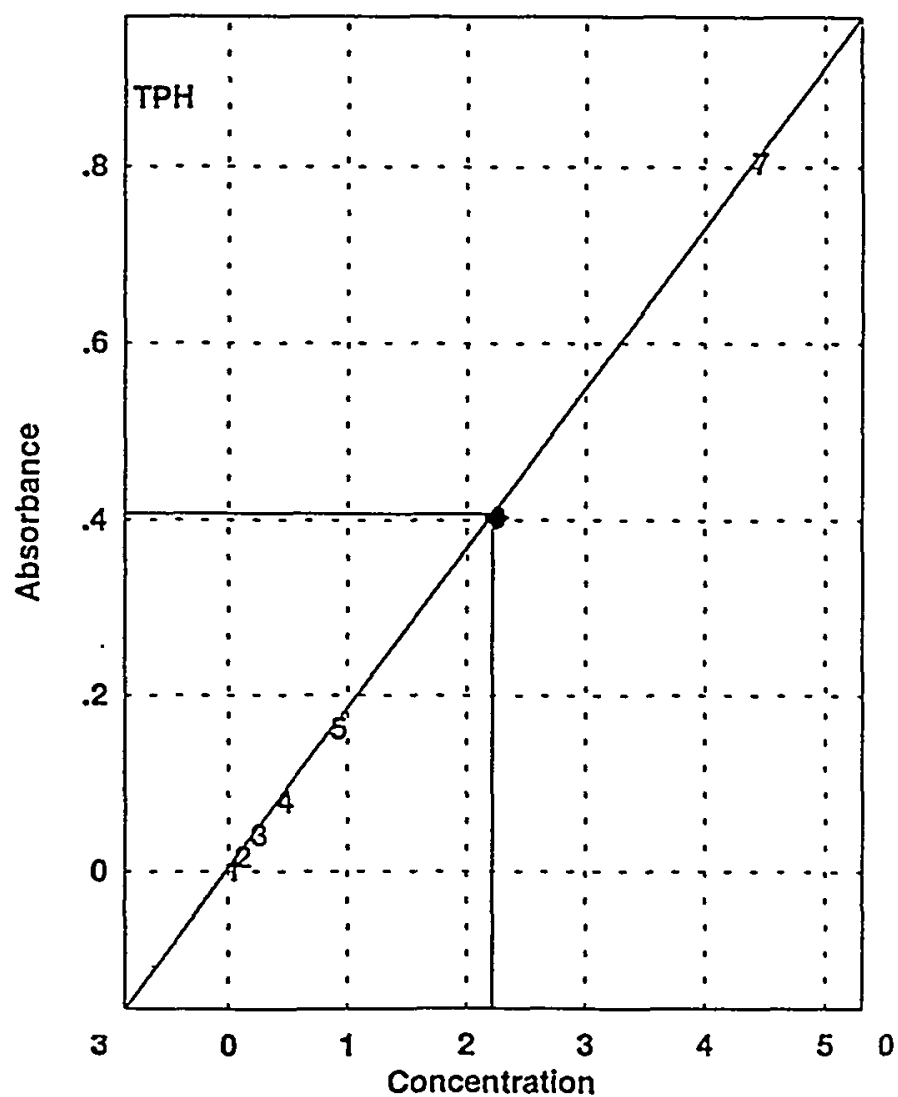


QuantBasic Report

Quant Type: Multipoint $\quad$ 7/15/97 5:03 pm

Method: C:PE1000GSIDATAL703GTPH.Q

Sample: c:pe1000gsidatal07036lcalcurve.spc

Analyst: TP

Sample 1D: CCB2

Component Concen R-Squared

TPH $\quad-.0268956 \quad .999991$

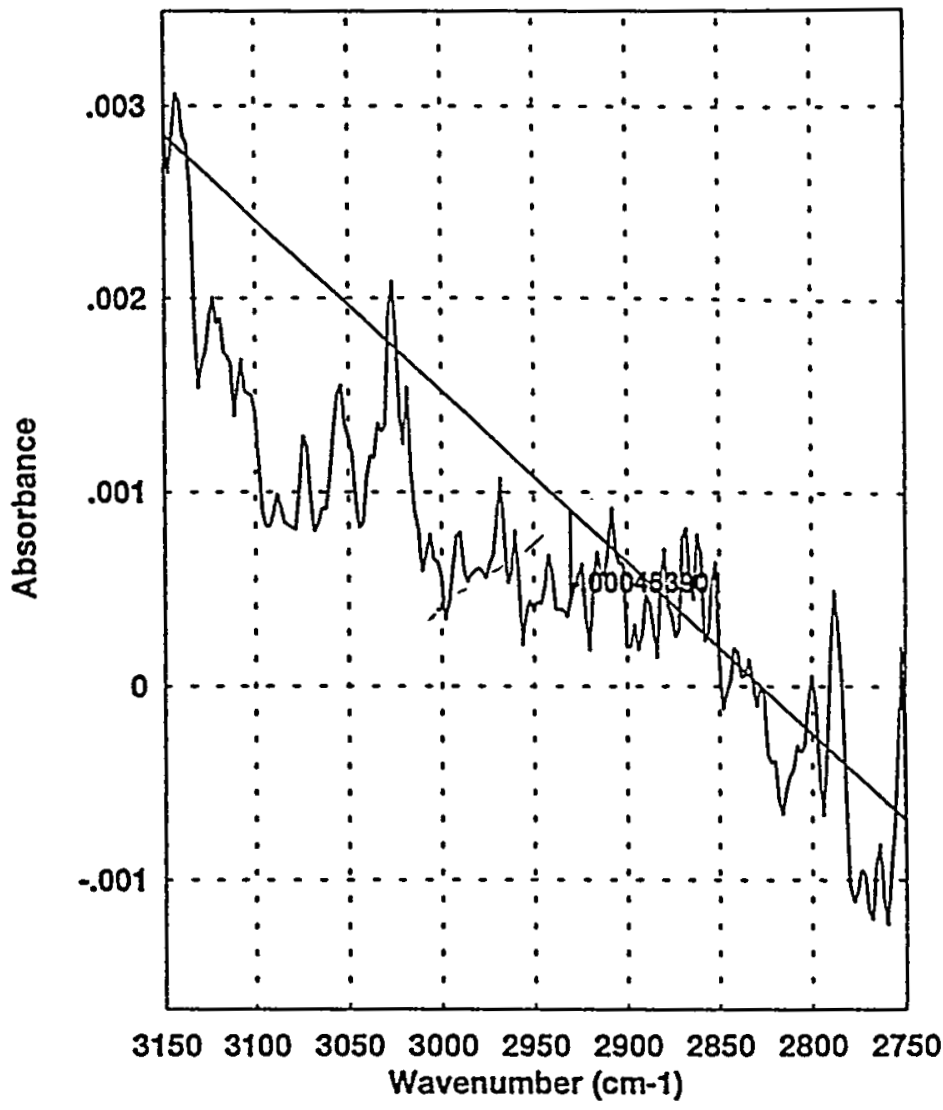

Instrument 1.D.: FTIR-1000C

Analyzed on: 09-JUL-1997

Date and time on printout is the day and time copy was printed.
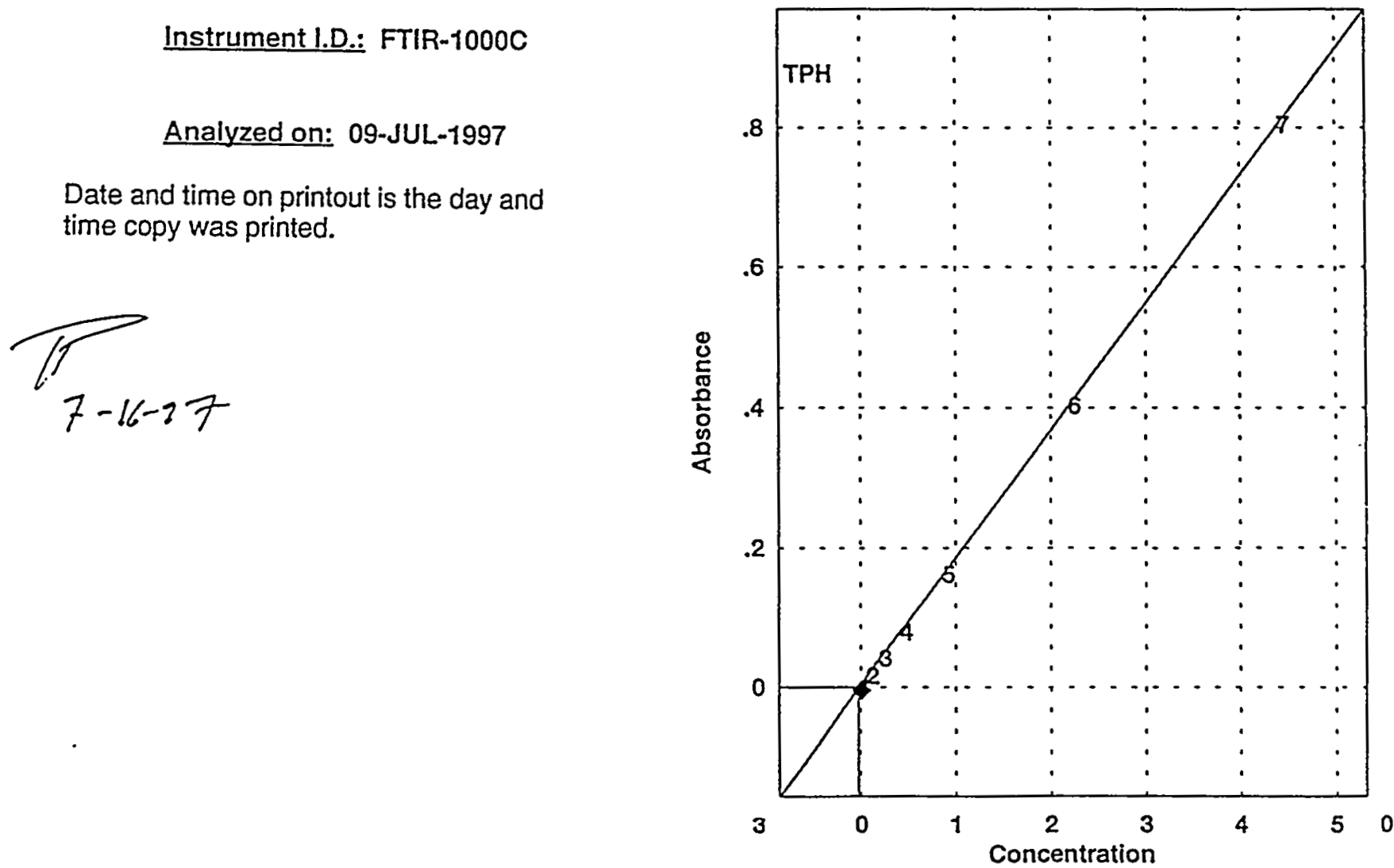


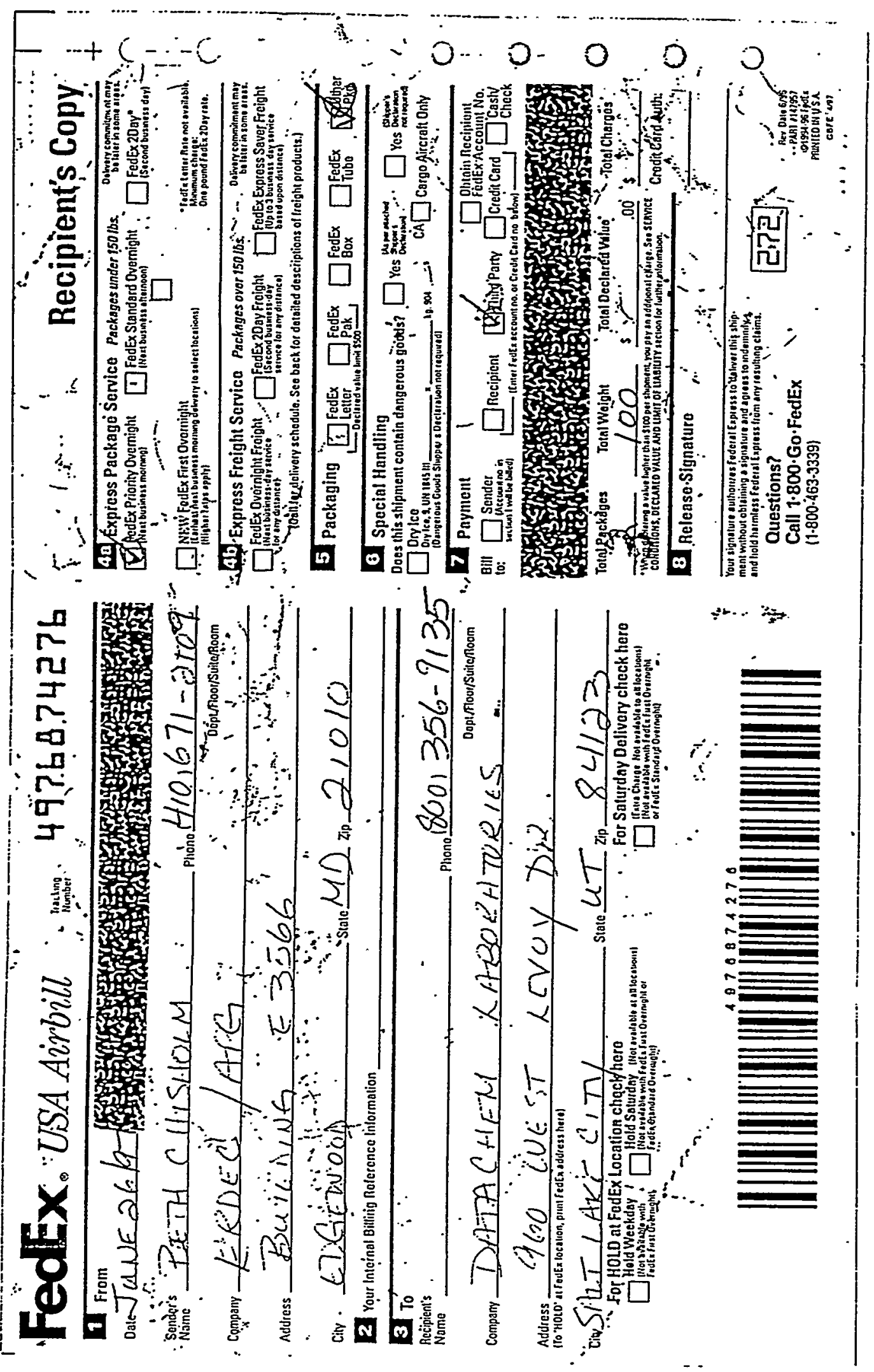




\section{DATACEEM LABORATORIES ClIENT-RELATED INFORMATION REPORT (CRIR) \\ CRIR No.}

\section{CoOler or Contaner INFORMation Checkist}

(Fill in or Cirrie)

\begin{tabular}{|c|c|}
\hline \multirow{2}{*}{\multicolumn{2}{|c|}{ Cient Neme: $\frac{S A L C}{\text { Duerime of Receipt: } 6-27-97 / 0930}$}} \\
\hline & \\
\hline Condition of Cooless. & Tooprabletionopable \\
\hline Custody Seals: & AresendabrenuNA \\
\hline & Gancieanu \\
\hline Treyer Evidut & Yestona \\
\hline Iec Present: & 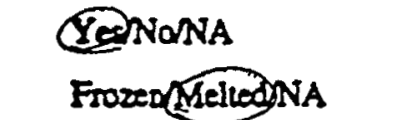 \\
\hline
\end{tabular}

Projece/Test/Site:

Number of Costes Received. (2) 2

Teure Courot

Location Teoperare Taken: Conouserween Sumples

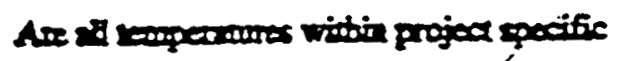

$$
\text { videlines? }
$$$$
\text { Yéngena }
$$

Are all aplicable pHis within specific

$$
\text { suidelines? YesNons }
$$

Cooler

Cooler Cooler

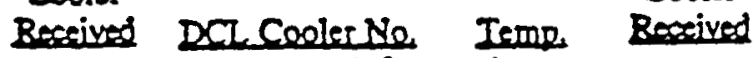

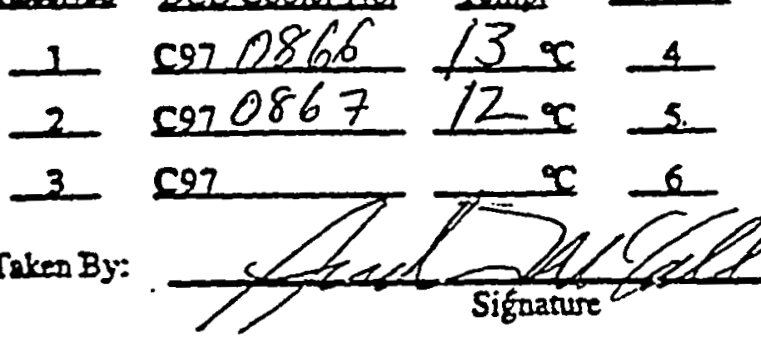

Daccooler No Teme Bexived

Dat Cooles Ne

cer

C97

\begin{tabular}{|c|c|c|c|c|}
\hline $\mathrm{C} 27$ & $x$ & 1 & $\mathrm{Cg}$ & $x$ \\
\hline 697 & $x$ & 8 & $\mathrm{Cg}$ & $x$ \\
\hline$c 27$ & $x$ & 9 & $\mathrm{C} 27$ & $x$ \\
\hline
\end{tabular}

C27
Temp.

\begin{tabular}{|c|c|c|c|}
\hline $\begin{array}{l}\text { ter } \\
\text { tions } \\
\text { rwork }\end{array}$ & $\begin{array}{l}\text { Missing Samples/Borles } \\
\square \text { Brokenleating Samples } \\
\square \text { Incorter Botle Type } \\
\text { Cooler Temperatures Out }\end{array}$ & $\begin{array}{l}0 \\
0 \\
0 \\
0\end{array}$ & $\begin{array}{l}\text { Incorrect Preservation } \\
\text { pH Criteria Not Met } \\
\text { Head Space in Borles } \\
\text { Insufficient Sample } \\
\text { Volume }\end{array}$ \\
\hline
\end{tabular}

\section{CLIENT-RELATED INFORMATION}

BRIETIY DESCRIBE TEE PROBLEM AND TEE ACTION TAKEN:

$N A P-R I-S Z-B(T R P H) ; N A P-K Z-S Z-A$ (TRPH);NAP-XP -SZA (SUK) sampls borken. Ice was also bnetted and femp was out of range.

Faxed 10 Cliem? Yes a No D (f yes, wact Fux Cover Sheet)

\section{Response Required Within 24 Bours}

\section{Project MaNAGEMENT}

PROJECT MANAGER COMMENTS: Per Art Shattuck direction (the client):

Note the temperature variance in the case narrative and proceed with analysis.

NAP-R1-S2-B - Cancel the SVOC analysis use container for TRPH.

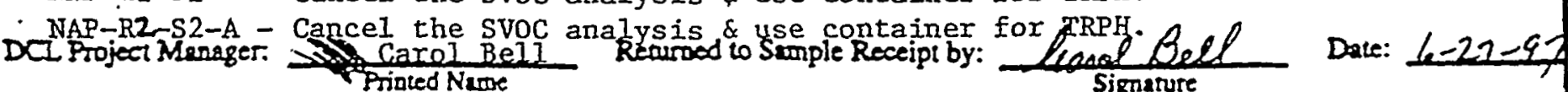
(Revised 7/3/96)

596 